

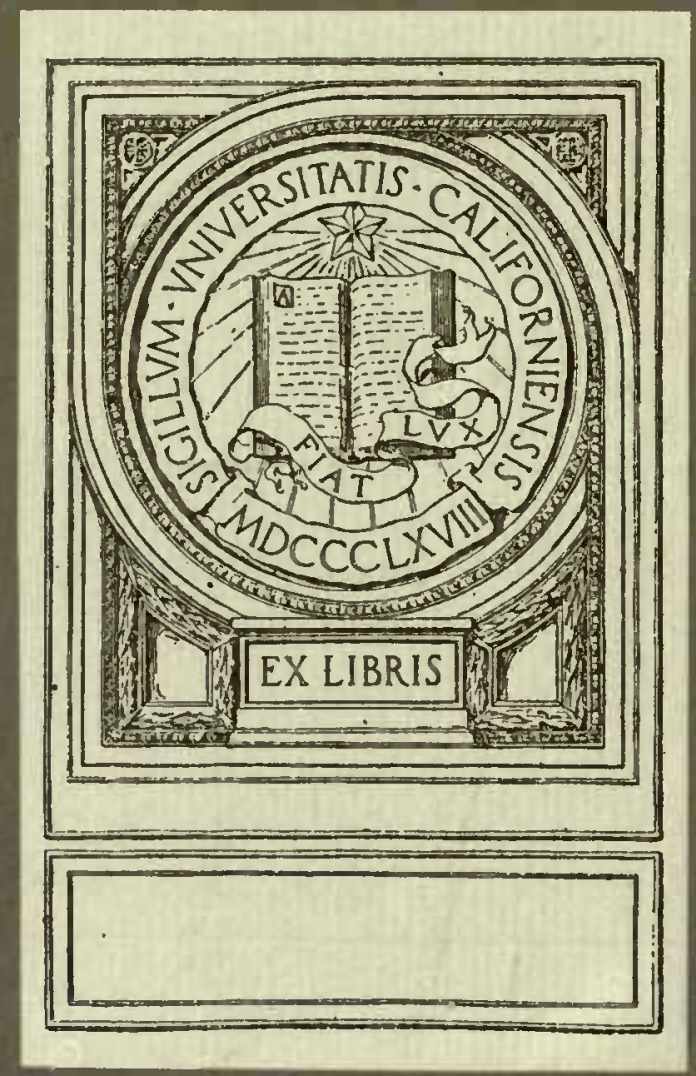






\section{S M I T H S 0 N I A N}

\section{CONTRIBUTIONS T0 KNOWLEDGE.}
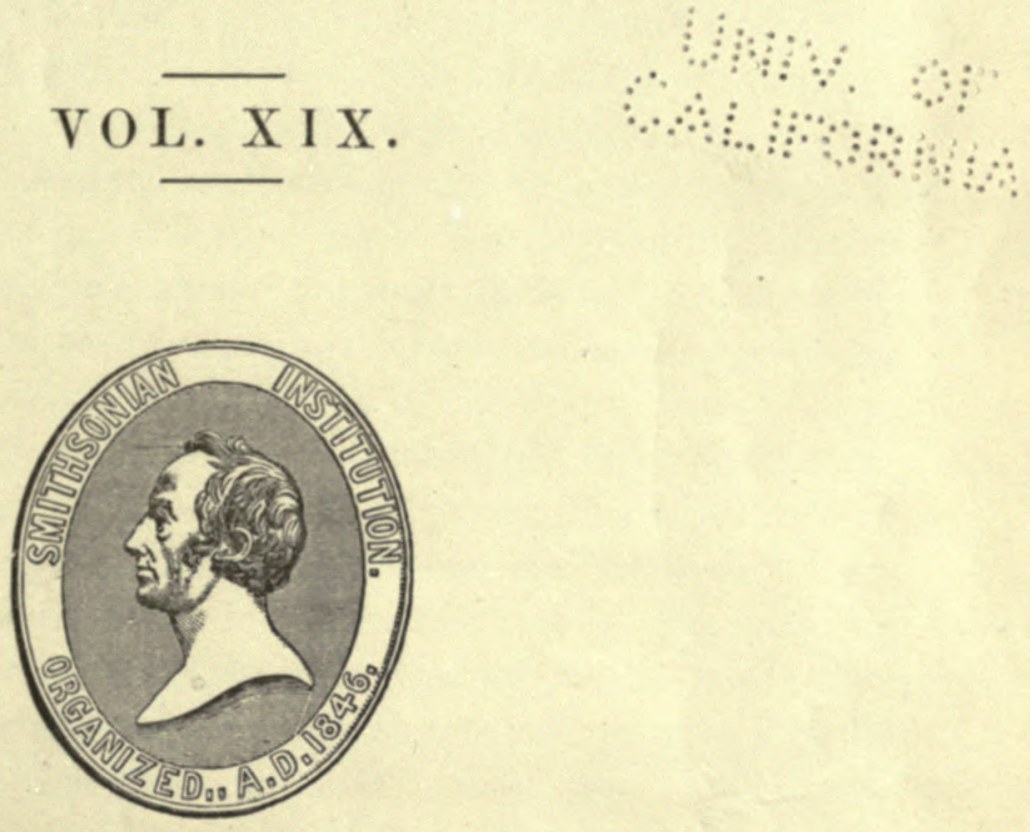

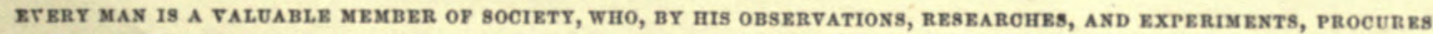
KXOWLEDGE FOR MEX.-SMTHSON.

\section{CITY OF WASHINGTON:}

PUBLISHED BY THE SMITHSONIAN INSTITUTION. MDCocixXrv. 
4.

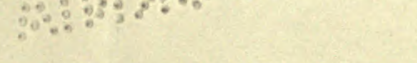

8947

COLLINS, PRINTER,

705 JAYNE ST. 


\section{A DVERTISEMENT.}

THIS volume forms the nineteenth of a series, composed of original memoirs on different branches of knowledge, published at the expense, and under the direction, of the Smithsonian Institution. The publication of this series forms part of a general plan adopted for carrying into effect the benevolent intentions of JAMES SMrthson, Esq., of England. This gentleman left his property in trust to the United States of America, to found, at Washington, an institution which should bear his own name, and have for its objects the "increase and diffusion of knowledge among men." This trust was accepted by the Government of the United States, and an Act of Congress was passed August 10, 1846, constituting the President and the other principal executive officers of the general government, the Chief Justice of the Supreme Court, the Mayor of Washington, and such other persons as they might elect honorary members, an establishment under the name of the "Sirthsonian INSTITUTION FOR THE INCREASE AND DIFfUSion OF KNOWLEDGE AMONG MEN." The members and honorary members of this establishment are to hold stated and special meetings for the supervision of the affairs of the Institution, and for the advice and instruction of a Board of Regents, to whom the financial and other affairs are intrusted.

The Board of Regents consists of three members ex officio of the establishment, namely, the Vice-President of the United States, the Chief Justice of the Supreme Court, and the Mayor of Washington, together with twelve other members, three of whom are appointed by the Senate from its own body, three by the House of Representatives from its members, and six persons appointed by a joint resolution of both houses. To this Board is given the power of electing a Secretary and other officers, for conducting the active operations of the Institution.

To carry into effect the purposes of the testator, the plan of organization should evidently embrace two objects: one, the increase of knowledge by the addition of new truths to the existing stock; the other, the diffusion of knowledge, thus increased, among men. No restriction is made in favor of any kind of knowledge; and, hence, each branch is entitled to, and should receive, a share of attention. 
The Act of Congress, establishing the Institution, directs, as a part of the plan of organization, the formation of a Library, a Museum, and a Gallery of Art, together with provisions for physical research and popular lectures, while it leaves to the Regents the power of adopting such other parts of an organization as they may deem best suited to promote the objects of the bequest.

After much deliberation, the Regents resolved to divide the annual income into two parts-one part to be devoted to the increase and diffusion of knowledge by means of original research and publications-the other part of the income to be applied in accordance with the requirements of the Act of Congress, to the gradual formation of a Library, a Museum, and a Gallery of Art.

The following are the details of the parts of the general plan of organization provisionally adopted at the meeting of the Regents, Dec. 8, 1847.

DETAILS OF THE FIRST PART OF THE PLAN.

\section{To INCREASE KNOWLEDGE.-It is proposed to stimulate research, by offering rewards for original memoirs on all subjects of investigation.}

1. The memoirs thus obtained, to be published in a series of volumes, in a quarto form, and entitled "Smithsonian Contributions to Knowledge."

2. No memoir, on subjects of physical science, to be accepted for publication, which does not furnish a positive addition to human knowledge, resting or original research; and all unverified speculations to be rejected.

3. Each memoir presented to the Institution, to be submitted for examination to a commission of persons of reputation for learning in the branch to which the memoir pertains; and to be accepted for publication only in case the report of this commission is fuvorable.

4. The commission to be chosen by the officers of the Institution, and the name of the author, as far as practicable, concealed, unless a favorable decision be made.

5. The volumes of the memoirs to be exchanged for the Transactions of literary and scientific societies, and copies to be given to all the colleges, and principal libraries, in this country. One part of the remaining copies may be offered for sale; and the other carefully preserved, to form complete sets of the work, to supply the demand from new institutions.

6. An abstract, or popular account, of the contents of these memoirs to be given to the public, through the annual report of the Regents to Congress. 
II. To INCREASE KNOWLEDGE.- $n$ is also proposed to appropriate a portion of the income, annually, to special objects of research, under the direction of suitable persons.

1. The objects, and the amount appropriated, to be recommended by counsellors of the Institution.

2. Appropriations in different years to different objects; so that, in course of time, each branch of knowledge may receive a share.

3. The results obtained from these appropriations to be published, with the memoirs before mentioned, in the volumes of the Smithsonian Contributions to Knowledge.

4. Examples of objects for which appropriations may be made:-

(1.) System of extended meteorological observations for solving the problem of American storms.

(2.) Explorations in descriptive natural history, and geological, mathematical, and topographical surveys, to collect material for the formation of a Physical Atlas of the United States.

(3.) Solution of experimental problems, such as a new determination of the weight of the earth, of the velocity of electricity, and of light; chemical analyses of soils and plants; collection and publication of articles of science, accumulated in the offices of Government.

(4.) Institution of statistical inquiries with reference to physical, moral, and political subjects.

(5.) Historical researches, and accurate surveys of places celebrated in American history.

(6.) Ethnological researches, particularly with reference to the different races of men in North Amerioa; also explorations, and accurate surveys, of the mounds and other remains of the ancient people of our country.

I. To DIFFUSE KNowledge.-It is proposed to publish a series of reports, giving an account of the nevo discoveries in science, and of the changes made from year to year in all branches of knowledge not strictly professional.

1. Some of these reports may be published annually, others at longer intervals, as the income of the Institution or the changes in the branches of knowledge may indicate.

2. The reports are to be prepared by collaborators, eminent in the different branches of knowledge. 
3. Each collaborator to be furnished with the journals and publications, domestic and foreign, necessary to the compilation of his report; to be paid a certain sum for his labors, and to be named on the title-page of the report.

4. The reports to be published in separate parts, so that persons interested in a particular branch, can procure the parts relating to it, without purchasing the whole.

5. These reports may be presented to Congress, for partial distribution, the remaining copies to be given to literary and scientific institutions, and sold to individuals for a moderate price.

The following are some of the subjects which may be embraced in the reports:-

\section{PHYSICAL CLASS.}

1. Physics, including astronomy, natural philosophy, cliemistry, and meteorology.

2. Natural listory, including botany, zoology, geology, \&c

3. Agriculture.

4. Application of science to arts.

\section{MORAL AND POLITICAL CLASS.}

5. Ethnology, including particular history, comparative philology, antiquities, \&c.

6. Statistics and political economy.

7. Mental and moral philosophy.

8. A survey of the political events of the world; penal reform, \&c.

\section{LITERATURE AND THE FINE ARTS.}

9. Modern literature.

10. The fine arts, and their application to the useful arts.

11. Bibliography.

12. Obituary notices of distinguished individuals.

II. To DIFFUSE KNOWLEDGE.-It is proposed to publish occasionally separate treatises on suljects of general interest.

1. These treatises may occasionally consist of valuable memoirs translated from foreign languages, or of articles prepared under the dircetion of the Institution, or procured by offering premiums for the best exposition of a given subject.

2. The treatises to be submitted to a commission cf cumpetent judges, previous to their publication. 


\section{DETAILS OF THE SECOND PART OF TIIE PLAN OF ORGANIZATION.} Art.

This part contemplates the formation of a Library, a Museum, and a Gallery of

1. To carry out the plan before described, a library will be required, consisting, 1st, of a complete collection of the transactions and proceedings of all the learned societies of the world; $2 \mathrm{~d}$, of the more important current periodical publications, and other works necessary in preparing the periodical reports.

2. The Institution should make special collections, particularly of objects to verify its own publications. Also a collection of instruments of research in all branches of experimental science.

3. With reference to the collection of books, other than those mentioned above, catalogues of all the different libraries in the United States should be procured, in order that the valuable books first purchased may be such as are not to be found elsewhere in the United States.

4. Also catalogues of memoirs, and of books in foreign libraries, and other materials, should be collected, for rendering the Institution a centre of bibliographical knowledge, whence the student may be directed to any work which he may require.

5. It is believed that the collections in natural history will increase by donation, as rapidly as the income of the Institution can make provision for their reception; and, therefore, it will seldom be necessary to purchase any article of this kind.

6. Attempts should be made to procure for the gallery of art, casts of the most celebrated articles of ancient and modern sculpture.

7. The arts may be encouraged by providing a room, free of expense, for the exhibition of the objects of the Art-Union, and other similar societies.

8. A small appropriation should annually be made for models of antiquity, such as those of the remains of ancient temples, \&c.

9. The Secretary and his assistants, during the session of Congress, will be required to illustrate new discoveries in science, and to exhibit new objects of art; distinguished individuals should also be invited to give lectures on subjects of general interest.

In accordance with the rules adopted in the programme of organization, each memoir in this volume has been favorably reported on by a Commission appointed 
for its examination. It is however impossible, in most cases, to verify the statements of an author; and, therefore, neither the Commission nor the Institution can be responsible for more than the general character of a memoir.

The following rules have been adopted for the distribution of the quarto volumes of the Smithsonian Contributions:-

1. They are to be presented to all learned societies which publish Transactions, and give copies of these, in exchange, to the Institution.

2. Also, to all foreign libraries of the first class, provided they give in exchange their catalogues or other publications, or an equivalent from their duplicate volumes.

3. To all the colleges in actual operation in this country, provided they furnish, in return, meteorological observations, catalogues of their libraries and of their students, and all other publications issued by them relative to their organization and history.

4. To all States and Territories, provided there be given, in return, copies of all documents published under their authority.

5. To all incorporated public libraries in this country, not included in any of the foregoing classes, now containing more than 10,000 volumes; and to smaller libraries, where a whole State or large district would be otherwise unsupplied. 


\section{O F F I C E R S}

\section{OF THK \\ SMITHSONIAN INSTITUTION.}

THE PRESIDENT OF THE UNITED STATES,

Ex-officio PRESIDING OFFICER OF THE INSTITUTION.

THE VICE-PRESIDENT OF THE UNITED STATES,

Ex-officio SECOND PRESIDING OFFICER.

MORRISON R. WAITE,

CHANCELLOR OF THE INSTITUTION.

JOSEPH HENRY,

SECRETARY OF THE INSTITUTION.

SPENCER F. BAIRD,

ASSISTANT SECRETARY.

PETER PARKER, JOHN MACLEAN, WILLIAM T. SHERMAN, 


\section{REGE N T S.}

Hentr Wilson, . . . . Vice-President of the United States.

Morrison R. Watte, . . . Chief Justice of the United States.

Hannibal Hamuin, . . . . Member of the Senate of the United States.

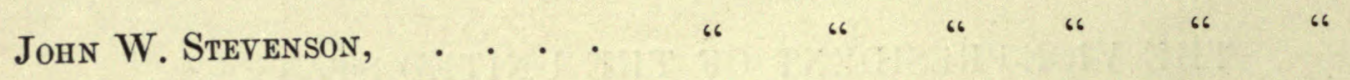

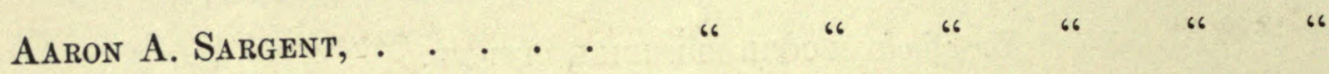

Samule S. Cox, . . . . . Member of the House of Representatives $U$. S.

Ebenezer R. Hoar, . . . . . " " " “ " “ “ " “

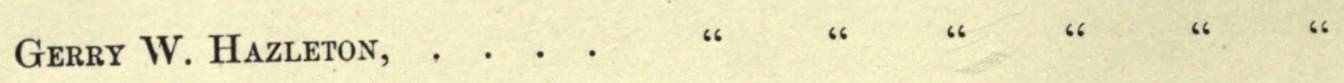

John Maclean, . . . . . Citizen of New Jersey.

Peter Parker, . . . . . “ of Wushington.

William T. Sherman, . . . . “ “

AsA Gray, . . . . . . " of Massachusetts.

J, D. DANA, . . . . . . " of Connecticut.

Hentr Coppée, . . . . . " of Pennsylvania. 


\section{MEMBERS EX-OFFICIO OF THE INSTITUTION.}

Ulysses S. Grant, .. . . . . President of the United Slates.

Henry Wilson, . . . . . Vice-President of the United Stutes.

Hamilton Fish, . . . . . . . Secretary of Slate.

B. H. BRistow, . . . . . . . Secretary of the Treasury.

W. W. Belknap, . . . . . Secretary of War.

George M. Robeson, . . . . . Secretary of the Navy.

J. A. J. Creswell, . . . . . Postmaster-General.

George H. Williams, . . . . . Attorney-General.

Morrison R. Waite, . . . . Chief Justice of the United Slales.

M. D. Leggatt, . . . . . . . Commissioner of Patents. 
\%

4. \% O 


\title{
ORBIT OF URANUS,
}

WITH GENERAL TABLES OF ITS MOTION.

\author{
BY \\ SIMON NEWCOMB, \\ PROFEsSOR OF MATHEMATICS, UNITED STATES NAVY.
}




\section{A D V ERTISE M ENT.}

In the investigation of the Orbit of Uranus which forms the subject of the accompanying memoir, as well as in that of the Orbit of Neptune previously published in the Smithsonian Contributions, a large amount of arithmetical computation has been required, especially in the reduction and comparison of observations. The cost of this, in accordance with the spirit of the Institution in advancing science, has been defrayed from the income of the Smithson fund.

As required by the rules of the Institution, the accompanying memoir was referred to competent authority for examination, and the persons selected for this purpose were Professor J. H. C. Coffin, of the Nautical Almanac Office, and Professor Asaph Hall, of the Naval Observatory.

JOSEPH HENRY,

Secretary S.I.

Washington, 1873. 


\section{P R E F A C E.}

THE present work was undertaken as far back as the year 1859. But the labor devoted to it at first amounted to little more than tentative efforts to obtain numerical data of sufficient accuracy, and to decide upon a satisfactory method of computing the general perturbations of the planet. The elements of Neptune employed in the earlier computations were found to deviate too widely from the truth to be used in computing the perturbations of Uranus with the first order of accuracy, and it became necessary to correct them. This was done during the years 1864 and 1865 , and the investigation was printed by the Smithsonian Institution in the latter year. It was then found that the adopted elements of Uranus also differed too widely from the truth to serve as the basis of the work, and they were provisionally corrected by a series of heliocentric longitudes derived from observations extending from 1781 to 1861 . Finally it was found that the adopted method of computing the perturbations, that of the "variation of elements," though not deserving of the disfavor into which it has fallen of late years, was practically inapplicable to the computation of the most difficult terms, namely, those of the second order with respect to the disturbing forces. Indeed, it appeared to the author that the only method of computing those terms which was at the same time general, practicable, and fully developed, was that of Hansen. But, were this method adopted, all that had previously been done would have been. useless, even for the purpose of comparison and verification, owing to the expression of the coordinates in terms of a disturbed mean anomaly. It appeared to the author that, although this form of theory led to expressions having fewer terms than the other, it was not without its relative disadvantages. Other considerations being equal, he conceived that astronomers generally would greatly prefer to see the perturbations expressed directly in terms of the time, owing to the ease with which the results of different investigators could then be compared, and with which corrections to the theory may be introduced.

Under these circumstances the method described in the first chapter of the present paper was worked out. The question how much it contains that is essentially new is one that the author has never closely examined: it is, however, certain 
that the mode of considering the subject is well known, being that employed by La Place, Herschel, De Pontécoulant, Encke, and perhaps others. The method of forming the required derivations of the perturbative function from the analytical development of that quantity, he has not seen elsewhere.

With these improved elements and methods the work was recommenced in 1868. The earlier investigations being merely provisional, it has not been deemed necessary to present them in the present work. Some of the results, corrected for errors of the older elements, are, however, given for the purpose of comparison.

Although this investigation has absorbed the greater part of the author's leisure for more than five years, it is only through the aid of the Smithsonian Institution and Nautical Almanac that he has been enabled to bring it to a conclusion within that time. At an early stage of the work Professor Henry responded farorably to a. request for aid by the employment of computers; it was, howcver, not found practicable to use such aid until the perturbations had been completed, and the provisional theory concluded. Then, the comparison of theory and obscrvation, and the construction of the tables, involved a large amount of mechanical computation, and on this part of the work a number of persons have been employed by the Institution at various times, among whom may be mentioned Professor F. W. Bardwell, of the University of Kansas, and Dr. C. L. F. Kampf, late of the Observatory of Leiden. Every part of the work has, however, been done under the author's immediate direction, and, as nearly as possible, in the same way as if he had done it himself, a result which, in one or two cases, has been attained only by the expenditure of an amount of labor approximating that saved by the employment of the computer.

In presenting the steps of the investigation, the end has been kept constantly in view to render as easy as possible the detection and correction of any error, or the introduction of any alteration in the elements or other data. It is, of course, impossible to present the steps of the computation with any approach to fulness without far transcending the limits of the printed work: The results given are, therefore, those which it was supposed would be most useful to the future investigator of the same subject. 'There is reason to believe that the original computations will ultimately become the property of the National Academy of Sciences, so that they may always be referred to for the clearing up of any difficulty in the printed text.

'The author's acknowledgments are due to Professor J. H. C. Coffin, Superintendent of the Nautical Almanac, and Mr. E. J. Loomis, of the Nautical Almanac Office, for reading the proof sheets of the last twelve tables during the absence of the former abroad. 


\section{TABLE OF CONTENTS.}

INTroduction

\section{CHAPTER I.}

METHOD OF DETERMINING THE PERTURBATIONS OF THE LONGITUDE, RADIUS VECTOR, AND LATITUDE OF A PLANET BY DIRECT INTEGRATION.

Notation and general differential formulæ

Formation of the required derivatives of the perturbative function $\quad . \quad+\quad . \quad . \quad . \quad 10$

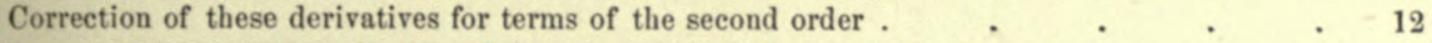

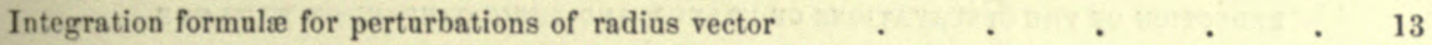

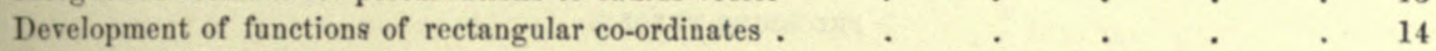

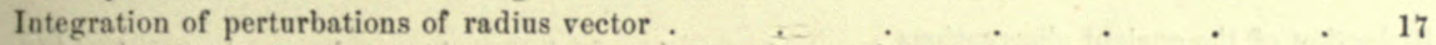

Formulæ for perturbations of longitude to terms of the second order $\quad$. $\quad . \quad 2 \quad . \quad 22$

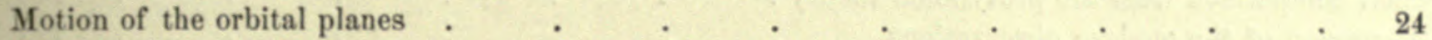

Perturbations of the second order depending on the motion of the orbital planes . $\quad 25$

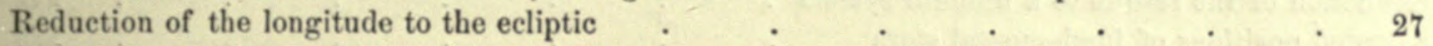

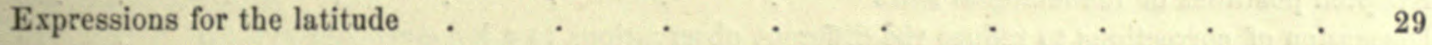

\section{CHAPTER II.}

APPLICATION OF THE PRECEDING METHOD TO THE COMPUTATION OF THE PERTURBATIONS OF URANUS BY SATURN.

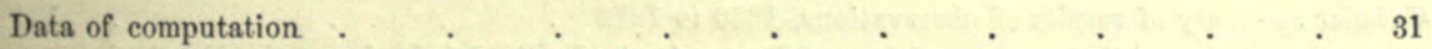

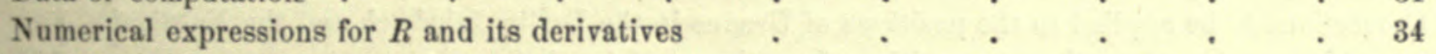

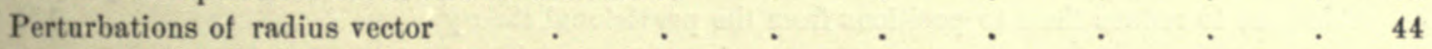

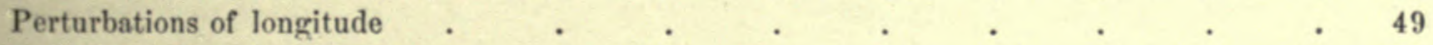

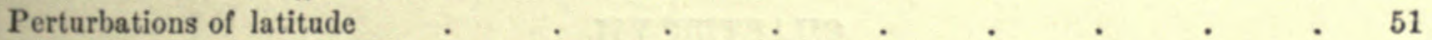

CHAPTER III.

PERTURBATIONS OF URANUS PRODUCED B́ NEPTUNE AND JUPITER.

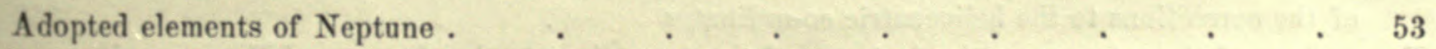

Development of $R$ and its derivatives for the action of Neptune $\quad . \quad . \quad . \quad . \quad 54$

The term of long period between Neptune and Uranus _ $\quad . \quad \ldots \quad$. $\quad . \quad 55$

Perturbations of the longitude produced by Neptune . . . . . . . . . 58

Perturbations of the radins vector prodnced by Neptune . . . . . 60

Perturbations of the latitude produced by Neptune . $\quad . \quad$. $\quad . \quad . \quad 61$

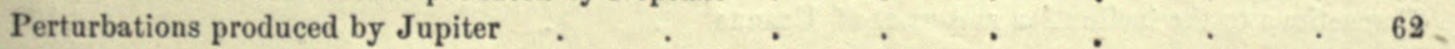




\section{CHAPTER IV.}

TERUS OF THE SECOND ORDFR DUE TO THE ACTION OF SATER.

Preliminary investigation of the orbit of Saturn

Perturbations of Saturn and Uranus.

Formation of the expressions for the terms of the second order

Perturbations depending on the square of the mass of Saturn

Perturbations depending on the product of the masses of Jupiter and Saturn

\section{CIAPTER. V.}

COLLECTION AND TRANSFoRMation OF THE PRECEDING PERTURBations OF URANUS.

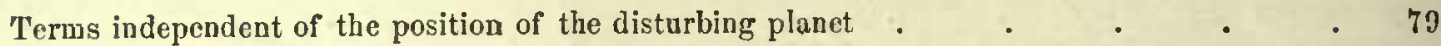

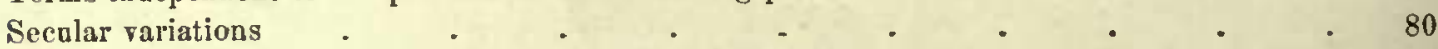

Auxiliary expressions on which the perturbations depend . $\quad . \quad$. $\quad . \quad$. 81

Reduced expressions for the latitude of Uranus . . . . . . . . . 93

Positions of Uranus resulting from the preceding theory $\quad . \quad \ldots \quad$. $\quad . \quad 98$

Elements III of Uranus

\section{CHAPTER VI.}

REDUCTION OF THE OBSERTATIONS OF URANUS, AND THEIR CONPARISON WITH THE PRECEDING TIIEORY.

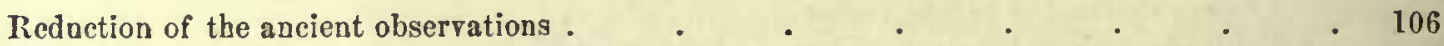

Their comparison with the provisional theory . $\quad . \quad$. . . . . 110

Discussion of the modern observations

Reduction of the results to a uniform system . $\quad . \quad \ldots \quad$. $\quad . \quad 111$

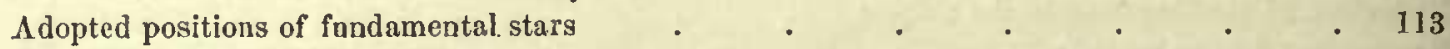

Discussion of corrections to reduce the different observations to a homogeneons system $\quad 115$

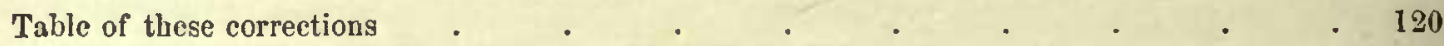

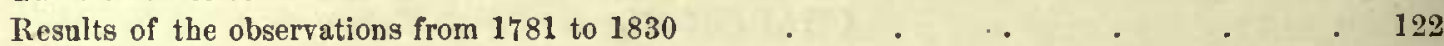

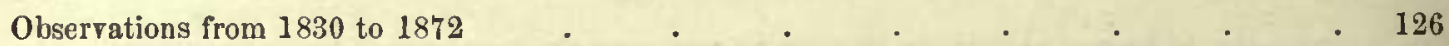

Table to convert errors of right ascension and declination of Uranus into crrors of longitude and latitude

Tabular summary of results of observations, 1830 to 1872 . . . . . .

Corrections to be applied to the positions of Uranus in the Berlin Jahrbuch and the Nautical

Almanac to reduce them to positions from the provisional theory

\section{CHAPTER. VII.}

\section{FORMATION AND SOLTTION OF THE EQUATIONS OF CONDITION RESULTING FROM THE PRECEDING COMPARISONS.}

Expressions of the observed corrections to the longitudes of the provisional theory in terms of the corrections to the heliocentric co-ordinates

Expressions of the same quantities in terms of the corrections to the elements of Uranns and the mass of Neptnne

Table to express errors of heliocentric co-ordinates as errors of elcments

Discussions and solutions of the equations thas formed

Concluded corrections to the elements of longitnde

Corrections to the inclination and node of Uranus 


\section{CHAPTER VIII.}

COMPLETION AND ARRANGEMENT OF THE THEORY TO FIT IT FOR PERMANENT USE.

Correction of the coefficients of the long inequality between Uranus and Neptune for the terms of the second order.

Concluded elements, or elements IV of Uranus

Mean elements of Uranus

Expressions for the concluded theory of Uranus

\section{CHAPTER IX.}

GENERAL TABLES OF URANUS.

Enumeration of the quantities contained in the several tables $\quad . \quad$. $\quad . \quad . \quad 190$

Precepts for the use of the tables . . . . . . . . . . . 195

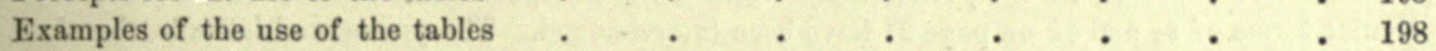

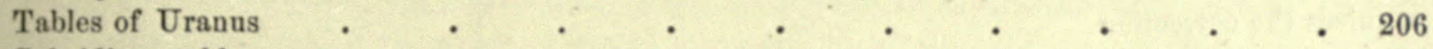

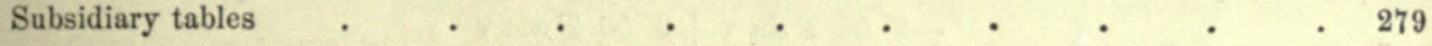


E R R A T A.

Pages 100 to 105 . In computing the latitude from the provisional theory the values of the secular terms of $\delta \eta$ and $\delta k$ on page 97 have been interchanged. The provisional latitude, therefore, requires the correction

where

$$
\begin{aligned}
& -0^{\prime \prime} .53 T \sin \mathrm{v}+0^{\prime \prime} .53 T \cos \mathrm{v} \\
& \mathrm{v}=g+12^{\circ} 45^{\prime}+2 e \sin g .
\end{aligned}
$$

This correction is not applied in the subsequent investigation. Its effect would have been to change the value of $b$ deduced on p. 176 by probably $0^{\prime \prime} .2$ or $0^{\prime \prime} .3$. The effect on the other elements of latitude would have been much smaller, and therefore unimportant.

Page 122, line 15. Add : the corrections in the sixth column being omitted.

Page 151. Add foot-note: In forming these comparisons the corrections to the heliocentric longitude in the sixth column of the provisional ephemeris, pages 100 to 105 , are not applied.

Page 159. Equation 7. In this equation the coefficients of $\delta \lambda$ and $\delta \rho$ have been multiplied by $\frac{1}{5}$, instead of $\frac{2}{5}$, the factor of $\delta l$. The effect of this error enters into all the subsequent results, but in the comparisons of theory and observation it is corrected.

Page 184. The element here represented by $x$ (kappa) is the same which, in the preceding chapters, has by mistake been represented by $k$, and which is defined on p. 24 . The $k$ of Chapter VIII is, therefore, not the same with that of preceding chapters. 


\section{ON THE ORBIT OF URANUS.}

\section{INTRODUCTION.}

THE connection of the planet Uranus with the most brilliant astronomical achievement of the century lends a peculiar interest to its theory. The researches of Adams and Le Verrier showed that the observed motions of that planet were represented, at least approximately, by the action of a theoretical planet having the longitude of Neptune. Peirce showed that the action of Neptune itself accounted for these motions within the limits of possible error of the observations used by Le Verrier. It remains to be seen whether the agreement between theory and observation still subsists when the comparatively few observations used by those investigators are reduced with the more refined data now at our disposal, and when the great mass of additional observations made both before and since the date of Le Verrier's researches are included.

The circumstances connected with the discovery of Neptune have been so exhaustively recounted by a number of authors that it would be difficult to add anything not already familiar to astronomers without transcending our present limits. I shall therefore confine myself to such an account of previous researches on the theory of Uranus as may give an idea of their nature and extent, and facilitate their comparison with the methods and results of the present investigation.

The perturbations used by Bouvard in his tables are those of the Mécanique Céleste. Although not affected with any striking error, the numerical methods adopted in their computation are necessarily too rough to allow of much interest attaching to their comparison with the results of the more recent researches.

It is essential to a clear understanding of subsequent researches that we classify the methods which have been or may be adopted in the computation of the general perturbations of the planets. This computation comprises two distinct operations: (1) the development of the disturbing forces, or some quantities of which these forces are functions; (2) the integration of the equations of motion under the influence of these forces. In each of these operations three methods have been employed.

In developing the perturbative function, we have first the purely analytic method used by the great geometers of the last century. In this method this function is developed in powers of the eccentricities and mutual inclination of the orbits of the two planets, and the numerical coefficients are found by substituting the values of the elements in these expressions. It is only applicable when the eccentricities 
and mutual inclination are sinall, and has for that reason fallen, of late, into a certain disrepute. The extended tables published by Le Verrier ${ }^{1}$ have, however, added so much to its facility for use that it is not wholly unworthy of attention.

At the other extreme stands the purely mechanical method, in which.special values of the disturbing force are computed for many combinations of the mean anomalies of the two planets, and the values of the coefficients in the general expression for the force thence deduced.

Between these two stands what I conceive we may designate as the CauchyHansen method, in which the development is made mechanically with respect to the one planet, but the cccentric anomaly of the other is retained as an undetermined quantity. The germ of this method is found in several papers, by Cauchy, in the earlier volumes of the Comptes Rendus of the French Academy, which have sinec been combined into a homogeneous memoir by Puiseux. ${ }^{2}$ The object had in view by these authors is only the computation of inequalities of long period. But Hansen has taken up the essential principle of the method, first, in his prize memoir on the perturbations of comets, crowned by the French Academy of Sciences, about 1848, and afterwards in his "Auseinandersetzung einer zueckmässigen Methode zur. Berechnung der Störungen der kleinen Planeten,"3 and applied it to the general development of perturbations.

Among the three methods of integration, the first in point of analytical elegance and generality, but the last in order of convenience in use, is that of the variation of elements, a method with which the name of La Grange is inseparably associated.

In the second the direct integration of the differential equations which express the perturbations of longitude, latitude, and radius vector is effected by special devices.

In the first of these methods the problem is presented in this form: 'The equations of motion being completely integrated for the action of the principal forces only, how must the arbitrary constants of integration vary in order that the same expressions may represent the motion of the planet under the influence of the disturbing forces? In the second method, the same thing being. presupposed, the question is, what expressions must be added to the integrals of undisturbed motion in order that the sum may represcit the integrals of the disturbed motion?

'The third is Hansen's method, in which the co-ordinates are partly expressed in terms of a certain function of the time known as the disturbed mean anomaly, determined by the condition that the true longitude in the disturbed orbit shall be the same function of the disturbed time that the longitude in the elliptic orbit is of the simple time.

Although the last two methods have a great advantage over the first in the computation of the periodic perturbations, I conceive the first to be best adapted to the computation of the secular variations, and perhaps, of terms of very long period in the mean longitude and the elements of the orbit.

- Annales de l'Observatoire Impérial de Paris. 'Tome I.

- Annales de l'Observatoire Impérial de Paris. Tome VII.

- Abhandlungen der Königlich Säehsischen Gesellschaft der Wissenschaften. Band V. VI, VII. 
In his researches on the motion of Uranus, the first thing done by Le Verrier was to recompute the perturbations by Jupiter and Saturn. It will sufficiently describe his method of doing this to say that in the developments he used the purely mechanical method for the action of Saturn, and the algebraic development of the perturbative function for the action of Jupiter, while in the integration he used the method of the variation of elements. After completing the perturbations of the first order he made the earliest attempt at a complete determination of those of the second order. Beginning with the terms of this order which arise from the secular variations of the elements, he determines them by recomputing the terms of the first order for the epoch 2300 , and assuming that the general term will then be given by interpolating between the two terms thus found, supposing them to increase uniformly with the time. This proceeding has the sanction of such high authority that it is worth while to call attention to its want of rigor. The differential coefficient of each element being given in the form

$$
\frac{d a}{d t}=k \cos b t
$$

$k$ being a function of the elcments, the perturbation of the first order will be

$$
\varepsilon a=\frac{k}{b} \sin b t .
$$

When we take into account the variation of $k$, and suppose it of the form $k_{0}+k^{\prime} t$, the process is equivalent to supposing that in this case

$$
\varepsilon a=\frac{k_{0}+k^{\prime} t}{b} \sin b t,
$$

whereas it really contains the additional term,

$$
\frac{k^{\prime}}{b^{2}} \cos b t
$$

which appears to be neglected in the process in question. It will be seen that the neglected coefficient is equal to the secular variation of the term during the time that its argument requires to increase by an amount equal to the unit radius. It is therefore the more important the longer the period of the inequality.

To obtain the periodic terms of the second order Le Verrier begins by determining the ten principal terms of the perturbations of the elements of Saturn produced by Jupiter. Next he takes up the terms in the mean longitude of Uranus which depend on the square of the mass of Saturn. The only sensible terms he finds are

$$
\begin{aligned}
& -1^{\prime \prime} .17 \sin \left(\zeta^{\prime \prime}-3 \zeta^{\prime}\right)-0^{\prime \prime} .35 \cos \left(\zeta^{\prime}-3 \zeta^{\prime \prime}\right) \\
& +0^{\prime \prime} .43 \sin \left(\zeta^{\prime \prime}-4 \zeta^{\prime \prime}+4 \zeta^{\prime \prime}-0^{\prime \prime} .21 \cos \left(\zeta^{\prime \prime}-4 \zeta^{\prime}+4 \zeta\right),\right.
\end{aligned}
$$

$\zeta, \zeta^{\prime \prime}$, and $\zeta^{\prime \prime}$ being the mean anomalies of Uranus, Saturn, and Jupiter, respectively. The terms depending on the product of the masses of Jupiter and Saturn are then taken up. Fifteen arguments are found the coefficients of which vary from a small fraction of a second to one or two seconds, while a single one of long period amounts to $32^{\prime \prime}$.

When the method of variation of elements is used, it is necessary not only to determine these variations to quantities of the second order, but, in the transforma- 
tion of the perturbations of the elements into perturbations of the co-ordinates, to carry this transformation to terms of the sccond order also. 'This Le Verrier avoids by showing that the terms of the lowest order with respect to the eccentricities thus introduced are destroyed by certain terms in the perturbations of the elements, so that it is only necessary to omit both classes of terms. 'These terms are of that fictitious class which disappear of themselves by a simple change of elements. When, instead of the eccentricity and longitude of the perihelion, we take $h$ and 7 , which represent the products of the eccentricity into the sine and cosine of this longitude respectively, these tcrms disappear of themselves both from the perturbations of the elements and of the co-ordinates. It is not likely that any of the neglected terms of this class cxceed $0^{\prime \prime} .1$.

As soon as the elements of Neptune were known, the nature of its general action on Uranus became of interest. 'This subject was taken up by Prof. Peirce, whose results are found in the Proceedings of the American Academy of Arts and Sciences, Vol. I, pp. 334-337. This paper is accompanied with a comparison of his theory of Uranus with observations, to which similar comparisons of the theories of Adams and Le Verrier are added. 'This comparative exhibit is of sufficient interest to be given here. The numbers given are probably excesses of computed over observed longitudes.

\begin{tabular}{|c|c|c|c|c|c|c|}
\hline \multirow[b]{2}{*}{ Year. } & \multirow{2}{*}{$\begin{array}{l}\text { From Le Ver- } \\
\text { rier's best ol- } \\
\text { bit of Uranus } \\
\text { from the mo- } \\
\text { dern observa- } \\
\text { tions without } \\
\text { any external } \\
\text { planet. }\end{array}$} & \multirow{2}{*}{$\begin{array}{l}\text { From Le Ver- } \\
\text { rier's original } \\
\text { theory with } \\
\text { his best orbit } \\
\text { of hypotheti- } \\
\text { cal planet, of } \\
\text { which the } \\
\text { mass is } \\
\text { gु' } \\
\text {. } 22 .\end{array}$} & \multirow{2}{*}{$\begin{array}{l}\text { From Adams's } \\
\text { original the- } \\
\text { ory with his } \\
\text { second hypo- } \\
\text { thetical planet } \\
\text { of which mass } \\
\text { is } \\
6665 .\end{array}$} & \multicolumn{3}{|c|}{$\begin{array}{c}\text { From Peirce's theory of Neptune adopting for its } \\
\text { mass }\end{array}$} \\
\hline & & & & $\begin{array}{l}\text { That of Struve } \\
\text { from his own } \\
\text { observations } \\
\text { of the satellite } \\
\text { It }\end{array}$ & $\begin{array}{c}\text { That deduced } \\
\text { by Peirce from } \\
\text { Bond's \& Las- } \\
\text { sel's observa- } \\
\text { tions combined } \\
18^{\frac{1}{3}} \text {. }\end{array}$ & $\begin{array}{l}\text { That deduced } \\
\text { by Peirce from } \\
\text { Bond's obser- } \\
\text { vations of Las- } \\
\text { sel's satellite } \\
\text { I98 } 40^{\circ}\end{array}$ \\
\hline & 11 & "I & "I & "1 & 11 & 11 \\
\hline 1690 & +289.0 & -19.9 & +50.0 & -124.7 & +13.0 & +0.8 \\
\hline 1715 & $\begin{array}{r}+2796 \\
+\end{array}$ & $\begin{array}{r}5.5 \\
+\quad 5\end{array}$ & -6.6 & $\begin{array}{l}-99.6 \\
\end{array}$ & +10.0 & $\begin{array}{l}+8.7 \\
\end{array}$ \\
\hline 1756 & +2309 & -40 & -4.0 & -102.4 & -12.7 & $\begin{array}{r}+4.0 \\
\end{array}$ \\
\hline 1769 & $\begin{array}{r}1233 \\
+\end{array}$ & $\begin{array}{r}3.7 \\
+\quad 3\end{array}$ & $\begin{array}{r}1.8 \\
+\quad 1 .\end{array}$ & $-\quad 67.0$ & -16.0 & -6.0 \\
\hline 1782 & $\begin{array}{r}205 \\
+\quad 20\end{array}$ & $\begin{array}{r}1 \\
+\quad 23\end{array}$ & 0.0 & -18.3 & $\begin{array}{l}-5.6 \\
-\quad 5\end{array}$ & -3.0 \\
\hline 1787 & $\begin{array}{r}2.0 \\
+\quad 2 .\end{array}$ & -1.2 & $-\quad 0.2$ & $\begin{array}{r}-\quad 4.7 \\
-\quad 1\end{array}$ & $-\quad 12$ & -0.5 \\
\hline 1792 & 7.8 & $+\quad 03$ & -1.1 & $\begin{array}{r}1.6 \\
+\quad 1.6\end{array}$ & $\begin{array}{r}05 \\
+\quad 05\end{array}$ & +0.3 \\
\hline 1797 & $-\quad 6.7$ & -1.0 & -0.5 & 33 & $\begin{array}{r}+\quad 0.8 \\
+\quad 0\end{array}$ & +0.3 \\
\hline 1803 & - $\quad 3.4$ & +0.8 & +1.6 & 3.2 & $\begin{array}{r}1.2 \\
+\quad 1.2\end{array}$ & +0.8 \\
\hline 1808 & 3.8 & $\begin{array}{r}0 \\
+\quad 0.8\end{array}$ & 0.0 & 13 & -0.6 & -0.4 \\
\hline 1813 & 4.5 & $-\quad 09$ & -1.0 & 2.3 & +1.1 & -0.3 \\
\hline 1819 & 3.8 & $+\quad 0.4$ & $-\quad 22$ & 0.9 & $\begin{array}{r}\quad 0.7 \\
+\quad 0 .\end{array}$ & +1.0 \\
\hline 1824 & -7.6 & -54 & $+\quad 1.7$ & 16 & $-\quad 1.9$ & -2.0 \\
\hline 1829 & 7.8 & -2.2 & $\begin{array}{r}+2.0 \\
+\quad 2\end{array}$ & 25 & +1.3 & +0.8 \\
\hline 1835 & -45. & $-\quad 0.8$ & -1.2 & 3.9 & $\begin{array}{r}14 \\
+\quad 24\end{array}$ & +2.0 \\
\hline 1840 & $+\quad 0.7$ & $\begin{array}{r}2.2 \\
+\quad 2\end{array}$ & $\begin{array}{r}1.3 \\
+\quad 1 .\end{array}$ & 1.3 & -1.3 & -1.1 \\
\hline 1845 & $\begin{array}{r}1 \\
+\quad 6.5\end{array}$ & -0.3 & $\ldots \ldots$ & 2.8 & -12 & -0.9 \\
\hline
\end{tabular}

In this paper Professor Peirce presents the results of a complete computation of the general perturbations of Uranus by Neptune in longitude and radius vector, 
but without any details vhatever of the investigation, or any statement of the methods employed. The minuteness of the residuals in the last column of the preceding table shows that employing these perturbations by Neptune, and those of Le Verrier by Jupiter and Saturn, we had a theory of Uranus from which quite accurate tables might have been constructed. But this never seems to have been done. The ephemeris of Uranus in the American Nautical Almanac was intended to be founded on this theory, but the proper definitive elements do not seem to have been adopted in the computations, as the ephemeris does not correspond with the theory.

Although twenty-five years have elapsed since the epoch of these researches, I am not aware of any published work of importance on the theory of Uranus during the interval. Mr. T. H. Safford has, however, made a very extended investigation of the subject, but has published nothing more than a brief general description of his work, which may be found in the Monthly Notices of the Royal Astronomical Society, Vol. 22. Like Professor Peirce, he took Le Verrier's perturbations by Jupiter and Saturn, but, instead of using general perturbations by Neptune, he computed the effect of the action of this planet by mechanical quadratures for the whole period of the observations of Uranus, and thus corrected the elements and the mass of Neptune from modern observations alone. The mass in question deduced was

\section{$1 \cdot$ \\ $\overline{20039}$}

Mr. Safford does not give the representation of the modern observations, but presents the following comparison of the ancient ones, alongside which we place for comparison the corresponding numbers of Peirce's theory and those of the present investigation.

\begin{tabular}{ccccc}
\multicolumn{5}{c}{ Excess of OBservation OVER Theory. } \\
Date. & No. of obs. & Safford. & Peirce. & Neweomb. \\
1690 & 1 & $+5^{\prime \prime} .0$ & $-0^{\prime \prime} .8$ & $-11^{\prime \prime}$ \\
1715 & 3 & -4.2 & -8.7 & -8 \\
1750 & 2 & -12 & $\ldots .$. & +2.9 \\
1753 & 1 & -0.2 & $\ldots . .3$ & \\
1756 & 1 & -0.9 & -4.0 & \\
1764 & 1 & +0.4 & & -1.4 \\
1769 & 8 & +4.5 & +6.0 & -1.4
\end{tabular}




\section{CHAPTER I.}

\section{METHOD OF DETERMINING THE PERTURBATIONS OF LONGITUDE, RADIUS} VECTOR, AND LATITUDE OF A PLANEI BY DIRECT INTEGRATION.

LET us conceive a plane determined by the condition that it shall pass through the sun and contain the tangent to the orbit of a planet at any moment. If the planet were acted on by the sun alone, the position of this plane would be invariable, but, under the influence of the disturbing forees of the other planets, it is subject, at each instant, to a motion of rotation around the radius vector of the planet. We may regard this as the instantaneous plane of the planet's orbit. 'The disturbing and the disturbed planet will each have its own instantaneous plane.

Let us now put:-

$v$, the longitude of a planet counted from a determinate point in the instantancons plane of its orbit.

$v$, its distance from the node of intersection of its own orbit with that of another planet.

$\gamma$, the mutual inclination of the two orbits.

$\sigma, \sin \frac{1}{2} \gamma$.

$r$, the radius vector of the planet.

$\rho$, its logarithm.

$\mu$, the attractive force of the sun upon unit of matter at unit distance.

$a$, the mean distance corresponding to the observed mean motion of the planct, determined by the condition

$$
a^{3}=\frac{\mu(1+m)}{n^{2}}
$$

$m$ and $n$ being as usual the mass and mean motion.

$\boldsymbol{a}_{0}$, the value of $a$ corrected for the constants introduced by the perturbations, so that, as in the elliptic motion, we have

$$
\rho=\log a+f(7, e, \varpi)
$$

we shall have in the disturbed motion

$$
\rho=\log a_{0}+f(l, e, \varpi)+\text { periodic terms only. }
$$

$a_{1}$, the mean distance of an outer planet, whether it be a disturbing or disturbed planet.

$x$, the logarithm of $a$.

$\alpha$, the ratio of two mean distances, taken less than unity.

$R$, the perturbative function. 
$h$, the coefficient of any term of $\frac{a_{1}}{m^{\prime}} R$, so that we have

$$
R=\Sigma \frac{m^{\prime} h}{a_{1}} \cos N
$$

$m^{\prime}$ being here the mass of the disturbing planet.

$\lambda$, the mean distance of the planet from the node, or the mean value of $\mathbf{v}$.

$\omega$, the distance of the perihelion from the node.

$g$, the mean anomaly.

$l$, the mean longitude, or the mean value of $v$.

$\psi$, the angle of eccentricity so that $e=\sin \psi$.

$r_{3}$, the radius of the planet in the undisturbed ellipse.

$r_{1}$, the quotient of $r_{0}$ divided by the mean distance, which is a function of the eccentricity and mean anomaly only.

$T$, the time after the epoch 1850, Jan. 0, Greenwich mean noon, counted in Julian centuries.

$v$, the integrating factors of the periodic terms, or the ratio $\frac{n}{N}, N$ being the change of the angle in unit of time.

$u$, the eccentric anomaly, and, in the tables, the argument of latitude.

We have for the value of $R$

$R=\frac{m^{\prime}}{\sqrt{r^{2}-2 r r^{\prime}\left(\cos \mathrm{v} \cos v^{\prime}+\sin \mathrm{v} \sin v^{\prime} \cos \gamma\right)+r^{\prime 2}}}-\frac{m^{\prime} r}{r^{\prime 2}}\left(\cos \mathrm{v} \cos \mathrm{v}^{\prime}+\sin \mathrm{v} \sin \mathrm{v}^{\prime} \cos \gamma\right)$

or, if we suppose $r$ replaced by its value in $\rho$, namely

we shall have

$$
r=c^{p}
$$

$$
R=m^{\prime} f\left(\mathrm{v}, \mathrm{v}^{\prime}, \rho, \rho^{\prime}, \gamma\right) .
$$

With this value of $R$ it is well known that the differential equations for the longitude and radius vector of a planet are

$$
\begin{aligned}
& r \frac{d^{3} r}{d t^{2}}-r^{2} \frac{d v^{2}}{d t^{2}}+\frac{\mu(1+m)}{r}=\mu \frac{\partial R}{\partial \rho} \\
& r^{2} \frac{d^{2} v}{d t^{2}}+2 r \frac{d r}{d t} \frac{d v}{d t}=\mu \frac{\partial R}{\partial \mathrm{v}} .
\end{aligned}
$$

If we multiply the first of these equations by $2 \frac{d \rho}{d t}$ and the second by $2 \frac{d v}{d t}$ and add them together, putting, for brevity,

$$
\frac{\partial R}{\partial \rho} \frac{d \rho}{d t}+\frac{\partial R}{\partial \mathrm{v}} \frac{d v}{d t}=D_{t} R
$$

and then integrate, we shall have

$$
\frac{d r^{2}}{d t^{2}}+r^{2} \frac{d v^{2}}{d t^{2}}-\frac{2 \mu(1+m)}{r}=2 \mu\left(C+\int D_{\mathrm{t}}^{\prime} R d t\right)
$$


$C$ being the arbitrary constant added to the integral. Adding this equation to the first of equations (1) we have

$$
\frac{1}{2} \frac{d^{2}\left(r^{2}\right)}{d t^{2}}-\frac{\mu(1+m)}{r}=\mu\left(2 C+2 \int D_{t}^{\prime} R d t+\frac{\partial R}{\partial \rho}\right)
$$

Let us now represent by $r_{0}$ that elliptic value of $r$ which satisfies the equation

$$
\frac{1}{2} \frac{d^{2}\left(r_{0}^{2}\right)}{d t^{2}}-\frac{\mu(1+m)}{r_{0}}=2 \mu C \text {. }
$$

Subtracting this equation from the last we have

$$
\frac{1}{2} \frac{d^{2}\left(r^{2}-r_{0}^{2}\right)}{d t^{2}}-\mu(1+m)\left(\frac{1}{r}-\frac{1}{r_{0}}\right)=\mu\left(2 \int D_{t}^{\prime} R d t+\frac{\partial R}{\partial \rho}\right) .
$$

in which no constant is to be added to the integral, and both sides of the equation are of the order of the disturbing forces. As there is a decided advantage in taking the logarithm of the radius vector as the variable instead of $r$ itself, we substitute for the latter its value

and put

Then

$$
r=c e, \quad r_{0}=c p_{0}
$$

$$
\delta \rho=\rho-\rho_{0}
$$

$$
\begin{aligned}
& r^{2}=c^{2 \rho_{0}}+2 \gamma_{\rho}=r_{0}{ }^{2} c^{2 \gamma_{\rho}}=r_{0}{ }^{2}\left(1+2^{r} \rho+\frac{2^{2}}{1.2} \delta \rho^{2}+\text { etc. }\right) \\
& \frac{1}{2}\left(r^{2}-r_{0}{ }^{2}\right)=r_{0}{ }^{2} \delta \rho+r_{0}{ }^{2} \delta \rho^{2}+\text { etc. } \\
& \frac{1}{r}-\frac{1}{r_{0}}=-\frac{\delta \rho}{r_{0}}+\frac{\delta \rho^{2}}{2 r_{0}}+\text { etc. }
\end{aligned}
$$

Substituting these values in the above equation, carrying the development only to terms of the second order, and transposing those terms to the right hand side of the equation, and putting $\mu^{\prime}=\mu(1+m)$, we find

$$
\frac{d^{2}\left(r_{0}^{2} \delta \rho\right)}{d t^{2}}+\frac{\mu^{\prime}}{r_{0}^{3}}\left(r_{0}^{2} \delta \rho\right)=\mu\left(2 \int D_{t}^{\prime} R d t+\frac{\partial R}{\partial \rho}\right)-\frac{d^{2}\left(r_{0}^{2} \delta \rho^{2}\right)}{d t^{2}}+\frac{\mu \delta \rho^{2}}{2 r_{0}}
$$

an equation which gives the perturbations of radius vector.

The general mode of solving this equation by successive approximation is familiar. The principles on which the successive approximations are made being the same, we shall begin by assuming that we have obtained first approximations to the values of $\delta v, \delta v^{\prime}, \delta \rho, \delta \rho^{\prime}, \delta \gamma$, and that from these we wish to pass to a second approximation. We must first carry this approximation into the functions of $R$ in the second member of (4). 'To effect this we must show how, from the development of $R$ in terms of the elements and the time, we may form its successive derivatives with respect to the quantities which enter into it. $R$, while originally a function of $\mathrm{v}, \mathrm{v}^{\prime}, \rho, \rho^{\prime}$, and $\gamma$, is, in its developed form, a function of $\lambda, \lambda^{\prime}, \omega, \omega^{\prime}, e, e^{\prime}, n, x,{ }^{\prime}$ and $\gamma$, the development being effected by substituting for the first set of quantitics their values in terms of the second. The substitution is as follows:

$$
\begin{aligned}
& \mathrm{v}=\lambda+F g, \\
& \mathrm{v}^{\prime}=\lambda^{\prime}+F g^{\prime}, \\
& \rho=x+\phi g, \\
& \rho^{\prime}=x^{\prime}+\phi g^{\prime},
\end{aligned}
$$


$F g$ being the equation of the centre, and $\phi g$ the part of $\rho$ depending on the eccentricity in the elliptic motion. It follows that if we express the developed expression for $R$ as a function of $\lambda, \lambda^{\prime}, g, g^{\prime}, n, x^{\prime}$, which we may do by putting

$$
\begin{aligned}
& \omega=\lambda-g, \omega^{\prime}=\lambda^{\prime}-g^{\prime} ; \\
& a=c^{\star}, \quad a^{\prime}=c^{\nu^{\prime}} ;
\end{aligned}
$$

we shall have by successive differentiation

$$
\begin{aligned}
& \frac{\partial R}{\partial \lambda}=\frac{\partial R}{\partial \mathrm{v}} \frac{\partial \mathrm{v}}{\partial \lambda}=\frac{\partial R}{\partial \mathrm{v}} \\
& \frac{\partial^{2} R}{\partial \lambda^{2}}=\frac{\partial^{2} R}{\partial \mathrm{v}^{2}} \frac{\partial \mathrm{v}}{\partial \lambda}=\frac{\partial^{2} R}{\partial \mathrm{v}^{2}} \\
& \frac{\partial R}{\partial x}=\frac{\partial R}{\partial \rho} \frac{\partial \rho}{\partial x}=\frac{\partial R}{\partial \rho} \\
& \frac{\partial^{2} R}{\partial x^{2}}=\frac{\partial^{2} R}{\partial \rho^{2}} \frac{\partial \rho}{\partial x}=\frac{\partial^{2} R}{\partial \rho^{2}} \\
& \text { etc. } \quad \text { etc. } \quad \text { etc. }
\end{aligned}
$$

and in general

$$
\frac{\partial^{m+n+m^{\prime}+n^{\prime}} R}{\partial \lambda^{m} \partial x^{n} \partial \lambda^{\prime m^{\prime}} \partial x^{n^{\prime}}}=\frac{\partial^{m+n+m^{\prime}+n^{\prime}} R}{\partial v^{m} \partial \rho^{n} \partial v^{m^{\prime}} \partial \rho^{m^{\prime}}}
$$

Thus, by expressing the developed $R$ in the above form, we may find the derivative of any order with respect to $\mathbf{v}, \mathbf{v}^{\prime}, \rho$ and $\rho^{\prime}$, by taking the corresponding derivative with respect to $\lambda, \lambda^{\prime}, n$ and $x^{\prime}$.

The developed $R$ is usually expressed in the form

$$
R=\Sigma \frac{m^{\prime} h}{a_{1}} \cos \left(i^{\prime} \lambda^{\prime}+i \lambda+j^{\prime} \omega^{\prime}+j \omega\right)
$$

$a_{1}$ being the mean distance of the outer planet, whether disturbing or disturbed, and $h$ a function of $e, e^{\prime}, \alpha$, and $\gamma$. Substituting for $\omega$ its value in $g$, this equation will become

Putting for brevity

$$
R=\Sigma \frac{m^{\prime} h}{a_{1}} \cos \left(\left(i^{\prime}+j^{\prime}\right) \lambda^{\prime}+(i+j) \lambda-j^{\prime} g^{\prime}-j g\right) .
$$

the formulæ (6) give

$$
N=i^{\prime} \lambda^{\prime}+i \lambda+j^{\prime \prime} \omega^{\prime}+j \omega
$$

$$
\begin{aligned}
& \frac{\partial R}{\partial \mathrm{v}}=-\Sigma \frac{m^{\prime} h}{a_{1}}(i+j) \sin N \\
& \frac{\partial^{2} R}{\partial \mathrm{v}^{2}}=-\Sigma \frac{m^{\prime} h}{a_{1}}(i+j)^{2} \cos N \\
& \frac{\partial R}{\partial \rho}=\Sigma m^{\prime} \frac{\partial \frac{h}{a_{1}}}{\partial n} \cos N
\end{aligned}
$$

and in general

$$
\frac{\partial^{u+u^{\prime}+n+n^{\prime}} R}{\partial \mathrm{v}^{u} \partial \mathrm{v}^{\prime u^{\prime}} \partial \rho^{n} \partial \rho^{\prime n^{\prime}}}=\Sigma \pm m^{\prime}(i+j)^{u}\left(i^{\prime}+j^{\prime}\right)^{u^{\prime}} \frac{\partial^{n+n^{\prime}} \frac{h}{a_{1}}}{\partial x^{n} \partial x^{\prime n^{\prime}}} \sin ^{\cos } N
$$

2 March, 1873, 
The formation of the derivatives in the second member of this equation demands attention. In the analytic development of the perturbative function each value of $h$ is composed of a series of terms each of the form

$$
E \times A \text {, }
$$

$E$ being a function of the eccentricities and mutual inclination, and $A$ a function of $\alpha$ of the form

$$
(0) \alpha^{s-1} b_{s}^{(i)}+(1) \alpha^{s+1} \frac{\partial b_{s}^{(i)}}{\partial \alpha}+(2) \alpha^{s+\frac{s}{2}} \frac{\partial^{2} b_{s}^{(i)}}{\partial \alpha^{2}}+\text { etc. }+\alpha^{\frac{2 s+2 n-1}{2}} \frac{\partial^{n} b_{s}^{(i)}}{\partial \alpha^{n}},
$$

$(0)$, (1), etc., being numerical coefficients connected with the coefficients $V^{(i)}$ tabulated by Le Verrier, in Tome I of his Amnales de l'Observatoire, by the relation

$$
(n)=\frac{V_{n}^{(i)}}{1.2 .3 \ldots n},
$$

and $b_{s}^{(i)}$ being, as usual, the coefficient of $\cos i \phi$ in the development of

$$
\left(1-2 \alpha \cos \phi+\alpha^{2}\right)^{-8}
$$

in multiples of $\cos \phi$, and $n-1$ the sum of the exponents of the eccentricities in $E$.

It would have been much more convenient if in effecting this devclopment the derivatives of $b_{s}^{(i)}$ had been taken with respect to $n$ instead of $\alpha$. In fact the derivative $\frac{\partial^{n} b^{(i)}}{\partial \alpha^{n}}$ when expressed in terms of the derivatives with respect to $n$ is of the form

$$
\alpha^{n} \frac{\partial^{n} b_{s}^{(i)}}{\partial \alpha^{n}}=n_{1} \frac{\partial b_{s}^{(i)}}{\partial x}+n_{2} \frac{\partial^{2} b_{s}^{(i)}}{\partial x^{2}}+\text { ctc. }+n_{n} \frac{\partial^{n} b_{s}^{(i)}}{\partial x^{n}}
$$

Therefore, when expressed in terms of the derivatives with respect to $x, A$ will be of the form

$$
\alpha^{s-1}\left((0)^{\prime} b_{s}^{(i)}+(1)^{\prime} \frac{\partial b_{s}^{(i)}}{\partial x}+(2)^{\prime} \frac{\partial^{2} b_{s}^{(i)}}{\partial x^{2}}+\text { etc. }\right)
$$

from which the derivatives $\frac{\partial A}{\partial x}, \frac{\partial^{2} A}{\partial x^{2}}$, etc., may be found with great facility.

As in the actual developments of $R$ which we possess, the values of $A$ are given in the form (8), we must find the expression for the first two derivatives of its several terms with respect to $n$, which we casily do by the application of the symbolic formulæ

$$
\begin{aligned}
& D_{v}=\alpha D_{a} \\
& D^{2}=\alpha\left(D_{a}+\alpha D_{a}^{2}\right) .
\end{aligned}
$$

Beginning with the case of $8=\frac{1}{2}$, we have

$$
\begin{aligned}
& \frac{\partial b_{i}^{(i)}}{\partial x}=\alpha \frac{\partial b_{i}^{(i)}}{\partial \alpha} \\
& \frac{\partial^{2} b_{i}^{(i)}}{\partial x^{2}}=\alpha \frac{\partial b_{i}^{(i)}}{\partial \alpha}+\alpha^{2} \frac{\partial^{2} b_{i}^{(i)}}{\partial \alpha^{2}}
\end{aligned}
$$




$$
\begin{gathered}
\frac{\partial\left(\alpha^{n} \frac{\partial^{n} b_{i}^{(i)}}{\partial \alpha^{n}}\right)}{\partial x}=n \alpha^{n} \frac{\partial^{n} b_{i}^{(i)}}{\partial \alpha^{n}}+\alpha^{n+1} \frac{\partial^{n+1} b_{i}^{(i)}}{\partial \alpha^{n+1}} \\
\frac{\partial^{2}\left(\alpha^{n} \frac{\partial^{n} b_{i}^{(i)}}{\partial \alpha^{n}}\right)}{\partial x^{2}}=n^{2} \alpha^{n} \frac{\partial^{n} b_{i}^{(i)}}{\partial \alpha^{n}}+(2 n+1) \alpha^{n+1} \frac{\partial^{n+1} b_{i}^{(i)}}{\partial \alpha^{n+1}}+\alpha^{n+2} \frac{\partial^{n+2} b_{i}^{(i)}}{\partial \alpha^{n+2}} \\
=n \frac{\partial\left(\alpha^{n} \frac{\partial^{n} b_{i}^{(i)}}{\partial \alpha^{n}}\right)}{\partial x}+\frac{\partial\left(\alpha^{n+1} \frac{\partial^{n+1} b_{i}^{(i)}}{\left.\partial \alpha^{n+1}\right)}\right.}{\partial x}
\end{gathered}
$$

consequently we have for the derivatives of $A$ from formulæ (8)

$$
\begin{aligned}
& \frac{\partial A}{\partial x}=(0) \alpha \frac{\partial b_{i}^{(i)}}{\partial \alpha}+(1)\left(\alpha \frac{\partial b_{i}^{(i)}}{\partial \alpha}+\alpha^{2} \frac{\partial^{2} b_{i}^{(i)}}{\partial \alpha^{2}}\right)+(2)\left(2 \alpha^{2} \frac{\partial^{2} b_{i}^{(i)}}{\partial \alpha^{2}}+\alpha^{3} \frac{\partial^{3} b_{i}^{(i)}}{\partial \alpha^{3}}\right)+\text { etc. } \\
& \frac{\partial^{2} A}{\partial x^{2}}=(0)\left(\alpha \frac{\partial b_{i}^{(i)}}{\partial \alpha}+\alpha^{2} \frac{\partial^{2} b_{i}^{(i)}}{\partial \alpha^{2}}\right)+(1)\left(\alpha \frac{\partial b_{i}^{(i)}}{\partial \alpha}+3 \alpha^{2} \frac{\partial^{2} b_{i}^{(i)}}{\partial \alpha^{2}}+\alpha^{3} \frac{\partial^{3} b_{i}^{(i)}}{\partial \alpha^{3}}\right)+\text { etc. }
\end{aligned}
$$

The derivatives of $A$ being formed in this way, those of $h$ are immediately deduced from the equations

$$
\begin{aligned}
& \frac{\partial h}{\partial x}=\Sigma E \frac{\partial A}{\partial x}, \\
& \frac{\partial^{2} h}{\partial x^{2}}=\Sigma E \frac{\partial^{2} A}{\partial x^{2}} .
\end{aligned}
$$

When $s$ is equal to $\frac{3}{2}, A$ is of the form

$$
\alpha\left\{\left(0^{\prime}\right) b_{\frac{1}{2}}^{(i)}+(1)^{\prime} \alpha \frac{\partial b_{\frac{3}{2}}^{(i)}}{\partial \alpha}+(2)^{\prime} \alpha^{3} \frac{\partial b_{\frac{3}{2}}^{(0)}}{\partial \alpha^{2}}+\text { etc. }\right\}
$$

The quantity within parentheses is of the same form with $A$, in the case of $s=\frac{1}{2}$. If we represent it by $A^{\prime}$ we shall have

$$
\begin{aligned}
& \frac{\partial A}{\partial x}=\alpha\left(\frac{\partial A^{\prime}}{\partial x}+A^{\prime}\right)=\alpha \frac{\partial A^{\prime}}{\partial x}+A \\
& \frac{\partial^{2} A}{\partial x^{3}}=A+2 \alpha \frac{\partial A^{\prime}}{\partial x}+\alpha \frac{\partial^{2} A^{\prime}}{\partial x^{2}}
\end{aligned}
$$

$A^{\prime}$ being the same form with $A$, the derivatives $\frac{\partial A^{\prime}}{\partial x}$ and $\frac{\partial^{2} A^{\prime}}{\partial x^{2}}$ will be of the form (9), substituting $\frac{3}{2}$ for the index $\frac{1}{2}$, and $(0)^{\prime},(1)^{\prime}$, etc., for $(0),(1)$, etc.

In the case of $8=\frac{5}{2}$ the derivatives are obtained in the same way, which is too simple to need elucidation.

We have now to pass from the derivatives of $h$ to those of $\frac{m h}{a_{1}}$, the coefficients of the perturbative function. The form of these derivatives will depend not on whether the planet is disturbing or disturbed, but on whether it is an outer or 
inner one. Let us then suppose for the present, that $a$ and $n$ refer to the inner planet, and put $n_{1}$ for the logarithm of the mean distance of the outer one. We then have for the derivatives relatively to $x$

$$
\frac{\partial^{n} \frac{h}{a_{1}}}{\partial x^{n}}=\frac{1}{a_{1}} \frac{\partial^{n} h}{\partial x^{n}},
$$

and for the first derivative relatively to $x$, using the symbolic notation,

$$
\frac{\partial \frac{h}{a_{1}}}{\partial x_{1}}=\frac{1}{a_{1}}\left(D_{a 1}-1\right) h .
$$

The symbols in the second member being distributive, we have by suecessive differentiation

$$
\frac{\partial^{n} \frac{h}{a_{1}}}{\partial n_{1}{ }^{n}}=\frac{1}{a_{1}}\left(D_{v_{1}}-1\right)^{n} h .
$$

The quantity $h$ is a function of $\alpha$, the ratio of the mean distances or of $C^{\infty}-\infty_{1}, C$ being the neperian base. Hence

$$
D_{v_{1}} h=-D_{v} h
$$

which substituted in the last equation gives

$$
\frac{\partial^{n} \frac{h}{a_{1}}}{\partial x_{1}{ }^{n}}=\frac{(-1)^{n}}{a_{1}}\left(D_{n}+1\right)^{n} \hbar
$$

This formula gives for the first two derivatives

$$
\begin{aligned}
& \frac{\partial \frac{h}{a_{1}}}{\partial x_{1}}=-\frac{1}{a_{1}}\left(h+\frac{\partial \hbar}{\partial x}\right) \\
& \frac{\partial^{2} \frac{h}{a_{1}}}{\partial x_{1}^{2}}=\frac{1}{a_{1}}\left(h+2 \frac{\partial h}{\partial x}+\frac{\partial^{2} h}{\partial x^{2}}\right) .
\end{aligned}
$$

Substituting in the general formula (7) these expressions for the derivatives relatively to $x$ and $x_{1}$ we have expressions for the derivatives of $R$ relatively to $v, v^{\prime}, \rho, \rho^{\prime}$, it being understood, however, that all the quantities are expressed in functions of the elements of elliptic motion.

In order to compute the perturbations of the second order we must carry $R$ and such of its derivatives as enter into the differential equations (1) to quantities of the first order with respect to the perturbations. Let us then represent by $\mathrm{v}_{0}, \mathrm{v}_{0}^{\prime}$, $\rho_{0}, \rho_{0}^{\prime}, \gamma_{0}$, the elliptic values of $v, v^{\prime}, \rho, \rho^{\prime}$, and $\gamma$, which we have assumed in the first approximation to the perturbations, and by $\delta v, \delta v^{\prime}$, etc., the quantitics to be 
added to $\mathbf{v}_{0}, \mathbf{v}_{0}^{\prime}$, etc., to make the true values of $\mathbf{v}, \mathbf{v}^{\prime}$, etc., whether perturbations or corrections of the elements. We shall then have

$$
\begin{gathered}
\delta R=\frac{\partial R_{0}}{\partial \mathbf{v}_{0}} \delta \mathbf{v}+\frac{\partial R_{0}}{\partial \mathbf{v}_{0}^{\prime}} \delta \mathbf{v}^{\prime}+\frac{\partial R_{0}}{\partial \rho_{0}} \delta \rho+\frac{\partial R_{0}}{\partial \rho_{0}^{\prime}} \delta \rho^{\prime}+\frac{\partial R_{0}}{\partial \gamma_{0}} \delta \gamma \\
\delta \frac{\partial R}{\partial \mathbf{v}}=\frac{\partial^{2} R_{0}}{\partial \mathbf{v}_{0}^{2}} \delta \mathbf{v}+\frac{\partial^{2} R_{0}}{\partial \mathbf{v}_{0} \partial \mathbf{v}_{0}^{\prime}} \delta \mathbf{v}^{\prime}+\frac{\partial^{2} R_{0}}{\partial \mathbf{v}_{0} \partial \rho_{0}} \delta \rho+\frac{\partial^{2} R_{0}}{\partial \mathbf{v}_{0} \partial \rho_{0}^{\prime}} \delta \rho^{\prime}+\frac{\partial^{2} R_{0}}{\partial \mathbf{v}_{0} \partial \gamma_{0}} \delta \gamma \\
\delta \frac{\partial R}{\partial \rho}=\frac{\partial^{2} R_{0}}{\partial \rho_{0} \partial \mathbf{v}_{0}} \delta \mathbf{v}+\frac{\partial^{2} R_{0}}{\partial \rho_{0} \partial \mathbf{v}_{0}^{\prime}} \delta \mathbf{v}^{\prime}+\frac{\partial^{2} R_{0}}{\partial \rho_{0}^{2}} \delta \rho+\frac{\partial^{2} R_{0}}{\partial \rho_{0} \partial \rho_{0}^{\prime}} \delta \rho^{\prime}+\frac{\partial^{2} R_{0}}{\partial \rho_{0} \delta \gamma_{0}} \delta \gamma
\end{gathered}
$$

The value of $D_{t}^{\prime} R$ may be found either by equation (2), or by differentiating with respect to the time as introduced by the co-ordinates of the disturbed planet. When quantities of the first order only are considered the latter operation is very simple, but it is different when terms of the second order come in, because the true longitude of the planet is then expressed in terms not only of its own mean longitude, but also of the mean longitude of all the disturbing planets. The result can still be obtained in the same way by separating all the mean longitudes introduced by the co-ordinates of the disturbed planet from those introduced by the co-ordinates of the other until after the differentiation relatively to $t$.

Let us now resume the equation (4), representing its second member by $\mu Q$, so that it becomes

where

$$
\frac{d^{2}\left(r_{0}{ }^{2} \delta \rho\right)}{d t^{2}}+\frac{\mu(1+m)}{r_{0}{ }^{3}} r_{0}{ }^{2} \delta \rho=\mu Q
$$

$$
Q=2 \int D_{t}^{\prime} R d t+\frac{\partial R}{\partial \rho}-\frac{1}{\mu} \frac{d^{2}\left(r_{0}{ }^{2} \delta \rho^{2}\right)}{d t^{2}}+\frac{1}{2} \frac{\delta \rho^{2}}{r_{0}}
$$

By the operations already given $Q$ has become a known function of the time.

It is well known that the integration of (12) may be effected by finding two values of $r_{0}{ }^{2} \delta \rho$ which satisfy this equation when the second member is neglected, or, in other words, by finding two variables $x$ and $y$ which satisfy the equations

$$
\begin{aligned}
& \frac{d^{2} x}{d t^{2}}+\frac{\mu(1+m)}{r_{0}{ }^{3}} x=0 \\
& \frac{d^{2} y}{d t^{2}}+\frac{\mu(1+m)}{r_{0}{ }^{3}} y=0
\end{aligned}
$$

when the required integral is

$$
r_{0}{ }^{2} \delta \rho=\frac{\mu}{x \frac{d y}{d t}-y \frac{d x}{d t}}\left\{y \int x Q d t-x \int y Q d t\right\} .
$$

The above differential equations are satisfied by the rectangular co-ordinates of the planet in its assumed elliptic orbit. The position of the axes of co-ordinates being arbitrary we shall take the line of apsides for the axis of $X$, the perihelion being on the positive side. If we put

we have

$$
e_{0}=\sin \psi,
$$

$$
x \frac{d y}{d t}-y \frac{d x}{d t}=\sqrt{a \mu(1+m)} \cos \psi=\frac{\mu(1+m) \cos \psi}{a n}
$$


Let us, for convenience, replace $x$ and $y$ by two other variables $\xi$ and $n$ connected with them by the equations

$$
\begin{aligned}
& x=a \xi, \\
& y=a_{n} \cos \psi
\end{aligned}
$$

$\xi$ and $\eta$ are then functions of the eccentricity and mean anomaly only, and may be developed according to the multiples of the latter. Substituting the last three expressions in the preceding value of $r_{0}^{2} \delta \rho$ it becomes

$$
r_{0}^{2} \delta_{\rho}=\frac{a^{3} n}{1+m}\left\{\eta \int \xi Q d t-\xi \int \eta Q d t\right\}
$$

If we put $r_{1}^{2}$ for the value of $r_{0}$ when the mean distance of the planet is put cqual to unity, so that $r_{1}$, like $\xi_{\xi}$ and $\eta$ contains ouly the eccentricity and mean anomaly, we shall have

$$
\delta \rho=\frac{n r_{1}^{-2}}{1+m}\left\{n \int \xi a Q d t-\xi \int r_{a} Q d t\right\}
$$

We must now express $\xi$ and $\eta$ in terms of the timc, or of the mean anomaly. Putting for the present $u$ for the eccentric and $v$ for the truc anomaly, we have, by the theory of the elliptic motion,

$$
\begin{aligned}
& x=r \cos v=a(\cos u-e), \\
& y=r \sin v=a \cos \psi \sin u,
\end{aligned}
$$

from which follow

$$
\begin{aligned}
& \xi=\cos u-e, \\
& \eta=\sin u .
\end{aligned}
$$

As $\xi$ and $\eta$ are to be expressed in the form

$$
\begin{aligned}
& \xi=\frac{1}{2} \Sigma p_{i} \cos i g, \\
& \eta=\frac{1}{2} \Sigma q_{i} \sin i g,
\end{aligned}
$$

the finite integrals extending to all valucs of $i$ from $-\propto$ to $+\propto$, we shall deducc general expressions from $p_{i}$ and $q_{i}$ arranged according to the power of the eccentricity. Since

$$
u=g+e \sin u
$$

we have by Lagrange's theorem

or

$$
\cos u=\cos g-e \sin ^{2} g-\frac{e^{2}}{2 !} \frac{\partial \sin ^{3} g}{\partial g}-\frac{e^{3}}{3 !} \frac{\partial^{2} \sin ^{4} g}{\partial g^{2}}-\text { etc. }
$$

using the notation

$$
\cos u=\frac{n=\infty}{n=0} \sum_{n} \frac{e^{n}}{n !} \frac{\partial^{n-1} \sin ^{n+1} g}{\partial g^{n-1}}
$$

We then have

$$
n !=1.2 .3 \ldots n=\Gamma(n+1) \text {. }
$$

$$
0 !=1 !=1
$$


Substituting in the general term of the above series for $\sin g$ its value in imaginary exponential functions

$$
2 \sin g=\sqrt{-1}\left(c^{-g \sqrt{-1}}-c^{g \sqrt{-1}}\right)
$$

we find by the binomial theorem, using the notation of combinations,

$$
\begin{aligned}
& \dot{C}=\frac{n(n-1) \ldots \ldots(n-s+1)}{1.2 .3 \ldots s}=\frac{n !}{s !(n-s) !} \\
& 2^{n+1} \sin ^{n+1} g=(\sqrt{-1})^{n+1}\left\{c^{-(n+1) g \sqrt{-1}}-C_{n+1}^{3} c^{-(n-1) g \sqrt{-1}}+{\stackrel{2}{C} c^{-(n-3) g} \sqrt{-1}}_{n+}-\ldots\right. \\
& \left.+(-1)^{n}{ }_{n+1}^{1} e^{(n-1) g \sqrt{-1}}+(-1)^{n+1} e^{(n+1) g \sqrt{-1}}\right\}
\end{aligned}
$$

Differentiating $n-1$ times with respect to $g$, and putting together the first and last terms, the one after the first, and that before the last, and so on, we find

$$
\begin{aligned}
& -2^{n+1} \frac{\partial^{n-1} \sin ^{n+1} g}{\partial g^{n-1}}=(n+1)^{n-1}\left(c^{(n+1) g \sqrt{-1}}+c^{-(n+1) g \sqrt{-1}}\right) \\
& -\stackrel{1}{C+1}_{n+1}^{1}(n-1)^{n-1}\left(c^{(n-1) g \sqrt{-1}}+c^{-(n-1) g} \sqrt{-1}\right)+\text { etc. }
\end{aligned}
$$

Substituting for the exponentials their values in circular functions, and dividing by $2^{n+1}$ we have

$$
\begin{aligned}
\frac{\partial^{n-1} \sin ^{n+1} g}{\partial g^{n-1}}=- & \frac{1}{2^{n}}\left\{(n+1)^{n-1} \cos (n+1) g-\stackrel{C}{n+1}_{(2}(n-1)^{n-1} \cos (n-1) g\right. \\
& \left.+\stackrel{C}{n+1}_{n}(n-3)^{n-1} \cos (n-3) g-\text { etc. }\right\}
\end{aligned}
$$

the series terminating at the last positive coefficient of $g$. Substituting this last value in the general term of the series which gives $\cos u$, we have

$$
\cos u=\sum_{n=0}^{n=\infty} \frac{e^{n}}{n ! 2^{n}}\left\{(n+1)^{n-1} \cos (n+1) g-\stackrel{C}{n+1}_{n}^{1}(n-1)^{n-1} \cos (n-1) g+\text { etc. }\right\}
$$

Let us now substitute for $n$ another variable $i$, putting in the first term of the last factor $i=n+1$, in the second $i=n-1$, in the third $i=n-3$, etc. The limits of finite integration with respect to $i$ will then be

$$
\begin{aligned}
& \text { in the first term, }+1 \text { to }+\propto, \\
& \text { in the second term, }-1 \text { to }+\propto \text {, } \\
& \text { in the third term, }-3 \text { to }+\propto, \\
& \text { etc. }
\end{aligned}
$$

But all the coefficients of $g$ will then be $i$, and the formula supposes the factor of $\cos i g$ to vanish whenever $i$ is zero or negative; whence, those elements of the finite integral in which $i$ is negative must be omitted, and all the terms must be taken between the limits +1 and $+\propto$. Making the proposed substitution we have 


$$
\begin{aligned}
\cos u & =\sum_{i=1}^{i=\alpha}\left\{\frac{i^{i-2}}{(i-1) ! 2^{i-1}} e^{i-1}-\frac{i^{i}}{(i+1) ! 2^{i+1}} \stackrel{1}{C+2}^{1} e^{i+1}+\frac{i^{i+2}}{(i+3) ! 2^{i+3}} \stackrel{2}{C+4}^{2} e^{i+3}-\text { etc. }\right\} \cos i g \\
= & \sum_{i=1}^{i=\infty} \frac{i^{i-2} e^{i-1}}{(i-1) ! 2^{i-1}}\left\{1-\frac{i^{2} e^{2}}{2^{2} i(i+1)} \stackrel{1}{C}_{i+2}^{1}+\frac{i^{4} e^{4}}{2^{4} i(i+1)(i+2)(i+3)} \stackrel{2}{C}_{i+4}^{i}-\text { e.c. }\right\} \cos i g
\end{aligned}
$$

We have, therefore, for all values of $i$ different from zero

$$
p_{i}=p_{-i}=\frac{i^{i-2} e^{i-1}}{(i-1) ! 2^{i-1}}\left\{1-\stackrel{1}{C}_{i+2} \frac{i e^{2}}{2^{2}(i+1)}+\stackrel{2}{C} \frac{i^{3} e^{4}}{2^{4}(i+1)(i+2)(i+3)}-\text { etc. }\right\}
$$

To obtain the value of $p_{0}$ we remark that the only constant term in cos $u$ arises from the term $-e \sin ^{2} g$; its value is therefore $-\frac{1}{2} e$. The constant term in $\xi=\cos u-e$ is therefore $\frac{8}{2} e$, whence

$$
p_{0}=-3 e \text {. }
$$

The values of $q_{i}$ may be obtained in a similar way by developing $\sin u$ by La Grange's theorem. But the development is rather more complex, and it is easier to derive them from $p_{i}$. Let us take up the equations

$$
\begin{aligned}
& \xi=\cos u-e \\
& \eta=\sin u \\
& u-e \sin u=g
\end{aligned}
$$

Considering $u$, like $\xi$ and $\eta$, as a funetion of the independent variables $e$ and $g$, we have by differentiation

$$
\begin{aligned}
& \frac{\partial u}{\partial e}-\frac{\partial(e \sin u)}{\partial e}=0 \\
\therefore & \frac{\partial u}{\partial e}=\frac{\partial(e n)}{\partial e}=\frac{\sin u}{1-e \cos u} \\
& \frac{\partial u}{\partial g}=\frac{1}{1-e \cos u} \\
\therefore & \frac{\partial u}{\partial e}=\sin u \frac{\partial u}{\partial g}=-\frac{\partial \xi}{\partial u} \frac{\partial u}{\partial g}=-\frac{\partial \xi}{\partial g}
\end{aligned}
$$

Comparing (a) and $(b)$

$$
\frac{\partial \xi}{\partial g}=-\frac{\partial(e n)}{\partial e}
$$

Putting in this equation for $\xi$ and $\eta$ their developed values this equation becomes

$$
\Sigma i p_{i} \sin i g=\Sigma \frac{\partial\left(e q_{i}\right)}{\partial e} \sin i g
$$

which gives by equating the coefficients of sin ig

$$
q_{i}=\frac{i}{e} \int p_{i} d e .
$$


The following are special values of $p_{i}$ and $q_{i}$, developed to the sixth power of the eccentricities, as derived from the preceding formulæ:

$$
\begin{aligned}
& p_{0}=-3 e \\
& p_{1}=1-\frac{3}{8} e^{2}+\frac{5}{192} e^{4}-\frac{7}{9216} e^{6} \\
& p_{2}=\frac{1}{2} e-\frac{1}{3} e^{3}+\frac{1}{16} e^{5} \\
& p_{3}=\frac{3}{8} e^{2}-\frac{45}{128} e^{4}+\frac{567}{5120} e^{6} \\
& p_{4}=\frac{1}{3} e^{3}-\frac{6}{15} e^{s} \\
& p_{\mathrm{s}}=\frac{125}{384} e^{4}-\frac{4375}{9216} e^{6} \\
& p_{6}=\frac{27}{80} e^{5} \\
& p_{7}=\frac{16807}{46080} e^{6} \\
& q_{1}=1-\frac{1}{8} e^{2}+\frac{1}{192} e^{4}-\frac{1}{9216} e^{6} \\
& q_{2}=\frac{1}{2} e-\frac{1}{6} e^{3}+\frac{1}{48} e^{5} \\
& q_{3}=\frac{3}{8} e^{2}-\frac{27}{128} e^{4}+\frac{243}{5120} e^{6} \\
& q_{4}=\frac{1}{3} e^{3}-\frac{4}{15} e^{5} \\
& q_{5}=\frac{125}{384} e^{4}-\frac{3125}{9216} e^{e^{6}} \\
& q_{6}=\frac{27}{80} e^{5} \\
& q_{7}=\frac{16807}{46080} e^{6}
\end{aligned}
$$

Having the developed $\xi$ and $\eta$ in terms of time, let us resume the equation (13). As only purely linear operations are performed on $Q$ in this equation, it follows that if we represent its several parts by $Q_{1}, Q_{2}$, etc., and by $\delta \rho_{1}, \delta \rho_{2}$, etc., the values $\delta \rho$ obtained by putting $Q=Q_{1}, Q=Q_{2}$, etc., we shall have

$$
\delta \rho=\delta \rho_{1}+\delta \rho_{2}+\text { etc. }
$$

We have, therefore, only to find the separate values of $r_{0}^{2} \delta \rho$ corresponding to the different terms of $Q$, and to take their sum. Let us then represent, as before, by

any one term of $R$.

$$
\frac{m^{\prime}}{a_{1}} h \cos \left(i^{\prime} \lambda^{\prime}+i \lambda+j^{\prime} \omega^{\prime}+j \omega\right)
$$

3 April, 1873. 
We then have, considering only terms of the first order with respect to the disturbing forces,

where we put for brevity,

$$
\begin{aligned}
& D_{t}^{\prime} R=-\frac{m^{\prime} i \hbar n}{a_{1}} \sin N \\
& \int D_{t} R=\frac{m^{\prime} i \hbar \nu}{a_{1}} \cos N \\
& \frac{\partial R}{\partial \rho}=m^{\prime} \frac{\partial \frac{h}{a_{1}}}{\partial x} \cos N
\end{aligned}
$$

$$
\begin{aligned}
& \nu=\frac{n}{i^{\prime} n^{\prime}+i n} \\
& N=i^{\prime} \lambda^{\prime}+i \lambda+j^{\prime \prime} \omega^{\prime}+j \omega .
\end{aligned}
$$

Let us represent by $Q_{0}$ the terms in $Q$ which are of the first order with respert to the disturbing forces, so that we have

$$
Q_{0}=2 \int D^{\prime} R_{0}+\frac{\partial R_{0}}{\partial \rho_{0}}
$$

The general term in $R$ will then give rise in $Q_{0}$ to the term

$$
m^{\prime}\left(\frac{2 i \hbar \nu}{a_{1}}+\frac{\partial \frac{\hbar}{a_{1}}}{\partial x}\right) \cos N \text {. }
$$

In the case of the action of an onter on an inner planet this expression becomes

$$
\frac{m^{\prime}}{a_{i}}\left(2 i v h+\frac{\partial h}{\partial x}\right) \cos N
$$

while in the contrary case it is

$$
\frac{m^{\prime}}{a_{1}}\left(2 i \nu h-h-\frac{\partial h}{\partial x^{\prime}}\right) \cos N,
$$

both derivatives being taken with respect to the logarithm of the mean distance of the inner planet.

In the integration it will be more convenient to substitute for $\lambda^{\prime}$ and $\lambda$ the mean longitudes counted from the perihelion of the disturbed planet. If we put

the angle $N$ will become,

$$
\begin{aligned}
& \lambda=g+\omega \\
& \lambda^{\prime}=l+\omega
\end{aligned}
$$

$$
i^{\prime} l^{\prime}+i g+j^{\prime \prime} \omega^{\prime}+\left(i+i^{\prime}+j\right) \omega
$$

Since corresponding to each set of values of $i$, and $i$ there are several values of $j^{\prime}$ and $j$, it will be convenient in the numerical computation to combine these different terms into a single one, because after forming the derivatives of $R$ there is no need that $\omega, \omega^{\prime}$ and the other elements should appear in an analytical form. If we put 
$k$ for the coefficient of $\frac{m^{\prime}}{a_{1}} \cos N$ in the preceding general term of $Q_{0}$, this term will become

If we put

$$
\begin{aligned}
Q_{0}= & \frac{m^{\prime}}{a_{1}} k \cos \left[j^{\prime} \omega^{\prime}+\left(i^{\prime}+i+j\right) \omega\right] \cos \left[i l^{\prime}+i g\right] \\
& -\frac{m^{\prime}}{a_{1}} k \sin \left[j^{\prime} \omega^{\prime}+\left(i^{\prime}+i+j\right) \omega\right] \sin \left[i l^{\prime}+i g\right]
\end{aligned}
$$

$$
\begin{aligned}
& k_{e}=\Sigma k \cos \left[j^{\prime} \omega^{\prime}+\left(i^{\prime}+i+j\right) \omega\right], \\
& k_{s}=\Sigma k \sin \left[j^{\prime} \omega^{\prime}+\left(i^{\prime}+i+j\right) \omega\right],
\end{aligned}
$$

the sign $\Sigma$ being extended so as to include all values of $j$ and $j^{\prime}$ which correspond to the given values of $i$ and $i^{\prime}$, we shall have for the general terms of $Q_{0}$

$$
\frac{m^{\prime}}{a_{1}}\left\{k_{e} \cos \left(i l^{\prime}+i g\right)+k_{s} \sin \left(i l^{\prime}+i g\right)\right\},
$$

or, when we represent the angle $i^{\prime} t^{\prime}+i g$ by $N_{1}$

$$
Q_{0}=\frac{m^{\prime}}{a_{1}}\left\{k_{e} \cos N_{1}+k_{e} \sin N_{1}\right\} .
$$

This we are to combine with the values of $\xi$ and $n$

$$
\begin{aligned}
& \xi=\frac{1}{2} \Sigma p_{i} \cos i g, * \\
& \eta=\frac{1}{2} \Sigma q_{j} \sin j g,
\end{aligned}
$$

in the general integral formula (13). If we substitute them in this formula, and represent by $\mu$ the coefficient of $t$ in the value of $N$ we shall have to integrate differentials of the form

$$
\sin \left(N_{1} \pm i g\right) \text {. }
$$

in which the coefficient of the time $t$ in the angle is $\mu+i n$. Let us represent by $v_{i}$ the integrating factor

$$
\frac{n}{\mu+i n} \text {. }
$$

The formula (13) will become by these substitutions, which, though a little complex, offer no difficulty,

$$
\begin{aligned}
& \delta \rho=\frac{1}{16} \frac{m^{\prime} a r_{1}^{-2}}{a_{1}(1+m)} \underset{-\infty}{\Sigma_{i, j}^{2}} p_{i} q_{j} \times
\end{aligned}
$$

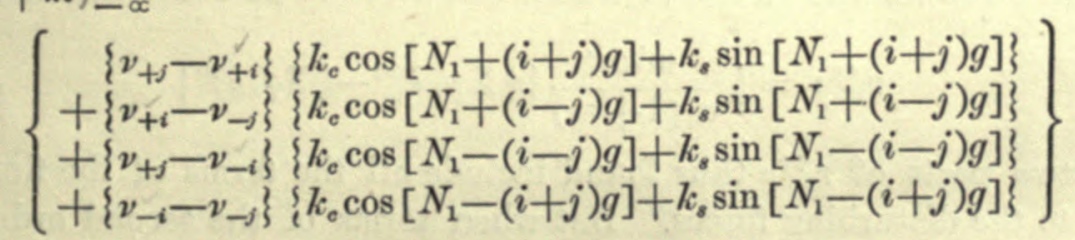

The sign $\Sigma$ of finite integration here includes the separate combination of every value of $i$ with every value of $j$, except those combinations which make the

* The indices $i$ and $j$, in these equations, are not to be confounded with the coefficients of $\lambda$ and $\omega$ in the general terms of $R$ and $Q$. We need not use the latter at present. 
coefficient of the time under the sign $\sin$ or cos vanish, and so render the corresponding value of $\nu$ infinite. 'These cases have to be treated separately.

To find, from the expression, the coefficient of the sine or cosine of cosine $N_{1}+u g$ in $r_{1}{ }^{2} \delta \rho$, we put, in the four lines of this equation, as follows:

$$
\begin{aligned}
& \text { In the first, } \quad i+j=u \cdot \therefore j=\quad u-i \\
& \text { " second, } i-j=u \cdot \therefore j=u-u ; \\
& \text { " third, }-i+j=u \therefore j=u+i \\
& \text { " fourth, }-i-j=u . \therefore j=-u-i .
\end{aligned}
$$

In the above expressions $i$ and $j$ being independent, and including all values from $-\propto$ to $+\propto, i$ and $u$ will also be independent, and include the same range of values. Substituting for $j$ its value in $u$ the coefficient of

becomes

$$
\frac{1}{16} \frac{m^{\prime} a r_{1}^{-2}}{a_{1}(1+m)}\left[k_{c} \cos \left(N_{1}+u g\right)+k_{s} \sin \left(N_{1}+u g\right)\right]
$$

$$
\Sigma\left\{\begin{array}{c}
p_{i} q_{(u-i)}\left(\nu_{(u-i)}-v_{i}\right) \\
+p_{i} q_{(i-u)}\left(v_{i}-v_{(u-i)}\right) \\
+p_{i} q_{(u+i)}\left(\nu_{(u+i)}-v_{-i}\right) \\
+p_{i} q_{-(u+i)}\left(v_{-i}-v_{(u+i)}\right)
\end{array}\right.
$$

Since $q_{j}=-q_{-j}$ this expression reduces immediately to

$$
2 \Sigma\left\{\begin{array}{r}
p_{i} q_{(i-u)}\left(v_{i}-v_{(u-i)}\right) \\
+p_{i} q_{(i+u)}\left(v_{(u+i)}-v_{-i}\right)
\end{array}\right.
$$

or, substituting $i-u$ for $i$ in the second line

$$
\begin{aligned}
& 2 \sum\left(p_{i} q_{(i-u)}+p_{(i-u)} q_{i}\right)\left(\nu_{i}-v_{u-i}\right) . \\
& \text { of } N_{1}
\end{aligned}
$$

Hence, writing $N$ instead of $N_{1}$,

$$
\delta \rho=\frac{1}{8} \frac{m^{\prime} a r_{1}^{-2}}{a_{1}(1+m)} \Sigma_{i, u}^{2}\left(p_{i} q_{(i-u)}+p_{(i-u)} q_{i}\right)\left(v_{i}-v_{(u-i)}\right)\left[k_{i} \cos (N+u g)+k_{s} \sin (N+u g)\right]
$$

This expression fails for the particular case $N=u g$, where the value of $\nu_{-u}$ will be infinite. If we take each term of $Q$ of the form

$$
\frac{m^{\prime}}{a_{1}}\left(k_{c}^{(u)} \cos u g+k_{s}^{(u)} \sin u g\right) \text {, }
$$

and substitute in the general expression (13) it will be found that the terms in $r_{1}^{2} \delta \rho$ which have the infinite values of $v$ as a factor are to be omitted, and replaced by

$$
r_{1}^{2} \delta \rho=\frac{1}{2} \frac{m^{\prime} a n t}{a_{1}(1+m)}\left\{\eta \Sigma p_{u} k_{c}^{(n)}-\xi \Sigma q_{u} k_{s}^{(n)}\right\}
$$

The two parts of $r_{1}^{2} \delta \rho$ thus found include all the terms of the first order with respect to the disturbing forces. But when terms of the second order are taken into account, we shall find terms in $Q$ proceeding from secular variation in which the time appears as a factor, outside the signs sin and cos. Let us represent such of these terms as depend on any angle $N$ by

$$
Q=\frac{m^{\prime} n t}{a_{1}}\left(k_{e} \cos N+k_{e} \sin N\right)
$$


and use the symbol $v_{i}$, as before, to represent the ratio of the mean motion of the planet to the coefficient of $t$ in the angle $N+i g$, so that if $\mu^{\prime}$ represents the coefficients of $t$ in $N$ we have

we find the expression

$$
\begin{aligned}
& v=\frac{n}{\mu^{\prime}} \\
& v_{i}=\frac{n}{\mu^{\prime}+i n}=\frac{1}{\frac{\mu^{\prime}}{n}+i}
\end{aligned}
$$

$$
\begin{aligned}
& \int \xi Q d t=\frac{1}{4} \frac{m^{\prime} \Sigma p_{i}}{a_{1} n}\left\{\left(\nu_{i}{ }^{2} k_{e}-v_{i} k_{e} n t\right) \cos (N+i g)+\left(\nu_{-i}^{2} k_{e}-\nu_{-i} k_{i} n t\right) \cos (N-i g)\right. \\
& \left.\left(v_{i}{ }^{2} k_{s}+v_{i} k_{c} n t\right) \sin (N+i g)+\left(\nu_{-i}^{2} k_{s}+v_{-i} k_{c} n t\right) \sin (N-i g)\right\} \\
& \int \eta Q d t=\frac{1}{4} \frac{m^{\prime} \Sigma q_{j}}{a_{1} n}\left\{\left(v_{j}{ }^{2} k_{e}-v_{j} k_{s} n t\right) \sin (N+j g)-\left(v_{-j}^{2} k_{e}-v_{-j} k_{s} n t\right) \sin (N-j g)\right. \\
& \left.-\left(v_{j}{ }^{2} k_{s}+v_{j} k_{c} n t\right) \cos (N+j g)+\left(\nu_{-j}^{2} k_{s}+v_{-j} k_{c} n t\right) \cos (N-j g)\right\} .
\end{aligned}
$$

If we now put for brevity

$$
\begin{aligned}
& v_{i}{ }^{2} k_{s}+v_{i} k_{e} n t=c_{i}, \\
& v_{i}{ }^{2} k_{c}-v_{i} k_{s} n t=s_{i},
\end{aligned}
$$

the general value of $r_{0}{ }^{2} \delta \rho$ becomes

$$
\begin{aligned}
& \delta \rho=\frac{1}{16} \frac{m^{\prime} a r_{1}^{-2}}{a_{1}(1+m)} \times \\
& \Sigma^{2} p_{i} q_{j}\left\{\begin{array}{r}
\left(c_{j}-c_{i}\right) \cos \left(N+\left(i+j^{\prime}\right) g\right)+\left(s_{i}-s_{j}\right) \sin (N+(i+j) g) \\
+\left(c_{i}-c_{-j}\right) \cos (N+(i-j) g)+\left(s_{-j}-s_{i}\right) \sin (N+(i-j) g) \\
+\left(c_{j}-c_{-i}\right) \cos (N-(i-j) g)+\left(s_{-i}-s_{j}\right) \sin (N-(i-j) g) \\
+\left(c_{-i}-c_{-j}\right) \cos (N-(i+j) g)+\left(s_{-j}-s_{-i}\right) \sin (N-(i+j) g)
\end{array}\right\}
\end{aligned}
$$

If, as before, we transform this expression by putting

$$
\begin{aligned}
& \text { in the first line } j=u-i \text {; } \\
& \text { in the second " } j=i-u \text {; } \\
& \text { in the third " } j=i+u \text {; } \\
& \text { in the fourth " } j=i-u
\end{aligned}
$$

the value of $r_{1}{ }^{2} \delta \rho$ reduces to

$$
\begin{aligned}
& \frac{1}{8} \frac{m^{\prime} a}{a_{1}(1+m)} \times \\
& \quad \sum_{i, u}^{2}\left\{\begin{array}{l}
p_{i} q_{(u-i)}\left(c_{(u-i)}-c_{i}\right) \cos (N+u g)+p_{i} q_{(u-i)}\left(s_{i}-s_{(u-i)}\right) \sin (N+u g) \\
p_{i} q_{(u+i)}\left(c_{(u+i)}-c_{-i}\right) \cos (N+u g)+p_{i} q_{(u+i)}\left(s_{i}-s_{(u+i)}\right) \sin (N+u g)
\end{array}\right\}
\end{aligned}
$$

or, putting $i-u$ for $i$ in the last line,

$$
\begin{aligned}
\delta \rho= & \frac{1}{8} \frac{m^{\prime} a r_{1}^{-2}}{a_{1}(1+m)} \times \\
& \Sigma_{u, i}^{2}\left\{p q_{(u-i)}-p_{(u-i} q_{i}\right\}\left\{\left(c_{(u-i)}-c_{i}\right) \cos (N+u g)+\left(s_{i}-s_{u-i}\right) \sin (N+u g)\right\} ;
\end{aligned}
$$


to which expression is to be added, in lieu of the terms which will have infinite values of $\nu$ as a factor.

$$
\frac{1}{4 a_{1}^{\prime} a r_{1}^{-2} n^{2} t^{2}}\left\{\eta \Sigma p_{u} k_{c}^{(u)}-\xi \Sigma q_{u} k_{c}^{(u)}\right\}
$$
$k_{c}^{(u)}$ and $k_{s}^{(u)}$ being the factors of $\frac{m^{\prime}}{a_{1}} n t \cos u g$ and $\frac{m^{\prime}}{a_{1}} n t \sin u g$ in the expression
for $Q$.

The formulæ $19,20,21$, and 22 give the complete expressions for the perturbations of the logarithm of radius vector by successively substituting in it all the terms of $Q$.

\section{Perturbutions of Longitude.}

We now pass to the perturbations of longitude. In the Mécanique Céleste (Première Partie, Liv. ii. Chap. vi.), Laplace gives an equation $(Y)$ by which the perturbations of longitude, which are of the first order, may be derived from those of the radius vector without the formation of any other derivatives of $R$ than those which enter into $Q$. But the formula does not seem easily adapted to the case in which the perturbations of the second order are taken into account, we shall therefore derive all the perturbations of longitude from the second of equations (1). By integration this equation gives

$$
\frac{d v}{d t}=\frac{\mu}{r^{2}}\left\{\int \frac{\partial R}{\partial \mathrm{v}} d t+C\right\}
$$

$C$ being the arbitrary constant of the integral. Representing, as before, by subscript zeros the values of the co-ordinates corresponding to the ellipse to which the orbit is supposed to reduce itself when the disturbing forces vanish, we have

$$
\frac{d v_{0}}{d t}=\frac{a^{2} n \cos \psi}{r_{0}^{2}}=\frac{\mu C}{r_{0}^{2}}
$$

because the constant to which the integral must reduce itself in the elliptic motion is $\frac{a^{2} n \cos \psi}{\mu}$. Subtracting the last equation from the preceding, and putting $v-v_{0}=\delta v$, we find

$$
\frac{d \delta v}{d t}=\frac{\mu}{r^{2}} \int_{\partial \mathrm{v}}^{\partial R} d t+\left(\frac{1}{r^{2}}-\frac{1}{r_{0}^{2}}\right) a^{2} n \cos \psi .
$$

Developing $\frac{1}{r^{2}}$ to terms of the second order with respect to the disturbing force

$$
\frac{1}{r^{2}}=\frac{1}{r_{0}^{2}}\left(1-2 \delta \rho+2 \delta \rho^{2}-\text { etc. }\right)
$$

which, being substituted in the last equation by putting

gives

$$
\begin{array}{r}
\mu=\frac{a^{3} n^{2}}{1+m}, \\
r_{0}{ }^{2}=a^{2} r_{1}{ }^{2},
\end{array}
$$

$$
r_{1}^{2} \frac{d \delta v}{d t}=\frac{a n^{2}}{1+m}(1-2 \delta \rho) \int \frac{\partial R}{\partial v} d t-2 n \cos \psi\left(\delta \rho-\delta \rho^{2}\right)
$$

which is rigorous to quantitics of the second order. 
The most convenient mode of making the numerical computation of the second order teruns by means of this equation will depend upon circumstances. If the perturbations of longitude and radius vector of both planets are already known with a sufficient degree of approximation for the computation of formula (11), it will be more convenient to form at once the complete values of ull the quantities which enter into the equations (12), (13), (19) to 22), and (23), so that no steps of the process shall have to be repeated. If such perturbations are not known, they must first be computed, and it will then be necessary to begin with the perturbations of the first order, and afterward add those of the sceond. 'There is, however, one class of terms of the second order which it will be most convenient to take account of from the beginning, namely, those arising from the constant term in i $p$ and $\delta \rho$ '. 'This is effected by correcting the mean distances for an approximate value of these constants at the beginning of the computation, and then proceeding in the usual way. This is in fact what we have supposed to be done in the preceding investigation. 'The values of $\delta v, \delta v^{\prime}, \delta \rho, \delta \rho^{\prime}$ in formula (11) will then contain only periodic terms.

In computing the terms of the first order we determine the value of $\delta \rho$ from the equations (19) and (20), using the value of $Q_{0}$ in (18). Then those of iv are obtained by integrating the equation

$$
\frac{d \delta v}{d t}=\frac{a n^{2} r_{1}^{-2}}{1+m} \int \frac{\partial R_{0}}{\partial \mathrm{v}} d t-2 n \cos \psi \frac{\delta \rho}{r_{1}^{2}} .
$$

Having found the values of $\delta v$ and $\delta \rho$ for both planets, they are to be substituted in (11), to obtain $\delta R, \delta \frac{\partial R}{\partial v}$ and $\delta \frac{\partial R}{\partial \rho}$. But, rigorously, $\delta v$ and $\delta v^{\prime}$ are not the same with $\delta v$ and $\delta v^{\prime}$, owing to the movement of the orbits of the planets, and the corrections for $\delta \gamma$ are also to be added. Considering, for the present, only the perturbations of the second orler, which depend on $i v, \delta v^{\prime}, \delta \rho$, and $\delta \rho^{\prime}$, we may use the following equation for $\delta R$, and similar ones for its derivatives:

$$
\delta R=\frac{\partial R}{\partial v} \delta v+\frac{\partial R}{\partial v^{\prime}} \delta v^{\prime}+\frac{\partial R}{\partial \rho} \delta \rho+\frac{\partial R}{\partial \rho^{\prime}} \delta \rho^{\prime} .
$$
Inving thus found $\delta R$, and hence $D_{t}^{\prime} \delta R$ by differentiation, and then $\delta \frac{\partial R}{\partial \rho}$, we form
the quantity

$$
\delta Q=2 \int D_{i}^{\prime} i R d t+\delta \frac{\partial R}{\partial \rho}-\frac{1}{\mu} \frac{t^{2}\left(r_{0}{ }^{2} \delta \rho^{2}\right)}{d t^{3}}+\frac{1}{2} \frac{\delta \rho^{2}}{r_{0}}
$$

which is the difference between the value of $Q_{0}$ in (18) and that of $Q$ in (12). The terms in $\delta \rho$ arising from $\delta Q$ are then to be computed by the formulx (19), $(20),(21)$, and $(22)$, when we shall have $\delta \rho$ accurate to quantitics of the second orler. I.et us represent these additional terms by $d^{2} \rho$. Subtracting (24) multiplied by $r_{1}{ }^{2}$ from (23), recollecting that the $\delta \rho$ which appears in the second term of the former is really $\delta \rho-\delta^{2} \rho$, we find, neglecting quantities of the third order,

$$
r_{1}{ }^{2} \frac{d \delta^{2} v}{d t}=a n^{2}\left\{\int \delta \frac{\partial R}{\partial \mathrm{v}} d t-2 \delta \rho_{0} \int \frac{\partial R_{0}}{\delta \mathrm{v}} d t\right\}-2 n \cos \psi\left(\delta^{2} \rho-\delta \rho^{2}\right)
$$


from which the terms of $\delta v$ of the sccond order are obtained by multiplying by $r_{1}^{-2}$ and integrating.

\section{Motion of the Orbital Planes.}

The general theory of the motion of the planes of reference, especially of the motion of the instantaneous orbit, has been so often treated that I can scarcely hope to add anything essentially new to it. I shall, however, endeavor to present the differential equations of the motion in a simple and general form, and one in which the geometrical conceptions of the problem shall be made as clear as possible.

'The orbital plane of each planet being at each moment osculatory to that part of the orbit which the planet is actually describing, its only motion is one of rotation around the radius vector of the planet as an instantaneous axis. 'This rotation may be resolved into two others around any pair of rectangular axes fixed in the moving plane. But the rotation produced by any one planet is most simply expressed when referred to axes, one of which coincides with the common node of the two orbits. The rotation produced by each separate planet must, therefore, be first referred to its node on the moving orbit, and then the combined rotations must be resolved into two aronnd axes assumed at plcasure. To effect this, let us suppose positive rotation around an axis to be such that an observer looking from the origin along the positive direction of the axis sees the right hand side of the plane move downwards, and the left hand side upwards. Lct us also denote the first axis in the order of longitude the principal axis, or that of $X$, and that $90^{\circ}$ farther advanced the secondary axis, or that of $Y$. Let us now put

$d q$, the instantaneous rotation around the axis of $X$;

$d p$, the instantaneous rotation around the axis of $Y$. Let us also put, relatively to any disturbing planet,

$d n$, the instantaneous rotation around the ascending node of the disturbing planct on the orbit of the disturbed one.

$d k$, that around the corresponding sccondary axis.

'Then, from the known equations for the perturbations of the inclination and node of an orbit, we find, that, if any torm of the perturbative function be represented, as before, by

$$
\frac{m^{\prime} h}{a_{1}} \cos \left(i^{\prime} \lambda^{\prime}+i \lambda+j^{\prime \prime} \omega^{\prime}+j \omega\right)
$$

the differential rotations $\eta$ and $k$ will be given by the equations

$$
\begin{aligned}
& \frac{d \eta}{d t}=-\frac{m^{\prime} \hbar}{a_{1}} \frac{a n}{\cos \psi}\left\{(i+j) \cot \gamma+\left(i^{\prime}+j^{\prime}\right) \operatorname{cosec} \gamma\right\} \sin \cdot N \\
& \frac{d k}{d t}=-\frac{m^{\prime} a n}{a_{1} \cos \psi} \frac{\partial h}{\partial \gamma} \cos N .
\end{aligned}
$$

As $R$ is actually developed, the mutual inclination $\gamma$ does not explicitly appear, but is replaced by

$$
\sigma=\sin \frac{1}{2} \gamma
$$


Making this substitution, and putting also

these equations become

$$
i+i+j+j=-\imath
$$

$$
\begin{aligned}
& \frac{d \eta}{d t}=\frac{m^{\prime} a n}{a_{1} \cos \psi \cos \frac{1}{2} \gamma}\left\{\frac{c h}{2 \sigma}+(i+j) \sigma h\right\} \sin N \\
& \frac{d k}{d t}=-\frac{m^{\prime} a n \cos \frac{1}{2} \gamma}{2 a_{1} \cos \psi} \frac{\partial h}{\partial \sigma} \cos N .
\end{aligned}
$$

To pass to the general rotations $d p$ and $d q$, let us represent by $\theta_{1}, \theta_{2}$, etc., the longitudes of the ascending nodes of the several orbits of the disturbing planets on that of the disturbed planet. We shall then have

$$
\begin{aligned}
& \frac{d q}{d t}=\Sigma \cos \theta_{i} \frac{d \eta_{i}}{d t}-\Sigma \sin \theta_{i} \frac{d k_{i}}{d t} \\
& \frac{d p}{d t}=\Sigma \cos \theta_{i} \frac{d l_{i}}{d t}+\Sigma \sin \theta_{i} \frac{d \eta_{i}}{d t} .
\end{aligned}
$$

These equations completely define the instantaneous motion of the orbital plane. They cannot, however, be rigorously integrated in their present form because $p$ and $q$ as integrals have no completely defined signification. To do this it is necessary to express the differential rotations $d p, d q$, etc., in terms, of the differentials of any elements we may select to define the position of the orbital plane, and then to integrate the equations thus formed. But, for the purpose of constructing tables of the planets we may consider $p, q$, etc., to represent small rotations of the planes of which the powers and products may be neglected, and the integration is then quite simple.

\section{Perturbations of the second order depending on the motion of the orbital planes.}

$R$ being a function of the five quantities of $r, r^{\prime}, \mathrm{v}, \mathrm{v}^{\prime}$, and $\gamma$, the motion of the orbital planes introduces terms of the second order by changing the values of $\mathbf{v}, \mathrm{v}^{\prime}$, and $\gamma$. These terms we have hitherto neglected. To investigate them let us refer the rotations of both planes as given by (28) to the node of the disturbing on the disturbed planet as the principal axis. If we represent by $d \eta, d k, d \eta^{\prime}$, and $d k^{\prime}$ the rotations corresponding to this axis, and designate by the subscript 1 , the quantities which refer to the disturbing planet whose action we are considering, and by 2,3 , etc., the other planets, the equations (28) will be replaced by these

$$
\begin{aligned}
& \frac{d \eta}{d t}=\frac{d \eta_{1}}{d t}+\Sigma \cos \left(\theta_{i}-\theta_{1}\right) \frac{d \eta_{i}}{d t}-\Sigma \sin \left(\theta_{i}-\theta_{1}\right) \frac{d k_{i}}{d t}, \\
& \frac{d k}{d t}=\frac{d k_{1}}{d t}+\Sigma \cos \left(\theta_{i}-\theta_{1}\right) \frac{d k_{i}}{d t}+\Sigma \sin \left(\theta_{i}-\theta_{1}\right) \frac{d \eta_{i}}{d t},
\end{aligned}
$$

the summation commencing with $i=2$.

By formulæ of the same kind we are to find the differential rotations $d \eta^{\prime}$ and $d k^{\prime}$ of the orbit of the disturbing planet, produced by the action of all the planets.

$$
4 \text { April, } 1873 .
$$


These rotations will be around the same principal axis with the rotations $d n$ and $d k$, but around a secondary axis in the plane of the disturbing orbit, and therefore making an angle $\gamma$ with the secondary axis of the disturbed orbit. A geometrical construction will now show quite simply that the infinitesimal rotations $\delta \eta, \delta k, \delta \eta^{\prime}$, and $\delta k^{\prime}$ will produce the following changes in $\mathrm{v}, \mathrm{v}^{\prime}$, and $\gamma$.

$$
\begin{aligned}
& \delta \mathrm{v}=\cot \gamma \delta z-\operatorname{cosec} \gamma \delta z^{\prime} \\
& \delta \mathrm{v}^{\prime}=\operatorname{cosec} \gamma \delta k-\cot \gamma \delta k^{\prime} \\
& \delta \gamma=\delta \eta^{\prime}-\delta \eta
\end{aligned}
$$

If we substitute these values in the general formulx (11) the terms of the second order added to $\delta R$ will be

$$
\begin{aligned}
\delta R & =\left(\frac{\partial R}{\partial \mathrm{v}} \cot \gamma+\frac{\partial R}{\partial \mathrm{v}^{\prime}} \operatorname{cosec} \gamma\right) \delta \hbar \\
& -\left(\frac{\partial R}{\partial \mathrm{v}} \operatorname{cosec} \gamma+\frac{\partial R}{\partial \mathrm{v}^{\prime}} \cot \gamma\right) \delta \pi^{\prime} \\
& +\frac{\partial R}{\partial \gamma}\left(\delta \eta^{\prime}-\delta \eta\right) .
\end{aligned}
$$

The first two terms of this expression may be put into the form

$$
\begin{aligned}
& \left\{\frac{1}{2}\left(\frac{\partial R}{\partial v}+\frac{\partial R}{\partial v^{\prime}}\right)(\operatorname{cosec} \gamma+\cot \gamma)-\frac{1}{2}\left(\frac{\partial R}{\partial v}-\frac{\partial R}{\partial v^{\prime}}\right)(\operatorname{cosec} \gamma-\cot \gamma)\right\} \delta k \\
- & \left\{\frac{1}{2}\left(\frac{\partial R}{\partial v}+\frac{\partial R}{\partial v^{\prime}}\right)(\operatorname{cosec} \gamma+\cot \gamma)+\frac{1}{2}\left(\frac{\partial R}{\partial v}-\frac{\partial R}{\partial v^{\prime}}\right)(\operatorname{cosec} \gamma-\cot \gamma)\right\} \delta k .
\end{aligned}
$$

But,

$$
\begin{aligned}
& \operatorname{cosec} \gamma+\cot \gamma=\cot \frac{1}{2} \gamma=\frac{\cos \frac{1}{2} \gamma}{\sigma} . \\
& \operatorname{cosec} \gamma-\cot \gamma=\tan \frac{1}{2} \gamma=\frac{\sigma}{\cos \frac{1}{2} \gamma} .
\end{aligned}
$$

and in the general term of $R$, by (7)

$$
\begin{aligned}
& \frac{\partial R}{\partial \mathrm{v}}=-\frac{m^{\prime} \hbar}{a_{1}}(i+j) \sin N \\
& \frac{\partial R}{\partial \mathrm{v}^{\prime}}=-\frac{m^{\prime} \hbar}{a_{1}}\left(i^{\prime}+j^{\prime}\right) \sin N .
\end{aligned}
$$

Making these substitutions, and putting, as before,

$$
i+j+i+j=-\imath
$$

the above value of $\delta R$ reduces to

$$
\begin{gathered}
\delta R=\frac{m^{\prime} \hbar}{2 a_{1}}\left\{\imath \cot \frac{1}{2} \gamma\left(\delta k-\delta k^{\prime}\right)+\left(i+j-i^{\prime}-j^{\prime}\right) \tan \frac{1}{2} \gamma\left(\delta k+\delta k^{\prime}\right)\right\} \sin N \\
+\frac{m^{\prime} \cos \frac{1}{2} \gamma}{2 a_{1}} \frac{\partial h}{\partial \sigma}\left(\delta \eta^{\prime}-\delta \eta\right) \cos N
\end{gathered}
$$


The corresponding terms of $\delta \frac{\partial R}{\partial \mathrm{v}}$ and $\delta \frac{\partial R}{\partial \rho}$, and may be obtained in the same way by substituting $\frac{\partial R}{\partial \mathrm{v}}$ and $\frac{\partial R}{\partial \rho}$ for $R$ in (30) and continuing the corresponding substitutions of the general terms of the derivatives of $R$ as given on page 9 .

The equation (31), besides being of the second order with respect to the disturbing forces, is also of the second order with respect to the mutual inclinations. For $\delta k, \delta k^{\prime}, \delta \eta$, and $\delta \eta^{\prime}$ are of the first order with respect to both quantities, and, whenever $\iota$ is not zero, $h$ is a quantity of the second order, containing $\sigma^{2}$ as a factor. It is, therefore, only in exceptional cases that the terms of the second order depending on the motion of the orbital planes can become sensible.

\section{Reduction of the longitude in the orbit to lo gitude on the ecliptic.}

The integration of (23) gives a value of $\delta v$, which, added to the longitude in orbit corresponding to the pure elliptic motion gives the longitude in the disturbed orbit, counted from a fixed point in the moving plane of that orbit. The position of this fixed point is completely determined by the condition that the instantaneous rotation of the plane in question around the axis perpendicular to itself is always zero, so that the motion of the point of reference is always perpendicular to the direction of the plane. But, although this instantaneous rotation is zero, the integrated rotation is not rigorously zero when we consider the terms of the second order. It follows that the value of $v$, the longitude in orbit, and the position of the plane of the orbit do not rigorously determine the position of the planet: we must also know how the fixed point of reference has changed its position in consequence of the motions which the plane has undergone. Let us consider the relative positions of this plane at two epochs. If the fixed point were equally distant from the common node of the two planes, the integrated rotation of the plane around its own axis would be zero. But, these distances not being equal, their difference is a correction to be applied to the longitude of the planet in its orbit. Suppose, now, that at the end of any time the inclination of the actual orbit to the primitive orbit is $\phi$, and the distance of its ascending node from the present position of the moving axis of $x$ is $\theta$. A rotation around the line of nodes will not change the quantity sought. But, if we represent the infinitesimal rotation around an axis perpendicular to it by $d r$ we shall have

$$
\cos \theta d p-\sin \theta d q=d r,
$$

$d q$ and $d l$ being the instantaneous rotations around the respective axes of $x$ and $\gamma$. By this rotation it is easy to see that the relative distance of any two fixed points, one on each plane, from the node, will be altered by the quantity,

$$
d r(\operatorname{cosec} \phi-\cot \phi)=d r \tan \frac{1}{2} \phi,
$$

the relative longitude of the fixed point on the moving plane being increased by this amount. The correction to the longitude in orbit from this cause is, therefore,

$$
d l=d r \tan \frac{1}{2} \phi=\tan \frac{1}{2} \phi(\cos \theta d p-\sin \theta d q) .
$$


Counting the integrated values of $p$ and $q$ in a direction perpendicular to the moving plane we have

$$
\begin{aligned}
& \sin \theta=\frac{\tan p}{\tan \phi} \\
& \cos \theta=\frac{\tan q}{\tan \phi}
\end{aligned}
$$

which, being substituted in the expression for $d l$, gives

$$
d l=\frac{\cos \phi}{1+\cos \phi}(\tan q d p-\tan p d q) .
$$

The approximate value of the integrated correction is therefore

$$
\delta l=\frac{1}{2} \int(q d p-p d q) .
$$

For every pair of periodic terms in $p$ and $q$, such as

$$
q=s \sin \mu t, p=s \cos \mu t,
$$

$\delta l$ will contain the secular term -- $\frac{1}{2} s^{2} \mu t$, which will be confounded with the mean motion, and, if it were not so confounded, would in few or none of the larger planets amount to a second in a thousand years. If the secular terms in $p$ and $q$ be

$$
q=s t ; p=s^{\prime} t
$$

$\delta l$ will vanish. We hence conclude that these terms are entirely unimportant in the present state of astronomy, and that, if we consider the positions of the plane of the orbit at two epochs, we may consider the points of departure in them to be equally distant from their common node.

We have therefore only to consider the motion of the inclination and node due to the change of the position of the orbit and of the ecliptic. If we put

$\phi$, the inclination of the orbit of the planct to the ecliptic,

$\theta$, the longitude of its node counted on the ecliptic,

$\tau$, the longitude of the same node counted from the same fixed point in the moving plane of the orbit from which $v$ is counted,

'Then, the longitude of the planet on the ecliptic, or $L$, will be given by the equation

$$
\tan (L-\theta)=\cos \phi \tan (v-\tau),
$$

or, when developed in powers of $\phi$,

$$
L=v+\theta-\tau+D
$$

where $D$ is the reduction to the ecliptic, the value of which is

$$
D=-\tan ^{2} \frac{1}{2} \phi \sin 2(v-\tau)+\frac{1}{2} \tan ^{4} \frac{1}{2} \phi \sin 4(v-\tau)-\text { etc. }
$$

Let us refer the instantaneous rotations of the orbit and of the ccliptic to the fixed points of reference in the two planes; $q$ being the rotation around an axis passing through the sun and the fixed point, and $p$ that around an axis in $90^{\circ}$ greater longitude, and the accented quantities referring to the ecliptic. We then have 


$$
\begin{gathered}
\frac{d \phi}{d t}=\cos \tau \frac{d q}{d t}+\sin \tau \frac{d p}{d t} \\
-\cos \theta \frac{d q^{\prime}}{d t}-\sin \theta \frac{d p^{\prime}}{d t} \\
\frac{d \theta}{d t}=\operatorname{cosec} \phi\left(-\sin \tau \frac{d q}{d t}+\cos \tau \frac{d p}{d t}\right) \\
+\cot \phi\left(\sin \theta \frac{d q^{\prime}}{d t}-\cos \theta \frac{d p^{\prime}}{d t}\right) \\
\frac{d \tau}{d t}=\cot \phi\left(-\sin \tau \frac{d q}{d t}+\cos \tau \frac{d p}{d t}\right) \\
+\operatorname{cosec} \phi\left(\sin \theta \frac{d q^{\prime}}{d t}-\cos \theta \frac{d p^{\prime}}{d t}\right)
\end{gathered}
$$

If we differentiate (33) and substitute these values of $\frac{d \theta}{d t}$ and $\frac{d \tau}{d t}$, we shall have

$$
\frac{d L}{d t}=\frac{d v}{d t}+\frac{d D}{d t}-\tan \frac{1}{2} \phi\left(\cos \tau \frac{d p}{d t}-\sin \tau \frac{d q}{d t}+\cos \theta \frac{d p^{\prime}}{d t}-\sin \theta \frac{d q^{\prime}}{d t}\right)
$$

If we consider only quantities of the first order with respect to the disturbing forces, we may, in integrating, suppose $\tau$ and $\theta$ equal and constant, and $\phi$ constant. The integral will then be

$$
L=v+D+\tan \frac{1}{2} \phi\left\{\cos \theta\left(\delta k+\delta k^{\prime}\right)-\sin \theta\left(\delta \eta+\delta \eta^{\prime}\right)\right\}
$$

In the case of Uranus, $\tan \phi$ is so small that this equation will be sufficient for a long time before and after our epoch.

In the application of the method to other planets the mode of operation must depend on the circumstances of each particular case. The differential equations (34) between $\theta, \tau$, and $\phi$ are rigorous, and their integrals may be approximated to in various ways, out of which that best applicable to the particular case must be selected.

\section{Expressions for the latitude.}

If the position of the orbital plane and of the ecliptic were each determined by the preceding formulæ, there would be no perturbations of the latitude, the latitude itself being given rigorously by the equation

$$
\begin{aligned}
\sin \beta & =\sin \phi \sin (v-\tau) . \\
& =\sin \phi \cos \tau \sin v-\sin \phi \sin \tau \cos v .
\end{aligned}
$$

But the instantaneous values of $\phi$ and $\tau$, or of $\sin \phi \cos \tau$ and $\sin \phi \sin \tau$, are troublesome to tabulate; it will therefore, in practice, be found more convenient to use only their mean values, and to consider their changes from this mean as perturbations of the latitude. Representing by the sign $\delta$ the deviations from the mean values, which are of course arbitrary, we have

$$
\cos \beta \delta \beta=\cos \phi \sin (v-\tau) \delta \phi-\sin \phi \cos (v-\tau) \delta \tau .
$$

Let us substitute for $\delta \phi$ and $\delta \tau$ their values given by the integration of (34) to 
quantities of the first order, in which case $\theta$ and $\tau$ may be assumed equal. 'These values are

$$
\begin{aligned}
\delta \phi & =\sin \tau \delta p+\cos \tau i q \\
\sin \phi \delta \tau & =\cos \phi(\cos \tau \delta p-\sin \tau \delta q)
\end{aligned}
$$

the terms dependent on $\delta p^{\prime}$ and $\delta q^{\prime}$ being omitted because, being purely secular, they may be included in the mean values of $\phi$ and $\tau$. Sulstituting in the expression for $\delta \beta$

$$
\cos \beta \delta \beta=\cos \phi\{\sin v\{q-\cos v i p\} .
$$

In the case of all the larger planets both $\cos \beta$ and $\cos \phi$ may here be put equal to unity, when the expression for $\delta \beta$ will become

$$
\delta \beta=\sin v \delta q-\cos v \delta p .
$$

To develop this expression in purely periodic terms we must substitute for $v$ its value in terms of the mean longitude or mean anomaly, namely,

$$
v=l+2 e \sin g+\frac{5}{4} e^{2} \sin 2 g+\text { etc. }
$$

suppose the terms of $\delta p$ and $\delta q$ depending on any argument, $N$ to be

$$
\begin{aligned}
& \delta p=-a_{s} \sin N-a_{c} \cos N \\
& \delta q=u_{s}^{\prime} \sin N+a_{c}^{\prime} \cos N
\end{aligned}
$$

and put $\pi$ for the longitude of the perihelion, so that

$$
l=\pi+g
$$

then, to terms of the first order with respect to the eccentricities, we have $\delta \beta=-e\left(\alpha_{s} \cos \pi+\alpha_{s}^{\prime} \sin \pi\right) \sin N-e\left(\alpha_{c} \cos \pi+a_{c}^{\prime} \sin \pi\right) \cos N$

$$
\begin{aligned}
& +\frac{1}{2}\left\{\left(a_{s}+a_{c}^{\prime}\right) \cos \pi+\left(a_{s}^{\prime}-a_{c}\right) \sin \pi\right\} \sin (N+g) \\
& +\frac{1}{2}\left\{\left(a_{c}-a_{s}^{\prime}\right) \cos \pi+\left(a_{c}^{\prime}+a_{s}\right) \sin \pi\right\} \cos (N+g) \\
& +\frac{1}{2}\left\{\left(a_{s}-a_{c}^{\prime}\right) \cos \pi+\left(a_{s}^{\prime}+a_{c}\right) \sin \pi\right\} \sin (N-g) \\
& +\frac{1}{2}\left\{\left(a_{c}+a_{s}^{\prime}\right) \cos \pi+\left(a_{c}^{\prime}-a_{s}\right) \sin \pi\right\} \cos (N-g) \\
& +\frac{1}{2} e\left\{\left(a_{s}+a_{c}^{\prime}\right) \cos \pi+\left(\alpha_{s}^{\prime}-a_{c}\right) \sin \pi\right\} \sin (N+2 g) \\
& +\frac{1}{2} e\left\{\left(a_{c}-a_{s}^{\prime}\right) \cos \pi+\left(a_{c}^{\prime}+a_{s}\right) \sin \pi\right\} \cos (N+2 g) \\
& +\frac{1}{2} e\left\{\left(a_{s}-a_{c}^{\prime}\right) \cos \pi+\left(a_{s}^{\prime}+a_{c}\right) \sin \pi\right\} \sin (N-2 g) \\
& +\frac{1}{2} e\left\{\left(a_{c}+a_{s}^{\prime}\right) \cos \pi+\left(a_{c}^{\prime}-a_{s}\right) \sin \pi\right\} \cos (N-2 g)
\end{aligned}
$$

The point of the orbit from which $\pi$ and $v$ are counted is entirely arbitrary, and, in considering the action of but a single planet, it will be most convenient to count them from the common node, in which case $\pi$ must be replaced by $\omega$, and $\delta p$ and $\delta q$ by $\delta \%$ and $\delta \eta$. Thus, deducing the perturbations of the latitude immediately from the formulæ (27), we shall have

$$
\delta \beta=\sin v \delta \eta-\cos v \delta \% \text {. }
$$




\section{CHAPTER II.}

\section{APPLICATION OF THE PRECEDING METHOD TO THE COMPUTATION OF THE PERTURBATIONS OF URANUS BY SATURN.}

\section{Data of Computation.}

THE elements of Uranus, adopted in this computation, were deduced from the comparison of nine normal heliocentric longitudes at intervals of 3697 days extending from 1781, December 26, to 1862 , December 18, with corresponding provisional places derived from the elements given in the "Investigation of the Orbit of Neptune," with perturbations produced by Jupiter, Saturn, and Neptune. As the perturbations are to be entirely re-computed, and the elements to be re-corrected from more extended series of observations, all the details of this first approximation will be omitted. The resulting elements of Uranus are given in the following table, together with the adopted elements of Saturn, which are nearly the same as those employed in the theory of Neptune, except that the inclination and longitude of the node have been corrected to agree with observations:-

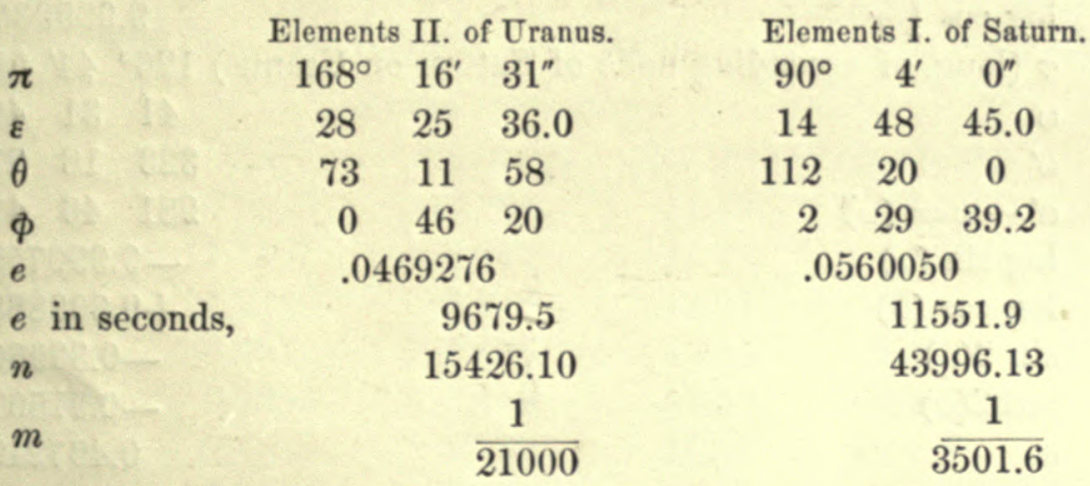

In computing the perturbations of the radius vector, one of the largest terms will be a constant. To avoid the necessity of computing separately the perturbations of the second order, which depend on this constant, we shall include an approximate value of it in the mean distance. This approximate value is, in the action of an outer or an inner planet, $\delta \log a=-\frac{1}{6} m^{\prime} M \alpha^{2} D_{a} b_{i}^{(0)}$. In the action of an inner or an outer planet, $\delta \log a^{\prime}=+\frac{1}{6} m M\left(b_{t}^{(0)}+\alpha D_{a} b_{t}^{(0)}\right) . \quad M$ being the modulus of the system of logarithms.

Using the values of $b_{i}^{(0)}$ and $\alpha D_{a} b_{i}^{(0)}$, which are found in different works relating to Celestial Mechanics, we find that the different planets produce the following changes in $6 \log a$, the units being those of the seventh place of decimals:- 


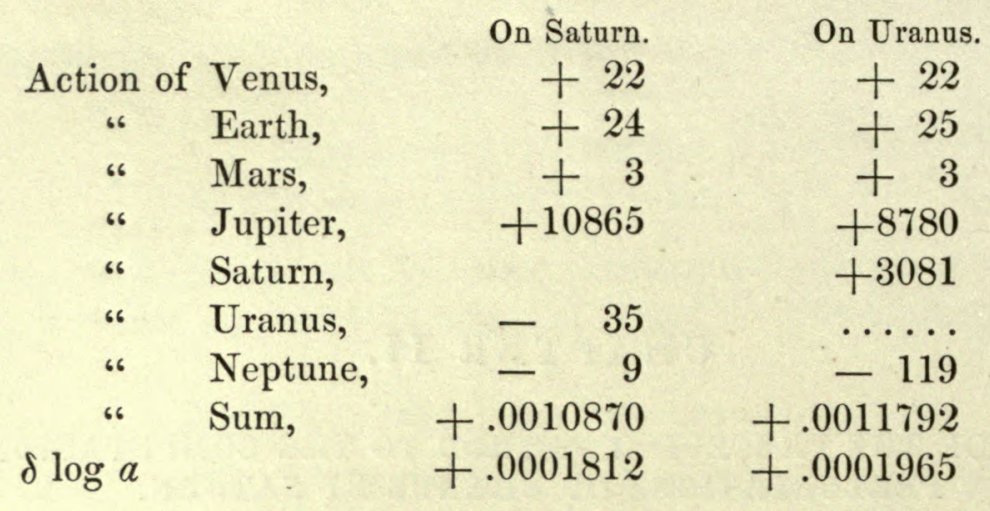

The uncorrected mean distance is deduced from the mean motion by the relation

We thus have

$$
a^{3}=\frac{\mu(1+m)}{n^{2}}
$$

$\begin{array}{lcc} & \text { Saturn. } & \text { Uranus. } \\ \text { Uncorrected mean dist. (log) } & 0.979496 & 1.282901 \\ \text { Action of the planets } & +181 & +197 \\ \text { Corrected } \log a & 0.979677 & 1.283098\end{array}$

The following functions of the elements are derived from the preceding elements by well known formulæ:-

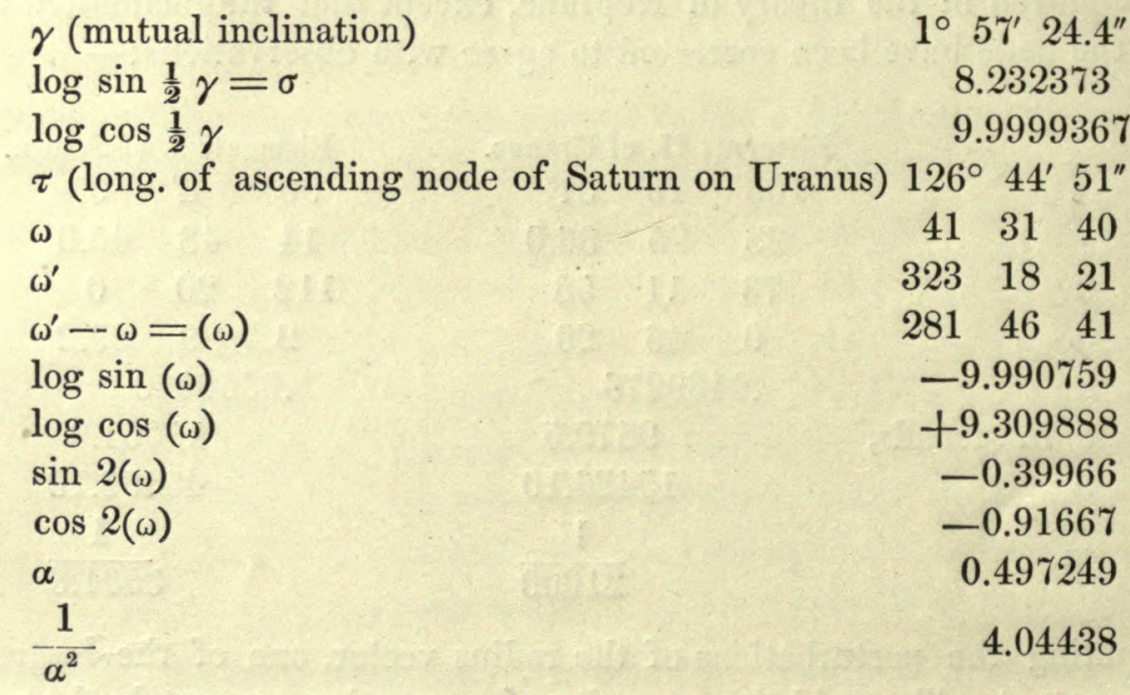

The following functions of $\alpha$, necessary in computing the coefficients $h$, are derived from Runkle's Tables, published by the Smithsonian Institution:- 
THE ORBIT OF URANUS.

Vulues of $\alpha^{n} D_{a}^{n} b$ ? .

\begin{tabular}{|c|c|c|c|c|c|c|}
\hline$i$ & $b_{?}^{\varphi}$ & $a D_{a} b_{i} \varphi$ & $a^{2} D_{a}^{2} b^{\varphi q}$ & $a^{3} D_{a}^{a} b^{\varphi}$ & $a^{4} D_{a}^{4} b_{b}^{4}$ & $a^{8} D_{a}^{s} b \varphi$ \\
\hline 0 & 2.14447 & 0.33969 & 0.5878 & 1.081 & 3.44 & 13.6 \\
\hline 1 & 0.55207 & .68314 & .4990 & 1.177 & 3.40 & 13.8 \\
\hline 2 & 0.20836 & .47198 & .7396 & 1.152 & 3.59 & 13.9 \\
\hline 3 & 0.08687 & .28491 & .7123 & 1.463 & 3.68 & 14.5 \\
\hline 4 & 0.03793 & .16270 & .5632 & 1.596 & 4.30 & 15.1 \\
\hline 5 & 0.01702 & .09010 & .3998 & 1.485 & 4.87 & 16.9 \\
\hline 6 & 0.00777 & .04896 & .2653 & 1.231 & 4.98 & 19.1 \\
\hline 7 & 0.00359 & .02624 & .1682 & 0.940 & 4.60 & 20.5 \\
\hline 8 & 0.00168 & .01392 & .1022 & 0.675 & 3.91 & 20.4 \\
\hline - & 0.00079 & 0.00733 & 0.0615 & 0.463 & 3.11 & 18.8 \\
\hline
\end{tabular}

Derivutives with respect to $(\log a=n)$ of $\alpha^{n} D_{\alpha}^{n} b_{?}^{(?)}$.

\begin{tabular}{|c|c|c|c|c|c|}
\hline$i$ & $\begin{array}{l}n=0 \\
D_{\mathbf{v}} b \varphi\end{array}$ & $\begin{array}{c}1 \\
D_{\mathrm{v}}\left(a D_{a} b_{i}^{(0)}\right)\end{array}$ & $\stackrel{2}{D_{\mathbf{v}}\left(\mathrm{a}^{2} D_{\alpha}^{2} b_{\varphi}^{(i)}\right)}$ & $\begin{array}{c}3 \\
D_{v}\left(a^{2} D_{a}^{a} b^{(!)}\right)\end{array}$ & $\stackrel{4}{D_{\mathbf{v}}: a^{4} D_{a^{4}}^{4} b^{\prime 9}}$ \\
\hline 0 & 0.33969 & 0.9275 & 2.257 & 6.68 & - 27.4 \\
\hline 1 & .68314 & 1.1821 & 2.175 & 6.93 & 27.4 \\
\hline 2 & .47198 & 1.2116 & 2.631 & 7.05 & 28.3 \\
\hline 3 & .28491 & 0.9972 & 2.888 & 8.07 & 29.2 \\
\hline 4 & .16270 & 0.7259 & 2.722 & 9.09 & 32.3 \\
\hline 5 & .09010 & 0.4899 & 2.285 & 9.33 & 36.4 \\
\hline 6 & .04896 & 0.3143 & 1.762 & 8.67 & 39.0 \\
\hline 7 & .02624 & 0.1944 & 1.276 & 7.41 & 38.9 \\
\hline 8 & .01392 & 0.1161 & 0.879 & 5.93 & 36.0 \\
\hline 9 & 0.00733 & 0.0688 & 0.586 & 4.50 & 31.2 \\
\hline
\end{tabular}

Second derivatives.

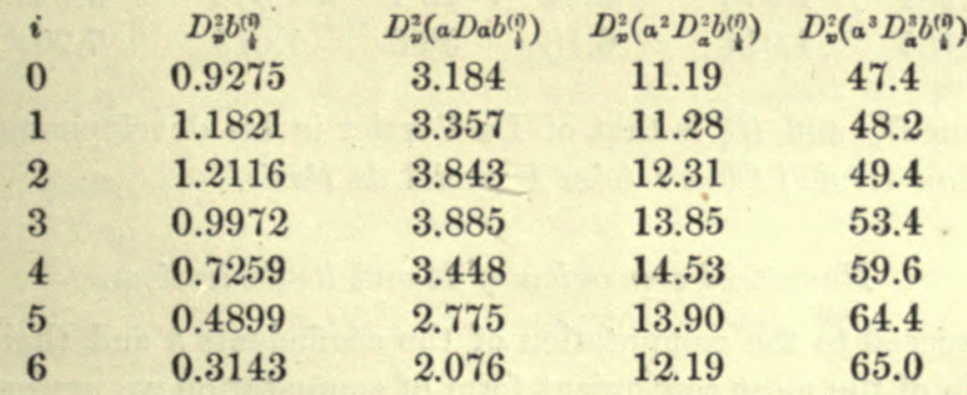

Vulues of $\alpha^{n+1} D_{a}^{n} b^{(0)}$

\begin{tabular}{|c|c|c|c|}
\hline$a b_{\frac{1}{2}}^{10}$ & $a^{2} D_{a} b_{\frac{1}{2}}^{(i)}$ & $a^{3} D_{a}^{2} b_{\frac{1}{2}}^{(0)}$ & $a^{4} D_{a}^{3} b_{\frac{9}{2}}$ \\
\hline 1.865 & 2.674 & 8.104 & 30.8 \\
\hline 1.267 & 2.844 & 7.77 & 30.8 \\
\hline 0.761 & 2.412 & 7.63 & 29.9 \\
\hline 0.433 & 1.790 & 6.92 & 28.7 \\
\hline 0.240 & 1.224 & 5.73 & 26.8 \\
\hline 0.130 & 0.792 & 4.41 & 23.5 \\
\hline 0.070 & 0.493 & 3.20 & 19.6 \\
\hline
\end{tabular}


Derivatives with respect to $\left(\log a=x_{\text {. }}\right)$

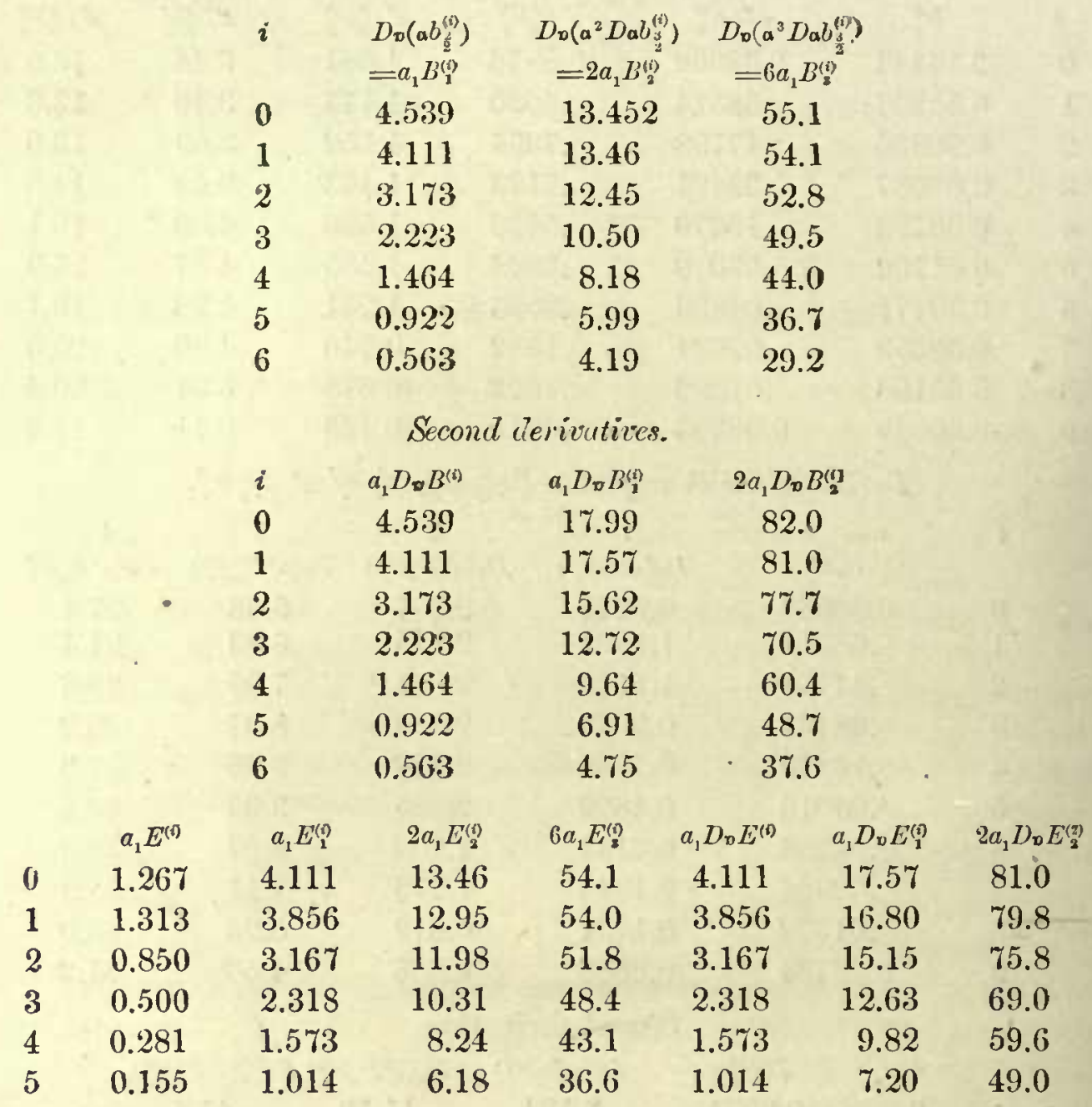

The notation $B_{n}^{(i)}$ and $E_{n}^{(i)}$ is that of Le Verrier in his development in the first volume of "Annales de l'Observataire Imperial de Puris."

Numericul expression of $R$ and its derivatives.

We next proceed to the computation of the coefficients $h$ and their derivatives. As an example of the most convenient form of computation we present in full that of the coefficient of $\frac{m^{\prime}}{a_{1}} \cos \left(i \lambda^{\prime}-(i-1) \lambda-\omega\right)$ in the expression of $R$ for the action of Saturn on Uranus. In this computation I use the tables given by Le Verrier in his "Annales de l'Observatoire," tome i, pages 358-383, comparing the development with that of Professor Peirce in the Astronomical Journal, vol. i, as a control. 


\begin{tabular}{|c|c|c|c|c|c|c|c|c|c|c|}
\hline & & & & $j=0$; & $j=1$ & $\cdot$ & & & & \\
\hline$i$ & -3 & -2 & -1 & 0 & +1 & 2 & 3 & 4 & 5 & 6 \\
\hline$i^{\prime}$ & +4 & 3 & 2 & 1 & 0 & -1 & -2 & -3 & -4 & 5 \\
\hline$(0) \times b(i)$ & +0.5212 & +0.8334 & +1.1041 & 0 & -1.10414 & -0.83344 & -0.5212 & -0.3034 & -0.1702 & -0.0929 \\
\hline (1) $\times a D_{a} b(9)$ & -0.2849 & -0.4720 & -0.6831 & -0.3397 & -0.68314 & -0.47198 & -0.2849 & -0.1627 & -0.0901 & -0.0490 \\
\hline$\Delta a_{1}(50)(i)$ & $\cdots \cdots$ & ...... & -16.1775 & $\ldots \ldots$ & -..... & $\cdots \cdots$ & $\ldots \ldots$ & $\ldots \ldots$ & ....... & $\ldots \ldots$ \\
\hline$a_{1}(50)(n)$ & +0.2363 & +0.3614 & -15.7565 & -0.3397 & -0.78728 & -1.30542 & -0.8061 & -0.4661 & -0.2603 & -0.1419 \\
\hline$(0) b(?)$ & -13.55 & -11.25 & -5.52 & 0.00 & 0.00 & +2.92 & +5.73 & +6.83 & +6.46 & \\
\hline (1) $\times a D_{a}$ & +8.54 & +7.78 & +4.78 & +0.51 & 0.00 & +1.18 & +2.56 & +3.25 & +3.06 & \\
\hline (2) $\times a^{2} D a$ & +1.43 & +0.74 & -0.00 & -0.59 & -1.00 & -2.22 & -2.85 & -2.82 & -2.40 & \\
\hline (3) $\times a^{3} D^{4}$ & -0.73 & -0.58 & -0.59 & -0.54 & -0.59 & -0.58 & -0.73 & -0.80 & -0.74 & \\
\hline$\Delta a_{1}(51)$ & $\ldots \ldots$ & $\ldots \ldots$ & +48.53 & $\ldots \ldots$ & -..... & $\ldots \ldots$ & $\cdots \cdots$ & $\cdots \cdots$ & $\ldots \ldots$ & \\
\hline$a_{1}(51)$ & -4.31 & -3.31 & +47.20 & -0.62 & -1.59 & +1.30 & +4.71 & +6.46 & +6.38 & \\
\hline$(0) \times b_{t}^{(i)}$ & -18.76 & -13.33 & -4.42 & 0.00 & +4.42 & +13.33 & +18.76 & +19.41 & +17.01 & \\
\hline (1) $\times a D_{a}$ & +13.10 & +10.38 & +4.10 & -0.68 & -1.37 & +2.83 & +6.27 & +7.48 & +7.02 & \\
\hline (2) $\times a^{2} D^{2} a$ & +1.43 & 0.00 & -1.00 & -2.35 & -3.00 & -5.92 & -7.13 & -6.76 & -5.60 & \\
\hline (3) $\times a^{3} D^{3} a$ & -1.46 & -1.15 & -1.18 & -1.08 & -1.18 & -1.15 & -1.46 & -1.60 & -1.48 & \\
\hline$\Delta a_{1}(52)$ & $\ldots \ldots$ & $\ldots \ldots$. & +32.36 & $\ldots \ldots$ & $\ldots . .$. & ....... & $\ldots \ldots$ & $\ldots . .$. & ...... & \\
\hline$a_{1}(52)$ & -5.69 & -4.10 & +29.86 & -4.11 & -1.13 & +9.09 & +16.44 & +18.53 & +16.95 & \\
\hline$(0) \times a E_{1}^{(i)}$ & -3.00 & -3.40 & -2.63 & 0.00 & +2.63 & +340 & +3.00 & +2.25 & +1.55 & \\
\hline (1) $\times a_{1} E_{1}(\theta)$ & +2.32 & +3.17 & +3.86 & +4.11 & +3.86 & +3.17 & +2.32 & +1.57 & +1.01 & \\
\hline$\Delta a_{1}(60)$ & 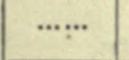 & $\ldots \ldots$ & +16.18 & $\ldots \ldots$ & ....... & $\ldots \ldots$ & ....... & $\ldots \ldots$ & ....... & \\
\hline$a_{1}(60)$ & -0.68 & -0.23 & +17.41 & +4.11 & +6.49 & +6.57 & +5.32 & +3.82 & +2.56 & \\
\hline $1 \cdot \times(50)$ & $+6.61^{1}$ & +10.12 & -441.22 & -9.51 & -50.049 & -36.556 & -22.573 & -13.05 & -7.28 & -3.97 \\
\hline$\frac{1}{4} e^{3} \times(51)$ & -0.09 & -0.07 & +1.04 & -0.01 & -0.035 & +0.028 & +0.103 & +0.14 & +0.14 & \\
\hline$\frac{1}{3} e e^{n} \times(52)$ & -0.08 & -0.06 & +0.45 & -0.06 & -0.017 & +0.138 & +0.248 & +0.28 & +0.26 & \\
\hline$\frac{1}{2} e c^{2} \times(60)$ & -0.05 & -0.02 & +0.14 & +0.03 & +0.051 & +0.052 & +0.043 & +0.03 & +0.02 & \\
\hline $\boldsymbol{h}$ & +6.39 & +9.97 & -439.59 & -955 & -50.050 & -36.338 & -22179 & -12.60 & -6.86 & -3.60 \\
\hline
\end{tabular}

The derivatives of $h$ with respect to (log. $a=n$ ) are computed in precisely the same way by simply substituting for $b_{i}^{(i)}, \alpha D \alpha b_{i}^{(i)}$, etc., their derivatives with respect to $x$ as given in the above table of constants.

The quantities $\Delta x_{1}(50)^{(i)}$, etc., which appear in the third series of terms above express that part of the perturbations of Uranus caused by the action of Saturn

In units of the third place of decimals. 
on the sun. They are each, of the form $N \times \alpha^{-2}, N$ being a numerical coefficient given by Le Verrier under the coefficient for each term. 'The derivative of this expression with respect to $n$ is $-2 N \times \alpha^{-2}$, so that for the corresponding terms in $D_{x} h$ and $D_{v}^{2} h$ we have

$$
\begin{aligned}
& \Delta D_{\imath} h=-2 \Delta h \\
& \Delta D_{v}^{2} h=+4 \Delta h
\end{aligned}
$$

The values of $h$ and its derivatives, corresponding to any one argument $i$ and $i$, are to be combined into two terms depending the one on the cosine, the other on the sine of the argument. Let us represent by $g$ the mean anomaly of Uranus, and let us put $l^{\prime}$ for the mean longitude of Saturn counted from the perihclion of Uranus, or, more exactly, for the arc $\lambda^{\prime}-\omega$. Put also

$$
\begin{aligned}
& N=i g+i^{\prime} l^{\prime}, \\
& i+i^{\prime}+j=j^{\prime \prime}, \\
& P=j \omega+j^{\prime} \omega^{\prime}, \\
& P^{\prime}=j^{\prime \prime} \omega+j^{\prime} \omega^{\prime} .
\end{aligned}
$$

'Then, for each value of $N$ there will be several values of $P$ corresponding to different powers and products of the eccentricities and inclinations in $h$. Distinguishing these values and the corresponding values of $h$ by subscript numerals, we shall have a series of terms of $R$ of the following form-

$$
R=\frac{m}{a_{1}}\left\{\begin{array}{c}
h_{1} \cos \left(N+P_{1}^{\prime}\right) \\
+h_{2} \cos \left(N+P_{2}^{\prime}\right) \\
+h_{3} \cos \left(N+P_{3}^{\prime}\right) \\
+ \text { etc. } \quad \text { etc. }
\end{array}\right\}
$$

and by putting

$$
\begin{aligned}
& h_{c}=h_{1} \cos P_{1}^{\prime}+h_{2} \cos P_{2}^{\prime}+h_{3} \cos P_{3}^{\prime}+\text { etc. } \\
& h_{s}=-h_{1} \sin P_{1}^{\prime}-h_{2} \sin P_{2}^{\prime}-h_{3} \sin P_{3}^{\prime}-\text { etc. }
\end{aligned}
$$

The above terms may be condensed into

$$
R=\frac{m}{a_{1}} h_{c} \cos N+\frac{m}{a_{1}} h_{s} \sin N,
$$

which are of the form supposed in the preceding theory.

In order that the derivative of $R$, with respect to the true longitude of Uranus, may be expressed in the form

we must, by (7), put

$$
\frac{\partial R}{\partial \mathrm{v}}=\frac{m}{a_{1}} v_{\mathrm{s}} \sin N+\frac{m}{a_{1}} v_{\mathrm{c}} \cos N
$$

$$
\begin{aligned}
& v_{s}=-\left(i+j_{1}\right) h_{1} \cos P_{1}^{\prime}-\left(i+j_{2}\right) h_{2} \cos P_{2}^{\prime}-\text { etc. } \\
& v_{c}=-\left(i+j_{1}\right) h_{1} \sin P_{1}^{\prime}-\left(i+j_{2}\right) h_{2} \sin P_{2}^{\prime}-\text { etc. }
\end{aligned}
$$

$j_{1}, j_{2}$, representing the several values of $j$ in the different terms which correspond to one and the same set of values of $i$ and $i$. 
To obtain the derivative with respect to $\gamma$ we notice that all the appreciable terms in the different values of $h$, which depend upon the mutual inclination, are of the form

$$
h=\sigma^{2} A,
$$

where $\sigma=\sin \frac{1}{2} \gamma$. These equations give

\section{Consequently}

$$
\frac{\partial h}{\partial \gamma}=\frac{\partial h}{\partial \sigma} \frac{\partial \sigma}{\partial \gamma}=A \sigma \cos \frac{\gamma}{2} \gamma=\frac{1}{2} A \sin \gamma
$$

$$
\frac{\partial R}{\partial \gamma}=\frac{1}{2} A \sin \gamma \cos \left(N+P^{\prime}\right)
$$

and the various terms depending on the same argument $(i, i)$ may be condensed into two, exactly as in the case of $R$ itself.

The different co-efficients $h$ and $D_{i} h$, computed in the way already described, are given in extenso in the following table. At the top of each individual column is given the value of $P$, or of $j \omega+j^{\prime} \omega^{\prime}$, corresponding to the values of $h$ below, and immediately under $P$ is given its modified value, or $P^{\prime}$, to be used in condensing the terms, putting for brevity

$$
(\omega)=\omega-\omega^{\prime} .
$$

$P$ and $P^{\prime}$ are therefore regarded as constant angles the numerical values of the sines and cosines of which may be obtained from the values of $\omega$ and $\omega^{\prime}$ already given.

The condensed $h_{c}$ and $h_{s}$ are given in the two right hand columns.

All the numbers are given in units of the third place of decimals.

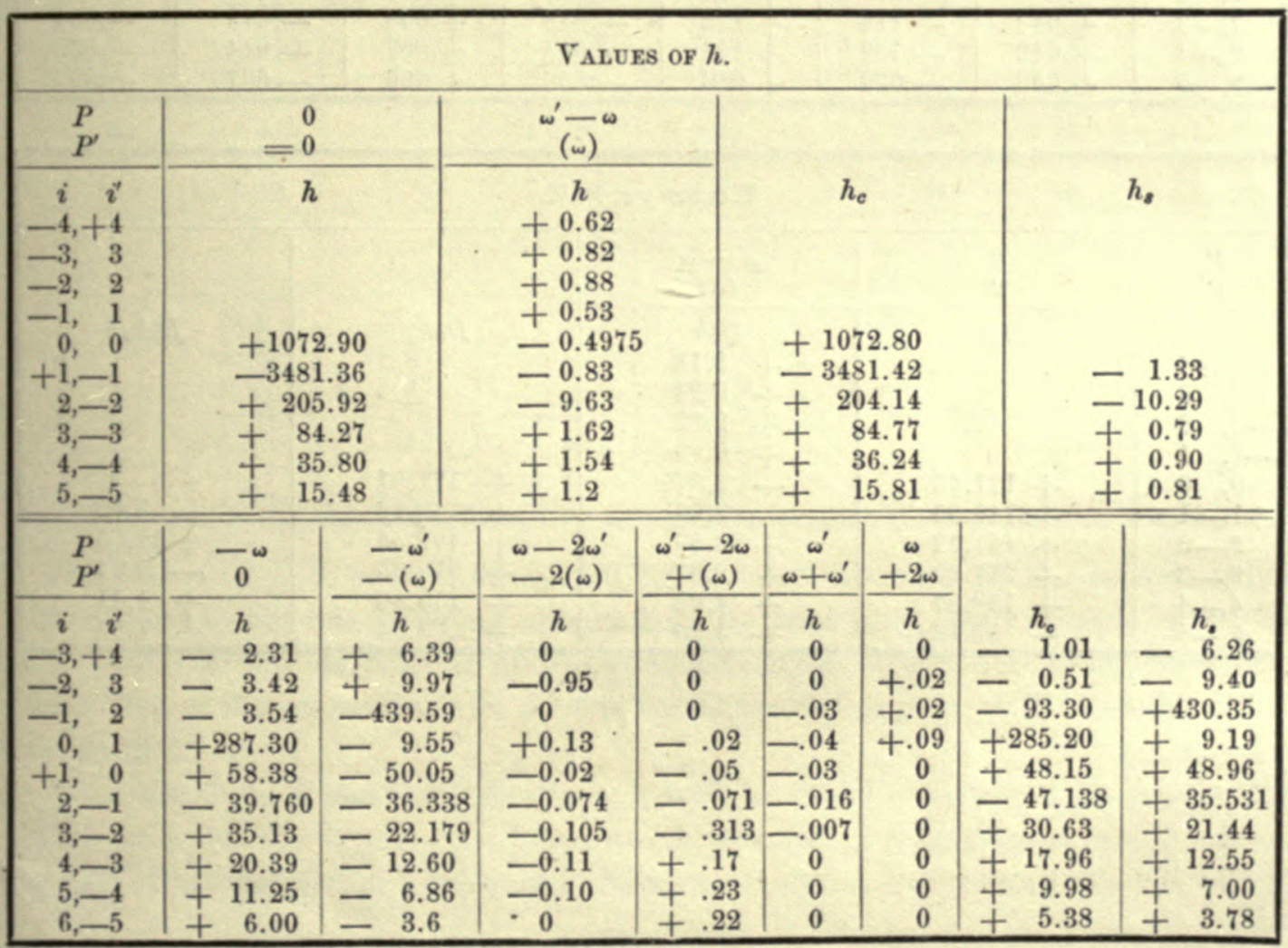


VALUES OF $h$.

\begin{tabular}{|c|c|c|c|c|c|c|c|c|c|}
\hline $\begin{array}{l}P \\
P^{\prime}\end{array}$ & $\begin{array}{c}-2 \omega \\
0\end{array}$ & \multicolumn{2}{|c|}{$\frac{-\omega^{\prime}-\omega}{-(\omega)}$} & \multicolumn{2}{|c|}{$\begin{array}{r}-2 \omega^{\prime} \\
-2(\omega)\end{array}$} & \multicolumn{2}{|l|}{$\begin{array}{r}0 \\
+2 \omega \\
\end{array}$} & \multirow[b]{2}{*}{$\begin{array}{l}h_{c} \\
+38.72 \\
+\quad 6.58 \\
+0.03 \\
+\quad 0.32 \\
+1.912 \\
+1.44 \\
+1.13 \\
+0.75 \\
+\quad 0.49 \\
\end{array}$} & \multirow[b]{2}{*}{$\begin{aligned} & h_{s} \\
&+17.03 \\
&+31.15 \\
&+ 2.15 \\
&+ 4.14 \\
&+ 5.216 \\
&+ 4.576 \\
&+ 3.39 \\
&+ 2.31 \\
&+ 1.49\end{aligned}$} \\
\hline $\begin{array}{rr}i & i^{\prime} \\
-1, & 3 \\
0, & 2 \\
+1, & 1 \\
2, & 0 \\
3,-1 \\
4,-2 \\
5,-3 \\
6,-4 \\
7,-5 \\
\end{array}$ & $\begin{array}{c}h \\
+0.04 \\
+0.06 \\
+0.74 \\
+3.09 \\
+1.268 \\
+4.16 \\
+3.13 \\
+2.11 \\
+1.35\end{array}$ & \multicolumn{2}{|c|}{$\begin{array}{l}h \\
-0.27 \\
+31.65 \\
-0.83 \\
-5.19 \\
-6.290 \\
-5.458 \\
-4.03 \\
-2.74 \\
-1.76 \\
\end{array}$} & $\begin{array}{l}-4 \\
- \\
+ \\
+ \\
+ \\
+ \\
+\end{array}$ & $\begin{array}{l}h \\
42.24 \\
0.04 \\
1.08 \\
1.89 \\
2.082 \\
1.767 \\
1.29 \\
0.87 \\
0.55 \\
\end{array}$ & \multicolumn{2}{|c|}{$\begin{array}{c}h \\
+0.11 \\
+0.18 \\
+0.91 \\
+0.18 \\
+0.111 \\
+0.061 \\
+0.04 \\
+0.02 \\
+0.01\end{array}$} & & \\
\hline $\begin{array}{l}P \\
P^{\prime} \\
\end{array}$ & $\begin{array}{c}-3 \omega \\
0\end{array}$ & $\begin{array}{c}-\omega^{\prime}-2 \omega \\
-(\omega)\end{array}$ & -2 & & $\begin{array}{r}-3 \omega^{\prime} \\
-3(\omega)\end{array}$ & \multicolumn{2}{|c|}{\begin{tabular}{c|c}
$-\omega^{\prime}$ & $-\omega$ \\
$3 \omega-\omega^{\prime}$ & $2 \omega$ \\
\end{tabular}} & \multirow[b]{2}{*}{$\begin{array}{c}h_{\mathrm{c}} \\
+.17 \\
0 \\
-.15 \\
-.325 \\
-.176 \\
-.145 \\
-.107 \\
-.083\end{array}$} & \multirow[b]{2}{*}{$\begin{array}{r}h_{s} \\
+.06 \\
+.04 \\
+.213 \\
+.416 \\
+.490 \\
+.460 \\
+.379 \\
+.289\end{array}$} \\
\hline $\begin{array}{r}i \quad i^{\prime} \\
+1,+2 \\
2, \quad 1 \\
3, \quad 0 \\
4,-1 \\
5,-2 \\
6,-3 \\
7,-4 \\
8,-5\end{array}$ & $\begin{aligned} & h \\
& 0 \\
&+.01 \\
&+.18 \\
&+.222 \\
&+.424 \\
&+.398 \\
&+.327 \\
&+.24 \\
&\end{aligned}$ & $\begin{array}{c}h \\
-.12 \\
-.07 \\
-.46 \\
-.760 \\
-.847 \\
-.773 \\
-.627 \\
-.47 \\
\end{array}$ & & & $\begin{array}{c}h \\
-.11 \\
-.02 \\
-.08 \\
-.117 \\
-.122 \\
-.107 \\
-.086 \\
-.06\end{array}$ & $\begin{array}{c}h \\
-.14 \\
-.02 \\
-.02 \\
-.016 \\
-.012 \\
-.008 \\
-.005 \\
0\end{array}$ & $\begin{array}{c}h \\
+.02 \\
+.01 \\
+.04 \\
+.029 \\
+.021 \\
+.014 \\
+.009 \\
0\end{array}$ & & \\
\hline $\begin{array}{l}A \\
P^{\prime}\end{array}$ & $\begin{array}{c}-4 \omega \\
0\end{array}$ & $\begin{array}{c}-\omega^{\prime}-3 \omega \\
-(\omega) \\
\end{array}$ & -2 & $\begin{array}{l}-2 \omega \\
0)\end{array}$ & $\begin{array}{l}-3 \omega^{\prime}-\omega \\
-3(\omega)\end{array}$ & - & $\begin{array}{l}-4 \omega^{\prime} \\
4(\omega)\end{array}$ & & \\
\hline $\begin{array}{l}i \quad i^{\prime} \\
4, \quad 0 \\
5,-1 \\
6,-2 \\
7,-3 \\
8,-4 \\
9,-5\end{array}$ & $\begin{array}{c}h \\
0 \\
+.02 \\
+.039 \\
+.043 \\
+.042 \\
+.035\end{array}$ & $\begin{array}{c}h \\
-.04 \\
-.08 \\
-.105 \\
-.114 \\
-.106 \\
-.090\end{array}$ & $\begin{array}{l}+ \\
+ \\
+ \\
+ \\
+ \\
+\end{array}$ & & $\begin{array}{c}h \\
-.02 \\
-.04 \\
-.047 \\
-.048 \\
-.044 \\
-.036\end{array}$ & $\begin{array}{r} \\
+ \\
+. \\
+. \\
+. \\
+ \\
+.\end{array}$ & $\begin{array}{l}h \\
0 \\
.01 \\
.008 \\
.008 \\
.007 \\
.006\end{array}$ & $\begin{array}{c}h_{s} \\
-.04 \\
-.03 \\
-.045 \\
-.049 \\
-.044 \\
-.037\end{array}$ & $\begin{array}{c}h_{c} \\
0 \\
+.003 \\
+.028 \\
+.034 \\
+.032 \\
+.029\end{array}$ \\
\hline
\end{tabular}

VALUes of $D_{\mathfrak{D}} h$.

\begin{tabular}{|c|c|c|c|c|}
\hline $\begin{array}{l}P \\
P^{\prime} \\
\end{array}$ & $\begin{array}{l}0 \\
0 \\
\end{array}$ & $\omega^{\prime}-\omega$ & & 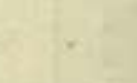 \\
\hline$i \quad i^{y}$ & $D_{\mathfrak{D}} h$ & $D_{v} h$ & $D_{w} h_{c}$ & $D_{\mathfrak{o}} h_{\mathfrak{s}}$ \\
\hline$-4,+4$ & & $\begin{array}{r}+3.18 \\
+\end{array}$ & & \\
\hline $\begin{array}{l}-2, \quad 2 \\
-2\end{array}$ & & $\begin{array}{r}+\quad 2.39 \\
+\quad 2.39\end{array}$ & & \\
\hline$-1, \quad 1$ & & $+\quad 0.40$ & " & \\
\hline $0, \quad 0$ & +171.93 & $-\quad 2.075$ & +171.51 & \\
\hline$+1,-1$ & +8749.59 & & +8749.12 & - 3.02 \\
\hline $\begin{array}{l}2,-2 \\
3-3\end{array}$ & $\begin{array}{r}+\quad 467.72 \\
+\quad 277.00\end{array}$ & $\begin{array}{r}+20.83 \\
+\quad 287\end{array}$ & $\begin{array}{r}+\quad 472.46 \\
+\quad 278.25\end{array}$ & $\begin{array}{r}18.05 \\
\end{array}$ \\
\hline $\begin{array}{l}3,-3 \\
4,-4\end{array}$ & $\begin{array}{r}+27.00 \\
+\quad 153.87\end{array}$ & $\begin{array}{r}+81 \\
+\quad 4.62\end{array}$ & $\begin{array}{r}213.20 \\
+\quad 155.46\end{array}$ & $+\quad 1.41$ \\
\hline $5,-5$ & $\begin{array}{r}82.12 \\
+\quad 8\end{array}$ & $\begin{array}{r}4.90 \\
+\quad 4\end{array}$ & $\begin{array}{r}1 \\
+\quad 83.54\end{array}$ & $\begin{array}{r}1.80 \\
+\quad 1.0\end{array}$ \\
\hline
\end{tabular}




\begin{tabular}{|c|c|c|c|c|c|c|c|c|}
\hline \multicolumn{9}{|c|}{ VALUES OF $L_{\mathrm{v}} h$. } \\
\hline $\begin{array}{l}P \\
P^{\prime}\end{array}$ & $-\omega$ & $\begin{array}{l}-\omega^{\prime} \\
-(\omega)\end{array}$ & $\begin{array}{l}\omega-2 \omega \\
-2(\omega) \\
\end{array}$ & $\begin{array}{c}\omega-2 \omega \\
+(\omega) \\
\end{array}$ & $\begin{array}{l}+\infty \\
++\infty \\
+\infty\end{array}$ & $2 \omega$ & & \\
\hline $\begin{array}{r}i \\
i \\
-3,+4 \\
-2, \quad 3 \\
-1, \quad 2 \\
-0, \quad 1 \\
1, \quad 0 \\
2,-1 \\
3,-2 \\
4,-3 \\
5,-4 \\
6,-5 \\
\end{array}$ & $\begin{aligned} & D_{\text {v } h} \\
&-\quad 9.28 \\
&-\quad 9.74 \\
&-\quad 4.63 \\
&-556.40 \\
&+\quad 30.16 \\
&+265.17 \\
&+83.10 \\
&+\quad 68.34 \\
&+49.04 \\
&+32.1 \\
&\end{aligned}$ & 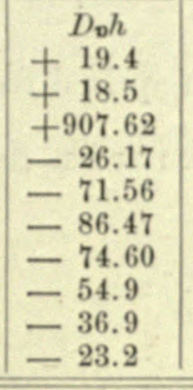 & $\begin{array}{c}D_{\mathrm{v}} h \\
0 \\
+2.05 \\
+.05 \\
-.10 \\
+.04 \\
-.19 \\
-.42 \\
-.55 \\
-.58 \\
-.53 \\
\end{array}$ & $\begin{array}{c}D_{\mathrm{v} h} \\
0 \\
-.06 \\
-.08 \\
-.09 \\
-.19 \\
-.26 \\
+.63 \\
+.29 \\
+.68 \\
+.90\end{array}$ & $\begin{array}{r}D_{\text {v } h} \\
0 \\
-.12 \\
-.13 \\
-.14 \\
-.11 \\
-.07 \\
-.04 \\
-.02 \\
-.01 \\
0\end{array}$ & $\begin{array}{r}D_{\text {v } h} \\
0 \\
+.08 \\
+.07 \\
+.12 \\
+.02 \\
0 \\
0 \\
0 \\
0 \\
0 \\
\end{array}$ & $\begin{array}{c}D_{v} h_{\circ} \\
-\quad 5.32 \\
-\quad 7.97 \\
+180.44 \\
-561.82 \\
+\quad 15.37 \\
+247.58 \\
+68.34 \\
+57.70 \\
+42.19 \\
+28.1 \\
\end{array}$ & $\begin{aligned} & D_{\text {v } h} \\
&- 19.00 \\
&- 17.43 \\
&- 888.65 \\
&+ 25.60 \\
&+ 69.86 \\
&+ 84.30 \\
&+ 73.49 \\
&+ 53.9 \\
&+ 36.7 \\
&+ 23.4 \\
&\end{aligned}$ \\
\hline $\begin{array}{l}P \\
P\end{array}$ & $\begin{array}{c}-2 \omega \\
0\end{array}$ & $\begin{array}{r}-\omega-\omega \\
-(\omega)\end{array}$ & -2 & & $\begin{array}{c}0 \\
+2 \omega \\
\end{array}$ & & & \\
\hline $\begin{array}{r}i \\
-1,+3 \\
0, \quad 2 \\
+1, \quad 1 \\
2, \quad 0 \\
3, \quad 1 \\
4,-2 \\
5,-3 \\
6,-4 \\
7,-5 \\
\end{array}$ & $\begin{array}{l}D_{\mathrm{v} h} \\
+0.16 \\
+0.32 \\
+\quad 3.27 \\
+\quad 2.56 \\
+13.745 \\
+10.25 \\
+10.76 \\
+9.39 \\
+7.3 \\
\end{array}$ & $\begin{array}{r}D_{\text {n }} h \\
-0.6 \\
-64.5 \\
-2.7 \\
-8.7 \\
-15.8 \\
-18.9 \\
-18.0 \\
-15.0 \\
-11.5 \\
\end{array}$ & $\begin{array}{l}D \\
+8 \\
+ \\
+ \\
+ \\
+ \\
+ \\
+ \\
+ \\
+\end{array}$ & $\begin{array}{l}\text { vh } \\
36.02 \\
0.15 \\
4.69 \\
5.00 \\
7.232 \\
7.85 \\
6.99 \\
5.59 \\
4.21 \\
\end{array}$ & $\begin{array}{c}D_{\mathrm{v}} h \\
+0.47 \\
+0.60 \\
+3.02 \\
+0.60 \\
+0.463 \\
+0.32 \\
+0.21 \\
+0.13 \\
+0.09 \\
\end{array}$ & & $\begin{array}{l}D_{\mathrm{v}} h_{\mathrm{e}} \\
-78.76 \\
-12.92 \\
-1.22 \\
-1.74 \\
+3.933 \\
+0.77 \\
+0.69 \\
+1.22 \\
+1.2 \\
\end{array}$ & $\begin{array}{c}D_{\text {v }}, \\
-34.19 \\
+62.54 \\
+2.26 \\
+6.00 \\
+12.153 \\
+15.07 \\
+14.68 \\
+12.4 \\
+9.6\end{array}$ \\
\hline $\begin{array}{l}P \\
P^{\prime}\end{array}$ & $\begin{array}{c}-3 \omega \\
0\end{array}$ & $\begin{array}{c}-2 \omega-\omega^{\prime} \\
-(\omega)\end{array}$ & $\begin{array}{c}-\omega-2 \omega^{\prime} \\
-2(\omega)\end{array}$ & $\begin{array}{l}-3 \omega^{\prime} \\
-3(\omega)\end{array}$ & $\left|\begin{array}{c}-\omega^{\prime} \\
3 \omega-\omega^{\prime}\end{array}\right|$ & $\begin{array}{l}-\omega \\
+2 \omega \\
\end{array}$ & . & \\
\hline $\begin{array}{r}i \\
i \\
+1,+2 \\
2, \quad 1 \\
3, \quad 0 \\
4,-1 \\
5,-2 \\
6,-3 \\
7,-4 \\
8,-5 \\
\end{array}$ & $\begin{array}{c}D_{\mathrm{v} h} \\
+0.03 \\
+0.12 \\
+0.21 \\
+0.89 \\
+1.08 \\
+1.40 \\
+1.46 \\
+1.32 \\
\end{array}$ & $\begin{array}{c}D_{\mathrm{v} h} h \\
+0.18 \\
-0.26 \\
-0.89 \\
-2.02 \\
-3.00 \\
-3.47 \\
-3.44 \\
-3.00 \\
\end{array}$ & $\begin{array}{c}D_{\mathrm{v} h} \\
+0.05 \\
+0.33 \\
+0.98 \\
+1.89 \\
+2.53 \\
+2.73 \\
+2.60 \\
+2.20 \\
\end{array}$ & $\begin{array}{c}D \text { D } h \\
+0.22 \\
-0.09 \\
-0.31 \\
-0.54 \\
-0.67 \\
-0.69 \\
-0.62 \\
-0.51\end{array}$ & $\begin{array}{c}D_{\text {v } h} \\
+.22 \\
-.08 \\
-.09 \\
-.09 \\
-.08 \\
-.07 \\
-.05 \\
0\end{array}$ & $\begin{array}{l}D \text { vh } \\
+.08 \\
+.17 \\
+.13 \\
+.13 \\
+.11 \\
+.09 \\
+.06 \\
+.04 \\
\end{array}$ & $\begin{array}{r}D_{\mathrm{p}} h_{\mathrm{c}} \\
-0.31 \\
-0.08 \\
-0.59 \\
-0.84 \\
-1.38 \\
-1.33 \\
-1.20 \\
-0.99 \\
\end{array}$ & $\begin{array}{r}D_{n} h \\
-0.17 \\
-0.09 \\
+0.13 \\
+0.69 \\
+1.30 \\
+1.68 \\
+1.78 \\
+1.62 \\
\end{array}$ \\
\hline $\begin{array}{l}P \\
P^{\prime}\end{array}$ & $\begin{array}{c}-4 \omega \\
0\end{array}$ & $-3 \omega-\omega^{\prime}$ & $\begin{array}{c}-2 \omega-2 \omega^{\circ} \\
-2(\omega) \\
\end{array}$ & $\begin{array}{l}-\omega-3 \omega^{\prime} \\
-3(\omega) \\
\end{array}$ & $\begin{array}{l}-4 \\
-4 \\
\end{array}$ & & & \\
\hline $\begin{array}{l}i \quad i^{7} \\
4, \quad 0 \\
5,-1 \\
6,-2 \\
7,-3 \\
8,-4 \\
9,-5 \\
\end{array}$ & $\begin{array}{r}D \text { Dh } \\
+.02 \\
+.05 \\
+.10 \\
+.15 \\
+.18 \\
+.19 \\
\end{array}$ & $\begin{array}{r}D_{\mathrm{v} h} \\
-.09 \\
-.22 \\
-.38 \\
-.52 \\
-.59 \\
-.59 \\
\end{array}$ & $\begin{array}{r}D \text { nh } \\
+.13 \\
+.30 \\
+.48 \\
+.62 \\
+.66 \\
+.65 \\
\end{array}$ & $\begin{array}{r}D_{\mathrm{p} h} \\
-.08 \\
-.18 \\
-.26 \\
-.32 \\
-.33 \\
-.31 \\
\end{array}$ & $\begin{array}{l}D \\
+. \\
+. \\
+. \\
+. \\
+. \\
+.\end{array}$ & $\begin{array}{l}\text { oh } \\
02 \\
04 \\
05 \\
06 \\
06 \\
05\end{array}$ & $\begin{array}{r}D_{\mathrm{v}} h_{\mathrm{e}} \\
-.06 \\
-.14 \\
-.24 \\
-.30 \\
-.32 \\
-.31 \\
\end{array}$ & $\begin{array}{l}D_{\mathrm{v}} h_{\mathrm{S}} \\
-.01 \\
-.02 \\
+.01 \\
+.04 \\
+.09 \\
+.11 \\
\end{array}$ \\
\hline
\end{tabular}

The values of $D_{i} h$, needed in computing the perturbations of the second order with respect to the masses being obtained in the same way, by the simple substitution of the second derivatives of the functions $b, \alpha D \alpha b$, etc., for those functions themselves in the expressions for $h$, it is not necessary to present the details of the computation.

After obtaining $h$ and its derivatives, it will be found convenient to change the arrangement of the terms. Hitherto we have kept in one series those in which the sum of the indices are a constant. Now, we shall put together all those in which 
the index of the disturbing planet has the same value, arranging the individual terms of each series according to the index of the disturbed planet. Thus, the index of the product of any term, as $h \cos N$, by any multiple of the mean anomaly of the disturbed planet, as $j y$, will be found in the same series with that of $N$ itself, and $j$ lines above and below.

The next process will be the formations of the required functions of the mean anomaly of Uranus, $\frac{d v}{d t}, \frac{d \rho}{d t}, \frac{a^{2}}{r^{2}}, \log r$. Their values are as follows:-

$$
\begin{array}{cccc}
r_{1}^{-2}= & \rho_{0}=n & \frac{d \rho_{0}}{n d t}= & \frac{d v_{0}}{n d t}=1 \\
1.001103 & +.0005507 & & \\
+.093933 \cos g & -.0468889 \cos g & +.0468889 \sin g & +.0938294 \cos g \\
+.005507 \cos 2 g & -.0016494 \cos 2 g & +.0032988 \sin 2 g & +.0055012 \cos 2 g \\
+.000336 \cos 3 g & -.0000732 \cos 3 g & +.0002196 \sin 3 g & +.0003357 \cos 3 g \\
+.000020 \cos 4 g & -.0000035 \cos 4 g & +.0000142 \sin 4 g & +.0000206 \cos 4 g
\end{array}
$$

Considering only those tcrms which are of the first order, the value of $D_{i}^{\prime} R$ may be found in two ways, the agreement of which will afford a clieck upon the entire development of the perturbative function, and upon the computations of $R$ and $\frac{\partial R}{\partial v}$. These are (1) by direct differentiation, with respect to the time as con. tained in the mean anomaly of a single planet, whereby each term in $R$ of tho form

$$
R=\frac{m}{a_{1}} h \cos N
$$

will produce in $D_{t}^{\prime} R$ the term

$$
D_{t}^{\prime} R=-\frac{m}{a_{1}} i n h \sin N
$$

and (2) by forming the expression

$$
D_{t}^{\prime} R=\frac{\partial R}{\partial \mathrm{v}} \frac{d v_{0}}{d t}+\frac{\partial R}{\partial \rho} \frac{d \rho_{0}}{d t}
$$

As several "mechanical multiplications," like those indicated in this last expression, are to be performed, the following example of the form of computation is presented. It exhibits the formation of the product of those terms of $\frac{\partial R}{\partial \mathrm{v}}$ in which $i^{\prime}=-1$ by $\frac{d v_{0}}{d t}$.

$$
\begin{aligned}
& \begin{array}{lllllllll}
i^{\prime} & -2 & -1 & 0 & +1 & 2 & 3 & 4 & 5
\end{array} \\
& \frac{a_{1}}{m} \frac{\partial R}{\partial v}(\sin )+.02+1.63-287.42+3481.14+54.42+6.988+1.18+0.10 \\
& \times .046915\left\{\begin{array}{ccc}
0 & 0 & +08-13.48+163.32+2.553+0.33+0.06 \\
+.08 & -13.48+163.32+2.55+0.33+0.056 & 0
\end{array}\right. \\
& \times .002750\left\{\begin{array}{c}
0 \\
0 \\
-.79+9.58+0.15+0.00-0.79+9.576+0.15+.02
\end{array}\right.
\end{aligned}
$$

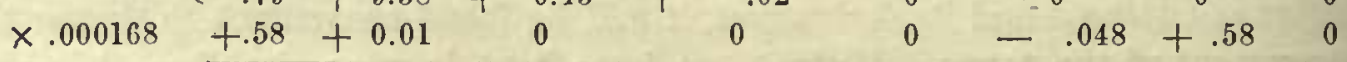

$$
\begin{aligned}
& \frac{a_{1}}{\eta^{\prime} n} \frac{\partial R}{\partial v} \frac{d v}{d t}(\mathrm{sin})-.11-2.26-123.87+3470.23+217.28+19.125+2.24+0.18
\end{aligned}
$$


The multipliers on the left are each one-lalf the coefficient $\cos j y$ in the expression for $\frac{d t_{0}}{d t}$, and each product is placed in the two columns corresponding respectively to $N+j g$ and $N-j y$.

All the derivations of $R_{0}$ necessary in the computation of the perturbations of the first order are giren in the following tables. First we have the values of $D_{i} R$ obtained by direct differentiation, as indicated in the preceding formulic. Next we have $\frac{\partial R}{\partial r}$ and $\frac{\partial R}{\partial \rho}$, obtained by the formulx (7) and (42). The products $\frac{\partial R}{\partial v}$ by $\frac{d x_{0}}{d t}$ and of $\frac{\partial R}{\partial \rho}$ by $\frac{\partial \rho}{d t}$, being formed in the simple way just pointed out, and with the values of the component factors just given, their sum is next slown. This sum should agree accurately with $\mathrm{D}_{b}^{\prime} R$. 'The discrepancies are shown in the next two columns. The only apparently large discrepancy is found in the argument $5 g^{\prime}-5$. It probably arises from the incompleteness of the computation of $R$ and $\frac{\partial R}{\partial r}$, so far as they depend on this argument. As the entire term does not amount to $0^{\prime \prime} .01, \mathrm{I}$ have not sought to correct it.

The great value of this clieck arises from the fact that it gives a complete control of the correctness of the development of the perturbative function, ab initio, since the two valves of $D_{i} R$ are derived from different terms of that development. It also controls all the computations except that of $\frac{\partial R}{\partial \rho}$. This quantity being multiplied by quantities of the order of the eccentricities in the second value of $D_{t} R$, an error in its value will produce a discrepancy of only $\frac{1}{40}$ its own amount in $D_{1}^{\prime} R$, and may therefore be orerlooked. The derivative in question must therefore be checked by a complete duplicate computation.

In the column next following are given the integrating factors $v$, for which the expression is

$$
v=\frac{n}{i^{\prime} n^{\prime}+i n}=\frac{1}{i+i \frac{n^{\prime}}{n}}
$$

For each value of $i$ the values of $v$ are therefore the reciprocals of a series of numbers in arithnetical progression, the common difference being unity. 


\begin{tabular}{|c|c|c|c|c|c|c|}
\hline \multirow[b]{2}{*}{$g \quad l^{\prime}$} & \multicolumn{2}{|c|}{$D_{i}^{\prime} R=\frac{m^{\prime}}{a_{1}} n \times$} & \multicolumn{2}{|c|}{$\frac{\partial R}{\partial \mathrm{v}}=\frac{m^{\prime}}{a_{1}} \times$} & \multicolumn{2}{|c|}{$\frac{\partial R}{\partial \rho}=\frac{m^{\prime}}{a_{1}} \times$} \\
\hline & $\sin$ & $\cos$ & $\sin$ & $\cos$ & $\cos$ & $\sin$ \\
\hline $0, \quad 0$ & 0 & 0 & . & $+\quad 0.487$ & -1244.31 & $\ldots$ \\
\hline 1 , & $-\quad 48.15$ & +48.96 & +10.20 & +49.07 & -63.52 & -118.82 \\
\hline 2 , & $-\quad 0.64$ & +8.28 & $+\quad 4.48$ & +3.20 & $+\quad 3.42$ & -10.14 \\
\hline 3 & $+\quad 0.45$ & $+\quad 0.64$ & 0.50 & -0.08 & 0.74 & $+\quad 0.34$ \\
\hline 4 & $+\quad 0.16$ & 0 & $+\quad 0.06$ & -0.06 & $+\quad 0.10$ & +0.01 \\
\hline$-2,-1$ & 0 & $+\quad 0.08$ & $+\quad 0.02$ & $-\quad 0.04$ & $+\quad 0.08$ & $-\quad 0.05$ \\
\hline-1 & $-\quad 0.03$ & $+\quad 2.15$ & $+\quad 1.63$ & +1.34 & $+\quad 1.25$ & -0.11 \\
\hline 0 & 0 & 0 & -287.42 & -0.10 & +276.62 & +34.79 \\
\hline 1 , & +3481.42 & -1.33 & +3481.14 & -1.04 & -5267.70 & +4.35 \\
\hline 2 , & +94.28 & +71.06 & +54.42 & +71.23 & -200.44 & -119.83 \\
\hline 3, & $+\quad 5.74$ & +15.65 & $+\quad 6.988$ & +9.490 & $-\quad 2.02$ & -17.37 \\
\hline 4 & $+\quad 1.30$ & +1.66 & $+\quad 1.18$ & +0.42 & $+\quad 1.16$ & -1.11 \\
\hline 5 , & $+\quad 0.15$ & +0.15 & $+\quad 0.10$ & 0 & $+\quad 0.17$ & -0.01 \\
\hline$-1,-2$ & 0.17 & +0.06 & 0.21 & -0.16 & 0.14 & -0.11 \\
\hline 0 & 0 & 0 & -6.58 & +30.99 & $+\quad 6.34$ & +31.39 \\
\hline 1 & +93.30 & -430.35 & +96.81 & -430.37 & -87.14 & -458.30 \\
\hline 2 & -408.28 & -20.58 & -410.42 & -12.01 & -676.60 & $-\quad 7.76$ \\
\hline 3 , & $-\quad 91.89$ & +64.32 & $-\quad 57.01$ & -64.98 & $-\quad 98.97$ & -94.93 \\
\hline 4 , & $-\quad 5.76$ & +18.30 & $+\quad 1.45$ & +12.96 & $-\quad 0.67$ & -19.65 \\
\hline 5 , & $+\quad 0.88$ & $+\cdot 2.45$ & $+\quad 1.30$ & +1.04 & $+\quad 1.56$ & $-\quad 1.79$ \\
\hline 6 , & $+\quad 0.27$ & +0.17 & $+\quad 0.20$ & -0.02 & $+\quad 0.28$ & -0.04 \\
\hline $1,-3$ & $-\quad 38.72$ & -17.03 & $-\quad 38.75$ & -17.29 & $+\quad 40.04$ & -17.16 \\
\hline 2, & $+\quad 1.02$ & +18.80 & $+\quad 5.31$ & +19.14 & $\begin{array}{l}8.48 \\
+\quad 8\end{array}$ & -26.83 \\
\hline 3 & -254.31 & $+\quad 2.37$ & -254.15 & -0.02 & -363.02 & $-\quad 0.43$ \\
\hline 4 & -71.84 & $\begin{array}{r}+50.20 \\
\end{array}$ & $-\quad 51.49$ & $\div 49.90$ & - 75.66 & -66.45 \\
\hline 5 , & $-\quad 5.65$ & +16.95 & $-\quad 0.23$ & +13.0 & $-\quad 1.82$ & -18.07 \\
\hline 6 , & $+\quad 0.87$ & $+\quad 2.76$ & $+\quad 1.29$ & $+\quad 1.5$ & $+\quad 1.48$ & $-\quad 2.14$ \\
\hline 7 & $+\quad 0.34$ & $+\quad 0.24$ & $+\quad 0.27$ & 0 & $+\quad 0.35$ & -0.07 \\
\hline $3,-4$ & $+\quad 3.03$ & +18.78 & $+\quad 1.34$ & +18.78 & $+\quad 6.33$ & -25.26 \\
\hline 4 & -144.96 & +3.60 & -144.78 & +1.5 & -191.70 & -2.31 \\
\hline 5 , & $-\quad 49.90$ & +35.00 & $-\quad 38.64$ & +34.58 & $-\quad 52.17$ & -43.7 \\
\hline 6 , & $-\quad 4.50$ & +13.86 & $-\quad 0.9$ & +11.2 & $-\quad 1.97$ & -14.7 \\
\hline 7 & $+\quad 0.75$ & +2.65 & 1.1 & +1.6 & $+\quad 1.31$ & $-\quad 2.2$ \\
\hline 8 & $+\quad 0.35$ & $+\quad 0.26$ & $+\quad 0.3$ & +0.1 & $+\quad 0.36$ & -0.1 \\
\hline $5,-5$ & $-\quad 79.05$ & +4.05 & $-\quad 78.9$ & $+\quad 2.4$ & -99.3 & $-\quad 2.1$ \\
\hline 6 & $-\quad 32.3$ & +22.7 & -26.3 & +22.3 & -33.5 & -27.2 \\
\hline 7 & - $\quad 3.4$ & +10.4 & $-\quad 1.1$ & $\begin{array}{l}+8.7 \\
\end{array}$ & $-\quad 1.7$ & -11.1 \\
\hline 8 & $+\quad 0.7$ & +2.3 & 0.9 & $+\quad 1.5$ & $+\quad 1.1$ & -1.0 \\
\hline 9 & 0.3 & $+\quad 0.3$ & $+\quad 0.3$ & +0.1 & $+\quad 0.3$ & $+\quad 0.1$ \\
\hline
\end{tabular}




\begin{tabular}{|c|c|c|c|c|c|c|c|}
\hline & $\begin{array}{r}\frac{\partial R}{\partial \mathrm{v}} \frac{d v_{0}}{d t} \\
\quad=\frac{m^{\prime}}{a_{3}}\end{array}$ & $\begin{array}{l}\frac{\partial R}{\partial p} \frac{d p_{0}}{d t} \\
\times\end{array}$ & Diser & ey. & v & k。 & k. \\
\hline$g \quad l^{\prime}$ & $\sin$ & $\cos$ & $\sin$ & $\cos$ & & & \\
\hline $0, \quad 0$ & 0 & -0.01 & 0 & -.01 & $\ldots$ & -1244.31 & \\
\hline 1 , & $-\quad 48.05$ & +48.92 & +.10 & -.04 & +1.0 & +32.78 & -20.90 \\
\hline 2 , & $-\quad 0.63$ & +8.27 & +.01 & -.01 & +0.5 & $+\quad 4.06$ & -1.86 \\
\hline 3 , & $+\quad 0.45$ & +0.65 & 0 & +.01 & $+\frac{1}{3}$ & $+\quad 0.44$ & $+\quad 0.08$ \\
\hline 4, & $+\quad 0.11$ & -0.03 & -.05 & -.03 & +0.25 & & \\
\hline$-2,-1$ & 0.03 & +0.02 & -.03 & -.06 & -0.206098 & $+\quad 0.08$ & -0.08 \\
\hline-1 & 0.05 & +2.16 & -.02 & +.01 & -0.259601 & $+\quad 1.23$ & -1.23 \\
\hline 0 , & $-\quad 0.06$ & $+\quad 0.09$ & -.06 & +.09 & -0.350623 & +276.62 & +34.79 \\
\hline+1 & +3481.41 & -1.33 & -.01 & 0 & -0.539942 & -1508.18 & +5.78 \\
\hline 2 , & $\begin{array}{l}94.20 \\
+\quad 9\end{array}$ & +71.06 & -.08 & 0 & -1.173630 & $+\quad 20.85$ & -286.59 \\
\hline 3 , & $+\quad 5.78$ & +15.63 & +.04 & -.02 & +6.75940 & - 79.56 & +194.17 \\
\hline 4, & +1.27 & $+\quad 1.66$ & -.03 & 0 & +0.871126 & $-\quad 1.10$ & $+\quad 1.79$ \\
\hline 5 , & $+\quad 0.14$ & +0.11 & -.01 & -.04 & +0.4656 & $+\quad 0.03$ & $+\quad 0.13$ \\
\hline$-1,-2$ & 0.16 & +0.10 & -.01 & +.04 & -0.149162 & $+\quad 0.19$ & -0.13 \\
\hline 0 , & $+\quad 0.01$ & $+\quad 0.07$ & +.01 & +.07 & -0.175312 & $+\quad 6.34$ & +31.39 \\
\hline+1 & +93.24 & -430.37 & -.06 & -.02 & -0.212580 & $-\quad 47.47$ & -275.33 \\
\hline 2 , & -408.28 & -20.60 & 0 & -.02 & -0.269971 & -897.05 & $+\quad 3.35$ \\
\hline 3 , & -91.90 & +64.33 & -.01 & +.01 & -0.369806 & -166.93 & -142.50 \\
\hline 4 & $-\quad 5.77$ & +18.17 & -.01 & -.13 & -0.586813 & $-\quad 7.44$ & -41.13 \\
\hline 5 , & $+\quad 0.91$ & $+\quad 2.45$ & +.03 & 0 & -1.42022 & $+\quad 4.05$ & -8.75 \\
\hline 6 , & $+\quad 0.29$ & +0.15 & +.02 & -.02 & +3.3797 & $-\quad 1.53$ & +1.09 \\
\hline $1,-3$ & $-\quad 38.78$ & -17.03 & -.06 & 0 & -0.132342 & $+\quad 29.78$ & -12.65 \\
\hline 2, & $+\quad 1.02$ & +18.68 & 0 & -12 & -0.152528 & $+\quad 8.79$ & -32.56 \\
\hline 3, & -254.29 & $+\quad 2.37$ & +.02 & 0 & -0.179981 & -454.55 & -1.28 \\
\hline 4 , & -71.88 & +50.13 & -.04 & -.07 & -0.219482 & -107.20 & -88.50 \\
\hline 5 , & $-\quad 5.69$ & +17.01 & -.04 & +.06 & -0.281202 & $-\quad 5.00$ & -27.61 \\
\hline 6 , & $+\quad 0.91$ & $+\quad 2.73$ & +.04 & -.03 & -0.391210 & 2.15 & -4.30 \\
\hline 7 , & $+\quad 0.35$ & +0.19 & +.01 & -.05 & -0.6426 & $+\quad 0.79$ & L 0.38 \\
\hline $3,-4$ & $+\quad 3.10$ & +18.78 & +.07 & 0 & +0.11893 & +7.05 & -29.73 \\
\hline 4, & -145.0 & +3.51 & -.04 & -.09 & -0.13500 & -230.83 & -3.28 \\
\hline 5 , & $-\quad 49.9$ & +34.85 & 0 & -.15 & -0.15605 & $-\quad 67.74$ & -54.60 \\
\hline 6 , & $-\quad 4.7$ & +13.95 & -.20 & +.09 & -0.18490 & $-\quad 3.63$ & -19.80 \\
\hline 7 , & $+\quad 0.8$ & +2.62 & +.05 & -.03 & -0.22685 & $+\quad 1.65$ & -3.36 \\
\hline 8, & $+\quad 0.3$ & +0.2 & -.05 & -.06 & -0.2934 & $+\quad 0.57$ & -0.27 \\
\hline $5,-5$ & -79.3 & $+\quad 2.7$ & -.25 & -1.35 & -0.1080 & -116.4 & -3.5 \\
\hline 6 , & -32.4 & +22.7 & -.10 & 0 & -0.1211 & -41.3 & -32.7 \\
\hline 7 , & $-\quad 3.5$ & +10.5 & -.10 & +.10 & -0.1377 & $-\quad 2.6$ & -13.9 \\
\hline 8 , & $+\quad 0.8$ & +2.3 & +.10 & 0 & -0.1597 & $+\quad 1.3$ & -2.6 \\
\hline 9, & $+\quad 0.3$ & +0.2 & 0 & -.10 & -0.1901 & $+\quad 0.5$ & -0.2 \\
\hline
\end{tabular}


The values of $\frac{a_{1}}{m} \int D_{t}^{\prime} R_{0} d t$ are formed from $\frac{a_{1}}{m^{\prime} n} D_{t}^{\prime} R$ by simple multiplication by $\nu$, and proper changes of sign. The values of $k_{c}$ and $k_{s}$ are then formed by adding the terms of $2 \frac{a_{1}}{m^{\prime}} \int D_{t}^{\prime} R_{0} d t$ to the corresponding terms of $\frac{a_{1}}{m^{\prime}} \frac{\partial R}{\partial \rho}$.

Perturbations of radius vector.

Let us now resume equation (19), and put for brevity

$$
M=\frac{m^{\prime} u}{a_{1}(1+m)} .
$$

If we give to $u$ the successive values $0,+1,-1,+2,-2,+3,-3$, we have

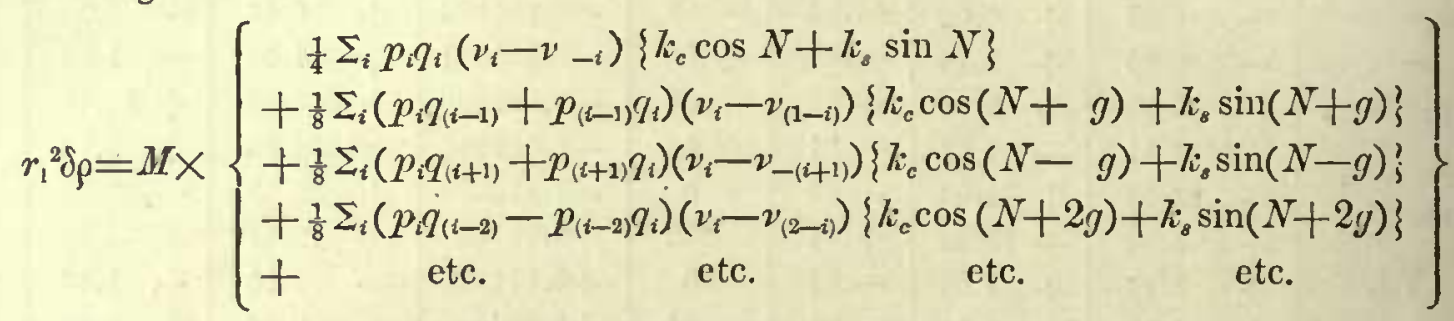

the finite integral being taken with respect to all valıes of $i$ from $-\propto$ to $+\propto$, and the terms in which the angles $N_{ \pm} u g$ vanish being omitted. Proceeding farther to expand with respect to $i$, if we collect similar terms we shall find the individual terms in $r^{2} \delta \rho$ to be as follows:

$$
\begin{aligned}
& r_{1}^{2} \delta \rho=\frac{1}{2} M\left\{\begin{array}{r}
p_{1} q_{1}\left(\nu_{1}-\nu_{-1}\right) \\
+p_{2} q_{2}\left(\nu_{2}-\nu_{-2}\right)
\end{array} . \quad\right\}\left\{k_{c} \cos N+k_{s} \sin N\right\} \\
& +\frac{1}{4} M\left\{\begin{array}{cc}
p_{0} q_{1} & \left(\nu_{1}-\nu_{0}\right) \\
+\left(p_{1} q_{2}+p_{2} q_{1}\right) & \left(\nu_{2}-\nu_{-1}\right) \\
+\left(p_{2} q_{3}+p_{3} q_{2}\right) & \left(\nu_{3}-\nu_{-2}\right) \\
+\quad \text { etc. } & \text { etc. }
\end{array}\right\}\left\{k_{c} \cos (N+g)+k_{\mathrm{s}} \sin (N+g)\right\} \\
& +\frac{1}{4} M\left\{\begin{array}{rc}
p_{0} q_{1} & \left(\nu_{0}-\nu_{-1}\right) \\
+\left(p_{1} q_{2}+p_{2} q_{1}\right) & \left(\nu_{1}-\nu_{-2}\right) \\
+\left(p_{2} q_{3}+p_{3} q_{2}\right) & \left(\nu_{2}-\nu_{-3}\right) \\
+\quad \text { etc. } & \text { etc. }
\end{array}\right\}\left\{k_{c} \cos (N-g)+k_{s} \sin (N-g)\right\} \\
& +\frac{1}{4} M\left\{\begin{array}{cc}
p_{0} q_{2} & \left(\nu_{2}-\nu_{0}\right) \\
+\left(p_{1} q_{3}+p_{3} q_{1}\right) & \left(\nu_{3}-\nu_{-1}\right) \\
+\quad \text { etc. } & \text { etc. }
\end{array}\right\}\left\{l_{c} \cos (N+2 g)+k_{s} \sin (N+2 g)\right\} \\
& +\frac{1}{4} M\left\{\begin{array}{cc}
p_{0} q_{2} & \left(\nu_{0}-\nu_{-2}\right) \\
+\left(p_{1} q_{3}+p_{3} q_{1}\right) & \left(\nu_{1}-\nu_{-3}\right) \\
+\quad \text { etc. } & \text { etc. }
\end{array}\right\}\left\{k_{c} \cos (N-2 g)+k_{b} \sin (N-2 g)\right\}
\end{aligned}
$$

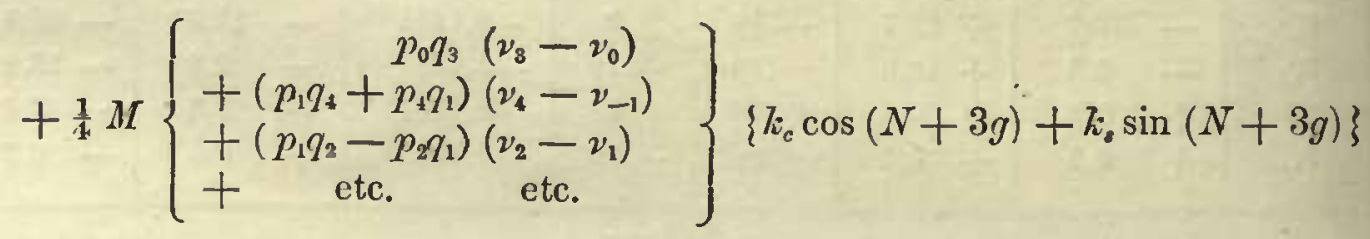




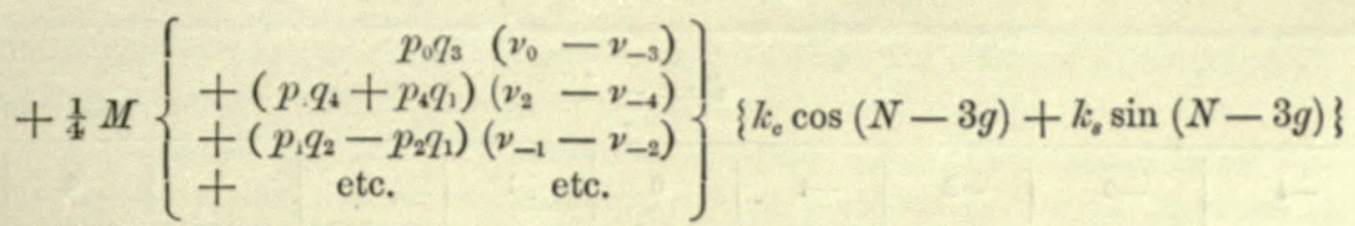

A law of the factors of $k_{e} \cos (N+u g)+k_{e} \sin (N+u g)$ which will be noticed in the above expression, is this: Representing this factor by $K_{u}$, we have

$$
K_{u}^{i}=K^{(i+u)} \text {, }
$$

the index $i$ representing the coefficient of $g$ in $N$, so that only half the values of $K$ need be separately computed.

As the computation of $r_{1}^{2} \delta \rho$ from these formulæ can be arranged in such a way as to be very simple, the computation of the terms in which the index $i^{\prime}$ is -1 is here presented quite fully. The logarithms only are omitted, being used only in the cases in which they are more convenient than a table of products. In practice I find it convenient to write them in red ink immediately under the numbers which they represent.

First, to find $M$, it will be noticed that in the expression $\frac{m^{\prime} a}{a_{1}(1+m)}$, the $a$ in the numerator represents the mean distance of the disturbed planet, as deduced from the observed mean motion by the equation $a^{3} n^{2}=\mu(1+m)$ while $a_{1}$ represents the mean motion of the outer planet. When the outer planet is the disturbed one, the ratio $\frac{a}{a_{1}}$ would be unity, but that, to avoid a large class of second order terms, $a_{1}$ has been corrected for perturbations in the beginning (p. 32). In the case of Uranus disturbed by Saturn, we have in consequence

Whence

$$
\log \frac{a}{a_{1}}=9.999803
$$

$$
M=285.44
$$

in units of the sixth place of decimals.

Computing the values of $p_{i}$ and $q_{i}$ from $(16)^{\prime}$ we find, for Uranus,

$$
\left.\begin{array}{llc}
\frac{1}{2} M p_{1} q_{1} & =+142.56 \\
\frac{1}{2} M p_{2} q_{2} & =+ & 0.0784 \\
\frac{1}{4} M p_{0} q_{1} & =- & 10.044 \\
\frac{1}{4} M\left(p q_{2}+p_{2} q_{1}\right) & =+ & 3.3433 \\
\frac{1}{4} M\left(p_{2} q_{3}+p_{3} q_{2}\right) & =+ & 0.0028 \\
\frac{1}{4} M p_{0} q_{2} & =- & 0.2358 \\
\frac{1}{4} M\left(p_{1} q_{3}+p_{3} q_{1}\right) & =+ & 0.118 \\
\frac{1}{4} M p_{0} q_{2} & =- & 0.008 \\
\frac{1}{4} M\left(p_{1} q_{4}+p_{4} q_{1}\right) & =+ & 0.005 \\
\frac{1}{4} M\left(p_{1} q_{2}-p_{2} q_{1}\right) & -+ & 0.003
\end{array}\right\} \begin{aligned}
& \text { In units of the sixth } \\
& \text { place of decimals. }
\end{aligned}
$$

In the computation the first three lines are copied from previous pages. 


$$
i^{\prime}=-1
$$

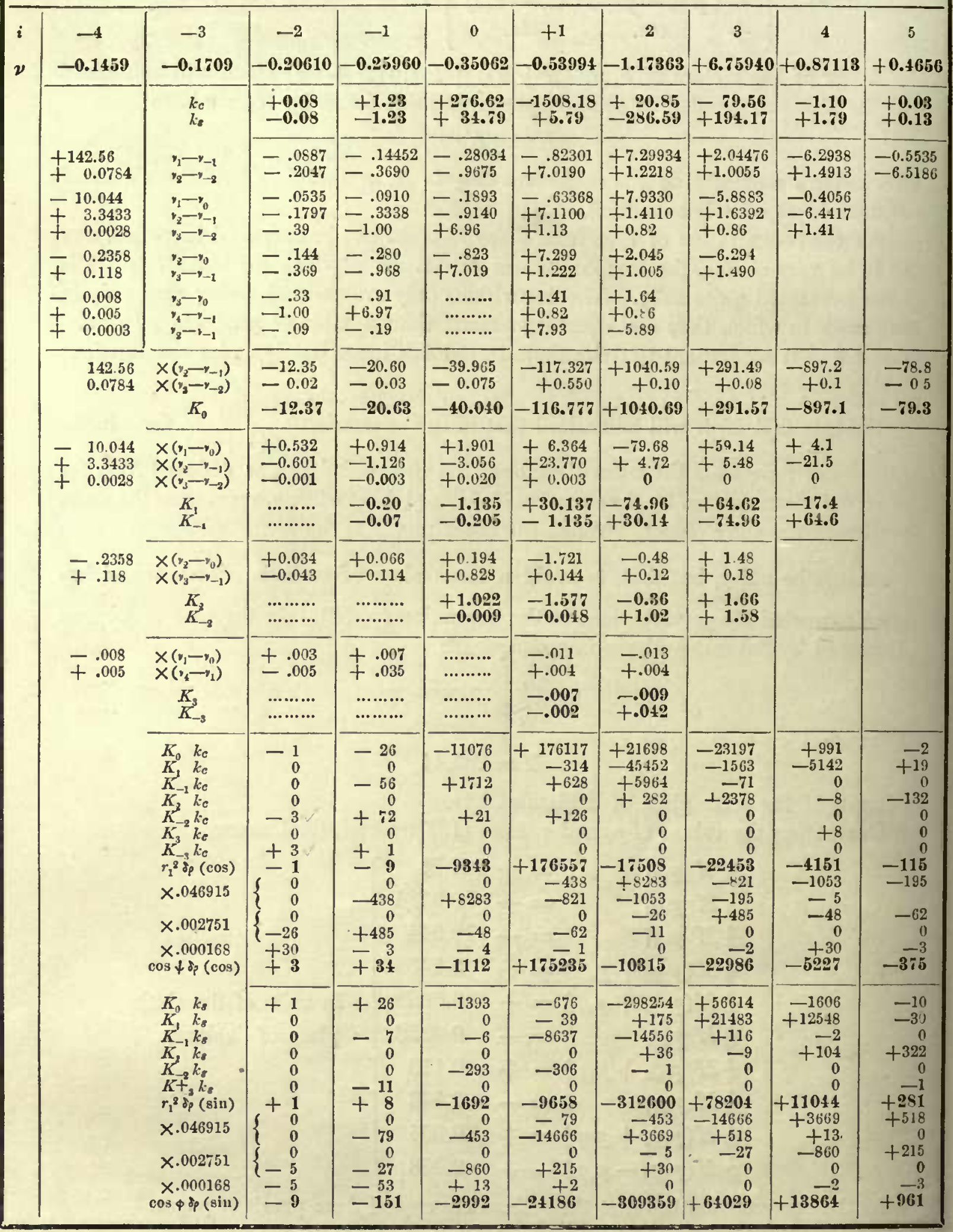


In forming the next ten lines, it will be noticed that the value of $v_{u}$ corresponding to any vertical column is found $u$ columns to the right. It is therefore necessary to extend the line $v$ two columns at each end. The extension on the right is, however, omitted for want of space. In performing the subtractions it will be convenient to copy the $\nu$ 's again on the lower edge of a horizontal strip of paper, and, in forming the differences $\nu_{u}-v_{u}{ }^{\prime}$ to lay the strip above the line of $\nu$ 's, and $u-u^{\prime}$ columns to the right.

On the left of each line of differences is written the factor by which that line is to be multiplied.

The mode of formation of the $K$ 's is evident from the formula.

It will be seen that the same computation which gives $K_{u}$ gives also $K_{-u}$, only the latter belongs $u$ columns to the right.

Each $k_{e}$ and $k_{s}$ is multiplied in succession by all the $K$ 's which lie below it in the same column, but the product by $K_{u}$ is to be written $u$ columns to the right, and that by $K_{-u} u$ columns to the left. The sum of the products in any one column-gires the coefficient of $\frac{\sin }{\sin }\left(i g+i^{\prime} l^{\prime}\right)$ in the development of $r_{1}^{2}{ }^{2} \rho$.

This quantity being multiplied by $\frac{d v_{0}}{n d t}=\frac{a^{2} \cos \psi}{r_{0}{ }^{2}}$ ave have $\cos \psi \delta \rho$, which only needs to be multiplied by sec $\psi=1.001103$ to give $\delta \rho$. The units of $r_{1}{ }^{2} \delta \rho$ and $\delta_{\rho}$ correspond to the ninth place of decimals.

All the periodic terms are to be treated in this manner, all the series of values of $k_{e}$ and $k_{s}$, including the constant term, being subjected to the same process. But, when $i^{\prime}$ and $i$ are both zero, $\nu$ will be infinite. Here we simply omit the $v$, treating it as if it were zero. We thus obtain the complete value of the terms with constant coefficients in $r_{1}{ }^{2} \delta \rho$ and $\delta \rho$ which are given in the following table. The terms multiplied by the time are still to be computed. They are derived from (20), which may be put in the form

$$
r_{1}^{2} \delta \rho=\frac{1}{2} M\left\{\eta \Sigma p_{u} k_{c}^{(n)}-\xi \Sigma q_{u} k_{s}^{(n)}\right\} n t .
$$

This expression is computed thus:

\begin{tabular}{|c|c|c|c|c|c|}
\hline$p_{u}$ & $k_{e}^{(u)}$ & $q_{u}$ & $k_{s}^{(u)}$ & $p_{u} k_{e}^{(u)}$ & $-q_{u} k_{s}^{(u)}$ \\
\hline-.140783 & -1244.31 & 0 & 0 & +175.18 & 0 \\
\hline+.99917 & $\begin{array}{l}+32.78 \\
\end{array}$ & +.99972 & -20.90 & +32.75 & +20.89 \\
\hline+.0234 & 4.06 & +.0234 & -1.86 & 0.09 & +0.04 \\
\hline
\end{tabular}

We have now

$$
\begin{array}{rlr}
\Sigma p_{u} k_{o}^{(u)}=+ & 208.02 ; & \frac{1}{2} M \Sigma p_{u} k_{c}^{(u)}=+29689 \\
-\Sigma q_{u} k_{s}^{(u)}=+ & 20.93 ; & \frac{1}{2} M \Sigma q_{u} k_{o}^{(u)}=+2988 \\
r_{1}{ }^{2} \delta \rho= & -210 n t & \\
& +2986 n t \cos g & +29681 n t \sin g \\
& +70 n t \cos 2 g & +697 n t \sin 2 g \\
& +2 n t \cos 3 g & +24 n t \sin 3 g,
\end{array}
$$

in units of the ninth place of decimals. The value of $\cos \psi \delta \rho$ is obtained from them by multiplying by $r_{1}^{-9}$, exactly as in the case of the constant terms. 


\begin{tabular}{|c|c|c|c|c|c|c|c|}
\hline \multirow{2}{*}{$\begin{array}{ll} & l^{\prime}\end{array}$} & \multicolumn{2}{|c|}{$r_{1}^{3} \delta_{p}$} & \multicolumn{2}{|c|}{$\cos \psi \delta \rho$} & $\log M I v$ & $\mathbf{v}_{\mathrm{o}}$ & $v_{0}$ \\
\hline & $\cos$ & $\sin$ & $\cos$ & $\sin$ & & & \\
\hline $0, \quad 0$ & $-210 n t$ & & $-70 n t$ & & & $+139 n^{\prime} t$ & \\
\hline 1, & $+2986 n \ell$ & $+29681 n t$ & $+2978 n t$ & $+29633 n \ell$ & & & \\
\hline 2 & $+70 n t$ & $+697 n t$ & $+209 n t$ & $+2084 n t$ & & & \\
\hline 3 & $+2 n t$ & $+24 n t$ & $+14 n t$ & $+139 n \ell$ & & & \\
\hline $0, \quad 0$ & -355045 & & -354347 & & $\propto$ & 0 & \\
\hline 1 & +14875 & -1503 & -18411 & -1496 & 2.45551 & -2912 & +14206 \\
\hline 2 & -283 & +49 & -1536 & -21 & 2.25448 & -639 & +457 \\
\hline 3. & -20 & -1 & -112 & -2 & 1.97839 & -46 & -8 \\
\hline$-2,-1$ & -1 & +1 & +3 & -9 & 1.7696 & +1 & +2 \\
\hline-1 & -10 & +8 & +34 & -151 & 1.8698 & +12 & -99 \\
\hline 0 & -9343 & -1692 & -1112 & -2992 & 2.00035 & -2876 & +10 \\
\hline+1 & +176557 & -9658 & +175235 & -24186 & 2.18786 & +536512 & +160 \\
\hline 2 & -17508 & -312600 & -10315 & -309359 & 2.52504 & +1823 & -23860 \\
\hline 3 & -22153 & +78204 & -22986 & +64029 & 3.28542 & -13482 & +18310 \\
\hline 4 & -4151 & +11044 & -5227 & +13864 & 2.39559 & -293 & +104 \\
\hline 5 & -115 & +281 & -373 & +961 & 2.1235 & -13 & 0 \\
\hline$-1,-2$ & -1 & +1 & +1 & -2 & 1.6292 & +9 & +7 \\
\hline 0 & -56 & -276 & +32 & -100 & 1.6993 & -329 & -1551 \\
\hline+1 & +710 & +3723 & +1675 & +3728 & 1.78303 & +5874 & +26114 \\
\hline 2 & +20180 & -13 & $\begin{array}{r}+20583 \\
\end{array}$ & +484 & 1.88683 & -31660 & +926 \\
\hline 3 & +7797 & +6512 & +8824 & +6796 & 2.02348 & -6019 & -6860 \\
\hline 4 & +1534 & +6202 & +2054 & +6230 & 2.22401 & +243 & -2172 \\
\hline 5 & +2071 & -5954 & +2134 & -5610 & 2.60786 & +527 & -421 \\
\hline 6 , & -653 & +755 & -550 & +494 & 2.98438 & -190 & -20 \\
\hline $1,-3$ & -151 & +64 & -137 & +75 & 1.5772 & -1464 & +653 \\
\hline 2 & -52 & +221 & +149 & +229 & 1.63886 & +231 & -833 \\
\hline 3 & +4348 & +15 & +4420 & +88 & 1.71074 & -13050 & +1 \\
\hline 4 & +1564 & +1280 & +1774 & +1314 & 1.79691 & -3225 & -3126 \\
\hline 5 , & +133 & +685 & +214 & +756 & 1.9045 & -18 & -1043 \\
\hline 6 & -111 & +232 & -109 & +272 & 2.0479 & +144 & -163 \\
\hline 7 & -163 & +87 & -168 & +100 & 2.2634 & +50 & -7 \\
\hline $3,-4$ & -28 & +121 & +31 & +122 & 1.5408 & +181 & -638 \\
\hline 4 & +1223 & +17 & +1245 & +41 & 1.5858 & -5578 & -58 \\
\hline 5 & +485 & +389 & +545 & +400 & 1.6488 & -1721 & -1540 \\
\hline 6 & +38 & +201 & +63 & +221 & 1.7225 & -50 & -590 \\
\hline 7 & -25 & +53 & -23 & +63 & 1.8012 & +70 & -100 \\
\hline 8 & -15 & +7 & -16 & +9 & 1.9229 & & \\
\hline $5,-5$ & +392 & +12 & +400 & +18 & 1.4889 & -2432 & -74 \\
\hline 6 & +175 & +138 & +194 & +142 & 1.5385 & -908 & -760 \\
\hline 7 , & +14 & +77 & +22 & +84 & 1.5946 & -40 & -340 \\
\hline 8 & -10 & +20 & -9 & +24 & 1.6589 & +40 & -70 \\
\hline 9 & -5 & +3 & -5 & +4 & 1.7345 & & \\
\hline
\end{tabular}




\section{Perturbations of Longitude.}

The perturbations of the longitude are now to be computed by formulæ (24). To do this in the most simple way we remark that the numbers given on page 42 , under the heading $\frac{\partial R}{\partial \mathrm{v}}$, are those represented in formula (42) by $v_{\mathrm{s}}$ and $v_{\mathrm{c}}$. If we put

$$
n t=t^{\prime}
$$

equation (24) may be put into the form

$$
\frac{d \delta v}{d t^{\prime}},=r_{1}^{-2}\left\{\frac{a}{1+m} \int \frac{\partial R}{\partial \mathrm{v}} d t^{\prime}-2 \cos \psi \delta \rho\right\}
$$

but we have from (42)

$$
\int \frac{\partial R}{\partial \mathrm{v}} d t=\Sigma\left(\frac{m^{\prime} v}{a_{1}} v_{e} \sin N-\frac{m^{\prime} v}{a_{1}} v_{s} \cos N\right) .
$$

If now we represent the numerical values of $\cos \psi \delta \rho$, already found, by

$$
\Sigma\left(\rho_{s} \sin N+\rho_{c} \cos N\right),
$$

and if we substitute these expressions in the above value of $\frac{d i v}{d t^{\prime}}$, the latter will become

$$
\frac{d i v}{d t^{\prime}}=r_{1}^{-2} \Sigma\left\{\left(\mathrm{v}_{s}-2 \rho_{s}\right) \sin N+\left(\mathrm{v}_{e}-2 \rho_{c}\right) \cos N\right\},
$$

where we put for brevity

$$
\begin{aligned}
& \mathbf{v}_{s}=M \nu v_{e}, \\
& \mathbf{v}_{c}=-M \nu v_{s} .
\end{aligned}
$$

The numerical expression for $r_{1}^{-2}$ is given on page 40, and by multiplying the quantities within brackets by this expression, after the manner explained on pages 40 and 41 , we form the terms of $\frac{d \delta v}{d t^{\prime}}$. Multiplying each of these terms by its corresponding value of $v$, changing $\cos$ to $\sin$ and $\sin$ to $\cos$, we have the coefficients in the expressions for $\delta v$ given on page 50 .

As previously mentioned, before commencing the above computation, I had computed all the perturbations of Uranus by the method of "perturbations of the elements," using the formulæ developed in my Investigation of the Orbit of Neptune. The two results are here placed side by side, for the purpose of comparison. The discrepancies in the various coefficients, expressed in thousandths of a second, are shown in the sixth and seventh columns.

It will be seen that the largest discrepancies, and indeed the only ones (with a single exception) exceeding one-tenth of a second, occur in the coefficients of the terms $2 j^{\prime}-l$ aug $3 g^{\prime}-l$. Here the errors are almost certainly in the computation from perturbations of the elements. Owing to the long period of the term $3 g-l$ they would not become sensible in the course of any one century. 
Perturbations of the Longitude of Uranus produced by the action of Saturn, and DEPENDINA ON THE FIRST POWER OF THE DISTURIBNG FURCES.

\begin{tabular}{|c|c|c|c|c|c|c|c|c|}
\hline \multirow[b]{2}{*}{$g, \quad l^{\prime}$} & \multicolumn{2}{|c|}{ From comp. preceding } & \multicolumn{2}{|c|}{$\begin{array}{l}\text { From pert. of clements } \\
\qquad v\end{array}$} & \multicolumn{2}{|c|}{ Discrepancy. } & \multicolumn{2}{|c|}{$0.434294 \delta p$} \\
\hline & $\sin ^{\prime \prime}$ & $\cos ^{\prime \prime}$ & $\sin ^{\prime \prime}$ & ${ }^{\cos }$ & $\sin ^{\prime \prime}$ & $\cos ^{\prime \prime}$ & $\cos$ & $\sin$ \\
\hline $0, \quad 0$ & $\ldots \ldots$ & $+10.9690 t$ & $\ldots \ldots$ & $+10.9645 t$ & 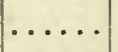 & $.0045 t$ & & \\
\hline 1 & $-1.230 n t$ & $+12.231 n t$ & $-1.228 u t$ & $+12.271 n t$ & $.002 n t$ & $.040 n t$ & $+13 n t$ & $t+128 n$ \\
\hline 2 & $-0.072 n t$ & $+0.717 n t$ & $-0.072 n t$ & $+0.720 n t$ & 0 & $.003 n t$ & $+1 n t$ & $+9 n t$ \\
\hline 3 & $-0.004 n t$ & $+0.043 n t$ & $-0.004 n t$ & $+0.043 n t$ & 0 & & 0 & 0 \\
\hline $0, \quad 0$ & $\ldots \ldots$ & $\cdots$ & $\ldots \ldots$ & 1 & $\cdots$ & . & -1541 & \\
\hline 1 , & +8.545 & $-\quad 4.735$ & $+\quad 2.844$ & +1.013 & $\cdots$ & $\cdots$ & -80 & 0) -6 \\
\hline 2 , & +0.461 & $-\quad 0.169$ & $+\quad 0.133$ & $+\quad 0.166$ & $\cdots$ & $\ldots$ & 一 & 0 \\
\hline 3 , & +0.028 & -0.005 & +0.013 & +0.014 & $\cdots$ & $\cdots$ & & 0 \\
\hline$-1,-1$ & +0.036 & $+\quad 0.039$ & $+\quad 0.032$ & $+\quad 0.005$ & 4 & 34 & 0 & - \\
\hline 0 & +1.282 & $+\quad 0.718$ & +1. & +0 & 2 & 1 & -5 & -13 \\
\hline 1, & -20.817 & +8.522 & -20.8 & +8.595 & 56 & 73 & +761 & -106 \\
\hline 2 , & -11.890 & +143.463 & -11.093 & +143.465 & 797 & 2 & -45 & -1351 \\
\hline 3 & +49.30 & +115.86 & +49.62 & +116.08 & 320 & 220 & -103 & +280 \\
\hline 4 & +2.133 & +5.616 & $+\quad 2.195$ & $+\quad 5.621$ & 62 & 5 & -24 & +61 \\
\hline 5 & +0.126 & +0.329 & +0.109 & +0.331 & 17 & 2 & -2 & + \\
\hline $0,-2$ & +0.017 & $-\quad 0.017$ & $+\quad 0.025$ & $-\quad 0.03$ & 8 & 16 & 0 & 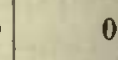 \\
\hline 1 , & +0.042 & $+\quad 0.814$ & +0.0 & +0.8 & 8 & 4 & +7 & +16 \\
\hline 2, & +4.110 & $-\quad 0.009$ & +4.103 & -0.012 & 7 & 3 & +89 & + \\
\hline 3 & +2.079 & $-\quad 1.607$ & +2.106 & -16 & 27 & 69 & +38 & $+\quad 30$ \\
\hline 4, & +0.648 & -1.830 & $+\quad 0.6$ & -1.9 & 5 & 72 & +9 & $+\quad 27$ \\
\hline 5 , & +1.163 & +2.956 & $+\quad 1.2$ & $+\quad 2.9$ & 111 & 35 & +9 & -21 \\
\hline 6 , & +0.503 & +0.378 & $+\quad 0.550$ & $+\quad 0.445$ & 53 & 67 & -2 & + \\
\hline $1,-3$ & +0.034 & $+\quad 0.012$ & $+\quad 0.0$ & +0.0 & 2 & 3 & 0 & 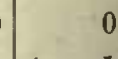 \\
\hline 2 & +0.037 & -0.041 & +0.0 & -0.0 & 23 & 9 & +1 & + \\
\hline 3 & +0.824 & $-\quad 0.019$ & +11.8 & - 0.0 & 12 & 2 & +19 & 0 \\
\hline 4, & +0.355 & -0.267 & +0.3 & -0.2 & 4 & 4 & $1+8$ & + \\
\hline 5 , & +0.047 & $-\quad 0.165$ & $+\quad 0.0$ & -0. & 8 & 26 & +1 & + \\
\hline 6 & -0.026 & $-\quad 0.063$ & $-\quad 0.0$ & -0.0 & 2 & 3 & 0 & + \\
\hline 7 & -0.053 & $-\quad 0.032$ & $-\quad 0.053$ & -0.018 & 0 & 14 & -1 & 0 \\
\hline $3,-4$ & +0.006 & $-\quad 0.022$ & $-\quad 0.0$ & $-\quad 0.0$ & 11 & 1 & 0 & 1 \\
\hline 4 & +0.228 & $-\quad 0.008$ & +0.2 & +0. & 7 & 10 & +5 & 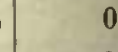 \\
\hline 5 & +0.103 & $-\quad 0.077$ & +0.08 & -0.0 & 19 & 2 & +2 & + \\
\hline 6 , & +0.013 & $-\quad 0.044$ & $+\quad 0.01$ & -0.0 & 0 & 13 & 0 & + \\
\hline 7 , & -0.005 & -0.013 & -0.001 & $-\quad 0.015$ & 4 & 2 & 0 & 0 \\
\hline 8, & -0.003 & $-\quad 0.002$ & 0 & $+\quad 0.002$ & 3 & 4 & 0 & 0 \\
\hline $5,-5$ & +0.074 & $-\quad 0.003$ & +0.07 & 0 & 3 & 3 & +2 & 0 \\
\hline 6 & +0.038 & $-\quad 0.027$ & $+\quad 0.023$ & $-\quad 0.026$ & 15 & 1 & +1 & + \\
\hline , & +0.005 & $-\quad 0.016$ & $+\quad 0.005$ & $-\quad 0.025$ & 0 & 9 & 0 & 0 \\
\hline 8, & -0.002 & -0.004 & 0 & -0.003 & 2 & 1 & 0 & 0 \\
\hline
\end{tabular}




\section{Perturbations of Latitude.}

These are computed from the formulæ (27) and (40), no reductions being made from $\delta k$ and $\delta \eta$ to $\delta p$ and $\delta q$, but the perturbations of the latitude being computed directly from the former by (40). We have only to represent the expressions for $\delta k$ and $\delta \eta$ by

$$
\begin{aligned}
& \delta k=-\Sigma a_{e} \cos N-\Sigma a_{s} \sin N \\
& \delta r_{i}=\Sigma a_{c}^{\prime} \cos N+\Sigma a_{c}^{\prime} \sin N
\end{aligned}
$$

and substitute $\omega$ for $\pi$ in the equations (40) from which $\delta \beta$ is computed.

The principal steps of the computation are shown quite fully in the following table. The values of

$$
\frac{\partial h}{\partial \gamma}=\frac{1}{2} \cos \frac{1}{2} \gamma \frac{\partial h}{\partial \sigma}
$$

are first formed from those terms of $h$, on pages 37 and 38 , which contain $\sigma$ as a coefficient. Then, having for each original term of $R$

$$
\frac{\partial R}{\partial \gamma}=\frac{m^{\prime}}{a_{1}} \frac{\partial h}{\partial \gamma} \cos N
$$

all the terms which have the same coefficients of $\lambda$ and $\lambda^{\prime}$ in $N$ are combined into two depending on $g$ and $l^{\prime}$ as shown in the case of $R$ on page 36. The coefficients of these terms, in units of the third place of decimals, are given in the columns headed $\frac{\partial R}{\partial \gamma}$.

The value of $\frac{t h}{2 \sigma} \sin N$ being formed for each term of $R$, all the terms depending on the same multiples of $\lambda$ and $\lambda^{\prime}$ are combined into two, of which the coefficients are given under the proper heading. The terms of $(i+j) \sigma h \sin N$ being formed in like manner, we have, by adding the last two expressions, all the quantities which enter into the formulæ (27). To integrate these equations thus forming the numerical values of $\delta k$ and $\delta \eta$ we have only to multiply each term in the second, third, eighth, and ninth columns of the table by the corresponding values of $\frac{m^{\prime} a \nu}{a_{1} \cos \psi}$, for which we may use the value of $\frac{M_{\nu}}{\sin 1^{\prime \prime}}$ already given.

The quantities given in the four columns under $\delta k$ and $-\delta \eta$ show the values of $-a_{s},-a_{e},-a_{c}^{\prime},-a_{s}^{\prime}$, corresponding to each argument. From these the terms of $\delta \beta$ are formed by equation (40) with the modification mentioned above. 


\begin{tabular}{|c|c|c|c|c|c|c|c|c|c|c|c|c|c|c|}
\hline \multicolumn{15}{|c|}{ Perturbations of the Latitude produced by Saturn. } \\
\hline \multirow{2}{*}{$\begin{array}{cc}g & l^{\prime} \\
i & i^{\prime}\end{array}$} & \multicolumn{2}{|c|}{$\frac{\partial R}{\partial \gamma}=\frac{m^{\prime}}{a_{1}} \times$} & \multicolumn{2}{|c|}{$\Sigma \frac{\hbar h}{2 \sigma} \sin N$} & \multicolumn{2}{|c|}{$\Sigma(i+j) \sigma h \sin N$} & \multicolumn{2}{|c|}{$\frac{d n}{d t}=M n X$} & \multicolumn{2}{|c|}{$\delta k$} & \multicolumn{2}{|r|}{$-\delta \eta$} & \multicolumn{2}{|c|}{$\delta \beta$} \\
\hline & $\cos$ & $\sin$ & $\sin$ & $\cos$ & $\sin$ & $\cos$ & $\sin$ & $\cos$ & $\sin _{/ 1}$ & $\cos _{1 \prime}$ & $\cos$ & $\sin _{\|}$ & $\sin _{\prime \prime}$ & $\cos$ \\
\hline 0,0 & -10.82 & 0 & 0 & 0 & 0 & +0.008 & 0.00 & +.008 & & & & 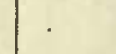 & & +0.192 \\
\hline 1, & -3.45 & -2.92 & -1.94 & -0.11 & +0.17 & +0.84 & -1.77 & +0.73 & $3+0.203$ & -0.172 & -0.104 & $4-0.043$ & +0.246 & $6+0.209$ \\
\hline 2 & +1.42 & -11.10 & +1.30 & +10.73 & +0.07 & +0.05 & +1.37 & +10.78 & -0.042 & -0.326 & +0.042 & $2-0.317$ & -0.049 & $9+0.015$ \\
\hline 3 & +1.31 & -1.71 & +1.31 & +1.71 & +0.01 & 0.00 & +1.32 & +1.71 & -0.026 & -0.033 & $3+0.026$ & $6-0.033$ & -0.008 & $8+0.003$ \\
\hline$-2,-1$ & +1.0 & +0.1 & -1.0 & -0.1 & 0.00 & 0.00 & -1.0 & -0.1 & +0.012 & -0.001 & +0.012 & $2-0.001$ & 0.000 & $\begin{array}{l}0.000 \\
0.0 .\end{array}$ \\
\hline-1 & -6.43 & -52.6 & +6.4 & -52.8 & +0.03 & +0.02 & +6.4 & -52.8 & -0.098 & +0.804 & -0.098 & $8-0.804$ & -0.004 & +0.018 \\
\hline 0 & -6.97 & +7.06 & +1.53 & +5.14 & -4.91 & 0.00 & -3.38 & +5.14 & -0.144 & -0.145 & +0.070 & $0+0.106$ & -0.661 & $+0.57 \mathrm{e}$ \\
\hline+1 & +46.67 & 0 & 0.00 & 0.00 & +59.45 & -0.02 & +59.45 & -0.02 & +1.482 & 0 & -1.887 & $7-0.001$ & $+0.08 t$ & $-0.02 \theta$ \\
\hline 2 , & -1.84 & -2.86 & -0.99 & -0.20 & +0.93 & +1.22 & -0.06 & +1.02 & -0.127 & +0.197 & +0.004 & $4+0.070$ & +2.218 & $8+1.920$ \\
\hline 3 , & +0.90 & -6.98 & +0.78 & +6.44 & +0.12 & +0.16 & +0.90 & +6.60 & -0.358 & -2781 & +0.358 & $8-2.630$ & +0.091 & $1-0.071$ \\
\hline 4 & +1.09 & -1.38 & +1.09 & +1.38 & +0.02 & +0.01 & +1.11 & +1.39 & -0.056 & -0.071 & +0.057 & $7-0.071$ & -0.048 & $8+0.056$ \\
\hline$-1,-2$ & +7.6 & -1.5 & -7.6 & +1.5 & 0.00 & 0.00 & -7.6 & +1.5 & +0.067 & +0.013 & +0.067 & $7+0.013$ & +0.003 & $3+0.004$ \\
\hline 0 & +1.3 & +10.9 & -1.3 & +10.7 & -0.11 & +0.53 & -1.4 & +11.2 & +0.013 & -0.112 & +0.014 & $4+0.116$ & +0.008 & $8+0.034$ \\
\hline+1 & -0.27 & +9.03 & +1.70 & +0.91 & +1.65 & -7.35 & +3.35 & -6.44 & -0.0013 & -0.112 & -0.042 & $2-0.080$ & +0.040 & -0.042 \\
\hline 2 , & -14.51 & 0 & 0.00 & 0.00 & -7.01 & -0.21 & -7.01 & -0.21 & -0.230 & 0 & +0.112 & $2-0.003$ & 0 & -0.029 \\
\hline 3 & -2.85 & -2.29 & -0.42 & -0.19 & -0.97 & +1.11 & -1.39 & +0.92 & -0.062 & +0.050 & +0.030 & $0+0.020$ & -0.063 & $3-0.054$ \\
\hline 4 & +0.43 & -4.23 & +0.44 & +3.67 & +0.02 & +0.22 & +0.46 & +3.89 & +0.015 & +0.146 & -0.016 & $6+0.134$ & -0.080 & $0-0.014$ \\
\hline 5 & +0.80 & -0.99 & +0.80 & +0.99 & +0.02 & +0.02 & +0.82 & +1.01 & +0.067 & +0.083 & -0.068 & $8+0.084$ & +0.008 & -0.001 \\
\hline $2,-3$ & -1.17 & +0.86 & +1.22 & +0.97 & +0.09 & +0.33 & +1.31 & +1.30 & -0.010 & -0.008 & -0.011 & $1+0.012$ & +0.050 & -0.046 \\
\hline 3 , & -8.54 & 0 & 0.00 & 0.00 & -4.34 & 0 & -4.34 & 0.00 & -0.090 & 0 & +0.046 & 0 & +0.004 & $4-0.010$ \\
\hline 4 & -2.08 & -1.65 & -0.13 & -0.15 & -0.88 & +0.85 & -1.01 & +0.70 & -0.026 & +0.022 & +0.013 & $3+0.009$ & -0.012 & $2-0.012$ \\
\hline 5 & +0.14 & -2.50 & +0.24 & +2.00 & 0.00 & +0.22 & +0.24 & +2.22 & +0.002 & +0.041 & -0.004 & $4+0.037$ & -0.010 & -0.002 \\
\hline 6 & +0.55 & -0.66 & +0.55 & +0.66 & +0.02 & +0.02 & +0.57 & +0.68 & +0.013 & +0.015 & -0.013 & $3+0.016$ & +0.004 & -0.001 \\
\hline $3,-4$ & -0.72 & +0.47 & +0.82 & +0.78 & +0.09 & +0.32 & +0.91 & +1.10 & -0.005 & -0.003 & -0.006 & $6+0.008$ & +0.022 & -0.020 \\
\hline 4 & $-4.80 \mid$ & 0 & 0.00 & 0.00 & -2.48 & -0.02 & -2.48 & -0.02 & -0.038 & 0 & +0.020 & 0 & +0.002 & -0.005 \\
\hline 5 & -1.41 & -1.09 & -0.01 & -0.10 & -0.66 & +0.59 & -0.67 & +0.49 & -0.013 & +0.010 & +0.006 & +0.004 & -0.004 & -0.003 \\
\hline 6 , & +0.12 & -1.3 & +0.12 & +1.0 & -0.02 & +0.19 & +0.10 & +1.2 & +0.001 & +0.014 & -0.001 & $1+0.013$ & +0.004 & +0.002 \\
\hline $5,-5$ & -2.64 & 0 & 0 & 0 & -1.35 & +0.04 & -1.35 & +0.04 & -0.017 & 0 & +0.008 & 80 & & \\
\hline & & & & & & & & & . & & & & & \\
\hline
\end{tabular}




\section{CHAPTER III.}

\section{PERTURBATIONS PRODUCED BY NEPTUNE AND JUPITER.}

ThE perturbations of Uranus by Neptune were originally computed with elements of both planets quite different from those finally adopted, But the last computations, on which the concluded values of the perturbations depend, were made with the concluded elements of Neptune found in my investigation of the orbit of that planet. ${ }^{1}$ They are as follows :

\begin{tabular}{|c|c|c|c|}
\hline & ○ & , & " \\
\hline$\pi$, & 43 & 17 & 30 \\
\hline$\theta$, & 130 & 7 & 33 \\
\hline$\varepsilon$, & 335 & 5 & 39 \\
\hline$\phi$, & 1 & 47 & 1.6 \\
\hline$n$, & \multicolumn{3}{|c|}{7864.935} \\
\hline$e$, & \multicolumn{3}{|c|}{0.0084962} \\
\hline $\log a$, & \multirow{2}{*}{\multicolumn{3}{|c|}{$\begin{array}{c}1.478141 \\
\overline{1} \frac{1}{0} 00\end{array}$}} \\
\hline & & & \\
\hline
\end{tabular}

Hence follow the following functions of the elements of Neptune and Uranus:

$$
\begin{aligned}
& \alpha=0.638195 \\
& \omega=12^{\circ} \quad 44^{\prime} \quad 58^{\prime \prime} \\
& \omega^{\prime}=247 \quad 45 \quad 20 \\
& \gamma=\begin{array}{lll}
\gamma & 30 & 29.6
\end{array} \\
& \sigma=\sin \frac{1}{2} \gamma=0.013161 \\
& M=37.522 \text { (in units of } 6 \text { th place of decimals). }
\end{aligned}
$$

From these values of the elements are obtained the following values of the various terms in the development of the perturbative function, and of $\nu$. As the developments have been formed on the same principle as in the case of Saturn, it is deemed unnecessary to give the details of the process. It is only necessary to remark that the indices $i^{\prime}$ and $i$ are the coefficients of $l^{\prime}$ and $g$ respectively, the mean longitude of Neptune, or $l$, being counted from the perihelion of Uranus. 


\begin{tabular}{|c|c|c|c|c|c|c|c|c|c|}
\hline \multirow[b]{3}{*}{$i^{\prime} \quad i$} & \multicolumn{9}{|c|}{ Action of Neptune. } \\
\hline & \multicolumn{2}{|c|}{$R=\frac{m^{\prime}}{a_{1}} \times$} & \multicolumn{2}{|c|}{$\frac{\partial R}{\partial v}=\frac{m^{\prime}}{a_{1}} \times$} & \multicolumn{2}{|c|}{$\frac{\partial R}{\partial p}=\frac{m^{\prime}}{a_{1}} \times$} & \multicolumn{2}{|c|}{$Q=\frac{m^{\prime}}{a_{1}} \times$} & \multirow[t]{2}{*}{$\nu$} \\
\hline & $h_{\mathrm{c}}$ & $h_{s}$ & vs & $v_{e}$ & $\cos$ & $\sin$ & $k_{c}$ & $k$ s & \\
\hline $\begin{array}{r}0, \quad 0 \\
+\quad 1 \\
+2 \\
+3\end{array}$ & $\begin{array}{r}+1135.63 \\
+\quad 18.17 \\
+\quad 0.41 \\
0\end{array}$ & $\begin{array}{r}0 \\
-1.25 \\
-0.03 \\
0\end{array}$ & {$\left[\begin{array}{ll} & 0 \\
+ & 1.01 \\
- & 0.6 \\
0 & \end{array}\right.$} & $\begin{aligned}+ & 0.202 \\
- & 1.23 \\
- & 0.1 \\
& 0\end{aligned}$ & $\begin{array}{r}+368.26 \\
+\quad 64.80 \\
+\quad 2.98 \\
0\end{array}$ & $\begin{aligned} & 0 \\
&- 5.89 \\
&+ 0.12 \\
& 0\end{aligned}$ & $\begin{aligned}+368.26 \\
+100.94 \\
+\quad 3.82 \\
0\end{aligned}$ & $\begin{array}{ll} & 0 \\
- & 8.39 \\
+ & 0.06 \\
& 0\end{array}$ & $\begin{array}{l}+1.0 \\
+0.5 \\
+0.3333\end{array}$ \\
\hline $\begin{array}{r}1,-3 \\
-2 \\
-1 \\
0 \\
+1 \\
+2\end{array}$ & $\begin{array}{rr}+ & 0.21 \\
& 5.21 \\
+ & 134.19 \\
& 25.45 \\
+ & 1.12 \\
-\quad 0.05\end{array}$ & $\begin{array}{r}-0.01 \\
-0.63 \\
+\quad 0.31 \\
-1143 \\
+1.19 \\
+0.01\end{array}$ & $\begin{array}{c}+\quad 0.7 \\
\pm \quad 4.93 \\
+133.99 \\
+18.06 \\
+\quad 0.5 \\
+\quad 0.02\end{array}$ & $\begin{array}{ll}- & 0.2 \\
+ & 1.27 \\
- & 0.08 \\
+ & 0.02 \\
+ & 0.1 \\
+ & 0.02\end{array}$ & $\begin{array}{r}+\quad 1.6 \\
-295 \\
+509.94 \\
+8457 \\
+\quad 5.53 \\
-\quad 0.24\end{array}$ & $\begin{array}{cc}+ & 0.2 \\
+ & 0.6 \\
+ & 0.77 \\
-11.47 \\
+ & 0.82 \\
0\end{array}$ & $\begin{array}{c}+2.1 \\
+43.5 \\
+1057.49 \\
\quad 84.57 \\
+\quad 7.31 \\
-\quad 0.32\end{array}$ & $\begin{array}{cc}+ & 0.1 \\
& 1.1 \\
+ & 1.99 \\
& 11.47 \\
+ & 1.07 \\
+ & 0.02\end{array}$ & $\begin{array}{r}-0.40158 \\
-\quad 0.67107 \\
+2.04023 \\
+1.96137 \\
+0.66232 \\
+\quad 0.39843\end{array}$ \\
\hline $\begin{array}{r}2,-4 \\
-3 \\
-2 \\
-1 \\
0 \\
+1\end{array}$ & $\begin{array}{cc}+ & 0.85 \\
+ & 13.53 \\
+ & 375.92 \\
& 60.090 \\
+ & 3.31 \\
- & 0.09\end{array}$ & $\begin{array}{l}-0.03 \\
-1.01 \\
+0.36 \\
-3.180 \\
+0.46 \\
-0.01\end{array}$ & $\begin{array}{l}+\quad 2.1 \\
+27.7 \\
+751.59 \\
-117903 \\
+\quad 5.9 \\
+\quad 0.09\end{array}$ & $\begin{aligned} & 0 \\
+ & 3.1 \\
+ & 0.73 \\
+ & 3.203 \\
- & 0.4 \\
+ & 0.02\end{aligned}$ & $\begin{array}{r}+\quad 2.5 \\
+\quad 18.7 \\
+97.82 \\
+171.79 \\
+\quad 12.24 \\
+\quad 0.51\end{array}$ & $\begin{array}{c}+0.1 \\
\pm 2.1 \\
+1.25 \\
-13.13 \\
+1.84 \\
-0.08\end{array}$ & $\begin{array}{r}+\quad 4.8 \\
+\quad 59.7 \\
+2491.69 \\
+5931.21 \\
+\quad 12.24 \\
+\quad 0.60\end{array}$ & $\begin{array}{cc} & 0 \\
-\quad 5.5 \\
+\quad 2.72 \\
+309.82 \\
+\quad 1.84 \\
-\quad 0.09\end{array}$ & $\begin{array}{l}-0.33554 \\
-0.50497 \\
-1.02009 \\
+50.7820 \\
+\quad 0.98069 \\
+\quad 0.49512\end{array}$ \\
\hline $\begin{array}{r}3,5 \\
-4 \\
-3 \\
-2 \\
-1 \\
0\end{array}$ & $\begin{array}{rr}+ & 0.83 \\
+ & 12.09 \\
+ & 200.37 \\
+\quad 5233 \\
+\quad 5.05 \\
+\quad 0.24\end{array}$ & $\begin{array}{r}0.04 \\
-0.96 \\
-0.04 \\
-9.88 \\
+\quad 1.56 \\
-0.07\end{array}$ & $\begin{array}{c}+\quad 27 \\
+\quad 36.9 \\
+601.13 \\
+150.02 \\
+\quad 13.8 \\
-\quad 0.58\end{array}$ & $\begin{array}{l}+0.2 \\
+3.8 \\
+0.42 \\
+19.81 \\
+3.1 \\
+\quad 0.15\end{array}$ & $\begin{array}{r}+\quad 3.0 \\
+\quad 34.5 \\
+717.94 \\
-190.33 \\
+\quad 20.12 \\
-\quad 1.11\end{array}$ & $\begin{array}{l}-0.1 \\
-3.6 \\
+\quad 0.81 \\
-27.51 \\
+4.65 \\
-0.30\end{array}$ & $\begin{array}{c}+\quad 5.5 \\
+73.6 \\
+1535.51 \\
-635.23 \\
+\quad 1.0 \\
-\quad 1.11\end{array}$ & $\begin{array}{cc}-\quad & 0.2 \\
-\quad & 6.7 \\
+\quad & 0.65 \\
-111.69 \\
-\quad 1.2 \\
-\quad & 0.30\end{array}$ & $\begin{array}{r}-0.28814 \\
-0.40478 \\
-0.68006 \\
-2.12559 \\
+1.88844 \\
+0.65378\end{array}$ \\
\hline $\begin{array}{r}4,6 \\
-5 \\
-4 \\
-3 \\
-2 \\
-1\end{array}$ & $\begin{array}{cc}+\quad 0.75 \\
+\quad 9.47 \\
+\quad 111.16 \\
+\quad 38.58 \\
+\quad 5.205 \\
-\quad 0.36\end{array}$ & $\begin{array}{l}-0.07 \\
-0.79 \\
-0.12 \\
-7.43 \\
+1.896 \\
-0.17\end{array}$ & $\begin{array}{r}+\quad 3.2 \\
+\quad 38.3 \\
+444.7 \\
149.10 \\
+\quad 19.54 \\
-\quad 1.28\end{array}$ & $\begin{array}{l}+\quad 0.3 \\
+\quad 4.0 \\
+0.18 \\
+22.37 \\
+5.50 \\
+\quad 1.49\end{array}$ & $\begin{array}{r}+3.4 \\
+\quad 38.5 \\
+512.23 \\
+178.15 \\
+\quad 25.30 \\
-\quad 1.90\end{array}$ & $\begin{array}{r}-0.2 \\
-3.9 \\
+\quad 0.35 \\
-27.95 \\
+\quad 7.19 \\
-0.70\end{array}$ & $\begin{array}{c}+\quad 5.7 \\
+\quad 70.4 \\
+965.7 \\
-419.12 \\
-503.3 \\
-\quad 1.21\end{array}$ & $\mid \begin{array}{ll}- & 0.4 \\
- & 6.6 \\
- & 0.1 \\
- & 74.36 \\
-185.4 \\
-\quad & 0.37\end{array}$ & $\begin{array}{r}-0.25249 \\
-0.33777 \\
-0.51004 \\
-1.14100 \\
+25.3910 \\
+0.96210\end{array}$ \\
\hline $\begin{array}{r}5,-7 \\
=6 \\
=5 \\
=4 \\
=3 \\
=2 \\
=1\end{array}$ & $\begin{array}{rr}+ & 0.65 \\
+ & 6.97 \\
+ & 63.03 \\
& 27.43 \\
+ & 4.84 \\
& 0.45 \\
+ & 0.03\end{array}$ & $\begin{array}{r}-0.07 \\
-0.59 \\
-0.16 \\
-5.32 \\
+1.81 \\
-0.24 \\
+0.02\end{array}$ & $\begin{array}{r}+3.5 \\
+\quad 35.1 \\
+315.3 \\
-133.4 \\
+\quad 23.0 \\
\bar{T} \quad 2.09 \\
+\quad 0.12\end{array}$ & $\begin{array}{l}+21.4 \\
+7.0 \\
+\quad 0.90 \\
+\quad 0.06\end{array}$ & $\begin{array}{r}+\quad 3.5 \\
+\quad 36.7 \\
+354.8 \\
-153.6 \\
+\quad 27.82 \\
+\quad 2.77 \\
+\quad 0.18\end{array}$ & $\begin{array}{c}-0.3 \\
-3.4 \\
-0.1 \\
-25.3 \\
+8.57 \\
+1.14 \\
+0.09\end{array}$ & $\begin{array}{r}+\quad 5.5 \\
+\quad 60.9 \\
+612.0 \\
-304.8 \\
+\quad 92.2 \\
+\quad 0.48 \\
+\quad 0.15\end{array}$ & $\begin{array}{cc}- & 0.5 \\
- & 5.5 \\
= & 0.7 \\
& 54.6 \\
+ & 32.7 \\
+ & 0.57 \\
+ & 0.07\end{array}$ & $\begin{array}{l}-0.22468 \\
-0.28984 \\
-0.40804 \\
-0.68929 \\
-2.21843 \\
+1.82073 \\
+0.6455\end{array}$ \\
\hline $\begin{array}{r}6,-7 \\
-6 \\
-5 \\
-4 \\
-3 \\
-2\end{array}$ & $\begin{array}{cc}+ & 4.93 \\
+ & 36.16 \\
& 19.04 \\
+ & 4.14 \\
+\quad 0.490 \\
+\quad 0.036\end{array}$ & $\begin{array}{l}-0.41 \\
-0.17 \\
-374 \\
+1.59 \\
-0.271 \\
+0.026\end{array}$ & $\begin{array}{r}+298 \\
+217.1 \\
-111.6 \\
+\quad 23.9 \\
+\quad 2.79 \\
+\quad 0.20\end{array}$ & $\begin{array}{r}+18.8 \\
+7.8 \\
+\quad 1.30 \\
-0.12\end{array}$ & $\begin{array}{r}+31.5 \\
+239.9 \\
-125.7 \\
+\quad 27.76 \\
+\quad 3.42 \\
+\quad 0.28\end{array}$ & $\begin{array}{r}-2.4 \\
+9.06 \\
+0.16\end{array}$ & $\begin{array}{r}+\quad 49.0 \\
+387.4 \\
+223.8 \\
+\quad 63.0 \\
+\quad 46.40 \\
+\quad 0.14\end{array}$ & $\begin{array}{l}-\quad 4.4 \\
-\quad 1.1 \\
-\quad 40.6 \\
+\quad 22.6 \\
+\quad 26.01 \\
+\quad 0.06\end{array}$ & $\begin{array}{r}-0.2538 \\
-0.3400 \\
-0.5152 \\
-1.0628 \\
+16.9273 \\
+0.9443\end{array}$ \\
\hline $\begin{array}{r}7-8 \\
-7 \\
-6 \\
=5 \\
=4 \\
-3\end{array}$ & $\begin{array}{cc}+\quad & 3.41 \\
+ & 20.90 \\
& 12.99 \\
+ & 3.40 \\
+\quad & 0.487 \\
+ & 0.044\end{array}$ & $\begin{array}{l}-0.28 \\
-0.16 \\
-2.54 \\
+1.31 \\
+0.276 \\
+0.034\end{array}$ & $\begin{array}{r}+24.0 \\
+146.4 \\
+89.2 \\
+\quad 23.0 \\
+\quad 3.25 \\
+\quad 0.25\end{array}$ & $\begin{array}{r}+2.1 \\
+0.4 \\
+15.4 \\
+7.7 \\
+1.61 \\
-0.19\end{array}$ & $\begin{array}{c}+25.5 \\
+1600 \\
+98.9 \\
+\quad 26.0 \\
+\quad 3.79 \\
+\quad 0.37\end{array}$ & $\begin{array}{r}-2.5 \\
-0.6 \\
-17.2 \\
+8.9 \\
-1.87 \\
+0.24\end{array}$ & $\begin{array}{r}+37.8 \\
+245.3 \\
+163.1 \\
+49.8 \\
-12.83 \\
-\quad 0.09\end{array}$ & $\begin{array}{cc}- & 3.5 \\
- & 1.3 \\
\bar{T} & 29.8 \\
\pm & 18.0 \\
- & 6.99 \\
- & 0.12\end{array}$ & $\begin{array}{r}-0.2257 \\
-0.2914 \\
-0.4113 \\
-0.6988 \\
-2.3198 \\
+\quad 1.7577\end{array}$ \\
\hline $\begin{array}{r}8-9 \\
-8 \\
-7 \\
-6 \\
-5 \\
-4\end{array}$ & $\begin{array}{cc}+ & 2.3 .5 \\
+ & 12.20 \\
& 8.8 \\
+ & 269 \\
+ & 045 \\
+ & 0.049\end{array}$ & $\begin{array}{l}-0.20 \\
-0.13 \\
-1.71 \\
+1.05 \\
-0.26 \\
+\quad 0.039\end{array}$ & $\begin{array}{c}+18.8 \\
+\quad 96.6 \\
-68.9 \\
+\quad 21.0 \\
+\quad 3.5 \\
+\quad 0.37\end{array}$ & $\begin{array}{l}+1.6 \\
+03 \\
+12.1 \\
+7.1 \\
+1.8 \\
-0.26\end{array}$ & $\begin{array}{c}75.5 \\
+\quad 23.2 \\
+\quad 3.90 \\
+\quad 0.45\end{array}$ & $\begin{array}{c}-13.1 \\
+8.1 \\
+1.99 \\
+\quad 0.31\end{array}$ & $\begin{array}{c}+28.1 \\
+154.8 \\
+117.7 \\
+40.0 \\
-\quad 8.8 \\
-\quad 4.50\end{array}$ & $\left|\begin{array}{cc}- & 1.2 \\
- & 1.3 \\
- & 21.3 \\
+ & 14.7 \\
- & 4.8 \\
- & 3.69\end{array}\right|$ & $\begin{array}{r}-0.2032 \\
-0.2550 \\
-0.3423 \\
-0.5205 \\
-1.0855 \\
+12.6955\end{array}$ \\
\hline $\begin{array}{r}9,-9 \\
-8 \\
-7 \\
-6 \\
-5\end{array}$ & $\begin{array}{ll}+ & 7.0 \\
& 5.9 \\
+ & 2.07 \\
+ & 0.39 \\
+ & 0.050\end{array}$ & $\begin{array}{ll}- & 0.1 \\
- & 1.13 \\
+ & 0.81 \\
- & 0.25 \\
+ & 0.042\end{array}$ & $\begin{array}{r}+\quad 63.4 \\
+519 \\
+\quad 18.1 \\
\quad 3.4 \\
+\quad 0.43\end{array}$ & $\begin{array}{r}+0.1 \\
+9.1 \\
+6.4 \\
+1.9 \\
-0.32\end{array}$ & $\begin{array}{c}+68.2 \\
+563 \\
+19.8 \\
+\quad 3.78 \\
+\quad 0.51\end{array}$ & $\begin{array}{r}-0.6 \\
-9.9 \\
+7.0 \\
+2.02 \\
+0.36\end{array}$ & $\begin{array}{c}+96.8 \\
+84.0 \\
+31.8 \\
+\quad 7.1 \\
+\quad 1.72\end{array}$ & $\mid \begin{array}{cc}- & 1.0 \\
- & 15.2 \\
+ & 11.7 \\
+ & 4.1 \\
+ & 1.38\end{array}$ & $\begin{array}{r}-0.2267 \\
-0.2931 \\
-0.4147 \\
=0.7085 \\
-2.4308\end{array}$ \\
\hline $\begin{array}{r}0,-10 \\
-9 \\
-8 \\
-7 \\
-6 \\
\end{array}$ & $\begin{array}{cc}+ & 4.1 \\
+ & 3.9 \\
+ & 1.6 \\
+ & 0.3 \\
+ & 0.08\end{array}$ & $\begin{array}{l}-0.1 \\
=0.8 \\
+0.6 \\
+0.2 \\
+\quad 0.04 \\
\end{array}$ & $\begin{array}{cc}+ & 43 . \\
& 40 . \\
+ & 14.7 \\
& 3.1 \\
+ & 0.46\end{array}$ & $\begin{array}{r}0 \\
+\quad 6 . \\
5.6 \\
+\quad 1.9 \\
-0.37\end{array}$ & $\begin{array}{c}+44.3 \\
+42.0 \\
+\quad 16.1 \\
+\quad 3.5 \\
+\quad 0.53\end{array}$ & $\begin{array}{l}-0.5 \\
-7.0 \\
+6.0 \\
+1.9 \\
+0.40\end{array}$ & $\begin{array}{c}+61 . \\
+60 . \\
+25.1 \\
+\quad 5.8 \\
+\quad 1.16\end{array}$ & $\begin{array}{cc}- & 0.8 \\
& 11 . \\
+ & 9.3 \\
& 3.4 \\
+ & 0.96\end{array}$ & $\begin{array}{r}-0.2040 \\
-0.2563 \\
-0.3446 \\
-0.5259 \\
-1.1092\end{array}$ \\
\hline
\end{tabular}




\section{The term of Long Period.}

From the expressions for the perturbations of Uranus, subsequently given, it will be seen that several of the terms hâve very large coefficients, that of $\sin (2 l-g)$ being nearly an entire degree. The magnitude of most of the terms in which ${ }^{\prime}$ ' is even arises from the near approach to commensurability in the mean motions of the two planets. Twice the mean, annual motion of Neptune exceeds that of Uranus by only $303^{\prime \prime} .8$. The elements of the orbits of both planets will therefore, in consequence of their mutual action, be affected with a slow oscillation, having a period of about 4266 years. The employment of these large terms and the great inconveniences to which they will give rise, especially in the corrections of the elements of Uranus, may be avoided by the device employed in the theory of Neptune. The following are the essential features of this method:

First, all the perturbations arising from that portion of the perturbative function in which the coefficient of the time is $2 n^{\prime}-n$ or its multiples are considered and developed as perturbations of the elements.

Secondly, the arbitrary constants to be added to the integrals of these perturbations are so taken that the perturbations shall vanish at the epoch 1850.0.

In other words, the perturbations in question will be treated as producing secular variations of the elements of the orbit, only, instead of being developed in powers of the time, these variations will be retained in their rigorous form.

The formulæ for the computations of the perturbations in question, are as follows:

Let

$$
\frac{m^{\prime}}{a_{1}} h \cos \left(i^{\prime} l^{\prime}+i l+j^{\prime} \omega^{\prime}+j \omega\right)=\frac{m^{\prime}}{a_{1}} \cos N
$$

be any term of the perturbative function, $h$ being a function of $\alpha, e$, and $\sigma$.

$$
\begin{aligned}
& \sin \psi=e \\
& g=\cos \psi \tan \frac{1}{2} \psi \\
& \iota=-\left(i^{\prime}+i+j^{\prime}+j\right) \\
& v=\frac{n}{i^{\prime} n^{\prime}+i n} .
\end{aligned}
$$

For each such term, compute

$$
\begin{aligned}
A & =2 i \hbar \\
L & =-3 i \nu h-2 \frac{\partial h}{\partial x}+\frac{\partial h}{\partial e} \\
e W & =\cos \psi \frac{\partial h}{\partial e} \\
E & =-h(i g+j \cot \psi) \\
T & =\frac{1}{2} \frac{\partial h}{\partial \sigma} \\
I & =\frac{1}{2} \iota \frac{h}{\sigma}+(i+j) \sigma h
\end{aligned}
$$


The corresponding perturbations of the elements may then be put into the form

$$
\begin{aligned}
\delta \log a & =M \nu A \cos N+\delta 0_{0}, \\
\delta l & =M \nu L \sin N+\delta l_{0}, \\
e \delta \pi & =M \nu \omega \sin N+e \delta \pi_{0}, \\
\delta e & =M \nu E \cos N+\delta e_{0}, \\
\delta \gamma & =M \nu I \cos N+\delta \gamma_{0} \\
\tan \gamma \delta \tau & =M \nu T \sin N+\tan \gamma \delta \tau_{0} .
\end{aligned}
$$

Here, $\delta x_{0}, \delta Z_{0}$, etc., are arbitrary constants so taken that $\delta \log a, \delta l_{\text {, etc., shall }}$ vanish at the fundamental epoch.

All the terms depending on the same values of $i^{\prime}$ and $i$ are to be combined into a single one. And it will save labor to make this combination at as carly a stage as possible in the computation; that is, to multiply the various values of $h$, $\frac{\partial \hbar}{\partial x}, \frac{\partial \hbar}{\partial e}, \frac{\partial h}{\partial \sigma}$, and $E$ by the sines and cosines of $j^{\prime} \omega^{\prime}+j \omega$, and afterward proceed with the sums of the products according to the proper modification of the formula.

Thus are obtained the following long period perturbations of the elements of Uranus :

$$
\begin{aligned}
& \delta l=-3474.32 \sin \left(2 l^{\prime}-g\right)+180.10 \cos \left(2 l^{\prime}-g\right) \\
& +146.72 \sin \left(4 l^{\prime}-2 g\right)-54.10 \cos \left(4 l^{\prime}-2 g\right) \\
& \text { - } 8.97 \sin \left(6 l^{\prime}-3 g\right)+5.03 \cos \left(6 l^{\prime}-3 g\right) \\
& +\quad 0.64 \sin \left(8 l^{\prime}-4 g\right)-0.53 \cos \left(8 l^{\prime}-4 g\right) \\
& + \text { constant }=3320^{\prime \prime} .18 \text {. }
\end{aligned}
$$

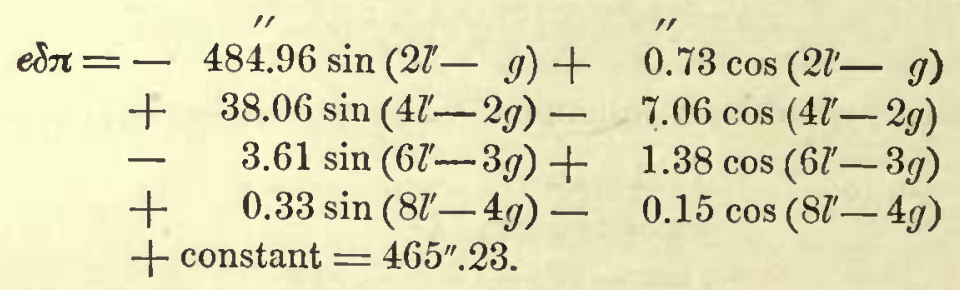

$$
\begin{aligned}
& =-484.21 \cos \left(2 l^{\prime}-g\right)-\quad 0.29 \sin \left(2 l^{\prime}-g\right) \\
& +\quad 38.21 \cos \left(4 l^{\prime}-2 g\right)+7.16 \sin \left(4 l^{\prime}-2 g\right) \\
& \text { - } 3.61 \cos \left(6 t^{\prime}-3 g\right)-1.40 \sin \left(6 t^{\prime}-3 g\right) \\
& +\quad 0.33 \cos \left(8 l^{\prime}-4 g\right)+0.15 \sin \left(8 l^{\prime}-4 g\right) \\
& \text { - constant }=158^{\prime \prime} .59 \text {. } \\
& \delta 0=+2277 \cos \left(2 l^{\prime}-g\right)+120 \sin \left(2 l^{\prime}-g\right) \\
& \text { - } \quad 198 \cos \left(4 l^{\prime}-2 g\right)-78 \sin \left(4 l^{\prime}-2 g\right) \\
& +18 \cos \left(6 l^{\prime}-3 g\right)+7 \sin \left(6 l^{\prime}-3 g\right) \\
& + \text { constant }=630 \text {. }
\end{aligned}
$$

The variations of the elements which fix the position of the plane of the orbit are here omitted, because their nature is such that it is indifferent in which form they are developed.

These expressions are reduced to perturbations of the co-ordinates by the follow- 
ing formulæ. Express the usual developments of the longitude and logarithm of radius vector in the form

$$
\begin{aligned}
& v=l+\Sigma V_{i} \sin i g ; \\
& \rho=x+\Sigma R_{i} \cos i g .
\end{aligned}
$$

Put also

$$
\begin{aligned}
& V_{i}^{\prime}=\frac{\partial V_{i}}{\partial e} \\
& V_{i}^{\prime \prime}=\frac{i}{e} V_{i} \\
& R_{i}^{\prime}=\frac{\partial R_{i}}{\partial e} \\
& R_{i}^{\prime \prime}=-\frac{i}{e} R_{i} .
\end{aligned}
$$

Express any set of corresponding terms of the preceding perturbations in the form

We shall then have

$$
\begin{aligned}
\imath l & =L_{s} \sin N+L_{c} \cos N ; \\
e \imath-e \delta \pi & =F_{s} \sin N+F_{e} \cos N ; \\
\delta e & =E_{c} \cos N+E_{s} \sin N ; \\
\delta \varpi & =A_{s} \cos N+A_{s} \sin N .
\end{aligned}
$$

$$
\begin{aligned}
2 \delta v & =\Sigma\left(V_{i i}^{\prime \prime} F_{s}+V_{i}^{\prime} E_{c}\right) \sin (N+i g)+\Sigma\left(V^{\prime \prime}{ }_{i} F_{s}-V_{i}^{\prime} E_{c}\right) \sin (N-i g) \\
& +\Sigma\left(V^{\prime \prime}{ }_{i} F_{c}-V_{i}^{\prime} E_{s}\right) \cos (N+i g)+\Sigma\left(V_{i}^{\prime \prime} F_{c}+V_{i}^{\prime} E_{s}\right) \cos (N-i g) \\
& +2 \delta l \\
2 \delta_{\rho} & =\Sigma\left(R_{i}^{\prime} E_{c}-R_{i}^{\prime \prime} F_{s}\right) \cos (N+i g)+\Sigma\left(R_{i}^{\prime} E_{c}+R_{i}^{\prime \prime} F_{s}\right) \cos (N-i g) \\
& +\Sigma\left(R_{i}^{\prime} E_{s}+R_{i}^{\prime \prime} F_{c}\right) \sin (N+i g)+\Sigma\left(R_{i} E_{s}-R_{i}^{\prime \prime} F_{c}\right) \sin (N-i g) \\
& +2 S_{0}
\end{aligned}
$$

The numerical values of $V^{\prime}, V^{\prime}, R^{\prime}$, and $R^{\prime \prime}$ are as follows:

$$
\begin{array}{ll}
V_{1}=1.99835 & V^{\prime \prime}{ }_{1}=1.99945 \\
V_{2}=0.11713 & V^{\prime \prime}{ }_{2}=0.11722 \\
V_{3}^{\prime}=0.00714 & V^{\prime \prime}{ }_{3}=0.00714 \\
V_{4}^{\prime}=0.00044 & V^{\prime \prime}=0.00044 \\
R_{0}^{\prime}=+0.02348 & \\
\dot{R}_{1}^{\prime}=-0.99753 & R_{1}^{\prime \prime}=+0.99917 \\
R_{2}^{\prime}=-0.07020 & R^{\prime \prime}{ }_{2}=+0.07030 \\
R_{3}=-0.00466 & R_{3}^{\prime \prime}=+0.00467
\end{array}
$$

The final results of the entire computations are given in the following table: In the columns $\delta v_{1}$, we have the complete perturbations of the longitude computed by the direct method from the values of $\frac{\partial R}{\partial \mathrm{v}}, \frac{\partial R}{\partial \rho}, Q$, etc., already given. Next we have, under the caption $i v_{2}$, the perturbations of the true longitude deduced from the long period perturbations of the elements, as set forth in the last paragraph, omitting the constants added to the perturbations. Under $\delta v_{3}$ we have the 
perturbations of the longitude deduced from all the remaining terms of the perturbations of the elements. The sum of the columns $\delta v_{2}$ and $\delta v_{3}$ shows the entire perturbations computed by the method of variation of elements. 'Thus, in $\delta v_{1}$ and $\delta v_{2}+\delta v_{3}$ we have two complete sets of perturbations computed by methods entirely independent. The differences of the results, expressed in thousandths of a sccond, are given in the last two columns of the table.

This comparison gives rise to remarks similar to those suggested by the perturbations of Saturn computed by the same methods. The only terms in which the difference of results amounts to as much as one-tenth of a second are those of very long period, and those very nearly the period of Uranus, where a more accurate value is not at present of great importance, because the error will be compensated by the corrections of the element during several centuries.

\begin{tabular}{|c|c|c|c|c|c|c|c|c|}
\hline \multirow[b]{3}{*}{$l^{\prime} g$} & \multicolumn{8}{|c|}{ Perturbations of the Longitude of Uranus producen by Neptune. } \\
\hline & \multicolumn{2}{|c|}{$\delta v_{1}$} & \multicolumn{2}{|c|}{$\delta v_{2}$} & \multicolumn{2}{|c|}{$\delta v_{3}$} & \multicolumn{2}{|c|}{ Discrepaney. } \\
\hline & $\sin ^{\prime}$ & $\stackrel{\cos }{\prime \prime}$ & $\sin$ & $\cos$ & $\sin$ & $\cos$ & $\sin$ & $\cos$ \\
\hline $\begin{array}{r}0, \quad 0 \\
-1 \\
-2\end{array}$ & $\begin{array}{l}-.065 n t \\
-.004 n t\end{array}$ & $\begin{array}{l}-0.4262 t \\
-1.181 n t \\
-0.069 n t\end{array}$ & & & & & & \\
\hline $\begin{array}{r}0, \quad 0 \\
-1 \\
-2 \\
-3\end{array}$ & $\begin{array}{r}+\quad 0.697 \\
+\quad 0.046 \\
+\quad 0.003\end{array}$ & $\begin{array}{l}-0.088 \\
-\quad 0.005\end{array}$ & & & & & & \\
\hline $\begin{array}{r}1,-3 \\
-2 \\
-1 \\
0 \\
-1 \\
-2\end{array}$ & $\begin{array}{rr}+\quad 0.147 \\
+\quad 2.509 \\
+\quad 39.658 \\
+\quad 4.257 \\
+\quad 0.280 \\
+\quad 0.017\end{array}$ & $\begin{array}{ll}- & 0.001 \\
- & 0.019 \\
- & 0.080 \\
- & 0.511 \\
- & 0.032 \\
- & 0.002\end{array}$ & $\begin{array}{l}\ldots \\
\ldots \\
\cdots \\
\cdots\end{array}$ & $\begin{array}{l}\ldots \\
\cdots \\
\cdots \\
\cdots\end{array}$ & $\begin{array}{r}+0.146 \\
+2.509 \\
+39.673 \\
+4249 \\
+\quad 0.275\end{array}$ & $\begin{array}{l}-0.002 \\
-0.010 \\
-0.081 \\
-0.478 \\
-0.032\end{array}$ & $\begin{array}{r}1 \\
0 \\
15 \\
8 \\
5\end{array}$ & $\begin{array}{r}1 \\
9 \\
1 \\
33 \\
0\end{array}$ \\
\hline $\begin{array}{r}2,-4 \\
-3 \\
-2 \\
-1 \\
0 \\
-1\end{array}$ & $\begin{array}{cc}+\quad 2.978 \\
+\quad 49.015 \\
+\quad 840.93 \\
-3475.4 \\
-\quad 162.07 \\
-\quad 9.447\end{array}$ & $\begin{array}{cc}+\quad & 0.027 \\
+\quad & 0.413 \\
+\quad & 7.388 \\
+ & 180.36 \\
+\quad & 8.01 \\
+\quad & 0.468\end{array}$ & $\begin{array}{r}+\quad 2.89 \\
+\quad 47.22 \\
+\quad 805.64 \\
-3474.32 \\
-\quad 161.96 \\
-\quad 9.50\end{array}$ & $\begin{array}{l}+\quad 0.03 \\
+\quad 0.44 \\
+\quad 7.43 \\
+180.10 \\
+\quad 8.01 \\
+\quad 0.47\end{array}$ & $\begin{array}{r}+\quad 0.098 \\
+\quad 1.797 \\
+35.355 \\
-0.700 \\
-0.067\end{array}$ & $\begin{array}{r}-0.002 \\
-0.021 \\
-0.028 \\
+0.095 \\
+0.015\end{array}$ & $\begin{array}{r}10 \\
2 \\
65 \\
380 \\
43\end{array}$ & $\begin{array}{r}1 \\
6 \\
14 \\
165 \\
15\end{array}$ \\
\hline $\begin{array}{l}3,-5 \\
-4 \\
-3 \\
-2 \\
-1 \\
0\end{array}$ & $\begin{array}{rr}- & 0.076 \\
- & 1.162 \\
- & 17.286 \\
- & 22.085 \\
- & 0.673 \\
- & 0.037\end{array}$ & $\begin{array}{r}0.000 \\
+\quad 0.000 \\
+\quad 0.228 \\
+\quad 4.037 \\
+\quad 0.082 \\
+\quad 0.006\end{array}$ & $\begin{array}{l}\ldots \\
\ldots \\
\ldots \\
\ldots \\
\ldots\end{array}$ & $\begin{array}{l}\ldots \\
\ldots \\
\cdots \\
\cdots \\
\cdots\end{array}$ & $\begin{array}{l}-0.077 \\
-1.153 \\
-17.285 \\
-22.077 \\
-0.682\end{array}$ & $\begin{array}{l}-0.003 \\
-0.011 \\
+0.229 \\
+4.020 \\
+0.079\end{array}$ & $\begin{array}{l}1 \\
9 \\
1 \\
8 \\
9\end{array}$ & $\begin{array}{r}3 \\
11 \\
1 \\
17 \\
3\end{array}$ \\
\hline $\begin{array}{l}4,6 \\
-5 \\
-4 \\
-3 \\
-2 \\
-1\end{array}$ & $\begin{array}{cc}- & 0.036 \\
- & 0.558 \\
- & 7.968 \\
- & 75.00 \\
+ & 146.78 \\
+\quad & 6.960\end{array}$ & $\begin{array}{rr}+\quad & 0.002 \\
+ & 0.037 \\
+ & 0.750 \\
+\quad 12.832 \\
+\quad 54.218 \\
-\quad 2.579\end{array}$ & $\begin{array}{l}-\quad 0.015 \\
-\quad 0.25 \\
-\quad 4.08 \\
-\quad 69.55 \\
+146.72 \\
+\quad 6.81\end{array}$ & $\begin{array}{l}+0.002 \\
+0.04 \\
+\quad 0.68 \\
+11.67 \\
-54.10 \\
-2.63\end{array}$ & $\begin{array}{r}-0.027 \\
-\quad 0.315 \\
-\quad 3.908 \\
-5.733 \\
+\quad 0.126\end{array}$ & $\begin{array}{r}0.003 \\
-0.007 \\
+0.059 \\
+1.067 \\
-0.079\end{array}$ & $\begin{array}{r}6 \\
7 \\
20 \\
283 \\
66\end{array}$ & $\begin{array}{r}3 \\
4 \\
11 \\
95 \\
39\end{array}$ \\
\hline
\end{tabular}




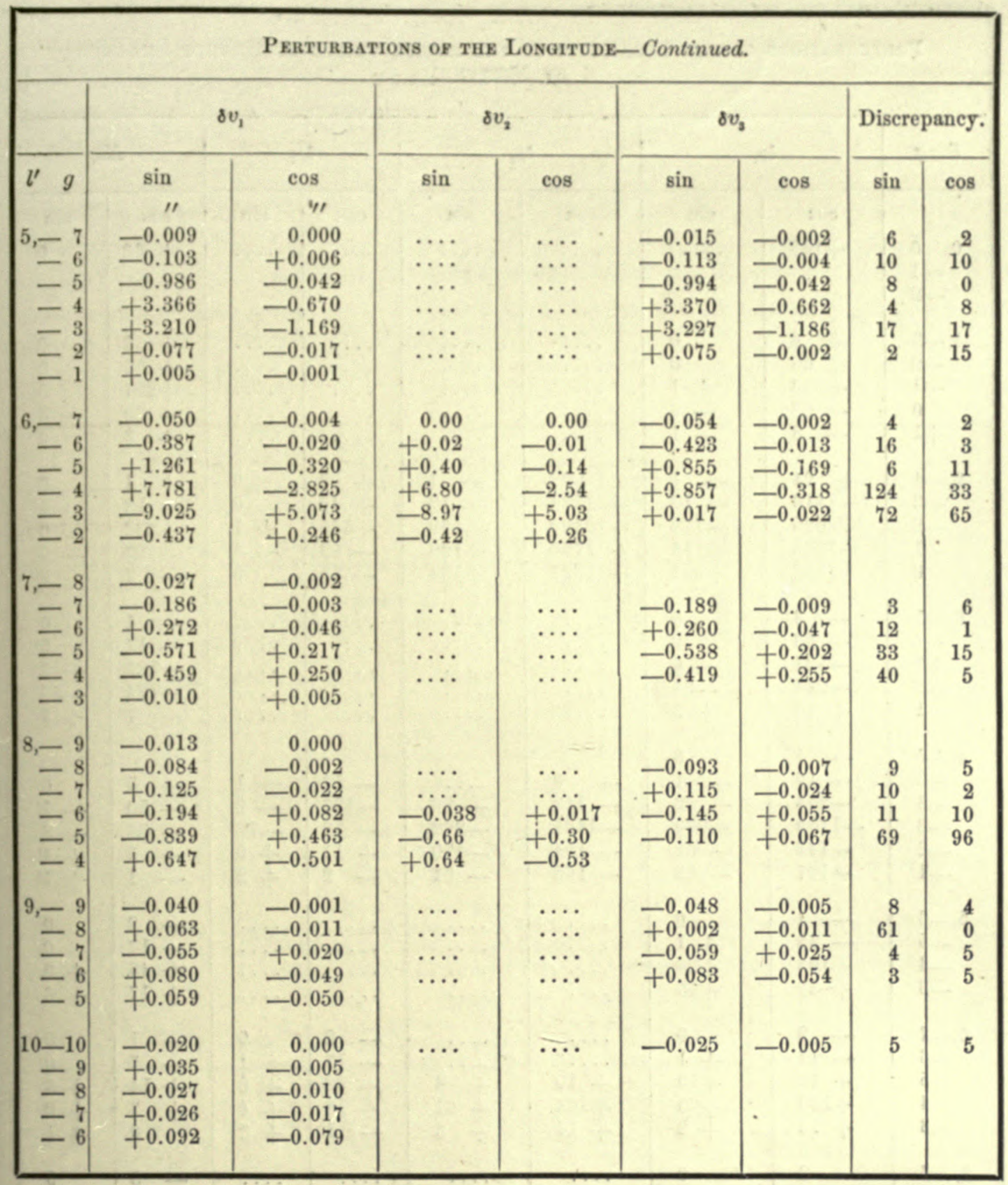

The perturbations of the logarithms of the radius vector are given in a form similar to those of the longitude. Under $\delta \rho_{1}$ we have the complete perturbation. Under $\delta \rho_{2}$ the effect of the perturbations of long period. But under $\delta \rho_{3}$ we have only the difference between $\delta \rho_{1}$ and $\delta \rho_{2}$, it being deemed unnecessary to present in full the perturbations of the radius vector as computed by the other method. $\delta \rho_{1}$ being employed in computing $\delta v_{1}$ may, in fact, be regarded as completely checked by its affording a correct yalue of the latter.

In the last two columns $\delta \rho_{3}$ is reduced to common logarithms by multiplying the coefficients by the modulus 0.434294 . 


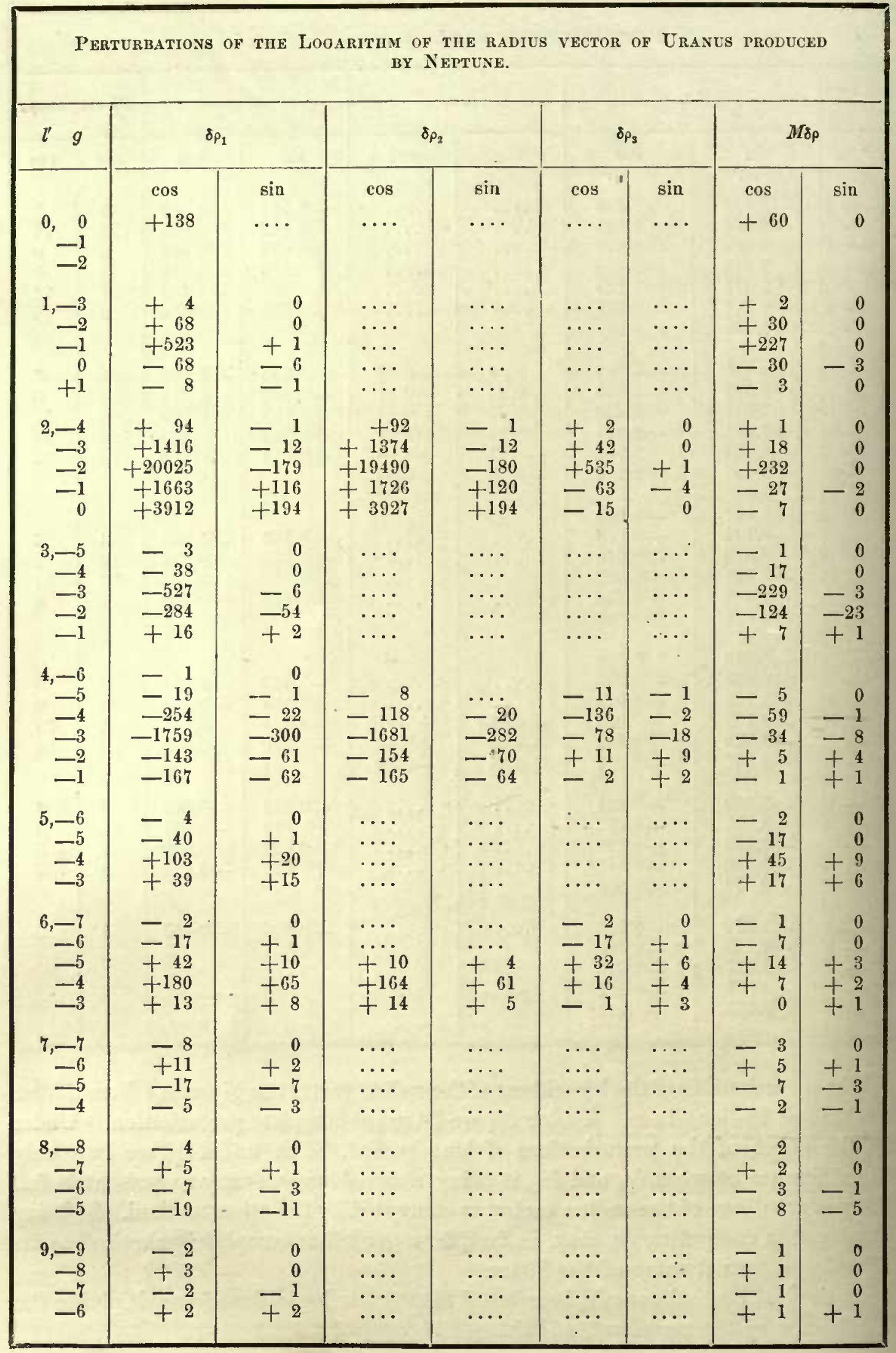




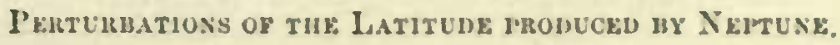

\begin{tabular}{|c|c|c|c|c|c|c|c|c|c|c|c|c|c|c|}
\hline \multirow[b]{2}{*}{$i^{\prime} \quad i$} & \multicolumn{2}{|c|}{$\frac{\partial R}{\partial r}=\frac{m^{\prime}}{a_{1}} \times$} & \multicolumn{2}{|c|}{$=\frac{\pi}{2 y} \sin V$} & \multicolumn{2}{|c|}{$\begin{array}{l}2(i+j) \\
\times h \text { แin } N\end{array}$} & \multicolumn{2}{|c|}{$\frac{d \eta}{d 6}=m^{\prime} a n x$} & \multicolumn{2}{|c|}{82} & \multicolumn{2}{|c|}{$\delta \eta$} & \multicolumn{2}{|c|}{83} \\
\hline & $\cos$ & $\sin$ & sin & $\cos$ & sin & $\cos$ & $\sin$ & $\cos$ & $\sin$ & cos & $\cos$ & $\sin$ & in & $\cos$ \\
\hline $\begin{array}{l}0 \\
1 \\
2\end{array}$ & $\begin{array}{r}21.65 \\
+\quad 1.18 \\
+19.32\end{array}$ & $\begin{array}{c}0 \\
+2.73 \\
-9.39 \\
\end{array}$ & $\begin{array}{c}0 \\
-4.00 \\
+19.32\end{array}$ & $\begin{array}{c}0 \\
-1.66 \\
+9.39\end{array}$ & $\begin{array}{c}0 \\
+.01 \\
-.01\end{array}$ & $\begin{array}{l}0.00 \\
-0.01 \\
0\end{array}$ & $\begin{array}{r}0 \\
-3.09 \\
+19.31\end{array}$ & $\begin{array}{c}0 \\
-1.67 \\
+8.39\end{array}$ & $\begin{array}{r}-0.008 \\
-0.073\end{array}$ & $\begin{array}{r}+0.021 \\
-0.036\end{array}$ & $\begin{array}{r}+0.030 \\
-0.073\end{array}$ & $\begin{array}{c}11 \\
-0.012 \\
+0.036\end{array}$ & $\begin{array}{r}0 \\
+.050 \\
+.016 \\
+.0\end{array}$ & $\begin{array}{r}.11 \\
-.012 \\
+.0120 \\
-.002\end{array}$ \\
\hline $\begin{array}{r}1,-3 \\
-2 \\
-1 \\
0 \\
+1\end{array}$ & $\begin{array}{l}+14.58 \\
-1.16 \\
-342 \\
+3.74 \\
+15.77\end{array}$ & $\begin{array}{c}+7.0 \\
-2.65 \\
0 \\
+2.32 \\
-7.87\end{array}$ & $\begin{array}{l}-14.7 \\
+4.08 \\
0 \\
-16.80 \\
+16.25\end{array}$ & $\begin{array}{c}+7.0 \\
-2.40 \\
0 \\
-1.45 \\
+7.75\end{array}$ & $\begin{array}{c}0 \\
-0.07 \\
+1.76 \\
-0.24 \\
0\end{array}$ & $\begin{array}{c}0 \\
+0.02 \\
0 \\
0 \\
0\end{array}$ & $\begin{array}{l}-147 \\
+397 \\
+1.76 \\
+3.04 \\
+16.25\end{array}$ & $\begin{array}{r}+7.0 \\
-3.4 \\
0 . .2 \\
-1.48 \\
+7.75\end{array}$ & $\begin{array}{l}+0.045 \\
-0.608 \\
-0.540 \\
-0.057 \\
-0.081\end{array}$ & $\begin{array}{c}-0.022 \\
+0.014 \\
0 \\
+0.035 \\
-0.040\end{array}$ & $\begin{array}{r}-0.043 \\
+0.020 \\
+0.028 \\
+0.048 \\
-0.063\end{array}$ & $\begin{array}{c}-0.022 \\
+0.012 \\
0 \\
-0.022 \\
+0.040\end{array}$ & \begin{tabular}{l}
+.007 \\
+.201 \\
\hdashline .008 \\
+.364 \\
+.060
\end{tabular} & $\begin{array}{r}-.002 \\
-.046 \\
-.037 \\
+.084 \\
+.007\end{array}$ \\
\hline $\begin{array}{r}2-4 \\
-3 \\
-2 \\
-1 \\
0\end{array}$ & $\begin{array}{r}+10.3 . \\
-3.60 \\
-33.35 \\
+\quad 3.51 \\
+18.79\end{array}$ & $\begin{array}{c}+5.06 \\
-2.38 \\
0 \\
+2.04 \\
-9.52\end{array}$ & $\begin{array}{c}-10.6 \\
+\quad 3.65 \\
0 \\
-2.12 \\
+19.56\end{array}$ & $\begin{array}{r}+5.0 \\
-2.28 \\
0 \\
-104 \\
+9.32\end{array}$ & $\begin{array}{r}0 \\
+0.38 \\
+9.85 \\
-1.53 \\
+0.08\end{array}$ & $\begin{array}{c}0 \\
+0.04 \\
0.01 \\
+0.04 \\
0\end{array}$ & $\begin{array}{r}-10.6 \\
+4.01 \\
+9.88 \\
+3.65 \\
+19.64\end{array}$ & $\begin{array}{l}+5.0 \\
-2.24 \\
-0.01 \\
-1.00 \\
+9.32\end{array}$ & $\begin{array}{l}+0.027 \\
-0.010 \\
-0.264 \\
{[-2.283]} \\
-0.143\end{array}$ & $\begin{array}{c}-0.013 \\
+0.009 \\
0 \\
+0.802] \\
-0.072\end{array}$ & $\left.\begin{array}{r}-0.027 \\
+0.016 \\
+0.078 \\
{[+1.431]}\end{array}\right]$ & $\left.\begin{array}{c}-0.013 \\
+0.009 \\
0 \\
-0.393\end{array}\right]$ & $\begin{array}{r}+.002 \\
+.062 \\
+.008 \\
+.326 \\
+.002\end{array}$ & $\begin{array}{c}-.001 \\
-.014 \\
-.014 \\
+.075 \\
0\end{array}$ \\
\hline $\begin{array}{r}3,-5 \\
-4 \\
-3 \\
-2 \\
-1 \\
0\end{array}$ & $\begin{array}{l}+7.4 \\
-3.06 \\
-24.4 \\
+6.55 \\
+13.66 \\
-1.51\end{array}$ & $\begin{array}{c}+3.6 \\
-2.00 \\
0 \\
+1.73 \\
-7.28 \\
+0.22\end{array}$ & $\begin{array}{l}-7.4 \\
+3.12 \\
0 \\
-1.20 \\
+14.65 \\
-1.51\end{array}$ & $\begin{array}{c}+3.5 \\
-2.0 \\
0 \\
-0.54 \\
+7.00 \\
-0.22\end{array}$ & $\begin{array}{c}0 \\
+0.48 \\
+7.87 \\
-1.98 \\
+0.18 \\
0\end{array}$ & $\begin{array}{c}0 \\
+0.05 \\
0 \\
+0.26 \\
-0.04 \\
0\end{array}$ & $\begin{array}{r}-7.4 \\
+3.60 \\
+7.87 \\
-3.18 \\
-14.86 \\
-1.31\end{array}$ & $\begin{array}{c}+3.5 \\
-2.0 \\
0 \\
-0.28 \\
+6.96 \\
-0.22\end{array}$ & $\begin{array}{r}+0.017 \\
-0.010 \\
-0.128 \\
+0.108 \\
-0.199 \\
+0.008\end{array}$ & $\begin{array}{c}-0.008 \\
+0.016 \\
0 \\
-0.029 \\
-0.106 \\
+0.001\end{array}$ & $\begin{array}{r}-0.017 \\
+0.011 \\
+0.041 \\
-0.053 \\
-0.217 \\
+0.008\end{array}$ & $\begin{array}{c}-0.008 \\
+0.006 \\
0 \\
+0.004 \\
+0.101 \\
-0.001\end{array}$ & $\begin{array}{r}+.002 \\
+.023 \\
.004 \\
+.303 \\
-.090 \\
-.013\end{array}$ & $\begin{array}{c}0 \\
-.004 \\
+.041 \\
+.074 \\
-.026 \\
0\end{array}$ \\
\hline $\begin{array}{r}4,-5 \\
-4 \\
-3 \\
-2 \\
-1\end{array}$ & $\begin{array}{r}-2.96 \\
-17.4 \\
+6.34 \\
+\quad 9.42 \\
-1.72\end{array}$ & $\begin{array}{c}-1.63 \\
0 \\
+1.34 \\
-3.34 \\
+0.32\end{array}$ & $\begin{array}{l}+2.53 \\
0 \\
-0.59 \\
+1057 \\
-1.72\end{array}$ & $\begin{array}{c}-1.7 \\
0 \\
-0.22 \\
+5.04 \\
-0.32\end{array}$ & $\begin{array}{l}+0.5 \\
+5.8 \\
-1.97 \\
+0.25 \\
+0.02\end{array}$ & $\begin{array}{c}0 \\
0 \\
+0.30 \\
-0.0 \overline{1} \\
+0.02\end{array}$ & $\begin{array}{r}+3.0 \\
+\quad 6.8 \\
-2.56 \\
+10.82 \\
-1.74\end{array}$ & $\begin{array}{c}-1.7 \\
0 \\
+0.08 \\
+4.97 \\
-0.30\end{array}$ & $\begin{array}{c}-0.008 \\
-0.070 \\
+0.051 \\
-1.852] \\
+0.013\end{array}$ & $\begin{array}{c}+0.004 \\
0 \\
-0.011 \\
{[-1.056]} \\
+0.002\end{array}$ & $\begin{array}{l}+0.008 \\
+0.023 \\
-0.020 \\
{[-2.127]} \\
+0.013\end{array}$ & $\left.\begin{array}{c}+0.004 \\
0 \\
-0.001 \\
{[+0.977]} \\
-0.002\end{array}\right]$ & $\begin{array}{r}+.022 \\
.008 \\
+.048 \\
-.047 \\
-.001\end{array}$ & $\begin{array}{c}.004 \\
+.006 \\
+.010 \\
0 \\
0\end{array}$ \\
\hline $\begin{array}{r}5,-6 \\
-5 \\
-4 \\
-3 \\
-2\end{array}$ & $\begin{array}{l}-2.4 \\
12.0 \\
+\quad 5.58 \\
+6.25 \\
-1.66\end{array}$ & $\begin{array}{c}-1.40 \\
0 \\
+1.01 \\
-3.89 \\
+0.33\end{array}$ & $\begin{array}{r}+1.8 \\
0 \\
-0.22 \\
+\quad 7.43 \\
-1.66\end{array}$ & $\begin{array}{c}-1.4 \\
0 \\
-0.04 \\
+334 \\
-0.33\end{array}$ & $\begin{array}{l}+0.5 \\
+4.0 \\
-1.75 \\
+0.30 \\
-0.13\end{array}$ & $\begin{array}{c}0 \\
0 \\
+0.28 \\
0.09 \\
+0.01\end{array}$ & $\begin{array}{l}+2.3 \\
+4.0 \\
+1.97 \\
+7.73 \\
-1.69\end{array}$ & $\begin{array}{c}-1.4 \\
0 \\
+0.24 \\
+3.45 \\
-0.32\end{array}$ & $\begin{array}{l}-0.005 \\
-0.038 \\
+0.030 \\
+0.107 \\
+0.024\end{array}$ & $\begin{array}{c}+0.003 \\
0 \\
-0.005 \\
+0.067 \\
+0.005\end{array}$ & $\begin{array}{r}+0.005 \\
+0.013 \\
-0.010 \\
+0.131 \\
+0.025\end{array}$ & $\begin{array}{c}+0.003 \\
0 \\
-0.001 \\
-0.059 \\
-0.004\end{array}$ & $\begin{array}{r}+.012 \\
-.012 \\
-.109 \\
-.038 \\
+.018\end{array}$ & $\begin{array}{c}-.003 \\
0 \\
-.028 \\
+.001 \\
0\end{array}$ \\
\hline $\begin{array}{r}6,-6 \\
-5 \\
-4 \\
-3\end{array}$ & $\begin{array}{r}-8.4 \\
+4.63 \\
+4.03 \\
-1.45\end{array}$ & $\begin{array}{c}0 \\
+0.76 \\
-2.79 \\
+0.31\end{array}$ & $\begin{array}{r}0 \\
-0.03 \\
+\quad 6.15 \\
-1.45 \\
\end{array}$ & $\begin{array}{c}0 \\
+0.04 \\
+2.45 \\
-0.31\end{array}$ & $\begin{array}{r}+2.8 \\
-1.46 \\
+0.31 \\
-0.04\end{array}$ & $\begin{array}{c}0 \\
+0.24 \\
-0.10 \\
+0.02\end{array}$ & $\begin{array}{r}+2.80 \\
+1.49 \\
+5.46 \\
1.49\end{array}$ & $\begin{array}{c}0 \\
+0.28 \\
+235 \\
-0.29\end{array}$ & $\begin{array}{c}-0.022 \\
+0.019 \\
+0.033 \\
{[+0.190]}\end{array}$ & $\begin{array}{c}0 \\
-0.003 \\
+0.022 \\
{[+0.041]}\end{array}$ & $\begin{array}{c}+0.007 \\
-0.006 \\
+0.045 \\
{[+0.195]}\end{array}$ & $\begin{array}{c}0 \\
-0.001 \\
-0.019 \\
{[-0.038]}\end{array}$ & $\begin{array}{l}-.010 \\
-.028 \\
.010 \\
+.015\end{array}$ & $\begin{array}{c}+.002 \\
-.008 \\
0 \\
0\end{array}$ \\
\hline $\begin{array}{r}7,-7 \\
-6 \\
-5 \\
-4\end{array}$ & $\begin{aligned} & 5.6 \\
+ & 3.63 \\
+ & 2.49 \\
- & 1.19\end{aligned}$ & $\begin{array}{c}0 \\
+0.61 \\
-2.00 \\
+0.27\end{array}$ & $\begin{array}{l}0 \\
0 \\
+3.52 \\
-1.19\end{array}$ & $\begin{array}{c}0 \\
0 \\
+1.68 \\
-0.27\end{array}$ & $\begin{array}{l}+1.9 \\
-1.17 \\
+0.30 \\
-0.04\end{array}$ & $\begin{array}{c}0 \\
+0.20 \\
-0.10 \\
+0.02\end{array}$ & $\begin{array}{l}+1.9 \\
+1.17 \\
+3.82 \\
-1.23\end{array}$ & $\begin{array}{c}0 \\
+0.20 \\
2+1.68 \\
-0.25\end{array}$ & $\begin{array}{r}-0.012 \\
+0.012 \\
+0.013 \\
-0.021\end{array}$ & $\begin{array}{c}0 \\
-0.002 \\
+0 .(111 \\
-0.605\end{array}$ & $\begin{array}{r}+0.004 \\
-0004 \\
+0.020 \\
-0.022\end{array}$ & $\begin{array}{c}0 \\
-0.001 \\
-1.008 \\
+0.004\end{array}$ & $\begin{array}{l}-.006 \\
-.008 \\
+.014 \\
+.002\end{array}$ & {$\left[\begin{array}{c}+.001 \\
-, 104 \\
0 \\
0\end{array}\right.$} \\
\hline $\begin{array}{l}8,-8 \\
-7 \\
-6 \\
-5\end{array}$ & $\begin{array}{l}-4.0 \\
+2.76 \\
+1.49 \\
-0.97\end{array}$ & $\begin{array}{r}0 \\
+0.50 \\
-1.41 \\
+0.22\end{array}$ & $\begin{aligned} & 0 \\
& 0 \\
+ & 2.38 \\
- & 0.97\end{aligned}$ & $\begin{array}{c}0 \\
0 \\
+1.14 \\
-0.22\end{array}$ & $\begin{array}{c}+1.3 \\
-0.91 \\
+0.27 \\
0\end{array}$ & $\begin{array}{c}0 \\
+0.16 \\
-0.09 \\
+0.02\end{array}$ & $\mid \begin{array}{l}+1.3 \\
0.91 \\
+2.65 \\
-0.97\end{array}$ & $\begin{array}{c}0 \\
+0.16 \\
+1.05 \\
-0.20 \\
\end{array}$ & $\begin{array}{r}-0.008 \\
+0.007 \\
+0.006 \\
-0.008\end{array}$ & $\begin{array}{l}0 \\
-0.002 \\
+0.000 \\
-0.002\end{array}$ & $\begin{array}{r}+0.003 \\
+0.002 \\
+0.011 \\
-0.008\end{array}$ & $\begin{array}{c}0 \\
0 \\
-0.004 \\
+0.002\end{array}$ & $\begin{array}{r}-.003 \\
-.003 \\
+.004 \\
+.002\end{array}$ & $\begin{array}{c}+.002 \\
-.002 \\
0 \\
0\end{array}$ \\
\hline & & & & & & & r term & & & & & & & \\
\hline
\end{tabular}

The terms $\delta \%$ and $\delta r_{1}$, which are inclosed in brackets, are of very long period, and are therefore omitted in forming the values of $\delta \beta$ in the last two columns 


\section{Perturbations produced by Jupiter.}

The series in which these perturbations are expressed converge so rapidly that I deem it unnecessary to present the details of the computation. They have been computed by both methods, and the separate and independent results are given in the following table, where $\delta v_{1}$ represents the perturbations computed by the method developed in Chapter $\mathrm{I}$, and $\delta v_{2}$ those computed by the method of variation of elements.

The apparently large diserepancy between the coefficients multiplied by the time arises from the circumstances that in the form of development the mean motion, and hence the mean anomaly, appears affected by the perturbation $31^{\prime \prime} .2 t$. Accordingly when we enter the table which gives the true longitude in terms of the mean anomaly in the form

$$
v=l+2 e \sin (l-\pi)+\text { etc., }
$$

we may consider this quantity $31^{\prime \prime} .2 t$ as a secular variation of $l-\pi$ producing in $v$ the term

$$
\varepsilon v=62^{n} .4 \text { et } \cos (1-\pi)
$$

In $\partial v_{1}$ this term is left in its primitive form, while in $\delta v_{2}$ the value of $l$ is supposed to include this term, and the secular terms are only those which arise from the secular variation of the eccentricity and perihelion.

It is also to be remarked that the terms which are independent of the mean longitude of Jupiter, or those in which $i^{\prime}=0$, are not comparable, as they correspond to slightly different elliptic elements in the two theories. 


\begin{tabular}{|c|c|c|c|c|c|c|c|c|c|c|}
\hline \multirow[b]{3}{*}{$g l$} & \multicolumn{10}{|c|}{ J'RRTURHATIONS OF URANES BY JUPITER. } \\
\hline & \multicolumn{2}{|c|}{$8 v_{1}$} & \multicolumn{2}{|c|}{80} & \multicolumn{2}{|c|}{ Difr. } & \multicolumn{2}{|c|}{$\cos \downarrow \delta \rho$} & \multicolumn{2}{|l|}{$M / \partial p$} \\
\hline & sin & $\begin{array}{c}\cos \\
\prime \prime\end{array}$ & $\sin ^{\prime \prime}$ & $\begin{array}{l}\cos \\
" \prime\end{array}$ & $\sin c$ & $\cos$ & $\cos$ & $\sin$ & $\cos$ & $\sin$ \\
\hline $\begin{array}{ll}0, & 0 \\
1 & \\
2 & \\
3 & \end{array}$ & $\begin{array}{l}\ldots .160 n t \\
-0.010 n t \\
\ldots\end{array}$ & $\begin{array}{l}+31.2116 t \\
+37.585 n t \\
+\quad 2.207 n t \\
+0.135 n t\end{array}$ & $\begin{array}{l}\cdots \\
-0.1622 n t \\
-0.0095 n t \\
-0.0006 n t\end{array}$ & $\left\{\begin{array}{l}+31.1952 t \\
-1.5406 \mathrm{nt} \\
-0.0899 \mathrm{nt} \\
-0.0054 \mathrm{nt}\end{array}\right.$ & $\begin{array}{l}2 \\
0 \\
0\end{array}$ & & & & & \\
\hline 0,0 & & $118=0$ & & & $\ldots$. & $\cdots$ & -10089 & 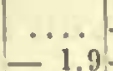 & $\begin{array}{r}4387 \\
-\quad 213.7\end{array}$ & $\begin{array}{l}0 \\
-0.8\end{array}$ \\
\hline $\begin{array}{l}1 \\
2\end{array}$ & $\begin{array}{r}+25.657 \\
+\quad 1.397\end{array}$ & $\begin{array}{r}-1.859 \\
-0.087\end{array}$ & $\begin{array}{r}1.346 \\
-0.030\end{array}$ & $\begin{array}{l}+1.361 \\
+0.086\end{array}$ & $\cdots$ & $\cdots$ & $\begin{array}{r}-491.3 \\
-32.8\end{array}$ & +1.1 & -14.2 & +0.5 \\
\hline 3 & +0.072 & -0.005 & $\ldots$ & $\ldots$ & $\cdots$ & $\cdots$ & -1.8 & 0 & -0.8 & 0 \\
\hline$-1,-1$ & +0.027 & +0.003 & +0.017 & +0.011 & 10 & 2 & 0.0 & -0.5 & 0.0 & -0.2 \\
\hline 0 & +1.269 & +0.002 & +1.232 & +0.001 & 37 & 1 & -59.8 & -0.5 & -25.6 & -0.2 \\
\hline 1 & -53.064 & -0.004 & -53.084 & -0.003 & 20 & 2 & +2585.7 & +0.1 & +1127.2 & 2. 0 \\
\hline 2 & -3.495 & -0.092 & -3.565 & -0.082 & 701 & 10 & +195.3 & +5.0 & +82.8 & $3+2.2$ \\
\hline 3 & -0.148 & -0.047 & -0.164 & -0.050 & 16 & 3 & +17.1 & +2.3 & +7.4 & +1.0 \\
\hline $0,-2$ & -0.027 & -0.011 & -0.031 & -0.014 & 4 & 3 & $+\quad 1.4$ & -0.6 & +0.6 & -0.3 \\
\hline 1 & +1.189 & +0.515 & +1.176 & +0.515 & 6 & 0 & -56.5 & +24.9 & -24.6 & +10.7 \\
\hline 2 & +0.277 & +0.036 & +0.263 & +0.037 & 14 & 1 & $\begin{array}{r}8.5 \\
\end{array}$ & +1.8 & +3.7 & +1.0 \\
\hline 3 & +0.074 & -0.005 & +0.083 & -0.008 & 9 & 3 & $+\quad 4.2$ & +0.5 & +1.8 & +0.8 \\
\hline 4 & +0.015 & -0.003 & +0.014 & -0.003 & 1 & 0 & $+\quad 0.9$ & +0.2 & +0.4 & +0.2 \\
\hline $1,-3$ & -0.032 & -0.034 & -0.025 & -0.025 & 7 & 9 & & & & \\
\hline 3 & -0.005 & 0 & -0.005 & -0.001 & 0 & 1 & & & & \\
\hline 3 & +0.025 & 0 & +0.015 & 0 & 10 & 0 & & & & \\
\hline 4 & +0.011 & -0.001 & +0.037 & -0.010 & 26 & 9 & & & & \\
\hline 5 & +0.003 & 0 & - & +0.004 & 20 & 4 & & & & \\
\hline
\end{tabular}

\begin{tabular}{|c|c|c|c|c|c|c|}
\hline \multicolumn{7}{|c|}{ Priturbations of tile Latitude. } \\
\hline \multirow{2}{*}{$\begin{array}{ll}g \quad l^{\prime} \\
i \quad i^{\prime}\end{array}$} & \multicolumn{2}{|c|}{$8 k$} & \multicolumn{2}{|c|}{$8 \eta$} & \multicolumn{2}{|c|}{$\delta \beta$} \\
\hline & $\sin$ & $\cos$ & $\cos$ & $\sin$ & $\cos$ & $\sin$ \\
\hline & " & $" 1$ & $\prime \prime$ & 11 & 11 & $\prime \prime$ \\
\hline $\begin{array}{ll}0 & 0\end{array}$ & $\cdots$ & $\cdots$ & .... & $\ldots$ & $\cdots$ & +.065 \\
\hline 1 & +0.071 & -0.041 & +0.030 & +0.036 & +.060 & $\begin{array}{r}+.047 \\
.007\end{array}$ \\
\hline $\begin{array}{l}2 \\
3\end{array}$ & $\begin{array}{l}-0.012 \\
-0.003\end{array}$ & $\begin{array}{l}-0.075 \\
-0.012\end{array}$ & $\begin{array}{l}-0.012 \\
-0.003\end{array}$ & $\begin{array}{l}+0.061) \\
+0.012\end{array}$ & -.010 & -.00 \\
\hline$-3-1$ & -0.006 & +0.012 & +0.006 & +0.042 & +.005 & -.004 \\
\hline-1 & -0.297 & +1.949 & +0.297 & +1.943 & +.001 & -.003 \\
\hline 0 & -0.161 & -0.191 & -0.189 & -0.190 & -.494 & +.420 \\
\hline 1 & +2.611 & 0 & +2.628 & 0 & -.024 & -.013 \\
\hline 2 & +0.070 & +0.021 & +0.066 & -0.002 & +.002 & +.019 \\
\hline$-3-2$ & +0.055 & -0.088 & -0.05 .5 & -0.088 & +.010 & -.003 \\
\hline 0 & +0.002 & -0.011 & -0.002 & -0.011 & +.017 & -.004 \\
\hline 1 & -0.110 & -0.050 & $-0.10 ?$ & +0.046 & -.009 & +.002 \\
\hline 2 & -0.014 & 0 & -0.011 & 0 & -.002 & 0 \\
\hline & & Seculnt & $\left\{\begin{array}{l}\partial k= \\
\partial \beta=\end{array}\right.$ & $\begin{array}{l}4 \mathrm{~T} \\
4 \mathrm{~T} \cos \mathrm{V}\end{array}$ & & . \\
\hline
\end{tabular}




\section{CHAP'TR I V.}

\section{TERMS OF THE SECOND ORDER PRODUCED BY TIIE ACTION OF SATURN.}

\section{Preliminary Investigation of the Orbit of Saturn.}

For the accurate determination of the perturbations of a planet it is essential that the functions of the time which are substituted for the co-ordinates of each planet in the expression of the disturbing forces should approximately represent the true places of the planet. 'The difference between the true place and that implicitly assumed in the investigation should be so small and of such a character that, when multiplied by the mass of the disturbing planet, and by the factors introduced by the process of integration, the result shall be insensible. If one of these factors is so large as to make a perturbation of an order of magnitude approximating that of the inequality which gives rise to it, it will represent an inequality of very long period in the elements, which, though apparently sensible, may be neglected for a great length of time.

The perturbations hitherto found have becn computed on the hypothesis that the disturbing action of Saturn on Uranus is the same as if both planets moved in the elliptic orbits corresponding to the adopted elements. We have given formulæ for the computation of the corrected perturbations when, to the co-ordinates of the two planets corresponding to the adopted ellipse, we add corrections represented by $\delta v, \delta v^{\prime}, \delta \rho$, etc. 'These corrections are now to be taken of such magnitude that when thus added they shall very nearly represent the actual motions of the planets.

Generally, it is considered sufficient to take for these corrections the perturbations of the first order. But this presupposes that the elliptic elements are nearly correct, which does not hold true in the case of the old elements of the outer planets. Bouvard's 'Tables of Saturn, the elements of which have been adopted, are subject to recurring errors amounting to $30^{\prime \prime}$ or more. Moreover, when we substitute the new and more accurate perturbations for the old and imperfect ones adopted in the tables, the chances are that the errors will be increased. Desiring that the theory shall be as far as possible free from doubt, we begin with a preliminary investigation of the orbit of Saturn, the design of which will be to give the co-ordinates of that body in terms of the time with sufficient ccrtainty and accuracy to serve for computing the perturbations both of Jupiter and Uranus. As usual, the first step in this investigation will be the determinations of the perturbations of the planet. 


\section{General Perturbations of Saturn.}

The perturbations produced by Jupiter will be taken from the exhaustive prize memoir of Hansen. ${ }^{1}$ As the perturbations required are those of the co-ordinates, it will be necessary to transform those of Hansen into the usual form. Hansen gives the true anomaly $v$ in the form

$$
v=g+n \varepsilon z+e_{1} \sin (g+n \varepsilon z)+e_{1} \sin 2(g+n \varepsilon z)+\text { etc., }
$$

$e_{1}, e_{2}$, etc., being the coefficients of the multiples of the mean anomaly in the usual development of the elliptic true anomaly. Whence, neglecting the second power of $n^{5} z$,

$$
\varepsilon v=n i z\left(1+e_{1} \cos g+2 e_{2} \cos 2 g+\text { etc. }\right) .
$$

To make the development sufficiently rigorous it is only necessary to increase $g$ by $\frac{1}{2} n i z$ in this expression. In the same way, we have for the perturbations of $\log r$,

$$
\delta \rho=\delta \rho_{0}+n i z\left(e^{(1)} \sin g+e^{(2)} \sin 2 g+\text { etc. }\right)
$$

$\delta \rho_{0}$ being Hansen's perturbation, and $e^{(i)}$ the negative coefficient of $\cos$ ig in the development of the elliptic $\log r$.

Hansen having adopted $\frac{1}{10 \frac{1}{7} \sigma .5}$ as the mass of Jupiter, it will be necessary to multiply his perturbations by 1.0216 to reduce them to Bessel's mass. Thus the perturbations by Jupiter hereafter given have been obtained.

The perturbations by Uranus and Neptune have been computed by the preceding general method, and are given in the following table. In the table $l^{\prime}$ is the mean longitude of the disturbing planet, Uranus or Neptune, counted from the perihelion of Saturn. $\delta \rho$ is the perturbation of the Naperian logarithm, in units of the seventh place of decimals.

\begin{tabular}{|c|c|c|c|c|c|c|c|c|c|}
\hline \multirow[b]{3}{*}{$\begin{array}{ll}l & g\end{array}$} & \multicolumn{4}{|c|}{ Action of Uranus. } & \multicolumn{5}{|c|}{ Action of Neptune. } \\
\hline & \multicolumn{2}{|r|}{$\delta v$} & \multicolumn{2}{|c|}{$\delta \rho$} & \multirow[b]{2}{*}{$t^{t} g$} & \multicolumn{2}{|c|}{$\delta v$} & \multicolumn{2}{|c|}{$\delta_{\rho}$} \\
\hline & $\sin ^{\prime \prime}$ & $\begin{array}{c}\cos \\
\prime \prime\end{array}$ & $\cos$ & $\sin$ & & $\begin{array}{c}\sin \\
\prime \prime\end{array}$ & $\begin{array}{c}\cos \\
\prime \prime\end{array}$ & $\cos$ & $\sin$ \\
\hline $\begin{array}{r}1, \quad 0 \\
-1\end{array}$ & $\begin{array}{r}+0.88 \\
+8.60\end{array}$ & $\begin{array}{r}+0.92 \\
+0.21\end{array}$ & $\begin{array}{r}-12.7 \\
+146.6\end{array}$ & $\begin{array}{r}+7.9 \\
-3.5\end{array}$ & $\begin{array}{r}1,-0 \\
-1\end{array}$ & $\begin{array}{r}+0.23 \\
+1.93\end{array}$ & $\begin{array}{r}-0.05 \\
0.00\end{array}$ & $\begin{array}{r}-2.2 \\
+39.9\end{array}$ & $\begin{array}{r}-0.2 \\
0.0\end{array}$ \\
\hline $\begin{array}{r}2, \quad 0 \\
-1 \\
-2\end{array}$ & $\begin{array}{r}-0.42 \\
-8.49 \\
-13.39\end{array}$ & $\begin{array}{r}-0.17 \\
-2.53 \\
-0.25\end{array}$ & $\begin{array}{r}+10.2 \\
-66.8 \\
-392.5\end{array}$ & $\begin{array}{r}3.7 \\
+21.4 \\
+\quad 6.6\end{array}$ & $\begin{array}{r}8, \quad 0 \\
-1 \\
-2\end{array}$ & $\begin{array}{r}+0.44 \\
-1.11 \\
-1.27\end{array}$ & $\begin{array}{r}+0.02 \\
+0.02 \\
0.00\end{array}$ & $\begin{array}{r}3.0 \\
-18.5 \\
-41.1\end{array}$ & $\begin{array}{r}+0.1 \\
-0.4 \\
0.0\end{array}$ \\
\hline $\begin{array}{r}3, \quad 0 \\
-1 \\
-2 \\
-3\end{array}$ & $\begin{array}{l}+0.06 \\
-2.9 \\
-10.61 \\
-2.05\end{array}$ & $\begin{array}{l}+1.62 \\
+28.1 \\
-20.88 \\
-1.47\end{array}$ & $\begin{array}{r}+\quad 0.5 \\
+\quad 10.8 \\
-247 . \\
-67.5\end{array}$ & $\begin{array}{r}+39.1 \\
+33.0 \\
+487 . \\
+42.8\end{array}$ & $\begin{array}{r}3,-1 \\
-2 \\
-3\end{array}$ & $\begin{array}{r}+0.02 \\
+0.10 \\
-0.10\end{array}$ & $\begin{array}{r}-0.03 \\
-0.04 \\
0.00\end{array}$ & $\begin{array}{r}+0.2 \\
+\quad 3.2 \\
-\quad 3.9\end{array}$ & $\begin{array}{r}+0.4 \\
+1.3 \\
0.0\end{array}$ \\
\hline $\begin{array}{r}4,-1 \\
-2 \\
-3 \\
-4\end{array}$ & $\begin{array}{r}+0.06 \\
-0.62 \\
+0.30 \\
-0.26\end{array}$ & $\begin{array}{r}+\quad 0.05 \\
+\quad 0.80 \\
+\quad 0.73 \\
+\quad 0.03\end{array}$ & $\begin{array}{rr}-\quad 0.1 \\
-\quad 10.5 \\
+\quad 10.5 \\
-10.6\end{array}$ & $\begin{array}{r}+0.6 \\
-12.5 \\
-23.4 \\
-0.8\end{array}$ & & & & & \\
\hline
\end{tabular}

General perturbations of the LoNGitude in ORBIT AND the LogarithM OF THE RADIUs VECTOR OF SATURN.

2 Untersuchungen über die gegenseitigen Störungen des Jupiters und Saturns. Von P. A. Hansen. Berlin, 1831.

$9 \quad$ April, 1873. 
I have submitted these perturbations to such duplicate computations and other checks as lead me to believe that none of the terms can be in error by more than a small fraction of a second, but, as they are not intended to form the basis of a definitive theory of Saturn, I do not vouch for their absolute precision.

In this provisional correction of the orbit of Saturn only heliocentric longitudes have been employed. 'These were derived for a series of dates from Airy's reduction of the Grcenwich observations, the modern Greenwich obscrvations, and the Washington observations.

For these dates the valuc of $n i z$ for Saturn was computed from the formulx found on pages 189 and 190 of the work of Hansen, already quoted, omitting all terms less than 1", and including only tenths of scconds in the results. The dates, the resulting values of $n £ z$, of the factor $e_{1} \cos \left(g+\frac{1}{2} n \varepsilon z\right)+2 e_{2} \cos 2\left(g+\frac{1}{2} n i z\right)$, and of the concluded $i v$ are as follows. The formulæ for $i v$ is

\begin{tabular}{|c|c|c|c|c|}
\hline \multicolumn{2}{|c|}{$\begin{array}{l}\text { Date } \\
\text { Gr. Mean Noon. }\end{array}$} & $n \delta z$ & Factor. & \\
\hline 1751 & May 31 & -1947.7 & -.0920 & -1792.7 \\
\hline 1757 & Aug. 7 & -2134.2 & -.0652 & -2038.2 \\
\hline 1758 & Aug. 27 & -2212.5 & -.0474 & -2153.2 \\
\hline 1761 & Oct. 6 & -2546.2 & +.0244 & -2664.5 \\
\hline 1763 & Nov. 1 & -2880.3 & +.0729 & -3157.1 \\
\hline 1765 & Nov. 23 & -3095.1 & +.1082 & -3504.0 \\
\hline 1773 & Feb. 26 & -3342.0 & +.0419 & -3557.2 \\
\hline 1780 & May 24 & -2858.2 & -.0956 & -2640.7 \\
\hline 1794 & Nov. 16 & -3321.1 & +.1017 & -3737.9 \\
\hline 1802 & Feb. 23 & -3184.7 & +.0529 & -3425.5 \\
\hline 1823 & Nov. 13 & -2716.3 & +.0944 & -3036.8 \\
\hline 1831 & Feb. 18 & -3378.5 & +.0639 & -3671.7 \\
\hline 1838 & May 19 & -2976.7 & -.0866 & -2777.9 \\
\hline 1845 & Aug. 17 & -2342.7 & -.0721 & -2220.9 \\
\hline 1852 & Nov. 15 & -2847.0 & +.0863 & -3159.3 \\
\hline 1860 & Feb. 14 & -3161.4 & +.0740 & -3468.3 \\
\hline 1867 & May 15 & -2373.1 & -.0812 & -2227.6 \\
\hline
\end{tabular}

The perturbations by Uranus and Neptune were computed from the values of their terms just given. The principal terms, the sum of which make up the heliocentric longitude resulting from the adopted elements, are shown in the first of the following tables.

In the next table we have after the date the heliocentric longitude from Bourard's Tables, as deduced from the longitudes given in Airy's reductions of the Grecnwich Observations, from the Astronomisches Jahrbuch for 1831, and from the Nautical Almanac. Then follow the corrections, roughly deduced from obscrvations made near the opposition. Adding these columns, we have the longitude 
from observation. To the right of these are the equations of condition for the correction of the elements.

\begin{tabular}{|c|c|c|c|c|c|c|c|c|c|c|}
\hline \multirow{2}{*}{$\begin{array}{c}\text { Long. of } \\
\text { Perihelion. }\end{array}$} & \multirow{2}{*}{\multicolumn{2}{|c|}{$\begin{array}{c}\text { Mean } \\
\text { anomaly. }\end{array}$}} & \multirow{2}{*}{\multicolumn{2}{|c|}{$\begin{array}{l}\text { Equation of } \\
\text { eentre. }\end{array}$}} & \multicolumn{3}{|c|}{ Perturbations by } & \multirow{2}{*}{$\begin{array}{c}\text { Red. to } \\
\text { Eeliptic. }\end{array}$} & \multirow{2}{*}{$\begin{array}{l}\text { Nuta- } \\
\text { tion. }\end{array}$} & \multirow{2}{*}{$\begin{array}{c}\text { True } \\
\text { longitude. }\end{array}$} \\
\hline & & & & & Jupiter. & \multirow{2}{*}{\begin{tabular}{|r} 
Uranus. \\
$\prime \prime$ \\
$j$
\end{tabular}} & \multirow{2}{*}{$\begin{array}{c}\begin{array}{c}\text { Nep- } \\
\text { tune. }\end{array} \\
\prime \prime\end{array}$} & & & \\
\hline 0,11 & - & " & ○ & 0,11 & $\prime \prime$ & & & 111 & $\prime \prime$ & 0,11 \\
\hline $\begin{array}{lll}88 & 41 & 27.5\end{array}$ & 159 & 5550.1 & +2 & 356.6 & -2952.7 & -45.7 & -2.7 & +136.9 & +15.8 & 2501225.8 \\
\hline $8846 \quad 41.2$ & 236 & $13 \quad 7.1$ & -5 & 738.1 & -3358.2 & -36.6 & +1.0 & -120.5 & -11.8 & $\begin{array}{llll}319 & 16 & 4.1\end{array}$ \\
\hline 884731.4 & 248 & 2553.1 & -5 & 4830.4 & -3553.2 & -35.2 & +1.7 & -135.8 - & -15.0 & 3304636.6 \\
\hline $\begin{array}{lll}8850 & 7.6\end{array}$ & 286 & 2629.8 & -6 & $\begin{array}{ll}16 & 1.3\end{array}$ & -4424.6 & -3.3 & +2.9 & $\begin{array}{llll}-0 & 43.5\end{array}$ & -14.1 & 81513.5 \\
\hline 8851516 & 311 & 4413.6 & -5 & 058.2 & -5237.1 & +11.9 & +2.4 & +043.3 & -4.9 & 344322.6 \\
\hline 885335.1 & 336 & 5556.1 & -2 & 4110.6 & -5824.0 & +19.2 & +1.9 & +136.6 & +6.7 & $\begin{array}{lll}62 & 12 & 1.0\end{array}$ \\
\hline $\begin{array}{llll}88 & 59 & 39.6\end{array}$ & 65 & $40 \quad 2.3$ & +6 & 035.9 & $-59 \quad 17.2$ & -32.9 & -0.8 & -137.1 & +4.81 & 1593854.6 \\
\hline $\begin{array}{lll}89 & 5 & 43.4\end{array}$ & 154 & $\begin{array}{ll}8 & 4.8\end{array}$ & +2 & 3757.7 & $\begin{array}{ll}-44 & 0.7\end{array}$ & -53.8 & -1.7 & $+137.5-$ & -14.52 & $\begin{array}{lll}245 & 8 & 13.2\end{array}$ \\
\hline 891750.7 & 331 & 610.4 & -3 & $\begin{array}{ll}18 & 5.2\end{array}$ & $-62 \quad 17.9$ & +9.6 & +3.5 & $+130.9-$ & -14.1 & $\begin{array}{lll}56 & 5 & 7.9\end{array}$ \\
\hline 892355.7 & 59 & $56 \quad 17.9$ & +5 & 4446.6 & $-57 \quad 5.5$ & -18.6 & +2.5 & $-1 \quad 37.3$ & +1.41 & $\begin{array}{lll}154 & 6 & 2.7\end{array}$ \\
\hline $\begin{array}{lll}8942 & 7.0\end{array}$ & 325 & 2226.0 & -3 & 35158.0 & -5036.8 & -27.9 & -3.0 & +121.6 & +14.7 & $\begin{array}{llll}50 & 23 & 3.6\end{array}$ \\
\hline $8948 \quad 11.9$ & 54 & 1033.1 & +5 & $\begin{array}{ll}25 & 2.6\end{array}$ & -6111.7 & +13.3 & +2.5 & -133.2 & -7.41 & 1482111.1 \\
\hline 895416.1 & 142 & 4437.0 & +3 & 4038.1 & $-46 \quad 17.9$ & -3.4 & +2.1 & $+129.7-$ & -4.32 & $235 \quad 34 \quad 37.4$ \\
\hline $\begin{array}{lll}90 & 0 & 20.4\end{array}$ & 231 & 1840.9 & -4 & 4736.6 & $\begin{array}{ll}-37 & 0.9\end{array}$ & -35.5 & +0.9 & -111.8 & +14.83 & 3155252.2 \\
\hline $\begin{array}{lll}90 & 6 & 24.6\end{array}$ & 319 & 5244.8 & -4 & +2153.0 & -5239.3 & +4.7 & -0.9 & +18.8 & -18.4 & $44 \quad 4531.3$ \\
\hline $90 \quad 12 \quad 28.7$ & 48 & 2648.7 & +5 & $5 \quad 146.4$ & $-5748.3 e$ & +30.3 & -3.4 & $-1 \quad 25.1$ & +14.11 & $\begin{array}{llll}142 & 42 & 31.4\end{array}$ \\
\hline $\begin{array}{llll}90 & 18 & 32.8\end{array}$ & 137 & 052.6 & +4 & 931.2 & $\begin{array}{ll}-37 & 7.6\end{array}$ & -8.2 & +1.3 & +121.6 & -4.12 & 2305259.7 \\
\hline \multicolumn{11}{|c|}{ 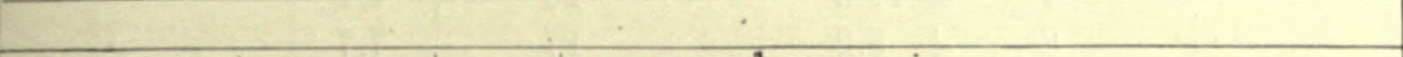 } \\
\hline \multicolumn{2}{|l|}{ Date. } & \multicolumn{2}{|c|}{$\begin{array}{c}\text { Tabular } \\
\text { longitude. }\end{array}$} & $\begin{array}{l}\text { Obs. } \\
\text { eor. }\end{array}$ & $\begin{array}{l}\text { Long. from } \\
\text { observation. }\end{array}$ & \multicolumn{5}{|c|}{ Equations of Condition. } \\
\hline & & & & & & " & & & & \\
\hline 1751 May & 31 & 250133 & 38.0 & -8.5 & $\begin{array}{llll}250 & 13 & 29.5\end{array}$ & $63.7=$ & +0.9 & $908 \varepsilon-448 n$ & $n+0.608 e$ & $\delta e+1.82 e \delta \omega$ \\
\hline 1757 Aug. & 27 & 31917 & 8.5 & -18.0 & 3191650.5 & 46.4 & 0.9 & $94-39$ & -1.53 & +1.14 \\
\hline 1758 Aug. & 27 & 330473 & 37.9 & -14.3 & $\begin{array}{llll}330 & 47 & 23.6\end{array}$ & 47.0 & 0.9 & $96-40$ & -1.76 & +0.80 \\
\hline 1761 Oet. & 6 & $8151:$ & 13.8 & +0.2 & 81514.0 & 0.5 & 1.0 & $03-39$ & -1.99 & -0.50 \\
\hline 1763 Nov. & 1 & 34434 & 12.3 & +15.3 & 344357.6 & 35.0 & 1.0 & $07 \quad-39$ & -1.63 & -1.31 \\
\hline 1765 Nov. & 23 & 62122 & 20.7 & +20.4 & 621241.1 & 40.1 & & $11-38$ & -0.88 & -1.88 \\
\hline 1773 Feb. & 26 & 15940 & 3.8 & +18.8 & 1594022.6 & 88.0 & 1.0 & $04-28$ & +1.93 & -0.79 \\
\hline 1780 May & 24 & $\begin{array}{lll}245 & 9 & 17\end{array}$ & 17.6 & $\begin{array}{r}+7.8 \\
\end{array}$ & $245 \quad 9 \quad 25.4$ & 72.2 & 0.9 & $90-18$ & +0.76 & +1.74 \\
\hline 1794 Nov. & 16 & $56 \quad 53$ & 88.8 & +14.7 & $\begin{array}{lll}56 & 5 & 53.5\end{array}$ & 45.6 & 1.1 & $10-6$ & -1.08 & -1.78 \\
\hline 1802 Feb. & 23 & 1547 & 2.7 & +10.8 & $\begin{array}{lll}154 & 7 & 13.5\end{array}$ & 70.8 & 1.0 & $05+2$ & +1.85 & -0.98 \\
\hline 1823 Nov. & 13 & 50233 & 34.5 & +21.3 & $\begin{array}{llll}50 & 23 & 55.8\end{array}$ & 52.2 & 1.0 & $09+26$ & -1.27 & -1.66 \\
\hline 1831 Feb. & 18 & 148223 & 38.5 & +3.0 & $148 \quad 22 \quad 41.5$ & 90.4 & 1.0 & $07+33$ & +1.75 & -1.16 \\
\hline 1838 May & 19 & 235361 & 11.2 & +3.4 & $\begin{array}{llll}235 & 36 & 14.6\end{array}$ & 97.2 & 0.9 & $91+35$ & +1.08 & +1.56 \\
\hline 1845 Aug. & 17 & 315534 & 12.2 & +15.7 & 3155357.9 & 65.7 & 0.9 & $93+42$ & -1.43 & +1.28 \\
\hline 1852 Nov. & 15 & 44461 & 15.4 & +5.9 & 444621.3 & 50.0 & 1.0 & $09+57$ & -1.42 & -1.53 \\
\hline 1860 Feb. & 14 & 142441 & 16.2 & -13.7 & $14244 \quad 2.5$ & 91.1 & 1.0 & $08+65$ & +1.63 & -1.35 \\
\hline 1867 May & 15 & 230544 & 47.8 & +5.1 & 2305452.9 & 113.2 & +0.9 & $92+62$ & +1.23 & +1.45 \\
\hline
\end{tabular}

A normal equation for $\delta \varepsilon$ is obtained by taking the sum of all the equations. That for $\delta n$ is formed by subtracting the sum of the first seven from the sum of the last seven, and those for ie and $e f_{\omega}$ by taking the sum of the equations in which the coefficients of $\varepsilon_{e}$ or $e \oint_{\omega}$ are greater than unity, after changing the signs of the equations in which they are negative. The normals thus obtained are 


$$
\begin{aligned}
& 17.19 \delta \varepsilon+31 \delta n-2.16 \delta e-3.15 e \delta \omega=+1069.3 \\
& 0.04+587+6.83-0.69+239.3 \\
& -2.14+207+21.58+2.19+208.1 \\
& -2.11-60+3.61+19.66+53.8
\end{aligned}
$$

These equations give

$$
\begin{aligned}
\delta \varepsilon & =+64.8(\text { Epoch, 1800.) } \\
\delta n & =+0.268 \\
\delta e & =+12.6 \\
e \delta \omega & =+8.2
\end{aligned}
$$

-Substituting these values in the scventeen equations of condition we have the following residuals, or excesses of theoretical over observed longitudes:

$\begin{array}{llll}1 & +7.2 & 10 & +10.7 \\ 2 & -4.3 & 11 & -6.4 \\ 3 & -10.2 & 12 & -1.1 \\ 4 & +21.7 & 13 & -0.4 \\ 5 & -9.0 & 14 & 0.0 \\ 6 & -7.1 & 15 & +3.7 \\ 7 & -11.3 & 16 & +4.1 \\ 8 & +8.2 & 17 & -7.7 \\ 9 & -6.4 & & \end{array}$

These residuals are much larger than they should be, and I scarcely know to what cause to attribute their magnitude. The results are however amply reliable for the purposes of the investigation, and lead to the following elements of Saturn:

$$
\begin{aligned}
& \pi, \quad 90 \quad 6 \quad 26 \\
& \varepsilon, \quad 1450 \quad 3.2 \\
& \theta, \quad 11220 \quad 0 \\
& \phi, \quad 229 \quad 39.2 \\
& n, \quad 43996.395 \\
& e, \quad .0560660 \\
& \log (a+\delta a), \quad 0.979676 \\
& \text { Epoch, 1850, Jan. 0, Greenwich mean noon. }
\end{aligned}
$$

It will be scen that the adopted position of the plane of Saturn's orbit is retained. It was corrected from observations before the perturbations were finally computed.

Of the above corrections, those of the epoch and mean motion need not be taken account of in the corrections of the co-ordinates, since the mean longitude remains in the formulæ as an arbitrary quantity to the end. The effect of the correction of the mean distance is insensible. The corrections of eccentricity aud perihelion are therefore alone to be retained. They are allowed for by adding to $\delta v$ and $\delta \rho$ the terms 


$$
\begin{aligned}
\delta v & =2 e^{\prime} \sin g-e \delta \omega \cos g \\
& =+25^{\prime \prime} .2 \sin g-16^{\prime \prime} .4 \cos g \\
\delta \rho & =-\delta e \cos g-e \delta \omega \sin g \\
& =-12^{\prime \prime} .6 \cos g-8^{\prime \prime} .2 \cos g .
\end{aligned}
$$

\section{Perturbations of Saturn and Uranus.}

The following expressions include, with these corrections, all the perturbations of Saturn and Uranus which can produce any appreciable perturbations of the second order in their mutual action. In these expressions the initial letter of each planet is put for its mean longitude counted from the perihelion of Uranus.

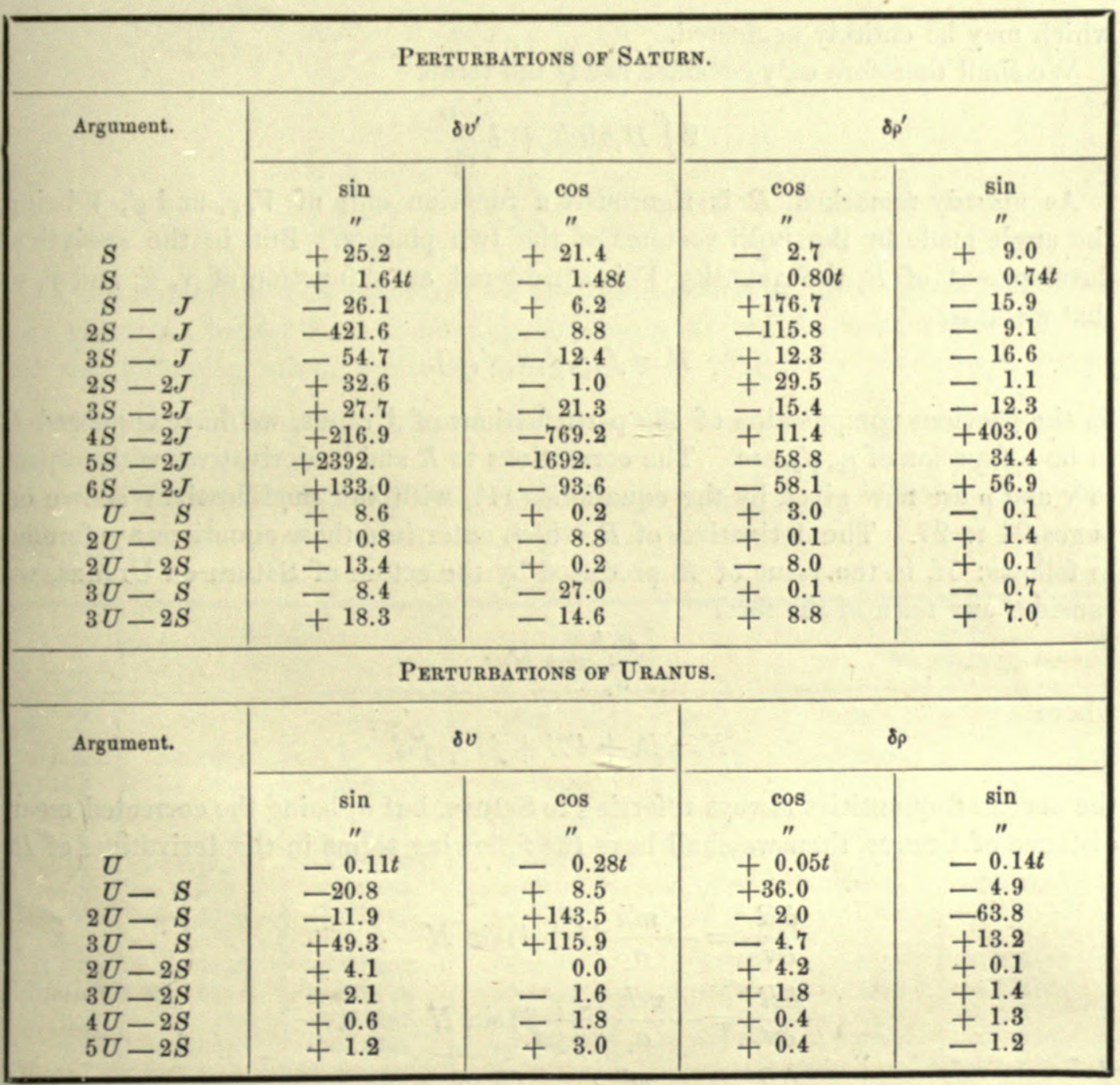

Let us now resume the equation

$$
\delta Q=2 \int D_{t}^{\prime} \delta R d t+\delta \frac{\partial R}{\partial \rho}-\frac{1}{\mu} D_{t}^{2}\left(r_{0}^{2} \delta \rho^{2}\right)+\frac{1}{2} \frac{\delta \rho^{2}}{r_{0}} .
$$


Beginning with the last two terms of this expression, it may be shown at the outset that they are quite insensible. 'The effect of the constant terms in $\delta \rho$ and $\delta \rho^{\prime}$ has already been included by correcting the lugarithm of the mean distance by their amount; they are therefore omitted. 'The largest remaining term is 64 ", the square of which is only $0^{\prime \prime} .02$. In the product $r_{1}^{2} \delta \rho^{2}$ the largest terms are

$$
\begin{aligned}
& \quad{ }^{\prime \prime} \\
& +0.014 \\
& -0.013 \sin g \\
& -0.011 \sin \left(3 g-2 l^{\prime}\right) \\
& -0.011 \cos \left(4 g-2 l^{\prime}\right)
\end{aligned}
$$

which may be entirely neglected.

We shall therefore only consider in $\delta Q$ the terms

$$
2 \int D_{t}^{\prime} \delta R d t+\delta \frac{\partial R}{\partial \rho}
$$

As already remarked, $R$ is rigorously a function only of $V, \rho$, and $\rho^{\prime}, V$ being the angle made by the radii vectores of the two planets. But, in the analytical development of $R$, the quantity $V$ is considered as a function of $v, v^{\prime}$, and $\gamma$, so that we have

$$
R=f\left(\rho, \rho^{\prime}, \mathrm{v}, \mathrm{v}^{\prime}, \gamma\right)
$$

In the previons computation of the perturbations of Uranus, we have supposed $R$ to be a function of $\rho_{0}, \rho_{0}^{\prime}$, etc. 'The corrections to $R$ and its derivatives with respect to $\mathrm{v}$ and $\rho$ are now given by the equations (11), with the modifications shown on pages 24 to 27 . The derivatives of $R_{0}$ which enter into these equations are formed as follows: If, in the value of $R$ produced by the action of Saturn on Uranus, we consider any term of the form

where

$$
\frac{m^{\prime} h}{a_{1}} \cos N
$$

$$
N=i \lambda+i^{\prime} \lambda^{\prime}+j \omega+j^{\prime} \omega^{\prime}
$$

the accented quantities always referring to Saturn, but $a_{1}$ being the corrected mean distance of Uranus, then we shall have the following terms in the derivatives of $R$.

$$
\begin{aligned}
& \frac{\partial R}{\partial \mathrm{v}}=-\frac{m^{\prime} h}{a_{1}}(i+j) \sin N \\
& \frac{\partial R}{\partial v^{\prime}}=-\frac{m^{\prime} h}{a_{1}}\left(i^{\prime}+j^{\prime}\right) \sin N \\
& \frac{\partial R}{\partial \rho}=-\frac{m^{\prime}}{a_{1}}\left(h+\frac{\partial h}{\partial x}\right) \cos N \\
& \frac{\partial R}{\partial \rho^{\prime}}=\frac{m^{\prime}}{a_{1}} \frac{\partial h}{\partial x} \cos N \\
& \frac{\partial^{2} R}{\partial v^{2}}=-\frac{m^{\prime} h}{a_{1}}(i+j)^{2} \cos N
\end{aligned}
$$




$$
\begin{aligned}
\frac{\partial^{2} R}{\partial \mathrm{v} \partial \mathrm{v}^{\prime}} & =-\frac{m^{\prime} h}{a_{1}}(i+j)\left(i^{\prime}+j^{\prime}\right) \cos N \\
\frac{\partial^{2} R}{\partial \mathrm{v} \partial \rho} & =\frac{m^{\prime}}{a_{1}}\left(h+\frac{\partial h}{\partial x}\right)(i+j) \sin N \\
& =-\frac{\partial R}{\partial \mathrm{v}}-\frac{\partial^{2} R}{\partial \mathrm{v} \partial \rho^{\prime}} \\
\frac{\partial^{2} R}{\partial \mathrm{v} \partial \rho^{\prime}} & =-\frac{m^{\prime}}{a_{1}} \frac{\partial h}{\partial x}(i+j) \sin N \\
\frac{\partial^{2} R}{\partial \rho \partial v^{\prime}} & =\frac{m^{\prime}}{a_{1}}\left(h+\frac{\partial h}{\partial x}\right)\left(i^{\prime}+j^{\prime}\right) \sin N \\
\frac{\partial^{2} R}{\partial \rho^{2}} & =\frac{m^{\prime}}{a_{1}}\left(h+2 \frac{\partial h}{\partial x}+\frac{\partial^{2} h}{\partial x^{2}}\right) \cos N \\
& =-\frac{\partial R}{\partial \rho}-\frac{\partial^{2} R}{\partial \rho \partial \rho^{\prime}} \\
\frac{\partial^{2} R}{\partial \rho \partial \rho^{\prime}} & =-\frac{m^{\prime}}{a_{1}}\left(\frac{\partial h}{\partial x}+\frac{\partial^{2} h}{\partial x^{2}}\right) \cos N
\end{aligned}
$$

\begin{tabular}{|c|c|c|c|c|c|c|}
\hline \multirow[b]{2}{*}{$g \quad l^{\prime}$} & \multicolumn{2}{|c|}{$\frac{a_{1}}{m^{\prime}} \frac{\partial R}{\partial v^{\prime}}$} & \multicolumn{2}{|c|}{$\frac{a_{1}}{m^{\prime}} \frac{\partial^{2} R}{\partial \mathrm{v} \partial^{\prime} v^{\prime}}$} & \multicolumn{2}{|c|}{$\frac{a_{1}}{m^{\prime}} \frac{\partial^{2} R}{\partial \rho \partial v^{\prime}}$} \\
\hline & $\sin$ & $\cos$ & $\sin$ & $\cos$ & $\sin$ & $\cos$ \\
\hline $\begin{array}{r}0,-1 \\
-2\end{array}$ & $\begin{array}{l}+0.2874 \\
+0.0066\end{array}$ & $\begin{array}{l}0 \\
-0.0310\end{array}$ & $\begin{array}{c}0 \\
+0.0310\end{array}$ & $\begin{array}{r}+0.2872 \\
+0.0067\end{array}$ & $\begin{array}{r}+0.2691 \\
+0.0060\end{array}$ & $\begin{array}{l}0 \\
-0.0322\end{array}$ \\
\hline $\begin{array}{r}1, \quad 0 \\
-1 \\
-2 \\
-3\end{array}$ & $\begin{array}{r}-0.0102 \\
-3.4811 \\
-0.0968 \\
+0.0388\end{array}$ & $\begin{array}{r}-0.0491 \\
-0.0010 \\
+0.4304 \\
+0.0173\end{array}$ & $\begin{array}{r}+0.0490 \\
-0.0021 \\
-0.4304 \\
-0.0179\end{array}$ & $\begin{array}{r}-0.0101 \\
-3.4810 \\
-0.1039 \\
+0.0389\end{array}$ & $\begin{array}{r}+0.0249 \\
-5.2684 \\
-0.0792 \\
+0.0398\end{array}$ & $\begin{array}{c}+0.1194 \\
0 \\
+0.4583 \\
+0.0163\end{array}$ \\
\hline $\begin{array}{r}2, \quad 0 \\
-1 \\
-2 \\
-3\end{array}$ & $\begin{array}{r}-0.0045 \\
-0.0544 \\
+0.4104 \\
-0.0053\end{array}$ & $\begin{array}{r}-0.0035 \\
-0.0712 \\
+0.0120 \\
-0.0191\end{array}$ & $\begin{array}{r}+0.0021 \\
+0.1426 \\
-0.0171 \\
+0.0390\end{array}$ & $\begin{array}{r}-0.0080 \\
-0.0689 \\
+0.8233 \\
-0.0226\end{array}$ & $\begin{array}{r}+0.0155 \\
-0.1760 \\
-1.3515 \\
+0.0288\end{array}$ & $\begin{array}{r}+0.0066 \\
+0.2403 \\
+0.0014 \\
+0.0547\end{array}$ \\
\hline $\begin{array}{r}3,-1 \\
-2 \\
-3\end{array}$ & $\begin{array}{r}-0.0070 \\
+0.0570 \\
+0.2542\end{array}$ & $\begin{array}{l}-0.0093 \\
-0.0650 \\
0\end{array}$ & $\begin{array}{r}+0.0171 \\
+0.1957 \\
-0.0065\end{array}$ & $\begin{array}{r}0.0210 \\
+0.1013 \\
+0.7624\end{array}$ & $\begin{array}{r}+0.0197 \\
-0.1791 \\
-1.0884\end{array}$ & $\begin{array}{r}+0.0304 \\
+0.2840 \\
-0.0071\end{array}$ \\
\hline $\begin{array}{r}4,-2 \\
-3 \\
-4\end{array}$ & $\begin{array}{r}-0.0014 \\
+0.0515 \\
+0.1448\end{array}$ & $\begin{array}{r}-0.0130 \\
-0.0499 \\
-0.0015\end{array}$ & $\begin{array}{r}+0.0368 \\
+0.1987 \\
-0.0016\end{array}$ & $\begin{array}{r}-0.0193 \\
+0.1450 \\
+0.5789\end{array}$ & $\begin{array}{r}+0.0213 \\
-0.2144 \\
-0.7644\end{array}$ & $\begin{array}{r}+0.0547 \\
+0.2647 \\
-0.0005\end{array}$ \\
\hline
\end{tabular}

All the numerical data necessary for the computation of these derivatives have been given in Chapter II. Combining the terms having the same argument, we find the following values, omitting those given in Chapter II, and those which are derived from the others by mere addition. The terms of $\frac{\partial^{2} R}{\partial \mathbf{v}^{2}}$ are also omitted, because they are sensibly the same with those of $\frac{\partial^{2} R}{\partial v \partial v^{\prime}}$, changing the algebraic sign. 


\begin{tabular}{|c|c|c|c|c|c|c|}
\hline \multirow{3}{*}{$\begin{array}{rr}g^{\prime} & l \\
0, & 0 \\
-1\end{array}$} & \multicolumn{2}{|c|}{$\frac{a_{1}}{m^{\prime}} \frac{\partial R}{\partial p^{\prime}}$} & \multicolumn{2}{|c|}{$\frac{a_{1}}{m^{\prime}} \frac{\partial^{2} R}{\partial{ }^{2} \partial p^{\prime}}$} & \multicolumn{2}{|c|}{ 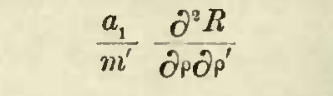 } \\
\hline & $\sin$ & $\cos$ & $\sin$ & $\cos$ & $\sin$ & $\cos$ \\
\hline & -0.026 & $\begin{array}{r}+0.172 \\
-0.562\end{array}$ & +0.556 & $+\underset{0}{+0.002}$ & +0.113 & $\begin{array}{l}-0.645 \\
-0.610\end{array}$ \\
\hline $1, \quad 0$ & +0.070 & +0.015 & +0.015 & +0.070 & -0.227 & -0.079 \\
\hline-1 & $\begin{array}{r}0.003 \\
+0.889\end{array}$ & +8.749 & -8.750 & -0.001 & +0.011 & +6.189 \\
\hline & +0.889 & +0.180 & -0.176 & +0.889 & +0.912 & +0.190 \\
\hline $\begin{array}{r}2,-1 \\
-2\end{array}$ & $\begin{array}{l}+0.084 \\
+0.018\end{array}$ & $\begin{array}{r}+0.248 \\
+0.472\end{array}$ & $\begin{array}{l}-0.230 \\
-0.941\end{array}$ & $\begin{array}{r}+0.169 \\
+0.013\end{array}$ & $\begin{array}{l}-0.322 \\
-0.006\end{array}$ & $\begin{array}{l}+0.020 \\
-1.685\end{array}$ \\
\hline $3,-1$ & +0.012 & +0.004 & +0.013 & +0.021 & -0.049 & +0.028 \\
\hline-2 & +0.073 & +0.068 & -0.122 & +0.219 & -0.344 & -0.244 \\
\hline-3 & 0 & +0.278 & -0.834 & ז & +0.010 & -1.260 \\
\hline $4,-2$ & +0.015 & -0.001 & +0.020 & +0.042 & -0.071 & +0.019 \\
\hline-4 & +0.001 & +0.150 & -0.619 & & +0.001 & -0.8 \\
\hline
\end{tabular}

'The derivations with respect to $\gamma$ and the node have been omitted because they are quite insensible. The terms of $\delta R$ depending on these derivatives are given by equation (31). In the case of Uranus disturbed by Saturn the largest values of the coefficients

$$
\frac{1}{2} \iota h \cot \frac{1}{2} \gamma ; \frac{1}{2} h \tan \frac{1}{2} \gamma, \frac{1}{2} \frac{\partial h}{\partial \sigma}
$$

are only about .05 , while the largest coefficients in $\delta k, \delta k$, and $\delta \gamma$ are less than $10^{\prime \prime}$. Hence the largest terms in (31) will be of the order of magnitude $0^{\prime \prime} .5$ multiplied by the mass of Saturn, and may therefore be omitted entirely. Omitting them the values of $\delta R, \delta \frac{\partial R}{\partial \mathrm{v}}$ and $\delta \frac{\partial R}{\partial \rho}$ become

$$
\begin{aligned}
\delta R & =\frac{\partial R}{\partial \mathrm{v}} \delta v+\frac{\partial R}{\partial \mathrm{v}^{\prime}} \delta v^{\prime}+\frac{\partial R}{\partial \rho} \delta \rho+\frac{\partial R}{\partial \rho^{\prime}} \delta \rho^{\prime} \\
\delta \frac{\partial R}{\partial \mathrm{v}} & =\frac{\partial^{2} R}{\partial \mathrm{v}^{2}} \delta v+\frac{\partial^{2} R}{\partial \mathrm{v} \partial \mathrm{v}^{\prime}} \delta v^{\prime}+\frac{\partial^{2} R}{\partial \mathrm{v} \partial \rho} \delta \rho+\frac{\partial^{2} R}{\partial \mathrm{v} \partial \rho^{\prime}} \delta \rho \\
\delta \frac{\partial R}{\partial \rho} & =\frac{\partial^{2} R}{\partial \mathrm{v} \partial \rho} \delta v+\frac{\partial^{2} R}{\partial \mathrm{v}^{\prime} \partial \rho} \delta v^{\prime}+\frac{\partial^{2} R}{\partial \rho^{2}} \delta \rho+\frac{\partial^{2} R}{\partial \rho \partial \rho^{\prime}} \delta \rho^{\prime}
\end{aligned}
$$

All the separate factors from which the second members of these equations are formed have already been given. Forming their products in the way described in Chapter II, we have the result given in the following tables.

The expressions for $\delta R$ are arranged so that the value of $D_{t}^{\prime} \delta R$ can be obtained from them by direct differentiation. This is done by distinguishing the time introduced into $R$ by the co-ordinates of Uranus from that introduced by the co-ordinates of Saturn. 


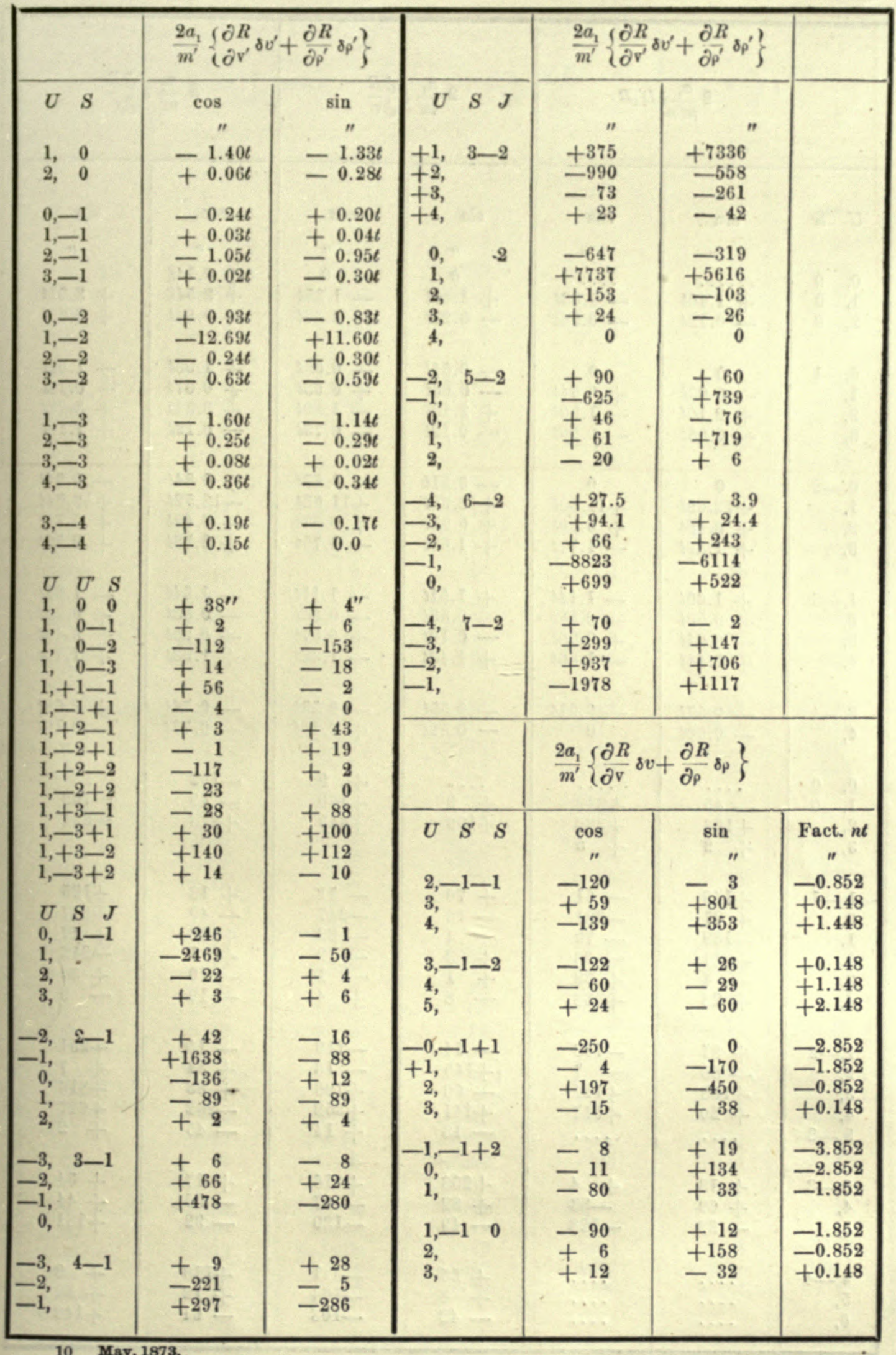




\begin{tabular}{|c|c|c|c|c|c|c|}
\hline \multirow[b]{2}{*}{$U \quad S$} & \multicolumn{2}{|c|}{$2 \frac{a_{1}}{m^{\prime} n} \delta D^{\prime} R$} & \multicolumn{2}{|c|}{$2 \frac{a_{1}}{m^{\prime}} \delta \frac{\partial R}{\partial \mathrm{v}}$} & \multicolumn{2}{|c|}{$2 \frac{a_{1}}{n^{\prime}} \delta \frac{\partial R}{\partial \rho}$} \\
\hline & $\sin$ & $\cos$ & $\sin$ & $\cos$ & $\cos$ & $\sin$ \\
\hline & $"$ & $n$ & " & $"$ & " & $"$ \\
\hline $\begin{array}{ll}0, & 0 \\
1 & 0\end{array}$ & & & & 0 & $+0.14 t$ & \\
\hline $\begin{array}{ll}1, & 0 \\
2, & 0\end{array}$ & $+1.40 t$ & $-1.33 t$ & $+1.40 t$ & $-1.35 t$ & $+3.54 t$ & $+3.21 t$ \\
\hline & $-0.12 t$ & $-0.56 t$ & -0.276 & $-0.43 t$ & $-0.34 t$ & $+0.90 t$ \\
\hline $0,-1$ & 0 & 0 & $-0.04 t$ & $-0.06 t$ & $+1.06 t$ & $-1.02 t$ \\
\hline & $-0.03 t$ & $+0.04 t$ & $-0.06 t$ & $+0.03 t$ & $+0.07 \imath$ & $-0.12 t$ \\
\hline $\begin{array}{l}2, \\
3,\end{array}$ & $+2.10 t$ & $-1.90 t$ & $+2.10 t$ & $-1.89 t$ & $+3.54 t$ & $+3.22 t$ \\
\hline 3 & $-0.06 t$ & $-0.90 t$ & $-0.19 t$ & $-0.73 t$ & $-0.19 t$ & $+1.18 t$ \\
\hline $0,-2$ & 0 & 0 & $-0.91 t$ & $-0.83 t$ & $+0.84 t$ & $-0.94 t$ \\
\hline & $+12.69 t$ & $+11.60 t$ & $+12.69 t$ & $+11.62 t$ & $-13.52 t$ & $+12.34 t$ \\
\hline $\begin{array}{l}2 \\
3\end{array}$ & $+0.48 t$ & $+0.60 t$ & $+0.21 t$ & $+0.37 t$ & $-0.42 t$ & $+0.14 t$ \\
\hline 3, & $+1.89 t$ & $-1.77 t$ & $+1.89 t$ & $-1.75 t$ & $+2.78 t$ & $+2.52 t$ \\
\hline $1,-3$ & $+1.60 t$ & $-1.14 t$ & $+1.61 t$ & $-1.11 t$ & $-1.64 t$ & $-1.21 t$ \\
\hline & $-0.50 t$ & $-0.58 t$ & $-0.53 t$ & $-0.56 t$ & $-0.83 t$ & $+0.75 t$ \\
\hline 3 & $-0.24 t$ & $+0.06 t$ & $-0.19 t$ & $+0.07 t$ & $-0.25 t$ & $-0.10 t$ \\
\hline & $+1.44 t$ & $-1.36 t$ & $+1.43 t$ & $-1.32 t$ & $+1.93 t$ & $+1.76 t$ \\
\hline $\begin{array}{l}3,-4 \\
4\end{array}$ & $-0.57 t$ & $-0.51 t$ & $-0.55 t$ & $-0.53 t$ & $-0.74 t$ & $+0.68 t$ \\
\hline 4 & $-0.60 t$ & 0 & $-0.52 t$ & $+0.10 t$ & $-0.73 t$ & $-0.10 t$ \\
\hline $0, \quad 0$ & $\ldots$ & & & +5 & -193 & \\
\hline $1, \quad 0$ & -45 & +319 & +9 & +160 & -49 & -709 \\
\hline $\begin{array}{l}2, \\
3,\end{array}$ & $\begin{array}{r}+168 \\
+\quad 2\end{array}$ & $\begin{array}{l}+383 \\
+\quad 5\end{array}$ & +199 & +449 & +278 & $-59 \mathrm{I}$ \\
\hline$-1,-1$ & +148 & -61 & +70 & -71 & +18 & +125 \\
\hline 0 & $\begin{array}{r}+27 \\
+\quad 27\end{array}$ & -381 & \pm 16 & 二 247 & $\begin{array}{l}+49 \\
-49\end{array}$ & $\begin{array}{r}+120 \\
+347\end{array}$ \\
\hline 1 , & -139 & -70 & -4 & -32 & +103 & +47 \\
\hline 2, & -21 & -36 & +3 & -12 & +1 & -215 \\
\hline 3, & -4 & +38 & +1 & -1 & -10 & +38 \\
\hline 4 , & +28 & +88 & -8 & +4 & -19 & \\
\hline $1,-2$ & +87 & -153 & -14 & -119 & -10 & +251 \\
\hline & -88 & -7 & +145 & -13 & +84 & \\
\hline & +108 & +121 & -60 & +796 & +83 & +816 \\
\hline $\begin{array}{l}4, \\
5,-2\end{array}$ & +20 & $\begin{array}{r}+517 \\
\ldots .\end{array}$ & $\begin{array}{r}+141 \\
-18\end{array}$ & $\begin{array}{l}+352 \\
+\quad 14\end{array}$ & $\begin{array}{l}-262 \\
-45\end{array}$ & $\begin{array}{r}+617 \\
+\quad 2\end{array}$ \\
\hline $3,-3$ & +18 & +4 & +208 & +16 & +17 & +34 \\
\hline & $\begin{array}{r}T \quad 69 \\
+\end{array}$ & -33 & $\begin{array}{l}+82 \\
+82\end{array}$ & -67 & \pm 35 & $\begin{array}{r}T \quad 4 \\
+\quad 44\end{array}$ \\
\hline 5 & -52 & -128 & -49 & -130 & -92 & +181 \\
\hline $4,-4$ & ... & $\cdots$ & +55 & -1 & +110 & \\
\hline 5 & 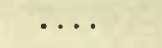 & $\ldots$ & +8 & $-\mathrm{i} 04$ & +12 & $\begin{array}{r}+52 \\
+\end{array}$ \\
\hline 6, & & $\cdots$ & -42 & -103 & -61 & +148 \\
\hline
\end{tabular}




\begin{tabular}{|c|c|c|c|c|c|c|}
\hline & \multicolumn{2}{|c|}{$2 \frac{a_{1}}{m^{\prime} n} \delta D^{\prime} R$} & \multicolumn{2}{|c|}{$2 \frac{a_{1}}{m^{\prime}} \delta \frac{\partial R}{\partial v}$} & \multicolumn{2}{|c|}{$2 \frac{a_{1}}{m^{\prime}} \delta \frac{\partial R}{\partial \rho}$} \\
\hline$U \quad S \quad \dot{J}$ & $\sin$ & $\cos$ & $\sin$ & $\cos$ & $\cos$ & $\sin$ \\
\hline & $"$ & " & $"$ & $"$ & " & " \\
\hline $\begin{array}{ll}0, & 1-1 \\
1, & -1\end{array}$ & $\begin{array}{r}0 \\
+2469\end{array}$ & $\begin{array}{r}0 \\
-50\end{array}$ & $\begin{array}{r}-185 \\
+2477\end{array}$ & $\begin{array}{r}2 \\
+\quad 50\end{array}$ & $\begin{array}{r}-84 \\
-2947\end{array}$ & $\begin{array}{r}13 \\
+\quad 69\end{array}$ \\
\hline 2 & $\begin{array}{r}+270 \\
+\quad 44\end{array}$ & $\begin{array}{r}8 \\
+\quad 8\end{array}$ & -7 & +13 & $\begin{array}{l}-2947 \\
-176\end{array}$ & $\begin{array}{l}-69 \\
-17\end{array}$ \\
\hline 3 , & -9 & +18 & -6 & +16 & -17 & -20 \\
\hline$-2, \quad 2-1$ & +84 & +32 & +42 & +29 & +9 & +49 \\
\hline-1 & +1638 & +88 & +1642 & +88 & +1231 & -143 \\
\hline $\begin{array}{l}\text { +1, } \\
\text { + }\end{array}$ & $\begin{array}{r}0 \\
+\quad 89\end{array}$ & $\begin{array}{r}0 \\
-89\end{array}$ & $\begin{array}{l}-114 \\
+83\end{array}$ & $\begin{array}{l}2 \\
-89\end{array}$ & $\begin{array}{l}+41 \\
-196\end{array}$ & $\begin{array}{l} \pm 11 \\
-62\end{array}$ \\
\hline+2 & -4 & +8 & -1 & +8 & -11 & -18 \\
\hline$-3, \quad 3-1$ & +18 & +24 & +25 & +28 & -42 & +41 \\
\hline-2 & $\begin{array}{l}+132 \\
\end{array}$ & -48 & +143 & -54 & -191 & -104 \\
\hline $\begin{array}{r}-1, \\
0\end{array}$ & $\begin{array}{r}+478 \\
0\end{array}$ & & $\begin{array}{l}+477 \\
-57\end{array}$ & $\begin{array}{r}+279 \\
-\quad 8\end{array}$ & $\begin{array}{r}+1529 \\
-60\end{array}$ & $\begin{array}{l}-279 \\
+44\end{array}$ \\
\hline$-3,4-1$ & +27 & -84 & +71 & -91 & -89 & -134 \\
\hline-2 & -442 & +10 & -449 & +7 & +776 & -8 \\
\hline-1 & +297 & +286 & +310 & +270 & +362 & -258 \\
\hline $1, \quad 3-2$ & -375 & +7336 & -393 & +7343 & $\begin{array}{l}+629 \\
\end{array}$ & +7722 \\
\hline $\begin{array}{l}2, \\
3,\end{array}$ & $\begin{array}{r}+1980 \\
+219\end{array}$ & $\begin{array}{l}-1116 \\
-783\end{array}$ & $\begin{array}{r}+1987 \\
+51\end{array}$ & $\begin{array}{l}-1271 \\
-672\end{array}$ & $\begin{array}{r}+3304 \\
+151\end{array}$ & $\begin{array}{l}+2544 \\
+1041\end{array}$ \\
\hline 4 , & -92 & -168 & -104 & -102 & -136 & $\begin{array}{r}+158 \\
+\end{array}$ \\
\hline $0, \quad 4-2$ & 0 & 0 & +669 & -485 & -601 & -966 \\
\hline 1, & -7737 & +5616 & -7733 & +5606 & +12136 & +8698 \\
\hline 2 , & -306 & -206 & -235 & -258 & +273 & $\begin{array}{r}910 \\
\end{array}$ \\
\hline 3 , & -72 & -78 & +59 & -52 & -60 & +109 \\
\hline$-2, \quad 5-2$ & +180 & -120 & +159 & +6 & -337 & -37 \\
\hline-1 & -625 & -739 & -620 & -740 & -1222 & -1217 \\
\hline 0, & 0 & $\begin{array}{r}0 \\
719\end{array}$ & +62 & $\begin{array}{r}56 \\
+725\end{array}$ & +167 & -60 \\
\hline $\begin{array}{l}+1 \\
+2\end{array}$ & $\begin{array}{l}\overline{61} \\
+40\end{array}$ & $\begin{array}{l}+719 \\
+\quad 12\end{array}$ & $\begin{array}{l}-63 \\
-13\end{array}$ & $\begin{array}{l}+725 \\
-\quad 6\end{array}$ & $\begin{array}{l}+72 \\
-17\end{array}$ & $\begin{array}{r}+1109 \\
+67\end{array}$ \\
\hline$-4, \quad 6-2$ & +110.0 & +15.6 & +86.1 & +32.9 & -127.5 & +39.4 \\
\hline-3, & +282.3 & -73.2 & +248.8 & -14.5 & -413.6 & -75.7 \\
\hline $\begin{array}{l}-2 \\
-1\end{array}$ & $\begin{array}{r}+132 \\
-8823\end{array}$ & $\begin{array}{r}-486 \\
+6114\end{array}$ & $\begin{array}{r}+237 \\
-8829\end{array}$ & $\begin{array}{r}-557 \\
+6121\end{array}$ & $\begin{array}{r}-1131 \\
-12973\end{array}$ & $\begin{array}{l}Z_{-9020}^{-1461} \\
-90\end{array}$ \\
\hline 0 & & $\begin{array}{r}+014 \\
0\end{array}$ & +746 & $\begin{array}{r}+0121 \\
-493\end{array}$ & +774 & +406 \\
\hline$-4, \quad 7-2$ & +280 & +8 & +207 & +45 & -216 & +48 \\
\hline-3 & $\begin{array}{r}+897 \\
\end{array}$ & -441 & $\begin{array}{r}+727 \\
\end{array}$ & -632 & -1128 & -977 \\
\hline-2 , & +1874 & -1412 & +1913 & -1374 & -3236 & -2195 \\
\hline -1, & $\begin{array}{r}-1978 \\
0\end{array}$ & -1117 & $\begin{array}{r}-1995 \\
+139\end{array}$ & $\begin{array}{r}-1097 \\
+\quad 90\end{array}$ & $\begin{array}{r}-2071 \\
+144\end{array}$ & $\begin{array}{l}+904 \\
-71\end{array}$ \\
\hline & & & & & & \\
\hline
\end{tabular}


In the terms of $\delta R$ introduced by the perturbations of Saturn, namely, $\frac{\partial R}{\partial v^{\prime}} \delta v^{\prime}+\frac{\partial R}{\partial \rho^{\prime}} \delta \rho^{\prime}$, the differentiation represented by $D_{t}^{\prime}$ should be performed by considering $\delta v^{\prime}$ and $\delta \rho^{\prime}$ as constant, although they are expressed as a function of the mean longitude of Uranus, as well as of Saturn. 'The mean longitude of Uranus thus introduced is therefore represented by $U^{\prime}$, which is regarded as constant in taking $D_{t}^{\prime} R$, and $U$ only supposed to vary.

Again, in the terms $\frac{\partial R}{\partial v} \delta v+\frac{\partial R}{\partial \rho} \delta \rho$, since $\delta v$ and $\delta \rho$ represent perturbations of Uranus, their complete derivatives, with respect to the time, are to be taken. But their expressions contain the mean longitude of Saturn as well as Uranus. The mean longitude of Saturn thus introduced is represented by $S^{\prime \prime}$, and is to be considered variable in obtaining $D_{t}^{\prime} \delta R$, while $S$ is considered constant. The ratio of the coefficient of $t$ to $n$ in the various terms of this part of $\delta R$ is given to the right of each corresponding term.

The value of $D_{t}^{\prime} \delta R$ being once obtained, there is no longer any distinction necessary between $U, U^{\prime}$, or between $S$ and $S^{\prime}$. 'The similar terms are therefore combined by putting $S^{\prime \prime}=S ; U^{\prime}=U$.

From the above values of $2 \delta D_{t}^{\prime} R$ and $2 \delta \frac{\partial R}{\partial \rho}$, we form the following value of

$$
\frac{a_{1}}{m} \delta Q=\frac{2 a_{1}}{m} \int \delta D_{t}^{\prime} R d t+\frac{a_{1}}{m} \delta \frac{\partial R}{\partial \rho}
$$

and of the other quantities which enter the perturbations of the co-ordinates. We shall begin with those terms which depend only on the mutual action of Saturn and Uranus, because they are few and small, and the only terms which are sensible are those in which the coefficient of the mean longitude of Saturn is -1 . We shall therefore confine ourselves to these. And, instead of employing the condensed formulæ, we shall make the computation in full by (13).

\begin{tabular}{|c|c|c|c|c|c|c|c|c|}
\hline \multicolumn{4}{|c|}{$\frac{a_{1}}{m^{\prime}} \delta Q=2 \frac{a_{1}}{m} \int \delta D^{\prime} R d t+\delta \frac{\partial R}{\partial p}$} & \multicolumn{2}{|c|}{$\frac{a_{1}}{m^{\prime}} \xi \delta Q$} & \multicolumn{3}{|c|}{$\frac{a_{1}}{m^{\prime}} \eta \delta Q$} \\
\hline $\begin{array}{l}g \quad l^{\prime} \\
-1,-1 \\
0 \\
1 \\
2 \\
3 \\
4\end{array}$ & $\begin{array}{c}\cos \\
\prime \prime \prime \prime \\
+47 \\
-14+0.53 t \\
-24+0.02 t \\
-60+4.23 t \\
-525+0.31 t \\
\cdots\end{array}$ & $\begin{array}{r} \\
+7 \\
+30 \\
+6 \\
-2 \\
+24\end{array}$ & $\begin{array}{l}11 \\
0.51 t \\
0.08 t \\
3.84 t \\
5.49 t \\
.\end{array}$ & $\begin{array}{c}\cos \\
\prime \prime \prime \\
-10 \\
+13+0.02 t \\
-41+2.37 t \\
-270-0.13 t \\
+\quad 7+2.09 t \\
-262+0.15 t\end{array}$ & $\begin{array}{c}\sin \\
\prime \prime \prime \prime \\
+148 \\
+49+0.05 t \\
+138+1.62 t \\
+157-3.05 t \\
+31+2.30 t \\
+120-2.74 t\end{array}$ & $\begin{array}{r} \\
+\quad \\
+3 \\
+2 \\
+25 \\
-3 \\
-26\end{array}$ & $\begin{array}{l}\sin \\
7 \\
6-0.06 t \\
9-1.85 t \\
0-0.13 t \\
0+2.11 t \\
2+0.15 t\end{array}$ & $\begin{array}{c}\cos \\
\prime \prime \prime \\
+153 \\
-\quad 8+0.01 t \\
-164+2.11 t \\
+85-2.70 t \\
+14-1.32 t \\
-120+2.74 t\end{array}$ \\
\hline & & & & & & & $\delta v$ & \\
\hline $\begin{array}{l}g \quad l^{\prime} \\
0,-1 \\
1, \\
2, \\
3, \\
4,\end{array}$ & $\begin{array}{r}\quad c o s \\
\quad " \prime \quad \\
+.001-.00 \\
+.024+.00 \\
+.333+.00 \\
-.142-.00 \\
-.071\end{array}$ & $\begin{array}{r}0001 t \\
0033 t \\
0437 t \\
0002 t\end{array}$ & $\begin{array}{r}11 \\
-.0 \\
-.0 \\
-.4 \\
+.0 \\
+.0\end{array}$ & $\begin{array}{c}\sin \\
\quad \prime \prime \\
23+.00002 t \\
36+.00033 t \\
06+.00432 t \\
79-.00169 t \\
19\end{array}$ & $\begin{array}{l}\text { sin } \\
\text { " } \\
+0.002+0 . \\
+0.043+0 . \\
+0.620+0 . \\
+2.69-0 . \\
+0.132\end{array}$ & $\begin{array}{r}0000 t \\
0006 t \\
0098 t \\
0011 t \\
0 t\end{array}$ & $\begin{array}{r}+0.0 \\
+0.0 \\
+0.8 \\
+0.8 \\
+0.0\end{array}$ & $\begin{array}{l}021+0.0000 t \\
058+0.0000 t \\
803-0.0097 t \\
830-0.0153 t \\
040+0.0000 t\end{array}$ \\
\hline
\end{tabular}


The computation of these terms being extremely complex, a check upon their accuracy is desirable. In the case of the secular variations of the coefficients, the coefficients of the time are easily obtained by substituting in the integrated perturbations the variations of the eccentricity and perihelion of Saturn. Thus I have found

$$
\begin{aligned}
\delta v= & +0.0103 t \sin \left(2 g-l^{\prime \prime}\right)-0.0094 t \cos \left(2 g-l^{\prime}\right) \\
& +0.0027 t \sin \left(3 j-l^{\prime}\right)-0.0138 t \cos \left(3 g-l^{\prime}\right)
\end{aligned}
$$

The greatest discrepancy is found in the coefficient of $\sin \left(3 g-l^{\prime}\right)$, and it amounts to $0^{\prime \prime} .0038 t$, or about $0^{\prime \prime} .4$ in a century. But, owing to the great period of this term, nearly 600 years, this difference, during any one century, will be nearly eliminated through the mean longitude and mean motion.

It may also be remarked that in this case the terms derived from the perturbations of the elements are undoubtedly the correct ones, and will therefore be employed.

The terms which the preceding integration fails to give, owing to the constant terms introduced into $\xi \delta Q$ and $\eta \delta Q$, are found by (22).

We thus have

$$
\begin{gathered}
n \Sigma p_{u} k_{e}^{(u)}=+0^{\prime \prime} .36 \\
n \Sigma q_{u} k_{s}^{(u)}=+0.27 \\
r_{1}^{2} \delta \rho=\frac{1}{4} M n t^{2}\left\{0^{\prime \prime} .36 \sin g-0^{\prime \prime} .27 \cos g\right\} \\
\delta v=\frac{1}{2} M n t^{2}\left\{0^{\prime \prime} .36 \cos g+0^{\prime \prime} .27 \sin g\right\} \\
=\quad t^{2}\left\{0^{\prime \prime} .0000038 \cos g+0^{\prime \prime} .0000029 \sin g\right\} .
\end{gathered}
$$

\begin{tabular}{|c|c|c|c|c|}
\hline & & - & 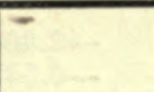 & \\
\hline $\begin{array}{r}g \\
l^{\prime} \\
-1,-1 \\
0,-1 \\
1,-1 \\
2,-1 \\
3,-1 \\
4,-1 \\
5,-1\end{array}$ & $\begin{array}{c}\text { sin } " \prime \\
\quad " 10 \\
+0.036 \\
+1.284 \\
-20.774+0.06 T \\
-11.270+1.03 T \\
+51.99+0.27 T \\
+2.265 \\
+0.126\end{array}$ & $\begin{array}{l}\quad c \quad c \quad " \\
\quad \quad " \quad 0.039 \\
+\quad 0.739 \\
+\quad 8.580 \\
+144.265-0.94 T \\
+116.69-1.38 T \\
+\quad 5.656 \\
+\quad 0.329\end{array}$ & $\begin{array}{r}\cos \\
+\quad 0 \\
-11 \\
+1753 \\
-87 \\
-237 \\
-55 \\
-\quad 4\end{array}$ & $\begin{array}{l}\sin \\
-\quad 2 \\
-31 \\
-244 \\
-3114 \\
+644 \\
+140 \\
+10\end{array}$ \\
\hline
\end{tabular}

The greatest effect of these terms amounts to less than one-twentieth of a second in a century. They may therefore be neglected in the present theory. The other terms containing the square of the time are yet smaller.

Applying the terms of the second order thus found to the terms of the first order depending on the corresponding arguments, the perturbations of Uranus by Saturn become

$T$ here represents the time counted in centuries from 1850.0 .

The other terms remain the same as given on page 50 .

Perturbations depending on the product of the masses of Jupiter and Siturn.

The values of $\delta D_{t}^{\prime} R, \delta \frac{\partial R}{\partial \mathrm{v}}$, and $\delta \frac{\partial R}{\partial \rho}$, depending on the products of the masses 
of Jupiter and Saturn, are given on page 74. The computation from these data being conducted in the same way as in the case of the terms of the first order, it is not necessary to give much more than the results. 'These are shown in the following table. 'The indices to the left represent the coefficients of the mean longitudes of Uranus, Saturn, and Jupiter, all counted from the perihelion of Uranus. Column $v$ gives the ratio of the mean motion of Uranus to the coefficient of the time in each argument. The perturbations of the common logarithm of the radius vector are expressed in units of the seventh place of decimals.

\begin{tabular}{|c|c|c|c|c|c|}
\hline . & $\nu$ & \multicolumn{2}{|c|}{$\delta v$} & \multicolumn{2}{|c|}{$0.4343 \delta \rho$} \\
\hline$U \quad S \quad J$ & & $\sin$ & $\cos$ & $\cos$ & $\sin$ \\
\hline $\begin{array}{ll}0, & 1,-1 \\
1, & \\
3, & \\
4 & \end{array}$ & $\begin{array}{l}-0.2364 \\
-0.3095 \\
-0.4480 \\
-0.8127\end{array}$ & $\begin{array}{l}+0.002 \\
+0.020 \\
+0.004 \\
+0.016\end{array}$ & $\begin{array}{c}0 \\
0 \\
-0.003 \\
-0.024\end{array}$ & $\begin{array}{r}0 \\
0 \\
0 \\
0\end{array}$ & $\begin{array}{l}0 \\
0 \\
0 \\
0\end{array}$ \\
\hline $\begin{array}{ll}-2, & 2,-1 \\
-1, & \\
0 & \\
1 & \\
2 & \end{array}$ & $\begin{array}{r}-0.2960 \\
-0.4204 \\
-0.7254 \\
-2.6420 \\
+1.6090\end{array}$ & $\begin{array}{r}-0.007 \\
-0.108 \\
-0.014 \\
+0.164 \\
+0.005\end{array}$ & $\begin{array}{l}-0.001 \\
-0.007 \\
-0.012 \\
-0.267 \\
-0.005\end{array}$ & $\begin{array}{r}0 \\
-2 \\
0 \\
+\quad 1 \\
0\end{array}$ & $\begin{array}{r}0 \\
0 \\
0 \\
+\quad 1 \\
0\end{array}$ \\
\hline $\begin{array}{ll}-3, & 3,-1 \\
-2, & \\
-1 & \\
0 & \end{array}$ & $\begin{array}{l}-0.6551 \\
-1.8997 \\
+2.1115 \\
+0.6786\end{array}$ & $\begin{array}{l}+0.012 \\
+0.175 \\
+0.078 \\
+0.007\end{array}$ & $\begin{array}{l}-0.002 \\
-0.015 \\
+0.512 \\
+0.024\end{array}$ & $\begin{array}{r}0 \\
+\quad 1 \\
-2 \\
0\end{array}$ & $\begin{array}{r}0 \\
0 \\
+\quad 3 \\
0\end{array}$ \\
\hline $\begin{array}{ll}-3, & 4,-1 \\
-2, & \\
-1 & \end{array}$ & $\begin{array}{l}+0.754 \\
+0.430 \\
+0.301\end{array}$ & $\begin{array}{r}-0.030 \\
+0.043 \\
+0.001\end{array}$ & $\begin{array}{l}+0.081 \\
+0.005 \\
-0.003\end{array}$ & $\begin{array}{r}0 \\
-1 \\
0\end{array}$ & $\begin{array}{r}1 \\
0 \\
0\end{array}$ \\
\hline $\begin{array}{ll}1, & 3,-2 \\
2, & \\
3, & \\
4, & \end{array}$ & $\begin{array}{l}-0.2170 \\
-0.2771 \\
-0.3833 \\
-0.6215\end{array}$ & $\begin{array}{l}-0.002 \\
-0.051 \\
-0.010 \\
+0.032\end{array}$ & $\begin{array}{l}-0.032 \\
+0.035 \\
+0.050 \\
+0.052\end{array}$ & $\begin{array}{r}0 \\
-1 \\
0 \\
0\end{array}$ & $\begin{array}{l}-1 \\
-1 \\
-1 \\
-1\end{array}$ \\
\hline $\begin{array}{ll}0, & 4,-2 \\
1, & \\
2, & \\
3 & \end{array}$ & $\begin{array}{l}-0.3627 \\
-0.5692 \\
-1.3210 \\
+4.1150\end{array}$ & $\begin{array}{r}-0.010 \\
+0.075 \\
-0.349 \\
-0.510\end{array}$ & $\begin{array}{l}-0.004 \\
-0.154 \\
-1.297 \\
-0.453\end{array}$ & $\begin{array}{r}0 \\
-5 \\
-\quad 3 \\
+\quad 2\end{array}$ & $\begin{array}{r}0 \\
-2 \\
+11 \\
-2\end{array}$ \\
\hline $\begin{array}{l}-2, \quad 5,-2 \\
-1, \\
0 \\
1, \\
2,\end{array}$ & $\begin{array}{r}-0.5250 \\
-1.1051 \\
+10.5152 \\
+0.9132 \\
+0.4773\end{array}$ & $\begin{array}{l}-0.253 \\
-4.433 \\
-0.546 \\
+0.206 \\
+0.012\end{array}$ & $\begin{array}{l}-0.034 \\
-0.617 \\
-0.032 \\
-3.254 \\
-0.192\end{array}$ & $\begin{array}{r}3 \\
-43 \\
+\quad 2 \\
-2 \\
0\end{array}$ & $\begin{array}{r}0 \\
+\quad 7 \\
0 \\
-36 \\
-2\end{array}$ \\
\hline $\begin{array}{l}-4, \quad 6,-2 \\
-3, \\
-2, \\
-1, \\
0\end{array}$ & $\begin{array}{r}-0.9497 \\
-18.9250 \\
+1.0558 \\
+0.5136 \\
+0.3393\end{array}$ & $\begin{array}{r}+1.824 \\
+40.650 \\
+6.237 \\
+0.467 \\
+0.007\end{array}$ & $\begin{array}{l}-0.519 \\
-10.500 \\
-7.866 \\
-0.539 \\
-0.017\end{array}$ & $\begin{array}{r}+19 \\
+32 \\
-63 \\
0 \\
0\end{array}$ & $\begin{array}{r}+6 \\
+10 \\
-79 \\
-\quad 2 \\
0\end{array}$ \\
\hline $\begin{array}{l}-4, \quad 7,-2 \\
-3, \\
-2, \\
-1 \\
-0\end{array}$ & $\begin{array}{r}+0.5558 \\
+0.3573 \\
+0.2632 \\
+0.2084 \\
+0.1724\end{array}$ & $\begin{array}{c}-0.050 \\
-0.046 \\
-0.045 \\
+0.006 \\
0\end{array}$ & $\begin{array}{c}-0.003 \\
+0.032 \\
+0.032 \\
+0.007 \\
0\end{array}$ & $\begin{array}{r}+1 \\
+\quad 1 \\
+1 \\
0 \\
0\end{array}$ & $\begin{array}{r}0 \\
+\quad 1 \\
+\quad 1 \\
0 \\
0\end{array}$ \\
\hline
\end{tabular}




\section{CH A P T E R V.}

\section{COLLECTION AND TRANSFORMATION OF THE PRECEDING PERTURBATIONS OF URANUS.}

THE terms of the perturbations which neither contain the elements of the disturbing planets, nor depend on the secular variations of the eccentricity and perihelion, admit of being greatly simplified by a slight change in the arbitrary elements. These terms are as follows:

(1) In the longitude of Uranus

$\begin{array}{cccccc}\text { Aetion of Jupiter, } & +31.2116 t & +25.657 \sin g & +1.397 \sin 2 g & -1.859 \cos g & -0.087 \cos 2 g \\ \text { Action of Saturn, } & +10.9690 t & +8.545 \sin g & +0.461 \sin 2 g & -4.735 \cos g & -0.169 \cos 2 g \\ \text { Aetion of Neptune, }-0.4262 t & +0.697 \sin g & +0.046 \sin 2 g & -0.088 \cos g & -0.005 \cos 2 g\end{array}$

(2) In the value of $\cos \psi \delta \rho$, units of 7 th place of decimals.

$\begin{array}{llllll}\text { Aetion of Jupiter, } & -10089 & -492 \cos g & -33 \cos 2 g & -2 \sin g & +1 \sin 2 g \\ \text { Action of Saturn, } & -3543 & -184 \cos g & -15 \cos 2 g & -15 \sin g & \\ \text { Action of Neptune, } & +138 & -1 \cos g & -1 \cos 2 g & +1 \sin g & \\ \quad \text { Total, } & -13494 & -677 \cos g & -49 \cos 2 g & -16 \sin g & +1 \sin 2 g\end{array}$

Let us first consider the first or constant term in the perturbation of each co-ordinate. If we suppose a change of $\delta \boldsymbol{n}$ in the mean motion of a planet, the corresponding change in $\delta \rho$ will be

$$
\delta \rho=-\frac{2}{3} \frac{\delta n}{n}
$$

If, then, we increase the mean motion of Uranus by $41^{\prime \prime} .754$, the corresponding change in $\delta \rho$ will be -18045 , and in $\cos \psi \delta \rho,-18025$. Subtracting these from the above perturbations, the secular term in the mean motion will disappear, and we shall have for the constant term of $\cos \psi \delta \rho$

$$
+4531
$$

This same change in the mean motion will produce a secular term in the equation of the centre of the same nature with that produced by the secular variation of the perihelion. The differences of the values of the secular terms, found by the two methods employed in Chapters II. and III., proceeds from the fact that in the one case the effect of the above term in the mean motion is included, and in the other excluded. 
If we subduct the effect in question when necessary, the remainder will be the effect of the secular variation of the longitude of the perihelion of Uranus, to which we shall revert presently.

Let us next introduce such a change in the eccentricity of Uranus as shall produce the term $34^{\prime \prime} .899 \sin g$, and ascertain its effect on the other terms. For this purpose we must determine ie by the condition

which gives

$$
\left(2-\frac{3}{4} e^{2}\right) \delta e=34^{\prime \prime} .899
$$

$$
\varepsilon_{e}=17^{\prime \prime} .464=.0000847 .
$$

A change of this amount in $\delta e$ will introduce the following terms in $\delta v$ and $\delta \rho$

$$
\begin{aligned}
\delta v & =34^{\prime \prime} .899 \sin g+2^{\prime \prime} .048 \sin 2 g \\
\cos \psi \delta \rho & =20-844 \cos g-59 \cos 2 g .
\end{aligned}
$$

Subtracting these terms from the expressions previously found we have

$$
\begin{gathered}
\delta v=-0^{\prime \prime} .144 \sin 2 g-6^{\prime \prime} .682 \cos g-0^{\prime \prime} .261 \cos 2 g . \\
\cos \psi \delta \rho=+4511+167 \cos g+10 \cos 2 g-16 \sin g+1 \sin 2 g .
\end{gathered}
$$

Again, let us put

$$
e \hat{\varepsilon} \pi=3^{\prime \prime} .342=.0000162,
$$

we shall have the elliptic terms

$$
\begin{aligned}
\delta v & =-6^{\prime \prime} .682 \cos g-0^{\prime \prime} .391 \cos 2 g \\
\cos \psi \delta \rho & =-162 \sin g-11 \sin 2 g .
\end{aligned}
$$

Subtracting these expressions the constant terms, independent of the mean longitude of the disturbing planets, are reduced to

$$
\begin{aligned}
\delta v & =-0^{\prime \prime} .144 \sin 2 g+0^{\prime \prime} .130 \cos 2 g . \\
\cos \psi \delta \rho & =4511+167 \cos g+10 \cos 2 g+146 \sin g+12 \sin 2 g . \\
0.43429 \delta \rho & =1969+73 \cos g+4 \cos 2 g+63 \sin g+5 \sin 2 g .
\end{aligned}
$$

In the last equation we have introduced the constant +.0000008 produced in $\delta \rho$ by the combiued action of Venus, the Earth, and Mars. 'The effect of cach planet is computed by the approximate formula

$$
\sum_{\rho}=\frac{1}{6} m^{\prime}\left(b_{i}^{(0)}+\alpha D \alpha b_{i}^{(0)}\right)
$$

\section{Secular Variations.}

The following inequalities result from the secular variations of the eccentricity and longitude of perihelion produced by each of the disturbing planets, $T$ being the time expressed in centuries.

From the variation of the eccentricity

$$
\begin{array}{lrrr}
\text { Action of Jupiter, } & \delta v=-1.216 T \sin g & -0.072 T \sin 2 g & -0.005 T \sin 3 g \\
\text { Action of Saturn, } & -9.182 T \sin g & -0.538 T \sin 2 g & -0.032 T \sin 3 g \\
\text { Action of Neptune, } & -0.502 T \sin g & -0.030 T \sin 2 g & -0.002 T \sin 3 g
\end{array}
$$


Action of Jupiter, A etion of Saturn, Action of Neptune,

$$
\begin{aligned}
& M / \delta_{p}=+13 T \cos g \quad+1 " T \cos 2 g \\
& +98 T \cos g \quad+7 T \cos 2 g
\end{aligned}
$$

The secular variation of the longitude of the perihelion is

$\begin{array}{cr}\text { Action of Jupiter, } & +122.1 T \\ \text { Aetion of Saturn, } & +118.4 T \\ \text { Action of Neptune, } & +51.1 T \\ \text { Total, } & \delta \pi=+291.6 T\end{array}$

\begin{tabular}{|c|c|c|c|}
\hline $\begin{array}{c}\text { Action of Jupiter, } \\
\text { Aetion of Saturn, } \\
\text { Action of Neptune, } \\
\text { Total, }\end{array}$ & 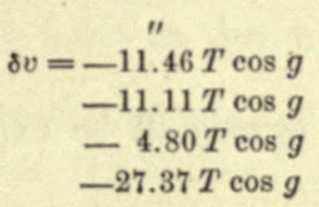 & $\begin{array}{l}\text { "0.671T } \cos 2 g \\
-0.651 T \cos 2 g \\
-0.281 T \cos 2 g \\
-1.603 T \cos 2 g\end{array}$ & $\begin{array}{l}\prime \prime \\
-0.047 T \cos 3 g \\
-0.039 T \cos 3 g \\
-0.016 T \cos 3 g \\
-0.102 T \cos 3 g\end{array}$ \\
\hline $\begin{array}{l}\text { ction of Jupiter, } \\
\text { tion of Saturn, } \\
\text { ction of Neptune, }\end{array}$ & $\begin{aligned} M \delta_{\rho}= & -120 T \sin g \\
& -117 T \sin g \\
& -50 T \sin g\end{aligned}$ & $\begin{array}{l}-8 T \sin 2 g \\
-8 T \sin 2 g \\
-3 T \sin 2 g\end{array}$ & \\
\hline
\end{tabular}

The effect of this secular variation on the longitude and radius vector is

For the purpose of conveniently tabulating the perturbations, we shall express them in a form similar to that adopted in the theory of Neptune. Let us select, from the terms of the periodic perturbations produced by any planet, all those in which the difference between the indices $i$ and $i^{\prime}$ is the same. For example, in the perturbations of the longitude produced by Jupiter, let us consider the terms

$$
\begin{array}{rlr}
\delta v= & +1.269 \sin (-l) & +0.002 \cos (-l) \\
& -3.495 \sin (2 g-l) & -0.092 \cos (2 g-l) \\
& +1.182 \sin (g-2 l) & +0.515 \cos (g-2 l) \\
& +0.074 \sin (3 g-2 l) & -0.005 \cos (3 g-2 l) \\
& -0.005 \sin (2 g-3 l) & \\
& +0.011 \sin (4 g-3 l) & -0.001 \cos (4 g-3 l)
\end{array}
$$

These terms may be expressed in the form

$$
\begin{array}{r}
i v=\sin g \times\left\{\begin{array}{cc}
+0.094 \sin (g-l) & -4.764 \cos (g-l) \\
+0.520 \sin 2(g-l) & -1.108 \cos 2(g-l) \\
& +0.016 \cos 3(g-l)
\end{array}\right\} \\
+\cos g \times\left\{\begin{array}{cc}
-2.226 \sin (g-l) & -0.090 \cos (g-l) \\
+1.256 \sin 2(g-l) & +0.510 \cos 2(g-l) \\
+0.006 \sin 3(g-l) &
\end{array}\right\}
\end{array}
$$


In general, a series of terms of the form

$$
\begin{array}{r}
\Sigma a_{i} \sin (i A+s g)+\Sigma b_{i} \cos (i A+s g) \\
+\Sigma a_{i}^{\prime} \sin (i A-s g)+\Sigma b_{i}^{\prime} \cos (i A-s g),
\end{array}
$$

may be put in the form

$$
\begin{aligned}
& \left\{\Sigma\left(a_{i}-a_{i}^{\prime}\right) \cos i A-\Sigma\left(b_{i}-b_{i}^{\prime}\right) \sin i A\right\} \sin 8 g \\
+ & \left\{\Sigma\left(a_{i}+a_{i}^{\prime}\right) \sin i A+\Sigma\left(b_{i}+b_{i}^{\prime}\right) \sin i A\right\} \cos 8 g .
\end{aligned}
$$

All the periodic terms containing only $g$ and $l$ in the arguments may be put into this form by taking

$$
A=g-l
$$

so that the coefficients of $\sin s g$ and $\cos s g$ may all be expressed as a function of the single variable argument $A$.

The perturbations of the elements may be reduced to perturbations of the co-ordinates expressed as the sum of several products of slowly varying functions into the sines and cosines of the multiples of $g$. We have, in fact,

$$
\begin{array}{rlrl}
\delta v= & \delta l & \\
& +\left(2-\frac{3}{4} e^{2}\right) \delta e \times \sin g & & +\left(2-\frac{1}{4} e^{2}\right) e \delta g \times \cos g \\
& +\left(\frac{5}{2} e-\frac{11}{6} e^{3}\right) i e \times \sin 2 g & & +\left(\frac{5}{2} e-\frac{11}{12} e^{3}\right) e \delta g \times \cos 2 g . \\
& + \text { etc. } & & + \text { etc. }
\end{array}
$$

It appears, therefore, that all the perturbations in which the arguments contain the mean longitudes of only two planets may be put in the form

$$
\begin{array}{r}
\delta v=(v . c .0)+(v . c .1) \cos g+(v . c .2) \cos 2 g+\text { etc. } \\
+(v . s .1) \sin g+(v . s .2) \sin 2 g+\text { etc. } \\
\begin{aligned}
M \delta \rho=(\rho . c .0) & +(\rho . c .1) \cos g+(\rho . c .2) \cos 2 g+\text { etc. } \\
& +(\rho . s .1) \sin g+(\rho . s .2) \sin 2 g+\text { etc. }
\end{aligned}
\end{array}
$$

We have next to reduce to the same form those terms which contain the mean longitudes of both Jupiter and Saturn, and which are given on page 78. We have here twenty-four terms, each greater than $0 " .04$. As most of these terms depend on three independent arguments, they cannot be included in a double entry table, while, if we include them as perturbations of the longitude in tables of single entry, we shall have to enter twenty-two tables with as many different arguments. But, by taking, for the argument $A$, the middle one in each series of arguments which depend on the same multiples of Jupiter and Saturn, and expressing the terms above and below it in each series as coefficients of $\sin g, \cos g, \sin 2 g$, and 
$\cos 2 g$, we may reduce the number of arguments to eight, and the number of tables to seventeen. Consider, for instance, the terms of the second series,

$$
\begin{aligned}
& -0.108 \sin (-g+2 S-J) \quad-0.007 \cos (-g+2 S-J) \\
& -0.014 \sin (2 S-J)-0.012 \cos (2 S-J) \\
& +0.164 \sin (g+2 S-J)-0.267 \cos (g+2 S-J) \text {. }
\end{aligned}
$$

These terms may be allowed for by adding to $(v . c .0),(v .8 .1),(v . c .1)$, the terms

$$
\begin{aligned}
& (\text { v.c.0 }=-\ddot{\prime \prime} 0.014 \sin (2 S-J)-0.012 \cos (2 S-J) \\
& (v .8 .1)=+0.260 \sin (2 S-J)+0.272 \cos (2 S-J) \\
& (v . c .1)=+0.056 \sin (2 S-J)-0.274 \cos (2 S-J),
\end{aligned}
$$

From the perturbations of longitude and radius vector already given, we readily find the following values of (v.c.0), (v.s.1), etc.

Acticn of Jupiter.

$$
\begin{aligned}
& A_{1}=l^{\prime}-g \\
& \text { (v.c.0) }=+53.064 \sin A_{1}-0.004 \cos A_{1} \\
& -0.277 \sin 2 A_{1}+0.036 \cos 2 A_{1} \\
& -0.025 \sin 3 A_{1} \\
& \text { (v.c.1) }=+2.226 \sin A_{1}-0.090 \cos A_{1} \quad(v .8 .1)=-0.094 \sin A_{1}-4.764 \cos A_{1} \\
& -1.256 \sin 2 A_{1}+0.510 \cos 2 A_{1} \quad-0.520 \sin 2 A_{1}-1.108 \cos 2 A_{1} \\
& -0.006 \sin 3 A_{1} \\
& -11^{\prime \prime} .46 T \\
& -1^{\prime \prime} .22 T \\
& +0.016 \cos 3 A_{1}
\end{aligned}
$$

(v.c.2) $=+0.121 \sin A_{1}-0.038 \cos A_{1}\left(\right.$ v.s.2) $=-0.056 \sin A_{1}-0.175 \cos A_{1}$

$+0.012 \sin 2 A_{1}-0.014 \cos 2 A_{1} \quad+0.008 \sin 2 A_{1}+0.042 \cos 2 A_{1}$

$+0.029 \sin 3 A_{1}-0.034 \cos 3 A_{1} \quad+0.034 \sin 3 A_{1}+0.035 \cos 3 A_{1}$ $-0^{\prime \prime} .67 T$

$$
(v . c .3)=-0.04 T \quad(v .8 .3)=-0.005 T
$$

$\begin{aligned}(\text { p.c. } 0)= & +1127 \cos A_{1} \\ & +\quad 4 \cos 2 A_{1}\end{aligned}$

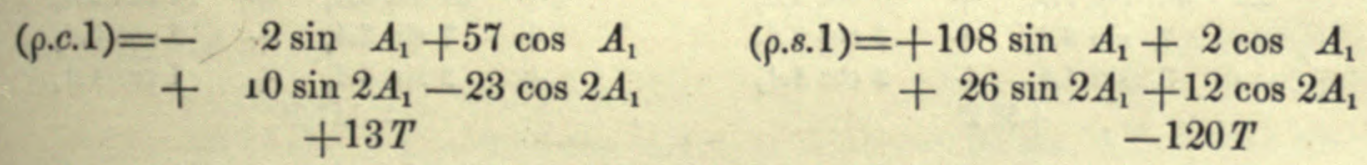

$\left(\right.$ ( .c.2) $=+\quad 7 \cos A_{1}+1 T \quad(\rho .8 .2)=+7 \sin A_{1}-8 T$ 


\section{Action of Saturn.}

$$
\begin{aligned}
& A_{2}=l-g \\
& \text { (v.c.0) }=\left(\begin{array}{c}
+20.774 \\
-0.06 T
\end{array}\right) \sin A_{3} \quad+8.580 \cos A_{2} \\
& -4.110 \sin 2 A_{3} \quad-0.009 \cos 2 A_{2} \\
& -0.824 \sin 3 A_{9} \quad-0.019 \cos 3 A_{2} \\
& -0.228 \sin 4 A_{3} \quad-0.008 \cos 4 A_{\mathrm{g}} \\
& \begin{array}{lll}
-0.074 \sin 5 A_{3} & -0.003 \cos 5 A_{3}
\end{array} \\
& -0.025 \sin 6 A_{2}
\end{aligned}
$$

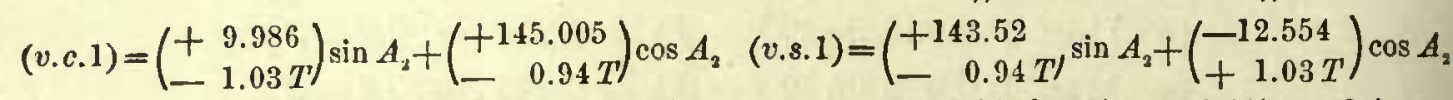

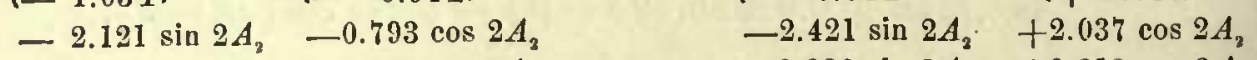

$$
\begin{aligned}
& -0.392 \sin 3 A_{2} \quad-0.308 \cos 3 A_{2} \quad-0.226 \sin 3 A_{2}+0.318 \cos 3 A_{2} \\
& -0.109 \sin 4 A_{2} \quad-0.099 \cos 4 A_{3} \quad-0.055 \sin 4 A_{3} \quad+0.097 \cos 4 A_{2} \\
& -0.038 \sin 5 A_{2} \quad-0.027 \cos 5 A_{2} \quad-0.027 \sin 5 A_{2} \quad+0.038 \cos 5 A_{2} \\
& -11^{\prime \prime} .11 T \\
& -9^{\prime \prime} .18 T \\
& \text { (v.c.2) }=\left(\begin{array}{c}
-52.026 \\
-0.27 T
\end{array}\right) \sin A_{2}+\left(\begin{array}{cc}
+116.73 \\
- & 1.38 T
\end{array}\right) \cos A_{3} \quad(v . s .2)=\left(\begin{array}{c}
+116.650 \\
-1.38 T
\end{array}\right) \sin A_{2}+\left(\begin{array}{r}
51.954 \\
+0.27 T
\end{array}\right) \cos A_{2} \\
& \begin{array}{lll}
-0.665 \sin 2 A_{3} & -1.847 \cos 2 A_{3}
\end{array}
\end{aligned}
$$

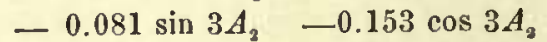

$$
\begin{aligned}
& -0.013 \sin 4 A_{2} \quad-0.044 \cos 4 A_{3} \\
& -0.005 \sin 5 A_{3}-0.016 \cos 5 A_{3} \\
& -0^{\prime \prime} .65 T \\
& \text { (v.c.3) }=-2.265 \sin A_{9}+5.656 \cos A_{3} \\
& -1.163 \sin 2 A_{2}+2.956 \cos 2 A_{9} \\
& +0.026 \sin 3 A_{2}-0.063 \cos 3 A_{2} \\
& +0.005 \sin 4 A_{2}-0.013 \cos 4 A_{2} \\
& +0.002 \sin 5 A_{9}-0.004 \cos 5 A_{3} \\
& -0^{\prime \prime} .04 T \\
& \text { (v.c.4) }=-0.126 \sin A_{2}+0.329 \cos A_{9} \\
& -0.503 \sin 2 A_{2}+0.378 \cos 2 A_{3} \\
& +0.053 \sin 3 A_{2}-0.032 \cos 3 A_{2} \\
& \text { (p.c.0) }=+106 \sin A_{2}+761 \cos A_{2} \\
& \begin{array}{r}
-2 \sin 2 A_{3}+89 \cos 2 A_{2} \\
+\quad 19 \cos 3 A_{2}
\end{array} \\
& +\quad 5 \cos 4 A_{3} \\
& \begin{array}{r}
(\rho . c .1)=+1364 \sin A_{3}-43 \cos A_{2} \\
-\quad 46 \sin 2 A_{3}+45 \cos 2 A_{2}
\end{array} \\
& \text { - } \quad 4 \sin 3 A_{3}+9 \cos 3 A_{2} \\
& \begin{array}{c}
-\quad 3 \sin 4 A_{3}+2 \cos 4 A_{3} \\
+98 T
\end{array}
\end{aligned}
$$

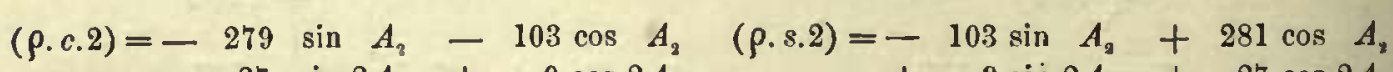

$$
\begin{aligned}
& \begin{array}{r}
-27 \sin 2 A_{3}+9 \cos 2 A_{3}+9 \sin 2 A_{9}+27 \cos 2 A_{2} \\
-\quad 3 \sin 3 A_{3}
\end{array} \\
& +7 T \quad-8 T
\end{aligned}
$$

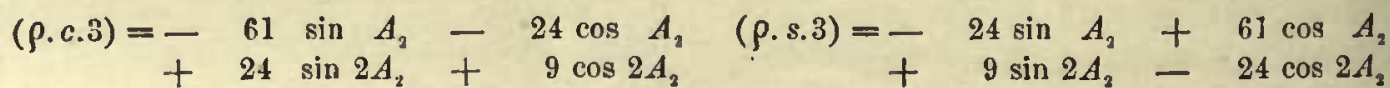

$$
\begin{aligned}
& \text { (v.s.3) }=+5.656 \sin A_{2}+2.265 \cos A_{2} \\
& \text { (p.s.1) }=-33 \sin A_{2}-1338 \cos A_{3} \\
& +\quad 31 \sin 2 A_{3}+14 \cos 2 A_{2} \\
& +\quad 7 \sin 3 A_{3}+5 \cos 3 A_{2} \\
& +\quad 2 \sin 4 A_{\mathrm{s}}+1 \cos 4 A_{\mathrm{s}} \\
& -117 T
\end{aligned}
$$


Action of Neptune.

$$
A_{3}=g-l
$$

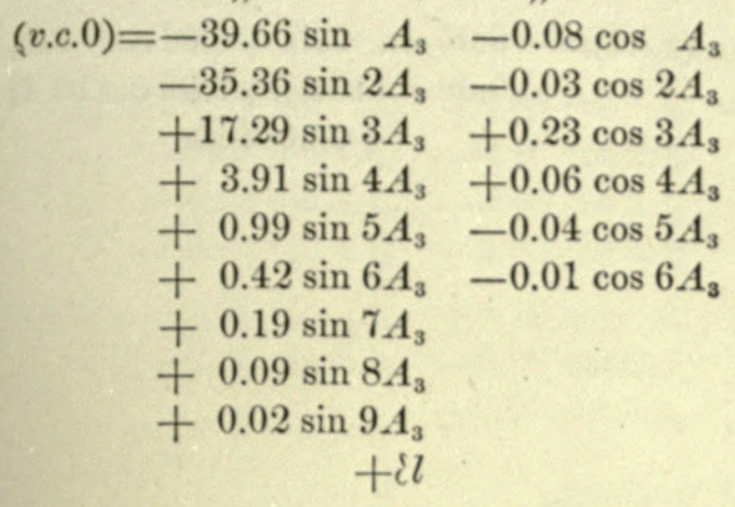

(v.c.1) $=-6.77 \sin \quad A_{3}-0.53 \cos A_{3}$ $-1.10 \sin 2 A_{3}+0.07 \cos 2 A_{3}$ $+23.25 \sin 3 A_{3}+4.04 \cos 3 A_{3}$ $+6.05 \sin 4 A_{3}+1.06 \cos 4 A_{3}$

$-3.26 \sin 5 A_{3}-0.66 \cos 5 A_{3}$

- $0.80 \sin 6 A_{3}-0.17 \cos 6 A_{3}$

$-0.24 \sin 7 A_{3}-0.05 \cos 7 A_{3}$

$-0.12 \sin 8 A_{3}-0.02 \cos 8 A_{3}$

$-0.06 \sin 9 A_{3}-0.01 \cos 9 A_{3}$

$-0.04 \sin 10 A_{3}$

$+1.99945 e \delta g$

(v.c. 2$)=-0.43 \sin \quad A_{3}-0.03 \cos \quad A_{3}$ $-0.03 \sin 2 A_{3}+0.01 \cos 2 A_{3}$ $+0.75 \sin 3 A_{3}+0.08 \cos 3 A_{3}$ $-0.10 \sin 4 A_{3}-0.08 \cos 4 A_{3}$ $-3.20 \sin 5 A_{3}-1.17 \cos 5 A_{3}$ $-0.83 \sin 6 A_{3}-0.32 \cos 6 A_{3}$ $+0.57 \sin 7 A_{3}+0.22 \cos 7 A_{3}$ $+0.14 \sin 8 A_{3}+0.06 \cos 8 A_{3}$ $+0.06 \sin 9 A_{3}+0.02 \cos 9 A_{3}$ $+0.03 \sin 10 A_{3}-0.01 \cos 10 A_{3}$ $+0.11722 e^{\varsigma} g$

(v.c.3) $=-0.02 \sin \quad A_{3}$

$+0.04 \sin 3 A_{3}+0.01 \cos 3 A_{3}$ $-0.15 \sin 4 A_{3}-0.05 \cos 4 A_{3}$ $-0.08 \sin 5 A_{3}-0.02 \cos 5 A_{3}$ $-0.02 \sin 6 A_{3}-0.02 \cos 6 A_{3}$ $+0.46 \sin 7 A_{3}+0.25 \cos 7 \mathrm{~A}_{3}$ $+0.11 \sin 8 A_{3}+0.07 \cos 8 A_{3}$ $-0.08 \sin 9 A_{3}-0.05 \cos 9 A_{3}$ $-0.03 \sin 10 A_{3}-0.02 \cos 10 A_{3}$ $+0.00714 e r g$

$$
\begin{aligned}
(v .8 .1)= & -0.49 \sin A_{3}+1.75 \cos A_{3} \\
& +0.12 \sin 2 A_{3}-2.48 \cos 2 A_{3} \\
& +4.04 \sin 3 A_{3}-20.92 \cos 3 A_{3} \\
& +1.07 \sin 4 A_{3}-5.42 \cos 4 A_{3} \\
& -0.68 \sin 5 A_{3}+3.47 \cos 5 A_{3} \\
& -0.17 \sin 6 A_{3}+0.91 \cos 6 A_{3} \\
& -0.04 \sin 7 A_{3}+0.30 \cos 7 A_{3} \\
& -0.02 \sin 8 A_{3}+0.12 \cos 8 A_{3} \\
& -0.01 \sin 9 A_{3}+0.06 \cos 9 A_{3} \\
& +0.04 \cos 10 A_{3}
\end{aligned}
$$
$+1.99835 \delta e$

$\begin{aligned}(v .8 .2)= & -0.03 \sin A_{3}+0.13 \cos A_{3} \\ & +0.02 \sin 2 A_{3}-0.16 \cos 2 A_{3} \\ & +0.08 \sin 3 A_{3}-0.60 \cos 3 A_{3} \\ & -0.08 \sin 4 A_{3}+0.15 \cos 4 A_{3} \\ & -1.17 \sin 5 A_{3}+3.22 \cos 5 A_{3} \\ & -0.32 \sin 6 A_{3}+0.86 \cos 6 A_{3} \\ & +0.22 \sin 7 A_{3}-0.57 \cos 7 A_{3} \\ & +0.06 \sin 8 A_{3}-0.14 \cos 8 A_{3} \\ & +0.02 \sin 9 A_{3}-0.06 \cos 9 A_{3} \\ & -0.01 \sin 10 A_{3}-0.03 \cos 10 A_{3} \\ & +0.11713 \delta e \\ (v .8 .3)= & +0.02 \cos A_{3} \\ & +0.01 \sin 3 A_{4}-0.04 \cos 3 A_{3} \\ & -0.05 \sin 4 A_{3}+0.15 \cos 4 A_{3} \\ & -0.02 \sin 5 A_{3}+0.08 \cos 5 A_{3} \\ & -0.02 \sin 6 A_{3}+0.02 \cos 6 A_{3} \\ & +0.25 \sin 7 A_{3}-0.46 \cos 7 A_{3} \\ & +0.07 \sin 8 A_{3}-0.11 \cos 8 A_{3} \\ & -0.05 \sin 9 A_{3}+0.08 \cos 9 A_{3} \\ & -0.02 \sin 10 A_{3}+0.03 \cos 10 A_{3} \\ & +0.00714 \delta e\end{aligned}$


Action of Neptune.-Continued.

$$
A_{3}=g-l^{\prime}
$$

(v.c.4) $=-0.06 \sin 9 A_{3}-0.05 \cos 9 A_{3} \quad(v . s .4)=-0.05 \sin 9 A_{3}+0.06 \cos 9 A_{3}$ $-0.09 \sin 10 A_{3}-0.08 \cos 10 A_{3}$ $+0.00044 e \AA g$ $-0.08 \sin 10 A_{3}+0.09 \cos 10 A_{3}$ +0.00044 de

$$
\begin{aligned}
& (\rho . c .0)= \\
& +227 \cos A_{3} \\
& +232 \cos 2 A_{3} \\
& +3 \sin 3 A_{3} \quad-229 \cos 3 A_{3} \\
& \text { - } 59 \cos 4 A_{3} \\
& -17 \cos 5 A_{3} \\
& \text { - } 7 \cos 6 A_{3} \\
& +0.0101 \overline{8} \dot{\delta} e \\
& \text { - } 3 \cos 7 A_{\mathrm{s}}
\end{aligned}
$$

$$
\begin{gathered}
(\text { p.c.1 })=+3 \sin A_{3} \\
+2 \sin 2 A_{3}-9 \cos 2 A_{3} \\
+23 \sin 3 A_{3}-141 \cos 3 A_{3} \\
+8 \sin 4 A_{3}-39 \cos 4 A_{3} \\
+9 \sin 5 A_{3}+43 \cos 5 A_{3} \\
-3 \sin 6 A_{3}+13 \cos 6 A_{3} \\
-1 \sin 7 A_{3}+5 \cos 7 A_{3} \\
-0.43322 e
\end{gathered}
$$

$$
\begin{aligned}
(\rho . s .1)= & -60 \sin A^{3}-3 \cos A_{3} \\
& -45 \sin 2 A_{3}-2 \cos 2 A_{3} \\
& -107 \sin 3 A_{3}-23 \cos 3 A_{3} \\
& -29 \sin 4 A_{3}-8 \cos 4 A_{3} \\
& +47 \sin 5 A_{3}+9 \cos 5 A_{3} \\
& +15 \sin 6 A_{3}+3 \cos 6 A_{3} \\
& +5 \sin 7 A_{3}+1 \cos 7 A_{3} \\
& +0.43394 e \delta g
\end{aligned}
$$

$$
\begin{aligned}
(\text { p.c.2 })= & -1 \cos A_{3} \\
& -6 \cos 2 A_{3} \\
& -1 \sin 3 A_{3}+6 \cos 3 A_{3} \\
& -4 \sin 4 A_{3}+5 \cos 4 A_{3} \\
& -6 \sin 5 A_{3}+17 \cos 5 A_{3} \\
& -2 \sin 6 A_{3}+7 \cos 6 A_{3} \\
& +3 \sin 7 A_{3}-7 \cos 7 A_{3} \\
-0.03048 \mathrm{e} e & \\
(\rho . c .3)= & -0.000202 \mathrm{e}
\end{aligned}
$$

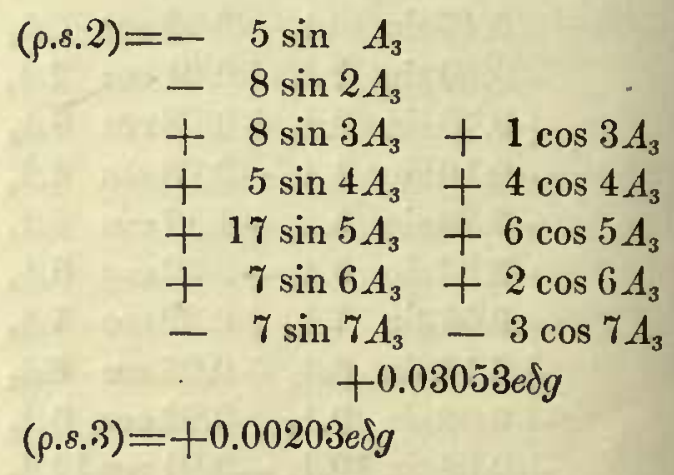

Perturbations of the Latitude.

(The secular terms being omitted.)

Action of Jupiter.

$$
\begin{aligned}
& \text { (b.c.0) }=0.024 \sin A_{1}-0.013 \cos A_{1} \\
& \text { (b.s.1) }=-0.420 \sin A_{1}+0.494 \cos A_{1} \\
& \text { (b.c.1) }=+0.494 \sin A_{1}+0.420 \cos A_{1} \\
& (\text { b.s.2 })=+0.004 \sin 2 A_{1} \quad-0.017 \cos 2 A_{1} \\
& \text { (b.c.2) }=-0.017 \sin 2 A_{1} \quad-0.004 \cos 2 A_{1}
\end{aligned}
$$


Action of Saturn.

$$
\begin{aligned}
& \text { (b.c.0) }=-0.08 \sin A_{2}-0.03 \cos A_{2} \\
& -0.03 \cos 2 A_{2} \\
& -0.01 \cos 3 A_{2} \\
& (\text { b.s.1 })=+1.34 \sin A_{2}+2.88 \cos A_{2} \quad(b . c .1)=-1.56 \sin A_{2}+2.50 \cos A_{2} \\
& -0.01 \sin 2 A_{2} \quad-0.10 \cos 2 A_{2} \quad+0.02 \sin 2 A_{2}-0.10 \cos 2 A_{2} \\
& \begin{array}{llll}
+0.03 \sin 3 A_{2} & -0.06 \cos 3 A_{2} & -0.04 \sin 3 A_{2} & -0.06 \cos 3 A_{2}
\end{array} \\
& \begin{array}{llll}
+0.02 \sin 4 A_{2} & -0.03 \cos 4 A_{2} \quad-0.02 \sin 4 A_{2} & -0.02 \cos 4 A_{2}
\end{array}
\end{aligned}
$$

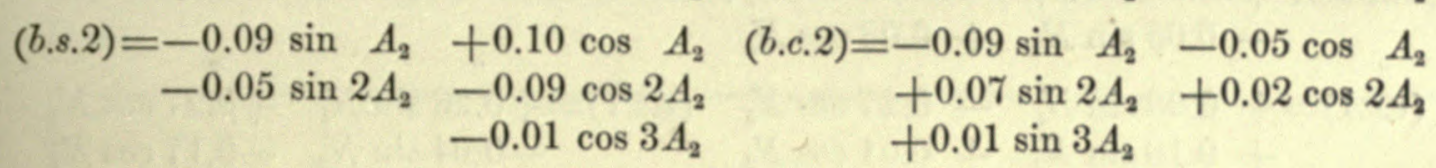

Action of Neptune.

$$
\begin{aligned}
(\text { b.c. } 0)= & +0.01 \sin A_{3} \quad-0.04 \cos A_{3} \\
& -0.01 \sin 2 A_{3}+0.00 \cos 2 A_{3} \\
& -0.01 \sin 3 A_{3}+0.04 \cos 3 A_{3} \\
& +0.01 \sin 4 A_{3}+0.01 \cos 4 A_{3} \\
& +0.01 \sin 5 A_{3} \\
& +0.01 \sin 6 A_{3}
\end{aligned}
$$

(b.s.1) $=+0.13 \sin A_{3}+0.16 \cos A_{3} \quad($ b.c. 1$)=-0.57 \sin A_{3}+0.04 \cos A_{3}$ $+0.09 \sin 2 A_{3}+0.26 \cos 2 A_{3}$ $+0.08 \sin 3 A_{3}+0.28 \cos 3 A_{3}$ $+0.01 \sin 4 A_{3}+0.03 \cos 4 A_{3}$ $\begin{array}{lll}-0.02 \sin 5 A_{3} & -0.12 \cos 5 A_{3}\end{array}$ $-0.01 \sin 6 A_{3} \quad-0.03 \cos 6 A_{3}$ $-0.01 \cos 7 A_{3}$ $-0.39 \sin 2 A_{3}+0.06 \cos 2 A_{3}$ $-0.33 \sin 3 A_{3}+0.07 \cos 3 A_{3}$ $-0.07 \sin 4 A_{3}+0.01 \cos 4 A_{3}$ $+0.10 \sin 5 A_{3} \quad-0.03 \cos 5 A_{3}$ $+0.03 \sin 6 A_{3}$ $+0.01 \sin 7 A_{3}$

(b.8.2) $=+0.01 \sin A_{3}+0.05 \cos A_{3} \quad(b . c .2)=-0.07 \sin A_{3}$ $\begin{array}{ll}-0.03 \sin 3 A_{3} & -0.09 \cos 3 A_{3}\end{array}$ $-0.05 \cos 4 A_{3}$ $+0.09 \sin 3 A_{3} \quad-0.03 \cos 3 A_{3}$ $+0.05 \sin 4 A_{3}$ $-0.04 \cos 5 A_{3}$ $+0.01 \cos 7 A_{3}$ $+0.04 \sin 5 A_{3}$ $+0.01 \sin 6 A_{3}$ $-0.01 \sin 7 A_{3}$

Action of Jupiter and Saturn.

(Terms multiplied by the product of their masses.)

$$
\begin{array}{lr}
N_{1}= & 2 S-J \\
N_{2}=-U+3 S-J \\
N_{3}=-2 U+4 S-J \\
N_{4}=3 U+3 S-2 J \\
N_{5}=2 U+4 S-2 J \\
N_{6}=r \\
N_{7}=-3 U+6 S-2 J \\
N_{8}=-3 U+7 S-2 J
\end{array}
$$


Action of Jupiter and Saturn-Continued.

(Terms multiplied by the product of their masses.)

$$
\begin{aligned}
& \text { (v.c.0) }=+0.08 \sin N_{2}+0.51 \cos N_{2} \\
& +0.04 \sin N_{3}+0.01 \cos N_{3} \\
& -0.01 \sin N_{4}+0.05 \cos N_{4} \\
& -0.35 \sin N_{5}-1.30 \cos N_{5} \\
& \left\{\begin{array}{ll}
-0.55 \sin N_{6} & -0.03 \cos N_{6} \\
+40.65 \sin N_{7} & -10.50 \cos N_{7}
\end{array}\right\} \\
& \text { - } 0.05 \sin N_{8}+0.03 \cos N_{8} \\
& \text { (v.c.1) }=+0.06 \sin N_{1}-0.27 \cos N_{1} \\
& +0.18 \sin N_{2}+0.01 \cos N_{2} \\
& -0.03 \sin N_{3}+0.08 \cos N_{3} \\
& -0.02 \sin N_{4}+0.09 \cos N_{4} \\
& -0.44 \sin N_{5}-0.61 \cos N_{5} \\
& \left\{\begin{array}{l}
-4.23 \sin N_{8}-3.87 \cos N_{6} \\
+8.06 \sin N_{7}-8.38 \cos N_{7}
\end{array}\right\} \\
& \text { - } 0.10 \sin N_{8}+0.03 \cos N_{8} \\
& \begin{aligned}
\text { (v.s.1 })= & +0.26 \sin N_{1} \quad+0.27 \cos N_{1} \\
& -0.04 \sin N_{2} \quad-0.17 \cos N_{2}
\end{aligned} \\
& +0.08 \sin N_{3}+0.03 \cos N_{3} \\
& -0.02 \sin N_{4}+0.08 \cos N_{4} \\
& +0.30 \sin N_{5}-0.58 \cos N_{5} \\
& \left\{+2.64 \sin N_{8}+4.64 \cos N_{6}\right\} \\
& \left\{+7.35 \sin N_{7}+4.41 \cos N_{7}\right\} \\
& -0.04 \sin N_{8}+0.00 \cos N_{8} \\
& (v . c .2)=\left\{\begin{array}{l}
-0.24 \sin N_{6}-0.22 \cos N_{6} \\
+0.47 \sin N_{7}-0.54 \cos N_{7}
\end{array}\right\} \quad(v .8,2)=\left\{\begin{array}{l}
+0.16 \sin N_{6}+0.26 \cos N_{6} \\
+0.54 \sin N_{7}+0.47 \cos N_{7}
\end{array}\right\} \\
& \text { (р.c.0) }=+11 \sin N_{5} \quad-3 \cos N_{5}
\end{aligned}
$$

Two of these arguments, namely, $5 S-2 J$, and $-3 g+6 S-2 J$, are of very long period, that of the first being about 880 , and that of the second about 1590 years. It will, therefore, be convenient to tabulate them both as functions of the time for the time during which the theory is to be used. To make their effect as small as possible during the period for which the provisional ephemeris is to be computed, we shall suppose the longitude of epoch, mean motion, and longitude of the perigee to be affected with the negative of the following corrections:

$$
\begin{aligned}
& \delta \varepsilon=+27.27 \\
& \delta \pi=+27.27 \\
& \delta n=-0.1172
\end{aligned}
$$

Reducing these corrections to corrections of the co-ordinates, and adding them to the terms of long period in the true longitude and logarithm of radius vector, we shall have for these terms,

$$
\begin{aligned}
& \text { (v.c.0) }=-0.546 \sin N_{6}-0.032 \cos N_{6} \\
& +40.650 \sin N-10.500 \cos N_{7}+27.27-11.72 T \\
& \text { (v.s.1) }=\quad 2.63 \sin N_{8}+4.64 \cos N_{6}+7.35 \sin N_{7}+4.42 \cos N_{7} \\
& \text { (v.c.1) }=-4.22 \sin N_{6}-3.87 \cos N_{6}+8.06 \sin N_{7}-8.39 \cos N_{7}-1.10 T
\end{aligned}
$$




$$
\begin{aligned}
& \text { (v.8.2) }=+0.16 \sin N_{6}+0.26 \cos N_{6}+0.54 \sin N_{7}+0.47 \cos N_{7} \\
& \text { (v.c. } 2)=-0.24 \sin N_{6}-0.22 \cos N_{6}+0.47 \sin N_{7}-0.54 \cos N_{7}-0^{\prime \prime} .07 T \\
& \left(\text { p.c.0) }=+2 \cos N_{6}+10 \sin N_{7}+32 \cos N_{7}+22\right. \\
& \text { (p.s.1) }=-41 \sin N_{6}-43 \cos N_{6}+82 \sin N_{7}-85 \cos N_{7}-12 T \\
& \text { ( } . \text {.c.1) }=-29 \sin N_{6}-45 \cos N_{6}-73 \sin N_{7}-44 \cos N_{7}
\end{aligned}
$$

The values of these and of the other secular terms and terms of long period for the period during which Uranus has been observed, are given in the following table:

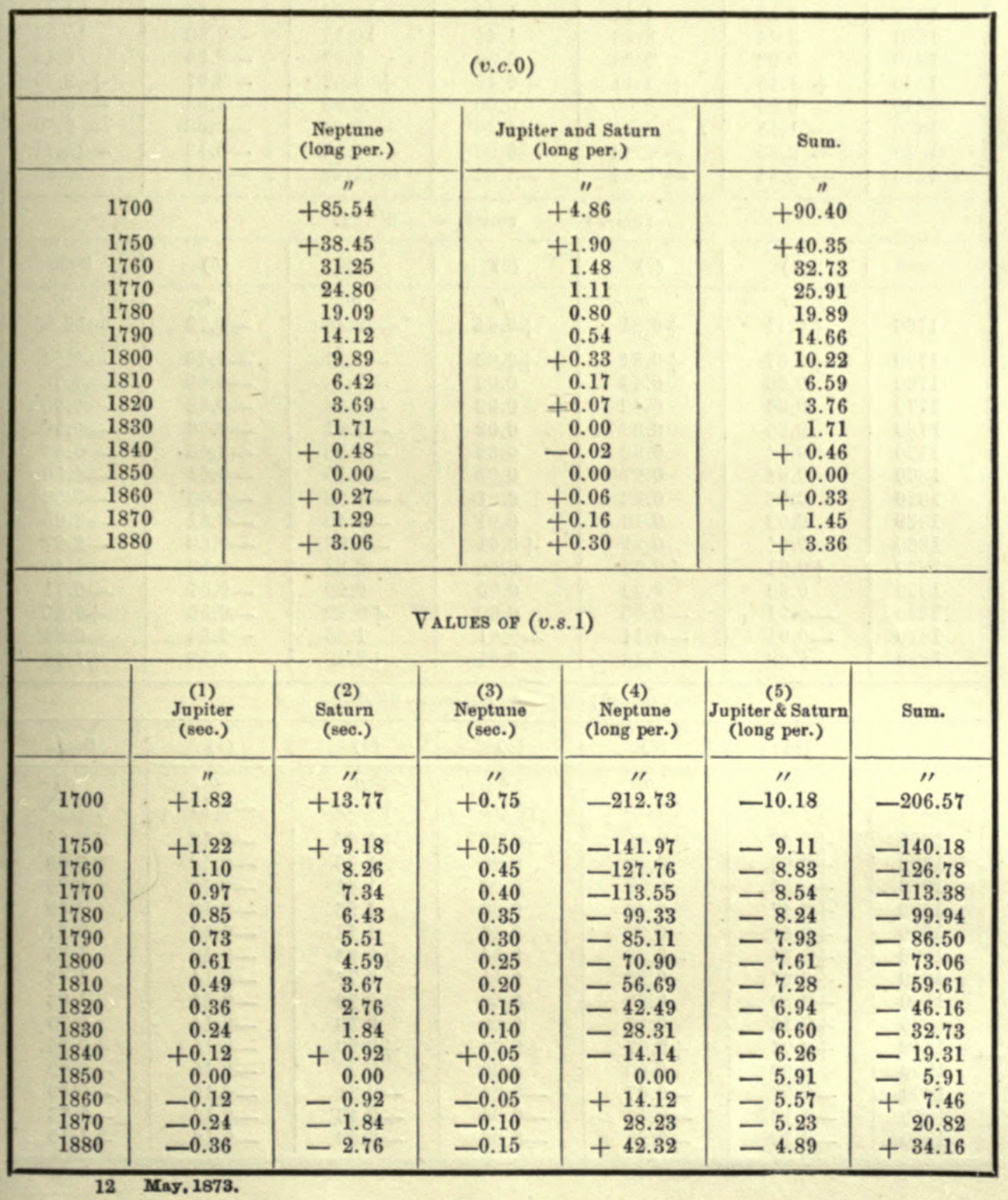




\begin{tabular}{|c|c|c|c|c|c|c|}
\hline \multicolumn{7}{|c|}{ VALUES OF (v.c.1) } \\
\hline & $\begin{array}{c}\text { (1) } \\
\text { Jupiter } \\
\text { (sec.) }\end{array}$ & $\begin{array}{c}(2) \\
\text { Saturn } \\
\text { (sec.) }\end{array}$ & $\begin{array}{l}\text { (3) } \\
\text { Neptune } \\
\text { (sec.) }\end{array}$ & $\begin{array}{c}(4) \\
\text { Neptune } \\
\text { (loug per.) }\end{array}$ & $\begin{array}{l}(5) \\
\text { Japiter \& Saturn } \\
\text { (long per.) }\end{array}$ & Sum. \\
\hline 1700 & $\begin{array}{c}\prime \prime \prime \\
+17.19\end{array}$ & $\begin{array}{c}\prime \prime \\
+16.67\end{array}$ & $\begin{array}{c}\prime \prime \\
+7.20\end{array}$ & $\begin{array}{c}\prime \prime \\
+37.63\end{array}$ & $\begin{array}{r}1 \prime \\
-1.98\end{array}$ & $\begin{array}{c}\prime \prime \prime \\
+76.71\end{array}$ \\
\hline $\begin{array}{l}1750 \\
1760 \\
1770 \\
1780 \\
1790 \\
1800 \\
1810 \\
1820 \\
1830 \\
1840 \\
1850 \\
1860 \\
1870 \\
1880\end{array}$ & $\begin{array}{r}+11.46 \\
10.31 \\
9.17 \\
8.02 \\
6.88 \\
5.73 \\
4.58 \\
3.44 \\
2.29 \\
+1.15 \\
0.00 \\
-1.15 \\
-2.29 \\
-3.44\end{array}$ & $\begin{array}{r}+11.11 \\
10.00 \\
8.89 \\
7.78 \\
6.67 \\
5.56 \\
4.44 \\
3.33 \\
2.22 \\
+1.11 \\
0.00 \\
-1.11 \\
-2.22 \\
-3.33\end{array}$ & $\begin{array}{r}+4.80 \\
4.32 \\
3.84 \\
3.36 \\
2.88 \\
2.40 \\
1.92 \\
1.44 \\
0.96 \\
+0.48 \\
0.00 \\
-0.48 \\
-0.96 \\
-1.44\end{array}$ & $\begin{array}{r}+28.67 \\
26.46 \\
24.10 \\
21.60 \\
18.95 \\
16.16 \\
13.23 \\
10.15 \\
6.92 \\
+3.53 \\
0.00 \\
-3.68 \\
-7.51 \\
-11.49\end{array}$ & $\begin{array}{r}-2.28 \\
-2.36 \\
-2.45 \\
-2.53 \\
-2.61 \\
-2.69 \\
-2.76 \\
-2.83 \\
-2.90 \\
-2.97 \\
-3.03 \\
-3.08 \\
-3.13 \\
-3.16\end{array}$ & $\begin{array}{r}+53.76 \\
48.73 \\
43.55 \\
38.23 \\
32.77 \\
27.16 \\
21.41 \\
15.53 \\
9.49 \\
+3.30 \\
-3.03 \\
-9.50 \\
-16.11 \\
-22.86\end{array}$ \\
\hline \multicolumn{7}{|c|}{$(v . s .2)$} \\
\hline & (1) & (2) & (3) & (4) & (5) & Sum. \\
\hline $\begin{array}{l}1700 \\
1750 \\
1760 \\
1770 \\
1780 \\
1790 \\
1800 \\
1810 \\
1820 \\
1830 \\
1840 \\
1850 \\
1860 \\
1870 \\
1880\end{array}$ & \begin{tabular}{r}
\multicolumn{1}{c}{$" \prime$} \\
+0.11 \\
+0.07 \\
0.06 \\
0.06 \\
0.05 \\
0.04 \\
0.04 \\
0.03 \\
0.02 \\
0.01 \\
+0.01 \\
0.00 \\
-0.01 \\
-0.01 \\
-0.02
\end{tabular} & $\begin{array}{r}\prime \prime \\
+0.81 \\
+0.54 \\
0.49 \\
0.43 \\
0.38 \\
0.32 \\
0.27 \\
0.22 \\
0.16 \\
0.11 \\
+0.05 \\
0.00 \\
-0.05 \\
-0.11 \\
-0.16\end{array}$ & \begin{tabular}{r}
\multicolumn{1}{c}{$" \prime$} \\
+0.04 \\
+0.03 \\
0.03 \\
0.02 \\
0.02 \\
0.02 \\
0.02 \\
0.01 \\
0.01 \\
+0.01 \\
0.00 \\
0.00 \\
0.00 \\
-0.01 \\
-0.01
\end{tabular} & $\begin{array}{r}\prime \prime \prime \\
-12.46 \\
-8.31 \\
-7.48 \\
-6.64 \\
-5.81 \\
-4.98 \\
-4.15 \\
-3.32 \\
-2.49 \\
-1.66 \\
-0.83 \\
0.00 \\
+0.82 \\
1.65 \\
+2.48\end{array}$ & $\begin{array}{c}\prime \prime \\
-0.72 \\
-0.70 \\
-0.69 \\
-0.68 \\
-0.66 \\
-0.65 \\
-0.64 \\
-0.63 \\
-0.61 \\
-0.60 \\
-0.58 \\
-0.57 \\
-0.56 \\
-0.54 \\
-0.53 \\
\end{array}$ & $\begin{array}{c}\prime \prime \\
-12.36 \\
-8.51 \\
-7.73 \\
-6.95 \\
-6.16 \\
-5.39 \\
-4.60 \\
-3.83 \\
-3.05 \\
-2.27 \\
-1.49 \\
-0.71 \\
+0.06 \\
0.84 \\
+1.62 \\
\end{array}$ \\
\hline \multicolumn{7}{|c|}{$(v . c .2)$} \\
\hline & (1) & (2) & (3) & (4) & (5) & Sum. \\
\hline 1700 & $\begin{array}{c}\prime \prime \prime \\
+1.00\end{array}$ & $\begin{array}{r}\prime \prime \prime \\
+0.98\end{array}$ & $\begin{array}{c}\prime \prime \prime \\
+0.42\end{array}$ & $\begin{array}{c}\prime \prime \\
+2.20\end{array}$ & -0.11 & $\begin{array}{c}\prime \prime \prime \\
+4.62\end{array}$ \\
\hline $\begin{array}{l}1750 \\
1760\end{array}$ & $\begin{array}{r}+0.67 \\
+0.60\end{array}$ & $\begin{array}{r}+0.65 \\
0.59\end{array}$ & $\begin{array}{r}+0.28 \\
0.25\end{array}$ & $\begin{array}{r}+1.68 \\
1.55\end{array}$ & $\begin{array}{l}-0.12 \\
-0.12\end{array}$ & $\begin{array}{r}+3.29 \\
3.00\end{array}$ \\
\hline 1770 & $\begin{array}{r}+0.60 \\
+0.54\end{array}$ & 0.52 & $\begin{array}{l}0.25 \\
0.22\end{array}$ & $\begin{array}{l}1.55 \\
1.41\end{array}$ & $\begin{array}{l}-0.12 \\
-0.12\end{array}$ & $\begin{array}{l}3.00 \\
2.70\end{array}$ \\
\hline 1780 & +0.47 & 0.46 & 0.20 & 1.26 & -0.13 & 2.39 \\
\hline 1790 & +0.40 & 0.39 & 0.17 & 1.11 & -0.13 & 2.07 \\
\hline 1800 & +0.34 & 0.33 & 0.14 & 0.94 & -0.13 & 1.75 \\
\hline 1810 & $\begin{array}{r}+0.27 \\
+0.90\end{array}$ & 0.26 & 0.11 & $\begin{array}{l}0.77 \\
0.59\end{array}$ & -0.14 & $\begin{array}{l}1.40 \\
1.06\end{array}$ \\
\hline 1830 & $\begin{array}{l}+0.20 \\
+0.13\end{array}$ & $\begin{array}{l}0.20 \\
0.13\end{array}$ & $\begin{array}{l}0.08 \\
0.06\end{array}$ & $\begin{array}{l}0.59 \\
0.39\end{array}$ & $\begin{array}{r}-0.14 \\
-0.14\end{array}$ & $\begin{array}{l}1.70 \\
0.70\end{array}$ \\
\hline 1840 & +0.07 & +0.07 & +0.03 & +0.20 & -0.15 & +0.35 \\
\hline 1850 & 0.00 & 0.00 & 0.00 & 0.00 & -0.15 & -0.02 \\
\hline 1860 & -0.07 & -0.07 & -0.03 & -0.21 & -0.15 & -0.40 \\
\hline $\begin{array}{l}1870 \\
1880\end{array}$ & -0.13 & -0.13 & -0.06 & -0.43 & -0.16 & -0.78 \\
\hline & & -0.20 & -0.08 & -0.68 & -0.16 & -1.19 \\
\hline
\end{tabular}




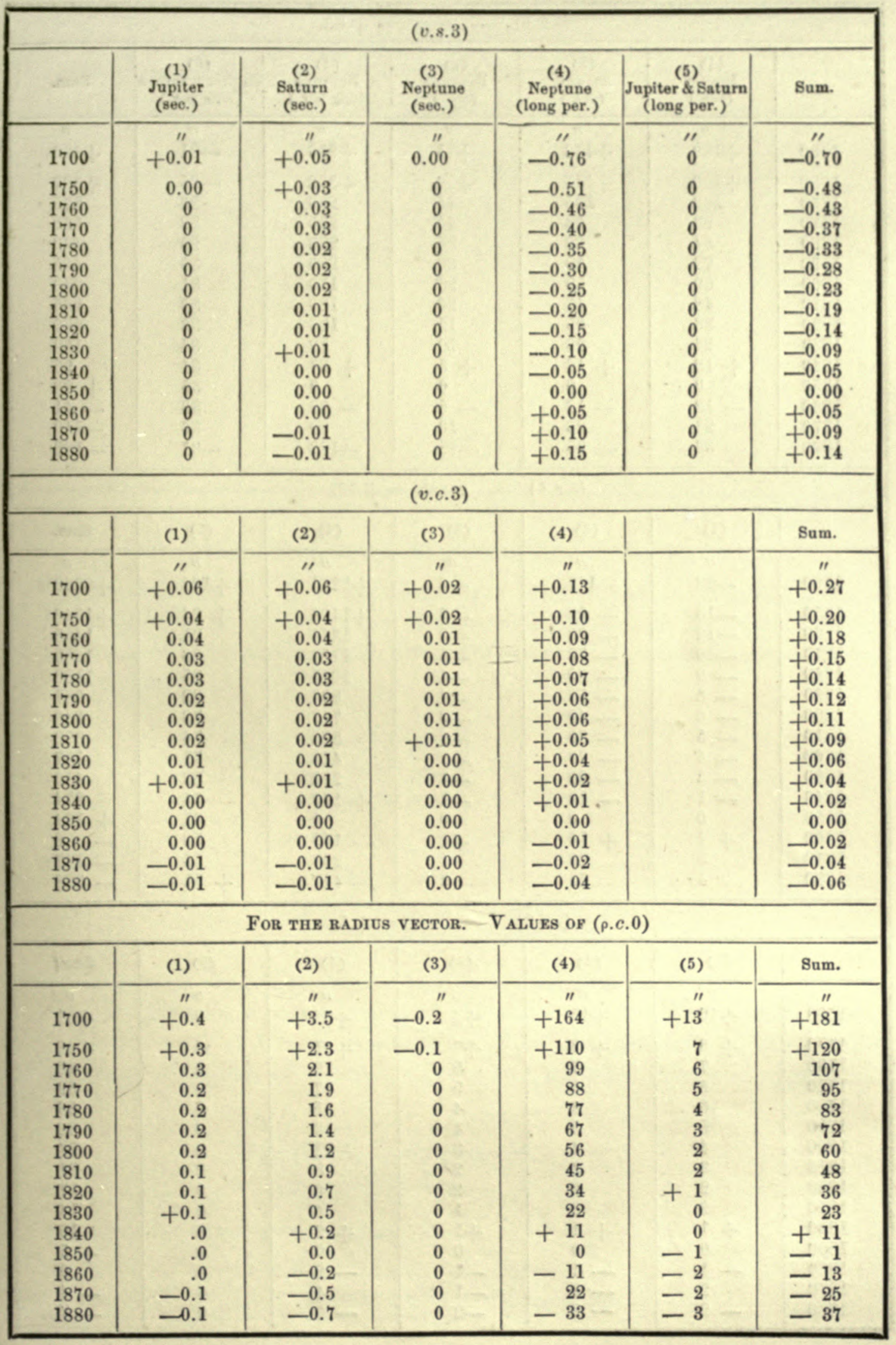




\begin{tabular}{|c|c|c|c|c|c|c|}
\hline \multicolumn{7}{|c|}{ const. $=+63$} \\
\hline & $\underset{\substack{\text { Jupiter } \\
\text { (seo.) }}}{(1)}$ & $\begin{array}{c}(2) \\
\text { Saturn } \\
\text { (sec.) }\end{array}$ & $\begin{array}{c}\text { (3) } \\
\text { Neptune } \\
\text { (see.) }\end{array}$ & $\begin{array}{c}(4) \\
\text { Neptune } \\
\text { (long per.) }\end{array}$ & $\begin{array}{l}(5) \\
\text { Jupiter \& Saturn } \\
\text { (long per.) }\end{array}$ & Sum. \\
\hline 1700 & $\begin{array}{r}\prime \prime \\
+180\end{array}$ & $\begin{array}{r}11 \\
+176\end{array}$ & $\begin{array}{r}\prime \prime \prime \\
+75\end{array}$ & $\begin{array}{r}\prime \prime \\
+396\end{array}$ & $\begin{array}{c}\prime \prime \\
-21\end{array}$ & $\begin{array}{r}\prime \prime \\
+869\end{array}$ \\
\hline $\begin{array}{l}1750 \\
1760 \\
1770 \\
1780 \\
1790 \\
1800 \\
1810 \\
1820 \\
1830 \\
1840 \\
1850 \\
1860 \\
1870 \\
1880 \\
\end{array}$ & $\begin{array}{r}+120 \\
108 \\
96 \\
84 \\
72 \\
60 \\
48 \\
36 \\
24 \\
+12 \\
-12 \\
-12 \\
-36 \\
\end{array}$ & $\begin{array}{r}+117 \\
105 \\
94 \\
-82 \\
70 \\
59 \\
47 \\
35 \\
23 \\
+12 \\
-12 \\
-12 \\
-35 \\
-35 \\
\end{array}$ & $\begin{array}{r}+50 \\
45 \\
40 \\
35 \\
30 \\
25 \\
20 \\
15 \\
10 \\
+5 \\
0 \\
-5 \\
10 \\
-15 \\
\end{array}$ & $\begin{array}{r}+302 \\
280 \\
255 \\
228 \\
200 \\
170 \\
139 \\
106 \\
72 \\
+\quad 37 \\
0 \\
-39 \\
80 \\
-122 \\
\end{array}$ & $\begin{array}{r}-25 \\
26 \\
28 \\
29 \\
30 \\
31 \\
32 \\
33 \\
34 \\
34 \\
35 \\
36 \\
36 \\
-37 \\
\end{array}$ & $\begin{array}{r}+627 \\
575 \\
520 \\
463 \\
405 \\
346 \\
285 \\
222 \\
159 \\
95 \\
+\quad 28 \\
-41 \\
-110 \\
-182\end{array}$ \\
\hline \multicolumn{7}{|c|}{ (p.c.1) } \\
\hline & (1) & (2) & (3) & (4) & (5) & Snm. \\
\hline 1700 & $\begin{array}{c}\prime \prime \\
-20\end{array}$ & $\begin{array}{r}\prime \prime \prime \\
-147\end{array}$ & ${ }^{\prime \prime}$ & $\begin{array}{r}\prime \prime \prime \\
+2241\end{array}$ & $\begin{array}{r}\prime \prime \\
+104\end{array}$ & $\begin{array}{r}\prime \prime \\
+2242\end{array}$ \\
\hline $\begin{array}{l}1750 \\
1760 \\
1770 \\
1780 \\
1790 \\
1800 \\
1810 \\
1820 \\
1830 \\
1840 \\
1850 \\
1860 \\
1870 \\
1880\end{array}$ & $\begin{array}{r}-13 \\
-12 \\
-10 \\
=9 \\
=8 \\
=6 \\
=5 \\
=4 \\
=3 \\
-1 \\
0 \\
+1 \\
3 \\
4\end{array}$ & $\begin{array}{r}-98 \\
=\quad 88 \\
=\quad 78 \\
=\quad 69 \\
=59 \\
=49 \\
=\quad 39 \\
=\quad 29 \\
=20 \\
-10 \\
0 \\
+\quad 10 \\
20 \\
29\end{array}$ & $\begin{array}{r}-6 \\
-5 \\
-5 \\
-4 \\
-4 \\
-4 \\
-3 \\
-2 \\
-2 \\
-1 \\
-1 \\
0 \\
+1 \\
1 \\
2\end{array}$ & $\begin{array}{r}+1496 \\
1346 \\
1197 \\
1047 \\
897 \\
747 \\
598 \\
448 \\
299 \\
+149 \\
0 \\
-149 \\
-298 \\
-447 \\
\end{array}$ & $\begin{array}{r}94 \\
91 \\
88 \\
85 \\
82 \\
78 \\
74 \\
71 \\
68 \\
64 \\
61 \\
58 \\
54 \\
+51 \\
\end{array}$ & $\begin{array}{r}+1546 \\
1405 \\
1265 \\
1123 \\
981 \\
840 \\
699 \\
557 \\
416 \\
274 \\
+\quad 134 \\
-\quad 6 \\
-147 \\
-288 \\
\end{array}$ \\
\hline \multicolumn{7}{|c|}{ (p.s.2) } \\
\hline & (1) & (2) & (3) & (4) & (5) & Sum. \\
\hline 1700 & $\begin{array}{r}\prime \prime \prime \\
+12\end{array}$ & $\dot{+}^{\prime \prime}$ & $\begin{array}{r}\prime \prime \\
+4\end{array}$ & $\begin{array}{r}\prime \prime \prime \\
+28\end{array}$ & " & $\begin{array}{r}\prime \prime \\
+61\end{array}$ \\
\hline $\begin{array}{l}1750 \\
1760 \\
1770 \\
1780 \\
1790 \\
1800 \\
1810 \\
1820 \\
1830 \\
1840 \\
1850 \\
1860 \\
1870 \\
1880\end{array}$ & $\begin{array}{r}+8 \\
7 \\
6 \\
6 \\
5 \\
4 \\
3 \\
2 \\
2 \\
+1 \\
+1 \\
0 \\
-1 \\
-2 \\
-2\end{array}$ & $\begin{array}{r}+8 \\
7 \\
6 \\
6 \\
5 \\
4 \\
3 \\
2 \\
2 \\
+1 \\
0 \\
-1 \\
=2 \\
-2\end{array}$ & $\begin{array}{r}+6 \\
5 \\
5 \\
4 \\
4 \\
3 \\
2 \\
2 \\
1 \\
+1 \\
0 \\
-1 \\
-1 \\
-2\end{array}$ & $\begin{array}{r}+21 \\
19 \\
17 \\
15 \\
13 \\
11 \\
8 \\
6 \\
4 \\
+2 \\
0 \\
-2 \\
4 \\
-6\end{array}$ & $\begin{array}{l}0 \\
0 \\
0 \\
0 \\
0 \\
0 \\
0 \\
0 \\
0 \\
0 \\
0 \\
0 \\
0 \\
0\end{array}$ & $\begin{array}{r}+48 \\
43 \\
39 \\
36 \\
32 \\
27 \\
21 \\
17 \\
14 \\
10 \\
+5 \\
0 \\
-4 \\
-7\end{array}$ \\
\hline
\end{tabular}




\begin{tabular}{|c|c|c|c|c|c|c|}
\hline \multicolumn{7}{|c|}{ (p.c.2) } \\
\hline & $\underset{\substack{\text { Jupiter } \\
\text { (sec.) }}}{(1)}$ & $\begin{array}{c}\text { (2) } \\
\text { Saturn } \\
\text { (sec.) }\end{array}$ & $\begin{array}{c}\text { (3) } \\
\text { Neptune } \\
\text { (sec.) }\end{array}$ & $\begin{array}{c}\text { (4) } \\
\text { Neptune } \\
\text { (long per.) }\end{array}$ & $\begin{array}{c}\text { (5) } \\
\text { Jupiter \& Saturn } \\
\text { (long per.) }\end{array}$ & Sum. \\
\hline 1700 & -1 & -10 & $\begin{array}{l}\prime \prime \\
0\end{array}$ & $\begin{array}{r}\prime \prime \prime \\
+157\end{array}$ & $\begin{array}{l}\prime \prime \\
0\end{array}$ & $\begin{array}{r}\prime \prime \prime \\
+150\end{array}$ \\
\hline 1750 & -1 & -7 & 0 & +105 & 0 & +101 \\
\hline 1760 & -1 & -6 & 0 & 95 & 0 & 92 \\
\hline 1770 & -1 & -6 & 0 & 84 & 0 & 81 \\
\hline 1780 & -1 & -5 & 0 & 74 & 0 & 72 \\
\hline 1790 & -1 & -4 & 0 & 63 & 0 & 62 \\
\hline 1800 & 0 & -3 & 0 & 52 & 0 & 53 \\
\hline 1810 & 0 & -3 & 0 & 42 & 0 & 43 \\
\hline 1820 & 0 & -2 & 0 & 31 & 0 & 33 \\
\hline 1830 & 0 & -1 & 0 & 21 & 0 & 24 \\
\hline 1840 & 0 & -1 & 0 & +10 & 0 & 13 \\
\hline 1850 & 0 & 0 & 0 & & 0 & +4 \\
\hline 1860 & 0 & +1 & 0 & -10 & 0 & -5 \\
\hline 1870 & 0 & 1 & 0 & 21 & 0 & -16 \\
\hline 1880 & 0 & +2 & 0 & -31 & 0 & -25 \\
\hline
\end{tabular}

\section{Reduced Expressions for the Latitude of Uranus.}

If we represent by $V_{1}, V_{2}, V_{3}$ the distances of Uranus from its descending nodes on the respective orbits of Jupiter, Saturn, and Uranus, we find the following perturbations of the latitude, which are independent of the mean longitude of the disturbing planets.

$$
\begin{aligned}
\delta \beta= & -0.0114 t \cos V_{1} \\
& -0.0477 t \cos V_{2} \\
& -0.0125 t \cos V_{3} \\
& +0.245 \quad{ }^{\prime \prime} \\
& +0.386 \sin g+0.266 \cos g \\
& -0.043 \sin 2 g+0.006 \cos 2 g .
\end{aligned}
$$

To find how far the last five terms may be represented by simple corrections to the elliptic elements, we first represent the effect of minute corrections to the inclination and node of Uranus as a function of its mean anomaly. Putting $u$ for the argument of latitude of Uranus, we have to a sufficient degree of approximation

$$
\begin{aligned}
& \delta \beta=\sin u \delta \phi-\sin \phi \cos u \delta \theta \\
& u=g+\omega+2 e \sin g
\end{aligned}
$$

$\sin u=-e \sin \omega$

$$
\begin{aligned}
& +\cos \omega \sin g+\sin \omega \cos g \\
& +e \cos \omega \sin 2 g+e \sin \omega \cos 2 g
\end{aligned}
$$




$$
\begin{aligned}
\cos u= & -e \cos \omega \\
& +\cos \omega \cos g-\sin \omega \sin g \\
& +e \cos \omega \cos 2 g-e \sin \omega \sin 2 g .
\end{aligned}
$$

Substituting these values of $\sin u$ and $\cos u$ in the expression for $\delta \beta$, and putting $\sin \phi \delta \theta=\delta^{\prime} \theta$, we have

$$
\begin{aligned}
& \delta \beta=e \cos \omega \delta^{\prime} \theta-e \sin \omega \delta \phi \\
& \quad+\left(\cos \omega \delta \phi+\sin \omega \delta^{\prime} \theta\right) \sin g+\left(\sin \omega \delta \phi-\cos \omega \delta^{\prime} \theta\right) \cos g \\
& \quad+\left(e \cos \omega \delta \phi+e \sin \omega \delta^{\prime} \theta\right) \sin 2 g+\left(e \sin \omega \delta \phi-e \cos \omega \delta^{\prime} \theta\right) \cos 2 g
\end{aligned}
$$

To represent the numerical coefficients of $\sin g$ and $\cos g$ in $\delta \beta$ we must put

$$
\begin{aligned}
& \cos \omega \delta \phi+\sin \omega^{\prime} \theta=0^{\prime \prime} .386 \\
& \sin \omega \delta \phi-\cos \omega^{\prime} \theta=0.266 .
\end{aligned}
$$

Since $\omega=95^{\circ} 3^{\prime}$, this gives

$$
\begin{aligned}
\delta \phi= & 0.231 ; \\
\delta^{\prime} \theta= & 0.409 ; \\
\delta \beta= & -0.013 \\
& +0.386 \sin g+0.266 \cos g \\
& +0.018 \sin 2 g+0.013 \cos 2 g
\end{aligned}
$$

Subtracting this expression from the corresponding terms of $\delta \beta$, we have left

$$
\delta \beta=+0^{\prime \prime} .258-0^{\prime \prime} .061 \sin 2 g-0^{\prime \prime} .007 \cos 2 g .
$$

The first term of this expression shows that the mean orbit of Uranus at the present time is a small circle of the sphere one-quarter of a second north of its parallel great circle.

If we put

$v=$ longitude of Uranus in its orbit, referred to the equinox and ecliptic of 1850, we have

$$
\begin{aligned}
& V_{1}=v-127^{\circ} 37^{\prime} \\
& V_{2}=v-12645 \\
& V_{3}=v-15532
\end{aligned}
$$

Substituting these values in the first three terms of $\delta \beta$, and multiplying the last term by the factor $(1+\mu)$ by which the adopted mass of Neptune, $\overline{1} \frac{1}{7} \frac{1}{0}$, must be multiplied to obtain the true mass, we find

$$
\delta \beta=\left(4^{\prime \prime} .69+1^{\prime \prime} .14 u\right) T \cos v-\left(5^{\prime \prime} .24+0^{\prime \prime} .52 u\right) T \sin v .
$$

To these terms must be added those which arise from the motion of the ecliptic.

In the absence of any exhaustive investigation of the obliquity and motion of the ecliptic, I adopt the elements of Hansen, employed in his "Tables du Soleil," because they are a mean between the results of others, and are very accordant with recent observations. The secular motion of the obliquity there employed is $-46^{\prime \prime} .78$. 
Hansen mentions $-5^{\prime \prime} .39$ as the corresponding motion at the equinox of 1850 , found by Olufsen, but I cannot reproduce this result from the secular diminution with any masses of Mercury, Venus, and Mars, which seem to me probable. The expressions in terms of the masses given by Le Verrier are (Annales de l'Observatoire Imperial de Puris, tome ii, p. 101),

$$
\begin{aligned}
& \text { Secular change }=-47.59-0.52 \nu-28.90 \nu^{\prime}-0.83 \nu^{\prime \prime \prime} \\
& \text { Mot. at equinox }=+5.89+0.62 \nu+7.57 \nu^{\prime}+0.73 \nu^{\prime \prime} \text {. }
\end{aligned}
$$

In this expression the masses of Mercury, Venus, and Mars are represented by $\frac{1+\nu}{3,000,000}, \frac{1+\nu^{\prime}}{401,847}$, and $\frac{1+\nu^{\prime \prime \prime}}{2,680,337}$, respectively. The influence of admissible changes in the masses of the other plants is insensible.

From the researches of Le Verrier on the motions of the four inner planets I conclude that the following are about the most probable distribution of the corrections of the masses necessary to produce the motion of the obliquity given by Hansen, namely,

$$
\begin{aligned}
& \nu=-\frac{2}{5} \\
& \nu^{\prime}=-.018 \\
& v^{\prime \prime \prime}=-\frac{1}{10}
\end{aligned}
$$

These values give for the motion at the equinox of 1850

$$
+5^{\prime \prime} .43
$$

Introducing the secular variation of these motions we have, for the change in the latitude of any celestial body near the ecliptic, arising from motion of the ecliptic,

$$
\delta \beta=\left(5^{\prime \prime} .43 T+0^{\prime \prime} .19 T^{2}\right) \cos v+\left(46^{\prime \prime} .78 T-0^{\prime \prime} .06 T^{2}\right) \sin v .
$$

Combining this with the change arising from the motion of the orbit of Uranus, we find

$$
\begin{aligned}
& \delta \beta=\left\{\left(10^{\prime \prime} .12+1^{\prime \prime} .14 \mu\right) T+0^{\prime \prime} .19 T^{2}\right\} \cos v \\
& \quad+\left\{\left(41^{\prime \prime} .54-0^{\prime \prime} .52 \mu\right) T-0^{\prime \prime} .06 T^{2}\right\} \sin v .
\end{aligned}
$$

We may represent these expressions in the usual way by secular variations of the inclination and node of Uranus. But, owing to the small inclination, and consequent rapid motion of the node, it will be necessary to include the coefficients of the second power of the time. On the other hand, no distinction between $\tau$ and $\theta$ is necessary. Putting $\phi$ for the inclination of the orbit, $\theta$ for the longitude of the node referred to the equinox of 1850 , and

$$
\begin{aligned}
& p=\sin \phi \sin \theta, \\
& q=\sin \phi \cos \theta ;
\end{aligned}
$$

we have

$$
\begin{aligned}
& \sin \beta=-p \cos v+q \sin v \\
& \cos \beta \delta \beta=-\delta p \cos v+\delta q \sin v
\end{aligned}
$$


From the expressions for $p$ and $q$ we obtain

$$
\begin{aligned}
& \cos \phi D_{t} \phi=\sin \theta D_{t} p+\cos \theta D_{t} q \\
& \sin \phi D_{t} \theta=\cos \theta D_{t} p-\sin \theta D_{t} q
\end{aligned}
$$

And, neglecting $\left(D_{t} \phi\right)^{2} \times \sin \phi$, we have farther,

$$
\begin{aligned}
& \cos \phi D_{t}^{2} \phi=\sin \phi\left(D_{t} \theta\right)^{2}+\sin \theta D^{2}{ }_{t} p+\cos \theta D^{2} q \\
& \sin \phi D_{t}^{2} \theta=-2 \cos \phi D_{t} \theta D_{t} \phi+\cos \theta D^{2}{ }_{t} p-\sin \theta L^{2}{ }_{t} q .
\end{aligned}
$$

Since $\phi$ is only $46^{\prime}$ we may put $\cos \phi$ and $\cos \beta$ both equal to unity in these expressions, while we have, for 1850 ,

$$
\begin{aligned}
\sin \theta & =0.9573 \\
\cos \theta & =0.2890 \\
D_{t} p & =-10^{\prime \prime} .12-1^{\prime \prime} .14 \mu \\
D_{t} \dot{q} & =+41.54-0.52 \mu \\
L^{2} p & =-0.38 \\
D^{2}{ }_{t} q & =-0.12 \\
\log \sin \phi & =8.129606 .
\end{aligned}
$$

The above formulæ then give

$$
\begin{gathered}
D_{t} \phi=+2^{\prime \prime} .31-1^{\prime \prime} .24 \mu \\
D_{t} \theta=-3167^{\prime \prime} .5+12^{\prime \prime} .6 \mu \\
D^{2} \phi=+0^{\prime \prime} .26 \\
D_{t}^{2} \theta=+5^{\prime \prime} .6 \\
\phi=\phi_{0}+\left(2^{\prime \prime} .31-1^{\prime \prime} .24 \mu\right) T+0^{\prime \prime} .13 T^{2} \\
\theta=\theta_{0}-\left(3167^{\prime \prime} .5-12^{\prime \prime} .6 \mu\right) T+2.8 T^{2},
\end{gathered}
$$

or, adding Struve's precession, we have when $\theta$ is counted from the mean equinox of date,

$$
\theta=\theta_{0}+\left(185 \tau^{\prime \prime} .7+12^{\prime \prime} .6 \mu\right) T+3^{\prime \prime} .9 T^{2} .
$$

Using the values of $\phi$ and 0 given by these expressions, the latitude, secular variation included, will be given by the expression

$$
\sin \beta=\sin \phi \sin (v-0) \text {. }
$$

If we take from a table, as the principal term of the latitude, the value of sin $\phi_{0} \sin (v-\theta)$, the secular term to be added will be

$$
\left\{\left(2^{\prime \prime} .31-1^{\prime \prime} .24 \mu\right) T+0^{\prime \prime} .13 T^{2}\right\} \sin (v-\theta) .
$$

If we represent, as before, by $\omega$ the variable distance of the perihelion from the node, this term will be allowed for by adding to $(b . s .1),(b . c .1)$, etc., the tcrms 
where

$$
\begin{array}{lr}
(b . c .0)= & -e \sin \omega \delta \phi, \\
(b . s .1)= & \cos \omega \delta \phi, \\
(b . c .1)= & \sin \omega \delta \phi, \\
(b . s .2)= & e \cos \omega \delta \phi, \\
(b . c .2)= & e \sin \omega \delta \phi ;
\end{array}
$$

$$
\delta \phi=\left(2^{\prime \prime} .31-1^{\prime \prime} .24 \cdot \imath\right) T+0^{\prime \prime} .13 T^{\prime}
$$

Putting in the above expressions

we find

$$
\begin{gathered}
\omega=95^{\circ} 3^{\prime}+3459^{\prime \prime} T, \\
\cos \omega=-.0880-.0167 T, \\
\sin \omega=+.9961-.0015 T,
\end{gathered}
$$

$$
\begin{aligned}
& (\text { b.c.0 })=-\left(0^{\prime \prime} .11-0^{\prime \prime} .06_{\Lambda}\right) T \\
& (b .8 .1)=-(0.20-0.11 \mu) T-07.05 T^{2} \\
& (\text { b.c.1 })=(2.30-1.24 \mu) T+0.12 T^{2} \\
& (b . s .2)=\quad-0.01 T \\
& (b . c .2)=(0.11-0.06 \cdot \iota) T .
\end{aligned}
$$

We have, finally, to consider the terms of long period in $\delta \eta$ and $i k$ which have been omitted from the periodic perturbations produced by Neptune, in computing the terms of $\delta \beta$ on page 61 , and which are as follows:

$$
\begin{aligned}
& i \eta=1^{\prime \prime} .43 \cos (2 l-g)-0^{\prime \prime} .39 \sin (2 l-g) \\
& -2.12 \cos \left(4 l^{\prime}-2 g\right)+1.00 \sin \left(4 l^{\prime}-2 g\right) \\
& +0.20 \cos (6 l-3 g)-0.04 \sin \left(6 l^{\prime}-3 g\right) \\
& + \text { constant }=0^{\prime \prime} .00 \\
& \varepsilon k=\quad 0^{\prime \prime} .80 \cos \left(2 l^{\prime}-g\right)-2^{\prime \prime} .28 \sin (2 l-g) \\
& -1.06 \cos \left(4 l^{\prime}-2 g\right)-1.85 \sin \left(4 l^{\prime}-2 g\right) \\
& +0.04 \cos (6 l-3 g)+0.19 \sin (6 l-3 g) \\
& + \text { constant }=0 \text { ". } 364 \text {. }
\end{aligned}
$$

For the period during which Uranus has been observed, these values of $\delta \eta$ and $\delta \varepsilon^{2}$ may be replaced by the following:

$$
\begin{aligned}
& \delta n=-0 " .80 T \\
& \delta z=+0.27 T
\end{aligned}
$$

which are to be multiplied by the factor $1+\mu$. The corresponding perturbation of the latitude will be

$$
\delta \beta=\sin \mathrm{v}_{i} \eta-\cos \mathrm{v} i
$$

Putting for $\mathrm{v}$ its approximate value

$$
\mathbf{v}=g+\omega+2 e \sin g
$$

and developing to quantities of the first order with respect to the eccentricities, we have

$$
\begin{aligned}
& \sin \mathrm{v}=\sin (g+\omega)+e \sin (2 g+\omega)-e \sin \omega \\
& \cos \mathrm{v}=\cos (g+\omega)+e \cos (2 g+\omega)-e \cos \omega .
\end{aligned}
$$

May, 1873. 
Substituting for $\omega$ its value, $12^{\circ} 45^{\prime}$, and for in and $\delta /$ their above values in the expression for $\delta \beta$, we find that the terms of $\delta_{\iota} \beta$ in question will add the following terms to (b.c.0), (b.s.1), etc.

$$
\begin{aligned}
& (b . c .0)=-.010 \delta \eta+.046 \delta \%=+0^{\prime \prime} .02 T(1+\mu) \\
& (b . s .1)=+.975 \delta \eta+.221 \delta \%=-0.72 T(1+\mu) \\
& (\text { b.c. } 1)=+.221 \delta \eta-.975 i z=-0.44 T(1+\mu) \\
& (b . s .2)=+.046 \delta \eta+.011 \delta \%=-0.04 T(1+\mu) \\
& (b . c .2)=-(b . c .0)=-0.02 T(1+\mu)
\end{aligned}
$$

These values will be employed in the construction of the provisional ephemeris, but not in the tables.

Collecting all three classes of terms discussed in this section, we have the following constant and secular terms in (b.c.0), (b.s.1), etc.

$$
\begin{aligned}
& (\text { b.c. } 0)=+0^{\prime \prime} .26+\left(-0^{\prime \prime} .09+0.08 \mu\right) T \\
& (\text { b.s.1 })=\left(-0^{\prime \prime} .92-0^{\prime \prime} .61 \mu\right) T-0^{\prime \prime} .05 T^{2} \\
& \text { (b.c. })=(+1.86-1.68 \mu) T+0.12 T^{2} \\
& (\text { b.s. } 2)=-0.06-0.05 T \\
& \left(\text { b.c.2) }=-0.01+\left(0.09-.08_{i \iota}\right) T\right.
\end{aligned}
$$

\section{Positions of Uranus resulting from the preceding theory.}

The next step in order is the preparation of an ephemeris of the planet for comparison with observations. As this provisional theory is, for future use, superseded by the tables appended to the present work, it seems unnecessary to enter very fully into the details of the computation of the ephemeris. The perturbations of the longitude, logarithm of radius vector, and latitude, were first computed by the formulæ already given.

$$
\begin{aligned}
& \delta v=(v . c .0)+(v . c .1) \cos g+(v . c .2) \cos 2 g+\text { etc. } \\
&+(v . s .1) \sin g+(v . s .2) \sin 2 g+\text { etc. } \\
& M \delta \rho=(\rho . c .0)+(\rho . c .1) \cos g+(\rho . c .2) \cos 2 g+\text { etc. } \\
&+(\text { p.s.1) } \sin g+(\rho . s .2) \sin 2 g+\text { etc. } \\
& \delta \beta=(b . c .0)+(b . c .1) \cos g+(b . s .1) \sin g .
\end{aligned}
$$

Each coefficient (v.c.0), (v.c.1), etc., is composed at most of the following quantities:

1. The five classes of secular, long period, or constant ternis, the separate values of which, with the sum of all, are given on pages 89 to 93 .

2. Periodic terms due to the action of Jupiter, Saturn, and Neptune, given on pages 83 to 87 .

3. Terms depending on the product of the masses of Jupiter and Saturn, given on page 88 , omitting those depending on $N_{6}$ and $N_{7}$, because they are given in column 5 of the terms of the first class.

'The sum of the perturbations thus computed is given in the third column of the following ephemeris.

An approximate value of the perturbations prodnced by Neptune alone is independently computed for every fourth date, and the result is given in the fourth 
column. The secular and long period terms are here taken from columns (3) and (4) of the tables on pages 89 to 93 .

The elliptic co-ordinates were then derived from the following elements, which are a little different from those employed in the computation of the perturbations.

Elements III. of Uranus.
\[ \begin{array}{rrrl}\pi, & 168^{\circ} & 15^{\prime} & 12^{\prime \prime} .0 \\ \varepsilon, & 28 & 25 & 29.5 \\ \theta, & 73 & 11 & 58.0 \\ \phi, & 0 & 46 & 20.0 \\ e, & & .0469436 \\ e,(\text { in sec.) } & 9682^{\prime \prime} .81 \\ n, & 15426.196 \\ \log a, & 1.2828989 \\ \text { Red. to Ecliptic, } & -9^{\prime \prime} .37 \sin 2(v-\theta)\end{array} \]

The longitudes thus found are corrected for lunar, but not for solar nutation, and the results are given in the fifth column.

The column "correction" arises in this way: after the comparison of the ephemeris with observations was nearly completed, it was found that some errors had crept into the former, the most important of which was the employment of a mean anomaly, $g$, corrected for secular variation of the perihelion in the computation of the perturbations from the preceding formulæ. As a large portion of the computations on the provisional ephemeris had been made by assistants furnished by the Smithsonian Institution and Nautical Almanac, I deemed it prudent to make a careful recomputation of the perturbations for every sixth date during the entire period of the modern observations. The longitudes actually printed in the fifth column are the results of the original incorrect computation, while the numbers in the next column show the several corrections to be applied to obtain the results of my final revised computation.

During the period of the modern observations the ephemeris is computed for intervals of 120 days, and the selected dates are all exact multiples of that interval before or after the fundamental epoch, 1850, Jan. 0, Greenwich mean noon. For convenience of reference the dates are numbered from an epoch earlier by 212 intervals, and the number is given in the second column.

Between 1796 and 1801 no observations worth using were made on Uranus, the ephemeris has, therefore, not been extended over this interval. 
Heliocentric Epiemerts of Uranus fron the preceding Provisional Tueory.

[The longitades are corrected for lanar bat not for solar nutation.]

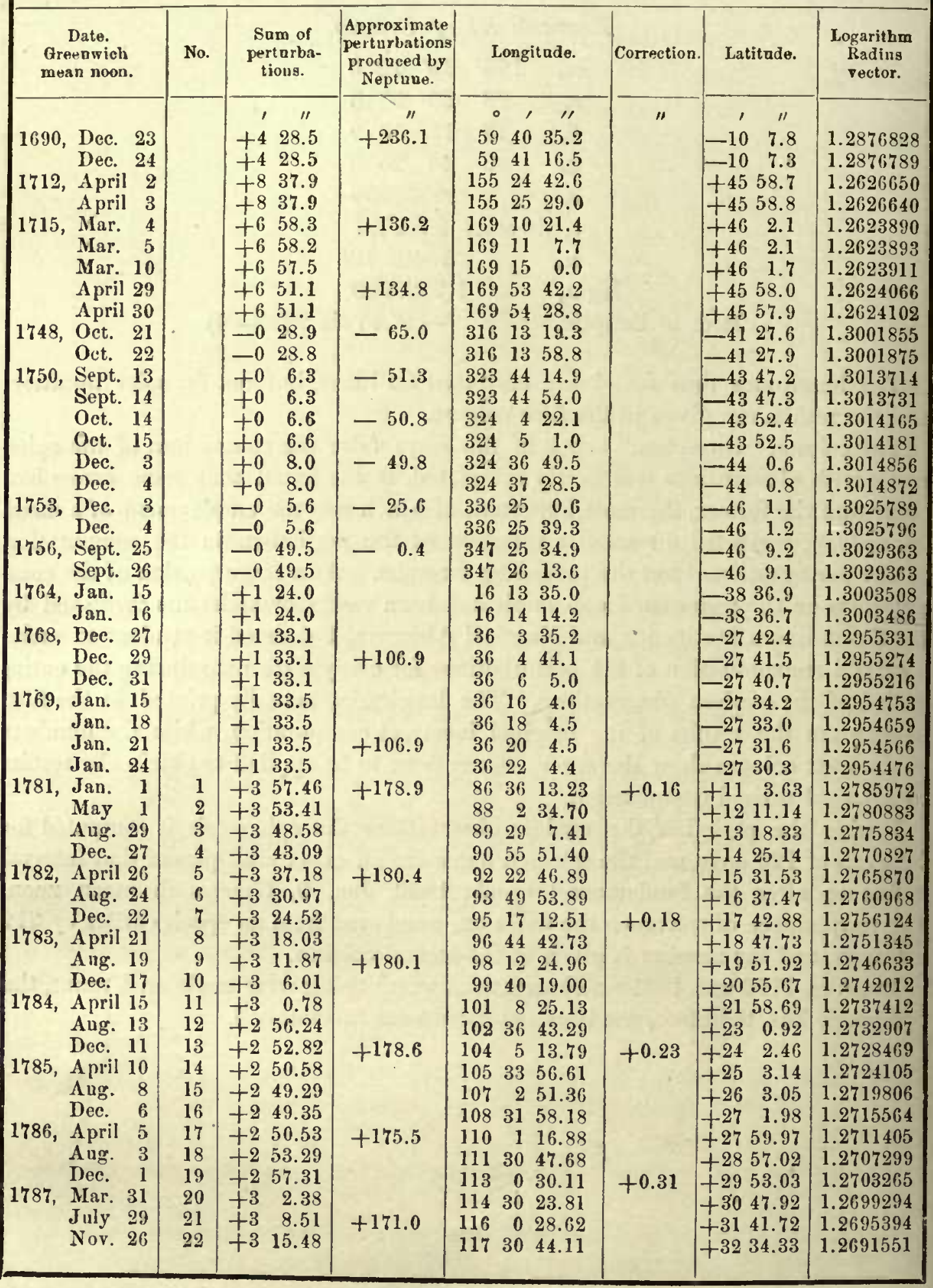




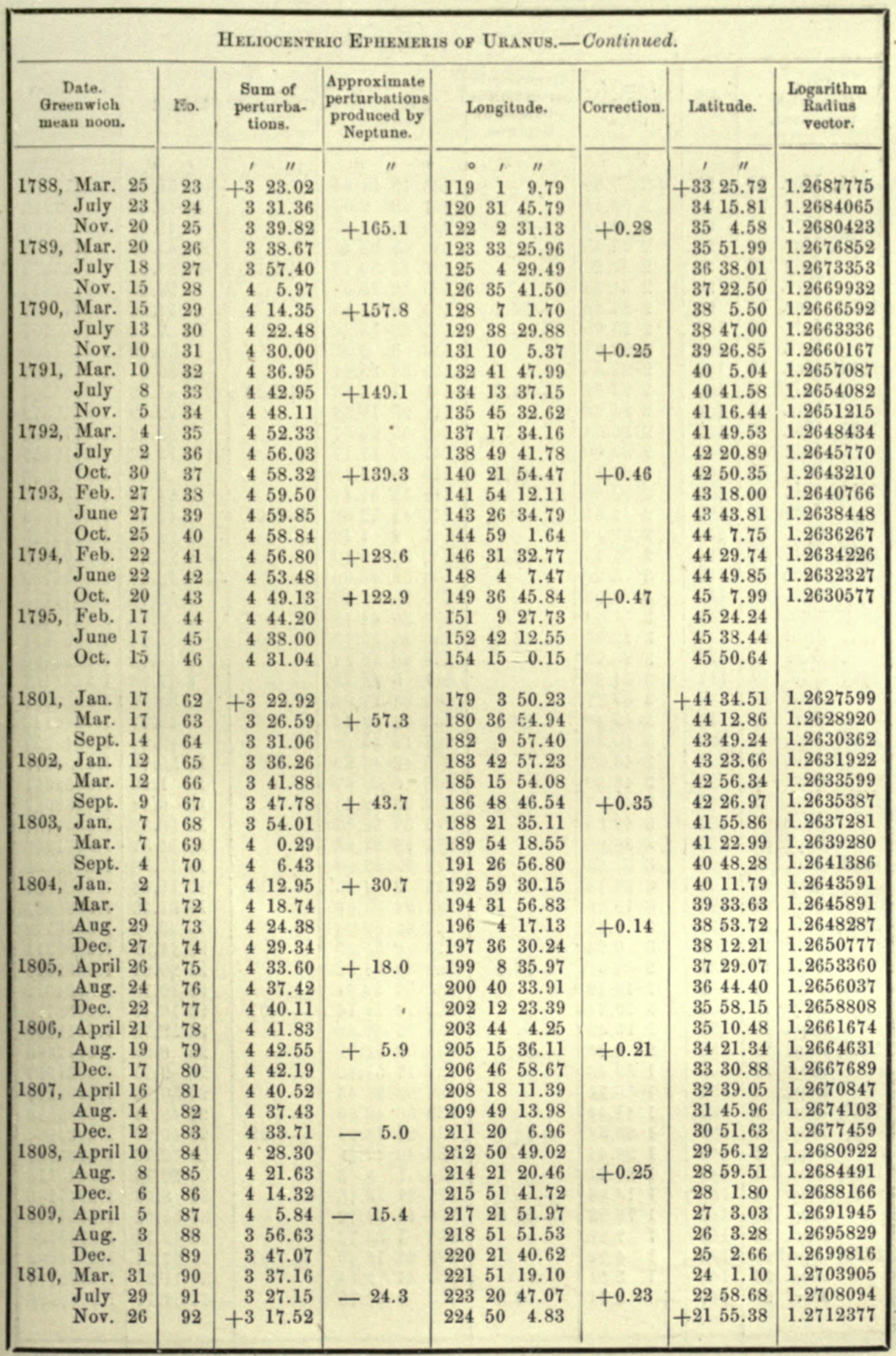




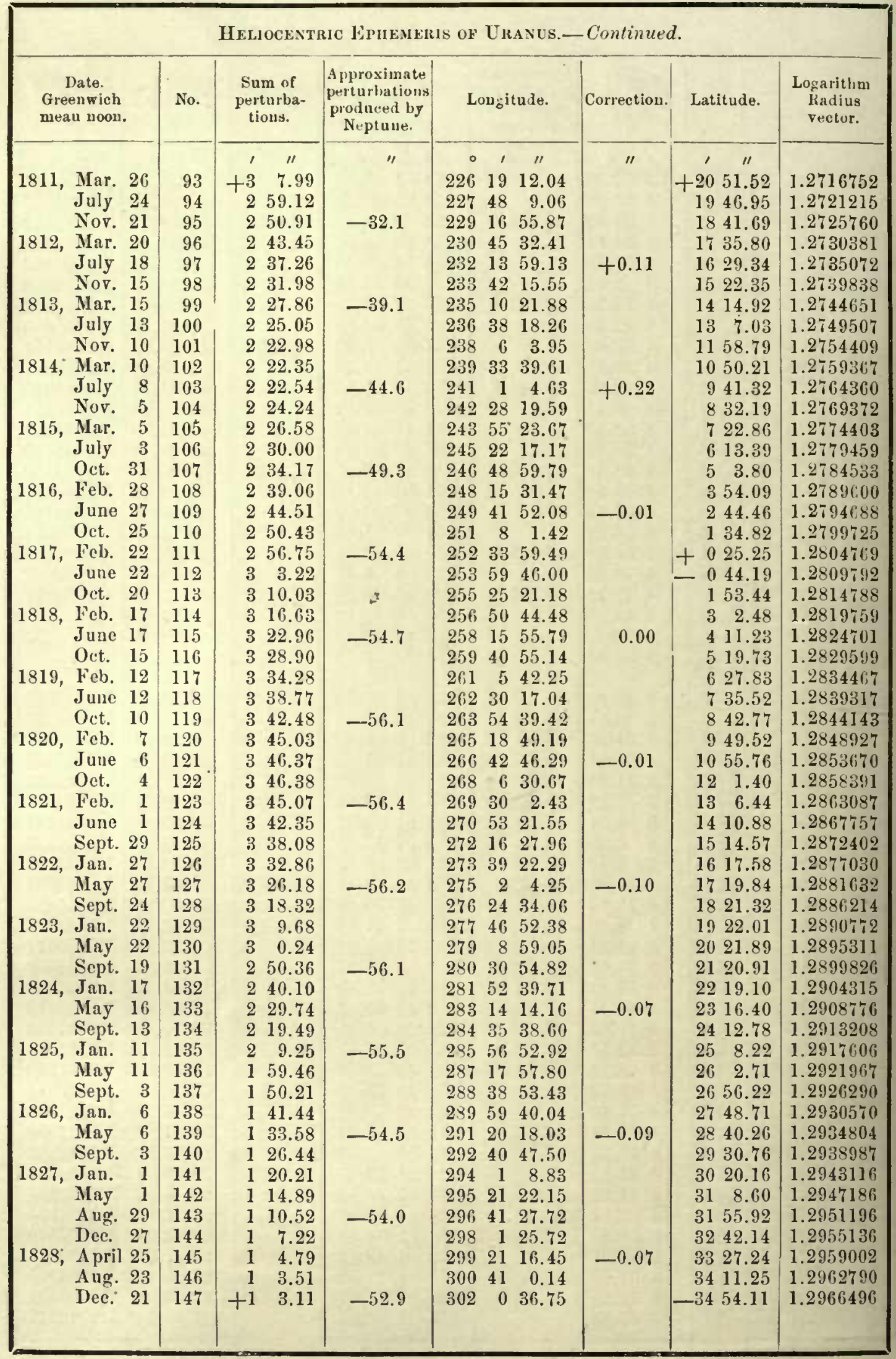




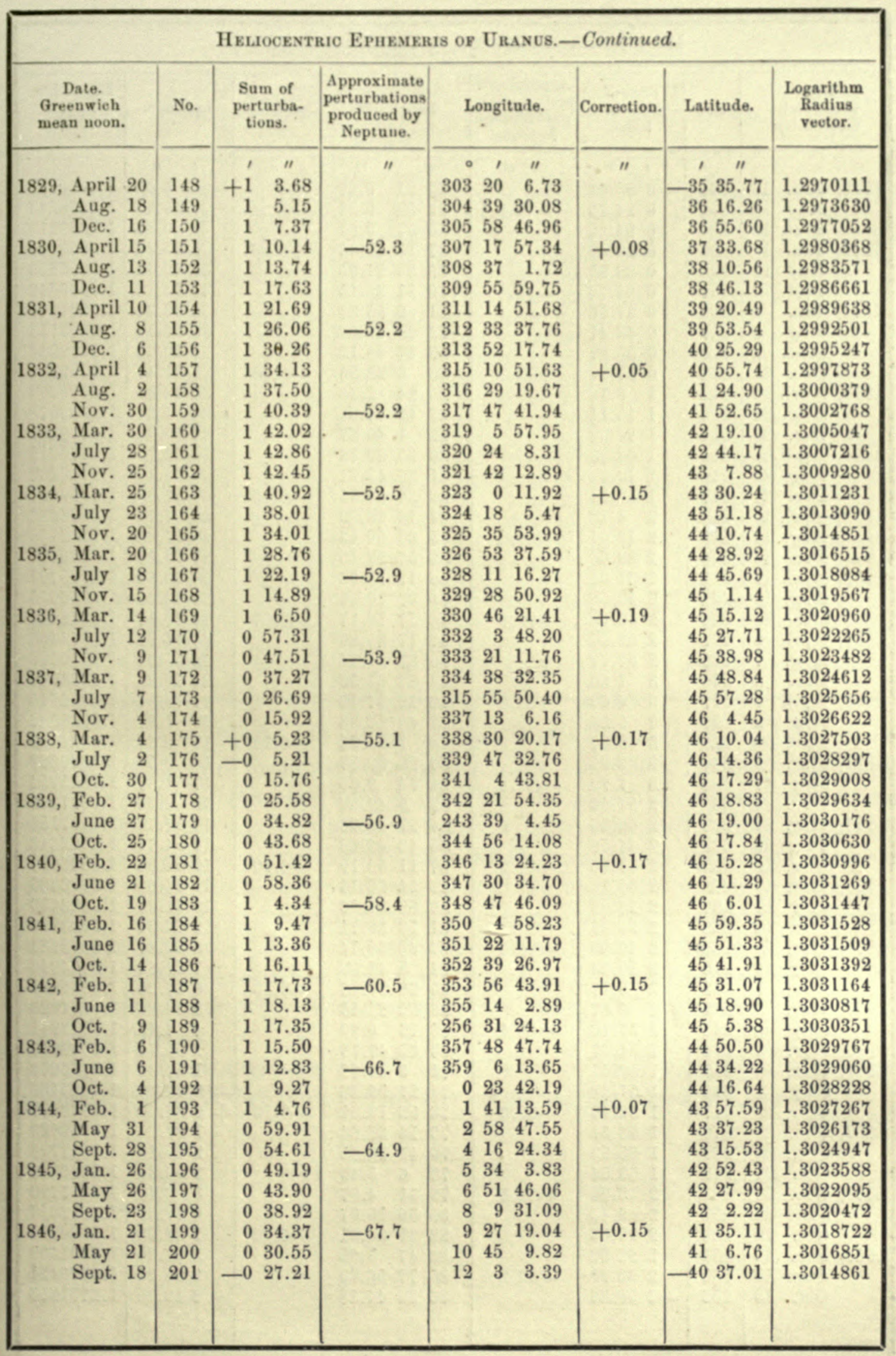




\begin{tabular}{|c|c|c|c|c|c|c|c|c|c|c|}
\hline & & & & & 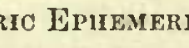 & OF & 1. & intiv & & \\
\hline $\begin{array}{r}\text { D } \\
\text { Gree } \\
\text { meat }\end{array}$ & $\begin{array}{l}\text { Date. } \\
\text { enwich } \\
\text { n noon. }\end{array}$ & & No. & $\begin{array}{c}\text { Sum of } \\
\text { perturba- } \\
\text { tions. }\end{array}$ & $\begin{array}{c}\text { Approximate } \\
\text { perturbations } \\
\text { produced by } \\
\text { Neptune. }\end{array}$ & Long & gitude. & Correction. & Latitude. & $\begin{array}{l}\text { Logarithm } \\
\text { Radius } \\
\text { rector. }\end{array}$ \\
\hline & & & & , 11 & " & $\circ$ & & $" \prime$ & & \\
\hline 1847 & Jan. & 16 & 202 & $-0 \quad 25.64$ & & 132 & $21 \quad 0.27$ & & $-40 \quad 6.03$ & 1.3012758 \\
\hline , & May & 16 & 203 & 024.73 & -70.2 & 143 & $\begin{array}{ll}39 & 0.21\end{array}$ & & 3933.75 & 1.3010544 \\
\hline & Sept. & 13 & 204 & 024.95 & & 155 & $7 \quad 3.61$ & & $39 \quad 0.23$ & 1.3008219 \\
\hline 1848 & Jan. & 11 & 205 & 026.43 & & - 171 & $15 \quad 10.46$ & +0.06 & 3825.40 & 1.3005790 \\
\hline & May & 10 & 206 & 028.95 & & 183 & 3321.30 & & 3749.42 & 1.3003263 \\
\hline & Sept. & 7 & 207 & $\begin{array}{ll}0 & 32.75\end{array}$ & 一 72.8 & 195 & 3136.15 & & 3712.18 & 0640 \\
\hline 49 & Jan. & 5 & 208 & $0 \quad 37.59$ & & 21 & 955.34 & & 3633.73 & 1.2997923 \\
\hline & May & 5 & 209 & $\begin{array}{ll}0 & 43.47\end{array}$ & & 222 & $\begin{array}{ll}28 & 19.02\end{array}$ & & 3554.16 & 1.2995115 \\
\hline & Sept. & 2 & 210 & 050.27 & & 234 & 1648.13 & & 3513.36 & 1.2992224 \\
\hline & Dec. & 31 & 211 & 057.74 & -75.3 & 25 & 522.56 & -0.08 & 3431.47 & 1.2989252 \\
\hline 1850 & April & 30 & 212 & 16.18 & & 262 & $4 \quad 2.38$ & & 3348.40 & 1.2986203 \\
\hline & Aug. & 28 & 213 & 115.11 & & 274 & 248.39 & & $133 \quad 4.37$ & 078 \\
\hline & Dec. & 26 & 214 & 124.38 & & 29 & 140.87 & & 3219.06 & 1.2 \\
\hline 1851 , & April & 25 & 215 & 134.33 & 一 ร.9 & 302 & 39.78 & & 3132.79 & 1.2976615 \\
\hline & Ang. & 23 & 216 & 144.28 & & 313 & $39 \quad 45.91$ & & $30 \quad 45.46$ & 1.2973287 \\
\hline & Dec. & 21 & 217 & 154.21 & & 325 & 859.55 & -0.09 & 2957.09 & 1.296 \\
\hline 1852, & April & 19 & 218 & $2 \quad 4.12$ & & 341 & 820.89 & & $\begin{array}{ll}29 & 7.71\end{array}$ & 436 \\
\hline & Aug. & 17 & 219 & 213.61 & -80.5 & 353 & 3750.43 & & 2817.33 & 316 \\
\hline & Dec. & 15 & 220 & 223.00 & & 365 & 728.19 & & 2725.95 & 38 \\
\hline 1853, & April & 14 & 221 & 231.54 & & 381 & 715.06 & & 2633.62 & 1.2955701 \\
\hline & Aug. & 12 & 222 & 239.58 & & 393 & 3710.77 & & 2540.35 & 1.2952015 \\
\hline & Dec. & 10 & 223 & 246.62 & -82.6 & 405 & 716.17 & -0.07 & 2446.17 & 3270 \\
\hline 1854 , & April & 9 & 224 & 252.64 & & $421^{\prime}$ & 731.26 & & 2351.08 & 458 \\
\hline & Aug. & 7 & 225 & 257.77 & & 433 & 756.24 & & 2255.08 & 83 \\
\hline & Dec. & 5 & 226 & $\begin{array}{ll}3 & 1.60\end{array}$ & & 4458 & 831.59 & & 2158.25 & 645 \\
\hline 1855 & A pril & 4 & 227 & 34.03 & -84.6 & 461 & $9 \quad 17.49$ & & $21 \quad 0.51$ & 1.2932645 \\
\hline & Aug. & 2 & 228 & $3 \quad 5.35$ & & 474 & $\begin{array}{ll}0 & 13.93\end{array}$ & & $\begin{array}{ll}20 & 1.97\end{array}$ & 1.2928582 \\
\hline & Nov. & 30 & 229 & $\begin{array}{ll}3 & 5.32\end{array}$ & & 491 & 121.26 & -0.11 & $19 \quad 2.66$ & 449 \\
\hline 1856, & Mar. & 29 & 230 & $\begin{array}{ll}3 & 3.95\end{array}$ & & 51) 2 & 2239.57 & & $18 \quad 2.55$ & 239 \\
\hline & July & 27 & 231 & $3 \quad 1.24$ & -86.1 & 514 & $4 \quad 9.08$ & & $17 \quad 1.67$ & 554 \\
\hline & Nor. & 24 & 232 & 257.62 & & 535 & $\begin{array}{ll}5 & 49.32\end{array}$ & & $\begin{array}{ll}16 & 0.03\end{array}$ & 1.2911592 \\
\hline 1857 & Mar. & 24 & 233 & 252.95 & & 542 & 7 40.66 & & 1457.72 & 1.2907155 \\
\hline & July & 22 & 234 & 247.58 & & 554 & 942.98 & & 1354.71 & 1.2902643 \\
\hline & Nor. & 19 & 235 & 241.65 & -87.9 & $57 \cdot 11$ & 156.10 & -0.10 & 1251.08 & 1.2898057 \\
\hline 1858, & Mar. & 19 & 236 & 235.18 & & 583 & 20.16 & & 1146.89 & 1.2893395 \\
\hline & July & 17 & 237 & 228.82 & & 5950 & 654.59 & & 1041.96 & 559 \\
\hline & Nor. & 14 & 238 & 222.46 & & 6119 & 939.71 & & 936.91 & 33853 \\
\hline 1859 & Mar. & 14 & 239 & 216.35 & -88.6 & 6242 & 235.14 & & 830.87 & 1.2878981 \\
\hline & July & 12 & 240 & 210.33 & & 64 & 5. 41.38 & . & 724.61 & 1.2874049 \\
\hline & Nov. & 9 & 241 & 25.17 & & 6528 & 857.59 & -0.13 & $\begin{array}{lll}6 & 17.90\end{array}$ & 1.2869061 \\
\hline 1860 & Mar. & 8 & 242 & 20.67 & & 6652 & 224.15 & & $\begin{array}{lll}5 & 10.84\end{array}$ & 1.2864023 \\
\hline & July & 6 & 243 & 157.10 & -89.1 & 6816 & $6 \quad 0.90$ & & $4 \quad 3.42$ & 8941 \\
\hline & Nov. & 3 & 244 & 154.37 & & 693 & 947.78 & & 255.74 & 1.2853816 \\
\hline 1861 & Mar. & & 245 & 152.61 & & 713 & 344.98 & & 147.83 & 1.2848658 \\
\hline & July & 1 & 246 & 151.92 & & 7227 & 752.38 & & 039.69 & 1.2843468 \\
\hline & Oct. & 29 & 247 & 152.43 & -89.3 & 735 & 210.31 & -0.19 & +028.60 & 1.2838257 \\
\hline 1862 & Feb. & 26 & 248 & 153.51 & & 7510 & $6 \quad 38.24$ & & 137.00 & 1.2833029 \\
\hline & June & 26 & 249 & 155.99 & & $76 \quad 41$ & 116.65 & & 245.46 & 1.2827788 \\
\hline & Oct. & 24 & 250 & 159.54 & & 78 & $6 \quad 5.46$ & & 353.95 & 1.2822542 \\
\hline 1863, & Feb. & 21 & 251 & 23.98 & -88.5 & 7931 & 14.97 & & $5 \quad 2.40$ & 1.2817290 \\
\hline & June & 21 & 252 & 29.43 & & 805 & 615.07 & & 610.80 & 1.2812047 \\
\hline & Oct. & 19 & 253 & $\begin{array}{ll}2 & 15.63\end{array}$ & & 8221 & 136.01 & -0.04 & 719.09 & 1.2806819 \\
\hline 1864 & Fcb. & 16 & 254 & 222.52 & & 834 & $7 \quad 7.95$ & & 827.25 & 1.2801609 \\
\hline & June & 15 & 255 & 230.20 & -87.2 & 851 . & 250.83 & & 935.22 & 1.2796421 \\
\hline & Oct. . & 13 & 256 & $-2 \quad 38.39$ & & 863 & 3844.88 & & +842.95 & 1.2791263 \\
\hline
\end{tabular}




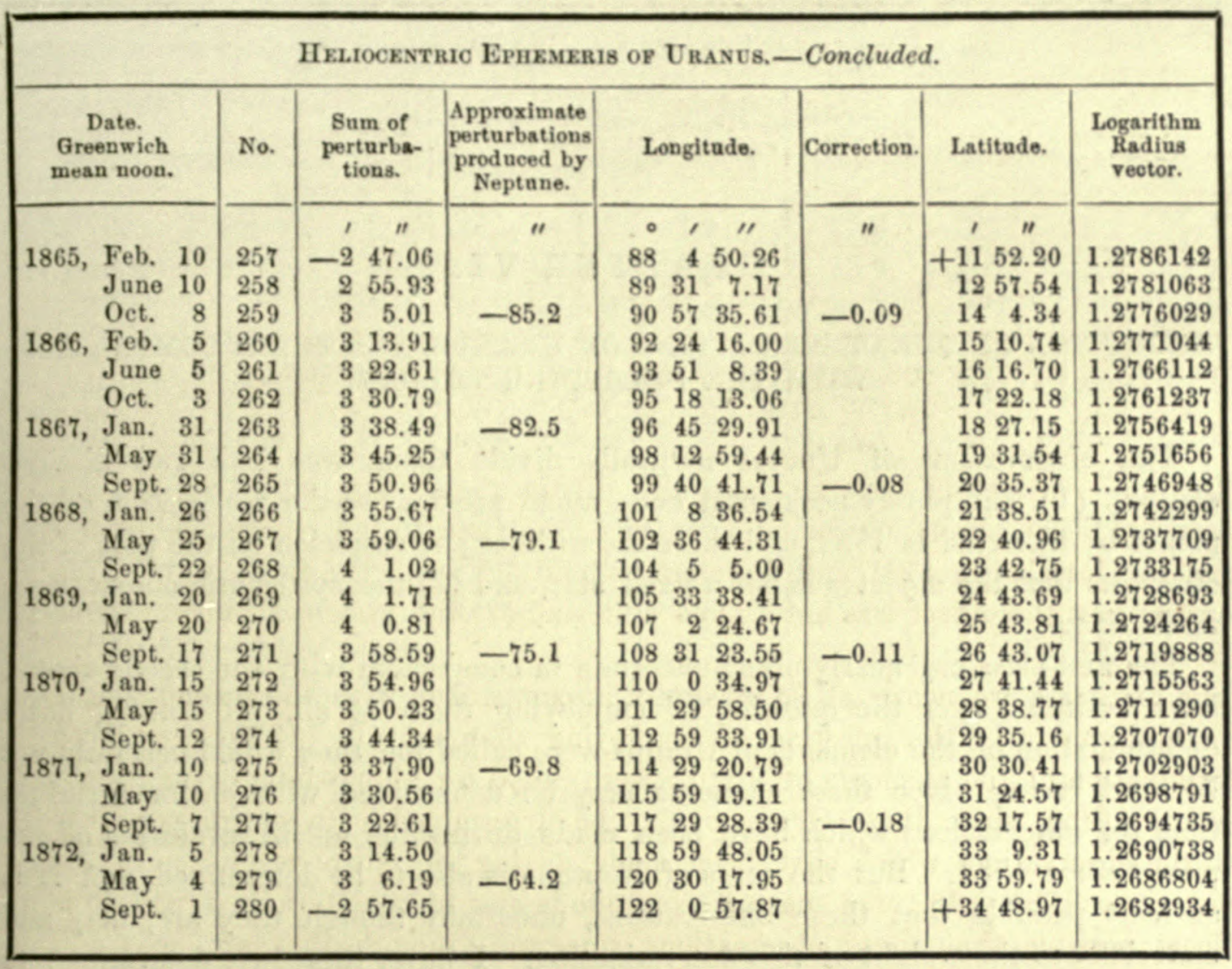

The next operation would be to interpolate these co-ordinates to intervals of time suitable for the computation of a geocentric ephemeris, to correct the longitudes for solar nutation, and then to compute the geocentric right ascension and declination. This operation has not, however, been completely carried out except for most of the observations before 1830, and for three of the oppositions observed since, the latter being computed only as a check upon the accuracy of the comparisons. As a general rule, it may be said that wherever a complete geocentric ephemeris, with the heliocentric ephemeris from which it was computed, were available, these ephemerides were made use of in a manner which will be more fully described hereafter, while, in all other cases, the geocentric places were computed directly.

It may also be stated here that Hansen's Tables du Soleil have been adopted as giving the places of the sun to be used in computing the geocentric places. 


\section{CHAPER VI.}

\section{REDUCTION OF THE OBSERVATIONS OF URANUS, AND THEIR COMPARISON WITII THE PRECEDING THEORY.}

THE observations of Uranus naturally divide themselves into two distinct classes. (1) The purely accidental ones, made previous to the recognition of the planet by Herschel in 1781, and therefore without any stuspicion on the part of the observers that the object was not a fixed star, and (2) the systematic observations made since.

The first class are nearly all so uncertain in comparison with the second that I have hesitated over the question of employing them at all. If nothing but a determination of the elements of Uranus were called for, they would certainly not be worth using, since these elements may be determined with entire certainty from the observations which have been made during the entire revolution of the planet since 1781. But the mass of Neptume is also to be determined, and it is at least possible that these observations, uncertain though they are, may add materially to the weight of this determination. I have, therefore, determined to include them all, re-reducing them when there seemed to be good reason so to do.

The earliest observations are those of Flamstead, published in the Historice Coelestis. The observations themselves, as printed, together with the principal elements for reduction, are given in the following tables.

The first column of the table gives the name of the star. The second gives the clock time of transit over the wire of the quadrant as given by Flamstead. The time, it will be seen, is only given to entire seconds. We must, therefore, expect to find a probable error, of which the mathematical minimum is $0^{5} .25$, and of which the minimum we can reasonably expect is much greater.

Next we have the apparent right ascensions of the stars as computed. For these data I am indebted to Prof. Coffin, Superintendent of the American Ephemeris. The mean places are mostly derived from the "Star Tables of the American Ephemeris," and from the two Greenwich Seven Year Catalogues, while the reduction to apparent place is made with the modern constants.

'The fourth column gives the apparent clock correction for sidereal time, in which is included the effect of deviation of the instrument from the meridian.

The clock keeping mean time, the errors are in the next column reduced to those of sidereal time at the moment of the transit of Uranus.

The next two columns give the corrections for clock rate, and for deviation of the instrument from the meridian, as inferred from the observations themselves, both being referred to the time and position of the transit of Uranus. 
In the last column we have the seconds of concluded correction for clock and instrument to be applied to the observed time of transit of Uranus.

\section{0, December 23. Right Ascension.}

Star. Time of Tr. R. A. of star. Cloek. $C^{\prime}$. R. Dev. $\mathbf{C}^{\prime \prime}$.

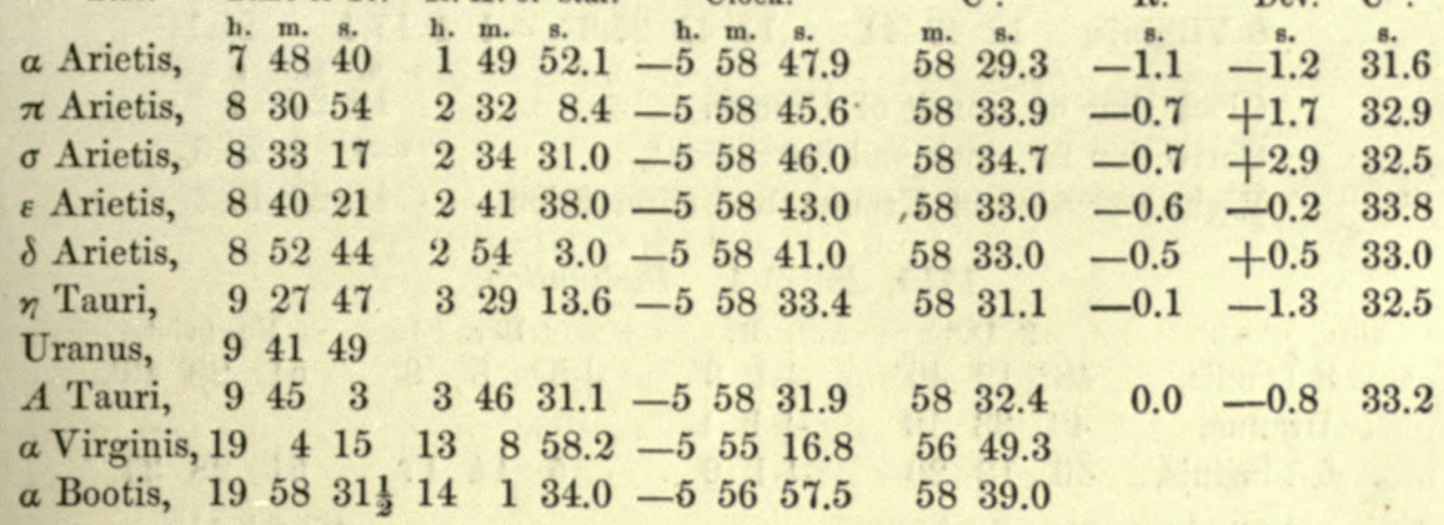

Hourly rate of clock,

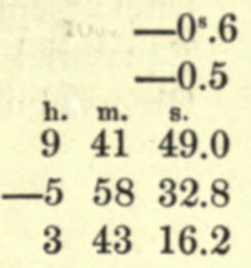

Transit of Uranus;

Correction for clock and instrument (mean),

Observed R. A. of the planet,

1690, December 23. Declination.

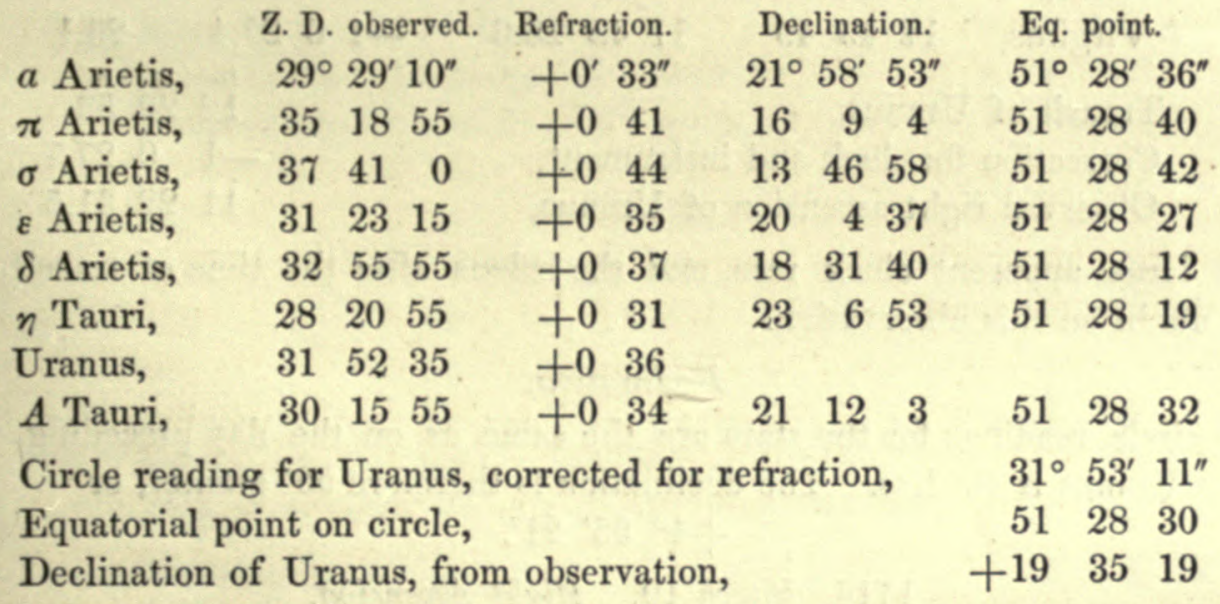

1712, April 2. Right Ascension.

\begin{tabular}{lrrrrrrrr} 
& \multicolumn{1}{c}{ h. } & m. & s. & & & & & \\
Uranus, & 9 & 35 & 19 & h. & m. & s. & m. & s. \\
$\varepsilon$ Virginis, & 12 & 0 & 19 & 12 & 47 & 52.4 & +47 & 33.4 \\
$e$ Virginis, & 12 & 14 & 51 & 13 & 2 & 31.5 & +47 & 40.5 \\
$\zeta$ Virginis, & 12 & 32 & 11 & 13 & 20 & 5.7 & +47 & 54.7
\end{tabular}

The discordance of clock errors, and the time which intervened between the transit of the planet and that of the first star, seem to render an accurate reduction impossible. 


\section{5, March 4. Right Ascension.}

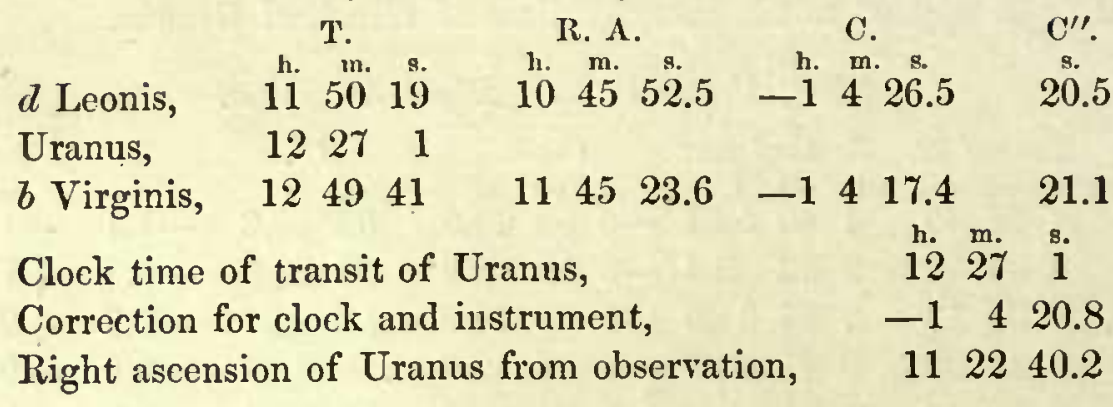

1715, March 4. Declination.

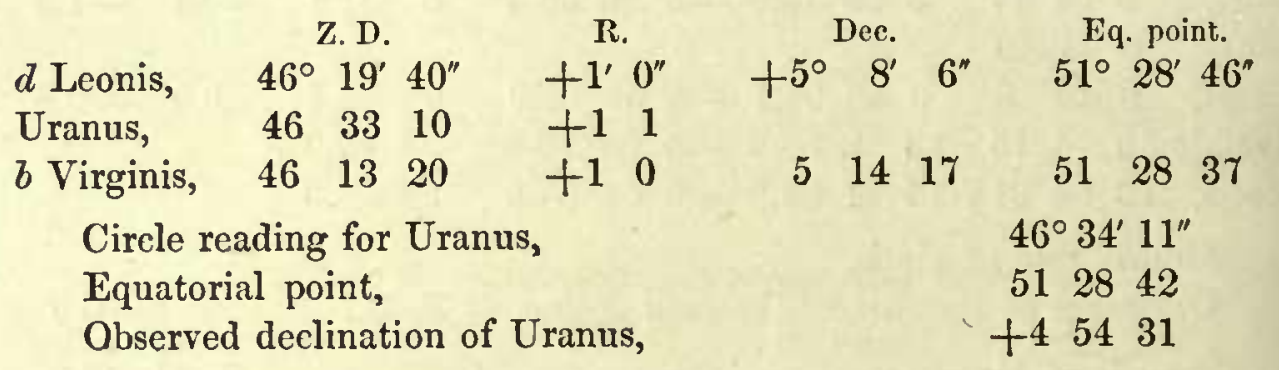
1715, Mirch 5. Right Ascension.

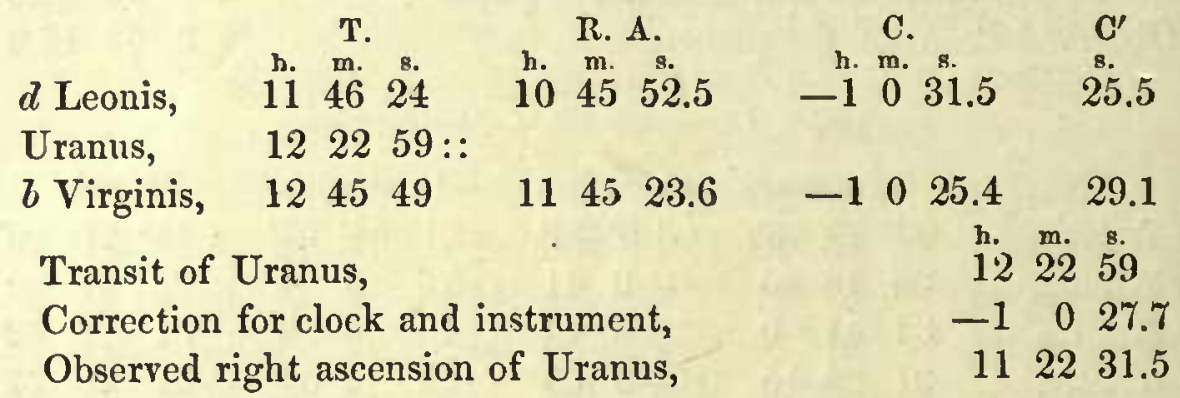

The large apparent clock rate, and the colons after the time of transit, both throw doubt on this observation.

\section{Declination.}

The circle readings for the stars are the same as on the day preceding, while that for Uranus is 50" less. The declination is therefore $50^{\prime \prime}$ greater, or $+4^{\circ} 55^{\prime} 21^{\prime \prime}$.

\section{5, March 10. Right Ascension.}
T.
R. A.
C. $\quad \mathrm{C}^{\prime}$.

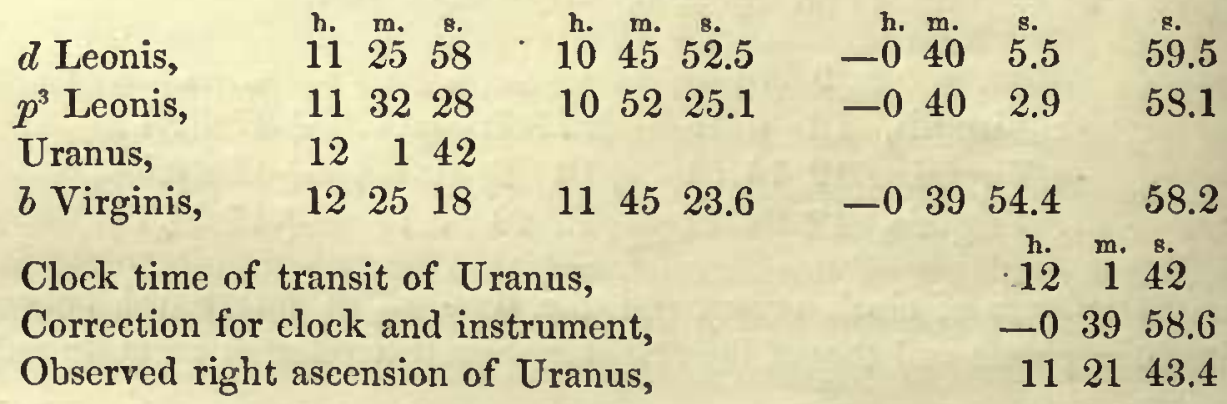


Declination.

\begin{tabular}{|c|c|c|c|c|c|c|c|c|}
\hline & & Z. D. & R. & Dec & & & 1. $\mathrm{Pt}$ & \\
\hline$d \mathrm{Le}$ & $46^{\circ}$ & $19^{\prime} 35^{\prime \prime}$ & $+1^{\prime} 1^{\prime \prime}$ & $5^{\circ} 8^{\prime}$ & $6^{\prime \prime}$ & $51^{\circ}$ & $28^{\prime}$ & $42^{\prime \prime}$ \\
\hline$p^{3}$ Leonis, & 47 & $58 \quad 35$ & +13 & 329 & 25 & 51 & 28 & 63 \\
\hline Uranus, & 46 & 27 & +11 & & & & & \\
\hline$b$ Virginis, & 46 & 1325 & +10 & 514 & 17 & 51 & 28 & 42 \\
\hline \multirow{3}{*}{\multicolumn{6}{|c|}{$\begin{array}{l}\text { Circle reading for Uranus, } \\
\text { Equatorial point on circle, } \\
\text { Observed declination of Uranus, }\end{array}$}} & 46 & 28 & 1 \\
\hline & & & & & & 51 & 28 & 49 \\
\hline & & & & & & +5 & 0 & 48 \\
\hline
\end{tabular}

1715. April 29. Right Ascension.

\begin{tabular}{|c|c|c|c|c|c|}
\hline Leonis, & 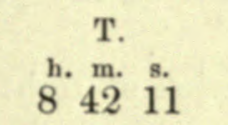 & $\begin{array}{l}\text { h. } \\
11\end{array}$ & $\begin{array}{l}\text { R. A. } \\
\text { m. s. } \\
628.3\end{array}$ & $\begin{array}{rl} & \text { c. } \\
\text { h. } & \text { m. g. } \\
+2 & 24.17 .3\end{array}$ & $\begin{array}{l}C^{\prime} . \\
18.7\end{array}$ \\
\hline 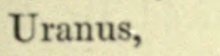 & $850 \quad 44$ & & & & \\
\hline$\nu$ Virginis, & $\begin{array}{lll}9 & 6 & 55\end{array}$ & 11 & 3114.1 & +22419.1 & 16.4 \\
\hline 17 Virginis, & $943 \quad 38:$ : & 12 & $8 \quad 6.5$ & +22428.5 & 19.8 \\
\hline$x$ Virginis, & 113248 . & 13 & $57 \quad 47.6$ & $\begin{array}{r}22459.6\end{array}$ & 33.1 \\
\hline
\end{tabular}

The discordance of the clock corrections makes a satisfactory determination of the right ascension very difficult. I deem it best to reject the doubtful observation of 17 Virginis, and the discordant one of $x$ Virginis. The result will then be

Observed transit of Uranus,

Correction for clock and instrument,

Observed right ascension of Uranus,
h. m. 8 .
22417.6
$\begin{array}{lll}11 & 15 & 1.6\end{array}$

Declination.

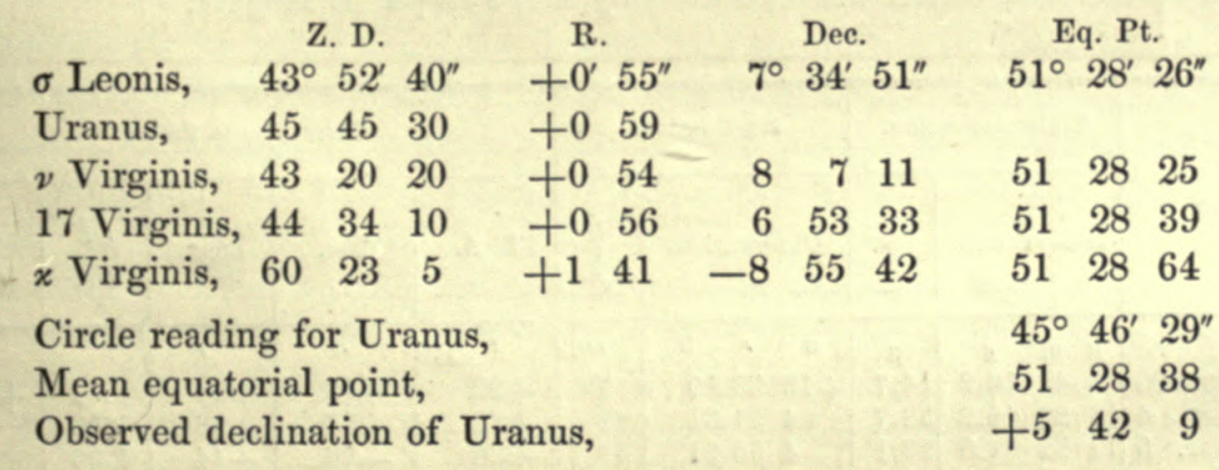

The next observations in the order of time are two by Bradley, discovered by Mr. Hugh Breen, but still unpublished. The following are the results as given by Mr. Breen in the Astronomische Nachrichten, No. 1463.
Mean Time.
R. A.
1748, October 21,

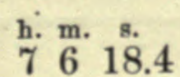 h. m. 8 . 1750, September 13, $10857.8 \quad 21 \quad 40 \quad 0.23$ N. P. D. 
Mr. Breen remarks: "The right ascensions are very accurate. It has been assumed that the N. P. D., on 1750 , September 13 , is identical with $\mu$ Capricorni, with which it was compared. The first observation was by the transit instrument, and the second by the quadrant."

No ground is given for the above assumption respecting the N.P.D. for the second observation; it may, therefore, be omitted as valueless.

In the year 1750 we have also two observations by Le Monnier at Paris. For these, and all the other observations by the same observer, I shall adopt the results given by Bouvard in the Connaissunce des Temps, for 1821, p. 341, with the corrections indicated by Le Verrier, in Connaissance des Temps, for 1849, pp. 125 and 126. The necessary uncertainty of the observations is such that, considering that Bouvard reduced them with the star positions of the "Fundamenta," scarcely anything will be gained by a new reduction.

1753 , December 3, we have another observation of right aseension by Bradley. I adopt the result kindly communicated by my distinguished friend, Dr. Auwers.

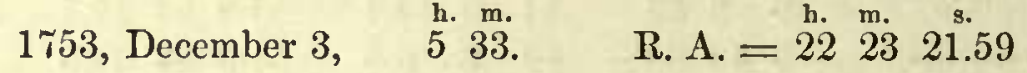

1756, September 25. Observation by Mayer, at Gottingen. I adopt the result given by Bessel, in Fundamenta Astronomia, p. 284.

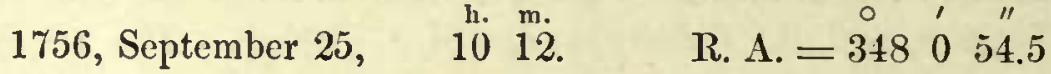

$$
\begin{aligned}
& \text { Dec. }=-6 \quad 149.4
\end{aligned}
$$

The following is a tabular summary of the preceding results, with their comparison with the provisional theory. In the computation of the geocentric place the places of the sun were derived from Hansen's Tables. I am indebted to Professor Coffin for a duplicate computation of the geocentric places from the provisional ephemeris, which was executed by Mr. Joseph A. Rogers.

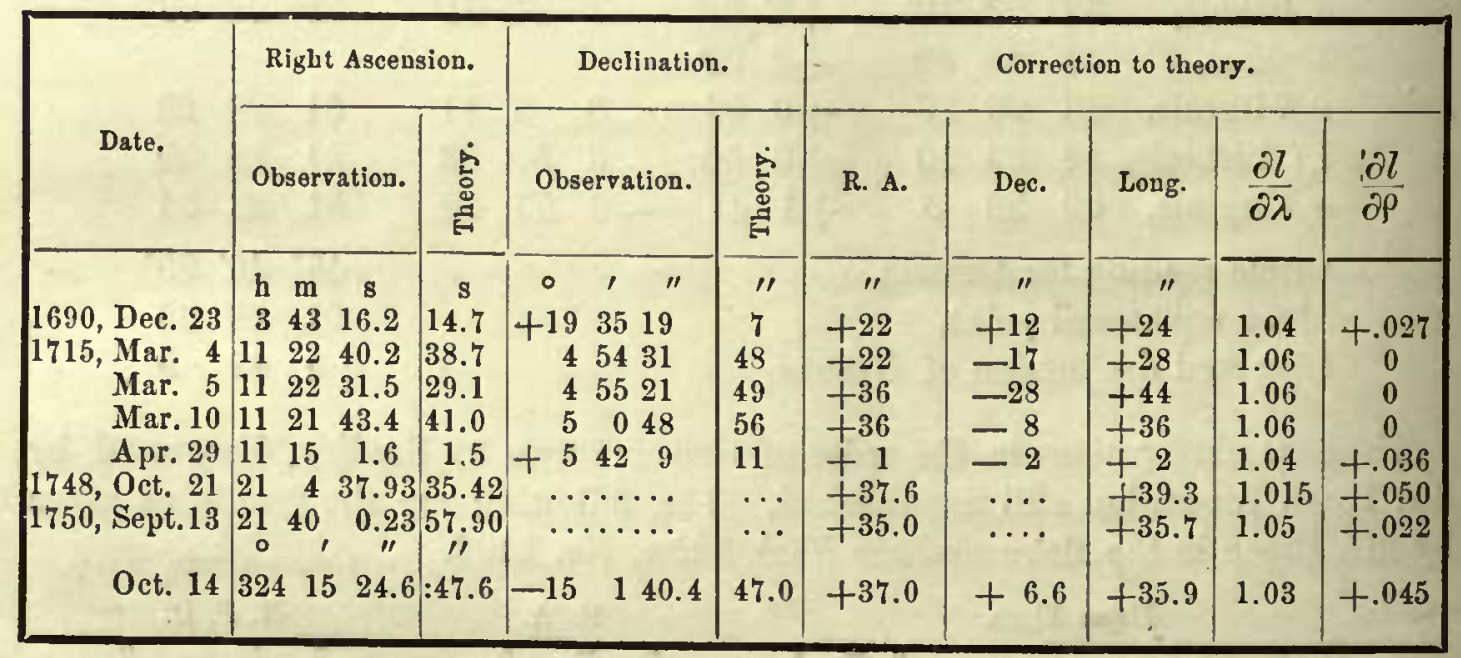




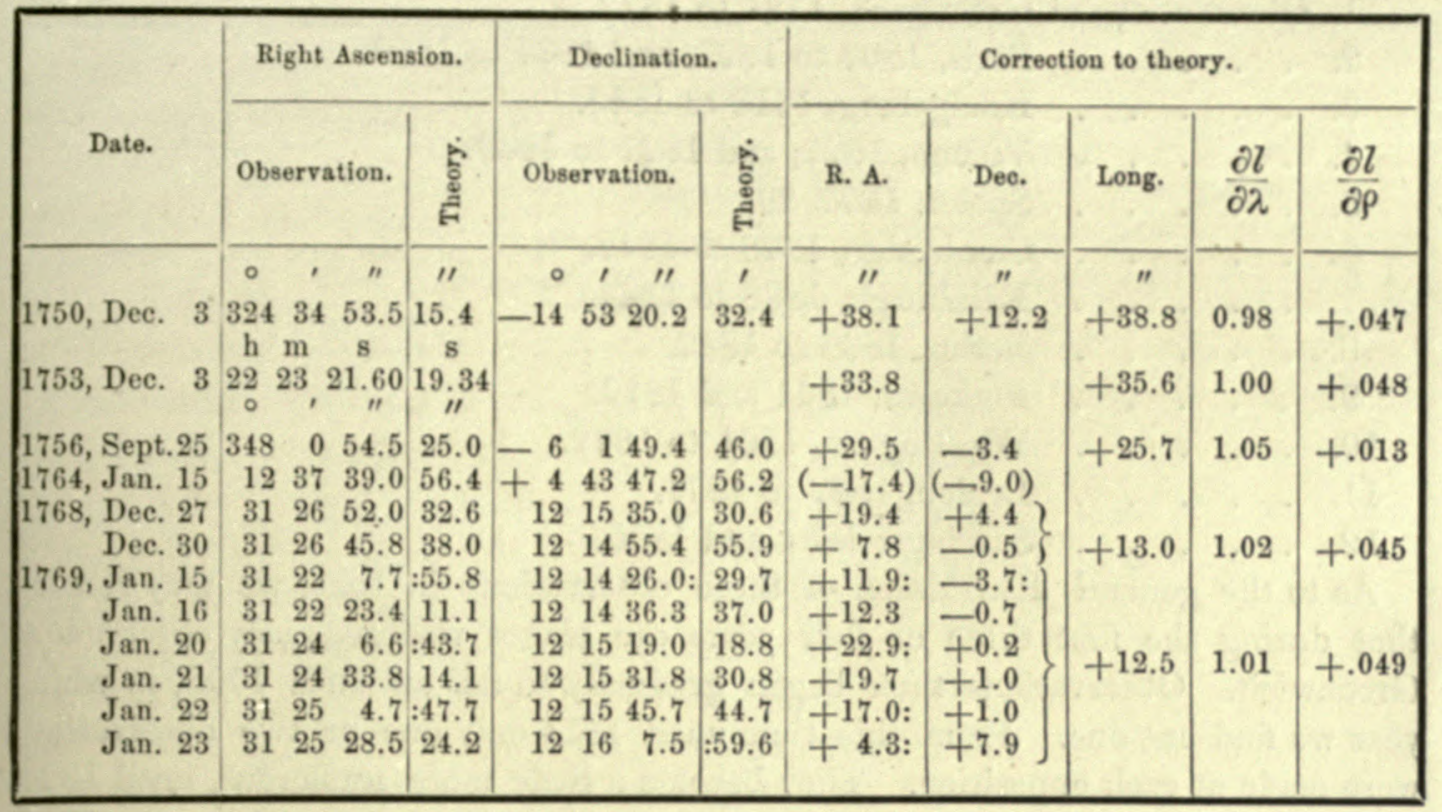

Where no declination has been observed the observed corrections in right ascension have been changed to corrections in longitude on the hypothesis that the theoretical latitude is correct. The approximate formula is

$$
\delta l=\frac{\delta \alpha \cos \delta}{\sin E}, \text { where }
$$

$\cos E=\sin \varepsilon \cos \alpha, \varepsilon$ being the obliquity.

\section{DISCUSSION OF THE MODERN OBSERVATIONS.}

\section{Reduction of the Published Results of Observations to a Uniform System.}

We have now to discuss the great mass of observations made at the principal observatories of the world since the discovery of the planet by Herschel, in 1781 . To make all the data of reduction rigorously homogeneous and uniform, it would be necessary to completely re-reduce the greater part of the observations made before 1850 , using the modern values of the constants of reduction, and to compare each observation separately with the geocentric place deduced from the prorisional theory. Such a reduction and comparison would be extremely desirable. Their execution would, however, involve an amount of labor far greater than it is now possible for the author to bestow upon the problem. We must, therefore, adopt the reductions which have been already made, applying such systematic corrections for reduction to a uniform system of star places as we have the means readily to determine. No reduced places are employed unless we can find data for some more or less accurate determination of these corrections, a rule which necessitates the rejection of a great mass of observations made at the minor observatories of the European continent, and published in the Astronomischen Nachrichten. We still have the following rich collection of materials at our disposal: 
1. Observations at Greenwich, 1781 to 1872 .

2. . . . Paris, 1802 to 1827 , and 1837 to 1869 .

3. . . . Königsberg, 1813 to 1835 .

4. . . . . Vienna, 1822, and 1827 to 1839.

5. . . . Speier, 1827-29.

6. . . . Cambridge, 1828 to 1842 .

7. . . . Edinburgh, 1836 to 1844 .

8. . . . Berlin, 1838 to 1842 .

9. . . . . Pulkowa, 1841 and 1842.

10. . . . . Washington, 1861 to 1872.

11. . . . . Leiden, 1863 to 1871 .

12. . . Santiago, 1854 and 1855.

As to the general distribution of these observations in time, we may remark that during the first three or four years the planet was zealously observed at Greenwich. Observations then began gradually to fall off until 1798, in which year we find but one. From this time until 1814 only one or two observations were made at each opposition. 'They become a little more numerous, until 1829, when there is a sudden increase. Few interruptions have occurred since. With regard to the other observatories it may be said that from 1802 until 1830 there is a gradual increase in the number of observations, and that since the latter year the number of observations is entirely satisfactory.

A great number of the observations were reduced with the star places of the Tabulce Regiomontanoe, and the entire Paris series are reduced with the star positions of Le Verrier, given in his "Annales de l'Observatoive Imperial de Paris," Tome II. As a preliminary to the discussion of the systematic corrections to the principal published reductions, I have prepared the following table, showing the corrections which must be applied to the places of the equatorial fundamental stars in the above catalogues to reduce them to the adopted standard, namely, Dr. Gould's coast survey list in right ascension, and Auwers' standard in declination.

In the table of right ascensions the first column after name of the star gives the annual variation of that co-ordinate for the epoch 1860.0, as derived from Le Verricr's tables of right ascensions just cited. Next we have the correction to this annual variation, expressed in units of the fourth place of decimals, to reduce it to that given in the "Star Tables of the American Ephemeris," the positions in which are founded on Dr. Gould's Catalogue. The fourth column gives the correction to the right ascensions of Le Verrier for 1860, in hundredths of a second of time. Subtracting from this column sixth-tenths of the preceding, we have the corresponding corrections for 1800 . The last four columns give the corresponding numbers for the right ascensions of the Tabuloe Regiomontance.

The table of declinations shows, for different epochs, the corrections necessary to reduce the tabular positions to those given by Auwers in his paper on the declinations of the fundamental stars 
I. Right Ascensions.

\begin{tabular}{|c|c|c|c|c|c|c|c|c|}
\hline \multirow{2}{*}{ Date. } & \multirow{2}{*}{$\begin{array}{c}\text { Le Verrier's } \\
\text { ann. var. } \\
1860 .\end{array}$} & \multicolumn{3}{|c|}{ Corrections to } & \multirow{2}{*}{$\begin{array}{c}\text { Ann. var. of } \\
\text { Tab. Reg. } \\
1860 \text {. }\end{array}$} & \multicolumn{3}{|c|}{ Corrections to } \\
\hline & & Ann. var. & $\begin{array}{l}\text { R. A. } \\
1860 \text {. }\end{array}$ & $\begin{array}{l}\text { R. A. } \\
1800 \text {. }\end{array}$ & & Ann: var. & $\begin{array}{l}\text { R. A. } \\
1860 \text {. }\end{array}$ & $\begin{array}{l}\text { R. A. } \\
1800 \text {. }\end{array}$ \\
\hline a Andromedæ, & $\begin{array}{r}8 \\
+3.0844\end{array}$ & +14 & +2 & -6 & ${ }^{8} .0840$ & +18 & +8 & -3 \\
\hline$\gamma$ Pegasi, & 3.0801 & +15 & +3 & -6 & 3.0824 & -8 & -9 & -4 \\
\hline a Arietis, & 3.3644 & +12 & 0 & -7 & 3.3636 & +20 & +6 & -6 \\
\hline a Ceti, & 3.1266 & +10 & +5 & -1 & 3.1267 & +9 & +7 & +2 \\
\hline a Tauri, & 3.4346 & -1 & -1 & 0 & 3.4335 & +10 & +7 & +1 \\
\hline$\beta$ Orionis, & 2.8797 & +6 & +2 & -2 & 2.8800 & +3 & +5 & +3 \\
\hline$\beta$ Tauri, & 3.7871 & -4 & +1 & +3 & 3.7888 & -21 & -4 & +9 \\
\hline a Orionis, & 3.2460 & +5 & +4 & +1 & 3.2464 & +1 & +3 & +2 \\
\hline a Geminorum, & 3.8409 & - 2 & 0 & +1 & 3.8386 & +21 & +18 & +5 \\
\hline a Canis Min. & 3.1462 & +5 & +9 & +6 & 3.1455 & +12 & +7 & 0 \\
\hline$\beta$ Geminorum, & 3.6828 & +5 & +3 & 0 & 3.6807 & +26 & +13 & -3 \\
\hline a Hydræ, & 2.9485 & +12 & +6 & -1 & 2.9469 & +28 & +16 & -1 \\
\hline a Leonis, & 3.2030 & +15 & +6 & -3 & 3.2014 & +31 & +13 & -6 \\
\hline$\beta$ Leonis, & 3.0654 & +11 & +4 & -3 & 3.0640 & +25 & +12 & -3 \\
\hline a Virginis, & 3.1495 & +20 & +5 & -7 & 3.1497 & +18 & +2 & -9 \\
\hline a Bootis, & 2.7325 & +16 & +3 & -7 & 2.7327 & +14 & +5 & -3 \\
\hline$a^{2}$ Libræ, & 3.3044 & +6 & +3 & -1 & 3.3074 & -24 & -6 & +8 \\
\hline a Coronæ, & 2.5378 & +12 & +1 & -6 & 2.5373 & +17 & +5 & -5 \\
\hline a Serpentis, & 2.9488 & +8 & +4 & -1 & 2.9513 & -17 & -7 & +3 \\
\hline a Scorpii, & 3.6654 & +16 & +4 & -6 & 3.6672 & -2 & -4 & -3 \\
\hline a Herculis, & 2.7322 & +7 & +2 & -2 & 2.7319 & +9 & 0 & -5 \\
\hline a Ophiuchi, & 2.7808 & +10 & 0 & -6 & 2.7783 & +34 & +15 & -5 \\
\hline a Lyræ, & 2.0312 & +2 & -1 & -2 & 2.0305 & +9 & +1 & -4 \\
\hline$\gamma$ Aquilæ, & 2.8520 & +9 & +2 & -3 & 2.8546 & -17 & -8 & +2 \\
\hline a Aquilæ, & 2.9281 & +4 & +1 & -1 & 2.9281 & +4 & -3 & -5 \\
\hline$\beta$ Aquilæ, & 2.9466 & +5 & +3 & 0 & 2.9496 & -25 & -12 & +3 \\
\hline$a^{2}$ Capriconii, & 3.3338 & +4 & +2 & 0 & 3.3349 & -7 & -6 & -2 \\
\hline a Aquarii, & 3.0829 & +13 & +4 & -4 & 3.0822 & +20 & +4 & -8 \\
\hline a Piscis Aust. & 3.3311 & +8 & +4 & -1 & 3.3326 & -7 & -16 & -12 \\
\hline a Pegasi, & 2.9828 & +11 & 0 & -7 & 2.9830 & +9 & -1 & -6 \\
\hline Sum, & & +254 & +81 & -72 & & +210 & +71 & -55 \\
\hline Mean, & & +8.5 & +.027 & -.024 & & +7.0 & +.024 & -.018 \\
\hline
\end{tabular}




\section{Dechivations.}

\begin{tabular}{|c|c|c|c|c|c|c|}
\hline & \multicolumn{4}{|c|}{ Corrections to Tabulæ Regiomontanæ. } & \multicolumn{2}{|c|}{ Corrections to Le Verrier. } \\
\hline & 1780. & 1800. & 1820. & 1840. & 1820. & 1840. \\
\hline & $\prime \prime$ & $" \prime$ & $\prime \prime$ & "I & " & " \\
\hline a Andromedx, & +0.3 & +0.2 & +0.1 & 0.0 & +0.2 & 0.0 \\
\hline$\gamma$ Pegasi, & -0.2 & +0.1 & +0.4 & +0.8 & +0.1 & +0.3 \\
\hline a Arietis, & -0.3 & 0.0 & +0.3 & +0.5 & +0.2 & +0.3 \\
\hline a Ceti, & -1.0 & -0.1 & +0.7 & +1.6 & +0.1 & +0.7 \\
\hline a Tauri, & 0.0 & +0.1 & +0.1 & +0.2 & +0.1 & 0.0 \\
\hline$\beta$ Orionis, & -0.5 & +0.1 & +0.6 & +1.2 & +0.3 & +0.7 \\
\hline $\beta$ Tauri, & -0.5 & 0.0 & +0.6 & +1.1 & +0.2 & +0.5 \\
\hline a Orionis, & -1.2 & -0.6 & 0.0 & +0.6 & -0.2 & +0.3 \\
\hline a Geminornm, & -0.8 & -0.5 & -0.1 & +0.3 & +0.5 & +1.0 \\
\hline a Canis Min. & -0.2 & 0.0 & +0.3 & +0.6 & +0.2 & +0.5 \\
\hline B Geminorum, & -0.2 & 0.0 & +0.3 & +0.6 & +0.2 & +0.5 \\
\hline a Hydræ, & -0.2 & +0.3 & +0.7 & +1.2 & +0.3 & +0.7 \\
\hline a Leonis, & +0.3 & +0.4 & +0.4 & +0.5 & +0.4 & +0.5 \\
\hline 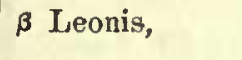 & +0.2 & +0.2 & +0.2 & +0.3 & +0.2 & +0.3 \\
\hline a Virginis, & +0.2 & +0.6 & +1.1 & +1.5 & +0.5 & +0.8 \\
\hline a Bootis, & +0.4 & +0.4 & +0.3 & +0.2 & +0.4 & +0.4 \\
\hline$a^{2}$ Libræ, & +0.5 & +0.5 & +0.6 & +0.6 & +0.5 & +0.6 \\
\hline a Coronæ, & +0.7 & +0.6 & +0.4 & +0.3 & +0.4 & +0.4 \\
\hline a Serpentis, & +0.2 & +0.6 & +1.1 & +1.5 & +0.5 & +0.9 \\
\hline a Scorpii, & +0.1 & +0.6 & +1.0 & +1.4 & +0.4 & +0.7 \\
\hline a Herculis, & +0.6 & +0.8 & +1.0 & +1.3 & +0.5 & +0.7 \\
\hline a Ophinchi, & +0.4 & +0.5 & +0.5 & +0.6 & +0.4 & +0.6 \\
\hline a Lyre, & +0.7 & +0.8 & +0.9 & +1.0 & +0.4 & +0.4 \\
\hline$\gamma$ Aquilæ, & 0.0 & +0.3 & +0.6 & +1.0 & +0.2 & +0.4 \\
\hline a Aquilæ, & +0.1 & +0.4 & +0.7 & +1.0 & +0.1 & +0.4 \\
\hline B Aquilæ, & +0.1 & +0.6 & +1.2 & +1.7 & +0.5 & +0.8 \\
\hline$a^{2}$ Capriconii, & +0.2 & +0.9 & +1.6 & +2.3 & +0.6 & +1.1 \\
\hline a Aquarii, & -0.5 & +0.1 & +0.8 & +1.5 & +0.5 & +1.0 \\
\hline a Piscis Aust. & +1.0 & +2.4 & +3.9 & +5.3 & +1.7 & +2.3 \\
\hline à Pegasi, & +0.4 & +0.3 & +0.2 & +0.1 & +0.3 & +0.2 \\
\hline Mean & +0.03 & +0.36 & +0.69 & +1.03 & +0.36 & +0.60 \\
\hline
\end{tabular}


The correction to the reductions to apparent place given in the Tabulæ Regiomontanx on account of the correction to the constant of Nutation is ; -

In right ascension:

$-0^{\prime \prime} .46 \sin 8-0^{\prime \prime} .18 \sin 8 \sin \alpha \tan \delta-0^{\prime \prime} .24 \sin 8 \cos \alpha \tan \delta$.

In declination:

$-0^{\prime \prime} 18 \sin 8 \cos \alpha+0.24 \cos 8 \sin \alpha$.

The terms which contain $\tan \delta$ as a factor may be entirely neglected, as they are small, periodic, and contain $\tan \delta$ as a factor which is sometimes positive and sometimes negative. I shall also neglect the corrections in declination, as their sum is sensibly

$$
0^{\prime \prime} .21 \sin (\alpha-8)
$$

the effect of which will generally be confounded with the accidental errors of observation.

The only correction we shall apply on account of nutation is, therefore,

$$
\delta \alpha=-0^{*} .030 \sin \Omega \text {. }
$$

The values of this expression at the dates when it is zero, a maximum, or a minimum, are as follows:-

\begin{tabular}{rrrr} 
y. & \multicolumn{1}{c}{ s. } & \multicolumn{1}{c}{ y. } & \multicolumn{1}{c}{ s. } \\
1778.5 & -.03 & 1820.3 & .00 \\
1783.1 & .00 & 1825.0 & +.03 \\
1787.7 & +.03 & 1829.6 & .00 \\
1792.4 & .00 & 1834.3 & -.03 \\
1797.0 & -.03 & 1838.9 & .00 \\
1801.7 & .00 & 1843.6 & +.03 \\
1806.3 & +.03 & 1848.2 & .00 \\
1811.0 & .00 & 1852.9 & -.03 \\
1815.6 & -.03 & 1857.5 & .00 \\
1820.3 & .00 & &
\end{tabular}

Having adopted this system of standard positions, we may adopt two ways of reducing the observations to it. One is to compare the positions of the stars adopted in the published reductions with the standard, and apply the mean difference to the reduced place of the planet. Another is to make a similar comparison of the standard catalogue with the positions of the fundamental stars which have been deduced from the observations by a system of reduction uniform with that employed in reducing the observations of the planet, and to regard the mean difference as a correction applicable to all the positions of the planet. If the standard catalogue and the observations are both free from systematic error, the results obtained in these two ways should be substantially identical. These are, however, conditions which we cannot expect to find fulfilled. In the following discussions I have sometimes used one, sometimes the other, and sometimes 
combined both, the choice being determined by circumstances. We shall consider the different scries of observations in succession.

\section{Greenwich Observations from 1781 to 1830.}

These observations are completely reduced by Airy and compared with Bouvard's Tables, in the work Reduction of the Observations of Planets made at the Royal Observatory, Gieenwich, from 1750 to 1830. London, 1845. The concluded positions given in this work depend mainly on the star places of the Tabulæ Regiomontanæ, both in right ascension and declination. If we consider the first four oppositions-1781-1785-as forming a single group of which the mean epoch is 1783, we find that the general correction to the Tabulæ Regiomontanæ for this epoch is

$$
\begin{aligned}
& \text { In right ascension, }-0^{\circ} .030 ; \\
& \text { In declination, } \quad+0^{\prime \prime} .08 \text {. }
\end{aligned}
$$

If, on the other hand, we consider only the particular stars compared with Uranus, the result will be a little different. 'The number of times each of the fundamental stars has been compared with Uranus, and the correction in right ascension corresponding to each star, are nearly as follows:-

$\begin{array}{lrrr}\alpha \text { Arietis, } \quad N=2 & \text { Cor. }=-0.09 & N \times C=-.18 \\ \alpha \text { Tauri, } & 2 & -.01 & -.02 \\ \gamma \text { Pegasi, } & 2 & -.03 & -.06 \\ \beta \text { Tauri, } & 19 & +.13 & +2.47 \\ \alpha \text { Orionis, } & 33 & +.02 & +0.66 \\ \alpha \text { Canis Minoris, } & 33 & -.02 & -0.66 \\ \beta \text { Geminorum, } & 34 & -.07 & -2.38 \\ \alpha \text { Leonis, } & 7 & -.11 & -.77 \\ \beta \text { Leonis, } & 2 & -.07 & -.14\end{array}$

'The mean correction from these data comes out $-0^{\circ} .008$, differing by $0^{\circ} .022$ from the general mean correction. Our choice between the two corrections depends on whether we are to consider the relative positions of the 'Tabulæ Regiomontanæ, or those of the standard catalogue, as nearest the truth at the epoch 1783, and particularly upon whether we are to consider the large correction to the proper motion of $\beta$ Tauri as real. In the absence of exact data for settling this question, the mean of the two results, or -09.020 , has been adopted.

A similar anomaly is exhibited by the declinations. It is probable that the declinations of Uranus during this period mainly depend on stars in the first twelve hours in right ascension, for which the mean correction is about $-0 " .30$ instead of $+0^{\prime \prime} .08$. I have adopted $-0 " .16$. Changing these corrections to longitude and latitude, we have, during the period 1781-1786:-

Correction to observed longitude, $=-0^{\prime \prime} .30$;

Correction to observed latitude, $\quad-0.19$. 
During the years 1788-1798 the above systematic difference in right ascension does not appear. The most probable correction seems to be
Whence

$$
\Delta \alpha=-0.025
$$$$
\Delta \text { long. }=-0^{\prime \prime} .34 \text {; }
$$$$
\Delta \delta=0 " .00 \text {. }
$$$$
\Delta \text { lat. }=-0^{\prime \prime} .10 \text {. }
$$

Between the years 1800 and 1823 the stars used for comparison are so widely scattered that I consider it safe to apply only the general mean correction for the epoch 1813 , which is

$$
\begin{array}{rrrr} 
& \Delta \alpha=-{ }^{\circ} .005 ; & \Delta \delta & =+0^{\prime \prime} .66 . \\
\text { Whence } & \Delta \text { long. }=0^{\prime \prime} .00 ; & \Delta \text { lat. }=+0.66 .
\end{array}
$$

From 1825 to 1830 more than half the weight of the right ascension comes upon the stars $\alpha, \beta$, and $\gamma$ Aquilæ, the mean correction to which, during this interval, is $-0^{*} .035$. The general mean correction at this epoch is $+0^{*} .002$. I think the right ascensions of these three stars in the Tabulæ Regiomontanæ are really too great at this epoch by the entire difference of these results. We may, in fact, hereafter regard the positions of the standard catalogue as sufficiently accurate. The mean corrections to be applied will then be

$$
\begin{aligned}
\Delta a & =-0^{\circ} .017 ; & \Delta \delta & =+0^{\prime \prime} .83 . \\
\text { Whence } & \Delta \text { long. } & =-0^{\prime \prime} .05 ; & \Delta \text { lat. }=+0^{\prime \prime} .86 .
\end{aligned}
$$

From the year 1831 until the present time the Greenwich obscrvations are regularly reduced in the several annual volumes of observations. But a reduction of the observations from 1831 to 1835 , executed by $\mathrm{Mr}$. Hugh Breen, is given in an appendix to the volume for the year 1864. The results here given differ from those published by Pond in the several annual volumes for the same interval. The right ascensions are altered only by applying the constant correction $-0^{*} .030$, which is found necessary to reduce Pond's right ascensions to those of the Tabulæ Regiomontanæ. This correction I have verified. The mean correction to reduce the right ascensions of the Tabulæ Regiomontanæ to our standard is at this time $+0^{*} .005$. On the other hand, when we compare the concluded right ascensions of stars within six hours of Uranus, as given by Pond in the Greenwich observations for 1834 , with our standard, we find a mean correction of -.034 to reduce his positions to the standard, which implies a correction - -.004 to Breen's reduction. The two results being $+^{*} .005$ and $-{ }^{*} .004$, I have applied no correction whatever.

In the paper in question the declinations are completely re-reduced, using improved data of reduction, but, so far as I see, making no changes in Pond's method. The results differ strikingly from those of Pond, and suggest the desirableness of a complete re-examination of all Pond's determinations of declination. Having no catalogue of observed declinations of standard stars reduced in this same way, we cannot directly determine the systematic correction to the declinations. I therefore proceed as follows: A comparison of Pond's observed declinations of standard stars with Auwers' normal catalogue show that the former require the following corrections near the parallel of Uranus: 


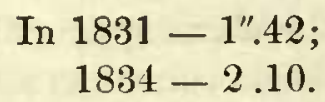

Then comparing Airy's reduced declinations of Uranus with Pond's, we find the following mean differences:

$$
\begin{aligned}
& \text { In 1831, Airy - Pond }=-3 " .18 \\
& \text { 1834, }-3.50 \text {. }
\end{aligned}
$$

To reduce Airy to Auvers we must there apply to the declinatious

$$
\text { In } \begin{array}{r}
1831+1^{\prime \prime} .76 \\
1834+1.40 .
\end{array}
$$

I have regarded the correction $+1^{\prime \prime} .60$ as applicable throughout the period in question.

$$
1836-72 .
$$

During this interval the corrections in right ascension have been derived by the following two sets of comparisons: (1) A comparison of the several collected six and seven year catalogues with Gould's standard, from which it appears that they require the following general corrections in right ascension :

$$
\begin{array}{ll}
\text { Six year catalogue of } 1840 & +0^{\circ} .047 \\
\text { Six year catalogue of } 1845 & +0.002 \\
\text { Seven year catalogue of } 1860 & +0.003 \\
\text { Seren year catalogue of } 1864 & +0.022
\end{array}
$$

(2) A comparison of the corrections applied to the right ascensions of the individual years to reduce them to the standard of the catalogue, as given in the introduction to each catalogue: The sum of these two numbers gives the corrections for each year.

A slightly different method is to regard the above correction for each catalogue as applicable to all right ascensions which depend fundamentally upon that catalogne. I have sometimes combined both methods so as to derive what secmed to be the most probable result, and sometimes used but one.

The corrections to the declinations during the interval in question have been derived from Auwers' "Tafeln zur Reduction der Declinationen verschiedener Sternverzeichnisse auf ein Fundamentılsystem," Astronomische Nachrichten, No. 1536. 'These tables include the Greenwich scven year catalogue for 1860, when the correction corresponding to the declination of Urauns is about $+0^{\prime \prime} .45$. The corrections for the previous catalogues vary between $0^{\prime \prime} .35$ and $0^{\prime \prime} .68$. The correction corresponding to the interval 1861-67 has been derived by a direct comparison with Auwers' declinations, and the result is $+0^{\prime \prime} .44$, agreeing with the two preceding catalogues. But, on making a similar comparison with the annual catalogue for 1869 , a considerable change was found, the correction being $-0 " .17$, a change of more than half a second. I shall use this correction for and after the beginning of 1868 , as the change is probably due to the introduction of a new constant of refraction in the reduction of the observations for 1868 and subsequent years. 


\section{Cambridge.}

An extended series of planetary observations was commenced here by Professor Airy, in 1827. The series was continued by him and Professor Challis, his successor, until 1842. During the first three or four years the combined right ascensions depend on a few special stars, and mainly on $\alpha^{2}$ Capricorni. Taking the mean correction to the adopted right ascensions of the stars actually compared as they are given in the introduction to each annual volume, giving to each star a weight proportional to the number of comparisons, the following corrections are deduced:

$$
\begin{array}{ll}
1828 & -0.10 \\
1829-31 & -0.16 \\
1832-37 & -0.19
\end{array}
$$

In the introduction to the volume for 1838 it is stated that the adopted right ascensions are diminished by the average amount of $0^{*} .083$, which would still leave a correction of $-0^{*} .107$. Actual comparisons in two subsequent years give

$$
\begin{aligned}
& 1840, \Delta \alpha=-0^{s} .087 \\
& 1842, \quad-0.069 .
\end{aligned}
$$

Although the positions deduced from each year's work were adopted for clock correction the year following, without any change of equinox, it seems that there was, effectively, a progressive change of about $0^{*} .01$ annually in the equinox as adopted.

No declinations were observed until 1830. On comparing the declinations deduced from several years' work with Auwers, it was evident that the correction increased with the polar distance of the star. The law of increase could be well enough represented by supposing the correction proportional to N.P.D. Thus the following corrections were deduced in three different years.

$$
\begin{array}{ll}
1834, \delta \text { dec. }= & -\frac{1^{\prime \prime} .78 \times \text { N.P.D. in degrees }}{100} \\
1840, & -\frac{1^{\prime \prime} .00 \times \text { N.P.D. in degrees }}{100} \\
1842, & -\frac{1^{\prime \prime} .03 \times \text { N.P.D. in degrees }}{100} .
\end{array}
$$

From which the correction for other years was deduced by interpolation. But, on applying these corrections, the results were found systematically different from those of other observatories, and on referring to Auwers' corrections to Airy's Cambridge Catalogue, it appeared that the mural circle required a large correction near the declination of Uranus during this period. The above results were therefore altered so as to conform as nearly as practicable to Auwers' law.

\section{Eilinburgh.}

In reducing the observations of 1836 Henderson uses the right ascensions of the Tabulæ Regiomontanæ, to which the general correction is at this epoch $+^{5} .007$. 
But, if we take only the stars near Uranus, with which the latter was necessarily most frequently compared, the corrections will be negative. Comparing the concluded positions of the stars from $\alpha$ Serpentis through $0^{\text {h }}$ to $\beta$ Orionis, we find the following mean corrections :

In right ascension, $-0^{\circ} .012$; in declination, $-0^{\prime \prime} .09$.

In subsequent years it is stated that the adopted positions of clock stars used each year are derived from the right ascensions observed at Greenwich, Cambridge, and Edinburgh, during the year or the two years preceding, without ally statement whether corrections were applied for difference of equinoxes. In some subsequent years the following corrections are deduced, sometimes from the adopted and sometimes from the concluded positions:

$$
\begin{aligned}
& \text { 1837, } \Delta \alpha=0.000 ; \\
& 1840, \Delta a=+0.015 ; \Delta \text { Dec. }=0^{\prime \prime} .00 ; \\
& 1844, \Delta \alpha=+0.070 ; \Delta \text { Dec. }=+0.49
\end{aligned}
$$

Paris.

All the positions of planets given by Le Verrier, in his "Annales de l'Observatoire Imperial de Paris: Observations"depend both in right ascension and N. P. D. on his adopted positions of fundamental stars, the corrections to which have already been given. As the corrections to the individual star places used by Le Verrier are not generally of a systematic character, the general mean correction is employed, which is:-

$$
\begin{aligned}
& \text { In right ascension }-0^{\circ} .024+0^{\circ} .085 T, \\
& \text { In declination }+0^{\prime \prime} .12+1^{\prime \prime} .20 T,
\end{aligned}
$$

$T$ being the fraction of a century after 1800 .

\begin{tabular}{|c|c|c|c|c|c|c|c|c|c|c|c|c|}
\hline \multirow{3}{*}{$\begin{array}{l}\text { Year. } \\
1830\end{array}$} & \multicolumn{2}{|c|}{ Greeuwich. } & \multicolumn{2}{|c|}{ Paris. } & \multicolumn{2}{|c|}{ Königsberg. } & \multicolumn{2}{|c|}{ Berlin. } & \multicolumn{2}{|c|}{ Cambridge. } & \multicolumn{2}{|c|}{ Edinburgh. } \\
\hline & $\begin{array}{c}\Delta a \\
\mathrm{~s}\end{array}$ & $\Delta_{1 \prime} \delta$ & $\begin{array}{c}\Delta a \\
\mathrm{~s}\end{array}$ & $\underset{\|}{\Delta \delta}$ & $\begin{array}{c}\Delta \alpha \\
\mathbf{s}\end{array}$ & ${ }_{\prime \prime}^{\prime \prime}$ & $\frac{\Delta a}{s}$ & $\Delta_{\prime \prime}$ & $\begin{array}{c}\Delta a \\
s\end{array}$ & ${ }_{\prime \prime} \delta$ & $\frac{\Delta \alpha}{\mathrm{s}}$ & ${ }_{11}^{\Delta \delta}$ \\
\hline & $\ldots$ & . & $\ldots$ & & .00 & & $\ldots$ & +0.9 & -.16 & -1.7 & .00 & $\ldots$ \\
\hline 1831 & .00 & +1.8 & .00 & +0.5 & -.02 & +0.9 & & +0.9 & -.16 & -1.6 & $\ldots$ & $\ldots$ \\
\hline 1832 & . . & +1.7 & $\ldots$ & $\ldots$ & -.02 & +0.9 & & $\ldots$ & -.19 & -1.5 & $\ldots$ & $\ldots$ \\
\hline 1833 & $\ldots$ & +1.6 & $\ldots$ & $\ldots$ & $\ldots$ & $\ldots$ & -.04 & ... & $\ldots$ & -1.4 & $\ldots$ & $\ldots$ \\
\hline 1834 & $\ldots$ & +1.4 & $\ldots$ & $\ldots$ & $\ldots$ & $\ldots$ & -.05 & $\ldots$ & $\ldots$ & -1.3 & $\ldots$ & $\ldots$ \\
\hline 1835 & $\cdots$ & +1.2 & $\ldots$ & $\ldots$ & $\ldots$ & $\ldots$ & -.05 & .. & $\ldots$ & -1.2 & $\cdots$ & $\cdots$ \\
\hline 1836 & -.04 & +1.0 & +.01 & +0.6 & -.02 & +1.0 & -.04 & +1.0 & $\ldots$ & -1.0 & -.01 & 0 \\
\hline 1837 & -.03 & +0.9 & $\ldots$ & $\ldots$ & & $\ldots$ & -.03 & & $\ldots$ & -0.8 & .00 & 0 \\
\hline 1838 & -.04 & +0.8 & $\ldots$ & $\ldots$ & $\ldots$ & & -.02 & $\ldots$ & -.11 & -0.5 & +.01 & 0 \\
\hline 1839 & $\ldots$ & +0.7 & ... & $\ldots$ & $\ldots$ & +1.0 & -.01 & $\ldots$ & -.10 & -0.3 & +.01 & 0 \\
\hline 1840 & . & +0.6 & ... & .. & $\ldots$ & $\ldots$ & .00 & $\ldots$ & -.09 & -0.2 & +.02 & +0.1 \\
\hline 1841 & & +0.5 & & . & & $\varepsilon$ & +.01 & & -.08 & -0.2 & -.04 & +0.2 \\
\hline
\end{tabular}

In 1854 a new and larger catalogue was introduced, and for this and the following years the correction in declination is derived from Auwers' tables.

A summary of the adopted corrections after 1830, as deduced from the preceding comparisons and discussions, is given in the following table:- 


\begin{tabular}{|c|c|c|c|c|c|c|c|c|c|c|c|c|}
\hline \multicolumn{13}{|c|}{ Table of A Dopted Systrematic Corketrons, - Continued. } \\
\hline \multirow[t]{2}{*}{ Year. } & \multicolumn{2}{|c|}{ Greenwieh. } & \multicolumn{2}{|c|}{ Paris. } & \multicolumn{2}{|c|}{ Königsberg. } & \multicolumn{2}{|c|}{ Berlin. } & \multicolumn{2}{|c|}{ Cambridge. } & \multicolumn{2}{|c|}{ Edinburgh. } \\
\hline & $\begin{array}{c}\Delta a \\
8\end{array}$ & $\Delta 8$ & $\begin{array}{c}\Delta a \\
\mathrm{~s}\end{array}$ & $\Delta s$ & $\begin{array}{c}\Delta a \\
g\end{array}$ & $\underset{\|}{\Delta \delta}$ & $\Delta a$ & $\Delta \delta$ & $\begin{array}{c}\Delta a \\
8\end{array}$ & $\Delta \delta$ & $\begin{array}{c}\Delta a \\
8\end{array}$ & $\Delta 8$ \\
\hline 1842 & -.02 & +0.4 & +.01 & +0.6 & & & +.03 & +1.1 & -.07 & -0.3 & -.02 & +0.3 \\
\hline 1843 & +.07 & +0.4 & +.01 & +0.6 & & $\ldots$ & +.04 & +1.1 & $\ldots$ & $\ldots$ & +.07 & +0.4 \\
\hline 1844 & +.06 & +0.4 & +.01 & +0.6 & & $\ldots$ & +.04 & +1.1 & $\ldots$ & $\ldots$ & +.07 & +0.5 \\
\hline 1845 & +.08 & +0.4 & +.01 & +0.6 & $\ldots$ & $\cdots$ & +.04 & +1.1 & $\ldots$ & $\ldots$ & & \\
\hline 1846 & +.04 & +0.4 & +.02 & +0.6 & $\ldots$ & $\cdots$ & +.03 & +1.1 & $\cdots$ & $\cdots$ & & \\
\hline 1847 & +.05 & +0.4 & +.02 & +06 & $\ldots$ & $\cdots$ & +.02 & +1.1 & $\cdots$ & $\ldots$ & & \\
\hline $\begin{array}{r}1848 \\
1840-53\end{array}$ & +.05 & +0.4 & +.02 & +0.6 & $\cdots \cdots$ & $\cdots$ & +.01 & +1.2 & $\cdots$ & $\cdots$ & & \\
\hline $1849-53$ & .00 & +0.4 & +.02 & +0.6 & * & $\cdots$ & .00 & +1.2 & Sant & iago & & \\
\hline $1854-55$ & .00 & +0.5 & +.02 & +0.2 & & & -.01 & +1.2 & .00 & +0.6 & \multirow{2}{*}{\multicolumn{2}{|c|}{ Washington }} \\
\hline $\begin{array}{c}1856-60 \\
1861\end{array}$ & -.01 & +0.5 & +.02 & +0.2 & $\cdots \cdots$ & $\cdots$ & $\cdots$ & $\cdots$ & $\ldots$ & $\cdots$ & & \\
\hline $\begin{array}{c}1861 \\
1862-65\end{array}$ & -.01 & +0.4 & +.03 & +0.2 & $\cdots$ & $\cdots$ & $\cdots$ & $\cdots$ & $\ldots$ & $\cdots$ & & \\
\hline$\left|\begin{array}{c}1862-65 \\
1866\end{array}\right|$ & .00 & +0.4 & +.03 & +0.2 & & $\cdots$ & $\cdots$ & $\ldots$ & $\cdots$ & $\ldots$ & .00 & -0.5 \\
\hline $\begin{array}{l}1866 \\
1867\end{array}$ & .00 & +0.4 & +.03 & $\begin{array}{l}+0.2 \\
+0.9\end{array}$ & & : & $\cdots$ & $\cdots$ & & $\ldots$ & .00 & +1.1 \\
\hline 1868 & .00 & $\begin{array}{r}+0.4 \\
-0.2\end{array}$ & $\begin{array}{r}+.05 \\
+.03\end{array}$ & $\begin{array}{r}+0.2 \\
+0.2\end{array}$ & & $\cdots$ & $\cdots$ & & . & & .00 & $\begin{array}{l}+1.1 \\
+1.2\end{array}$ \\
\hline 1869 & .00 & -0.2 & +.03 & +0.2 & . & $\ldots$ & $\ldots$ & & & & .00 & $\begin{array}{r}+1.2 \\
+0.6\end{array}$ \\
\hline 1870 & +.01 & -0.2 & $\ldots$ & $\ldots$ & . & $\ldots$ & $\ldots$ & & & & .00 & +0.4 \\
\hline 1871 & +.01 & -0.2 & $\ldots$ & $\ldots$ & & $\ldots$ & $\cdots$ & $\ldots$ & ... & $\ldots$ & .00 & +0.4 \\
\hline 1872 & +.02 & -0.2 & $\ldots$ & $\ldots$ & & $\cdots$ & $\ldots$ & $\ldots$ & ... & $\ldots$ & .00 & +0.4 \\
\hline
\end{tabular}

Applying the preceding corrections to the positions of the planet as originally reduced and published, we have a series of observed positions as nearly homogeneous as it is possible to make them with the means now at our command. The next step in order will be the computation of the geocentric place of the planet from the provisional theory for the moment of every observation, to be compared with the results of the latter. The complete execution of this labor, ab initio, is, however, at present impracticable, and it is proposed to diminish it by making use of the published comparisons with the older tables. This can be done without danger of serious error, and with all the more ease that owing to the great distance of Uranus the errors of the solar tables are, for the most part, without appreciable effect upon the computed geocentric place of the planet. The method of making the comparison is different with different series of observations, and each series must therefore be described and discussed separately. The general plan, however, has been to replace observed and computed absolute positions by observed and computed corrections to the geocentric positions deduced from Bouvard's Tables. To carry out this plan it is necessary to have at our disposal an ephemeris both of the heliocentric and geocentric positions derived from these tables. The corrections to the latter given by the observations are then given by direct comparison. To obtain the corrections given by the provisional theory, the heliocentric longitudes, latitudes, and radii vectores given by that theory are interpolated to the dates of the heliocentric ephemeris from Bouvard's Tables, and compared with that ephemeris. The differences are then changed to differences of geocentric place by the usual differential formulæ, and thus the corrections given by theory are derived. The difference between the two sets of corrections is the difference 16 May, 1873. 
between the provisional theory and observation. A condensed summary of the results for each of the principal series of observations is lere presented.

\section{Greenwich, 1781-1830.}

In Airy's reductions, already referred to, we have given for the moment of each individual observation a heliocentric place computed from Bonvard's 'Tables, and the geocentric longitudes and latitudes thence deduced. 'The observed right ascensions and declinations are then changed to longitudes and latitudes, and the apparent error of the tables thence dednced. The means of these errors are taken for groups of observations, and expressed in terms of the errors of heliocentric longitude, raclius vector, and latitude. The mode in which these means have been treated is fully shown in the following table. 'The first column gives the mean date of each individual group of observations. The next three give the mean excesses of the co-ordinates interpolated from the heliocentric ephemeris, p. 100, and corrected for solar nutation, over those printed in the "Computations of tabular place, etc.," in the Greenwich reductions. In the fifth column these corrections are changed to corrections of geocentric longitude. In the next two columns we have the mean corrections to Bouvard's geocentric places given by observation. It is the negative of the mean error of tabular place printed in the "Reductions," corrected by the numbers already given to reduce the star places to a uniform system. 'I'hen we have the difference between these two sets of corrections, or, the mean correction to the geocentric place of the provisional theory as given by observation. Lastly, we have the differential coefficients for expressing the errors of geocentric in terms of the errors of heliocentric co-ordinates taken without change from the Greenwich volume.

\begin{tabular}{|c|c|c|c|c|c|c|c|c|c|c|c|}
\hline \multirow{3}{*}{ Mean Date. } & \multicolumn{4}{|c|}{ From Provisional Theory. } & \multicolumn{3}{|c|}{ From Observations. } & \multirow{2}{*}{\multicolumn{2}{|c|}{$\begin{array}{l}\text { Correction to } \\
\text { Prov. Theory. }\end{array}$}} & \multirow{3}{*}{$\frac{\partial l}{\partial \lambda}$} & \multirow{3}{*}{$\frac{\partial l}{\partial \rho}$} \\
\hline & \multicolumn{4}{|c|}{$\begin{array}{l}\text { Correction to tabnlar position in } \\
\text { Greenwich Reductions. }\end{array}$} & \multicolumn{3}{|c|}{ Correctiou to } & & & & \\
\hline & Long. & $\begin{array}{l}\text { Log. } \\
\text { R. } .\end{array}$ & Lat. & $\begin{array}{l}\text { Geoc. } \\
\text { long. }\end{array}$ & $\begin{array}{l}\text { Geoo. } \\
\text { long. }\end{array}$ & $\begin{array}{l}\text { Hel. } \\
\text { lat. }\end{array}$ & $\mid \begin{array}{l}\text { No.of } \\
\text { ous. }\end{array}$ & $\begin{array}{l}\text { Geoc. } \\
\text { long. }\end{array}$ & $\begin{array}{l}\text { Hel. } \\
\text { lat. }\end{array}$ & & \\
\hline & & & $" \prime$ & & 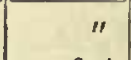 & & & $"$ & & & \\
\hline $\begin{array}{r}1781, \text { Oct. } 10 \\
\text { Nov. } 13\end{array}$ & $\begin{array}{r}-7.8 \\
-7.5\end{array}$ & $\cdots$ & $\begin{array}{l}+1.1 \\
+1.1\end{array}$ & $\begin{array}{l}-7.5 \\
-7.5\end{array}$ & $\begin{array}{l}-6.4 \\
-4.9\end{array}$ & $\begin{array}{l}+2.6 \\
-1.6\end{array}$ & $\begin{array}{l}4 \\
4\end{array}$ & $\begin{array}{l}+1.1 \\
+2.6\end{array}$ & $\begin{array}{l}+1.5 \\
-2.7\end{array}$ & $\begin{array}{l}1.01 \\
1.04\end{array}$ & $\begin{array}{r}-9 \\
-6\end{array}$ \\
\hline Dec. 27 & -7.3 & -159 & $\begin{array}{l}1.0 \\
+1.0\end{array}$ & -7.7 & -5.6 & -2.3 & 5 & $\begin{array}{r}2.1 \\
\end{array}$ & -3.3 & 1.05 & +1 \\
\hline 1782, Jan. 31 & -7.4 & $\ldots$ & $\begin{array}{r}+0.9 \\
+\end{array}$ & -8.0 & -4.9 & -1.1 & 4 & +3.1 & -2.0 & 1.04 & +6 \\
\hline Mar. 4 & -6.6 & $\ldots$ & +0.9 & $-7: 1$ & -4.6 & -0.6 & 4 & +2.5 & -1.5 & 1.01 & +9 \\
\hline $\begin{array}{l}\text { Oct. } 10 \\
\text { Nov. } 26\end{array}$ & -5.6 & $\cdots$ & $\begin{array}{l}+0.4 \\
+0.3\end{array}$ & -5.3 & -3.6 & -4.0 & 4 & +1.7 & -4.4 & 1.01 & -9 \\
\hline $\begin{array}{r}\text { Nov. } 26 \\
1783, \text { Jan. } 11\end{array}$ & $\begin{array}{r}-5.3 \\
-5.0\end{array}$ & $\cdots$ & $\begin{array}{l}+0.3 \\
+0.3\end{array}$ & $\begin{array}{r}-5.3 \\
-5.4\end{array}$ & $\begin{array}{r}-6.0 \\
-3.9\end{array}$ & $\begin{array}{l}-5.0 \\
-3.7\end{array}$ & $\begin{array}{l}3 \\
3\end{array}$ & $\begin{array}{l}-0.7 \\
+1.4\end{array}$ & $\begin{array}{r}-5.3 \\
-4.0\end{array}$ & $\begin{array}{l}1.04 \\
1.05\end{array}$ & $\begin{array}{l}-5 \\
+3\end{array}$ \\
\hline Feb. 24 & -4.4 & & +0.2 & -48 & -2.2 & -2.4 & 3 & $\begin{array}{r}+2.6 \\
+2.6\end{array}$ & -2.6 & 1.02 & +9 \\
\hline Oct. 10 & -3.5 & $\ldots$ & -0.3 & -3.2 & -0.3 & -1.0 & 2 & +2.9 & -0.7 & 1.00 & -10 \\
\hline Nov. 1 & -3.2 & $\ldots$ & -0.3 & -3.0 & -1.1 & -2.3 & 2 & +1.9 & -2.0 & 1.02 & -9 \\
\hline Dec. 15 & -3.3 & -121 & -0.4 & -3.4 & -2.2 & -3.8 & 3 & +1.2 & -3.4 & 1.05 & -3 \\
\hline 1784, Jan. 29 & -3.1 & $\cdots$ & -0.4 & -3.4 & -2.4 & -2.4 & 3 & +1.0 & -2.0 & 1.05 & +5 \\
\hline Mar. 12 & -2.6 & $\cdots$ & -0.5 & -2.9 & -0.3 & -4.3 & 3 & +2.6 & -3.8 & 1.01 & +9 \\
\hline Oct. 30 & -1.8 & $\ldots$ & -0.8 & -1.5 & -0.7 & +1.4 & 2 & $\overline{+0.8}$ & +2.2 & 1.02 & -9 \\
\hline Dec. 14 & -1.5 & -128 & -0.9 & -1.5 & -2.4 & +2.7 & 2 & -0.9 & +3.6 & 1.05 & -4 \\
\hline
\end{tabular}




\begin{tabular}{|c|c|c|c|c|c|c|c|c|c|c|c|}
\hline \multirow{3}{*}{ Mean Date. } & \multicolumn{4}{|c|}{ Frota Provisional Theory. } & \multicolumn{3}{|c|}{ From Observations. } & \multirow{2}{*}{\multicolumn{2}{|c|}{$\begin{array}{l}\text { Correotion to } \\
\text { Prov. Theory. }\end{array}$}} & \multirow{3}{*}{$\frac{\partial l}{\partial \lambda}$} & \multirow{3}{*}{$\frac{\partial l}{\partial \rho}$} \\
\hline & \multicolumn{4}{|c|}{$\begin{array}{l}\text { Correction to tabular position in } \\
\text { Greenwieh observations. }\end{array}$} & \multicolumn{3}{|c|}{ Correetion to } & & & & \\
\hline & Long. & $\begin{array}{l}\text { Log. } \\
\text { R. V. }\end{array}$ & Lat. & $\begin{array}{l}\text { Geoc. } \\
\text { long. }\end{array}$ & $\begin{array}{l}\text { Geoe. } \\
\text { long. }\end{array}$ & $\begin{array}{l}\text { Hel. } \\
\text { lat. }\end{array}$ & $\left|\begin{array}{c}\text { No.of } \\
\text { Obs. }\end{array}\right|$ & $\begin{array}{l}\text { Geoe. } \\
\text { long. }\end{array}$ & $\begin{array}{l}\text { Hel. } \\
\text { lat. }\end{array}$ & & \\
\hline & & & & & & & & & & & \\
\hline 1785, Jan. 16 & -1.2 & & -1.0 & -1.3 & -1.4 & -1.4 & 2 & -0.1 & -0.4 & 1.05 & +2 \\
\hline $\begin{array}{l}\text { Feb. } 13 \\
\text { Mar. } 20\end{array}$ & $\begin{array}{l}-1.0 \\
-0.6\end{array}$ & $\ldots$ & $\begin{array}{l}-1.1 \\
-1.1\end{array}$ & $\begin{array}{l}-1.3 \\
-0.9\end{array}$ & $\begin{array}{l}-1.2 \\
-2.1\end{array}$ & $\begin{array}{l}-1.1 \\
-5.2\end{array}$ & $\begin{array}{l}2 \\
2\end{array}$ & $\begin{array}{l}+0.1 \\
-1.2\end{array}$ & $\begin{array}{r}0.0 \\
-4.1\end{array}$ & $\begin{array}{l}1.04 \\
1.01\end{array}$ & +10 \\
\hline Nov. 8 & +0.2 & & -1.5 & +0.5 & +1.2 & -1.7 & 2 & +0.7 & -0.2 & 1.02 & - \\
\hline $1788, \mathrm{M}$ & +2.1 & -187 & -3.2 & +1.8 & +4.4 & -4.9 & 3 & +2.6 & -1.7 & 1.03 & + \\
\hline Oct. 26 & +3.4 & & -3.6 & +3.9 & +3.0 & -2.2 & 2 & -0.9 & +1.4 & 1.00 & -10 \\
\hline 1789, Jan. 18 & +4.2 & -224 & $\begin{array}{r}-3.7 \\
3.8\end{array}$ & +4.5 & +10 & -14.5 & 1( & $(+5.9)$ & $(-10.8)$ & 1.06 & -1 \\
\hline Apr. 8 & +4.7 & $\cdots$ & -3.8 & +4.2 & +9.4 & -3.7 & 4 & +5.2 & +0.1 & 1.01 & +10 \\
\hline Oet. 31 & +4.8 & $\cdots$ & -4.2 & +5.4 & +4.7 & -1.1 & 2 & -0.7 & +3.1 & 1.00 & -10 \\
\hline 1790 , Jan. 24 & +4.9 & $\cdots \cdots$ & -4.4 & +5.2 & +3.8 & -2.6 & 2 & -1.4 & +1.8 & 1.06 & - \\
\hline Nov. 5 & +5.1 & -254 & -4.8 & +5.8 & +5.0 & -2.3 & 3 & -0.8 & +2.5 & 1.00 & -10 \\
\hline 1791, Jan. 29 & +5.1 & -258 & -5.0 & +5.4 & +4.5 & -3.8 & 3 & - & +1.2 & 1.06 & \\
\hline Apr. 14 & +5.0 & $\cdots$ & -5.2 & +4.5 & +2.7 & -4.8 & 1 & -1.8 & +0.4 & 1.01 & +10 \\
\hline Nov. 10 & +5.0 & -234 & -5.4 & +5.6 & +5.7 & -4.0 & 2 & +0.1 & +1.4 & 1.00 & -10 \\
\hline $1792, \mathrm{~F}$ & +5.2 & & -5.6 & +5.5 & +4.2 & -4.0 & 1 & -1.3 & +1.6 & 1.06 & \\
\hline Nov. 15 & +5.6 & & -6.0 & +6.2 & +3.7 & -2.5 & 3 & -2.5 & +3.5 & 1.00 & -10 \\
\hline 1793, Feb. & +5.4 & . & -6.1 & +5.7 & +8.5 & -5.0 & 2 & +2.8 & +1.1 & 1.06 & \\
\hline Nov. 14 & +5.6 & -135 & -6.4 & +5.9 & +10.4 & -6.3 & 1 & +4.5 & +0.1 & 0.99 & -10 \\
\hline 1794, Feb. 15 & +5.7 & -100 & -6.5 & +6.0 & +7.3 & -6.3 & 2 & 3 & +0.2 & 1.06 & \\
\hline Nov. 19 & +5.4 & $-\quad 67$ & -6.7 & +5.6 & +4.1 & -5.1 & 4 & -1.5 & +1.6 & 1.00 & -10 \\
\hline 1795, Feb. 20 & +5.9 & - 46 & -6.9 & +6.3 & +6.0 & -8.0 & 3 & -0.3 & -1.1 & 1.06 & 0 \\
\hline Nov. 29 & +5.4 & -13 & -7.0 & +5.5 & +3.9 & -3.4 & 2 & -1.6 & +3.6 & 1.00 & -10 \\
\hline 1796, Feb. 24 & +5.3 & - & -7.2 & +5.6 & +4.5 & -4.5 & 2 & -1.1 & +2.7 & 1.06 & \\
\hline 1797, Feb. 27 & +5.1 & +62 & -7.5 & +5.4 & +4.7 & -4.2 & 3 & -0.7 & +3.3 & 1.06 & \\
\hline 1800, Mar. 14 & +4.6 & +224 & -8.4 & +4.9 & +4.9 & -8.7 & 2 & 0.0 & -0.3 & 1.06 & \\
\hline 1814, May 22 & -1.2 & +217 & -2.2 & -1.3 & +1.1 & +0.2 & 2 & +2.4 & +2.4 & 1.06 & \\
\hline 1815, May 25 & -1.1 & +214 & -1.5 & -1.2 & +1.4 & -1.0 & 4 & +2.6 & +0.5 & 1.06 & \\
\hline 1818 , June 10 & -0.5 & +413 & -0.8 & -0.5 & -1.1 & +4.8 & 2 & -0.6 & +5.6 & 1.06 & \\
\hline , June 14 & -0.4 & +483 & +1.5 & -0.4 & -1.4 & +3.7 & 4 & -1.0 & -2.2 & 1.06 & \\
\hline , June 16 & -01 & +498 & +2.2 & -0.1 & -2.6 & +4.0 & 2 & -2.5 & +1.8 & 1.06 & \\
\hline 3, July 1 & 0.0 & +538 & +4.4 & 0.0 & +0.2 & +5.0 & 4 & +0.2 & +0.6 & 1.05 & \\
\hline 1825 , July 11 & -2.4 & +567 & +5.8 & -2.5 & -4.8 & +7.8 & 2 & -2.3 & +2.0 & 1.05 & \\
\hline 1826 , July 16 & -4.5 & $\begin{array}{r}+582 \\
\end{array}$ & +6.6 & -4.7 & -3.6 & +8.3 & 4 & +1.1 & +1.7 & 1.05 & \\
\hline 1827, July 20 & -6.6 & +626 & +7.2 & -6.9 & -7.2 & +9.6 & 6 & -0.3 & +2.4 & 1.05 & \\
\hline 1828, July 23 & -9.8 & +710 & +7.9 & -10.3 & -6.9 & +6.9 & 3 & +3.4 & -1.0 & 1.05 & \\
\hline 9 , Aug. 7 & -13.4 & +835 & +8.5 & -13.7 & -16.3 & +10.0 & 14 & -2.6 & +1.5 & 1.08 & +2 \\
\hline Oet. 4 & -14.2 & +865 & +8.6 & -12.5 & -13.0 & +9.9 & 8 & -0.5 & +1.3 & 1.02 & + \\
\hline 1830 , July 30 & -17.8 & $\begin{array}{r}+970 \\
\end{array}$ & +9.0 & -18.8 & -21.4 & +11.1 & 3 & -2.6 & +2.1 & & \\
\hline A ug. 29 & -18.2 & $\begin{array}{r}+982 \\
\end{array}$ & +9.1 & -17.9 & -20.0 & +10.2 & 5 & -2.1 & +1.1 & & \\
\hline Sept. 20 & -18.5 & +992 & +9.1 & -17.2 & -19.1 & +-9.1 & 12 & -1.9 & 0.0 & & \\
\hline Oct. 14 & -18.9 & +1009 & +9.1 & -16.8 & -17.3 & +9.9 & 11 & -0.5 & +0.8 & & \\
\hline Nov. 13 & -19.3 & +1009 & +9.2 & -16.8 & -17.8 & +9.3 & 8 & -1.0 & +0. & & \\
\hline
\end{tabular}




\section{Paris, 1801-1827.}

A complete reduction of this series is fonnd in Le Verrier's Anuales de l'Olservatoire Imperial de Paris, Observations, tome 1. No comparison with any ephemeris is given here, nor is there any complete ephemeris to compare them with. A complete geocentric ephemeris was therefore computed from the provisional theory for the principal groups of the Paris observations. 'The individual observations being compared with it, the resulting mean corrections are given in the following table :

\begin{tabular}{|c|c|c|c|c|c|c|}
\hline Mean date. & $\alpha$ & $\Delta \delta$ & $N$. & Mean dat & $\Delta \alpha$ & $\Delta \delta$ \\
\hline h 24, & $-{ }^{8} .02$ & $+1^{\prime \prime} .2$ & 2 & 1813, Мay 20, & +.19 & $+1^{\prime \prime} .8$ \\
\hline 302, Apr & +.08 & +0.6 & 13 & 1814, May 27, & +.21 & $\begin{array}{r}+0.8 \\
+\end{array}$ \\
\hline $305, A$ & +.10 & +2.2 & 13 & 1815, May 24, & -.02 & +2.2 \\
\hline $306, A$ & - . & -1.6 & 5 & $1816, \mathrm{~J}$ & - & +0.8 \\
\hline 307, Apr & +.17 & +0.4 & 16 & $1817, J_{1}$ & - . & +1.6 \\
\hline 308, & +.02 & +1.4 & 6 & $1818, \mathrm{~J}$ & + . & +2.2 \\
\hline 309,1 & +.20 & +0.1 & 9 & $1819, \mathrm{~J}$ & -.07 & +1.8 \\
\hline 310, & +.22 & +2.6 & 16 & $1820, \mathrm{~J}$ & -.20 & -2.4 \\
\hline 811,1 & +.21 & +2.2 & 3 & $1821, J$ & +.05 & +1.0 \\
\hline 311, May 17, & +.14 & +2.6 & 8 & $1823, \mathrm{~J}$ & +.02 & +1.8 \\
\hline 312, Febr'y 16 & +.28 & & 2 & 1824 , July 13 , & +.04 & 0.0 \\
\hline $12, \mathrm{M}$ & +.16 & +3.0 & 6 & 1827, July 25, & -.05 & +0.6 \\
\hline 813, & +.44 & & & & & \\
\hline
\end{tabular}

Total number of observations in right ascension, 175 .

The observations in this series exhibit numbers of discordances of that class which leave the astronomer in doubt whether the observation should be retained or rejected. This renark applies more especially to the declinations. If we dctermine the probable error of an observation in declination by the condition that it is that amount which the error falls short of as often as it exceeds, it is found to be about $2^{\prime \prime}$. Then, if the errors followed the commonly assumed law of probability, only about one in six of the errors should exceed 4", and one in twentythree 6". But errors of these magnitudes are much more numerous, the deviations often amounting to six or eight seconds. I have rejected only a few in which the discordances approached $10^{\prime \prime}$.

\section{Bessel's Königsberg Observations, 1814-1835.}

I have made a complete re-reduction of the right ascensions of this important series, and of most of the declinations. In order to avoid the necessity of applying systematic corrections, Dr. Gould's right ascensions and Dr. Auwers' declinations were used throughout in these reductions. In this work a selection of the fundamental stars observed by Bessel was made for each observation of the planet, to be used for clock error. These were chosen so that the mean of their right ascensions and declinations should be as near as practicable to those of Uranus, a condition, however, which could not generally be fulfilled for the declinations, owing to the southern position of the planet. Bessel's instrumental cor- 
rections were applied to his observed times of transit over the mean wire, and the resulting time was employed as that of transit. Each time, compared with the computed right ascension of the star gave a value of the clock correction, which was reduced to the time of transit of the planet by the known daily rate. If the instrumental errors were always accurately determined, the mean of these clock corrections would be used to obtain the right ascension of Uranus. But it was frequently found that the clock error varied systematically with the declination of the star, so that it was deemed advisable to add to the clock correction a term varying as the simple declination, which was deduced from all the stars, and used to reduce the correction to the parallel of Uranus.

It was intended to give the results of this reduction for each observation, but on comparing the results with those of Fleming in the Astronomische Nachrichten, Band 30, it appeared that the results were not materially better than his. It does not, therefore, seem necessary to give more than the mean results for each opposition.

From Bessel's declinations, with the old Cary circle, I was unable to obtain any satisfactory results, owing, apparently, to a want of knowledge of some peculiarity of the instrument. Fleming's reductions were therefore adopted. They are designated by the letter $F$ in the following list.

\section{Mean Corrections to the Provisional. Ephemeris given by Bessel's Observations at} Königsberg, 1814-1829.

\begin{tabular}{cccr|lccr} 
Mean date. & $\Delta \alpha$ & $\Delta \delta$ & $N$. & Mean date. & $\Delta \alpha$ & $\Delta \delta$ & $N$. \\
1814, May 22, & +.11 & $+2^{\prime \prime} .5 F$ & 9 & 1822 , June 24, & +.10 & $+1^{\prime \prime} .8$ & 7 \\
1815, May 25, & +.13 & $+1.8 F$ & 11 & 1823 , July 4, & -.05 & $-1.6 F$ & 2 \\
1816, May 27, & +.06 & $+1.2 F$ & 11 & 1824 , July 6, & +.01 & $-1.0 F$ & 5 \\
1817, June 6, & +.13 & $+2.3 F$ & 8 & 1825 , July 16, & +.01 & $-2.4 F$ & 5 \\
1818, June 8, & +.02 & $+4.3 F$ & 13 & 1826 , July 18, & -.01 & $-3.0 F$ & 7 \\
1820, June 21, & +.02 & +4.1 & 4 & 1828 , July 25, & -.15 & $-3.5 F$ & 7 \\
1821, June 23, & +.12 & +1.5 & 5 & 1829, Aug. 1, & -.10 & $-1.0 F$ & 9
\end{tabular}

Total numbers of observations, 103.

Results of Observations at various Observatories, from 1827 to 1829 inclusive.

During these three years we have, besides the observations already quoted, the following:-

1. Observations by Schwerd, at Speier, of which the originals are given in Astronomische Beobachtungen angestellt auf der Sternwarte des Königl. Lyzeums in Speyer von F. M. Schwerd, Speyer, 1829-30, and of which the reduced results are found in the Astronomische Nachrichten, Band 8, S. 264.

2. The series by Airy, at Cambridge, commenced in 1828 , and found in the Cambridge Observations.

3. Littrow's Vienna Observations, found in the first series of Annalen der $K . K$. Sternwarte in Wien.

The mean corrections to the provisional ephemeris given by these series are shown in the following table. The observations have been divided in the usual 
way into groups of about a month each, and the mean date and mean correction found for each group. The Paris and Königsberg results are repeated for the sake of clearness. The small figures sliow, as usual, the number of observations employed in forming the mean.

\begin{tabular}{|c|c|c|c|c|}
\hline \multirow{5}{*}{ 1827, } & & & $\Delta \alpha$ & $\Delta \delta$ \\
\hline & $\begin{array}{l}\text { Date. } \\
\text { July } 22,\end{array}$ & $\begin{array}{l}\text { Observatory. } \\
\text { Speier, }\end{array}$ & $\begin{array}{c}\text { Original. Corrected. } \\
-0^{\circ} .16_{8}-0^{\circ} .14\end{array}$ & \multirow{4}{*}{$\begin{array}{r}+0^{\prime \prime} .5_{3} \\
0.0_{14} \\
-2.2_{6}\end{array}$} \\
\hline & July 25 & Paris, & $-0.03_{5}-0.05$ & \\
\hline & September 15 , & Vienna, & $-0.11_{11}-0.10$ & \\
\hline & October 14 & Viemna, & $-0.18_{s}-0.17$ & \\
\hline \multirow[t]{8}{*}{1828} & July 25 & Kiönigsberg, & $-0.15_{7}-0.15$ & $-3.5_{7}$ \\
\hline & July 29 & Vienna, & $-0.2 t_{2}-0.20$ & $-1.4_{2}$ \\
\hline & August 14, & Vienna, & $-0.13_{10}-0.09$ & $+1.1_{10}$ \\
\hline & August 27, & Speier, & $-0.10_{8}-0.09$ & \\
\hline & September 18, & Vienna, & $-0.03_{9}+0.01$ & $+1.0_{9}$ \\
\hline & September 25 , & Cambridge, & $-0.05_{0}-0.16$ & \\
\hline & October 17 , & Viemna, & $-0.13_{14}-0.09$ & $0.0_{14}$ \\
\hline & October 17, & Cambridge, & $-0.02_{10}-0.12$ & \\
\hline \multirow[t]{4}{*}{1829} & August 1, & IIönigsberg, & $-0.10_{9}-0.10$ & \multirow{4}{*}{$\begin{array}{l}-1.0 \\
-1.1_{17}\end{array}$} \\
\hline & Augnst 6, & Cambridge, & $+0.11_{s}-0.08$ & \\
\hline & $\begin{array}{l}\text { Angust 28, } \\
\text { Sentember } 23\end{array}$ & $\begin{array}{l}\text { Speier, } \\
\text { Cambridre }\end{array}$ & $-0.04_{5}-0.04$ & \\
\hline & $\begin{array}{l}\text { September } 23 \text {, } \\
\text { November } 6 \text {, }\end{array}$ & $\begin{array}{l}\text { Cambridge, } \\
\text { Cambridge, }\end{array}$ & $\begin{array}{l}+0.21_{10}+0.05 \\
+0.25_{6}+0.09\end{array}$ & \\
\hline
\end{tabular}

Since the year 1830 heliocentric and geocentric eplemerides of Uranus computed from Bouvard's 'Tables are at our disposal. We make use of those in the Berlin Astronomisches Jahrbuch for the years 1830 to 1833, and of those in the Nautical Almanac from 1834 forward. The system of comparison is the same as that already explained. That is to say, we deduce separately:

(1) Mean corrections to the geocentric longitude and latitude of Uranus in the ephemeris as derived from observation.

(2) Mean corrections to the same, given by the provisional theory, as derired from a comparison of the heliocentric positions of that theory with the heliocentric positions in the ephemeris.

Then (1) - (2) is the correction to the provisional theory given by observation. The process of forming (1) and (2) is shown quite fully in the following pages. Each individual printed observation was first compared with the printed ephemeris, and a correction to the latter was thence deduced. When this correction was given with the observations themselves, it was of course not recomputed, unless in some doubtful cases. 'The observations were then divided into groups, usually of about a month each, and coinciding in time with the gronping of the Greenwich results. The mean of the dates and the mean of the corrections were then taken separately for each group and each observatory. 'The separate' results are shown 
in the proper columns of the following table, under the head "Mean dates," Mean cor. in R. A., and Mean cor. in Dec. These means are those given by the observations as printed, without the application of the systematic corrections on pages 120 and 121. In the columns "Corrected mean" these corrections are applied; this column would therefore exhibit no systematic differences between the results of the different observatories, unless the observations of Uranus were affected by errors different from those which affect the positions of the fundamental stars. A careful cormparison of the differences in various parts of the table shows that this is unfortunately the case. A weight is next assigned to each individual result depending on the number of observations, the general sufficiency of the data of reduction, the mean discordance of the individual observations, and the quality of the instruments. The critical reader will notice a lack of homogeneity among the weights assigned, of which I shall speak presently. The mean of the separate group-results is then taken with regard to these weights, and also the mean of the mean dates, using for the latter the relative weights adopted for the several right ascensions. Thus, we have a mean result derived from all the observations for each month, or other group-period, which is written under the horizontal lines.

These corrections to right ascension and declination are next changed to corrections of longitude and latitude, using for this purpose the following table, which is computed from the formulæ of Gauss :

$$
\begin{aligned}
\cos E & =\sin \varepsilon \cos \alpha \sec b=\sin \varepsilon \cos l \sec \delta \\
\Delta l & =\frac{\sin E \cos \delta}{\cos b} \Delta a+\frac{\cos E}{\cos b} \Delta \delta \\
\Delta b & =-\cos E \cos \delta \Delta \alpha+\sin E \Delta \delta .
\end{aligned}
$$

The differential coefficients in this table are expressed as a function of the right ascension of Uranus only, which may be done because, owing to the small inclination and great distance of the planet, its geocentric position on the celestial sphere is never more than about $2^{\prime}$ from some point of the projection of its heliocentric orbit. The coefficients of $\Delta \alpha$ are multiplied by 15 , that the right ascension may be expressed in time.

To Convert Errors of Right Asoension and Dechination of Uranus into errors of

\begin{tabular}{|c|c|c|c|c|c|c|c|c|c|c|}
\hline \multirow[b]{2}{*}{ R. A. } & \multicolumn{6}{|c|}{ Logarithms of } & \multirow[b]{2}{*}{$\frac{\partial l}{\partial \alpha}$} & \multirow[b]{2}{*}{$\frac{\partial b}{\partial \alpha}$} & \multirow[b]{2}{*}{$\frac{\partial l}{\partial \delta}$} & \multirow[b]{2}{*}{$\frac{\partial b}{\partial \delta}$} \\
\hline & $\frac{\partial l}{\partial \alpha}$ & $\frac{\partial b}{\partial \alpha}$ & & $\frac{\partial l}{\partial \delta}$ & & $\frac{\partial b}{\partial \delta}$ & & & & \\
\hline $\begin{array}{c}0^{\mathrm{h}} 0^{\mathrm{m}} \\
10 \\
20 \\
30 \\
40 \\
50\end{array}$ & $\begin{array}{l}1.1386 \\
1.1387 \\
1.1388 \\
1.1389 \\
1.1390 \\
1.1391\end{array}$ & $\begin{array}{l}-0.7761 \\
-0.7757 \\
-0.7743 \\
-0.7720 \\
-0.7687 \\
-0.7643\end{array}$ & $\begin{array}{l}-\quad 4 \\
-14 \\
-\quad 23 \\
-33 \\
-44 \\
-55\end{array}$ & $\begin{array}{r}+9.6000 \\
+9.5996 \\
+9.5983 \\
+9.5963 \\
+9.5934 \\
+9.5896\end{array}$ & $\begin{array}{r}-\quad 4 \\
-13 \\
-20 \\
-29 \\
-38 \\
-47\end{array}$ & $\begin{array}{l}9.9626 \\
9.9626 \\
9.9628 \\
9.9632 \\
9.9637 \\
9.9645\end{array}$ & $+13.8+$ & $\begin{array}{c}-5.97+ \\
5.96 \\
5.95 \\
5.92 \\
5.87 \\
5.81\end{array}$ & $\begin{array}{c}+0.40- \\
0.40 \\
0.40 \\
0.40 \\
0.39 \\
0.38\end{array}$ & $+0.92+$ \\
\hline 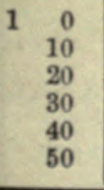 & $\begin{array}{l}1.1392 \\
1.1393 \\
1.1394 \\
1.1394 \\
1.1395 \\
1.1395\end{array}$ & $\begin{array}{l}-0.7588 \\
-0.7525 \\
-0.7451 \\
-0.7365 \\
-0.7270 \\
-0.7162\end{array}$ & $\begin{array}{r}-63 \\
-74 \\
-86 \\
-95 \\
-108 \\
-118\end{array}$ & $\begin{array}{r}+9.5849 \\
+9.5794 \\
+9.5730 \\
+9.5656 \\
+9.5573 \\
+9.5479\end{array}$ & $\begin{array}{r}-55 \\
-64 \\
-74 \\
-83 \\
-94 \\
-104\end{array}$ & $\begin{array}{l}9.9653 \\
9.9662 \\
9.9673 \\
9.9685 \\
9.9698 \\
9.9711\end{array}$ & $+13.8+$ & $\begin{array}{c}-5.74+ \\
5.66 \\
5.56 \\
5.45 \\
5.33 \\
5.20\end{array}$ & $\begin{array}{c}+0.38- \\
0.38 \\
0.37 \\
0.37 \\
0.36 \\
0.35\end{array}$ & $+0.92+$ \\
\hline
\end{tabular}
Longitude AND Latitude.

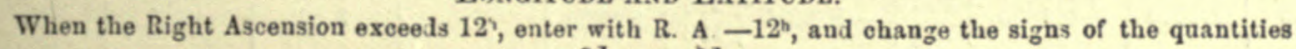


To Convent Errors of Riont Ascension and Declination.-Continucd.

\begin{tabular}{|c|c|c|c|c|c|c|c|c|c|}
\hline \multirow[b]{2}{*}{ R. A. } & \multicolumn{5}{|c|}{ Logarithms of } & \multirow[b]{2}{*}{$\frac{\partial l}{\partial \alpha}$} & \multirow[b]{2}{*}{$\frac{\partial b}{\partial \alpha}$} & \multirow[b]{2}{*}{$\frac{\partial l}{\partial \delta}$} & \multirow[b]{2}{*}{$\frac{\partial b}{\partial \delta}$} \\
\hline & $\frac{\partial l}{\partial \alpha}$ & $\frac{\partial b}{\partial \alpha}$ & & $\frac{\partial l}{\partial \delta}$ & $\frac{\partial b}{\partial J}$ & & & & \\
\hline $\begin{array}{c}2^{\mathrm{h}} 0 \\
10 \\
20 \\
30 \\
40 \\
50\end{array}$ & $\begin{array}{l}1.1395 \\
1.1395 \\
1.1395 \\
1.1395 \\
1.1395 \\
1.1394\end{array}$ & $\begin{array}{l}-0.7044 \\
-0.6915 \\
-0.6773 \\
-0.6618 \\
-0.6450 \\
-0.6266\end{array}$ & $\begin{array}{l}-129 \\
-142 \\
-155 \\
-168 \\
-184 \\
-197\end{array}$ & $\begin{array}{r}+9.5375-115 \\
+9.5260-126 \\
+9.5134-139 \\
+9.4995-152 \\
+0.4843-167 \\
+9.4676-181\end{array}$ & $\begin{array}{l}9.9725 \\
9.9740 \\
9.9756 \\
9.9772 \\
9.9788 \\
9.9804\end{array}$ & $+13.8+$ & $\begin{array}{c}-5.06+ \\
4.01 \\
4.75 \\
4.58 \\
4.41 \\
4.23\end{array}$ & $\begin{array}{c}+0.34 \\
0.33 \\
0.32 \\
0.31 \\
0.30 \\
0.29\end{array}$ & $+0.93+$ \\
\hline $\begin{array}{r}30 \\
10 \\
20 \\
30 \\
40 \\
50\end{array}$ & $\begin{array}{l}1.1394 \\
1.1394 \\
1.1393 \\
1.1393 \\
1.1392 \\
1.1391\end{array}$ & $\begin{array}{l}-0.6069 \\
-0.5854 \\
-0.5621 \\
-0.5368 \\
-0.5094 \\
-0.4795\end{array}$ & $\begin{array}{l}-215 \\
-233 \\
-253 \\
-274 \\
-299 \\
-327\end{array}$ & $\begin{array}{r}+9.4495 \\
+9.4297-198 \\
+9.4081-216 \\
+9.3844-237 \\
+9.3586-258 \\
+9.3302-284 \\
+312\end{array}$ & $\begin{array}{l}9.9821 \\
9.9837 \\
9.9853 \\
9.9869 \\
9.9883 \\
9.9893\end{array}$ & $+13.8+$ & $\begin{array}{l}-4.05+ \\
3.86 \\
3.66 \\
3.45 \\
3.24 \\
3.02\end{array}$ & $\begin{array}{c}+0.28- \\
0.27 \\
0.26 \\
0.24 \\
0.23 \\
0.21\end{array}$ & $+0.96+$ \\
\hline $\begin{array}{r}0 \\
10 \\
20 \\
30 \\
40 \\
50\end{array}$ & $\begin{array}{l}1.1390 \\
1.1390 \\
1.1389 \\
1.1388 \\
1.1387 \\
1.1386\end{array}$ & $\begin{array}{l}-0.4468 \\
-0.4109 \\
-0.3710 \\
-0.3267 \\
-0.2769 \\
-0.2198\end{array}$ & $\begin{array}{l}-359 \\
-399 \\
-443 \\
-498 \\
-571 \\
-659\end{array}$ & $\begin{array}{r}+9.2990 \\
+9.2644-346 \\
+9.2259-385 \\
+9.1828-431 \\
+9.1341-487 \\
+90781-560 \\
\quad-651\end{array}$ & $\begin{array}{l}9.9912 \\
9.9925 \\
9.9938 \\
9.9949 \\
9.9959 \\
9.9969\end{array}$ & $+13.8+$ & $\begin{array}{c}-2.80+ \\
2.58 \\
2.36 \\
2.14 \\
1.91 \\
1.67\end{array}$ & $\begin{array}{c}+0.20- \\
0.18 \\
0.17 \\
0.15 \\
0.13 \\
0.12\end{array}$ & $+0.98+$ \\
\hline $\begin{array}{r}0 \\
10 \\
20 \\
30 \\
40 \\
50\end{array}$ & $\begin{array}{l}1.1385 \\
1.1384 \\
1.1383 \\
1.1382 \\
1.1381 \\
1.1380\end{array}$ & $\begin{array}{l}-0.1539 \\
-0.0753 \\
-9.979 \\
-9.854 \\
-9.679 \\
-9.378\end{array}$ & $\begin{array}{r}-786 \\
-96 \\
-125 \\
-175 \\
-301 \\
\ldots \ldots\end{array}$ & $\begin{array}{l}+9.0130-777 \\
+8.9353-956 \\
+8.8397-1240 \\
+8.7157-1757 \\
+8.540-300 \\
+8.240 \quad \ldots \ldots\end{array}$ & $\begin{array}{l}9.9977 \\
9.9984 \\
9.9990 \\
9.9994 \\
9.9997 \\
9.9999\end{array}$ & $+13.8+$ & $\begin{array}{c}-1.43+ \\
1.19 \\
0.95 \\
0.71 \\
0.48 \\
0.24\end{array}$ & $\begin{array}{c}+0.10- \\
0.08 \\
0.07 \\
0.05 \\
0.03 \\
0.02\end{array}$ & $+0.99+$ \\
\hline $\begin{array}{r}0 \\
10 \\
20 \\
30 \\
40 \\
50\end{array}$ & $\begin{array}{l}1.1379 \\
1.1378 \\
1.1377 \\
1.1376 \\
1.1375 \\
1.1374\end{array}$ & $\begin{array}{l}-\propto \\
+9.378 \\
+9.679 \\
+9.854 \\
+9.978 \\
+0.0743\end{array}$ & $\begin{array}{r}\ldots 301 \\
+301 \\
+175 \\
+124 \\
+96 \\
+783\end{array}$ & $\begin{array}{ll}\propto & \\
-8.240 & \ldots \ldots \\
-8.540 & +300 \\
-8.7157 & +1757 \\
-8.8397 & +1240 \\
-8.9353 & +956 \\
& +777\end{array}$ & $\begin{array}{c}0 \\
9.9999 \\
9.9997 \\
9.9994 \\
9.9990 \\
9.9984\end{array}$ & $+13.7+$ & $\begin{array}{c}0.00 \\
-0.24+ \\
0.48 \\
0.71 \\
0.95 \\
1.18\end{array}$ & $\begin{array}{l}0.00 \\
-0.02+ \\
0.03 \\
0.05 \\
0.07 \\
0.08\end{array}$ & $+1.00+$ \\
\hline $\begin{array}{r}7 \quad 0 \\
10 \\
20 \\
30 \\
40 \\
50\end{array}$ & $\begin{array}{l}1.1373 \\
1.1373 \\
1.1372 \\
1.1371 \\
1.1371 \\
1.1370\end{array}$ & $\begin{array}{l}+0.1526 \\
+0.2184 \\
+0.2753 \\
+0.3250 \\
+0.3692 \\
+0.4089\end{array}$ & $\begin{array}{l}+658 \\
+569 \\
+497 \\
+442 \\
+397 \\
+359\end{array}$ & $\begin{array}{l}-9.0130+651 \\
-9.0781+560 \\
-9.1341+487 \\
-9.1828+431 \\
-9.2259+385 \\
-9.2644+346\end{array}$ & $\begin{array}{l}9.9977 \\
9.9969 \\
9.9959 \\
9.9949 \\
9.9938 \\
9.9925\end{array}$ & $+13.7+$ & $\begin{array}{c}+1.42- \\
1.65 \\
1.88 \\
2.11 \\
2.34 \\
2.56\end{array}$ & $\begin{array}{l}-0.10+ \\
0.12 \\
0.13 \\
0.15 \\
0.17 \\
0.18\end{array}$ & $+0.99+$ \\
\hline $\begin{array}{r}80 \\
10 \\
20 \\
30 \\
40 \\
50\end{array}$ & $\begin{array}{l}1.1370 \\
1.1370 \\
1.1370 \\
1.1370 \\
1.1370 \\
1.1370\end{array}$ & $\begin{array}{l}+0.4448 \\
+0.4773 \\
+0.5071 \\
+0.5344 \\
+0.5597 \\
+0.5830\end{array}$ & $\begin{array}{l}+325 \\
+298 \\
+273 \\
+253 \\
+233 \\
+214\end{array}$ & $\begin{array}{l}-9.2990+312 \\
-9.3302+284 \\
-9.3586+258 \\
-9.3844+237 \\
-9.4081+216 \\
-9.4297+198\end{array}$ & $\begin{array}{l}9.9912 \\
9.9898 \\
9.9883 \\
9.9869 \\
9.9853 \\
99837\end{array}$ & $+13.7+$ & $\begin{array}{c}+2.78- \\
3.00 \\
3.22 \\
3.43 \\
3.63 \\
3.83\end{array}$ & $\begin{array}{l}-0.20+ \\
0.21 \\
0.23 \\
0.24 \\
0.26 \\
0.27\end{array}$ & $+0.98+$ \\
\hline $\begin{array}{r}90 \\
10 \\
20 \\
30 \\
40 \\
50\end{array}$ & $\begin{array}{l}1.1370 \\
1.1370 \\
1.1370 \\
1.1371 \\
1.1371 \\
1.1372\end{array}$ & $\begin{array}{l}+0.6044 \\
+0.6241 \\
+0.6425 \\
+0.6594 \\
+0.6749 \\
+0.6892\end{array}$ & $\begin{array}{l}+197 \\
+184 \\
+169 \\
+155 \\
+143 \\
+131\end{array}$ & $\begin{array}{l}-9.4495+181 \\
-9.4676+167 \\
-9.4843+152 \\
-9.4995+139 \\
-9.5134+126 \\
-9.5260+115\end{array}$ & $\begin{array}{l}9.9821 \\
9.9804 \\
9.9788 \\
9.9772 \\
9.9756 \\
9.9740\end{array}$ & $+15.7+$ & $\begin{array}{c}+4.02- \\
4.20 \\
4.38 \\
4.55 \\
4.72 \\
4.38\end{array}$ & $\begin{array}{c}-0.28+ \\
0.29 \\
0.30 \\
0.31 \\
0.32 \\
0.33\end{array}$ & $+0.96+$ \\
\hline $\begin{array}{r}0 \\
0 \\
10 \\
20 \\
30 \\
40 \\
50\end{array}$ & $\begin{array}{l}1.1373 \\
1.1374 \\
1.1375 \\
1.1376 \\
1.1377 \\
1.1378\end{array}$ & $\begin{array}{l}+0.7023 \\
+0.7142 \\
+0.7251 \\
+0.7347 \\
+0.7434 \\
+0.7510\end{array}$ & $\begin{array}{l}+119 \\
+109 \\
+96 \\
+87 \\
+76 \\
+\quad 65\end{array}$ & $\begin{array}{l}-9.5375+104 \\
-9.5479+94 \\
-9.5573+83 \\
-9.5656+74 \\
-9.5730+64 \\
-9.5794^{\circ}+55\end{array}$ & $\begin{array}{l}9.9725 \\
9.9711 \\
99698 \\
9.9685 \\
9.9673 \\
9.9662\end{array}$ & $+13.7+$ & $\begin{array}{c}+5.04 \\
5.18 \\
5.31 \\
5.43 \\
5.54 \\
5.64\end{array}$ & $\begin{array}{l}-0.34+ \\
0.35 \\
0.36 \\
0.37 \\
0.37 \\
0.38\end{array}$ & $+0.93+$ \\
\hline $11 \begin{array}{r}0 \\
10 \\
20 \\
30 \\
40 \\
50\end{array}$ & $\begin{array}{l}1.1379 \\
1.1380 \\
1.1381 \\
1.1382 \\
1.1383 \\
1.1385\end{array}$ & $\begin{array}{l}+0.7575 \\
+0.7632 \\
+0.7678 \\
+0.7713 \\
+0.7737 \\
+0.7754\end{array}$ & $\begin{array}{l}+57 \\
+46 \\
+35 \\
+24 \\
+\quad 17 \\
+\quad 7\end{array}$ & $\begin{array}{l}-9.5849+47 \\
-9.5896+38 \\
-9.5934+29 \\
-9.5963+20 \\
-9.5983+13 \\
-9.5996+4\end{array}$ & $\begin{array}{l}9.9653 \\
9.9645 \\
9.9637 \\
9.9632 \\
9.9628 \\
9.9626\end{array}$ & $13.7+$ & $\begin{array}{c}+5.72- \\
5.79 \\
5.85 \\
6.90 \\
5.94 \\
5.96\end{array}$ & $\begin{array}{c}-0.38+ \\
0.38 \\
0.39 \\
0.39 \\
0.39 \\
0.40\end{array}$ & $+0.92+$ \\
\hline 0 & 1.1386 & +0.7761 & $\ldots . .$. & $-9.6000 \quad \ldots \ldots$ & 9.9626 & $+13.8+$ & $+5.97-$ & $-0.40+$ & $+0.92+$ \\
\hline
\end{tabular}


We thus have, for the interval occupied by each group of observations, a mean correction to the geocentric longitude and latitude of the planet given by observations, which are found in the ninth and tenth columns of the table, on the same horizontal line with the mean corrections in right ascension and declination from which they are derived. The next step is to obtain the corresponding corrections given by the provisional ephemeris.

This correction has been first obtained for every twentieth day of each of the forty-two oppositions included in the table. The heliocentric longitude, latitude, and radius vector were interpolated to the most convenient twenty-day intervals, and compared with the corresponding co-ordinates in the heliocentric ephemeris. This ephemeris was of course the one corresponding to that with which the observations were compared, namely, the Berliner Jahrbuch for the years 1830-33, and the Nautical Almanac for subsequent years. These comparisons are fully given at the end of this chapter, and the resulting corrections to the printed ephemeris are given in the proper columns of the table.

These corrections to the heliocentric co-ordinates were then changed to corrections of geocentric longitude and latitude by the following formulæ. Put

$r^{\prime}$, the projection of the planet's radius vector on the ecliptic;

$\rho$, the projection of the planet's distance from the earth on the same plane;

$\rho$, this distance itself;

$\lambda, \beta$, the planet's heliocentric longitude and latitude;

$L$, the sun's geocentric longitude;

$R$, its radius vector;

$M$, the modulus of the common logarithms;

$\delta l, \delta b$, the corrections to the geocentric longitude and latitude;

$\delta \rho$, the correction to the common logarithm of the radius vector.

Then

$$
\begin{aligned}
\delta l= & \frac{r^{\prime 2}}{\rho^{\prime 2}}\left\{1+\frac{R}{r^{\prime}} \cos (L-\lambda)\right\} \delta \lambda \\
& \quad-\frac{R r^{\prime}}{\rho^{\prime 2}} \sin (L-\lambda) \frac{\delta \rho}{M \sin 1^{\prime \prime}} \\
+ & \frac{R r^{\prime}}{\rho^{\prime 2}} \sin (L-\lambda) \tan \beta \delta \beta \\
\delta b= & \frac{r^{\prime}}{\rho^{\prime}}\left\{1+\frac{r^{\prime} R}{\rho^{2}} \tan ^{2} \beta \cos (L-\lambda)\right\} \delta \beta \\
& \quad-\frac{r^{\prime 2} R}{\rho^{\prime} \rho^{2}} \tan \beta \sin (L-\lambda) \delta \lambda \\
& \quad+\frac{r R^{2}}{\rho^{\prime} \rho^{2}}\left\{1+\frac{r^{\prime}}{R} \cos (L-\lambda)\right\} \sin \beta \frac{\delta \rho}{M \sin 1^{\prime \prime} .}
\end{aligned}
$$

The last term in $\delta l$ and the last two terms of $\delta b$ have been omitted in the computation, as they scarcely ever exceed a few hundredths of a second.

17 May, 1873. 
The values of $\delta l$ and $\delta b$ are printed in the last two columns of the table. The formula for $\delta b$ might have contained the additional term

$$
\delta b=\sin l i \omega
$$

$\delta \omega$ being the correction to the obliquity of the ecliptic adopted in the ephemeris to reduce it to that employed in the provisional theory. This correction is, however, deferred until we come to form the equations of condition.

From the values of $\varepsilon l$ and $\delta b$ thus obtained we are to find the mean values during each group of observations. If these quantities varied uniformly, the proper value would be that corresponding to the mean date of each group. But the second differences are so large that this value would generally be in error by one- or two-tenths of a second. Owing to the minuteness of this difference, it has been considered that when the mean date was near the middle of a twenty-day interval, the correction $\delta l$ interpolated to that date without regard to second differences would furnish a sufficient approximation to the required mean value of $\delta l$ during an interval of about 30 days. In other case the value of $\delta l$ was interpolated to 5-day intervals through the period of each group of observations, and the mean value taken.

During the years 1850-1863 the sun's longitude employed in the ephemeris required a gradually increasing correction, amounting at the latter date to about $3^{\prime \prime}$. A small correction of which the maximum value is about $0^{\prime \prime}, 15$ was applied to il to reduce it to the value it would have had if Hansen's tables had been employed.

The corrected mean values of $i l$ and $i b$ thus obtained are given in the last two columns of the following table, being inclosed in brackets and printed immediately above the values of $\Delta l$ and $\Delta b$ derived from observation.

I deem it proper to mention that the mechanical labor of constructing these tables of comparisons, in the manner just described, was in great part performed by Dr. C. L. F. Kampf, who was employed by the Smithsonian Institution to assist me in the work. Before using it I subjected the whole of the work to a careful revision, altering especially the relative weights of the corrected means in many cases. $\Lambda \mathrm{s}$ the assigned weiglits now stand, each set of results which are combined into a single mean has its own unit of weight, which does not necessarily coincide with that of any other set. The use of a uniform scale of weights through this series of observations, and the assignment to every final mean of a weight equal to tlie sum of the weights of the quantities whose mean was taken, would have led to weights in many cases quite fictitions, owing to the obvious presence of systematic errors in the results. For this reason I have made no further use of the weights found in this table, and their lack of homogeneousness therefore does no harm. 
Mran Corrections to the Ephemeris of Uranus in the Berliner Jahrbuch and the Nautical almanac.

\begin{tabular}{|c|c|c|c|c|c|c|c|c|c|}
\hline \multirow{2}{*}{$\begin{array}{l}\text { Observatory. } \\
\text { [R.A. of } \\
\text { Uranus.] }\end{array}$} & \multirow{2}{*}{ Mean dates. } & \multicolumn{3}{|c|}{ Observed eorrections in R.A. } & \multicolumn{3}{|c|}{ Observed corrections in Dee. } & \multicolumn{2}{|c|}{ Corr. to Geocentrio } \\
\hline & & Mean. & $\begin{array}{l}\text { No. of } \\
\text { obs. }\end{array}$ & $\begin{array}{c}\text { Corrected } \\
\text { mean. }\end{array}$ & Mean. & $\begin{array}{l}\text { No. of } \\
\text { obs. }\end{array}$ & $\begin{array}{c}\text { Corrected } \\
\text { mean. }\end{array}$ & Longitude. & Latitude. \\
\hline $\begin{array}{l}\text { Kōnigsberg, } \\
\text { Cambridge, }\end{array}$ & \begin{tabular}{cc}
\multicolumn{1}{c}{1830} \\
July & 29 \\
July & 29 \\
\end{tabular} & $\begin{array}{c}\mathrm{s} \\
-1.56 \\
-1.51\end{array}$ & $\begin{array}{l}7 \\
6\end{array}$ & $\begin{array}{c}\mathrm{s} \\
-1.56_{\mathrm{s}} \\
-1.67_{3}\end{array}$ & $\begin{array}{r}\prime \prime \prime \\
+4.6\end{array}$ & 7 & $\begin{array}{r}\prime \prime \\
+4.6\end{array}$ & [-10 9า & $51 \quad 0.57$ \\
\hline$\left[\begin{array}{ll}20^{\mathrm{b}} & 40^{\mathrm{m}}\end{array}\right]$ & July $\quad 29$ & $\ldots$. & $\therefore$ & -1.60 & … & $\cdots$ & & {$\left[\begin{array}{c}-19.2 \\
-20.8\end{array}\right]$} & {$\left[\begin{array}{r}+9.5 \\
+10.3\end{array}\right]$} \\
\hline $\begin{array}{l}\text { Kōnigsberg, } \\
\text { Cambridge, }\end{array}$ & $\begin{array}{l}\text { Aug. } 12 \\
\text { Aug. } 25 \\
\end{array}$ & $\begin{array}{l}-1.65 \\
-1.36\end{array}$ & $\begin{array}{l}2 \\
4\end{array}$ & $\begin{array}{r}-1.65_{2} \\
-1.52_{2} \\
\end{array}$ & +3.6 & 2 & +3.6 & & \\
\hline$\left[\begin{array}{ll}20^{\mathrm{h}} & 36^{\mathrm{m}}\end{array}\right]$ & Ang. 19 & $\cdots$ & $\cdots$ & $-1,58$ & $\cdots$ & $\cdots$ & & {$\left[\begin{array}{l}-18.5 \\
-20.8 \\
-17\end{array}\right]$} & {$\left[\begin{array}{c}++9.5] \\
+9.1\end{array}\right.$} \\
\hline $\begin{array}{l}\text { Cambridge, } \\
{\left[\begin{array}{lll}20^{\mathrm{t}} & 37^{\mathrm{m}}\end{array}\right]}\end{array}$ & Sept. 19 & -1.46 & 7 & -1.62 & .... & $\cdots$ & ... & {$\left[\begin{array}{l}{[-17.2} \\
-21.2\end{array}\right]$} & \\
\hline $\begin{array}{l}\text { Cambridge, } \\
{\left[\begin{array}{ll}20^{\mathrm{s}} & 36^{\mathrm{m}}\end{array}\right]}\end{array}$ & Oct. $\quad 17$ & -1.34 & 8 & -1.50 & .. & $\ldots$ & $\cdots$ & {$\left[\begin{array}{c}-16.7 \\
-19.5\end{array}\right]$} & \\
\hline $\begin{array}{l}\text { Cambridge, } \\
{\left[\begin{array}{ll}20^{\mathrm{a}} & 37^{\mathrm{m}}\end{array}\right]}\end{array}$ & $\begin{array}{c}\text { Nov, } 14 \\
1831\end{array}$ & -1.36 & 8 & -1.52 & .. & $\cdots$ & & {$\left[\begin{array}{l}{[-16.6} \\
-19.8\end{array} \mid\right.$} & \\
\hline $\begin{array}{l}\text { Greenwich, } \\
\text { Cambridge, }\end{array}$ & $\begin{array}{ll}\text { Aug. } & 3 \\
\text { A ug. } & 8 \\
\end{array}$ & $\begin{array}{l}-1.72 \\
-1.70\end{array}$ & $\begin{array}{r}11 \\
9\end{array}$ & $\begin{array}{l}-1.72_{4} \\
-1.86_{4} \\
\end{array}$ & +2.5 & 11 & +4.3 & $-23.87[$ r & \\
\hline$\left[\begin{array}{ll}20^{\mathrm{h}} & 58^{\mathrm{m}}\end{array}\right]$ & Aug. $\quad 6$ & $\ldots$ & $\ldots$ & -1.79 & $\cdots$ & $\cdots$ & & -23.4 & $\begin{array}{l}{[+10.1]} \\
+11.1\end{array}$ \\
\hline $\begin{array}{l}\text { Greenwich, } \\
\text { Cambridge, }\end{array}$ & $\begin{array}{lr}\text { Sept. } & 7 \\
\text { Sept. } & 15 \\
\end{array}$ & $\begin{array}{l}-1.67 \\
-1.60\end{array}$ & $\begin{array}{l}5 \\
6\end{array}$ & $\begin{array}{l}-1.67_{\mathrm{s}} \\
-1.76_{\mathrm{s}}\end{array}$ & +3.5 & 5 & +5.3 & & \\
\hline$\left[\begin{array}{ll}20^{\mathrm{h}} & 52^{\mathrm{m}}\end{array}\right]$ & Sept. 11 & $\ldots$ & $\ldots$ & -1.72 & $\cdots$ & $\cdots$ & & {$\left[\begin{array}{l}{[-22.0} \\
-22.2\end{array}\right]$} & {$\left[\begin{array}{l}+10.0] \\
+11.8\end{array}\right.$} \\
\hline $\begin{array}{l}\text { Greenwich, } \\
\text { Cambridge, }\end{array}$ & $\begin{array}{lr}\text { Nov. } & 4 \\
\text { Oet. } & 26 \\
\end{array}$ & $\begin{array}{l}-1.48 \\
-1.54\end{array}$ & $\begin{array}{r}7 \\
16\end{array}$ & $\begin{array}{l}-1.48_{4} \\
-1.70_{8} \\
\end{array}$ & +3.1 & 7 & +4.9 & {$[-20.7] \mid$} & \\
\hline$\left[\begin{array}{ll}20^{\mathrm{h}} & 50^{\mathrm{m}}\end{array}\right]$ & $\begin{array}{c}\text { Oet. } 31 \\
1832\end{array}$ & $\ldots$. & $\ldots$ & -1.63 & . & $\cdots$ & & & L†, \\
\hline $\begin{array}{l}\text { Green wich, } \\
\text { Königsberg, } \\
\text { Cambridge, } \\
\text { Vienna, }\end{array}$ & $\begin{array}{lr}\text { Aug. } & 9 \\
\text { Aug. } & 10 \\
\text { A ug. } & 15 \\
\text { Aug. } & 3\end{array}$ & $\begin{array}{l}-2.02 \\
-2.24 \\
-1.99 \\
-2.33\end{array}$ & $\begin{array}{l}3 \\
3 \\
9 \\
3\end{array}$ & $\begin{array}{l}-2.02_{2} \\
-2.24_{4} \\
-2.18_{8} \\
-2.33_{1}\end{array}$ & +1.7 & 2 & $+3.4_{2}$ & & \\
\hline$\left[\begin{array}{ll}21^{\mathrm{h}} & 17^{\mathrm{m}}\end{array}\right]$ & Aug. 12 & $\ldots$ & $\cdots$ & -2.19 & $\cdots$ & $\cdots$ & +2.5 & $\begin{array}{l}{[-28.4]} \\
-29.2 \\
{[-26.97}\end{array}$ & 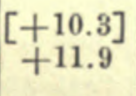 \\
\hline $\begin{array}{c}\text { Cambridge, } \\
{\left[\begin{array}{ll}21^{\mathrm{h}} & 12^{\mathrm{m}}\end{array}\right]}\end{array}$ & Sept. 12 & -1.97 & 10 & -2.16 & & ... & & $\left|\begin{array}{l}-26.9 \\
-28.8\end{array}\right|$ & \\
\hline $\begin{array}{l}\text { Cambridge, } \\
\text { Vienna, }\end{array}$ & $\begin{array}{lr}\text { Oet. } & 6 \\
\text { Oet. } & 12 \\
\end{array}$ & $\begin{array}{l}-1.91 \\
-1.90\end{array}$ & $\begin{array}{l}13 \\
15\end{array}$ & $\begin{array}{r}-2.09 \\
-1.90 \\
\end{array}$ & +1.9 & 15 & +2.8 & & \\
\hline$\left[\begin{array}{ll}21^{\mathrm{h}} & 9^{\mathrm{m}}\end{array}\right]$ & Oct. $\quad 7$ & $\cdots$ & $\cdots$ & -2.01 & & ". & & -26.6 & \\
\hline $\begin{array}{l}\text { Cambridge, } \\
\text { Vienna, }\end{array}$ & $\begin{array}{lr}\text { Nov. } & 16 \\
\text { Nov. } & 9 \\
\end{array}$ & $\begin{array}{l}-1.85 \\
-1.89\end{array}$ & $\begin{array}{l}7 \\
2\end{array}$ & $\begin{array}{r}-2.04 \\
-1.89_{1} \\
\end{array}$ & +1.1 & $\cdots$ & +2.0 & {$[-25.2]$} & {$[+1$} \\
\hline$\left[\begin{array}{lll}21^{\mathrm{h}} & 10^{\mathrm{m}}\end{array}\right]$ & Nov. 15 & $\cdots$ & $\cdots$ & -2.02 & $\cdots$ & $\cdots$ & .. & -27.4 & +10.5 \\
\hline
\end{tabular}




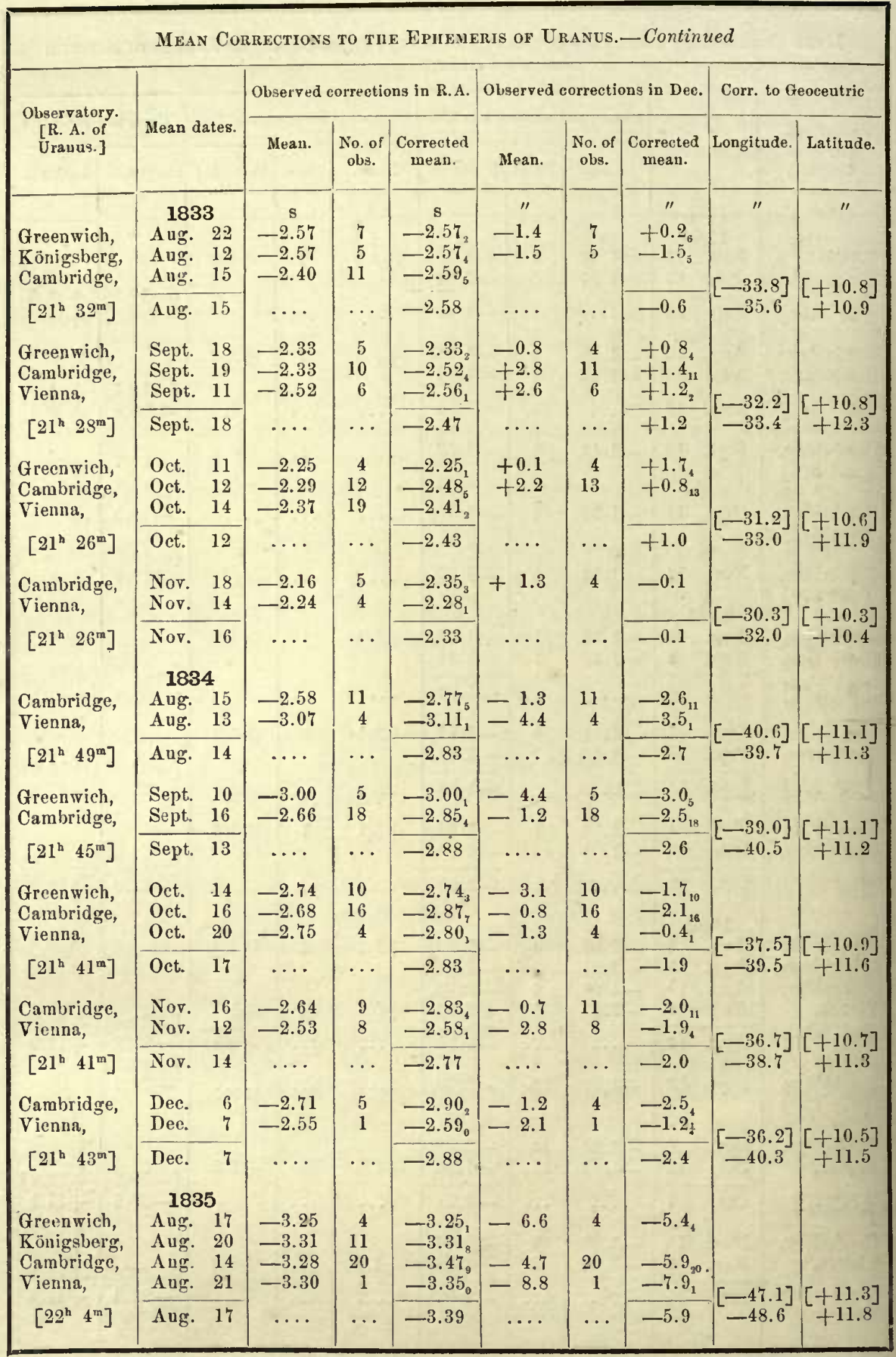




\begin{tabular}{|c|c|c|c|c|c|c|c|c|c|}
\hline \multicolumn{10}{|c|}{ Mran Corrections to the Ephemeris of Uranus.-Continued. } \\
\hline \multirow{2}{*}{$\begin{array}{l}\text { Observatory. } \\
\text { [R. A. of } \\
\text { Urauus.] }\end{array}$} & \multirow[b]{2}{*}{ Mean dates. } & \multicolumn{3}{|c|}{ Observed corrections in R.A. } & \multicolumn{3}{|c|}{ Observed corrections in Dee. } & \multicolumn{2}{|c|}{ Corr. to Geocentrio } \\
\hline & & Mean. & $\begin{array}{l}\text { No. of } \\
\text { obs. }\end{array}$ & $\begin{array}{l}\text { Corrected } \\
\text { mean. }\end{array}$ & Mean. & $\begin{array}{l}\text { No. of } \\
\text { obs. }\end{array}$ & $\begin{array}{l}\text { Corrected } \\
\text { mean. }\end{array}$ & Longitude. & Latitude. \\
\hline \multirow{4}{*}{$\begin{array}{l}\text { Cambridge, } \\
\text { Vienna, } \\
\qquad\left[22^{\mathrm{h}} 1^{\mathrm{m}}\right]\end{array}$} & 1835 & 8 & & & $" \prime$ & & $"$ & \multirow{4}{*}{$\begin{array}{l}{[-45.8]} \\
-48.1\end{array}$} & \multirow{4}{*}{$\begin{array}{l}+11.2] \\
+11.6\end{array}$} \\
\hline & Sept. 15 & -3.17 & 9 & -3.36 & -4.6 & 9 & -5.8 & & \\
\hline & Sept. 14 & -3.30 & 9 & $-3.35_{1}$ & -6.7 & 9 & -5.8 & & \\
\hline & Sept. 15 & $\ldots$ & $\cdots$ & -3.36 & $\cdots$ & $\cdots$ & -5.8 & & \\
\hline \multirow{3}{*}{$\begin{array}{l}\text { Greenwieh, } \\
\text { Cambridge, } \\
{\left[21^{\mathrm{h}} 57^{\mathrm{m}} \mid\right.}\end{array}$} & Oct. 10 & -3.27 & 4 & -3.27 & -6.53 & 4 & $-5.3_{4}$ & \multirow{3}{*}{$\begin{array}{l}-44.4] \\
-46.9\end{array}$} & \multirow{3}{*}{$\begin{array}{c}+11.1] \\
+11.4\end{array}$} \\
\hline & Oet. 17 & -3.11 & 8 & -3.30 & -4.1 & 8 & $-5.3_{8}$ & & \\
\hline & Oet. 15 & $\cdots$ & $\cdots$ & -3.29 & $\cdots$ & $\cdots$ & -5.3 & & \\
\hline \multirow{3}{*}{$\begin{array}{l}\text { Greenwich, } \\
\text { Cambridge, } \\
{\left[21^{\mathrm{h}} 57^{\mathrm{m}}\right]}\end{array}$} & Nov. 27 & -3.21 & 6 & $-3.21_{2}$ & -5.6 & 7 & -4.4 & \multirow{3}{*}{$\begin{array}{l}{[-43.0]} \\
-45.6\end{array}$} & \multirow{3}{*}{$\begin{array}{c}+10.7] \\
+11.2\end{array}$} \\
\hline & Nov. 26 & -3.00 & 10 & -3.19 & -4.5 & 9 & -5.7 & & \\
\hline & Nov. 26 & $\ldots$ & $\cdots$ & -3.20 & $\ldots$ & $\cdots$ & -5.1 & & \\
\hline \multirow{3}{*}{$\begin{array}{l}\text { Greenwich, } \\
\text { Cambridge, } \\
{\left[\begin{array}{l}\left.22^{\mathrm{h}} 24^{\mathrm{m}}\right]\end{array}\right.}\end{array}$} & July $_{22} 1836$ & -3.80 & 7 & $-3.84 a$ & -10.5 & 8 & -9.5 & \multirow{3}{*}{$\begin{array}{l}-55.3] \\
-56.5\end{array}$} & \multirow{3}{*}{$\begin{array}{c}+11.5] \\
+11.8\end{array}$} \\
\hline & July 25 & -3.60 & 3 & $\begin{array}{r}-3.84_{2} \\
-3.89_{1} \\
\end{array}$ & $\begin{array}{l}-10.5 \\
-8.9\end{array}$ & 2 & $\begin{array}{l}-9.5_{8} \\
-9.9_{2}\end{array}$ & & \\
\hline & July 23 & $\cdots$ & $\cdots$ & -3.86 & $\ldots$ & $\cdots$ & -9.6 & & \\
\hline \multirow{6}{*}{$\begin{array}{l}\text { Greenwieh, } \\
\text { Königsberg, } \\
\text { Cambridge, } \\
\text { Edinburgh, } \\
\text { Vienna, } \\
{\left[22^{\mathrm{h}} 20^{\mathrm{m}}\right]}\end{array}$} & A ug. 24 & -3.78 & 7 & $-3.82_{\mathrm{a}}$ & -9.8 & 8 & $-8.8_{8}$ & \multirow{6}{*}{$\begin{array}{l}{[-54.6]} \\
-56.6\end{array}$} & \multirow{6}{*}{$\begin{array}{c}+11.6] \\
+11.5\end{array}$} \\
\hline & Aug. 30 & -3 . & 5 & $-3.63_{4}$ & & & & & \\
\hline & Aug. 16 & -3.78 & 12 & $-3.97_{\mathrm{s}}$ & -9.3 & 12 & $-10.3_{12}$ & & \\
\hline & Aug. 19 & -4.09 & $\begin{array}{l}9 \\
1\end{array}$ & $-4.10_{3}$ & $\begin{array}{r}-9.3 \\
-195\end{array}$ & 7 & -9.3, & & \\
\hline & Aug. 20 & -3.77 & 1 & $-3.81_{0}$ & -12.5 & 1 & -11.5 & & \\
\hline & A ug. 22 & $\cdots$ & $\cdots$ & -3.87 & $\cdots$ & $\cdots$ & -9.6 & & \\
\hline \multirow{5}{*}{$\begin{array}{l}\text { Greenwich, } \\
\text { Cambridge, } \\
\text { Edinburgh, } \\
\text { Vieuna, } \\
{\left[22^{\mathrm{h}} 16^{\mathrm{m}}\right]}\end{array}$} & Sept. 13 & -3.77 & 7 & $-3.81_{3}$ & -8.8 & 6 & $-7.8_{6}$ & \multirow{5}{*}{$\begin{array}{l}-53.4] \\
-56.2\end{array}$} & \multirow{5}{*}{$\begin{array}{c}+11.6] \\
+12.1\end{array}$} \\
\hline & Sept. 16 & -3.70 & 10 & -3.89 & -8.4 & 10 & $-9.4_{10}$ & & \\
\hline & Sept. 16 & -4.01 & $\begin{array}{l}8 \\
9\end{array}$ & -4.02 & -8.6 & 8 & $-8.6_{8}$ & & \\
\hline & Sept. 15 & -3.59 & 9 & -3.63, & -10.4 & 9 & $-9.4_{3}$ & & \\
\hline & Sept. 15 & . & $\cdots$ & -3.87 & $\cdots$ & $\cdots$ & $-8: 8$ & & \\
\hline & Oet. $\quad 12$ & -3.67 & 7 & $-3.71_{2}$ & -8.6 & 8 & $-7.6_{8}$ & & \\
\hline & $\begin{array}{ll}\text { Oct. } & 16 \\
\text { Oct. } & 15\end{array}$ & $\begin{array}{l}-3.51 \\
-4.05\end{array}$ & 12 & $-3.70_{5}$ & -8.5 & 11 & $-9.5_{11}$ & & \\
\hline $\begin{array}{l}\text { Edinburgh, } \\
\text { Vienna, }\end{array}$ & $\begin{array}{ll}\text { Oct. } & 15 \\
\text { Oet. } & 11\end{array}$ & $\begin{array}{l}-4.05 \\
-3.57\end{array}$ & $\begin{array}{r}8 \\
10\end{array}$ & $\begin{array}{l}-4.06_{2} \\
-3.61_{1}\end{array}$ & $\begin{array}{l}7.9 \\
-10.2\end{array}$ & $\begin{array}{r}4 \\
10\end{array}$ & $\begin{array}{l}-7.9_{4} \\
-9.2_{3}\end{array}$ & {$[-5$} & \\
\hline$\left[\begin{array}{ll}22^{\mathrm{h}} & 13^{\mathrm{m}}\end{array}\right]$ & Oet. 15 & & $\ldots$ & -3.77 & $\cdots$ & $\cdots$ & -8.6 & -54.8 & $\begin{array}{c}{[+11.5]} \\
+11.7\end{array}$ \\
\hline Greenwich, & Nov. 13 & -3.59 & 11 & $-3.63_{5}$ & -9.1 & 11 & $-8.1_{11}$ & & \\
\hline Cambridge, & Nov. 13 & -3.37 & 8 & -3.56 & -7.9 & 7 & $-8.9_{7}$ & & \\
\hline & $\begin{array}{lr}\text { Nov. } & 17 \\
\text { Nov. } & 9\end{array}$ & $\begin{array}{l}-3.78 \\
-3.56\end{array}$ & $\begin{array}{l}8 \\
3\end{array}$ & $\begin{array}{l}-3.79_{2} \\
-3.60_{1}\end{array}$ & -10.2 & 3 & -9 & & \\
\hline$\left[\begin{array}{ll}22^{\mathrm{h}} & 12^{\mathrm{m}}\end{array}\right]$ & Nov. 13 & & $\ldots$ & -3.63 & -20.2 & $\ldots$ & -8.5 & $\begin{array}{c}{[-50.6]} \\
-52.8\end{array}$ & {$\left[\begin{array}{c}{[+11.1]} \\
+11.0\end{array}\right.$} \\
\hline Cambridge, & Dec. 12 & -3.40 & 7 & $-3.59_{\mathrm{g}}$ & -8.0 & 7 & -9.0 & & \\
\hline Edinburgh, & Dec. 12 & -3.29 & 5 & $-3.30_{1}$ & -8.8 & 3 & $-8.8_{3}$ & {$[-50.0]$} & {$[+10.9]$} \\
\hline$\left[\begin{array}{ll}22^{\mathrm{h}} & 13^{\mathrm{m}}\end{array}\right]$ & Dec. 12 & ... & $\ldots$ & -3.52 & $\cdots$ & $\cdots$ & -8.9 & -51.5 & +10.1 \\
\hline & 1837 & & & & & & & {$[-62.9]$} & {$[+11.5]$} \\
\hline $\begin{array}{l}\text { Greenwich, } \\
{\left[\begin{array}{ll}22^{\mathrm{b}} & 40^{\mathrm{m}}\end{array}\right]}\end{array}$ & July 22 & -4.23 & 4 & $-4.26_{1}$ & -13.4 & 4 & -12.5 & -63.2 & \\
\hline
\end{tabular}




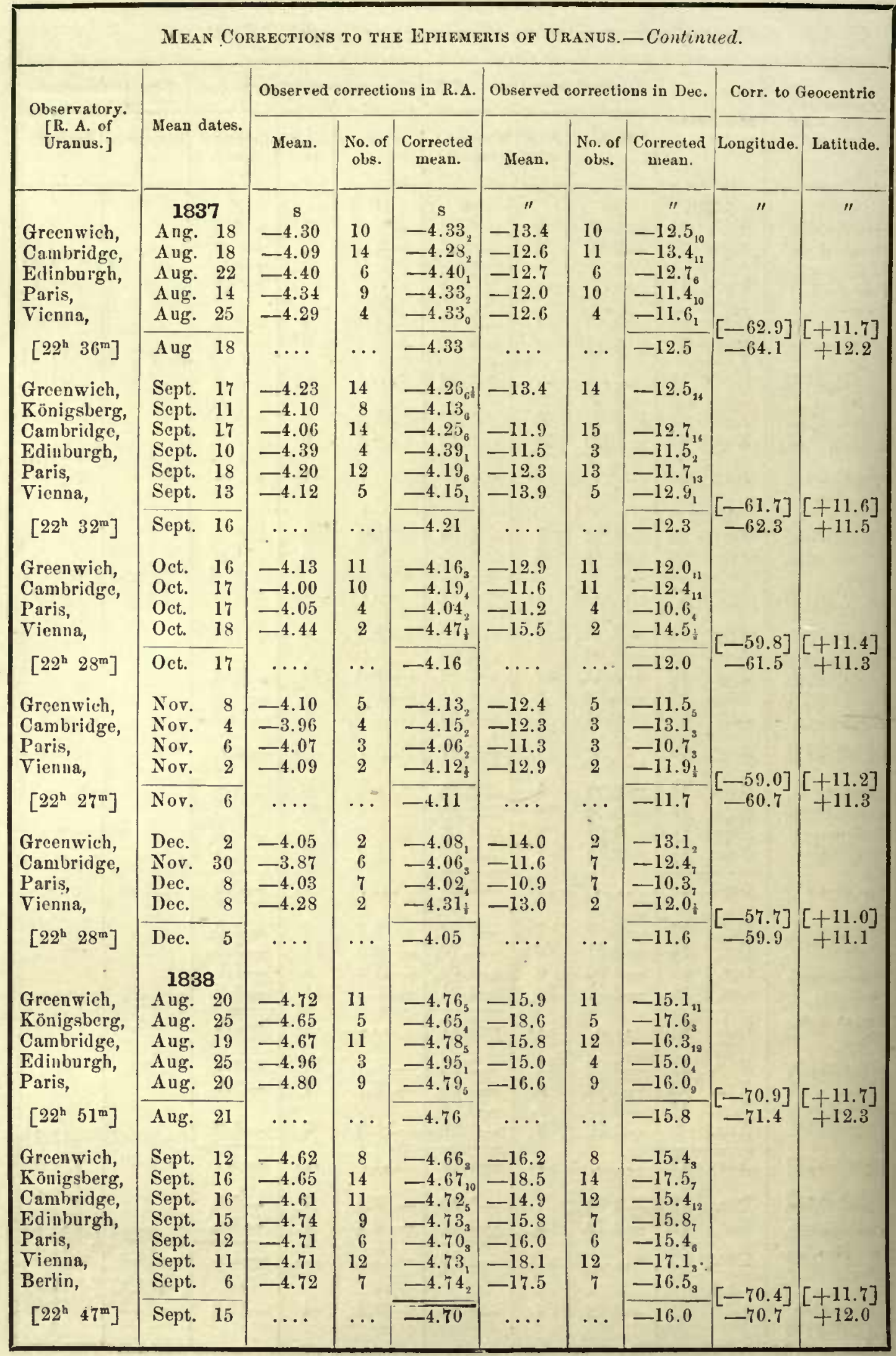




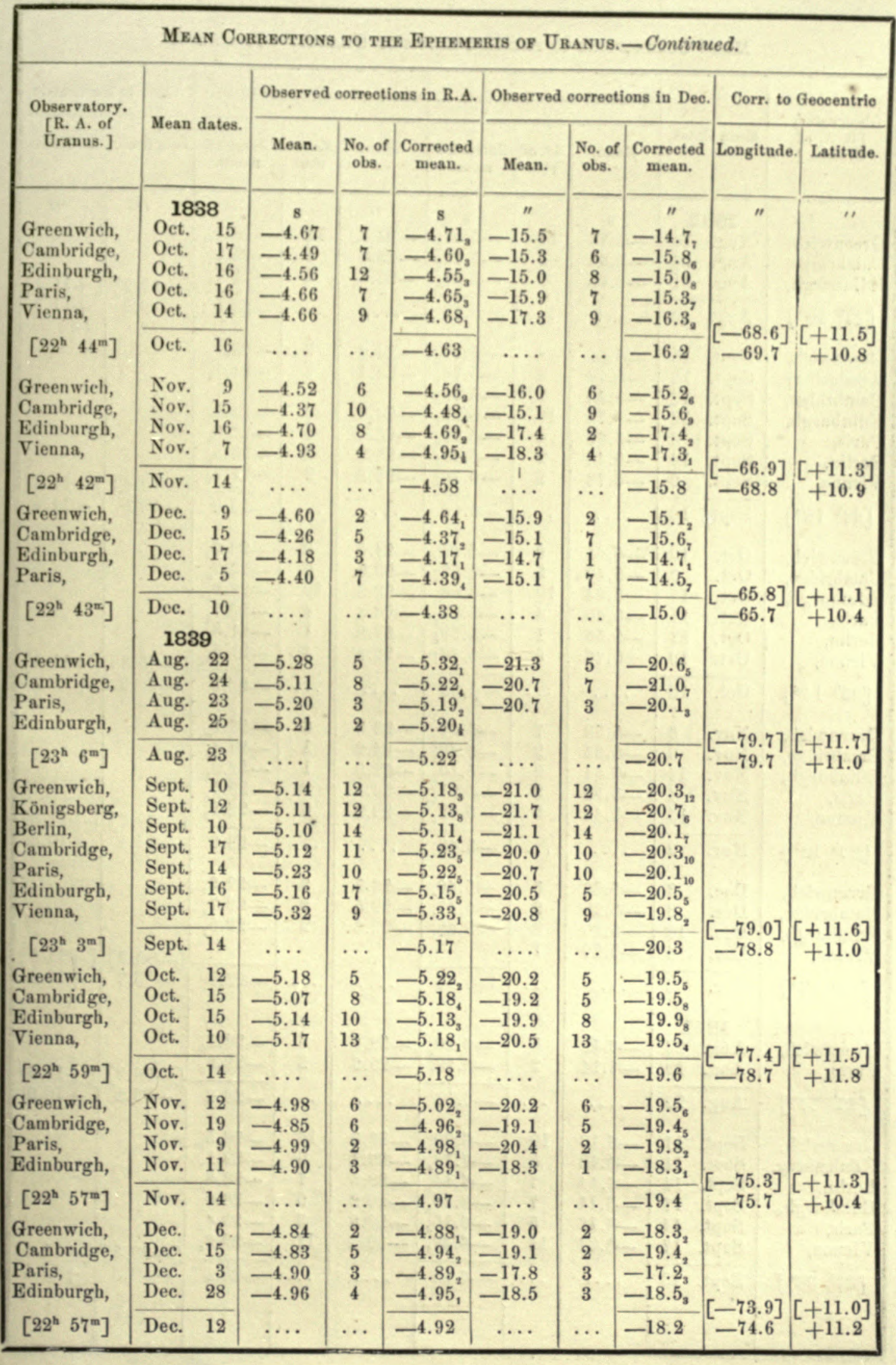




\begin{tabular}{|c|c|c|c|c|c|c|c|c|c|}
\hline \multicolumn{10}{|c|}{ Mean Corrections to the Epheneris of Uranus.-Continued. } \\
\hline \multirow{2}{*}{$\begin{array}{l}\text { Observatory. } \\
\text { [R. A. of } \\
\text { Uranus.] }\end{array}$} & \multirow[b]{2}{*}{ Mean dates. } & \multicolumn{3}{|c|}{ Observed corrections in R. A. } & \multicolumn{3}{|c|}{ Observed corrections in Dec. } & \multicolumn{2}{|c|}{ Corr. to Geocentric } \\
\hline & & Mean. & $\begin{array}{c}\text { No. of } \\
\text { obs. }\end{array}$ & $\begin{array}{c}\text { Corrected } \\
\text { mean. }\end{array}$ & Mean. & $\begin{array}{c}\text { No. of } \\
\text { obs. }\end{array}$ & $\begin{array}{c}\text { Corrected } \\
\text { neau. }\end{array}$ & Longitude. & Latitude. \\
\hline $\begin{array}{l}\text { Greenwich, } \\
\text { Cambridge, } \\
\text { Edinburgh, }\end{array}$ & \begin{tabular}{ll}
\multicolumn{2}{c}{1840} \\
Aug. 14 \\
Allg. 14 \\
Aug. & 31 \\
\end{tabular} & $\begin{array}{c}\mathrm{s} \\
-5.77 \\
-5.64 \\
-5.65\end{array}$ & $\begin{array}{r}11 \\
1 \\
1\end{array}$ & $\begin{array}{c}\mathrm{s} \\
-5.81_{3} \\
-5.73_{1} \\
-5.64_{1} \\
\end{array}$ & $\begin{array}{r}\prime \prime \\
-24.5 \\
-25.0 \\
-23.8\end{array}$ & $\begin{array}{r}11 \\
1 \\
1\end{array}$ & $\begin{array}{r}\prime \prime \\
-23.9_{11} \\
-25.2_{1} \\
-23.7_{1} \\
\end{array}$ & {$[-87.9]$} & {$[+11.4]$} \\
\hline$\left[\begin{array}{ll}23^{\mathrm{h}} & 20^{\mathrm{m}}\end{array}\right]$ & Aug. 16 & $\ldots$ & $\ldots$ & -5.78 & $\ldots$ & $\cdots$ & -23.9 & -88.8 & +1 \\
\hline $\begin{array}{l}\text { Greenwich, } \\
\text { Königsberg, } \\
\text { Cambridge, } \\
\text { Edinburgh, } \\
\text { Paris, } \\
\text { Berlin, } \\
\text { Vienna, }\end{array}$ & $\begin{array}{lr}\text { Scpt. } & 15 \\
\text { Sept. } & 13 \\
\text { Sept. } & 12 \\
\text { Sept. } & 16 \\
\text { Sept. } & 6 \\
\text { Sept. } & 14 \\
\text { Sept. } & 9 \\
\end{array}$ & $\begin{array}{l}-5.66 \\
-5.74 \\
-5.43 \\
-5.70 \\
-5.60 \\
-5.45 \\
-5.76\end{array}$ & $\begin{array}{r}8 \\
6 \\
1 \\
13 \\
4 \\
2 \\
2\end{array}$ & $\begin{array}{l}-5.70_{3} \\
-5.76_{4} \\
-5.52_{1} \\
-5.69_{4} \\
-5.59_{2} \\
-5.45_{1} \\
-5.76_{4} \\
\end{array}$ & $\begin{array}{l}-24.0 \\
-25.1 \\
-24.6 \\
-23.6 \\
-26.3 \\
-22.2\end{array}$ & $\begin{array}{l}8 \\
6 \\
\\
9 \\
4 \\
2 \\
2\end{array}$ & $\begin{array}{l}-23.4_{8} \\
-24.1_{3} \\
-24.5_{9} \\
-23.0_{4} \\
-25.3_{1} \\
-21.2_{1}\end{array}$ & & \\
\hline$\left[\begin{array}{ll}23^{\mathrm{h}} & 18^{\mathrm{m}}\end{array}\right]$ & Sept. 13 & . & $\ldots$ & -5.69 & 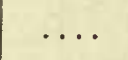 & $\cdots$ & -23.8 & -87.5 & +11.4 \\
\hline $\begin{array}{l}\text { Greenwich, } \\
\text { Cambridge, } \\
\text { Edinburgh, } \\
\text { Paris, } \\
\text { Bcrlin, } \\
\text { Vienna, }\end{array}$ & $\begin{array}{lr}\text { Oct. } & 10 \\
\text { Oct. } & 9 \\
\text { Oct. } & 15 \\
\text { Oct. } & 11 \\
\text { Oct. } & 27 \\
\text { Oct. } & 19 \\
\end{array}$ & $\begin{array}{l}-5.61 \\
-5.49 \\
-5.64 \\
-5.60 \\
-5.50 \\
-5.76\end{array}$ & $\begin{array}{r}9 \\
5 \\
12 \\
4 \\
1 \\
5\end{array}$ & $\begin{array}{l}-5.65_{3} \\
-5.58_{3} \\
-5.63_{3} \\
-5.59_{2} \\
-5.50_{4}^{4} \\
-5.76_{1}\end{array}$ & $\begin{array}{l}-24.5 \\
-23.5 \\
-23.3 \\
-23.3 \\
-22.8 \\
-22.5\end{array}$ & $\begin{array}{r}9 \\
5 \\
10 \\
4 \\
1 \\
4\end{array}$ & $\begin{array}{l}-23.9_{9} \\
-23.7_{5} \\
-23.2_{10} \\
-22.7_{4} \\
-21.8_{1} \\
-21.5_{1}\end{array}$ & , & 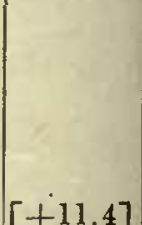 \\
\hline$\left[\begin{array}{ll}23^{\mathrm{b}} & 14^{\mathrm{m}}\end{array}\right]$ & Oct. $\quad 12$ & & $\ldots$ & -5.62 & 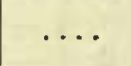 & $\cdots$ & -23.3 & -86.3 & \\
\hline $\begin{array}{l}\text { Greenwich, } \\
\text { Cambridgc, } \\
\text { Edinburgh, } \\
\text { Paris, } \\
\text { Vienna, }\end{array}$ & $\begin{array}{lr}\text { Nov. } & 6 \\
\text { Nov. } & 3 \\
\text { Nov. } & 17 \\
\text { Nov. } & 4 \\
\text { Nov. } & 15 \\
\end{array}$ & $\begin{array}{r}-5.58 \\
-5.34 \\
--5.41 \\
-5.52 \\
-5.42\end{array}$ & $\begin{array}{l}7 \\
2 \\
8 \\
2 \\
6\end{array}$ & $\begin{array}{r}-5.62_{\mathrm{a}} \\
-5.43_{1} \\
-5.40_{\mathrm{g}} \\
-5.51_{1} \\
-5.42_{1} \\
\end{array}$ & $\begin{array}{l}-23.5 \\
-24.2 \\
-22.9 \\
-26.1 \\
-23.2\end{array}$ & $\begin{array}{l}6 \\
1 \\
1 \\
2 \\
6\end{array}$ & $\begin{array}{l}-22.9_{6} \\
-24.4_{1} \\
-22.8_{1} \\
-25.5_{2} \\
-22.2_{2} \\
\end{array}$ & & \\
\hline$\left[\begin{array}{ll}23^{\mathrm{h}} & 12^{\mathrm{m}}\end{array}\right]$ & Nor. 9 & & $\cdots$ & -5.48 & & .. & -23.3 & -84.4 & \\
\hline $\begin{array}{l}\text { Greenwich, } \\
\text { Canibridge, } \\
\text { Edinburgh, } \\
\text { Vienna, }\end{array}$ & $\begin{array}{lr}\text { Dec. } & 3 \\
\text { Dec. } & 3 \\
\text { Dec. } & 15 \\
\text { Dec. } & 4 \\
\end{array}$ & $\begin{array}{r}-5.36 \\
-5.38 \\
-5.49 \\
-5.50\end{array}$ & $\begin{array}{l}8 \\
2 \\
3 \\
1\end{array}$ & $\begin{array}{l}-5.40_{2} \\
-5.47_{1} \\
-5.48_{1}\end{array}$ & $\begin{array}{l}-23.5 \\
-22.5 \\
-22.6\end{array}$ & $\begin{array}{l}9 \\
2 \\
2\end{array}$ & $\begin{array}{l}-22.9_{9} \\
-22.7_{2} \\
-22.5_{2}\end{array}$ & & \\
\hline$\left[\begin{array}{ll}23^{h} & 12^{\mathrm{m}}\end{array}\right]$ & $\begin{array}{l}\text { Dec. } 6 \\
1841\end{array}$ & & .. & -5.44 & & $\cdots$ & -22.8 & {$\left[\begin{array}{l}-82.2 \\
-83.7\end{array}\right.$} & +10.4 \\
\hline $\begin{array}{l}\text { Greenwich, } \\
\text { Paris, }\end{array}$ & $\begin{array}{ll}\text { Aug. } & 20 \\
\text { Aug. } 19 \\
\end{array}$ & $\begin{array}{l}-6.16 \\
-6.14\end{array}$ & $\begin{array}{l}5 \\
2\end{array}$ & $\begin{array}{l}-6.20_{2} \\
-6.13_{1} \\
\end{array}$ & $\begin{array}{l}-28.6 \\
-29.6\end{array}$ & $\begin{array}{l}5 \\
2\end{array}$ & $\begin{array}{l}-28.1_{3} \\
-29.0_{2} \\
\end{array}$ & & \\
\hline$\left[23^{\mathrm{h}} 37^{\mathrm{m}}\right]$ & Aug. 20 & & $\cdots$ & -6.18 & & $\cdots$ & -28.3 & -96.2 & +10.6 \\
\hline $\begin{array}{l}\text { Greenwich, } \\
\text { Königsberg, } \\
\text { Berlin, } \\
\text { Edinburgh, } \\
\text { Paris, } \\
\text { Vienna, }\end{array}$ & $\begin{array}{ll}\text { Sept. } & 11 \\
\text { Sept. } & 11 \\
\text { Sept. } & 14 \\
\text { Sept. } & 17 \\
\text { Sept. } & 14 \\
\text { Sept. } & 17 \\
\end{array}$ & $\begin{array}{l}-6.16 \\
-6.18 \\
-6.14 \\
-6.11 \\
-6.16 \\
-6.37\end{array}$ & $\begin{array}{r}10 \\
5 \\
7 \\
7 \\
5 \\
11\end{array}$ & $\begin{array}{l}-6.20_{4} \\
-6.18^{4} \\
-6.14_{3}^{2} \\
-6.15_{2} \\
-6.15_{3} \\
-6.37_{1} \\
\end{array}$ & $\begin{array}{l}-29.0 \\
-30.1 \\
-29.6 \\
-28.2 \\
-29.0 \\
-30.3\end{array}$ & $\begin{array}{r}10 \\
5 \\
7 \\
5 \\
3 \\
11\end{array}$ & $\begin{array}{l}-28.5_{10} \\
-29.1_{2} \\
-28.6_{3} \\
-28.0_{5} \\
-28.4_{3} \\
-29.3_{3} \\
\end{array}$ & - & {$[+1$} \\
\hline$\left[\begin{array}{ll}23^{\mathrm{h}} & 33^{\mathrm{m}}\end{array}\right]$ & Sept. 13 & & & -6.18 & $\cdots$ & $\cdots$ & -28.5 & -96.3 & +10.4 \\
\hline
\end{tabular}


THE ORBIT OF URANUS.

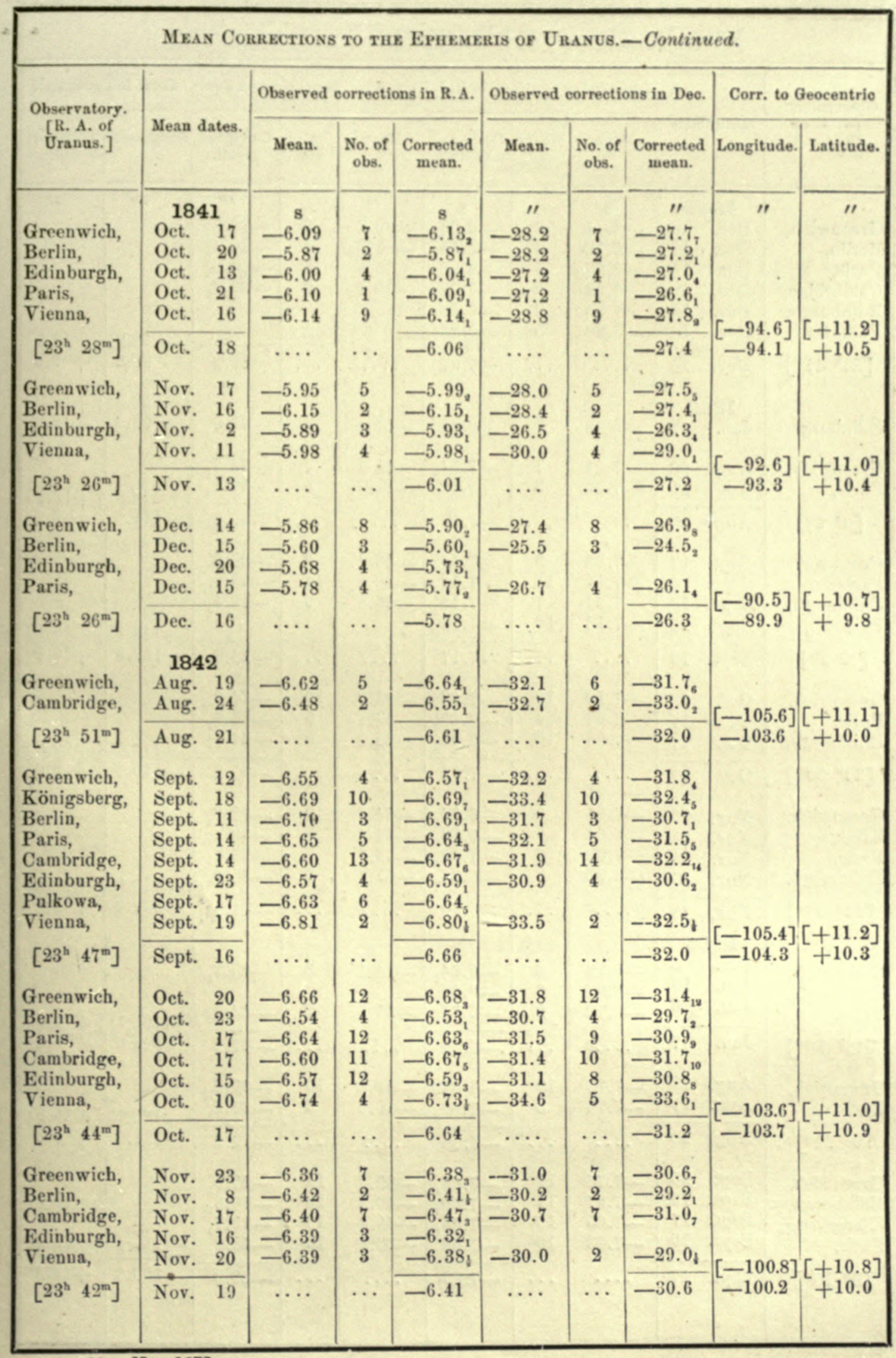




\begin{tabular}{|c|c|c|c|c|c|c|c|c|c|}
\hline \multirow{3}{*}{$\begin{array}{l}\text { Observatory. } \\
\text { [R. A. of } \\
\text { Uranus.] }\end{array}$} & \multicolumn{9}{|c|}{ Mean Corrections to the Ephemeris of Uranus. - Continued. } \\
\hline & \multirow{2}{*}{ Mean dates. } & \multicolumn{3}{|c|}{ Observed corrections in R.A. } & \multicolumn{3}{|c|}{ Observed corrections in Dec. } & \multicolumn{2}{|c|}{ Corr. to Geocentric } \\
\hline & & Mean. & $\begin{array}{c}\text { No. of } \\
\text { obs. }\end{array}$ & $\begin{array}{c}\text { Corrected } \\
\text { mean. }\end{array}$ & Mean. & $\begin{array}{l}\text { No. of } \\
\text { ous. }\end{array}$ & $\begin{array}{c}\text { Corrected } \\
\text { mean. }\end{array}$ & Longitude. & Latitnde. \\
\hline $\begin{array}{l}\text { Greenwich, } \\
\text { Berlin, } \\
\text { Paris, } \\
\text { Cambridge, } \\
\text { Edinburgh, } \\
\text { Pulkowa, }\end{array}$ & \begin{tabular}{|cc|}
\multicolumn{2}{c|}{1842} \\
Dec. & 16 \\
Dec. & 10 \\
Dec. & 14 \\
Dee. & 16 \\
Dec. & 13 \\
Dec. & 13 \\
\end{tabular} & $\begin{array}{c}\mathrm{s} \\
-6.31 \\
-6.16 \\
-6.23 \\
-6.22 \\
-6.25 \\
-6.28\end{array}$ & $\begin{array}{l}8 \\
4 \\
5 \\
9 \\
2 \\
3\end{array}$ & $\begin{array}{c}\mathrm{s} \\
-6.33_{3} \\
-6.15_{1} \\
-6.22_{3} \\
-6.29_{4} \\
-6.18_{1} \\
-6.28_{2} \\
\end{array}$ & $\begin{array}{r}\prime \prime \\
-30.2 \\
-31.4 \\
-30.9 \\
-30.1\end{array}$ & $\begin{array}{l}8 \\
4 \\
5 \\
8\end{array}$ & $\begin{array}{c}\prime \prime \\
-29.8_{8} \\
-30.4_{9} \\
-30.3_{3} \\
-30.4_{8}\end{array}$ & $\prime \prime$ & $\prime \prime$ \\
\hline$\left[\begin{array}{ll}23^{\mathrm{h}} & 41^{\mathrm{m}}\end{array}\right]$ & Dec. 15 & & $\cdots$ & -6.27 & & $\therefore$ & -30.2 & -98.2 & +9.6 \\
\hline Edinburgh, & $\begin{array}{c}1843 \\
\operatorname{Jan} .\end{array}$ & -6.32 & 7 & -6.25 & -31.4 & 4 & -31.0 & {$\left[\begin{array}{l}-97.5 \\
-98.2\end{array}\right.$} & {$\left[\begin{array}{l}+10.3] \\
+8.6\end{array}\right.$} \\
\hline $\begin{array}{l}\text { Greenwich, } \\
\text { Paris, }\end{array}$ & $\begin{array}{l}\text { Aug. } 20 \\
\text { Aug. } 21\end{array}$ & $\begin{array}{r}-7.11 \\
-7.20\end{array}$ & $\begin{array}{l}7 \\
3\end{array}$ & $\begin{array}{l}-7.04_{3} \\
-7.19_{9}\end{array}$ & $\begin{array}{l}-35.1 \\
-37.5\end{array}$ & $\begin{array}{l}7 \\
5\end{array}$ & $\begin{array}{l}-34.7_{4} \\
-37.1_{5}\end{array}$ & & \\
\hline$\left[\begin{array}{ll}0^{\mathrm{h}} & 6^{\mathrm{m}}\end{array}\right]$ & Aug. 20 & & $\cdots$ & -7.10 & & .. & -35.7 & -111.9 & +9.6 \\
\hline $\begin{array}{l}\text { Greenwich, } \\
\text { Paris, } \\
\text { Edinburgh, } \\
\text { Pulkowa, }\end{array}$ & $\begin{array}{ll}\text { Sept. } & 17 \\
\text { Sept. } & 15 \\
\text { Sept. } & 18 \\
\text { Sept. } & 22\end{array}$ & $\begin{array}{l}-7.23 \\
-7.17 \\
-7.26 \\
-7.18\end{array}$ & $\begin{array}{r}9 \\
11 \\
7 \\
10\end{array}$ & $\begin{array}{l}-7.16_{3} \\
-7.16_{6} \\
-7.19_{2} \\
-7.18_{10}\end{array}$ & $\begin{array}{l}-35.4 \\
-36.8 \\
-36.8 \\
\end{array}$ & $\begin{array}{r}9 \\
14 \\
5\end{array}$ & $\begin{array}{l}-35.0_{3} \\
-36.2_{14} \\
-36.4_{3}\end{array}$ & & \\
\hline$\left[\begin{array}{ll}0^{\mathrm{h}} & 3^{\mathrm{m}}\end{array}\right]$ & Sept. 19 & & $\cdots$ & -7.17 & & $\ldots$ & -35.8 & -112.9 & +10.0 \\
\hline $\begin{array}{l}\text { Greenwich, } \\
\text { Paris, } \\
\text { Edinburgh, }\end{array}$ & $\begin{array}{ll}\text { Oct. } & 18 \\
\text { Oct. } & 17 \\
\text { Oct. } & 17\end{array}$ & $\begin{array}{r}-7.10 \\
-7.07 \\
-7.05\end{array}$ & $\begin{array}{r}10 \\
6 \\
12\end{array}$ & $\begin{array}{r}-7.03_{3} \\
-7.06_{3} \\
-6.98_{3} \\
\end{array}$ & $\begin{array}{r}-35.3 \\
-37.1 \\
-35.1\end{array}$ & $\begin{array}{r}10 \\
5 \\
5\end{array}$ & $\begin{array}{l}-34.9_{10} \\
-36.5_{5} \\
-34.7_{5} \\
\end{array}$ & .67 & {$[+10.5]$} \\
\hline$\left[\begin{array}{ll}23^{\mathrm{b}} & 59^{\mathrm{m}}\end{array}\right]$ & Oct. 17 & $\cdots$ & $\cdots$ & -7.02 & 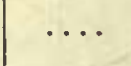 & $\cdots$ & -35.2 & -110.6 & +9.6 \\
\hline $\begin{array}{l}\text { Greenwich, } \\
\text { Königsberg, } \\
\text { Paris, } \\
\text { Edinburgh, }\end{array}$ & $\begin{array}{ll}\text { Nov. } & 20 \\
\text { Nov. } & 11 \\
\text { Nov. } & 15 \\
\text { Nov. } & 15 \\
\end{array}$ & $\begin{array}{l}-7.01 \\
-6.84 \\
-7.03 \\
-6.93\end{array}$ & $\begin{array}{l}9 \\
5 \\
3 \\
8\end{array}$ & $\begin{array}{r}-6.94_{3} \\
-6.83_{4} \\
-7.02_{3} \\
-6.86_{2} \\
\end{array}$ & $\begin{array}{l}-34.3 \\
-34.4 \\
-36.3 \\
-35.7\end{array}$ & $\begin{array}{l}9 \\
5 \\
3 \\
6\end{array}$ & $\begin{array}{r}-33.9_{9} \\
-33.4_{3} \\
-35.7_{3} \\
-35.3_{6} \\
\end{array}$ & {$[-]$} & {$[+10.4]$} \\
\hline$\left[\begin{array}{ll}23^{\mathrm{h}} & 56^{\mathrm{m}}\end{array}\right]$ & $\begin{array}{c}\text { Nov. } 15 \\
1844\end{array}$ & & $\cdots$ & -6.90 & & - & -34.5 & -108.6 & +9.5 \\
\hline $\begin{array}{l}\text { Greenwich, } \\
\text { Edinburgl, } \\
\text { Paris, }\end{array}$ & $\begin{array}{lr}\operatorname{Jan} . & 3 \\
\operatorname{Jan} . & 10 \\
\operatorname{Jan} . & 3 \\
\end{array}$ & $\begin{array}{l}-6.70 \\
-6.67 \\
-6.72\end{array}$ & $\begin{array}{l}3 \\
6 \\
2\end{array}$ & $\begin{array}{l}-6.64_{1} \\
-6.60_{2} \\
-6.71_{1}\end{array}$ & $\begin{array}{l}-34.1 \\
-35.5\end{array}$ & $\begin{array}{l}3 \\
2\end{array}$ & $\begin{array}{l}-33.7_{3} \\
-34.9_{2} \\
\end{array}$ & & \\
\hline$\left[\begin{array}{ll}23^{\mathrm{h}} & 56^{\mathrm{m}}\end{array}\right]$ & Jan. 7 & ( & $\cdots$ & -6.64 & & 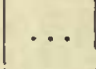 & -34.2 & -105.0 & +8.2 \\
\hline $\begin{array}{l}\text { Grcenwich, } \\
\text { Paris, }\end{array}$ & $\begin{array}{ll}\text { Aug. } & 19 \\
\text { Aug. } & 17 \\
\end{array}$ & $\begin{array}{l}-7.73 \\
-7.70\end{array}$ & $\begin{array}{r}10 \\
3\end{array}$ & $\begin{array}{r}-7.67_{3} \\
-7.69^{3}\end{array}$ & $\begin{array}{l}-39.6 \\
-40.0\end{array}$ & $\begin{array}{r}10 \\
3\end{array}$ & $\begin{array}{l}-39.2_{10} \\
-39.4_{3}\end{array}$ & & \\
\hline$\left[\begin{array}{ll}0^{\mathrm{h}} & \left.22^{\mathrm{m}}\right]\end{array}\right.$ & Aug. 18 & & . & -7.68 & & $\cdots$ & -39.2 & -121.2 & +9.6 \\
\hline $\begin{array}{l}\text { Greenwich, } \\
\text { Edinburgh, } \\
\text { Königsberg, } \\
\text { Paris, }\end{array}$ & $\begin{array}{ll}\text { Sept. } & 25 \\
\text { Sept. } & 19 \\
\text { Sept. } & 17 \\
\text { Sept. } & 10\end{array}$ & $\begin{array}{l}-7.74 \\
-7.67 \\
-7.65 \\
--7.63\end{array}$ & $\begin{array}{l}11 \\
11 \\
10 \\
10\end{array}$ & $\begin{array}{r}-7.68_{4} \\
-7.60_{3} \\
-7.64_{4} \\
-7.62_{5} \\
\end{array}$ & $\begin{array}{l}-39.9 \\
-40.3 \\
-40.7 \\
-39.4\end{array}$ & $\begin{array}{l}11 \\
10 \\
10 \\
15\end{array}$ & $\begin{array}{r}-39.5_{11} \\
-39.8_{10} \\
-39.7_{5} \\
-38.8_{15} \\
\end{array}$ & {$\left[\begin{array}{c}\cdot \\
{[-122.6]}\end{array}\right.$} & {$[+10.3]$} \\
\hline$\left[\begin{array}{ll}0^{\mathrm{h}} & 18^{\mathrm{m}}\end{array}\right]$ & Sept. 17 & $\cdots$ & $\cdots$ & -7.63 & $\because$ & $\cdots$ & -39.3 & $\rightarrow 120.6$ & +9.3 \\
\hline
\end{tabular}




\begin{tabular}{|c|c|c|c|c|c|c|c|c|c|}
\hline \multirow{3}{*}{$\begin{array}{c}\text { Observatory. } \\
\text { [R. A. of } \\
\text { Uranus.] }\end{array}$} & \multicolumn{9}{|c|}{ Mran Corrections to the Ephemeris of Uranus.-Continued. } \\
\hline & \multirow{2}{*}{ Mean dates. } & \multicolumn{3}{|c|}{ Observed corrections in R.A. } & \multicolumn{3}{|c|}{ Observed corrections in Dec. } & \multicolumn{2}{|c|}{ Corr. to Geocentrio } \\
\hline & & Mean. & $\begin{array}{c}\text { No. of } \\
\text { obs. }\end{array}$ & $\begin{array}{c}\text { Corrected } \\
\text { mean. }\end{array}$ & Mean. & $\begin{array}{c}\text { No.of } \\
\text { obs. }\end{array}$ & $\begin{array}{c}\text { Corrected } \\
\text { mean. }\end{array}$ & Longitude. & Latitude. \\
\hline \multirow{4}{*}{$\begin{array}{c}\text { Greenwieh, } \\
\text { Edinburgh, } \\
{\left[\begin{array}{ll}0^{\mathrm{h}} & 15^{\mathrm{m}}\end{array}\right]}\end{array}$} & 1844 & $\mathbf{s}$ & & & $n$ & & $"$ & $"$ & " \\
\hline & Oet. 17 & -7.70 & 9 & $-7.64_{3}$ & -39.2 & 9 & -38.8 & & \\
\hline & Oet. 13 & -7.58 & 10 & $-7.51_{2}$ & -4 & 1 & $-40.0_{1}$ & {$[-121.5]$} & {$[+10.2]$} \\
\hline & Oet. 15 & $\cdots$ & $\cdots$ & -7.59 & $\cdots$ & $\cdots$ & -39.0 & -120.0 & +9.4 \\
\hline \multirow{2}{*}{$\begin{array}{r}\text { Greenwieh, } \\
\text { Edinburgh, } \\
{\left[\begin{array}{ll}0^{\mathrm{b}} & 12^{\mathrm{m}}\end{array}\right]}\end{array}$} & $\begin{array}{ll}\text { Nov. } & 26 \\
\text { Nov. } & 19\end{array}$ & $\begin{array}{r}-7.44 \\
-7.41\end{array}$ & $\begin{array}{l}9 \\
7\end{array}$ & $\begin{array}{l}-7.38_{\mathrm{s}} \\
-7.35_{\mathrm{a}}\end{array}$ & -38.7 & 9 & $-38.3_{9}$ & & \\
\hline & Nov. 23 & & & -7.37 & 政 & & -38.3 & {$\left[\begin{array}{l}-118.2 \\
-116.6\end{array}\right]$} & $\begin{array}{c}{[+9.9]} \\
+8.8\end{array}$ \\
\hline \multirow{3}{*}{$\begin{array}{l}\text { Edinburgh, } \\
\text { Paris, } \\
\qquad\left[\begin{array}{ll}0^{\mathrm{h}} & 10^{\mathrm{m}}\end{array}\right]\end{array}$} & Dec. 18 & -7.24 & 5 & & & 0 & & & \\
\hline & Dee. 22 & -7.10 & 2 & $-7.09_{1}$ & -39.3 & 3 & $-38.7_{3}$ & {$[-115.7]$} & {$[+9.6]$} \\
\hline & Dec. 20 & $\cdots$ & $\cdots$ & -7.14 & $\cdots$ & . & -38.7 & -113.7 & +7.1 \\
\hline \multirow{3}{*}{$\begin{array}{c}\text { Greenwich, } \\
\text { Edinburgh, } \\
{\left[\begin{array}{lll}0^{\mathrm{b}} & 11^{\mathrm{m}}\end{array}\right]}\end{array}$} & 1845 & & & & & & & & \\
\hline & $\begin{array}{ll}\text { Jan. } & 13 \\
\text { Jan. } & 14\end{array}$ & $\begin{array}{l}-7.18 \\
-7.16\end{array}$ & $\begin{array}{l}1 \\
8\end{array}$ & $\begin{array}{l}-7.10_{1} \\
-7.08_{4}\end{array}$ & -38.4 & 1 & -38.0 & & \\
\hline & Jan. $\quad 14$ & & . & -7.08 & $\cdots$ & .. & -38.0 & -112.5 & $\begin{array}{l}1+7.3 \\
+73\end{array}$ \\
\hline \multirow{5}{*}{$\begin{array}{l}\text { Greenwieh, } \\
\text { Greenwich, } \\
\text { Königsberg, } \\
\text { Paris, } \\
{\left[\begin{array}{ll}0^{\mathrm{h}} & 33^{\mathrm{m}}\end{array}\right]}\end{array}$} & Ang. 25 & -8.25 & 5 & $-8.17_{2}$ & -42.7 & 6 & -42.3 & {$\left[\begin{array}{c}-131.0 \\
-129.1\end{array}\right]$} & $\begin{array}{c}{[+9.6]} \\
+9.2\end{array}$ \\
\hline & Sept. 18 & -8.21 & 10 & $-8.13_{i}$ & -42.6 & 10 & $-42.2_{10}$ & & \\
\hline & Sept. 30 & -8 & 5 & -8.15 & -43.4 & 5 & -42 & & \\
\hline & Sept. 14 & -8.24 & 5 & $-8.23_{3}$ & -43.9 & 3 & $-43.3_{3}$ & {$\left[\begin{array}{lll}-131 & 6\end{array}\right]$} & {$[+9.9]$} \\
\hline & Sept. 22 & $\cdots$ & $\cdots$ & -8.17 & $\cdots$ & $\cdots$ & -42.5 & -129.2 & +9.1 \\
\hline \multirow{2}{*}{$\begin{array}{l}\text { Greenwich, } \\
\text { Paris, } \\
\qquad\left[\begin{array}{ll}0^{\mathrm{h}} & 29^{\mathrm{m}}\end{array}\right]\end{array}$} & $\begin{array}{ll}\text { Oct. } & 17 \\
\text { Oct. } & 20\end{array}$ & $\begin{array}{l}-8.22 \\
-8.01\end{array}$ & $\begin{array}{r}10 \\
6\end{array}$ & $\begin{array}{l}-8.14_{4} \\
-8.00_{3}\end{array}$ & $\begin{array}{l}-42.2 \\
-43.7\end{array}$ & $\begin{array}{r}10 \\
6\end{array}$ & $\begin{array}{l}-41.8_{10} \\
-43.1_{6}\end{array}$ & & \\
\hline & Oet. 18 & $\ldots$ & $\ldots$ & -8.08 & $\ldots$ & $\because$ & -42.3 & {$\left[\begin{array}{c}-130.2] \\
-128.0\end{array}\right.$} & {$\left[\begin{array}{l}+9.8] \\
+8.9\end{array}\right.$} \\
\hline \multirow{2}{*}{$\begin{array}{c}\text { Greenwich, } \\
{\left[\begin{array}{ll}0^{\mathrm{h}} & 27^{\mathrm{m}}\end{array}\right]}\end{array}$} & Nov. 9 & -8.06 & 6 & -7.98 & -43.3 & 7 & -42.9 & {$[-128.4]$} & {$[+9.6]$} \\
\hline & & & & & $\cdots$ & $\cdots$ & & -126.9 & L \\
\hline \multirow{2}{*}{$\begin{array}{c}\text { Greenwich, } \\
{\left[\begin{array}{ll}0^{\mathrm{h}} & 25^{\mathrm{m}}\end{array}\right]}\end{array}$} & Dee. 14 & -7.85 & 8 & -7.77 & -49.2 & 8 & -39.8 & {$[-124.8]$} & {$[+9.3]$} \\
\hline & $\cdots \cdots$ & & $\cdots$ & & & $\cdots$ & $\cdots$ & & $\begin{array}{l}+9 . \\
\end{array}$ \\
\hline \multirow{3}{*}{$\begin{array}{l}\text { Greenwieh, } \\
\text { Paris, } \\
{\left[\begin{array}{ll}0^{\mathrm{h}} & 50^{\mathrm{m}}\end{array}\right]}\end{array}$} & $\begin{array}{l}1846 \\
\text { Sept. }\end{array}$ & -8.80 & 8 & $-8.76_{3}$ & -46.2 & 8 & $-45.8_{8}$ & & \\
\hline & Sept. 12 & -8.73 & 19 & & -46.9 & 14 & & {$[-1$} & {$[+9.2]$} \\
\hline & Sept. 11 & & & -8.73 & & . & -46.1 & -138.2 & +8.3 \\
\hline \multirow{3}{*}{$\begin{array}{l}\text { Greenwich, } \\
\text { Königsberg, } \\
\text { Paris, } \\
{\left[\begin{array}{ll}0^{\mathrm{h}} & 46^{\mathrm{m}}\end{array}\right]}\end{array}$} & Oct. $\quad 8$ & -8.74 & 7 & $-8.70_{3}$ & -46.8 & 7 & $\begin{array}{l}-46.4 \\
-43.8\end{array}$ & & \\
\hline & $\begin{array}{lr}\text { Oet. } & 4 \\
\text { Oet. } & 14\end{array}$ & $\begin{array}{l}-8.61 \\
-8.69\end{array}$ & $\begin{array}{l}11 \\
13\end{array}$ & $\begin{array}{l}-8.61_{8} \\
-8.68_{7}\end{array}$ & $\begin{array}{l}-44.8 \\
-46.7\end{array}$ & $\begin{array}{l}11 \\
13\end{array}$ & $-43.8_{6}$ & & \\
\hline & Oet. $\quad 9$ & & $\cdots$ & -8.65 & 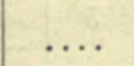 & $\cdots$ & -45.8 & {$[-137.1$} & $\begin{array}{l}+9.2] \\
+8.3\end{array}$ \\
\hline \multirow{3}{*}{$\begin{array}{l}\text { Greenwich, } \\
\text { Paris, } \\
\qquad\left[\begin{array}{ll}0^{\mathrm{h}} & 41^{\mathrm{m}}\end{array}\right]\end{array}$} & Nov. 10 & -8.62 & 6 & $-8.58_{2}$ & -46.8 & 6 & $-46.4_{6}$ & & \\
\hline & Nov. 16 & -8.48 & 0 & & -46.4 & 9 & & {$[-136.8]$} & {$[+9.0]$} \\
\hline & Nov. 14 & $\cdots$ & $\cdots$ & -8.50 & $\cdots$ & . & -46.0 & -135.1 & +7.6 \\
\hline
\end{tabular}




\begin{tabular}{|c|c|c|c|c|c|c|c|c|c|}
\hline \multirow{2}{*}{$\begin{array}{c}\text { Observatory. } \\
\text { [R. A. of } \\
\text { Uravus.] }\end{array}$} & \multirow[b]{2}{*}{ Mean dates. } & \multicolumn{3}{|c|}{ Observed corrections in R.A. } & \multicolumn{3}{|c|}{ Observed corrections in Dec. } & \multicolumn{2}{|c|}{ Corr. to Geocentric } \\
\hline & & Mean. & $\begin{array}{l}\text { No. of } \\
\text { olss. }\end{array}$ & $\begin{array}{c}\text { Corrected } \\
\text { weau. }\end{array}$ & Mean. & $\begin{array}{l}\text { No.of } \\
\text { olss. }\end{array}$ & $\begin{array}{c}\text { Corrected } \\
\text { mean. }\end{array}$ & Longitude. & Latitude. \\
\hline $\begin{array}{l}\text { Greenwich, } \\
\text { Paris, }\end{array}$ & 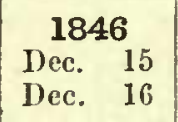 & $\begin{array}{c}\mathrm{s} \\
-8.31 \\
-8.25\end{array}$ & $\begin{array}{l}9 \\
6\end{array}$ & $\begin{array}{c}\mathrm{s} \\
-8.27_{3} \\
-8.24_{3}\end{array}$ & $\begin{array}{c}\prime \prime \\
-46.0 \\
-46.1\end{array}$ & $\begin{array}{r}10 \\
4\end{array}$ & $\begin{array}{c}\prime \prime \\
-45.6_{10} \\
-45.5^{4}\end{array}$ & " & " \\
\hline$\left[\begin{array}{ll}0^{\mathrm{h}} & 39^{\mathrm{m}}\end{array}\right]$ & $\begin{array}{c}\text { Dec. } 16 \\
1847\end{array}$ & .. & .. & -8.26 & & $\because$ & -45.6 & {$\left[\begin{array}{l}{[-134.5} \\
-131.7\end{array}\right.$} & $\begin{array}{l}8.9] \\
6.5\end{array}$ \\
\hline $\begin{array}{l}\text { Green wich, } \\
\text { Paris, }\end{array}$ & $\begin{array}{ll}\text { Jan. } & 13 \\
\text { Jan. } & 11 \\
\end{array}$ & $\begin{array}{l}-8.25 \\
-8.14\end{array}$ & $\begin{array}{r}4 \\
6\end{array}$ & $\begin{array}{r}-8.20_{2} \\
-8.12_{3} \\
\end{array}$ & $\begin{array}{l}-43.1 \\
-45.4\end{array}$ & $\begin{array}{l}4 \\
6\end{array}$ & $\begin{array}{l}-42.7_{4} \\
-44.8_{6} \\
\end{array}$ & {$[-130.9]$} & 8.67 \\
\hline$\left[\begin{array}{ll}0^{\mathrm{h}} & 40^{\mathrm{m}}\end{array}\right]$ & Jan. 12 & .. & . & -8.15 & .. & . & -44.0 & -129.6 & \\
\hline $\begin{array}{c}\text { Greenwich, } \\
{\left[\begin{array}{ll}1^{\mathrm{b}} & 6^{\mathrm{m}}\end{array}\right]}\end{array}$ & $\begin{array}{c}\text { Sept. } 3 \\
\cdots\end{array}$ & $\begin{array}{c}-9.21 \\
\ldots\end{array}$ & $\begin{array}{c}8 \\
\cdots\end{array}$ & $\begin{array}{c}-9.16 \\
\ldots\end{array}$ & $\begin{array}{c}-49.4 \\
\ldots\end{array}$ & $\begin{array}{c}8 \\
\cdots\end{array}$ & $\begin{array}{c}-49.0 \\
\ldots\end{array}$ & {$\left[\begin{array}{l}{[-148.4]} \\
-144.9\end{array}\right.$} & $\begin{array}{c}{\left[\begin{array}{l}+8.7] \\
+6.8\end{array}\right.}\end{array}$ \\
\hline $\begin{array}{l}\text { Greenwich, } \\
\text { Paris, }\end{array}$ & $\begin{array}{lr}\text { Oet. } & 1 \\
\text { Oct. } & 12 \\
\end{array}$ & $\begin{array}{r}-9.25 \\
-9.23\end{array}$ & $\begin{array}{r}6 \\
18\end{array}$ & $\begin{array}{r}-9.20_{3} \\
-9.21_{10} \\
\end{array}$ & $\begin{array}{l}-49.0 \\
-49.2\end{array}$ & $\begin{array}{r}6 \\
16\end{array}$ & $\begin{array}{r}-48.6_{6} \\
-48.6_{16} \\
\end{array}$ & $\Gamma$ & \\
\hline$\left[\begin{array}{ll}1^{\mathrm{h}} & 2^{\mathrm{m}}\end{array}\right]$ & Oet. 10 & ... & $\cdots$ & -9.21 & . & $\cdots$ & -48.6 & -145.5 & -7.8 \\
\hline $\begin{array}{l}\text { Greenwich, } \\
\text { Paris, }\end{array}$ & $\begin{array}{lr}\text { Nov. } & 3 \\
\text { Nov. } & 12\end{array}$ & $\begin{array}{r}-9.10 \\
-9.08\end{array}$ & $\begin{array}{r}9 \\
10\end{array}$ & $\begin{array}{r}-9.05_{3} \\
-9.06_{5} \\
\end{array}$ & $\begin{array}{l}-49.2 \\
-49.3\end{array}$ & $\begin{array}{l}9 \\
9\end{array}$ & $\begin{array}{l}-48.8_{y} \\
-48.7_{9} \\
\end{array}$ & {$[-146.2]$} & {$[+8.5]$} \\
\hline$\left[\begin{array}{ll}0^{\mathrm{h}} & 57^{\mathrm{m}}\end{array}\right]$ & Nov. 8 & $\ldots$ & .. & -9.06 & $\cdots$ & . & -48.8 & -142.6 & +7.2 \\
\hline $\begin{array}{l}\text { Greenwich, } \\
\text { Paris, }\end{array}$ & $\begin{array}{rr}\text { Dec. } & 3 \\
\text { Dec. } & 12 \\
\end{array}$ & $\begin{array}{l}-8.96 \\
-8.84\end{array}$ & $\begin{array}{l}7 \\
9\end{array}$ & $\begin{array}{l}-8.91_{2} \\
-8.82_{5} \\
\end{array}$ & $\begin{array}{l}-49.0 \\
-48.5\end{array}$ & $\begin{array}{l}7 \\
7\end{array}$ & $\begin{array}{r}-48.6_{7} \\
-47.9_{7} \\
\end{array}$ & {$[-$} & \\
\hline$\left[\begin{array}{ll}0^{\mathrm{h}} & 54^{\mathrm{m}} 7\end{array}\right.$ & $\begin{array}{l}\text { Dec. } \\
1848\end{array}$ & ... & $\cdots$ & -8.85 & 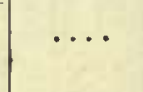 & . & -48.2 & -140.6 & \\
\hline $\begin{array}{l}\text { Greenwich, } \\
\text { Paris, }\end{array}$ & $\begin{array}{ll}\text { Jan. } & 10 \\
\text { J an. } & 11 \\
\end{array}$ & $\begin{array}{l}-8.83 \\
-8.53\end{array}$ & $\begin{array}{l}2 \\
5\end{array}$ & $\begin{array}{r}-8.78_{2} \\
-8.51_{5} \\
\end{array}$ & $\begin{array}{l}-47.1 \\
-47.6\end{array}$ & $\begin{array}{l}2 \\
5\end{array}$ & $\begin{array}{l}-46.7_{2} \\
-47.0_{5} \\
\end{array}$ & {$[-139.4]$} & {$[+8.1]$} \\
\hline$\left[\begin{array}{ll}0^{\mathrm{h}} & 54^{\mathrm{m}}\end{array}\right]$ & Jan. 11 & $\cdots$ & $\cdots$ & -8.57 & $\cdots$ & . & -46.9 & -136.2 & $\begin{array}{l}1 \\
+6.0\end{array}$ \\
\hline $\begin{array}{l}\text { Greenwich, } \\
\text { Paris, }\end{array}$ & $\begin{array}{lr}\text { Sept. } & 8 \\
\text { Sept. } & 22 \\
\end{array}$ & $\begin{array}{r}-9.84 \\
-9.82\end{array}$ & $\begin{array}{r}7 \\
11\end{array}$ & $\begin{array}{r}-9.79^{7} \\
-9.80_{11} \\
\end{array}$ & $\begin{array}{l}-51.1 \\
-51.5\end{array}$ & $\begin{array}{l}7 \\
9\end{array}$ & $\begin{array}{r}-50.7 \\
-50.9_{8} \\
\end{array}$ & . & \\
\hline$\left[\begin{array}{ll}1^{\mathrm{h}} & 19^{\mathrm{m}}\end{array}\right]$. & Sept. 17 & . & . & -9.80 & & - & -50.8 & {$\left[\begin{array}{l}{[-156.4} \\
-154.1\end{array}\right.$} & $\begin{array}{c}{\left[\begin{array}{l}+8.0 \\
+7.5\end{array}\right.} \\
+7\end{array}$ \\
\hline $\begin{array}{l}\text { Green wieh, } \\
\text { Paris, }\end{array}$ & $\begin{array}{ll}\text { Oct. } & 19 \\
\text { Oct. } & 16 \\
\end{array}$ & $\begin{array}{r}-9.82 \\
-9.79\end{array}$ & $\begin{array}{r}7 \\
11\end{array}$ & $\begin{array}{r}-9.77_{7} \\
-9.77_{11} \\
\end{array}$ & $\begin{array}{l}-52.2 \\
-52.2\end{array}$ & $\begin{array}{r}7 \\
10\end{array}$ & $\begin{array}{l}-51.8_{7} \\
-51.6_{10} \\
\end{array}$ & & \\
\hline$\left[\begin{array}{ll}1^{\mathrm{h}} & 15^{\mathrm{m}}\end{array}\right]$ & Oet. 17 & & $\cdots$ & -9.77 & & $\cdots$ & -51.7 & {$\left[\begin{array}{c}{[-156.4]} \\
-154.2\end{array}\right.$} & $\begin{array}{c}{\left[\begin{array}{l}+8.0] \\
+6.9\end{array}\right.} \\
\end{array}$ \\
\hline $\begin{array}{l}\text { Greenwich, } \\
\text { Paris, }\end{array}$ & $\begin{array}{ll}\text { Nov. } 13 \\
\text { Nov. } 13\end{array}$ & $\begin{array}{r}-9.68 \\
-9.55\end{array}$ & $\begin{array}{l}7 \\
4\end{array}$ & $\begin{array}{r}-9.63_{7} \\
-9.53_{4} \\
\end{array}$ & $\begin{array}{l}-52.3 \\
-52.2\end{array}$ & $\begin{array}{l}7 \\
3\end{array}$ & $\begin{array}{r}-51.9_{7} \\
-51.6_{3} \\
\end{array}$ & & \\
\hline$\left[\begin{array}{ll}1^{\mathrm{b}} & 11^{\mathrm{m}}\end{array}\right]$ & Nov. 13 & & & -9.59 & & . & -51.8 & {$\left[\begin{array}{l}-154.6] \\
-151.8\end{array}\right.$} & {$\left[\begin{array}{c}{[+8.0]} \\
+6.3\end{array}\right.$} \\
\hline $\begin{array}{l}\text { Greenwich, } \\
\text { Paris, }\end{array}$ & $\begin{array}{lr}\text { Dee. } & 14 \\
\text { Dee. } & 9 \\
\end{array}$ & $\begin{array}{r}-9.41 \\
-9.43\end{array}$ & $\begin{array}{l}5 \\
5\end{array}$ & $\begin{array}{r}-9.36_{5} \\
-9.41_{5} \\
\end{array}$ & $\begin{array}{r}-51.2 \\
-51.3\end{array}$ & $\begin{array}{l}5 \\
6\end{array}$ & $\begin{array}{l}-50.8_{5} \\
-50.7_{6} \\
\end{array}$ & & \\
\hline$\left[\begin{array}{ll}1^{\mathrm{h}} & 9^{\mathrm{m}}\end{array}\right]$ & $\begin{array}{c}\text { Dee. } 12 \\
1849\end{array}$ & $\ldots$ & 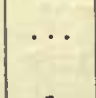 & -9.38 & & & -50.7 & {$\left[\begin{array}{l}-150.2] \\
-148.6\end{array}\right.$} & $\begin{array}{c}{[+7.7]} \\
+6.2\end{array}$ \\
\hline $\begin{array}{l}\text { Greenwich, } \\
\text { Paris, }\end{array}$ & $\begin{array}{rr}\text { Jan. } & 6 \\
\text { Jan. } & 18 \\
\end{array}$ & $\begin{array}{l}-9.37 \\
-9.11\end{array}$ & $\begin{array}{l}1 \\
1\end{array}$ & $\begin{array}{r}-9.37_{1} \\
-9.09_{1} \\
\end{array}$ & $\begin{array}{l}-51.2 \\
-50.0\end{array}$ & $\begin{array}{l}1 \\
1\end{array}$ & $\begin{array}{r}-50.8_{1} \\
-49.4_{1} \\
\end{array}$ & $1 \cdot$ & {$[+7.5]$} \\
\hline$\left[\begin{array}{ll}1^{\mathrm{h}} & 9^{\mathrm{m}}\end{array}\right]$ & Jan. 12 & $\ldots$ & 0 & -9.23 & $\cdots$ & . & -50.1 & -146.2 & $\begin{array}{r}1 \\
+6.0 \\
\end{array}$ \\
\hline
\end{tabular}




\begin{tabular}{|c|c|c|c|c|c|c|c|c|c|}
\hline \multicolumn{10}{|c|}{ Mean Corrections to the Ephemeris of Uranus.-Continued. } \\
\hline \multirow{2}{*}{$\begin{array}{c}\text { Observatory. } \\
\text { [R. A. of } \\
\text { Uranus.] }\end{array}$} & \multirow[b]{2}{*}{ Mean dates. } & \multicolumn{3}{|c|}{ Observed corrections in R.A. } & \multicolumn{3}{|c|}{ Observed corrections in Dee. } & \multicolumn{2}{|c|}{ Corr. to Geocentrio } \\
\hline & & Mean. & $\begin{array}{l}\text { No. of } \\
\text { obs. }\end{array}$ & $\begin{array}{c}\text { Corrected } \\
\text { mean. }\end{array}$ & Mean. & $\begin{array}{l}\text { No.of } \\
\text { obs. }\end{array}$ & $\begin{array}{c}\text { Corrected } \\
\text { mean. }\end{array}$ & Longitude. & Latitude. \\
\hline $\begin{array}{c}\text { Greenwich, } \\
{\left[\begin{array}{ll}1^{\mathrm{h}} & 34^{\mathrm{m}}\end{array}\right]}\end{array}$ & $\begin{array}{l}1849 \\
\text { Sept. } 16 \\
\ldots \ldots\end{array}$ & $\begin{array}{c}\mathrm{s} \\
-10.27 \\
\ldots .\end{array}$ & $\begin{array}{c}3 \\
\cdots\end{array}$ & $\begin{array}{c}8 \\
-10.27 \\
\cdots\end{array}$ & $\begin{array}{c}\prime \\
-53.3 \\
\ldots .\end{array}$ & $\begin{array}{c}3 \\
\cdots\end{array}$ & $\begin{array}{c}\prime \prime \\
-52.9 \\
\ldots\end{array}$ & $\begin{array}{c}" \\
{[+165.1]} \\
+160.8\end{array}$ & $\begin{array}{c}\prime \prime \\
{[+7.3]} \\
+6.2\end{array}$ \\
\hline $\begin{array}{l}\text { Greenwich, } \\
\text { Puris, }\end{array}$ & $\begin{array}{ll}\text { Oet. } & 28 \\
\text { Oet. } & 21 \\
\end{array}$ & $\begin{array}{l}-10.24 \\
-10.26\end{array}$ & $\begin{array}{l}6 \\
5\end{array}$ & $\begin{array}{l}-10.24 \\
-10.24_{6}\end{array}$ & $\begin{array}{r}-53.9 \\
-54.8\end{array}$ & $\begin{array}{l}6 \\
5\end{array}$ & $\begin{array}{l}-53.5_{6} \\
-54.2_{5} \\
\end{array}$ & {$[-164.4]$} & {$[+7.4]$} \\
\hline$\left[\begin{array}{ll}1^{\mathrm{h}} & \left.30^{\mathrm{m}}\right]\end{array}\right.$ & Oet. 25 & $\cdots$ & $\cdots$ & -10.24 & $\cdots$ & - & -53.8 & -161.0 & \\
\hline $\begin{array}{l}\text { Greenwich, } \\
\text { Paris, }\end{array}$ & $\begin{array}{ll}\text { Nov. } & 16 \\
\text { Nov. } & 17 \\
\end{array}$ & $\begin{array}{l}-10.12 \\
-10.22\end{array}$ & $\begin{array}{l}3 \\
5\end{array}$ & $\begin{array}{r}-10.12_{3} \\
-10.20_{s}\end{array}$ & $\begin{array}{l}-54.4 \\
-54.1\end{array}$ & $\begin{array}{l}3 \\
4\end{array}$ & $\begin{array}{l}-54.0_{3} \\
-53.5_{4} \\
\end{array}$ & & \\
\hline$\left[\begin{array}{ll}1^{\mathrm{h}} & 26^{\mathrm{m}}\end{array}\right]$ & Nov. 17 & $\ldots$ & $\cdots$ & -10.17 & ... & . & -53.7 & {$\left[\begin{array}{l}{[-162.5]} \\
-160.1\end{array}\right.$} & $\begin{array}{r}{[+7.3]} \\
+6.0\end{array}$ \\
\hline $\begin{array}{l}\text { Greenwich, } \\
\text { Paris, }\end{array}$ & $\begin{array}{ll}\text { Dec. } & 13 \\
\text { Dec. } & 16 \\
\end{array}$ & $\begin{array}{l}-9.95 \\
-9.88\end{array}$ & $\begin{array}{l}6 \\
2\end{array}$ & $\begin{array}{r}-9.95_{6} \\
-9.86_{\mathrm{s}} \\
\end{array}$ & $\begin{array}{l}-53.1 \\
-54.4\end{array}$ & $\begin{array}{l}9 \\
2\end{array}$ & $\begin{array}{r}-52.7, \\
-53.8_{2} \\
\end{array}$ & & \\
\hline$\left[\begin{array}{ll}1^{\mathrm{h}} & 24^{\mathrm{m}}\end{array}\right]$ & $\begin{array}{c}\text { Dec. } 14 \\
1850\end{array}$ & . & $\ldots$ & -9.93 & $\ldots$ & . & -52.9 & {$\left[\begin{array}{l}{[-160.4} \\
-156.6\end{array}\right]$} & $\begin{array}{l}1 \\
7\end{array}$ \\
\hline $\begin{array}{l}\text { Greenwich, } \\
\text { Paris, }\end{array}$ & $\begin{array}{ll}\text { Jan. } & 7 \\
\text { Jan. } & 5 \\
\end{array}$ & $\begin{array}{l}-9.71 \\
-9.77\end{array}$ & $\begin{array}{l}1 \\
2\end{array}$ & $\begin{array}{r}-9.71_{1} \\
-9.75_{2} \\
\end{array}$ & $\begin{array}{r}-53.0 \\
-53.0\end{array}$ & $\begin{array}{l}1 \\
3\end{array}$ & $\begin{array}{r}-52.6_{1} \\
-52.4_{3} \\
\end{array}$ & {$[-156.8]$} & {$[+6.9]$} \\
\hline$\left[\begin{array}{ll}1^{\mathrm{h}} & 24^{\mathrm{m}}\end{array}\right]$ & $\begin{array}{ll}\text { Jan. } \quad 6 \\
\end{array}$ & $\cdots$ & $\cdots$ & -9.74 & $\ldots$ & . & -52.5 & -153.8 & +6.2 \\
\hline $\begin{array}{c}\text { Greenwich, } \\
{\left[\begin{array}{ll}1^{\mathrm{h}} & 52^{\mathrm{m}}\end{array}\right]}\end{array}$ & $\begin{array}{c}\text { Sept. } \quad 6 \\
\ldots \ldots\end{array}$ & $\begin{array}{c}-10.83 \\
\cdots\end{array}$ & $\begin{array}{c}9 \\
\cdots\end{array}$ & $\begin{array}{c}-10.83 \\
\ldots\end{array}$ & $\begin{array}{c}-53.9 \\
\ldots\end{array}$ & $\begin{array}{l}9 \\
\cdots\end{array}$ & $\begin{array}{c}-53.5 \\
\ldots\end{array}$ & {$\left[\begin{array}{l}-171.7] \\
-168.1\end{array}\right]$} & $\begin{array}{c}{[+6.5]} \\
+5.9\end{array}$ \\
\hline $\begin{array}{l}\text { Greenwieh, } \\
\text { Paris, }\end{array}$ & $\begin{array}{ll}\text { Oet. } & 11 \\
\text { Oct. } & 17 \\
\end{array}$ & $\begin{array}{l}-10.92 \\
-19.87\end{array}$ & $\begin{array}{l}8 \\
7\end{array}$ & $\begin{array}{r}-10.92_{8} \\
-10.85 \\
\end{array}$ & $\begin{array}{l}-55.3 \\
-56.3\end{array}$ & $\begin{array}{l}7 \\
5\end{array}$ & $\begin{array}{l}-54.9, \\
-55.7 \\
\end{array}$ & & \\
\hline$\left[\begin{array}{ll}1^{\mathrm{h}} & 47^{\mathrm{m}}\end{array}\right]$ & Oet. 16 & $\ldots$ & $\cdots$ & -10.87 & $\ldots$ & . & -55.3 & -169.6 & $\begin{array}{l}0.4] \\
+5.3\end{array}$ \\
\hline $\begin{array}{l}\text { Greenwich, } \\
\text { Paris, }\end{array}$ & $\begin{array}{lr}\text { Nov. } & 6 \\
\text { Nov. } & 10 \\
\end{array}$ & $\begin{array}{r}-10.79 \\
-10.89\end{array}$ & $\begin{array}{l}5 \\
3\end{array}$ & $\begin{array}{r}-10.79_{5} \\
-10.87_{5}\end{array}$ & $\begin{array}{l}-55.7 \\
-56.1\end{array}$ & $\begin{array}{l}6 \\
4\end{array}$ & $\begin{array}{l}-55.3_{6} \\
-55.5_{4} \\
\end{array}$ & {$[-172.4]$} & {$[+6.4]$} \\
\hline$\left[\begin{array}{ll}1^{\mathrm{h}} & 43^{\mathrm{m}}\end{array}\right]$ & Nov. 8 & $\ldots$ & $\ldots$ & -10.82 & $\ldots$ & . & -55.4 & -169.1 & $\begin{array}{r}5.6 \\
+5\end{array}$ \\
\hline $\begin{array}{l}\text { Greenwich, } \\
\text { Königsberg, }\end{array}$ & $\begin{array}{lr}\text { Dec. } & 7 \\
\text { Dec. } & 15 \\
\end{array}$ & $\begin{array}{l}-10.56 \\
-10.50\end{array}$ & $\begin{array}{l}8 \\
2 .\end{array}$ & $\begin{array}{r}-10.56_{3} \\
-10.48_{2} \\
\end{array}$ & $\begin{array}{r}-55.0 \\
-56.0\end{array}$ & $\begin{array}{l}9 \\
2\end{array}$ & $\begin{array}{c}-54.6_{9} \\
\ldots\end{array}$ & {$[-1$} & {$[+6.3]$} \\
\hline$\left[\begin{array}{ll}1^{\mathrm{h}} & 41^{\mathrm{m}}\end{array}\right]$ & $\begin{array}{c}\text { Dec. } 9 \\
1851\end{array}$ & & $\ldots$ & -10.54 & $\ldots$ & . & -54.6 & {$\left[\begin{array}{l}{[-169.0} \\
-165.0\end{array}\right]$} & $\begin{array}{c}{[+6.3]} \\
+5.2\end{array}$ \\
\hline $\begin{array}{c}\text { Greenwich, } \\
{\left[\begin{array}{ll}1^{\mathrm{b}} & 41^{\mathrm{m}}\end{array}\right]}\end{array}$ & $\begin{array}{c}\text { Jan. } 19 \\
\ldots . . .\end{array}$ & $\begin{array}{c}-10.21 \\
\cdots\end{array}$ & $\begin{array}{c}4 \\
\cdots\end{array}$ & $\begin{array}{c}-10.21 \\
\cdots\end{array}$ & $\begin{array}{c}-54.8 \\
\cdots\end{array}$ & $\begin{array}{c}4 \\
\cdots\end{array}$ & $\begin{array}{l}-54.4 \\
\cdots\end{array}$ & {$\left[\begin{array}{l}-163.5] \\
-160.4\end{array}\right.$} & $\begin{array}{c}{[+6.0]} \\
+3.5\end{array}$ \\
\hline $\begin{array}{l}\text { Greenwich, } \\
\text { Königsberg, } \\
\text { Paris, }\end{array}$ & $\begin{array}{ll}\text { Sept. } & 11 \\
\text { Sept. } & 19 \\
\text { Sept. } 14 \\
\end{array}$ & $\begin{array}{l}-11.42 \\
-11.41 \\
-11.59\end{array}$ & $\begin{array}{l}2 \\
2 \\
1\end{array}$ & $\begin{array}{r}-11.42_{2} \\
-11.41_{1} \\
-11.57_{1} \\
\end{array}$ & $\begin{array}{c}-55.7 \\
\ldots \ldots \\
-57.5\end{array}$ & $\begin{array}{l}2 \\
\dddot{1}\end{array}$ & $\begin{array}{c}-55.3_{2} \\
-\ldots .9_{1} \\
\end{array}$ & {$[-180.0]$} & {$[+5.7]$} \\
\hline$\left[\begin{array}{ll}2^{\mathrm{h}} & 7^{\mathrm{m}}\end{array}\right]$ & Sept. 14 & $\ldots$ & $\cdots$ & -11.46 & $\cdots$ & .. & -55.8 & -176.9 & +4.4 \\
\hline $\begin{array}{l}\text { Königsberg, } \\
\text { Paris, }\end{array}$ & $\begin{array}{ll}\text { Oct. } & 22 \\
\text { Oet. } & 22\end{array}$ & $\begin{array}{l}-11.75 \\
-11.46\end{array}$ & $\begin{array}{r}3 \\
3\end{array}$ & $\begin{array}{l}-11.75_{2} \\
-11.44_{3}\end{array}$ & $\begin{array}{c}-58.4 \\
\ldots\end{array}$ & $\begin{array}{l}3 \\
\cdots\end{array}$ & $\begin{array}{c}-57.4 \\
\ldots\end{array}$ & & 4 \\
\hline$\left[\begin{array}{ll}2^{\mathrm{h}} & 2^{\mathrm{m}}\end{array}\right]$ & Oet. 23 & $\ldots$ & $\cdots$ & -11.56 & $\ldots$ & . & -57.4 & {$\left[\begin{array}{l}-181.8] \\
-179.1\end{array}\right.$} & $\begin{array}{l}{[+5.8]} \\
+4.3\end{array}$ \\
\hline
\end{tabular}




\begin{tabular}{|c|c|c|c|c|c|c|c|c|c|}
\hline \multicolumn{10}{|c|}{ Mean Conrections to the Ephemeris of Unanus. - Continued. } \\
\hline \multirow{2}{*}{$\begin{array}{c}\text { Observatory. } \\
\text { [R.A. of } \\
\text { Uranus.] }\end{array}$} & \multirow[b]{2}{*}{ Mean dates. } & \multicolumn{3}{|c|}{ Observed corrections in R.A. } & \multicolumn{3}{|c|}{ Observed corrections in Dec. } & \multicolumn{2}{|c|}{ Corr. to Geooentrio } \\
\hline & & Mean. & $\begin{array}{c}\text { No. of } \\
\text { obs. }\end{array}$ & $\begin{array}{c}\text { Corrected } \\
\text { mean. }\end{array}$ & Mean. & $\begin{array}{l}\text { No. of } \\
\text { obs. }\end{array}$ & $\begin{array}{c}\text { Corrected } \\
\text { mean. }\end{array}$ & Longitude. & Latitude. \\
\hline . & 1851 & \multirow{3}{*}{$\begin{array}{c}\mathrm{s} \\
-11.50 \\
-11.48\end{array}$} & \multirow{3}{*}{$\begin{array}{l}5 \\
2\end{array}$} & 3 & \multirow{3}{*}{$\begin{array}{c}\prime \prime \\
-57.5 \\
-58.1\end{array}$} & \multirow{3}{*}{$\begin{array}{l}5 \\
1\end{array}$} & $"$ & $"$ & \multirow[t]{2}{*}{$"$} \\
\hline \multirow{3}{*}{$\begin{array}{l}\text { Areenwich, } \\
\text { Paris, } \\
\qquad\left[\begin{array}{ll}2^{\mathrm{h}} & 0^{\mathrm{m}}\end{array}\right]\end{array}$} & Nov. 2 & & & $-11.50_{5}$ & & & $-57.1_{5}$ & \multirow{3}{*}{$\begin{array}{l}-181.4] \\
-178.1\end{array}$} & \\
\hline & Nov. & & & $-11.46^{\circ}$ & & & $-57.5_{1}$ & & \multirow{2}{*}{$\begin{array}{l}{[+5.7]} \\
+4.5\end{array}$} \\
\hline & Nov. 2 & $\cdots$ & $\cdots$ & -11.49 & & $\cdots$ & -57.2 & & \\
\hline \multirow{3}{*}{$\begin{array}{l}\text { Greenwich, } \\
\text { Paris, } \\
{\left[1^{\text {h }} 58^{\mathrm{m}}\right]}\end{array}$} & Nov. 22 & \multirow{2}{*}{$\begin{array}{r}-11.43 \\
-11.39\end{array}$} & \multirow{2}{*}{$\begin{array}{l}5 \\
1\end{array}$} & $-11.43_{5}$ & \multirow{2}{*}{$\begin{array}{c}-58.3 \\
\ldots\end{array}$} & 5 & $-57.9_{5}$ & \multirow{3}{*}{$\begin{array}{l}{[-179.9]} \\
-177.6\end{array}$} & \multirow{3}{*}{$\begin{array}{c}{[+5.6]} \\
+3.8\end{array}$} \\
\hline & Nov. 14 & & & $-11.37_{1}$ & & $\cdots$ & $\cdots$ & & \\
\hline & Nov. 21 & $\ldots$ & ... & -11.42 & . & . & $-57.9_{5}$ & & \\
\hline \multirow{3}{*}{$\begin{array}{l}\text { Greenwich, } \\
\text { Paris, } \\
\qquad\left[\begin{array}{ll}1^{\mathrm{h}} & 54^{\mathrm{m}}\end{array}\right]\end{array}$} & Dec. $\quad 22$ & \multirow{2}{*}{$\begin{array}{l}-11.15 \\
-11.00\end{array}$} & \multirow{2}{*}{$\begin{array}{l}3 \\
3\end{array}$} & $-11.15_{3}$ & \multirow{2}{*}{$\begin{array}{l}-57.2 \\
-57.2\end{array}$} & \multirow{2}{*}{$\begin{array}{l}3 \\
3\end{array}$} & $-56.8_{3}$ & & \\
\hline & Dec. 25 & & & -10.98 & & & $-56.6_{3}$ & {$[-175.7]$} & {$[+5.3]$} \\
\hline & $\begin{array}{c}\text { Dec. } 24 \\
1852\end{array}$ & .. & $\ldots$ & -11.06 & . & . & -56.7 & -172.3 & 8 \\
\hline $\begin{array}{l}\text { Greenwich, } \\
\text { Paris, }\end{array}$ & $\begin{array}{ll}\text { Jan. } & 11 \\
\text { Jan. } & 14\end{array}$ & $\begin{array}{l}-10.85 \\
-10.89\end{array}$ & $\begin{array}{l}7 \\
5\end{array}$ & $\begin{array}{l}-10.85 \\
-10.87\end{array}$ & $\begin{array}{l}-57.2 \\
-56.5\end{array}$ & $\begin{array}{l}7 \\
5\end{array}$ & $-56.8_{7}$ & & \\
\hline$\left[\begin{array}{ll}1^{\mathrm{h}} & 54^{\mathrm{m}}\end{array}\right]$ & Jan. 12 & & & $\frac{-10.015}{-10.86}$ & -30 & & $\frac{-05.9_{5}}{-56.4}$ & {$\left[\begin{array}{l}{[-173.1]} \\
-169.4\end{array}\right.$} & $\begin{array}{c}+5.2] \\
+3.1\end{array}$ \\
\hline $\begin{array}{c}\text { Greenwich, } \\
{\left[\begin{array}{ll}2^{h} & 22^{\mathrm{m}}\end{array}\right]}\end{array}$ & $\begin{array}{c}\text { Sept. } 12 \\
\ldots \ldots\end{array}$ & $\begin{array}{c}-12.06 \\
\ldots\end{array}$ & $\begin{array}{c}7 \\
\cdots\end{array}$ & $\begin{array}{c}-12.06 \\
\ldots\end{array}$ & $\begin{array}{c}-56.5 \\
\cdots\end{array}$ & $\begin{array}{l}7 \\
\cdots\end{array}$ & $\begin{array}{c}-56.1 \\
\ldots\end{array}$ & {$\left[\begin{array}{l}{[-187.4]} \\
-184.5\end{array}\right.$} & $\begin{array}{c}{[+4.9]} \\
+3.9\end{array}$ \\
\hline Greenwich, & Oct. 17 & -12.23 & 8 & $-12.23_{8}$ & -58.6 & 8 & $-58.2_{k}$ & & \\
\hline Paris, & Oct. $\quad 23$ & -12 & 3 & $-12.04_{3}$ & -59.6 & 3 & $-59.0_{3}$ & {$[-18 ?$} & {$[+$} \\
\hline$\left[\begin{array}{ll}2^{\mathrm{h}} & 18^{\mathrm{m}}\end{array}\right]$ & Oct. 19 & . & $\cdots$ & -12.18 & $\ldots$ & . & -58.4 & -187.2 & $\begin{array}{l}1+1 \\
+3.1\end{array}$ \\
\hline Greenwich, & Nor. 13 & -12.00 & 5 & $-12.00_{5}$ & -59.7 & 5 & $-59.3_{5}$ & & \\
\hline Paris, & Nov. 15 & -12 & 5 & $-12.06_{5}^{\circ}$ & -60.2 & 4 & $-59.6_{4}^{\circ}$ & {$[-188.9]$} & {$[+4$} \\
\hline$\left[\begin{array}{ll}2^{\mathrm{h}} & 14^{\mathrm{m}}\end{array}\right]$ & Nov. 14 & 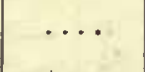 & . & -12.03 & $\cdots$ & . & -59.4 & -185.6 & $\begin{array}{r}+4 \\
+2\end{array}$ \\
\hline $\begin{array}{l}\text { Green wich, } \\
\text { Königsberg, }\end{array}$ & $\begin{array}{ll}\text { Dec. } & 19 \\
\text { Dec. } & 16\end{array}$ & $\begin{array}{r}-11.72 \\
-11.67\end{array}$ & 7 & -11.72 & -58.7 & 7 & -58.3 & & \\
\hline Paris, & Dec. 19 & -11.77 & 5 & $-11.75_{5}$ & $\begin{array}{l}-59.3 \\
-59.0\end{array}$ & $\begin{array}{l}3 \\
5\end{array}$ & $\begin{array}{l}-58.3_{3} \\
-58.4_{5}\end{array}$ & & \\
\hline$\left[\begin{array}{ll}2^{\mathrm{h}} & 10^{\mathrm{m}}\end{array}\right]$ & $\begin{array}{cc}\text { Dec. } & 19 \\
1853\end{array}$ & & & -11.73 & . & . & -58.3 & {$\left[\begin{array}{l}{[-184.8]} \\
-181.3\end{array}\right.$} & $\begin{array}{c}{[+4.6]} \\
+2.8\end{array}$ \\
\hline $\begin{array}{l}\text { Greenwich, } \\
\text { Paris, }\end{array}$ & $\begin{array}{ll}\text { Jan. } & 12 \\
\text { Jan. } & 15\end{array}$ & $\begin{array}{l}-11.42 \\
-11.46\end{array}$ & 4 & $\begin{array}{l}-11.42 \\
-11.44\end{array}$ & -57.5 & 7 & -57.1 & & \\
\hline$\left[\begin{array}{ll}2^{\mathrm{h}} & 9^{\mathrm{m}}\end{array}\right]$ & Jan. 13 & & $\cdots$ & -11.43 & & $\begin{array}{l}\cdots \\
\cdots\end{array}$ & $\frac{\cdots}{-57.1}$ & {$\left[\begin{array}{l}-181.0] \\
-176.8\end{array}\right.$} & $\begin{array}{c}{[+4.4]} \\
+2.6\end{array}$ \\
\hline Greenwich, & Sept. 17 & -12.69 & 4 & -12.69 & -56.7 & 4 & -5 & & \\
\hline Paris, & Sept. 16 & -12.58 & 2 & -12.56 & -57.0 & 2 & $-56.4_{2}^{4}$ & {$[-$} & {$[+$} \\
\hline$\left[\begin{array}{ll}2^{\mathrm{h}} & 39^{\mathrm{m}}\end{array}\right]$ & Sept. 17 & 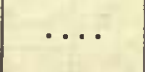 & $\cdots$ & -12.65 & $t_{1}=x^{2}$ & . & -56.3 & {$[-18$} & \\
\hline $\begin{array}{l}\text { Greenwich, } \\
\text { Paris, }\end{array}$ & $\begin{array}{ll}\text { Oct. } & 15 \\
\text { Oct. } & 27\end{array}$ & $\begin{array}{r}-12.69 \\
-12.66\end{array}$ & 7 & -12.69 & -58.8 & 7 & -58.4 & & \\
\hline & Uct. 26 & -1 & 6 & $-12.64_{9}$ & -59.0 & 6 & $-58.4_{\mathrm{n}}$ & {$[-196.7]$} & {$[+3.8]$} \\
\hline$\left[2^{n}\right.$ & Oct. 22 & $\cdots$ & $\ldots$ & -12.66 & $\cdots$ & $\therefore$ & -58.4 & -192.7 & +1.7 \\
\hline
\end{tabular}




\begin{tabular}{|c|c|c|c|c|c|c|c|c|c|}
\hline \multicolumn{10}{|c|}{ Mean Corrections to the EpHzMeris of UranUs.-Continued. } \\
\hline \multirow{2}{*}{$\begin{array}{c}\text { Observatory. } \\
\text { [R. A. of } \\
\text { Uranus.] }\end{array}$} & \multirow{2}{*}{ Mean dates. } & \multicolumn{3}{|c|}{ Observed eorrections in R.A. } & \multicolumn{3}{|c|}{ Observed eorrections in Dee. } & \multicolumn{2}{|c|}{ Corr. to Geocentrie } \\
\hline & & Mean. & $\begin{array}{c}\text { No. of } \\
\text { obs. }\end{array}$ & $\begin{array}{c}\text { Corrected } \\
\text { mean. }\end{array}$ & Mean. & $\begin{array}{c}\text { No.of } \\
\text { obs. }\end{array}$ & $\begin{array}{c}\text { Correoted } \\
\text { mean. }\end{array}$ & Longitude. & Latitude. \\
\hline \multirow{4}{*}{$\begin{array}{l}\text { Greenwich, } \\
\text { Paris, } \\
\qquad\left[2^{\mathrm{h}} 31^{\mathrm{m}}\right]\end{array}$} & 1853 & 8 & & 8 & $\prime \prime$ & & $\prime \prime$ & $" \prime$ & . \\
\hline & $\begin{array}{ll}\text { Nov, } 12 \\
\text { Nov. } 15\end{array}$ & -12.65 & 14 & $-12.65_{14}$ & -59.4 & 14 & $-59.0_{14}$ & & \\
\hline & $\frac{\text { Nov, } 15}{\text { Nov } \quad 14}$ & -12.67 & & $-12.65_{10}$ & -59.2 & 8 & $\frac{-58.6_{8}}{58.9}$ & {$[-196.0]$} & {$[+3.7]$} \\
\hline & Nov. 14 & & $\cdots$ & -12.65 & $\cdots$ & $\because$ & -58.9 & -192.9 & +1.9 \\
\hline \multirow{2}{*}{$\begin{array}{l}\text { Paris, } \\
{\left[2^{\mathrm{h}}, 27^{\mathrm{m}}\right]}\end{array}$} & $\begin{array}{c}\text { Dec. } 12 \\
\ldots \ldots\end{array}$ & $\begin{array}{c}-12.28 \\
\ldots\end{array}$ & $\begin{array}{l}1 \\
\ldots\end{array}$ & -12.26 & -59.0 & 1 & -58.4 & {$\left[\begin{array}{c}-193.4 \\
-187.9\end{array}\right]$} & 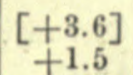 \\
\hline & 1854 & & & & & & & & \\
\hline \multirow{2}{*}{$\begin{array}{l}\text { Greenwich, } \\
\text { Paris, } \\
\qquad\left[\begin{array}{ll}2^{\mathrm{h}} & 25^{\mathrm{m}}\end{array}\right]\end{array}$} & $\begin{array}{ll}\text { Jan. } & 13 \\
\text { Jan. } & 20 \\
\end{array}$ & $\begin{array}{r}-12.02 \\
-11.87\end{array}$ & $\begin{array}{l}4 \\
2\end{array}$ & $\begin{array}{r}-12.02, \\
-11.85_{2} \\
\end{array}$ & $\begin{array}{l}-59.7 \\
-58.1\end{array}$ & $\begin{array}{l}4 \\
1\end{array}$ & $\begin{array}{r}-59.2_{4} \\
-57.9_{1}\end{array}$ & {$[-188.1]$} & $-3.5]$ \\
\hline & Jan, 15 & $\ldots$ & $\cdots$ & -11.96 & $\cdots$ & $\cdots$ & -58.9 & {$[-183.9$} & -0.1 \\
\hline $\begin{array}{l}\text { Paris, } \\
{\left[2^{\mathrm{b}} 26^{\mathrm{m}}\right]}\end{array}$ & $\begin{array}{c}\text { Feb. } 8 \\
\ldots \ldots\end{array}$ & $\begin{array}{c}-11.89 \\
\cdots\end{array}$ & $\begin{array}{l}1 \\
\cdots\end{array}$ & $\begin{array}{c}-11.87 \\
\cdots\end{array}$ & $\begin{array}{c}-57.8 \\
\ldots\end{array}$ & $\begin{array}{l}1 \\
\cdots\end{array}$ & $\begin{array}{c}-57.6 \\
\cdots\end{array}$ & {$\left[\begin{array}{l}{[-184.5]} \\
-182.1\end{array}\right.$} & $\begin{array}{c}{[+3.5]} \\
+0.7\end{array}$ \\
\hline $\begin{array}{c}\text { Greenwich, } \\
{\left[\begin{array}{ll}2^{\mathrm{h}} & 53^{\mathrm{m}}\end{array}\right]}\end{array}$ & $\begin{array}{c}\text { Sept. } 21 \\
\ldots \ldots\end{array}$ & $\begin{array}{c}-13.12 \\
\cdots \cdots\end{array}$ & $\begin{array}{c}6 \\
\cdots\end{array}$ & $\begin{array}{c}-13.12 \\
\ldots\end{array}$ & $\begin{array}{c}-56.0 \\
\cdots\end{array}$ & $\begin{array}{r}6 \\
\cdots\end{array}$ & $\begin{array}{c}-55.5 \\
\ldots\end{array}$ & {$\left[\begin{array}{l}-201.3] \\
-196.9\end{array}\right.$} & $\begin{array}{c}{[+3.0]} \\
+1.7\end{array}$ \\
\hline \multirow{2}{*}{$\begin{array}{l}\text { Greenwich, } \\
\text { Paris, } \\
\qquad\left[\begin{array}{ll}2^{\mathrm{h}} & 50^{\mathrm{m}}\end{array}\right]\end{array}$} & $\begin{array}{ll}\text { Oet. } & 26 \\
\text { Oet. } & 29 \\
\end{array}$ & $\begin{array}{l}-13.24 \\
-13.12\end{array}$ & 7 & $\begin{array}{r}-13.24_{7} \\
-13.10_{1} \\
\end{array}$ & $\begin{array}{r}-58.0 \\
-57.2\end{array}$ & $\begin{array}{l}7 \\
3\end{array}$ & $\begin{array}{r}-57.5_{\mathrm{a}} \\
-57.0_{\mathrm{1}} \\
\end{array}$ & {$[-203.7]$} & {$[+$} \\
\hline & Oet. 27 & & $\cdots$ & -13.22 & ...' & $\cdots$ & -57.3 & -199.0 & \\
\hline \multirow{3}{*}{$\begin{array}{l}\text { Greenwich, } \\
\text { Paris, } \\
\text { Santiago, } \\
\qquad\left[\begin{array}{ll}2^{\mathrm{h}} & \left.48^{\mathrm{m}}\right]\end{array}\right.\end{array}$} & $\begin{array}{ll}\text { Nov. } & 12 \\
\text { Nov. } & 16\end{array}$ & $\begin{array}{l}-13.22 \\
-13.21\end{array}$ & $\begin{array}{l}5 \\
6\end{array}$ & $\begin{array}{r}-13.22_{5} \\
-13.19_{9}\end{array}$ & $\begin{array}{l}-58.2 \\
-58.7\end{array}$ & $\begin{array}{l}5 \\
1\end{array}$ & $\begin{array}{r}-57.5_{\mathrm{s}} \\
-58.5\end{array}$ & & \\
\hline & $\begin{array}{ll}\text { Nov. } 17 \\
\end{array}$ & -13.20 & $\begin{array}{l}6 \\
4\end{array}$ & $\begin{array}{l}-13.19_{6} \\
-13.20_{3} \\
\end{array}$ & $\begin{array}{l}-58.7 \\
-58.7\end{array}$ & $\begin{array}{l}1 \\
3\end{array}$ & $\begin{array}{l}-58.0_{1} \\
-58.1_{2} \\
\end{array}$ & {$[-$} & {$[+2.7]$} \\
\hline & Nov. 15 & , & $\ldots$ & -13.20 & . & $\cdots$ & -57.8 & -199.1 & \\
\hline \multirow{4}{*}{$\begin{array}{l}\text { Greenwich } \\
\text { Paris, } \\
\text { Santiago, } \\
\quad\left[\begin{array}{ll}2^{\mathrm{h}} & 44^{\mathrm{m}}\end{array}\right]\end{array}$} & Dec. 14 & -12.93 & 7 & -12.93 & -58.6 & 7 & $-58.1_{2}$ & & \\
\hline & $\begin{array}{lr}\text { Dec. } & 9 \\
\text { Dee. } & 15\end{array}$ & -13.14 & 1 & -1 & -5 & 4 & -56 & & \\
\hline & $\begin{array}{ll}\text { Dec. } & 15 \\
\text { Dea } & 14\end{array}$ & -12.85 & & -12.85, & 7 & & $-59.1_{1}$ & {$[-200.1]$} & {$[+2.6]$} \\
\hline & $\begin{array}{c}\text { Dec. } 14 \\
1855\end{array}$ & & $\ldots$ & -12.90 & $\ldots$ & . & -58.0 & -1 & +0.7 \\
\hline $\begin{array}{l}\text { Greenwich, } \\
{\left[\begin{array}{ll}2^{\mathrm{k}} & 42^{\mathrm{m}}\end{array}\right]}\end{array}$ & $\begin{array}{c}\text { Jan. } 18 \\
\ldots .\end{array}$ & $\begin{array}{c}-12.55 \\
\cdots\end{array}$ & $\begin{array}{l}2 . \\
\cdots\end{array}$ & $\begin{array}{c}-12.55_{2} \\
\cdots\end{array}$ & $\begin{array}{c}-56.9 \\
\ldots\end{array}$ & $\begin{array}{r}2 \\
\cdots\end{array}$ & $\begin{array}{c}-56.4 \\
\ldots\end{array}$ & {$\left[\begin{array}{l}-194.6] \\
-190.1\end{array}\right.$} & 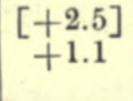 \\
\hline \multirow{2}{*}{$\begin{array}{l}\text { Greenwich, } \\
\text { Paris, } \\
\qquad\left[\begin{array}{ll}3^{\mathrm{h}} & 9^{\mathrm{m}}\end{array}\right]\end{array}$} & $\begin{array}{ll}\text { Oet. } & 13 \\
\text { Oet. } & 25 \\
\end{array}$ & $\begin{array}{l}-13.71 \\
-13.93\end{array}$ & . 6 & $\begin{array}{r}-13.71_{6} \\
-13.91_{3} \\
\end{array}$ & $\begin{array}{l}-55.7 \\
-55.8\end{array}$ & $\begin{array}{l}6 \\
2\end{array}$ & $\begin{array}{r}-55.2_{3} \\
-55.6_{1} \\
\end{array}$ & {$[-$} & {$[+]$} \\
\hline & Oet. 17 & & $\ldots$ & -13.78 & $\cdots$ & . & -55.3 & -204.9 & \\
\hline \multirow{3}{*}{$\begin{array}{l}\text { Greenwich, } \\
\text { Paris, } \\
\text { Santiago, } \\
\qquad\left[\begin{array}{ll}3^{\mathrm{h}} 5^{\mathrm{m}}\end{array}\right]\end{array}$} & $\begin{array}{ll}\text { Nov. } & 11 \\
\text { Nov. } & 17\end{array}$ & $\begin{array}{r}-13.85 \\
-13.62\end{array}$ & $\begin{array}{l}4 \\
4\end{array}$ & $\begin{array}{l}-13.85 \\
-13.60\end{array}$ & $\begin{array}{r}-57.6 \\
-56.6\end{array}$ & $\begin{array}{l}5 \\
4\end{array}$ & $\begin{array}{l}-57.1_{s} \\
-56.4\end{array}$ & & \\
\hline & Nov. 16 & -13.82 & 5 & $-13.82_{4}$ & -57.2 & 5 & $-56.6_{4}^{4}$ & {$[-$} & {$[+1.7]$} \\
\hline & Nov. 15 & $\cdots$ & $\cdots$ & -13.76 & $\cdots$ & $\cdots$ & -56.7 & -205.3 & -0.2 \\
\hline \multirow{3}{*}{$\begin{array}{l}\text { Greenwich, } \\
\text { Paris, } \\
\text { Santiago, } \\
\quad\left[\begin{array}{ll}3^{\mathrm{h}} & 0^{\mathrm{m}}\end{array}\right]\end{array}$} & Dec. 18 & -13.48 & 10 & $-13.48_{10}$ & -57.3 & 10 & $-56.8_{\mathrm{s}}$ & & \\
\hline & $\begin{array}{ll}\text { Dee. } & 16 \\
\text { Dec. } & 18 \\
\end{array}$ & $\begin{array}{l}-13.52 \\
-13.53\end{array}$ & $\begin{array}{l}2 \\
6\end{array}$ & $\begin{array}{l}-13.50_{2} \\
-13.53_{4}\end{array}$ & $\begin{array}{l}-56.7 \\
-58.1\end{array}$ & $\begin{array}{l}3 \\
6\end{array}$ & $\begin{array}{l}-56.5_{1} \\
-57.5_{2}\end{array}$ & & \\
\hline & Dec. 18 & & $\ldots$ & -13.50 & $\ldots$ & $\cdots$ & -57.0 & -202.1 & -0.1 \\
\hline
\end{tabular}




\begin{tabular}{|c|c|c|c|c|c|c|c|c|c|}
\hline \multicolumn{10}{|c|}{ Mean Corrections to tire EpHemeris of Uranus.-Continued. } \\
\hline \multirow{2}{*}{$\begin{array}{l}\text { Observatory. } \\
\text { [R.A. of } \\
\text { Uranus.] }\end{array}$} & \multirow{2}{*}{ lean dates. } & \multicolumn{3}{|c|}{ Observed corrections in R.A. } & \multicolumn{3}{|c|}{ Observed corrections in Dec. } & \multicolumn{2}{|c|}{ Corr. to Geocentric } \\
\hline & & Mean. & $\begin{array}{c}\text { No. of } \\
\text { obs. }\end{array}$ & $\begin{array}{c}\text { Corrected } \\
\text { wean. }\end{array}$ & Mean. & $\begin{array}{c}\text { No of } \\
\text { obs. }\end{array}$ & $\begin{array}{c}\text { Corrected } \\
\text { meall. }\end{array}$ & Longitude. & Latitude. \\
\hline & 1000 & 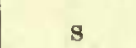 & & 5 & $\prime \prime$ & & $"$ & $"$ & 11 \\
\hline Greenwich, & Jan. 22 & -12.97 & 6 & -12.97. & -56.1 & 6 & $-55.6_{3}$ & & \\
\hline Paris, & Feb. 10 & -12.87 & 2 & $-12.85_{2}$ & -5 & 2 & -54 & {$[-$} & {$[+1.4]$} \\
\hline$\left[\begin{array}{ll}2^{\mathrm{h}} & 58^{\mathrm{m}}\end{array}\right]$ & Jall. 27 & $\ldots$ & $\cdots$ & -12.94 & 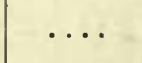 & $\cdots$ & -55.4 & & \\
\hline $\begin{array}{l}\text { Grecnwich, } \\
\text { Paris, }\end{array}$ & $\begin{array}{ll}\text { Oct. } & 16 \\
\text { Oct. } & 16\end{array}$ & $\begin{array}{r}-14.36 \\
-14.38\end{array}$ & $\begin{array}{l}4 \\
2\end{array}$ & $\begin{array}{r}-14.36_{4} \\
-11.36_{3}\end{array}$ & -53.8 & 4 & -53.3 & & \\
\hline$\left[\begin{array}{ll}3^{b} & 27^{m}\end{array}\right]$ & $\overline{\text { Oct. } \quad 16}$ & 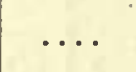 & $\cdots$ & -14.36 & . & $\cdots$ & -53.3 & -211.0 & {$\left[\begin{array}{c}{[+0.7]} \\
-1.4\end{array}\right.$} \\
\hline $\begin{array}{l}\text { Green wich, } \\
\text { Paris, }\end{array}$ & $\begin{array}{ll}\text { Nov. } & 18 \\
\text { Nov. } & 17\end{array}$ & $\begin{array}{r}-14.23 \\
-14.32\end{array}$ & $\begin{array}{l}8 \\
6\end{array}$ & $\begin{array}{r}-14.23_{\mathrm{s}} \\
-14.30_{6}\end{array}$ & $\begin{array}{l}-54.8 \\
-54.0\end{array}$ & $\begin{array}{l}8 \\
7\end{array}$ & $\begin{array}{l}-54.3_{8} \\
-53.8_{7}\end{array}$ & & 0.77 \\
\hline$\left[\begin{array}{ll}3^{\mathrm{h}} & 22^{\mathrm{m}}\end{array}\right]$ & Nov. 18 & 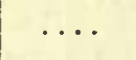 & $\cdots$ & -14.26 & 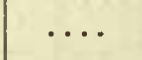 & $\cdots$ & -54.1 & -210.2 & -0.9 \\
\hline $\begin{array}{l}\text { Areenwich, } \\
\text { Paris, }\end{array}$ & $\begin{array}{ll}\text { Dec. } & 18 \\
\text { Dec. } & 19 \\
\end{array}$ & $\begin{array}{l}-13.92 \\
-14.11\end{array}$ & $\begin{array}{l}5 \\
7\end{array}$ & $\begin{array}{r}-13.93_{3} \\
-14.09_{7} \\
\end{array}$ & $\begin{array}{l}-55.9 \\
-55.0\end{array}$ & $\begin{array}{l}5 \\
6\end{array}$ & $\begin{array}{l}-55.4_{s} \\
-54.8_{6}\end{array}$ & & \\
\hline$\left[\begin{array}{ll}3^{\mathrm{b}} & 17^{\mathrm{m}}\end{array}\right]$ & $\begin{array}{c}\text { Dec. } 19 \\
1857\end{array}$ & . & $\ldots$ & -14.03 & & . & -55.1 & - & 2 \\
\hline $\begin{array}{l}\text { Greenwich, } \\
\text { Paris, }\end{array}$ & $\begin{array}{ll}\text { Jan. } & 21 \\
\text { Jan. } & 12 \\
\end{array}$ & $\begin{array}{l}-13.59 \\
-13.87\end{array}$ & $\begin{array}{r}10 \\
.3\end{array}$ & $\begin{array}{l}-13.60_{10} \\
-13.85_{3} \\
\end{array}$ & $\begin{array}{r}-53.4 \\
-55.3\end{array}$ & $\begin{array}{l}8 \\
3\end{array}$ & $\begin{array}{l}-52.9_{3} \\
-55.1_{1}\end{array}$ & & \\
\hline$\left[\begin{array}{ll}3^{\mathrm{h}} & 15^{\mathrm{m}}\end{array}\right]$ & Jan. 19 & & .. & -13.66 & & - & -53.5 & -202.3 & -0.4 \\
\hline $\begin{array}{c}\text { Greenwich, } \\
{\left[\begin{array}{ll}3^{\mathrm{h}} & 46^{\mathrm{m}}\end{array}\right]}\end{array}$ & $\begin{array}{c}\text { Oet. } \quad 8 \\
\ldots \ldots\end{array}$ & $\begin{array}{c}-14.68 \\
\ldots\end{array}$ & 4 & $\begin{array}{c}-14.69 \\
\ldots\end{array}$ & $\begin{array}{c}-49.1 \\
\ldots\end{array}$ & 4 & $\begin{array}{c}-48.6 \\
\ldots\end{array}$ & {$\left[\begin{array}{l}{[-216.8]} \\
-213.0\end{array}\right.$} & $\begin{array}{c}{[-0.3]} \\
-1.9\end{array}$ \\
\hline $\begin{array}{l}\text { Greenwich, } \\
\text { Paris, } \\
\text { Königsberg, }\end{array}$ & $\begin{array}{lr}\text { Nov. } & 6 \\
\text { Nov. } & 10 \\
\text { Nov. } & 19 \\
\end{array}$ & $\begin{array}{r}-14.80 \\
-14.83 \\
-14.77\end{array}$ & $\begin{array}{l}4 \\
9 \\
3\end{array}$ & $\begin{array}{r}-14.81^{4} \\
-14.81_{9} \\
-14.75_{4} \\
\end{array}$ & $\begin{array}{l}-51.3 \\
-51.5 \\
-52.7\end{array}$ & $\begin{array}{l}5 \\
8 \\
3\end{array}$ & $\begin{array}{l}-50.8_{5} \\
-51.3_{8} \\
-51.5_{2}\end{array}$ & & - \\
\hline$\left[\begin{array}{ll}3^{\mathrm{h}} & 41^{\mathrm{m}}\end{array}\right]$ & Nov. 11 & & 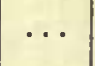 & -14.80 & & - & -51.2 & -215.5 & -2.4 \\
\hline $\begin{array}{l}\text { Greenwich, } \\
\text { Paris, } \\
\text { Königsberg, }\end{array}$ & $\begin{array}{lr}\text { Dec. } & 11 \\
\text { Dec. } & 16 \\
\text { Dec. } & 8\end{array}$ & $\begin{array}{r}-14.55 \\
-14.48 \\
-14.56\end{array}$ & $\begin{array}{l}6 \\
7 \\
1\end{array}$ & $\begin{array}{r}-14.56_{6} \\
-14.46_{7} \\
-14.54_{2} \\
\end{array}$ & $\begin{array}{r}-53.2 \\
-52.8 \\
-53.7\end{array}$ & $\begin{array}{l}5 \\
6 \\
1\end{array}$ & $\begin{array}{l}-52.7^{5} \\
-52.6_{6}^{6} \\
-52.5_{1}\end{array}$ & & \\
\hline$\left[\begin{array}{ll}3^{\mathrm{h}} & 36^{\mathrm{m}}\end{array}\right]$ & $\begin{array}{c}\text { Dec. } 13 \\
1858\end{array}$ & & 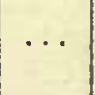 & -14.51 & & - & -52.6 & -212.2 & {$\left[\begin{array}{l}{[-0.5} \\
-3.0\end{array}\right]$} \\
\hline $\begin{array}{l}\text { Greenwich, } \\
\text { Paris, }\end{array}$ & $\begin{array}{ll}\text { Jan. } & 17 \\
\text { Jan. } & 16 \\
\end{array}$ & $\begin{array}{l}-14.13 \\
-14.19\end{array}$ & $\begin{array}{r}13 \\
8\end{array}$ & $\begin{array}{l}-14.14_{13} \\
-14.17_{6} \\
\end{array}$ & $\begin{array}{l}-52.8 \\
-52.8\end{array}$ & $\begin{array}{r}13 \\
9\end{array}$ & $\begin{array}{l}-52.3_{18} \\
-52.6\end{array}$ & & \\
\hline$\left[\begin{array}{ll}3^{\mathrm{h}} & 32^{\mathrm{m}} \cdot .2\end{array}\right]$ & Jan. 17 & & & -14.15 & & 0 & -52.4 & -2 & -2.8 \\
\hline $\begin{array}{l}\text { Greenwich, } \\
\text { Paris, }\end{array}$ & $\begin{array}{ll}\text { Feb. } & 11 \\
\text { Feb. } & 12\end{array}$ & $\begin{array}{l}-13.81 \\
-13.91\end{array}$ & $\begin{array}{r}10 \\
1\end{array}$ & $\begin{array}{l}-13.82_{10} \\
-13.89_{1}\end{array}$ & $\begin{array}{l}-52.1 \\
-52.7\end{array}$ & $\begin{array}{r}10 \\
1\end{array}$ & $\begin{array}{l}-51.6_{10} \\
-52.5\end{array}$ & & \\
\hline$\left[\begin{array}{ll}3^{\mathrm{h}} & 31^{\mathrm{m}} \cdot 9\end{array}\right]$ & Feb. 11 & & $\cdots$ & -13.83 & & $\cdots$ & -51.7 & {$\left[\begin{array}{l}-207.0] \\
-203.1\end{array}\right.$} & {$\left[\begin{array}{l}{[-0.6]} \\
-3.1\end{array}\right.$} \\
\hline $\begin{array}{l}\text { Greenwich, } \\
{\left[\begin{array}{ll}4^{\mathrm{h}} & 3^{\mathrm{m}} \cdot 3\end{array}\right]}\end{array}$ & $\begin{array}{c}\text { Oct. } 17 \\
\ldots \ldots\end{array}$ & $\begin{array}{c}-15.06 \\
\ldots\end{array}$ & $\begin{array}{l}5 \\
\cdots\end{array}$ & -15.06 & $\begin{array}{c}-46.4 \\
\ldots\end{array}$ & 5 & $\begin{array}{c}-45.9 \\
\ldots\end{array}$ & {$\left[\begin{array}{l}{[-220.6]} \\
-216.2\end{array}\right]$} & $\begin{array}{l}{[-1.4]} \\
-4.0\end{array}$ \\
\hline $\begin{array}{l}\text { Grecnwich, } \\
\text { Paris, }\end{array}$ & $\begin{array}{ll}\text { Nov. } 14 \\
\text { Nov. } 20 \\
\end{array}$ & $\begin{array}{r}-15.16 \\
-15.19\end{array}$ & $\begin{array}{r}10 \\
9\end{array}$ & $\begin{array}{l}-15.17_{10} \\
-15.17_{0}\end{array}$ & $\begin{array}{l}-48.2 \\
-47.8\end{array}$ & $\begin{array}{l}11 \\
10\end{array}$ & $\begin{array}{l}-47.7_{11} \\
-47.6_{10}\end{array}$ & & \\
\hline$\left[\begin{array}{ll}3^{h} & 58^{m} .5\end{array}\right]$ & Nov. 17 & tons & . & -15.17 & & $\cdots$ & -47.7 & - & - -1.0$]$ \\
\hline
\end{tabular}




\begin{tabular}{|c|c|c|c|c|c|c|c|c|c|}
\hline \multirow{3}{*}{$\begin{array}{l}\text { Observatory. } \\
\text { [R. A. of } \\
\text { Uranus.] }\end{array}$} & \multicolumn{9}{|c|}{ Mean Corrections to the Ephemeris of Uranus.-Continued. } \\
\hline & \multirow[b]{2}{*}{ Mean dates. } & \multicolumn{3}{|c|}{ Observed corrections in R.A. } & \multicolumn{3}{|c|}{ Observed corrections in Dee. } & \multicolumn{2}{|c|}{ Corr. to Geocentric } \\
\hline & & Mean. & $\begin{array}{l}\text { No. of } \\
\text { obs. }\end{array}$ & $\begin{array}{c}\text { Corrected } \\
\text { mean. }\end{array}$ & Mean. & $\begin{array}{l}\text { No.of } \\
\text { olis. }\end{array}$ & $\begin{array}{c}\text { Corrected } \\
\text { mean. }\end{array}$ & Latitude. & Longitude. \\
\hline \multirow{4}{*}{$\begin{array}{l}\text { Greenwich, } \\
\text { Paris, } \\
{\left[\begin{array}{ll}3^{\mathrm{b}} & \left.53^{\mathrm{m}} \cdot 6\right]\end{array}\right.}\end{array}$} & 1858 & $\therefore$ & & 8 & $\prime \prime$ & & $\prime \prime$ & " & $\prime \prime$ \\
\hline & Dee. 16 & -14.96 & 6 & $-14.97_{6}$ & -49.2 & 6 & $-48.7_{2}$ & & \\
\hline & Dee. 16 & -14.78 & 5 & $-14.76_{s}$ & -49.5 & 3 & $-49.3_{1}$ & {$[-220.7]$} & {$[-1.6]$} \\
\hline & Dec. 16 & $\cdots$ & $\therefore$ & -14.87 & $\cdots$ & $\cdots$ & -48.9 & -215.1 & -4.1 \\
\hline \multirow{3}{*}{$\begin{array}{l}\text { Greenwich,. } \\
\text { Paris, } \\
{\left[3^{\mathrm{h}} 50^{\mathrm{m}} .0^{\mathrm{y}}\right]}\end{array}$} & $\begin{array}{c}1859 \\
\text { Jan. } 19\end{array}$ & -14.56 & 8 & -1 & & 8 & & \multirow{3}{*}[\begin{array}{l}{[-216.1]}\\
{-211.5}\end{array}]{} & \multirow{3}{*}{$\begin{array}{l}-1.5] \\
-3.1\end{array}$} \\
\hline & Jan. 10 & -14.72 & 4 & $-14.70_{8}^{8}$ & $\begin{array}{l}-48.9 \\
-48.2\end{array}$ & 4 & $\begin{array}{l}-48.4_{2} \\
-48.0_{1}\end{array}$ & & \\
\hline & Jan. 16 & $\cdots$ & $\cdots$ & -14.61 & 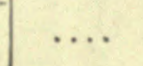 & . & -48.3 & & \\
\hline \multirow{3}{*}{$\begin{array}{l}\text { Greenwich, } \\
\text { Paris, } \\
{\left[\begin{array}{ll}3^{\mathrm{h}} & 49^{\mathrm{m}}, 3\end{array}\right]}\end{array}$} & Feb. $\quad 18$ & -14.18 & .5 & $-14.19_{3}$ & -48.1 & 5 & $-47.6_{s}$ & \multirow{3}{*}[\begin{array}{l}{[-210.9]}\\
{-207.1}\end{array}]{} & \multirow{3}{*}{$\begin{array}{c}{[-1.6]} \\
-3.4\end{array}$} \\
\hline & Feb. 11 & -14.37 & & $-14.35_{6}$ & -48.3 & 4 & -48.14 & & \\
\hline & Feb. 14 & . & $\cdots$ & -14.28 & $\cdots$ & . & -47.8 & & \\
\hline \multirow{2}{*}{$\begin{array}{l}\text { Greenwich, } \\
{\left[\begin{array}{ll}4^{\mathrm{h}} & 20^{\mathrm{m}} \cdot 8\end{array}\right]}\end{array}$} & Oet. 25 & -15.43 & 6 & -15.44 & -42.6 & 6 & -42.1 & \multirow{2}{*}{$\begin{array}{l}{[-223.2]} \\
-219.6\end{array}$} & \multirow{2}{*}{$\begin{array}{l}{[-2.2]} \\
-5.5\end{array}$} \\
\hline & & & . & 1 & $\cdots$ & $\cdots$ & $\cdots$ & & \\
\hline \multirow{2}{*}{$\begin{array}{l}\text { Greenwich, } \\
\text { Paris, } \\
{\left[\begin{array}{ll}4^{\mathrm{h}} & 17^{\mathrm{m}} \cdot 2\end{array}\right]}\end{array}$} & $\begin{array}{ll}\text { Nov. } 18 \\
\text { Nov. } 17\end{array}$ & $\begin{array}{l}-15.56 \\
-15.61\end{array}$ & $\begin{array}{l}6 \\
8\end{array}$ & $\begin{array}{l}-15.57_{6} \\
-15.59_{8}\end{array}$ & $\begin{array}{l}-43.3 \\
-43.0\end{array}$ & $\begin{array}{l}5 \\
9\end{array}$ & $\begin{array}{l}-42.8_{\mathrm{s}} \\
-42.8_{\mathrm{g}}\end{array}$ & \multirow{2}{*}{$\begin{array}{l}{[-224.7]} \\
-221.9\end{array}$} & \multirow{2}{*}{$\begin{array}{c}{[-2.2]} \\
-4.6\end{array}$} \\
\hline & Nov, 17 & $\cdots$ & $\cdots$ & -15.58 & $\ldots$ & $\cdots$ & -42.8 & & \\
\hline \multirow{3}{*}{$\begin{array}{l}\text { Greenwich, } \\
\text { Paris, } \\
{\left[\begin{array}{ll}4^{\mathrm{h}} & \left.12^{\mathrm{m}} \cdot 5\right]\end{array}\right.}\end{array}$} & Dec. 16 & -15.43 & $\begin{array}{l}9 \\
5\end{array}$ & $-15.44_{9}$ & -45.2 & 8 & $-44.7_{8}$ & \multirow{3}{*}{$\begin{array}{l}-223.9] \\
-220.7\end{array}$} & \multirow{3}{*}{$\begin{array}{l}{[-2.3]} \\
-4.9\end{array}$} \\
\hline & Dec. 11 & -15 & 5 & -15 & -44.5 & 5 & $-44.3_{s}$ & & \\
\hline & Dec. 14 & . & $\cdots$ & -15.44 & $\ldots$ & . & -44.5 & & \\
\hline \multirow{2}{*}{$\begin{array}{l}\text { Greenwich, } \\
\text { Paris, } \\
{\left[\begin{array}{ll}4^{\mathrm{h}} & \left.8^{\mathrm{m}} \cdot 0\right]\end{array}\right.}\end{array}$} & \begin{tabular}{ll}
\multicolumn{2}{c}{1860} \\
Jan. & 16 \\
Jan. & 15 \\
\end{tabular} & $\begin{array}{l}-15.08 \\
-15.08\end{array}$ & $\begin{array}{r}* 8 \\
8\end{array}$ & $\begin{array}{l}-15.09_{8} \\
-15.06_{8}\end{array}$ & $\begin{array}{l}-45.8 \\
-44.3\end{array}$ & $\begin{array}{l}8 \\
5\end{array}$ & $\begin{array}{l}-45.3_{8} \\
-44.1_{s}\end{array}$ & \multirow{2}{*}{$\begin{array}{c}{[-219.6]} \\
-216.1\end{array}$} & \multirow{2}{*}{$\begin{array}{l}{[-2.3]} \\
-4.5\end{array}$} \\
\hline & Jan. 16 & $\cdots$ & $\cdots$ & -15.08 & & .. & -44.8 & & \\
\hline \multirow{3}{*}{$\begin{array}{l}\text { Greenwich, } \\
\text { Paris, } \\
{\left[\begin{array}{ll}4^{\mathrm{n}} & 7^{\mathrm{m}} .0\end{array}\right]}\end{array}$} & Feb. 17 & -14.64 & $\begin{array}{r}12 \\
2\end{array}$ & $-14.65_{12}$ & -45.0 & 12 & -44.5 & \multirow{3}{*}[\begin{array}{l}{-213.9}\\
{-210.4}\end{array}]{} & \\
\hline & $\begin{array}{lr}\text { Feb. } & 6 \\
\text { Feb } & 15\end{array}$ & & & $-14.77_{2}$ & & & & & {$[-2.3]$} \\
\hline & 0. 15 & & $\cdots$ & & (s. & $\cdots$ & & & -0.0 \\
\hline $\begin{array}{l}\text { Greenwich, } \\
{\left[4^{\mathrm{h}} 40^{\mathrm{m}} \cdot 9\right]}\end{array}$ & Oct. 13 & -15.52 & 2 & -15.52 & -35.2 & 3 & -34.7 & {$\left[\begin{array}{l}-223.0 \\
-218.3\end{array}\right]$} & $\begin{array}{c}{[-2.9} \\
-5.4\end{array}$ \\
\hline & & & & 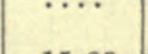 & 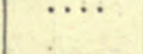 & $\cdots$ & 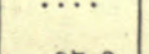 & & \\
\hline $\begin{array}{l}\text { Greenwich, } \\
\text { Paris, }\end{array}$ & $\begin{array}{ll}\text { Nov. } 15 \\
\text { Nov. } 22 \\
\end{array}$ & $\begin{array}{l}-15.88 \\
-15.79\end{array}$ & $\begin{array}{l}9 \\
5\end{array}$ & $\begin{array}{r}-15.89_{0} \\
-15.77_{5} \\
\end{array}$ & $\begin{array}{l}-38.3 \\
-37.3\end{array}$ & $\begin{array}{l}9 \\
5\end{array}$ & $\begin{array}{r}-37.8_{2} \\
-37.1_{1} \\
\end{array}$ & {$[-$} & {$[-$} \\
\hline$\left[\begin{array}{ll}4^{\mathrm{h}} & \left.35^{\mathrm{m}} \cdot 8\right]\end{array}\right]$ & Nov. 18 & . & $\cdots$ & -15.85 & . & . & -37.6 & & -5.8 \\
\hline $\begin{array}{l}\text { Greenwich, } \\
\text { Paris. }\end{array}$ & Dec. 12 & -15.77 & 4 & -15 & -41.5 & 5 & $-41.0_{5}$ & & \\
\hline & Dec. 12 & -15.79 & 3 & $-15.77_{3}$ & -38.5 & 3 & $-38.3_{3}$ & {$[-226.2]$} & {$[-3.2]$} \\
\hline$\left[\begin{array}{ll}4^{\mathrm{h}} & 31^{\mathrm{m}} \cdot 6\end{array}\right]$ & Dec. 12 & $\ldots$ & $\cdots$ & -15.78 & . & . & -40.0 & -223.2 & $2 \quad 88^{-}$ \\
\hline $\begin{array}{l}\text { Greenwich, } \\
\text { Paris, }\end{array}$ & \begin{tabular}{ll}
\multicolumn{2}{c}{1861} \\
Jan. 13 \\
Jan. 13
\end{tabular} & $\begin{array}{l}-15.45 \\
-15.52\end{array}$ & $\begin{array}{r}10 \\
6\end{array}$ & $\begin{array}{l}-15.46_{10} \\
-15.49_{6}\end{array}$ & $\begin{array}{l}-40.5 \\
-40.2\end{array}$ & $\begin{array}{r}10 \\
3\end{array}$ & $\begin{array}{l}-40.1_{3} \\
-40.0_{1}\end{array}$ & & \\
\hline$\left[\begin{array}{ll}4^{\mathrm{h}} & \left.26^{\mathrm{m}} \cdot 8\right]\end{array}\right.$ & $\begin{array}{ll}\text { Jan. } 13 \\
13\end{array}$ & $\cdots$ & $\cdots$ & -15.47 & $\cdots$ & . & -40.1 & {$\left[\begin{array}{l}-222.4 \\
-219.3\end{array}\right.$} & {$\left[\begin{array}{l}{[-3.2]} \\
-5.8\end{array}\right.$} \\
\hline
\end{tabular}




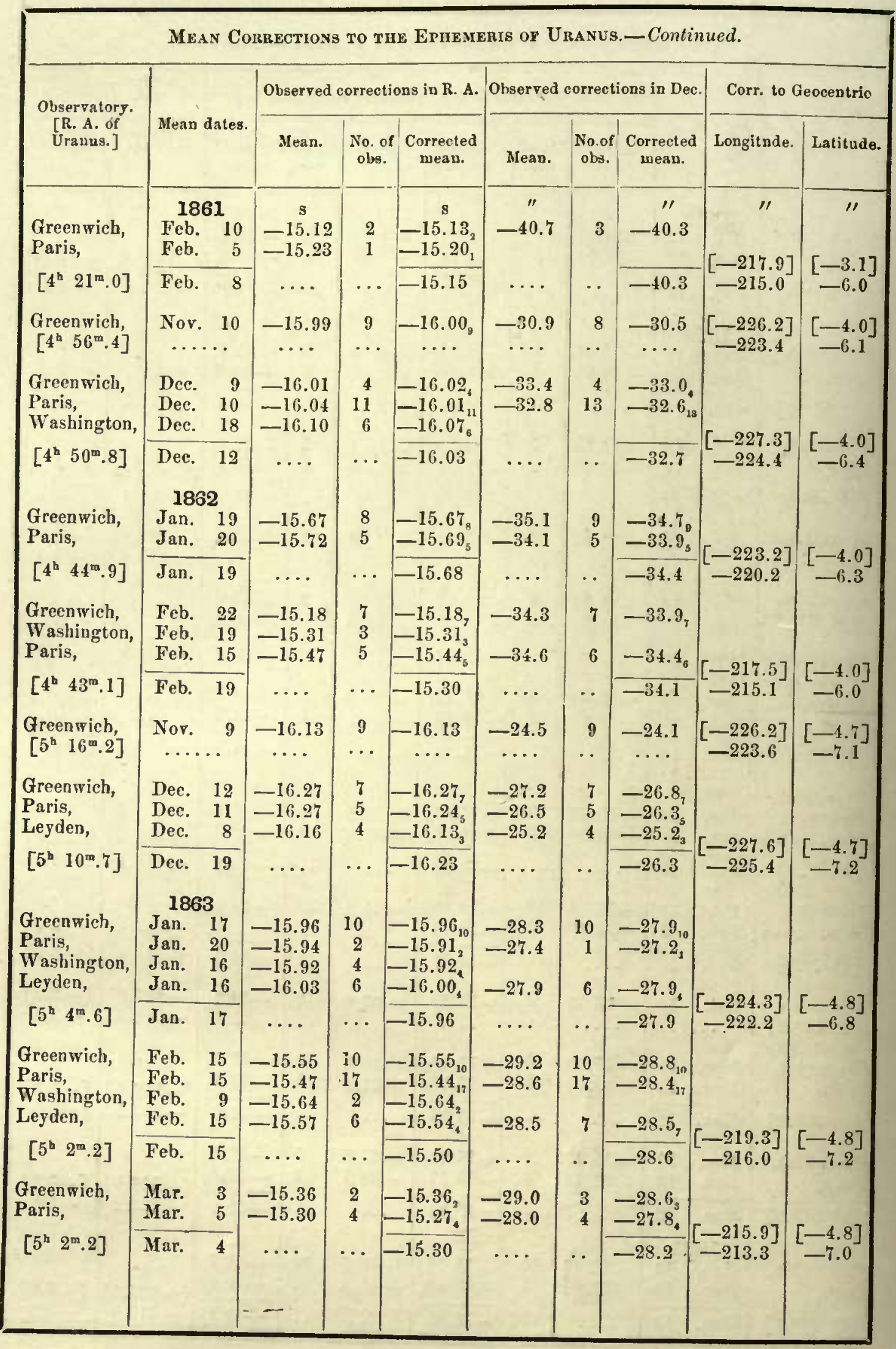


Mean Corrections to the Ephemeris of Uranus,-Continued.

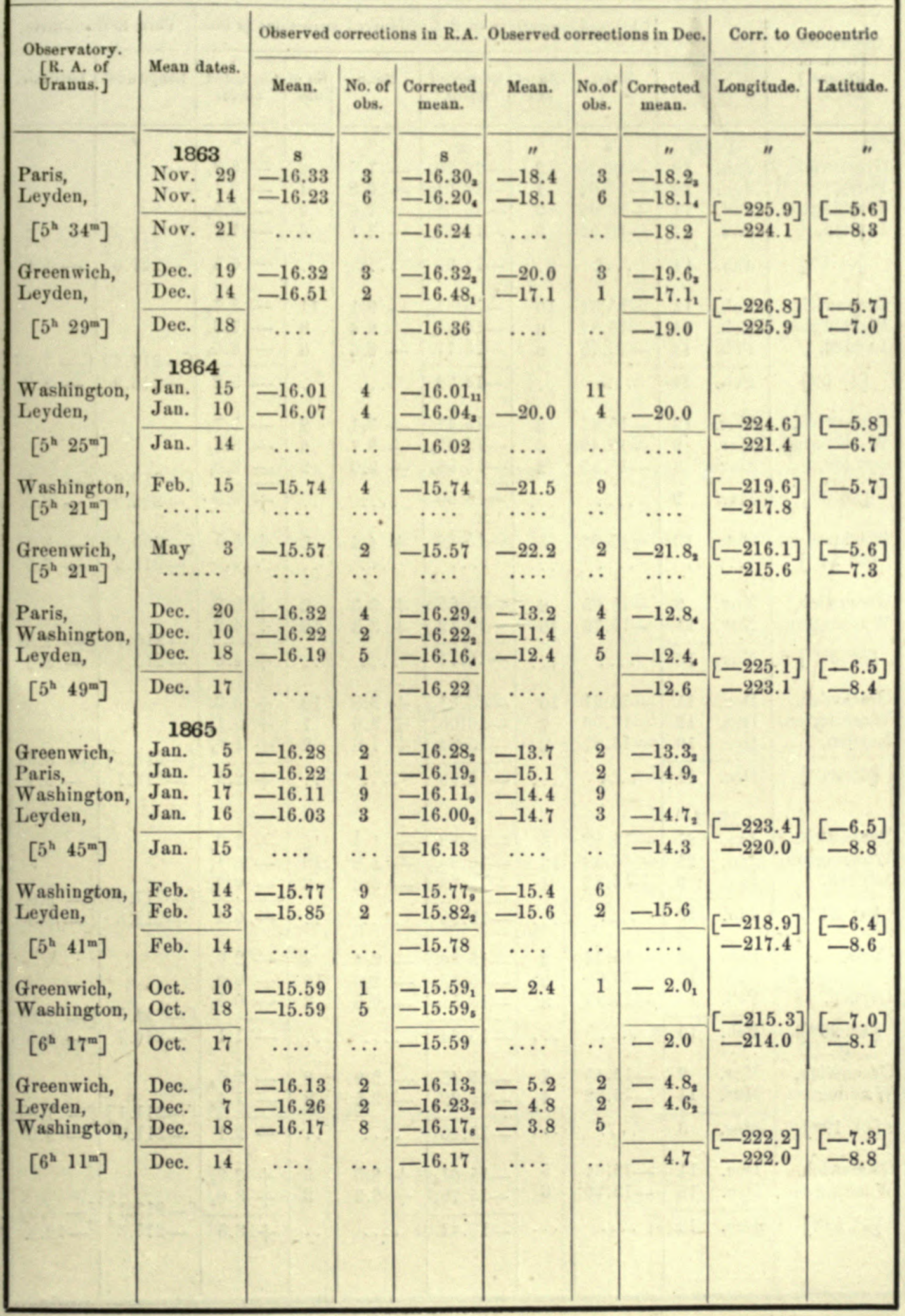




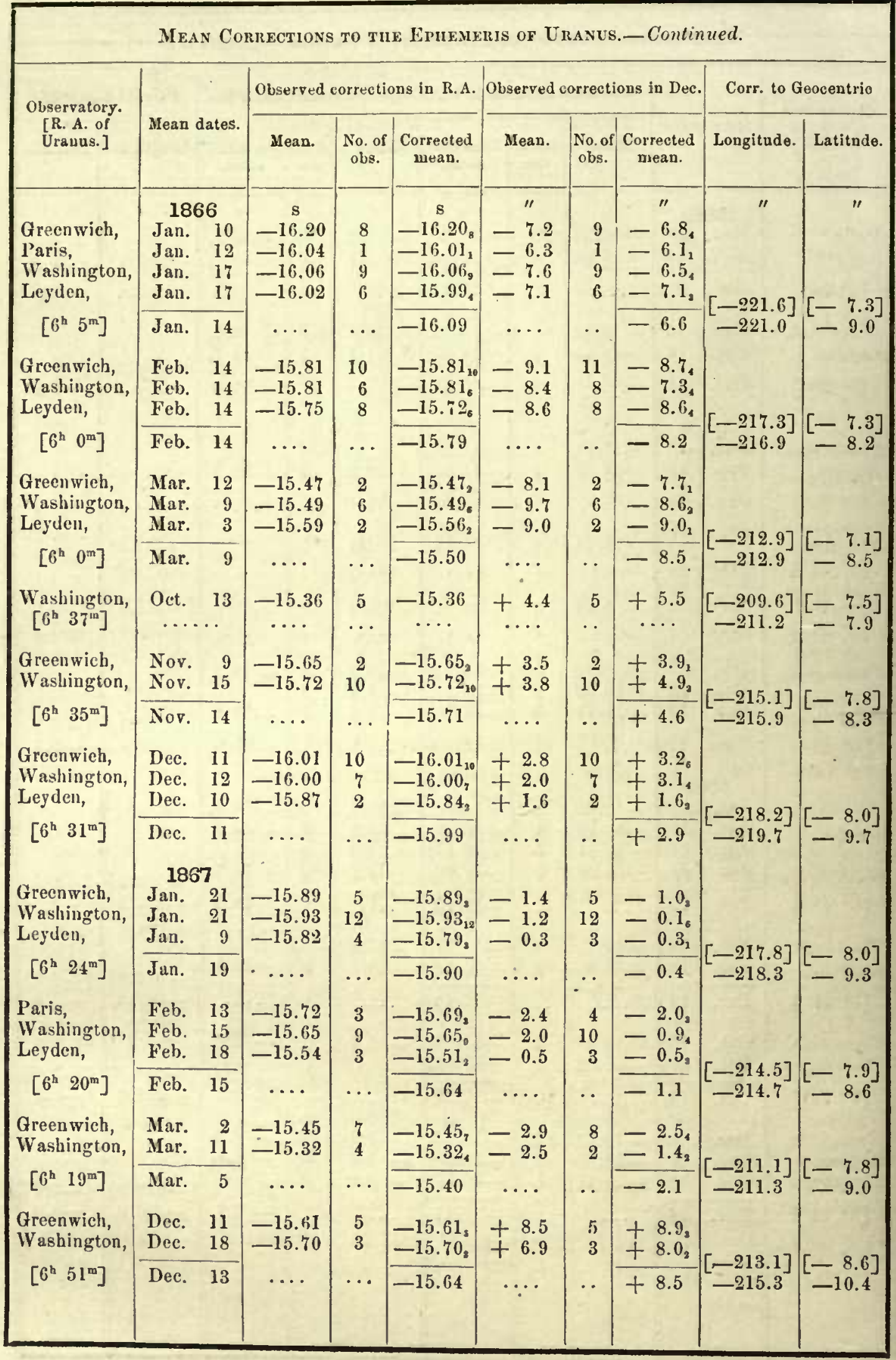




\begin{tabular}{|c|c|c|c|c|c|c|c|c|c|}
\hline \multirow{3}{*}{$\begin{array}{c}\text { Observatory. } \\
\text { [H. A. of } \\
\text { Urauas.] }\end{array}$} & \multicolumn{9}{|c|}{ Mean Counetions to tur kipheneris of Unasus. - Conlinued. } \\
\hline & \multirow{2}{*}{ Mlean dates. } & \multicolumn{3}{|c|}{ Observed correctlous in $\mathbf{R} . \mathbf{A}$. } & \multicolumn{3}{|c|}{ Observed corroctlons in Deo. } & \multicolumn{2}{|c|}{ Corr. to Aeoceutrio } \\
\hline & & Meau. & $\begin{array}{c}\text { No. of } \\
\text { obs. }\end{array}$ & $\begin{array}{c}\text { Corrected } \\
\text { mean. }\end{array}$ & Mean. & $\begin{array}{c}\text { Na.of } \\
\text { obs. }\end{array}$ & $\begin{array}{c}\text { Corrected } \\
\text { weau. }\end{array}$ & Longltado. & Letitade. \\
\hline $\begin{array}{c}\text { Grecenich, } \\
{\left[0^{b}+5^{m}\right]}\end{array}$ & $\begin{array}{c}1868 \\
\text { Jan. } 13 \\
\ldots . .\end{array}$ & $\begin{array}{c}8 \\
-15.67 \\
\ldots\end{array}$ & $\begin{array}{l}t \\
\cdots\end{array}$ & $\begin{array}{c}8 \\
-15.67 \\
\ldots\end{array}$ & $\begin{array}{c}" 1 \\
+6.9 \\
\ldots .\end{array}$ & $\begin{array}{l}4 \\
\cdots\end{array}$ & $\begin{array}{c}\prime \prime \\
+6.7 . \\
\ldots\end{array}$ & {$\left[\begin{array}{c}\prime \prime \\
{[-213.4]} \\
-215.5\end{array}\right.$} & {$\left[\begin{array}{c}\prime \prime \\
-8.7] \\
-10.1\end{array}\right.$} \\
\hline $\begin{array}{l}\text { Greenwich, } \\
\text { J,eyden, } \\
\text { Wishington, } \\
\text { Puris, }\end{array}$ & $\begin{array}{lr}\text { leb. } & 15 \\
\text { leb. } & 5 \\
\text { Feb. } & 13\end{array}$ & $\begin{array}{r}-15.43 \\
-15.43 \\
-15.50 \\
-15.41\end{array}$ & $\begin{array}{l}8 \\
3 \\
7 \\
7\end{array}$ & $\begin{array}{r}-15.43 \\
-15.40 \\
-15.50^{\circ} \\
-15.39\end{array}$ & $\begin{array}{r}+4.8 \\
+\quad 5.9 \\
+\quad 4.9\end{array}$ & $\begin{array}{l}8 \\
3 \\
6\end{array}$ & $\begin{array}{l}+4.6 \\
+5.92 \\
+6.02\end{array}$ & & \\
\hline$\left[6^{\mathrm{h}}+1^{\mathrm{m}}\right]$ & Feb. 14 & $\cdots$ & $\cdots$ & -15.44 & $\because \cdots$ & $\cdots$ & +5.3 & -212.3 & {$\left[\begin{array}{l}{[-8.6} \\
-9.7\end{array}\right]$} \\
\hline $\begin{array}{l}\text { I,eyden, } \\
\text { Washington, }\end{array}$ & $\begin{array}{ll}\text { Mar. } & 11 \\
\text { Mar. } & 19 \\
\end{array}$ & $\begin{array}{l}-15.02 \\
-14.91\end{array}$ & $\begin{array}{l}2 \\
1\end{array}$ & $\begin{array}{r}-14.99_{1} \\
-14.91_{1} \\
\end{array}$ & $\begin{array}{l}+4.6 \\
+\quad 3.4\end{array}$ & 2 & $\begin{array}{r}+4.6 \\
+\quad 4.5_{1} \\
\end{array}$ & {$[-205.0]$} & {$[-8.5]$} \\
\hline$\left[\begin{array}{ll}6^{\mathrm{h}} & 39^{\mathrm{m}}\end{array}\right]$ & Mar. 15 & $\cdots$ & $\cdots$ & -14.95 & $\cdots$ & $\cdots$ & +4.6 & -205.5 & -9.2 \\
\hline $\begin{array}{c}\text { Washington, } \\
{\left[\begin{array}{lll}7^{*} & 16^{\mathrm{m}}\end{array}\right]}\end{array}$ & $\begin{array}{c}\text { Oet. } 1 \mathrm{~s} \\
\ldots .\end{array}$ & $\begin{array}{c}-14.57 \\
\ldots\end{array}$ & $\begin{array}{c}6 \\
\cdots\end{array}$ & $\begin{array}{c}-14.57 \\
\ldots\end{array}$ & $\begin{array}{c}+12.3 \\
\ldots\end{array}$ & $\begin{array}{l}5 \\
\cdots\end{array}$ & $\begin{array}{c}+13.3 \\
\ldots\end{array}$ & {$\left[\begin{array}{l}{[-198.3]} \\
-201.5\end{array}\right.$} & 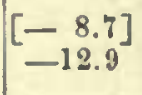 \\
\hline $\begin{array}{c}\text { Washinglon, } \\
{\left[\begin{array}{ll}7^{\mathrm{h}} & 16^{\mathrm{m}}\end{array}\right]}\end{array}$ & $\begin{array}{c}\text { Nor. } \\
\ldots\end{array}$ & $\begin{array}{c}-14.83 \\
\ldots\end{array}$ & $\begin{array}{c}5 \\
\cdots\end{array}$ & $\begin{array}{c}-14.83 \\
\ldots\end{array}$ & $\begin{array}{c}+16.2 \\
\ldots\end{array}$ & $\begin{array}{r}5 \\
\cdots\end{array}$ & $\begin{array}{c}+17.3 \\
\ldots\end{array}$ & {$\left[\begin{array}{l}{[-202.0} \\
-205.6\end{array}\right]$} & 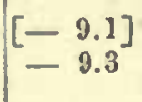 \\
\hline $\begin{array}{l}\text { Greenwich, } \\
\text { Washington, }\end{array}$ & $\begin{array}{ll}\text { Nec. } & 25 \\
\text { Iec. } & 12 \\
\end{array}$ & $\begin{array}{l}-15.24 \\
-15.16\end{array}$ & $\begin{array}{l}5 \\
6\end{array}$ & $\begin{array}{r}-15.24_{1} \\
-15.16 \\
\end{array}$ & $\begin{array}{r}+15.1 \\
+14.6\end{array}$ & $\begin{array}{l}5 \\
4\end{array}$ & $\begin{array}{l}+14.9 \\
+15.6 \\
\end{array}$ & 6.57 & {$[-9.2]$} \\
\hline$\left[\begin{array}{ll}7^{\mathrm{h}} & 10^{\mathrm{m}}\end{array}\right]$ & $\begin{array}{c}\text { Dce. } 18 \\
1869\end{array}$ & & $\cdots$ & -15.20 & . & . & +15.2 & -210.3 & -10.0 \\
\hline $\begin{array}{l}\text { Greenwich, } \\
\text { Washingtou, }\end{array}$ & $\begin{array}{ll}\text { Jan. } & 19 \\
\text { Jan. } & 18 \\
\end{array}$ & $\begin{array}{l}-15.28 \\
-15.31\end{array}$ & $\begin{array}{l}1 \\
8\end{array}$ & $\begin{array}{r}-15.28_{1} \\
-15.31_{1} \\
\end{array}$ & $\begin{array}{l}+12.6 \\
+12.9\end{array}$ & $\begin{array}{l}1 \\
8\end{array}$ & $\begin{array}{r}+12.4_{1} \\
+13.4 \\
\end{array}$ & {$[-207.1]$} & {$[-9.3]$} \\
\hline$\left[\begin{array}{ll}7^{2} & 5\end{array}\right]$ & Jan. 18 & $\cdots$ & $\cdots$ & -15.31 & . & - & +13.3 & -211.4 & -10.3 \\
\hline $\begin{array}{l}\text { Areenwich, } \\
\text { Washington, } \\
\text { Paris, }\end{array}$ & $\begin{array}{ll}\text { Feb. } & 14 \\
\text { Feb. } & 14 \\
\text { Feb. } & 10\end{array}$ & $\begin{array}{r}-15.04 \\
-15.13 \\
-15.09\end{array}$ & $\begin{array}{l}10 \\
13 \\
10\end{array}$ & $\begin{array}{r}-15.04_{10} \\
-15.13_{13} \\
-15.06_{9} \\
\end{array}$ & $\begin{array}{r}+11.1 \\
+11.2 \\
+11.7\end{array}$ & $\begin{array}{l}10 \\
13 \\
10\end{array}$ & $\begin{array}{l}+10.9_{10} \\
+11.7_{11} \\
+12.2_{10} \\
\end{array}$ & {$[-2$} & \\
\hline$\left[\begin{array}{ll}i^{b} & 1^{\mathrm{m}}\end{array}\right]$ & Feb. 13 & & . & -15.08 & . & . & +11.7 & -208.1 & {$\left[\begin{array}{l}{[-10.2} \\
-10\end{array}\right.$} \\
\hline $\begin{array}{l}\text { Greenwich, } \\
\text { Washington, }\end{array}$ & $\begin{array}{ll}\text { Mar. } & 3 \\
\text { Mar. } & 7 \\
\end{array}$ & $\begin{array}{l}-14.81 \\
-14.87\end{array}$ & $\begin{array}{l}2 \\
2\end{array}$ & $\begin{array}{r}-14.81, \\
-14.87, \\
\end{array}$ & $\begin{array}{r}+10.8 \\
+11.4\end{array}$ & $\begin{array}{l}2 \\
2\end{array}$ & $\begin{array}{r}+10.6 \\
+11.9 \\
\end{array}$ & {$[-201.5]$} & {$[-9.2]$} \\
\hline$\left[\begin{array}{ll}6^{\mathrm{h}} & 59^{\mathrm{m}}\end{array}\right]$ & Mar. 5 & 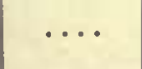 & $\cdots$ & -14.84 & $\ldots$ & .. & +11.3 & -204.8 & -9.3 \\
\hline $\begin{array}{l}\text { Greenwich, } \\
{\left[\begin{array}{ll}7^{\mathrm{t}} & 32^{m}\end{array}\right]}\end{array}$ & $\begin{array}{c}\text { Dec. } 8 \\
\ldots \ldots\end{array}$ & $\begin{array}{c}-14.50 \\
\cdots\end{array}$ & $\begin{array}{c}2 \\
\cdots\end{array}$ & $\begin{array}{c}-14.50 \\
\cdots\end{array}$ & $\begin{array}{c}+21.1 \\
\ldots\end{array}$ & $\begin{array}{l}2 \\
\cdots\end{array}$ & $\begin{array}{c}+20.9 \\
\ldots\end{array}$ & {$\left[\begin{array}{l}{[-197.1]} \\
-202.1\end{array}\right]$} & {$\left[\begin{array}{l}-9.8 \\
-10.3\end{array}\right]$} \\
\hline $\begin{array}{c}\text { Green wich, } \\
{\left[\begin{array}{ll}7^{\natural} & 25^{\varpi}\end{array}\right]}\end{array}$ & $\begin{array}{c}1870 \\
\text { Jan. } 19 \\
\ldots \ldots\end{array}$ & $\begin{array}{c}-14.73 \\
\ldots\end{array}$ & $\begin{array}{l}10 \\
\cdots\end{array}$ & $\begin{array}{c}-14.72 \\
\ldots\end{array}$ & $\begin{array}{c}+19.0 \\
\ldots\end{array}$ & $\begin{array}{l}10 \\
\cdots\end{array}$ & $\begin{array}{c}+18.8 \\
\ldots\end{array}$ & {$\left[\begin{array}{l}-199.3 \\
-204.5\end{array}\right]$} & {$\left[\begin{array}{l}-9.9] \\
-10.8\end{array}\right.$} \\
\hline $\begin{array}{l}\text { Greenwich, } \\
\text { Washington, }\end{array}$ & $\begin{array}{ll}\text { Feb. } & 16 \\
\text { Feb. } & 16\end{array}$ & $\begin{array}{r}-14.58 \\
-14.49\end{array}$ & $\begin{array}{l}7 \\
1\end{array}$ & $\begin{array}{r}-14.57_{2} \\
-14.49_{1}\end{array}$ & $\begin{array}{l}+17.2 \\
+16.7\end{array}$ & $\begin{array}{l}7 \\
2\end{array}$ & $\begin{array}{r}+17.0 \\
+17.1\end{array}$ & & \\
\hline$\left[\begin{array}{ll}7^{b} & 21^{\varpi}\end{array}\right]$ & Feb. 16 & $\ldots$ & .. & -14.55 & $\ldots$ & $\cdots$ & +17.0 & - -201.9 & {$\left[\begin{array}{l}{[-10.9} \\
-10.9\end{array}\right.$} \\
\hline
\end{tabular}




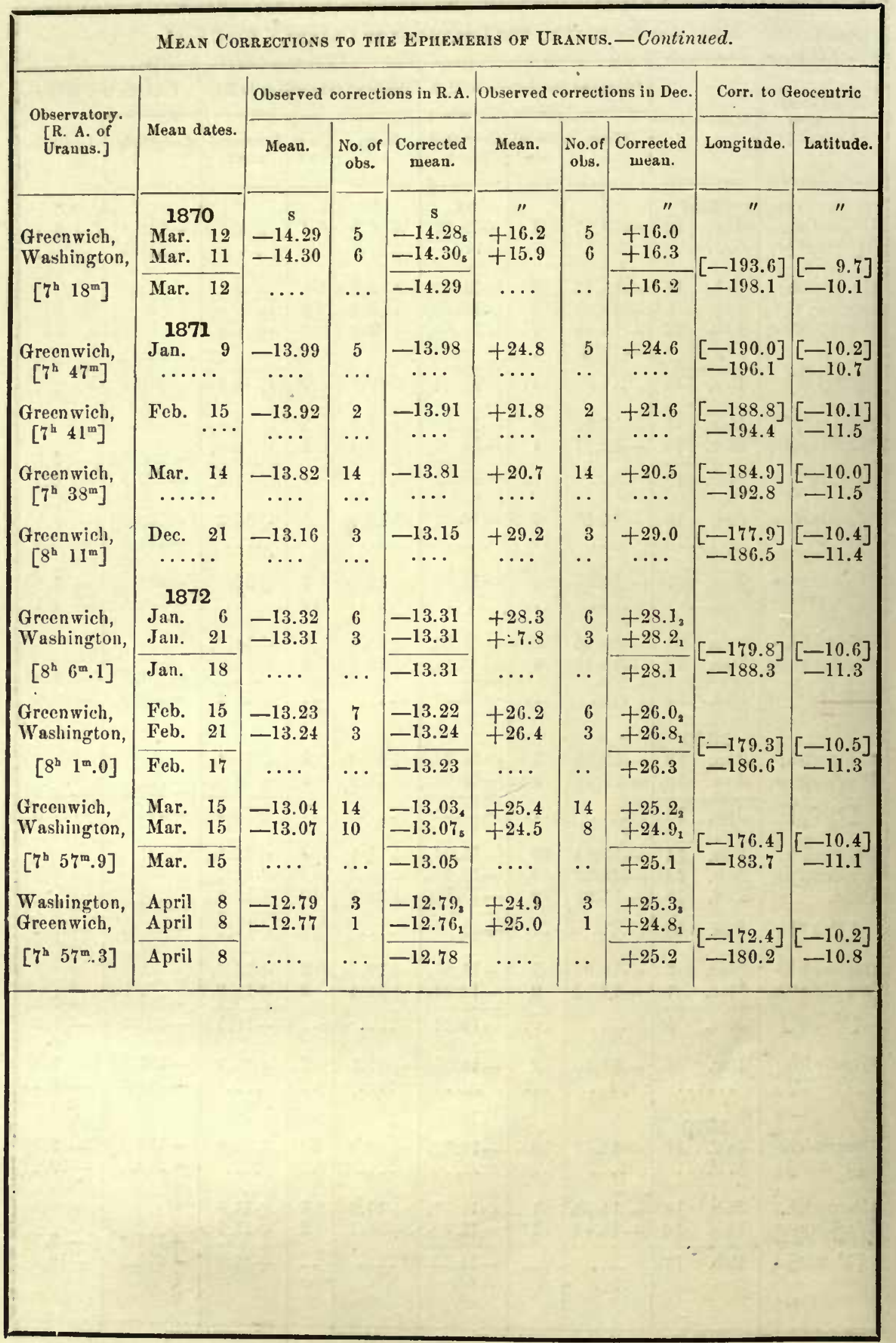




\begin{tabular}{|c|c|c|c|c|c|c|}
\hline \multicolumn{2}{|c|}{ Date. } & \multicolumn{3}{|c|}{ Heliocentrio. } & \multicolumn{2}{|c|}{ Geoeentrio. } \\
\hline \multirow{8}{*}{1830} & & $\delta \lambda_{n}$ & $M \delta_{\rho}$ & $\delta \beta_{\prime \prime}$ & $\delta l$ & $\delta b$ \\
\hline & July 24 & -18.0 & +1001 & +9.0 & -19.3 & +9.5 \\
\hline & Aug. 13 & 18.3 & 1007 & 9.0 & 18.8 & 9.5 \\
\hline & Sept. 2 & 18.4 & 1014 & 9.1 & 17.9 & 9.5 \\
\hline & Sept. 22 & 18.5 & 1020 & 9.1 & 17.1 & 9.4 \\
\hline & Oet. 12 & 18.7 & 1027 & 9.1 & 16.7 & 9.3 \\
\hline & Nov. 1 & 19.0 & 1031 & $\begin{array}{r}9.2 \\
\end{array}$ & 16.5 & 9.2 \\
\hline & Nov. 21 & -19.3 & +1038 & +9.2 & -16.7 & +9.1 \\
\hline \multirow{8}{*}{1831} & July 19 & -22.5 & +1112 & +9.5 & -24.5 & +10.0 \\
\hline & Aug. 8 & 22.6 & 1119 & 9.5 & 23.7 & 10.0 \\
\hline & Aug. 28 & 22.7 & 1123 & 9.6 & 22.7 & 10.1 \\
\hline & Sept. 17 & 22.8 & 1128 & 9.6 & 21.7 & 10.0 \\
\hline & Oet. 7 & 22.9 & 1133 & 9.6 & 20.9 & 9.9 \\
\hline & Oet. 27 & 23.2 & 1138 & 9.7 & 20.6 & 9.8 \\
\hline & Nov. 16 & 23.5 & 1142 & 9.7 & 20.6 & 9.6 \\
\hline & Dec. 6 & -23.8 & +1146 & +9.7 & -21.0 & 9.5 \\
\hline \multirow[t]{7}{*}{1832} & Aug. $\quad 2$ & -26.9 & +1198 & +9.8 & -28.7 & +10.3 \\
\hline & Aug. 22 & 27.2 & 1201 & 9.9 & 27.9 & 10.4 \\
\hline & Sept. 11 & 27.4 & 1202 & 9.9 & 26.9 & 10.3 \\
\hline & Oet. 1 & 27.6 & 1205 & 9.9 & 26.1 & 10.2 \\
\hline & Oet. 21 & 27.8 & 1208 & 10.0 & 25.4 & 10.2 \\
\hline & Nov. 10 & 28.0 & 1209 & 10.1 & 25.1 & 10.1 \\
\hline & Nov. 30 & -28.3 & +1209 & +10.2 & -25.2 & +10.0 \\
\hline \multirow[t]{7}{*}{1833} & July 28 & -31.9 & +1233 & +10.2 & -34.5 & +10.7 \\
\hline & Aug. 17 & 32.2 & 1236 & 10.3 & 33.7 & 10.8 \\
\hline & Sept. 6 & 32.4 & 1240 & 10.3 & 32.7 & 10.8 \\
\hline & Sept. 26 & 32.6 & 1242 & 10.3 & 31.8 & 10.7 \\
\hline & Oct. 16 & 32.9 & 1245 & 10.3 & 31.0 & 10.5 \\
\hline & Nov. 5 & 33.2 & 1247 & 10.4 & 30.4 & 10.4 \\
\hline & Nov. 25 & -33.5 & +1250 & +10.4 & -30.2 & +10.3 \\
\hline \multirow[t]{8}{*}{1834} & July 23 & -38.0 & +1264 & +10.5 & -41.2 & +11.0 \\
\hline & Aug. 12 & 38.4 & 1266 & 10.5 & 40.7 & 11.1 \\
\hline & Sept. 1 & 38.4 & 1268 & 10.6 & 39.6 & 11.1 \\
\hline & Sept. 21 & 38.7 & 1270 & 10.6 & 38.6 & 11.1 \\
\hline & Oet. 11 & 39.1 & 1275 & 10.6 & 37.7 & 10.9 \\
\hline & Oct. 31 & 39.4 & 1277 & 10.6 & 36.9 & 10.8 \\
\hline & Nov. 20 & 39.7 & 1282 & 10.7 & 36.6 & 10.7 \\
\hline & Dec. 10 & -40.1 & +1282 & +10.7 & -36.1 & +10.5 \\
\hline \multirow[t]{8}{*}{1835 , } & July 18 & -43.9 & +1309 & +10.8 & -47.7 & +11.3 \\
\hline & Aug. 7 & 44.5 & 1311 & 10.7 & 47.6 & 11.3 \\
\hline & Aug. 27 & 44.5 & 1313 & 10.7 & 46.6 & 11.3 \\
\hline & Sept. 16 & 45.1 & 1316 & 10.7 & 45.8 & 11.2 \\
\hline & Oct. 6 & 45.4 & 1318 & 10.8 & 44.8 & 11.2 \\
\hline & Oct. 26 & 45.9 & 1321 & 10.7 & 44.0 & 10.9 \\
\hline & Nov. 15 & 46.5 & 1324 & 10.8 & 43.6 & 10.8 \\
\hline & Dec. 5 & -46.6 & +1327 & +10.7 & -42.5 & +10.6 \\
\hline \multirow[t]{4}{*}{1836} & July 12 & -51.0 & +1361 & +11.0 & -55.3 & +11.4 \\
\hline & Aug. 1 & 51.3 & 1364 & 11.0 & 55.2 & 11.5 \\
\hline & Ang. 21 & 51.5 & 1365 & $\begin{array}{r}11.1 \\
+11.0\end{array}$ & $\begin{array}{r}54.6 \\
-53.7\end{array}$ & $\begin{array}{r}11.7 \\
+11.6\end{array}$ \\
\hline & Sept. 10 & -51.9 & +1368 & +11.0 & & \\
\hline
\end{tabular}


Corrections to be Applied to the Positions of Uranus-Continued.

\begin{tabular}{|c|c|c|c|c|c|c|c|}
\hline \multicolumn{3}{|c|}{ Date. } & \multicolumn{3}{|c|}{ Helioceutric. } & \multicolumn{2}{|c|}{ Geocentric. } \\
\hline & & & $\delta 2$ & $M \delta \rho$ & $\delta \beta$ & ${ }^{\prime \prime}$ & $\delta b$ \\
\hline \multirow[t]{5}{*}{1836} & Sept. 3 & 30 & -52.2 & +1370 & +11.0 & -52.4 & +11.5 \\
\hline & Oct. 2 & 20 & 52.9 & 1372 & 11.0 & 51.6 & 11.3 \\
\hline & Nov. & 9 & 53.1 & 1375 & 11.0 & 50.5 & 11.1 \\
\hline & Nov. 2 & 29 & 53.9 & 1377 & 11.0 & 50.4 & 11.0 \\
\hline & Dec. 1 & 19 & -53.8 & +1380 & +11.1 & -49.8 & +10.9 \\
\hline \multirow{9}{*}{1837} & July & 7 & -58.3 & +1387 & +11.0 & -62.9 & +11.4 \\
\hline & July ? & 27 & 58.4 & 1387 & 11.0 & 62.9 & 11.5 \\
\hline & Aug. 1 & 16 & 59.2 & 1388 & 11.1 & 63.1 & 11.7 \\
\hline & Sept. & 5 & 59.2 & 1389 & 11.1 & 62.0 & 11.7 \\
\hline & Scpt. 2 & 25 & 60.0 & 1391 & 11.0 & 61.3 & 11.5 \\
\hline & Oct. 1 & 15 & 60.0 & 1393 & 11.0 & 59.6 & 11.4 \\
\hline & Nov. & 4 & 61.0 & 1394 & 11.0 & 59.1 & 11.2 \\
\hline & Nov. 2 & 24 & 61.3 & 1394 & 11.0 & 58.2 & 11.0 \\
\hline & Dec. 1 & 14 & -62.1 & +1393 & +11.0 & -57.1 & +10.9 \\
\hline \multirow{8}{*}{1838} & Aug. 1 & 11 & -66.4 & +1395 & +11.1 & -71.1 & +11.6 \\
\hline & Aug. 3 & 31 & 67.2 & 1396 & 11.1 & 71.0 & 11.7 \\
\hline & Sept. ? & 20 & 67.7 & 1395 & 11.1 & 70.2 & 11.7 \\
\hline & Oct. 1 & 10 & 68.1 & 1392 & 11.1 & 68.9 & 11.6 \\
\hline & Oct. 3 & 30 & 68.5 & 1390 & 11.1 & 67.6 & 11.4 \\
\hline & Nor. 1 & 19 & 68.9 & 1388 & 11.1 & 66.4 & 11.2 \\
\hline & Dec. & 9 & 69.4 & 1388 & 11.1 & 65.8 & 11.1 \\
\hline & Dec. 2 & 29 & -69.7 & +1389 & +11.2 & -65.4 & +11.0 \\
\hline \multirow{8}{*}{1839} & Ang. & 6 & -74.7 & +1382 & +11.1 & -80.0 & +11.6 \\
\hline & Aug. 2 & 26 & 75.1 & 1381 & 11.1 & 79.7 & 11.7 \\
\hline & Sept. 1 & 15 & 75.5 & 1380 & 11.0 & 79.0 & 11.6 \\
\hline & Oct. & 5 & 76.0 & 1379 & 11.1 & 77.9 & 11.6 \\
\hline & Oct. 2 & 25 & 76.4 & 1379 & 11.1 & 76.5 & 11.5 \\
\hline & Nov. 1 & 14 & 76.8 & 1379 & 11.1 & 75.2 & 11.3 \\
\hline & Dec. & 4 & 77.2 & 1378 & 11.1 & 74.0 & 11.1 \\
\hline & Dec. 2 & 24 & 77.6 & 1377 & 11.1 & - 73.5 & 10.9 \\
\hline \multirow[t]{9}{*}{1840} & Jan. 1 & 13 & -78.0 & +1376 & +11.0 & -73.1 & +10.7 \\
\hline & June 2 & 24 & -81.9 & +1376 & +11.0 & -86.0 & +11.1 \\
\hline & Aug. 2 & 20 & 82.7 & 1374 & 11.0 & 87.9 & 11.5 \\
\hline & Sept. & 9 & 83.4 & 1376 & 11.0 & 87.8 & 11.6 \\
\hline & Sept. 2 & 29 & 83.5 & 1377 & 11.0 & 86.4 & 11.5 \\
\hline & Oct. 1 & 19 & 84.2 & 1376 & 11.0 & 85.5 & 11.4 \\
\hline & Nov. & 8 & 84.4 & 1376 & 11.0 & 83.5 & 11.3 \\
\hline & Nov. 1 & 13 & 85.3 & 1377 & 11.0 & 82.7 & 11.1 \\
\hline & Dec. 1 & 13 & 85.5 & 1378 & 11.0 & 81.5 & 10.9 \\
\hline \multirow[t]{9}{*}{1841} & Jan. & 7 & -86.1 & +1377 & +10.9 & -81.3 & +10.7 \\
\hline & Junc 1 & 16 & -89.8 & +1378 & +10.8 & -93.2 & +10.8 \\
\hline & Aug. 1 & 15 & 91.1 & 1384. & 10.8 & 97.0 & 11.3 \\
\hline & Sept. & 4 & 91.5 & 1385 & 10.8 & 96.9 & 11.4 \\
\hline & Sept. 2 & 24 & 91.9 & 1398 & 10.7 & 96.1 & 11.3 \\
\hline & Oct. 1 & 14 & 92.3 & 1390 & 10.7 & 94.8 & 11.2 \\
\hline & Nor. & 3 & 92.9 & 1392 & 10.7 & 93.4 & 11.1 \\
\hline & Nor. 2 & 23 & 93.4 & 1393 & 10.8 & 91.8 & 10.9 \\
\hline & Dec. 1 & 13 & 93.8 & 1395 & 10.7 & 90.5 & 10.7 \\
\hline \multirow[t]{2}{*}{1842} & Jan. & 2 & 94.3 & 1398 & 10.8 & 89.7 & 10.6 \\
\hline & Fcb. 1 & 11 & -95.0 & +1404 & +10.7 & -89.6 & +10.3 \\
\hline
\end{tabular}




\begin{tabular}{|c|c|c|c|c|c|c|}
\hline \multicolumn{7}{|c|}{ Corrections to be Applied to the Posirions of Uranus-Continued. } \\
\hline \multicolumn{2}{|r|}{ Date. } & \multicolumn{3}{|c|}{ Helioeentrio. } & \multicolumn{2}{|c|}{ Geoeentrie. } \\
\hline & & $\delta \lambda_{\prime \prime}$ & $M \delta \rho$ & ${ }_{n}^{\delta}{ }_{n}$ & $\ell_{n}$ & ${ }^{8 b}$ \\
\hline \multirow[t]{9}{*}{1842} & June 11 & -96.7 & +1409 & +10.6 & -98.5 & +10.5 \\
\hline & Aug. 10 & 99.1 & 1408 & 10.6 & 105.3 & 10.0 \\
\hline & Aug. 30 & 99.6 & 1409 & 10.7 & 105.8 & 10.2 \\
\hline & Sept. 19 & 99.9 & 1409 & 10.6 & 105.2 & 10.2 \\
\hline & Oct. $\quad 9$ & 100.5 & 1410 & 10.5 & 104.2 & 10.0 \\
\hline & Oet. 29 & 100.9 & 1412 & 10.5 & 102.6 & 10.9 \\
\hline & Nov. 18 & $101.3^{-}$ & 1413 & 10.5 & 100.8 & 10.8 \\
\hline & Dec. 8 & 101.8 & 1414 & 10.5 & 99.2 & 10.6 \\
\hline & Dec. 28 & 102.2 & 1415 & 10.5 & 98.0 & 10.4 \\
\hline \multirow[t]{9}{*}{ 1843, } & Jan. 17 & -102.5 & +1417 & +10.4 & -97.2 & +10.2 \\
\hline & Aug. 5 & -107.2 & +1422 & +10.3 & -113.7 & +10.7 \\
\hline & Aug. 25 & 107.7 & 1420 & 10.2 & 114.4 & 10.7 \\
\hline & Sept. 14 & 108.0 & 1420 & 10.1 & 114.3 & 10.6 \\
\hline & Oct. 4 & 108.5 & 1420 & 10.1 & 113.4 & 10.6 \\
\hline & Oet. 24 & 109.0 & 1419 & 10.1 & 112.1 & 10.5 \\
\hline & Nov. 13 & 109.4 & 1418 & 10.1 & 110.2 & 10.4 \\
\hline & Dec. 3 & 110.0 & 1418 & 10.1 & 108.6 & 10.3 \\
\hline & Dec. 23 & 110.4 & 1417 & 10.1 & 106.9 & 10.1 \\
\hline \multirow[t]{9}{*}{1844} & Jan. 12 & -110.8 & +1418 & +10.0 & -105.9 & +9.8 \\
\hline & July 30 & -115.1 & +1406 & +9.7 & -121.2 & +10.0 \\
\hline & Aug. 19 & 115.4 & 1403 & 9.8 & 122.3 & 10.2 \\
\hline & Sept. 8 & 115.8 & 1400 & 9.8 & 122.7 & 103 \\
\hline & Sept. 28 & 116.3 & 1397 & 9.8 & 122.4 & 10.3 \\
\hline & Oet. 18 & 116.9 & 1396 & 9.7 & 121.3 & 10.2 \\
\hline & Nov. 7 & 117.4 & 1392 & 9.6 & 119.7 & 10.0 \\
\hline & Nov. 27 & 117.8 & 1389 & 9.6 & 117.6 & 9.8 \\
\hline & Dec. 17 & 118.3 & 1384 & 9.6 & 115.7 & 9.7 \\
\hline \multirow[t]{9}{*}{1845} & Jan. 6 & 118.6 & 1381 & 9.5 & 114.3 & 9.4 \\
\hline & Jan. 26 & -119.1 & +1378 & +9.6 & -113.3 & +9.4 \\
\hline & Aug. 14 & -123.5 & +1340 & +9.1 & -130.4 & +9.4 \\
\hline & Sept. 3 & 123.9 & 1335 & 9.4 & 131.4 & 9.8 \\
\hline & Sept. 23 & 124.4 & 1330 & 9.4 & 131.7 & 9.9 \\
\hline & Oct. 13 & 124.9 & 1327 & 9.3 & 130.6 & $\begin{array}{r}9.8 \\
\end{array}$ \\
\hline & Nov. 2 & 125.3 & 1322 & 9.3 & 129.0 & 9.7 \\
\hline & Nov. 22 & 125.7 & 1318 & 9.2 & 127.0 & 9.5 \\
\hline & Dec. 12 & 126.2 & 1313 & 9.2 & 125.0 & 9.3 \\
\hline \multirow[t]{9}{*}{1846 , } & Jan. 1 & 126.7 & 1309 & 9.2 & 123.2 & 9.2 \\
\hline & Jan. 21 & -127.1 & +1303 & +9.2 & -121.9 & -9.0 \\
\hline & Aug. 29 & -132.0 & +1253 & +8.8 & -139.5 & +9.2 \\
\hline & Sept. 18 & 132.3 & 1249 & 8.8 & 139.9 & 9.2 \\
\hline & Oet. 8 & 132.8 & 1246 & 8.8 & 139.8 & 9.3 \\
\hline & Oct. 28 & 133.2 & 1242 & 8.7 & 138.4 & 9.1 \\
\hline & Nov. 17 & 133.5 & 1238 & 8.7 & 136.4 & 9.0 \\
\hline & Dec. 7 & 134.1 & 1234 & 8.7 & 134.4 & 8.9 \\
\hline & Dec. 27 & 134.6 & 1233 & 8.6 & 132.3 & 8.7 \\
\hline \multirow[t]{5}{*}{1847} & Jan. 16 & -134.9 & +1230 & +8.6 & -130.5 & +8.5 \\
\hline & Aug. 24 & -139.5 & +1197 & +8.3 & -146.7 & +8.6 \\
\hline & Sept. 13 & 140.0 & 1194 & 8.3 & 148.0 & 8.7 \\
\hline & Oet. 3 & 140.5 & 1191 & 8.3 & 148.3 & 8.7 \\
\hline & Oet. 23 & -140.9 & +1188 & +8.2 & -147.4 & +8.6 \\
\hline
\end{tabular}


Corrections to be Applied to the Postrions of Uranus-Continued.

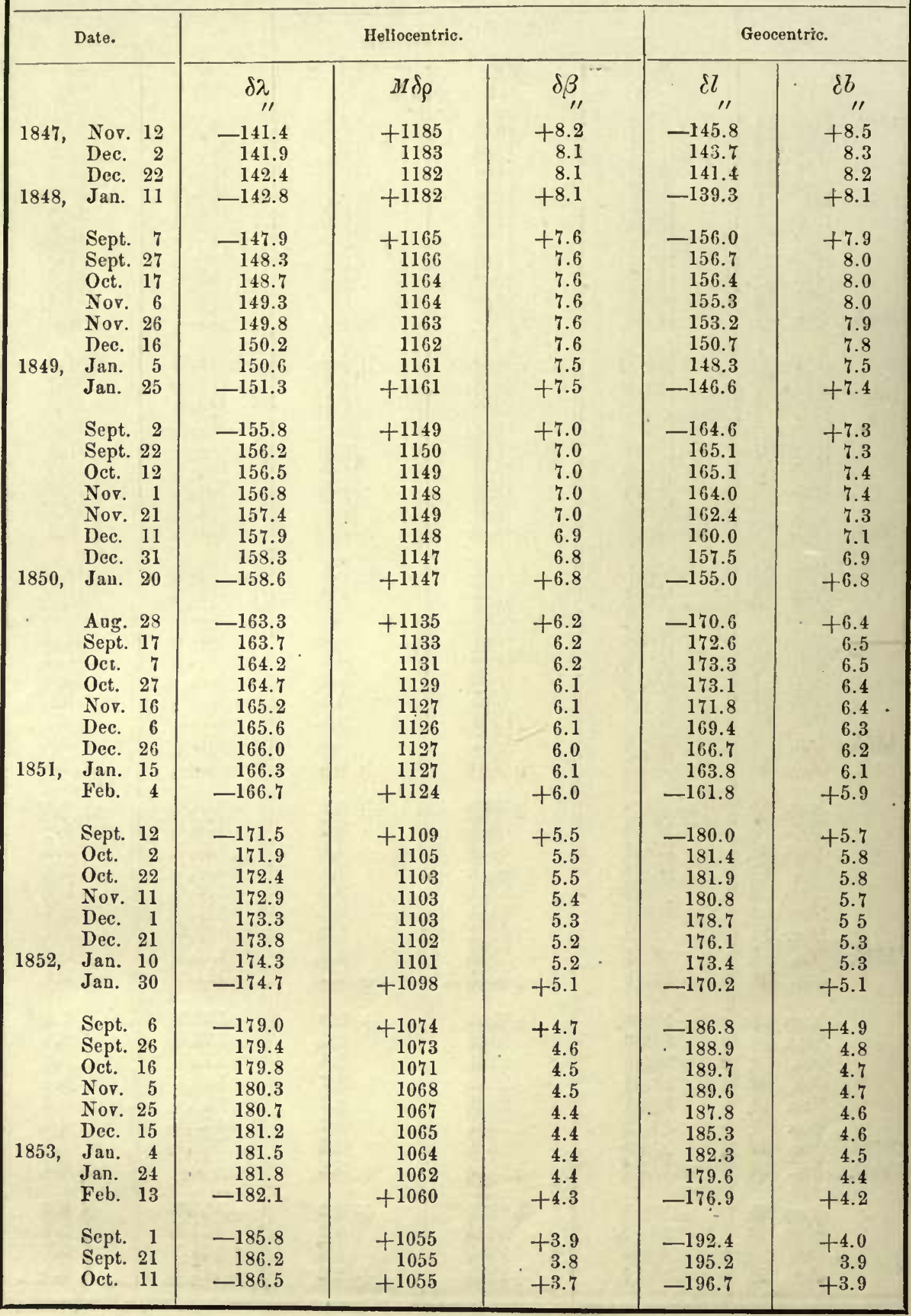




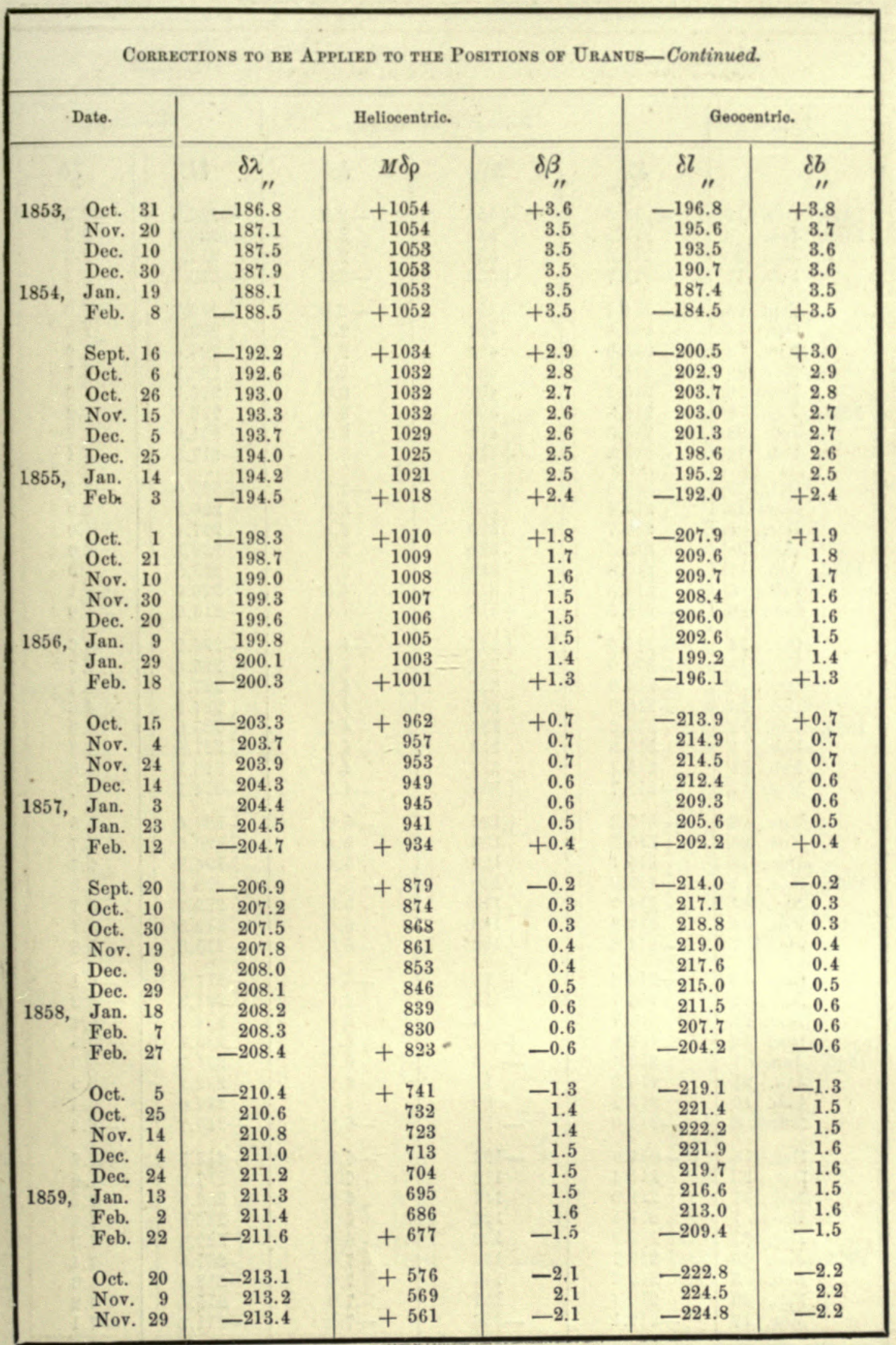


Corrections to be Applied to the Pusitions of Uranus-Continued.

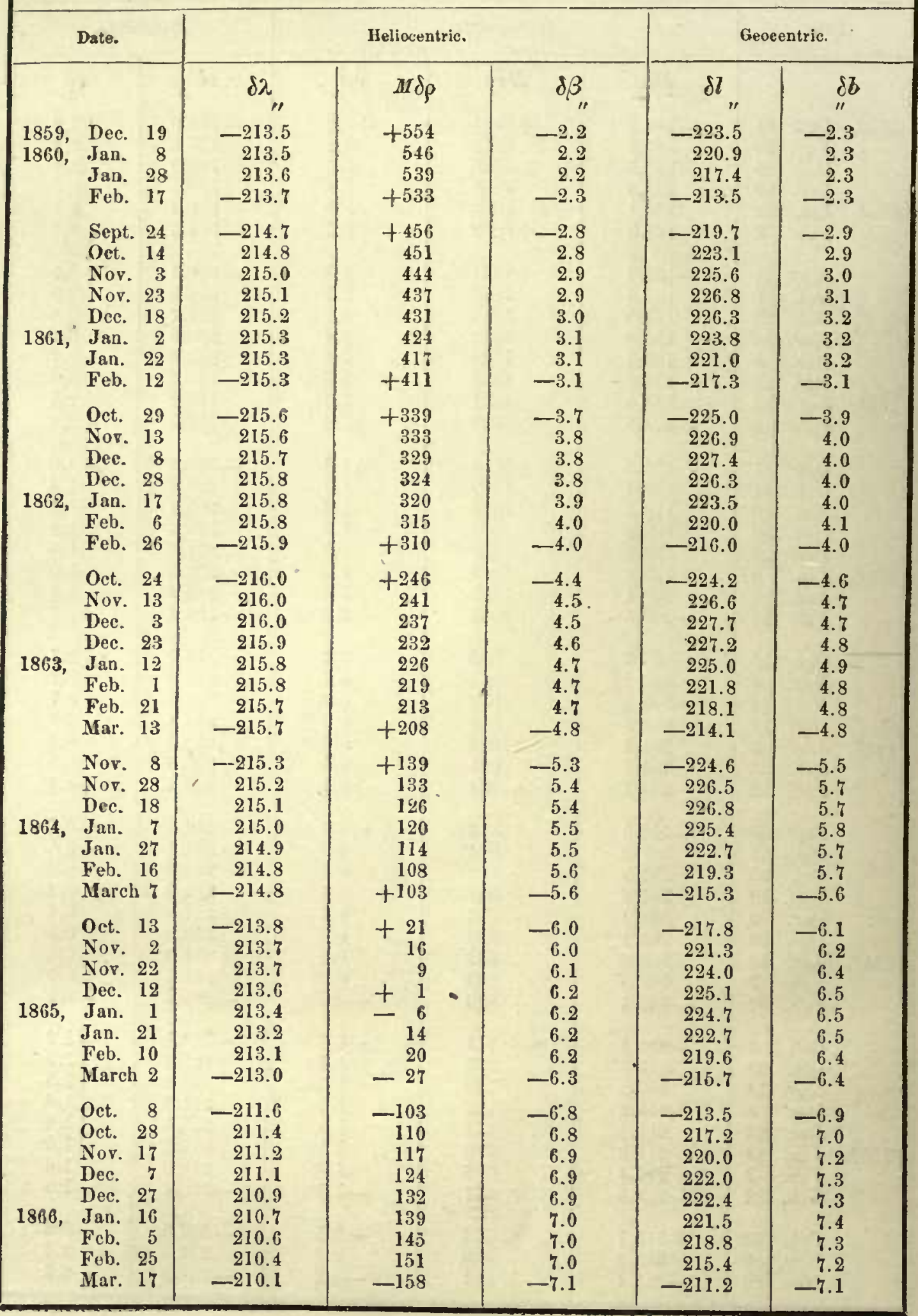


Corrections to be Applied to the Positions of Uranus-Continued.

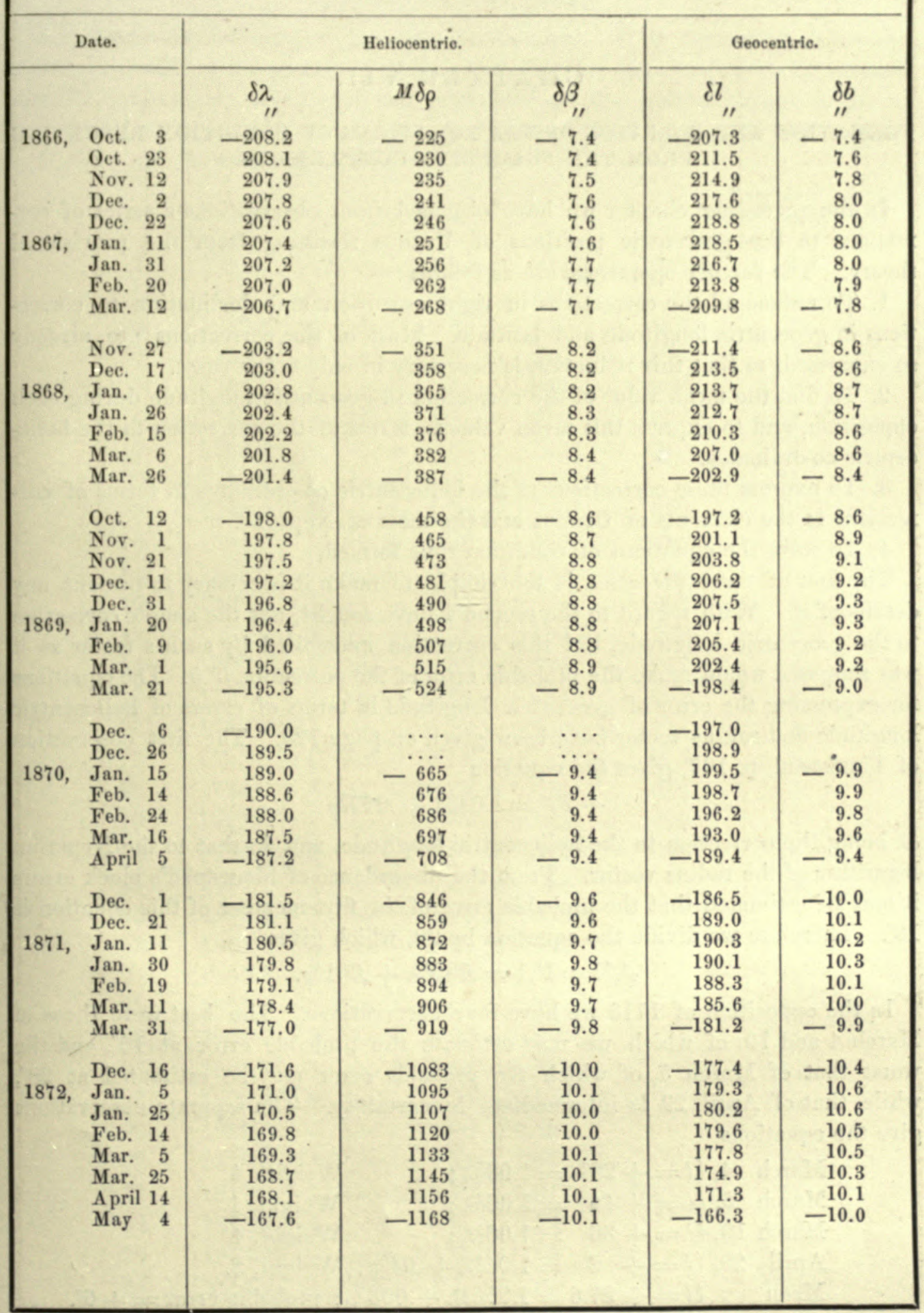


C II $\Lambda$ P T E R VII.

\section{FORMATION AND SOLUTION OF THE FQUATIONS OF CONDITION RESULTING FROM THE PRECEDING COMPARISONS.}

Is the preceding chapter we have obtained from obscrvations a series of corrections to the geocentric positions of Uranus resulting from the provisional theory. The further operations are as follows:-

1. To reduce all the corrections in right ascension and declination to corrections in geocentric longitude and latitude. Most of the corrections are already so expressed, so that this reduction is necessary in only a few cases.

2. 'To find the mean value of the correction in geocentric longitude during each opposition, and to express this mean value in terms of the correction to the heliocentric co-ordinates.

3. 'To express these corrections to the heliocentric co-ordinates in terms of corrections to the elements of Uranus and the mass of Neptunc.

4. 'To solve the equations of condition thus formed.

The first of these processes is too simple to make it necessary to present any details of it. With regard to the second I have sought, not the simple correction to the geocentric longitude, but this correction multiplied by such a factor as it was supposed would make the probable error of the correction $0^{\prime \prime} .5$. The equations for expressing the error of geocentric longitude in terms of errors of heliocentric longitude and radius vector have been given on page 129 . The first observation of Flamistead, p. 107, gives the equation

$$
+22^{\prime \prime}=1.04 \delta \lambda+.027 \delta \rho
$$

$\delta \lambda$ being the correction to the heliocentric longitude, and $\delta \rho$ that to the Neperian logarithm of the radius vector. From the discordance of Flamstead's clock errors it may be estimated that the probable error of the first member of this equation is 10 ". 'Therefore we divide the equation by 20 , which gives

$$
{ }_{2}^{\frac{1}{\delta}} \delta l=1^{\prime \prime} .1=.052 \delta \lambda+.001 \delta \rho .
$$

In the opposition of 1715 we have four observations. The best were those of March 4 and 10, of which we may estimate the probable crror at $10^{\prime \prime}$, and the worst that of March 5, of which the probable error may be estimated at $20^{\prime \prime}$, while that of April 29 is intcrmediate in certainty. The scparate obscrvations give the equations

$$
\begin{array}{lrl}
\text { March } 4, i l=+28^{\prime \prime}=1.06 \delta \lambda ; & & \text { Weight, } 4 \\
\text { March } 5, \delta l=+44=1.06 \delta \lambda ; & & \text { Weight, } 1 \\
\text { March 10, } 1 l=+36=1.06 \delta \lambda ; & & \text { Weight, } 4 \\
\text { April 29, } 2 l=+2=1.04 \delta \lambda+.04 \delta_{\rho} ; & \text { Weight, } 2 . \\
\text { Mean } \delta l=+27.6 & =1.056 \delta \lambda+.003 \delta_{\rho} ; \text { probable error }= \pm 6^{\prime \prime} .
\end{array}
$$


Applying the correction $-1^{\prime \prime} .1$ for equinox, and dividing by 12 , the equation of condition becomes

$$
\frac{1}{12} i l=+2^{\prime \prime} .2=0.088 \delta \lambda .
$$

In this way the following equations were obtained. It is deemed unnecessary to give the details of the process, as it is one which every one can go over for himself from the data already given, and can reproduce all the results, except so far as they depend on the relative weights assigned to the different groups of observations during one and the same opposition.

\begin{tabular}{|c|c|c|c|c|c|}
\hline No. & Date. & & $\begin{array}{c}\text { Equations. } \\
\|\end{array}$ & & $\begin{array}{c}\text { Number of } \\
\text { observations } \\
\text { in R. A. }\end{array}$ \\
\hline 1 & 1691.0 ; & $\frac{1}{2} \delta 8$ & $=+1.1=.052 \delta \lambda$ & $+.001 \delta \rho$ & 1 \\
\hline 2 & 1715.2 & $\frac{1}{12}$ & $=+2.2=.088$ & & 4 \\
\hline 3 & 1748.8 & $\frac{1}{3}$ & $=+12.8=.338$ & +.017 & 1 \\
\hline 4 & 1750.8 & $\frac{1}{3}$ & $=+11.8=.345$ & +.010 & 3 \\
\hline 5 & 1753.9 & $\frac{i}{3}$ & $=+11.6=.333$ & +.016 & 1 \\
\hline 6 & 1756.7 & $\frac{1}{5}$ & $=+5.0=.210$ & +.003 & 1 \\
\hline 7 & 1769.0 & $\frac{2}{5}$ & $=+4.8=.203$ & +.010 & 8 \\
\hline 8 & 1782.0 & $\frac{4}{3}$ & $=+3.0=1.3 \% 0$ & & 21 \\
\hline 9 & 1783.0 & 1 & $=+1.25=1.030$ & -.002 & 13 \\
\hline 10 & 1784.0 & 1 & $=+1.92=1.026$ & -.008 & 13 \\
\hline 11 & 1785.0 & 1 & $=-0.26=1.034$ & +.006 & 10 \\
\hline 12 & 1788.0 & $\frac{2}{3}$ & $=+1.23=0.684$ & +.006 & 5 \\
\hline 13 & 1789.0 & $\frac{1}{2}$ & $=+1.58=0.504$ & +.008 & 6 \\
\hline 14 & 1790.0 & $\frac{1}{2}$ & $=-0.52=0.514$ & -.013 & 4 \\
\hline 15 & 1791.0 & $\frac{2}{3}$ & $=-0.56=0.684$ & -.010 & 7 \\
\hline 16 & 1792.0 & $\frac{1}{3}$ & $=-0.12=0.340$ & -.011 & 3 \\
\hline 17 & 1793.0 & $\frac{1}{2}$ & $=-0.19=0.512$ & -.015 & 5 \\
\hline 18 & 1794.0 & $\frac{1}{3}$ & $=+0.79=0.344$ & -.006 & 3 \\
\hline 19 & 1795.0 & $\frac{2}{3}$ & $=-0.66=0.683$ & -.019 & 7 \\
\hline 20 & 1796.0 & $\frac{1}{2}$ & $=-0.68=0.514$ & -.015 & 4 \\
\hline 21 & 1797.1 & $\frac{1}{2}$ & $=-0.35=0.528$ & & 3 \\
\hline 22 & 1800.2 & $\frac{1}{3}$ & $0.00=0.352$ & & 2 \\
\hline 23 & 1801.2 & $\frac{1}{3}$ & $=-0.26=0.352$ & & 2 \\
\hline 24 & 1802.3 & 1 & $=+0.85=1.05$ & & 13 \\
\hline 25 & 1805.3 & 1 & $=+0.69=1.05$ & & 13 \\
\hline 26 & 1806.3 & $\frac{1}{2}$ & $=+0.20=0.52$ & & 5 \\
\hline 27 & -1807.3 & 1 & $=+2.34=1.045$ & & 16 \\
\hline 28 & 1808.3 & $\frac{1}{2}$ & $=-0.04=0.52$ & & 6 \\
\hline 29 & 1809.3 & $\frac{2}{3}$ & $=+1.80=0.70$ & & 9 \\
\hline 30 & 1810.3 & 1 & $=+2.39==1.05$ & & 16 \\
\hline 31 & 1811.3 & 1 & $=+1.49=1.04$ & -0.01 & 11 \\
\hline 32 & 1812.4 & $\frac{1}{2}$ & $=+0.8=0.53$ & & 8 \\
\hline 33 & 1813.4 & $\frac{1}{2}$ & $=+1.2=0.53$ & & 9 \\
\hline 34 & 1814.4 & 1 & $=+1.7=1.05$ & & 15 \\
\hline
\end{tabular}




\begin{tabular}{|c|c|c|c|c|c|c|}
\hline No. & Date. & & $\underset{\prime \prime}{\text { Equations. }}$ & & & $\begin{array}{c}\text { Number of } \\
\text { observations } \\
\text { in R. A. }\end{array}$ \\
\hline 35 & 1815.4 & $\frac{3}{2} d z$ & $=+2.1$ & $=1.58 \delta \lambda$ & & 20 \\
\hline 36 & $1818.4^{*}$ & $\frac{3}{2}$ & $=+0.3$ & $=1.58$ & & 24 \\
\hline 37 & 1819.4 & 1 & $=-0.8$ & $=1.05$ & & 11 \\
\hline 38 & 1820.5 & 1 & $=-1.3$ & $=1.05$ & & 14 \\
\hline 39 & 1821.5 & $\frac{2}{3}$ & $=+0.9$ & $=0.70$ & & 10 \\
\hline 40 & 1822.5 & $\frac{2}{3}$ & $=+0.9$ & $=0.70$ & & 7 \\
\hline 41 & 1823.5 & $\frac{2}{3}$ & $=\quad 0.0$ & $=0.70$ & & 11 \\
\hline 42 & 1824.5 & 1 & $=+0.5$ & $=1.05$ & & 12 \\
\hline 43 & 1825.5 & $\frac{2}{3}$ & $=-0.4$ & $=0.70$ & & 7 \\
\hline 44 & 1826.5 & 1 & $=+0.3$ & $=1.05$ & & 11 \\
\hline 45 & 1827.7 & 2 & $=-2.7$ & $=2.07$ & $+0.04 \delta \rho$ & 37 \\
\hline 46 & 1828.7 & $2 \frac{1}{2}$ & $=-2.7$ & $=2.57$ & +0.07 & 67 \\
\hline 47 & 1829.7 & $2 \frac{1}{2}$ & $=-2.4$ & $=2.59$ & +0.04 & 61 \\
\hline 48 & 1830.7 & $2 \frac{1}{2}$ & $=-4.9$ & $=2.56$ & +0.07 & 73 \\
\hline 49 & 1831.7 & 2 & $=\quad 0.0$ & $=2.06$ & +0.05 & 54 \\
\hline 50 & 1832.7 & 2 & $=-2.2$ & $=2.07$ & +0.05 & 65 \\
\hline 51 & 1833.8 & $2 \frac{1}{2}$ & $=-4.1$ & $=2.58$ & +0.08 & 88 \\
\hline 52 & 1834.8 & $2 \frac{1}{2}$ & $=-3.9$ & $=2.57$ & +0.08 & 91 \\
\hline 53 & 1835.8 & $2 \frac{1}{2}$ & $=-5.1$ & $=2.59$ & +0.05 & 82 \\
\hline 54 & 1836.8 & 3 & $=-7.0$ & $=3.11$ & +0.08 & 157 \\
\hline 55 & 1837.8 & 3 & $=-3.6$ & $=3.11$ & +0.04 & 162 \\
\hline 56 & 1838.8 & 3 & $=-1.6$ & $=3.11$ & +0.06 & 193 \\
\hline 57 & 1839.8 & 3 & $=-1.2$ & $=3.11$ & +0.06 & 170 \\
\hline 58 & 1840.8 & 3 & $=-1.5$ & $=3.11$ & +0.06 & 124 \\
\hline 59 & 1841.8 & 3 & $=+0.8$ & $=3.10$ & +0.06 & 108 \\
\hline 60 & 1842.8 & 3 & $=+1.6$ & $=3.10$ & +0.06 & 169 \\
\hline 61 & 1843.8 & 3 & $=+4.7$ & $=3.12$ & +0.04 & 111 \\
\hline 62 & 1844.9 & 3 & $=+5.1$ & $=3.11$ & +0.03 & 106 \\
\hline 63 & 1845.9 & 2 & $=+4.2$ & $=2.08$ & +0.02 & 5.5 \\
\hline 64 & 1846.9 & 3 & $=+6.3$ & $=3.10$ & +0.04 & 98 \\
\hline 65 & 1847.9 & 2 & $=+5.8$ & $=2.07$ & +0.03 & 74 \\
\hline 66 & 1848.9 & 2 & $=+4.4$ & $=2.08$ & +0.02 & 59 \\
\hline 67 & 1849.9 & 2 & $=+6.6$ & $=2.08$ & +0.04 & 33 \\
\hline 68 & 1850.9 & 2 & $=+7.2$ & $=2.08$ & +0.01 & 46 \\
\hline 69 & 1851.9 & 2 & $=+6.3$ & $=2.07$ & +0.04 & 42 \\
\hline 70 & 1852.9 & 2 & $=+6.6$. & $=2.07$ & +0.03 & 54 \\
\hline 71 & 1853.9 & 2 & $=+7.9$ & $=2.09$ & +0.01 & 49 \\
\hline 72 & 1854.9 & 2 & $=+8.9$ & $=2.09$ & +0.02 & 49 \\
\hline 74 & 1855.9 & 2 & $=+8.5$ & $=2.08$ & +0.04 & 48 \\
\hline 75 & 1856.9 & 2 & $=+7.9$ & $=2.08$ & +0.04 & 45 \\
\hline 76 & 1858.0 & $2 \frac{1}{2}$ & $=+10.3$ & $=2.61$ & +0.09 & 66 \\
\hline
\end{tabular}

* The results for 1816 and 1817 were onitted in this list through oversight. 


\begin{tabular}{|c|c|c|c|c|c|c|}
\hline No. & Date. & & Equations. & & & $\begin{array}{l}\text { Number of } \\
\text { observations } \\
\text { in R. A. }\end{array}$ \\
\hline 77 & 1859.0 & $2 \frac{1}{3}$ & $\varepsilon l=+10.6$ & $=2.60 \delta \lambda$ & $+0.03 \delta \rho$ & \\
\hline 78 & 1860.0 & $2 \frac{1}{2}$ & $=+8.2$ & $=2.60$ & +0.05 & 64 \\
\hline 79 & 1861.0 & 2 & $=+6.3$ & $=2.09$ & +0.03 & 41 \\
\hline 80 & 1862.0 & $2 \frac{1}{2}$ & $=+7.0$ & $=2.60$ & +0.05 & 60 \\
\hline 81 & 1863.0 & $2 \frac{1}{2}$ & $=+6.6$ & $=2.59$ & +0.08 & 88 \\
\hline 82 & 1864.0 & 2 & $=+4.3$ & $=2.09$ & +0.03 & 35 \\
\hline 83 & $180 ิ 5.0$ & $1 \frac{1}{2}$ & $=+3.9$ & $=1.57$ & +0.04 & 37 \\
\hline 84 & 1866.0 & $2 \frac{1}{2}$ & $=+1.1$ & $=2.60$ & +0.06 & 76 \\
\hline 85 & 1867.0 & $2 \frac{1}{2}$ & $=-1.8$ & $=2.60$ & +0.02 & 83 \\
\hline 86 & 1868.0 & 2 & $=-3.8$ & $=2.09$ & +0.04 & 40 \\
\hline 87 & 1869.0 & $2 \frac{1}{2}$ & $=-9.1$ & $=2.61$ & +0.02 & 68 \\
\hline 88 & 1870.0 & 2 & $=-9.6$ & $=2.084$ & +0.054 & 31 \\
\hline 89 & 1871.0 & $1 \frac{1}{2}$ & $=-10.6$ & $=1.560$ & +0.040 & 21 \\
\hline 90 & 1872.1 & $2 \frac{1}{2}$ & $=-19.1$ & $=2.600$ & +0.070 & 50 \\
\hline
\end{tabular}

Total number of observations in R. A., . . . . . . . . 3763.

We have next to express the values of $\delta \lambda$ and $i \rho$ in terms of the corrections to the elements. Differentiating the expressions

$\lambda=l+\left(2 e-\frac{1}{4} e^{3}\right) \sin (l-\pi)+\frac{5}{4} e^{2} \sin (2 l-2 \pi)+\frac{1}{1} \frac{3}{2} e^{3} \sin e^{3} \sin (3 l-3 \pi)+$ etc. $\rho=n+\frac{1}{4} e^{2}+\left(-e+\frac{3}{8} e^{3}\right) \cos (l-\pi)-\frac{3}{4} e^{2} \cos (2 l-\pi)-$ etc.,

with respect to $l, e$, and $\pi$, and reducing the coefficients to numbers, we find

$$
\begin{aligned}
& \frac{\partial \lambda}{\partial l}=1+0.0939 \cos g+0.0055 \cos 2 g \\
& \frac{\partial \lambda}{\partial e}=1.999 \sin g+0.117 \sin 2 g+0.007 \sin 3 g \\
& \frac{\partial \lambda}{\partial \pi}=-2.000 \cos g-0.117 \cos 2 g-0.007 \cos 3 g
\end{aligned}
$$

We have here put $l$ for the mean longitude, or

whence

$$
l=n t+\varepsilon
$$

$$
\begin{aligned}
& \frac{\partial \lambda}{\partial \varepsilon}=\frac{\partial \lambda}{\partial l} \\
& \frac{\partial \lambda}{\partial n}=t \frac{\partial \lambda}{\partial l}
\end{aligned}
$$

Also, from the expression for $\rho$

$$
\begin{aligned}
& \frac{\partial \rho}{\partial l}=-\frac{\partial \rho}{\partial x}=\left(e-\frac{3}{8} e^{3}\right) \sin g+\frac{s}{4} e^{2} \sin 2 g+\ldots \\
& \frac{\partial \rho}{\partial e}=\frac{1}{2} e-\left(1-\frac{3}{8} e^{2}\right) \cos g-\frac{3}{2} e \cos 2 g-\text { etc. } \\
& \frac{\partial \rho}{\partial n}=t \frac{\partial \rho}{\partial l}-\frac{2}{3 n}
\end{aligned}
$$

The values of these coefficients which depend only on $g$ are shown in the following table: 


\begin{tabular}{|c|c|c|c|c|c|c|}
\hline$g$ & $\frac{\partial \lambda}{\partial \varepsilon}$ & $\frac{\partial \lambda}{\partial e}$ & $\frac{\partial \lambda}{e d \pi}$ & $\frac{\partial \rho}{\partial \varepsilon}$ & $\frac{\partial \rho}{\partial e}$ & - $\frac{\partial \rho}{e d \pi}$ \\
\hline $0^{\circ}$ & +1.099 & 0 & -2.124 & 0 & -1.00 & 0 \\
\hline 1 & 1.099 & +0.039 & 2.124 & +0.001 & 1.00 & -0.02 \\
\hline 2 & 1.099 & 0.079 & 2.123 & 0.002 & 1.00 & 0.03 \\
\hline 3 & 1.099 & 0.118 & 2.121 & 0.002 & 1.00 & 0.05 \\
\hline 4 & 1.099 & 0.158 & 2.118 & 0.003 & 1.00 & 0.07 \\
\hline 5 & +1.099 & +0.196 & -2.114 & +0.004 & -1.00 & -0.09 \\
\hline 6 & 1.099 & 0.235 & 2.110 & 0.005 & 0.99 & 0.10 \\
\hline 7 & 1.099 & 0.274 & 2.105 & 0.006 & 0.99 & 0.12 \\
\hline 8 & 1.098 & 0.313 & 2.099 & 0.006 & 0.99 & 0.14 \\
\hline 9 & 1.098 & 0.352 & 2.092 & 0.007 & 0.99 & 0.16 \\
\hline 10 & +1.098 & +0.391 & -2.085 & +0.008 & -0.98 & -0.17 \\
\hline 11 & 1.097 & 0.430 & 2.077 & 0.009 & 0.98 & 0.19 \\
\hline 12 & 1.097 & 0.468 & 2.069 & 0.010 & 0.98 & 0.21 \\
\hline 13 & 1.097 & 0.505 & 2.059 & 0.011 & 0.97 & 0.22 \\
\hline 14 & 1.096 & 0.544 & 2.049 & 0.011 & 0.97 & 0.24 \\
\hline 15 & +1.096 & +0.581 & -2.038 & +0.012 & -0.97 & -0.26 \\
\hline 16 & 1.095 & 0.618 & 2.027 & 0.013 & 0.96 & 0.28 \\
\hline 17 & 1.095 & 0.655 & 2.014 & 0.014 & 0.96 & 0.29 \\
\hline 18 & 1.094 & 0.693 & 2.001 . & 0.015 & 0.95 & 0.31 \\
\hline 19 & 1.094 & 0.729 & -1.987 & 0.015 & 0.95 & 0.33 \\
\hline 20 & +1.093 & +0.765 & -1.973 & $+i) .016$ & -0.94 & -0.34 \\
\hline 21 & 1.092 & 0.801 & 1.957 & 0.017 & 0.93 & 0.36 \\
\hline 22 & 1.091 & 0.836 & 1.941 & 0.018 & 0.93 & 0.37 \\
\hline 23 & 1.090 & 0.872 & 1.925 & 0.018 & 0.92 & 0.39 \\
\hline 24 & 1.090 & 0.907 & 1.907 & 0.019 & 0.91 & 0.41 \\
\hline 25 & +1.089 & +0.942 & -1.890 & +0.020 & -0.91 & -0.42 \\
\hline 26 & 1.088 & 0.976 & 1.872 & 0.021 & 0.90 & 0.44 \\
\hline 27 & 1.087 & 1.010 & 1.852 & 0.021 & 0.89 & 0.45 \\
\hline 28 & 1.086 & 1.043 & 1.832 & 0.022 & 0.88 & 0.47 \\
\hline 29 & 1.085 & 1.076 & 1.811 & 0.023 & 0.87 & 048 \\
\hline 30 & +1.084 & +1.108 & -1.790 & +0.023 & -0.87 & -0.50 \\
\hline 31 & 1.084 & 1.140 & 1.769 & 0.024 & 0.86 & 0.51 \\
\hline 32 & 1.083 & 1.172 & 1.747 & 0.025 & 0.85 & 0.53 \\
\hline 33 & 1.082 & 1.2113 & 1.724 & 0.026 & 0.84 & 0.54 \\
\hline 34 & 1.080 & 1.233 & 1.700 & 0.026 & 0.83 & 0.56 \\
\hline 35 & +1.079 & +1.264 & -1.676 & +0.027 & -0.82 & -0.57 \\
\hline 36 & 1.078 & 1.294 & 1.651 & 0.028 & 0.81 & 0.59 \\
\hline 37 & 1.077 & 1.323 & 1.626 & 0.028 & 0.80 & 0.60 \\
\hline 38 & 1.076 & 1.351 & 1.601 & 0.029 & 0.79 & 0.62 \\
\hline 39 & 1.074 & 1.379 & 1.574 & 0.030 & 0.78 & 0.63 \\
\hline 40 & +1.073 & +1.407 & -1.548 & +0.030 & -0.77 & -0.64 \\
\hline 41 & 1.072 & 1.434 & 1.521 & 0.031 & 0.76 & 0.66 \\
\hline 42 & 1.070 & 1.460 & 1.494 & 0.031 & 0.74 & 0.67 \\
\hline 43 & 1.069 & 1.486 & 1.466 & 0.032 & 0.73 & 0.68 \\
\hline 44 & 1.068 & 1.511 & 1.438 & 0.033 & 0.72 & 0.70 \\
\hline 45 & +1.067 & +1.536 & -1.409 & +0.033 & -0.71 & -0.71 \\
\hline 46 & 1.065 & 1.561 & 1.380 & 0.034 & 0.70 & 0.71 \\
\hline 47 & 1.064 & 1.584 & 1.351 & 0.034 & 0.68 & 0.72 \\
\hline 48 & 1.063 & 1.606 & 1.320 & 0.035 & 0.67 & 0.74 \\
\hline 49 & 1.061 & 1.629 & 1.290 & 0.036 & 0.66 & 0.75 \\
\hline 50 & +1.060 & +1.651 & -1.260 & +0.036 & -0.64 & -0.76 \\
\hline 51 & 1.058 & 1.672 & 1.229 & 0.037 & 0.63 & 0.77 \\
\hline 52 & 1.057 & 1.692 & 1.197 & 0.037 & 0.62 & 0.78 \\
\hline 53 & 1.055 & 1.712 & 1.165 & 0.037 & 0.60 & 0.79 \\
\hline 54 & 1.054 & 1.731 & 1.133 & 0.038 & 0.59 & 0.80 \\
\hline 55 & +1.052 & +1.750 & -1.100 & +0.038 & -0.57 & -0.81 \\
\hline 56 & 1.051 & 1.768 & 1.067 & 0.039 & 0.56 & 0.82 \\
\hline 57 & 1.049 & 1.785 & 1.034 & 0.039 & 0.54 & 0.83 \\
\hline 58 & 1.047 & 1.802 & 1.002 & 0.040 & 0.53 & 0.84 \\
\hline 59 & +1.046 & +1.817 & -0.968 & +0.040 & -0.51 & -0.85 \\
\hline
\end{tabular}


THE ORBIT OF URANUS.

\begin{tabular}{|c|c|c|c|c|c|c|}
\hline$g$ & $\frac{\partial \lambda}{\partial \varepsilon}$ & $\frac{\partial \lambda}{\partial e}$ & $\frac{\partial \lambda}{e d \pi}$ & $\frac{\partial \rho}{\partial \varepsilon}$ & $\frac{\partial \rho}{\partial e}$ & $\frac{\partial \rho}{e d \pi}$ \\
\hline $60^{\circ}$ & +1.044 & +1.833 & -0.935 & +0.041 & -0.50 & -0.86 \\
\hline 61 & 1.043 & 1.848 & 0.901 & 0.041 & 0.49 & 0.87 \\
\hline 62 & 1.041 & 1.862 & 0.867 & 0.041 & 0.47 & 0.87 \\
\hline 63 & 1.040 & 1.875 & 0.832 & 0.042 & 0.45 & 0.88 \\
\hline 64 & 1.038 & 1.888 & 0.798 & 0.042 & 0.44 & 0.89 \\
\hline 65 & +1.036 & +1.901 & -0.763 & +0.043 & -0.42 & -0.90 \\
\hline 66 & 1.034 & 1.912 & 0.728 & 0.043 & 0.41 & 0.91 \\
\hline 67 & 1.033 & 1.922 & 0.693 & 0.043 & 0.39 & 0.91 \\
\hline 68 & 1.031 & 1.932 & 0.659 & 0.044 & 0.37 & 0.92 \\
\hline 69 & 1.030 & 1.942 & 0.624 & 0.044 & 0.36 & 0.93 \\
\hline 70 & +1.028 & +1.950 & -0.588 & +0.044 & -0.34 & -0.94 \\
\hline 71 & 1.027 & 1.959 & 0.553 & 0.044 & 0.33 & 0.95 \\
\hline 72 & 1.025 & 1.967 & 0.518 & 0.045 & 031 & 0.95 \\
\hline 73 & 1.023 & 1.974 & 0.483 & 0.045 & 0.29 & 0.96 \\
\hline 74 & 1.021 & 1.980 & 0.447 & 0.045 & 0.28 & 0.96 \\
\hline 75 & +1.019 & +1.985 & -0.412 & +0.045 & -0.26 & -0.97 \\
\hline 76 & 1.018 & 1.990 & 0.376 & 0.046 & 0.24 & 0.97 \\
\hline 77 & 1.016 & 1.994 & 0.341 & 0.046 & 0.22 & 0.97 \\
\hline 78 & 1.014 & 1.997 & 0.305 & 0.046 & 0.21 & 0.98 \\
\hline 79 & 1.013 & 2.000 & 0.269 & 0.046 & 0.19 & 0.98 \\
\hline 80 & +1.011 & +2.003 & -0.233 & +0.046 & -0.17 & -0.98 \\
\hline 81 & 1.010 & 2.005 & 0.198 & 0.046 & 0.16 & 0.99 \\
\hline 82 & 1.008 & 2.007 & 0.163 & 0.046 & 0.14 & 0.99 \\
\hline 83 & 1.006 & 2.007 & 0.128 & 0.047 & 0.12 & 0.99 \\
\hline 84 & 1.005 & 2,096 & 0.093 & 0.047 & 0.11 & 0.99 \\
\hline 85 & +1.003 & +2.005 & -0.057 & +0.047 & -0.09 & -1.00 \\
\hline 86 & 1.002 & 2.004 & -0.022 & 0.047 & 0.07 & 1.00 \\
\hline 87 & 1.000 & 2.002 & +0.013 & 0.047 & 0.05 & 1.00 \\
\hline 88 & 0.998 & 2.000 & 0.048 & 0.047 & 0.03 & 1.00 \\
\hline 89 & 0.997 & 1.997 & 0082 & 0.047 & -0.02 & 1.00 \\
\hline 90 & +0.995 & +1.993 & +0.117 & +0.047 & 0.00 & -1.00 \\
\hline 91 & 0.993 & 1.989 & 0.152 & 0.047 & +0.02 & 1.00 \\
\hline 92 & 0.992 & 1.984 & 0.186 & 0.047 & 0.03 & 1.00 \\
\hline 93 & 0.990 & 1.978 & 0.220 & 0.047 & 0.05 & 1.00 \\
\hline 94 & 0.988 & 1.972 & 0.254 & 0.047 & 0.07 & 1.00 \\
\hline 95 & +0.987 & +1.965 & +0.287 & +0.047 & +0.09 & -1.00 \\
\hline 96 & 0.985 & 1.958 & 0.321 & 0.047 & 0.11 & 0.99 \\
\hline 97 & 0.984 & 1.950 & 0.354 & 0.047 & 0.12 & 0.99 \\
\hline 98 & 0.982 & 1.943 & 0.387 & 0.046 & 0.14 & 0.99 \\
\hline 99 & 0.980 & 1.933 & 0.421 & 0.046 & 0.16 & 0.99 \\
\hline 100 & +0.979 & +1.924 & +0.453 & +0.046 & +0.17 & -0.98 \\
\hline 101 & 0.977 & 1.914 & 0.487 & 0.046 & 0.19 & 0.98 \\
\hline 102 & 0.976 & 1.903 & 0.519 & 0.046 & 0.21 & 0.98 \\
\hline 103 & 0.974 & 1.893 & 0.551 & 0.046 & 0.22 & 0.97 \\
\hline 104 & 0.972 & 1.882 & 0.582 & 0.046 & 0.24 & 0.97 \\
\hline 105 & +0.971 & +1.869 & +0.614 & +0.045 & +0.26 & -0.97 \\
\hline 106 & 0.969 & 1.857 & 0.645 & 0.045 & 0.28 & 0.96 \\
\hline 107 & 0.968 & 1.844 & 0.677 & 0.045 & 0.29 & 0.96 \\
\hline 108 & 0.967 & 1.829 & 0.707 & 0.045 & 0.31 & 0.95 \\
\hline 109 & 0.965 & 1.815 & 0.737 & 0.044 & 0.33 & 0.95 \\
\hline 110 & +0.964 & +1.800 & +0.768 & +0.044 & +0.34 & -0.94 \\
\hline 111 & 0.962 & 1.786 & 0.798 & 0.044 & 0.36 & 0.93 \\
\hline 112 & 0.961 & 1.770 & 0.827 & 0.044 . & 0.37 & 0.92 \\
\hline 113 & 0.959 & 1.754 & 0.855 & $0.043^{\circ}$ & 0.39 & 0.91 \\
\hline 114 & 0.958 & 1.738 & 0.884 & 0.043 & 0.41 & 0.91 \\
\hline 115 & 0.956 & +1.721 & +0.913 & +0.043 & +0.42 & -0.90 \\
\hline 116 & 0.955 & -1.704 & 0.942 & 0.042 & 0.44 & 0.89 \\
\hline 117 & 0.954 & 1.686 & 0.970 & 0.042 & 0.45 & 0.88 \\
\hline 118 & 0.953 & 1.668 & 0.997 & 0.041 & 0.47 & 0.87 \\
\hline 119 & +0.951 & +1.650 & +1.025 & +0.041 & +0.49 & -0.87 \\
\hline
\end{tabular}




\begin{tabular}{|c|c|c|c|c|c|c|}
\hline$g$ & $\frac{\partial \lambda}{\partial \varepsilon}$ & $\begin{array}{l}\partial \lambda \\
\partial \boldsymbol{e}\end{array}$ & $\frac{\partial \lambda}{e c l \pi}$ & $\frac{\partial \rho}{\partial \varepsilon}$ & $\frac{\partial \rho}{\partial e}$ & $\frac{\partial \rho}{e d x}$ \\
\hline $120^{\circ}$ & +0.950 & +1.631 & +1.051 & +0.041 & +0.50 & -0.86 \\
\hline 121 & 0.949 & 1.611 & 1.077 & 0.040 & 0.51 & 0.85 \\
\hline 122 & 0.948 & 1.592 & 1.104 & 0.040 & 0.53 & 0.84 \\
\hline 123 & 0.947 & 1.571 & 1.129 & 0.039 & 0.54 & 0.83 \\
\hline 124 & 0.945 & 1.551 & 1.154 & 0.039 & 0.56 & 0.82 \\
\hline 125 & +0.944 & +1.530 & +1.180 & +0.038 & +0.57 & -0.81 \\
\hline 126 & 0.943 & 1.509 & 1.205 & 0.038 & 0.59 & 0.80 \\
\hline 127 & 0.942 & 1.488 & 1.229 & 0.037 & 0.60 & 0.79 \\
\hline 128 & 0.941 & 1.466 & 1.253 & 0.037 & 062 & 0.78 \\
\hline 129 & 0.940 & 1.443 & 1.277 & 0.037 & 0.63 & 0.77 \\
\hline 130 & +0.939 & +1.421 & +1.300 & +0.036 & +0.64 & -0.76 \\
\hline 131 & 0.937 & 1.397 & 1.322 & 0.036 & 0.66 & 0.75 \\
\hline 132 & 0.936 & 1.374 & 1.344 & 0.035 & 0.67 & 0.74 \\
\hline 133 & 0.936 & 1.350 & 1.367 & 0.034 & 0.68 & 0.72 \\
\hline 134 & 0.935 & 1.327 & 1.388 & 0.034 & 0.70 & 0.71 \\
\hline 135 & +0.934 & +1.302 & +1.409 & +0.033 & +0.71 & -0.71 \\
\hline 136 & 0.933 & 1.277 & 1.430 & 0.033 & 0.71 & 0.70 \\
\hline 137 & 0.932 & 1.253 & 1.451 & 0.032 & 0.72 & 0.68 \\
\hline 138 & 0.931 & 1.228 & 1.470 & 0.031 & 0.74 & 0.67 \\
\hline 139 & 0.930 & 1.202 & 1.489 & 0.031 & 0.75 & 0.66 \\
\hline 140 & +0.929 & +1.177 & +1.508 & +0.030 & +0.76 & -0.64 \\
\hline 141 & 0.928 & 1.151 & 1.527 & 0.030 & 0.77 & 0.63 \\
\hline 142 & 0.927 & 1.124 & 1.545 & 0.029 & 0.78 & 0.62 \\
\hline 143 & 0.927 & 1.099 & 1.562 & 0.028 & 0.79 & 0.60 \\
\hline 144 & 0.926 & 1.072 & 1.580 & 0.028 & 0.80 & 0.59 \\
\hline 145 & +0.923 & +1.044 & +1.596 & +0.027 & +0.81 & -0.57 \\
\hline 146 & 0.924 & 1.016 & 1.613 & 0.026 & 0.82 & 0.56 \\
\hline 147 & 0.923 & 0.989 & 1.629 & 0.026 & 0.83 & 0.54 \\
\hline 148 & 0.922 & $0: 962$ & 1.644 & 0.025 & 0.84 & 0.53 \\
\hline 149 & 0.922 & 0.934 & 1.659 & 0.024 & 0.85 & 0.51 \\
\hline 150 & +0.921 & +0.906 & +1.674 & +0.023 & +0.86 & -0.50 \\
\hline 151 & 0.921 & 0.878 & 1.688 & 0.023 & 0.87 & 0.49 \\
\hline 152 & 0.920 & 0.849 & 1.702 & 0.022 & 0.87 & 0.47 \\
\hline 153 & 0.919 & 0.820 & 1.714 & 0.021 & 0.88 & 0.45 \\
\hline 154 & 0.919 & 0.792 & 1.727 & 0.021 & 0.89 & 0.44 \\
\hline 155 & +0.919 & +0.762 & +1.740 & +0.020 & +0.90 & -0.42 \\
\hline 156 & 0.918 & 0.733 & 1.751 & 0.019 & 0.91 & 0.41 \\
\hline 157 & 0.918 & 0.704 & 1.763 & 0.018 & 0.91 & 0.39 \\
\hline 158 & 0.917 & 0.674 & 1.773 & 0.018 & 0.92 & 0.37 \\
\hline 159 & 0.916 . & 0.645 & 1.783 & 0.017 & 0.93 & 0.36 \\
\hline 160 & +0.916 & +0.615 & +1.793 & +0.016 & +0.94 & -0.34 \\
\hline 161 & 0.915 & 0.585 & 1.803 & 0.015 & 0.95 & 0.33 \\
\hline 162 & 0.915 & 0.555 & 1.811 & 0.015 & 0.95 & 0.31 \\
\hline 163 & 0.915 & 0.525 & 1.820 & 0.014 & 0.96 & 0.29 \\
\hline 164 & 0.915 & 0.494 & 1.829 & 0.013 & 0.96 & 0.28 \\
\hline 165 & +0.914 & +0.465 & +1.836 & +0.012 & +0.97 & -0.26 \\
\hline 166 & 0.914 & 0.435 & 1.843 & 0.011 & 0.97 & 0.24 \\
\hline 167 & 0.914 & 0.403 & 1.849 & 0.011 & 0.97 & 0.22 \\
\hline 168 & 0.913 & 0.373 & 1.855 & 0.010 & 0.98 & 0.21 \\
\hline 169 & 0.913 & 0.343 & 1.860 & 0.009 & 0.98 & 0.19 \\
\hline 170 & +0.913 & +0.311 & +1.865 & +0.008 & +0.98 & -0.17 \\
\hline 171 & 0.912 & 0.290 & 1.870 & 0.007 & 0.99 & 0.16 \\
\hline 172 & 0.912 & 0.249 & 1.875 & 0.006 & 0.99 & 0.14 \\
\hline 173 & 0.912 & 0.219 & 1.879 & 0.006 & 0.99 & 0.12 \\
\hline 174 & 0.912 & 0.187 & 1.882 & 0.005 & 0.99 & 0.11 \\
\hline 175 & +0.911 & +0.156 & +1.884 & +0.004 & +1.00 & -0.09 \\
\hline 176 & 0.911 & 0.126 & 1.886 & 0.003 & 1.00 & 0.07 \\
\hline 177 & 0.911 & 0.094 & 1.888 & 0.002 & 1.00 & 0.05 \\
\hline 178 & 0.911 & 0.063 & 1.889 & 0.002 & 1.00 & 0.03 \\
\hline 179 & +0.911 & +0.031 & +1.890 & +0.001 & +1.00 & -0.02 \\
\hline
\end{tabular}


In the equations of condition ten years has been adopted for the unit of time, in order to make the general value of the coefficients as nearly equal as possible, and the time has been counted from the epoch 1830.0 , in order to have the positive and negative values of $t$ in the equations more nearly balanced. To distinguish these values of $\delta \varepsilon$ and $\delta n$ they are marked with an accent. This unit of time gives 0.8914 for the value of $\frac{2}{3 n}$ in arc, whence

$$
\frac{\partial \rho}{\partial n^{\prime}}=t \frac{\partial \rho}{\partial l}-0.891
$$

The equations of condition are now formed by putting in the preceding equations for heliocentric longitude and radius vector

$$
\begin{aligned}
& \delta \lambda=\frac{\partial \lambda}{\partial \varepsilon} \delta \varepsilon+\frac{\partial \lambda}{\partial v} \delta v+\frac{\partial \lambda}{\partial e} \delta e+\frac{\partial \lambda}{e \partial \pi} e i \pi+\frac{\partial \lambda}{\partial \mu} \delta \mu^{\prime} \\
& \varepsilon_{\rho}=\frac{\partial \rho}{\partial \varepsilon} \delta \varepsilon+\frac{\partial \delta}{\partial n} \delta n+\frac{\partial \rho}{\partial e} \delta e+\frac{\partial \rho}{e \partial \pi} e \delta \pi .
\end{aligned}
$$

For the coefficients $\frac{\partial \lambda}{\partial \mu^{\prime}}$ have been taken one-hundredth the perturbations of longitude produced by Neptune, as given in the heliocentric ephemeris at the end of Chapter V. The corrected mass of Neptune will then be

$$
\frac{1}{17000}\left(1+\frac{\delta \mu^{\prime}}{100}\right)
$$

Finally, I remark that all the preceding comparisons are made with the heliocentric ephemeris as printed, without the correction indicated in the column adjoining it, but in the following equations this correction is for the first time introduced.

Equations of condition given by the Corrections in Longitude.

\begin{tabular}{r|llllll}
1 & $0.05 \delta \varepsilon^{\prime}$ & $-0.70 i n^{\prime}$ & $-0.10 \delta e$ & $+0.03 e \delta \pi+0.12 \delta \mu^{\prime}$ & $=+1$ \\
2 & 0.10 & -1.11 & +0.01 & -0.19 & +0.12 & $=+2.2$ \\
3 & 0.31 & -2.55 & +0.33 & +0.51 & -0.22 & $=+12.8$ \\
4 & 0.32 & -2.52 & +0.27 & +0.58 & -0.18 & $=+11.8$ \\
5 & 0.30 & -2.32 & +0.12 & +0.59 & -0.09 & $=+11.6$ \\
6 & 0.19 & -1.40 & -0.01 & +0.39 & 0.00 & $=+5.0$ \\
7 & 0.19 & -1.18 & -0.29 & +0.23 & +0.22 & $=+4.8$ \\
8 & 1.41 & -6.75 & -2.69 & -0.75 & +2.47 & $=+2.8$ \\
9 & 1.06 & -5.01 & -1.98 & -0.72 & +1.85 & $=+1.1$ \\
10 & 1.07 & -4.91 & -1.94 & -0.86 & +1.85 & $=+1.7$ \\
11 & 1.09 & -4.87 & -1.86 & -1.04 & +1.85 & $=-0.5$ \\
12 & 0.73 & -3.06 & -1.04 & -0.97 & +1.16 & $=+1.0$ \\
13 & 0.54 & -2.20 & -0.71 & -0.78 & +0.82 & $=+1.4$ \\
14 & 0.55 & -2.19 & -0.68 & -0.81 & +0.81 & $=-0.6$ \\
15 & 0.73 & -2.86 & -0.82 & -1.16 & +1.03 & $=-0.9$ \\
16 & 0.37 & -1.39 & -0.36 & -0.60 & +0.49 & $=-0.2$ \\
17 & 0.56 & -2.05 & -0.47 & -0.95 & +0.70 & $=-0.4$ \\
18 & 0.37 & -1.33 & -0.26 & -0.65 & +0.44 & $=+0.6$ \\
19 & 0.75 & -2.59 & -0.38 & -1.34 & +0.82 & $=-1.0$
\end{tabular}




\begin{tabular}{|c|c|c|c|c|c|c|}
\hline 20 & $0.56 \int_{E^{\prime}}$ & $-1.89 g^{\top} u^{\prime}$ & $-0.22 \delta e$ & $-1.04 e \delta \pi$ & $+0.58 f \mu^{\prime}$ & $=-0^{\prime \prime} .9$ \\
\hline 21 & 0.58 & -1.92 & -0.13 & -1.12 & +0.55 & $=-0.5$ \\
\hline ?.? & 0.38 & -1.15 & +0.09 & -0.74 & +0.24 & $=-0.2$ \\
\hline & 0.38 & -1.11 & +0.15 & -0.73 & +0.21 & $=-0.3$ \\
\hline 4 & 1.15 & -3.18 & +0.65 & -2.13 & +0.50 & $=+0.6$ \\
\hline & 1.13 & -2.81 & +1.11 & -1.91 & +0.19 & $=+0.5$ \\
\hline & 0.56 & -1.33 & +0.62 & -0.89 & +0.05 & $=+0.1$ \\
\hline & 1.12 & -2.51 & +1.38 & -1.68 & 0.00 & $=+2.1$ \\
\hline & 0.56 & -1.21 & +0.75 & -0.79 & -0.04 & $=-0.1$ \\
\hline & 0.70 & -1.55 & +1.09 & -0.97 & -0.10 & $=+1.6$ \\
\hline & 1.11 & -2.19 & +1.73 & -1.32 & -0.23 & $=+2.2$ \\
\hline & 1.09 & -2.04 & +1.81 & -1.11 & -0.29 & $=+1.3$ \\
\hline & 0.56 & -0.98 & +0.96 & $-0 . \tilde{0} 1$ & -0.18 & $=+0.7$ \\
\hline & 0.55 & -0.91 & +1.00 & -0.43 & -0.21 & $=+1.1$ \\
\hline & 1.08 & -169 & +2.03 & -0.71 & -0.46 & $=+1.5$ \\
\hline & 1.63 & -2.37 & +3.11 & -0.82 & -0.74 & $=+2.0$ \\
\hline & 1.07 & -1.45 & +2.08 & -0.38 & -0.52 & $=+0.4$ \\
\hline $5^{\prime \prime}$ & 1.06 & -1.33 & +2.10 & -0.22 & -0.55 & $=+0.8$ \\
\hline 3 & 1.58 & -1.83 & +3.18 & -0.09 & -0.85 & $=+0.3$ \\
\hline & 1.05 & -1.11 & +2.10 & +0.08 & -0.59 & $=-0.8$ \\
\hline & 1.04 & -0.99 & +2.07 & +0.26 & -0.59 & $=-1.3$ \\
\hline & 0.69 & -0.58 & +1.36 & +0.27 & -0.39 & $=+0.8$ \\
\hline 4 & 0.69 & -0.51 & +1.33 & +0.38 & -0.39 & $=+0.8$ \\
\hline 4 & 0.68 & -0.44 & +1.29 & +0.47 & -0.39 & 0.0 \\
\hline 4 & 1.01 & -0.56 & +1.88 & +0.84 & -0.59 & $=+0.3$ \\
\hline 43 & 0.67 & -0.30 & +1.20 & +0. & -0.38 & $=-0.5$ \\
\hline 44 & 1.00 & -0.35 & +1.72 & +1.09 & -0.57 & $=+0.2$ \\
\hline 4 & 1.95 & -0.50 & +3.21 & +2.39 & -1.12 & $=-2.5$ \\
\hline 46 & 2.42 & -0.37 & +3.77 & +3.21 & -1.36 & $=-2.6$ \\
\hline 1 & 2.42 & -0.12 & +3.53 & $+3 . \overline{2} 2$ & -1.37 & $=-2.4$ \\
\hline & 2.39 & +0.12 & +3.22 & +3.69 & -1.33 & $=-4.9$ \\
\hline 4. & 1.91 & +0.28 & +2.37 & +3.14 & -1.07 & $=-0.1$ \\
\hline 50 & 1.91 & +0.48 & +2.15 & +3.30 & -1.09 & $=-2.4$ \\
\hline 5 & 2.37 & +0.83 & +2.31 & +4.32 & -1.34 & $=-4.5$ \\
\hline 5 & 2.36 & +1.06 & +2.02 & +4.44 & -1.36 & $=-4.3$ \\
\hline 5 & 2.37 & +1.32 & +1.68 & +4.62 & -1.37 & 5.5 \\
\hline 5 & 2.85 & +1.86 & +1.66 & +5.64 & -1.68 & $=-7.5$ \\
\hline 5 & 2.84 & +2.17 & +1.22 & +5.74 & -1.71 & $=-4.1$ \\
\hline 5 & 2.84 & +2.44 & +081 & +5.84 & -1.74 & $=-2.1$ \\
\hline 5 & 2.83 & +2.71 & +0.40 & +5.88 & -1.78 & $=-1.7$ \\
\hline 5 & 2.83 & +3.00 & -0.06 & +5.88 & -1.82 & -2.0 \\
\hline$\sigma$ & 2.82 & +3.28 & -0.44 & +5.84 & -1.86 & +0.3 \\
\hline 60 & 2.83 & +3.57 & -0.84 & +5.81 & -1.91 & $=+1.2$ \\
\hline 6 & 2.85 & +3.90 & -1.27 & +5.78 & -1.98 & $=+4.4$ \\
\hline 6 & 2.85 & +4.21 & -1.74 & +5.64 & -2.02 & $=+4.8$ \\
\hline 6 & 1.91 & +3.02 & -1.42 & +3.69 & -1.39 & $=+4.0$ \\
\hline 6 & 2.85 & +4.78 & -2.50 & +5.32 & -2.14 & $=+6.0$ \\
\hline 6 & 1.91 & +3.39 & -1.92 & +3.46 & -1.47 & $=+5.6$ \\
\hline 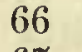 & 1.92 & +3.61 & -2.18 & +3.32 & -1.52 & $=+4.3$ \\
\hline C & 1.93 & +3.82 & -2.40 & +3.17 & -1.56 & $=+6.6$ \\
\hline 68 & 1.94 & +4.05 & -2.65 & +2.98 & -1.60 & 7.3 \\
\hline 69 & 1.94 & +4.20 & -2.83 & +2.80 & -1.64 & $=+6.5$ \\
\hline
\end{tabular}




\begin{tabular}{l|llllll}
70 & $1.95 \delta \varepsilon^{\prime}$ & $+4.44 \delta n^{\prime}$ & $-3.04 i e$ & $+2.59 e \delta \pi$ & $-1.68 \delta \mu$ & $=+6 " .8$ \\
71 & 1.98 & +4.72 & -3.23 & +2.37 & -1.73 & $=+8.1$ \\
72 & 1.99 & +4.93 & -3.44 & +2.15 & -1.76 & $=+9.1$ \\
73 & 1.99 & +5.13 & -3.58 & +1.91 & -1.78 & $=+8.7$ \\
74 & 2.00 & +5.35 & -3.73 & +1.64 & -1.80 & $=+8.1$ \\
75 & 2.53 & +7.02 & -4.83 & +1.73 & -2.30 & $=+10.6$ \\
76 & 2.54 & +7.34 & -4.96 & +1.33 & -2.29 & $=+10.9$ \\
77 & 2.56 & +7.64 & -5.07 & +0.97 & -2.31 & $=+8.2$ \\
78 & 2.07 & +6.38 & -4.14 & +0.47 & -1.86 & $=+6.3$ \\
79 & 2.59 & +8.25 & -5.20 & +0.21 & -2.32 & $=+7.0$ \\
80 & 2.60 & +8.51 & -5.21 & -0.15 & -2.29 & $=+6.6$ \\
81 & 2.11 & +7.14 & -4.19 & -0.51 & -1.83 & $=+4.3$ \\
82 & 1.60 & +5.56 & -3.13 & -0.58 & -1.36 & $=+3.9$ \\
83 & 2.67 & +9.59 & -5.12 & -1.37 & -2.20 & $=+1.1$ \\
84 & 2.68 & +9.92 & -5.00 & -1.80 & -2.14 & $=-1.8$ \\
85 & 2.17 & +8.22 & -3.92 & -1.75 & -1.67 & $=-3.8$ \\
86 & 2.73 & +10.61 & -4.97 & -2.59 & -2.01 & $=-9.0$ \\
87 & 2.20 & +8.78 & -3.63 & -2.33 & -1.52 & $=-9.5$ \\
88 & 1.65 & +6.74 & -258 & -1.96 & -1.08 & $=-10.5$ \\
89 & 2.77 & +11.61 & -4.04 & -3.61 & -1.69 & $=-19.9$
\end{tabular}

The following are the approximate normals to which these equations give rise. Inaccuracies being detected in several of the equations of condition after these normals were formed, they do not accurately correspond to those equations as written.

$\begin{array}{rlllll}283.64 \delta \varepsilon^{\prime} & +414.36 \delta n^{\prime} & -151.63 i e & +247.23 e \delta \pi & -176.03 \delta \mu^{\prime} & =+123^{\prime \prime} .5 \\ 414.36 & +1619.44 & -689.11 & +260.26 & -436.02 & =+103.2 \\ -151.63 & -689.11 & +557.82 & +38.45 & +122.88 & =-399.8 \\ 247.23 & +260.26 & +38.45 & +618.45 & -194.60 & =+267.3 \\ -176.03 & -436.02 & +122.88 & -194.60 & +163.13 & =-128.1\end{array}$

The values of the unknown quantities deduced from these normals were substituted in the equations of condition, and a farther approximation was made by solving the equations given by the residuals. The following are the first approximations given by the normals, and the finally concluded corrections

$$
\begin{aligned}
& \text { Preliminary. Final. } \\
& \begin{array}{lll}
\delta \mu^{\prime}, & -15.00 & -13.44
\end{array} \\
& e \delta \pi, \quad-0.52-0.36 \\
& \text { Se, } \quad-4.25 \quad-4.04 \\
& 10 \delta n=\delta n^{\prime},-4.73 \quad-4.33 \\
& \delta \varepsilon^{\prime}, \quad-3.78 \quad-3.44
\end{aligned}
$$

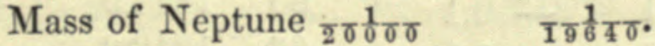

The final values of the corrections being substituted in the equations leave the following system of residuals, or outstanding excesses of the observed longitudes over theory. Column $f \delta l$ gives the residual of the equation itself; the probable error of which has always been judged to be $0 . " 5$, while in column $i l$ this residual is divided by $f$ to obtain the residual correction of the longitude itself. The values of the factors $f$ are found with the original equations on pages 159 and 160 . 


\begin{tabular}{|c|c|c|c|c|c|c|c|}
\hline No. of Eq. & Year. & $f \delta_{\prime \prime}$ & $\delta_{11}$ & No. of Eq & Year. & $f_{11}$ & $\delta l$ \\
\hline 1 & 1691.0 & -0.5 & -10 & $35^{\prime \prime}$ & 1817.4 & -0.3 & -0.3 \\
\hline & & & & 36 & I818.4 & -0.7 & -0.5 \\
\hline 2 & 1715.2 & -0.8 & -10. & 37 & 1819.4 & -1.4 & -1.4 \\
\hline 3 & 1748.8 & $+1.5^{\circ}$ & +4.5 & 38 & 1820.5 & -1.4 & -1 \\
\hline 4 & 1750.8 & +0.8 & +2.4 & 39 & 1821.5 & +0.9 & + \\
\hline 5 & 1753.9 & +2.2 & +6.6 & 40 & 1822.5 & +1.4 & +2 \\
\hline 6 & 1756.7 & -0.2 & -1.0 & 41 & 1823.5 & +0.6 & $+c$ \\
\hline 7 & 1769.0 & -0.2 & -1.0 & 42 & 1821.5 & +1.2 & + \\
\hline & & & & 43 & 1825.5 & +0.7 & + \\
\hline 8 & 1782.0 & +0.1 & +0.2 & 44 & 1826.5 & +2.0 & +2 \\
\hline 9 & 1783.0 & -0.5 & -0.5 & & & & \\
\hline 10 & 1784.0 & +0.6 & +0.6 & 45 & 1827.7 & +1.0 & +0.5 \\
\hline 11 & 1785.0 & -1.0 & -1.0 & 46 & 1828.7 & +2.5 & +1.0 \\
\hline 12 & 1788.0 & +1.2 & +1.8 & $\begin{array}{l}41 \\
48\end{array}$ & $\begin{array}{l}182 \\
183\end{array}$ & $\begin{array}{l}+2.8 \\
+0.4\end{array}$ & + \\
\hline 13 & 1789.0 & +1.7 & +3.4 & 49 & 1831.7 & $\begin{array}{r}T .1 \\
+4.1\end{array}$ & $\begin{array}{r} \\
+\quad 2.0\end{array}$ \\
\hline 14 & 1790.0 & -0.5 & -1.0 & & & & \\
\hline 15 & 1791.0 & -0.7 & -1.0 & 50 & 1832.7 & +1.8 & +0.9 \\
\hline 16 & 1792.0 & 0.0 & 0.0 & 51 & 1833.8 & +0.5 & +0.2 \\
\hline & & & & 52 & 1834.8 & +0.1 & 0.0 \\
\hline 17 & 1793.0 & -0.1 & -0.2 & 53 & 1835.8 & -1.4 & -0.6 \\
\hline 18 & 1794.0 & +0.5 & +1.5 & 54 & 1836.8 & -3.4 & -1 \\
\hline 19 & 1795.0 & -0.6 & -0.9 & & & & \\
\hline 20 & 1796.0 & -0.5 & -1.0 & 55 & 1837.8 & -0.6 & -0.2 \\
\hline 21 & 1797.1 & -0.6 & -1.2 & 56 & 1838.8 & +0.5 & +0.2 \\
\hline 22 & 1800.2 & -1.4 & & 57 & 1839.8 & -0.4 & -0.1 \\
\hline 23 & 1801.2 & -1.4 & $\begin{array}{r}-4.2 \\
-\quad 49\end{array}$ & 58 & 1840.8 & -1.7 & -0.6 \\
\hline 24 & 1802.3 & -0.6 & $\begin{array}{r}-4.2 \\
-0.6\end{array}$ & 59 & 1841.8 & -0.3 & -0.1 \\
\hline 25 & 1805.3 & -1.4 & -1.4 & 60 & 1842.8 & -0.5 & -0.2 \\
\hline 26 & 1806.3 & -1.0 & -2.0 & 61 & 1843.8 & +1.6 & +0.5 \\
\hline 27 & 1807.3 & -0.1 & -0.1 & 62 & 1844.9 & +0.7 & +0.2 \\
\hline 28 . & 1808.3 & -1.0 & -2.0 & 63 & 1845.9 & +0.6 & +0.3 \\
\hline & 10 & & & 64 & 1846.9 & -0.4 & -0 \\
\hline 29 & 1809.3 & +0.1 & +0.2 & & 18479 & +06 & \\
\hline 30 & 1810.3 & -0.1 & -0.1 & $\begin{array}{l}65 \\
66\end{array}$ & 18489 & -16 & + \\
\hline 31 & 1811.3 & -0.9 & -0.9 & $\begin{array}{l}66 \\
67\end{array}$ & $\begin{array}{l}1040.9 \\
18499\end{array}$ & $\begin{array}{r}-1.0 \\
+02\end{array}$ & $\overline{+}$ \\
\hline 32 & 181 & 0 & & $\begin{array}{l}01 \\
68\end{array}$ & 1850.9 & $\begin{array}{r}0.3 \\
\end{array}$ & $\begin{array}{l}+ \\
+\end{array}$ \\
\hline 33 & 1813.4 & $\begin{array}{l}-0.3 \\
+0.1\end{array}$ & -0.6 & $\begin{array}{l}00 \\
69\end{array}$ & 1851.9 & -1.2 & T \\
\hline 34 & 1814.4 & $\begin{array}{l}+0.1 \\
-0.4\end{array}$ & +0.2 & & & & \\
\hline 35 & 1815.4 & -0.3 & $\begin{array}{r}-0 . \pm \\
-0.2\end{array}$ & 70 & 1852.9 & -1.2 & -0.6 \\
\hline $35^{\prime}$ & 1816.4 & -0.8 & -0.8 & 71 & 1853.9 & -0.2 & -0.1 \\
\hline
\end{tabular}




\begin{tabular}{|c|c|c|c|c|c|c|c|}
\hline No. of $\mathrm{Eq}$ & . Year. & $f_{\prime \prime}$ & 87 & No. of Eq & Year. & $f \delta l$ & $\delta_{11} 8$ \\
\hline 72 & 1854.9 & +0.5 & +0.2 & 81 & 1864.0 & +0.7 & +0.4 \\
\hline 73 & 1855.9 & 0.0 & 0.0 & 82 & 1865.0 & +2.2 & +1.5 \\
\hline 74 & 1856.9 & -0.5 & -0.2 & 83 & 1866.0 & +1.0 & +0.4 \\
\hline 75 & 1858.0 & -0.2 & -0.1 & 84 & 1867.0 & +0.5 & +0.2 \\
\hline 76 & 1859.0 & +0.9 & +0.4 & & & & \\
\hline 77 & 1860.0 & -1.1 & -0.4 & 85 & 1868.0 & +0.4 & 2 \\
\hline 78 & 1861.0 & -0.6 & -0.3 & 86 & 1869.9 & -1.9 & 0.8 \\
\hline 79 & 1862.0 & -0.6 & -0.2 & 87 & 1870.0 & -0.1 & 0.0 \\
\hline 80 & 1863.0 & +0.2 & +0.1 & $\begin{array}{l}80 \\
89\end{array}$ & $\begin{array}{l}1871.0 \\
1872.1\end{array}$ & $\begin{array}{l}-1.2 \\
+0.4\end{array}$ & +0.2 \\
\hline
\end{tabular}

A simple glance at the course of the residuals shows (1) that their probable value is considerably greater than the probable error attributed to the equations of condition, being more nearly $0^{\prime \prime} .7$ than $0^{\prime \prime} .5$, and yet larger in the later years; (2) that during certain periods they are of a systematic character. During the years 1748 to 1753 the observations show a decided positive correction to the theory of a magnitude greater than we can consider probable, amounting to about one-third of a second of time in the mean of Bradley's two observations of 1748 and 1753. About 1800 the correction becomes negative, and so continues for 20 years with an average value of about $1^{\prime \prime}$. In 1821 it suddenly becomes positive, and so continues until 1833. From this year forward the residuals are not systematic in character.

In order to show clearly the general course of the outstanding corrections, they have been divided into groups, generally including about five years each. The mean outstanding correction for each group, taken with respect to the weights indicated by the factors $f$, is as follows. In the column $\varepsilon$ is shown what the probable error of the residual should be if the weights assigned to the sereral equations were strictly correct, and no systematic errors were present either in theory or observation.

\begin{tabular}{|c|c|c|c|c|c|}
\hline Year. & $\iota_{\|}$ & - $\quad \underset{I \prime}{E}$ & Year. & ${ }_{\prime \prime}^{\delta l}$ & $\underset{\prime \prime}{\varepsilon}$ \\
\hline 1715.2 & -10 & \pm 6 & 1824.8 & +1.50 & \pm 0.16 \\
\hline 1751.1 & +3.7 & \pm 0.8 & 1829.7 & +0.91 & \pm 0.10 \\
\hline 1769.0 & -1.0 & \pm 2.5 & 1835.2 & -0.27 & \pm 0.09 \\
\hline 1783.3 & -0.18 & \pm 0.23 & 1839.8 & -0.17 & \pm 0.07 \\
\hline 1790.0 & +0.62 & \pm 0.40 & 1844.8 & +0.14 & \pm 0.03 \\
\hline 1795.0 & -0.55 & \pm 0.41 & 1849.9 & -0.21 & \pm 0. \\
\hline 1802.0 & -1.25 & \pm 0.45 & 1854.9 & -0.14 & \pm 0.1 \\
\hline 1806.5 & -1.00 & \pm 0.31 & 1860.0 & -0.13 & \pm 0.0 \\
\hline 1810.5 & -0.37 & \pm 0.32 & 1865.0 & +0.36 & \pm 0 \\
\hline 1814.5 & -0.37 & \pm 0.23 & 1870.0 & -0.21 & \pm 0 . \\
\hline 1819.5 & -0.37 & \pm 0.18 & & & \\
\hline
\end{tabular}


A simple glance at the residuals $\delta l$ shows that they are much greater than the purely accidental residnals resulting from the theory of least squares. We may divide the possible causes of these systematic errors into three classes.

1. Systematic Errors of Observation.-These may result from deviation of the line of collimation of the instrument from a true great circle, or from any peculiarity of the observer which leads to his registering the transit of Uranus earlicr or later than that of a fixed star. If we compare the corrections derived from the work of different observatories as given in the last chapter, we shall find frequent cases not only of systematic differences between the results of different observatories, but between those of the same observatory in two successive years. An instance which particularly attracted my attention on first preparing the comparisons of theory and observation is that of the Greenwich observations for 1831, which, as compared with observations at the same observatory during the years preceding and following, seem to be affected with some constant error in $R$. A. of about $2^{\prime \prime}$. I find that this discrepancy can be attributed only to the original observations.

2. Errors in the Theory compared.-These may arise from errors in the preceding theoretical computations, from the omission of the terms of the second order produced by Neptune, from the adoption of an crroncous mass of Saturn, or from the attraction of an unknown planet. With regard to the probability of these different sources of error it may be remarked that errors of computation seem possible only in the terms of the second order, that the mass of Saturn is taken from the exhaustive discussion of the Saturnian system by Bessel, in which an error sufficient to influence the theory of Uranus seems highly improbable, and that a transNeptunian planet large enough to prodnce a sensible deviation of the orbit of Uranus from an ellipse in the course of a century would be too large to have cscaped detection. The choice of the elliptic elements of Uranus and Neptune is such that the terms of the second order, due to the action of Neptune, can scarcely become sensible within a century of the epoch.

3. Errors in the various Reductions by which Theory and Observation are compared. - In the method adopted for comparing theory and observation a number of small uncertainties incident to the imperfections of the older data of reduction necessarily creep in. In the early observations the imperfections arise principally from the uncertainty of the instrumental corrections, and the errors in the adopted positions of the fundamental stars, and indeed in nearly all the data of reduction. In the late years they arise principally from the great magnitude of the correction to Boavard's tables, and the consequent rapid change of the corrections to the geocentric ephemerides, which make the determination of the corrections $\Delta l$ and $\delta l$ from theory and observation somewhat uncertain. Errors from this source will necessarily be in part of a systematic character, and, in view of their possibility, I regret not having been able to completely re-reduce all the observations before $18 \pm 0$, and to compare all since directly with ephemerides computed from the provisional theory. In order, however, to test the question whether they are sensible, I have prepared an ephemeris from the provisional theory for the three recent oppositions of $1861-2,1862-3$, and 1872 , and compared it directly with 
the observations. The mean corrections in geocentric longitude for groups of observations are given in columns (2), column (1) showing the correction given by the work of the last chapter.

Opposition 1861-2

(1)

$\begin{array}{rr}+2^{\prime} .8 & +2^{\prime \prime} .4_{1} \\ 2.9 & 2.4_{3} \\ 3.0 & 3.1_{2} \\ 2.4 & 2.4_{2}\end{array}$
Mean $\overline{+2.79+2.62}$
$.4_{3}$

$.4_{2}$

\begin{tabular}{rr}
\multicolumn{2}{c}{$1862-3$} \\
$(1)^{(2)}$ \\
$+2^{\prime} .6$ & $+2^{\prime} .6_{1}$ \\
2.3 & $2.3_{2}$ \\
2.1 & $2.0_{3}$ \\
3.3 & $2.9_{4}$ \\
2.6 & $2.7_{1}$ \\
\hline+2.65 & +2.50
\end{tabular}

(1)<smiles>C[Se][Se][Se]</smiles>$$
-8.5-8.1_{2}
$$$$
-7.3-7.5_{3}
$$$$
-7.3-7.7^{\circ}
$$$$
\frac{-7.8-7.6_{1}}{-7.65-7.82}
$$

A systematic difference of $0^{\prime \prime} .16$ would seem to be indicated, and on account of it a correction of $0^{\prime \prime} .10$ was applied to the comparisons of the last few years in forming the equations of condition.

In view of the possibility of systematic errors from this source it may be considered that too great relative weight has been assigned to the results of the later observations. If the residuals arise from errors of comparison and of theory, their probable magnitude is nearly as great at one epoch as at another. It may therefore be interesting to inquire what result we should get if, instead of assigning such different weights to the comparisons at different epochs, we sought only for the best general agreement with observations during the period the planet has been observed. The preceding system of mean residuals will enable us to discuss this question quite easily. In the first solution we shall reject the results from Flamstead's observations, owing to their assured uncertainty, and those from Le Monnier's of 1769 , owing to the possible maladjustment of his quadrant. The equations from the remaining residuals will be the following:

$\begin{array}{lllllll}1.0 \delta^{2} \varepsilon^{\prime} & -7.6 \delta^{2} n^{\prime} & +0.8 \delta^{2} e & +1.7 e \delta^{2} \pi-0.5 \delta^{2} \mu^{\prime}=+3.7 & \text { Wt. } \frac{1}{2} \\ 1.1 & -5.0 & -2.0 & -0.8 & +1.8 & =-0.18 & 2 \\ 1.1 & -4.4 & -1.4 & -1.6 & +1.6 & =+0.62 & 1 \\ 1.1 & -3.9 & -0.6 & -2.0 & +1.2 & =-0.55 & 1 \\ 1.2 & -3.2 & +0.6 & -2.1 & +0.5 & =-1.25 & 1 \\ 1.1 & -2.6 & +1.3 & -1.8 & 0.0 & =-1.00 & 2 \\ 1.1 & -2.2 & +1.7 & -1.3 & -0.2 & =-0.37 & 2 \\ 1.1 & -1.7 & +2.0 & -0.7 & -0.5 & =-0.37 & 2 \\ 1.0 & -1.1 & +2.1 & +0.1 & -0.6 & =-0.37 & 2 \\ 1.0 & -0.5 & +1.8 & +0.9 & -0.6 & =+1.50 & 2 \\ 1.0 & 0.0 & +1.4 & +1.4 & -0.5 & =+0.91 & 3 \\ 0.9 & +0.5 & +0.8 & +1.8 & -0.5 & =-0.27 & 3 \\ 0.9 & +0.9 & +0.1 & +2.0 & -0.6 & =-0.17 & 3 \\ 1.0 & +1.4 & -0.6 & +1.9 & -0.7 & =+0.14 & 3 \\ 1.0 & +1.9 & -1.2 & +1.6 & -0.8 & =-0.21 & 3 \\ 1.0 & +2.5 & -1.7 & +1.1 & -0.9 & =-0.14 & 3 \\ 1.0 & +3.1 & -2.0 & +0.4 & -0.9 & =-0.13 & 3 \\ 1.1 & +3.7 & -2.1 & -0.4 & -0.9 & =+0.36 & 3 \\ 1.1 & +4.4 & -1.8 & -1.2 & -0.8 & =-0.21 & 3\end{array}$


Giving these nineteen equations equal weights, we have the second of the following solutions, and the second of the series of residuals the first corresponding to the primitive solution. Solving them again and assigning the weights attached to the respective equations, which I judge to be tnose to which they are entitled when a liberal allowance is made for systematic errors of observation and of comparison of theory with obscrvations, adding also the equations given by the observations of Flamstead and Le Monnier, which are as follows:

$$
\begin{aligned}
& 16900.0 \delta^{2} \varepsilon^{\prime}-0.7 \delta^{2} n^{\prime}-0.1 \delta^{2} e+0.0 e \delta^{2} \pi+0.1 \delta^{2} \mu^{\prime}=-0^{\prime \prime} .5 ; f, \frac{1}{2} ; \mathrm{Wt}, 1 \\
& \begin{array}{lllllllll}
1715 & 0.1 & -1.1 & 0.0 & -0.2 \quad+0.1 & =-0.5 & \frac{1}{12} & 1
\end{array} \\
& 1769-0.2-1.2-0.3+0.2+0.2=+2.2 \quad \frac{1}{6} \quad 1
\end{aligned}
$$

\begin{tabular}{|c|c|c|c|}
\hline & (1) & (2) & (3) \\
\hline$\delta^{2} \varepsilon_{1}$ & 0 & $-0^{\prime \prime} .39$ & -0.21 \\
\hline$\delta^{2} n_{1}$ & 0 & -0.38 & -0.19 \\
\hline$\delta^{2} e$ & 0 & -0.33 & -0.15 \\
\hline$e \delta^{2} \pi$ & 0 & +0.25 & +0.19 \\
\hline$\delta^{2} \mu^{\prime}$ & 0 & -1.02 & -0.49 \\
\hline$m^{\prime}$ & $1 \frac{1}{6} \frac{1}{40}$ & $\overline{1} \frac{1}{8} \div \overline{0}$ & $\overline{1} \frac{1}{9} \overline{5} 0$ \\
\hline
\end{tabular}

we have the sccond solution, and the third series of residuals.

Year.

1691.0

1715.2

1751.1

1769.0

1793.3

1790.0

1795.0

1802.0

1806.5

1810.5

1814.5

1819.5

1824.8

1829.7

1835.2

1839.8

1844.8

1849.9

1854.9

1860.0

1865.0

1870.0
$-0.19$

$-0.15$

$+0.19$

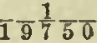

liesiduals.

\begin{tabular}{ccc}
\hline$\Delta_{1} l$ & $\Delta_{2} l$ & $\Delta_{3} l$ \\
${ }^{\prime \prime}$ & & \\
-10. & -14. & -12. \\
-10. & -9. & -7. \\
+3.7 & -0.5 & +2.0 \\
-1.0 & -0.30 & -1.9 \\
-0.18 & +0.93 & -0.17 \\
+0.62 & -0.09 & -0.89 \\
-0.55 & -0.78 & -0.87 \\
-1.25 & -0.69 & -0.73 \\
-1.00 & -0.10 & -0.05 \\
-0.37 & -0.26 & -0.28 \\
-0.37 & +0.34 & -0.37 \\
-0.37 & +0.90 & +1.41 \\
+1.50 & -0.43 & -0.81 \\
+0.91 & -0.56 & -0.46 \\
-0.27 & -0.31 & -0.18 \\
-0.17 & -0.70 & -0.52 \\
+0.14 & -0.54 & -0.36 \\
-0.21 & -0.23 & -0.14 \\
-0.14 & +0.70 & +0.62 \\
-0.13 & +0.80 & +0.44 \\
+0.36 & &
\end{tabular}


It will be seen that the effect of these changes of weights is, that the older observations are a little better, and the later a little worse represented. I conceive that our choice must lie between the first and third solutions, the first being the more probable if we conceive the outstanding residuals to be due to errors of observation only, and the third if we suppose them equally due to errors of computation. On the whole, I consider the mean of the two to be about the most probable, and this will give the mass of Neptune very near the round number

$\overline{19700}$

which will be adopted as the definitive value. The definitive corrections to Elements III (p. 99) will then be

$\begin{array}{cc}\delta \varepsilon^{\prime}(1830) & -3^{\prime \prime} .56 \\ \delta \varepsilon(1850) & -12.45 \\ 10 \delta n & -4.44 \\ \delta e & -4.12 \\ e \delta \pi & -0.25 \\ \delta \mu & -0.137\end{array}$

\section{Corrections to the Inclination and Nude.}

These corrections have been derived entirely from the modern observations, the ancient ones being too uncertain to add anything to the weight of the result. The mode in which the correction to the latitude of the provisional ephemeris has been concluded from the observations has been sufficiently explained: it is only necessary to add that the immediate results from the data of the preceding chapter require two corrections, namely:

(1) A correction to the theoretical latitude for the change in the adopted mass of Neptune. The value of this correction, as derived from the data of Chapter V, is with sufficient approximation

$$
\delta \beta=0^{\prime \prime} .25 T \cos g .
$$

(2) A correction to the observed latitude on account of the difference between the obliquity of the ecliptic adopted in the various ephemerides compared, and that of Hansen's Tables du Soleil, which having been adopted in the theory should be used throughout.

Applying the correction (2) - (1) to all the observed latitudes, we have the following corrections to the latitude of the provisional ephemeris derived from all the observations of each opposition since 1781. The third column gives the number of observations in declination. These numbers may, however, in some cases be inaccurate. The fourth and fifth columns give the sine and cosine of the argument of latitude, to be used in forming the equations of condition. 


\begin{tabular}{|c|c|c|c|c|c|c|c|c|c|}
\hline Year. & $\delta \beta$ & No. of ols. & $\sin u$ & $\cos u$ & Year. & $\delta \beta^{\circ}$ & No. of obs. & $\sin u$ & $\cos 2$ \\
\hline & " & & & & & 100 & & & \\
\hline 1782.0 & -0.4 & 21 & +0.31 & +0.95 & 1830.7 & $\begin{array}{l}+0.2 \\
+1.0\end{array}$ & $\begin{array}{l}48 \\
23\end{array}$ & $\begin{array}{r}-0.82 \\
0.87\end{array}$ & $\begin{array}{r}-0.57 \\
0.50\end{array}$ \\
\hline $\begin{array}{l}1783.0 \\
17840\end{array}$ & -3.5 & 13 & $\begin{array}{l}0.38 \\
0.45\end{array}$ & $\begin{array}{l}0.92 \\
0.89\end{array}$ & 1831.7 & +1.0 & $\begin{array}{ll}23 \\
90\end{array}$ & $\begin{array}{l}0.81 \\
090\end{array}$ & \\
\hline $\begin{array}{l}1784.0 \\
1785.0\end{array}$ & $\begin{array}{l}-2.4 \\
-0.1\end{array}$ & $\begin{array}{l}13 \\
10\end{array}$ & 0.52 & 0.85 & $\begin{array}{l}1832.7 \\
1833.7\end{array}$ & $\begin{array}{r}+0.9 \\
+0.6\end{array}$ & $\begin{array}{l}20 \\
54\end{array}$ & $\begin{array}{r}-0.90 \\
0.93\end{array}$ & $\begin{array}{r}-0.41 \\
0.37\end{array}$ \\
\hline 1788.0 & -1.0 & 5 & +0.71 & +0.71 & 1834.7 & +0.3 & 92 & 0.95 & 0.31 \\
\hline 1789.0 & +0.9 & 6 & 0.77 & 0.64 & 1835.7 & +0.2 & 71 & -9.97 & -0.24 \\
\hline 1790.0 & +2.6 & 4 & 0.81 & 0.58 & 1836.7 & +0.1 & 135 & 0.98 & 0.17 \\
\hline 1791.0 & +1.9 & 7 & 0.86 & 0.51 & 1837.8 & 0.0 & 154 & 0.99 & 0.10 \\
\hline 1792.0 & +1.8 & 3 & +0.90 & +0.44 & 1838.8 & -0.2 & 182 & -1.00 & -0.03 \\
\hline 1793.0 & +2.9 & 5 & 0.93 & 0.37 & 1839.8 & -0.4 & 142 & 1.00 & +0.03 \\
\hline 1794.0 & +0.5 & 3 & 0.95 & 0.30 & 1840.8 & -0.3 & 106 & 0.99 & 0.10 \\
\hline 1795.0 & +0.7 & 7 & +0.97 & +0.22 & 1841.8 & -0.7 & 101 & -0.98 & +0.17 \\
\hline 1796.0 & +3.6 & 4 & 0.99 & 0.14 & 1842.8 & -0.7 & 145 & 0.97 & 0.24 \\
\hline 1797.0 & +3.6 & 3 & 1.00 & +0.05 & 1843.8 & -0.9 & 88 & 0.95 & 0.31 \\
\hline 1800.2 & 0.0 & 2 & +0.98 & -0.21 & 1844.9 & -1.0 & 87 & -0.93 & +0.37 \\
\hline 1801.2 & +1.2 & 2 & 0.97 & 0.26 & 1845.9 & -0.6 & 55 & 0.90 & 0.44 \\
\hline 1802.3 & +1.4 & 13 & 0.94 & 0.34 & 1846.9 & -1.1 & 92 & 0.87 & 0.50 \\
\hline 1805.3 & +1.1 & 3 & +0.83 & -0.56 & 1847.9 & -1.1 & 69 & -0.83 & +0.56 \\
\hline 1806.3 & -1.4 & 5 & 0.78 & 0.63 & 1848.9 & -0.9 & 56 & 0.79 & 0.62 \\
\hline 1807.3 & +1.6 & 16 & 0.72 & 0.69 & 1849.9 & -1.0 & 36 & 0.74 & 0.67 \\
\hline 1808.3 & +1.8 & 6 & +0.67 & -0.74 & 1850.9 & -0.7 & 46 & -0.69 & +0.72 \\
\hline 1809.3 & +1.3 & 9 & 0.60 & 0.80 & 1851.9 & -1.2 & 35 & 0.64 & 0.77 \\
\hline 1810.3 & +3.7 & 16 & 0.53 & 0.85 & 1852.9 & -1.3 & 49 & 0.59 & 0.81 \\
\hline 1811.3 & +3.4 & 11 & +0.45 & -0.89 & 1853.9 & -1.8 & 48 & -0.53 & +0.85 \\
\hline 1812.4 & +3.6 & 6 & 0.38 & 0.93 & 1854.9 & -1.0 & 47 & 0.47 & 0.88 \\
\hline 1813.4 & +2.4 & 6 & 0.29 & 0.96 & 1855.9 & -1.3 & 49 & 0.41 & 0.91 \\
\hline 1814.4 & +2.5 & 13 & +0.22 & -0.98 & 1856.9 & -1.1 & 41 & -0.34 & +0.94 \\
\hline 1815.4 & +2.2 & 16 & 0.14 & 0.99 & 1858.0 & -1.7 & 65 & 0.26 & 0.97 \\
\hline 1816.4 & +1.3 & 18 & 0.06 & 1.00 & 1859.0 & -1.7 & 56 & 0.19 & 0.98 \\
\hline 1817.5 & +2.4 & 13 & -0.01 & -1.00 & 1860.0 & -1.9 & 58 & -0.12 & +0.99 \\
\hline 1818.5 & +4.2 & 22 & 0.09 & 1.00 & 1861.0 & -2.3 & 41 & 0.05 & 1.00 \\
\hline 1819.5 & +1.7 & 7 & 0.17 & 0.99 & 1862.0 & -1.6 & 52 & +0.02 & 1.00 \\
\hline 1820.5 & +1.0 & 9 & -0.24 & -0.97 & 1863.0 & -1.7 & 83 & +0.10 & +0.99 \\
\hline 1821.5 & +1.3 & 10 & 0.31 & 0.95 & 1864.0 & -2.2 & 39 & 0.18 & 0.99 \\
\hline 1822.5 & +1.8 & 7 & 0.38 & 0.92 & 1865.0 & -1.7 & 37 & 0.25 & 0.97 \\
\hline 1823.5 & +0.6 & 7 & -0.45 & -0.89 & 1866.0 & -0.9 & 72 & +0.33 & +0.95 \\
\hline 1824.5 & -0.2 & 11 & 0.51 & 0.86 & 1867.0 & -0.6 & 83 & 0.40 & 0.92 \\
\hline 1825.5 & +1.5 & 5 & 0.57 & 0.82 & 1668.1 & -0.9 & 32 & 0.47 & 0.88 \\
\hline 1826.5 & +1.2 & 7 & -0.63 & -0.78 & 1869.1 & -0.8 & 65 & +0.54 & +0.84 \\
\hline 1827.6 & +2.0 & 5 & 0.68 & 0.73 & 1870.1 & -0.4 & 32 & 0.60 & 0.80 \\
\hline 18 & -1.0 & 7 & $0=0$ & 0.68 & 1871.1 & -0.7 & 21 & +0.66 & 0.75 \\
\hline 1829.7 & +0.9 & 9 & -0.78 & -0.63 & 1872.1 & -0.4 & 47 & +0.72 & +0.69 \\
\hline
\end{tabular}

It will be remembered that the observed declinations have, as far as possible, been reduced to Auvers' standard. We have no positive proof that this standard is correct. If it be affected by a constant error, the result will be that the orbit of the planet on the celestial sphere, as deduced from observation, instead of being a great circle, as we know the real orbit to be, will be a small one, and the comparison of a uniform series of observations extending through an entire revolution of the planet, after making the best correction to the position of the orbit, will leave a constant residual. Now, we can best determine this residual by including it as an unknown quantity in our equations. 
Again, the error of the stamlarl is not necessarily constant, but may contuin a term proportional to the time, arising from erroneous proper motions of the stundard stars. Therefore, instead of supposing the residual constant, we shall suppose it of the form $a+b \ell$. Each obserred correction to the theoretical lutitude will then give the equation,

$$
\sin \imath \omega \phi-\cos \imath \phi \delta \theta+a+\iota T=\delta \beta .
$$

'To fucilitate the solution of these equations they have been divided into groups, each group usually comprehending three oppositions, and combined into a single equation multiplied by such a factor as would make its probable error half a second. 'The factor by which the correction of the latitule is multiplied in the equation is the same with the cocfficient of $a$. The year 1840.0 is taken as the epoch for $b$. 'Thus we have the following:

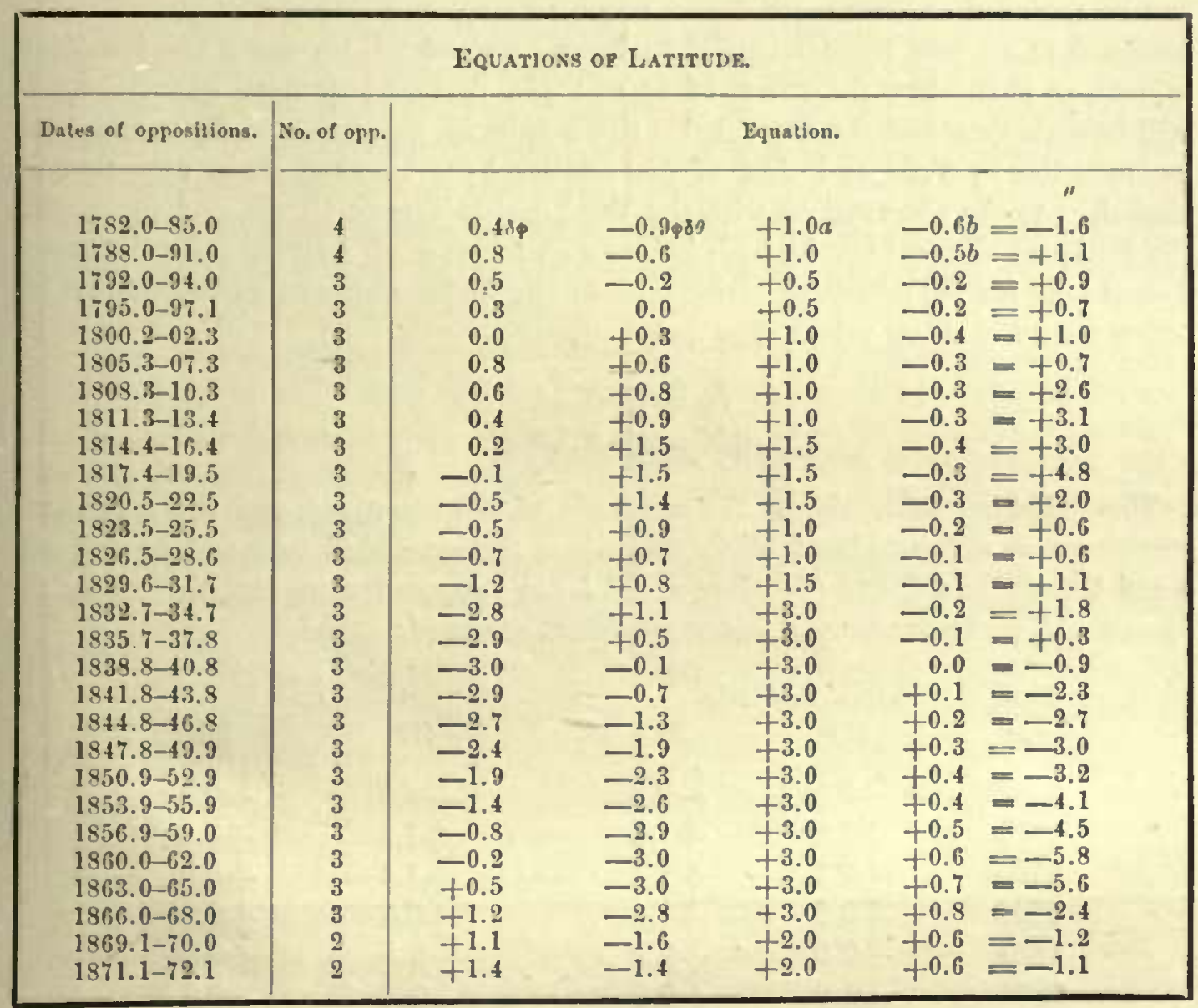

Treating these equations by the method of least squares, we find the normal equations

$$
\begin{array}{r}
63.60 \delta \phi+6.45 \phi \delta 0-52.10 a-1.08 b=+28.33 \\
6.45 \delta \phi+69.59 \phi \delta 0-52.60 a-14.25 b=+11.02 \\
-52.10 \delta \phi-52.60 \phi \delta \theta+133.50 a+8.95 b=-76.55 \\
-1.08 \delta \phi-14.25 \phi \delta 0+8.95 a+4.49 b=-23.69
\end{array}
$$


The solution of these equations gives

$$
\begin{aligned}
\delta \phi & =+0^{\prime \prime} .28+0^{\prime \prime} .75 a=+0^{\prime \prime} .54 \\
\phi \delta \theta & =+1.57+0.686 a+0.205 b=+1^{\prime \prime} .75 \\
a & =+0.35 \\
b & =-0.28
\end{aligned}
$$

These values of $a$ and $b$ indicate that at the epoch 1810 Auwers' equatorial declinations are too great, or his north polar distances are too small by $0 " .35$, and that this error is diminishing at the rate of $0^{\prime \prime} .28$ per century. If the older measures in declination had been comparable in precision with those made at the present time, and if the possible periodic error in the reduced right ascensions had been carefully eliminated, I should regard this determination as entitled to considerable weight. In view of the great uncertainty of the declinations previous to 1820 , it can be regarded as little more than a rough attempt at a determination. For this reason the first two normal equations have been solved, leaving $a$ and $b$ indeterminate, so as to show the valves of $\delta \phi$ and $\phi \delta \theta$ in terms of these quantities. It will be seen that had we neglected $a$ and $b$ entirely, the value of $\delta \phi$ would have been smaller by $0^{\prime \prime} .26$, and that of $\phi \delta \theta$ smaller by $0^{\prime \prime} .18$ than those actually concluded. As the observations with the Washington Transit Circle, and those with the Pulkowa Vertical Circle, both indicate an increase of Auwers' polar clistances, I shall take for the definitive corrections to the inclination and node those which follow from the above values of $a$ and $b$, or,

$$
\begin{aligned}
\delta \phi & =+0^{\prime \prime} .54 \\
\phi \delta \theta & =+1.75 .
\end{aligned}
$$

The following table shows the residuals of the equations, and the mean outstanding corrections to the latitude, (1) when the concluded values of $\delta \phi$ and $\phi \delta 0$ $a$ and $b$ are all used, and (2) when $a$ and $b$ are supposed zero, and the values of $\delta \phi$ and $\phi \delta \theta$, corresponding to this supposition, are used:

\begin{tabular}{lcccc} 
Year. & \multicolumn{2}{c}{ Residuals. } & & $\delta \beta$ \\
& $(1)$ & $(2)$ & $(1)$ & $(2)$ \\
1783 & -0.8 & -0.3 & -0.8 & -0.3 \\
1789 & +1.2 & +1.8 & +1.2 & +1.8 \\
1793 & +0.7 & +1.1 & +1.4 & +2.2 \\
1796 & +0.3 & +0.6 & +0.6 & +1.2 \\
1801 & -0.5 & +0.3 & -0.5 & +0.3 \\
1806 & -1.2 & -0.5 & -1.2 & -0.5 \\
1809 & +0.5 & +1.2 & +0.5 & +1.2 \\
1812 & +0.9 & +1.6 & +0.9 & +1.6 \\
1815 & -0.3 & +0.6 & -0.2 & +0.4 \\
1818 & +1.6 & +2.5 & +1.1 & +1.7 \\
1821 & -0.7 & -0.1 & -0.5 & -0.1 \\
1824 & -1.3 & -0.7 & -1.3 & -0.7 \\
1827 & -0.6 & -0.3 & -0.6 & -0.3
\end{tabular}




\begin{tabular}{|c|c|c|c|c|}
\hline \multirow[t]{2}{*}{ Year. } & \multicolumn{2}{|c|}{ Residuals. } & \multicolumn{2}{|c|}{$\delta \beta$} \\
\hline & (1) & (2) & (1) & (2) \\
\hline 1830 & -0.2 & +0.2 & -0.1 & +0.1 \\
\hline 1833 & +0.3 & +0.9 & +0.10 & +0.30 \\
\hline 1836 & 0.0 & +0.3 & 0.00 & +0.10 \\
\hline 1839 & -0.1 & +0.1 & -0.03 & +0.03 \\
\hline 1842 & -0.5 & -0.4 & -0.17 & -0.13 \\
\hline 1845 & +0.1 & 0.0 & +0.03 & 0.00 \\
\hline 1848 & +0.6 & +0.7 & +0.20 & +0.23 \\
\hline 1851 & +0.9 & +0.9 & +0.30 & +0.30 \\
\hline 1854 & +0.3 & +0.3 & +0.10 & +0.10 \\
\hline 1858 & +0.1 & +0.3 & +0.03 & +0.10 \\
\hline 1861 & -1.4 & -1.0 & -0.47 & -0.33 \\
\hline 1864 & -1.5 & -1.0 & -0.50 & -0.33 \\
\hline 1867 & +1.1 & +1.7 & +0.37 & +0.57 \\
\hline 1869 & +0.5 & +1.0 & +0.17 & +0.33 \\
\hline 1871 & 0.0 & +0.7 & 0.00 & +0.23 \\
\hline
\end{tabular}

The sum of the squares of the residuals is in the first case 17".94, and in the second $25^{\prime \prime} .41$, so that the introduction of $a$ and $b$ makes a decided improvement in the representation of the observations.

I have not attempted a rigorous investigation of the probable error of any of these results for the reason that the values of the probable error deducible by the method of least squares would, in a case like the present, be entirely untrustworthy. It is, however, very desirable that we should be able to form some judgment of the uncertainty of the mass of Neptune. From the last system of equations of condition the value of $\mu^{\prime}$ comes out with the weight 3.13 , or nearly that assigned to the mean result of each five years of modern observations. Regarding these results as independent, their mean error would be about $0^{\prime \prime} .5$, so that the probable error of $\mu^{\prime}$ would be 0.5 , and that of $\mu$ would be .005 , or about $\frac{1}{2} \frac{1}{0}$ the entire mass of Neptune. A probable error derived from the original equations would have been much smaller, and when, in the last equations, we allow for the systematic character of the residuals, it will be larger. If we suppose the theory to be perfect, I conceive we may fairly estimate the probable error of the mass of Neptune to be $\frac{1}{10} \frac{1}{0}$ of its entire amount, and its possible error two or three times greater. If there is any error or imperfection in the theory, the error may be much larger. 


\section{CHAP'TER VIII.}

\section{COMPLETION AND ARRANGEMENT OF THE THEORY TO FIT IT FOR PERMANENT USE.}

In the preceding discussions the terms of the second order due to the action of Neptune have been neglected, the elements of Uranus and Neptune being so chosen that these terms can scarcely become sensible within a century of the epoch. But this very choice will make them larger in the course of centuries than if mean elements had been chosen. They will be most sensible in the case of the great inequality of 4300 years between Uranus and Neptune, an inequality which will make centuries of observation necessary to an accurate determination of the mcan elements of the two orbits. 'The uncertainty arising from the great inequality is probably of the same order of magnitude with the omitted terms of the second order, and, such being the case, the theory would really be made but little more accurate by the addition of those terms. I conceive, however, that the theory will be made much more satisfactory by the computation of at least the largest of the terms in question, if only to arrive at a certain determination of their order of magnitude, and of their effect on the planet during the period in which it has been observed.

The term in question, being of very long period, may be most advantagcously treated by the method of variation of elements, more especially as it has in the theory been already treated as such a perturbation. The largest of the perturbations in question are those of the mean longitude which are multiplied by the square of the integrating factor $v$, which is nearly 51, but which also contain the eccentricities as factors, and those of the eccentricity and perihelion which are independent of the eccentricities, but are multiplied by only the first power of $\nu$. 'These terms will probably comprise nearly or quite nine-tenths of those arising from the term of long period.

Let us begin with the perturbations of mean longitude. These are given by the integration of the equation

$$
\frac{d^{2} l}{d t^{2}}=-3 m^{\prime} \alpha n^{2}\left\{e k_{1} \sin \left(2 l^{\prime}-l-\pi\right)+e^{\prime} k_{2} \sin \left(2 l^{\prime}-l-\pi^{\prime}\right)\right\}
$$

$k_{1}$ and $k_{2}$ being functions of the ratio of the mean distances, or $\alpha$. If we integrate this cquation, supposing all the quantities in the second member except $l^{\prime}$ and $l$ to be constant, and these two to be of the form $n t+\varepsilon, n$ and $\varepsilon$ being constants, we shall reproduce the principal term of long period already found. But in the second approximation we must suppose all the elements variable. It is not, however, necessary to take into account the variations of $a, n$, and $k$, because these are 
of a lower order of magnitude. The perturbations to be added will be those of ${ }^{*}$ $l, l, e, e^{\prime}, \pi$, and $\pi^{\prime}$.

The point from which the longitudes are counted being arbitrary, we shall take the position of the perihelion of Uranus for 1850.0 as the origin, and put, as before, $g$ for the mean longitude of Uranus counted from this point, and let $l^{\prime}$ represent the mean longitude of Neptune counted from the same point. The terms of $\frac{d^{2} l}{d t^{2}}$ within the brackets will thus become

$$
e k_{1} \sin \left(2 l^{\prime}-g-\delta \pi\right)+e^{\prime} k_{2} \sin \left(2 l^{\prime}-g-\left(\pi^{\prime}-\pi\right)\right)
$$

or, if we put

$$
\begin{aligned}
& 2 l^{\prime}-g=N \\
& e^{\prime} \sin \left(\pi^{\prime}-\pi\right)=h^{\prime} \\
& e^{\prime} \cos \left(\pi^{\prime}-\pi\right)=k^{\prime}
\end{aligned}
$$

and notice, that to terms of the first order we have, $\sin \delta \pi=\delta \pi, \cos \delta \pi=1$, we shall have

$$
\frac{d^{2} l}{d t^{2}}=-3 m^{\prime} \alpha n^{2}\left\{\left(e k_{1}+k^{\prime} k_{2}\right) \sin N-\left(e k_{1} \delta \pi+k^{\prime} k_{2}\right) \cos N\right\}
$$

differentiating the quantities, of which the perturbations are to be considered with respect to the sign $\delta$, we find for the terms of the second order.

$$
\begin{aligned}
\delta \frac{d^{2} l}{d t^{2}}=-3 m^{\prime} \alpha n^{2} & \left\{\left(k_{1} \delta e+k_{2} \delta l^{\prime}+h^{\prime} k_{2} \delta N\right) \sin N\right. \\
& \left.+\left(\left(e k_{1}+k^{\prime} k_{2}\right) \delta N-e k_{1} \delta \pi-k_{2} \delta h^{\prime}\right) \cos N\right\} .
\end{aligned}
$$

We have now to substitute in this expression the numerical values of the quantities within parentheses. Those of the perturbations of Uranus have already been given in Chapter III, but it is necessary to diminish them by the factor $0.145^{*}$ for the altered mass of Neptune. Those of Neptune are taken from my investigation of the orbit of that planet (p. 38). The mass of Uranus there adopted is $\frac{1}{2} \frac{1}{0} \sigma \sigma$, while the investigation of Dr. Von Asten, $\uparrow$ from the observations of Struve and others, shows it to be $\frac{\overline{2}}{2} \frac{1}{\delta \tau}$. The perturbations are therefore diminished by $\frac{1}{2} \frac{2}{2}$. In accordance with the system adopted throughout both investigations, constants are added to all the perturbations to make them vanish at the epoch 1850.0. A term is also added to make $\frac{d l}{d t}$ also vanish at the epoch; this corresponds to the constant which ought to be added to $\delta a$. The numerical values thus obtained, are:

* This factor was adopted before the mass of Neptune to be employed had been finally decided upon. Hence the difference between it and that in the preceding chapter.

$†$ Mémoires de l'Académie de St. Pétersbourg, tome xviii, vii série. 


$$
\begin{aligned}
& \delta N=+7260^{\prime \prime} \sin N-6658^{\prime \prime}+4^{\prime \prime} .26 t \\
& e \delta \pi=-414 \sin N+380 \\
& \delta e=-414 \cos N-165 \\
& i l^{\prime}=+120 \sin N-110 \\
& \delta l^{\prime}=+120 \cos N+48 \\
& l_{1}=-1.234 \\
& l_{2}=+0.452 \\
& l^{\prime}=+0.00695 \\
& l^{\prime}=-0.00486
\end{aligned}
$$

Substituting these values in the cxpression for $\delta \frac{d^{2} l}{d t^{2}}$ and integrating twice, we find, putting $b$ for the coefficient of the time in $N$, of which the value, taking the century as the unit, is +0.1472 , and putting $T$ for the time in centuries,

$$
\begin{gathered}
\delta l=\frac{3}{2} m^{\prime} \alpha \nu^{2}\left\{\left(411^{\prime \prime}+\frac{102^{\prime \prime} .8}{b}+2^{\prime \prime} .6 T\right) \sin N+\left(1837^{\prime \prime}+\frac{5^{\prime \prime} .3}{b}-51 . " 4 T\right) \cos N\right. \\
\left.-109^{\prime \prime} .3 \sin 2 N-5^{\prime \prime} .5 \cos 2 N\right\} \\
-16^{\prime \prime} .5 m^{\prime} \alpha \nu^{2} t^{2}+c T+c^{\prime}
\end{gathered}
$$

$c$ and $c^{\prime}$ being the arbitrary constants of integration, which are to be chosen so that both $\delta l$ and its first differential coefficient shall vanish at the epoch. Reducing to numbers, we find

the last two terms being arbitrary.

$$
\begin{gathered}
\delta l=\left(140^{\prime \prime} .70+0^{\prime \prime} .32 T\right) \sin N \\
+(232.60-6.37 T) \cos N \\
-\quad 13.60 \sin 2 N \\
-\quad 0.70 \cos 2 N \\
-\quad 0.03 T^{2} \\
+\quad 34.27 T \\
-\quad 46.76
\end{gathered}
$$

When we carry the perturbations of the eccentricity and perihclion to quantities of the second order, we are troubled by the introduction of large terms depending on the square of the disturbing force, which disappear from the rigorous expressious for the co-ordinates. . These may be avoided by substituting for the cccentricity and perihelion the quantities $\hbar$ and $k$ determined by the condition

$$
\begin{aligned}
& h=e \sin \pi \\
& k=e \cos \pi
\end{aligned}
$$

If, as before, we count the longitudes from the perihelion of Uranus at the epoch 1850 , we should substitute $\delta \pi$ for $\pi$ in thesc expressions. The values of $h$ and $\%$ will then be given by the integration of the equations

$$
\begin{aligned}
& \frac{d \hbar}{d t}=m^{\prime} \alpha n k_{1} \cos N \\
& \frac{d k}{d t}=-m^{\prime} \alpha n k_{i_{1}} \sin N .
\end{aligned}
$$


Differentiating with respect to $\delta$, we find for the terms of the second order

$$
\begin{aligned}
& \delta \frac{d h}{d t}=-m^{\prime} \alpha n k_{1} \sin N \delta N \\
& \delta_{d l}^{d t}=-m^{\prime} \alpha n k_{1} \cos N \delta N .
\end{aligned}
$$

Substituting for $\delta N$ its numerical value just given and integrating, we find

$$
\begin{aligned}
& i h=m^{\prime} \alpha \nu k_{1}\left\{-2895^{\prime \prime} \sin N-\left(6658^{\prime \prime}-4^{\prime \prime} .26 t\right) \cos N+1815^{\prime \prime} \sin 2 N\right\} \\
& -3630^{\prime \prime} m^{\prime} \alpha k_{1} n t+\text { constant; } \\
& \left\{k=m^{\prime} \alpha \nu k_{1}\left\{-2895^{\prime \prime} \cos N+\left(6658^{\prime \prime}-4^{\prime \prime} .26 t\right) \sin N+1815^{\prime \prime} \cos 2 N\right\}\right. \\
& + \text { constant; }
\end{aligned}
$$

the constants being so chosen that $\delta h$ and $\delta k$ shall vanish at the epoch.

Reducing the values of $\delta h$ and $\delta k$ to numbers, they become

$$
\begin{aligned}
& i h=5^{\prime \prime} .82 \sin N+\left(13^{\prime \prime} .40-0^{\prime \prime} .86 T\right) \cos N-3^{\prime \prime} .65 \sin 2 N+1^{\prime \prime} .08 T-2^{\prime \prime} .67, \\
& \delta l=5.82 \cos N-(13.40-0.86 T) \sin N-3.65 \cos 2 N
\end{aligned}
$$

the last two terms being arbitrary constants.

Computing the values of these terms of $\delta l, \delta h$, and $\delta l$, for intervals of 50 years, from 1600 to 2000 , we find them to be as follows:

\begin{tabular}{lrrr} 
Year & \multicolumn{1}{c}{$\delta l$} & \multicolumn{1}{c}{$\delta$} & \multicolumn{1}{c}{$\delta l$} \\
1600 & $-1^{\prime \prime} .34$ & $+0^{\prime \prime} .10$ & $-00^{\prime \prime} .02$ \\
1650 & -0.71 & +0.05 & -0.02 \\
1700 & -0.31 & +0.02 & -0.01 \\
1750 & -0.10 & 0.00 & -0.01 \\
1800 & -0.01 & 0.00 & 0.00 \\
1850 & 0.00 & 0.00 & 0.00 \\
1900 & 0.00 & 0.00 & 0.00 \\
1950 & +0.04 & 0.00 & -0.01 \\
2000 & +0.18 & -0.01 & -0.02
\end{tabular}

We see that although the ultimate effect of these terms is very considerable,

\begin{tabular}{|c|c|c|c|}
\hline$\pi$ & $168^{\circ}$ & $15^{\prime}$ & \\
\hline , & 28 & 25 & \\
\hline$\theta$, & 73 & 14 & \\
\hline$\phi$, & 0 & 46 & \\
\hline e, & .04 & 6923 & \\
\hline
\end{tabular}
their effect, during the period that Uranus has been observed, is insignificant.

\section{Concluded Elements and Perturbations of Uranus.}

The corrections found in the last chapter being applied to the final provisional elements (p. 99) give the following elements for 1850, affected by the great inequality produced by Neptune:

\section{Elements IV of Uranus.}

Epoch, 1850, Jan. 0, Greenwich mean noon. 


$$
\begin{array}{cc}
e \text { (in sec.) }, & 9678^{\prime \prime} .69 \\
n, & 15425.752 \\
\log a, & 1.2829072 \\
\log a_{1}, & 1.2831044
\end{array}
$$

$\log a_{1}$ includes, as before, the constant term in the perturbations of the logarithm of the radius vector which, with the corrected mass of Neptune, is +.0001972 .

'I'o find the corresponding elements at any other epoch, the following secular and long-period perturbations are to be applied. Those produced by Neptune are derived from the expressions in Chapter III by correcting them for the new mass of Neptune, and for the change in the value of the small divisor $2 n^{\prime}-n$ produced by the correction of the elements of Uranns. The logarithms of the factors for correction are,

\begin{tabular}{|c|c|}
\hline Correction of mass of Neptune & 9.93598 \\
\hline Correction of divisor & 0.00051 \\
\hline Log. factor for $\delta l$ & 9.93496 \\
\hline Log. factor for $\delta e, \delta \pi, \delta x$ & 9.93547 \\
\hline
\end{tabular}

Including the perturbations of the second order just found, we have, by putting

$$
\begin{aligned}
& N=2 l^{\prime}-y, \\
& =113^{\circ} 30^{\prime} 46^{\prime \prime} .0+8^{\circ} 26^{\prime} 51^{\prime \prime} .9 T \text {, } \\
& \varepsilon l=\left(-2850^{\prime \prime} .41+0^{\prime \prime} .32 T\right) \sin N+\left(38 \tau^{\prime \prime} .67-6^{\prime \prime} .37 T\right) \cos N \\
& +112.72 \sin 2 N-47.28 \cos 2 N \\
& -7.72 \sin 3 N+4.33 \cos 3 N \\
& +\quad 0.55 \sin 4 N \quad-\quad 0.46 \cos 4 N \\
& \text { - } 0.03 T^{2}-83^{\prime \prime} .78 T+2811^{\prime \prime} .41 \text {. } \\
& \delta /=-412^{\prime \prime} .18 \sin N+\left(14^{\prime \prime} .03-0^{\prime \prime} .86 T\right) \cos N \\
& +29.20 \sin 2 N-6.09 \cos 2 N \\
& \text { - } 3.11 \sin 3 N+1.19 \cos 3 N \\
& +\quad 0.28 \sin 4 N-0.13 \cos 4 N \\
& +14.76 \mathrm{~T}+398.33 \\
& \delta k=-411^{\prime \prime} .53 \cos N-\left(13^{\prime \prime} .65-0^{\prime \prime} .86 T\right) \sin N \\
& +29.33 \cos 2 N+6.17 \sin 2 N \\
& -\quad 3.12 \cos 3 N-1.21 \sin 3 N \\
& +\quad 0.29 \cos 4 N+\quad 0.13 \sin 4 N \\
& \text { - } 5.453 T-124.72
\end{aligned}
$$

$\delta x$ (in units of the 7 th place of decimals).

$$
\begin{gathered}
=1963 \cos N+103 \sin N \\
-\quad 171 \cos 2 N-67 \sin 2 N \\
+\quad 15 \cos 3 N+6 \sin 3 N \\
+511.0 .
\end{gathered}
$$

The perturbations of $e \delta \pi$ and $\delta e$ are here replaced by those of $\hbar$ and $k$, defined by the equations

$$
\begin{aligned}
& h=e \sin \left(\pi-\pi_{0}\right) \\
& k=e \cos \left(\pi-\pi_{0}\right)
\end{aligned}
$$


$\pi_{0}$ representing the perihelion of $1850=168^{\circ} 15^{\prime} 6^{\prime \prime} .7$. We then have for the eccentricity and longitude of perihelion at any epoch

$$
\begin{aligned}
& e \sin \left(\pi-\pi_{0}\right)=\delta \hbar \\
& e \cos \left(\pi-\pi_{0}\right)=e_{0}+\delta k .
\end{aligned}
$$

In the above terms multiplied by the time we have included the secular variations produced by Jupiter and Saturn. If the perturbations of the elements due to each particular planet are required, we have

$$
\begin{aligned}
& \text { Action of Jupiter, } \\
& \qquad \delta h=+5^{\prime \prime} .73 T ; \delta k=-0^{\prime \prime} .608 T .
\end{aligned}
$$

Action of Saturn,

$$
\imath h=+5^{\prime \prime} .56 T ; \delta k=-4.589 T .
$$

Subtracting these from the above expressions all the remaining terms will be due to the action of Neptune. The values of $\delta l$ and $\delta x$ are due entirely to the action of Neptune.

For the sake of rigor, we may suppose the perturbations produced by each planet to be multiplied by a factor representing the number by which the adopted mass of the planet must be multiplied to obtain the true mass.

It will add to the homogeneousness of the theory to express the perturbations of long period, which are multiplied by the product of the masses of Jupiter and Saturn, as perturbations of the elements. These terms, as found on page 88 , are

$$
\begin{aligned}
& \text { (v.c.0) }=-0^{\prime \prime} .55 \sin N_{6}-0^{\prime \prime} .03 \cos N_{6} \\
& +40.65 \sin N_{7}-10.50 \cos N_{7} \\
& (v . s .1)=+2.64 \sin N_{6}+4.64 \cos N_{6} \\
& +7.35 \sin N_{7}+4.41 \cos N_{7} \\
& \text { (v.c.1) }=-4.23 \sin N_{6}-3.87 \cos N_{6} \\
& +8.06 \sin N_{7}-8.38 \cos N_{7}
\end{aligned}
$$

These terms, together with the arbitrary corrections of the elements which have been applied to make them very small at the epoch, may be replaced by the following corrections to the elements:

$$
\begin{aligned}
\delta l= & -0^{\prime \prime} .55 \sin N_{6}-0^{\prime \prime} .03 \cos N_{6} \\
& +40.65 \sin N_{7}-10.50 \cos N_{7} \\
& +27^{\prime \prime} .27-11^{\prime \prime} .72 T . \\
\delta l= & +2.09 \sin N_{6}+1.94 \cos N_{6} \\
& \quad 2.13 \sin N_{7}+3.71 \cos N_{7} \\
& +1^{\prime \prime} .28 . \\
\delta k= & +1.32 \sin N_{6}+2.32 \cos N_{6} \\
& +3.68 \sin N_{7}+2.21 \cos N_{7}
\end{aligned}
$$

$$
\delta n=27 \sin N_{7}+104 \cos N_{7}+76 \text { (in units of the } 7 \text { th decimal). }
$$

The amount of the perturbations of the elements for every half century, from the year 1000 to 2200 , is given in the following table. Column(1) gives the perturbations by Neptune, Saturn, and Jupiter, computed from the expressions 
on page 182; column (2) those just given depending on the product of the masses of Jupiter and Saturn.

\begin{tabular}{|c|c|c|c|c|c|c|c|c|c|c|}
\hline Year. & \multicolumn{2}{|c|}{$\delta l$} & \multicolumn{2}{|c|}{$\delta h$} & \multicolumn{2}{|c|}{$\delta k$} & \multicolumn{2}{|c|}{$\delta x$} & $\delta x$ & $\delta \eta$ \\
\hline & $(1)_{11}$ & (2) & $(1)$ & (2) & $(1)^{\prime \prime}$ & (2) & (1) & (2) & & \\
\hline 1000 & +2050.31 & +160.69 & +42.66 & +1.94 & -389.51 & +6.08 & +1955 & -140 & -2.06 & +1.20 \\
\hline 1050 & 1841.17 & 149.08 & 27.79 & 3.60 & 375.65 & 6.68 & 1882 & 156 & 2.10 & 1.37 \\
\hline 1100 & 1638.76 & 136.39 & 14.03 & 4.99 & 360.31 & 6.82 & 1802 & 169 & 2.12 & 1.54 \\
\hline 50 & 1444.87 & 122.87 & $+\quad 1.45$ & 6 & 343.49 & 6.47 & 1717 & 178 & 2.08 & 1.66 \\
\hline & 1260.24 & 108.85 & - 9.90 & 6.60 & 325.27 & 5.64 & 1626 & 183 & 2.02 & 1.77 \\
\hline 1250 & +1085.76 & +94.67 & -19.84 & +6.75 & -305.69 & +4.42 & +1529 & +183 & -1.94 & +1.86 \\
\hline 1300 & 921.76 & 80.67 & 28.34 & 6.49 & 284.90 & 2.86 & 1426 & 180 & 1.84 & 1.91 \\
\hline 1350 & 769.32 & 67.12 & 35.27 & 5. & 252.90 & +1.16 & 13 & $1 \%$ & 1.70 & 1.92 \\
\hline 1. & 00 & 54.35 & 40.56 & 5. & 239.78 & -0.57 & 1205 & 161 & 1.54 & 1.91 \\
\hline 0 & 501.39 & 42.62 & 44.11 & 4.16 & 215.68 & 2.17 & 1086 & 147 & 1.37 & 1.85 \\
\hline 00 & 387.18 & +32.11 & 45.83 & +3.24 & -190.66 & -3.52 & +963 & +129 & -1.19 & +1.74 \\
\hline 1550 & 286.65 & 22.78 & 45.63 & 2.42 & 164.84 & 4.52 & 835 & 110 & 0.99 & 1.60 \\
\hline 1600 & 200.65 & 15.38 & 43.46 & 1.80 & 138.31 & 5.12 & 704 & 90 & 0.80 & 1.42 \\
\hline 1650 & 129.43 & 9.34 & 39.20 & 1.41 & 111.22 & 5.30 & 568 & 68 & 0.61 & 1.20 \\
\hline 17 & .46 & 4.86 & 32.79 & 1.23 & 83.70 & 5.09 & 430 & 48 & 0.44 & 0.95 \\
\hline 50 & 33.06 & +1.90 & 24.16 & +1.23 & -55.90 & -4.56 & +288 & +29 & -0.27 & +0.66 \\
\hline 1 & 8.51 & 0.33 & -13.26 & 1.36 & -27.93 & 3.81 & +145 & +11 & -0.12 & +0.34 \\
\hline & 0.00 & 0.00 & 0.00 & 1.52 & 0.00 & 2.96 & 0 & -5 & 0.00 & 0.00 \\
\hline 1900 & 7.64 & 0.71 & +15.65 & 1.63 & +27.74 & 2.10 & -145 & 18 & +0.10 & -0.36 \\
\hline 1950 & 31.47 & 2.23 & 33.70 & 1.59 & 55.09 & 1.34 & 290 & 26 & 0.16 & 0.73 \\
\hline 2000 & $+\quad 71.43$ & $+\quad 4.25$ & +54.20 & +1.34 & +81.81 & -0.73 & -433 & -32 & +0.20 & -1.10 \\
\hline & 127.33 & 6.52 & 77.12 & 0.85 & 107.77 & 0.34 & 574 & 30 & 0.20 & 1.47 \\
\hline & 198.86 & 8.75 & 102.46 & +0.13 & 132.67 & 0.14 & 711 & 27 & 0.17 & 1.83 \\
\hline 2150 & 285.72 & 10.59 & 130.15 & -0.77 & 156.33 & 0.11 & 843 & 20 & 0.11 & 2.17 \\
\hline 2200 & 387.11 & +11.77 & +160.15 & -1.76 & +178.48 & -0.19 & 968 & -9 & +0.02 & -2.48 \\
\hline
\end{tabular}

\section{Mean Elements of Uranus.}

If, instead of the elements of Uranus affected by the great inequality, we wish the absolute mean elements, these are to be obtained by adding to the elements already given the constants applied to the perturbations $\delta l, \delta \hbar, \delta k$, and $\delta x$ to makc the perturbations vanish at the epoch 1850.0, and also the corrections (p. 113) which we have subtracted from the elements and added to the perturbations to reduce the latter to a small quantity during the period for which the tables are likely to be used. We thus find the following mean elements :

Elements V of Uranus. Epoch, 1850, Jan. 0, Greenwich mean noon.

Longitude of the perihelion

Mean longitude at epoch,

Longitude of the node,

Inclination of the orbit,

Eccentricity,

Eccentricity in seconds,

Mean motion,

Log mean distance (uncorrected),

'The same corrected,

True mass of Neptune, $170^{\circ} 38^{\prime} 48^{\prime \prime} .7+8698^{\prime \prime} . \mu$

$291243.73+2811.4$ ॥

$731437.6+29.6 u$

$04620.92+0.38 \mu$

$.0463592-5236 \mu$

$9562^{\prime \prime} .27-108^{\prime \prime} .0 \mu$

$15424.797-0^{\prime \prime} .838 \mu$

$1.2829251+179 \mu$

$1.2831223+179 \mu$

$1+\mu$

19700 
Supposing the mass of Neptune to be uncertain by one-fiftieth of its entire amount, which is quite possible, it will be seen the longitude of the mean perihelion is from this cause uncertain by more than two minutes, the mean longitude of Uranus itself by nearly a minute, and the mean motion by nearly two seconds in a century.

It will be seen that the logarithm of the mean distance just given does not accurately correspond to that of elements IV plus the constant term of $\delta_{0} \times 0.4343$, as it should. This difference arises from the rejection of the terms of the second order in $\delta x$, which can not affect the geocentric longitude of the planet by a tenth of a second for a number of centuries.

It is to be remarked that these mean elements are those to be used in the general theory of the secular variation of the planetary orbits.

\section{Concluded Theory of Uranus.}

The elliptic longitude and radius vector of Uranus, affected by the secular and long period perturbations of the elements, will be given by the following equations. Put

$$
\begin{aligned}
& l_{0}=n_{0} t+\varepsilon_{0}, \\
& l=l_{0}+\imath l, \\
& g=l \quad-\pi_{0}, \\
& h=\delta h, \\
& l=e_{0}+\delta l, \\
& e^{2}=h^{2}+k^{2},
\end{aligned}
$$

the zeros indicating elements IV, and $i \hbar, \delta l$, and $\delta l$ being the perturbations of these three elements just given. Then

Elliptic longitude in orbit $=l$

$$
\begin{aligned}
& +\left\{2-\frac{1}{4} e^{2}+\frac{5}{96} e^{4}\right\}\{k \sin g-h \cos g\} \\
& +\left\{\frac{5}{4}-\frac{11}{24} e^{2}\right\}\left\{\left(k^{2}-h^{2}\right) \sin 2 \jmath-2 h k \cos 2 g\right\} \\
& +\left\{\frac{13}{12}-643 e^{2}\right\}\left\{\left(k^{3}-3 k^{2} k\right) \sin 3 g-\left(3 h k^{2}-h^{3}\right) \cos 3 g\right\} \\
& +\frac{103}{96}\left\{\left(k^{1}-6 k^{2} h^{2}+h^{1}\right) \sin 4 \jmath-\left(4 k^{3} h-4 k h^{3}\right) \cos 4 \jmath\right\} \\
& +\frac{1097}{960}\left\{\left(k^{3}-10 k^{3} h^{2}+5 k h^{1}\right) \sin 5 g-\left(5 k^{1} h-10 k^{2} h^{3}+k^{5} \cos 5 g\right\}\right.
\end{aligned}
$$

Neperian logarithm of $r=n+\frac{1}{4} e^{2}+\frac{1}{32} e^{4}$

$$
\begin{aligned}
& -\left\{1-\frac{3}{8} e^{2}\right\}\{l \cdot \cos g+h \sin g\} \\
& -\left\{\frac{3}{4}-\frac{11}{24} e^{2}\right\}\left\{\left(k^{2}-h^{2}\right) \cos 2 g+2 h k \sin 2 g\right\}
\end{aligned}
$$




$$
\begin{aligned}
& -\frac{17}{24}\left\{\left(h^{3}-3 h \cdot h^{2}\right) \cos 3 g+\left(3 h^{2} h-h^{3}\right) \sin 3 g\right\} \\
& -\frac{71}{96}\left\{\left(\hbar^{4}-6 h^{2} h^{2}+h^{4}\right) \cos 4 g+\left(4 l^{3} h-47 h^{3}\right) \sin 4 g\right\}
\end{aligned}
$$

In computing these expressions it will be sufficient for several centuries before or after 1850 to develop $h, \delta l$, and $i l$ to their first dimensions: it will, however, be more convenient to correct the mean anomaly $g$ for the perturbation $\delta l$ before obtaining the equation of the centre. Developing the perturbations of $h$ and $k$ to terms of the first order, we have for the effects of the pcrturbations of those elements:

$$
\begin{aligned}
& (v .8 .1)=\left(2-\frac{3}{4} e^{2}\right) \delta z^{2} \\
& \text { (v.c.1) }=-\left(2-\frac{1}{4} e_{0}^{2}\right) i \hbar \\
& \text { (v.s. } 2)=\left(\begin{array}{l}
5 \\
2^{e_{0}}-\frac{11}{6} e_{0}^{3}
\end{array}\right) \delta k \\
& \text { (v.c. } 2)=-\left(\frac{5}{2^{e_{0}}}-\frac{11}{24} e_{0}^{3}\right) \delta \hbar \\
& (v . s .3)=\frac{13}{4} e_{0}^{2} \delta \% \\
& (v . c .3)=-\frac{13}{4} e_{0}^{2} \delta \hbar \\
& (v . s .4)=\frac{103}{24} e_{0}^{3} \delta k \\
& (v . c .4)=-\frac{103}{24} e_{0}{ }^{3} i \hbar \\
& (\rho . c .0)=\delta x+\frac{1}{2} e_{0} \delta z \\
& (\rho .8 .1)=-\left(1-\frac{3}{8} e^{2}\right) \delta / \\
& (\rho . c .1)=-\left(1-\frac{9}{8} e^{2}\right) \delta k^{2} \\
& \left(\text { p.s.2) }=-\frac{3}{2}^{e_{0} i \hbar}\right. \\
& \text { (р.c.2) }=-\frac{3}{2} e_{0} \delta z \\
& (\rho . s .3)=-\frac{17}{8} e_{0}^{2} \delta \hbar \\
& \left(\text { p.c.3) }=-\frac{17}{8} e_{0}^{2} \delta \%\right.
\end{aligned}
$$

These coefficients for $\rho$ must, of course, be multiplied by the modulus 0.434294 to reduce the perturbations to those of the common logarithm of the radius vector. 
Among the elliptic terms may be included the effect of the following minute constants introduced by the perturbations.

$$
\begin{aligned}
(v . s .2) & =-0^{\prime \prime} .144 \\
(v . c .2) & =+0.130 \\
0.4343(\rho . c .0) & =+1972 \text { in units of the } 7 \text { th place } \\
0.4343(\rho . s .1) & =+63 \quad \text { of decimals. } \\
0.4343(\rho . c .1) & =+73 \\
0.4343(\rho . s .2) & =+5 \\
0.4343(\rho . c .2) & =+4
\end{aligned}
$$

This term (p.c.0) is that added as a correction to the logarithm of the mean distance.

To the coefficients $(v . s .1),(v . c .1)$, etc., are still to be added the following periodic terms:-

1. The periodic terms due to the action of Jupiter, given in Chapter $\mathrm{V}$, omitting the terms multiplied by $T$, which are included in the perturbations of the elements.

2. The periodic terms produced by Saturn, including those terms multiplied both by $T$ and by $\sin A_{2}$ or $\cos A_{2}$, but omitting those multiplied by $T$ only for the same reason as in the case of Jupiter.

3. The periodic terms produced by Neptune, multiplied by the factor 0.86294 on account of the correction to the mass of that planet, and omitting the terms multiplied by $\delta l, \delta e$, and $e \delta g$.

4. The periodic terms multiplied by the product of the masses of Jupiter and Saturn, given on page 88 , omitting the terms multiplied by the sine and cosine of $N_{6}$ and $N_{7}$, because they are replaced by the terms of $\delta l, \delta h$, and $\delta k$, given on page 183 , and tabulated in the columns headed (2) on page 184. The result will be the same whether we employ the terms of $(v . c .0),(v . s .1)$, etc., given at the bottom of page 88 and the top of page 89 , omitting the numbers in the columns 2 on page 184 from the expressions on page 186, or whether we include the latter and omit the former.

The true anomaly of Uranus will then be:

$g_{0}+\delta l+$ (equation of centre from elements IV, using for mean anomaly $g_{0}+\delta l$ )

$$
+\Sigma(v . s . i) \sin i g+\Sigma(v . c . i) \cos i g .
$$

The logarithm of the radius vector will be:

$$
\begin{aligned}
& \log r \text { in elliptic orbit from elements IV. } \\
& \quad+\Sigma(\rho .8 . i) \sin i g+\Sigma(\rho . c . i) \cos i g
\end{aligned}
$$

care being taken to multiply the coefficients by the modulus where that has not already been done. All the terms in Chapter $\mathrm{V}$ are so multiplied.

To pass from the true anomaly to the true longitude we must investigate the secular motion of the planes of the orbit and of the ecliptic. The effect of this motion on $\phi, \theta$, and $\tau$ will be found by successive approximations from the formulæ 
(34), correcting the data for the new mass of Neptune. We shall also use the same motion of the ecliptic adopted on p. 95. We have thus:

$$
\begin{aligned}
& \frac{d p}{d t}=-4^{\prime \prime} .53 \\
& \frac{d p^{\prime}}{d t}=+5.43+0^{\prime \prime} .38 T . \\
& \frac{d q}{d t}=-5.17 \\
& \frac{d q^{\prime}}{d t}=-46.78+0.12 T .
\end{aligned}
$$

As a first approximation we liave

$$
\begin{aligned}
& \theta=\tau=73^{\circ} 14^{\prime} \quad 8^{\prime \prime} \quad-3169^{\prime \prime} .2 T \\
& \phi \quad=\quad \begin{array}{llll}
0 & 46 & 20.54+2.48 T
\end{array}
\end{aligned}
$$

Substituting these values in (34) and integrating we find

$$
\begin{aligned}
& \phi=\phi_{0}+2^{\prime \prime} .47 T+0^{\prime \prime} .13 T^{2} \\
& \theta=\theta_{0}-3168.42 T+3.00 T^{2} \\
& \tau=\tau_{0}-3168.76 T+3.00 T^{2}
\end{aligned}
$$

For tabulating we shall use, instead of $\theta$ and $\tau$, the distance of the perihelion from the ascending node, or $\pi-\tau$, and the value of $\theta$ corrected for Struve's precession. Since the mean motion has been derived without making any distinction between $\tau$ and $\theta$, it will be necessary to correct the motion of mean anomaly by the difference of those quantities. We thus obtain for the values of the three principal arguments :

$$
\begin{aligned}
& g=220^{\circ} 10^{\prime} 10^{\prime \prime} .35+1542574^{\prime \prime} .86 T+\delta \tau \\
& \omega=95 \quad 058.70+3168.76 T-3.00 T^{2} \\
& \theta=73148.00+1856.82 T+4.12 T^{2}
\end{aligned}
$$

If we represent all the inequalities of the true longitude by $\Delta l$, so that we shall have for the true anomaly

the argument of latitude will be

$$
f=g+\Delta l
$$

$$
u=f+\omega \text {. }
$$

The reduction to the ecliptic will then be

$$
R=-\left(9^{\prime \prime} .37+0^{\prime \prime} .016 T\right) \sin 2 u,
$$

the true longitude on the ecliptic referred to the mean equinox of date,

and the sine of the elliptic latitude,

$$
\lambda=\imath+\theta+R
$$

$$
\sin \beta_{0}=\sin \phi_{0} \sin u \text {. }
$$

The perturbations of the latitude will be

$$
(\text { b.c. } 0)+(\text { b.c. } 1) \cos g+(\text { b.s.1) } \sin g+\text { etc. }
$$


The periodic terms of (b.c.0), (b.s.1), (b.c.1), etc., are given in Chapter $\mathrm{V}$, on pages 86 and 87 , and are to be taken without any farther modification than the multiplication of those due to the action of Neptune by the factor 0.863 . The constant, secular; and long period terms are

$$
\begin{aligned}
& \text { b.c. } 0=+0^{\prime \prime} .26-0^{\prime \prime} .12 T-0.011 \delta \eta+0.046 \delta x \\
& (b . s .1)=-0.22 T-0.05 T^{2}+0.975 \delta \eta+0.221 \delta x \\
& (b . c .1)=+2.47 T+0.12 T^{2}+0.221 \delta \eta-0.975 \delta x \\
& (b . s .2)=-0.06-0.01 T+0.046 \delta \eta+0.011 \delta x \\
& (b . c .2)=-0.01+0.12 T+0.011 \delta \eta-0.046 \delta x
\end{aligned}
$$

The values of $\delta \eta$ and $\delta x$ to be used in these expressions are those the expressions for which are given on page 97 , and which are tabulated in the last two columns of the table on page 184 .

The following tables are based on the elements and theory laid down in this chapter. 


\section{CHAPTER IX.}

GENERAL TABLES OF URANUS.

Enumeration of the Quantities contained in the several Tables.

THE first six tables are designed to give the values of the three arguments of the elliptic motion, $g, \omega$, and $\theta$, and of the ninc arguments of the tables of perturbations. 'The argument $\omega$ is, however, diminished by 3 ', the sums of the constants added to the perturbations of (v.c.0) to make these quantities positive, and $\theta$ by $10^{\prime \prime}$, the constant added to the reduction to ecliptic. The expressions for the arguments of perturbations are as follows, the mean longitude of each planet, counted from the perihelion of Uranus, being represented by the initial letter of the planet. All thesc arguments are expressed in units, of which 600 make an entire circumference, so that each unit is $36^{\prime}$. The time $t$ is counted in Julian ycars from the fundamental epoch,

1850, January 0, Green wich mean noon.

$$
\begin{array}{rlr}
\operatorname{Arg} .1 & =J-U & =219.190+43.44028 t \\
2 & =S-U & =577.349+13.22717 t \\
3 & =U-N & =88.884+3.50035 t \\
4 & =J-2 S & =497.6+9.8445 t \\
5 & =3 S-U-J=79.8+3.3825 t \\
6 & =4 S-2 U-J=57.1+16.610 t \\
7 & =2 J-3 S-3 U=238.7+18.633 t \\
8 & =2 J-4 S-2 U=261.3+5.4058 t \\
9 & =7 S-2 J-3 U=136.9+19.992 t
\end{array}
$$

Table I gives the corrections which must be applied to the values of the arguments at any time during the nineteenth century to reduce them to the corresponding time in any preceding or following century between the Cliristian era and the year 2300. Since $\omega$ and $\theta$ each contains a term proportional to the square of the time, the correction for these quantities is not constant during each century, but is of the form

$$
\omega+\omega^{\prime} T
$$

$\omega$ and $\omega$ ' being constant during each century, and $T$ being the fraction of the century counted from its beginning.

Table II gives the value of $g, \omega-3^{\prime}, \theta-10^{\prime \prime}$, and the above nine arguments for Greenwich mean noon of Jan. 0 of each leap year from 1752 to 1948 , and for January -1 of the years 1800 and 1900 , corresponding to December 30 of the years 1799 and 1899. The corrections for the perturbations of long period are not 
applied in this table. The numbers at the bottom of this table, in the line $\Delta_{i 20}^{(1)}$, show the variation of the corresponding quantity in 120 days, for the epoch 1850.0 . In the line "Factor $T$ " is given the change of this variation in a century, while $\Delta_{120}^{(2)}$ is the second difference for intervals of 120 days. By means of these numbers, when the arguments are computed for any date, their values for other dates at intervals of 120 days may be found by successive addition.

Table III gives the motion of the several arguments between the epochs of the preceding table and the zero day of each month in the course of a four-year cycle. The variable motions, $\omega$ and $\theta$, correspond to the epoch 1850 , and rigorously they each require a correction for any other four-year cycle than that between 1848 and 1852. But, owing to the small inclination of the orbit of Uranus it is not necessary that either $\omega$ or $\theta$ should be exact, if only their sum is exact. The column $\theta$ of this table, therefore, gives the correction which must be applied to the motion of $\theta$ at the end of a century (1950) in order that, being applied to $\theta$ alone, $\omega+\theta$ may be exact. This correction is, in fact, that for the secular variation of the precession.

Tables IV and V give the motion of the arguments for days and hours. The motion for hours is, however, not necessary in the case of any argument but $g$, as all the others can be readily enough interpolated to fractions of a day.

Table VI gives the corrections to the arguments on account of the terms of long period from 1000 to 2200 . The terms in question are, in the case of Jupiter, the great inequality produced by the action of Saturn, in the case of Neptune the great inequality produced by Uranus, and, in the case of Uranus, the inequalities in the mean longitude tabulated in the preceding chapter. The numerical expressions are

$$
\begin{aligned}
& \delta J=0.535 \sin \left(116^{\circ} 21^{\prime}+40^{\circ} 45^{\prime} 20^{\prime \prime} T\right) \\
& \delta U=\imath l \\
& \delta N=-0.75 \imath l .
\end{aligned}
$$

The corrections to the several arguments are

$$
\begin{aligned}
& \delta g=\imath l \\
& \delta \text { arg. } 1=\delta J-\imath l \\
& \delta \text { arg. } 2=-i l \\
& \delta \arg .3=\quad \delta l-\delta N=1.75 \delta l
\end{aligned}
$$

No correction to the mean longitude of Saturn is applied, all its inequalities being taken account of in the terms of the second order.

The corrections, expressed in seconds, have been reduced to units of the argument by dividing them by $2160^{\prime \prime}$.

Outside the limits of the table these corrections must be computed from their formulæ.

Table VII gives the equation of the centre, and the elliptic part of the logarithm of the radius vector. No constant is applied to the former, but the latter is diminished by .0003400 , the sum of the constants added to (p.c.0) in Tables VIII, IX, $\mathrm{X}$ and $\mathrm{XVII}$. 
The formula for the Tables are

$$
\begin{aligned}
\text { Equation of centre }= & 19352^{\prime \prime} .06 \sin \\
& +567.24 \sin \\
& +\quad 23.05 \sin 3 g \\
& +\quad 1.07 \sin 4 g \\
& +\quad 0.05 \sin 5 g \\
\text { Elliptic log. } v= & 1.2833435 \\
& -.0003400 \\
& -.0203618 \cos g \\
& -.0007165 \cos 2 g \\
& -.0000318 \cos 3 g \\
& -.0000016 \cos 4 g .
\end{aligned}
$$

Table VIII gives the cocfficients (v.c.0), (v.c.1), etc., for the perturbations of the longitude and logarithm of radius vector produced by the action of Jupiter. 'They are computed from the periodic terms of the formule on page 8:3, with the addition of the following constants to make all the numbers of the table positive:

$$
\begin{aligned}
& \text { Constant of }(v . c .0)=55^{\prime \prime} \text {. } \\
& (v . s .1)=6 \text {. } \\
& (v . c .1)=4 \text {. } \\
& (v . s 2)=0.20 \\
& \text { (v.c.2) }=0.20 \\
& \text { (p.c.0) }=1200 \\
& (\rho . s .1)=150 \\
& (\text { p.c.1) }=100 \\
& (\rho . s .2)=10 \\
& (\text { p.c.2) }=10
\end{aligned}
$$

Table IX gives the periodic part of the coefficients due to the action of Saturn, taken without change from the expressions on page 84, together with the secular variations, the latter including only the terms of (v.s.1), (v.c.1), (v.s.2), and (v.c.2), which are multiplied by $T$ and by $\sin A_{2}$ or $\cos A_{2}$. The coefficients of $T$ are given in the columns Sec. Var. and each number is increased by the constant $1^{\prime \prime} .50$ to make it positive. 'The term $-0^{\prime \prime} .06 T \sin A_{2}$ in (v.c.0) is omitted entirely, as it will not amount to a tenth of a second until after the year 2000. The constant terms added to the quantities of these tables to make all the numbers positive, are:

$$
\begin{aligned}
& \text { Constant of }(v . c .0)=30 . \\
&(v . s .1)=150 .+1.50 T \\
&(v . c .1)=150 .+1.50 T \\
&(v . s .2)=130 .+1.50 T \\
&(v . c .2)=130 .+1.50 T \\
&(v . s .3)=8 . \\
&(v . c .3)=6 . \\
&(v . s .4)=1 . \\
&(v . c .4)=1 .
\end{aligned}
$$




$$
\begin{array}{r}
\text { Constant of }(\rho . c .0)=800 \\
(\rho . s .1)=1500 \\
(\rho . c .1)=1500 \\
(\rho .8 .2)=400 \\
(\rho . c .2)=400 \\
(\rho . s .3)=100 \\
(\rho . c .3)=100
\end{array}
$$

Table $\mathrm{X}$ gives the coefficients produced by the action of Neptune, computed from the periodic terms on pages 85 and 86 without any other change than the multiplication of all the numbers by the factor 0.863 to reduce them to the new mass of Neptune. The constants added to the several quantities, are

$$
\begin{aligned}
& \text { Constant of }(v . c .0)=92.85 \\
& (v . s .1)=20.00 \\
& (\text { v.c.1) }=\quad 31.00 \\
& (v .8 .2)=5.00 \\
& (\text { c.c.2) }=\quad 5.00 \\
& (v . s .3)=1.00 \\
& (\text { v.c.3 })=1.00 \\
& (v .8 .4)=-1.00 \\
& (\text { v.c.4) }=-1.00 \\
& \text { Constant of }(\rho . c .0)=400 \\
& (\text { p.s.1) }=200 \\
& (\text { p.c.1) }=200 \\
& (\rho .8 .2)=\quad 40 \\
& (\rho . c .2)=\quad 40
\end{aligned}
$$

Tables XI to XVI give the terms of the second order and of short period which contain the products of the masses of Jupiter and Saturn, which, with the constants added to the numbers of the several tables, are as follows:

$$
\begin{aligned}
& \text { (v.c.0 })=+0^{\prime \prime} .03 \sin A_{5}+0^{\prime \prime} .51 \cos A_{5} ; \text { Table XII; const }=0^{\prime \prime} .60 \\
& +0.04 \sin A_{6}+0.01 \cos A_{6} ; \quad \text { XIII; } \quad=0.05 \\
& -0.01 \sin A_{7}+0.05 \cos A_{7} ; \quad \text { XIV; } \quad=0.05
\end{aligned}
$$

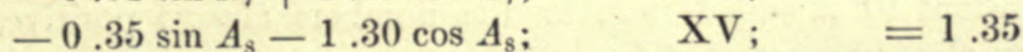

$$
\begin{aligned}
& -0.05 \sin A_{0}+0.03 \cos A_{9} ; \quad \mathrm{XVI} ; \quad=0.10 \\
& \text { Sum of constants added to these tables } \quad 2.15
\end{aligned}
$$

$$
\begin{aligned}
& \text { (v.8.1) }=+0^{\prime \prime} .26 \sin A_{1}+0^{\prime \prime} .27 \cos A_{1} ; \quad \text { Table XI; const }=0^{\prime \prime} .40 \\
& -0.04 \sin A_{5}-0.17 \cos \cdot A_{5} ; \quad \mathrm{XII} ; \quad=0.20 \\
& +0.08 \sin A_{6}+0.03 \cos A_{6} ; \quad \text { XIII; } \quad=0.10 \\
& -0.02 \sin A_{7}+0.08 \cos A_{7} ; \quad \text { XIV; } \quad=0.10 \\
& +0.30 \sin A_{8}-0.58 \cos A_{8} ; \quad \text { XV; } \quad 0.75 \\
& -0.04 \sin A_{9} \text {; } \quad \text { XVI; } \quad 0.10
\end{aligned}
$$

25 Juns, 1873. 


$$
\begin{aligned}
& \text { (v.c.1) }=+0^{\prime \prime} .06 \sin A_{4}-0^{\prime \prime} .27 \cos A_{4} ; \quad \text { Table XI; const }=0^{\prime \prime} .40 \\
& +0.18 \sin A_{5}+0.01 \cos A_{j} ; \quad \mathrm{XII} ; \quad 0.20 \\
& -0.03 \sin A_{6}+0.08 \cos A_{6} ; \quad \text { XIII; } \quad 0.10 \\
& -0.02 \sin A_{7}+0.09 \cos A_{7} ; \quad \mathrm{XIV} ; \quad 0.10 \\
& -0.44 \sin A_{8}-0.61 \cos A_{8} ; \quad X V ; \quad 0.75 \\
& -0.10 \sin A_{9}+0.03 \cos A_{9} ; \quad \mathrm{XVI} ; \quad 0.10
\end{aligned}
$$

'The term of $(\rho . c .0)$

$$
11 \sin A_{s}-3 \cos A_{\mathrm{s}}
$$

\begin{tabular}{|c|c|c|c|}
\hline 01 & Pert. Const. & Tables VIII to XVI. & $\begin{array}{c}(1)-(2) \\
-177^{\prime \prime} 65-1^{\prime \prime} 50 T\end{array}$ \\
\hline$(v . c .1)$ & 0 & $186.65+1.50 \mathrm{~T}$ & $-186.65-1.50 \mathrm{~T}$ \\
\hline$(v . s .2)$ & $-0^{\prime \prime} .14$ & $135.20+1.50 T$ & $-135.34-1.50 T$ \\
\hline$(v . c .2)$ & +0.13 & $135.20+1.50 T$ & $-135.07-1.50 T$ \\
\hline$(v .8 .3)$ & 0 & 9.00 & $-\quad 9.00$ \\
\hline (v.c.3) & 0 & 7.00 & 7.00 \\
\hline$($ p.c.0) & {$[+1972]$} & -1000 & +1000 \\
\hline ( $(. s .1)$ & $\begin{array}{l}+\quad 63 \\
\end{array}$ & 1850 & -1787 \\
\hline (p.c.1) & +73 & 1800 & -1727 \\
\hline$(\rho . s .2)$ & + & 450 & -445 \\
\hline$(\rho . c .2)$ & + & 450 & -446 \\
\hline
\end{tabular}

is omitted from the tables entirely.

Tables XVII $a$ and XVII $b$ give the coisstant, secular, and long-period terms of (v.s.1) (v.c.1), computed from the formulæ p. 186, with the following additions:

1. The constant terms introduced by the perturbations, given on p. 187.

2. 'The negatives of the constants added to the tables VII to XVI inclusive to make the numbers of those tables positive. The values of these terms are

The perturbation constant of ( $\rho . c .0$ ), being added to $\log a$ in forming the elliptic radius vector, is not included in this table.

Table XVIII gives the reduction to the ecliptic

$$
-9^{\prime \prime} .37 \sin 2 u \text {. }
$$

The constant $10^{\prime \prime}$ is added to make the numbers always positive, which constant has been already subtracted from $\theta$.

Table XIX gives the principal term of the latitude

$$
46^{\prime} 20^{\prime \prime} .54 \times \sin u \text {. }
$$

Table XX gives the coefficients $(b . s .1)$ and $(b . c .1)$ for the perturbations of the latitude produced by Jupiter. They are given by the formula

$$
\begin{aligned}
& (\text { b.s.1 })=0^{\prime \prime} .65 \cos \left(J-U+40^{\circ}\right) \\
& (\text { b.c.1 })=0.65 \sin \left(J-U+40^{\circ}\right)
\end{aligned}
$$

The constant $0^{\prime \prime} .70$ is added to make all the numbers of the table positive. 
Table XXI gives the corresponding coefficients for the action of Saturn, computed from the expressions on p. 87 with the addition of the following constants.

$$
\begin{array}{r}
\text { Const. of }(b . c .0)=0 " .10 \\
(b . s .1)=3.30 \\
(b . c .1)=3.10 \\
(b . s .2)=0.20 \\
(b . c .2)=0.20
\end{array}
$$

Table XXII gives the coefficients for the action of Neptune from the formulæ on p. 87 , all the numbers being multiplied by the factor 0.863 to reduce them to the adopted mass of Neptune. The following constants are added:

$\begin{array}{rrr}\text { To }(b . c .0) & \ldots & 0^{\prime \prime} .06 \\ (\text { b.s.1) } & & 1.00 \\ (\text { b.c.1) } & & 1.20 \\ (b . s .2) & & 0.20 \\ (\text { b.c.2) } & & 0.20\end{array}$

Table XXIII gives the secular and long-period terms for various epochs computed from the formulæ of p. 189. The sums of the several constants added in the three preceding tables are here subtracted again so that these expressions become

$$
\begin{gathered}
\text { b.c. } 0-\Sigma c=00^{\prime \prime} .10-0^{\prime \prime} .12 T-.011 \delta \eta+.046 \delta x \\
(b . s .1)-\Sigma c=-5.00-0.22 T-0^{\prime \prime} .05 T^{2}+.975 \delta \eta+.221 \delta x \\
(b . c .1)-\Sigma c=-5.00+2.47 T+0.12 T^{2}+.221 \delta \eta-.975 \delta x \\
(b . s .2)-\Sigma c=-0.46-0.01 T+.046 \delta \eta+.011 \delta x \\
(b . c .2)-\Sigma c=-0.41+0.12 T+.011 \delta \eta-.046 \delta x \\
\text { Precepts for the use of the Tables. }
\end{gathered}
$$

Express the date for which the position of Uranus is required in years, months, days, and hours of Greenwich mean time, according to the Julian Calendar if the date is earlier than 1500, according to the Gregorian Calendar if it is later than 1600 , and according to either calendar between these epochs.

Enter Table I with the beginning of the century, and take out the values of $g$, $\omega, \omega^{\prime}, \theta, \theta^{\prime}$, and arguments 1 to 9 . Multiply $\omega^{\prime}$ and $\theta^{\prime}$ by the fraction of a century corresponding to the date, and write the products with their proper algebraic signs under $\omega$ and $\theta$. If the calendar is the Julian, the century marked $J$ must be taken, and if the Gregorian, that marked $G$. Between the dates 1752 and 1951 it is not necessary to enter Table I at all.

If Table I was not entered, enter Table II with the year, or the first preceding year found therein. If Table I was entered, enter Table II between the year 1800 and 1896 as if the number of the century were changed to 18 . Take out the values of $g, \omega, \theta$, and the arguments, and write them under the corresponding quantities from Table I.

Enter Table III with the excess of the actual year over that with which Table II was entered, and with the month. Write the corresponding values of $g, \omega, \theta$, and the arguments under the previous values. Multiply $\theta^{\prime}$ by the fraction of a 
cen ury after 1850 , corresponding to the date with which Table II was entered, and write the product under $\theta$, or add it to it in writing $\theta$. If Table 11 was entered with a date before 1850 , this product is negative.

Enter 'Table IV with the day of the month and write down the corresponding values of $g, \omega$, etc., under the former values.

If the date does not correspond to Greenwich mean noon, the motion of $g$ for the hours must be computed from Table $\mathrm{V}$, and the other quantities must be interpolated to the fraction of a day in entering Table IV.

Enter Table VI with the year, find by interpolation the values of $g$, and arguments 1,2, and 3, corresponding to the date, and write them under the former values.

Add up all the partial values of $g, \omega, \theta$, and the arguments, attending to the algebraic signs of the products. Subtract from the arguments as many times 600 as possible, and the results will be the final values of those quantities.

Enter Table VII with $g$ as the argument, the seconds being first reduced to fractions of a minute, and interpolate the quantities $E$ and $\log r$. When $g$ exceeds $180^{\circ}$ the former quantity is to receive the negative sign; the latter is always positive.

Enter 'Tables VIII to XVI inclusive with their respective arguments, and take out the values of the quantities (v.c.0), (v.s.1), (v.c.1), etc., (.$c .0),(\rho . s .1)$, etc., so far as they are found in the tables, writing the quantities having the same designation under each other. In 'Table IX the quantities Sec. Var. must be multiplied by the centuries and fraction of a century of the actnal date after 1850, and the product must be included with the corresponding quantities, (v.s.1), (v.c.1), etc. Before 1850 this product will always be negative; afterward always positive. All the quantities taken from these tables are positive except (v.s.4) and (v.c.4) in Table 1X, which are negative.

Add up all the partial values of (v.c.0), (v.s.1), etc., thus obtained from Tables VIII to XVI, and from their sum take the corresponding quantities obtained from 'Table XVII by interpolating to the date. 'The required quantities are all given in 'Table XVII $b$; 'Table XVII $a$ being only an expansion of a part of XVII $b$ for the present century. The final values of $(v . s .1),(v . c .1),(v . s .2)$, etc., (.$s .1),(\rho . c .1)$, etc., thus obtained are to be multiplied by the sines and cosines of the corresponding multiples of $g$, in doing which four place logarithms are sufficient if the computation is carefully made. The products are then all added together, and to $\eta, \omega, E$, and $(v . c .0)$; in the case of $v$, and to $\log . r,(\rho . c .0)$ in the case of $\rho$. That is, we are to form the expressions:

$$
\begin{aligned}
u=g+\omega+E+(v . c .0) & +(v . s .1) \sin g+(v . c .1) \cos g \\
& +(v . s .2) \sin 2 J+(v . c .2) \cos 2 g \\
& + \text { etc. }+ \text { etc. } \\
\log r= & \log r(\text { from Table VII })+(\rho . c .0) \\
& +(\rho . s .1) \sin g)+(\rho . c .1) \cos g \\
& +(\rho . s .2) \sin 2 g)+(\rho . c .2) \cos 2 g \\
& +(\rho . s .3) \sin 3 g)+(\rho . c .3) \cos 3 g .
\end{aligned}
$$


" will then be the true argument of latitude, and $\log r$ the logarithm of the radius rector with seven places of decimals.

Under $u$ write 0 ; cuter Iuble XVIII with the argument $u$ and take out the reduction to the ccliptic. Add it to $u$ and 0 , and the sum of the three quantities will be the heliocentric longitude of Uranus referred to the mean equinox and ecliptic of the date. Applying nutation the longitude will be reduced to the true equinox.

linter Table XIX with $u$ as the argument, or, when $u$ exceeds $180^{\circ}$, with $u-180^{\circ}$, and take out the principal term of the latitude, which will be positive when $u$ is less than $180^{\circ}$, and negative when it is greater.

Enter lables XX, XXI, XXII, and XXIII with their respectire arguments, the argument for the last being the date, and add up the rarious quantities having the same designation, noticing that in the first three tables all the quunntities are positive, while in the last they are all negative except (b.c.0). Then form the expression,

$$
\text { (b.c.0) }+(\text { b.8.1) } \sin g+(\text { b.c. } 1) \cos g+(\text { (b.8.2) } \sin 2 \jmath+(\text { b.c. } 2) \cos 2 g,
$$

and add it to the principal term of the latitude, with regard to the algebraic signs. The sum will be the heliocentric latitude of Uranus above the ecliptic of the date.

When an ephemeris of Uranus is to be computed for a series of years, some modifications may be introduced, which will save the computer labor. In the first place an equidistant series of dates being selected for computation, it will be suffi cient to compute $g, \omega, 0$, and the arguments for every sixth, eighth, or tenth date, and to fill in the arguments for the intermediate dates by adding the nearly constant differences corresponding to the adopted intervals. The agreement of the uumbers thus obtained for the last date with those found by the original computation will prove the whole process. This interval may be as great as 120 days withont detracting from the accuracy with which the places for the immediate dates can be interpolated, and the differences for this interval may be deduced from the numbers at the bottom of Table II. If these numbers are used without change the values of $\omega$ and 0 for the last date may not always come out right. But these crrors, if less than a second, will be of no importance if the one quantity comes out as much too great as the other is too small, and they may be avoided entirely by making a small change in the constant difference to be added.

Tables XI to XVI, inchusive, need be entered only for every third or fourth date, and the sums of the quantities can be then interpolated to every date, and added up with the corresponding quantities from the other tables.

Again, it will be found convenient to compute the sum of the small terms $(v .8 .3) \sin 3 g+(v . c .3) \cos 3 g+(v .8 .4) \sin 4 g+(v . c .4) \cos 4 g$, as well as the corre sponding terms of the radius vector, and all the terms of the latitude, not for the dates adopted, but for every fourth entire degree of $g$. Haring a series of values computed in this way, the sum can be interpolated to the value of $g$ corresponding to the date. 'To facilitate the formation of the smaller products for entire deyrees of $g$, a table of products of numbers by the sine and cosine of every degree is appended to these tables, by which the products in question can be formed at sight 
whenever the coefficient to be multiplied is less than $32^{\prime \prime}$. 'The values of these coefficients, $(v . s .3),(v . c .3)$, etc., corresponding to the entire degrees of $y$, may be either formed by interpolation at sight from those corresponding to the dates of computation, or the ralues of the argunents 2 and 3 corresponding to the required degrees of $g$ may be computed, and the values of (v.s.3), ctc., corresponding to these values of the arguments may be taken from ' lables IX and $\mathrm{X}$, while Table XVII must be entered with the corresponding dates.

If the heliocentric ephemeris is computed for ten years at a time, the last of these modifications in the mode of computation will greatly facilitate the computation of the smaller terms. We first find the date, and the values of arguments 1 , 2 , and 3 , to one place of decimals, for some entire degree of $g$ preceding that which corresponds to the first date, and then find the dates and the values of the arguments corresponding to successive values of $g$, differing by $2^{\circ}$ or $4^{\circ}$, until we pass the last date of computation. We then take out the values of (v.s.3), (v.c.3), (v.s.4), (v.c.4), $(\rho . s .3),(\rho . c .3),(b . c .0),(b . s .1),(b . c .1),(b . s .2)$, and (b.c.2), with these values of the dates and arguments, form their products by the sines and cosines of the corresponding multiples of $g$ by means of the supplementary tables, and arld the proper products together so as to form three small tables with $g$ as the argument. 'These terms are then interpolated to the values of $g$ corresponding to the original dates of computation.

As a first cxample of the use of the Tables we will compute the heliocentric co-ordinates of Uranus for Greenwich mean noon of the date 1753, Dec. 3 . In computing the arguments we shall make use of 'lable $I$, though it is not necessary to do so. 'The computation of the arguments is as follows:

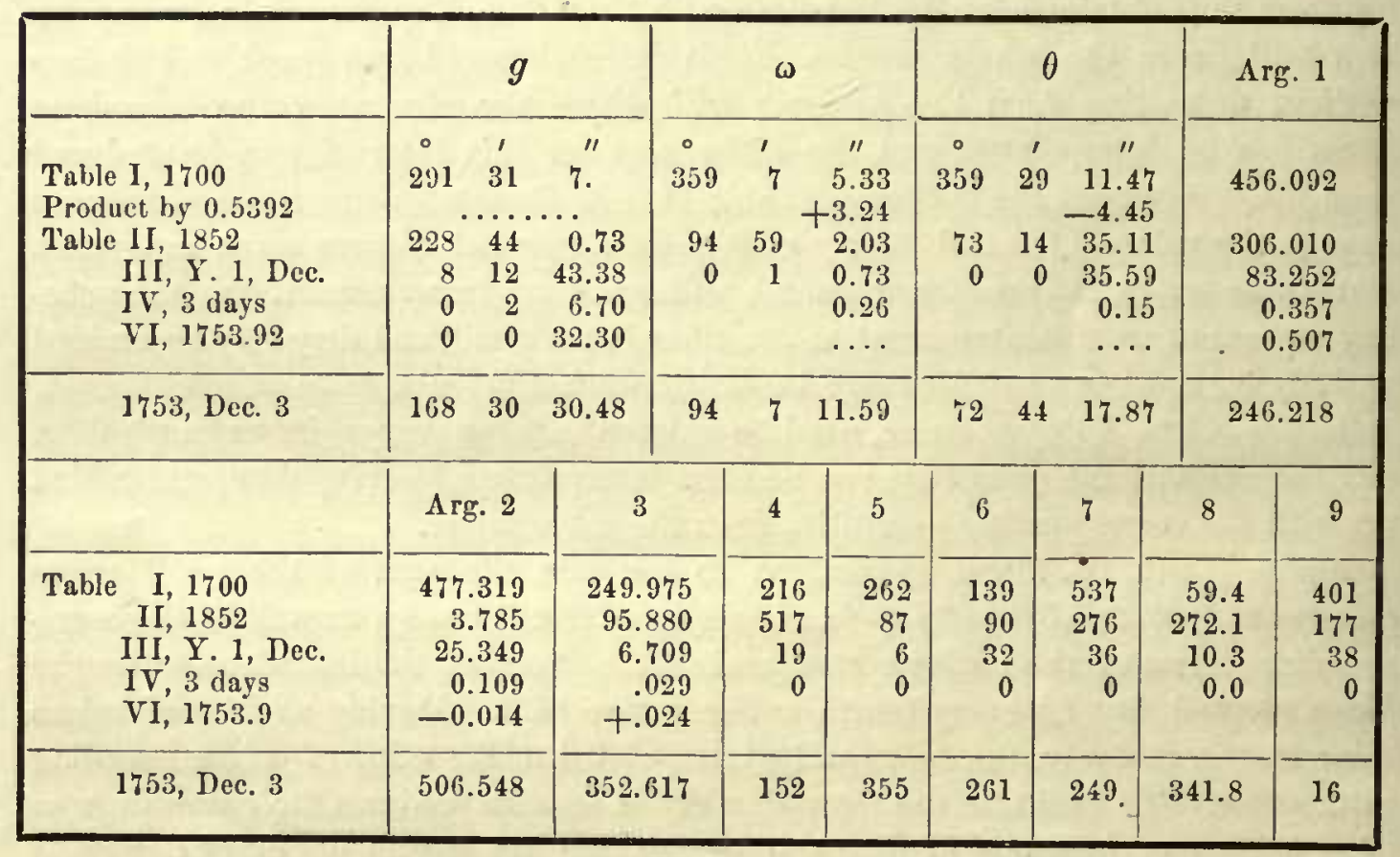




\begin{tabular}{|c|c|c|c|c|c|c|c|c|c|c|}
\hline & & $\begin{array}{c}(v, c .0) \\
\text { " }\end{array}$ & $\begin{array}{c}(v, s, 1) \\
" \prime\end{array}$ & $\begin{array}{c}\text { (c.c.1) } \\
" \prime\end{array}$ & $(v .8 .2)$ & $(v, c .2)$ & $\begin{array}{l}(v .8 .3) \\
\prime \prime\end{array}$ & $(v . c .3)$ & $(v .8 .4)$ & $(v, c .4)$ \\
\hline \multirow[t]{9}{*}{ Table } & VIII & 83.58 & 9.97 & 6.61 & 0.37 & 0.31 & & & & \\
\hline & IX & 21.32 & 22.24 & 223.49 & 60.92 & 238.89 & 1.42 & 11.07 & 0.32 & 1.64 \\
\hline & $\mathrm{x}$ & 71.08 & 19.02 & 18.30 & 6.64 & 5.38 & 0.70 & 1.19 & -0.95 & -1.04 \\
\hline & $\mathrm{XI}$ & .. & .13 & .47 & & & & & & \\
\hline & XII & .13 & .36 & .10 & - & & & & & \\
\hline & XIII & .06 & 11 & .01 & & & & & & \\
\hline & XIV & .01 & .04 & .03 & & & & & & \\
\hline & XV & 2.38 & 1.40 & 1.12 & & & & & & \\
\hline & XVI & .12 & .09 & .11 & & & & & & \\
\hline \multirow{4}{*}{$\underset{\text { Table }}{\Sigma}$} & & 178.68 & 53.36 & 250.24 & 67.93 & 244.58 & 2.12 & 12.26 & & \\
\hline & XVII & & -292.55 & -140.91 & -140.73 & -131.03 & -9.41 & -6.85 & & \\
\hline & & & -239.19 & +109.35 & -72.80 & +113.55 & -7.29 & +5.41 & -0.63 & +0.60 \\
\hline & & $(\rho . c .0)$ & ( $\rho$. & $(p .8 .1)$ & (p.c.1) & $(p .8 .2)$ & & (p.c.2) & $(p .8 .3)$ & (p.c.3) \\
\hline \multirow[t]{6}{*}{ Table } & VIII & 251 & & 188 & 32 & 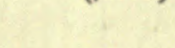 & & & & \\
\hline & IX & 1086 & & 741 & 360 & 621 & & 597 & 154 & 111 \\
\hline & $\mathrm{x}$ & 326 & & 259 & 222 & 21 & & 42 & & \\
\hline & & 1663 & & 1188 & 614 & 642 & & 639 & 154 & 111 \\
\hline & XVII & 1104 & & -1321 & -500 & -412 & & -360 & -98 & -94 \\
\hline & & 2767 & - & 133 & +114 & +230 & & +279 & +56 & +17 \\
\hline
\end{tabular}

$\begin{array}{lllll}\log (v .8 .1)-2.3787 & \log (v . c .1)+2.0388 & \log (v . s .2)-1.8621 & \log (v . c .2+2.0552 \\ \log \sin +9.2994 & \log \cos g-9.9912 & \log \sin 2 g-9.5916 & \log \cos 2 g+9.9641 \\ \log (\text { p.s.1)-2.124 } & \log (\text { o.c.1) + 2.057 } & \log (\text { p.s.2) + 2.362 } & \log (\text { p.c.2)+2.446 }\end{array}$

\begin{tabular}{|c|c|c|c|c|c|c|c|c|}
\hline & ○ & " & & & (b.8.1) & (b.c.1) & (b.8.2) & (b.c.2) \\
\hline$g$ & 168 & $\begin{array}{ll}30 & 30.48\end{array}$ & Table & $\mathrm{XX}$ & $" .06$ & $0^{\prime \prime} .62$ & & \\
\hline$\omega$ & 94 & $7 \quad 11.59$ & & XXI & 3.91 & 5.88 & 0.41 & 0.17 \\
\hline E & 1 & $\begin{array}{ll}0 & 46.21\end{array}$ & & XII & .84 & 1.36 & 0.18 & 0.17 \\
\hline$(v . c .0)$ & & 258.68 & & & 4.81 & 7.86 & 0.59 & 0.34 \\
\hline$(v . s .1) \sin g$ & & -47.66 & $\mathrm{XX}$ & KIII & -4.28 & -6.88 & -0.43 & -0.51 \\
\hline (v.c.1) $\cos g$ & & $-1 \quad 47.15$ & & & +0.53 & +0.98 & +0.16 & -0.17 \\
\hline$(v .8 .2) \sin 2 g$ & & 28.43 & $\%$ & & & & & \\
\hline (v.c.2) $\cos 2 g$ & & 144.55 & & & & & & \\
\hline$(v .8 .3) \sin 3 g$ & & -4.13 & & $\log r$ & & & & \\
\hline (v.c.3) $\cos 3 g$ & & -4.46 & Table VII & 023222 & $\beta_{0}$ & Table XIX & $-0 \quad 46^{\prime}$ & 3."73 \\
\hline$(v .8 .4) \sin 4 g$ & & 0.47 & (p.c.0) & 2767 & & (b.c.0) Tabl & le XXI & 0.16 \\
\hline (v.c.4) $\cos 4 g$ & & 0.41 & $(p .8 .1) \sin g$ & -26 & & & XXII & 0.09 \\
\hline$u$ & 263 & $40 \quad 57.42$ & (p.c.1) $\cos g$ & -112 & & & XXIII & 0.20 \\
\hline$\theta$ & 72 & $44 \quad 17.87$ & $(\rho . s .2) \sin 2 g$ & -90 & & $(b .8 .1) \sin g$ & & +0.11 \\
\hline I & & 7.95 & $($ (p.c.2) $\cos 2 g$ & +257 & & (b.c.1) $\cos g$ & & -0.96 \\
\hline ong. mean Eq & .336 & $25 \quad 23.24$ & $(\mu .8 .3) \sin 3 g$ & +31 & & $($ b.8.2) $\sin 2 g$ & & -0.06 \\
\hline Nutation & & +6.51 & (p.c.3) $\cos 3 g$ & -14 & & (b.c.2) $\cos 2 g$ & & -0.16 \\
\hline Long. true $\mathrm{E}$ & 36 & $25 \quad 29.75$ & $\log r$ & 326035 & & Latitude & $-0 \quad 46$ & 4.35 \\
\hline
\end{tabular}

As a second example we will take the computation of an ephemeris for the years 1876 and 1877. We take as the extreme dates 1875 , December 15, and 1878, April 3 , between which are seven intervals of 120 days each, which we adopt as those of computation. We first form the arguments for the extreme dates as follows: 


\begin{tabular}{|c|c|c|c|c|c|c|c|c|c|}
\hline & \multicolumn{2}{|c|}{$g$} & \multicolumn{3}{|c|}{$\omega$} & \multicolumn{2}{|r|}{$\theta$} & \multicolumn{2}{|c|}{ Arg. 1} \\
\hline $\begin{array}{l}\text { Table 1I, } 1872 \\
\text { III, } 3 \text { Y. Dec. } \\
\text { IV, } 15 \text { days } \\
\text { VI, } 1875.96\end{array}$ & $\begin{array}{rr}0 & 1 \\
314 & 25 \\
16 & 46 \\
0 & 10\end{array}$ & $\begin{array}{c}\prime \prime \\
55.70 \\
33.70 \\
33.50 \\
2.20\end{array}$ & $\begin{array}{r}\circ \\
95 \\
0\end{array}$ & $\begin{array}{r}1 \\
9 \\
2 \\
\ldots\end{array}$ & $\begin{array}{c}\prime \prime \\
35.64 \\
4.06 \\
1.30 \\
\ldots\end{array}$ & $\begin{array}{c}\circ \\
73 \\
0 \\
\\
\end{array}$ & 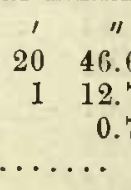 & $\begin{array}{r}57 \\
17 \\
+\end{array}$ & $\begin{array}{l}.815 \\
.072 \\
.784 \\
.426\end{array}$ \\
\hline For 1875 , Dec. 15 & $331 \quad 23$ & 5.16 & 95 & 11 & 41.00 & 73 & 22 & & .097 \\
\hline & Arg. 2 & 3 & & 4 & 5 & 6 & 7 & 8 & 9 \\
\hline $\begin{array}{l}\text { Table II, } 1872 \\
\text { III, 3 Y. Dcc. } \\
\text { IV, } 15 \text { days } \\
\text { VI, } 1875.96\end{array}$ & $\begin{array}{r}268.328 \\
51.785 \\
0.543 \\
-\quad 1\end{array}$ & $\begin{array}{r}165.88 \\
13.70 \\
0.14 \\
+\end{array}$ & & $\begin{array}{r}114 \\
39 \\
0\end{array}$ & $\begin{array}{r}154 \\
13 \\
0\end{array}$ & $\begin{array}{r}423 \\
65 \\
1\end{array}$ & $\begin{array}{r}49 \\
73 \\
1\end{array}$ & $\begin{array}{r}380.3 \\
21.1 \\
0.2\end{array}$ & $\begin{array}{r}577 \\
78 \\
1\end{array}$ \\
\hline For 1875, Dec. 15 & 320.655 & 179.73 & & 153 & 167 & 489 & 123 & 401.6 & 56 \\
\hline
\end{tabular}

\begin{tabular}{|c|c|c|c|c|c|c|c|c|c|}
\hline - & \multicolumn{2}{|c|}{$g$} & \multicolumn{3}{|c|}{$\omega$} & \multicolumn{2}{|r|}{$\theta$} & \multicolumn{2}{|c|}{ Arg. 1} \\
\hline $\begin{array}{l}\text { Table II, } 1876 \\
\text { III, } 2 \text { Y. April } \\
\text { IV, 3d } \\
\text { VI, } 1878.26\end{array}$ & $\begin{array}{rr}\circ & \text { ' } \\
331 & 34 \\
9 & 37 \\
& 2\end{array}$ & $\begin{array}{c}\prime \prime \\
18.70 \\
53.62 \\
6.70 \\
2.62\end{array}$ & $\begin{array}{l}\circ \\
95 \\
0\end{array}$ & $\begin{array}{r}1 \\
11 \\
1 \\
\ldots\end{array}$ & $\begin{array}{l}1 " \\
42.33 \\
11.23 \\
0.26 \\
\therefore\end{array}$ & $\begin{array}{c}0 \\
73 \\
0 \\
.\end{array}$ & $\begin{array}{rr}1 & \\
22 & 1 . \\
0 & 41 . \\
& 0 . \\
\ldots . . . & \end{array}$ & & $\begin{array}{l}.576 \\
.643 \\
.357 \\
.421\end{array}$ \\
\hline \multirow[t]{2}{*}{ For $1878, \Lambda$ pril 3} & $341 \quad 14$ & 21.64 & 95 & 12 & 53.82 & 73 & 2242. & & .997 \\
\hline & Arg. 2 & \multicolumn{2}{|l|}{3} & 4 & 5 & 6 & 7 & 8 & 9 \\
\hline $\begin{array}{l}\text { Table II, } 1876 \\
\text { III, 2 Y. April } \\
\text { IV, 3d } \\
\text { VI, } 1878.26\end{array}$ & $\begin{array}{r}321.237 \\
29.732 \\
.109 \\
-.001\end{array}$ & \multicolumn{2}{|c|}{$\begin{array}{r}179.889 \\
7.868 \\
+\quad .029 \\
+\quad .002\end{array}$} & $\begin{array}{r}153 \\
22 \\
0\end{array}$ & $\begin{array}{r}168 \\
7 \\
0\end{array}$ & $\begin{array}{r}489 \\
37 \\
0\end{array}$ & $\begin{array}{r}123 \\
42 \\
0\end{array}$ & $\begin{array}{r}401.9 \\
12.1 \\
0\end{array}$ & $\begin{array}{r}57 \\
45 \\
0\end{array}$ \\
\hline For 1878 , April 3 & 351.077 & \multicolumn{2}{|c|}{187.788} & 175 & 175 & 526 & 165 & 414.0 & 102 \\
\hline
\end{tabular}

We now fill in the values of $g$, the arguments $1-9$, and the times with which Table XVII is to be entered, for the intermediate dates, by adding the nearly constant differences deduced from the numbers at the bottom of Table II. The seconds of $g$ are first reduced to fractions of a minute, with which to enter Table VII. In making the subsequent computation we have used none of the devices previously described except in the case of the small longitude terms, as follows: 


\begin{tabular}{lrrrrrrr}
$g$ & $\circ$ & $\circ$ & $\circ$ & $\circ$ & $\circ$ & $\circ$ & $\circ$ \\
$3 g$ & 230 & 332 & 334 & 336 & 338 & 340 & 342 \\
$4 g$ & 240 & 276 & 282 & 288 & 294 & 300 & 306 \\
& $\prime \prime$ & $\prime \prime$ & 256 & 264 & 272 & 280 & 288 \\
$(v .8 .3)$ & -1.14 & -1.12 & -1.13 & -1.16 & -1.23 & -1.33 & -1.48 \\
$(v . c .3)$ & -2.46 & -2.56 & -2.66 & -2.76 & -2.88 & -3.01 & -3.16 \\
$(v . s .4)$ & +0.59 & +0.60 & +0.59 & +0.55 & +0.50 & +0.43 & +0.36 \\
$(v . c .4)$ & -0.10 & -0.20 & -0.30 & -0.40 & -0.47 & -0.57 & -0.64 \\
$(v .8 .3) \sin 3 g$ & +1.14 & +1.12 & +1.11 & +1.11 & +1.12 & +1.15 & +1.20 \\
$(v . c .3) \cos 3 g$ & .00 & -0.26 & -0.55 & -0.85 & -1.17 & -1.50 & -1.86 \\
$(v . s .4) \sin 4 g$ & -.51 & -.56 & -.57 & -.55 & -.50 & -.42 & -.34 \\
$(v . c .4) \cos 4 g$ & +.05 & +.07 & +.07 & +.04 & -.02 & -.10 & -.19 \\
\hline $\operatorname{Sum}$ & +0.68 & +0.37 & +0.06 & -0.25 & -0.57 & -0.87 & -1.19
\end{tabular}

It will be seen that we have here computed twice as many numbers as are necessary to interpolate with all attainable accuracy.

The rest of the computation is fully given on the four following pages. First we have the values of $g$ and the nine arguments for the intermediate dates, filled in by successive addition of the nearly constant difference. The arguments thus obtained for the last date may be compared with those just computed on the preceding page.

The numerals in the first columns of the sections of computation following indicate the arguments with which tables are entered to obtain the separate values of the quantities (v.c.0), (v.s.1), (v.c.1), etc. The negative terms in Table XVII being taken from the sum of all the periodic terms from Tables VIII to XVI with argument 1 to 9 , we have the final values of (v.c.0), (v.s.1), etc.

The final computation of the products $(v .8 . i) \sin i g$, etc., and the addition of the separate terms which make up the three co-ordinates, are shown on page 205 . The expressions $c .0,8.1$, etc., are employed for brevity, instead of $(v . c .0),(v . s .1) \sin g$, etc.

The longitude finally given by the tables is referred to the mean equinox, and must therefore be corrected for nutation before being used to compute the geocentric place. 


\begin{tabular}{|c|c|c|c|c|c|c|c|c|}
\hline Date, \{ & $\begin{array}{c}\text { 1875, Dec. } 15 \\
1875.955 .\end{array}$ & $\begin{array}{c}1876, \text { Apr. } 13 \\
1876.253 .\end{array}$ & $\begin{array}{c}\text { Ang. 11 } \\
1876.612 .\end{array}$ & $\begin{array}{l}\text { Dec. } 9 \\
1876.940 .\end{array}$ & $\begin{array}{c}1877, \text { Apr. } 8 \\
1877.269 .\end{array}$ & $\begin{array}{c}\text { Aug. } 6 \\
1877.597 .\end{array}$ & $\begin{array}{l}\text { Deo. } 4 \\
1877.925 .\end{array}$ & $\begin{array}{c}1878, \text { Apr. }^{3} \\
1878.254 .\end{array}$ \\
\hline & $\mid \begin{array}{cc}\circ & \prime \\
331 & 23.086\end{array}$ & $\mid \begin{array}{cc}\circ & \prime \\
332 & 47.554\end{array}$ & $\mid \begin{array}{cc}\circ & \prime \\
334 & 12.021\end{array}$ & $\begin{array}{cc}\circ & \prime \\
335 & 36.489\end{array}$ & $\begin{array}{cc}\circ & \prime \\
337 & 0.957\end{array}$ & $\left|\begin{array}{cc}\circ & \prime \\
338 & 25.425\end{array}\right|$ & $\mid \begin{array}{cc}\circ & \prime \\
339 & 49.893\end{array}$ & $\begin{array}{cc}\circ & \prime \\
341 & 14.361\end{array}$ \\
\hline $\begin{array}{r}\text { Arg. } 1 \\
2 \\
3 \\
4 \\
5 \\
6 \\
7 \\
8 \\
9 \\
\end{array}$ & $\begin{array}{c}147.097 \\
320.655 \\
179.739 \\
153 . \\
167 . \\
489 . \\
123 . \\
401.6 \\
56 . \\
\end{array}$ & $\begin{array}{l}161.368 \\
325.001 \\
180.889 \\
156 . \\
\ldots 3 . \\
494 . \\
129 . \\
403.4 \\
63 . \\
\end{array}$ & $\begin{array}{c}175.639 \\
329.346 \\
182.038 \\
159 . \\
\ldots \ldots . \\
500 . \\
135 . \\
405.2 \\
69 . \\
\end{array}$ & $\begin{array}{l}189.910 \\
333.692 \\
183.188 \\
163 . \\
\ldots \ldots . \\
505 . \\
141 . \\
407.0 \\
75 . \\
\end{array}$ & $\begin{array}{c}204.182 \\
338.038 \\
184.338 \\
166 . \\
\ldots \ldots . \\
511 . \\
147 . \\
408.7 \\
82 . \\
\end{array}$ & $\begin{array}{l}218.453 \\
342.383 \\
185.488 \\
169 . \\
\ldots \ldots . \\
516 . \\
153 . \\
410.5 \\
88 . \\
\end{array}$ & $\begin{array}{l}232.724 \\
346.729 \\
186.638 \\
172 . \\
\ldots \ldots \ldots \\
521 . \\
160 . \\
412.3 \\
95 . \\
\end{array}$ & $\begin{array}{l}246.995 \\
351.074 \\
187.788 \\
175 . \\
175 . \\
527 . \\
166 . \\
414.1 \\
101 . \\
\end{array}$ \\
\hline $\begin{array}{r}(v . c .0) \\
1 \\
2 \\
3 \\
5 \\
\mathbf{5} \\
\mathbf{6} \\
7 \\
8 \\
9\end{array}$ & $\begin{array}{r}108.01 \\
15.90 \\
72.43 \\
.59 \\
.02 \\
.07 \\
1.68 \\
.09 \\
\end{array}$ & $\begin{array}{r}107.74 \\
14.71 \\
73.60 \\
.58 \\
.02 \\
.07 \\
1.65 \\
.09 \\
\end{array}$ & $\begin{array}{r}106.29 \\
13.66 \\
74.77 \\
.58 \\
.02 \\
.06 \\
1.63 \\
.09 \\
\end{array}$ & $\begin{array}{r}103.68 \\
12.65 \\
75.94 \\
.57 \\
.03 \\
.06 \\
1.60 \\
.09 \\
\end{array}$ & $\begin{array}{r}99.98 \\
11.68 \\
77.10 \\
.56 \\
.03 \\
.06 \\
1.58 \\
.08 \\
\end{array}$ & $\begin{array}{r}95.25 \\
10.76 \\
78.26 \\
.56 \\
.03 \\
.06 \\
1.55 \\
.08 \\
\end{array}$ & $\begin{array}{r}89.62 \\
9.90 \\
79.42 \\
.55 \\
.03 \\
.05 \\
1.53 \\
.08 \\
\end{array}$ & $\begin{array}{r}83.21 \\
9.09 \\
80.56 \\
.54 \\
.03 \\
.05 \\
1.50 \\
.07 \\
\end{array}$ \\
\hline$(v . c .0)$ & 198.69 & 198.46 & 197.10 & 194.62 & 191.07 & 186.55 & 181.18 & 175.05 \\
\hline 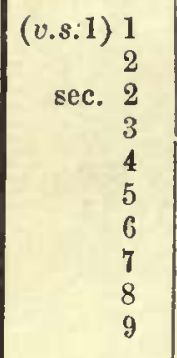 & $\begin{array}{r}6.84 \\
132.20 \\
.18 \\
1.09 \\
.13 \\
.19 \\
.04 \\
.14 \\
1.28 \\
.09 \\
\end{array}$ & $\begin{array}{r}7.68 \\
125.49 \\
.20 \\
1.07 \\
.12 \\
.19 \\
.04 \\
.14 \\
1.28 \\
.08\end{array}$ & $\begin{array}{r}8.40 \\
118.82 \\
.22 \\
1.08 \\
.11 \\
.19 \\
.04 \\
.13 \\
1.27 \\
.08 \\
\end{array}$ & $\begin{array}{r}8.99 \\
112.21 \\
.23 \\
1.12 \\
.10 \\
.19 \\
.05 \\
.13 \\
1.26 \\
.08\end{array}$ & $\begin{array}{r}9.44 \\
105.67 \\
.25 \\
1.19 \\
.10 \\
.19 \\
.05 \\
.12 \\
1.26 \\
.07 \\
\end{array}$ & $\begin{array}{r}9.74 \\
99.24 \\
.27 \\
1.29 \\
.09 \\
.20 \\
.06 \\
.12 \\
1.25 \\
.07 \\
\end{array}$ & $\begin{array}{r}9.91 \\
92.89 \\
.29 \\
1.41 \\
.09 \\
.20 \\
.06 \\
.11 \\
1.24 \\
.07 \\
\end{array}$ & $\begin{array}{r}9.98 \\
86.69 \\
.31 \\
1.56 \\
.08 \\
.20 \\
.07 \\
.10 \\
1.23 \\
.07 \\
\end{array}$ \\
\hline$\stackrel{\Sigma}{\mathbf{\Sigma}}$ & $\begin{array}{r}142.17 \\
-154.21 \\
\end{array}$ & $\begin{array}{r}136.28 \\
-153.84 \\
\end{array}$ & $\begin{array}{r}130.34 \\
-153.47 \\
\end{array}$ & $\begin{array}{r}124.36 \\
-153.10 \\
\end{array}$ & $\begin{array}{r}118.34 \\
-152.73 \\
\end{array}$ & $\begin{array}{r}112.33 \\
-152.36 \\
\end{array}$ & $\begin{array}{r}106.27 \\
-151.99 \\
\end{array}$ & $\begin{array}{r}100.29 \\
-151.62 \\
\end{array}$ \\
\hline$(v . s .1)$ & -12.04 & -17.56 & -23.13 & -28.74 & -34.39 & -40.03 & -45.72 & -51.33 \\
\hline $\begin{array}{r}\text { (v.c.1) } 1 \\
2 \\
\text { sec. } 2 \\
3 \\
4 \\
5 \\
6 \\
7 \\
8 \\
9\end{array}$ & $\begin{array}{r}5.64 \\
5.01 \\
.69 \\
23.20 \\
.47 \\
.37 \\
.16 \\
.15 \\
0.66 \\
.07 \\
\end{array}$ & $\begin{array}{r}6.03 \\
600 \\
.71 \\
24.09 \\
.48 \\
.37 \\
.17 \\
.14 \\
0.64 \\
.07 \\
\end{array}$ & $\begin{array}{r}6.38 \\
7.31 \\
.72 \\
24.95 \\
.49 \\
.37 \\
.17 \\
.13 \\
0.63 \\
.06 \\
\end{array}$ & $\begin{array}{r}6.67 \\
8.90 \\
.74 \\
25.82 \\
.50 \\
.37 \\
.18 \\
.13 \\
0.61 \\
.06 \\
\end{array}$ & $\begin{array}{r}6.85 \\
10.80 \\
.75 \\
26.68 \\
.50 \\
.37 \\
.18 \\
.12 \\
0.60 \\
.05 \\
\end{array}$ & $\begin{array}{r}6.91 \\
13.00 \\
.77 \\
27.53 \\
.51 \\
.37 \\
.18 \\
.12 \\
0.59 \\
.04 \\
\end{array}$ & $\begin{array}{r}6.82 \\
15.49 \\
.79 \\
28.37 \\
.52 \\
.37 \\
.18 \\
.11 \\
0.57 \\
.04 \\
\end{array}$ & $\begin{array}{r}6.59 \\
18.25 \\
.80 \\
29.19 \\
.52 \\
.37 \\
.18 \\
.10 \\
0.56 \\
.03 \\
\end{array}$ \\
\hline$\stackrel{\Sigma}{\operatorname{Tab.XVII}}$ & $\begin{array}{r}36.42 \\
-205.86 \\
\end{array}$ & $\begin{array}{r}38.70 \\
-206.08 \\
\end{array}$ & $\begin{array}{r}41.21 \\
-206.29 \\
\end{array}$ & $\begin{array}{r}43.98 \\
-206.51 \\
\end{array}$ & $\begin{array}{r}46.90 \\
-206.73 \\
\end{array}$ & $\begin{array}{r}50.02 \\
-206.94 \\
\end{array}$ & $\begin{array}{r}53.26 \\
-207.15 \\
\end{array}$ & $\begin{array}{r}56.59 \\
-207.37 \\
\end{array}$ \\
\hline$(v . c .1)$ & -169.44 & -167.38 & -165.08 & -162.53 & -159.83 & -156.92 & -153.89 & -150.78 \\
\hline $\begin{array}{rr}(v . s .2) & 1 \\
2 \\
\text { sec. } & 2 \\
3\end{array}$ & $\begin{array}{r}.06 \\
54.12 \\
.39 \\
2.05 \\
\end{array}$ & $\begin{array}{r}.10 \\
49.37 \\
.42 \\
2.19 \\
\end{array}$ & $\begin{array}{r}.15 \\
44.78 \\
.44 \\
2.31 \\
\end{array}$ & $\begin{array}{r}.21 \\
40.37 \\
.47 \\
2.45 \\
\end{array}$ & $\begin{array}{r}.26 \\
36.15 \\
.49 \\
2.60 \\
\end{array}$ & $\begin{array}{r}.31 \\
32.11 \\
.51 \\
2.76\end{array}$ & $\begin{array}{r}.34 \\
28.30 \\
.53 \\
2.91 \\
\end{array}$ & $\begin{array}{r}.37 \\
24.70 \\
.56 \\
3.07 \\
\end{array}$ \\
\hline \multirow{2}{*}{$\begin{array}{l}{ }^{\Sigma} \\
\text { Tab.XVII } \\
(v . s .2)\end{array}$} & $\begin{array}{r}56.62 \\
-134.34 \\
\end{array}$ & $\begin{array}{r}52.08 \\
-134.32 \\
\end{array}$ & $\begin{array}{r}47.68 \\
-134.31 \\
\end{array}$ & $\begin{array}{r}43.50 \\
-134.29 \\
\end{array}$ & $\begin{array}{r}39.50 \\
-134.27 \\
\end{array}$ & $\begin{array}{r}35.69 \\
-134.26 \\
\end{array}$ & $\begin{array}{r}32.08 \\
-134.24 \\
\end{array}$ & $\begin{array}{r}28.70 \\
-134.22 \\
\end{array}$ \\
\hline & -77.72 & -82.24 & -86.63 & -90.79 & -94.77 & -98.57 & -102.16 & -105.52 \\
\hline
\end{tabular}




\begin{tabular}{|c|c|c|c|c|c|c|c|c|}
\hline Date, $\{1$ & $\begin{array}{c}\text { 1875, Deo. } 15 \\
1875.955 .\end{array}$ & $\begin{array}{c}1876, A \text { pr. } 13 \\
1876.283 .\end{array}$ & $\begin{array}{c}\text { Aug. 11 } \\
1876.612 .\end{array}$ & $\begin{array}{c}\text { Dee. } 9 \\
1867.940 .\end{array}$ & $\begin{array}{c}1877, \text { Apr. } 8 \\
1877.269 .\end{array}$ & $\begin{array}{c}\text { Ang. } 6 \\
1877.597 .\end{array}$ & $\begin{array}{c}\text { Dee. } 4 \\
1877.925 .\end{array}$ & $\begin{array}{c}\text { 1878, Apr. } 3 \\
1878.254 .\end{array}$ \\
\hline \multirow{9}{*}{ 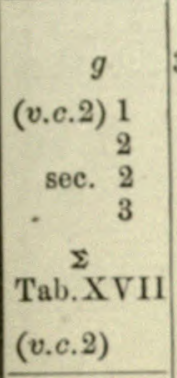 } & $\begin{array}{cc}\circ & 1 \\
221 & 02086\end{array}$ & $\begin{array}{ll}0 & 1 \\
020 & 2\end{array}$ & - ' & ० 1 & 0 & - 1 & - ' & $\circ \quad 1$ \\
\hline & 33123.086 & $332 \quad 47.554$ & $334 \quad 12.021$ & $\begin{array}{ll}335 & 36.489\end{array}$ & $337 \quad 0.057$ & $338 \quad 25.425$ & $5 \quad 339 \quad 49.893$ & $341 \quad 14.361$ \\
\hline & .31 & .30 & .29 & .29 & .29 & .30 & .30 & .31 \\
\hline & 25.34 & 28.93 & 32.71 & 36.73 & 40.93 & 45.35 & 49.93 & 54.69 \\
\hline & $\begin{array}{r}.76 \\
.99\end{array}$ & .76 & .77 & .78 & .79 & .79 & .80 & .80 \\
\hline & 6.23 & 6.40 & $\begin{array}{l}. \\
\end{array}$ & 6.70 & 6.85 & 6.99 & 7.10 & 7.20 \\
\hline & 32.64 & $\begin{array}{r}36.39 \\
\end{array}$ & 40.33 & 44.50 & 48.86 & 53.43 & 58.13 & 63.00 \\
\hline & -136.57 & -136.59 & -136.60 & -136.62 & -136.64 & -136.65 & -136.67 & -136.69 \\
\hline & -103.93 & -100.20 & -96.27 & -92.12 & -87.78 & - 83.22 & -78.54 & -73.69 \\
\hline \multirow{5}{*}{$\begin{array}{l}(v . s .3) \frac{2}{3} \\
\text { Tab.XVII } \\
(v . s .3)\end{array}$} & 6.92 & 6.89 & 6.84 & 6.78 & 6.70 & 6.61 & 6.51 & 6.37 \\
\hline & 0.87 & 0.91 & 0.94 & 0.98 & 1.01 & 1.04 & 1.08 & 1.11 \\
\hline & 7.79 & 7.80 & 7.78 & 7.76 & 7.71 & 7.65 & 7.59 & 7.48 \\
\hline & -8.92 & -8.92 & -8.91 & -8.91 & -8.91 & -8.91 & -8.91 & -8.91 \\
\hline & -1.13 & -1.12 & -1.13 & -1.15 & -1.20 & -1.26 & -1.32 & -1.43 \\
\hline \multirow{5}{*}{$\begin{array}{l}(v . c .3) \frac{2}{3} \\
\text { Tab.X XII } \\
(v . c .3)\end{array}$} & 3.19 & 3.12 & 3.05 & 2.98 & 2.90 & 2.82 & 2.75 & 2.67 \\
\hline & 1.34 & 1.34 & 1.84 & 1.34 & 1.33 & 1.33 & 1.31 & 1.29 \\
\hline & 4.53 & 4.46 & 4.39 & 4.32 & 4.23 & 4.15 & 4.06 & 3. \\
\hline & -7.06 & -7.06 & -7.06 & -7.06 & -7.06 & -7.06 & -7.06 & -7.06 \\
\hline & -2.53 & -2.60 & -2.67 & -2.74 & -2.83 & -2.91 & -3.00 & -3.10 \\
\hline \multirow{3}{*}{$\begin{array}{l}(v .8 .4)^{2} \\
3 \\
(v .8 .4)\end{array}$} & 1.48 & 1.48 & 1.47 & 1.45 & 1.43 & 1.40 & 3.36 & 1.33 \\
\hline & -0.88 & -0.88 & -0.88 & -0.89 & -0.89 & -0.91 & -0.93 & -.94 \\
\hline & +0.60 & +0.60 & +0.59 & +0.56 & +0.54 & +0.49 & +0.43 & +0.39 \\
\hline \multirow{3}{*}{$\begin{array}{l}(v . c .4)^{2} \\
3 \\
(v . c .4)\end{array}$} & 0.83 & 0.77 & 0.72 & 0.66 & 0.61 & 0.57 & 0.51 & 0.47 \\
\hline & -1.00 & -1.02 & -1.03 & -1.04 & -1.05 & -1.06 & -1.07 & -1.08 \\
\hline & -0.17 & -0.25 & -0.31 & -0.38 & -0.44 & -0.49 & -0.56 & -0.61 \\
\hline \multirow{5}{*}{$\begin{array}{ll}(\rho . c .0) & 1 \\
& 2 \\
3 \\
\text { Tab.X X II } \\
(\rho . c .0)\end{array}$} & 1230 & 1063 & 899 & 741 & 594 & 460 & 344 & 246 \\
\hline & 98 & 99 & 101 & 104 & 108 & 113 & 118 & 126 \\
\hline & 11 & 10 & 9 & 9 & 8 & 8 & 8 & $\theta$ \\
\hline & 968 & 968 & 968 & 967 & 967 & 967 & 966 & 966 \\
\hline & 2307 & 2140 & 1977 & 1821 & 1677 & 1548 & 1436 & 1347 \\
\hline \multirow{6}{*}{ 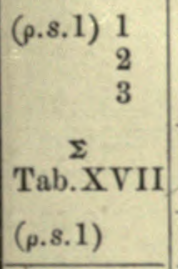 } & 248 & 240 & 230 & 221 & 211 & 203 & 194 & 187 \\
\hline & 2835 & 2822 & 2807 & 2789 & 2769 & 2748 & 2723 & 2696 \\
\hline & 173 & 168 & 165 & 161 & 158 & 155 & 151 & 148 \\
\hline & 3256 & 3230 & 3202 & 3171 & 3138 & 3106 & 3068 & 3031 \\
\hline & -1984 & -1987 & -1989 & -1991 & -1993 & -1996 & -1998 & 2000 \\
\hline & +1272 & 1243 & 1213 & 1180 & 1145 & 1110 & 1070 & 1031 \\
\hline \multirow{6}{*}{ 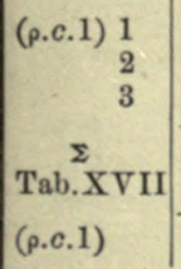 } & 123 & 112 & 97 & 83 & 69 & 55 & 41 & 31 \\
\hline & 1266 & 1202 & 1138 & 1074 & 1009 & 947 & 886 & 827 \\
\hline & 66 & 66 & 67 & 67 & 68 & 70 & 72 & 74 \\
\hline & 1455 & 1380 & 1302 & 1224 & 1146 & 1072 & 999 & 932 \\
\hline & -1977 & -1981 & -1986 & -1990 & -1994 & -1998 & -2002 & 2006 \\
\hline & -522 & -601 & -684 & -766 & -848 & -926 & -1003 & -1074 \\
\hline
\end{tabular}




\begin{tabular}{|c|c|c|c|c|c|c|c|c|}
\hline Date, \{ & $\left|\begin{array}{c}1875, \text { Deo. } 15 \\
1875.955 .\end{array}\right|$ & $\mid \begin{array}{c}1876, \text { A pr. } 13 \\
1876.283 .\end{array}$ & $\begin{array}{c}\text { Ang. } 11 \\
1876.612 .\end{array}$ & $\begin{array}{l}\text { Dec. } 9 \\
1867.940 .\end{array}$ & $\begin{array}{c}4877, \text { Apr. } 8 \\
1877.269 .\end{array}$ & $\begin{array}{c}\text { Aug. } 6 \\
1877.597 .\end{array}$ & $\begin{array}{c}\text { Dec. } 4 \\
1877.925 .\end{array}$ & $\begin{array}{c}1878, \mathrm{Apr}^{3}{ }^{3} \\
1878.254 .\end{array}$ \\
\hline & $\begin{array}{ll}0 & 1\end{array}$ & 10 & 1 & $\begin{array}{ll}0 & 1\end{array}$ & $0 \quad 1$ & 101 & 01 & $0 \quad 1$ \\
\hline$g$ & 33123.086 & 33247.554 & $334 \quad 12.021$ & $335 \quad 36.489$ & $337 \quad 0.957$ & $\begin{array}{ll}338 & 25.425\end{array}$ & $339 \quad 49.893$ & $341 \quad 14.361$ \\
\hline $\begin{array}{r}(p . s .2) \\
3 \\
3\end{array}$ & $\begin{array}{r}174 \\
26 \\
\end{array}$ & $\begin{array}{r}181 \\
25 \\
\end{array}$ & $\begin{array}{r}189 \\
25 \\
\end{array}$ & $\begin{array}{r}196 \\
24 \\
\end{array}$ & $\begin{array}{r}204 \\
24 \\
\end{array}$ & $\begin{array}{r}213 \\
24 \\
\end{array}$ & $\begin{array}{r}223 \\
23 \\
\end{array}$ & $\begin{array}{r}232 \\
23\end{array}$ \\
\hline $\mathrm{Tab}^{\Sigma} \mathrm{XVII}$ & $\begin{array}{r}200 \\
-459 \\
\end{array}$ & $\begin{array}{r}206 \\
-459 \\
\end{array}$ & $\begin{array}{r}214 \\
-459 \\
\end{array}$ & $\begin{array}{r}220 \\
-459 \\
\end{array}$ & $\begin{array}{r}228 \\
-460 \\
\end{array}$ & $\begin{array}{r}237 \\
-460 \\
\end{array}$ & $\begin{array}{r}246 \\
-460 \\
\end{array}$ & $\begin{array}{r}255 \\
-460 \\
\end{array}$ \\
\hline (p.s.2) & -259 & -253 & -245 & -239 & -232 & -223 & -214 & -205 \\
\hline (p.c.2) $\frac{2}{3}$ & $\begin{array}{r}559 \\
33 \\
\end{array}$ & $\begin{array}{r}568 \\
33 \\
\end{array}$ & $\begin{array}{r}576 \\
34\end{array}$ & $\begin{array}{r}584 \\
35\end{array}$ & $\begin{array}{r}592 \\
36\end{array}$ & $\begin{array}{r}600 \\
37\end{array}$ & $\begin{array}{r}607 \\
39\end{array}$ & $\begin{array}{r}614 \\
40\end{array}$ \\
\hline Tab.XVII & $\begin{array}{r}592 \\
-464 \\
\end{array}$ & $\begin{array}{r}601 \\
-464 \\
\end{array}$ & $\begin{array}{r}610 \\
-464 \\
\end{array}$ & $\begin{array}{r}619 \\
-465 \\
\end{array}$ & $\begin{array}{r}628 \\
-465 \\
\end{array}$ & $\begin{array}{r}637 \\
-465 \\
\end{array}$ & $\begin{array}{r}646 \\
-465 \\
\end{array}$ & $\begin{array}{r}654 \\
-466 \\
\end{array}$ \\
\hline (p.c.2) & +128 & 137 & 146 & 154 & 163 & 172 & 181 & 188 \\
\hline $\begin{array}{l}\text { (p.s.3) } 2 \\
\text { Tab.XVII }\end{array}$ & $\begin{array}{r}28 \\
-101 \\
\end{array}$ & $\begin{array}{r}31 \\
-101 \\
\end{array}$ & $\begin{array}{r}34 \\
-101 \\
\end{array}$ & $\begin{array}{r}39 \\
-101 \\
\end{array}$ & $\begin{array}{r}43 \\
-101 \\
\end{array}$ & $\begin{array}{r}47 \\
-101 \\
\end{array}$ & $\begin{array}{r}52 \\
-101\end{array}$ & $\begin{array}{r}57 \\
-101\end{array}$ \\
\hline (p.s.3) & -73 & -70 & -67 & -62 & -58 & -54 & -49 & -44 \\
\hline $\begin{array}{l}\text { (p.c. } 3) 2 \\
\text { Tab.XVII }\end{array}$ & $\begin{array}{r}155 \\
-102 \\
\end{array}$ & $\begin{array}{r}159 \\
-102 \\
\end{array}$ & $\begin{array}{r}162 \\
-102 \\
\end{array}$ & $\begin{array}{r}166 \\
-102 \\
\end{array}$ & $\begin{array}{r}169 \\
-102 \\
\end{array}$ & $\begin{array}{r}172 \\
-102 \\
\end{array}$ & $\begin{array}{r}175 \\
-102 \\
\end{array}$ & $\begin{array}{r}177 \\
-102 \\
\end{array}$ \\
\hline (p.c.3) & +53 & 57 & 60 & 64 & 67 & 70 & 73 & 75 \\
\hline $\begin{array}{c}(b . c .0) \\
2 \\
3 \\
\text { Tab.XXIII }\end{array}$ & $\begin{array}{l}0.12 \\
0.11 \\
0.07 \\
\end{array}$ & $\begin{array}{l}0.12 \\
0.11 \\
0.07 \\
\end{array}$ & $\begin{array}{l}0.13 \\
0.11 \\
0.07\end{array}$ & $\begin{array}{l}0.13 \\
0.11 \\
0.07 \\
\end{array}$ & $\begin{array}{l}0.14 \\
0.11 \\
0.07\end{array}$ & $\begin{array}{l}0.14 \\
0.11 \\
0.07\end{array}$ & $\begin{array}{l}0.15 \\
0.11 \\
0.07 \\
\end{array}$ & $\begin{array}{l}0.15 \\
0.11 \\
0.07 \\
\end{array}$ \\
\hline (b.c.0) & 0.30 & 0.30 & 0.31 & 0.31 & 0.32 & 0.32 & 0.33 & 0.33 \\
\hline $\begin{array}{r}(b . s .1) \\
1 \\
2 \\
3\end{array}$ & $\begin{array}{l}0.30 \\
0.12 \\
1.11 \\
\end{array}$ & $\begin{array}{l}0.22 \\
0.09 \\
1.11 \\
\end{array}$ & $\begin{array}{l}0.16 \\
0.06 \\
1.12 \\
\end{array}$ & $\begin{array}{l}0.12 \\
0.05 \\
1.12 \\
\end{array}$ & $\begin{array}{l}0.08 \\
0.04 \\
1.13 \\
\end{array}$ & $\begin{array}{l}0.06 \\
0.04 \\
1.13 \\
\end{array}$ & $\begin{array}{l}0.05 \\
0.05 \\
1.14 \\
\end{array}$ & $\begin{array}{l}0.06 \\
0.06 \\
1.14 \\
\end{array}$ \\
\hline Tab.XXIII & $\begin{array}{r}1.53 \\
-5.23 \\
\end{array}$ & $\begin{array}{r}1.42 \\
-5.24 \\
\end{array}$ & $\begin{array}{r}1.34 \\
-5.24 \\
\end{array}$ & $\begin{array}{r}1.29 \\
-5.24 \\
\end{array}$ & $\begin{array}{r}1.25 \\
-5.24 \\
\end{array}$ & $\begin{array}{r}1.23 \\
-5.25 \\
\end{array}$ & $\begin{array}{r}1.24 \\
-5.25 \\
\end{array}$ & $\begin{array}{r}1.26 \\
-5.25 \\
\end{array}$ \\
\hline (b.s.1) & -3.70 & -3.82 & -3.90 & -3.95 & -3.99 & -4.02 & -4.01 & -3.99 \\
\hline $\begin{array}{r}(b . c .1) \\
1 \\
2 \\
3 \\
\end{array}$ & $\begin{array}{l}1.21 \\
0.96 \\
1.01 \\
\end{array}$ & $\begin{array}{r}1.14 \\
1.06 \\
1.00 \\
\end{array}$ & $\begin{array}{l}1.06 \\
1.17 \\
1.00 \\
\end{array}$ & $\begin{array}{r}0.98 \\
1.28 \\
0.99 \\
\end{array}$ & $\begin{array}{l}0.90 \\
1.39 \\
0.99 \\
\end{array}$ & $\begin{array}{r}0.80 \\
1.51 \\
0.99 \\
\end{array}$ & $\begin{array}{l}0.70 \\
1.63 \\
0.98 \\
\end{array}$ & $\begin{array}{l}0.61 \\
1.75 \\
0.98 \\
\end{array}$ \\
\hline Tab. $\stackrel{\Sigma}{X} X I I I$ & $\begin{array}{r}3.13 \\
-4.45 \\
\end{array}$ & $\begin{array}{r}3.20 \\
-4.44 \\
\end{array}$ & $\begin{array}{r}3.23 \\
-4.43 \\
\end{array}$ & $\begin{array}{r}3.25 \\
-4.43 \\
\end{array}$ & $\begin{array}{r}3.28 \\
-4.49 \\
\end{array}$ & $\begin{array}{r}3.30 \\
-4.41 \\
\end{array}$ & $\begin{array}{r}3.31 \\
-4.41 \\
\end{array}$ & $\begin{array}{r}3.34 \\
-4.40 \\
\end{array}$ \\
\hline (b.c.1) & -1.27 & -1.24 & -1.20 & -1.18 & -1.14 & -1.11 & -1.10 & -1.06 \\
\hline $\begin{array}{c}(\text { b.s.2) } 2 \\
3 \\
\text { Tab.XXIII }\end{array}$ & $\begin{array}{r}0.02 \\
0.16 \\
-0.48 \\
\end{array}$ & $\begin{array}{r}0.02 \\
0.16 \\
-0.48 \\
\end{array}$ & $\begin{array}{r}0.03 \\
0.16 \\
-0.48 \\
\end{array}$ & $\begin{array}{r}0.04 \\
0.15 \\
-0.48 \\
\end{array}$ & $\begin{array}{r}0.04 \\
0.15 \\
-0.48 \\
\end{array}$ & $\begin{array}{r}0.05 \\
0.15 \\
-0.48 \\
\end{array}$ & $\begin{array}{r}0.06 \\
0.15 \\
-0.48 \\
\end{array}$ & $\begin{array}{r}0.07 \\
0.15 \\
-0.49 \\
\end{array}$ \\
\hline (b.s.2) & -0.30 & -0.30 & -0.29 & -0.29 & -0.29 & -0.28 & -0.27 & -0.26 \\
\hline $\begin{array}{cc}(b . c .2) & 2 \\
& 3 \\
\text { Tab.XXIII } \\
\end{array}$ & $\begin{array}{r}0.31 \\
0.10 \\
-0.38 \\
\end{array}$ & $\begin{array}{r}0.32 \\
0.10 \\
-0.38 \\
\end{array}$ & $\begin{array}{r}0.33 \\
0.10 \\
-0.38 \\
\end{array}$ & $\begin{array}{r}0.34 \\
0.10 \\
-0.38 \\
\end{array}$ & $\begin{array}{r}0.34 \\
0.11 \\
-0.38 \\
\end{array}$ & $\begin{array}{r}0.35 \\
0.11 \\
-0.38 \\
\end{array}$ & $\begin{array}{r}0.36 \\
0.11 \\
-0.38\end{array}$ & $\begin{array}{r}0.36 \\
0.11 \\
-0.38 \\
\end{array}$ \\
\hline (b.c. 2) & +0.03 & +0.04 & +0.05 & +0.06 & +0.07 & +0.08 & +0.09 & +0.09 \\
\hline
\end{tabular}


THE ORBIT OF URANUS.

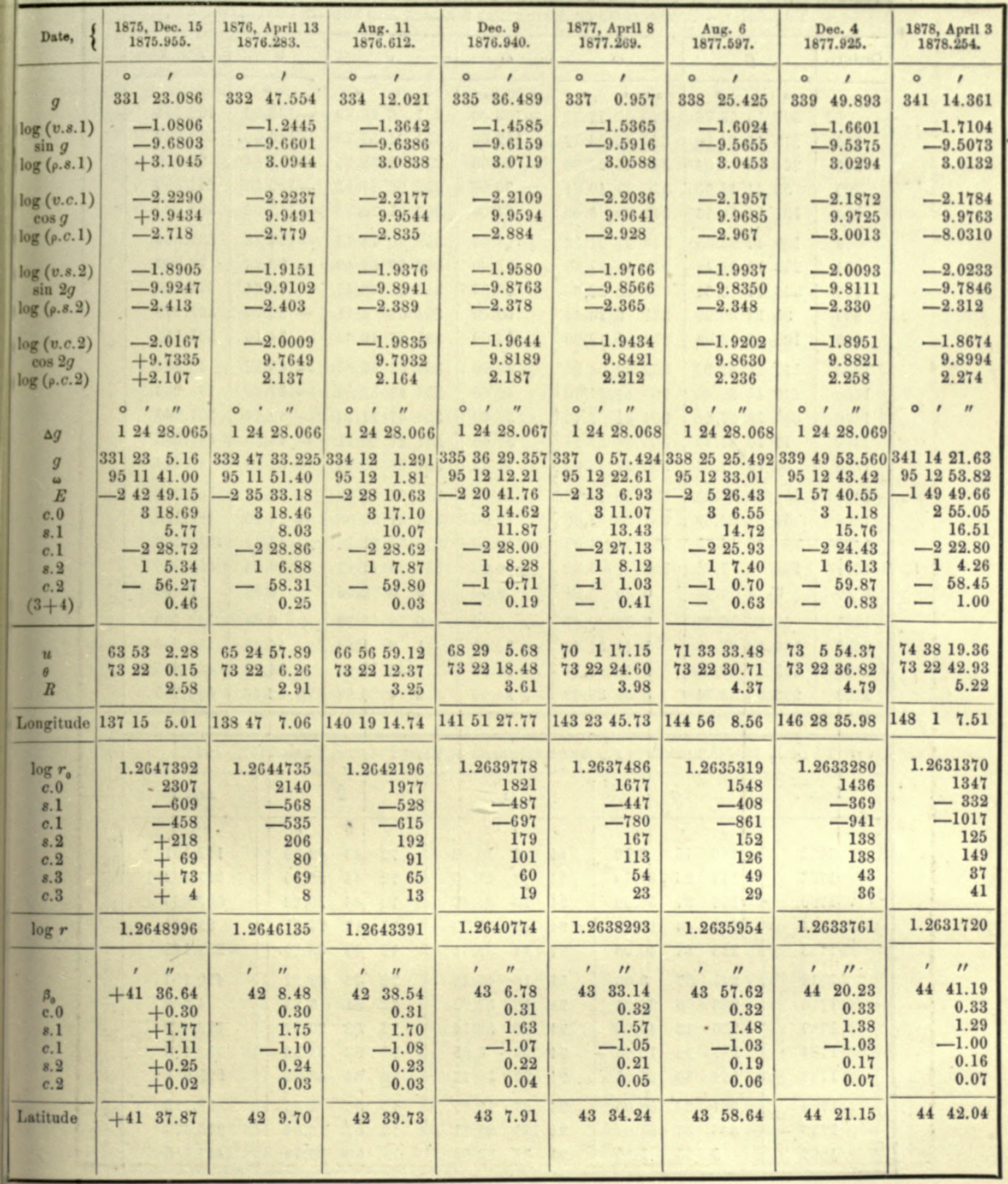


TABLE I.-Corrections of Arguments For past and Future Centuries.

\begin{tabular}{|c|c|c|c|c|c|c|c|c|c|c|c|c|}
\hline Century. & & $g$ & & & $\omega$ & & $\omega$ & & $\theta$ & & $\theta^{\prime}$ & Arg. 1 \\
\hline & $\circ$ & ' & $"$ & $\circ$ & , & " & $"$ & 。 & ， & " & $" \prime$ & \\
\hline $0 \mathrm{~J}$ & 207 & 15 & 59.32 & 343 & 52 & 17.36 & +108.00 & 351 & 6 & 26.89 & -148.32 & 408.924 \\
\hline 100 & 275 & 45 & 34.18 & 344 & 46 & 54.12 & 102.00 & 351 & 34 & 55.39 & -140.08 & 552.952 \\
\hline 200 & 344 & 15 & 9.04 & 345 & 41 & 24.88 & 96.00 & 352 & 3 & 32.13 & -131.84 & 96.980 \\
\hline 300 & 52 & 44 & 43.90 & 346 & 35 & 49.64 & 90.00 & 352 & 32 & 17.11 & -123.60 & 241.008 \\
\hline 400 & 121 & 14 & 18.76 & 347 & 30 & 8.40 & 84.00 & 353 & 1 & 10.33 & -115.36 & 385.036 \\
\hline 500 & 189 & 43 & 53.62 & 348 & 24 & 21.16 & +.78 .00 & 353 & 30 & 11.79 & -107.12 & 529.064 \\
\hline 600 & 258 & 13 & 28.48 & 349 & 18 & 27.92 & 72.00 & 353 & 59 & 21.49 & -98.88 & 73.092 \\
\hline 700 & 326 & 43 & 3.34 & 350 & 12 & 28.68 & 66.00 & 354 & 28 & 39.43 & -90.64 & 217.120 \\
\hline 800 & 35 & 12 & 38.20 & 351 & 6 & 23.44 & 60.00 & 354 & 58 & 5.61 & -82.40 & 361.148 \\
\hline 900 & 103 & 42 & 13.06 & 352 & 0 & 12.20 & 54.00 & 355 & 27 & 40.03 & -74.16 & 505.176 \\
\hline 1000 & 172 & 11 & 47.92 & 352 & 53 & 54.96 & +48.00 & 355 & 57 & 22.69 & -65.92 & 49.204 \\
\hline 1100 & 240 & 41 & 22.78 & 353 & 47 & 31.72 & 42.00 & 356 & 27 & 13.59 & -57.68 & 193.232 \\
\hline 1200 & 309 & 10 & 57.64 & 354 & 41 & 2.48 & 36.00 & 356 & 57 & 12.73 & -49.44 & 337.260 \\
\hline 1300 & 17 & 40 & 32.50 & 355 & 34 & 27.24 & 30.00 & 357 & 27 & 20.11 & -41.20 & 481.288 \\
\hline 1400 & 86 & 10 & 7.36 & 356 & 27 & 46.00 & 24.00 & 357 & 57 & 35.73 & -32.96 & 25.316 \\
\hline $1500 \mathrm{~J}$ & 154 & 39 & 42.22 & 357 & 20 & 58.76 & +18.00 & 358 & 27 & 59 & -24.72 & 44 \\
\hline 15000 & 154 & 32 & 39.89 & 357 & 20 & 57.89 & 18.00 & 358 & 27 & 59.08 & -24.72 & 168.155 \\
\hline 1600 & $22: 3$ & 2 & 14.75 & 358 & 14 & 4.65 & 12.00 & 358 & 58 & $31.18^{\circ}$ & -16.48 & .312 .183 \\
\hline 1700 & 291 & 31 & 7.37 & 359 & 7 & 5.33 & 6.00 & 359 & 29 & 11.47 & -8.24 & 456.092 \\
\hline 1800 & 0 & 0 & 0.00 & 0 & 0 & 0.00 & 0.00 & 0 & 0 & 0.00 & 0.00 & 0.000 \\
\hline 1900 & 68 & 28 & 52.63 & 0 & 52 & 48.67 & -6.00 & 0 & 30 & 56.77 & +8.24 & 143.908 \\
\hline 2000 & 136 & 58 & 27.49 & 1 & 45 & 31.43 & -12.00 & 1 & 2 & 1.83 & 16.48 & 287.936 \\
\hline 2100 & 205 & 27 & 20.11 & 2 & 38 & 8.11 & -18.00 & 1 & 33 & 15.08 & 24.72 & 431.815 \\
\hline 2200 & 273 & 56 & 12.74 & 3 & 30 & 38.78 & -24.00 & 2 & 4 & 36.57 & +32.96 & 575.755 \\
\hline
\end{tabular}

TABLE II.-Arguments for the beginning of each Fourth Year 1752-1948.

\begin{tabular}{|c|c|c|c|c|c|c|c|c|c|c|}
\hline \multirow[t]{2}{*}{ Year. } & \multicolumn{3}{|c|}{$g$} & \multicolumn{3}{|c|}{$\omega$} & \multicolumn{3}{|c|}{$\theta$} & Arg. 1 \\
\hline & $\circ$ & ' & $"$ & $\circ$ & ' & $"$ & $\circ$ & ; & $"$ & \\
\hline 1752 & 160 & 15 & 8.10 & 94 & 6 & 10.48 & 72 & 43 & 42.30 & 162.101 \\
\hline 1756 & 177 & 23 & 31.10 & 94 & 8 & 17.46 & 72 & 44 & 56.25 & 335.862 \\
\hline 1760 & 194 & 31 & 54.09 & 94 & 10 & 24.43 & 72 & 46 & 10.22 & 509.623 \\
\hline 1764 & 211 & 40 & 17.09 & 94 & 12 & 3 k. 39 & 72 & 47 & 24.21 & 83.384 \\
\hline 1768 & 228 & 48 & 40.08 & 94 & 14 & 38.34 & 72 & 48 & 38.20 & 257.145 \\
\hline 1772 & 245 & 57 & 3.08 & 94 & 16 & 45.28 & 72 & 49 & 52.21 & 430.907 \\
\hline 1776 & 263 & 5 & 26.07 & 94 & 18 & 52.22 & 72 & 51 & 6.23 & 4.668 \\
\hline 1780 & 280 . & 13 & 49.06 & 94 & 20 & 59.14 & 72 & 52 & 20.27 & 178.429 \\
\hline 1784 & 297 & 22 & 12.06 & 94 & 23 & 6.05 & 72 & 53 & 31.32 & 352.190 \\
\hline 1788 & 314 & 30 & 35.05 & 94 & 25 & 12.96 & 72 & 54 & 48.38 & 525.951 \\
\hline 1792 & 331 & 38 & 58.05 & 94 & 27 & 19.85 & 72 & 56 & 2.46 & 99.712 \\
\hline 1796 & 348 & 47 & 21.04 & 94 & 29 & 26.74 & 72 & 57 & 16.54 & 273.473 \\
\hline 1800 & 5 & 55 & 1.80 & 94 & 31 & 33.53 & 72 & 58 & 30.59 & 447.115 \\
\hline
\end{tabular}




\begin{tabular}{|c|c|c|c|c|c|c|c|c|}
\hline Century. & 2 & 3 & 4 & 5 & 6 & -7 & 8 & 9 \\
\hline $0 \mathrm{~J}$ & 191.528 & 299.485 & 280 & 512 & 102 & 61 & 470.0 & 15 \\
\hline 100 & 314.245 & 49.520 & 64 & 250 & 563 & 124 & 410.6 & 214 \\
\hline 200 & 436.962 & 399.555 & 449 & 589 & 424 & 188 & 351.2 & 413 \\
\hline 300 & 559.679 & 149.590 & 233 & 327 & 285 & 251 & 291.7 & 13 \\
\hline 400 & 82.396 & 499.625 & 18 & 65 & 146 & 314 & 232.3 & 212 \\
\hline 500 & 205.113 & 249.660 & 402 & 403 & 7 & 378 & 172.9 & 411 \\
\hline 600 & 327.830 & 599.695 & 187 & 142 & 468 & 441 & 113.5 & 10 \\
\hline 700 & 450.547 & 349.730 & 571 & 480 & 329 & 504 & 54.1 & 209 \\
\hline 800 & 573.264 & 99.765 & 356 & 218 & 190 & 567 & 594.6 & 409 \\
\hline 900 & 95.981 & 449.800 & 140 & 556 & 51 & 31 & 535.2 & 8 \\
\hline 1000 & 218.698 & 199.835 & 524 & 295 & 512 & 94 & 475.8 & 207 \\
\hline 1100 & 341.415 & 549.870 & 309 & 33 & 373 & 157 & 416.4 & 406 \\
\hline 1200 & 464.132 & 299.905 & 93 & 371 & 234 & 221 & 357.0 & 5 \\
\hline 1300 & 586.849 & 49.940 & 478 & 109 & 95 & 284 & 297.5 & 205 \\
\hline 1400 & 109.566 & 399.975 & 262 & 448 & 556 & 347 & 238.1 & 404 \\
\hline $1500 \mathrm{~J}$ & 232.283 & 150.010 & 47 & 186 & 417 & 411 & 178.6 & 3 \\
\hline $1500 \mathrm{G}$ & 231.921 & 149.914 & 47 & 186 & 417 & 410 & 178.3 & 2 \\
\hline 1600 & 354.638 & 499.949 & 431 & 524 & 278 & 473 & 118.8 & 201 \\
\hline 1700 & 477.319 & 249.975 & 216 & 262 & 139 & 537 & 59.4 & 401 \\
\hline 1800 & 0.000 & 0.000 & 0 & 0 & 0 & 0 & 0 & 0 \\
\hline 1900 & 122.681 & 350.025 & 385 & 338 & 461 & 63 & 540.6 & 199 \\
\hline 2000 & 245.398 & 100.060 & 169 & 76 & 322 & 127 & 481.2 & 398 \\
\hline 2100 & 368.079 & 450.086 & 554 & 415 & 183 & 190 & 421.8 & 597 \\
\hline 2200 & 490.760 & 200.111 & 338 & 153 & 44 & 253 & 362.3 & 196 \\
\hline
\end{tabular}

TABLE II.-Continued.

\begin{tabular}{|c|c|c|c|c|c|c|c|c|}
\hline Year. & 2 & 3 & 4 & 5 & 6 & 7 & 8 & 9 \\
\hline 1752 & 481.104 & 345.855 & 133 & 348 & 229 & 213 & 331.6 & 578 \\
\hline 1756 & 534.013 & 359.856 & 172 & 362 & 296 & 287 & 353.2 & 58 \\
\hline 1760 & 586.921 & 373.858 & 212 & 375 & 362 & 362 & 374.8 & 138 \\
\hline 1764 & 39.830 & 387.859 & 251 & 389 & 429 & 436 & 396.4 & 218 \\
\hline 1768 & 92.739 & 401.860 & 290 & 402 & 495 & 511 & 418.1 & 298 \\
\hline 1772 & 145.647 & 415.862 & 330 & 416 & 562 & 585 & 439.7 & 377 \\
\hline 1776 & 198.556 & 429.863 & 369 & 429 & 28 & 60 & 461.3 & 457 \\
\hline 1780 & 251.465 & 443.865 & 408 & 443 & 95 & 134 & 482.9 & 537 \\
\hline 1784 & 304.373 & 457.866 & 448 & 457 & 161 & 209 & 504.6 & 17 \\
\hline 1788 & 357.282 & 471.868 & 487 & 470 & 227 & 283 & 526.2 & 97 \\
\hline 1792 & 410.191 & 485.869 & 527 & 484 & 294 & 358 & 547.8 & 177 \\
\hline 1796 & 463.100 & 499.870 & 566 & 497 & 360 & 433 & 569.4 & 257 \\
\hline 1800 & 515.972 & 513.862 & 5 & 511 & 427 & 507 & 591.1 & 337 \\
\hline
\end{tabular}




\begin{tabular}{|c|c|c|c|c|c|c|c|c|c|c|}
\hline \multicolumn{11}{|c|}{ TABLE II._Continued. } \\
\hline \multirow[t]{2}{*}{ Year. } & \multicolumn{3}{|c|}{$g$} & \multicolumn{3}{|c|}{$\omega$} & \multicolumn{3}{|c|}{$\theta$} & \multirow[t]{2}{*}{ Arg. 1.} \\
\hline & 。 & , & " & $\circ$ & , & " & ○ & ' & $"$ & \\
\hline 1800 & 5 & 55 & 1.80 & 94 & 31 & 33.53 & 72 & 58 & 30.59 & 447.115 \\
\hline 1804 & $23^{\circ}$ & 3 & 24.80 & 94 & 33 & 40.39 & 72 & 59 & 44.71 & 20.876 \\
\hline 1808 & 40 & 11 & 47.79 & 94 & 35 & 47.25 & 73 & 0 & 58.84 & 194.638 \\
\hline 1812 & 57 & 20 & 10.79 & 94 & 37 & 54.09 & 73 & 2 & 12.98 & 368.399 \\
\hline 1816 & $\uparrow 4$ & 28 & 33.78 & 94 & 40 & 0.93 & 73 & 3 & 27.13 & 542.160 \\
\hline 1820 & 91 & 36 & 56.77 & 94 & 42 & 7.76 & 73 & 4 & 41.30 & 115.921 \\
\hline 1824 & 108 & 45 & 19.77 & 94 & 44 & 14.58 & 73 & 5 & 55.48 & 289.683 \\
\hline 1828 & 125 & 53 & 42.76 & 94 & 46 & 21.38 & 73 & 7 & 9.67 & 463.444 \\
\hline 1832 & 143 & 2 & 5.76 & 94 & 48 & 28.18 & 73 & 8 & 23.88 & 37.205 \\
\hline 1836 & 160 & 10 & 28.75 & 94 & 50 & 34.97 & 73 & 9 & 38.10 & 210.966 \\
\hline 1840 & 177 & 18 & 51.75 & 94 & 52 & 41.75 & 73 & 10 & 52.33 & 384.727 \\
\hline 1844 & 194 & 27 & 14.74 & 94 & 54 & 48.52 & 73 & 12 & 6.58 & 558.488 \\
\hline 1848 & 211 & 35 & 37.74 & 94 & 56 & 55.28 & 73 & 13 & 20.84 & 132.249 \\
\hline 1852 & 228 & 44 & 0.73 & 94 & 59 & 2.03 & 73 & 14 & 35.11 & 306.010 \\
\hline 1856 & 245 & 52 & 23.75 & 95 & 1 & 8.77 & 73 & 15 & 49.40 & 479.771 \\
\hline 1860 & 263 & 0 & 46.72 & 95 & 3 & 15.50 & 73 & 17 & 3.70 & 53.532 \\
\hline 1864 & 280 & 9 & 9.71 & 95 & 5 & 22.22 & 73 & 18 & 18.01 & 227.293 \\
\hline 1868 & 297 & 17 & 32.71 & 95 & 7 & 28.94 & 73 & 19 & 32.34 & 401.054 \\
\hline 1872 & 314 & 25 & 55.70 & 95 & 9 & 35.64 & 73 & 20 & 46.67 & 574.815 \\
\hline 1876 & 331 & 34 & 18.70 & 95 & 11 & 42.33 & 73 & 22 & 1.03 & 148.576 \\
\hline 1880 & 318 & 42 & 41.69 & 95 & 13 & 49.01 & 73 & 23 & 15.39 & 322.337 \\
\hline 1884 & 5 & 51 & 4.69 & 95 & 15 & 55.69 & 73 & 24 & 29.77 & 496.098 \\
\hline 1888 & 22 & 59 & 27.68 & 95 & 18 & 2.35 & 73 & 25 & 44.16 & 69.860 \\
\hline 1892 & 40 & 7 & 50.68 & 95 & 20 & 9.01 & 73 & 26 & 58.57 & 243.621 \\
\hline 1896 & 57 & 16 & 13.67 & 95 & 22 & 15.65 & 73 & 28 & 12.98 & 417.382 \\
\hline 1900 & 74 & 23 & 54.43 & 95 & 24 & 22.20 & 73 & 29 & 27.36 & 591.024 \\
\hline 1904 & 91 & 32 & 17.43 & 95 & 26 & 28.83 & 73 & 30 & 41.81 & 164.785 \\
\hline 1908 & 108 & 40 & 40.42 & 95 & 28 & 35.44 & 73 & 31 & 56.26 & 338.546 \\
\hline 1912 & 125 & 49 & 3.41 & 95 & 30 & 42.05 & 73 & 33 & 10.74 & 512.307 \\
\hline 1916 & 142 & 57 & 26.41 & 95 & 32 & 48.64 & 73 & 34 & 25.22 & 86.068 \\
\hline 1920 & 160 & 5 & 49.40 & 95 & 34 & 55.23 & 73 & 35 & 39.72 & 259.830 \\
\hline 1924 & 177 & 14 & 12.40 & 95 & 37 & 1.81 & 73 & 36 & 54.23 & 433.591 \\
\hline 1928 & 194 & 22 & 35.39 & 95 & 39 & 8.38 & 73 & 38 & 8.75 & 7.352 \\
\hline 1932 & 211 & 30 & 58.39 & 95 & 41 & 14.94 & 73 & 39 & 23.29 & 181.113 \\
\hline 1936 & 228 & 39 & 21.38 & 95 & 43 & 21.48 & 73 & 40 & 37.84 & 354.874 \\
\hline 1940 & 245 & 47 & 44.38 & 95 & 45 & 28.02 & 73 & 41 & 52.40 & 528.635 \\
\hline 1944 & 262 & 56 & 7.37 & 95 & 47 & 34.55 & 73 & 43 & 6.97 & 102.396 \\
\hline 1948 & 280 & 4 & 30.36 & 95 & 49 & 41.07 & 73 & 44 & 21.56 & 276.158 \\
\hline$\Delta_{103}^{(1)}$ & 1 & 24 & 28.007 & & & 10.411 & & & 6.100 & 14.2715 \\
\hline Factor $T$ & & & +0.222 & & & -.020 & & & +.027 & -.0012 \\
\hline$\Delta_{1>0}^{(2)}$ & & & +.0007 & & & -.0001 & & & +.0001 & 0 \\
\hline
\end{tabular}




\begin{tabular}{|c|c|c|c|c|c|c|c|c|}
\hline & 2 & 3 & 4 & 5 & 6 & 7 & 8 & 9 \\
\hline 1800 & 515.972 & 513.862 & 5 & 511 & 427 & 507 & 591.1 & 337 \\
\hline 1804 & 568.881 & 527.863 & 45 & 524 & 493 & 582 & 12.7 & 417 \\
\hline 1808 & 21.790 & 541.865 & 84 & 538 & 560 & 56 & 34.3 & 497 \\
\hline 1812 & 74.698 & 555.866 & 124 & 551 & 26 & 131 & 55.9 & 577 \\
\hline 1816 & 127.607 & 569.868 & 163 & 565 & 93 & 205 & 77.5 & 57 \\
\hline 1820 & 180.516 & 583.869 & 202 & 578 & 159 & 280 & 99.2 & 137 \\
\hline 1824 & 233.424 & 597.870 & 242 & 592 & 225 & 354 & 120.8 & 217 \\
\hline 1828 & 286.333 & 11.872 & 281 & 6 & 292 & 429 & 142.4 & 297 \\
\hline 1832 & 339.242 & 25.874 & 320 & 19 & 358 & 503 & 164.0 & 377 \\
\hline 1836 & 392.150 & 39.875 & 360 & 32 & 425 & 578 & 185.7 & 457 \\
\hline 1840 & 445.059 & 53.876 & 399 & 46 & 491 & 52 & 207.3 & 537 \\
\hline 1844 & 497.968 & 67.878 & 439 & 59 & 557 & 127 & 228.9 & 17 \\
\hline 1848 & 550.876 & 81.879 & 478 & 73 & 24 & 201 & 250.5 & 97 \\
\hline 1852 & 8.785 & 95.880 & 517 & 87 & 90 & 276 & 272.1 & 177 \\
\hline 1856 & 56.694 & 109.882 & 557 & 100 & 157 & 350 & 293.8 & 257 \\
\hline 1860 & 109.602 & 123.883 & 596 & 114 & 223 & 425 & 315.4 & 337 \\
\hline 1864 & 162.511 & 137.885 & 35 & 127 & 290 & 499 & 337.0 & 417 \\
\hline 1868 & 215.420 & 151.886 & 75 & 141 & 356 & 574 & 358.6 & 497 \\
\hline 1872 & 268.328 & 165.888 & 114 & 154 & 423 & 49 & 380.3 & 577 \\
\hline 1876 & 321.237 & 179.889 & 153 & 168 & 489 & 123 & 401.9 & 57 \\
\hline 1880 & 374.146 & 193.890 & 193 & 181 & 555 & 198 & 423.5 & 137 \\
\hline 1884 , & 427.054 & 207.892 & 232 & 195 & 22 & 272 & 445.1 & 217 \\
\hline 1888 & 479.963 & 221.893 & 271 & 208 & 88 & 347 & 466.7 & 297 \\
\hline 1892 & 532.872 & 235.895 & 311 & 222 & 155 & 421 & 488.4 & 376 \\
\hline 1896 & 585.780 & 249.896 & 350 & 235 & 221 & 496 & 510.0 & 456 \\
\hline 1900 & 38.653 & 263.889 & 390 & 249 & 287 & 570 & 531.6 & 536 \\
\hline 1904 & 91.562 & 277.890 & 429 & 262 & 354 & 45 & 553.2 & 16 \\
\hline 1908 & 144.470 & 291.891 & 468 & 276 & 420 & 119 & 574.9 & 96 \\
\hline 1912 & 197.379 & 305.892 & 508 & 290 & 487 & 194 & 596.5 & 176 \\
\hline 1916 & 250.288 & 319.894 & 547 & 303 & 553 & 268 & 18.1 & 256 \\
\hline 1920 & 303.197 & 333.895 & 597 & 317 & 20 & 343 & 39.7 & 336 \\
\hline 1924 & 356.105 & 347.897 & 26 & 330 & 86 & 417 & 61.4 & 416 \\
\hline 1928 & 409.014 & 361.898 & 65 & 344 & 152 & 492 & 83.0 & 496 \\
\hline 1932 & 461.923 & 375.900 & 105 & 357 & 219 & 566 & 104.6 & 576 \\
\hline 1936 & 514.831 & 389.901 & 144 & 371 & 285 & 41 & 126.2 & 56 \\
\hline 1940 & 567.740 & 403.902 & 184 & 384 & 352 & 116 & 147.8 & 136 \\
\hline 1944 & 20.649 & 417.904 & 223 & 398 & 418 & 190 & 169.5 & 216 \\
\hline \multirow[t]{4}{*}{1948} & 73.557 & 431.905 & 262 & 411 & 485 & 265 & 191.1 & 296 \\
\hline & 4.3457 & 1.1500 & 3.2 & 1.1 & 5.4 & 6.1 & 1.78 & 6.6 \\
\hline & -.0001 & +.0002 & 0 & 0 & 0 & 0 & 0 & 0 \\
\hline & 0 & & 0 & 0 & 0 & 0 & 0 & 0 \\
\hline
\end{tabular}




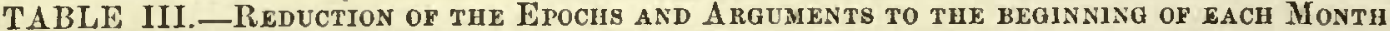
iN A CYCLE OF FOUR YeARS.

\begin{tabular}{|c|c|c|c|c|c|c|c|c|c|c|c|c|}
\hline & & \multicolumn{3}{|c|}{$g$} & \multicolumn{3}{|c|}{$\omega$} & \multicolumn{3}{|c|}{$\theta$} & \multirow{2}{*}{$\frac{\theta^{\prime}}{\prime \prime}$} & \multirow[t]{2}{*}{ Arg. 1} \\
\hline \multicolumn{2}{|l|}{ Year 0} & o & ' & $"$ & $\circ$ & ' & " & 0 & , & $\prime \prime$ & & \\
\hline January & 0 & 0 & 0 & 0.00 & 0 & 0 & 0.00 & 0 & 0 & 0.00 & 0.00 & 0.000 \\
\hline February & 0 & 0 & 21 & 49.24 & 0 & 0 & 2.69 & 0 & 0 & 1.58 & 0.00 & 3.687 \\
\hline Mareh & 0 & 0 & 42 & 14.00 & 0 & 0 & 5.21 & 0 & 0 & 3.05 & 0.00 & 7. 136 \\
\hline April & 0 & 1 & 4 & 3.24 & 0 & 0 & 7.89 & 0 & 0 & 4.63 & 0.01 & 10.823 \\
\hline May & 0 & 1 & 25 & 10.24 & 0 & 0 & 10.50 & 0 & 0 & 6.15 & 0.01 & 14.391 \\
\hline June & 0 & 1 & 46 & 59.48 & 0 & 0 & 13.19 & 0 & 0 & 7.73 & 0.01 & 18.078 \\
\hline July & 0 & 2 & 8 & 6.48 & 0 & 0 & 15.79 & 0 & 0 & 9.25 & 0.01 & 21.647 \\
\hline August & 0 & 2 & 29 & 55.71 & 0 & 0 & 18.48 & 0 & 0 & 10.83 & 0.01 & 25.333 \\
\hline September & 0 & 2 & 51 & 44.95 & 0 & 0 & 21.17 & 0 & 0 & 12.40 & 0.01 & 29.019 \\
\hline October & 0 & 3 & 12 & 51.95 & 0 & 0 & 23.77 & 0 & 0 & 13.93 & 0.02 & 32.587 \\
\hline November & 0 & 3 & 34 & 41.19 & 0 & 0 & 26.46 & 0 & 1) & 15.51 & 0.02 & 36.274 \\
\hline December & 0 & 3 & 55 & 48.19 & 0 & 0 & 29.06 & 0 & 0 & 17.03 & 0.02 & 39.842 \\
\hline \multirow{2}{*}{\multicolumn{13}{|c|}{ Year 1}} \\
\hline January & & 4 & 17 & 37.42 & 0 & 0 & 31.75 & 0 & 0 & 18.61 & 0.02 & 43.529 \\
\hline February & 0 & 4 & 39 & 26.66 & 0 & 0 & 34.44 & 0 & 0 & 20.18 & 0.02 & 47.216 \\
\hline March & 0 & 4 & 59 & 9.12 & 0 & 0 & 36.87 & 0 & 0 & 21.61 & 0.03 & 50.546 \\
\hline April & 0 & 5 & 20 & 58.43 & 0 & 0 & 39.56 & 0 & 0 & 23.18 & 0.03 & 54.233 \\
\hline May & 0 & 5 & 42 & 5.43 & 0 & 0 & 42.16 & 0 & 0 & 24.71 & 0.03 & 57.801 \\
\hline June & 0 & 6 & 3 & 54.67 & 0 & 0 & 44.85 & 0 & 0 & 26.28 & 0.03 & 61.488 \\
\hline July & 0 & 6 & 25 & 1.67 & 0 & 0 & 47.46 & 0 & 0 & 27.81 & 0.03 & 65.056 \\
\hline August & 0 & 6 & 46 & 50.90 & 0 & 0 & 50.14 & 0 & 0 & 29.38 & 0.04 & 68.742 \\
\hline September & 0 & 7 & 8 & 40.14 & 0 & 0 & 52.83 & 0 & 0 & 30.96 & 0.04 & 72.429 \\
\hline Oetober & 0 & 7 & 29 & 47.14 & 0 & 0 & 55.44 & 0 & 0 & 32.48 & 0.04 & 75.997 \\
\hline November & 0 & 7 & 51 & 36.38 & 0 & 0 & 58.13 & 0 & 0 & 34.06 & 0.04 & 79.684 \\
\hline December & 0 & 8 & 12 & 43.38 & 0 & 1 & 0.73 & 0 & (1) & 35.59 & 0.04 & 83.252 \\
\hline \multicolumn{13}{|l|}{ Year 2} \\
\hline January & 0 & 8 & 34 & 32.61 & 0 & 1 & 3.43 & 0 & 0 & 37.16 & 0.04 & 86.939 \\
\hline February & 0 & 8 & 56 & 21.85 & 0 & 1 & 6.11 & 0 & 0 & 38.74 & 0.05 & 90.626 \\
\hline March & 0 & 9 & 16 & 4.38 & 0 & 1 & 8.54 & 0 & 0 & 40.16 & 0.05 & 93.956 \\
\hline April & 0 & 9 & 37 & 53.62 & 0 & 1 & 11.23 & 0 & 0 & 41.74 & 0.05 & 97.643 \\
\hline May & 0 & 9 & 59 & 0.62 & 0 & 1 & 13.83 & 0 & 0 & 43.26 & 0.05 & 101.211 \\
\hline June & 0 & 10 & 20 & 49.86 & 0 & 1 & 16.52 & 0 & 0 & 44.84 & 0.05 & 104.898 \\
\hline July & 0 & 10 & 41 & 56.86 & 0 & 1 & 19.12 & 0 & 0 & 46.36 & 0.06 & 108.466 \\
\hline August & 0 & 11 & 3 & 46.09 & 0 & 1 & 21.81 & 0 & 0 & 47.94 & 0.06 & 112.153 \\
\hline September & 0 & 11 & 25 & 35.33 & 0 & 1 & 24.50 & 0 & 0 & 49.52 & 0.06 & 115.840 \\
\hline October & 0 & 11 & 46 & 42.33 & 0 & 1 & 27.10 & 0 & 0 & 51.04 & 0.06 & 119.407 \\
\hline November & 0 & 12 & 8 & 31.57 & 0 & 1 & 29.79 & 0 & 0 & 52.62 & 0.06 & 123.094 \\
\hline December & 0 & 12 & 29 & 38.57 & 0 & 1 & 32.40 & 0 & 0 & 54.14 & 0.07 & 126.662 \\
\hline \multicolumn{13}{|c|}{ Year 3} \\
\hline January & 0 & 12 & 51 & 27.80 & 0 & 1 & 35.08 & 0 & 0 & 55.72 & 0.07 & 130.349 \\
\hline February & 0 & 13 & 13 & 17.04 & 0 & 1 & 37.77 & 0 & 0 & 57.29 & 0.07 & 134.036 \\
\hline March & 0 & 13 & 32 & 59.57 & 0 & 1 & 40.20 & 0 & 0 & 58.72 & 0.07 & 137.366 \\
\hline April & 0 & 13 & 54 & 48.81 & 0 & 1 & 42.89 & 0 & 1 & 0.29 & 0.07 & 141.053 \\
\hline May & 0 & 14 & 15 & 55.81 & 0 & 1 & 45.50 & 0 & 1 & 1.82 & 0.08 & 144.621 \\
\hline June & 0 & 14 & 37 & 45.05 & 0 & 1 & 48.18 & 0 & 1 & 3.40 & 0.08 & 148.308 \\
\hline July & 0 & 14 & 58 & 52.05 & 0 & 1 & 50.79 & 0 & 1 & 4.92 & 0.08 & 151.876 \\
\hline August & 0 & 15 & 20 & 41.28 & 0 & 1 & 53.48 & 0 & 1 & 6.50 & 0.08 & 155.563 \\
\hline September & 0 & 15 & 42 & 30.52 & 0 & 1 & 56.17 & 0 & 1 & 8.07 & 0.08 & 159.249 \\
\hline October & 0 & 16 & 3 & 37.52 & 0 & 1 & 58.77 & 0 & 1 & 9.60 & 0.08 & 162.817 \\
\hline November & 0 & 16 & 25 & 26.76 & 0 & 2 & 1.46 & 0 & 1 & 11.17 & 0.09 & 166.504 \\
\hline Deeember & 0 & 16 & 46 & 33.76 & 0 & 2 & 4.06 & 0 & 1 & 12.70 & 0.09 & 170.072 \\
\hline
\end{tabular}




\begin{tabular}{|c|c|c|c|c|c|c|c|c|}
\hline & 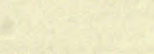 & & BLE II & - Cont & ed. & & & \\
\hline & 2 & 3 & 4 & 5 & 6 & 7 & 8 & 9 \\
\hline \begin{tabular}{ll}
\multicolumn{1}{c}{ Year 0 } \\
January & 0 \\
February & 0 \\
Mareh & 0 \\
April. & 0
\end{tabular} & $\begin{array}{l}0.000 \\
1.123 \\
2.209 \\
3.259\end{array}$ & $\begin{array}{l}0.000 \\
0.297 \\
0.575 \\
0.872\end{array}$ & $\begin{array}{l}0 \\
1 \\
2 \\
2\end{array}$ & $\begin{array}{l}0 \\
0 \\
1 \\
1\end{array}$ & $\begin{array}{l}0 \\
1 \\
3 \\
4\end{array}$ & $\begin{array}{l}0 \\
1 \\
3 \\
4\end{array}$ & $\begin{array}{l}0.0 \\
0.5 \\
0.9 \\
1.3\end{array}$ & $\begin{array}{l}0 \\
2 \\
3 \\
5\end{array}$ \\
\hline $\begin{array}{ll}\text { May } & 0 \\
\text { June } & 0 \\
\text { July } & 0 \\
\text { August } & 0\end{array}$ & $\begin{array}{l}4.382 \\
5.505 \\
6.591 \\
7.714\end{array}$ & $\begin{array}{l}1.159 \\
1.457 \\
1.745 \\
2.042\end{array}$ & $\begin{array}{r}3 \\
4 \\
-\quad 5 \\
6\end{array}$ & $\begin{array}{l}1 \\
1 \\
2 \\
2\end{array}$ & $\begin{array}{r}5 \\
7 \\
8 \\
10\end{array}$ & $\begin{array}{r}6 \\
7 \\
9 \\
10\end{array}$ & $\begin{array}{l}1.8 \\
2.2 \\
2.7 \\
3.1\end{array}$ & $\begin{array}{r}7 \\
8 \\
10 \\
12\end{array}$ \\
\hline $\begin{array}{ll}\text { September } & 0 \\
\text { October } & 0 \\
\text { November } & 0 \\
\text { December } & 0\end{array}$ & $\begin{array}{r}8.837 \\
9.923 \\
11.046 \\
12.132\end{array}$ & $\begin{array}{l}2.339 \\
2.626 \\
2.923 \\
3.211\end{array}$ & $\begin{array}{l}7 \\
7 \\
8 \\
9\end{array}$ & $\begin{array}{l}2 \\
3 \\
3 \\
3\end{array}$ & $\begin{array}{l}11 \\
12 \\
14 \\
15\end{array}$ & $\begin{array}{l}12 \\
14 \\
15 \\
17\end{array}$ & $\begin{array}{l}3.6 \\
4.0 \\
4.5 \\
4.9\end{array}$ & $\begin{array}{l}13 \\
15 \\
17 \\
18\end{array}$ \\
\hline \begin{tabular}{ll}
\multicolumn{1}{c}{ Year 1 } \\
January & 0 \\
February & 0 \\
March & 0 \\
April & 0
\end{tabular} & $\begin{array}{r}13.254 \\
14.377 \\
-15.391 \\
16.514\end{array}$ & $\begin{array}{l}3.508 \\
3.805 \\
4.073 \\
4.370\end{array}$ & $\begin{array}{l}10 \\
11 \\
11 \\
12\end{array}$ & $\begin{array}{l}3 \\
4 \\
4 \\
4\end{array}$ & $\begin{array}{l}17 \\
18 \\
19 \\
21\end{array}$ & $\begin{array}{l}19 \\
20 \\
22 \\
23\end{array}$ & $\begin{array}{l}5.4 \\
5.9 \\
6.3 \\
6.7\end{array}$ & $\begin{array}{l}20 \\
29 \\
23 \\
25\end{array}$ \\
\hline $\begin{array}{ll}\text { May } & 0 \\
\text { June } & 0 \\
\text { July } & 0 \\
\text { August } & 0\end{array}$ & $\begin{array}{l}17.600 \\
18.723 \\
19.809 \\
20.932\end{array}$ & $\begin{array}{l}4.658 \\
4.955 \\
5.242 \\
5.539\end{array}$ & $\begin{array}{l}13 \\
14 \\
15 \\
15\end{array}$ & $\begin{array}{l}4 \\
5 \\
5 \\
5\end{array}$ & $\begin{array}{l}22 \\
24 \\
25 \\
26\end{array}$ & $\begin{array}{l}25 \\
27 \\
28 \\
30\end{array}$ & $\begin{array}{l}7.2 \\
7.6 \\
8.1 \\
8.5\end{array}$ & $\begin{array}{l}27 \\
28 \\
30 \\
32\end{array}$ \\
\hline $\begin{array}{ll}\text { September } & 0 \\
\text { October } & 0 \\
\text { November } & 0 \\
\text { December } & 0\end{array}$ & $\begin{array}{l}22.054 \\
23.140 \\
24.263 \\
25.349\end{array}$ & $\begin{array}{l}5.836 \\
6.124 \\
6.421 \\
6.709\end{array}$ & $\begin{array}{l}16 \\
17 \\
18 \\
19\end{array}$ & $\begin{array}{l}6 \\
6 \\
6 \\
6\end{array}$ & $\begin{array}{l}28 \\
29 \\
30 \\
32\end{array}$ & $\begin{array}{l}31 \\
33 \\
34 \\
36\end{array}$ & $\begin{array}{r}9.0 \\
9.4 \\
9.9 \\
10.3\end{array}$ & $\begin{array}{l}33 \\
35 \\
37 \\
38\end{array}$ \\
\hline \begin{tabular}{ll}
\multicolumn{1}{c}{ Year 2 } \\
January & 0 \\
February & 0 \\
Mareh & 0 \\
April & 0
\end{tabular} & $\begin{array}{l}26.472 \\
27.595 \\
28.609 \\
29.732\end{array}$ & $\begin{array}{l}7.006 \\
7.303 \\
7.571 \\
7.868\end{array}$ & $\begin{array}{l}20 \\
20 \\
21 \\
22\end{array}$ & $\begin{array}{l}7 \\
7 \\
7 \\
7\end{array}$ & $\begin{array}{l}33 \\
34 \\
36 \\
37\end{array}$ & $\begin{array}{l}37 \\
39 \\
40 \\
42\end{array}$ & $\begin{array}{l}10.8 \\
11.3 \\
11.7 \\
12.1\end{array}$ & $\begin{array}{l}40 \\
42 \\
43 \\
45\end{array}$ \\
\hline $\begin{array}{ll}\text { May } & 0 \\
\text { June } & 0 \\
\text { July } & 0 \\
\text { August } & 0\end{array}$ & $\begin{array}{l}30.818 \\
31.941 \\
33.027 \\
34.150\end{array}$ & $\begin{array}{l}8.156 \\
8.453 \\
8.741 \\
9.038\end{array}$ & $\begin{array}{l}23 \\
24 \\
25 \\
25\end{array}$ & $\begin{array}{l}8 \\
8 \\
8 \\
9\end{array}$ & $\begin{array}{l}38 \\
40 \\
41 \\
43\end{array}$ & $\begin{array}{l}44 \\
45 \\
47 \\
48\end{array}$ & $\begin{array}{l}12.6 \\
13.0 \\
13.5 \\
13.9\end{array}$ & $\begin{array}{l}47 \\
48 \\
50 \\
52\end{array}$ \\
\hline $\begin{array}{ll}\text { September } & 0 \\
\text { October } & 0 \\
\text { November } & 0 \\
\text { December } & 0\end{array}$ & $\begin{array}{l}35.272 \\
36.358 \\
37.481 \\
38.567\end{array}$ & $\begin{array}{r}9.335 \\
9.622 \\
9.919 \\
10.207\end{array}$ & $\begin{array}{l}26 \\
27 \\
28 \\
29\end{array}$ & $\begin{array}{r}9 \\
9 \\
9 \\
10\end{array}$ & $\begin{array}{l}44 \\
45 \\
47 \\
48\end{array}$ & $\begin{array}{l}50 \\
51 \\
53 \\
55\end{array}$ & $\begin{array}{l}14.4 \\
14.8 \\
15.3 \\
15.7\end{array}$ & $\begin{array}{l}53 \\
55 \\
57 \\
58\end{array}$ \\
\hline \begin{tabular}{ll}
\multicolumn{1}{c}{ Year 3 } \\
January & 0 \\
February & 0 \\
March & 0 \\
April & 0
\end{tabular} & $\begin{array}{l}39.690 \\
40.813 \\
41.827 \\
42.950\end{array}$ & $\begin{array}{l}10.504 \\
10.801 \\
11.070 \\
11.367\end{array}$ & $\begin{array}{l}30 \\
30 \\
31 \\
32\end{array}$ & $\begin{array}{l}10 \\
10 \\
11 \\
11\end{array}$ & $\begin{array}{l}50 \\
51 \\
52 \\
54\end{array}$ & $\begin{array}{l}56 \\
58 \\
59 \\
61\end{array}$ & $\begin{array}{l}16.2 \\
16.7 \\
17.1 \\
17.5\end{array}$ & $\begin{array}{l}60 \\
62 \\
63 \\
65\end{array}$ \\
\hline $\begin{array}{ll}\text { May } & 0 \\
\text { June } & 0 \\
\text { July } & 0 \\
\text { A ugust } & 0\end{array}$ & $\begin{array}{l}44.036 \\
45.159 \\
46.245 \\
47.368\end{array}$ & $\begin{array}{l}11.654 \\
11.951 \\
12.239 \\
12.536\end{array}$ & $\begin{array}{l}33 \\
34 \\
35 \\
35\end{array}$ & $\begin{array}{l}11 \\
11 \\
12 \\
12\end{array}$ & $\begin{array}{l}55 \\
57 \\
58 \\
59\end{array}$ & $\begin{array}{l}62 \\
64 \\
65 \\
67\end{array}$ & $\begin{array}{l}18.0 \\
18.4 \\
18.9 \\
19.3\end{array}$ & $\begin{array}{l}67 \\
68 \\
70 \\
72\end{array}$ \\
\hline $\begin{array}{ll}\text { September } & 0 \\
\text { October } & 0 \\
\text { November } & 0 \\
\text { December } & 0\end{array}$ & $\begin{array}{l}48.490 \\
49.576 \\
50.699 \\
51.785\end{array}$ & $\begin{array}{l}12.833 \\
13.121 \\
13.418 \\
13.705\end{array}$ & $\begin{array}{l}36 \\
37 \\
38 \\
39\end{array}$ & $\begin{array}{l}12 \\
12 \\
13 \\
13\end{array}$ & $\begin{array}{l}61 \\
62 \\
64 \\
65\end{array}$ & $\begin{array}{l}68 \\
70 \\
72 \\
73\end{array}$ & $\begin{array}{l}19.8 \\
20.2 \\
20.7 \\
21.1\end{array}$ & $\begin{array}{l}73 \\
75 \\
77 \\
78\end{array}$ \\
\hline
\end{tabular}




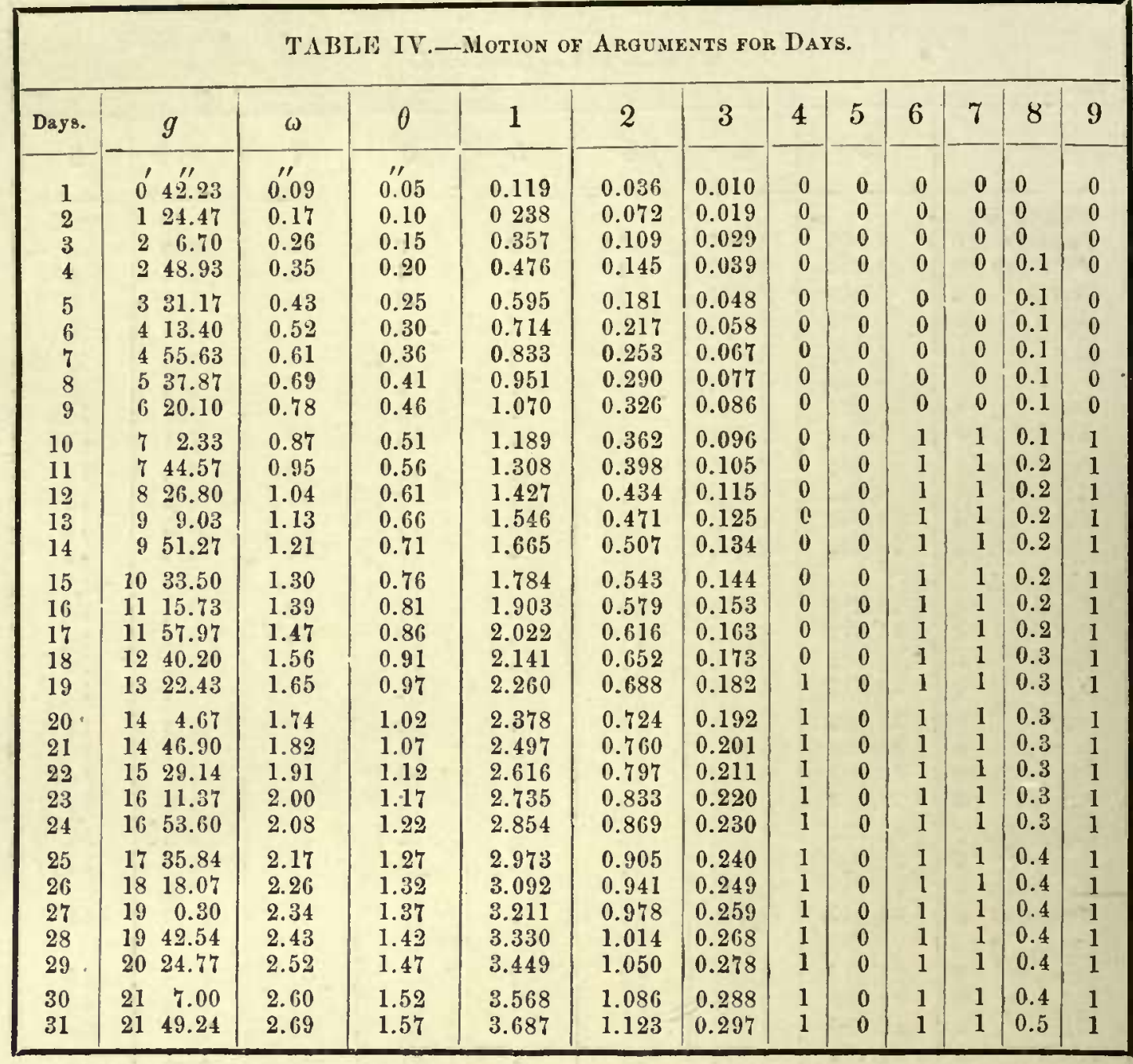

\begin{tabular}{|c|c|c|c|c|c|c|c|}
\hline \multicolumn{8}{|c|}{ TABLE V.-Motion of $g$ Fon Hours. } \\
\hline Hours. & $g$ & IIours. & $g$ & Ilours. & $g$ & Ilours. & $g$ \\
\hline $\begin{array}{l}0 \\
1 \\
9 \\
3 \\
4 \\
5 \\
6\end{array}$ & $\begin{array}{c}11 \\
0.00 \\
1.76 \\
3.52 \\
5.28 \\
7.04 \\
8.80 \\
10.56\end{array}$ & $\begin{array}{r}6 \\
7 \\
8 \\
9 \\
10 \\
11 \\
12\end{array}$ & $\begin{array}{c}\prime \prime \\
10.56 \\
12.32 \\
14.08 \\
15.84 \\
17.60 \\
19.36 \\
21.12\end{array}$ & $\begin{array}{l}13 \\
13 \\
14 \\
15 \\
16 \\
17 \\
18\end{array}$ & $\begin{array}{c}\prime \prime \\
21.12 \\
22.88 \\
24.64 \\
26.40 \\
28.16 \\
29.92 \\
31.67\end{array}$ & $\begin{array}{l}18 \\
19 \\
20 \\
21 \\
22 \\
23 \\
24\end{array}$ & $\begin{array}{c}\prime \prime \\
31.67 \\
33.43 \\
35.19 \\
36.95 \\
38.71 \\
40.47 \\
42.23\end{array}$ \\
\hline
\end{tabular}

The period of arguments 1 to 9 is 600 .

In January and February of those years which, though divisible by 4 , are not leap years, namely, 1700, 1800, 1900, 2100, etc., Table IV must be entered with a number 1 greater than the real day of the month. 


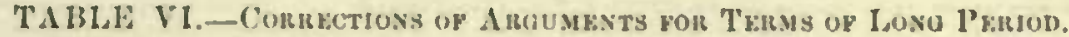

\begin{tabular}{|c|c|c|c|c|c|c|c|c|c|c|}
\hline Yoar. & \multicolumn{2}{|r|}{$y$} & 1 & 2 & 3 & Year. & I & 1 & 2 & 3 \\
\hline & 1 & $\prime \prime$ & & & & & 111 & & & \\
\hline 1000 & +36 & 51.00 & -0.614 & -1.024 & +1.792 & 1550 & +59.73 & -0.200 & -0.144 & +0.252 \\
\hline 1010 & 36 & 6.49 & 0.621 & 1.003 & 1.756 & 1560 & 449.70 & 0.158 & 0.134 & 0.236 \\
\hline 1020 & 3i) & 2.14 & 0.629 & 0.983 & 1.720 & 1570 & 430.32 & 0.106 & 0.125 & 0.219 \\
\hline 1030 & 34 & 37.97 & 0.638 & 0.962 & 1.tist & 1580 & 411.57 & $0.06,0$ & 0.117 & 0.205 \\
\hline 1040 & 33 & 54.00 & 0.645 & 0.942 & 1.649 & 1590 & 353.48 & -0.013 & 0.108 & 0.189 \\
\hline 1050 & +83 & 10.25 & -0.659 & -0.922 & +1.614 & 1600 & +336.03 & +0.034 & -0.100 & +0.175 \\
\hline 1060 & 32 & $21 i .71$ & 0.672 & 0.902 & 1.579 & 1610 & 319.26 & 0.078 & 0.092 & $0.16,1$ \\
\hline 1070 & 31 & 43.41 & 0.686 & 0.882 & 1.544 & 1620 & 33.14 & 0.120 & 0.085 & 0.148 \\
\hline 1080 & 31 & 0.37 & 0.702 & 0.862 & 1.509 & 1630 & 247.69 & 0.162 & 0.078 & 0.135 \\
\hline 1090 & 30 & 17.61 & 0.718 & 0.812 & 1.474 & 1640 & 232.83 & 0.203 & 0.071 & 0.123 \\
\hline 1100 & +29 & 35.15 & -0.735 & -0.822 & +1.439 & 1650 & +218.77 & +0.242 & -0.064 & +0.112 \\
\hline 1110 & 23 & 53.01 & 0.752 & 0. & 1.405 & 1660 & 25.32 & 78 & 58 & 01 \\
\hline 1120 & 2.8 & 11.20 & 0.770 & 0.783 & 1.370 & 1670 & 152.55 & 0.312 & 0.052 & 0.091 \\
\hline 1130 & 27 & 29.11 & 0.790 & 0.764 & 1.337 & 1680 & 140.46 & 0.345 & 0.046 & 0.081 \\
\hline $11+0$ & 26 & 48.56 & 0.809 & $0.7 \$ 5$ & 1.304 & 1690 & 129.05 & 0.375 & 0.011 & 0.072 \\
\hline 1150 & +26 & 7.74 & -0.829 & -0.726 & +1.270 & 1700 & +118.32 & +0.403 & -0.036 & +0.063 \\
\hline 1160 & 25 & 27.27 & 0.848 & 0.707 & 1.237 & 1710 & 18.23 & 0.429 & 0.031 & 0.054 \\
\hline 1170 & 24 & 47.16 & 0.866 & 0.689 & 1.206 & 1720 & 058.92 & 0.452 & 0.027 & 0.046 \\
\hline 1180 & 24 & 7.42 & 0.883 & 0.6 & 1.172 & 1730 & 050.24 & 0.472 & 3 & 0.039 \\
\hline 1190 & 23 & 28.06 & 0.899 & 0.652 & 1.141 & 1740 & 042.26 & 0.489 & 0.019 & 0.032 \\
\hline 1200 & +22 & 49.09 & -0.914 & -0.634 & +1.110 & 1750 & to 34.96 & +0.503 & -0.015 & +0.026 \\
\hline 1210 & 22 & 10 & 0. & b & 1.075 & 1760 & 028.436 & 14 & 2 & 21 \\
\hline 1220 & 21 & 32 & 0.9 & 0.598 & 1.046 & 1770 & 022.44 & 0.522 & 0.0 & 0.017 \\
\hline 1230 & 20 & 54 & 0.950 & 0.5 & 1.017 & 1780 & $\begin{array}{lll}0 & 17.22\end{array}$ & $0.52 i$ & 0.007 & 0.013 \\
\hline 1240 & 20 & 17.23 & 0.960 & 0.564 & 0.986 & 1790 & +012.68 & 0.529 & 0.005 & 0.010 \\
\hline 1250 & +19 & 40.31 & -0.967 & -0.547 & +0.957 & 1800 & +8.84 & +0.528 & -0.004 & +0.007 \\
\hline 1260 & 19 & & 0.972 & 0.530 & 0.928 & 1801 & $8.49^{-.35}$ & 0.528 & 0.004 & 07 \\
\hline 1270 & 18 & 27.80 & 0.975 & 0.513 & 0.898 & 18 & $8.15 \cdot 34$ & 0.528 & is. 004 & 0.007 \\
\hline 1280 & 17 & 52.21 & 0.977 & 0.497 & 0.870 & 1803 & 7.82 & $0.52 i$ & 4 & 0.007 \\
\hline 1290 & 17 & 17.09 & 0.977 & 0.481 & 0.842 & 1804 & 7.50 & 0.527 & 0.004 & 0.007 \\
\hline 1300 & +16 & 42.43 & -0.974 & -0.465 & +0.814 & 1805 & +7.18 & $+0.52 i$ & -0.003 & +0.006 \\
\hline 1310 & 16 & & 0.967 & 19 & 0.786 & 1806 & $6.87^{-.31}$ & 0.526 & 03 & 6 \\
\hline 1320 & 15 & 34.55 & 0.959 & 3 & 0.753 & 18 & $6.57 \quad \cdot 30$ & 0.526 & 0. & 0.006 \\
\hline 1330 & 15 & 1.34 & 0. & 0 & 0.730 & 1808 & 6.27 & 0.525 & $0 . c$ & 0.006 \\
\hline 1340 & 14 & 29.63 & 0.936 & 0.402 & 0.704 & 1809 & 5.98 & 0.525 & 0.003 & 0.006 \\
\hline 1350 & +13 & 56.44 & -0.921 & -0.387 & +0.677 & 1810 & +5.69 & +0.524 & 03 & +0 \\
\hline 1360 & 13 & & 0.903 & & 0.651 & 1811 & $5.41-$ & 0.523 & 0.003 & 0.005 \\
\hline $1: 370$ & 12 & 53.61 & 0.883 & 0.358 & 0.626 & 1812 & 5.14 & 0.523 & 0.003 & 0.005 \\
\hline 1380 & 12 & 22.99 & 0.861 & 0.344 & 0.602 & 1813 & 4.88 & 0.522 & 3 & 0.005 \\
\hline 1390 & 11 & 52.90 & 0.837 & 0.330 & 0.578 & 1814 & 4.62 & 0.522 & 0.002 & 0.005 \\
\hline 1400 & +11 & 23.35 & -0.810 & -0.317 & +0.555 & 1815 & +4.37 & +0521 & -0.002 & +0.004 \\
\hline 1410 & 10 & & 0.780 & & 0.530 & 1816 & $4.13^{-}$ & 0.520 & 2 & 0. \\
\hline 1420 & 10 & 25.92 & 0.748 & 0 & 0.508 & 1817 & 3.89 & 0.5 & 0. & 0.004 \\
\hline 1430 & 9 & 59.04 & 0.714 & 0.2 & 0.485 & 1818 & 3.66 & 0.519 & 0.002 & 0.004 \\
\hline 1440 & 0 & 30.74 & 0.678 & 0.264 & 0.462 & 1819 & 3.44 & & 0. & 0.004 \\
\hline 1450 & +9 & 4.01 & -0.641 & -0.252 & +0.441 & 1820 & +3.23 & +0.517 & -0.002 & +0.003 \\
\hline 1460 & 8 & 37.89 & 0.601 & 0.240 & 0.420 & 1821 & $3.02^{-}$ & 0.516 & 0.002 & 0.003 \\
\hline 1470 & 8 & 12.34 & 0.560 & 0.228 & 0.399 & 1822 & 2.82 & 0.515 & 0.002 & 0.003 \\
\hline 1480 & 7 & 47.39 & 0.518 & 0.216 & 0.378 & 1823 & 2.63 & 0.514 & 0.002 & 0.003 \\
\hline 1490 & & 23.04 & 0.475 & 0.205 & 0.359 & 1824 & 2.44 & 0.513 & 0.002 & 0.003 \\
\hline 1500 & & 59.29 & -0.431 & -0.194 & +0.340 & 1825 & +2.26 & +0.512 & -0.001 & +0.002 \\
\hline 1510 & 6 & 36.14 & 0.386 & 0.183 & 0.322 & 1826 & $2.09^{-}$ & 0.511 & 0.001 & 0.002 \\
\hline 1520 & & & 0.340 & & 0.303 & & 1.82 & 0.510 & 0.001 & 0.002 \\
\hline 1530 & & 51.69 & 0.293 & 0.162 & 0.284 & 1829 & 1.76 & 0.509 & 0.001 & 0.002 \\
\hline 1540 & 5 & & -0.247 & -0.153 & +0.268 & 1829 & $+1.61-.15$ & $-0.50 s$ & -0.001 & +0.002 \\
\hline
\end{tabular}


TABLE VI.-Continued.

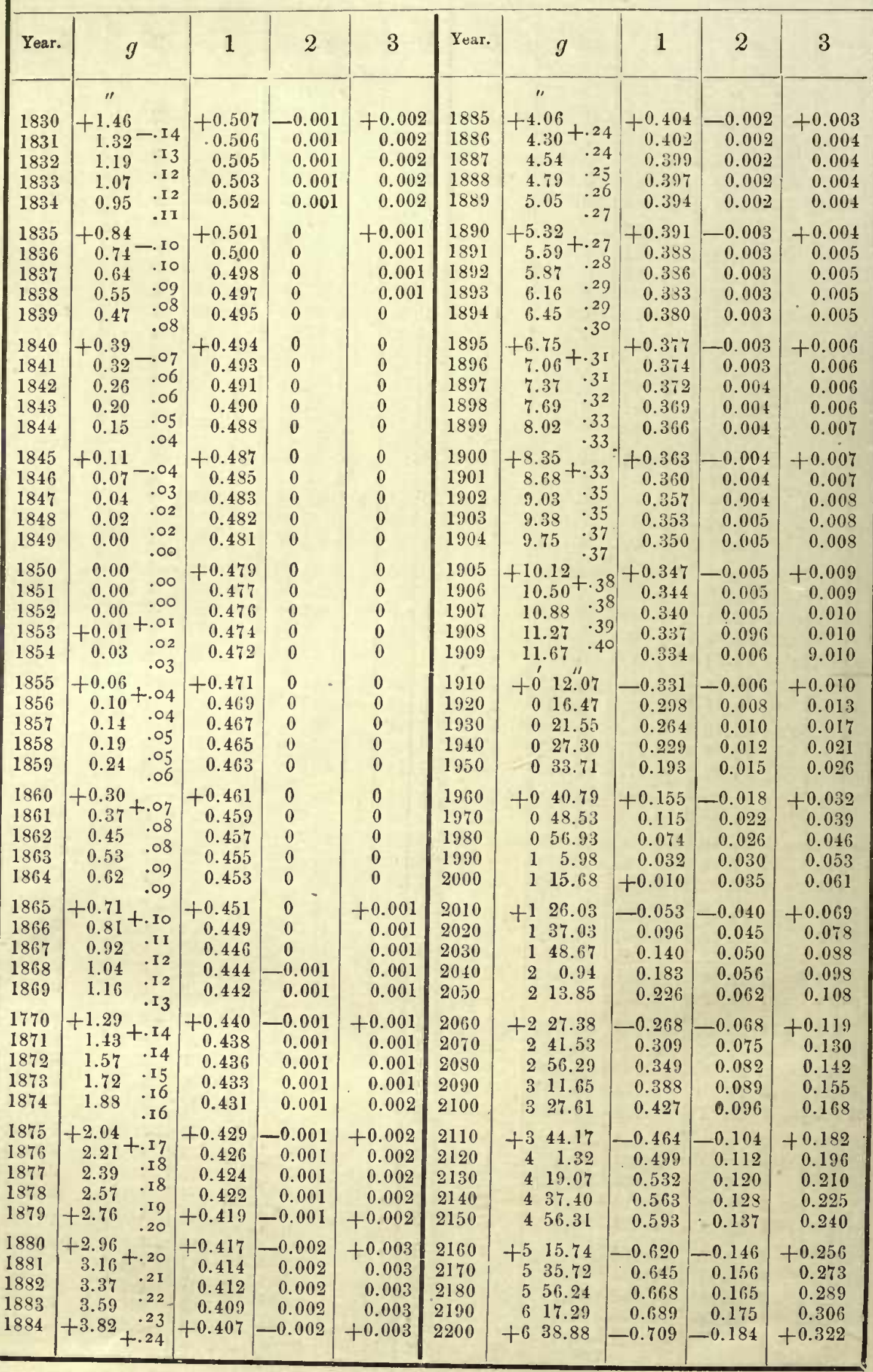




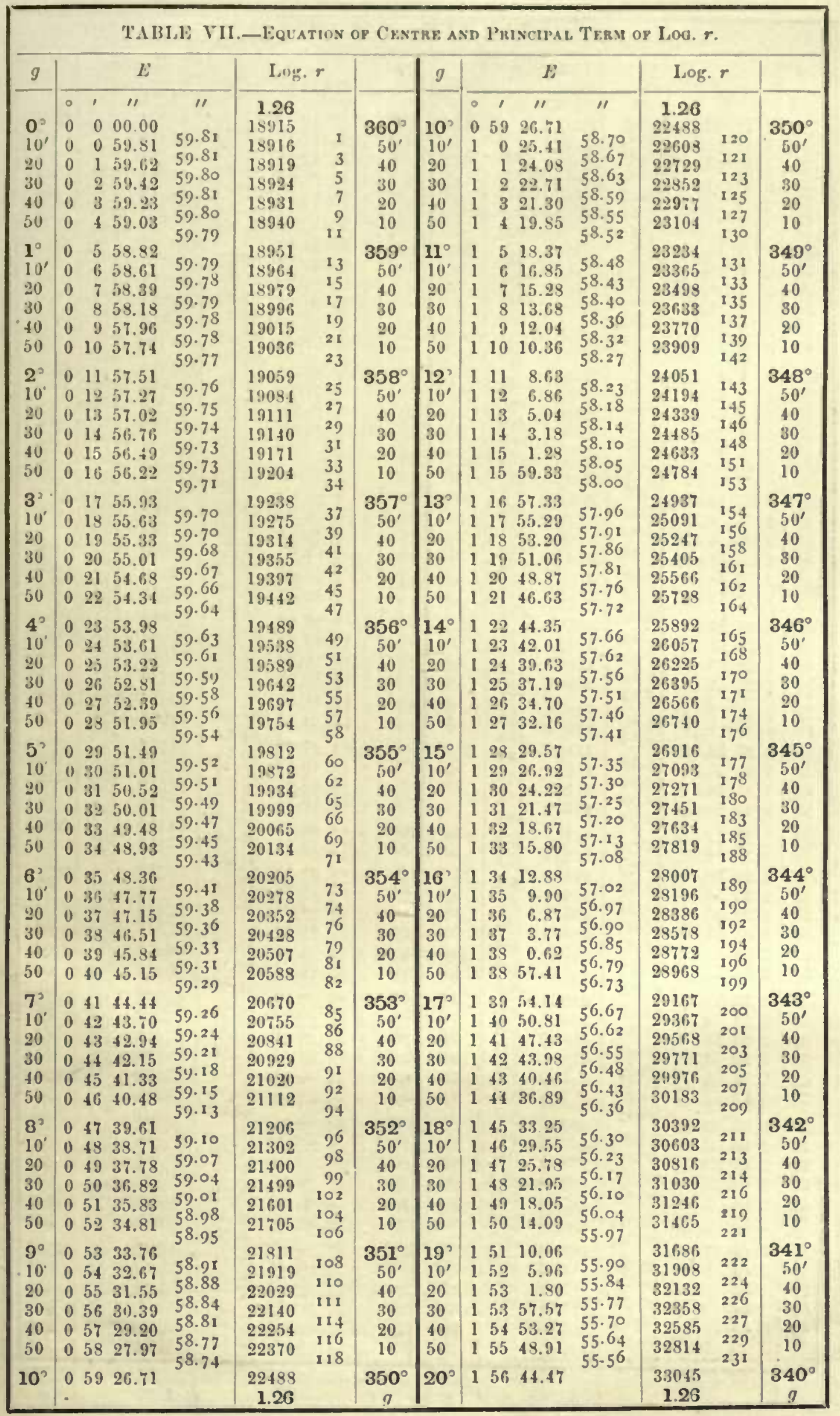




\begin{tabular}{|c|c|c|c|c|c|c|c|c|c|c|c|c|c|}
\hline \multicolumn{14}{|c|}{ TABLE VII._Continued. } \\
\hline$g$ & \multicolumn{3}{|c|}{$E$} & \multicolumn{2}{|c|}{$\log \cdot r$} & & $g$ & \multicolumn{3}{|c|}{$E$} & \multicolumn{2}{|c|}{$\log \cdot r$} & \\
\hline $\begin{array}{c}20^{\circ} \\
10^{\prime} \\
20 \\
30 \\
40 \\
50\end{array}$ & $\begin{array}{ll} & 0 \\
1 & 5 \\
1 & 5 \\
1 & 5 \\
1 & 58 \\
2 & 0 \\
2 & 1\end{array}$ & \begin{tabular}{lc} 
& \multicolumn{1}{c}{ " } \\
56 & 44.47 \\
57 & 39.96 \\
58 & 35.39 \\
99 & 30.74 \\
0 & 26.02 \\
1 & 21.23
\end{tabular} & $\begin{array}{c}\prime \prime \\
55.49 \\
55.43 \\
55.35 \\
55.28 \\
55.21 \\
55.13\end{array}$ & $\begin{array}{r}1.26 \\
33045 \\
33278 \\
33513 \\
33749 \\
33987 \\
34227\end{array}$ & $\begin{array}{l}233 \\
235 \\
236 \\
238 \\
240 \\
241\end{array}$ & $\begin{array}{c}340^{\circ} \\
50^{\prime} \\
40 \\
30 \\
20 \\
10\end{array}$ & $\begin{array}{l}30^{\circ} \\
10^{\prime} \\
20 \\
30 \\
40 \\
50\end{array}$ & \begin{tabular}{|ll} 
& \\
& \\
2 & 4 \\
2 & 5 \\
2 & 5 \\
2 & 5 \\
2 & 5 \\
2 & 5
\end{tabular} & \begin{tabular}{lc} 
& \multicolumn{1}{c}{$\prime \prime$} \\
49 & 51.28 \\
50 & 41.63 \\
51 & 31.87 \\
52 & 22.01 \\
53 & 12.06 \\
54 & 2.00
\end{tabular} & $\begin{array}{c}\prime \prime \\
50.35 \\
50.24 \\
50.14 \\
50.05 \\
49.94 \\
49.83\end{array}$ & $\begin{array}{r}1.26 \\
50121 \\
50457 \\
50795 \\
51134 \\
51475 \\
51818\end{array}$ & $\begin{array}{l}336 \\
338 \\
339 \\
34 \mathrm{I} \\
343 \\
344\end{array}$ & $\begin{array}{c}330^{\circ} \\
50^{\prime} \\
40 \\
30 \\
20 \\
10\end{array}$ \\
\hline $\begin{array}{c}21^{\circ} \\
10^{\prime} \\
20 \\
30 \\
40 \\
50\end{array}$ & $\begin{array}{ll}2 & 2 \\
2 & 3 \\
2 & 4 \\
2 & 5 \\
2 & 5 \\
2 & 6\end{array}$ & $\begin{array}{rr}2 & 16.36 \\
3 & 11.42 \\
4 & 6.41 \\
5 & 1.33 \\
5 & 56.17 \\
6 & 50.93\end{array}$ & $\begin{array}{l}53.13 \\
55.06 \\
54.99 \\
54.92 \\
54.84 \\
54.76 \\
54.68\end{array}$ & $\begin{array}{l}34468 \\
34711 \\
34956 \\
35204 \\
35454 \\
35705\end{array}$ & $\begin{array}{l}243 \\
245 \\
248 \\
250 \\
251 \\
252\end{array}$ & $\begin{array}{c}339^{\circ} \\
50^{\prime} \\
40 \\
30 \\
20 \\
10\end{array}$ & $\begin{array}{l}31^{\circ} \\
10^{\prime} \\
20 \\
30 \\
40 \\
50\end{array}$ & $\begin{array}{ll}2 & 5 \\
2 & 5 \\
2 & 5 \\
2 & 5 \\
2 & 5 \\
2 & 5\end{array}$ & $\begin{array}{ll}54 & 51.83 \\
55 & 41.56 \\
56 & 31.18 \\
57 & 20.70 \\
58 & 10.12 \\
58 & 59.43\end{array}$ & $\begin{array}{l}49 \cdot 73 \\
49.62 \\
49 \cdot 5^{2} \\
49 \cdot 42 \\
49 \cdot 31 \\
49.20\end{array}$ & $\begin{array}{l}52162 \\
52507 \\
52854 \\
53203 \\
53553 \\
53905\end{array}$ & $\begin{array}{l}345 \\
347 \\
349 \\
350 \\
352 \\
353\end{array}$ & $\begin{array}{c}329^{\circ} \\
50^{\prime} \\
40 \\
30 \\
20 \\
10\end{array}$ \\
\hline $\begin{array}{c}22 \\
10^{\prime} \\
20 \\
30 \\
40 \\
50\end{array}$ & $\begin{array}{rr}2 & 7 \\
2 & 8 \\
2 & 9 \\
2 & 10 \\
2 & 11 \\
2 & 12\end{array}$ & $\begin{array}{ll}7 & 45.61 \\
8 & 40.22 \\
9 & 34.76 \\
0 & 29.22 \\
1 & 23.60 \\
2 & 17.90\end{array}$ & $\begin{array}{l}54.00 \\
54.61 \\
54.54 \\
54.46 \\
54.38 \\
54.30 \\
54.22\end{array}$ & $\begin{array}{l}35957 \\
36211 \\
36467 \\
36726 \\
36986 \\
37248\end{array}$ & $\begin{array}{l}254 \\
256 \\
259 \\
260 \\
262 \\
263\end{array}$ & $\begin{array}{c}338^{\circ} \\
50^{\prime} \\
40 \\
30 \\
20 \\
10\end{array}$ & $\begin{array}{l}32^{\prime} \\
10^{\prime} \\
20 \\
30 \\
40 \\
50\end{array}$ & $\begin{array}{ll}2 & 5 \\
3 \\
3 \\
3 \\
3 \\
3\end{array}$ & $\begin{array}{rr}59 & 48.63 \\
0 & 37.73 \\
1 & 26.73 \\
2 & 15.62 \\
3 & 4.41 \\
3 & 53.09\end{array}$ & $\begin{array}{l}49.10 \\
4900 \\
48.89 \\
48.79 \\
48.68 \\
48.57\end{array}$ & $\begin{array}{l}54258 \\
54613 \\
54970 \\
55329 \\
55689 \\
56050\end{array}$ & $\begin{array}{l}353 \\
355 \\
357 \\
359 \\
300 \\
361 \\
363\end{array}$ & $\begin{array}{c}328^{\circ} \\
50^{\prime} \\
40 \\
30 \\
20 \\
10\end{array}$ \\
\hline $\begin{array}{c}23^{\circ} \\
10^{\prime} \\
20 \\
30 \\
40 \\
50\end{array}$ & $\begin{array}{ll}2 & 13 \\
2 & 14 \\
2 & 15 \\
2 & 15 \\
2 & 16 \\
2 & 17\end{array}$ & $\begin{array}{rr}3 & 12.12 \\
4 & 6.26 \\
5 & 0.32 \\
5 & 54.30 \\
6 & 48.20 \\
7 & 42.02\end{array}$ & $\begin{array}{l}54.14 \\
54.06 \\
53.98 \\
53.90 \\
53.82 \\
53.73\end{array}$ & $\begin{array}{l}37511 \\
37776 \\
38043 \\
38311 \\
38581 \\
38853\end{array}$ & $\begin{array}{l}265 \\
267 \\
268 \\
270 \\
272 \\
275\end{array}$ & $\begin{array}{c}337^{\circ} \\
50^{\prime} \\
40 \\
30 \\
20 \\
10\end{array}$ & $\begin{array}{l}33^{\prime} \\
10^{\prime} \\
20 \\
30 \\
40 \\
50\end{array}$ & $\begin{array}{l}3 \\
3 \\
3 \\
3 \\
3 \\
3\end{array}$ & $\begin{array}{rr}4 & 41.66 \\
5 & 30.12 \\
6 & 18.47 \\
7 & 6.72 \\
7 & 54.85 \\
8 & 42.87\end{array}$ & $\begin{array}{l}48.46 \\
48.35 \\
48.25 \\
48.13 \\
48.02 \\
47.91\end{array}$ & $\begin{array}{l}56413 \\
56777 \\
57143 \\
57510 \\
57879 \\
58249\end{array}$ & $\begin{array}{l}364 \\
366 \\
367 \\
369 \\
37^{\circ} \\
37^{2}\end{array}$ & $\begin{array}{c}327^{\circ} \\
50^{\prime} \\
40 \\
30 \\
20 \\
10\end{array}$ \\
\hline $\begin{array}{c}24^{\circ} \\
10^{\prime} \\
20 \\
30 \\
40 \\
50 \\
\end{array}$ & $\begin{array}{ll}2 & 18 \\
2 & 19 \\
2 & 20 \\
2 & 21 \\
2 & 22 \\
2 & 23 \\
& 0\end{array}$ & $\begin{array}{rr}8 & 35.75 \\
9 & 29.40 \\
0 & 22.97 \\
1 & 16.46 \\
2 & 9.87 \\
3 & 3.19\end{array}$ & $\begin{array}{l}53.49 \\
53.41 \\
53.32 \\
53.23\end{array}$ & $\begin{array}{l}39128 \\
39404 \\
39681 \\
39960 \\
40241 \\
40524\end{array}$ & $\begin{array}{l}276 \\
277 \\
279 \\
281 \\
283 \\
284\end{array}$ & $\begin{array}{c}336 \\
50^{\prime} \\
40 \\
30 \\
20 \\
10\end{array}$ & $\begin{array}{l}34 \\
10 \\
20 \\
30 \\
40 \\
50\end{array}$ & $\begin{array}{ll}3 & \\
3 & 1 \\
3 & 1 \\
3 & 1 \\
3 & 1 \\
3 & 1\end{array}$ & $\begin{array}{rr}9 & 30.78 \\
10 & 13.58 \\
11 & 6.26 \\
11 & 53.83 \\
13 & 41.29 \\
13 & 28.64\end{array}$ & $\begin{array}{l}47.80 \\
47.68 \\
47.57 \\
47.46 \\
47.35 \\
47.23\end{array}$ & $\begin{array}{l}58621 \\
58994 \\
59369 \\
59746 \\
60124 \\
60503\end{array}$ & $\begin{array}{l}373 \\
375 \\
377 \\
378 \\
379 \\
381\end{array}$ & $\begin{array}{c}326^{\circ} \\
50^{\prime} \\
40 \\
30 \\
20 \\
10\end{array}$ \\
\hline $\begin{array}{r}25^{\circ} \\
10^{\prime} \\
20 \\
30 \\
40 \\
50\end{array}$ & $\begin{array}{ll}2 & 23 \\
2 & 24 \\
2 & 25 \\
2 & 26 \\
2 & 27 \\
2 & 28\end{array}$ & $\begin{array}{ll}3 & 56.42 \\
4 & 49.57 \\
5 & 42.63 \\
6 & 35.61 \\
7 & 28.50 \\
8 & 21.30\end{array}$ & $\begin{array}{l}53.15 \\
53.06 \\
52.98 \\
52.89 \\
52.80 \\
52.71\end{array}$ & $\begin{array}{l}40808 \\
41094 \\
41382 \\
41671 \\
41962 \\
42255\end{array}$ & $\begin{array}{l}286 \\
288 \\
289 \\
291 \\
293 \\
295\end{array}$ & $\begin{array}{c}335^{\prime} \\
50^{\prime} \\
40 \\
30 \\
20 \\
10\end{array}$ & $\begin{array}{l}35^{\circ} \\
10^{\prime} \\
20 \\
30 \\
40 \\
50\end{array}$ & $\begin{array}{ll}3 & 1 \\
3 & 1 \\
3 & 1 \\
3 & 1 \\
3 & 1 \\
3 & 1\end{array}$ & $\begin{array}{rr}14 & 15.87 \\
15 & 2.99 \\
15 & 50.00 \\
16 & 36.89 \\
17 & 23.67 \\
18 & 10.33\end{array}$ & $\begin{array}{l}47.12 \\
47.01 \\
46.89 \\
46.78 \\
46.66 \\
46.55\end{array}$ & $\begin{array}{l}60884 \\
61266 \\
61650 \\
62035 \\
62422 \\
62810\end{array}$ & $\begin{array}{l}3^{82} \\
3^{84} \\
3^{85} \\
3^{87} \\
3^{88} \\
390\end{array}$ & $\begin{array}{c}325^{\circ} \\
50^{\prime} \\
40 \\
30 \\
20 \\
10\end{array}$ \\
\hline \begin{tabular}{c|}
$26^{\prime}$ \\
$10^{\prime}$ \\
20 \\
30 \\
40 \\
50
\end{tabular} & $\begin{array}{ll}2 & 29 \\
2 & 30 \\
2 & 30 \\
2 & 31 \\
2 & 32 \\
2 & 33\end{array}$ & $\begin{array}{rr}9 & 14.01 \\
0 & 6.63 \\
0 & 59.16 \\
1 & 51.60 \\
2 & 43.95 \\
3 & 36.21\end{array}$ & $\begin{array}{l}52.44 \\
52.35 \\
52.26 \\
52.17\end{array}$ & $\begin{array}{l}42550 \\
42846 \\
43144 \\
43444 \\
43745 \\
44048\end{array}$ & $\begin{array}{l}296 \\
298 \\
300 \\
301 \\
303 \\
305\end{array}$ & $\begin{array}{c}334^{2} \\
50^{\prime} \\
40 \\
30 \\
20 \\
10\end{array}$ & $\begin{array}{l}36^{\prime} \\
10^{\prime} \\
20 \\
30 \\
40 \\
50\end{array}$ & $\begin{array}{ll}3 & 1 \\
3 & 1 \\
3 & 2 \\
3 & 2 \\
3 & 2 \\
3 & 2\end{array}$ & $\begin{array}{rr}18 & 56.88 \\
19 & 43.31 \\
20 & 29.63 \\
21 & 15.83 \\
22 & 1.91 \\
22 & 47.89\end{array}$ & $\begin{array}{l}46.43 \\
46.3^{2} \\
46.20 \\
46.08 \\
45.97 \\
45.84\end{array}$ & $\begin{array}{l}63200 \\
63592 \\
63985 \\
64350 \\
64776 \\
65173\end{array}$ & $\begin{array}{l}392 \\
393 \\
395 \\
396 \\
397 \\
399\end{array}$ & $\begin{array}{c}324^{\circ} \\
50^{\prime} \\
40 \\
30 \\
20 \\
10\end{array}$ \\
\hline $\begin{array}{r}27^{\circ} \\
10^{\prime} \\
20 \\
30 \\
40 \\
50\end{array}$ & $\begin{array}{ll}2 & 34 \\
2 & 35 \\
2 & 36 \\
2 & 37 \\
2 & 37 \\
2 & 38\end{array}$ & $\begin{array}{rr}4 & 29.38 \\
5 & 20.46 \\
6 & 12.44 \\
7 & 4.34 \\
7 & 56.14 \\
8 & 47.85\end{array}$ & $\begin{array}{l}52.08 \\
51.98 \\
51.90 \\
51.80 \\
51.71 \\
51.61\end{array}$ & $\begin{array}{l}44353 \\
44659 \\
44967 \\
45277 \\
45588 \\
45901\end{array}$ & $\begin{array}{l}306 \\
308 \\
310 \\
311 \\
313 \\
315\end{array}$ & $\begin{array}{c}333^{\circ} \\
50^{\prime} \\
40 \\
30 \\
20 \\
10\end{array}$ & $\begin{array}{l}37 \\
10 \\
20 \\
30 \\
40 \\
50\end{array}$ & & $\begin{array}{rr}23 & 33.72 \\
24 & 19.44 \\
25 & 5.04 \\
25 & 50.53 \\
26 & 35.89 \\
27 & 21.13\end{array}$ & $\begin{array}{l}45.72 \\
45.60 \\
45.49 \\
45 \cdot 3^{6} \\
45.24 \\
45.12\end{array}$ & $\begin{array}{l}65572 \\
65472 \\
66373 \\
66776 \\
67180 \\
67585\end{array}$ & $\begin{array}{l}400 \\
401 \\
403 \\
404 \\
405 \\
407\end{array}$ & $\begin{array}{c}323^{\circ} \\
50^{\prime} \\
40 \\
30 \\
20 \\
10\end{array}$ \\
\hline $\begin{array}{c}28^{\circ} \\
10^{\prime} \\
20 \\
30 \\
40 \\
50\end{array}$ & $\begin{array}{ll}2 & 39 \\
2 & 40 \\
2 & 41 \\
2 & 42 \\
2 & 43 \\
2 & 43\end{array}$ & $\begin{array}{rr}9 & 39.46 \\
0 & 30.98 \\
1 & 22.40 \\
2 & 13.72 \\
3 & 4.95 \\
3 & 56.08\end{array}$ & $\begin{array}{l}51.01 \\
51.52 \\
51.42 \\
51.32 \\
51.23 \\
51.13 \\
51.04\end{array}$ & $\begin{array}{l}46216 \\
46532 \\
46850 \\
47170 \\
47491 \\
47814\end{array}$ & $\begin{array}{l}316 \\
318 \\
320 \\
321 \\
323 \\
324\end{array}$ & $\begin{array}{c}332^{\prime} \\
50^{\prime} \\
40 \\
30 \\
20 \\
10\end{array}$ & $\begin{array}{l}38^{\circ} \\
10^{\prime} \\
20 \\
30 \\
40 \\
50\end{array}$ & $\begin{array}{ll}3 & 2 \\
3 & 2 \\
3 & 2 \\
3 & 3 \\
3 & 3 \\
3 & 3\end{array}$ & $\begin{array}{rr}28 & 6.25 \\
28 & 51.25 \\
29 & 36.13 \\
30 & 20.90 \\
31 & 5.54 \\
31 & 50.05\end{array}$ & $\begin{array}{l}45.00 \\
44.88 \\
44.77 \\
44.64 \\
44.52 \\
44.40\end{array}$ & $\begin{array}{l}67992 \\
68400 \\
68810 \\
69221 \\
69633 \\
70047\end{array}$ & $\begin{array}{l}408 \\
410 \\
411 \\
412 \\
414 \\
416\end{array}$ & $\begin{array}{c}322^{\circ} \\
50^{\prime} \\
40 \\
30 \\
20 \\
10\end{array}$ \\
\hline $\begin{array}{c}29^{\circ} \\
10^{\prime} \\
20 \\
30 \\
40 \\
50\end{array}$ & $\begin{array}{ll}2 & 4 \\
2 & 4 \\
2 & 4 \\
2 & 4 \\
2 & 4 \\
2 & 4\end{array}$ & $\begin{array}{rr}4 & 47.12 \\
5 & 38.06 \\
6 & 28.90 \\
7 & 19.64 \\
8 & 10.29 \\
9 & 0.83\end{array}$ & $\begin{array}{l}50.94 \\
50.84 \\
50.74 \\
50.65 \\
50.54 \\
50.45\end{array}$ & $\begin{array}{l}48138 \\
48464 \\
48792 \\
49122 \\
49453 \\
49786\end{array}$ & $\begin{array}{l}326 \\
328 \\
330 \\
331 \\
333 \\
335\end{array}$ & $\begin{array}{c}331^{\circ} \\
50^{\prime} \\
40 \\
30 \\
20 \\
10\end{array}$ & $\begin{array}{l}39^{2} \\
10^{\prime} \\
20 \\
30 \\
40 \\
50\end{array}$ & $\begin{array}{ll}3 & 3 \\
3 & 3 \\
3 & 3 \\
3 & 3 \\
3 & 3 \\
3 & 3\end{array}$ & $\begin{array}{rr}32 & 34.45 \\
33 & 18.72 \\
34 & 2.86 \\
34 & 46.88 \\
35 & 30.78 \\
36 & 14.55\end{array}$ & $\begin{array}{l}44.40 \\
44.27 \\
44.02 \\
43.90 \\
43.77 \\
43.64\end{array}$ & $\begin{array}{r}70463 \\
70880 \\
71298 \\
71717 \\
72138 \\
72560\end{array}$ & $\begin{array}{l}417 \\
418 \\
419 \\
420 \\
422 \\
424\end{array}$ & $\begin{array}{c}321^{\circ} \\
50^{\prime} \\
40 \\
30 \\
20 \\
10\end{array}$ \\
\hline $30^{2}$ & 24 & 951.28 & & $\begin{array}{r}50121 \\
1.26 \\
\end{array}$ & & $\begin{array}{c}330^{\circ} \\
g\end{array}$ & $40^{2}$ & 33 & 3658.19 & & $\begin{array}{r}72984 \\
1.26\end{array}$ & & $\begin{array}{c}320^{\circ} \\
g\end{array}$ \\
\hline
\end{tabular}




\begin{tabular}{|c|c|c|c|c|c|c|c|c|c|c|c|c|c|c|}
\hline \multirow[b]{2}{*}{$g$} & \multicolumn{11}{|c|}{ TABI.F VII.-Continued. } & \multirow{2}{*}{\multicolumn{2}{|c|}{ Log. $r$}} & \\
\hline & \multicolumn{4}{|c|}{$E$} & \multicolumn{2}{|c|}{ I,og. r } & & $g$ & \multicolumn{3}{|c|}{$\boldsymbol{E}$} & & & \multirow[b]{3}{*}{$310^{\circ}$} \\
\hline & $\circ$ & 1 & $"$ & $"$ & 1.26 & & & & 01 & $1 "$ & $"$ & 1.27 & & \\
\hline $40^{\circ}$ & 33 & $36:$ & 59.19 & & 72994 & & $320^{\circ}$ & $50^{\circ}$ & 410 & 634.27 & & 00687 & & \\
\hline $10^{\prime}$ & 33 & 37 & $\$ 1.71$ & $\begin{array}{l}43 \cdot 5^{2} \\
43 \cdot 39\end{array}$ & 73409 & $\begin{array}{l}425 \\
426\end{array}$ & $50^{\prime}$ & $10^{\prime}$ & +17 & $\begin{array}{ll}7 & 9.62\end{array}$ & $35 \cdot 35$ & 01183 & 490 & $50^{\prime}$ \\
\hline 20 & 33 & 39 & 25.10 & $\begin{array}{l}43.39 \\
43.27\end{array}$ & 73835 & 420 & 10 & 20 & 417 & 744.82 & & 01681 & 498 & 10 \\
\hline 30 & 33 & 39 & 8.37 & $\begin{array}{l}43.27 \\
43.14\end{array}$ & $\begin{array}{l}7262 \\
-4691\end{array}$ & $\begin{array}{l}427 \\
429\end{array}$ & 30 & 30 & 418 & 819.88 & 35 & 02180 & $\begin{array}{l}499 \\
500\end{array}$ & 30 \\
\hline 40 & 33 & 39 : & 51.51 & 43.01 & 74691 & $\begin{array}{l}429 \\
430\end{array}$ & 20 & 40 & 418 & 854.80 & 34.77 & 02680 & 501 & 20 \\
\hline 50 & 34 & 40 & 34.52 & 42.88 & 75121 & $43^{2}$ & 10 & 50 & +19 & 929.57 & 34.62 & 03181 & 502 & 10 \\
\hline $\begin{array}{l}41^{\circ} \\
10^{\prime}\end{array}$ & 34 & 11 & $\begin{array}{r}17.40 \\
0.16\end{array}$ & 42.76 & & 433 & $\begin{array}{r}319^{\circ} \\
50^{\prime}\end{array}$ & $51^{\circ}$ & 420 & $\begin{array}{r}4.19 \\
39.67\end{array}$ & 34.48 & 03683 & 503 & $309^{\circ}$ \\
\hline $\begin{array}{l}10^{\prime} \\
20\end{array}$ & 34 & 42 & $\begin{array}{r}0.16 \\
42.79\end{array}$ & 42.63 & $\begin{array}{l}75986 \\
76420\end{array}$ & 434 & $50^{\circ}$ & $10^{\prime}$ & 420 & $\begin{array}{ll}0 & 35 \\
1 & 13\end{array}$ & 34 & & 504 & $50^{\prime}$ \\
\hline $\begin{array}{l}20 \\
30\end{array}$ & 34 & 12 & $\begin{array}{l}42.79 \\
25.29\end{array}$ & 42.50 & 78956 & $43^{6}$ & 40 & 20 & +21 & 13 & 34.18 & 0 & 505 & 40 \\
\hline $\begin{array}{l}30 \\
40\end{array}$ & 34 & 43 & $\begin{array}{r}23.29 \\
7.666\end{array}$ & 42.37 & 77293 & 437 & 20 & & 421 & 14 & 34.04 & 5 & & 80 \\
\hline $\begin{array}{l}40 \\
50\end{array}$ & 34 & 3 & $\begin{array}{r}7.66 \\
\mathbf{4 9 . 9 1}\end{array}$ & 42.25 & 77731 & $43^{8}$ & $\begin{array}{l}20 \\
10\end{array}$ & 10 & +22 & $\begin{array}{ll}2 & 21 \\
2 & 55\end{array}$ & 33.89 & 00 & 506 & 20 \\
\hline 42 & 34 & 45 & 32.02 & 42. & 78171 & 440 & 0 & 50 & 422 & 255.11 & $33 \cdot 74$ & 062 & 507 & 10 \\
\hline $\begin{array}{l}42 \\
10^{\prime}\end{array}$ & $\begin{array}{ll}3 & 4 \\
34 & 4\end{array}$ & 46 & 14.01 & $\begin{array}{l}41.99 \\
41.85\end{array}$ & 75611 & 440 & $50^{\circ}$ & $\begin{array}{l}52 \\
10^{\prime}\end{array}$ & $\begin{array}{l}423 \\
+24\end{array}$ & $\begin{array}{r}328.85 \\
4 \quad 2.44\end{array}$ & 33.59 & $\begin{array}{l}06714 \\
07229\end{array}$ & 508 & $308^{\circ}$ \\
\hline 20 & 34 & 16 & 55.86 & $\begin{array}{l}41.05 \\
41.72\end{array}$ & 79053 & 442 & 40 & 20 & 424 & $4 \quad 35.88$ & & & 510 & $\begin{array}{l}50^{\prime} \\
10\end{array}$ \\
\hline 30 & 34 & 47 & 37.59 & $\begin{array}{l}4 \\
4\end{array}$ & 79496 & 443 & 30 & 30 & 42.5 & $5 \quad 9.18$ & 33 & 3 & 511 & 10 \\
\hline 40 & 34 & 49 & 19.17 & $\begin{array}{r}41.59 \\
41.46\end{array}$ & 79041 & $\begin{array}{l}4.45 \\
4.16\end{array}$ & 20 & $\$ 0$ & +25 & $\begin{array}{ll}5 & 42.32\end{array}$ & & 08754 & 5 & 20 \\
\hline 50 & 34 & & 0.63 & 41.33 & s03st & $\begin{array}{l}4.40 \\
448\end{array}$ & 10 & 50 & 420 & $6 \quad 15.32$ & & 09266 & 512 & $\begin{array}{l}20 \\
10\end{array}$ \\
\hline $43^{\circ}$ & 34 & 19 & 41.96 & & 80835 & & $317^{\circ}$ & $53^{\circ}$ & 426 & 648.16 & & 09780 & 5 & $307^{\circ}$ \\
\hline $10^{\prime}$ & 35 & 0 & 23.16 & 41.20 & 81284 & 449 & $50^{\prime}$ & $10^{\prime}$ & 427 & & $3^{2.69}$ & & 514 & $50^{\prime}$ \\
\hline 20 & 35 & 51 & 4.22 & 41. & 81733 & 449 & 40 & 20 & 427 & 753 & 32.54 & & 515 & 40 \\
\hline 30 & 35 & 51 & 45.15 & 40.93 & 82183 & $45^{\circ}$ & 30 & 30 & +28 & 825 & 32.4 & 1 & & 30 \\
\hline to & 35 & 32 & 25.94 & $\begin{array}{l}40.79 \\
40.66\end{array}$ & 826,35 & $45^{2}$ & 20 & 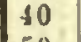 & 429 & 855 & 3 & & 7 & 20 \\
\hline 50 & 35 & 53 & 6.60 & $\begin{array}{l}40.00 \\
40.53\end{array}$ & 83089 & $\begin{array}{l}454 \\
455\end{array}$ & 11 & 50 & 429 & 930.12 & & 12361 & 30 & 10 \\
\hline $44^{\circ}$ & 35 & 53 & 47.13 & 40.39 & 83544 & 456 & $316^{\circ}$ & $54^{\circ}$ & 430 & $\begin{array}{ll}0 & 2.06\end{array}$ & & 12881 & & $306^{\circ}$ \\
\hline 2 & 35 & 54 & $\begin{array}{r}27.52 \\
7.78\end{array}$ & 40.26 & $\begin{array}{l}8 \\
8\end{array}$ & 457 & $F_{2}$ & ' & 4 & 03 & 4 & 1 & 521 & $50^{\prime}$ \\
\hline$\frac{2}{3}$ & 35 & 55 & $\begin{array}{r}7.79 \\
+7.90\end{array}$ & 40.12 & $\begin{array}{l}8 \\
8\end{array}$ & 457 & 4 & 12 & 431 & 15 & 31.49 & & 522 & $\begin{array}{l}40 \\
30\end{array}$ \\
\hline $\begin{array}{l}30 \\
40\end{array}$ & 35 & 55 & $\begin{array}{l}47.90 \\
27.89\end{array}$ & 39.99 & $\begin{array}{l}84914 \\
85373\end{array}$ & 459 & $\begin{array}{l}30 \\
20\end{array}$ & 3 & 431 & $\begin{array}{rr}1 & 36 \\
2 & 8\end{array}$ & 31.33 & & 522 & $\begin{array}{l}30 \\
20\end{array}$ \\
\hline $\begin{array}{l}10 \\
50\end{array}$ & $\begin{array}{ll}3 & 5 \\
3 & 5\end{array}$ & 56 & $\begin{array}{r}27.89 \\
7.75\end{array}$ & 39.86 & 85834 & 461 & $\begin{array}{l}20 \\
10\end{array}$ & $\begin{array}{l}40 \\
50\end{array}$ & $\begin{array}{r}432 \\
432\end{array}$ & $\begin{array}{rr}2 & 8.31 \\
2 & 39.50\end{array}$ & 31 & $\begin{array}{l}14966 \\
15490\end{array}$ & 524 & $\begin{array}{l}20 \\
10\end{array}$ \\
\hline $45^{\circ}$ & 35 & 57 & 47.47 & $39 \cdot 7$ & 86297 & 463 & $315^{\circ}$ & $55^{\circ}$ & 433 & $3 \quad 10.53$ & $3^{1}$ & 16015 & 525 & 10 \\
\hline $10^{\circ}$ & 35 & 59 & 27.05 & $39.5^{8}$ & 86760 & $46_{3}$ & $50^{\prime}$ & $10^{\prime}$ & $\begin{array}{r}43 \\
+\quad 3\end{array}$ & 34 & $3^{\circ}$ & & 525 & $50{ }^{\prime}$ \\
\hline 20 & 35 & 59 & 6.50 & $39 \cdot 45$ & 87224 & 464 & 40 & 20 & 434 & 412 & 3 & & 7 & 10 \\
\hline 30 & 35 & 59 & 45.81 & 39. & 87690 & $\begin{array}{l}466 \\
466\end{array}$ & 3 & 30 & 434 & +42 & 7 & 1 & & 30 \\
\hline$\$ 0$ & 4 & 0 & 24.99 & 39. & 88156 & $\begin{array}{l}466 \\
468\end{array}$ & 20 & 40 & 435 & $\begin{array}{ll}5 & 13.13\end{array}$ & & 14 & 28 & 20 \\
\hline 50 & 4 & 1 & 4.01 & $\begin{array}{l}39.03 \\
38.89\end{array}$ & 89624 & $\begin{array}{l}408 \\
469\end{array}$ & 1 & 50 & 435 & $5 \quad 43.10$ & $\begin{array}{l}30.27 \\
30.12\end{array}$ & 18652 & $\begin{array}{l}529 \\
530\end{array}$ & 10 \\
\hline $46^{\circ}$ & 4 & 1 & 42.90 & & 89093 & & $314^{\circ}$ & $56^{\circ}$ & 430 & 613.52 & & 19182 & & $304^{\circ}$ \\
\hline $10^{\prime}$ & 4 & 2 & 21.65 & $\begin{array}{l}30 \\
38\end{array}$ & 89564 & $\begin{array}{l}471 \\
471\end{array}$ & 50 & $10^{\prime}$ & 430 & 643 & 29. & & 31 & $50^{\prime}$ \\
\hline 20 & 4 & 3 & 0.27 & & 90035 & $\begin{array}{l}47 \\
47\end{array}$ & 4 & 20 & 437 & 713.29 & & 3 & & 40 \\
\hline 30 & 1 & 3 & 39.75 & & 90507 & 47 & 3 & 3 & 437 & $74:$ & & 6 & & 30 \\
\hline 40 & 4 & 4 & 17.09 & 38. & 90930 & 47 & 2 & 4 & 438 & 812 & & $21:$ & 533 & 20 \\
\hline 50 & 4 & 4 & 55.30 & 38.06 & 91455 & $\begin{array}{l}475 \\
476\end{array}$ & 1 & 50 & 438 & $8+1.77$ & 29.1 & 21842 & 534 & 10 \\
\hline $47^{\circ}$ & 4 & 5 & 33.36 & & 91931 & & $313^{\circ}$ & $57^{\circ}$ & 439 & 910.96 & & 22376 & & $303^{\circ}$ \\
\hline $10^{\prime}$ & 4 & 6 & 11 & $\begin{array}{l}3 \\
3\end{array}$ & 9 & $\begin{array}{l}47 \\
47\end{array}$ & 1 & $10^{\prime}$ & 439 & 939.99 & $\begin{array}{l}29 \\
28\end{array}$ & & 535 & $50^{\prime}$ \\
\hline 20 & 1 & f & 49.07 & & 92896 & $\begin{array}{l}478 \\
479\end{array}$ & 4 & 20 & 440 & ) 8.57 & & & & 40 \\
\hline$\ddot{s}$ & 4 & 1 & 26.72 & & 03365 & $\begin{array}{l}479 \\
480\end{array}$ & 3 & 3 & 440 & 037.59 & & 6 & & 80 \\
\hline 40 & 4 & 8 & 4.22 & 37 & 93345 & $\begin{array}{l}480 \\
482\end{array}$ & 20 & 4 & 441 & 16.16 & & & 53 & 20 \\
\hline 50 & 4 & 8 & 41.58 & 37.2 & & 483 & & 5 & 441 & 134.57 & 28.26 & 25063 & 540 & 10 \\
\hline $48^{\circ}$ & 4 & 9 & 19.90 & 37. & $94 \$ 10$. & $4 \varepsilon$ & $312^{\circ}$ & $58^{\circ}$ & 442 & 2.83 & 28.10 & 25603 & & $302^{\circ}$ \\
\hline 2 & 4 & 9 & 55.88 & $3^{6}$ & & 48 & $50^{\prime}$ & $10^{\prime}$ & 445 & $\begin{array}{l}230.93 \\
258.87\end{array}$ & 2 & 98 & 541 & 50 \\
\hline $\begin{array}{l}2 \\
3\end{array}$ & 11 & 10 & 32.81 & & $\begin{array}{l}5779 \\
6265\end{array}$ & 486 & $\begin{array}{l}4 \\
3\end{array}$ & 2 & 442 & $\begin{array}{ll}2 & 58.87 \\
3 & 26.66\end{array}$ & 27 & 27226 & 5 & 4 \\
\hline $\begin{array}{l}3 \\
4\end{array}$ & 41 & 11 & $\begin{array}{r}9.60 \\
46.25\end{array}$ & $3^{6}$. & $\begin{array}{l}96265 \\
96752\end{array}$ & 487 & $\begin{array}{l}3 \\
2\end{array}$ & 3 & $44 i$ & $\begin{array}{ll}3 & 26.66 \\
3 & 54.29\end{array}$ & & 27 & 542 & 30 \\
\hline $\begin{array}{l}40 \\
50\end{array}$ & 4 & 11 & $\begin{array}{l}46.25 \\
22.76\end{array}$ & 36. & $\begin{array}{l}96752 \\
97240\end{array}$ & 488 & $\begin{array}{l}2 \\
1\end{array}$ & 4 & $44:$ & $\begin{array}{r}354.29 \\
+\quad 21.76\end{array}$ & 27.47 & & 543 & $\begin{array}{l}20 \\
10\end{array}$ \\
\hline $49^{\circ}$ & 4 & & & & 96070 & 490 & 110 & & 44 & $\begin{array}{r}+21.76 \\
+49.07\end{array}$ & $27 \cdot 31$ & & 544 & 10 \\
\hline $10^{\prime}$ & 4 & 13 & 35.34 & $3^{6.22}$ & 38 & 490 & $\begin{array}{r}311 \\
50\end{array}$ & $59^{\circ}$ & 44 & $\begin{array}{ll}4 & 49.07 \\
5 & 16.23\end{array}$ & 27.16 & 29855 & & $\begin{array}{r}301^{\circ} \\
50^{\prime}\end{array}$ \\
\hline 20 & 41 & 14 & 11.41 & $3^{6.07}$ & 98711 & 491 & $\begin{array}{l}50^{\circ} \\
40\end{array}$ & & +4 & 3 & & & 54 & $\begin{array}{l}50^{\prime} \\
40\end{array}$ \\
\hline 30 & 4 & 14 & 47.34 & 35.93 & 99203 & $49^{2}$ & 30 & & 44 & $\begin{array}{ll}5 & 43.22 \\
6 & 10.06\end{array}$ & 26.84 & 1 & 547 & $\begin{array}{l}40 \\
30\end{array}$ \\
\hline 40 & 4 & 15 & 23.13 & 35.79 & 99636 & 493 & 20 & 4 & 44 & $\begin{array}{l}10.065 \\
36.75\end{array}$ & 26.69 & & 547 & 20 \\
\hline 50 & 4 & 15 & 58.77 & $\begin{array}{l}35.64 \\
35.50\end{array}$ & $\neq 00191$ & 495 & 10 & $\begin{array}{l}40 \\
50\end{array}$ & $\begin{array}{ll}4 & 4 \\
4 & 4\end{array}$ & $\begin{array}{rr}6 & 36.79 \\
7 & 3.27\end{array}$ & 26.52 & 31587 & 549 & $\begin{array}{l}20 \\
10\end{array}$ \\
\hline $50^{2}$ & 4 & 16 & 34.27 & & $\neq 0$ & & $310^{\circ}$ & $60^{2}$ & 44 & 729.64 & & 32137 & 50 & $300^{\circ}$ \\
\hline & & & & & & & & & & & & 1.21 & & 9 \\
\hline
\end{tabular}

28 Juno, 1873. 
TABLE VII.-Continued.

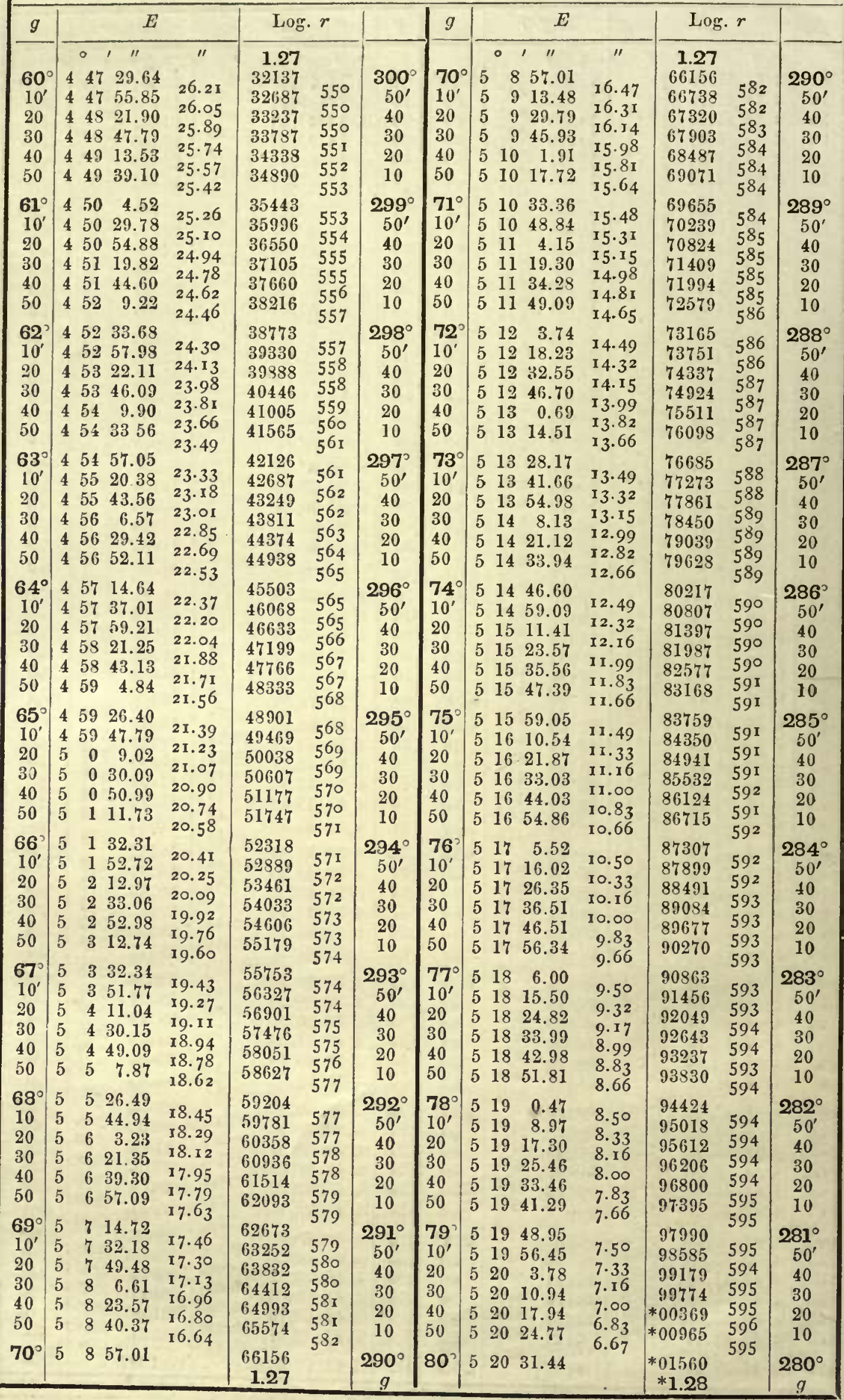




\begin{tabular}{|c|c|c|c|c|c|c|c|c|c|c|c|c|c|c|}
\hline \multirow[b]{2}{*}{$g$} & \multicolumn{14}{|c|}{ TABI. FII.-Continued. } \\
\hline & & & $b$ & & Log. & & & $g$ & & $E$ & & Log. & $r$ & \\
\hline $\begin{array}{l}80^{\circ} \\
10^{\prime} \\
20 \\
30 \\
40 \\
50\end{array}$ & $\begin{array}{l}\circ \\
5 \\
5 \\
5 \\
5 \\
5 \\
5\end{array}$ & $\begin{array}{l}. \\
20 \\
20 \\
20 \\
20 \\
20 \\
21\end{array}$ & $\begin{array}{c}11 \\
31.41 \\
37.94 \\
44.23 \\
50.45 \\
56.40 \\
2.30\end{array}$ & $\begin{array}{c}11 \\
6.50 \\
6.34 \\
6.17 \\
6.01 \\
5.84 \\
5.66\end{array}$ & $\begin{array}{l}1.28 \\
01560 \\
02155 \\
02750 \\
03346 \\
03941 \\
04536\end{array}$ & $\begin{array}{l}595 \\
595 \\
596 \\
595 \\
595 \\
595\end{array}$ & $\begin{array}{c}280^{\circ} \\
50^{\circ} \\
40 \\
30 \\
20 \\
10\end{array}$ & $\begin{array}{l}90^{\circ} \\
10^{\prime} \\
20 \\
30 \\
40 \\
50\end{array}$ & $\begin{array}{lc}0 & 1 \\
5 & 22 \\
5 & 22 \\
5 & 22 \\
5 & 21 \\
5 & 21 \\
5 & 21\end{array}$ & $\begin{array}{l}\not 1 \\
9.05 \\
5.68 \\
2.15 \\
59.45 \\
54.59 \\
50.58\end{array}$ & $\begin{array}{l}3.37 \\
3.53 \\
3.70 \\
3.86 \\
4.01 \\
4.18\end{array}$ & $\begin{array}{r}1.28 \\
37187 \\
37776 \\
39365 \\
39954 \\
39543 \\
40131\end{array}$ & $\begin{array}{l}589 \\
589 \\
589 \\
589 \\
588 \\
588\end{array}$ & $\begin{array}{c}270^{\circ} \\
50 \\
40 \\
30 \\
20 \\
10\end{array}$ \\
\hline $\begin{array}{l}81^{\circ} \\
10^{\prime} \\
20 \\
30 \\
40 \\
50\end{array}$ & $\begin{array}{l}5 \\
5 \\
5 \\
5 \\
5 \\
5\end{array}$ & $\begin{array}{l}21 \\
21 \\
21 \\
21 \\
21 \\
21\end{array}$ & $\begin{array}{l}7.96 \\
13.46 \\
18.80 \\
23.97 \\
28.97 \\
33.81\end{array}$ & $\begin{array}{l}5.50 \\
5.34 \\
5.17 \\
5.00 \\
4.84 \\
4.67\end{array}$ & $\begin{array}{l}05131 \\
05726 \\
06322 \\
06917 \\
07512 \\
08107\end{array}$ & $\begin{array}{l}595 \\
596 \\
595 \\
595 \\
595 \\
595\end{array}$ & $\begin{array}{c}279^{\circ} \\
50^{\circ} \\
40 \\
30 \\
20 \\
10\end{array}$ & $\begin{array}{l}91^{\circ} \\
10^{\circ} \\
20 \\
30 \\
40 \\
50\end{array}$ & $\begin{array}{ll}5 & 21 \\
5 & 21 \\
5 & 21 \\
5 & 21 \\
5 & 21 \\
5 & 21\end{array}$ & $\begin{array}{l}46.40 \\
42.06 \\
37.57 \\
32.91 \\
28.09 \\
23.11\end{array}$ & $\begin{array}{l}4.34 \\
4.49 \\
4.66 \\
4.82 \\
4.98 \\
5.14\end{array}$ & $\begin{array}{l}40719 \\
41307 \\
41894 \\
42482 \\
43069 \\
43655\end{array}$ & $\begin{array}{l}588 \\
587 \\
588 \\
587 \\
586 \\
587\end{array}$ & $\begin{array}{c}269^{\circ} \\
50 \\
10 \\
30 \\
20 \\
10\end{array}$ \\
\hline $\begin{array}{l}82^{\circ} \\
10^{\prime} \\
20 \\
30 \\
40 \\
50\end{array}$ & $\begin{array}{l}5 \\
5 \\
5 \\
5 \\
5 \\
5\end{array}$ & $\begin{array}{l}21 \\
21 \\
21 \\
21 \\
21 \\
21\end{array}$ & $\begin{array}{l}38.49 \\
42.99 \\
47.33 \\
51.50 \\
55.51 \\
59.36\end{array}$ & $\begin{array}{l}4.51 \\
4.34 \\
4.17 \\
4.01 \\
3.85 \\
3.68\end{array}$ & $\begin{array}{l}09702 \\
09297 \\
09893 \\
10493 \\
11093 \\
11679\end{array}$ & $\begin{array}{l}595 \\
596 \\
595 \\
595 \\
596\end{array}$ & $\begin{array}{c}278^{\circ} \\
50^{\circ} \\
40 \\
30 \\
20 \\
10\end{array}$ & $\begin{array}{l}92^{\circ} \\
10^{\prime} \\
20 \\
30 \\
40 \\
50\end{array}$ & $\begin{array}{ll}5 & 21 \\
5 & 21 \\
5 & 21 \\
5 & 21 \\
5 & 20 \\
5 & 20\end{array}$ & $\begin{array}{r}17.97 \\
12.67 \\
7.20 \\
1.58 \\
55.79 \\
49.85\end{array}$ & $\begin{array}{l}5.30 \\
5.47 \\
5.62 \\
5.79 \\
5.94 \\
6.11\end{array}$ & $\begin{array}{l}44242 \\
44928 \\
45414 \\
46000 \\
46585 \\
47170\end{array}$ & $\begin{array}{l}586 \\
586 \\
586 \\
585 \\
585 \\
585\end{array}$ & $\begin{array}{c}268^{\circ} \\
50^{\prime} \\
40 \\
30 \\
20 \\
10\end{array}$ \\
\hline $\begin{array}{c}83^{\circ} \\
10^{\prime} \\
20 \\
30 \\
40 \\
50\end{array}$ & $\begin{array}{l}5 \\
5 \\
5 \\
5 \\
5 \\
5\end{array}$ & $\begin{array}{l}22 \\
22 \\
22 \\
22 \\
22 \\
22\end{array}$ & $\begin{array}{r}3.04 \\
6.56 \\
9.91 \\
13.09 \\
16.11 \\
18.97\end{array}$ & $\begin{array}{l}3.52 \\
3.35 \\
3.18 \\
3.02 \\
2.86 \\
2.69\end{array}$ & $\begin{array}{l}12274 \\
12369 \\
13465 \\
14060 \\
14655 \\
15250\end{array}$ & $\begin{array}{l}595 \\
596 \\
595 \\
595 \\
595 \\
596\end{array}$ & $\begin{array}{c}277^{\circ} \\
50^{\circ} \\
40 \\
30 \\
20 \\
10\end{array}$ & $\begin{array}{l}93^{\circ} \\
10^{\prime} \\
20 \\
30 \\
40 \\
50\end{array}$ & $\begin{array}{ll}5 & 20 \\
5 & 20 \\
5 & 20 \\
5 & 20 \\
5 & 20 \\
5 & 20\end{array}$ & $\begin{array}{l}43.74 \\
37.49 \\
31.05 \\
24.47 \\
17.72 \\
10.82\end{array}$ & $\begin{array}{l}0.11 \\
6.26 \\
6.43 \\
6.58 \\
6.75 \\
6.90 \\
7.06\end{array}$ & & $\begin{array}{l}585 \\
584 \\
584 \\
584 \\
583 \\
5^{8} 8 \\
583\end{array}$ & $\begin{array}{c}267^{\circ} \\
50^{\prime} \\
40 \\
30 \\
20 \\
10\end{array}$ \\
\hline $\begin{array}{l}84^{\circ} \\
10^{\prime} \\
20 \\
30 \\
40 \\
50 \\
0=0\end{array}$ & $\begin{array}{l}5 \\
5 \\
5 \\
5 \\
5 \\
5\end{array}$ & $\begin{array}{l}22 \\
22 \\
22 \\
22 \\
22 \\
23\end{array}$ & $\begin{array}{l}21.66 \\
24.19 \\
26.55 \\
29.75 \\
30.78 \\
32.64\end{array}$ & $\begin{array}{l}2.53 \\
2.36 \\
2.20 \\
2.03 \\
1.86 \\
1.70\end{array}$ & $\begin{array}{l}15946 \\
16441 \\
17036 \\
17631 \\
19226 \\
18820\end{array}$ & $\begin{array}{l}595 \\
595 \\
595 \\
595 \\
594 \\
595\end{array}$ & $\begin{array}{c}276^{\circ} \\
50^{\circ} \\
40 \\
30 \\
20 \\
10\end{array}$ & $\begin{array}{l}34^{\circ} \\
10^{\prime} \\
20 \\
30 \\
40 \\
50\end{array}$ & $\begin{array}{ll}5 & 20 \\
5 & 19 \\
5 & 19 \\
5 & 19 \\
5 & 19 \\
5 & 19\end{array}$ & $\begin{array}{r}3.76 \\
56.54 \\
49.16 \\
41.62 \\
33.93 \\
26.07\end{array}$ & $\begin{array}{l}7.22 \\
7.38 \\
7.54 \\
7.69 \\
7.86 \\
8.01\end{array}$ & $\begin{array}{l}51256 \\
51839 \\
52420 \\
53002 \\
53593 \\
5 \$ 163\end{array}$ & $\begin{array}{l}503 \\
582 \\
582 \\
582 \\
581 \\
580 \\
580\end{array}$ & $\begin{array}{c}266^{3} \\
50^{\prime} \\
40 \\
30 \\
20 \\
10\end{array}$ \\
\hline $\begin{array}{l}85^{\circ} \\
10^{\prime} \\
20 \\
30 \\
40 \\
50\end{array}$ & $\begin{array}{l}5 \\
5 \\
5 \\
5 \\
5 \\
5\end{array}$ & $\begin{array}{l}22 \\
22 \\
22 \\
22 \\
22 \\
23\end{array}$ & $\begin{array}{l}34.34 \\
35.87 \\
37.24 \\
39.44 \\
39.49 \\
40.35\end{array}$ & $\begin{array}{l}0.87 \\
0.71\end{array}$ & $\begin{array}{l}19415 \\
20010 \\
20604 \\
21199 \\
21792 \\
22396\end{array}$ & $\begin{array}{l}595 \\
594 \\
594 \\
594 \\
594 \\
594\end{array}$ & $\begin{array}{c}275^{\circ} \\
50^{\prime} \\
40 \\
30 \\
20 \\
10\end{array}$ & $\begin{array}{l}95^{\circ} \\
10^{\prime} \\
20 \\
30 \\
40 \\
50\end{array}$ & $\begin{array}{ll}5 & 19 \\
5 & 19 \\
5 & 19 \\
5 & 18 \\
5 & 18 \\
5 & 18\end{array}$ & $\begin{array}{r}18.06 \\
9.89 \\
1.56 \\
53.06 \\
44.43 \\
35.61\end{array}$ & $\begin{array}{l}8.17 \\
8.33 \\
8.50 \\
8.64 \\
8.81 \\
8.96\end{array}$ & & $\begin{array}{l}580 \\
579 \\
579 \\
579 \\
578 \\
578\end{array}$ & $\begin{array}{c}265^{\circ} \\
50^{\prime} \\
40 \\
30 \\
20 \\
10\end{array}$ \\
\hline $\begin{array}{l}86^{\circ} \\
10^{\prime} \\
20 \\
30 \\
40 \\
50\end{array}$ & $\begin{array}{l}5 \\
5 \\
5 \\
5\end{array}$ & $\begin{array}{l}22 \\
22 \\
22 \\
22 \\
22 \\
22 \\
92\end{array}$ & $\begin{array}{l}41.06 \\
41.60 \\
41.99 \\
42.21 \\
42.26 \\
42.15\end{array}$ & $\begin{array}{l}0.54 \\
0.39 \\
0.22 \\
0.05 \\
0.11 \\
0.27\end{array}$ & $\begin{array}{l}22990 \\
23574 \\
24167 \\
24761 \\
25354 \\
25948\end{array}$ & $\begin{array}{l}594 \\
593 \\
594 \\
593 \\
594 \\
593\end{array}$ & $\begin{array}{c}274^{\circ} \\
50^{\prime} \\
40 \\
30 \\
20 \\
10\end{array}$ & $\begin{array}{l}96^{\circ} \\
10^{\prime} \\
20 \\
30 \\
40 \\
50\end{array}$ & $\begin{array}{ll}5 & 18 \\
5 & 18 \\
5 & 18 \\
5 & 17 \\
5 & 17 \\
5 & 17\end{array}$ & $\begin{array}{r}26.65 \\
17.53 \\
8.26 \\
59.83 \\
49.24 \\
39.50\end{array}$ & $\begin{array}{l}9.12 \\
9.27 \\
9.43 \\
9.59 \\
9.74 \\
9.90\end{array}$ & & $\begin{array}{l}578 \\
577 \\
577 \\
577 \\
576 \\
576\end{array}$ & $\begin{array}{c}264^{\circ} \\
50^{\prime} \\
40 \\
30 \\
20 \\
10\end{array}$ \\
\hline $\begin{array}{l}87^{\circ} \\
10^{\prime} \\
20 \\
30 \\
40 \\
50\end{array}$ & $\begin{array}{l}5 \\
5 \\
5 \\
5 \\
5 \\
5\end{array}$ & $\begin{array}{l}22 \\
22 \\
22 \\
22 \\
22 \\
22\end{array}$ & $\begin{array}{l}41.89 \\
41.45 \\
40.85 \\
40.09 \\
39.16 \\
38.07\end{array}$ & $\begin{array}{l}0.43 \\
0.60 \\
0.76 \\
0.93 \\
1.09 \\
1.25\end{array}$ & $\begin{array}{l}26,541 \\
27134 \\
27726 \\
29319 \\
23911 \\
29504\end{array}$ & $\begin{array}{l}593 \\
592 \\
593 \\
592 \\
593 \\
592\end{array}$ & $\begin{array}{c}273^{\prime} \\
50^{\circ} \\
40 \\
30 \\
20 \\
10\end{array}$ & $\begin{array}{l}97^{\circ} \\
10^{\prime} \\
20 \\
30 \\
40 \\
50\end{array}$ & $\begin{array}{ll}5 & 17 \\
5 & 17 \\
5 & 17 \\
5 & 16 \\
5 & 16 \\
5 & 16\end{array}$ & $\begin{array}{r}29.60 \\
19.54 \\
9.33 \\
58.96 \\
48.44 \\
37.76\end{array}$ & $\begin{array}{l}10.06 \\
10.21 \\
10.37 \\
10.52 \\
10.68 \\
10.84\end{array}$ & $\begin{array}{l}61677 \\
62252 \\
62827 \\
63401 \\
63975 \\
64548\end{array}$ & $\begin{array}{l}575 \\
575 \\
574 \\
574 \\
573\end{array}$ & $\begin{array}{c}263^{\circ} \\
50^{\prime} \\
10 \\
30 \\
20 \\
10\end{array}$ \\
\hline $\begin{array}{l}88^{\circ} \\
10^{\prime} \\
20 \\
30 \\
40 \\
50\end{array}$ & $\begin{array}{l}5 \\
5 \\
5 \\
5 \\
5 \\
5\end{array}$ & $\begin{array}{l}22 \\
22 \\
22 \\
22 \\
22 \\
22\end{array}$ & $\begin{array}{l}36.82 \\
35.40 \\
33.82 \\
32.03 \\
30.17 \\
28.10\end{array}$ & $\begin{array}{l}1.42 \\
1.58 \\
1.74 \\
1.91 \\
2.07 \\
2.24\end{array}$ & $\begin{array}{l}30096 \\
30689 \\
31290 \\
31872 \\
32464\end{array}$ & $\begin{array}{l}592 \\
592 \\
592 \\
592 \\
592 \\
591 \\
592\end{array}$ & $\begin{array}{c}272^{\circ} \\
50^{\circ} \\
40 \\
30 \\
20 \\
10\end{array}$ & $\begin{array}{l}98^{\circ} \\
10^{\prime} \\
20 \\
30 \\
40 \\
50\end{array}$ & $\begin{array}{ll}5 & 16 \\
5 & 16 \\
5 & 16 \\
5 & 15 \\
5 & 15 \\
5 & 15\end{array}$ & $\begin{array}{r}26.92 \\
15.93 \\
4.78 \\
53.48 \\
42.02 \\
30.41\end{array}$ & $\begin{array}{l}10.99 \\
11.15 \\
11.30 \\
11.46 \\
11.61 \\
11.77\end{array}$ & $\begin{array}{l}65120 \\
65692 \\
66264 \\
66936 \\
67407 \\
67977\end{array}$ & $\begin{array}{l}572 \\
572 \\
572 \\
571 \\
570 \\
570\end{array}$ & $\begin{array}{c}262^{\circ} \\
50^{\prime} \\
40 \\
30 \\
20 \\
10\end{array}$ \\
\hline $\begin{array}{c}89^{2} \\
10^{\prime} \\
20 \\
30 \\
40 \\
50\end{array}$ & $\begin{array}{l}5 \\
5 \\
5 \\
5 \\
5 \\
5\end{array}$ & $\begin{array}{l}22 \\
22 \\
22 \\
22 \\
22 \\
22\end{array}$ & $\begin{array}{l}25.96 \\
23.47 \\
20.91 \\
18.19 \\
15.31 \\
12.26\end{array}$ & $\begin{array}{l}2.39 \\
2.56 \\
2.72 \\
2.89 \\
3.05 \\
3.21\end{array}$ & $\begin{array}{l}33647 \\
34238 \\
34828 \\
35418 \\
36008 \\
36597\end{array}$ & $\begin{array}{l}591 \\
590 \\
590 \\
590 \\
589 \\
590\end{array}$ & $\begin{array}{c}271^{\circ} \\
50^{\circ} \\
40 \\
30 \\
20 \\
10\end{array}$ & $\begin{array}{l}99^{\prime} \\
10^{\prime} \\
20 \\
30 \\
40 \\
50\end{array}$ & $\begin{array}{ll}5 & 15 \\
5 & 15 \\
5 & 14 \\
5 & 14 \\
5 & 14 \\
5 & 14\end{array}$ & $\begin{array}{r}18.64 \\
6.73 \\
54.64 \\
42.41 \\
30.03 \\
17.49\end{array}$ & $\begin{array}{l}11.92 \\
12.08 \\
12.23 \\
12.38 \\
12.54 \\
12.69\end{array}$ & $\begin{array}{l}68547 \\
69117 \\
69686 \\
70255 \\
70823 \\
71391\end{array}$ & $\begin{array}{l}570 \\
569 \\
569 \\
568 \\
568 \\
567\end{array}$ & $\begin{array}{c}261^{\circ} \\
50^{\prime} \\
40 \\
30 \\
20 \\
10\end{array}$ \\
\hline $30^{2}$ & 5 & 22 & 9.05 & & $\begin{array}{r}371.97 \\
128\end{array}$ & & $\begin{array}{c}270^{\circ} \\
g\end{array}$ & $100^{\circ}$ & (5) 14 & 4.80 & & $\begin{array}{r}11959 \\
1.28\end{array}$ & & $\begin{array}{c}260^{\circ} \\
g\end{array}$ \\
\hline
\end{tabular}




\begin{tabular}{|c|c|c|c|c|c|c|c|c|c|c|c|c|c|}
\hline \multicolumn{14}{|c|}{ TABLE VII.-Continued. } \\
\hline$g$ & & $E$ & & log. & $r$ & & $g$ & & $E$ & & Log. & $r$ & \\
\hline $\begin{array}{c}100^{\circ} \\
10^{\prime} \\
20 \\
30 \\
40 \\
50\end{array}$ & \begin{tabular}{|ll} 
& 0 \\
5 & 14 \\
5 & 13 \\
5 & 13 \\
5 & 13 \\
5 & 13 \\
5 & 12
\end{tabular} & \begin{tabular}{lr} 
& \multicolumn{1}{c}{$\prime \prime$} \\
4 & 4.80 \\
3 & 51.96 \\
3 & 38.96 \\
3 & 25.81 \\
3 & 12.51 \\
2 & 59.06
\end{tabular} & $\begin{array}{c}11 \\
\text { I } 2.84 \\
13.00 \\
\text { I3.1 } 5 \\
\text { 13.30 } \\
\text { I3.45 } \\
\text { 13.61 }\end{array}$ & $\begin{array}{r}1.28 \\
71958 \\
72525 \\
73091 \\
73656 \\
74221 \\
74785\end{array}$ & $\begin{array}{l}567 \\
566 \\
565 \\
565 \\
564 \\
563\end{array}$ & $\begin{array}{c}260^{\circ} \\
50^{\prime} \\
40 \\
30 \\
20 \\
10\end{array}$ & $\begin{array}{r}110^{\circ} \\
10^{\prime} \\
20 \\
30 \\
40 \\
50\end{array}$ & \begin{tabular}{ll}
\multicolumn{2}{c}{} \\
4 & 56 \\
4 & 56 \\
4 & 56 \\
4 & 55 \\
4 & 55 \\
4 & 55
\end{tabular} & \begin{tabular}{rr}
\multicolumn{1}{c}{$\prime \prime$} \\
6 & 49.88 \\
6 & 28.20 \\
6 & 6.39 \\
5 & 44.44 \\
5 & 22.34 \\
5 & 0.10
\end{tabular} & $\begin{array}{l}11 \\
21.68 \\
21.81 \\
21.95 \\
22.10 \\
22.24 \\
22.38\end{array}$ & $\begin{array}{r}1.29 \\
04888 \\
05416 \\
05944 \\
06471 \\
06997 \\
07522\end{array}$ & $\begin{array}{l}528 \\
528 \\
527 \\
526 \\
525 \\
525\end{array}$ & $\begin{array}{c}250^{\circ} \\
50^{\prime} \\
40 \\
30 \\
20 \\
10\end{array}$ \\
\hline $\begin{array}{c}101^{\circ} \\
10^{\circ} \\
20 \\
30 \\
40 \\
50\end{array}$ & $\begin{array}{ll}5 & 12 \\
5 & 12 \\
5 & 12 \\
5 & 12 \\
5 & 11 \\
5 & 11\end{array}$ & $\begin{array}{rr}2 & 45.45 \\
2 & 31.69 \\
2 & 17.78 \\
2 & 3.72 \\
1 & 49.50 \\
1 & 35.14\end{array}$ & $\begin{array}{l}13.76 \\
13.91 \\
14.06 \\
14.22 \\
14.3^{6} \\
14.5^{2}\end{array}$ & $\begin{array}{l}75348 \\
75911 \\
76474 \\
77036 \\
77598 \\
78159\end{array}$ & $\begin{array}{l}563 \\
563 \\
562 \\
5^{62} \\
561 \\
561\end{array}$ & $\begin{array}{c}259^{\circ} \\
50^{\prime} \\
40 \\
30 \\
20 \\
10\end{array}$ & $\begin{array}{r}111^{\circ} \\
10^{\prime} \\
20 \\
30 \\
40 \\
50\end{array}$ & $\begin{array}{ll}4 & 54 \\
4 & 54 \\
4 & 53 \\
4 & 53 \\
4 & 53 \\
4 & 52\end{array}$ & $\begin{array}{rr}4 & 37.72 \\
4 & 15.21 \\
3 & 52.55 \\
3 & 29.76 \\
3 & 6.83 \\
2 & 43.76\end{array}$ & $\begin{array}{l}22.51 \\
22.66 \\
22.79 \\
22.93 \\
23.07 \\
23.21\end{array}$ & $\begin{array}{l}08047 \\
08571 \\
09094 \\
09616 \\
10137 \\
10658\end{array}$ & $\begin{array}{l}523 \\
524 \\
523 \\
522 \\
521 \\
521 \\
520\end{array}$ & $\begin{array}{c}249^{\circ} \\
50^{\prime} \\
40 \\
30 \\
20 \\
10\end{array}$ \\
\hline $\begin{array}{c}102^{\circ} \\
10^{\prime} \\
20 \\
30 \\
40 \\
50\end{array}$ & $\begin{array}{ll}5 & 11 \\
5 & 11 \\
5 & 10 \\
5 & 10 \\
5 & 10 \\
5 & 10\end{array}$ & $\begin{array}{rr}1 & 20.62 \\
1 & 5.95 \\
0 & 51.13 \\
0 & 36.16 \\
0 & 21.04 \\
0 & 5.77\end{array}$ & $\begin{array}{l}14.67 \\
14.82 \\
14.97 \\
15.12 \\
15.27\end{array}$ & $\begin{array}{l}78720 \\
79280 \\
79839 \\
80398 \\
80956 \\
81514\end{array}$ & $\begin{array}{l}560 \\
559 \\
559 \\
55^{8} \\
55^{8}\end{array}$ & $\begin{array}{c}258^{\circ} \\
50^{\prime} \\
40 \\
30 \\
20 \\
10\end{array}$ & $\begin{array}{c}112^{\circ} \\
10^{\prime} \\
20 \\
30 \\
40 \\
50\end{array}$ & $\begin{array}{ll}4 & 52 \\
4 & 51 \\
4 & 51 \\
4 & 51 \\
4 & 50 \\
4 & 50\end{array}$ & $\begin{array}{ll}2 & 20.55 \\
1 & 57.20 \\
1 & 33.72 \\
1 & 10.10 \\
& 46.34 \\
& 22.44\end{array}$ & $\begin{array}{l}23.35 \\
23.48 \\
23.62 \\
23.76 \\
23.90 \\
24.03\end{array}$ & $\begin{array}{l}11178 \\
11697 \\
12215 \\
12732 \\
13248 \\
13764\end{array}$ & $\begin{array}{l}519 \\
518 \\
517 \\
516 \\
516\end{array}$ & $\begin{array}{c}248^{\circ} \\
50^{\prime} \\
40 \\
30 \\
20 \\
10\end{array}$ \\
\hline $\begin{array}{c}103^{\circ} \\
10^{\prime} \\
20 \\
30 \\
40 \\
50\end{array}$ & $\begin{array}{rr}\mathbf{5} & 9 \\
\mathbf{5} & 9 \\
\mathbf{5} & \mathbf{9} \\
\mathbf{5} & 9 \\
\mathbf{5} & 8 \\
\mathbf{5} & 8 \\
& 8\end{array}$ & $\begin{array}{rr}9 & 50.35 \\
9 & 34.78 \\
9 & 19.06 \\
9 & 3.19 \\
8 & 47.17 \\
8 & 31.01\end{array}$ & $\begin{array}{l}15.57 \\
15.72 \\
15.87 \\
16.02 \\
16.16 \\
16.32\end{array}$ & $\begin{array}{l}82071 \\
82628 \\
83184 \\
83739 \\
84294 \\
84849\end{array}$ & $\begin{array}{l}557 \\
556 \\
555 \\
555 \\
555 \\
554\end{array}$ & $\begin{array}{c}257^{\circ} \\
50 \\
40 \\
30 \\
20 \\
10\end{array}$ & $\begin{array}{r}113^{\circ} \\
10^{\prime} \\
20 \\
30 \\
40 \\
50\end{array}$ & $\begin{array}{ll}4 & 49 \\
4 & 49 \\
4 & 49 \\
4 & 48 \\
4 & 48 \\
4 & 47\end{array}$ & $\begin{array}{r}58.41 \\
34.24 \\
9.94 \\
45.50 \\
20.92 \\
56.21\end{array}$ & $\begin{array}{l}24.03 \\
24.17 \\
24.30 \\
24.44 \\
24.5^{8} \\
24.71 \\
24.85\end{array}$ & & $\begin{array}{l}514 \\
513 \\
5112 \\
511 \\
5111 \\
510\end{array}$ & $\begin{array}{c}247^{\circ} \\
50^{\prime} \\
40 \\
30 \\
20 \\
10\end{array}$ \\
\hline $\begin{array}{c}104^{\circ} \\
10^{\prime} \\
20 \\
30 \\
40 \\
50\end{array}$ & $\begin{array}{ll}5 & 8 \\
5 & 7 \\
5 & 7 \\
5 & 7 \\
5 & 7 \\
5 & 6\end{array}$ & $\begin{array}{rr}8 & 14.69 \\
7 & 58.22 \\
7 & 41.61 \\
7 & 24.85 \\
7 & 7.94 \\
6 & 50.88\end{array}$ & $\begin{array}{l}16.76 \\
16.91 \\
17.06 \\
17.21\end{array}$ & $\begin{array}{l}85403 \\
85956 \\
86508 \\
87059 \\
87610 \\
88160\end{array}$ & $\begin{array}{l}553 \\
55^{2} \\
55^{1} \\
55 \mathrm{I} \\
550 \\
549\end{array}$ & $\begin{array}{c}256^{\circ} \\
50^{\prime} \\
40 \\
30 \\
20 \\
10\end{array}$ & $\begin{array}{r}114^{\circ} \\
10^{\prime} \\
20 \\
30 \\
40 \\
50\end{array}$ & $\begin{array}{ll}4 & 47 \\
4 & 47 \\
4 & 46 \\
4 & 46 \\
4 & 45 \\
4 & 45\end{array}$ & $\begin{array}{r}31.36 \\
6.38 \\
41.26 \\
16.00 \\
50.61 \\
25.09\end{array}$ & & & $\begin{array}{l}509 \\
508 \\
507 \\
506 \\
506 \\
505\end{array}$ & $\begin{array}{c}246^{\circ} \\
50^{\prime} \\
40 \\
30 \\
20 \\
10\end{array}$ \\
\hline $\begin{array}{c}105^{\circ} \\
10^{\prime} \\
20 \\
30 \\
40 \\
50\end{array}$ & $\begin{array}{ll}\mathbf{5} & 6 \\
\mathbf{5} & 6 \\
5 & 5 \\
5 & 5 \\
\mathbf{5} & \mathbf{5} \\
\mathbf{5} & 5\end{array}$ & $\begin{array}{r}33.67 \\
16.32 \\
58.82 \\
41.17 \\
23.37 \\
5.42\end{array}$ & $\begin{array}{l}17.35 \\
17.50 \\
17.65 \\
17.80 \\
17.95 \\
18.09\end{array}$ & $\begin{array}{l}90902 \\
91449\end{array}$ & & $\begin{array}{c}255^{\circ} \\
50^{\prime} \\
40 \\
30 \\
20 \\
10\end{array}$ & $\begin{array}{c}115^{\circ} \\
10^{\prime} \\
20 \\
30 \\
40 \\
50\end{array}$ & $\begin{array}{ll}4 & 44 \\
4 & 44 \\
4 & 44 \\
4 & 43 \\
4 & 43 \\
4 & 42\end{array}$ & $\begin{array}{r}59.44 \\
33.66 \\
7.74 \\
41.69 \\
15.50 \\
49.19\end{array}$ & $\begin{array}{l}20.31 \\
26.45\end{array}$ & & $\begin{array}{l}504 \\
503 \\
502 \\
501 \\
500 \\
500\end{array}$ & $\begin{array}{c}245^{\circ} \\
50^{\prime} \\
40 \\
30 \\
20 \\
10\end{array}$ \\
\hline $\begin{array}{c}106^{\circ} \\
10^{\prime} \\
20 \\
30 \\
40 \\
50\end{array}$ & $\begin{array}{ll}5 & 4 \\
5 & 4 \\
5 & 4 \\
5 & 3 \\
5 & 3 \\
5 & 3 \\
5 & \end{array}$ & $\begin{array}{l}47.33 \\
42.10 \\
10.72 \\
52.19 \\
33.52 \\
14.70\end{array}$ & $\begin{array}{l}1823 \\
18.3^{2} \\
18.53 \\
18.67 \\
18.82 \\
18.96\end{array}$ & $\begin{array}{l}93085 \\
93629 \\
94172 \\
94715\end{array}$ & $\begin{array}{l}540 \\
545 \\
545 \\
544 \\
543 \\
543 \\
542\end{array}$ & $\begin{array}{c}254^{\circ} \\
50^{\prime} \\
40 \\
30 \\
20 \\
10\end{array}$ & $\begin{array}{r}116^{\circ} \\
10^{\prime} \\
20 \\
30 \\
40 \\
50\end{array}$ & $\begin{array}{ll}4 & 42 \\
4 & 41 \\
4 & 41 \\
4 & 41 \\
4 & 40 \\
4 & 40\end{array}$ & $\begin{array}{r}22.74 \\
56.16 \\
29.45 \\
2.60 \\
35.62 \\
8.52\end{array}$ & $\begin{array}{l}20.45 \\
26.58 \\
26.71 \\
26.85 \\
26.98 \\
27.10 \\
27.24\end{array}$ & $\begin{array}{l}23401 \\
23899 \\
24397 \\
24894 \\
25390 \\
25885\end{array}$ & $\begin{array}{l}498 \\
498 \\
497 \\
496 \\
495 \\
495\end{array}$ & $\begin{array}{c}244^{\circ} \\
50^{\prime} \\
40 \\
30 \\
20 \\
10\end{array}$ \\
\hline $\begin{array}{c}107^{\circ} \\
10^{\prime} \\
20 \\
30 \\
40 \\
50\end{array}$ & $\begin{array}{ll}5 & 2 \\
5 & 2 \\
5 & 2 \\
5 & 1 \\
5 & 1 \\
5 & 1\end{array}$ & $\begin{array}{l}55.74 \\
26.63 \\
17.38 \\
57.99 \\
38.45 \\
18.77\end{array}$ & $\begin{array}{l}19.11 \\
19.25 \\
19.39 \\
19.54 \\
19.68 \\
19.83\end{array}$ & $\begin{array}{l}95257 \\
95798 \\
96338 \\
96878 \\
97417 \\
97955\end{array}$ & $\begin{array}{l}540 \\
539 \\
538 \\
538\end{array}$ & $\begin{array}{c}253^{\circ} \\
50^{\prime} \\
40 \\
30 \\
20 \\
10\end{array}$ & $\begin{array}{c}117^{\circ} \\
10^{\prime} \\
20 \\
30 \\
40 \\
50\end{array}$ & $\begin{array}{ll}4 & 39 \\
4 & 39 \\
4 & 38 \\
4 & 38 \\
4 & 37 \\
4 & 37\end{array}$ & $\begin{array}{l}41.28 \\
13.91 \\
46.41 \\
18.78 \\
51.02 \\
23.13\end{array}$ & $\begin{array}{l}27.76 \\
27.89 \\
28.01\end{array}$ & $\begin{array}{l}26380 \\
26874 \\
27366 \\
27857 \\
28348 \\
28837\end{array}$ & $\begin{array}{l}494 \\
49^{2} \\
491 \\
491 \\
489 \\
488\end{array}$ & $\begin{array}{c}243^{\circ} \\
50^{\prime} \\
40 \\
30 \\
20 \\
10\end{array}$ \\
\hline $\begin{array}{c}108^{\circ} \\
10^{\circ} \\
20 \\
30 \\
40 \\
50 \\
109^{\circ}\end{array}$ & $\begin{array}{rr}5 & 0 \\
5 & 0 \\
5 & 0 \\
4 & 59 \\
4 & 59 \\
4 & 59 \\
4 & 58\end{array}$ & $\begin{array}{l}58.94 \\
38.97 \\
18.86 \\
58.60 \\
38.21 \\
17.67 \\
56.98\end{array}$ & $\begin{array}{l}19.97 \\
20.11 \\
20.26 \\
20.39 \\
20.54 \\
20.69\end{array}$ & $\begin{array}{r}98493 \\
99030 \\
99566 \\
* 00101\end{array}$ & $\begin{array}{l}535 \\
535 \\
534 \\
533\end{array}$ & $\begin{array}{c}252^{\circ} \\
50^{\prime} \\
40 \\
30 \\
20 \\
10 \\
57\end{array}$ & $\begin{array}{r}118^{\circ} \\
10^{\prime} \\
20 \\
30 \\
40 \\
50\end{array}$ & $\begin{array}{ll}4 & 36 \\
4 & 36 \\
4 & 35 \\
4 & 35 \\
4 & 35 \\
4 & 34\end{array}$ & $\begin{array}{r}55.12 \\
26.98 \\
58.71 \\
30.31 \\
1.78 \\
33.13\end{array}$ & $\begin{array}{l}28.14 \\
28.27 \\
28.40 \\
28.53 \\
28.65 \\
28.78\end{array}$ & $\begin{array}{l}29325 \\
29812 \\
30299 \\
30785 \\
31270 \\
31754\end{array}$ & $\begin{array}{l}487 \\
487 \\
486 \\
485 \\
484 \\
483\end{array}$ & $\begin{array}{c}242^{\circ} \\
50^{\prime} \\
40 \\
30 \\
20 \\
10\end{array}$ \\
\hline $\begin{array}{c}109^{\circ} \\
10^{\prime} \\
20 \\
30 \\
40 \\
50\end{array}$ & $\begin{array}{ll}4 & 58 \\
4 & 58 \\
4 & 58 \\
4 & 57 \\
4 & 57 \\
4 & 57\end{array}$ & $\begin{array}{l}56.98 \\
36.15 \\
15.18 \\
54.07 \\
32.81 \\
11.42\end{array}$ & $\begin{array}{l}20.83 \\
20.97 \\
21.11 \\
21.26 \\
21.39 \\
21.54\end{array}$ & $\begin{array}{l}* 03299 \\
* 03829 \\
* 04359\end{array}$ & $\begin{array}{l}533 \\
533 \\
532 \\
531 \\
530 \\
530 \\
529\end{array}$ & $\begin{array}{c}251^{\circ} \\
50^{\prime} \\
40 \\
30 \\
20 \\
10\end{array}$ & $\begin{array}{c}119^{\circ} \\
10^{\prime} \\
20 \\
30 \\
40 \\
50\end{array}$ & $\begin{array}{ll}4 & 34 \\
4 & 33 \\
4 & 33 \\
4 & 32 \\
4 & 32 \\
4 & 31\end{array}$ & $\begin{array}{r}4.35 \\
35.44 \\
6.41 \\
37.25 \\
7.96 \\
38.55\end{array}$ & $\begin{array}{l}28.91 \\
29.03 \\
29.16 \\
29.29 \\
29.41 \\
29.54\end{array}$ & $\begin{array}{l}32237 \\
32719 \\
33200 \\
33681 \\
34161 \\
34640\end{array}$ & $\begin{array}{l}482 \\
481 \\
481 \\
480 \\
479 \\
477\end{array}$ & $\begin{array}{c}241^{\circ} \\
50^{\prime} \\
40 \\
30 \\
20 \\
10\end{array}$ \\
\hline $110^{2}$ & 456 & 49.88 & & $\begin{array}{r}* 04888 \\
* 1.29\end{array}$ & & $\begin{array}{c}250^{\circ} \\
g\end{array}$ & $120^{\circ}$ & 431 & 9.01 & & & & $\begin{array}{c}240^{\circ} \\
g\end{array}$ \\
\hline
\end{tabular}




\begin{tabular}{|c|c|c|c|c|c|c|c|c|c|c|c|c|c|}
\hline$g$ & & $E$ & & log. & $r$ & & $g$ & & $E$ & & 1.0g. & $r$ & 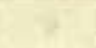 \\
\hline $\begin{array}{r}120^{\circ} \\
10^{\prime} \\
20 \\
30 \\
40 \\
50\end{array}$ & $\begin{array}{l}431 \\
+30 \\
+30 \\
+29 \\
429 \\
+\quad 23\end{array}$ & $\begin{array}{r}9.01 \\
39.35 \\
9.56 \\
39.65 \\
9.61 \\
39.45\end{array}$ & $\begin{array}{l}29.66 \\
29.79 \\
29.91 \\
30.04 \\
30.16 \\
30.29\end{array}$ & $\begin{array}{l}1.29 \\
35117 \\
35593 \\
36069 \\
36544 \\
37018 \\
37491\end{array}$ & $\begin{array}{l}476 \\
476 \\
475 \\
474 \\
473 \\
472\end{array}$ & $\begin{array}{c}240^{\circ} \\
50^{\prime} \\
40 \\
30 \\
20 \\
10\end{array}$ & $\begin{array}{r}130^{\circ} \\
10^{\circ} \\
20 \\
30 \\
40 \\
50\end{array}$ & $\begin{array}{ll}3 & 57 \\
3 & 57 \\
3 & 56 \\
3 & 56 \\
3 & 55 \\
3 & 54\end{array}$ & $\begin{array}{r}57.75 \\
21.10 \\
44.35 \\
7.48 \\
30.51 \\
53.43\end{array}$ & $\begin{array}{l}3^{6.65} \\
3^{6.75} \\
3^{6.87} \\
36.97 \\
37.08 \\
37.18\end{array}$ & $\begin{array}{r}1.29 \\
61903 \\
62317 \\
62729 \\
63140 \\
63550 \\
63959\end{array}$ & $\begin{array}{l}414 \\
412 \\
411 \\
410 \\
409 \\
407\end{array}$ & $\begin{array}{c}230^{\circ} \\
50^{\prime} \\
40 \\
80 \\
20 \\
10\end{array}$ \\
\hline $\begin{array}{c}121^{\circ} \\
10^{\prime} \\
20 \\
30 \\
40 \\
50\end{array}$ & $\begin{array}{l}428 \\
427 \\
+227 \\
+26 \\
+26 \\
+25\end{array}$ & $\begin{array}{r}9.16 \\
35.75 \\
8.22 \\
37.56 \\
6.75 \\
35.88\end{array}$ & $\begin{array}{l}30.41 \\
30.53 \\
30.66 \\
30.78 \\
30.90 \\
31.03\end{array}$ & $\begin{array}{l}37963 \\
38434 \\
35904 \\
39372 \\
39940 \\
40306\end{array}$ & $\begin{array}{l}471 \\
470 \\
468 \\
469 \\
466 \\
465\end{array}$ & $\begin{array}{c}239^{\circ} \\
50^{\prime} \\
40 \\
30 \\
20 \\
10\end{array}$ & $\begin{array}{c}131^{\circ} \\
10^{\prime} \\
20 \\
30 \\
40 \\
50\end{array}$ & $\begin{array}{ll}3 & 54 \\
3 & 53 \\
3 & 53 \\
3 & 52 \\
3 & 51 \\
3 & 51\end{array}$ & $\begin{array}{r}16.25 \\
39.97 \\
1.58 \\
24.08 \\
46.49 \\
8.78\end{array}$ & $\begin{array}{l}37.28 \\
37.39 \\
37.50 \\
37.60 \\
37.70 \\
37.81\end{array}$ & $\begin{array}{l}64366 \\
64773 \\
65179 \\
65553 \\
65957 \\
66389\end{array}$ & $\begin{array}{l}407 \\
406 \\
404 \\
404 \\
402 \\
401\end{array}$ & $\begin{array}{c}229^{\circ} \\
50^{\circ} \\
40 \\
30 \\
26 \\
10\end{array}$ \\
\hline $\begin{array}{c}122^{\circ} \\
10^{\circ} \\
20 \\
30 \\
40 \\
50\end{array}$ & $\begin{array}{l}425 \\
+24 \\
+24 \\
423 \\
+22 \\
+22\end{array}$ & $\begin{array}{r}4.55 \\
33.71 \\
2.44 \\
31.05 \\
59.54 \\
27.91\end{array}$ & $\begin{array}{l}31.14 \\
31.27 \\
31.39 \\
31.51 \\
31.63 \\
31.75\end{array}$ & $\begin{array}{l}40771 \\
41236 \\
41700 \\
42163 \\
42625 \\
43086\end{array}$ & $\begin{array}{l}465 \\
464 \\
463 \\
462 \\
461 \\
460\end{array}$ & $\begin{array}{c}238^{\circ} \\
50^{\prime} \\
40 \\
30 \\
20 \\
10\end{array}$ & $\begin{array}{r}132 \\
10^{\prime} \\
20 \\
30 \\
40 \\
50\end{array}$ & $\begin{array}{ll}3 & 50 \\
3 & 49 \\
3 & 49 \\
3 & 48 \\
3 & 47 \\
3 & 47\end{array}$ & $\begin{array}{l}39.97 \\
53.06 \\
15.04 \\
36.92 \\
58.10 \\
20.38\end{array}$ & $\begin{array}{l}37.91 \\
38.02 \\
38.12 \\
38.22 \\
38.32 \\
38.43\end{array}$ & $\begin{array}{l}66790 \\
67190 \\
67588 \\
67985 \\
68882 \\
68777\end{array}$ & $\begin{array}{l}400 \\
398 \\
397 \\
397 \\
395 \\
394\end{array}$ & $\begin{array}{c}228^{\circ} \\
50^{\prime} \\
40 \\
80 \\
20 \\
10\end{array}$ \\
\hline $\begin{array}{r}123^{\circ} \\
10^{\circ} \\
20 \\
30 \\
40 \\
50\end{array}$ & $\begin{array}{l}+21 \\
+21 \\
+20 \\
+20 \\
419 \\
+19\end{array}$ & $\begin{array}{l}56.16 \\
24.29 \\
52.29 \\
20.19 \\
47.94 \\
15.59\end{array}$ & $\begin{array}{l}31.87 \\
32.00 \\
32.11 \\
32.24 \\
32.35 \\
32.47\end{array}$ & $\begin{array}{l}43546 \\
44005 \\
44463 \\
44919 \\
45375 \\
45829\end{array}$ & $\begin{array}{l}459 \\
45^{8} \\
45^{6} \\
45^{6} \\
454 \\
453\end{array}$ & $\begin{array}{c}237^{\circ} \\
50^{\circ} \\
40 \\
30 \\
20 \\
10\end{array}$ & $\begin{array}{c}133^{\circ} \\
10^{\prime} \\
20 \\
30 \\
40 \\
50\end{array}$ & $\begin{array}{ll}3 & 46 \\
3 & 46 \\
3 & 45 \\
3 & 44 \\
3 & 44 \\
3 & 43\end{array}$ & $\begin{array}{r}41.95 \\
3.42 \\
24.79 \\
46.06 \\
7.23 \\
29.30\end{array}$ & $\begin{array}{l}3^{8.53} \\
3^{8.63} \\
3^{8.73} \\
3^{8.83} \\
3^{8.93} \\
39.03\end{array}$ & $\begin{array}{l}69171 \\
69564 \\
69955 \\
70345 \\
70734 \\
71122\end{array}$ & $\begin{array}{l}394 \\
393 \\
391 \\
390 \\
389 \\
388 \\
387\end{array}$ & $\begin{array}{c}227^{\circ} \\
50^{\prime} \\
40 \\
30 \\
20 \\
10\end{array}$ \\
\hline $\begin{array}{r}124^{\circ} \\
10^{\prime} \\
20 \\
30 \\
40 \\
50\end{array}$ & $\begin{array}{ll}+18 \\
+118 \\
+117 \\
417 \\
+16 \\
+15\end{array}$ & $\begin{array}{r}43.12 \\
10.53 \\
37.83 \\
5.01 \\
32.07 \\
59.01\end{array}$ & $\begin{array}{l}32.59 \\
32.70 \\
32.82 \\
32.94 \\
33.06 \\
33.17\end{array}$ & $\begin{array}{l}46252 \\
46735 \\
47186 \\
47636 \\
45086 \\
48534\end{array}$ & $\begin{array}{l}453 \\
451 \\
450 \\
450 \\
448 \\
447\end{array}$ & $\begin{array}{c}236^{\prime} \\
50^{\prime} \\
40 \\
30 \\
20 \\
10\end{array}$ & $\begin{array}{r}134^{\circ} \\
10^{\prime} \\
20 \\
30 \\
40 \\
50\end{array}$ & $\begin{array}{ll}3 & 42 \\
3 & 42 \\
3 & 41 \\
3 & 40 \\
3 & 40 \\
3 & 39\end{array}$ & $\begin{array}{l}49.27 \\
10.14 \\
30.91 \\
51.59 \\
12.15 \\
32.62\end{array}$ & $\begin{array}{l}39.13 \\
39.23 \\
39 \cdot 33 \\
39.43 \\
39.53 \\
39.62\end{array}$ & $\begin{array}{l}71509 \\
71895 \\
72279\end{array}$ & $\begin{array}{l}3^{86} \\
3^{84} \\
3^{83} \\
3^{82} \\
3^{81} \\
3^{80}\end{array}$ & $\begin{array}{c}226^{\circ} \\
50^{\prime} \\
40 \\
30 \\
20 \\
10\end{array}$ \\
\hline $\begin{array}{r}125^{\circ} \\
10^{\circ} \\
-20 \\
30 \\
40 \\
50\end{array}$ & $\begin{array}{ll}+ & 15 \\
4 & 14 \\
4 & 14 \\
413 \\
+13 \\
+12\end{array}$ & $\begin{array}{l}25.84 \\
52.55 \\
19.14 \\
45.62 \\
11.99 \\
38.22\end{array}$ & $\begin{array}{l}33.39 \\
33.41 \\
33 \cdot 52 \\
33.64 \\
33.76 \\
33.87\end{array}$ & $\begin{array}{l}48981 \\
49428 \\
49373 \\
50317 \\
50761 \\
51204\end{array}$ & $\begin{array}{l}447 \\
445 \\
444 \\
444 \\
443 \\
441\end{array}$ & $\begin{array}{c}235^{\circ} \\
50^{\circ} \\
40 \\
30 \\
20 \\
10\end{array}$ & $\begin{array}{r}135^{\circ} \\
10^{\prime} \\
20 \\
30 \\
40 \\
50\end{array}$ & $\begin{array}{ll}3 & 39 \\
3 & 38 \\
3 & 37 \\
3 & 36 \\
3 & 36 \\
3 & 35\end{array}$ & $\begin{array}{l}53.00 \\
13.28 \\
33.46 \\
53.54 \\
13.52 \\
33.41\end{array}$ & $\begin{array}{l}39.72 \\
39.82 \\
39.92 \\
40.02 \\
40.11 \\
40.20\end{array}$ & $\begin{array}{l}73805 \\
7 \$ 184 \\
74561 \\
74937 \\
75312 \\
75686\end{array}$ & $\begin{array}{l}379 \\
379 \\
377 \\
376 \\
375 \\
374 \\
373\end{array}$ & $\begin{array}{c}225^{\circ} \\
50^{\circ} \\
40 \\
30 \\
20 \\
10\end{array}$ \\
\hline $\begin{array}{c}126^{\circ} \\
10^{\prime} \\
20 \\
30 \\
40 \\
50\end{array}$ & $\begin{array}{rr}4 & 12 \\
4 & 11 \\
4 & 10 \\
4 & 10 \\
4 & 9 \\
4 & 9\end{array}$ & $\begin{array}{r}4.35 \\
30.37 \\
56.27 \\
22.06 \\
47.73 \\
13.29\end{array}$ & $\begin{array}{l}33.99 \\
34.10 \\
34.21 \\
34.33 \\
34.44 \\
34.55\end{array}$ & $\begin{array}{l}51645 \\
52035 \\
52524 \\
52962 \\
53399 \\
53934\end{array}$ & $\begin{array}{l}440 \\
439 \\
438 \\
437 \\
435 \\
434\end{array}$ & $\begin{array}{c}234^{\circ} \\
50^{\circ} \\
40 \\
30 \\
20 \\
10 \\
00\end{array}$ & $\begin{array}{c}136^{\circ} \\
10^{\prime} \\
20 \\
30 \\
40 \\
50\end{array}$ & $\begin{array}{ll}3 & 34 \\
3 & 34 \\
3 & 33 \\
3 & 32 \\
3 & 32 \\
3 & 31\end{array}$ & $\begin{array}{l}53.21 \\
12.90 \\
32.50 \\
52.01 \\
11.42 \\
30.73\end{array}$ & $\begin{array}{l}40.31 \\
40.40 \\
40.49 \\
40.59 \\
40.69 \\
40.78\end{array}$ & $\begin{array}{l}76059 \\
76430 \\
76800 \\
77169 \\
77537 \\
77903\end{array}$ & $\begin{array}{l}371 \\
370 \\
369 \\
368 \\
366 \\
365\end{array}$ & $\begin{array}{c}224^{\circ} \\
50^{\circ} \\
40 \\
30 \\
20 \\
10\end{array}$ \\
\hline $\begin{array}{c}127^{\circ} \\
10^{\prime} \\
20 \\
30 \\
40 \\
50\end{array}$ & $\begin{array}{ll}4 & 8 \\
4 & 8 \\
4 & 7 \\
4 & 6 \\
4 & 6 \\
+ & 5\end{array}$ & $\begin{array}{r}38.74 \\
4.07 \\
29.29 \\
54.40 \\
19.40 \\
44.29\end{array}$ & $\begin{array}{l}34.67 \\
34.78 \\
34.89 \\
35.00 \\
35.11 \\
35.23\end{array}$ & $\begin{array}{l}54268 \\
54702 \\
55134 \\
55565 \\
55995 \\
56424\end{array}$ & $\begin{array}{l}434 \\
432 \\
431 \\
430 \\
429 \\
428\end{array}$ & $\begin{array}{c}233^{\circ} \\
50^{\prime} \\
40 \\
30 \\
20 \\
10\end{array}$ & $\begin{array}{c}137^{\circ} \\
1 \theta^{\prime} \\
20 \\
30 \\
40 \\
50\end{array}$ & $\begin{array}{ll}3 & 30 \\
3 & 30 \\
3 & 29 \\
3 & 28 \\
3 & 29 \\
3 & 27\end{array}$ & $\begin{array}{r}49.95 \\
11.08 \\
23.11 \\
47.05 \\
5.90 \\
24.66\end{array}$ & $\begin{array}{l}40.87 \\
40.97 \\
41.06 \\
41.15 \\
41.24 \\
41.34\end{array}$ & $\begin{array}{l}78268 \\
78632 \\
78995 \\
79356 \\
79716 \\
80075\end{array}$ & $\begin{array}{l}364 \\
363 \\
361 \\
360 \\
359 \\
358\end{array}$ & $\begin{array}{c}223^{\circ} \\
50^{\prime} \\
40 \\
30 \\
20 \\
10\end{array}$ \\
\hline $\begin{array}{r}128^{\circ} \\
10^{\prime} \\
20 \\
30 \\
40 \\
50\end{array}$ & $\begin{array}{ll}4 & 5 \\
4 & 4 \\
4 & 3 \\
4 & 3 \\
4 & 2 \\
4 & 2\end{array}$ & $\begin{array}{r}9.06 \\
33.72 \\
53.27 \\
22.72 \\
47.05 \\
11.27\end{array}$ & $\begin{array}{l}35 \cdot 34 \\
35 \cdot 45 \\
35 \cdot 55 \\
35.67 \\
35 \cdot 78 \\
35.89\end{array}$ & $\begin{array}{l}56852 \\
57279 \\
57705 \\
58130 \\
58554 \\
58977\end{array}$ & $\begin{array}{l}427 \\
426 \\
425 \\
424 \\
423 \\
422\end{array}$ & $\begin{array}{c}232^{\circ} \\
50^{\prime} \\
40 \\
30 \\
20 \\
10\end{array}$ & $\begin{array}{c}138^{\circ} \\
10^{\prime} \\
20 \\
30 \\
40 \\
50\end{array}$ & $\begin{array}{ll}3 & 26 \\
3 & 26 \\
3 & 25 \\
3 & 24 \\
3 & 23 \\
3 & 23\end{array}$ & $\begin{array}{r}43.32 \\
1.89 \\
20.37 \\
38.76 \\
57.05 \\
15.26\end{array}$ & $\begin{array}{l}41.43 \\
41.52 \\
41.61 \\
41.71 \\
41.79 \\
41.88\end{array}$ & $\begin{array}{l}80433 \\
80789 \\
81144 \\
81498 \\
81851 \\
82202\end{array}$ & $\begin{array}{l}356 \\
355 \\
354 \\
353 \\
351 \\
350\end{array}$ & $\begin{array}{c}222^{\circ} \\
50 \\
40 \\
30 \\
20 \\
10\end{array}$ \\
\hline $\begin{array}{c}129^{\circ} \\
10^{\circ} \\
20 \\
30 \\
40 \\
50\end{array}$ & $\begin{array}{rr}4 & 1 \\
4 & 0 \\
4 & 0 \\
3 & 59 \\
3 & 59 \\
3 & 59\end{array}$ & $\begin{array}{l}35.38 \\
59.38 \\
23.27 \\
47.05 \\
10.73 \\
34.29\end{array}$ & $\begin{array}{l}3^{6.00} \\
3^{6.111} \\
36.22 \\
3^{6.32} \\
3^{66.44} \\
3^{6.54}\end{array}$ & $\begin{array}{l}59399 \\
59920 \\
60239 \\
606.57 \\
61074 \\
61489\end{array}$ & $\begin{array}{l}421 \\
419 \\
418 \\
417 \\
415 \\
414\end{array}$ & $\begin{array}{c}231^{\circ} \\
50^{\circ} \\
40 \\
30 \\
20 \\
10\end{array}$ & $\begin{array}{c}139^{\circ} \\
10^{\prime} \\
20 \\
30 \\
40 \\
50\end{array}$ & $\begin{array}{ll}3 & 22 \\
3 & 21 \\
3 & 21 \\
3 & 20 \\
3 & 19 \\
3 & 19\end{array}$ & $\begin{array}{r}33.38 \\
51.41 \\
9.35 \\
27.20 \\
44.96 \\
2.63\end{array}$ & $\begin{array}{l}41.97 \\
42.06 \\
42.15 \\
42.24 \\
42.33 \\
42.41\end{array}$ & $\begin{array}{l}82552 \\
82901 \\
83249 \\
83595 \\
83940 \\
84284\end{array}$ & $\begin{array}{l}349 \\
348 \\
346 \\
345 \\
344 \\
343\end{array}$ & $\begin{array}{c}221^{\circ} \\
50^{\circ} \\
40^{\prime} \\
30 \\
20 \\
10\end{array}$ \\
\hline $130^{\circ}$ & 357 & 57.75 & & $\begin{array}{r}61.203 \\
1.29\end{array}$ & & $\begin{array}{c}230^{\circ} \\
g\end{array}$ & $140^{\circ}$ & $\begin{array}{ll}318 \\
\end{array}$ & 20.22 & & $\begin{array}{r}84627 \\
1.29\end{array}$ & & $220^{\circ}$ \\
\hline
\end{tabular}




\begin{tabular}{|c|c|c|c|c|c|c|c|c|c|c|c|c|c|}
\hline \multicolumn{14}{|c|}{ TABLE VII._Continued. } \\
\hline$g$ & \multicolumn{3}{|c|}{$\boldsymbol{E}$} & \multicolumn{2}{|c|}{$\log \cdot r$} & & $g$ & \multicolumn{3}{|c|}{$E$} & \multicolumn{2}{|c|}{ Log. $r$} & \\
\hline $\begin{array}{c}140^{\circ} \\
10^{\prime} \\
20 \\
30 \\
40 \\
50\end{array}$ & $\begin{array}{ll} & 0 \\
3 & 18 \\
3 & 17 \\
3 & 10 \\
3 & 10 \\
3 & 15 \\
3 & 14\end{array}$ & \begin{tabular}{lc}
\multicolumn{1}{c}{$\prime \prime$} \\
18 & 20.22 \\
7 & 37.72 \\
6 & 55.13 \\
6 & 12.45 \\
5 & 29.68 \\
4 & 46.83
\end{tabular} & $\begin{array}{c}\prime \prime \\
42.50 \\
42.59 \\
42.68 \\
42.77 \\
42.85 \\
42.94\end{array}$ & $\begin{array}{r}1.29 \\
84627 \\
84968 \\
85308 \\
85647 \\
85985 \\
86321\end{array}$ & $\begin{array}{l}34 \mathrm{I} \\
340 \\
339 \\
338 \\
33^{6} \\
335\end{array}$ & $\begin{array}{c}220^{\circ} \\
50^{\prime} \\
40 \\
30 \\
20 \\
10\end{array}$ & $\begin{array}{c}150^{\circ} \\
10^{\prime} \\
20 \\
30 \\
40 \\
50\end{array}$ & \begin{tabular}{ll}
\multicolumn{2}{c}{} \\
2 & 33 \\
2 & 32 \\
2 & 31 \\
2 & 31 \\
2 & 30 \\
2 & 29
\end{tabular} & \begin{tabular}{lr}
$l$ & \multicolumn{1}{c}{,} \\
33 & 26.93 \\
32 & 39.79 \\
31 & 52.59 \\
1 & 5.32 \\
30 & 17.98 \\
9 & 30.58
\end{tabular} & $\begin{array}{l}47 \cdot 14 \\
47 \cdot 20 \\
47.27 \\
4734 \\
47.40 \\
47.47\end{array}$ & $\begin{array}{r}\mathbf{1 . 3 0} \\
02798 \\
03060 \\
03320 \\
03579 \\
03837 \\
04093\end{array}$ & $\begin{array}{l}262 \\
260 \\
259 \\
258 \\
256 \\
255\end{array}$ & $\begin{array}{c}210^{\circ} \\
50^{\prime} \\
40 \\
30 \\
20 \\
10\end{array}$ \\
\hline $\begin{array}{c}141^{\circ} \\
10^{\prime} \\
20 \\
30 \\
40 \\
50\end{array}$ & $\begin{array}{ll}3 & 1 \\
3 & 1 \\
3 & 12 \\
3 & 11 \\
3 & 11 \\
3 & 10\end{array}$ & $\begin{array}{rr}4 & 3.89 \\
3 & 20.87 \\
2 & 37.76 \\
1 & 54.56 \\
1 & 11.28 \\
0 & 27.92\end{array}$ & $\begin{array}{l}43.02 \\
43.11 \\
43 \cdot 20 \\
43.28 \\
43 \cdot 36 \\
43.45\end{array}$ & $\begin{array}{l}86656 \\
86990 \\
87322 \\
87653 \\
87983 \\
88312\end{array}$ & $\begin{array}{l}334 \\
332 \\
331 \\
330 \\
329 \\
327\end{array}$ & $\begin{array}{c}219^{\circ} \\
50^{\prime} \\
40 \\
30 \\
20 \\
10\end{array}$ & $\begin{array}{c}151^{\circ} \\
10^{\prime} \\
20 \\
30 \\
40 \\
50\end{array}$ & $\mid \begin{array}{ll}2 & 28 \\
2 & 27 \\
2 & 27 \\
2 & 26 \\
2 & 25 \\
2 & 24\end{array}$ & $\begin{array}{rr}8 & 43.11 \\
7 & 55.58 \\
7 & 7.98 \\
6 & 20.32 \\
5 & 32.59 \\
4 & 44.80\end{array}$ & $\begin{array}{l}47.41 \\
47.53 \\
47.60 \\
47.66 \\
47.73 \\
47.79 \\
47.85\end{array}$ & $\begin{array}{l}04348 \\
04602 \\
04854 \\
05105 \\
05355 \\
05604\end{array}$ & $\begin{array}{l}254 \\
252 \\
251 \\
250 \\
249 \\
247\end{array}$ & $\begin{array}{c}209^{\circ} \\
50^{\prime} \\
40 \\
30 \\
20 \\
10\end{array}$ \\
\hline $\begin{array}{c}142^{\circ} \\
10^{\prime} \\
20 \\
30 \\
40 \\
50\end{array}$ & $\begin{array}{ll}3 & 9 \\
3 & 9 \\
3 & 8 \\
3 & 7 \\
3 & 6 \\
3 & 6\end{array}$ & $\begin{array}{rr}9 & 44.47 \\
9 & 0.94 \\
8 & 17.32 \\
7 & 33.62 \\
6 & 49.83 \\
6 & 5.97\end{array}$ & $\begin{array}{l}43 \cdot 53 \\
43.62 \\
43 \cdot 70 \\
43.79 \\
43.86 \\
43.95\end{array}$ & $\begin{array}{l}88639 \\
88965 \\
89290 \\
89614 \\
89936 \\
90257\end{array}$ & $\begin{array}{l}326 \\
325 \\
324 \\
322 \\
321 \\
320\end{array}$ & $\begin{array}{c}218^{\circ} \\
50^{\prime} \\
40 \\
30 \\
20 \\
10\end{array}$ & $\begin{array}{c}152^{\circ} \\
10^{\prime} \\
20 \\
30 \\
40 \\
50\end{array}$ & & $\begin{array}{rr}3 & 56.95 \\
3 & 9.04 \\
2 & 21.06 \\
1 & 33.02 \\
0 & 44.92 \\
9 & 56.76\end{array}$ & $\begin{array}{l}47.91 \\
47.98 \\
48.04 \\
48.10 \\
48.16 \\
48.22\end{array}$ & $\begin{array}{l}05851 \\
06096 \\
06340 \\
06583 \\
06824 \\
07064\end{array}$ & $\begin{array}{l}245 \\
244 \\
243 \\
241 \\
240 \\
238\end{array}$ & $\begin{array}{l}208^{\circ} \\
50^{\prime} \\
40 \\
30 \\
20 \\
10\end{array}$ \\
\hline $\begin{array}{c}143^{\circ} \\
10^{\prime} \\
20 \\
30 \\
40 \\
50\end{array}$ & $\begin{array}{ll}3 & 5 \\
3 & 4 \\
3 & 3 \\
3 & 3 \\
3 & 2 \\
3 & 1\end{array}$ & $\begin{array}{rr}5 & 22.02 \\
4 & 37.99 \\
3 & 53.88 \\
3 & 9.69 \\
2 & 25.42 \\
1 & 41.08\end{array}$ & $\begin{array}{l}43.95 \\
44.03 \\
44.1 \text { I }\end{array}$ & $\begin{array}{l}90577 \\
90895 \\
91212 \\
91528 \\
91842 \\
92155\end{array}$ & $\begin{array}{l}3^{20} \\
3 \text { I } 8 \\
3 \text { I } 7 \\
3 \text { I } 6 \\
3^{I} 4 \\
313 \\
3 \text { I }\end{array}$ & $\begin{array}{c}217^{\circ} \\
50^{\prime} \\
40 \\
30 \\
20 \\
10\end{array}$ & $\begin{array}{c}153^{\circ} \\
10^{\prime} \\
20 \\
30 \\
40 \\
50\end{array}$ & $\begin{array}{ll}2 & 19 \\
2 & 18 \\
2 & 17 \\
2 & 16 \\
2 & 15 \\
2 & 15\end{array}$ & $\begin{array}{rr}9 & 8.54 \\
8 & 20.26 \\
7 & 31.92 \\
6 & 43.51 \\
5 & 55.05 \\
5 & 6.53\end{array}$ & & $\begin{array}{l}07302 \\
07539 \\
07775 \\
08009 \\
08242 \\
08473\end{array}$ & $\begin{array}{l}238 \\
237 \\
236 \\
234 \\
233 \\
231 \\
230\end{array}$ & $\begin{array}{c}207^{\circ} \\
50^{\prime} \\
40 \\
30 \\
20 \\
10\end{array}$ \\
\hline $\begin{array}{c}144^{\circ} \\
10^{\prime} \\
20 \\
30 \\
40 \\
50\end{array}$ & $\begin{array}{rr}3 & 0 \\
3 & 0 \\
2 & 59 \\
2 & 59 \\
2 & 57 \\
2 & 57\end{array}$ & $\begin{array}{ll}0 & 56.65 \\
0 & 12.14 \\
9 & 27.55 \\
8 & 42.89 \\
7 & 58.14 \\
7 & 13.32\end{array}$ & $\begin{array}{l}44.43 \\
44.51 \\
44.59 \\
44.66 \\
44.75 \\
44.82\end{array}$ & $\begin{array}{l}92466 \\
92776 \\
93085 \\
93393 \\
93700 \\
94005\end{array}$ & $\begin{array}{l}308 \\
3 \circ 7 \\
3 \circ 5 \\
304\end{array}$ & $\begin{array}{c}216^{\circ} \\
50^{\prime} \\
40 \\
30 \\
20 \\
10\end{array}$ & $\begin{array}{c}154^{\circ} \\
10^{\prime} \\
20 \\
30 \\
40 \\
50\end{array}$ & $\begin{array}{ll}2 & 14 \\
2 & 13 \\
2 & 12 \\
2 & 11 \\
2 & 11 \\
2 & 10\end{array}$ & $\begin{array}{rr}4 & 17.95 \\
3 & 29.31 \\
2 & 40.62 \\
1 & 51.86 \\
1 & 3.05 \\
0 & 14.18\end{array}$ & & & $\begin{array}{l}228 \\
227 \\
226 \\
224 \\
223 \\
222\end{array}$ & $\begin{array}{c}206^{\circ} \\
50^{\prime} \\
40 \\
30 \\
20 \\
10\end{array}$ \\
\hline $\begin{array}{c}145^{\circ} \\
10 \\
20 \\
30 \\
40 \\
50\end{array}$ & $\begin{array}{ll}2 & 56 \\
2 & 55 \\
2 & 54 \\
2 & 54 \\
2 & 53 \\
2 & 52\end{array}$ & $\begin{array}{ll}6 & 28.42 \\
5 & 43.44 \\
4 & 58.39 \\
4 & 13.26 \\
3 & 29.05 \\
2 & 42.77\end{array}$ & $\begin{array}{l}44.90 \\
44.98 \\
45.05 \\
45.13\end{array}$ & $\begin{array}{l}94309 \\
94612 \\
94913 \\
95213 \\
95511 \\
95808\end{array}$ & $\begin{array}{l}303 \\
301 \\
300 \\
298 \\
297 \\
296\end{array}$ & $\begin{array}{r}215^{\circ} \\
50^{\prime} \\
40 \\
30 \\
20 \\
10\end{array}$ & $\begin{array}{c}155^{\circ} \\
10^{\prime} \\
20 \\
30 \\
40 \\
50\end{array}$ & $\begin{array}{ll}2 & 9 \\
2 & 8 \\
2 & 7 \\
2 & 6 \\
2 & 6 \\
2 & 5\end{array}$ & $\begin{array}{rr}9 & 25.26 \\
8 & 36.28 \\
7 & 47.24 \\
6 & 58.15 \\
6 & 9.01 \\
5 & 19.81\end{array}$ & & & $\begin{array}{l}222 \\
220 \\
219 \\
217 \\
216 \\
214 \\
213\end{array}$ & $\begin{array}{c}205^{\circ} \\
50^{\prime} \\
40 \\
30 \\
20 \\
10\end{array}$ \\
\hline $\begin{array}{c}146^{\circ} \\
10^{\circ} \\
20 \\
30 \\
40 \\
50\end{array}$ & $\begin{array}{ll}2 & 51 \\
2 & 51 \\
2 & 50 \\
2 & 49 \\
2 & 48 \\
2 & 48\end{array}$ & $\begin{array}{rr}1 & 57.41 \\
1 & 11.97 \\
0 & 26.46 \\
9 & 40.87 \\
8 & 55.21 \\
8 & 9.48\end{array}$ & $45 \cdot 44$ & $\begin{array}{l}96104 \\
96398 \\
96691 \\
96983 \\
97273 \\
97562\end{array}$ & $\begin{array}{l}292 \\
290 \\
289 \\
288\end{array}$ & $\begin{array}{c}214^{\circ} \\
50^{\prime} \\
40 \\
30 \\
20 \\
10\end{array}$ & $\begin{array}{c}156^{\circ} \\
10^{\prime} \\
20 \\
30 \\
40 \\
50\end{array}$ & $\begin{array}{ll}2 & 4 \\
2 & 3 \\
2 & 2 \\
2 & 2 \\
2 & 1 \\
2 & 0\end{array}$ & $\begin{array}{rr}4 & 30.55 \\
3 & 41.24 \\
2 & 51.88 \\
2 & 2.47 \\
1 & 13.00 \\
0 & 23.48\end{array}$ & $\begin{array}{l}49 \cdot 41 \\
49 \cdot 47 \\
49 \cdot 52 \\
49 \cdot 57\end{array}$ & & $\begin{array}{l}212 \\
210 \\
209 \\
208 \\
206 \\
205\end{array}$ & $\begin{array}{c}204^{\circ} \\
50^{\prime} \\
40 \\
30 \\
20 \\
10\end{array}$ \\
\hline $\begin{array}{c}147^{\circ} \\
10^{\prime} \\
20 \\
30 \\
40 \\
50\end{array}$ & $\begin{array}{ll}2 & 47 \\
2 & 46 \\
2 & 45 \\
2 & 45 \\
2 & 44 \\
2 & 43 \\
\end{array}$ & $\begin{array}{rr}7 & 23.67 \\
6 & 37.79 \\
5 & 51.83 \\
5 & 5.80 \\
4 & 19.70 \\
3 & 33.53\end{array}$ & $\begin{array}{l}45.01 \\
45.88 \\
45.96\end{array}$ & $\begin{array}{l}97850 \\
98136 \\
98421 \\
98705 \\
98988 \\
99269\end{array}$ & $\begin{array}{l}288 \\
286 \\
285 \\
284 \\
293 \\
281 \\
280\end{array}$ & $\begin{array}{r}213^{\circ} \\
50^{\prime} \\
40 \\
30 \\
20 \\
10\end{array}$ & $\begin{array}{c}157^{\circ} \\
10^{\prime} \\
20 \\
30 \\
40 \\
50\end{array}$ & $\begin{array}{ll}1 & 59 \\
1 & 58 \\
1 & 57 \\
1 & 57 \\
1 & 56 \\
1 & 55\end{array}$ & $\begin{array}{rr}9 & 33.91 \\
8 & 44.29 \\
7 & 54.61 \\
7 & 4.88 \\
6 & 15.10 \\
5 & 25.28\end{array}$ & $\begin{array}{l}49.57 \\
49.62 \\
49.68 \\
49.73 \\
49.78 \\
49.82 \\
49.88\end{array}$ & $\begin{array}{l}12602 \\
12805 \\
13007 \\
13207 \\
13405 \\
13602\end{array}$ & $\begin{array}{l}205 \\
203 \\
202 \\
200 \\
198 \\
197 \\
196\end{array}$ & $\begin{array}{c}203^{\circ} \\
50^{\prime} \\
40 \\
30 \\
20 \\
10\end{array}$ \\
\hline $\begin{array}{c}148^{\circ} \\
10^{\prime} \\
20 \\
30 \\
40 \\
50 \\
110^{\circ}\end{array}$ & $\begin{array}{ll}2 & 49 \\
2 & 42 \\
2 & 41 \\
2 & 40 \\
2 & 39 \\
2 & 38 \\
& 20\end{array}$ & $\begin{array}{rr}2 & 47.29 \\
2 & 0.98 \\
1 & 14.59 \\
0 & 28.14 \\
9 & 41.61 \\
8 & 55.01 \\
& \end{array}$ & & $\begin{array}{r}99549 \\
99827 \\
* 00104 \\
* 00380 \\
* 00654 \\
* 00926\end{array}$ & $\begin{array}{l}280 \\
278 \\
277 \\
276 \\
274 \\
272 \\
271\end{array}$ & $\begin{array}{c}212^{\circ} \\
50^{\prime} \\
40 \\
30 \\
20 \\
10\end{array}$ & $\begin{array}{c}158^{\circ} \\
10^{\prime} \\
20 \\
30 \\
40 \\
50\end{array}$ & $\begin{array}{ll}1 & 54 \\
1 & 53 \\
1 & 5 \% \\
1 & 52 \\
1 & 51 \\
1 & 50\end{array}$ & $\begin{array}{rr}4 & 35.40 \\
3 & 45.47 \\
4 & 55.50 \\
2 & 5.48 \\
1 & 15.41 \\
0 & 25.29\end{array}$ & $\begin{array}{l}49.88 \\
49.93 \\
49.97 \\
50.02 \\
50.07 \\
50.12 \\
50.17\end{array}$ & $\begin{array}{l}13798 \\
13992 \\
14185 \\
14377 \\
14567 \\
14756\end{array}$ & $\begin{array}{l}192 \\
190 \\
189 \\
188\end{array}$ & $\begin{array}{c}202^{\circ} \\
50^{\prime} \\
40 \\
30 \\
20 \\
10\end{array}$ \\
\hline $\begin{array}{c}149^{\circ} \\
10^{\prime} \\
20 \\
30 \\
40 \\
50\end{array}$ & $\begin{array}{ll}2 & 35 \\
2 & 35 \\
2 & 36 \\
2 & 35 \\
2 & 35 \\
2 & 34\end{array}$ & $\begin{array}{rr}8 & 8.35 \\
7 & 21.62 \\
6 & 34.81 \\
5 & 47.94 \\
5 & 1.01 \\
4 & 14.00\end{array}$ & $\begin{array}{l}73 \\
81 \\
87\end{array}$ & $\begin{array}{l}* 01197 \\
* 01467 \\
* 01736 \\
* 02004 \\
* 02270 \\
* 02535\end{array}$ & $\begin{array}{l}271 \\
270 \\
269 \\
268 \\
266 \\
265 \\
263\end{array}$ & $\begin{array}{r}211^{\circ} \\
50^{\prime} \\
40 \\
30 \\
20 \\
10\end{array}$ & $\begin{array}{c}159^{\circ} \\
10^{\prime} \\
20 \\
30 \\
40 \\
50\end{array}$ & $\begin{array}{ll}1 & 49 \\
1 & 48 \\
1 & 47 \\
1 & 47 \\
1 & 46 \\
1 & 45\end{array}$ & $\begin{array}{rr}9 & 35.12 \\
8 & 44.91 \\
7 & 54.65 \\
7 & 4.34 \\
6 & 13.98 \\
5 & 23.58\end{array}$ & & $\begin{array}{l}14944 \\
15130 \\
15315 \\
15498 \\
15680 \\
15860\end{array}$ & $\begin{array}{l}186 \\
185 \\
183 \\
182 \\
180 \\
179\end{array}$ & $\begin{array}{c}201^{\circ} \\
50^{\prime} \\
40 \\
30 \\
20 \\
10\end{array}$ \\
\hline $150^{\circ}$ & 233 & 326.93 & 7.07 & $\begin{array}{r}* 02798 \\
* 1.30\end{array}$ & & $\begin{array}{c}210^{\circ} \\
g\end{array}$ & $160^{\circ}$ & 144 & $4 \quad 33.14$ & 4 & & 9 & $200^{\circ}$ \\
\hline
\end{tabular}




\begin{tabular}{|c|c|c|c|c|c|c|c|c|c|c|c|c|c|}
\hline \multicolumn{14}{|c|}{ TABLL: VII._Continued. } \\
\hline$g$ & & $F$ & & Log. & $r$ & & $g$ & & $E$ & & Log. & $r$ & \\
\hline $\begin{array}{c}160^{\circ} \\
10^{\prime} \\
20 \\
30 \\
40 \\
50\end{array}$ & $\begin{array}{lc}0 & 1 \\
1 & 44 \\
1 & 43 \\
1 & 42 \\
1 & 42 \\
1 & 41 \\
1 & 40\end{array}$ & $\begin{array}{c}" 1 \\
33.14 \\
42.65 \\
52.11 \\
1.53 \\
10.91 \\
20.24\end{array}$ & $\begin{array}{l}11 \\
50.49 \\
50.54 \\
50.58 \\
50.62 \\
50.67 \\
50.71\end{array}$ & $\begin{array}{r}1.30 \\
16039 \\
16216 \\
16392 \\
16566 \\
16739 \\
16910\end{array}$ & $\begin{array}{l}177 \\
176 \\
174 \\
173 \\
171 \\
170\end{array}$ & $\begin{array}{c}200^{\circ} \\
50^{\circ} \\
40 \\
30 \\
20 \\
10\end{array}$ & $\begin{array}{c}170^{\circ} \\
10^{\circ} \\
20 \\
30 \\
40 \\
50\end{array}$ & $\begin{array}{ll}0 & \\
0 & 5 \\
0 & 5 \\
0 & 5 \\
0 & 5 \\
0 & 1 \\
0 & 1\end{array}$ & \begin{tabular}{lr}
$\prime$ & \multicolumn{1}{c}{$"$} \\
52 & 57.32 \\
52 & 4.81 \\
51 & 12.28 \\
50 & 19.72 \\
$\$ 9$ & 27.14 \\
$\$ 5$ & 34.54
\end{tabular} & $\begin{array}{l}11 \\
52.51 \\
52.53 \\
52.56 \\
52.58 \\
52.60 \\
52.62\end{array}$ & $\begin{array}{r}1.30 \\
24057 \\
24176 \\
24264 \\
24350 \\
24434 \\
24517\end{array}$ & $\begin{array}{l}89 \\
88 \\
86 \\
84 \\
83 \\
82\end{array}$ & $\begin{array}{c}130^{\circ} \\
60^{\prime} \\
10 \\
30 \\
20 \\
10\end{array}$ \\
\hline $\begin{array}{c}161^{\circ} \\
10^{\prime} \\
20 \\
30 \\
10 \\
50\end{array}$ & $\begin{array}{ll}1 & 39 \\
1 & 39 \\
1 & 37 \\
1 & 36 \\
1 & 36 \\
1 & 35\end{array}$ & $\begin{array}{r}29.53 \\
39.78 \\
47.95 \\
57.14 \\
6.25 \\
15.33\end{array}$ & $\begin{array}{l}50.75 \\
50.80 \\
50.84 \\
50.89 \\
50.92 \\
50.96\end{array}$ & $\begin{array}{l}17090 \\
17248 \\
17415 \\
17581 \\
17745 \\
17908\end{array}$ & $\begin{array}{l}168 \\
167 \\
166 \\
164 \\
163 \\
162\end{array}$ & $\begin{array}{c}199^{\circ} \\
50^{\prime} \\
40 \\
30 \\
20 \\
10\end{array}$ & $\begin{array}{c}171^{\circ} \\
10^{\circ} \\
20 \\
30 \\
10 \\
50\end{array}$ & $\begin{array}{ll}0 & 4 \\
0 & 4 \\
0 & 4 \\
0 & 4 \\
0 & 4 \\
0 & 4\end{array}$ & $\begin{array}{rr}47 & 11.92 \\
46 & 49.25 \\
45 & 56.62 \\
45 & 3.93 \\
44 & 11.23 \\
43 & 18.51\end{array}$ & $\begin{array}{l}52.64 \\
52.66 \\
52.69 \\
52.70 \\
52.72 \\
52.74\end{array}$ & $\begin{array}{l}24599 \\
24679 \\
24758 \\
24935 \\
24910 \\
24934\end{array}$ & $\begin{array}{l}80 \\
79 \\
77 \\
75 \\
74 \\
73\end{array}$ & $\begin{array}{r}189^{\circ} \\
50^{\prime} \\
40 \\
80 \\
20 \\
10\end{array}$ \\
\hline $\begin{array}{r}162^{\circ} \\
10^{\prime} \\
20 \\
30 \\
40 \\
50\end{array}$ & $\begin{array}{ll}1 & 34 \\
1 & 33 \\
1 & 32 \\
1 & 31 \\
1 & 31 \\
1 & 30\end{array}$ & $\begin{array}{r}24.37 \\
33.37 \\
42.33 \\
51.24 \\
0.12 \\
8.96\end{array}$ & $\begin{array}{l}51.00 \\
51.04 \\
51.09 \\
51.12 \\
51.16 \\
51.20\end{array}$ & $\begin{array}{l}18070 \\
18230 \\
18395 \\
18545 \\
18700 \\
19854\end{array}$ & $\begin{array}{l}160 \\
158 \\
157 \\
155 \\
154 \\
153\end{array}$ & $\begin{array}{c}198^{\circ} \\
50^{\prime} \\
40 \\
30 \\
20 \\
10\end{array}$ & $\begin{array}{r}172^{3} \\
10^{\prime} \\
20 \\
30 \\
40 \\
50\end{array}$ & $\begin{array}{ll}0 & 4 \\
0 & 4 \\
0 & 4 \\
0 & 3 \\
0 & 3 \\
0 & 3\end{array}$ & $\begin{array}{rr}42 & 25.77 \\
41 & 33.01 \\
40 & 40.24 \\
39 & 47.45 \\
39 & 54.64 \\
39 & 1.82\end{array}$ & $\begin{array}{l}52.76 \\
52.77 \\
52.79 \\
52.81 \\
52.82 \\
52.84\end{array}$ & $\begin{array}{l}25057 \\
25128 \\
25198 \\
25267 \\
25334 \\
25399\end{array}$ & $\begin{array}{l}71 \\
70 \\
69 \\
67 \\
65 \\
64\end{array}$ & $\begin{array}{c}188^{\circ} \\
50^{\prime} \\
40 \\
30 \\
20 \\
10\end{array}$ \\
\hline $\begin{array}{r}163^{\circ} \\
10^{\prime} \\
20 \\
30 \\
40 \\
50\end{array}$ & $\begin{array}{ll}1 & 29 \\
1 & 29 \\
1 & 27 \\
1 & 26 \\
1 & 25 \\
1 & 25\end{array}$ & $\begin{array}{r}17.76 \\
26.52 \\
35.25 \\
+3.93 \\
52.53 \\
1.19\end{array}$ & $\begin{array}{l}51.24 \\
51.27 \\
51.32 \\
51.35 \\
51.39 \\
51.42\end{array}$ & $\begin{array}{l}19007 \\
19158 \\
19305 \\
19456 \\
19603 \\
19749\end{array}$ & $\begin{array}{l}150 \\
151 \\
150 \\
148 \\
147 \\
146 \\
144\end{array}$ & $\begin{array}{r}197^{\circ} \\
50^{\prime} \\
40 \\
30 \\
20 \\
10\end{array}$ & $\begin{array}{c}173^{\circ} \\
10^{\prime} \\
20 \\
30 \\
40 \\
50\end{array}$ & $\begin{array}{ll}0 & 3 \\
0 & 3 \\
0 & 3 \\
0 & 3 \\
0 & 3 \\
0 & 3\end{array}$ & $\begin{array}{rr}37 & 8.98 \\
36 & 16.12 \\
35 & 23.25 \\
34 & 30.36 \\
33 & 37.45 \\
32 & 44.53\end{array}$ & $\begin{array}{l}52.04 \\
52.86 \\
52.87 \\
52.89 \\
52.91 \\
52.92 \\
52.93\end{array}$ & $\begin{array}{l}25463 \\
25525 \\
25596 \\
25645 \\
25703 \\
25759\end{array}$ & $\begin{array}{l}62 \\
61 \\
59 \\
58 \\
56 \\
55\end{array}$ & $\begin{array}{r}187^{\circ} \\
50^{\prime} \\
10 \\
30 \\
20 \\
10\end{array}$ \\
\hline $\begin{array}{c}164^{\circ} \\
10^{\prime} \\
20 \\
30 \\
10 \\
50\end{array}$ & $\begin{array}{ll}1 & 21 \\
1 & 23 \\
i & 22 \\
1 & 21 \\
1 & 20 \\
1 & 19\end{array}$ & $\begin{array}{r}9.77 \\
13.31 \\
26.81 \\
35.28 \\
43.71 \\
52.11\end{array}$ & $\begin{array}{l}51.46 \\
51.50 \\
51.53 \\
51.57 \\
51.60 \\
51.64\end{array}$ & $\begin{array}{l}19993 \\
20035 \\
20176 \\
20316 \\
20454 \\
20590\end{array}$ & $\begin{array}{l}142 \\
141 \\
140 \\
138 \\
136 \\
135\end{array}$ & $\begin{array}{r}196^{\circ} \\
50^{\prime} \\
40 \\
30 \\
20 \\
10\end{array}$ & $\begin{array}{c}174^{\circ} \\
10^{\prime} \\
20 \\
30 \\
40 \\
50\end{array}$ & $\begin{array}{ll}0 & 3 \\
0 & 3 \\
0 & 3 \\
0 & 2 \\
0 & 2 \\
0 & 2\end{array}$ & $\begin{array}{rr}31 & 51.60 \\
30 & 58.66 \\
30 & 5.70 \\
29 & 12.73 \\
29 & 19.75 \\
27 & 26.76\end{array}$ & $\begin{array}{l}52.95 \\
52.94 \\
52.96 \\
52.97 \\
52.98 \\
52.99 \\
53.00\end{array}$ & $\begin{array}{l}25814 \\
25967 \\
25919 \\
25969 \\
26017 \\
26064\end{array}$ & $\begin{array}{l}50 \\
53 \\
52 \\
50 \\
48 \\
47 \\
46\end{array}$ & $\begin{array}{c}186^{\circ} \\
50^{\prime} \\
10 \\
30 \\
20 \\
10\end{array}$ \\
\hline $\begin{array}{c}165^{\circ} \\
10^{\prime} \\
20 \\
30 \\
40 \\
50\end{array}$ & $\begin{array}{ll}1 & 19 \\
1 & 19 \\
1 & 17 \\
1 & 16 \\
1 & 15 \\
1 & 14\end{array}$ & $\begin{array}{r}0.47 \\
8.80 \\
17.10 \\
25.36 \\
33.59 \\
41.79\end{array}$ & $\begin{array}{l}51.67 \\
51.70 \\
51.74 \\
51.77 \\
51.80 \\
51.83\end{array}$ & $\begin{array}{l}20725 \\
20858 \\
20990 \\
21121 \\
21250 \\
21377\end{array}$ & $\begin{array}{l}133 \\
133 \\
132 \\
131 \\
129 \\
127 \\
126\end{array}$ & $\begin{array}{c}195^{\circ} \\
50^{\prime} \\
40 \\
30 \\
20 \\
10\end{array}$ & $\begin{array}{c}175^{\circ} \\
10^{\prime} \\
20 \\
30 \\
40 \\
50\end{array}$ & $\begin{array}{ll}0 & 2 \\
0 & 2 \\
0 & 2 \\
0 & 2 \\
0 & 2 \\
0 & 2\end{array}$ & $\begin{array}{lr}26 & 33.76 \\
25 & 40.75 \\
24 & 47.72 \\
23 & 54.68 \\
23 & 1.64 \\
22 & 8.58\end{array}$ & $\begin{array}{l}53.01 \\
53.03 \\
53.04 \\
53.04 \\
53.06 \\
53.07\end{array}$ & $\begin{array}{l}26110 \\
26154 \\
26197 \\
26239 \\
26279 \\
26318\end{array}$ & $\begin{array}{l}44 \\
43 \\
42 \\
40 \\
39 \\
37\end{array}$ & $\begin{array}{c}185^{\circ} \\
50^{\prime} \\
10 \\
30 \\
20 \\
10\end{array}$ \\
\hline $\begin{array}{c}166^{\circ} \\
10^{\prime} \\
20 \\
30 \\
10 \\
50\end{array}$ & $\begin{array}{rr}1 & 13 \\
1 & 12 \\
1 & 12 \\
1 & 11 \\
1 & 10 \\
1 & 9\end{array}$ & $\begin{array}{r}49.96 \\
53.10 \\
6.20 \\
14.27 \\
22.31 \\
30.33\end{array}$ & $\begin{array}{l}51.86 \\
51.90 \\
51.93 \\
51.96 \\
51.98 \\
52.02\end{array}$ & $\begin{array}{l}21503 \\
21628 \\
21751 \\
21873 \\
21993 \\
22112\end{array}$ & $\begin{array}{l}125 \\
123 \\
122 \\
120 \\
119 \\
118\end{array}$ & $\begin{array}{c}194^{\circ} \\
50^{\prime} \\
40 \\
30 \\
20 \\
10\end{array}$ & $\begin{array}{c}178^{\circ} \\
10^{\prime} \\
20 \\
30 \\
40 \\
50\end{array}$ & $\begin{array}{ll}0 & 2 \\
0 & 2 \\
0 & 1 \\
0 & 1 \\
0 & 1 \\
0 & 1\end{array}$ & $\begin{array}{ll}21 & 15.51 \\
20 & 22.43 \\
19 & 29.35 \\
19 & 36.25 \\
17 & 43.15 \\
16 & 50.03\end{array}$ & $\begin{array}{l}53.08 \\
53.08 \\
53.10 \\
53.10 \\
53.12 \\
53.12\end{array}$ & & $\begin{array}{l}35 \\
34 \\
32 \\
30 \\
29 \\
28\end{array}$ & $\begin{array}{c}184^{\circ} \\
50^{\prime} \\
10 \\
30 \\
20 \\
10\end{array}$ \\
\hline $\begin{array}{r}167^{\circ} \\
10^{\circ} \\
20 \\
30 \\
40 \\
50\end{array}$ & $\begin{array}{ll}1 & 8 \\
1 & 7 \\
1 & 6 \\
1 & 6 \\
1 & 5 \\
1 & 4\end{array}$ & $\begin{array}{r}39.31 \\
46.27 \\
54.19 \\
2.09 \\
9.94 \\
17.78\end{array}$ & $\begin{array}{l}52.04 \\
52.08 \\
52.11 \\
52.14 \\
52.16 \\
52.19\end{array}$ & $\begin{array}{l}22230 \\
22346 \\
22460 \\
22573 \\
22684 \\
22793\end{array}$ & $\begin{array}{l}116 \\
114 \\
113 \\
111 \\
109 \\
108\end{array}$ & $\begin{array}{c}193^{\circ} \\
50^{\prime} \\
40 \\
30 \\
20 \\
10\end{array}$ & $\begin{array}{c}177^{\circ} \\
10^{\circ} \\
20 \\
30 \\
10 \\
50\end{array}$ & $\begin{array}{ll}0 & 1 \\
0 & 1 \\
0 & 1 \\
0 & 1 \\
0 & 1 \\
0 & 1\end{array}$ & $\begin{array}{rr}15 & 56.91 \\
15 & 3.78 \\
14 & 10.65 \\
13 & 17.51 \\
12 & 24.36 \\
11 & 31.21\end{array}$ & $\begin{array}{l}53.13 \\
53.13 \\
53.14 \\
53.15 \\
53.15 \\
53.15\end{array}$ & $\begin{array}{l}26543 \\
26569 \\
26594 \\
26617 \\
26639 \\
26659\end{array}$ & $\begin{array}{l}26 \\
25 \\
23 \\
22 \\
20 \\
19\end{array}$ & $\begin{array}{c}183^{\circ} \\
50^{\prime} \\
10 \\
30 \\
20 \\
10\end{array}$ \\
\hline $\begin{array}{c}169^{\circ} \\
10^{\prime} \\
20 \\
30 \\
40 \\
50\end{array}$ & $\begin{array}{rr}1 & 3 \\
1 & 2 \\
1 & 1 \\
1 & 0 \\
0 & 59 \\
0 & 59\end{array}$ & $\begin{array}{r}25.59 \\
33.37 \\
41.13 \\
49.86 \\
56.57 \\
4.25\end{array}$ & $\begin{array}{l}52.22 \\
52.24 \\
52.27 \\
52.29 \\
52.32 \\
52.35\end{array}$ & $\begin{array}{l}22901 \\
23008 \\
23113 \\
23217 \\
23320 \\
23421\end{array}$ & $\begin{array}{l}107 \\
105 \\
104 \\
103 \\
101 \\
100\end{array}$ & $\begin{array}{c}192^{\circ} \\
50^{\circ} \\
40 \\
30 \\
20 \\
10\end{array}$ & $\begin{array}{c}178^{\prime} \\
10^{\prime} \\
20 \\
30 \\
40 \\
50\end{array}$ & $\begin{array}{ll}0 & 1 \\
0 \\
0 \\
0 \\
0 \\
0\end{array}$ & $\begin{array}{rr}10 & 35.06 \\
9 & 44.90 \\
8 & 51.74 \\
7 & 58.53 \\
7 & 5.41 \\
6 & 12.24\end{array}$ & $\begin{array}{l}53.16 \\
53.16 \\
53.16 \\
53.17 \\
53.17 \\
53.17\end{array}$ & $\begin{array}{l}26678 \\
26695 \\
26711 \\
26726 \\
26739 \\
26750\end{array}$ & $\begin{array}{l}17 \\
16 \\
15 \\
13 \\
11 \\
10\end{array}$ & $\begin{array}{c}182^{\circ} \\
50^{\prime} \\
40 \\
30 \\
20 \\
10\end{array}$ \\
\hline $\begin{array}{c}169^{\circ} \\
10^{\circ} \\
20 \\
30 \\
10 \\
50\end{array}$ & $\begin{array}{ll}0 & 58 \\
0 & 57 \\
0 & 56 \\
0 & 55 \\
0 & 54 \\
0 & 53\end{array}$ & $\begin{array}{l}11.90 \\
19.53 \\
27.13 \\
34.71 \\
42.27 \\
49.81\end{array}$ & $\begin{array}{l}52.35 \\
52.37 \\
52.40 \\
5^{2.42} \\
52.44 \\
52.46 \\
52.49\end{array}$ & $\begin{array}{l}23521 \\
23619 \\
23716 \\
23811 \\
23904 \\
23996\end{array}$ & $\begin{array}{l}98 \\
97 \\
95 \\
93 \\
92 \\
91\end{array}$ & $\begin{array}{c}191^{\circ} \\
50^{\prime} \\
10 \\
30 \\
20 \\
10\end{array}$ & $\begin{array}{c}179^{\circ} \\
10^{\prime} \\
20 \\
30 \\
40 \\
50\end{array}$ & $\begin{array}{l}0 \\
0 \\
0 \\
0 \\
0 \\
0\end{array}$ & $\begin{array}{ll}5 & 19.07 \\
4 & 25.89 \\
3 & 32.72 \\
2 & 39.54 \\
1 & 46.36 \\
0 & 53.18\end{array}$ & $\begin{array}{l}53.18 \\
53.18 \\
53.17 \\
53.18 \\
53.18 \\
53.18 \\
53.18\end{array}$ & $\begin{array}{l}26760 \\
26768 \\
26775 \\
26780 \\
26784 \\
26786\end{array}$ & $\begin{array}{l}8 \\
7 \\
5 \\
4 \\
2 \\
1\end{array}$ & $\begin{array}{c}181^{\circ} \\
50^{\prime} \\
40 \\
30 \\
20 \\
10\end{array}$ \\
\hline $170^{\circ}$ & 052 & 57.32 & & $\begin{array}{r}24097 \\
1.30\end{array}$ & & $\begin{array}{c}190^{\circ} \\
g\end{array}$ & $180^{\circ}$ & 0 & $0 \quad 0.00$ & & $\begin{array}{r}26787 \\
1.30\end{array}$ & & $\begin{array}{c}180^{\circ} \\
g\end{array}$ \\
\hline
\end{tabular}


TABLE VIII, Ara. 1.-ACtion of Jupiter.

\begin{tabular}{|c|c|c|c|c|c|c|c|c|c|}
\hline Arg. & $(v . c .0)$ & Diff. & (v.s.1) & (v.c.1) & $(v . s .2)$ & (v.c.2) & $(\rho . c .0)$ & (p.s.1) & (p.c.1) \\
\hline & " & $"$ & " & $"$ & " & $"$ & & & \\
\hline 0 & 55.03 & & 0.14 & 4.42 & 0.10 & 0.11 & 2331 & 164 & 134 \\
\hline 1 & 55.58 & +0.55 & 0.13 & 4.42 & 0.10 & 0.12 & 2331 & 166 & 134 \\
\hline 2 & 56.13 & $\begin{array}{l}0.55 \\
0.55\end{array}$ & 0.12 & 4.41 & 0.10 & 0.12 & 2330 & 167 & 134 \\
\hline 3 & 56.68 & 0.55 & 0.11 & 4. 41 & 0.10 & 0.12 & 2330 & 169 & 134 \\
\hline-4 & 57.23 & $\begin{array}{l}0.55 \\
0.55\end{array}$ & 0.11 & 4.41 & 0.10 & 0.12 & 2330 & 171 & 135 \\
\hline 5 & 57.78 & +0.54 & 0.10 & 4.40 & 0.10 & 0.13 & 2329 & 172 & 135 \\
\hline 6 & 58.32 & $\begin{array}{r}0.54 \\
0.55\end{array}$ & 0.09 & 4.40 & 0.11 & 0.13 & 2328 & 174 & 135 \\
\hline 7 & 58.87 & $\begin{array}{l}0.55 \\
0.55\end{array}$ & 0.09 & 4.39 & 0.11 & 0.13 & 2328 & 176 & 135 \\
\hline 8 & 59.42 & $\begin{array}{l}0.55 \\
0.55\end{array}$ & 0.08 & 4.39 & 0.11 & 0.13 & 2327 & 177 & 135 \\
\hline 9 & 59.97 & $\begin{array}{l}0.55 \\
0.54\end{array}$ & 0.08 & 4.38 & 0.11 & 0.14 & 2326 & 179 & 136 \\
\hline 10 & 60.51 & & 0.08 & 4.38 & 0.11 & 0.14 & 2324 & 180 & 136 \\
\hline 11 & 61.06 & $\begin{array}{r}+0.55 \\
0.54\end{array}$ & 0.07 & 4.37 & 0.11 & 0.14 & 2323 & 182 & 136 \\
\hline 12 & 61.60 & $\begin{array}{l}0.54 \\
0.55\end{array}$ & 0.07 & 4.37 & 0.11 & 0.15 & .2321 & 184 & 137 \\
\hline 13 & 62.15 & $\begin{array}{l}0.55 \\
0.54\end{array}$ & 0.07 & 4.36 & 0.11 & 0.15 & 2320 & 185 & 137 \\
\hline 14 & 62.69 & $\begin{array}{l}0.54 \\
0.54\end{array}$ & 0.08 & 4.36 & 0.11 & 0.15 & 2318 & 187 & 137 \\
\hline 15 & 63.23 & & 0.08 & 4.35 & 0.11 & 0.15 & 2316 & 188 & 137 \\
\hline 16 & 63.78 & $\begin{array}{r}+0.55 \\
0.54\end{array}$ & 0.08 & 4.35 & 0.11 & 0.16 & 2314 & 190 & 137 \\
\hline 17 & 64.32 & 0.54 & 0.09 & 4.34 & 0.11 & 0.16 & 2312 & 191 & 138 \\
\hline 18 & 64.86 & 0.54 & 0.09 & 4.34 & 0.11 & 0.16 & 2310 & 193 & 138 \\
\hline 19 & 65.40 & $\begin{array}{l}0.54 \\
0.53\end{array}$ & 0.10 & 4.33 & 0.11 & 0.17 & 2308 & 194 & 138 \\
\hline 20 & 65.93 & & 0.11 & 4.33 & 0.11 & 0.17 & 2305 & 196 & 138 \\
\hline 21 & 66.47 & +0.54 & 0.12 & 4.32 & 0.11 & 0.17 & 2303 & 198 & 139 \\
\hline 22 & 67.00 & 0.53 & 0.13 & 4.32 & 0.11 & 0.17 & 2300 & 199 & 139 \\
\hline 23 & 67.54 & 0.54 & 0.14 & 4.31 & 0.11 & 0.18 & 2297 & 200 & 139 \\
\hline 24 & 68.07 & $\begin{array}{l}0.53 \\
0.53\end{array}$ & 0.15 & 4.30 & 0.11 & 0.18 & 2294 & 202 & 140 \\
\hline 25 & 68.60 & & 0.16 & 4.30 & 0.10 & 0.18 & 2291 & 203 & 140 \\
\hline 26 & 69.14 & +0.54 & 0.18 & 4.29 & 0.10 & 0.19 & 2288 & 205 & 140 \\
\hline 27 & 69.66 & $0.5^{2}$ & 0.20 & 4.29 & 0.10 & 0.19 & 2281 & 206 & 140 \\
\hline 28 & 70.19 & 0.53 & 0.21 & 4.28 & 0.10 & 0.19 & 2281 & 208 & 140 \\
\hline 29 & 70.72 & $\begin{array}{l}0.53 \\
0.52\end{array}$ & 0.23 & 4.28 & 0.10 & 0.20 & 2277 & 209 & 141 \\
\hline 30 & 71.24 & & 0.25 & 4.27 & 0.10 & 0.20 & 2274 & 210 & 141 \\
\hline 31 & 51.76 & +0.52 & 0.27 & 4.27 & 0.10 & 0.20 & 2270 & 212 & 141 \\
\hline 32 & 72.28 & $\begin{array}{l}0.52 \\
0.52\end{array}$ & 0.29 & 4.26 & 0.10 & 0.21 & 2266 & 213 & 141 \\
\hline 33 & 72.80 & $\begin{array}{l}0.5^{2} \\
0.52\end{array}$ & 0.31 & 4.26 & 0.10 & 0.21 & 2262 & 214 & 142 \\
\hline 34 & 73.32 & $\begin{array}{l}0.52 \\
0.51\end{array}$ & 0.33 & 4.25 & 0.10 & 0.21 & 2258 & 216 & 142 \\
\hline 35 & 73.83 & & 0.35 & 4.25 & 0.10 & 0.22 & 2254 & 217 & 142 \\
\hline 36 & $74.34^{-1}$ & & 0.38 & 4.21 & 0.10 & 0.22 & 2249 & 218 & 142 \\
\hline 37 & 74.85 & $0.5 \mathrm{I}$ & 0.40 & 4.24 & 0.10 & 0.22 & 2245 & 219 & 143 \\
\hline 38 & 75.36 & $\begin{array}{l}0.51 \\
0.51\end{array}$ & 0.43 & 4.23 & 0.10 & 0.23 & 2240 & 221 & 143 \\
\hline 39 & 75.87 & $\begin{array}{l}0.51 \\
0.50\end{array}$ & 0.46 & 4.23 & 0.09 & 0.23 & 2236 & 222 & 143 \\
\hline 40 & 76.37 & +0.50 & 0.49 & 4.22 & 0.09 & 0.23 & 2231 & 223 & 143 \\
\hline 41 & 76.87 & $\begin{array}{r}+0.50 \\
0.50\end{array}$ & 0.52 & 4.22 & 0.09 & 0.23 & 2226 & 224 & 144 \\
\hline 42 & 77.37 & $\begin{array}{l}0.50 \\
0.50\end{array}$ & 0.55 & 4.22 & 0.09 & 0.24 & 2221 & 225 & 144 \\
\hline 43 & 77.87 & 0.50 & 0.58 & 4.21 & 0.09 & 0.24 & 2216 & 227 & 144 \\
\hline 44 & 78.37 & 0.49 & 0.61 & 4.21 & 0.09 & 0.24 & 2210 & 228 & 144 \\
\hline 45 & 78.86 & & 0.64 & 4.21 & 0.09 & 0.25 & 2205 & 229 & 144 \\
\hline 46 & 79.35 & +0.49 & 0.68 & 4.20 & 0.09 & 0.25 & 2200 & 230 & 145 \\
\hline 47 & 79.84 & $\begin{array}{l}0.49 \\
0.48\end{array}$ & 0.71 & 4.20 & 0.09 & 0.25 & 2194 & 231 & 145 \\
\hline 48 & 80.32 & $\begin{array}{l}0.48 \\
0.48\end{array}$ & 0.75 & 4.20 & 0.09 & 0.26 & 2188 & 232 & 145 \\
\hline 49 & 80.80 & & 0.79 & 4.20 & 0.09 & 0.26 & 2182 & 233 & 145 \\
\hline 50 & 81.28 & 0.48 & 0.82 & 4.20 & 0.08 & 0.26 & 2176 & 234 & 146 \\
\hline 51 & 81.76 & $\begin{array}{r}+0.48 \\
0.47\end{array}$ & 0.86 & 4.19 & 0.08 & 0.26 & 2170 & 235 & 146 \\
\hline 52 & 82.23 & 0.47 & 0.90 & 4.19 & 0.08 & 0.26 & 2164 & 236 & 146 \\
\hline 53 & 82.70 & 0.47 & 0.94 & 4.19 & 0.08 & 0.27 & 2158 & 237 & 146 \\
\hline 54 & 83.17 & $\begin{array}{l}0.47 \\
0.46\end{array}$ & 0.98 & 4.19 & 0.08 & 0.27 & 2151 & 238 & 146 \\
\hline 55 & 83.63 & & 1.02 & 4.19 & 0.07 & 0.27 & 2145 & 239 & 146 \\
\hline 56 & $84.10^{-}$ & +0.47 & 1.07 & 4.19 & 0.07 & 0.28 & 2138 & 240 & 146 \\
\hline 57 & 84.55 & $\begin{array}{l}0.45 \\
0.46\end{array}$ & 1.11 & 4.19 & 0.07 & 0.28 & 2132 & 241 & 146 \\
\hline 58 & 85.01 & $\begin{array}{l}0.46 \\
0.45\end{array}$ & 1.16 & 4.19 & 0.07 & 0.28 & 2125 & 242 & 147 \\
\hline 59 & 85.46 & $\begin{array}{l}0.45 \\
0.45\end{array}$ & 1.20 & 4.19 & 0.07 & 0.28 & 2118 & 243 & 147 \\
\hline 60 & 85.91 & & 1.25 & 4.19 & 0.07 & 0.29 & 2111 & 241 & 147 \\
\hline
\end{tabular}


THE ORBIT OF URANUS.

\begin{tabular}{|c|c|c|c|c|c|c|c|c|c|}
\hline & & & TABI & VIII, & a. 1. & ntinued. & & & \\
\hline \multirow[t]{2}{*}{ Arg. } & $(v, c .0)$ & Diff. & $(v, 8,1)$ & $(v, c, 1)$ & $(v .8 .2)$ & $(v . c .2)$ & $(p . c .0)$ & $(p, 8,1)$ & $(\rho, c, 1)$ \\
\hline & $"$ & $"$ " & $" \prime$ & $" \prime$ & $"$ & $"$ & & & \\
\hline 60 & 85.91 & & 1.25 & 4.19 & 0.07 & 0.29 & 2111 & 244 & 147 \\
\hline 61 & 86.36 & $\begin{array}{r}+0.45 \\
0.44\end{array}$ & 1.30 & 4.19 & 0.07 & 0.29 & 2104 & 244 & 147 \\
\hline 69 & 86.80 & 0.44 & 1.34 & 4.20 & 0.06 & 0.29 & 2097 & 245 & 148 \\
\hline 63 & 87.24 & 0.44 & 1.39 & 4.20 & 0.06 & 0.29 & 2090 & 246 & 148 \\
\hline 64 & 87.68 & $\begin{array}{l}0.44 \\
0.43\end{array}$ & 1.44 & 4.20 & 0.06 & 0.29 & 2082 & 247 & 148 \\
\hline 65 & 88.11 & $+0,42$ & 1.49 & 4.20 & 0.06 & 0.30 & 2075 & 248 & 148 \\
\hline 66 & $88.53^{\top}$ & $\begin{array}{r}+0.42 \\
0.43\end{array}$ & 1.54 & 4.21 & 0.06 & 0.30 & 2067 & 248 & 143 \\
\hline 67 & 88.96 & $\begin{array}{l}0.43 \\
0.42\end{array}$ & 1.60 & 4.21 & 0.06 & 0.30 & 2059 & 249 & 148 \\
\hline 68 & 89.38 & $\begin{array}{l}0.42 \\
0.42\end{array}$ & 1.65 & 4.21 & 0.05 & 0.30 & 2052 & 250 & 148 \\
\hline 69 & 89.80 & $\begin{array}{l}0.4^{2} \\
0.41\end{array}$ & 1.70 & 4.22 & 0.05 & 0.31 & 2044 & 250 & 148 \\
\hline 70 & 90.21 & & 1.75 & 4.22 & 0.05 & 0.31 & 2036 & 251 & 149 \\
\hline 71 & $90.62^{\dagger}$ & +0.41 & 1.81 & 4.23 & 0.05 & 0.31 & 2028 & 252 & 149 \\
\hline 72 & 91.03 & $0.4 \mathrm{r}$, & 1.86 & 4.23 & 0.05 & 0.31 & 2020 & 252 & 149 \\
\hline 73 & 91.43 & 0.40 & 1.92 & 4.24 & 0.05 & 0.31 & 2012 & 253 & 149 \\
\hline 74 & 91.83 & $\begin{array}{l}0.40 \\
0.39\end{array}$ & 1.98 & 4.24 & 0.04 & 0.32 & 2003 & 253 & 149 \\
\hline 75 & 99.22 & & 2.03 & 4.25 & 0.04 & 0.32 & 1995 & 254 & 149 \\
\hline 76 & $92.62^{\dagger}$ & +0.43 & 2.09 & 4.26 & 0.04 & 0.32 & 1986 & 254 & 149 \\
\hline 77 & 93.00 & $\begin{array}{l}0.33 \\
0.28\end{array}$ & 2.15 & 4.26 & 0.04 & 0.32 & 1978 & 255 & 149 \\
\hline 73 & 93.38 & $\begin{array}{l}0.3^{8} \\
0.38\end{array}$ & 2.21 & 4.27 & 0.04 & 0.32 & 1969 & 255 & 149 \\
\hline 79 & 93.76 & $\begin{array}{l}0.3^{8} \\
0.3^{8}\end{array}$ & 2.27 & 4.28 & 0.04 & 0.32 & 1960 & 256 & 149 \\
\hline 80 & 94.14 & & 2.33 & 4.29 & 0.04 & 0.32 & 1952 & 256 & 149 \\
\hline 81 & $94.51^{\dagger}$ & +0.37 & 2.39 & 4.30 & 0.03 & 0.32 & 1943 & 257 & 149 \\
\hline 82 & 94.87 & $\begin{array}{l}0.36 \\
0.36\end{array}$ & 2.45 & 4.31 & 0.03 & 0.33 & 1934 & $25 i$ & 149 \\
\hline 83 & 95.23 & $\begin{array}{l}0.3^{0} \\
0.3^{6}\end{array}$ & 2.51 & 4.32 & 0.03 & 0.33 & 1925 & 257 & 149 \\
\hline 84 & 95.59 & $\begin{array}{l}0.3^{\circ} \\
0.3^{6}\end{array}$ & 2.57 & 4.33 & 0.03 & 0.33 & 1916 & 258 & 149 \\
\hline 85 & 95.95 & & 2.64 & 4.34 & 0.03 & 0.33 & 1906 & 258 & 149 \\
\hline 86 & $96.30^{-}$ & $\begin{array}{r}+0.35 \\
0.34\end{array}$ & 2.70 & 4.35 & 0.03 & 0.33 & 1897 & 258 & 149 \\
\hline 87 & 93.64 & $\begin{array}{l}0.34 \\
0.34\end{array}$ & 2.76 & 4.36 & 0.03 & 0.33 & 1888 & 259 & 149 \\
\hline 88 & 96.93 & $\begin{array}{l}0.34 \\
0.34\end{array}$ & 2.83 & 4.37 & 0.03 & 0.83 & 1878 & 259 & 149 \\
\hline 89 & 97.32 & $\begin{array}{l}0.34 \\
0.33\end{array}$ & 2.89 & 4.38 & 0.02 & 0.33 & 1869 & 259 & 149 \\
\hline 90 & 97.65 & & 2.96 & 4.39 & 0.02 & 0.33 & 1859 & 260 & 148 \\
\hline 91 & 97.97 & $\begin{array}{r}+0.3^{2} \\
0.32\end{array}$ & 3.02 & 4.41 & 0.02 & 0.33 & 1850 & 260 & 148 \\
\hline 92 & 98.29 & $\begin{array}{l}0.3^{2} \\
0.3^{2}\end{array}$ & 3.09 & 4.42 & 0.02 & 0.33 & 1840 & 260 & 148 \\
\hline 93 & 98.61 & $\begin{array}{l}0.3^{2} \\
0.3^{\mathrm{I}}\end{array}$ & 3.15 & 4.43 & 0.02 & 0.33 & 1830 & 260 & 148 \\
\hline 94 & 98.92 & $0.3 \mathrm{t}$ & 3.22 & 4.45 & 0.02 & 0.33 & 1820 & 260 & 148 \\
\hline 95 & $99.23+$ & +0.30 & 3.29 & 4.46 & 0.02 & 0.33 & 1810 & 261 & 148 \\
\hline 96 & $99.53^{\dagger}$ & $\begin{array}{r}+0.30 \\
0.30\end{array}$ & 3.35 & 4.48 & 0.02 & 0.34 & 1800 & 261 & 148 \\
\hline 97 & 99.83 & 0.29 & 3.49 & 4.49 & 0.02 & 0.34 & 1790 & 261 & 148 \\
\hline 98 & 100.12 & 0.29 & 3.49 & 4.51 & 0.01 & 0.34 & 1780 & 261 & 147 \\
\hline 99 & 100.41 & 0.29 & 3.56 & 4.52 & 0.01 & 0.34 & 1770 & 261 & 147 \\
\hline 100 & 100.70 & +0.27 & 3.63 & 4.54 & 0.01 & 0.34 & 1760 & 261 & 147 \\
\hline 101 & $100.97^{\dagger}$ & 0.28 & 3.69 & 4.56 & 0.01 & 0.34 & 1750 & 261 & 147 \\
\hline 102 & 101.25 & 0.27 & 3.76 & 4.57 & 0.01 & 0.34 & 1739 & 261 & 147 \\
\hline 103 & 101.52 & 0.26 & 3.83 & 4.59 & 0.01 & 0.34 & 1729 & 261 & 146 \\
\hline 104 & 101.78 & 0.26 & 3.90 & 4.61 & 0.01 & 0.34 & 1718 & 261 & 146 \\
\hline 105 & $102.04+$ & +0.25 & 3.97 & 4.63 & 0.01 & 0.34 & 1708 & 261 & 146 \\
\hline 106 & $102.29^{\dagger}$ & 0.25 & 4.04 & 4.65 & 0.01 & 0.34 & 1697 & 261 & 145 \\
\hline 107 & 102.54 & 0.24 & 4.11 & 4.67 & 0.01 & 0.34 & 1686 & 261 & 145 \\
\hline 108 & 102.78 & 0.24 & 4.18 & 4.69 & 0.01 & 0.34 & 1676 & 261 & 145 \\
\hline 109 & 103.02 & 0.23 & 4.25 & 4.71 & 0.01 & 0.34 & 1665 & 261 & 145 \\
\hline 110 & 103.25 & +0.23 & 4.32 & 4.73 & 0.01 & 0.34 & 1654 & 261 & 144 \\
\hline 111 & $103.48^{t}$ & $\begin{array}{r}+0.23 \\
0.22\end{array}$ & 4.39 & 4.75 & 0.01 & 0.34 & 1643 & 261 & 144 \\
\hline 112 & 103.70 & 0.22 & 4.46 & 4.77 & 0.01 & 0.34 & 1632 & 261 & 144 \\
\hline 113 & 103.92 & 0.21 & 4.53 & 4.79 & 0.01 & 0.34 & 1622 & 260 & 143 \\
\hline 114 & 104.13 & $\begin{array}{l}0.21 \\
0.21\end{array}$ & 4.60 & 4.81 & 0.01 & 0.34 & 1611 & 260 & 143 \\
\hline 115 & 104.34 & +0.20 & 4.67 & 4.83 & 0.01 & 0.33 & 1600 & 260 & 142 \\
\hline 116 & $104.54^{\dagger}$ & $\begin{array}{r}+0.20 \\
0.20\end{array}$ & 4.74 & 4.85 & 0.01 & 0.33 & 1588 & 260 & 142 \\
\hline 117 & 104.74 & 0.19 & 4.81 & 4.87 & 0.01 & 0.33 & 1577 & 260 & 142 \\
\hline 118 & 104.93 & o. 18 & 4.88 & 4.90 & 0.01 & 0.33 & 1566 & 259 & 141 \\
\hline 119 & 105.11 & 0.18 & 4.95 & 4.92 & 0.01 & 0.33 & 1555 & 259 & 141 \\
\hline 120 & 105.29 & & 5.01 & 4.94 & 0.02 & 0.33 & 1544 & 259 & 140 \\
\hline
\end{tabular}

29 July, 1873. 
TABLE VIII, ARa. 1.-Continued.

\begin{tabular}{|c|c|c|c|c|c|c|c|c|c|}
\hline Arg. & $(u . c .0)$ & Diff. & (v.s.1) & $(r . c .1)$ & $(v . s .2)$ & $(v . c .2)$ & $(\rho . c .0)$ & (p.s.l) & $(\rho . c .1)$ \\
\hline & $"$ & $"$ & " & $"$ & $"$ & $"$ & & & \\
\hline 120 & 105.29 & & 5.01 & 4.94 & 0.02 & 0.33 & 1544 & 259 & 140 \\
\hline 121 & $105.46^{+}$ & †. 17 & 5.08 & 4.97 & 0.02 & 0.33 & 1533 & 259 & 140 \\
\hline 122 & 105.63 & $\begin{array}{l}0.17 \\
0.16\end{array}$ & 5.15 & 4.99 & 0.02 & 0.33 & 1521 & 253 & 139 \\
\hline 123 & 105.79 & 0.16 & 5.22 & 5.01 & 0.02 & 0.33 & 1510 & 258 & 139 \\
\hline 124 & 105.95 & $\begin{array}{l}0.10 \\
0.15\end{array}$ & 5.29 & 5.04 & 0.02 & 0.33 & 1499 & 258 & 139 \\
\hline 125 & 106.10 & +0.15 & 5.36 & 5.06 & 0.02 & 0.33 & 1487 & 257 & 138 \\
\hline 126 & $106.25^{7}$ & $\begin{array}{r}+0.15 \\
0.14\end{array}$ & 5.43 & 5.09 & 0.02 & 0.33 & 1476 & 257 & 137 \\
\hline 127 & 106.39 & $\begin{array}{l}0.14 \\
0.14\end{array}$ & 5.50 & 5.11 & 0.02 & 0.33 & 1464 & 257 & 137 \\
\hline 128 & 106.53 & $\begin{array}{l}.14 \\
0.12\end{array}$ & 5.57 & 5.14 & 0.02 & 0.33 & 1453 & 256 & 136 \\
\hline 129 & 106.65 & 0.13 & 5.64 & 5.16 & 0.02 & 0.33 & 1441 & 256 & 135 \\
\hline 130 & 106.78 & & 5.71 & 5.19 & 0.02 & 0.32 & 1430 & 256 & 135 \\
\hline 131 & 106.89 & HO. 11 & 5.78 & 5.21 & 0.03 & 0.32 & 1418 & 255 & 134 \\
\hline 132 & 107.01 & O. I 2 & 5.85 & 5.24 & 0.03 & 0.32 & 1407 & 255 & 134 \\
\hline 133 & 107.11 & 0.10 & 5.91 & 5.26 & 0.03 & 0.32 & 1395 & 254 & 133 \\
\hline 134 & 107.22 & $\begin{array}{l}0.11 \\
0.09\end{array}$ & 5.98 & 5.29 & 0.03 & 0.32 & 1384 & 254 & 132 \\
\hline 135 & 107.31 & to.0n & 6.05 & 5.32 & 0.03 & 0.32 & 1372 & 254 & 132 \\
\hline 136 & $107.40^{\dagger}$ & F-0.09 & 6.11 & 5.34 & 0.04 & 0.32 & 1360 & 253 & 131 \\
\hline 137 & 107.49 & $\begin{array}{l}0.09 \\
0.09\end{array}$ & 6.18 & 5.37 & 0.04 & 0.32 & 1349 & 253 & 131 \\
\hline 138 & 107.57 & 0.05 & 6.25 & 5.40 & 0.04 & 0.32 & 1337 & 252 & 130 \\
\hline 139 & 107.64 & $\begin{array}{l}0.07 \\
0.06\end{array}$ & 6.31 & 5.42 & 0.04 & 0.32 & 1325 & 252 & 129 \\
\hline 140 & 107.70 & & 6.38 & 5.45 & 0.04 & 0.32 & 1314 & 251 & 129 \\
\hline 141 & $107.76^{-}$ & $\begin{array}{r}-0.06 \\
0.06\end{array}$ & 6.44 & 5.48 & 0.05 & 0.31 & 1302 & 251 & 128 \\
\hline 142 & 107.82 & 0.06 & 6.51 & 5.51 & 0.05 & 0.31 & 1290 & 250 & 127 \\
\hline 143 & 107.87 & $\begin{array}{l}0.05 \\
0.04\end{array}$ & 6.57 & 5.53 & 0.05 & 0.31 & 1278 & 250 & 126 \\
\hline 144 & 107.91 & $\begin{array}{l}0.04 \\
0.04\end{array}$ & 6.64 & 5.56 & 0.05 & 0.31 & 1267 & 249 & 126 \\
\hline 145 & 107.95 & -0.03 & 6.70 & 5.59 & 0.05 & 0.31 & 1255 & 249 & 125 \\
\hline 146 & $107.98^{t}$ & $\begin{array}{r}0.03 \\
0.03\end{array}$ & 6.76 & 5.61 & 0.06 & 0.31 & 1243 & 248 & 124 \\
\hline 147 & 108.01 & $\begin{array}{l}0.03 \\
0.02\end{array}$ & 6.83 & 5.64 & 0.06 & 0.31 & $12: 31$ & 248 & 123 \\
\hline 148 & 109.03 & 0.02 & 6.89 & 5.67 & 0.06 & 0.31 & 1220 & 247 & 123 \\
\hline 149 & 108.05 & 0.00 & 6.95 & 5.69 & 0.06 & 0.31 & 1208 & 247 & 122 \\
\hline 150 & 108.05 & LO., & 7.01 & 5.72 & 0.07 & 0.31 & 1196 & 246 & 121 \\
\hline 151 & $108.06^{t}$ & $\begin{array}{l}-0.01 \\
-0.01\end{array}$ & 7.09 & 5.75 & 0.07 & 0.31 & 1184 & 246 & 120 \\
\hline 152 & $108.05^{-}$ & $\begin{array}{l}0.01 \\
0.01\end{array}$ & 7.14 & 5.78 & 0.07 & 0.31 & 1172 & 245 & 119 \\
\hline 153 & 108.04 & $\begin{array}{l}0.01 \\
0.01\end{array}$ & 7.20 & 5.80 & 0.07 & 0.30 & 1161 & 244 & 118 \\
\hline 154 & 108.03 & $\begin{array}{l}0.01 \\
0.02\end{array}$ & 7.25 & 5.83 & 0.08 & 0.30 & 1149 & 244 & 118 \\
\hline 155 & 108.01 & -0.03 & 7.31 & 5.86 & 0.08 & 0.30 & 1137 & 243 & 117 \\
\hline 156 & 107.98 & & 7.37 & 5.88 & 0.08 & 0.30 & 1126 & 243 & 116 \\
\hline 157 & 107.95 & $\begin{array}{l}0.03 \\
0.04\end{array}$ & 7.43 & 5.91 & 0.09 & 0.30 & 1114 & 242 & 115 \\
\hline 158 & 107.91 & $\begin{array}{l}0.04 \\
0.04\end{array}$ & 7.49 & 5.94 & 0.09 & 0.30 & 1102 & 241 & 114 \\
\hline 159 & 107.87 & 0.05 & 7.54 & 5.97 & 0.09 & 0.30 & 1090 & 241 & 113 \\
\hline 160 & 107.82 & -0.05 & 7.60 & 5.99 & 0.10 & 0.30 & 1079 & 240 & 112 \\
\hline 161 & 107.77 & 0.07 & 7.66 & 6.02 & 0.10 & 0.30 & 1067 & 240 & 112 \\
\hline 162 & 107.70 & 0.06 & 7.71 & 6.04 & 0.10 & 0.30 & 1055 & 239 & 111 \\
\hline 163 & 107.64 & 0.08 & 7.77 & 6.07 & 0.11 & 0.30 & 1044 & 238 & 110 \\
\hline 164 & 107.56 & 0.07 & 7.82 & 6.10 & 0.11 & 0.29 & 1032 & 238 & 109 \\
\hline 165 & 107.49 & & 7.87 & 6.12 & 0.11 & 0.29 & 1020 & 237 & 108 . \\
\hline 166 & $107.40^{-}$ & & T. 93 & 6.15 & 0.12 & 0.29 & 1009 & 236 & $10\}$ \\
\hline 167 & 107.31 & 0.09 & 7.98 & 6.17 & 0.12 & 0.29 & 997 & 236 & 106 \\
\hline 168 & 107.22 & 0.11 & 8.03 & 6.20 & 0.12 & 0.29 & 996 & 235 & 105 \\
\hline 169 & 107.11 & 0.10 & 8.08 & 6.22 & 0.13 & 0.29 & 974 & 234 & 104 \\
\hline 170 & 107.01 & & 8.13 & 6.25 & 0.13 & 0.29 & 963 & 234 & 103 \\
\hline 171 & $106.89^{-}$ & $\begin{array}{r}-0.12 \\
0.12\end{array}$ & 8.18 & 6.27 & 0.14 & 0.29 & 951 & 233 & 102 \\
\hline 172 & 106.77 & $\begin{array}{l}0.12 \\
0.12\end{array}$ & 8.23 & 6.29 & 0.14 & 0.29 & 940 & .232 & 101 \\
\hline 173 & 106.65 & $\begin{array}{l}0.12 \\
0.13\end{array}$ & 8.28 & 6.32 & 0.14 & 0.29 & 929 & 232 & 100 \\
\hline 174 & 106.52 & $\begin{array}{l}0.13 \\
0.14\end{array}$ & 8.33 & 6.34 & 0.15 & 0.29 & 917 & 231 & 99 \\
\hline 175 & 106.38 & $=0.14$ & 8.37 & 6.36 & 0.15 & 0.29 & 906 & 230 & 98 \\
\hline 176 & $106.24^{-}$ & $\begin{array}{r}0.14 \\
0.14\end{array}$ & 8.42 & 6.39 & 0.15 & 0.29 & 894 & 230 & 97 \\
\hline 177 & 106.10 & $\begin{array}{l}0.14 \\
0.16\end{array}$ & 8.46 & 6.41 & 0.16 & 0.29 & 883 & 229 & 96 \\
\hline 178 & 105.94 & $\begin{array}{l}0.10 \\
0.16\end{array}$ & 8.51 & 6.43 & 0.16 & 0.29 & 872 & 228 & 95 \\
\hline 179 & 105.78 & $\begin{array}{l}0.10 \\
0.16\end{array}$ & 8.55 & 6.45 & 0.17 & 0.29 & 861 & 228 & 94 \\
\hline 180 & 105.62 & & 8.60 & 6.47 & 0.17 & 0.29 & 850 & 227 & 93 \\
\hline
\end{tabular}


TABLE VIII, Ang. 1.-Continued.

\begin{tabular}{|c|c|c|c|c|c|c|c|c|c|}
\hline Arg. & $(v . c .0)$ & Diff & (v.8.1) & $(v . c .1)$ & $(v, 8.2)$ & $(v . c .2)$ & $(p . c .0)$ & (p.s.1) & (p.e.1) \\
\hline & " & " & $"$ & " & " & " & & & \\
\hline 180 & 105.62 & & 8.60 & 6.47 & 0.17 & 0.29 & 850 & 227 & 93 \\
\hline 181 & 105.45 & $\begin{array}{r}-0.17 \\
0.18\end{array}$ & 8.64 & 6.50 & 0.17 & 0.29 & 839 & 226 & 92 \\
\hline 182 & 105.27 & $\begin{array}{l}0.10 \\
0.18\end{array}$ & 8.68 & 6.52 & 0.18 & 0.29 & 828 & 226 & 91 \\
\hline 183 & 105.09 & 0.19 & 8.72 & 6.54 & 0.18 & 0.29 & 816 & 225 & 90 \\
\hline 184 & 104.90 & 0.19 & 8.76 & 6.56 & 0.19 & 0.29 & 805 & 224 & 89 \\
\hline 185 & 104.71 & -0.20 & 8.80 & 6.57 & 0.19 & 0.29 & 794 & 224 & 88 \\
\hline 186 & 104.51 & 0.20 & 8.84 & 6.59 & 0.19 & 0.29 & 784 & 223 & 87 \\
\hline 187 & 104.31 & 0.21 & 8.88 & 6.61 & 0.20 & 0.29 & 773 & 222 & 86 \\
\hline 188 & 104.10 & 0.21 & 8.92 & 6.63 & 0.20 & 0.29 & 762 & 222 & 85 \\
\hline 189 & 103.89 & 0.22 & 8.96 & 6.65 & 0.21 & 0.29 & 751 & 221 & 84 \\
\hline 190 & 103.67 & -0.23 & 8.99 & 6.67 & 0.21 & 0.29 & 740 & 221 & 83 \\
\hline 191 & 103.44 & 0.23 & 9.03 & 6.68 & 0.21 & 0.29 & 730 & 220 & 82 \\
\hline 192 & 103.21 & 0.23 & 9.07 & 6.70 & 0.22 & 0.29 & 719 & 219 & 81 \\
\hline 193 & 102.98 & 0.24 & 9.10 & 6.71 & 0.22 & 0.29 & 708 & 219 & 80 \\
\hline 194 & 102.74 & 0.24 & 9.13 & 6.73 & 0.22 & 0.29 & 698 & 218 & 79 \\
\hline 195 & 102.49 & -0.25 & 9.17 & 6.74 & 0.23 & 0.29 & 688 & 217 & 78 \\
\hline 196 & 102.24 & $\begin{array}{l}-0.25 \\
0.26\end{array}$ & 9.20 & 6.76 & 0.23 & 0.29 & 677 & 216 & 77 \\
\hline 197 & 101.98 & $\begin{array}{l}0.20 \\
0.26\end{array}$ & 9.23 & 6.77 & 0.24 & 0.29 & 667 & 216 & 76 \\
\hline 198 & 101.72 & 0.27 & 9.26 & 6.78 & 0.24 & 0.29 & 657 & 215 & 75 \\
\hline 199 & 101.45 & 0.27 & 9.29 & 6.79 & 0.24 & 0.29 & 646 & 215 & 74 \\
\hline 200 & 101.18 & -0.28 & 9.32 & 6.81 & 0.25 & 0.29 & 636 & 214 & 73 \\
\hline 201 & 100.90 & 0.28 & 9.35 & 6.82 & 0.25 & 0.29 & 626 & 213 & 72 \\
\hline 202 & 100.62 & 0.29 & 9.38 & 6.83 & 0.25 & 0.29 & 616 & 213 & 71 \\
\hline 203 & 100.33 & 0.29 & 9.41 & 6.84 & 0.26 & 0.29 & 606 & 212 & 70 \\
\hline 204 & 100.04 & $\begin{array}{l}0.29 \\
0.30\end{array}$ & 9.43 & 6.85 & 0.26 & 0.29 & 596 & 211 & 69 \\
\hline 205 & 99.74 & & 9.46 & 6.86 & 0.26 & 0.29 & 586 & 211 & 68 \\
\hline 206 & 99.44 & $\begin{array}{l}-0.3^{0} \\
0.31\end{array}$ & 9.48 & 6.86 & 0.27 & 0.29 & 577 & 210 & 67 \\
\hline 207 & 99.13 & $\begin{array}{l}0.31 \\
0.31\end{array}$ & 9.51 & 6.87 & 0.27 & 0.29 & 567 & 210 & 66 \\
\hline 208 & 98.82 & 0.32 & 9.53 & 6.88 & 0.27 & 0.29 & 557 & 209 & 65 \\
\hline 209 & 98.50 & $0.3^{2}$ & 9.55 & 6.88 & 0.28 & 0.29 & 548 & 208 & 64 \\
\hline 210 & 98.18 & -0.33 & 9.58 & 6.89 & 0.28 & 0.29 & 538 & 208 & 63 \\
\hline 211 & 97.85 & $\begin{array}{r}-0.33 \\
0.33\end{array}$ & 9.60 & 6.89 & 0.28 & 0.29 & 529 & 207 & 62 \\
\hline 212 & 97.52 & $\begin{array}{l}0.33 \\
0.34\end{array}$ & 9.62 & 6.90 & 0.29 & 0.29 & 520 & 206 & 61 \\
\hline 213 & 97.18 & $\begin{array}{l}0.34 \\
0.34\end{array}$ & 9.64 & 6.90 & 0.29 & 0.29 & 510 & 206 & 60 \\
\hline 214 & 96.84 & 0.35 & 9.66 & 6.90 & 0.29 & 0.29 & 501 & 205 & 59 \\
\hline 215 & 96.49 & & 9.68 & 6.91 & 0.30 & 0.29 & 492 & 205 & 58 \\
\hline 216 & 96.14 & $\begin{array}{r}0.35 \\
0.35\end{array}$ & 9.70 & 6.91 & 0.30 & 0.29 & 483 & 204 & 57 \\
\hline 217 & 95.79 & 0.36 & 9.71 & 6.91 & 0.30 & 0.29 & 474 & 204 & 56 \\
\hline 218 & 95.43 & 0.37 & 9.73 & 6.91 & 0.31 & 0.29 & 464 & 203 & 55 \\
\hline 219 & 95.06 & 0.37 & 9.75 & 6.91 & 0.31 & 0.30 & 456 & 202 & 54 \\
\hline 220 & 94.69 & & 9.76 & 6.91 & 0.31 & 0.30 & 448 & 202 & 53 \\
\hline 221 & 94.32 & $\begin{array}{l}-0.37 \\
0.28\end{array}$ & 9.78 & 6.90 & 0.31 & 0.30 & 439 & 201 & 52 \\
\hline 222 & 93.94 & $\begin{array}{l}0.38 \\
0.38\end{array}$ & 9.79 & 6.90 & 0.32 & 0.30 & 430 & 201 & 51 \\
\hline 223 & 93.56 & $\begin{array}{l}0.3^{\circ} \\
0.30\end{array}$ & 9.81 & 6.90 & 0.32 & 0.30 & 422 & 200 & 50 \\
\hline 224 & 93.17 & $\begin{array}{l}.39 \\
0.39\end{array}$ & 9.82 & 6.90 & 0.32 & 0.30 & 413 & 200 & 49 \\
\hline .225 & 92.78 & -0.3 & 9.83 & 6.89 & 0.33 & 0.30 & 405 & 199 & 48 \\
\hline 226 & 92.39 & $\begin{array}{l}-0.39 \\
0.40\end{array}$ & 9.85 & 6.88 & 0.33 & 0.30 & 398 & 198 & 47 \\
\hline 227 & 91.99 & $\begin{array}{l}0.40 \\
0.40\end{array}$ & 9.86 & 6.88 & 0.33 & 0.30 & 390 & 198 & 46 \\
\hline 228 & 91.59 & $\begin{array}{l}0.40 \\
0.41\end{array}$ & 9.87 & 6.87 & 0.33 & 0.30 & 382 & 197 & 46 \\
\hline 229 & 91.18 & $\begin{array}{l}0.41 \\
0.4 \mathrm{I}\end{array}$ & 9.88 & 6.86 & 0.34 & 0.30 & 374 & 197 & 45 \\
\hline 230 & 90.77 & & 9.89 & 6.85 & 0.34 & 0.30 & 365 & 196 & 44 \\
\hline 231 & 90.36 & $-0.4 \mathrm{I}$ & 9.90 & 6.84 & 0.34 & 0.30 & 357 & $196=$ & 43 \\
\hline 232 & 89.94 & 0.42 & 9.91 & 6.83 & 0.34 & 0.30 & 349 & 195 & 42 \\
\hline 233 & 89.51 & 0.43 & 9.91 & 6.82 & 0.34 & 0.30 & 342 & 194 & 41 \\
\hline 234 & 89.09 & $\begin{array}{l}0.42 \\
0.44\end{array}$ & 9.92 & 6.81 & 0.35 & 0.30 & 334 & 194 & 41 \\
\hline 235 & 88.65 & & 9.93 & 6.80 & 0.35 & 0.30 & 327 & 194 & 40 \\
\hline 236 & 88.22 & -0.43 & 9.94 & 6.79 & 0.35 & 0.30 & 320 & 193 & 39. \\
\hline 237 & 87.78 & 0.44 & 9.94 & 6.78 & 0.35 & 0.30 & 312 & 192 & 88 \\
\hline 238 & 87.34 & 0.44 & 9.95 & 6.76 & 0.35 & 0.30 & 305 & 192 & 38 \\
\hline 239 & 86.89 & $\begin{array}{l}0.45 \\
0.45\end{array}$ & 9.95 & 6,74 & 0.36 & 0.30 & 298 . & 191 & 37 \\
\hline 240 & 86.44 & & 9.96 & 6.73 & 0.36 & 0.31 & 291 & 191 & 36 \\
\hline
\end{tabular}




\begin{tabular}{|c|c|c|c|c|c|c|c|c|c|}
\hline \multicolumn{10}{|c|}{ TABLE V111, ARG. 1.-Continued. } \\
\hline Arg. & $(v . c .0)$ & Diff. & $(v . s .1)$ & $(v . c .1)$ & $(v . s .2)$ & $(v . c .2)$ & $(\rho . c .0)$ & $(\rho . s .1)$ & $(\rho . c .1)$ \\
\hline & $"$ & $"$ & $" \prime$ & $"$ & $" 1$ & " & & & \\
\hline 240 & 86.44 & & 9.96 & 6.73 & 0.36 & 0.31 & 291 & 191 & 36 \\
\hline 241 & $85.99^{-}$ & -0.45 & 9.96 & 6.71 & 0.36 & 0.31 & 284 & 190 & 35 \\
\hline 242 & 85.53 & 0.46 & 9.96 & 6.69 & 0.36 & 031 & 278 & 190 & 35 \\
\hline 243 & 85.08 & 0.45 & 9.97 & 6.67 & 0.36 & 0.31 & 271 & 189 & 34 \\
\hline 244 & 84.61 & $\begin{array}{l}0.47 \\
0.46\end{array}$ & 9.97 & 6.66 & 0.37 & 0.31 & 265 & 189 & 33 \\
\hline 245 & 84.15 & & 9.97 & 6.64 & 0.37 & 0.31 & 258 & 188 & 33 \\
\hline 246 & 83.68 & -0.47 & 9.97 & 6.61 & 0.37 & 0.31 & 252 & 188 & 32 \\
\hline 247 & 83.21 & 0.47 & 9.98 & 6.59 & 0.37 & 0.31 & 246 & 187 & 31 \\
\hline 248 & 82.73 & $\begin{array}{l}0.48 \\
0.48\end{array}$ & 9.98 & 6.57 & 0.37 & 0.31 & 240 & 187 & 31 \\
\hline 249 & 82.25 & $\begin{array}{l}0.48 \\
0.48\end{array}$ & 9.98 & 6.55 & 0.37 & 0.31 & 234 & 186 & 30 \\
\hline 250 & 81.77 & & 9.98 & 6.53 & 0.37 & 0.31 & 228 & 186 & 29 \\
\hline 251 & 81.28 & -0.49 & 9.98 & 6.51 & 0.38 & 0.31 & 222 & 185 & 29 \\
\hline 252 & 80.80 & 0.48 & 9.98 & 6.48 & 0.38 & 0.31 & 216 & 185 & 28 \\
\hline 253 & 80.30 & 0.50 & 9.97 & 6.46 & 0.38 & 0.31 & 211 & 184 & 28 \\
\hline 254 & 79.81 & $\begin{array}{l}0.49 \\
0.50\end{array}$ & 9.97 & 6.43 & 0.38 & 0.31 & 205 & 184 & 27 \\
\hline 255 & 79.31 & & 9.97 & 6.40 & 0.38 & 0.31 & 200 & 183 & 27 \\
\hline 256 & $78.82^{-}$ & -0.49 & 9.97 & 6.37 & 0.38 & 0.30 & 195 & 183 & 26 \\
\hline 257 & 78.31 & 0.51 & 9.96 & 6.34 & 0.38 & 0.30 & 190 & 182 & 26 \\
\hline 258 & 77.81 & 0.50 & 9.96 & 6.31 & 0.38 & 0.30 & 184 & 182 & 25 \\
\hline 259 & 77.30 & $\begin{array}{l}0.5 \mathrm{I} \\
0.5 \mathrm{I}\end{array}$ & 9.96 & 6.28 & 0.38 & 0.30 & 179 & 181 & 25 \\
\hline 260 & 76.79 & & 9.95 & 6.25 & 0.38 & 0.30 & 175 & 181 & 24 \\
\hline 261 & $76.28^{-}$ & -0.51 & 9.95 & 6.22 & 0.38 & 0.30 & 170 & 180 & 24 \\
\hline 262 & 75.77 & $0.5^{I}$ & 9.95 & 6.19 & 0.38 & 0.30 & 165 & 180 & 24 \\
\hline 263 & 75.25 & 0.52 & 9.94 & 6.16 & 0.38 & 0.30 & 161 & 179 & 23 \\
\hline 264 & 74.73 & $\begin{array}{l}0.52 \\
0.52\end{array}$ & 9.94 & 6.13 & 0.38 & 0.30 & 156 & 179 & 23 \\
\hline 265 & 74.21 & & 9.93 & 6.10 & 0.38 & 0.30 & 152 & 178 & 22 \\
\hline 266 & $73.69^{-}$ & $-0.5^{2}$ & 9.93 & 6.06 & 0.38 & 0.30 & 148 & 178 & 22 \\
\hline 267 & 73.16 & 0.53 & 9.92 & 6.03 & 0.38 & 0.30 & 144 & 177 & 22 \\
\hline 268 & 72.64 & 0.52 & 9.92 & 5.99 & 0.38 & 0.30 & 140 & 177 & 21 \\
\hline 269 & 72.11 & $\begin{array}{l}0.53 \\
0.54\end{array}$ & 9.91 & 5.96 & 0.38 & 0.30 & 137 & 176 & 21 \\
\hline 270 & 71.57 & & 9.90 & 5.92 & 0.39 & 0.30 & 133 & 176 & 21 \\
\hline 271 & 71.04 & -0.53 & 9.90 & 5.88 & 0.39 & 0.30 & 129 & 175 & 20 \\
\hline 272 & 70.51 & 0.53 & 9.89 & 5.85 & 0.39 & $0: 30$ & 126 & 175 & 20 \\
\hline 273 & 69.97 & 0.54 & 9.88 & 5.81 & 0.39 & 0.30 & 122 & 174 & 20 \\
\hline 274 & 69.43 & $\begin{array}{l}0.54 \\
0.54\end{array}$ & 9.88 & 5.77 & 0.39 & 0.30 & 119 & 174 & 20 \\
\hline 275 & 68.89 & & 9.87 & 5.73 & 0.39 & 0.30 & 116 & 174 & 20 \\
\hline 276 & $68.35^{-}$ & -0.54 & 9.86 & 5.69 & 0.39 & 0.29 & 113 & 173 & 19 \\
\hline 277 & 67.81 & 0.54 & 9.85 & 5.65 & 0.39 & 0.29 & 110 & 172 & 19 \\
\hline 278 & 67.26 & $\begin{array}{l}0.55 \\
0.54\end{array}$ & 9.84 & 5.61 & 0.39 & 0.29 & 107 & 172 & 19 \\
\hline 279 & 66.72 & $\begin{array}{l}0.54 \\
0.55\end{array}$ & 9.84 & 5.57 & 0.39 & 0.29 & 105 & 171 & 19 \\
\hline 280 & 66.17 & & 9.83 & 5.52 & 0.39 & 0.29 & 102 & 171 & 19 \\
\hline 2881 & 65.62 & -0.55 & 9.82 & 5.48 & 0.39 & 0.29 & .100 & 170 & 19 \\
\hline 282 & 65.07 & 0.55 & 9.81 & 5.44 & 0.39 & 0.29 & 97 & 170 & 18 \\
\hline 283 & 64.52 & 0.55 & 9.80 & 5.40 & 0.39 & 0.29 & 95 & 169 & 18 \\
\hline 284 & 63.97 & $\begin{array}{l}0.55 \\
0.56\end{array}$ & 9.79 & 5.35 & 0.39 & 0.29 & 93 & 169 & 18 \\
\hline 285 & 63.41 & & 9.78 & 5.31 & 0.39 & 0.28 & 91 & 168 & 18 \\
\hline 286 & $62.86^{-}$ & -0.55 & 9.77 & 5.26 & 0.39 & 0.28 & 90 & 168 & 18 \\
\hline 287 & 62.31 & 0.55 & 9.77 & 5.22 & 0.39 & 0.28 & 88 & 167 & 18 \\
\hline 288 & 61.75 & 0.56 & 9.76 & 5.17 & 0.39 & 0.28 & 86 & 167 & 18 \\
\hline 289 & 61.19 & $\begin{array}{l}0.5^{6} \\
0.55\end{array}$ & 9.75 & 5.13 & 0.38 & 0.28 & 85 & 166 & 18 \\
\hline 290 & 60.64 & & 9.74 & 5.08 & 0.38 & 0.28 & 84 & 166 & 18 \\
\hline 291 & 60.08 & -0.56 & 9.73 & 5.03 & 0.38 & 0.27 & 82 & 165 & 19 \\
\hline 292 & 59.52 & $\begin{array}{l}0.56 \\
0.56\end{array}$ & 9.72 & 4.99 & 0.38 & 0.27 & 81 & 164 & 19 \\
\hline 293 & 58.96 & $\begin{array}{l}0.56 \\
0.56\end{array}$ & 9.71 & 4.94 & 0.38 & 0.27 & 80 & 164 & 19 \\
\hline 294 & 58.40 & $\begin{array}{l}0.5^{6} \\
0.56\end{array}$ & 9.70 & 4.89 & 0.38 & 0.27 & 80 & 163 & 19 \\
\hline 295 & 57.84 & & 9.69 & 4.84 & 0.38 & 0.27 & 79 & 163 & 19 \\
\hline 296 & $57.28^{-}$ & $\begin{array}{r}-0.56 \\
0.56\end{array}$ & 9.68 & 4.80 & 0.38 & 0.27 & 78 & 162 & 19 \\
\hline 297 & 56.72 & $\begin{array}{l}0.56 \\
0.56\end{array}$ & 9.67 & 4.75 & 0.38 & 0.26 & 78 & 162 & 19 \\
\hline 298 & 56.16 & $\begin{array}{l}0.50 \\
0.56\end{array}$ & 9.66 & 4.70 & 0.38 & 0.26 & 77 & 161 & 20 \\
\hline 299 & 55.60 & $\begin{array}{l}0.50 \\
0.56\end{array}$ & 9.65 & 4.65 & 0.38 & 0.26 & 77 & 161 & 20 \\
\hline 300 & 55.04 & & 9,64 & 4.60 & 0.39 & 0.26 & 77 & 160 & 20 \\
\hline
\end{tabular}


TABLE VIII, Aве. 1.-Continued.

\begin{tabular}{|c|c|c|c|c|c|c|c|c|c|}
\hline Arg. & $(v \cdot c .0)$ & Diff. & $(v, 8,1)$ & (v.e.1) & $(v, 8,2)$ & (v.c.2) & $(p, c, 0)$ & $(p, 8,1)$ & $(\rho . c .1)$ \\
\hline & $" \prime$ & $"$ & $"$ & " & " & " & & & \\
\hline 300 & 55.04 & -0.56 & 9.64 & 4.60 & 0.38 & 0.26 & 77 & 160 & 20 \\
\hline 301 & 54.48 & $\begin{array}{r}-0.56 \\
0.56\end{array}$ & 9.63 & 4.55 & 0.38 & 0.26 & 77 & 159 & 20 \\
\hline 302 & 53.92 & $\begin{array}{l}0.56 \\
0.56\end{array}$ & 9.62 & 4.50 & 0.38 & 0.25 & 77 & 159 & 20 \\
\hline 303 & 53.36 & $\begin{array}{l}0.5^{\circ} \\
0.56\end{array}$ & 9.61 & 4.45 & 0.38 & 0.25 & 77 & 158 & 21 \\
\hline 304 & 52.80 & $\begin{array}{l}0.50 \\
0.56\end{array}$ & 9.60 & 4.40 & 0.38 & 0.25 & 78 & 158 & 21 \\
\hline 305 & 52.24 & -0.56 & 9.59 & 4.35 & 0.38 & 0.25 & 78 & 157 & 21 \\
\hline 306 & 51.68 & $\begin{array}{r}-0.5^{\circ} \\
0.56\end{array}$ & 9.58 & 4.30 & 0.38 & 0.25 & 79 & 156 & 22 \\
\hline 307 & 51.12 & $\begin{array}{l}0.5^{\circ} \\
0.56\end{array}$ & 9.57 & 4.25 & 0.38 & 0.24 & 80 & 156 & 22 \\
\hline 308 & 50.56 & $\begin{array}{l}0.50 \\
0.56\end{array}$ & 9.56 & 4.20 & 0.38 & 0.24 & 81 & 155 & 22 \\
\hline 309 & 50.00 & $\begin{array}{l}0.5^{0} \\
0.5^{6}\end{array}$ & 9.55 & 4.15 & 0.38 & 0.24 & 82 & 154 & 23 \\
\hline 310 & 49.44 & & 9.54 & 4.10 & 0.38 & 0.24 & 83 & 154 & 23 \\
\hline 311 & 48.88 & $-0.5^{6}$ & 9.53 & 4.05 & 0.38 & 0.23 & 84 & 153 & 24 \\
\hline 312 & 48.33 & 0.55 & 9.52 & 4.01 & 0.38 & 0.23 & 85 & 152 & 24 \\
\hline 313 & 47.77 & 0.56 & 9.51 & 3.94 & 0.38 & 0.23 & 87 & 152 & 24 \\
\hline 314 & 47.22 & $\begin{array}{l}0.55 \\
0.56\end{array}$ & 9.50 & 3.89 & 0.38 & 0.23 & 88 & 151 & 25 \\
\hline 315 & 46.66 & & 9.49 & 3.84 & 0.38 & 0.23 & 90 & 150 & 25 \\
\hline 316 & $46.11^{-}$ & -0.55 & 9.48 & 3.79 & 0.38 & 0.22 & 92 & 150 & 26 \\
\hline 317 & 45.56 & 0.55 & 9.47 & 3.74 & 0.38 & 0.22 & 94 & 149 & 26 \\
\hline 318 & 45.01 & 0.55 & 9.46 & 3.69 & 0.38 & 0.22 & 96 & 148 & 27 \\
\hline 319 & 44.46 & $\begin{array}{l}0.55 \\
0.55\end{array}$ & 9.45 & 3.63 & 0.38 & 0.22 & 98 & 148 & 27 \\
\hline 320 & 43.91 & 0.55 & 9.44 & 3.58 & 0.38 & 0.21 & 100 & 147 & 28 \\
\hline 321 & 43.36 & -0.55 & 9.43 & 3.53 & 0.38 & 0.21 & 103 & 146 & 28 \\
\hline 322 & 42.81 & 0.55 & 9.42 & 3.48 & 0.38 & 0.21 & 105 & 146 & 29 \\
\hline 323 & 42.27 & 0.54 & 9.41 & 3.43 & 0.38 & 0.21 & 108 & 145 & 29 \\
\hline 324 & 41.72 & $\begin{array}{l}0.55 \\
0.54\end{array}$ & 9.40 & 3.38 & 0.38 & 0.20 & 111 & 144 & 30 \\
\hline 325 & 41.18 & & 9.40 & 3.33 & 0.38 & 0.20 & 114 & 144 & 30 \\
\hline 326 & 40.64 & -0.54 & 9.39 & 3.28 & 0.38 & 0.20 & 117 & 143 & 31 \\
\hline 327 & 40.10 & 0.54 & 9.38 & 3.23 & 0.38 & 0.20 & 120 & 142 & 32 \\
\hline 328 & 39.56 & 0.54 & 9.37 & 3.18 & 0.38 & 0.19 & 123 & 141 & 32 \\
\hline 329 & 39.03 & $\begin{array}{l}0.53 \\
0.54\end{array}$ & 9.36 & 3.14 & 0.37 & 0.19 & 127 & 140 & 33 \\
\hline 330 & 38.49 & 0.54 & 9.35 & 3.07 & 0.37 & 0.19 & 130 & 140 & 34 \\
\hline 331 & 37.96 & -0.53 & 9.34 & 3.02 & 0.37 & 0.19 & 134 & $139^{\circ}$ & 34 \\
\hline 332 & 37.43 & 0.53 & 9.33 & 2.98 & 0.37 & 0.18 & 138 & 138 & 35 \\
\hline 333 & 36.90 & 0.53 & 9.32 & 2.93 & $0.37 \cdot$ & 0.18 & 141 & 137 & 36 \\
\hline 334 & 36.37 & $\begin{array}{l}0.53 \\
0.52\end{array}$ & 9.31 & 2.88 & 0.37 & 0.18 & 145 & 136 & 36 \\
\hline 335 & 35.85 & & 9.30 & 2.83 & 0.37 & 0.18 & 150 & 136 & 37 \\
\hline 336 & 35.33 & $-0.5^{2}$ & 9.29 & 2.78 & 0.37 & 0.17 & 154 & 135 & 38 \\
\hline 337 & 34.81 & $0.5^{2}$ & 9.29 & 2.73 & 0.37 & 0.17 & 158 & 134 & 38 \\
\hline 338 & 34.29 & $0.5^{2}$ & 9.28 & 2.69 & 0.37 & 0.17 & 162 & 133 & 39 \\
\hline 339 & 33.78 & $\begin{array}{l}0.5^{I} \\
0.52\end{array}$ & 9.27 & 2.64 & 0.37 & 0.17 & 167 & 132 & 40 \\
\hline 340 & 33.26 & & 9.26 & 2.59 & 0.37 & 0.17 & 172 & 132 & 41 \\
\hline 341 & 32.75 & $\begin{array}{r}-0.5^{I} \\
0.5 \mathrm{I}\end{array}$ & 9.25 & 2.54 & 0.37 & 0.16 & 176 & 131 & 42. \\
\hline 342 & 32.24 & $0.5 \mathrm{I}$ & 9.24 & 2.50 & 0.37 & 0.16 & 181 & 130 & 42 \\
\hline 343 & 31.74 & $0.5^{\circ}$ & 9.23 & 2.45 & 0.37 & 0.16 & 186 & 129 & 43 \\
\hline 344 & 31.23 & $\begin{array}{l}0.51 \\
0.50\end{array}$ & 9.22 & 2.41 & 0.37 & 0.16 & 191 & 128 & 44 \\
\hline 345 & 30.73 & & 9.21 & 2.36 & 0.37 & 0.15 & 197 & 127 & 45 \\
\hline 346 & 30.24 & -0.49 & 9.20 & 2.31 & 0.37 & 0.15 & 202 & 126 & 46 \\
\hline 347 & 29.74 & $0.5^{\circ}$ & 9.20 & 2.27 & 0.37 & 0.15 & 207 & 126 & 46 \\
\hline 348 & 29.25 & 0.49 & 9.19 & 2.23 & 0.37 & 0.15 & 213 & 125 & 47 \\
\hline 349 & 28.76 & $\begin{array}{l}0.49 \\
0.49\end{array}$ & 9.18 & 2.18 & 0.37 & 0.15 & 218 & 124 & 48 \\
\hline 350 & 28.27 & & 9.17 & 2.14 & 0.37 & 0.14 & 224 & 123 & 49 \\
\hline 351 & 27.79 & -0.48 & 9.16 & 2.09 & 0.37 & 0.14 & 230 & 122 & 50 \\
\hline 352 & 27.31 & 0.48 & 9.15 & 2.05 & 0.37 & 0.14 & 236 & 121 & 51 \\
\hline 353 & 26.83 & $0.4^{8}$ & 9.14 & 2.01 & 0.37 & 0.14 & 242 & 120 & 51 \\
\hline 354 & 26.36 & $\begin{array}{l}0.47 \\
0.47\end{array}$ & 9.13 & 1.97 & 0.37 & 0.14 & 248 & 119 & 52 \\
\hline 355 & 25.89 & & 9.12 & 1.93 & 0.37 & 0.14 & 255 & 118 & 53 \\
\hline 356 & 25.42 & -0.47 & 9.12 & 1.89 & 0.37 & 0.13 & 261 & 117 & 54 \\
\hline 357 & 24.96 & 0.46 & 9.11 & 1.85 & 0.37 & 0.13 & 268 & 116 & 55 \\
\hline 358 & 24.50 & 0.46 & 9.10 & 1.81 & 0.37 & 0.13 & 274 & 115 & 56 \\
\hline 359 & 24.04 & $\begin{array}{l}0.46 \\
0.46\end{array}$ & 9.09 & 1.77 & 0.37 & 0.13 & 281 & 114 & 57 \\
\hline 360 & 23.58 & & 9.08 & 1.73 & 0.38 & 0.13 & 288 & 113 & 58 \\
\hline
\end{tabular}


TABLE VIII, ARG. 1.-Continued.

\begin{tabular}{|c|c|c|c|c|c|c|c|c|c|}
\hline Arg. & $($ v.c. 0$)$ & Diff. & $(v . s .1)$ & (v.c.1) & $(v . s .2)$ & $(v . c .2)$ & $(\rho . c .0)$ & (n.s.1) & $(\rho . c .1)$ \\
\hline & "I & $" \prime$ & " & " & " & $"$ & & & \\
\hline 360 & 23.58 & & 9.08 & 1.73 & 0.38 & 0.13 & 298 & 113 & 58 \\
\hline 361 & 23.13 & -0.45 & 9.07 & 1.70 & 0.38 & 0.13 & 294 & 112 & 58 \\
\hline 362 & 22.69 & 0.44 & 9.06 & 1.66 & 0.38 & 0.13 & 301 & 111 & 59 \\
\hline 363 & 22.24 & 0.45 & 9.05 & 1.62 & 0.38 & 0.13 & 308 & 110 & 60 \\
\hline 364 & 21.80 & $\begin{array}{l}0.44 \\
0.43\end{array}$ & 9.04 & 1.59 & 0.38 & 0.12 & 316 & 109 & 61 \\
\hline 365 & 21.37 & & 9.03 & 1.55 & 0.37 & 0.12 & 323 & 108 & 62 \\
\hline 366 & $20.94^{-}$ & $\begin{array}{r}-0.43 \\
0.43\end{array}$ & 9.02 & 1.52 & 0.37 & 0.12 & 330 & 107 & 63 \\
\hline 367 & 20.51 & & 9.01 & 1.48 & 0.37 & 0.12 & $338^{\circ}$ & 106 & 64 \\
\hline 368 & 20.08 & $\begin{array}{l}0.43 \\
0.42\end{array}$ & 9.00 & 1.45 & 0.37 & 0.12 & 345 & 105 & 65 \\
\hline 369 & 19.66 & $0.4^{2}$ & 8.99 & 1.42 & 0.37 & 0.11 & 353 & 104 & 66 \\
\hline 370 & 19.24 & & 8.98 & 1.39 & 0.37 & 0.11 & 361 & 103 & 66 \\
\hline 371 & $18.83^{-}$ & $-0.4 \mathrm{I}$ & 8.97 & 1.36 & 0.37 & 0.11 & 369 & 102 & 67 \\
\hline 372 & 18.42 & $0.4 \mathrm{I}$ & 8.96 & 1.32 & 0.37 & 0.11 & 377 & 101 & 68 \\
\hline 373 & 18.02 & 0.40 & 8.95 & 1.29 & 0.37 & 0.11 & 385 & 100 & 69 \\
\hline 374 & 17.62 & $\begin{array}{l}0.40 \\
0.40\end{array}$ & 8.94 & 1.27 & 0.37 & 0.11 & 393 & 99 & 70 \\
\hline 375 & 17.22 & & 8.93 & 1.24 & 0.37 & 0.11 & 401 & 98 & 71 \\
\hline 376 & $16.83^{-}$ & -0.39 & 8.92 & 1.21 & 0.37 & 0.11 & 410 & 97 & 72 \\
\hline 377 & 16.44 & 0.39 & 8.91 & 1.18 & 0.37 & 0.11 & 418 & 96 & 73 \\
\hline 378 & 16.06 & 0.38 & 8.89 & 1.16 & 0.37 & 0.11 & 426 & 95 & 74 \\
\hline 379 & 15.68 & $\begin{array}{l}0.3^{8} \\
0.3^{8}\end{array}$ & 8.88 & 1.13 & 0.37 & 0.11 & 435 & 94 & 75 \\
\hline $38 \mathrm{v}$ & 15.30 & & 8.87 & 1.11 & 0.37 & 0.10 & 444 & 93 & 76 \\
\hline 381 & $14.93^{-}$ & -0.37 & 8.86 & 1.08 & 0.37 & 0.10 & 452 & 92 & $77^{\circ}$ \\
\hline 382 & $14.5 ?$ & $0.3^{6}$ & 8.85 & 1.06 & 0.37 & 0.10 & 461 & 91 & 78 \\
\hline 383 & 14.21 & $0.3^{6}$ & 8.83 & 1.04 & 0.37 & 0.10 & 470 & 90 & 78 \\
\hline 384 & 13.85 & $\begin{array}{l}0.3^{6} \\
0.35\end{array}$ & 8.82 & 1.02 & 0.37 & 0.10 & 479 & 89 & 79 \\
\hline 385 & 13.50 & 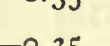 & 8.81 & 1.00 & 0.37 & 0.10 & 488 & 88 & 80 \\
\hline 386 & $13.15^{-}$ & -0.35 & 8.79 & 0.98 & 0.37 & 0.10 & 497 & 87 & 81 \\
\hline 387 & 12.80 & 0.35 & 8.78 & 0.96 & 0.37 & 0.10 & 506 & 86 & 82 \\
\hline 388 & 12.47 & $\begin{array}{l}0.33 \\
0.34\end{array}$ & 8.77 & 0.94 & 0.37 & 0.10 & 514 & 85 & 83 \\
\hline 389 & 12.13 & $\begin{array}{l}0.34 \\
0.3^{2}\end{array}$ & 8.75 & 0.92 & 0.37 & 0.10 & 525 & 84 & 84 \\
\hline 390 & . 11.81 & -0.33 & 8.74 & 0.90 & 0.37 & 0.10 & 534 & 82 & 85 \\
\hline 391 & $11.48^{-}$ & -0.33 & 8.72 & 0.88 & 0.37 & 0.10 & 544 & 81 & 86 \\
\hline 392 & 11.16 & $\begin{array}{l}0.32 \\
0.21\end{array}$ & 8.71 & 0.87 & 0.37 & 0.10 & 554 & 80 & 86 \\
\hline 393 & 10.85 & $\begin{array}{l}0.3 I \\
0.3 I\end{array}$ & 8.70 & 0.85 & 0.36 & 0.10 & 564 & 79 & 87 \\
\hline 394 & 10.54 & $\begin{array}{l}0.31 \\
0.31\end{array}$ & 8.68 & 0.84 & 0.36 & 0.10 & 573 & 78 & 88 \\
\hline 395 & 10.23 & & 8.67 & 0.83 & 0.36 & 0.10 & 583 & 77 & 89 \\
\hline 396 & $9.93^{-}$ & -0.30 & 8.65 & 0.82 & 0.36 & 010 & 593 & 76 & 90 \\
\hline 397 & 9.64 & 0.29 & 8.63 & 0.80 & 036 & 0.10 & 603 & 75 & 91 \\
\hline 398 & 9.35 & $\begin{array}{l}0.29 \\
0.28\end{array}$ & 8.62 & 0.79 & 0.36 & 0.10 & 613 & 74 & 92 \\
\hline 399 & 9.07 & $\begin{array}{l}0.28 \\
0.28\end{array}$ & 8.60 & 0.78 & 0.36 & 0.10 & 623 & 73 & 93 \\
\hline 400 & 8.79 & -0.27 & 8.59 & 0.77 & 0.36 & 0.10 & 633 & 72 & 93 \\
\hline 401 & 8.52 & 0.27 & 8.57 & 0.76 & 0.36 & 0.10 & 643 & 71 & 94 \\
\hline 402 & 8.25 & 0.27 & 8.55 & 0.76 & 0.36 & 0.10 & 653 & 70 & 95 \\
\hline 403 & 7.98 & 0.25 & 8.53 & 0.75 & 0.36 & 0.10 & 664 & 69 & 96 \\
\hline 404 & 7.73 & 0.26 & 8.51 & 0.74 & 0.35 & 0.10 & 674 & 68 & 97 \\
\hline 405 & 7.47 & & 8.49 & 0.74 & 0.35 & 0.10 & 684 & 67 & 98 \\
\hline 406 & $7.22-$ & -0.25 & 8.47 & 0.74 & 0.35 & 0.10 & 695 & 66 & 98 \\
\hline 407 & 6.98 & 0.24 & 8.46 & 0.73 & 0.35 & 0.10 & 706 & 65 & 99 \\
\hline 408 & 6.75 & 0.23 & 8.44 & 0.73 & 0.35 & 0.10 & 716 & 64 & 100 \\
\hline 409 & 6.51 & $\begin{array}{l}0.24 \\
0.22\end{array}$ & 8.42 & 0.73 & 0.34 & 0.10 & 727 & 63 & 101 \\
\hline 410 & 6.29 & & 8.39 & 0.73 & 0.34 & 0.10 & 737 & 62 & 101 \\
\hline 411 & $6.07-$ & $=0.22$ & 8.37 & 0.73 & 0.34 & 0.10 & 748 & 61 & 102 \\
\hline 412 & 5.85 & 0.22 & 8.35 & 0.73 & 0.34 & 0.10 & 759 & 60 & 103 \\
\hline 413 & 5.64 & $\begin{array}{l}0.21 \\
0.20\end{array}$ & 8.33 & 0.73 & 0.34 & 0.10 & 770 & 59 & 104 \\
\hline 414 & 5.44 & $\begin{array}{l}0.20 \\
0.20\end{array}$ & 8.31 & 073 & 0.34 & 0.10 & 781 & 58 & 104 \\
\hline 415 & 5.24 & & 8.29 & 0.73 & 0.34 & 0.10 & 792 & 57 & 105 \\
\hline 416 & 5.04 & $\begin{array}{r}-0.20 \\
0.18\end{array}$ & 8.26 & 0.74 & 0.33 & 0.10 & 803 & 56 & 106 \\
\hline 417 & 4.86 & $\begin{array}{l}0.18 \\
0.10\end{array}$ & 8.24 & 0.74 & 0.33 & 0.10 & 814 & 55 & 107 \\
\hline 418 & 4.67 & 0.19 & 8.21 & 0.74 & 0.33 & 0.10 & 825 & 54 & 107 \\
\hline 419 & 4.50 & $\begin{array}{l}0.17 \\
0.17\end{array}$ & 8.19 & 0.75 & 0.33 & 0.10 & 836 & 53 & 108 \\
\hline 420 & 4.33 & & 8.17 & 0.76 & 0.33 & 0.10 & 847 & 52 & 109 \\
\hline
\end{tabular}




\begin{tabular}{|c|c|c|c|c|c|c|c|c|c|}
\hline \multicolumn{10}{|c|}{ TABLE VIII, Arg. 1.-Continued. } \\
\hline \multirow[t]{2}{*}{ Arg. } & $(v . e .0)$ & Diff. & $(v, 8.1)$ & $(v . e .1)$ & $(v .8 .2)$ & $(v . c .2)$ & $(\rho . e .0)$ & $(\rho .8 .1)$ & $(p . c .1)$ \\
\hline & " & " & " & $"$ & " & $"$ & & & \\
\hline 420 & 4.33 & $-0,17$ & 8.17 & 0.76 & 0.33 & 0.10 & 847 & 52 & 109 \\
\hline 421 & 4.16 & $\begin{array}{l}-0.17 \\
0.16\end{array}$ & 8.14 & 0.76 & 0.32 & 0.10 & 858 & 51 & 110 \\
\hline 422 & 4.00 & 0.15 & 8.11 & 0.77 & 0.32 & 0.11 & 870 & 50 & 110 \\
\hline 423 & $\begin{array}{l}3.85 \\
3.70\end{array}$ & 0.15 & $\begin{array}{l}8.09 \\
8.06\end{array}$ & 0.78 & 0.32 & 0.11 & 881 & 50 & 111 \\
\hline 424 & 3.70 & 0.14 & 8.06 & 0.79 & 0.32 & 0.11 & 892 & 49 & 111 \\
\hline 425 & 3.56 & -0.14 & 8.04 & 0.80 & 0.32 & 0.11 & 904 & 48 & 112 \\
\hline 426 & 3.42 & $\begin{array}{l}-0.14 \\
0.13\end{array}$ & 8.01 & 0.81 & 0.31 & 0.11 & 916 & 47 & 113 \\
\hline 427 & 3.29 & $\begin{array}{l}.13 \\
0.13\end{array}$ & 7.98 & 0.82 & 0.31 & 0.11 & 927 & 46 & 113 \\
\hline 428 & 3.16 & 0.12 & 7.95 & 0.83 & 0.31 & 0.11 & 938 & 45 & 114 \\
\hline 429 & 3.04 & 0.11 & 7.92 & 0.85 & 0.31 & 0.11 & 950 & 44 & \\
\hline 430 & 2.93 & -0.111 & 7.89 & 0.86 & 0.31 & 0.11 & 961 & 44 & 115 \\
\hline 431 & $2.82-$ & $\begin{array}{r}-0.11 \\
0.10\end{array}$ & 7.86 & 0.87 & 0.30 & 0.11 & 973 & 43 & 116 \\
\hline 432 & 2.72 & $\begin{array}{l}0.10 \\
0.10\end{array}$ & 7.83 & 0.89 & 0.30 & 0.11 & 984 & 42 & 116 \\
\hline 433 & 2.62 & $\begin{array}{l}0.10 \\
0.09\end{array}$ & 7.80 & 0.90 & 0.30 & 0.11 & 996 & 41 & 117 \\
\hline 434 & 2.53 & $\begin{array}{l}0.09 \\
0.08\end{array}$ & 7.77 & 0.92 & 0.29 & 0.11 & 1008 & 40 & 118 \\
\hline 435 & 2.45 & & 7.74 & 0.94 & 0.29 & 0.11 & 1019 & 40 & 118 \\
\hline 436 & 2.37 & $\begin{array}{r}-0.08 \\
0.08\end{array}$ & 7.71 & 0.95 & 0.29 & 0.12 & 1031 & 39 & 119 \\
\hline 437 & 2.29 & $\begin{array}{l}0.08 \\
0.06\end{array}$ & 7.67 & 0.97 & 0.29 & 0.12 & 1043 & 38 & 119 \\
\hline 438 & 2.23 & $\begin{array}{l}0.00 \\
0.07\end{array}$ & 7.64 & 0.99 & 0.28 & 0.12 & 1054 & 38 & 120 \\
\hline 439 & 2.16 & 0.05 & 7.60 & 1.01 & 0.28 & 0.12 & 1066 & 37 & 120 \\
\hline 440 & 2.11 & -0.05 & 7.57 & 1.03 & 0.28 & 0.12 & 1078 & 36 & 121 \\
\hline 441 & $2.06-$ & $\begin{array}{l}0.05 \\
0.04\end{array}$ & $\begin{array}{l}7.54 \\
7.50\end{array}$ & 1.05 & 0.28 & 0.12 & 1089 & 35 & 121 \\
\hline 442 & 2.02 & $\begin{array}{l}0.04 \\
0.04\end{array}$ & 7.50 & 1.07 & 0.27 & 0.12 & 1100 & 35 & 122 \\
\hline 443 & 1.98 & $\begin{array}{l}0.04 \\
0.03\end{array}$ & $\begin{array}{l}7.47 \\
7.43\end{array}$ & 1.09 & 0.27 & 0.12 & 1113 & 34 & 122 \\
\hline 444 & 1.95 & $\begin{array}{l}0.03 \\
0.03\end{array}$ & 7.43 & 1.11 & 0.27 & 0.12 & 1124 & 33 & 122 \\
\hline 445 & 1.92 & -0.02 & 7.39 & 1.14 & 0.26 & 0.12 & 1137 & 33 & 123 \\
\hline 446 & $1.90^{-}$ & $\begin{array}{l}-0.02 \\
0.02\end{array}$ & 7.36 & 1.16 & 0.26 & 0.12 & 1149 & 32 & 123 \\
\hline 447 & 1.88 & $\begin{array}{l}0.02 \\
0.01\end{array}$ & 7.32 & 1.18 & 0.26 & 0.12 & 1160 & 32 & 124 \\
\hline 448 & 1.87 & 0.00 & 7.28 & 1.21 & 0.26 & 0.12 & 1172 & 31 & 124 \\
\hline 449 & 1.87 & 0.00 & 7.24 & 1.23 & 0.25 & 0.12 & 1184 & 30 & 125 \\
\hline 450 & $1.87+$ & & 7.20 & 1.26 & 0.25 & 0.12 & 1196 & 30 & 125 \\
\hline 451 & $1.88^{+}$ & 0.02 & 7.16 & 1.28 & 0.25 & 0.12 & 1208 & 30 & 125 \\
\hline 452 & 1.90 & 0.02 & 7.12 & 1.31 & 0.24 & 0.12 & 1220 & 29 & 126 \\
\hline 453 & 1.92 & 0.03 & 7.08 & 1.33 & 0.24 & 0.12 & 1232 & 28 & 126 \\
\hline 454 & 1.95 & 0.03 & 7.04 & 1.36 & 0.24 & 0.12 & 1243 & 28 & 127 \\
\hline 455 & 1.98 & & 7.00 & 1.39 & 0.23 & 0.12 & 1255 & 28 & 127 \\
\hline 456 & $2.01^{+}$ & $\begin{array}{l}0.03 \\
0.05\end{array}$ & 6.96 & 1.42 & 0.23 & 0.12 & 1267 & 27 & 127 \\
\hline 457 & 206 & 0.05 & 6.91 & 1.45 & 0.23 & 0.12 & 1279 & 27 & 128 \\
\hline 458 & 2.11 & 0.05 & $\begin{array}{l}6.87 \\
6.83\end{array}$ & 1.47 & 0.22 & 0.12 & 1291 & 26 & 128 \\
\hline 459 & 2.16 & 0.07 & 6.83 & 1.50 & 0.22 & 0.12 & 1302 & 26 & 128 \\
\hline .460 & $2.23+$ & -0.06 & 6.78 & 1.53 & 0.22 & 0.12 & 1314 & 26 & 128 \\
\hline 461. & $2.29^{+}$ & 0.08 & 6.74 & 1.56 & 0.21 & 0.12 & 1326 & 25 & 129 \\
\hline 462 & 2.37 & 0.07 & 6.69 & 1.59 & 0.21 & 0.12 & 1338 & 25 & 129 \\
\hline 463 & 2.44 & 0.09 & 6.65 & 1.62 & 0.21 & 0.12 & 1350 & 25 & 129 \\
\hline 464 & 2.53 & 0.09 & 6.60 & 1.65 & 0.20 & 0.12 & 1361 & 25 & 130 \\
\hline 465 & 2.62 & & 6.56 & 1.68 & 0.20 & 0.12 & 1373 & 24 & 130 \\
\hline 466 & $2.71^{\dagger}$ & $\begin{array}{r}-0.09 \\
0.11\end{array}$ & 6.51 & $1.72^{\circ}$ & 0.20 & 0.12 & 1385 & 24 & 130 \\
\hline 467 & 2.82 & 0.10 & 6.46 & 1.75 & 0.19 & 0.13 & 1396 & 24 & 130 \\
\hline 468 & 2.92 & 0.12 & 6.41 & 1.78 & 0.19 & 0.13 & 1408 & 24 & 130 \\
\hline 469 & 3.04 & 0.11 & 6.37 & 1.81 & 0.19 & 0.13 & 1420 & 23 & 131 \\
\hline 470 & 3.15 & & 6.32 & 1.84 & 0.18 & 0.13 & 1431 & 23 & 131 \\
\hline 471 & $3.28+$ & $-0.3^{-1}$ & 6.27 & 1.88 & 0.18 & 0.13 & 1443 & 23 & 131 \\
\hline 472 & 3.41 & 0.13 & 6.22 & 1.91 & 0.18 & 0.12 & 1455 & 23 & 131 \\
\hline 473 & 3.55 & 0.14 & 6.17 & 1.94 & 0.17 & 0.12 & 1466 & 23 & 131 \\
\hline 474 & 3.69 & $\begin{array}{l}0.14 \\
\text { O.14 }\end{array}$ & 6.12 & 1.98 & 0.17 & 0.12 & 1478 & 23 & 132 \\
\hline 475 & 3.83 & & 6.07 & 2.01 & 0.17 & 0.12 & 1489 & 23 & 132 \\
\hline 476 & $3.98^{+}$ & $\begin{array}{r}+0.15 \\
\end{array}$ & 6.02 & 2.04 & 0.16 & 0.12 & 1501 & 23 & 132 \\
\hline 477 & 4.14 & 0.10 & 5.96 & 2.07 & 0.16 & 0.12 & 1512 & 23 & 132 \\
\hline 478 & 4.31 & 0.17 & 5.91 & 2.11 & 0.16 & 0.12 & 1524 & 23 & 132 \\
\hline 479 & 4.48 & $\begin{array}{l}0.17 \\
0.17\end{array}$ & 5.86 & 2.14 & 0.15 & 0.12 & 1535 & 23 & 132 \\
\hline 480 & 4.65 & & 5.81 & 2.18 & 0.15 & 0.12 & 1546 & 23 & 132 \\
\hline
\end{tabular}


TABLE VIII, ARG. 1.-Continued.

\begin{tabular}{|c|c|c|c|c|c|c|c|c|c|}
\hline Arg. & $(v . c .0)$ & Diff. & (v.s.1) & (v.c.1) & (v.s.2) & (v.c.2) & $(\rho . c .0)$ & (p.s.1) & (p.c.1) \\
\hline 7 & " & $"$ & $" \prime$ & $"$ & " & $"$ & & & \\
\hline 480 & 4.65 & +0.18 & 5.81 & 2.18 & 0.15 & 0.12 & 1546 & 23 & 132 \\
\hline 481 & 4.83 & $\begin{array}{r}+0.10 \\
0.19\end{array}$ & $5: 75$ & 2.21 & 0.15 & 0.12 & 1558 & 23 & 132 \\
\hline 482 & 5.02 & 0.19 & 5.70 & 2.24 & 0.15 & 0.12 & 1569 & 23 & 132 \\
\hline 483 & 5.21 & 0.19 & 5.65 & 2.28 & 0.14 & 0.12 & 1580 & 23 & 132 \\
\hline 484 & 5. 40 & 0.21 & 5.59 & 2.31 & 0.14 & 0.12 & 1591 & 23 & 133 \\
\hline 485 & 5.61 & +0.20 & 5.54 & 2.35 & 0.14 & 0.12 & 1602 & 24 & 133 \\
\hline 486 & 5.81 & 0.22 & 5.48 & 2.38 & 0.13 & 0.12 & 1613 & 24 & 133 \\
\hline 487 & 6.03 & $0.2 \mathrm{I}$ & 5.43 & 2.43 & 0.13 & 0.12 & 1624 & 24 & 133 \\
\hline $\begin{array}{l}488 \\
489\end{array}$ & $\begin{array}{l}6.24 \\
6.47\end{array}$ & 0.23 & $\begin{array}{l}5.37 \\
5.32\end{array}$ & $\begin{array}{l}2.45 \\
2.48\end{array}$ & 0.13 & 0.11 & 1635 & 24 & $\begin{array}{l}134 \\
134\end{array}$ \\
\hline 489 & 0.48 & 0.23 & 0.32 & 2.48 & 0.12 & 0.11 & 1646 & 25 & 134 \\
\hline $\begin{array}{l}490 \\
491\end{array}$ & $\begin{array}{l}6.70 \\
6.93\end{array}$ & +0.23 & 5.25 & 2.52 & 0.12 & 0.11 & 1657 & 25 & 133 \\
\hline $\begin{array}{l}491 \\
492\end{array}$ & $\begin{array}{l}6.93^{-} \\
7.17\end{array}$ & 0.24 & 5.20 & 2.55 & 0.12 & 0.11 & 1668 & 25 & 133 \\
\hline $\begin{array}{l}492 \\
493\end{array}$ & $\begin{array}{l}7.17 \\
7.41\end{array}$ & 0.24 & 5.15 & 2.59 & 0.12 & 0.11 & 1679 & 25 & 133 \\
\hline $\begin{array}{l}493 \\
494\end{array}$ & $\begin{array}{l}7.41 \\
7.66\end{array}$ & 0.25 & 5.09 & 2.62 & 0.11 & 0.11 & 1690 & 25 & 133 \\
\hline 494 & 7.66 & 0.26 & 5.03 & 2.66 & 0.11 & 0.11 & 1700 & 26 & 133 \\
\hline $\begin{array}{l}495 \\
496\end{array}$ & $\begin{array}{l}7.92 \\
8.18\end{array}$ & +0.26 & 4.98 & 2.69 & 0.11 & 0.11 & 1711 & 27 & 133 \\
\hline $\begin{array}{l}496 \\
497\end{array}$ & $\begin{array}{l}8.18 \\
8.44\end{array}$ & 0.26 & $\begin{array}{l}4.92 \\
4.86\end{array}$ & 2.72 & 0.11 & $0.1 \mathrm{~L}$ & 1721 & 27 & 133 \\
\hline $\begin{array}{l}497 \\
498\end{array}$ & $\begin{array}{l}8.44 \\
8.71\end{array}$ & 0.27 & $\begin{array}{l}4.86 \\
480\end{array}$ & 2.76 & 0.10 & 0.11 & 1732 & 28 & 133 \\
\hline $\begin{array}{l}498 \\
499\end{array}$ & $\begin{array}{l}8.71 \\
8.99\end{array}$ & 0.28 & $\begin{array}{l}4.80 \\
4.75\end{array}$ & 2.79 & 0.10 & 0.11 & 1742 & 28 & $\begin{array}{l}133 \\
133\end{array}$ \\
\hline 499 & 8.93 & 0.27 & 4.75 & 2.83 & 0.10 & 0.11 & 1753 & 28 & 133 \\
\hline $\begin{array}{l}500 \\
501\end{array}$ & $\begin{array}{l}9.26 \\
9.55\end{array}$ & +0.29 & 4.69 & 2.86 & 0.10 & 0.11 & 1763 & 29 & 133 \\
\hline $\begin{array}{l}501 \\
502\end{array}$ & $\begin{array}{l}9.55 \\
9.84\end{array}$ & 0.29 & 4.63 & 2.89 & 0.10 & 0.11 & 1774 & 30 & 133 \\
\hline $\begin{array}{l}502 \\
503\end{array}$ & $\begin{array}{r}9.84 \\
10.13\end{array}$ & 0.29 & 4.57 & 2.93 & 0.09 & 0.10 & 1784 & 30 & 133 \\
\hline $\begin{array}{l}503 \\
504\end{array}$ & $\begin{array}{l}10.13 \\
10.43\end{array}$ & 0.30 & 4.51 & 2.95 & 0.09 & 0.10 & 1794 & $3 I$ & 133 \\
\hline $\begin{array}{l}504 \\
505\end{array}$ & 10.43 & 0.31 & 4.44 & 2.29 & 0.09 & 0.10 & 1804 & 31 & 133 \\
\hline $\begin{array}{l}505 \\
506\end{array}$ & $\begin{array}{l}10.74 \\
11.05+\end{array}$ & +0.31 & 4.39 & 3.02 & 0.09 & 0.10 & 1814 & 32 & 133 \\
\hline $\begin{array}{l}506 \\
507\end{array}$ & $\begin{array}{l}11.05^{+} \\
11.36\end{array}$ & $0.3^{1}$ & 4.33 & 3.06 & 0.09 & 0.10 & 1824 & 32 & 133 \\
\hline $\begin{array}{l}507 \\
508\end{array}$ & $\begin{array}{l}11.36 \\
11.68\end{array}$ & $0.3^{2}$ & 4.28 & 309 & 0.08 & 0.10 & 1834 & 33 & 133 \\
\hline $\begin{array}{l}508 \\
509\end{array}$ & $\begin{array}{l}11.68 \\
12.00\end{array}$ & $0.3^{2}$ & 422 & 3.12 & 0.08 & 0.10 & 1844 & 34 & 133 \\
\hline 509 & 12.00 & 0.33 & 4.16 & 3.15 & 0.08 & 0.10 & 1853 & 35 & 133 \\
\hline 510 & $12.33+$ & +0.33 & 4.10 & 3.18 & 0.08 & 0.09 & 1863 & 35 & 133 \\
\hline 5 II & $12.66^{+}$ & $\begin{array}{r}0.33 \\
0.34\end{array}$ & 4.04 & 3.21 & 0.08 & 0.09 & 1873 & 36 & 133 \\
\hline 512 & 13.00 & $\begin{array}{l}.34 \\
0.34\end{array}$ & 3.98 & 3.25 & 0.07 & 0.09 & 1882 & 37 & 133 \\
\hline $\begin{array}{l}513 \\
514\end{array}$ & $\begin{array}{l}13.34 \\
13.68\end{array}$ & 0.34 & 3.92 & 3.28 & 0.07 & 0.09 & 1892 & 38 & 133 \\
\hline & $\begin{array}{l}13.68 \\
1403\end{array}$ & 0.35 & 3.86 & 3.31 & 0.07 & 0.09 & 1901 & 39 & 133 \\
\hline $\begin{array}{l}515 \\
516\end{array}$ & $\begin{array}{l}14.03 \\
14.39\end{array}+$ & +o. $3^{6}$ & 3.80 & 3.34 & 0.07 & 0.09 & 1910 & 40 & 133 \\
\hline $\begin{array}{l}516 \\
517\end{array}$ & $\begin{array}{l}14.39^{\top} \\
14.75\end{array}$ & $0.3^{6}$ & 3.74 & 3.37 & 0.07 & 0.09 & 1920 & 40 & 133 \\
\hline $\begin{array}{l}517 \\
518\end{array}$ & $\begin{array}{l}14.75 \\
15.11\end{array}$ & $0.3^{6}$ & 3.68 & 3.40 & 0.07 & 0.09 & 1929 & 41 & 133 \\
\hline 519 & $\begin{array}{l}15.11 \\
15.48\end{array}$ & 0.37 & 3.62 & 3.43 & 0.07 & 0.09 & 1938 & 42 & 133 \\
\hline & $\begin{array}{l}15.48 \\
15.85\end{array}$ & 0.37 & 3.56 & 3.46 & 0.07 & 0.08 & 1947 & 43 & 133 \\
\hline 521 & $\begin{array}{l}10.85 \\
16.23\end{array}+$ & to. $3^{8}$ & 3.50 & 3.49 & 0.06 & 0.08 & 1956 & 44 & 132 \\
\hline 522 & 16.61 & $0.3^{8}$ & 3.44 & 3.51 & 0.06 & 0.08 & 1964 & 45 & 132 \\
\hline 523 & 16.99 & $0.3^{8}$ & $\begin{array}{l}3.38 \\
3.29\end{array}$ & 3.54 & 0.06 & 0.08 & 1973 & 46 & 132 \\
\hline 524 & 17.38 & $\begin{array}{l}0.39 \\
0.30\end{array}$ & $\begin{array}{l}3.32 \\
3.27\end{array}$ & $\begin{array}{l}3.57 \\
3.60\end{array}$ & $\begin{array}{l}0.06 \\
0.06\end{array}$ & 0.08 & 1982 & 47 & 132 \\
\hline 525 & 17.77 & 0.39 & 3.21 & 3.62 & $\begin{array}{l}0.00 \\
0.06\end{array}$ & 0.08 & 1990 & 48 & 132 \\
\hline 526 & $18.17+$ & +0.40 & 3.15 & 3.65 & $\begin{array}{l}0.06 \\
0.06\end{array}$ & 0.08 & 1999 & 49 & 132 \\
\hline 527 & 18.57 & 0.40 & 3.09 & 3.67 & $\begin{array}{l}0.06 \\
0.06\end{array}$ & 0.08 & 2007 & 50 & 132 \\
\hline 528 & 18.97 & 0.40 & 3.03 & 3.70 & $\begin{array}{l}0.06 \\
0.06\end{array}$ & $\begin{array}{l}0.08 \\
0.07\end{array}$ & 2016 & 51 & $\begin{array}{l}132 \\
139\end{array}$ \\
\hline 529 & 19.38 & 0.41 & 2.97 & 3.73 & 0.05 & $\begin{array}{l}0.07 \\
0.07\end{array}$ & $\begin{array}{l}2024 \\
2032\end{array}$ & $\begin{array}{l}52 \\
53\end{array}$ & $\begin{array}{l}132 \\
132\end{array}$ \\
\hline 530 & 19.79 & 0.41 & 2.91 & 3.75 & 0.05 & 0.07 & 2040 & 55 & 132 \\
\hline $53 \mathrm{I}$ & $20.21+$ & +0.42 & 2.86 & 3.77 & 0.05 & 0.07 & 2048 & 56 & 132 \\
\hline 532 & 20.62 & $0.4 \mathbf{I}$ & 280 & 3.80 & 0.05 & 0.07 & 2056 & 57 & 132 \\
\hline 533 & 21.05 & 0.43 & 2.75 & 3.82 & 0.05 & 0.07 & 2063 & 58 & 132 \\
\hline 534 & 21.47 & $\begin{array}{l}0.42 \\
0.43\end{array}$ & 2.69 & 3.81 & 0.05 & 0.07 & 2071 & 59 & 131 \\
\hline 535 & 21.90 & & 2.63 & 3.87 & 0.05 & 0.07 & 2079 & 61 & 131 \\
\hline 536 & $22.34^{t}$ & +0.44 & 2.57 & 3.89 & 0.05 & 0.07 & 2086 & 62 & 131 \\
\hline 537 & 22.77 & 0.43 & 2.52 & 3.91 & 0.05 & 0.07 & 2094 & 63 & 131 \\
\hline 538 & 23.21 & 0.44 & 2.46 & 3.93 & 0.05 & 0.07 & 2101 & 64 & 131 \\
\hline 539 & 23.66 & $\begin{array}{l}0.45 \\
0.44\end{array}$ & 2.40 & 3.96 & 0.05 & 0.06 & 2108 & 66 & 131 \\
\hline 540 & 24.10 & & 2.35 & 3.98 & 0.05 & 0.06 & 2115 & 67 & 131 \\
\hline
\end{tabular}




\begin{tabular}{|c|c|c|c|c|c|c|c|c|c|}
\hline \multicolumn{10}{|c|}{ TABLE VIII, Arg. 1.-Concluded. } \\
\hline \multirow[t]{2}{*}{ Arg. } & $(v . c .0)$ & Diff. & $(v .8 .1)$ & (v.c.1) & $(v .8 .2)$ & (v.c.2) & $(\rho . c .0)$ & $(p .8 .1)$ & (p.c.1) \\
\hline & $"$ & $"$ & $"$ & $"$ & $" \prime$ & " & & & \\
\hline 540 & 24.10 & Lo, 46 & 2.35 & 3.98 & 0.05 & 0.06 & 2115 & 67 & 131 \\
\hline 541 & $24.56^{-}$ & $1-0.46$ & 2.29 & 4.00 & 0.05 & 0.06 & 2122 & 68 & 131 \\
\hline 542 & 25.01 & $\begin{array}{l}0.45 \\
0.46\end{array}$ & 2.24 & 4.02 & 0.05 & 0.06 & 2129 & 70 & 131 \\
\hline 543 & 25.47 & $\begin{array}{l}0.46 \\
0.46\end{array}$ & 2.18 & 4.04 & 0.05 & 0.06 & 2135 & 71 & 130 \\
\hline 544 & 25.93 & $\begin{array}{l}0.40 \\
0.46\end{array}$ & 2.13 & 4.06 & 0.05 & 0.06 & 2142 & 73 & 130 \\
\hline 545 & 26.39 & -0.46 & 2.08 & 4.07 & 0.05 & 0.06 & 2149 & 74 & 130 \\
\hline 546 & $26.85^{-}$ & $\begin{array}{l}0.40 \\
0.47\end{array}$ & 2.02 & 4.09 & 0.05 & 0.06 & 2155 & 75 & 130 \\
\hline 547 & 27.32 & 0.47 & 1.97 & 4.11 & 0.05 & 0.06 & 2162 & 77 & 130 \\
\hline 548 & 27.79 & $\begin{array}{l}0.47 \\
0.48\end{array}$ & 1.92 & 4.13 & 0.05 & 0.06 & 2168 & 78 & 130 \\
\hline 549 & 28.27 & 0.48 & 1.87 & 4.14 & 0.05 & 0.06 & 2174 & 80 & 130 \\
\hline 550 & 28.75 & & 1.82 & 4.16 & 0.05 & 0.06 & 2180 & 81 & 130 \\
\hline 551 & 29.23 & $\begin{array}{r}-0.48 \\
0.48\end{array}$ & 1.77 & 4.17 & 0.05 & 0.06 & 2186 & 83 & 130 \\
\hline 552 & 29.71 & 0.48 & 1.72 & 4.19 & 0.06 & 0.06 & 2191 & 84 & 130 \\
\hline 553 & 30.20 & 0.49 & 1.67 & 4.20 & 0.06 & 0.06 & 2197 & 86 & 130 \\
\hline 554 & 30.69 & $\begin{array}{l}0.49 \\
0.49\end{array}$ & 1.62 & 4.22 & 0.06 & 0.06 & 2203 & 87 & 130 \\
\hline 555 & 31.18 & & 1.57 & 4.23 & 0.06 & 0.06 & 2208 & 89 & 130 \\
\hline 556 & 31.67 & -0.49 & 1.52 & 4.25 & 0.06 & 0.06 & 2214 & 90 & 130 \\
\hline 557 & 32.16 & 0.49 & 1.48 & 4.26 & 0.06 & 0.06 & 2219 & 92 & 130 \\
\hline 558 & 32.66 & $0.5^{\circ}$ & 1.43 & 4.27 & 0.06 & 0.06 & 2224 & 94 & 130 \\
\hline 559 & 33.16 & $\begin{array}{l}0.5^{0} \\
0.5^{1}\end{array}$ & 1.38 & 4.28 & 0.06 & 0.06 & 2229 & 95 & 130 \\
\hline 560 & 33.67 & -0.50 & 1.34 & 4.29 & 0.06 & 0.06 & 2234 & 97 & 130 \\
\hline 561 & $34.17^{-}$ & Fo. $5^{\circ}$ & 1.29 & 4.31 & 0.06 & 0.06 & 2238 & 98 & 130 \\
\hline 562 & 34.68 & $\begin{array}{l}0.5 \mathrm{I} \\
0.5 \mathrm{I}\end{array}$ & 1.25 & 4.32 & 0.07 & 0.06 & 2243 & 100 & 130 \\
\hline 563 & 35.19 & $\begin{array}{l}0.5^{1} \\
0.5^{1}\end{array}$ & 1.20 & 4.32 & 0.07 & 0.06 & 2248 & 101 & 130 \\
\hline 564 & 35.70 & $\begin{array}{l}0.5^{1} \\
0.5^{1}\end{array}$ & 1.16 & 4.33 & 0.07 & 0.06 & 2252 & 103 & 130 \\
\hline 565 & 36.21 & & 1.12 & 4.34 & 0.07 & 0.06 & 2256 & 105 & 130 \\
\hline 566 & $36.73^{-}$ & $-0.5^{2}$ & 1.08 & 4.35 & 0.07 & 0.06 & 2261 & 106 & 130 \\
\hline 567 & 37.25 & $0.5^{2}$ & 1.03 & 4.36 & 0.07 & 0.06 & 2265 & 108 & 130 \\
\hline 568 & 37.76 & 0.51 & 0.99 & 4.37 & 0.07 & 0.06 & 2269 & 110 & 130 \\
\hline 569 & 38.29 & $\begin{array}{l}0.53 \\
0.52\end{array}$ & 0.96 & 4.38 & 0.07 & 0.06 & 2272 & 111 & 130 \\
\hline 570 & 38.81 & -0.53 & 0.92 & 4.38 & 0.07 & 0.06 & 2276 & 113 & 130 \\
\hline 571 & 39.34 & $\begin{array}{r}+0.53 \\
0.52\end{array}$ & 0.88 & 4.39 & 0.07 & 0.06 & 2280 & 115 & 130 \\
\hline 572 & 39.86 & $\begin{array}{l}0.5^{2} \\
0.53\end{array}$ & 0.84 & 4.40 & 0.07 & 0.07 & 2283 & 116 & 130 \\
\hline 573 & 40.39 & $\begin{array}{l}0.53 \\
0.53\end{array}$ & 0.80 & 4.40 & 0.08 & 0.07 & 2287 & 118 & 130 \\
\hline 574 & 40.92 & $\begin{array}{l}0.53 \\
0.53\end{array}$ & 0.77 & 4.41 & 0.08 & 0.07 & 2290 & 120 & 131 \\
\hline 575 & 41.45 & & 0.73 & 4.41 & 0.08 & 0.07 & 2293 & 121 & 131 \\
\hline 576 & 41.98 & $\begin{array}{r}+0.53 \\
0.54\end{array}$ & 0.70 & 4.42 & 0.08 & 0.07 & 2296 & 123 & 131 \\
\hline 577 & 42.52 & $\begin{array}{l}0.54 \\
0.53\end{array}$ & 0.67 & 4.42 & 0.08 & 0.07 & 2299 & 125 & 131 \\
\hline 578 & 43.05 & $\begin{array}{l}0.53 \\
0.54\end{array}$ & 0.63 & 4.42 & 0.08 & 0.07 & 2302 & 126 & 131 \\
\hline 579 & 43.59 & $\begin{array}{l}0.54 \\
0.53\end{array}$ & 0.60 & 4.43 & 0.08 & 0.07 & 2304 & 128 & 131 \\
\hline 580 & 44.12 & & 0.57 & 4.43 & 0.08 & 0.07 & 2307 & 130 & 131 \\
\hline 581 & $44.66^{-}$ & +0.54 & 0.54 & 4.43 & 0.08 & 0.08 & 2309 & 132 & 131 \\
\hline 582 & 45.20 & 0.54 & 0.51 & 4.44 & 0.09 & 0.08 & 2312 & 133 & 131 \\
\hline 583 & 45.74 & 0.54 & 0.48 & 4.44 & 0.09 & 0.08 & 2314 & 135 & 131 \\
\hline 584 & 46.28 & $\begin{array}{l}0.54 \\
0.55\end{array}$ & 0.46 & 4.44 & 0.09 & 0.08 & 2316 & 137 & 132 \\
\hline 585 & 46.83 & & 0.43 & 4.44 & 0.09 & 0.08 & 2318 & 138 & 132 \\
\hline 586 & 47.37 & +0.54 & 0.40 & 4.44 & 0.09 & 0.08 & 2319 & 140 & 132 \\
\hline 587 & 47.91 & 0.54 & 0.38 & 4.44 & 0.09 & 0.09 & 2321 & 142 & 132 \\
\hline 588 & 48.46 & 0.55 & 0.36 & 4.44 & 0.09 & 0.09 & 2322 & 144 & 132 \\
\hline 589 & 49.00 & $\begin{array}{l}0.54 \\
0.55\end{array}$ & 0.33 & 4.44 & 0.09 & 0.09 & 2324 & 145 & 132 \\
\hline 590 & 49.55 & & 0.31 & 4.44 & 0.09 & 0.09 & 2325 & 147 & 132 \\
\hline 591 & $50.10^{-t}$ & +0.55 & 0.29 & 4.44 & 0.09 & 0.09 & 2326 & 149 & 132 \\
\hline 592 & 50.64 & 0.54 & 0.27 & 4.44 & 0.10 & 0.09 & 2327 & 150 & 133 \\
\hline 593 & 51.19 & $\begin{array}{l}0.55 \\
0.55\end{array}$ & 0.25 & 4.44 & 0.10 & 0.10 & 2328 & 152 & 133 \\
\hline 594 & 51.74 & $\begin{array}{l}0.55 \\
0.55\end{array}$ & 0.23 & 4.43 & 0.10 & 0.10 & 2329 & 154 & 133 \\
\hline 595 & 52.29 & & 0.22 & 4.43 & 0.10 & 0.10 & 2330 & 155 & 133 \\
\hline 596 & $52.84^{+}$ & -0.55 & 0.20 & 4.43 & 0.10 & 0.10 & 2330 & 157 & 133 \\
\hline 597 & 53.38 & 0.54 & 0.18 & 4.43 & 0.10 & 0.11 & 2330 & 159 & 133 \\
\hline 598 & 53.93 & 0.55 & 0.17 & 4.43 & 0.10 & 0.11 & 2331 & 161 & 134 \\
\hline 599 & 54.48 & $\begin{array}{l}0.55 \\
0.55\end{array}$ & 0.16 & 4.42 & 0.10 & 0.11 & 2331 & 162 & 134 \\
\hline 600 & 55.03 & & 0.14 & 4.42 & 0.10 & 0.11 & 2331 & 164 & 134 \\
\hline
\end{tabular}


TABLE 1X, Ara. 2.-ACtion of Saturn.

Arg. (v.c.0) Diff. (v.s.1) Diff. Sec.var. (v.c.1) Diff. Scc.var. (v.s.2) Diff. Sec.var. (v.c.2) Diff. Sec.var

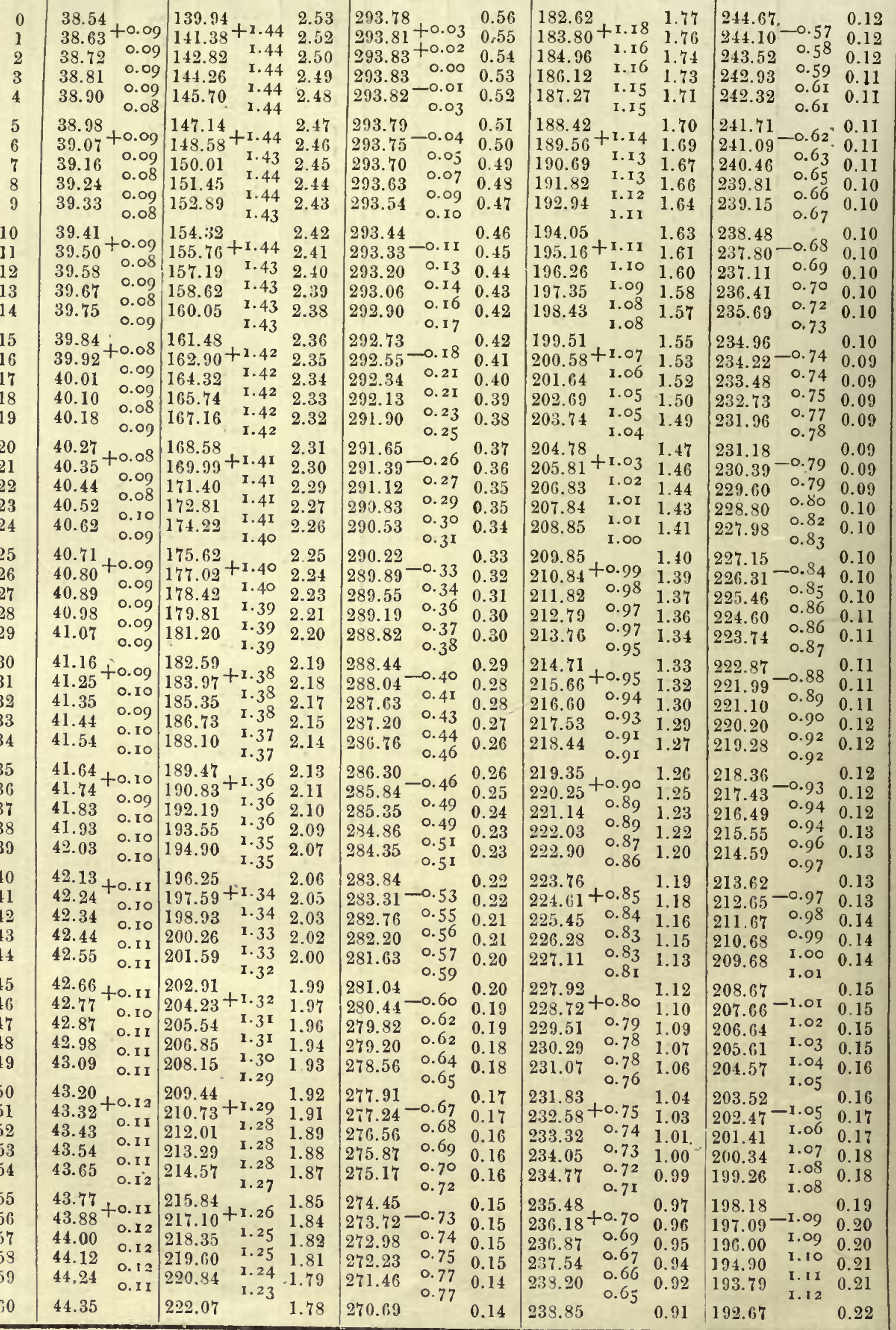




\begin{tabular}{|c|c|c|c|c|c|c|c|c|c|c|c|}
\hline Arg. & (v.x.3) & (v.c.3) & $(v .8 .1)$ & $(v . c .4)$ & $(p . c .0)$ & $($ (p.8.1) & $(p . c .1)$ & $(p .8 .2)$ & (p.c.2) & $($ (p.8.3) & (p.c.3) \\
\hline & $"$ & " & $"$ & $"$ & & & & & & & \\
\hline 0 & 11.40 & 14.53 & 1.59 & 1.68 & 1678 & 182 & 1513 & 711 & $30 G$ & 137 & 85 \\
\hline 1 & 11.52 & 14.49 & 1.59 & 1.66 & 1679 & 182 & 1526 & 711 & 302 & 137 & 85 \\
\hline 2 & 11.64 & 14.43 & 1.60 & 1.65 & 16,50 & 183 & 1539 & 710 & 298 & 137 & 85 \\
\hline 3 & 11.75 & 14.38 & 1.61 & 1.64 & 1680 & 184 & 1551 & 709 & 295 & 137 & 85 \\
\hline 4 & 11.87 & 14.33 & 1.62 & 1.63 & 1681 & 185 & 1564 & 703 & 291 & 137 & 85 \\
\hline 5 & 11.99 & 11.29 & 1.63 & 1.62 & 1682 & 187 & 1577 & 707 & 289 & 137 & 85 \\
\hline 6 & 12.10 & 14.22 & 1.64 & 1.61 & 1632 & 188 & 1590 & 706 & 284 & 137 & 85 \\
\hline 7 & 12.21 & 14.16 & 1.64 & 1.60 & 1683 & 190 & 1603 & 704 & 281 & 138 & 84 \\
\hline 8 & 12.32 & 14.10 & 1.65 & 1.50 & 1683 & 191 & 1615 & 703 & 278 & 138 & 84 \\
\hline 9 & 12.43 & 14.04 & 1.66 & 1.58 & 1682 & 193 & 1623 & 701 & 274 & 138 & 84 \\
\hline 10 & 12.54 & 13.97 & 1.67 & 1.50 & 1682 & 195 & 1641 & 700 & 271 & 139 & 84 \\
\hline 11 & 12.65 & 13.91 & 1.68 & 1.55 & 1681 & 197 & 1654 & 699 & 267 & 138 & 84 \\
\hline 12 & 12.76 & 13.81 & 1.69 & 1.54 & 1680 & 199 & 1606 & 695 & 264 & 139 & 84 \\
\hline 13 & 12.87 & 13.77 & 1.69 & 1.53 & 1679 & 201 & 1679 & 696 & 260 & 138 & 81 \\
\hline 14 & 12.97 & 13.70 & 1.70 & 1.52 & 1678 & 203 & 1691 & 695 & 257 & 137 & 83 \\
\hline 15 & 13.07 & 13.63 & 1.71 & 1.50 & 1677 & 20 s & 1704 & 691 & 253 & 137 & 83 \\
\hline 10 & 13.18 & 13.56 & 1.71 & 1.49 & 1675 & 203 & 1716 & 692 & 250 & 137 & 83 \\
\hline 17 & 13.28 & 13.49 & 1.73 & 1.49 & 1674 & 211 & 1729 & 691 & 246 & 137 & 83 \\
\hline 18 & 13.39 & 13.41 & 1.73 & 1.47 & 1672 & 211 & 1741 & 6.89 & 243 & 137 & 82 \\
\hline 19 & 13.49 & 13.33 & 1.74 & 1.45 & 1670 & 217 & 1754 & 687 & 239 & 137 & 82 \\
\hline 20 & 13.58 & 13.25 & 1.74 & 1.44 & 1668 & 220 & 1706 & 685 & 236 & 137 & 82 \\
\hline 21 & 13.68 & 13.17 & 1.75 & 1.43 & 1660 & $2 \pm 3$ & 1778 & 683 & $23: 3$ & 137 & 82 \\
\hline 22 & 13.78 & 13.09 & 1.75 & 1.42 & 1664 & 2.7 & 1720 & 681 & 230 & 137 & 81 \\
\hline 23 & 13.87 & 13.01 & 1.76 & 1.40 & 1662 & 230 & 1802 & 673 & 229 & 137 & 81 \\
\hline 24 & 13.97 & 12.92 & 1.76 & 1.39 & 1660 & 231 & 1814 & C7T & 223 & 137 & 81 \\
\hline 25 & 14.06 & 12.83 & 1.77 & 1.37 & 1658 & 237 & 1826 & 675 & 220 & 137 & 81 \\
\hline 26 & 14.15 & 12.74 & 1.79 & 1.30 & 1656 & 241 & 1839 & 673 & 217 & 137 & 81 \\
\hline 27 & 14.24 & 12.65 & 1.79 & 1.35 & 1653 & 245 & 1850 & 6it & 214 & 137 & 80 \\
\hline 23 & 14.33 & 12.56 & 1.79 & 1.34 & 1651 & 249 & 1862 & 6ifs & 211 & 137 & 80 \\
\hline 29 & 14.41 & 12.47 & 1.79 & 1.32 & 1648 & 2.33 & 1874 & 666 & 203 & 137 & 80 \\
\hline 30 & 14.50 & 12.37 & 1.79 & 1.31 & 1645 & 259 & 1896 & 601 & 205 & 137 & 79 \\
\hline 31 & 14.58 & 12.29 & 1.80 & 1.30 & 1642 & 262 & 1895 & 60,3 & 202 & 137 & 78 \\
\hline 32 & 14.66 & 12.19 & 1.80 & 1.29 & 1638 & 267 & 1910 & 659 & 199 & 137 & 78 \\
\hline 33 & 14.74 & 12.09 & 1.80 & 1.27 & 1635 & 272 & 1922 & 657 & 196 & 137 & 78 \\
\hline 34 & 14.82 & 11.99 & 1.81 & 1.25 & 1631 & 277 & 193. & 654 & 193 & 137 & 78 \\
\hline 3.5 & 14.90 & 11.89 & 1.81 & 1.24 & 1627 & 282 & 1946 & 652 & 190 & 137 & 77 \\
\hline 36 & 14.97 & 11.79 & 1.81 & 1.22 & 1623 & 297 & 1958 & 650 & 187 & 137 & 77 \\
\hline 37 & 15.05 & 11.69 & 1.81 & 1.21 & 1619 & 292 & 1970 & 647 & 184 & 137 & 77 \\
\hline 33 & 15.12 & 11.59 & 1.82 & 1.20 & 1615 & $2 ?$ & 1982 & 645 & 181 & 137 & 76 \\
\hline 39 & 15.19 & 11.49 & 1.82 & 1.18 & 1611 & 302 & 1993 & 642 & 179 & 137 & 76 \\
\hline 40 & 15.26 & 11.38 & 1.82 & 1.17 & 1609 & 309 & 2005 & 610 & 170 & $13 \uparrow$ & 76 \\
\hline+1 & 15.33 & 11.28 & 1.82 & 1.15 & 1602 & 313 & 2016 & 637 & 173 & 137 & 70 \\
\hline 42 & 15.39 & 11.17 & 1.82 & 1.14 & 1597 & 313 & 2028 & 635 & 171 & 137 & 76 \\
\hline 43 & 15.46 & 11.06 & 1.82 & 1.13 & 1592 & 325 & 2039 & 6,32 & 16,8 & 137 & 75 \\
\hline 44 & 15.52 & 10.95 & 1.82 & 1.11 & 1587 & 331 & 2051 & 630 & 160 & 137 & 74 \\
\hline 45 & 15.59 & 10.81 & 1.82 & 1.10 & 1582 & 337 & $20<2$ & 6.27 & 16.3 & 137 & 74 \\
\hline 16 & 15.64 & 10.73 & 1.82 & 1.08 & 1577 & 343 & 2073 & 624 & 160 & 137 & 74 \\
\hline 47 & 15.70 & 10.62 & 1.92 & 1.07 & 1578 & 349 & 2081 & c,21 & 159 & 137 & 73 \\
\hline 48 & 15.76 & 10.51 & 1.82 & 1.06 & 1566 & 355 & 2035 & 619 & 155 & 137 & 73 \\
\hline 49 & 15.81 & 10.40 & 1.82 & 1.04 & 1561 & 362 & $210 \mathrm{C}$ & 616 & 153 & 137 & 73 \\
\hline 50 & 15.80 & 10.28 & 1.82 & 1.03 & 1556 & 368 & 2117 & 613 & 150 & 137 & 72 \\
\hline 51 & 15.91 & 10.17 & 1.82 & 1.01 & 1550 & 375 & 2129 & 610 & 143 & 137 & 72 \\
\hline 52 & 15.96 & 10.05 & 1.92 & 1.00 & 1545 & 391 & 2139 & 607 & 145 & ז 13 & 72 \\
\hline 53 & 10.01 & 9.34 & 1.91 & 0.99 & 1539 & 389 & 2149 & 604 & 143 & 137 & 72 \\
\hline 54 & 16.05 & 9.82 & 1.81 & 0.97 & 1533 & 394 & 2159 & 601 & 140 & 137 & 72 \\
\hline 55 & 16.09 & 9.70 & 1.81 & 0.96 & -1527 & 401 & 2170 & 599 & 139 & 137 & 71 \\
\hline 56 & 16.13 & 9.59 & 1.81 & 0.94 & 1521 & 408 & 2180 & 595 & 135 & 137 & 71 \\
\hline 57 & 16.17 & 9.47 & 1.80 & 0.93 & 1515 & $\$ 15$ & 2191 & 592 & 133 & 137 & 71 \\
\hline 58 & 16.20 & 9.35 & 1.80 & 0.92 & 1509 & 122 & 2201 & 599 & 131 & 137 & 71 \\
\hline 59 & 16.23 & 9.23 & 1.80 & 0.90 & 1502 & 430 & 2212 & 586 & 129 & 137 & 71 \\
\hline 60 & 16.26 & 9.11 & 1.80 & 0.89 & 1490 & 437 & 2222 & 583 & 127 & 137 & 71 \\
\hline
\end{tabular}




\begin{tabular}{|c|c|c|c|c|c|c|c|c|c|c|c|c|c|c|}
\hline \multicolumn{15}{|c|}{ TABLE IX, ARa. 2.-Continued. } \\
\hline \multirow[t]{2}{*}{ Arg. } & $(v . c .0)$ & Diff. & $(0.8 .1)]$ & Diff. $\mathrm{s}$ & Sec.var. & $(v . c .1)$ & Diff, $\mathrm{S}$ & Seevar. & $(v . s .2)$ & Diff. $s$ & Sec.var. & $(v . c .2)$ & Diff. $S$ & See.var. \\
\hline & $" 1$ & " & $"$ & $"$ & $"$ & " & " & $"$ & $" \prime$ & $\prime \prime$ & " & $"$ & " & " \\
\hline 60 & 44.35 & +0.12 & 222.07 & +1.23 & 1.78 & 270.69 & -0.80 & 0.14 & 238.85 & & 0.91 & 192.67 & & 0.22 \\
\hline 61 & $44.47^{+}$ & $\begin{array}{r}+0.12 \\
0.12\end{array}$ & $223.30^{-1}$ & $\begin{array}{r}+1.23 \\
1.22\end{array}$ & 1.76 & 269.89 & $\begin{array}{r}-0.80 \\
0.80\end{array}$ & 0.13 & $239.49^{-1}$ & $\begin{array}{r}+0.64 \\
0.62\end{array}$ & 0.90 & 191.55 & -1.12 & 0.23 \\
\hline 62 & 44.59 & $\begin{array}{l}0.12 \\
0.12\end{array}$ & 224.52 & $\begin{array}{l}1.22 \\
1.21\end{array}$ & 1.75 & 269.09 & $\begin{array}{l}0.80 \\
0.81\end{array}$ & 0.13 & 240.12 & 0.63 & 0.98 & 190.42 & I. 3 & 0.23 \\
\hline 63 & 44.71 & 0.12 & 225.73 & $\begin{array}{l}1.21 \\
1.21\end{array}$ & 1.73 & 268.28 & $\begin{array}{l}0.81 \\
0.83\end{array}$ & 0.13 & $2 \pm 0.74$ & 0.02 & 0.87 & 189.29 & I. I 3 & 0.24 \\
\hline 64 & 44.83 & 0.12 & 226.94 & 1.20 & 1.72 & 267.45 & $\begin{array}{l}0.83 \\
0.84\end{array}$ & 0.13 & 241.35 & $\begin{array}{l}0.01 \\
0.60\end{array}$ & 0.86 & 188.14 & $\begin{array}{l}\text { I. I } 5 \\
\text { 1. I } 5\end{array}$ & 0.25 \\
\hline 65 & 44.95 & -O. 12 & 228.14 & +1.19 & 1.70 & 266.61 & -0.85 & 0.12 & 241.95 & $+0.5^{8}$ & & 186.99 & & 0.25 \\
\hline 66 & & 0.12 & 229.33 & I. 18 & 1. & 76 & 0.87 & $\begin{array}{l}0.12 \\
0.12\end{array}$ & & 0.57 & $\begin{array}{l}3 \\
6\end{array}$ & 185.83 & 1.16 & 0.26 \\
\hline 67 & 12 & O.I 2 & $\begin{array}{l}230.51 \\
231.69\end{array}$ & I. 18 & $\begin{array}{l}1.67 \\
1.66\end{array}$ & $\begin{array}{l}204.89 \\
264.02\end{array}$ & 0.87 & $\begin{array}{l}0.12 \\
0.12\end{array}$ & $\begin{array}{l}243 \\
243\end{array}$ & 0.57 & 0 & 184.67 & 1.16 & 0.27 \\
\hline 68 & $\begin{array}{l}31 \\
44\end{array}$ & 0.13 & $\begin{array}{l}231.6 \mathrm{~J} \\
232.86\end{array}$ & I. 17 & 1.64 & 263.13 & 0.89 & 0.11 & $\begin{array}{l}243.67 \\
244.22\end{array}$ & 0.55 & $\begin{array}{l}0.81 \\
0.79\end{array}$ & 18 & I. 17 & 0.28 \\
\hline 6 & & 0.12 & We.00 & I. I 6 & & - & 0.90 & & 244.22 & 0.54 & 0.79 & 182.34 & I. I 8 & 0.28 \\
\hline $\begin{array}{l}70 \\
71\end{array}$ & $\begin{array}{l}45.56 \\
45.68\end{array}$ & -0.12 & $\begin{array}{l}234.02 \\
235.17\end{array}+$ & +1.15 & $\begin{array}{l}1.63 \\
1.62\end{array}$ & $\begin{array}{l}262.23 \\
261.32\end{array}$ & -0.91 & $\begin{array}{l}0.11 \\
0.11\end{array}$ & $\left.\begin{array}{l}244.76 \\
245.28\end{array}\right\}$ & $+0.5^{2}$ & $\begin{array}{l}0.78 \\
0.77\end{array}$ & 181.16 & -1. I9 & .29 \\
\hline 72 & 45.80 & 0.12 & 236.31 & I. I 4 & $\begin{array}{l}1.02 \\
1.60\end{array}$ & 260.39 & 0.93 & 0.11 & 245.79 & 0.51 & $\begin{array}{l}0.77 \\
0.75\end{array}$ & $\begin{array}{l}179.97 \\
178.78\end{array}$ & I. I9 & \\
\hline 73 & 45.92 & 0.12 & 237.45 & I. I 4 & 1.59 & 259.46 & 0.93 & 0.11 & 246.29 & $0.5^{\circ}$ & 0.74 & 177.59 & 1.19 & $\begin{array}{l}1.31 \\
0.32\end{array}$ \\
\hline 74 & 46.04 & $\begin{array}{l}0.12 \\
0.12\end{array}$ & 238.58 & $\begin{array}{l}\text { I. I3 } \\
\text { I. I } 2\end{array}$ & 1.57 & 258.52 & $\begin{array}{l}0.94 \\
0.06\end{array}$ & 0.11 & 246.78 & $\begin{array}{l}0.49 \\
0.48\end{array}$ & 0.73 & 176.40 & $\begin{array}{l}1.19 \\
1.20\end{array}$ & 0.33 \\
\hline 75 & 46.16 & -0.13 & $239.70_{+}$ & +1. I I & 1.56 & 257.56 & & 0.11 & 247.26 & & 0.71 & 175.20 & & 0.34 \\
\hline 76 & & 0.12 & 240. & I.10 & 1.55 & $256.59^{-}$ & -0.97 & 0.11 & 24 & +0.47 & 0.70 & $99^{-}$ & -1.21 & 0.34 \\
\hline 77 & 46 & 0.12 & 241.91 & 1.09 & 1.53 & 255.61 & 0.98 & 0.11 & 24 & 0.45 & 0. & 17 & I. 21 & 0.35 \\
\hline 7 & 40 & 0.12 & 243.00 & 1.09 & 1.52 & 54.62 & 0.99 & 0.11 & 24 & $\begin{array}{l}9.44 \\
0.43\end{array}$ & 0. & 56 & $\begin{array}{l}1.22 \\
1.22\end{array}$ & 0.36 \\
\hline 79 & 46.65 & 0.12 & 244.09 & 1.08 & 1.50 & 253.62 & $\begin{array}{l}1.00 \\
1.01\end{array}$ & 0.11 & 249.05 & $\begin{array}{l}0.43 \\
0.42\end{array}$ & 0.66 & 170.34 & $\begin{array}{l}1.22 \\
1.23\end{array}$ & 0.37 \\
\hline 80 & 46.77 & -0.12 & 245. & +1.07 & 1.49 & 252.61 & & 0.11 & 249.47 & 0.40 & 0.65 & 169.11 & & 0.38 \\
\hline 81 & 46 & 0.12 & 24 & 1.06 & 1.48 & $251.59^{-}$ & $\begin{array}{r}-1.02 \\
1.03\end{array}$ & 0.11 & $249.87^{t}$ & $\begin{array}{l}-0.40 \\
0.39\end{array}$ & 0 . & $16^{r}$ & & 0.39 \\
\hline 82 & 47 & 0.12 & 247 & 1.05 & 1.46 & 250.56 & $\begin{array}{l}1.03 \\
1.05\end{array}$ & 0.11 & 250.26 & $\begin{array}{l}.39 \\
0.38\end{array}$ & 0 . & 160 & $\begin{array}{l}1.23 \\
x .24\end{array}$ & 0 \\
\hline 83 & 4 & O. I I & $\begin{array}{l}248.33 \\
240.38\end{array}$ & 1.03 & 1.45 & 249.51 & $\begin{array}{l}1.05 \\
1.06\end{array}$ & 0.11 & 250.64 & $\begin{array}{l}.30 \\
0.37\end{array}$ & 0 . & 165.41 & $\begin{array}{l}\text { 1. } 24 \\
\text { I. } 24\end{array}$ & 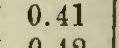 \\
\hline 84 & 4 & 0.12 & 249.58 & 1.03 & 1.43 & 24 & I.07 & 0.11 & 251. & 0.36 & 0.1 & 164.17 & $\begin{array}{l}1.24 \\
1.25\end{array}$ & 0.42 \\
\hline 85 & $\begin{array}{l}47.36 \\
47.48\end{array}$ & -0.12 & 250. & +1.02 & 1.42 & 247.38 & 8 & 0.11 & 251.37 & & 0.60 & 162.92 & I. & 0.43 \\
\hline $\begin{array}{l}86 \\
87\end{array}$ & $\begin{array}{l}47 . \\
47 .\end{array}$ & 0.1 I & 251 & 1.01 & 1.40 & 246. & $\begin{array}{r}-1.00 \\
1.09\end{array}$ & 0.12 & $1^{+}$ & $\begin{array}{l}0.34 \\
0.33\end{array}$ & 0 & 16 & & 3 \\
\hline $\begin{array}{l}87 \\
88\end{array}$ & $\begin{array}{l}47.59 \\
47.71\end{array}$ & 0.12 & 292,44 & 1.00 & 1.39 & 245.21 & 1.09 & 0.12 & 252.04 & $\begin{array}{l}0.33 \\
0.32\end{array}$ & 0 . & 16 & 5 & 0.44 \\
\hline 8 & 47.82 & 0.11 & $\begin{array}{l}230.44 \\
254.43\end{array}$ & 0.99 & 1.38 & $\begin{array}{l}244.12 \\
243.01\end{array}$ & I. I I & 0.12 & 252.36 & 0.31 & 0 . & 15 & 1.26 & 45 \\
\hline & & 0.12 & 204.40 & 0.98 & 1.36 & 243.01 & 1.12 & 0.12 & 252.67 & 0.29 & 0.55 & 157.90 & 1.27 & 0.46 \\
\hline $\begin{array}{l}90 \\
91\end{array}$ & $\begin{array}{l}47.94 \\
48.05\end{array}+$ & -0.11 & & +0.97 & 1.35 & 241.89 & -1.13 & 0.12 & 252.96 & +0.28 & 0.54 & 156.63 & 7 & 0.47 \\
\hline $\begin{array}{l}91 \\
92\end{array}$ & 48.16 & 0.11 & $\begin{array}{l}256.38 \\
257.34\end{array}$ & 0.96 & 1.33 & $240.76^{-}$ & $\begin{array}{r}-1.13 \\
1.14\end{array}$ & 0.12 & $253.24^{\dagger}$ & $\begin{array}{r}0.20 \\
0.26\end{array}$ & 0 . & 15 & 1.27 & 8 \\
\hline $\begin{array}{l}92 \\
93\end{array}$ & 48.27 & 0.11 & $\begin{array}{l}237.34 \\
258.29\end{array}$ & 0.95 & 1.32 & $\begin{array}{l}2 \\
8\end{array}$ & I. I 4 & 0.12 & 253.50 & 0.25 & 0. & 15 & 1.27 & 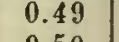 \\
\hline 94 & 48.38 & 0.11 & 259.23 & .94 & $\begin{array}{l}1.30 \\
1.29\end{array}$ & $\begin{array}{l}238.48 \\
237.33\end{array}$ & I. I 5 & 0.13 & 253 & 0.23 & 0.51 & 15 & 1.27 & 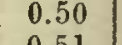 \\
\hline 95 & 48.49 & O. I I I & 260.16 & 0.93 & 1.29 & $\begin{array}{l}237.33 \\
236.16\end{array}$ & 1.17 & & 53 & 0.23 & 0.50 & [15] & 1.28 & \\
\hline 96 & $48.59^{-}$ & -0.10 & $261.08^{+}$ & -0.92 & 1.28 & $\begin{array}{l}236.16 \\
234.98\end{array}$ & -1.18 & 0.13 & 254.21 & 0.22 & 0.49 & 150 & -1.28 & .52 \\
\hline 97 & 48.70 & 0.11 & 261.99 & $0.9 \mathrm{I}$ & $\begin{array}{l}1.26 \\
1.25\end{array}$ & \begin{tabular}{|l}
234.98 \\
233.79
\end{tabular} & 1. I9 & & 254. & 0.20 & 0. & 14 & 1.28 & 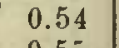 \\
\hline 98 & 48.80 & 0. & 262.88 & .89 & $\begin{array}{l}1.23 \\
1.23\end{array}$ & 232.60 & I.19 & 0.13 & 3 & 0.19 & 0. & 147.71 & I. 29 & \\
\hline 99 & 48.91 & & 263.76 & $\begin{array}{l}.88 \\
87\end{array}$ & 1.22 & 231.39 & 1.21 & & 254.82 & 0.17 & 0.46 & 14 & 1.29 & 56 \\
\hline 100 & 49.01 & & 264. & 0.87 & 1.20 & 230.18 & I. 2 I & & 254 & 0.16 & 0 & 145 & 1.29 & 7 \\
\hline 1 & & -0.10 & 265.49 & +0.86 & $\begin{array}{l}1.20 \\
1.19\end{array}$ & $228.96-$ & $-1: 22$ & 0.14 & 255.15 & 5 & 0.44 & 143.84 & 1.29 & 0.58 \\
\hline $18+2-$ & 4 & ? & 266.34 & 0.85 & 1.17 & 227.73 & 1.23 & 0.14 & 255 & 0.13 & 0 & 5 & 1.29 & 9 \\
\hline 10 & 4 & o & 267.18 & 0.84 & 1.16 & 22 & 1.23 & $\begin{array}{l}0.15 \\
0.15\end{array}$ & 255.43 & 0.12 & 0 & 6 & 1.29 & 0 \\
\hline 104 & 49.40 & $\begin{array}{l}0.09 \\
0.10\end{array}$ & 268.00 & & 1.14 & 225.25 & 1.25 & $\begin{array}{l}0.15 \\
0.16\end{array}$ & 255 & 0.1 & 0. & 13 & 1.30 & 1 \\
\hline 105 & 49.50 & & 268 & & 1.13 & 223 & & & Jor & 0.09 & & 13 & 1.30 & \\
\hline 106 & $49.59^{-}$ & & $269.61^{+}$ & +0.80 & 1.11 & 222.73 & -1.26 & $\begin{array}{l}0.16 \\
0.16\end{array}$ & $\begin{array}{l}255.75 \\
255.83\end{array}$ & +0.0 & $\begin{array}{l}0.39 \\
0.39\end{array}$ & 137.37. & 0 & 0.63 \\
\hline 107 & 49.68 & & 270.41 & $0-78$ & 1.10 & 21.46 & 1.27 & 0.17 & 255 & 0.07 & & & 1.30 & \\
\hline 108 & 49.77 & $\begin{array}{l}0.09 \\
0.8\end{array}$ & 271.19 & .78 & 1.09 & 220.18 & 1.28 & 0.17 & 255.95 & 0.05 & 0. & 7 & 1.30 & 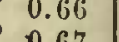 \\
\hline 109 & 49.85 & $\begin{array}{l}0.00 \\
0.09\end{array}$ & 271.96 & .77 & 1.07 & 218.88 & 1.30 & 0.18 & 255.99 & 0.04 & & & 1.50 & \\
\hline 110 & 49 & & 272.7 & & 1.06 & 217.58 & I. 3 & 0.18 & & & & 12 & 1.30 & \\
\hline & & & 273.45 & & 1.05 & 16.28 & -1.30 & 0.18 & $\begin{array}{l}256.02 \\
256.04\end{array}+$ & +0.0 & 0.35 & & -1.30 & , \\
\hline 112 & 50.11 & & 274.18 & 0.73 & 1.03 & 214.97 & I. 31 & 0.1 & & & & & I. 31 & \\
\hline 1 & 50 & & 274.90 & .72 & 1.02 & 213.65 & $1 \cdot 3^{2}$ & 0.19 & 256.03 & -0.0 & 0 & & 1.30 & \\
\hline 114 & 50.26 & $\begin{array}{l}07 \\
08\end{array}$ & 275.60 & & 1.00 & 212.33 & $3^{2}$ & 0.20 & 256.00 & 0 & $\begin{array}{l}0.3 \\
0.3\end{array}$ & 25.66 & I. & \\
\hline 115 & 50.34 & & 276.29 & & 0.99 & & 3 & & & & & & 1 & \\
\hline 116 & 50.41 & & $276.97+$ & +0.68 & 0.98 & $209.65-$ & -1.34 & $\begin{array}{l}0.20 \\
0.21\end{array}$ & $\begin{array}{l}255.96 \\
255.91\end{array}$ & -0.0 & 0.31 & 124.36 & $-1 \cdot 31$ & \\
\hline 117 & & 07 & 277.63 & 0.66 & 0.97 & 208.31 & I. 34 & 0.21 & 255. & 0.0 & 0. & & 1.30 & 0. \\
\hline 118 & 50.55 & .07 & 278.28 & 0.65 & 0.96 & 206.96 & I. 35 & 0.22 & 255. & 0.08 & 0.2 & $\begin{array}{l}75 \\
45\end{array}$ & 1.30 & 0.78 \\
\hline 119 & 50.62 & & 278.92 & 0.63 & 0.94 & 205.60 & 36 & 0.22 & 255.67 & 0.09 & $\begin{array}{l}0.28 \\
0.28\end{array}$ & $\begin{array}{l}120.45 \\
119.15\end{array}$ & 1.30 & 0.80 \\
\hline 120 & 50.69 & & 279.55 & & 0.93 & 204.23 & & 0.23 & 255.56 & & 0.27 & 117.85 & & 0.81 \\
\hline
\end{tabular}




\begin{tabular}{|c|c|c|c|c|c|c|c|c|c|c|c|}
\hline Arg. & $(\bullet . s .3)$ & $(v, c, 3)$ & $(c .8 .4)$ & (v.c.4) & $(p \cdot c .0) \mid$ & $(p .8 .1)$ & $(p, c .1)$ & $(0.8 .2) !$ & $(p . c .2)$ & $(p .8 .3)$ & (p.c.s) \\
\hline & " & " & " & $"$ & & & & & & & \\
\hline 60 & 16.26 & 9.11 & 1.80 & 0.89 & 1496 & 437 & 2222 & 583 & 127 & 137 & 71 \\
\hline 61 & 16. 98 & 9.00 & 1.79 & 0.88 & 1189 & 445 & 2233 & 580 & 125 & 137 & 70 \\
\hline 62 & 16.82 & 8.83 & 1.79 & 0.87 & 1483 & 453 & 2243 & 577 & 123 & 137 & 70 \\
\hline (j3) & 16.35 & 8.76 & 1.78 & 0.85 & 1477 & 460 & $2: 54$ & 674 & 121 & 137 & 70 \\
\hline 64 & 16.38 & 8.64 & 1.78 & 0.84 & 1470 & 408 & 2264 & 570 & 119 & 137 & 69 \\
\hline 65 & 16.41 & 8.52 & 1.78 & 0.83 & 1464 & 476 & 2275 & 667 & 117 & 136 & 69 \\
\hline 66) & 16.43 & 8.40 & 1.77 & 0.81 & 1457 & 184 & 2285 & 564 & 115 & 136 & 68 \\
\hline 67 & 16.45 & 8.28 & 1.77 & 0.80 & 1451 & 482 & 2295 & 560 & 113 & 136 & 68 \\
\hline 68 & 16.47 & 8.16 & 1.76 & 0.79 & 1444 & 501 & 2306 & 557 & 112 & 136 & 67 \\
\hline 69 & 16.49 & 8.04 & 1.75 & 0.78 & 1438 & 509 & 2316 & 553 & 110 & 136 & 67 \\
\hline 70 & 16.50 & 7.91 & 1.75 & 0.76 & 1431 & 518 & 2326 & 550 & 109 & 136 & 66 \\
\hline 71 & 16.51 & 7.79 & 1.74 & 0.75 & 1424 & 527 & 2336 & 546 & $10 \%$ & 136 & 66 \\
\hline 72 & 16.52 & 7.67 & 1.74 & 0.74 & 1417 & 536 & 2346 & 543 & 105 & 136 & 66 \\
\hline 73 & 16.53 & 7.55 & 1.73 & 0.73 & 1410 & 545 & 2356 & 540 & 103 & 135 & 65 \\
\hline it & 16.54 & 7.43 & 1.72 & 0.71 & 1403 & 554 & 2365 & 536 & 102 & 135 & 65 \\
\hline 75 & 16.54 & 7.31 & 1.72 & 0.70 & 1396 & 563 & 2375 & 533 & 100 & 135 & 64 \\
\hline 76 & 16.54 & 7.19 & 1.71 & 0.69 & 1389 & 572 & 2384 & 530 & 93 & 135 & 64 \\
\hline 77 & 16.54 & 7.07 & 1.70 & 0.68 & 1382 & 681 & 2394 & 526 & 99 & 135 & 64 \\
\hline 73 & 16.54 & 6.95 & 1.70 & 0.67 & 1374 & 530 & 2403 & 523 & 96 & 135 & 63 \\
\hline 79 & 16.53 & 6.83 & 1.69 & 0.65 & 1367 & 599 & 2413 & 519 & 95 & 135 & 63 \\
\hline so & 16.53 & 6.71 & 1.68 & 0.64 & 1360 & 608 & 2422 & 516 & 94 & 135 & 62 \\
\hline 81 & 16.52 & 6.60 & 1.67 & 0.63 & 1353 & 618 & 2431 & 512 & 93 & 135 & 62 \\
\hline 82 & 16.52 & 6.48 & 1.66 & 0.62 & 1346 & 627 & 2440 & 508 & 92 & 134 & 61 \\
\hline 83 & 16.51 & 6.36 & 1.65 & 0.61 & 1339 & 637 & $24+9$ & 505 & 91 & 134 & 61 \\
\hline 84 & 16.50 & 6.24 & 1.64 & 0.60 & 1332 & 646 & 2457 & 501 & 89 & 134 & 60 \\
\hline 85 & 16.48 & 6.13 & 1.63 & 0.59 & 1325 & 656 & 2466 & $4 ! 9$ & 88 & 134 & 60 \\
\hline 86 & 16.46 & 6.01 & 1.62 & 0.58 & $131 \mathrm{~s}$ & 664 & 2475 & 494 & 87 & 134 & 59 \\
\hline 87 & 16.41 & 5.89 & 1.61 & 0.57 & 1311 & 670 & 2483 & 490 & 86 & 134 & 59 \\
\hline 83 & 16.42 & 5.78 & 1.60 & 0.56 & 1304 & 684 & 2492 & 487 & 85 & 134 & 58 \\
\hline 89 & 16.40 & 5.66 & 1.59 & 0.55 & 1296 & 636 & 2500 & 483 & 84 & 133 & 58 \\
\hline 90 & 16.38 & 5.55 & 1.58 & 0.54 & 1289 & 706 & $250 ?$ & 480 & 84 & 133 & 57 \\
\hline 91 & 16.35 & 5.44 & 1.57 & 0.53 & 1291 & 717 & 2517 & 476 & 83 & 133 & 57 \\
\hline 93 & 16.33 & 5.32 & 1.56 & 0.52 & 1273 & 727 & 2525 & 472 & 82 & 133 & 56 \\
\hline 93 & 16.30 & 5.21 & 1.55 & 0.51 & 1266 & 738 & 2534 & 469 & 82 & 132 & 56 \\
\hline 94 & 16.27 & 5.09 & 1.54 & 0.50 & 1258 & 749 & $25+2$ & 465 & 81 & 132 & 55 \\
\hline 95 & 16.24 & 4.99 & 1.53 & 0.50 & 1250 & 760 & 2550 & 40,1 & 81 & 132 & 55 \\
\hline 96 & 16.20 & 4.87 & 1.52 & 0.49 & 1242 & 771 & 2558 & 457 & 80 & 131 & 54 \\
\hline 97 & 16.17 & 4.76 & 1.51 & 0.49 & 1234 & 782 & 2566 & 454 & 80 & 131 & 54 \\
\hline 98 & 16.13 & 4.64 & 1.50 & 0.48 & 1226 & 793 & 2574 & 450 & 80 & 131 & 53 \\
\hline 99 & 16.03 & 4.54 & 1.49 & 0.47 & 1218 & 804 & 25.92 & 446 & 73 & 130 & 53 \\
\hline 100 & 16.05 & 4.44 & 1.49 & 0.46 & 1210 & 815 & 25.20 & 442 & 79 & 130 & 52 \\
\hline 101 & 16.01 & 4.33 & 1.46 & 0.46 & 1202 & $821 ;$ & 25.99 & 438 & 79 & 130 & 51 \\
\hline 102 & 15.96 & 4.23 & 1.45 & 0.45 & 1194 & 839 & 2605 & 135 & 79 & 129 & 51 \\
\hline 103 & 15.92 & 4.13 & 1.41 & 0.44 & 1186 & 849 & 2613 & 431 & 78 & 129 & 50 \\
\hline 104 & 15.87 & 4.02 & 1.43 & 0.44 & 1178 & 861 & 2620 & 428 & 78 & 129 & 50 \\
\hline 105 & 15.83 & 3.92 & 1.42 & 0.43 & 1170 & 872 & 2627 & 424 & 79 & 128 & 49 \\
\hline 106 & 15.78 & 3.82 & 1.41 & 0.43 & 1162 & 893 & 2634 & 420 & 78 & 129 & $4 ! !$ \\
\hline 107 & 15.73 & 3.72 & 1.39 & 0.42 & 1154 & 89.5 & 2641 & 416 & 78 & 128 & 48 \\
\hline 108 & 15.68 & 3.62 & 1.39 & 0.42 & 1146 & 907 & 26,47 & 413 & 78 & 127 & 48 \\
\hline 109 & 15.62 & 3.52 & 1.37 & 0.41 & 1139 & 919 & 2654 & 409 & 78 & 127 & 17 \\
\hline 110 & 15.56 & 3.43 & 1.36 & 0.41 & 1130 & 931 & 2601 & 405 & 79 & 126 & 46 \\
\hline 111 & 15.51 & 3.34 & 1.34 & 0.40 & 1122 & 943 & 2668 & 402 & 78 & 126 & 46 \\
\hline 112 & 15.45 & 3.24 & 1.33 & 0.40 & 1114 & 955 & 2674 & 393 & $7 ?$ & 125 & 15 \\
\hline 113 & 15.39 & 3.15 & 1.32 & 0.40 & 1106 & 967 & 26,91 & 324 & $7 ?$ & 125 & 45 \\
\hline 114 & 15.33 & 3.06 & 1.31 & 0.39 & 1098 & 979 & 2687 & 391 & 79 & 124 & 44 \\
\hline 115 & 15.27 & 2.97 & 1.29 & 0.39 & 1090 & 991 & 2693 & 397 & 80 & 124 & 14 \\
\hline 110 & 15.21 & 2.88 & 1.29 & 0.39 & 1082 & 1003 & 26.99 & 393 & 80 & 123 & 44 \\
\hline 117 & 15.14 & 2.80 & 1.26 & 0.38 & 1074 & 1016 & 2705 & 390 & 80 & 122 & 43 \\
\hline 118 & 15.08 & 2.71 & 1.25 & 0.39 & 1066 & 1028 & 2711 & 376 & 81 & 122 & 13 \\
\hline 113 & 15.01 & 2.62 & 1.24 & 0.38 & 1059 & 1041 & 2716 & 373 & 81 & 121 & 42 \\
\hline 120 & 14.94 & 2.54 & 1.23 & 0.37 & 1051 & 1053 & 2722 & 370 & 82 & 120 & 42 \\
\hline
\end{tabular}




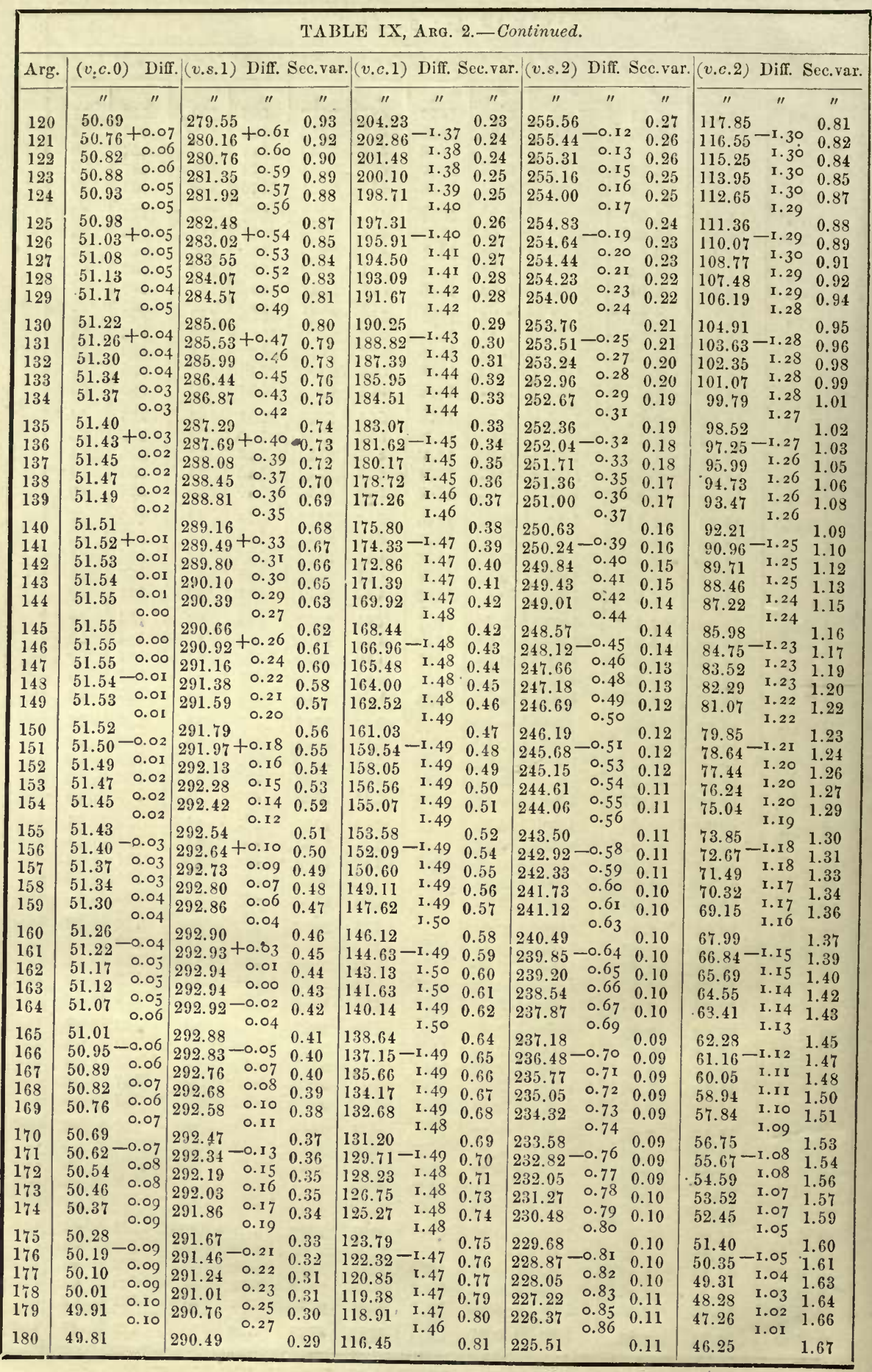




\begin{tabular}{|c|c|c|c|c|c|c|c|c|c|c|c|}
\hline Arg. & $(v .8 .3)$ & $(v . c .3)$ & $(v .8 .4)$ & $(v . c .4)$ & $(p, c .0)$ & $(p, 8,1)$ & (p.c.1) & $($ (p.8.2) & $(p, c, 2)$ & $(\rho, 8.3)$ & $(p . c .3)$ \\
\hline & " & $"$ & $"$ & " & 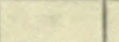 & $x$ & 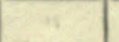 & & & & \\
\hline 120 & 14.94 & 2.54 & 1.23 & 0.37 & 1051 & 1053 & 2722 & 370 & 82 & 120 & 42 \\
\hline 121 & 14.87 & 2.46 & 1.22 & 0.37 & 1043 & 1065 & 2727 & 366 & 82 & 120 & 42 \\
\hline 122 & 14.80 & 2.38 & 1.20 & 0.37 & 1035 & 1078 & 2732 & 362 & 83 & 120 & 41 \\
\hline 123 & 14.73 & 2.30 & 1.19 & 0.37 & 1028 & 1090 & 2737 & 359 & 83 & 119 & 41 \\
\hline 124 & 14.66 & 2.22 & 1.18 & 0.37 & 1020 & 1103 & 2742 & 355 & 84 & 119 & 40 \\
\hline 125 & 14.59 & 2.15 & 1.17 & 0.37 & 1012 & 1116 & 2747 & 352 & 85 & 119 & 40 \\
\hline 126 & 14.52 & 2.07 & 1.15 & 0.37 & 1004 & 1129 & 2751 & 348 & 85 & 118 & 39 \\
\hline 127 & 14.44 & 2.00 & 1.14 & 0.37 & 996 & 1141 & 2756 & 344 & 86 & 118 & 39 \\
\hline 128 & 14.37 & 1.93 & 1.13 & 0.37 & 989 & 1154 & 2760 & 341 & 87 & 117 & 38 \\
\hline 129 & 14.29 & 1.86 & 1.11 & 0.37 & $9 \$ 1$ & 1167 & 2765 & 337 & 88 & 117 & 38 \\
\hline 130 & 14.21 & 1.79 & 1.10 & 0.37 & 973 & 1180 & 2769 & 334 & 89 & 116 & 37 \\
\hline 131 & 14.13 & 1.72 & 1.09 & 0.37 & 965 & 1193 & 2773 & 330 & 90 & 115 & 37 \\
\hline 132 & 14.06 & 1.66 & 1.07 & 0.37 & 958 & 1206 & 2777 & 327 & 91 & 115 & 36 \\
\hline 133 & 13.98 & 1.59 & 1.06 & 0.37 & 950 & 1219 & 2781 & 324 & 92 & 114 & 36 \\
\hline 134 & 13.90 & 1.53 & 1.05 & 0.38 & 942 & 1232 & 2785 & 321 & 93 & 113 & 35 \\
\hline 135 & 13.82 & 1.47 & 1.04 & 0.38 & 935 & 1245 & 2789 & 318 & 94 & 112 & 35 \\
\hline 136 & 13.74 & 1.41 & 1.02 & 0.38 & 927 & 1258 & 2793 & 314 & 95 & 112 & 35 \\
\hline 137 & 13.66 & $1.35-$ & 1.01 & 0.38 & 920 & 1271 & 2796 & 311 & 96 & 111 & 34 \\
\hline 138 & 13.57 & 1,30 & 1.00 & 0.39 & 912 & 1284 & 2800 & 308 & 97 & 110 & 34 \\
\hline 139 & 13.49 & 1.24 & 0.99 & 0.39 & 905 & 1298 & 2803 & 305 & 99 & 109 & 33 \\
\hline 140 & 13.40 & 1.19 & 0.97 & 0.39 & 897 & 1311 & 2806 & 302 & 100 & 108 & 33 \\
\hline 141 & 13.32 & 1.14 & 0.96 & 0.40 & 889 & 1325 & 2809 & 298 & 101 & 108 & 33 \\
\hline 142 & 13.24 & 1.09 & 0.95 & 0.40 & 882 & 1338 & 2811 & 295 & 103 & 107 & 32 \\
\hline 143 & 13.15 & 1.04 & 0.94 & 0.41 & 875 & 1352 & 2814 & 292 & 104 & 106 & 32 \\
\hline 144 & 13.07 & 0.99 & 0.93 & 0.41 & 867 & 1366 & 2816 & 289 & 106 & 105 & 32 \\
\hline 145 & 12.98 & 0.94 & 0.91 & 0.41 & 859 & 1379 & 2819 & 286 & 107 & 105 & 31 \\
\hline 146 & 12.90 & 0.90 & 0.90 & 0.42 & 852 & 1393 & 2821 & 282 & 109 & 104 & 31 \\
\hline 147 & 12.81 & 0.86 & 0.89 & 0.42 & 844 & 1407 & 2823 & 279 & 110 & 103 & 31 \\
\hline 148 & 12.73 & 0.82 & 0.88 & 0.43 & 837 & 1420 & 2825 & 276 & 112 & 102 & 30 \\
\hline 149 & 12.64 & 0.78 & 0.87 & 0.44 & 829 & 1433 & 2826 & 273 & 113 & 101 & 30 \\
\hline 150 & 12.55 & 0.74 & 0.86 & 0.44 & 822 & 1447 & 2828 & 270 & 115 & 100 & 30 \\
\hline 151 & 12.46 & 0.70 & 0.85 & 0.45 & 815 & 1461 & 2829 & 267 & 117 & 99 & 30 \\
\hline 152 & 12.38 & 0.67 & 0.84 & 0.46 & 807 & 1474 & 2831 & 264 & 118 & 98 & 29 \\
\hline 153 & 12.29 & 0.64 & 0.83 & 0.46 & 800 & 1488 & 2832 & 261 & 120 & 97 & 29 \\
\hline 154 & 12.20 & 0.61 & 0.82 & 0.47 & 793 & 1502 & 2834 & 258 & 122 & 96 & 29 \\
\hline 155 & 12.11 & 0.58 & 0.81 & 0.48 & 786 & 1515 & 2835 & 256 & 124 & 95 & 28 \\
\hline 156 & 12.03 & 0.55 & 0.80 & 0.49 & 778 & 1529 & 2836 & 253 & 126 & 94 & 28 \\
\hline 157 & 11.94 & 0.52 & 0.79 & 0.49 & 771 & 1543 & 2837 & 250 & 128 & 93 & 28 \\
\hline 158 & 11.86 & 0.50 & 0.78 & 0.50 & 764 & 1556 & 2837 & 247 & 129 & 92 & 28 \\
\hline 159 & 11.77 & 0.47 & 0.77 & 0.51 & 756 & 1569 & 2838 & 244 & 131 & 91 & 27 \\
\hline 160 & 11.68 & 0.45 & 0.76 & 0.52 & 749 & 1583 & 2838 & 242 & 133 & 90 & 27 \\
\hline 161 & 11.59 & 0.43 & 0.75 & 0.52 & 742 & 1597 & 2838 & 239 & 135 & 90 & 27 \\
\hline 162 & 11.51 & 0.41 & 0.74 & 0.53 & 735 & 1611 & 2838 & 236 & 137 & 89 & 27 \\
\hline 163 & 11.42 & 0.40 & 0.73 & 0.54 & 728 & 1624 & 2837 & 233 & 139 & 88 & 27 \\
\hline 164 & 11.33 & 0.38 & 0.72 & 0.55 & 721 & 1638 & 2837 & 230 & 141 & 87 & 27 \\
\hline 165 & 11.24 & 0.37 & 0.71 & 0.56 & 714 & 1652 & 2836 & 228 & 143 & 86 & 27 \\
\hline 166 & 11.16 & 0.36 & 0.71 & 0.57 & 707 & 1665 & 2835 & 225 & 145 & 85 & 27 \\
\hline 167 & 11.07 & 0.35 & 0.70 & 0.58 & 700 & 1679 & 2834 & 222 & 147 & 84 & 27 \\
\hline 168 & 10.99 & 0.34 & 0.69 & 0.59 & 693 & 1693 & 2833 & 219 & 149 & 83 & 27 \\
\hline 169 & 10.91 & 0.33 & 0.68 & 0.60 & 686 & 1706 & 2833 & 216 & 151 & 82 & 27 \\
\hline 170 & 10.82 & 0.32 & 0.68 & 0.61 & 679 & 1720 & 2831 & 214 & 153 & 82 & 27 \\
\hline 171 & 10.74 & 0.32 & 0.67 & 0.62 & 672 & 1734 & 2829 & 211 & 156 & 81 & 27 \\
\hline 172 & 10.66 & 0.31 & 0.66 & 0.63 & 665 & 1747 & 2828 & 208 & 158 & 80 & 27 \\
\hline 173 & 10.57 & 0.31 & 0.66 & 0.64 & 659 & 1761 & 2826 & 206 & 160 & 79 & 27 \\
\hline 174 & 10.49 & 0.31 & 0.65 & 0.65 & 652 & 1775 & 2824 & 204 & 162 & 78 & 28 \\
\hline 175 & 10.41 & 0.31 & 0.65 & 0.66 & 645 & 1788 & 2822 & 202 & 165 & 77 & 28 \\
\hline 176 & 10.33 & 0.31 & 0.64 & 0.67 & 638 & 1802 & 2820 & 199 & 167 & 76 & 28 \\
\hline 177 & 10.25 & 0.31 & 0.63 & 0.68 & 632 & 1815 & 2817 & 197 & 170 & 75 & 28 \\
\hline 178 & 10.17 & 0.32 & 0.63 & 0.69 & 625 & 1829 & 2815 & 195 & 173 & 74 & 28 \\
\hline 179 & 10.09 & 0.32 & 0.63 & 0.70 & 618 & 1842 & 2812 & 192 & 175 & 73 & 28 \\
\hline 180 & 10.01 & 0.33 & 0.62 & 0.71 & 611 & 1855 & 2810 & 190 & 178 & 72 & 28 \\
\hline
\end{tabular}


TABLE IX, Ara. 2.-Continued.

\begin{tabular}{|c|c|c|c|c|c|c|c|c|c|c|c|c|c|c|}
\hline$\Delta \mathrm{rg}$ & $(v . c .0)$ & Diff. & $($ (v.s.1) $]$ & Diff. &.$v \varepsilon$ & $(v . c .1)$ & Diff & r. & $\cdot(v . s .2) \mathrm{I}$ & Ditl & s & v.c.2) & 1 & ec.va \\
\hline & " & $" \prime$ & $" \prime$ & $"$ & " & " & " & $"$ & " & " & $" \prime$ & " & $" \prime$ & " \\
\hline 180 & 49.81 & & 290.49 & & 0.29 & 116.45 & & 0.81 & 225.51 & & 0.11 & 46.25 & & 1.67 \\
\hline & 49.71 & $\begin{array}{r}-0.10 \\
0.11\end{array}$ & $290.20^{-}$ & $\begin{array}{r}-0.29 \\
0.30\end{array}$ & 0.28 & & $\begin{array}{r}-1.46 \\
1.46\end{array}$ & 0.82 & 224.64 & $\begin{array}{l}-0.87 \\
0.87\end{array}$ & 0.11 & 45.24 & $\begin{array}{r}-1.01 \\
1.00\end{array}$ & 1.68 \\
\hline & 49.60 & $\begin{array}{l}0.11 \\
0.11\end{array}$ & 289.90 & $\begin{array}{l}0.30 \\
0.31\end{array}$ & 0.28 & & $\begin{array}{l}1.46 \\
1.45\end{array}$ & & 77 & $\begin{array}{l}0.87 \\
0.88\end{array}$ & 0.11 & & 0 & \\
\hline 183 & 49.49 & & 239.59 & $\begin{array}{l}0.31 \\
0.33\end{array}$ & 0.27 & & $\begin{array}{l}1.45 \\
\text { I. } 45\end{array}$ & & & & & & 9 & \\
\hline 184 & 49 & $\begin{array}{l}0.12 \\
0.12\end{array}$ & 299.26 & $\begin{array}{l}0.33 \\
0.35\end{array}$ & 0.26 & 3 & $\begin{array}{l}1.45 \\
1.45\end{array}$ & 0.86 & 221.99 & $\begin{array}{l}0.90 \\
0.91\end{array}$ & 12 & 28 & $\begin{array}{l}0.97 \\
0.97\end{array}$ & 73 \\
\hline $\begin{array}{l}185 \\
186\end{array}$ & $\begin{array}{l}49.25 \\
49.13\end{array}$ & -0.12 & 298 & $-0.3^{6}$ & 0.26 & 109.18 & .44 & 0.88 & 231.08 & .992 & 0.12 & 41.31 & & \\
\hline 187 & $\begin{array}{l}49.13 \\
49.00\end{array}$ & 0.13 & $\begin{array}{l}288.55 \\
288.17\end{array}$ & $0.3^{8}$ & & & I. 44 & & & 0.93 & & 4 & & \\
\hline 188 & 48.87 & 0.13 & 287.78 & 0.39 & $\begin{array}{l}0.24 \\
0.24\end{array}$ & & I. 43 & & & 0.94 & & & & \\
\hline 189 & 48.74 & $\begin{array}{l}\text { o. I3 } \\
0.13\end{array}$ & 287.37 & $\begin{array}{l}0.4 \mathrm{I} \\
0.42\end{array}$ & 0.23 & & $\begin{array}{l}\text { I. } 43 \\
\text { I. } 42\end{array}$ & 0.93 & & $\begin{array}{l}0.95 \\
0.96\end{array}$ & 0.13 & $\begin{array}{l}38.46 \\
37.53\end{array}$ & $\begin{array}{l}0.93 \\
0.02\end{array}$ & $\begin{array}{l}1.78 \\
1.80\end{array}$ \\
\hline 190 & 48.6 & -0.14 & 95 & & 0.22 & 102.02 & & 0.94 & 216.38 & & 0.13 & 36.61 & & 1.81 \\
\hline & & $\begin{array}{r}-0.14 \\
0.14\end{array}$ & & $\begin{array}{r}-0.44 \\
0.46\end{array}$ & $\begin{array}{ll}021 \\
0\end{array}$ & & & & & $\begin{array}{l}-0.97 \\
0.07\end{array}$ & & $70^{-}$ & & \\
\hline 1 & & $\begin{array}{l}.14 \\
0.14\end{array}$ & 5 & $\begin{array}{l}0.40 \\
0.47\end{array}$ & 0.21 & & $\begin{array}{l}1.41 \\
I .4 I\end{array}$ & & & $\begin{array}{l}0.97 \\
0.98\end{array}$ & 0 & & & \\
\hline 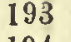 & 9 & $\begin{array}{l}0.14 \\
0.14\end{array}$ & 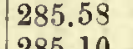 & $\begin{array}{l}0.47 \\
0.48\end{array}$ & 0.20 & 8 & $\begin{array}{l}1.41 \\
1.40\end{array}$ & 0 & & $\begin{array}{l}0.98 \\
1.00\end{array}$ & & & & \\
\hline 194 & & 0.15 & & 0.50 & 0. & & 1.40 & 10 & & $\begin{array}{l}1.00 \\
1.01\end{array}$ & 0. & 2 & $\begin{array}{l}0.89 \\
0.87\end{array}$ & \\
\hline 195 & 47.8 & & 284.60 & & 0.19 & 94.98 & & 1.01 & 211.45 & & 0.15 & 32.15 & & 1.88 \\
\hline 197 & & $\begin{array}{r}-0.15 \\
0.15\end{array}$ & & $\begin{array}{r}-0.5 \mathrm{I} \\
0.53\end{array}$ & & & & & & & & & & \\
\hline 97 & & $\begin{array}{l}0.16 \\
0.16\end{array}$ & 6 & $\begin{array}{l}0.53 \\
0.55\end{array}$ & 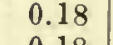 & & $\begin{array}{l}1.39 \\
\text { I. } 38\end{array}$ & & & $\begin{array}{l}1.03 \\
1.03\end{array}$ & 0 . & & & \\
\hline $\begin{array}{l}198 \\
199\end{array}$ & & 0.16 & 1 & $\begin{array}{l}.55 \\
0.56\end{array}$ & 0. & & $\begin{array}{l}1.30 \\
\text { I. } 38\end{array}$ & & & $\begin{array}{l}1.03 \\
1.04\end{array}$ & 0 & & & a \\
\hline 00 & & 0.16 & 2 & 0.57 & 0.17 & 8 & 1.37 & 1.07 & 3 & $\begin{array}{l}1.04 \\
1.05\end{array}$ & 0 & & $\begin{array}{l}0.03 \\
0.82\end{array}$ & 1.94 \\
\hline 200 & $\begin{array}{l}47.12 \\
46.95\end{array}$ & -0.17 & 981 & -0.59 & 17 & $88 .(1$ & $-I$ & 08 & $20 f_{-1}$ & -1.06 & 0.16 & 27.95 & & 6 \\
\hline 1 & & 0.17 & 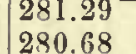 & 0.61 & & & $\begin{array}{r}-1.30 \\
\text { I. } 35\end{array}$ & & & 1.07 & 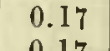 & & & \\
\hline Ju & & 0.17 & 0.06 & 0.62 & & & I. 35 & & & 1.08 & & & & \\
\hline 04 & 46.44 & $\begin{array}{l}0.17 \\
0.18\end{array}$ & 279.43 & 0.63 & 0 & 66 & I. 35 & & & 1.09 & & & & \\
\hline 05 & 46.26 & 0.8 & 278. & & 0.1 & 32 & I. 34 & 1.15 & 200.89 & 1.09 & 0.1 & & 0.77 & \\
\hline 06 & & -0.18 & & -0.65 & & 9 & -1.33 & & & -1.10 & 0.19 & 1 & & \\
\hline 7 & & 0.18 & 2 & 0.6 & 0. & & I. $3^{2}$ & & & I.II & & & & \\
\hline & & $\begin{array}{l}0.19 \\
0.19\end{array}$ & 5 & 0.6 & 0.1 & & I. & & & I. I 2 & & & & \\
\hline 09 & & $\begin{array}{l}.19 \\
0.19\end{array}$ & 04 & $\begin{array}{l}0.71 \\
0.72\end{array}$ & 0.14 & 76 & $\begin{array}{l}\text { I. } 3 \text { I } \\
\text { I. } 30\end{array}$ & 1.2 & 196.43 & I. I 3 & & & $0.7^{2}$ & \\
\hline 10 & 45.33 & & 275.32 & & 0.14 & 5 & & 1.22 & 19 & . I3 & 2 & 1.01 & 0. & \\
\hline & & & $8^{-}$ & & & & & & & -I.I 4 & & & -0. & \\
\hline & & 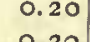 & & 0.75 & & & I & & & .15 & & & & \\
\hline & & 0.20 & & 0.77 & 0 . & & I. 2 & & & I.I 6 & & & 0. & \\
\hline 214 & 4 & $\begin{array}{l}0.20 \\
0.20\end{array}$ & 272.29 & 8 & 0.13 & 69.64 & $\begin{array}{l}\text { I. } \\
\text { I. }\end{array}$ & 1.28 & 190.69 & I. I 6 & 0.25 & & 0.1 & \\
\hline 15 & 44.34 & -0 & 271.49 & 0.8 & 0.12 & 68.37 & & .29 & 18 & & & 0 & & \\
\hline & & 0.21 & & $\begin{array}{l}-0.81 \\
0.82\end{array}$ & & & & & & 18 & & 7 & 10 & \\
\hline & & $0.2 \mathrm{I}$ & 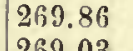 & 0.82 & 0.12 & & & & & I. I 9 & & & & \\
\hline & & $0.2 \mathrm{I}$ & & $\begin{array}{l}0.03 \\
0.85\end{array}$ & 0.12 & & I. 2 & & & I. 19 & & & & \\
\hline 19 & & 0.22 & & $\begin{array}{l}0.05 \\
0.86\end{array}$ & 0.12 & 6 & $\begin{array}{l}1.2 \\
1.2\end{array}$ & 1. & 184.76 & $\begin{array}{l}1.20 \\
1.20\end{array}$ & 0. & 14.53 & & \\
\hline 20 & 43.28 & 0.22 & 267.32 & & .11 & 62. & & 1.3 & 83 & & 0. & 1 & & \\
\hline & & $\begin{array}{l}0.22 \\
0.22\end{array}$ & $44^{-}$ & -0.88 & & & & & & $\mathbf{I}$ & & & & \\
\hline & & 0.22 & & 0.89 & & & 1.19 & & & I & & & & \\
\hline & & 0.22 & & 0.90 & 0.1 & & I. & & & I. 22 & & & 0. & \\
\hline 84 & 42.40 & 0.23 & 26 & $\begin{array}{l}0.92 \\
0.93\end{array}$ & 0.1 & 57.43 & & 1.43 & 110 & $\begin{array}{l}1.23 \\
1.23\end{array}$ & 0. & 1 & $\begin{array}{l}0.54 \\
0.52\end{array}$ & \\
\hline 25 & 1 & & 262. & .9 & 0.1 & 5 & & 1.4 & ' & & 0 & 11.21 & & \\
\hline & & 3 & & 0.95 & & & 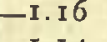 & & & 4 & & & & \\
\hline & & 3 & & 96 & & & I. & & & & & & & \\
\hline & & & & 0.97 & & & I. I 4 & & & 5 & & & 8 & \\
\hline 29 & & 4 & 2 & $\begin{array}{l}0.98 \\
1.00\end{array}$ & 0.11 & 51.69 & I. I 3 & 1.50 & 172.46 & & 0.37 & 9.25 & 0.47 & 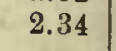 \\
\hline 30 & 41.00 & & 70 & & 0.1 & 50 & & 1 & 17 & & $0.3^{2}$ & 8.79 & & \\
\hline & & & & or & & & -I. I & & & .27 & & & & \\
\hline & & 24 & & OI & & & 1.0 & & & 1.27 & & & & \\
\hline & & .24 & & 03 & 0. & & & & & & & & 0. & \\
\hline 234 & & 5 & & 04 & 0. & 4 & I. $\mathrm{C}$ & $\pi$ & 100 & .24 & 0. & 7. & 0. & 2.39 \\
\hline 32 & & & 1 & & 0.1 & 4 & & 1.5 & 164.80 & & 0.4 & 6.70 & & 2. \\
\hline & & .25 & & & & & 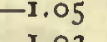 & & & & & & & \\
\hline & & & & 08 & & & 1.03 & & & 9 & & & 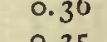 & \\
\hline & $\begin{array}{l}3 \\
3\end{array}$ & 6 & & I. IO & & & 1.03 & & & I. 30 & & & 0.3 & \\
\hline & 3851 & 0.26 & & .12 & 0. & 0 & $\begin{array}{l}1.02 \\
1.02\end{array}$ & 1.64 & 159.02 & $\begin{array}{l}1.30 \\
1.31\end{array}$ & .80 & 0.21 & $\begin{array}{l}0.34 \\
0.32\end{array}$ & \\
\hline & 38.51 & & 247.33 & & 0.12 & 39.98 & & 1.66 & 158.31 & & 0.47 & 4.95 & & 2.4 \\
\hline
\end{tabular}




\begin{tabular}{|c|c|c|c|c|c|c|c|c|c|c|c|}
\hline A rg. & $(11.8 .3)$ & $(v, c .3)$ & $(v .8 .4)$ & $(v . c .4)$ & $(p . c .0)$ & $(p .8 .1)$ & $(p . c .1)$ & $(p .8 .2)$ & $(p . c .2)$ & $($ p.8.3) & $(p \cdot c .3)$ \\
\hline & $"$ & " & " & " & & & & & & & \\
\hline 190 & 10.01 & 0.33 & 0.63 & 0.71 & 611 & 1855 & 2810 & 190 & 178 & 72 & 28 \\
\hline 181 & 9.93 & 0.34 & 0.62 & 0.72 & 604 & 1862 & 2807 & 188 & 180 & 71 & 28 \\
\hline 199 & 9.85 & 0.35 & 0.61 & 0.73 & 538 & 1853 & 2901 & 186 & 183 & 70 & 28 \\
\hline 183 & 9.78 & 0.36 & 0.61 & 0.75 & 591 & 1895 & 2801 & 184 & 185 & 63 & 28 \\
\hline 184 & 9.70 & 0.37 & 0.61 & 0.76 & 584 & 1908 & 2798 & 182 & 195 & 68 & 28 \\
\hline 195 & 9.63 & 0.38 & 0.60 & 0.77 & 577 & 1921 & 2794 & 180 & 190 & 67 & 29 \\
\hline 156 & 9.55 & 0.39 & 0.60 & 0.78 & 571 & 1935 & 2791 & 178 & 192 & 66 & 29 \\
\hline 157 & $9 .+7$ & 0.41 & 0.60 & 0.79 & 564 & 1948 & 2787 & 176 & 195 & 65 & 29 \\
\hline 188 & 9.40 & 0.42 & 0.60 & 0.80 & 557 & 1961 & 2783 & 174 & 197 & 64 & 29 \\
\hline 189 & 9.33 & 0.44 & 0.59 & 0.81 & 551 & 1974 & 2779 & 172 & 200 & 63 & 30 \\
\hline 190 & 9.26 & 0.45 & 0.59 & 0.83 & 544 & 1987 & 2775 & 171 & 202 & 62 & 30 \\
\hline 191 & 9.19 & 0.47 & 0.59 & 0.84 & 538 & 2000 & 2771 & 169 & 205 & 61 & 30 \\
\hline 192 & 8.12 & 0.50 & 0.59 & 0.85 & 531 & 2013 & 2766 & 167 & 207 & 60 & 30 \\
\hline 193 & 9.05 & 0.52 & 0.53 & 0.86 & 525 & 2025 & 2762 & 165 & 210 & 59 & 31 \\
\hline 194 & 8.93 & 0.54 & 0.53 & 0.87 & 519 & 2039 & 2757 & 163 & 212 & 58 & 31 \\
\hline 195 & 8.31 & 0.56 & 0.59 & 0.89 & 512 & 2051 & 2752 & 161 & 215 & 67 & 32 \\
\hline 196 & 8.34 & 0.59 & 0.59 & 0.90 & 506 & 2064 & 2747 & 159 & 218 & 56 & 32 \\
\hline 197 & 8.79 & 0.61 & 0.59 & 0.91 & 500 & 2076 & $27 \pm 2$ & 157 & 220 & 55 & 82 \\
\hline 198 & 8.71 & 0.64 & 0.59 & 0.92 & 494 & 2059 & 2737 & 156 & 223 & 54 & 33 \\
\hline 199 & 8.65 & 0.66 & 0.59 & 0.93 & 198 & 2101 & 2731 & 154 & 225 & 53 & 33 \\
\hline 200 & 8.59 & 0.69 & 0.59 & 0.94 & 482 & 2113 & 2726 & 152 & 228 & 52 & 34 \\
\hline 201 & 8.53 & 0.72 & 0.59 & 0.95 & 476 & 2126 & 2720 & 151 & 231 & 51 & 34 \\
\hline 203 & 3.47 & 0.75 & 0.59 & 0.97 & 470 & 2139 & 2714 & 150 & 233 & 50 & 35 \\
\hline 203 & 8.41 & 0.79 & 0.59 & 0.98 & 46,4 & 2150 & 2708 & 149 & 230 & 49 & 35 \\
\hline 204 & 8.36 & 0.81 & 0.60 & 0.29 & 453 & 2162 & 2702 & 147 & 239 & 48 & 86 \\
\hline 205 & 8.30 & 0.84 & 0.60 & 1.00 & 453 & 2174 & 2635 & 146 & 242 & 47 & 36 \\
\hline $20 G$ & 8.25 & 0.87 & 0.60 & 1.01 & 147 & 2186 & 2681 & 144 & 244 & 47 & 37 \\
\hline 207 & 8.19 & 0.90 & 0.61 & 1.02 & 441 & 2198 & 2682 & 142 & 247 & 16 & 37 \\
\hline 209 & 8.14 & 0.23 & 0.61 & 1.03 & 436 & 2210 & 2675 & 141 & 250 & 45 & 38 \\
\hline 209 & 8.08 & 0.96 & 0.61 & 1.04 & 430 & 2222 & 2668 & 140 & 253 & 14 & 30 \\
\hline 210 & 9.03 & 1.00 & 0.63 & 1.05 & 425 & 2234 & 2661 & 138 & 256 & 43 & 39 \\
\hline 211 & 7.98 & 1.03 & 0.62 & 1.06 & 419 & 2245 & 2654 & 137 & 259 & 43 & 40 \\
\hline 212 & 7.93 & 1.06 & 0.62 & 1.07 & 413 & 2257 & 2646 & 135 & 262 & 42 & 11 \\
\hline 213 & 7.89 & 1.10 & 0.63 & 1.08 & 408 & 2268 & 2633 & 134 & 265 & 41 & 42 \\
\hline 214 & 7.84 & 1.13 & 0.63 & 1.09 & 402 & 2280 & 2631 & 133 & 268 & 40 & 43 \\
\hline 215 & 7.79 & 1.17 & 0.64 & 1.10 & 397 & 2291 & 2624 & 132 & 271 & 39 & 43 \\
\hline 216 & 7.74 & 1.21 & 0.64 & 1.11 & 392 & 2302 & 2610 & 130 & 274 & 39 & 44 \\
\hline 217 & 7.70 & 1.21 & 0.65 & 1.12 & 386 & 2313 & 2608 & 129 & 277 & 38 & 14 \\
\hline 218 & 7.65 & 1.29 & 0.66 & 1.13 & 380 & 2324 & 2600 & 123 & 290 & 37 & 45 \\
\hline 219 & 7.61 & 1.32 & 0.66 & 1.14 & 375 & 2335 & 2592 & 127 & 283 & 36 & 46 \\
\hline 220 & 7.57 & 1.35 & 0.67 & 1.15 & 870 & 2346 & 2584 & 126 & 286 & 35 & 46 \\
\hline 221 & 7.53 & 1.39 & 0.68 & 1.16 & 364 & 2357 & 2575 & 125 & 250 & 35 & 47 \\
\hline 222 & 7.49 & 1.43 & 0.69 & 1.17 & 359 & 2367 & 2567 & 125 & 292 & 34 & 47 \\
\hline 223 & 7.45 & 1.46 & 0.69 & 1.18 & 354 & 2379 & 2558 & 124 & 295 & 33 & 48 \\
\hline 224 & 7.41 & 1.50 & 0.70 & 1.19 & 343 & 2389 & 2550 & 123 & 298 & 32 & 49 \\
\hline 225 & 7.38 & 1.54 & 0.71 & 1.20 & 344 & 2393 & 2541 & 122 & 301 & 32 & 50 \\
\hline 226 & 7.34 & 1.58 & 0.71 & 1.20 & 339 & 2409 & 2532 & 121 & 304 & 31 & 50 \\
\hline 227 & 7.31 & 1.62 & 0.72 & 1.21 & 334 & 2420 & 2523 & 121 & 307 & 30 & 51 \\
\hline 228 & 7.28 & 1.66 & 0.73 & 1.22 & 329 & 2430 & 2513 & 120 & 310 & 30 & 52 \\
\hline 220 & 7.25 & 1.70 & 0.74 & 1.23 & 324 & 2440 & 2504 & 110 & 313 & 29 & 53 \\
\hline 230 & 7.22 & 1.74 & 0.75 & 1.24 & 319 & 2450 & 2494 & 118 & 316 & 29 & 54 \\
\hline 231 & 7.19 & 1.78 & 0.75 & 1.24 & 314 & 2460 & 2484 & 118 & 318 & 29 & 54 \\
\hline 232 & 7.17 & 1.81 & 0.76 & 1.25 & 310 & 2470 & 2474 & 117 & 321 & 27 & 55 \\
\hline 233 & 7.14 & 1.85 & 0.77 & 1.25 & 305 & 2480 & 2464 & 116 & 324 & 27 & 56 \\
\hline 234 & 7.11 & 1.89 & 0.78 & $1.2 \mathrm{G}$ & 300 & 2489 & 2454 & 116 & 327 & 26 & 57 \\
\hline 235 & 7.09 & 1.93 & 0.79 & $1.2 T$ & 296 & 2499 & 2444 & 115 & 330 & 25 & 58 \\
\hline 236 & 7.07 & 1.97 & 0.80 & 1.27 & 291 & 2508 & 2433 & 114 & 333 & 25 & 59 \\
\hline 237 & 7.05 & 2.00 & 0.81 & 1.29 & $2 S 7$ & 2518 & 2423 & 114 & 336 & 24 & CO \\
\hline 238 & 7.03 & 2.04 & 0.83 & 1.28 & 283 & 2527 & 2412 & 113 & 339 & 23 & 61 \\
\hline 239 & 7.01 & 2.08 & 0.83 & 1.29 & 278 & 2536 & 2401 & 113 & 342 & 22 & 62 \\
\hline 240 & 6.99 & 2.18 & 0.84 & 1.30 & 274 & 2545 & 2390 & 113 & 345 & 21 & 63 \\
\hline
\end{tabular}




\begin{tabular}{|c|c|c|c|c|c|c|c|c|c|c|c|c|c|c|}
\hline Arg. & $(v, c, 0)$ & Diff. & $(v . s .1)$ & Diff. $\mathrm{S}$ & Sec.var. & $(v . c .1)$ & Diff. S & Sec.var. & $(v . s .2)$ & Diff. $S$ & Sec.var. & $(v . c .2) \mathrm{D}$ & Diff. S & ec.var. \\
\hline & " & " & $"$ & $"$ & " & " & " & $"$ & $"$ & $"$ & $"$ & " & " & "I \\
\hline 240 & 38.51 & & 247.33 & 13 & 0.12 & 39.98 & & 1.66 & 158.31 & & 0.47 & 4.95 & & 2.46 \\
\hline $2+1$ & & $\begin{array}{r}-0.26 \\
0.26\end{array}$ & $246.20^{-}$ & $\begin{array}{l}-1.13 \\
1.14\end{array}$ & 0.12 & $38.98^{-}$ & $\begin{array}{r}-1.00 \\
0.08\end{array}$ & $\begin{array}{ll}3 & 1.67\end{array}$ & $157.00^{-}$ & $-\mathrm{I} \cdot 3^{\mathrm{I}}$ & 0.48 & 4.64 & -0.31 & 2.47 \\
\hline 242 & 37.99 & 0.26 & 245.06 & $\begin{array}{l}\text { I.I } 4 \\
\text { I. } 15\end{array}$ & 0.12 & 38.00 & 0.98 & 1.69 & 155.69 & 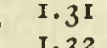 & 0.49 & 4.34 & $\begin{array}{l}0.30 \\
0.28\end{array}$ & 2.48 \\
\hline 243 & 37.73 & 0.27 & 243.91 & $\begin{array}{l}\text { 1.15 } \\
\text { I. } 16\end{array}$ & 0.13 & 37.03 & $\begin{array}{l}0.97 \\
0.96\end{array}$ & $\begin{array}{ll}7 & 1.70\end{array}$ & 154.37 & $\begin{array}{l}1.32 \\
1.32\end{array}$ & 0.50 & 4.06 & $\begin{array}{l}0.28 \\
0.27\end{array}$ & 49 \\
\hline 244 & 37.46 & 0.26 & 242.75 & 1.1 8 & 0.13 & 36.07 & 0.95 & 1.71 & 153.05 & $\begin{array}{l}1.3^{2} \\
1.3^{2}\end{array}$ & 0.51 & 3.79 & 0.25 & 2.50 \\
\hline 245 & 37.20 & & 241.57 & -1.18 & 0.13 & 35.12 & & 1.73 & 151.73 & & 0.53 & 3.54 & -0.24 & 2.51 \\
\hline 246 & 36.93 & $\begin{array}{r}-0.27 \\
0.27\end{array}$ & 240.39 & $\begin{array}{r}-1.18 \\
1.19\end{array}$ & 0.13 & $34.19^{-}$ & $\begin{array}{r}-0.93 \\
0.92\end{array}$ & $\begin{array}{ll}3 & 1.74 \\
2 & \end{array}$ & $150.40^{-}$ & $\begin{array}{r}-1.33 \\
1.33\end{array}$ & 0 & $3.30^{\circ}$ & $\begin{array}{r}-0.24 \\
0.23\end{array}$ & \\
\hline 247 & 36.66 & $\begin{array}{l}0.27 \\
0.27\end{array}$ & 239.20 & $\begin{array}{l}1.19 \\
1.21\end{array}$ & 0.13 & 33.27 & & 1.75 & 149.07 & $\begin{array}{l}1.33 \\
1.33\end{array}$ & 0 . & 07 & & \\
\hline 248 & 36.39 & 0.27 & 237.99 & 1.22 & 0.14 & 32.36 & $\begin{array}{l}0.91 \\
0.90\end{array}$ & 1.76 & 147.74 & 1. & 0.56 & 2.86 & & 2 \\
\hline 249 & 36 & 0.27 & 23 & I. 23 & 0.14 & 31.46 & 0.89 & 1.78 & 41 & $\begin{array}{l}1.33 \\
1.34\end{array}$ & 0.57 & 2.66 & $\begin{array}{l}0.20 \\
0.18\end{array}$ & \\
\hline 250 & 35.85 & -0.27 & 235.54 & -1.24 & 0.14 & & -0.87 & 1.79 & 145.07 & -1.34 & 0.58 & 2.48 & & 2.56 \\
\hline $\begin{array}{l}251 \\
252\end{array}$ & & 0.27 & $\begin{array}{l}0 \\
5\end{array}$ & 1.25 & 0 & & 0.86 & 1.80 & 143.73 & $\begin{array}{r}34 \\
1.34\end{array}$ & 59 & 2.31 & 0.15 & 7 \\
\hline $\begin{array}{l}202 \\
253\end{array}$ & 3 & 0.28 & $\begin{array}{l}233.05 \\
231.80\end{array}$ & 1.25 & 0.15 & $\begin{array}{l}4 \\
9\end{array}$ & 0.85 & 51. & $14 \%$ & $\begin{array}{l}1.34 \\
\end{array}$ & $\begin{array}{l}0 \\
+\end{array}$ & 6 & 0.14 & 3 \\
\hline 254 & 34.75 & $\begin{array}{l}0.28 \\
0.28\end{array}$ & 230.54 & 1.26 & 0.16 & $\begin{array}{l}27.99 \\
27.15\end{array}$ & 0.84 & $\begin{array}{ll}4 & 1.83 \\
4 & 1.85\end{array}$ & 141. & $x \cdot 34$ & $\begin{array}{r}+\quad 0.61 \\
+\quad 062\end{array}$ & 2.02 & o. 13 & 9 \\
\hline 255 & 34.47 & 28 & 200.07 & I. 28 & 0.16 & 26.33 & 0.82 & 1.85 & 139. & I. 35 & 0.62 & 1. & 0.11 & 30 \\
\hline 256 & & -0.28 & $\begin{array}{l}229.26 \\
227.98\end{array}$ & -1.28 & $\begin{array}{l}0.16 \\
0.16\end{array}$ & $\begin{array}{l}26.35 \\
25.52\end{array}$ & $-0.8 \mathrm{I}$ & $\begin{array}{l}1.86 \\
1.88\end{array}$ & 138.36 & -1.35 & 0.64 & 1.78 & & .61 \\
\hline 257 & 1 & 0.28 & 226.68 & I. 30 & 0.17 & 24.73 & 0.79 & $\begin{array}{l}1.88 \\
1.89\end{array}$ & 135 & 1. 35 & & & 0.08 & \\
\hline 258 & 33.63 & 0.28 & 225.37 & I. 3 I & 0.17 & 23.95 & 0.78 & $\begin{array}{ll}3.01 \\
1.91\end{array}$ & 134.31 & I. 35 & & & 0.07 & \\
\hline 259 & 33.34 & $\begin{array}{l}0.29 \\
0.28\end{array}$ & 224.06 & I. 3 I & 0.18 & 23.18 & 0.77 & 1.92 & 132.96 & I. 35 & $\begin{array}{l}0.67 \\
0.68\end{array}$ & $\begin{array}{l}1.53 \\
1.47\end{array}$ & 0.06 & \\
\hline 260 & 33.06 & & 222.74 & & 0.18 & 22.43 & & 1.94 & 131.61 & I. 35 & 8.00 & 1.46 & 0.04 & \\
\hline 261 & $32.78^{-}$ & -0.28 & 221.41 & -1.33 & 0.18 & $21.69^{\circ}$ & -0.74 & $\begin{array}{ll}7 & 1.95\end{array}$ & 13 & $-\mathrm{I} \cdot 35$ & & 1.43 & $-0 . c \quad-1$ & 5 \\
\hline 262 & 32.49 & 0.29 & 220.07 & I. 34 & 0.19 & 20.97 & 0.72 & 1.97 & 128.90 & 1.36 & 0. & & & \\
\hline 263 & 32.21 & 0.28 & 218.72 & 1. 35 & 0.19 & 20.26 & 0.71 & 1.98 & 127 & I. 35 & 0.73 & & +0. & \\
\hline 264 & 31.92 & $\begin{array}{l}0.29 \\
0.29\end{array}$ & 217.36 & $\begin{array}{l}1.3^{6} \\
1.37\end{array}$ & 0.20 & 19.56 & $\begin{array}{l}0.70 \\
0.68\end{array}$ & 1.99 & 126.20 & $\begin{array}{l}\text { I. } 35 \\
1.35\end{array}$ & 0.74 & $\begin{array}{l}1.40 \\
1.42\end{array}$ & 0. & \\
\hline 265 & 31.63 & & 215.99 & & 0.20 & 18.88 & 67 & 2.01 & 124.85 & & 0.75 & 1.45 & & 60 \\
\hline 266 & $31.34^{-}$ & -0.29 & 214.62 & -1.37 & 0.21 & $18.21^{-}$ & -0.67 & 2.02 & $123.50^{-}$ & -1.35 & 0 . & $1.50^{+}$ & +0.05 & 0 \\
\hline 267 & 31.05 & 0.29 & 213.24 & I. $3^{8}$ & 0.21 & 17.56 & $\begin{array}{l}0.65 \\
0.64\end{array}$ & 2.03 & 122. & 1.36 & 0 & 1.56 & 0.06 & 2.71 \\
\hline 268 & 6 & 0.29 & 211. & I. 39 & 0.22 & 16.92 & 0.0 & 2. & 12 & 1. & & 3 & 0.07 & 2.71 \\
\hline 269 & 30. & $\begin{array}{l}0.29 \\
0.29\end{array}$ & 210 & $\begin{array}{l}1.40 \\
1.40\end{array}$ & 0.22 & 16.29 & $\begin{array}{l}0.03 \\
0.61\end{array}$ & 2.06 & 119.44 & $\begin{array}{l}1.35 \\
1.35\end{array}$ & 0.80 & 1.72 & $\begin{array}{l}0.09 \\
0.10\end{array}$ & 2.72 \\
\hline 270 & 30.18 & -0.30 & 209.05 & & 0.23 & 15.68 & -0.60 & 2.07 & 118.09 & -1.35 & 0.81 & $1.82+$ & +0.12 & 2.73 \\
\hline 271 & & $\begin{array}{l}-.30 \\
0.29\end{array}$ & 207.64 & $\begin{array}{r}-1.41 \\
1.42\end{array}$ & 0.24 & 15 & $\begin{array}{l}-0.00 \\
0.5^{8}\end{array}$ & 2.08 & 116 & $\begin{array}{r}-1.35 \\
1.35\end{array}$ & 0 . & $4^{t}$ & 0.13 & \\
\hline 2 & 29.59 & 0.30 & 206.22 & $\begin{array}{l}1.42 \\
1.43\end{array}$ & 0.24 & 14.50 & $\begin{array}{l}0.50 \\
0.56\end{array}$ & 2.10 & 115 & $\begin{array}{r}1.35 \\
1.35\end{array}$ & 0. & 2.07 & $\begin{array}{l}0.13 \\
0.14\end{array}$ & \\
\hline 2 & 29.29 & 0.29 & 204 & $\begin{array}{l}1.43 \\
1.43\end{array}$ & 0.2 & 13.94 & 0.55 & 2.11 & 114.04 & $\begin{array}{l}1.35 \\
\text { I. } 34\end{array}$ & 0.85 & 2. & $\begin{array}{l}0.14 \\
0.16\end{array}$ & \\
\hline 274 & 29.00 & 0.2 & 203 & 1.43 & 0.2 & 13. & $\begin{array}{l}.55 \\
0.54\end{array}$ & 2.13 & 112. & $\begin{array}{l}1.34 \\
1.35\end{array}$ & 0.87 & 37 & 0.18 & 2.75 \\
\hline 275 & 29.71 & -0.30 & 201.92 & -1.4 & 0.26 & 12.85 & -0 & 2.14 & 111.35 & -1. & 0.88 & $2.55+$ & +0.19 & 2.76 \\
\hline 276 & $\begin{array}{l}23.41 \\
28.12\end{array}$ & 0.29 & 200 & $\begin{array}{l}1.44 \\
1.45\end{array}$ & & 1 & 0.50 & 2. & & $\begin{array}{r}-1.34 \\
1.34\end{array}$ & 0 . & $2.74^{+}$ & $\begin{array}{r}+0.19 \\
0.21\end{array}$ & 2 \\
\hline 277 & $\begin{array}{l}2 \\
2\end{array}$ & 0.29 & 197. & I. 46 & 098 & 11 & 0.49 & 2. & 108.67 & $\begin{array}{l}1.34 \\
1.34\end{array}$ & 0 & 2.95 & 0.22 & 2.77 \\
\hline 278 & 2 & 0.29 & 96.1 & 1.46 & $0.2>>$ & 11.34 & $\begin{array}{l}0.49 \\
0.48\end{array}$ & 2. & 10 & $\begin{array}{l}.34 \\
1.34\end{array}$ & 0 . & 3.17 & 0.23 & 2.78 \\
\hline 279 & & 0.29 & & 1.47 & & 10 & 0.46 & 2.20 & 105 & $\begin{array}{l}1.33 \\
\text { I }\end{array}$ & 0.94 & $?$ & 0.25 & 8 \\
\hline 280 & $\begin{array}{l}27.2 \\
26.9\end{array}$ & -0.30 & $\begin{array}{l}194.64 \\
193.17\end{array}$ & -1.47 & 0.29 & 10.40 & -0.45 & 2.21 & 104.66 & -1.33 & 0.95 & $3.65+$ & +0.26 & 2.79 \\
\hline & $\begin{array}{l}2 \\
2\end{array}$ & 0.29 & $\begin{array}{l}93.17 \\
91.69\end{array}$ & $\begin{array}{r}-1.4 ! \\
1.48\end{array}$ & 0 & $\begin{array}{l}9.95 \\
952\end{array}$ & 0.43 & $?$ & 3 & $\begin{array}{r}.33 \\
1.33\end{array}$ & & 3. & 0.28 & \\
\hline 283 & 36 & 0.30 & $\begin{array}{l}91.09 \\
90.20\end{array}$ & 1.49 & & 9. & 0.42 & 2. & 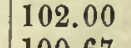 & 1. 33 & 0 & 9 & 0. & \\
\hline 284 & 26.07 & $\begin{array}{l}0.29 \\
0.29\end{array}$ & 188.71 & I. 49 & 0 & 9. & 0.40 & 2. & 10 & 1.3 & 0. & 4 & 0.3 & \\
\hline 285 & 25.78 & 0.29 & 187.22 & r. 49 & & & 0.39 & 0 & & I. 3 & 1.0 & 4.78 & $0.3^{2}$ & 2.81 \\
\hline & & 30 & $185.72-$ & -1.50 & 0.34 & 8.31 & & 2.27 & 98.02 & & 1.02 & 5.10 & & 2.81 \\
\hline 2 & 19 & .29 & 184.21 & 1.51 & & & $\begin{array}{l}-0.37 \\
0.35\end{array}$ & 2.2 & & & & & & \\
\hline 288 & 89 & 0.30 & 182. & 1.5 & $\begin{array}{l}0 \\
0\end{array}$ & & $\begin{array}{l}0.35 \\
0.34\end{array}$ & 2. & 95 & & & & 0.3 & \\
\hline 289 & 24.60 & 0.29 & 181.19 & 1.51 & & 6 & $0.3^{2}$ & 2. & 94 & $I_{2} 2+3$ & 1. & & $0.3^{6}$ & \\
\hline 290 & 24.31 & & 179.67 & 52 & & 6.9 & 0.30 & ? & ? & $\begin{array}{l}\text { I.3I } \\
1.30\end{array}$ & 1.08 & & $\begin{array}{l}0.37 \\
0.39\end{array}$ & 2 . \\
\hline & & 0.29 & 178. & $-1.5^{2}$ & $\begin{array}{l}0.38 \\
0.39\end{array}$ & 6.63 & & 2.32 & 91.47 & & 1.09 & 6.90 & & 2.84 \\
\hline & & 29 & 1 & $1.5^{2}$ & & & & 2 & & & 1.10 & $7.30^{+}$ & & 2. \\
\hline 2 & 2 & & 175. & 1.53 & $\begin{array}{l}0.4 \\
0.4\end{array}$ & 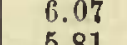 & 0.26 & 2.3 & & 1.30 & 1.12 & & & 2. \\
\hline 294 & 23.15 & & 173.57 & I. 53 & $\begin{array}{l}0.41 \\
0.42\end{array}$ & . & 0.24 & 2.3 & & $\begin{array}{l}1.29 \\
1.20\end{array}$ & 1.13 & 8.15 & 0.43 & 2.85 \\
\hline 295 & 22.86 & & & $5=$ & & & 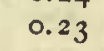 & 98 & $86-2>$ & 1.28 & 15 & & $\begin{array}{l}3.44 \\
0.46\end{array}$ & \\
\hline & 22.57 & & $173.50^{1}$ & -1.54 & $\begin{array}{l}0.43 \\
0.43\end{array}$ & $\begin{array}{l}5.34 \\
5.13\end{array}$ & -0.21 & $\begin{array}{l}2.38 \\
0.30\end{array}$ & 85.01 & & 1.16 & 9.05 & & 2.86 \\
\hline 2 & 22.29 & 28 & 168.96 & I. 54 & 0. & 4.9 & 0.20 & & & & 1.7 & $9.52^{-}$ & & 2.8 \\
\hline 2 & 22.00 & 29 & 167.42 & I. 54 & 0. & 4.75 & 0.18 & & & 1.27 & 1.1 & & $y$ & 2.8 \\
\hline 299 & $21.7 \mathrm{I}$ & & 165.88 & $7+2+3$ & 0.16 & 4.59 & 0.16 & & & 1.27 & & & 0.51 & 2.87 \\
\hline 300 & 21.42 & & 164. & & 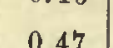 & 11 & 0.15 & 2.4 & 19. & 1. 26 & 1.22 & to. & 0.53 & 2.88 \\
\hline & & & & & & & & 2.44 & 78.67 & & 1.23 & 11.55 & & 2.88 \\
\hline
\end{tabular}


TII ORBIT OF URANUS.

\begin{tabular}{|c|c|c|c|c|c|c|c|c|c|c|c|}
\hline Arg. & $(v .8,3)$ & $(v . c .3)$ & $(v .8 .1)$ & $(v . c .4)$ & $(p . c .0)$ & (p.6.I) & (p.c.1) & (p.8.2) & (p.c.2) & $(p .8 .3)$ & $(p, c .3)$ \\
\hline & $"$ & $"$ & $"$ & $"$ & & & & & & & \\
\hline 240 & 6.99 & 2.13 & 0.84 & 1.30 & 274 & 2545 & 2390 & 113 & 345 & 21 & 63 \\
\hline 241 & 6.97 & 2.16 & 0.85 & 1.81 & 270 & 2554 & 2379 & 113 & 348 & 21 & 64 \\
\hline 243 & 6.96 & 2.20 & 0.86 & 1.31 & 265 & 2562 & 2368 & 113 & 350 & 20 & 65 \\
\hline 243 & 6.94 & 2.24 & 0.87 & 1.31 & 261 & 2571 & 2357 & 112 & 353 & 19 & 66 \\
\hline 244 & 6.92 & 2.23 & 0.88 & 1.31 & 257 & 2579 & 2346 & 112 & 856 & 19 & 67 \\
\hline 245 & 6.90 & 2.31 & 0.90 & 1.32 & 253 & 2587 & 2384 & 112 & 859 & 18 & 68 \\
\hline 216 & 6.88 & 2.35 & 0.91 & 1.32 & 249 & 2595 & 2322 & 112 & 862 & 18 & 69 \\
\hline 247 & 6.87 & 2.38 & 0.32 & 1.32 & 245 & 2603 & 2311 & 112 & 365 & 17 & 70 \\
\hline 218 & 6.85 & 2.42 & 0.93 & 1.32 & 243 & 2611 & 2299 & 112 & 368 & 16 & 72 \\
\hline 243 & 6.84 & 2.45 & 0.94 & 1.33 & 238 & 2618 & 2287 & 111 & 371 & 16 & 73 \\
\hline 250 & 6.83 & 2.48 & 0.95 & 1.33 & 234 & 2626 & 2275 & 111 & 374 & 15 & 74 \\
\hline 251 & 6.82 & 2.52 & 0.96 & 1.33 & 230 & 2633 & 2263 & 111 & 377 & 15 & 75 \\
\hline 252 & 6.82 & 2.55 & 0.97 & 1.33 & 227 & 2641 & 2251 & 111 & 380 & 14 & 76 \\
\hline 253 & 6.81 & 2.58 & 0.98 & 1.33 & 223 & 2648 & 2239 & 111 & 383 & 14 & 77 \\
\hline 254 & 6.80 & 2.61 & 0.99 & 1.34 & 219 & 2656 & 2226 & 111 & 386 & 13 & 78 \\
\hline 255 & 6.79 & 2.64 & 1.00 & 1.34 & 215 & 2663 & 2214 & 111 & 389 & 13 & 79 \\
\hline 256 & 6.79 & 2.67 & 1.02 & 1.34 & 212 & 2670 & 2201 & 111 & 392 & 13 & 80 \\
\hline 2.57 & 6.78 & 2.70 & 1.03 & 134 & 208 & 2677 & 2189 & 112 & 395 & 12 & 82 \\
\hline 258 & 6.78 & 2.73 & 1.04 & 1.34 & 204 & 2684 & 2176 & 112 & 393 & 12 & 83 \\
\hline 259 & 6.77 & 2.76 & 1.05 & 1.34 & 201 & 2691 & 2164 & 112 & 401 & 12 & 84 \\
\hline 260 & 6.77 & 2.79 & 1.06 & 1.34 & 198 & 2698 & 2151 & 112 & 404 & 11 & 85 \\
\hline 261 & 6.77 & 2.82 & 1.07 & 1.34 & 195 & 2704 & 2138 & 112 & 407 & 11 & 86 \\
\hline 262 & 0.77 & 2.85 & 1.09 & 1.33 & 192 & 2711 & 2125 & 113 & 410 & 11 & 87 \\
\hline 263 & 6.77 & 2.88 & 1.10 & 1.33 & 189 & 2717 & 2112 & 113 & 413 & 11 & 89 \\
\hline 261 & 6.77 & 2.90 & 1.11 & 1.33 & 186 & $2\lceil 23$ & 2098 & 113 & 416 & 11 & 90 \\
\hline 265 & 6.75 & 2.93 & 1.12 & 1.33 & 183 & 2729 & 2085 & 114 & 419 & 11 & 91 \\
\hline 266 & 6.77 & 2.95 & 1.13 & 1.32 & 180 & 2735 & 2072 & 114 & 422 & 11 & 92 \\
\hline 267 & 6.77 & 2.98 & 1.14 & 1.32 & 177 & 2740 & 2058 & 115 & 425 & 11 & 93 \\
\hline 269 & 6.77 & 3.00 & 1.15 & 1.32 & 174 & $27 \$ 5$ & 2045 & 115 & 427 & 11 & 94 \\
\hline 269 & 6.77 & 3.03 & 1.16 & 1.32 & 172 & 2751 & 2031 & 116 & 430 & 11 & 96 \\
\hline 270 & 6.77 & 3.05 & 1.17 & 1.31 & 169 & 2756 & 2018 & 116 & 433 & 11 & 97 \\
\hline 271 & 6.77 & 3.07 & 1.18 & 1.31 & 166 & 2761 & 2004 & 117 & 436 & 11 & 98 \\
\hline 272 & 6.71 & 3.09 & 1.20 & 1.31 & 164 & 2766 & 1990 & 117 & 439 & 10 & 99 \\
\hline 273 & 6.79 & 3.11 & 1.21 & 1.30 & $16 L$ & 2711 & 1977 & 118 & 442 & 10 & 100 \\
\hline $27 t$ & 6.78 & 3.13 & 1.22 & 1.30 & 159 & 2776 & 1963 & 119 & 444 & 10 & 102 \\
\hline 275 & 6.78 & 3.15 & 1.23 & 1.29 & $15 i$ & 2781 & 1949 & 119 & 447 & 10 & 103 \\
\hline 276 & 6.79 & 3.17 & 1.24 & 1.28 & 154 & 2785 & 1935 & 120 & 450 & 10 & 104 \\
\hline 277 & 6.79 & 3.19 & 1.25 & 1.28 & 151 & 2790 & 1921 & 121 & 452 & 10 & 105 \\
\hline 278 & 6.80 & 3.20 & 1.26 & 1.27 & 149 & 2794 & 1907 & 121 & 455 & 10 & 106 \\
\hline 279 & 6.81 & 3.22 & 1.27 & 1.27 & 146 & 2798 & 1892 & 122 & 457 & 10 & 107 \\
\hline 280 & 6.81 & 3.23 & 1.29 & 1.26 & 144 & 2802 & 1878 & 123 & 460 & 10 & 108 \\
\hline 281 & 6.82 & 3.25 & 1.28 & 1.25 & 142 & 2806 & 1864 & 124 & 463 & 10 & 110 \\
\hline 282 & 6.83 & 3.26 & 1.29 & 1.25 & 139 & 2809 & 1849 & 124 & $\$ 65$ & 10 & 111 \\
\hline 283 & 6.83 & 3.27 & 1.30 & 1.24 & 137 & 2813 & 1835 & 125 & 468 & 10 & 112 \\
\hline 284 & 6.81 & 3.28 & 1.31 & 1.23 & 135 & 2816 & 1820 & 126 & 471 & 11 & 113 \\
\hline 285 & 6.84 & 3.29 & 1.33 & 1.22 & 133 & 2819 & 1805 & 127 & 474 & 11 & 115 \\
\hline 286 & 6.85 & 3.30 & 1.33 & 1.22 & 131 & 2822 & 1791 & 127 & 176 & 11 & 116 \\
\hline 287 & 6.85 & 3.31 & 1.34 & 1.21 & 129 & 2825 & 1776 & 129 & 179 & 11 & 117 \\
\hline 288 & 6.86 & 3.33 & 1.34 & 1.20 & 127 & 2828 & 1761 & 129 & 482 & 11 & 118 \\
\hline 289 & 6.86 & 3.32 & 1.35 & 1.19 & 126 & 2830 & 1746 & 130 & 184 & 12 & 120 \\
\hline 290 & 6.97 & 3.33 & 1.36 & 1.18 & 124 & 2833 & 1731 & 131 & 487 & 12 & 121 \\
\hline 291 & 6.88 & 3.33 & 1.37 & 1.17 & 123 & 2835 & 1716 & 132 & 490 & 12 & 122 \\
\hline 292 & 6.88 & 3.34 & 1.38 & 1.16 & 121 & 2837 & 1702 & 133 & 492 & 12 & 123 \\
\hline 293 & 6.99 & 3.34 & 1.38 & 1.15 & 119 & 2838 & 1686 & 134 & 495 & 13 & 125 \\
\hline 294 & 6.89 & 3.35 & 1.39 & 1.14 & 118 & 2840 & 1671 & 136 & 497 & 13 & 126 \\
\hline 295 & 6.90 & 3.35 & 1.40 & 1.13 & 116 & 2841 & 1656 & 137 & 500 & 13 & 127 \\
\hline 296 & 6.90 & 3.35 & 1.40 & 1.12 & 115 & 2943 & 1641 & 138 & 503 & 13 & 128 \\
\hline 297 & 6.90 & 3.35 & 1.41 & 1.11 & 114 & 2944 & 1626 & 139 & 505 & 14 & 130 \\
\hline 298 & 6.91 & 3.35 & 1.42 & 1.10 & 112 & $29+5$ & 1611 & 141 & 508 & 14 & 131 \\
\hline 299 & 6.91 & 3.35 & 1.42 & 1.09 & 111 & 2847 & 1596 & 142 & 510 & 14 & 132 \\
\hline 300 & 6.92 & 3.35 & 1.13 & 1.09 & 110 & 2849 & 1581 & 143 & 512 & 15 & 133 \\
\hline
\end{tabular}


TABLE IX, ARo. 2.-Continued.

\begin{tabular}{|c|c|c|c|c|c|c|c|c|c|c|c|c|c|c|}
\hline Arg. & $. c .0)$ & Diff. & v.s.1) & If. $\mathrm{s}$ & . & c.1) & -1 & C.va & $.8 .2)$ & & & .2 & & \\
\hline & " & $"$ & $"$ & " & " & " & $"$ & " & " & & $"$ & $"$ & $"$ & $"$ \\
\hline 300 & 21.42 & & 164 & & 7 & 4.44 & & 2.44 & 78.67 & & 1.23 & 11.55 & & 2.88 \\
\hline & & & 2.78 & & & & - & & & & & & & \\
\hline 2 & & & 1.23 & I. & & & 0.12 & & & $\begin{array}{l}1.25 \\
\times\end{array}$ & & & & 88 \\
\hline & & 8 & 9.68 & I. & 0 & & 0. & & & 4 & & & & 89 \\
\hline 304 & $\theta$ & $\begin{array}{l}0.28 \\
0.28\end{array}$ & & $\begin{array}{l}1.50 \\
1.55\end{array}$ & & & & & & $\begin{array}{l}1.23 \\
1.23\end{array}$ & & & $\begin{array}{l}0.5^{8} \\
0.59\end{array}$ & 89 \\
\hline 05 & 20.01 & -0.28 & 156.57 & & .53 & .94 & & & & & & & 0 & \\
\hline & & 0.28 & & I. 55 & 0.55 & & 0.03 & & & 2 & & & & \\
\hline $\begin{array}{l}07 \\
08\end{array}$ & & 0.28 & $\begin{array}{l}6 \\
0\end{array}$ & 1.56 & 0.56 & 3.85 & 0. & & 8.82 & I. 2 I & & & & \\
\hline 309 & 18.90 & 0.27 & 150.34 & 1.56 & 0.57 & 3.84 & to. & 2. & 67.62 & o & 1.3 & & & \\
\hline 310 & 18.63 & & 9 & & 5 & 3.85 & & 2.54 & 66.42 & & 1.37 & 17.54 & & 90 \\
\hline 11 & & & & $\begin{array}{r}-1.50 \\
1.56\end{array}$ & & & & & & & & & & 90 \\
\hline 312 & & 0. & & 1.55 & & & 0. & & & & & & & 90 \\
\hline 314 & & $\begin{array}{l}0.27 \\
0.27\end{array}$ & & $\begin{array}{l}1.55 \\
1.56\end{array}$ & & & 0. & & & & & & & \\
\hline 314 & 17 & 0.27 & 6 & 1.56 & & 6 & 0.10 & & & I. 16 & & & $\begin{array}{l}0.71 \\
0.72\end{array}$ & 90 \\
\hline 315 & 17.28 & & 41.00 & & 0.64 & 4.16 & & .59 & 60.54 & & 1.45 & 1.03 & & .91 \\
\hline 317 & & -0.26 & & -1.50 & & & & & & & & & & 91 \\
\hline 317 & & 0.27 & & & & & 0.13 & & & & & & & 91 \\
\hline 319 & & 6 & & 1. & & & 6 & & & & & & & 1 \\
\hline 319 & & o. & & $\begin{array}{l}1.50 \\
1.55\end{array}$ & & 4. & 0.18 & 2 & 7 & 3 & 1.51 & 04 & & 91 \\
\hline 320 & & -0. & 1 & & & 9 & & 8 & 5 & & 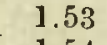 & & & 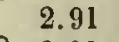 \\
\hline & & o. & & & & & $0.2 \mathrm{I}$ & & & & & & & 1 \\
\hline & & 0.25 & & 1 & & & 0.22 & & & & & & & 1 \\
\hline 324 & & 0.25 & & $\begin{array}{l}1.53 \\
1.55\end{array}$ & & & 0.24 & & & 1.09 & & & & \\
\hline 25 & & & & 54 & & & & & & I. & & & o. 85 & \\
\hline 20 & & & & $-I$ & & & 27 & & & 7 & & & & 90 \\
\hline 27 & & 0 & & 1.5 & & & 0.29 & & & 1.07 & & & & \\
\hline 28 & & 0. & & 1.5 & & & 0. & & & I. & & & & \\
\hline 329 & & $\begin{array}{l}0 \\
0\end{array}$ & 119 & 1. & 0.8 & 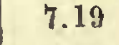 & & 2. & & 1.04 & & & o. & 9 \\
\hline 30 & 51 & & 117.82 & & 0.81 & 7.52 & & 2.71 & 44 & & 1.67 & 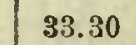 & & .89 \\
\hline & & & & & & & 0. & & & 3 & & & +o & \\
\hline 32 & & 0 & & 1. & & & & & & & & & o & \\
\hline 333 & & 0.23 & & 1.5 & & & & & & & & & o & \\
\hline 334 & & 0.23 & 111 & $\begin{array}{l}1.5^{2} \\
1.51\end{array}$ & & 9.02 & & & & & 1.73 & & & 5 \\
\hline 35 & 1 & -0.2 & - & & 0.8 & & +0. & & 39. & & 1.74 & & & 88 \\
\hline 336 & & 0.22 & & $\begin{array}{r}-1.51 \\
1.50\end{array}$ & & & & & & & & & & \\
\hline 337 & & 0.22 & & $\begin{array}{l}1.50 \\
1.49\end{array}$ & & & & & & & & & & \\
\hline 338 & & 0.22 & & 1. 49 & & & 0 . & & & & & & & \\
\hline 339 & & 0.21 & & $\begin{array}{l}1.49 \\
1.49\end{array}$ & & & 0. & & & & 1.80 & 41.89 & & 2.87 \\
\hline 340 & 6 & & 1 & & 0.94 & & & 2.78 & & & & & & 37 \\
\hline 11 & & & & & & & & & & & & 2 & - & 37 \\
\hline 342 & & & & & & & & & & & & & & \\
\hline 343 & & 0. & & $\begin{array}{l}1.47 \\
1.47\end{array}$ & & & 0 . & & & & & & & \\
\hline 344 & & & & 1.46 & 1.00 & 1 & 0. & 2.80 & & 8 & 1.87 & & $\begin{array}{l}\text { I.04 } \\
\text { I.06 }\end{array}$ & .86 \\
\hline 345 & 1 & & 9 & & 1 & 14.46 & & 2.8 & 29.80 & & 188 & & & 85 \\
\hline 16 & & & & & & & & & & & & & & \\
\hline 21 & & & & & & & & & & & & & & \\
\hline if & & & & & & & & & & 0. & & & & \\
\hline 349 & & & & $\begin{array}{l}1.43 \\
1.42\end{array}$ & 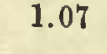 & & & & & $\begin{array}{l}0.04 \\
0.82\end{array}$ & & & & .8 \\
\hline 5 & 9 & & & & & & & 8 & 25.57 & - a. & & 5 & & 84 \\
\hline & & 0. & & I. & & & 0. & & & & & & & \\
\hline & & 0.18 & & 1.40 & & & $\begin{array}{l}0.00 \\
0.60\end{array}$ & & & & & & & \\
\hline & & 0.1 & & 1.40 & & & & & & & & & & \\
\hline 35 & & & & 1.39 & & & & & & & & & 1. & 3 \\
\hline 355 & 8.40 & & 81.19 & & 1.15 & & & 2 & 21 & & 2.0 & 59.14 & & 2.81 \\
\hline & & & & & & & & & & & & & & \\
\hline & & 0.1 & & I. & & & 5 & & & & & & & \\
\hline & & 0.16 & & 1. 36 & ] & & 0.78 & & & & & & 7 & \\
\hline 359 & & .15 & & 1. 35 & & & 0.79 & & & & 2.08 & 63.80 & $\begin{array}{l}1.10 \\
1.18\end{array}$ & \\
\hline 360 & 7.60 & & 74.36 & & 1.22 & 24.82 & & 2.8 & 18.01 & & 2.09 & 64.98 & & 2.78 \\
\hline
\end{tabular}




\begin{tabular}{|c|c|c|c|c|c|c|c|c|c|c|c|}
\hline Arg. & $(\llcorner, 8,3)$ & (v.c.3) & $(0.8 .4)$ & $|(v, c .1)|$ & $(p . c .0)$ & $(0.8 .1)$ & $(p . c .1)$ & $(p .8 .2)$ & (p.c.2) & (p.8.3) & (p.c.3) \\
\hline & $"$ & $"$ & $"$ & & & & & & & & \\
\hline 300 & 6.22 & 3.35 & 1.43 & 1.09 & 110 & $38+9$ & 1581 & 143 & 512 & 15 & 133 \\
\hline 801 & 6.92 & 3.35 & 1.43 & 1.07 & 109 & 2949 & 1566 & 144 & 515 & 15 & 134 \\
\hline 302 & 6. 93 & 3.35 & 1.44 & 1.06 & 108 & 2849 & 15.51 & 146 & 517 & 15 & 135 \\
\hline 303 & 6.93 & 3.34 & 1.44 & 1.05 & 107 & 2850 & 15.35 & 147 & 519 & 16 & 137 \\
\hline 304 & 6.33 & 3.34 & 1.45 & 1.04 & 106 & 2850 & 1520 & 149 & 622 & 16 & 188 \\
\hline 305 & 6.94 & 3.34 & 1.45 & 1.03 & 105 & 2850 & 1505 & 150 & 524 & 17 & 139 \\
\hline 306 & 6.94 & 3.33 & 1.46 & 1.01 & 104 & 2850 & $14: 0$ & 152 & 520 & 17 & 140 \\
\hline 307 & 6.94 & 3.33 & 1.46 & 1.00 & 103 & 2850 & 147 & 153 & 528 & 18 & 141 \\
\hline 3018 & 6.94 & 3.32 & 1.47 & 0.99 & 103 & 2850 & 1459 & 154 & 531 & 18 & $1+2$ \\
\hline 309 & 6.95 & 3.31 & 1.47 & 0.98 & 102 & 2549 & 1444 & 156 & 533 & $: 9$ & 143 \\
\hline 310 & 6.95 & 3.31 & 1.47 & 0.96 & 102 & 2949 & 1429 & 157 & 5.35 & 20 & $1+4$ \\
\hline 311 & 6.95 & 3.30 & 1.47 & 0.95 & 101 & 2848 & 1413 & 158 & 538 & 20 & 145 \\
\hline 312 & 6.95 & 3.29 & 1.48 & 0.94 & 101 & 2847 & 1398 & 160 & 540 & 21 & 146 \\
\hline 813 & 6.95 & 3.28 & $1.45^{3}$ & 0.93 & 100 & 2847 & 1383 & 162 & $5+2$ & 22 & 147 \\
\hline 314 & 6.94 & 3.27 & 1.48 & 0.92 & 100 & 2846 & 1368 & 163 & 545 & 23 & 148 \\
\hline 315 & 6.94 & 3.26 & 1.48 & 0.90 & 99 & 2845 & 1352 & 165 & 547 & 24 & 149 \\
\hline 316 & 6.94 & 3.25 & 1.48 & 0.89 & 99 & 2943 & 1337 & 167 & $5+9$ & 24 & 150 \\
\hline 317 & 6.94 & 8. 23 & 1.48 & 0.88 & 99 & 2842 & 1322 & 168 & 551 & 25 & 151 \\
\hline 318 & 6.93 & 3.22 & 1.48 & 0.86 & 93 & 2840 & 1307 & 170 & 554 & 26 & 152 \\
\hline 319 & 6.93 & 3.21 & 1.48 & 0.85 & 98 & 2938 & 1291 & 171 & 556 & 27 & 153 \\
\hline 320 & 6.92 & 3.20 & 1.48 & 0.84 & 98 & 2836 & 1276 & 173 & 558 & 27 & 154 \\
\hline 321 & 6.92 & 3.18 & 1.48 & 0.83 & 98 & 2834 & 1261 & 175 & 560 & 28 & 155 \\
\hline 322 & 6.91 & 3.17 & 1.49 & 0.81 & 98 & 2831 & 1246 & 176 & 562 & 29 & 156 \\
\hline 323 & 6.91 & 3.15 & 1.48 & 0.80 & 98 & 2928 & 1231 & 178 & 564 & 29 & 157 \\
\hline 324 & 6.90 & 3.14 & 1.48 & 0.79 & 99 & 2825 & 1216 & 180 & 566 & 30 & 158 \\
\hline 325 & 6.89 & 3.12 & 1.48 & 0.77 & 99 & 2822 & 1202 & 181 & 568 & 31 & 159 \\
\hline 326 & 6.88 & 3.11 & 1.48 & 0.76 & 99 & 2819 & 1187 & 183 & 570 & 32 & 159 \\
\hline 321 & 6.87 & 3.10 & 1.47 & 0.75 & 100 & 2815 & 1172 & 185 & 572 & 33 & 160 \\
\hline 328 & 6. 86 & 3.08 & 1.47 & 0.74 & 100 & 2912 & 1157 & 186 & 573 & 33 & 161 \\
\hline 329 & 6.85 & 3.06 & 1.47 & 0.72 & 101 & 2808 & 1143 & 188 & 575 & 34 & 162 \\
\hline 330 & 6.83 & 3.04 & 1.46 & 0.71 & 101 & 2904 & 1128 & 190 & 577 & 35 & 163 \\
\hline 331 & 6.82 & 3.03 & 1.46 & 0.70 & 102 & 2900 & 1113 & 192 & 579 & .36 & 164 \\
\hline 332 & 6.81 & 3.01 & 1.46 & 0.69 & 103 & 2796 & 1098 & 193 & 581 & 37 & 165 \\
\hline 333 & 6.79 & 2.99 & 1.45 & 0.67 & 103 & 2792 & 1084 & 195 & 583 & 38 & 165 \\
\hline 334 & 6.77 & 2.97 & 1.45 & 0.66 & 104 & 2788 & 1069 & 197 & 585 & 39 & 166 \\
\hline 335 & 6.75 & 2.96 & 1.44 & 0.65 & 105 & 2784 & 1054 & 199 & 587 & 40 & 167 \\
\hline 336 & 6.74 & 2.94 & 1.44 & 0.64 & 106 & 2773 & 1039 & 201 & 589 & 41 & 168 \\
\hline 337 & 6.72 & 2.92 & 1.43 & 0.63 & 107 & 2775 & 1025 & 203 & 591 & 42 & 169 \\
\hline 338 & 6.70 & 2.90 & 1.43 & $0.6 i 1$ & 103 & 2770 & 1010 & 204 & 592 & 43 & 169 \\
\hline 339 & 6.68 & 2.88 & 1.42 & 0.60 & 103 & 2765 & 996 & 206 & 594 & 44 & 170 \\
\hline 340 & 6.66 & 2.87 & 1.42 & 0.59 & 110 & 2760 & 981 & 208 & 596 & 45 & 171 \\
\hline 341 & 6.64 & 2.85 & 1.41 & 0.58 & 111 & 2755 & 967 & 210 & 598 & 16 & 171 \\
\hline 343 & 6.62 & 2.83 & 1.40 & 0.57 & 112 & 27.50 & 953 & 212 & 599 & 47 & 172 \\
\hline 343 & 6.59 & 2.81 & 1.40 & 0.56 & 114 & 2744 & 938 & 214 & 601 & 48 & 173 \\
\hline 344 & 6.57 & 2.79 & 1.39 & 0.55 & 115 & 2739 & 924 & 217 & 603 & 49 & 173 \\
\hline 345 & 6.55 & 2.78 & 1.39 & 0.53 & 116 & $2 \div 33$ & 910 & 219 & 605 & 50 & 174 \\
\hline 346 & 6.52 & 2.76 & 1.37 & 0.52 & 117 & 2727 & 896 & 221 & 606 & 51 & 174 \\
\hline 347 & 6.50 & 2.74 & 1.36 & 0.51 & 119 & 2721 & 882 & 223 & 608 & 52 & 175 \\
\hline 348 & 6.47 & 2.72 & 1.36 & 0.50 & 121 & 2715 & 868 & 226 & 610 & 53 & 176 \\
\hline 349 & 6.44 & 2.70 & 1.35 & 0.49 & 122 & 2709 & 855 & 228 & 611 & 54 & 176 \\
\hline 350 & 6.41 & 2.69 & 1.34 & 0.48 & 124 & 2703 & 841 & 230 & 613 & 55 & 177 \\
\hline 351 & 6.37 & 2.67 & 1.33 & 0.47 & 126 & 2696 & 829 & 2.32 & 614 & 57 & 177 \\
\hline 352 & 6.34 & 2.65 & 1.32 & 0.16 & 128 & 2689 & 814 & 235 & 616 & 58 & 178 \\
\hline 353 & 6.31 & 2.64 & 1.31 & 0.45 & 130 & 2682 & 801 & 237 & 617 & 53 & 178 \\
\hline 354 & 6.28 & 2.62 & 1.30 & 0.44 & 132 & 2675 & 788 & 239 & 619 & 60 & 178 \\
\hline 355 & 6.24 & 2.60 & 1.29 & 0.43 & 134 & 2668 & 775 & 241 & 620 & 61 & 179 \\
\hline 356 & 6.21 & 2.59 & 1.28 & 0.42 & 136 & 26,60 & 762 & 244 & 621 & 62 & 179 \\
\hline 357 & 6. 18 & 2.57 & 1.26 & 0.41 & 139 & 2653 & 749 & 246 & 623 & 63 & 180 \\
\hline 358 & 6.14 & 2.56 & 1.25 & 0.40 & 141 & 26,45 & 736 & 248 & 624 & 64 & 180 \\
\hline 353 & 6.10 & 2.54 & 1.24 & 0.39 & 144 & 26.37 & 723 & 250 & 626 & 65 & 180 \\
\hline 360 & 6.07 & 2.53 & 1.23 & 0.38 & 146 & 2629 & 710 & 252 & 627 & 67 & 181 \\
\hline
\end{tabular}




\begin{tabular}{|c|c|c|c|c|c|c|c|c|c|c|c|c|c|c|}
\hline \multicolumn{15}{|c|}{ TABLE IX, ARa. 2.-Continued. } \\
\hline \multirow[t]{2}{*}{ Arg. } & \multirow{2}{*}{$\frac{(c . c .0)}{\prime \prime}$} & Diff. & $(v . s .1)$ & Diff. & \multirow{2}{*}{$\frac{\text { Seevar. }}{\prime \prime}$} & \multirow{2}{*}{$\frac{(v . c .1)}{\prime \prime}$} & Diff. S & \multirow{2}{*}{$\frac{\text { Sec.var. }}{\prime \prime}$} & $(v . s .2)$ & Diff. $S$ & Sec.var. & $(v . c .2)$ & Diff. $s$ & Sec.var. \\
\hline & & $" \prime$ & $"$ & $"$ & & & " & & " & " & " & " & $"$ & $"$ \\
\hline 360 & 7.60 & & 74.36 & & 1.22 & 24.82 & & 2.86 & 18.01 & & 2.09 & 64.98 & & 2.78 \\
\hline 361 & 7.45 & -O.I5 & 73.02 & -1.34 & 1.23 & 25.63 & $\begin{array}{r}+0.81 \\
0.82\end{array}$ & 2.86 & 17.33 & $\begin{array}{r}-0.68 \\
0.67\end{array}$ & 2.10 & 66.17 & $\begin{array}{r}+1.19 \\
1.20\end{array}$ & 2.77 \\
\hline 362 & 7.30 & 0.15 & 71.69 & 1. 33 & 1.25 & 26.45 & 0.82 & 2.87 & 16.66 & $\begin{array}{l}0.07 \\
0.66\end{array}$ & 2.12 & 67.37 & 1.20 & 2.77 \\
\hline 363 & 7.15 & 0.15 & 70.37 & I. 32 & 1.26 & 27.29 & $\begin{array}{l}0.84 \\
0.85\end{array}$ & 2.87 & 16.00 & $\begin{array}{l}0.66 \\
0.65\end{array}$ & 2.13 & 68.58 & 1.21 & 2.76 \\
\hline 364 & 7.01 & $\begin{array}{l}0.14 \\
0.14\end{array}$ & 69.05 & $\begin{array}{l}1 \cdot 3^{2} \\
1 \cdot 3^{\circ}\end{array}$ & 1.28 & 28.14 & $\begin{array}{l}0.85 \\
0.86\end{array}$ & 2.87 & 15.35 & $\begin{array}{l}0.65 \\
0.64\end{array}$ & 2.15 & 69.79 & $\begin{array}{l}1.21 \\
1.22\end{array}$ & 2.75 \\
\hline 365 & 6.87 & -0.13 & 67.75 & & 1.29 & 29.00 & +0.88 & 2.88 & 14.71 & -0.62 & 2.16 & 71.01 & +1.22 & 2.74 \\
\hline 366 & & $\begin{array}{l}-0.13 \\
0.13\end{array}$ & 66.46 & $\begin{array}{r}-1.29 \\
1.28\end{array}$ & 1.31 & & 0.90 & $\begin{array}{l}2.88 \\
2.88\end{array}$ & & 0.61 & 2.17 & 72.23 & $\begin{array}{r}T .22 \\
1.23\end{array}$ & 2.74 \\
\hline 367 & 1 & $\begin{array}{l}0.13 \\
0.13\end{array}$ & 65.18 & r. 28 & 1.32 & 30.78 & $0.9 \mathrm{I}$ & $\begin{array}{l}2.88 \\
2.88\end{array}$ & 13.48 & 0.60 & $\begin{array}{l}2.18 \\
0.19\end{array}$ & 73.46 & & 2.73 \\
\hline 5 & 8 & 0.12 & 63.90 & 1.27 & $\begin{array}{l}1.34 \\
135\end{array}$ & 31.69 & 0.92 & $\begin{array}{l}2.88 \\
2.89\end{array}$ & 12.88 & 0.59 & 2.19 & 70 & $\begin{array}{l}1.24 \\
1.25\end{array}$ & 2.72 \\
\hline 369 & 6 & 0.12 & 62.63 & 1.25 & 1.35 & 32.61 & 0.93 & 2.89 & 12.29 & 0.57 & 2.21 & 75.95 & 1.26 & 2.71 \\
\hline 370 & 6.24 & -0.12 & 61.38 & -1.24 & $\begin{array}{l}1.37 \\
1.38\end{array}$ & $\begin{array}{l}33.54 \\
34.49^{-}\end{array}$ & +0.95 & $\begin{array}{l}2.89 \\
2.89\end{array}$ & $\begin{array}{l}11.72 \\
11.17\end{array}$ & -0.55 & $\begin{array}{l}2.22 \\
2.23\end{array}$ & 77.21 & +1.26 & \\
\hline 372 & 6.00 & 0.12 & $\begin{array}{l}60.14 \\
58.91\end{array}$ & I.23 & 1.40 & 35.45 & 0.96 & $\begin{array}{l}2.09 \\
2.89\end{array}$ & 10.63 & 0.54 & $\begin{array}{l}2.25 \\
2.25\end{array}$ & & 1.27 & \\
\hline 373 & 5.89 & O.I I & 57.68 & 1.23 & 1.41 & 36.42 & 0.97 & 2.89 & 10.10 & 0.53 & 2.26 & 81 & 1.27 & 89 \\
\hline 374 & 5.78 & O.II & 56.46 & $\begin{array}{l}1.22 \\
1.20\end{array}$ & 1.43 & 37.40 & $\begin{array}{l}0.98 \\
1.00\end{array}$ & 2.89 & 9.59 & $\begin{array}{l}0.51 \\
0.50\end{array}$ & 2.27 & 82.28 & $\begin{array}{l}1.27 \\
1.28\end{array}$ & 2.67 \\
\hline 375 & 5.68 & & 55.26 & & 1.44 & 38.40 & & 2.89 & 9.09 & & 2.29 & 83.56 & & 2.66 \\
\hline 376 & 5 & -O. I I & 54.07 & -1.19 & 1.45 & 39. & $\begin{array}{r}+1.01 \\
1.02\end{array}$ & 2.89 & 8.60 & -0.49 & 2.30 & & & 2.66 \\
\hline 377 & 5.47 & O. I0 & 52.89 & & 1.47 & 40 & $\begin{array}{l}1.02 \\
1.04\end{array}$ & 2.89 & 8.12 & 0.48 & 2.31 & 86 & 9 & 2.65 \\
\hline 378 & 5 & 0.09 & 51.72 & $\begin{array}{l}7 \\
5\end{array}$ & 1.48 & 41 . & $\begin{array}{l}\text { I.04 } \\
\text { x.05 }\end{array}$ & 2.89 & 7. & 0.40 & 2.32 & 43 & & .64 \\
\hline 379 & 30 & $\begin{array}{l}0.08 \\
0.08\end{array}$ & 50.57 & $\begin{array}{l}\text { T.15 } \\
\text { 1.15 }\end{array}$ & 1.50 & 42.52 & $\begin{array}{l}1.05 \\
1.06\end{array}$ & 2.89 & 1 & $\begin{array}{l}0.45 \\
0.43\end{array}$ & 2.34 & 3 & $\begin{array}{l}\text { I. } 30 \\
1.31\end{array}$ & .63 \\
\hline 380 & 5.22 & & 49.42 & & 1.51 & 43.58 & & 2.89 & 6.78 & & 2.35 & 90.04 & & 2.62 \\
\hline 1 & & $\begin{array}{r}-0.08 \\
0.07\end{array}$ & & $\begin{array}{r}-1.13 \\
1.12\end{array}$ & 1.52 & $5^{-1}$ & $\begin{array}{r}1.09 \\
1.09\end{array}$ & $\begin{array}{l}2.89 \\
2.89\end{array}$ & 6 & $\begin{array}{l}0.42 \\
0.40\end{array}$ & 2.36 & $5^{-}$ & & 1 \\
\hline 32 & 5 & $\begin{array}{l}0.07 \\
0.08\end{array}$ & 47.17 & Y.X I & 1.54 & 45. & I. I0 & $\begin{array}{l}2.89 \\
2.89\end{array}$ & 6 & $\begin{array}{l}0.40 \\
0.39\end{array}$ & 2.37 & 7 & & 0 \\
\hline 3 & & 0.08 & 46.06 & 1.10 & 1.5 & $\begin{array}{l}4 \\
5\end{array}$ & I. II & $\begin{array}{l}2.89 \\
2.89\end{array}$ & 5 & $\begin{array}{l}.39 \\
0.38\end{array}$ & 2 & 9 & & 9 \\
\hline 384 & 4.91 & 0.07 & 44.96 & 1.09 & 1.57 & 47.95 & I. 12 & 2.89 & 5.19 & $0.3^{6}$ & 2.39 & 95.31 & 1.33 & 2.58 \\
\hline 385 & 4.84 & -0.07 & 43.87 & -1.07 & 1.58 & 49.07 & fI.I3 & 2.89 & 4.83 & & 2.40 & 96.64 & & 2.57 \\
\hline 6 & 4 & 0.06 & 42.80 & 1.06 & 1.60 & $0^{-}$ & $\begin{array}{r}+1.13 \\
1.14\end{array}$ & $\begin{array}{l}2.89 \\
289\end{array}$ & 8 & $\begin{array}{r}-0.35 \\
0.33\end{array}$ & 2.42 & & +1 & 7 \\
\hline 7 & 4 & 0.06 & 41.74 & 1.05 & 1.61 & 4 & $\begin{array}{l}1.14 \\
\text { I. } 16\end{array}$ & 2.88 & & $\begin{array}{l}.33 \\
0.32\end{array}$ & 2.43 & & & 6 \\
\hline 8 & 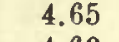 & 0.05 & 40.69 & I.04 & 1.63 & 0 & $\begin{array}{l}1.10 \\
1.17\end{array}$ & 2.88 & & $\begin{array}{l}0.3^{2} \\
0.31\end{array}$ & 2.44 & 10 & 4 & 5 \\
\hline 389 & 60 & 0.04 & 65 & x.02 & 1.64 & 7 & $\begin{array}{l}1.18 \\
1.18\end{array}$ & 2.88 & 3.52 & $\begin{array}{l}.31 \\
0.29\end{array}$ & 2.45 & 101.98 & $\begin{array}{l}1.34 \\
1.35\end{array}$ & 2.54 \\
\hline 390 & 4.56 & & 38.63 & $-\mathrm{I} . \mathrm{OI}$ & 1.66 & 54.85 & & 2.88 & 3.23 & 0.27 & 2.46 & 3 & & 2.53 \\
\hline 1 & & $\begin{array}{l}0.04 \\
0.04\end{array}$ & 37.62 & 0.99 & 1.67 & & $\begin{array}{r}1.19 \\
1.21\end{array}$ & 2.88 & & $\begin{array}{l}-0.27 \\
0.26\end{array}$ & 2.47 & & 5 & 2 \\
\hline 392 & 8 & $\begin{array}{l}0.03 \\
0.03\end{array}$ & 36.63 & 0.98 & 1.69 & 5 & I.2I & 2.88 & 2.70 & 0.25 & 2.48 & 3 & 5 & .51 \\
\hline 5 & 4 & 0.04 & 35.65 & 0.98 & 1.70 & 58.46 & 1.22 & 2.87 & 2.45 & 0.23 & 2.49 & 10 & & 2.50 \\
\hline 394 & 1 & 0.03 & 34.67 & 0.96 & 1 & 59 & 1.23 & 2.87 & 22 & 0.21 & 50 & 108 & $1.3^{6}$ & 2.49 \\
\hline 395 & 4.38 & & 33.71 & & 1.73 & $60.91+$ & $+r$ & 2.87 & 2.01 & 0.20 & 2.51 & 110.10 & & 2.47 \\
\hline $\begin{array}{l}96 \\
97\end{array}$ & 3 & 0.02 & & 0.92 & 1.7 & 62. & $\begin{array}{l}1.25 \\
\text { I. }\end{array}$ & 2.87 & & 0.19 & 52 & $66^{-}$ & I. & 46 \\
\hline $\begin{array}{l}3 \\
3 \\
3\end{array}$ & 3 & 0.02 & 3. & 0.91 & 1.75 & $6:$ & I. 27 & 2.87 & 2 & 0.17 & 3 & 22 & r. 36 & .45 \\
\hline 399 & & 0.01 & 30.94 & 0.91 & 1.7 & 6 & 1.28 & 2.86 & 1. & 0.16 & 2 & 8 & 1.37 & 4 \\
\hline 399 & 4 & 0.01 & 3 & 0.89 & & & 1.28 & .86 & 1.29 & 0.15 & -2.55 & 115 & 1.37 & 2.43 \\
\hline 400 & 4.29 & -0.01 & 29.14 & -0.87 & 1.79 & 67.23 & -1.29 & 2.86 & 1.14 & 0.13 & 2.56 & 2 & 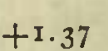 & 2.4 \\
\hline $\begin{array}{l}401 \\
402\end{array}$ & & 0.00 & 27 & 0.85 & 1.80 & 6 & I. 30 & 2.86 & & O.I I & 2 & & I. & 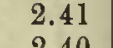 \\
\hline $\begin{array}{l}402 \\
403\end{array}$ & & 0.00 & 27.42 & 0.85 & 1.82 & 69.82 & I. 3 I & 2.8 & & 0.10 & & & I. & 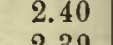 \\
\hline $\begin{array}{l}40 \\
40\end{array}$ & & 0.00 & $\begin{array}{l}26.57 \\
25.73\end{array}$ & 0.84 & 1.83 & 71.13 & 1.32 & 2. & & 0.08 & 9 & & $1.3^{8}$ & 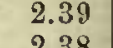 \\
\hline 404 & & to. & & 0.82 & 1.8 & 7 & 1.33 & 2.84 & & 0.07 & 0 & & 1.37 & 8 \\
\hline 405 & 4.29 & & 24.91 & -0.80 & 1.86 & 73 & & 2.84 & 0.65 & & 2.60 & 8 & & 2.36 \\
\hline $\begin{array}{l}406 \\
407\end{array}$ & & 0.02 & & 0.78 & 1. & $75.12^{-}$ & $\begin{array}{r}+1.34 \\
1.35\end{array}$ & 2.84 & $0.60^{\circ}$ & & 2.61 & 16 & & 2.35 \\
\hline 4 & & 0.02 & 23 & 0.77 & 1. & 76.47 & $\begin{array}{l}1.35 \\
1.36\end{array}$ & 2.83 & 0.56 & 0.04 & 2.62 & 126.53 & 8 & 2.34 \\
\hline & & 0.02 & 2 & 0.76 & 1. & 77 & I. 36 & 2.83 & 0.53 & 0.01 & 2.63 & 1 & & 2.33 \\
\hline 409 & (3) & 0.03 & 2 & 0.74 & 1.9 & 79.19 & I. 37 & 2.82 & 0.52 & 0.00 & 2.64 & 129.28 & & 2.32 \\
\hline 410 & 4.39 & & 21.06 & & 1.94 & 80.5 & & 2.82 & 0. & & 2.65 & 13 & & 2.31 \\
\hline 411 & & & 20.33 & & 1.9 & & $\begin{array}{r}+1.38 \\
1.30\end{array}$ & 2.82 & & -0.02 & 2.66 & 2 & $\mathrm{~T}$ & 0 \\
\hline 412 & & $\begin{array}{l}0.04 \\
0.04\end{array}$ & 19.62 & & 1.9 & 83. & $\begin{array}{l}\text { I. } 39 \\
\text { I. } 40\end{array}$ & 2.8 & & 5 & 2.67 & 1 & & 299 \\
\hline 41 & & $\begin{array}{l}0.04 \\
0.04\end{array}$ & 18.93 & 0.68 & 1.9 & 81.73 & $\begin{array}{l}1.40 \\
1.41\end{array}$ & 2.81 & & $\begin{array}{l}0.05 \\
0.06\end{array}$ & & & 1.37 & 2.27 \\
\hline 414 & $A$ & $\begin{array}{l}0.05 \\
0.05\end{array}$ & 1895 & 0.67 & 1.9 & 86.14 & $\begin{array}{l}1.4 \mathrm{I} \\
1.4 \mathrm{I}\end{array}$ & 2.80 & 0.68 & 0.08 & 3 & 136 & $\begin{array}{l}1.37 \\
1.37\end{array}$ & 2.26 \\
\hline 415 & 4.59 & & 17.58 & & 2.01 & 87.55 & & 2.80 & 0.76 & & 2.69 & 1 & & 2.25 \\
\hline 416 & & $\begin{array}{l}-0.05 \\
0.06\end{array}$ & 16.93 & $\begin{array}{r}-0.05 \\
0.63\end{array}$ & 2.02 & & 42 & 2.79 & 0.85 & & 2.70 & $38^{-}$ & +1.37 & 2.24 \\
\hline 417 & 4 & & 16.30 & 0.63 & 2.0 & 90.39 & 1.42 & 2.79 & 0.96 & 0.11 & 2.71 & & 1.37 & 2.22 \\
\hline 418 & 4.76 & 0.00 & 15.67 & $0.6 \mathrm{I}$ & 2.0 & & I. 43 & 2.78 & 1.08 & 0.12 & 2.71 & 14 & 1.37 & 2.21 \\
\hline 419 & 4.82 & 0.06 & 10.0 & 0.59 & 2.06 & 93.26 & $\begin{array}{l}\text { I. } 44 \\
\text { I. } 45\end{array}$ & 2.78 & 1.22 & $\begin{array}{l}0.14 \\
0.15\end{array}$ & 2.72 & 142.99 & $\begin{array}{l}1.37 \\
\text { I. } 36\end{array}$ & 2.20 \\
\hline 420 & 4.88 & & 14.47 & & 2.07 & 94.71 & & 2.77 & 1.37 & & 2.73 & 144.35 & & 2.19 \\
\hline
\end{tabular}




\begin{tabular}{|c|c|c|c|c|c|c|c|c|c|c|c|}
\hline Arg. & $(v .8 .3)$ & $(v, c .3)$ & $(10,8,4)$ & $(v, c .4)$ & $(p . c .0)$ & (p.8.1) & $($ (p.c.1) & $($ p.6.2) & (p.c.2) & $(\rho .8 .3)$ & $(p . c .3)$ \\
\hline & " & $"$ & " & $"$ & & & & & & & \\
\hline 36,0 & 6.07 & 2.53 & 1.23 & 0.38 & 146 & 26,29 & 710 & 252 & 627 & 67 & 181 \\
\hline 361 & 6.02 & 2.51 & 1.22 & 0.38 & 149 & 2621 & $6 ! 17$ & 254 & 623 & 68 & 181 \\
\hline 362 & 5.98 & 2.50 & 1.21 & 0.37 & 151 & 2613 & 685 & 256 & 629 & 69 & 182 \\
\hline 363 & 5.24 & 2.49 & 1.20 & 0.36 & 153 & 2605 & $6: 2$ & 259 & 631 & 70 & 182 \\
\hline 364 & 5.90 & 2.48 & 1.19 & 0.35 & 156 & 2596 & 660 & $26 \mathrm{I}$ & 632 & 71 & 182 \\
\hline 365 & 5.55 & 2.46 & 1.17 & 0.35 & 159 & 2588 & 647 & 263 & 633 & 72 & 183 \\
\hline 366 & 5.81 & 2.45 & 1.16 & 0.34 & $16 ; 2$ & 2579 & 635 & 265 & 634 & 74 & 183 \\
\hline 367 & 5.76 & 2.44 & 1.15 & 0.34 & 164 & 2570 & 623 & 267 & 635 & 75 & 183 \\
\hline 368 & 5.72 & 2.44 & 1.14 & 0.33 & 167 & 2562 & 610 & 270 & 636 & 76 & 183 \\
\hline 369 & 5.67 & 2.43 & 1.12 & 0.32 & 170 & 2553 & 598 & 272 & 639 & 77 & 184 \\
\hline 370 & 5.63 & 2.42 & 1.11 & 0.32 & 173 & 2544 & 586 & 274 & 639 & 78 & 184 \\
\hline 371 & 5.58 & 2.41 & 1.10 & 0.31 & 176 & 2535 & 574 & 276 & 640 & 79 & 184 \\
\hline 372 & 5.53 & 2.41 & 1.08 & 0.31 & 180 & 2526 & 562 & 279 & 641 & 80 & 184 \\
\hline 373 & 5.48 & 2.40 & 1.07 & 0.30 & 183 & 2516 & 550 & 281 & 642 & 81 & 184 \\
\hline 374 & 5.43 & 2.39 & 1.05 & 0.30 & 186 & 2507 & 539 & 283 & 643 & 82 & 184 \\
\hline 375 & 5.39 & 2.39 & 1.04 & 0.29 & 190 & 2498 & 528 & 285 & 645 & 83 & 184 \\
\hline 376 & 5.33 & 2.39 & 1.03 & 0.29 & 194 & 2488 & 517 & 298 & 646 & 84 & 184 \\
\hline 377 & 5.28 & 2.38 & 1.01 & 0.29 & 197 & 2479 & 506 & 290 & 647 & 85 & 184 \\
\hline 378 & 522 & 2.38 & 1.00 & 0.28 & 201 & 2469 & 495 & 292 & 648 & 86 & 184 \\
\hline 379 & 5.17 & 2.38 & 0.98 & 0.28 & 205 & 2460 & 485 & 294 & 649 & 87 & 184 \\
\hline 380 & 5.12 & 2.39 & 0.97 & 0.28 & 209 & 2450 & 474 & 297 & 650 & 83 & 184 \\
\hline 331 & 5.07 & 2.39 & 0.96 & 0.28 & 212 & 2440 & 463 & 299 & 651 & 89 & 184 \\
\hline 382 & 5.01 & 2.39 & 0.34 & 0.27 & 216 & 2429 & 453 & 302 & 653 & 90 & 184 \\
\hline 343 & 4.96 & 2.39 & 0.93 & 0.27 & 220 & 2419 & 443 & 304 & 653 & 91 & 181 \\
\hline 394 & 4.90 & 2.39 & 0.91 & 0.27 & 224 & 2408 & 433 & 306 & 654 & 92 & 184 \\
\hline 355 & 4.94 & 2.39 & 0.90 & 0.27 & 228 & 2397 & 423 & 309 & 655 & 94 & 184 \\
\hline 386 & 4.79 & 2.40 & 0.88 & 0.27 & 232 & 2386 & 413 & 312 & 656 & 95 & 184 \\
\hline 357 & 4.73 & 2.40 & 0.86 & 0.27 & 2.36 & 2374 & 404 & 314 & 657 & 96 & 184 \\
\hline 388 & 4.67 & $2 .+1$ & 0.85 & 0.27 & 240 & 2363 & 394 & 317 & 657 & 97 & 183 \\
\hline $35 ?$ & 4.61 & 2.42 & 0.84 & 0.27 & 245 & 2851 & 384 & 319 & 658 & 93 & 183 \\
\hline 390 & 4.55 & 2.43 & 0.82 & 0.27 & 249 & 2340 & 375 & 322 & 659 & 99 & 183 \\
\hline 391 & 449 & 2.44 & 0.81 & 0.27 & 253 & 2328 & 366 & 324 & 660 & 100 & 183 \\
\hline 392 & 4.43 & 2.46 & 0.79 & 0.27 & 258 & 2316 & 357 & 327 & 661 & 102 & 183 \\
\hline 393 & 4.37 & 2.47 & 0.78 & 0.27 & 262 & 2305 & 319 & 329 & 662 & 103 & $18 \%$ \\
\hline 394 & 4.31 & 2.48 & 0.76 & 0.27 & 266 & 2293 & 339 & 332 & 662 & 104 & 182 \\
\hline 395 & 4.24 & 2.49 & 0.75 & 0.27 & 271 & 2291 & 330 & 334 & 663 & 105 & 182 \\
\hline 396 & 4.19 & 2.51 & 0.73 & 0.28 & 276 & 2269 & 322 & 336 & 664 & 106 & 182 \\
\hline 397 & 4.12 & 2.53 & 0.72 & 0.28 & 280 & 2257 & 313 & 339 & 665 & 107 & 182 \\
\hline 398 & 4.06 & 2.55 & 0.70 & 0.28 & 295 & 2245 & 304 & $3+1$ & 666 & 109 & 181 \\
\hline 399 & 4.00 & 2.57 & 0.69 & 0.28 & 290 & 2232 & 296 & 344 & 667 & 110 & 181 \\
\hline 400 & 3.93 & 2.59 & 0.67 & 0.29 & 294 & 2220 & 289 & 346 & 667 & 111 & 181 \\
\hline 401 & 3.87 & 2.62 & 0.66 & 0.29 & 299 & 2207 & 290 & 348 & 668 & 112 & 181 \\
\hline 402 & 3.80 & 2.64 & 0.65 & 0.30 & 304 & 2195 & 273 & 351 & 668 & 113 & 180 \\
\hline 403 & 3.74 & 2.66 & 0.63 & 0.30 & 309 & 2182 & 265 & 353 & 669 & 114 & 180 \\
\hline 404 & 3.68 & 2.69 & 0.63 & 0.31 & 314 & 2170 & 258 & 355 & 669 & 115 & 180 \\
\hline 405 & 3.62 & 2.72 & 0.60 & 0.31 & 320 & 2157 & 251 & 358 & 669 & 116 & 179 \\
\hline 406 & 3.55 & 2.75 & 0.59 & 0.31 & 325 & 2144 & 244 & 361 & 669 & 116 & 179 \\
\hline 407 & 3.49 & 2.78 & 0.57 & 0.32 & 331 & 2131 & 237 & 363 & 670 & 117 & 179 \\
\hline 408 & 3.43 & 2.31 & 0.56 & 0.33 & 337 & 2113 & 230 & 365 & 670 & 118 & 178 \\
\hline 409 & 3.36 & 2.84 & 0.55 & 0.33 & 342 & 2106 & 224 & 368 & 670 & 119 & 178 \\
\hline 410 & 3.30 & 2.88 & 0.53 & 0.34 & 348 & 2093 & 217 & 371 & 670 & 120 & 178 \\
\hline 411 & 3.24 & 2.92 & 0.52 & 0.35 & $35 t$ & 2080 & 211 & 373 & 670 & 121 & 177 \\
\hline 412 & 3.17 & 2.95 & 0.50 & 0.36 & 359 & 2066 & 205 & 376 & 670 & 122 & 177 \\
\hline 413 & 3.11 & 2.99 & 0.49 & 0.36 & 36.5 & 2053 & 1.99 & 378 & 671 & 122 & 176 \\
\hline 414 & 3.04 & 3.03 & 0.48 & 0.37 & 371 & 2039 & 193 & 381 & 671 & 123 & 176 \\
\hline 415 & 2.99 & 3.07 & 0.46 & 0.39 & 377 & 2026 & 187 & 382 & 671 & 124 & 175 \\
\hline 416 & 2.92 & 3.11 & 0.45 & 0.39 & 383 & 21112 & 181 & 386 & 671 & 125 & 174 \\
\hline 417 & 2.85 & 3.15 & 0.44 & 0.39 & 399 & 1998 & 176 & 388 & 671 & 126 & 174 \\
\hline 418 & 2.79 & 3.20 & 0.42 & 0.40 & 395 & 1985 & 170 & 391 & 671 & 126 & 173 \\
\hline 419 & 2.73 & 3.24 & 0.41 & 0.41 & 101 & 1971 & 165 & 393 & 672 & 127 & 173 \\
\hline 120 & 2.67 & 3.29 & 0.40 & 0.42 & 407 & 1957 & 160 & 396 & 672 & 128 & 172 \\
\hline
\end{tabular}




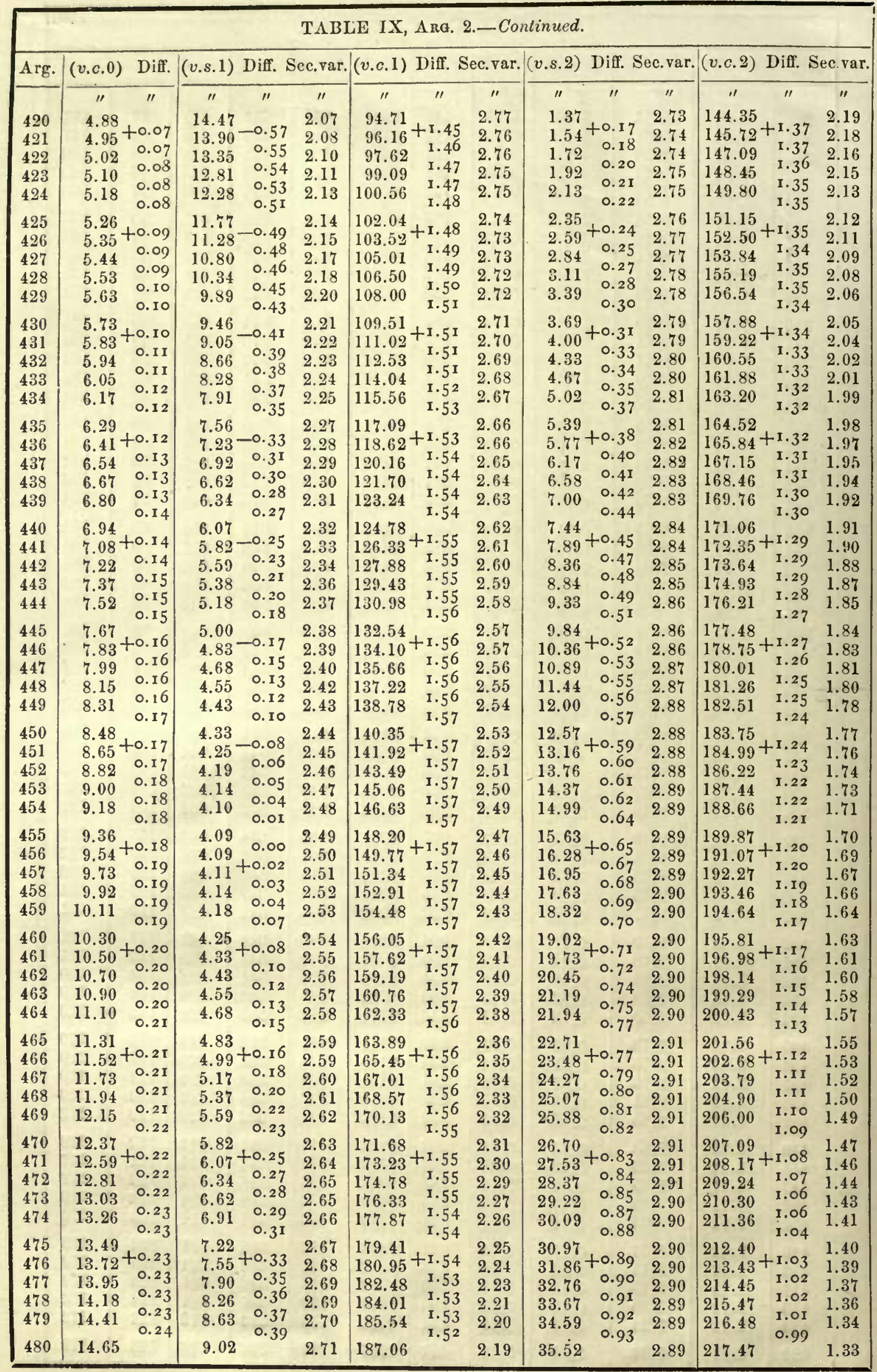


TIF OIBI' OF URANUS.

\begin{tabular}{|c|c|c|c|c|c|c|c|c|c|c|c|}
\hline Arg. & $(v .8 .3)$ & $(v . c .3)$ & $(0.8 .4)$ & $(v . c .4)$ & $(p . c .0)$ & $(p .8 .1)$ & $(p . c .1)$ & $(0.8 .2)^{\prime}$ & $(\rho, c, 2)$ & $(p .8 .3)$ & $(p, c .3)$ \\
\hline & " & $"$ & " & " & & & & & & & \\
\hline 420 & 2.67 & 3.29 & 0.40 & 0.42 & 407 & 1957 & 160 & 396 & 672 & 128 & 172 \\
\hline $4: 21$ & 2.61 & 3.31 & 0.39 & 0.43 & 413 & 1943 & 155 & 399 & 672 & 129 & 172 \\
\hline 422 & 2.55 & 3.39 & 0.39 & 0.44 & 419 & 1930 & 150 & 402 & 673 & 120 & 171 \\
\hline 423 & 2.49 & 3.44 & 0.36 & 0.45 & 425 & 1916 & 146 & 405 & 673 & 130 & 171 \\
\hline 424 & 2.43 & 3.50 & 0.35 & 0.46 & 431 & 1903 & 141 & 407 & 673 & 131 & 171 \\
\hline 425 & 2.37 & 3.55 & 0.34 & 0.47 & $\$ 37$ & 1859 & 136 & 410 & 673 & 132 & 170 \\
\hline 426 & 2.31 & 3.61 & 0.33 & 0.49 & 443 & 1875 & 132 & 413 & 673 & 133 & 170 \\
\hline 427 & 2.25 & 3.66 & 0.32 & 0.50 & 450 & 1862 & 128 & 416 & 673 & 133 & 160 \\
\hline 423 & 2.20 & 3.73 & 0.31 & 0.51 & 456 & 1843 & 124 & 418 & 673 & 134 & 169 \\
\hline 429 & 2.14 & 3.78 & 0.30 & 0.52 & 463 & 1834 & 120 & 421 & 673 & 135 & 168 \\
\hline 430 & 2.09 & 3.84 & 0.29 & 0.53 & 469 & 1820 & 117 & 424 & 673 & 136 & 167 \\
\hline 431 & 2.03 & 3.90 & 0.28 & 0.54 & 476 & 1906 & 114 & 426 & 673 & 136 & 167 \\
\hline 432 & 1.97 & 3.96 & 0.27 & 0.55 & 482 & 1791 & 111 & 423 & 673 & 137 & 166 \\
\hline 4333 & 1.92 & 4.03 & 0.26 & 0.57 & 489 & 1777 & 108 & 432 & 673 & 138 & 165 \\
\hline 434 & 1.86 & 4.10 & 0.25 & 0.58 & 496 & 1762 & 105 & 434 & 673 & 139 & 165 \\
\hline 435 & 1.81 & 4.16 & 0.24 & 0.59 & 503 & 1748 & 102 & 437 & 672 & 139 & $16 t$ \\
\hline 436 & 1.76 & 4.23 & 0.23 & 0.60 & 510 & 1733 & 100 & 440 & 672 & 140 & 163 \\
\hline $4: 37$ & 1.70 & 4.30 & 0.22 & 0.63 & 517. & 1719 & 98 & 442 & 672 & 141 & 163 \\
\hline $4: 39$ & 1.65 & 4.37 & 0.22 & 0.63 & 524 & 1704 & 96 & 445 & 671 & 112 & 162 \\
\hline 439 & 1.60 & 4.45 & 0.21 & 0.64 & 531 & 1690 & 94 & 447 & 671 & 142 & 161 \\
\hline 440 & 1.55 & 4.53 & 0.20 & 0.66 & 539 & 1675 & 92 & 450 & 671 & 143 & 160 \\
\hline $4+1$ & 1.50 & 4.60 & 0.19 & 0.67 & 546 & 1660 & 91 & 45.3 & 670 & 144 & 159 \\
\hline 442 & 1.46 & 4.67 & $0.1 \mathrm{~s}$ & 0.68 & 553 & 1645 & 90 & 455 & 670 & 144 & 159 \\
\hline 443 & 1.41 & 4.75 & 0.18 & 0.70 & 5 fio & 1631 & $8 ?$ & 459 & 669 & 145 & 158 \\
\hline 444 & 1.37 & 4.83 & 0.17 & 0.71 & 567 & 1616 & $8 S$ & 460 & 669 & 145 & 157 \\
\hline 445 & 1.32 & 4.81 & 0.17 & 0.73 & 574 & 16,01 & 87 & 463 & 669 & 146 & 156 \\
\hline 416 & 1.23 & 4.99 & 0.16 & 0.74 & 592 & $15 \mathrm{Si}$ & 87 & 466 & 668 & 140 & 156 \\
\hline $4+7$ & 1.24 & 5.07 & 0.16 & 0.75 & 589 & 1571 & 80 & 469 & 663 & 147 & 155 \\
\hline 449 & 1.20 & 5.15 & 0.15 & 0.77 & 596 & 1557 & 86 & 471 & 667 & 147 & 154 \\
\hline 449 & 1.16 & 5.24 & 0.14 & 0.79 & 603 & 1542 & 86 & 473 & 667 & 147 & 153 \\
\hline 450 & 1.12 & 5.32 & 0.14 & 0.80 & 610 & 1527 & 86 & 476 & nicit & 149 & 152 \\
\hline 4.) 1 & 1.08 & 5.40 & 0.13 & 0.82 & 618 & 1512 & 87 & 479 & 666 & 148 & 152 \\
\hline 452 & 1.04 & 5.49 & 0.13 & 0.83 & 625 & 1497 & 87 & 481 & 666 & 149 & 151 \\
\hline 4.3 & 1.01 & 5.57 & 0.12 & 0.85 & 633 & 1483 & 89 & 494 & 665 & 149 & 150 \\
\hline 454 & 0.97 & 5.66 & 0.12 & 0.86 & 640 & 1463 & 88 & 486 & 665 & 149 & 149 \\
\hline 455 & 0.94 & 5.75 & 0.12 & 0.88 & 648 & 1453 & 89 & 499 & 664 & 150 & 148 \\
\hline 4.56 & 0.91 & 5.81 & 0.11 & 0.89 & 656 & 1439 & 90 & 492 & 66,4 & 150 & 148 \\
\hline 457 & 0.88 & 5.94 & 0.11 & 0.90 & 664 & 1423 & 92 & 494 & 663 & 150 & 147 \\
\hline 458 & 0.85 & 6. $0: 3$ & 0.11 & 0.92 & 671 & 1409 & 93 & 497 & 602 & 151 & 146 \\
\hline 459 & 0.82 & 6.13 & 0.10 & 0.94 & 679 & 1394 & 95 & 499 & 661 & 151 & 145 \\
\hline 460 & 0.80 & 6.23 & 0.10 & 0.95 & 687 & 1379 & 96 & 502 & 661 & 151 & 144 \\
\hline 461 & 0.77 & 6.32 & 0.10 & 0.97 & 695 & 1364 & 98 & 505 & 66,0 & 151 & 144 \\
\hline 462 & 0.75 & 6.49 & 0.10 & 0.99 & 703 & 1349 & 100 & 507 & 660 & 152 & 143 \\
\hline $4 f i 3$ & 0.73 & 6.51 & 0.10 & 1.00 & 711 & 1335 & 102 & 510 & 659 & 152 & 142 \\
\hline 464 & 0.71 & 6.61 & 0.10 & 1.02 & 710 & 1320 & 105 & 512 & 658 & 152 & 142 \\
\hline 465 & 0.69 & 6.71 & 0.09 & 1.03 & 727 & 1305 & 108 & 515 & 657 & 152 & 141 \\
\hline+66 & 0.69 & 6.81 & 0.09 & 1.05 & 735 & 1290 & 110 & 517 & 657 & 153 & 140 \\
\hline 467 & 0.66 & 6.91 & 0.09 & 1.06 & $i 43$ & 1275 & 113 & 520 & 656 & 153 & 139 \\
\hline 465 & 0.64 & 7.01 & 0.09 & 1.08 & 751 & 1261 & 116 & 523 & 655 & 153 & 139 \\
\hline 469 & 0.63 & 7.11 & 0.09 & 1.10 & 759 & 1246 & 119 & 525 & 654 & 154 & 139 \\
\hline 470 & 0.62 & 7.21 & 0.09 & 1.11 & 767 & 1232 & 123 & 529 & 653 & 154 & 137 \\
\hline 471 & 0.62 & 7.31 & 0.10 & 1.13 & 775 & 1217 & 127 & 531 & 652 & 154 & 136 \\
\hline 479 & 0.61 & 7.42 & 0.10 & 1.15 & 784 & 1203 & 131 & 533 & 651 & 155 & 136 \\
\hline 473 & 0.60 & 7.52 & 0.10 & 1.16 & 792 & 1189 & 135 & 536 & 649 & 155 & 135 \\
\hline 474 & 0.60 & 7.62 & 0.10 & 1.18 & 800 & 1175 & 139 & 539 & 648 & 155 & 134 \\
\hline 475 & 0.60 & 7.72 & 0.10 & 1.10 & 809 & 1161 & 143 & 542 & 647 & 156 & 133 \\
\hline 476 & 0.60 & 7.83 & 0.11 & 1.21 & 817 & $11+7$ & 148 & 545 & $6+6$ & 156 & 133 \\
\hline 477 & 0.60 & 7.94 & 0.11 & 1.23 & 826 & 1133 & 153 & 549 & 644 & 156 & 132 \\
\hline 478 & 0.60 & 8.04 & 0.11 & 1.24 & 834 & 1119 & 158 & 551 & 643 & 156 & 131 \\
\hline 479 & 0.61 & 8.15 & 0.12 & 1.25 & 843 & 1105 & 163 & 551 & 642 & 156 & 130 \\
\hline 490 & 0.61 & 8.26 & 0.12 & 1.27 & 851 & 1091 & 168 & 556 & 640 & 156 & 130 \\
\hline
\end{tabular}

32 July, 1873. 
TABLE IX, ARa. 2.-Continued.

\begin{tabular}{|c|c|c|c|c|c|c|c|c|c|c|c|c|c|c|}
\hline Arg. & $(v . c .0)$ & Diff. & $(v . s .1)$ & Diff. $s$ & Sec.var & (v.c.1) & Diff. \& & c.var & $(v . s .2)$ & Diff & ecvar. & .c.2) & Diff. & c.. \\
\hline & $" 1$ & " & " & " & " & " & $"$ & " & $"$ & " & " & " & " & " \\
\hline 480 & 14.65 & -0.24 & 9.02 & $0.4 \mathrm{I}$ & 2.71 & 187.06 & $+15 \mathrm{I}$ & 2.19 & 35.52 , & +0.04 & 2.89 & 217.47 & & 1.33 \\
\hline & $14.89^{\dagger}$ & $\begin{array}{r}+0.24 \\
0.24\end{array}$ & & $\begin{array}{r}\mathbf{H}^{0} .41 \\
0.42\end{array}$ & 2.72 & & $\begin{array}{r}+1.5 I \\
\text { I. } 51\end{array}$ & & 36.46 & 0.05 & 2.89 & & & \\
\hline 45 & 15.13 & $\begin{array}{l}0.24 \\
0.24\end{array}$ & 9.85 & $\begin{array}{l}0.42 \\
0.44\end{array}$ & 72 & 190 & $\begin{array}{l}\text { I. } 5 \text { I } \\
\text { I. } 51\end{array}$ & & 37.41 & $\begin{array}{l}0.95 \\
0.06\end{array}$ & 89 & 219.44 & $\begin{array}{l}0.98 \\
0.06\end{array}$ & 1. \\
\hline 48 & 15.37 & $\begin{array}{l}0.24 \\
0.24\end{array}$ & 10.29 & $\begin{array}{l}0.44 \\
0.45\end{array}$ & 2.73 & 191.59 & $\begin{array}{l}1.5^{1} \\
\text { I. } 50\end{array}$ & 2.15 & 38.37 & $\begin{array}{l}0.96 \\
0.98\end{array}$ & 2.88 & 220.40 & $\begin{array}{l}0.9^{\circ} \\
0.95\end{array}$ & 18 \\
\hline & & 0 & 701 & 0.47 & 74 & 193.09 & $\begin{array}{l}1.50 \\
\text { I. }\end{array}$ & .14 & 39.35 & $\begin{array}{l}0.98 \\
0.98\end{array}$ & .88 & 22 & 0.95 & \\
\hline 485 & $15.85+$ & +0.24 & $11.21+$ & +0.48 & 2.74 & $194.59+$ & $+\mathbf{r} .49$ & 2.12 & $40.33+$ & +0.99 & 2.88 & $222.29+$ & 0.03 & 1.26 \\
\hline & $\begin{array}{l}16.09 \\
16.33\end{array}$ & 0.24 & 12.19 & $0.5^{\circ}$ & $\begin{array}{l}2.75 \\
2.76\end{array}$ & & $\begin{array}{r}1.49 \\
\text { I. } 49\end{array}$ & & & & & & & 1.25 \\
\hline & $\begin{array}{l}16.55 \\
16.58\end{array}$ & 0.25 & 12.19 & 0.52 & $\begin{array}{l}2.76 \\
2.77\end{array}$ & $\begin{array}{l}197 \\
193\end{array}$ & I. 48 & & $\begin{array}{l}32 \\
33\end{array}$ & I.OI & 88 & 13 & $\begin{array}{l}0.91 \\
0.91\end{array}$ & 1.23 \\
\hline 489 & 16.83 & $\begin{array}{l}0.25 \\
0.25\end{array}$ & 13.24 & 0.53 & 2.77 & 200.53 & I. 48 & 2.07 & $\begin{array}{l}45.55 \\
44.35\end{array}$ & 1.02 & & & 0.90 & $\begin{array}{l}1.22 \\
1.20\end{array}$ \\
\hline 490 & 17.08 & & 13.79 & & 2.78 & 202.00 & & 2.06 & 45.38 & & 2.87 & 226.83 & & $\begin{array}{l}1.20 \\
1.19\end{array}$ \\
\hline & 17.3 & +0.25 & & +o. & 2.79 & & +1.46 & & & -I.04 & & & 8 & 1. \\
\hline 9 & 17.58 & 0.25 & 14.93 & $0.5^{8}$ & 2.79 & 204.92 & I. 46 & 2.03 & & r.04 & & & 87 & 1.1 \\
\hline 49 & 17.83 & 0.25 & 15.52 & 0.59 & 2.80 & 206.37 & I. & & & 1.05 & 2.86 & 22 & 85 & 1.1 \\
\hline $\begin{array}{l}494 \\
495\end{array}$ & 18. & 0.26 & 16.13 & $\begin{array}{l}0.6 \\
0.6\end{array}$ & 2.80 & 207 & $\begin{array}{l}\text { I. } 44 \\
\text { I. } 43\end{array}$ & 2 & 49.58 & $\begin{array}{l}1.07 \\
1.07\end{array}$ & 2.86 & 23 & $\begin{array}{l}0.84 \\
0.83\end{array}$ & 1.13 \\
\hline 95 & $\begin{array}{l}18.34 \\
18.60\end{array}$ & 0.26 & 16.75 & +0.6 & 2.81 & $\begin{array}{l}209.24 \\
210.67\end{array}$ & +1.43 & 1.99 & 50.65 & 08 & 2.85 & $0_{1}$ & 8 & 1.12 \\
\hline 0 & $\begin{array}{l}18.0 \\
18.8\end{array}$ & 0.25 & $\begin{array}{l}17 \\
18\end{array}$ & $\begin{array}{r}+0.04 \\
0.65\end{array}$ & $\begin{array}{l}2.81 \\
2.82\end{array}$ & $\begin{array}{l}210 \\
218\end{array}$ & $\begin{array}{r}1.43 \\
\text { I. } 42\end{array}$ & & & $\begin{array}{r}1.009 \\
1.09\end{array}$ & & & $\begin{array}{l}0.82 \\
0.8 \mathrm{I}\end{array}$ & 1.11 \\
\hline$\theta$ & 19. & 0.26 & $\begin{array}{l}18.04 \\
18.71\end{array}$ & 0.67 & $\begin{array}{l}2.82 \\
2.82\end{array}$ & 213 & I. $4 \mathrm{I}$ & & & $\begin{array}{l}1.09 \\
1.10\end{array}$ & & & 89 & 1.09 \\
\hline 499 & 19.36 & 0.25 & 19.39 & 0.68 & 2.83 & 214.90 & I. 40 & 1.93 & $\begin{array}{l}53.92 \\
55.03\end{array}$ & I. II & & & 0.78 & 1.07 \\
\hline 0 & 19.62 & & 20.09 & & 2.83 & 216.30 & & 1.92 & 56. & I. I I & 0.04 & 204.00 & 0.77 & 1.06 \\
\hline & & $\begin{array}{r}+0.26 \\
0.26\end{array}$ & & +0.71 & & & $\begin{array}{r}+1.39 \\
\end{array}$ & & & 12 & & & 76 & 1.04 \\
\hline 0 & 20.14 & 0.26 & 21.52 & 0.72 & 2.84 & $21 \%$ & I. 38 & & & & & & 0.75 & .0 \\
\hline & & 0.26 & 22.26 & 0.74 & 008 & & I. 37 & 1. & & $\begin{array}{l}1.14 \\
\end{array}$ & & & 0.73 & 1.0 \\
\hline 504 & 2 & & 23.01 & $\begin{array}{l}0.75 \\
0.76\end{array}$ & 2.84 & 221 & $\begin{array}{l}\text { I. } 37 \\
\text { I. } 36\end{array}$ & 1.8 & 60.67 & $\begin{array}{l}\text { I.15 } \\
\text { I.15 }\end{array}$ & 2.82 & $23 \varepsilon$ & $\begin{array}{l}0.72 \\
0.71\end{array}$ & 0.9 \\
\hline 505 & $\begin{array}{l}20.92 \\
21.18+\end{array}$ & 26 & $\begin{array}{l}23.7 \\
24.5\end{array}$ & +0.78 & $\begin{array}{l}2.85 \\
2.85\end{array}$ & $\begin{array}{l}223.17 \\
224.52+\end{array}$ & & & & $+\mathrm{r} .16$ & 81 & 23 & 40 & 0.97 \\
\hline & 21.44 & 0.26 & \begin{tabular}{l}
24.53 \\
\hdashline .34
\end{tabular} & 0.79 & & $\begin{array}{l}224 \\
225\end{array}$ & I. 33 & & & I.1 6 & & & 0.68 & 0. \\
\hline 003 & 21 & & 26.15 & & 2. & & & 1. & & & & & 0.67 & \\
\hline 509 & 21.5 & & 26.97 & $\begin{array}{l}82 \\
84\end{array}$ & 2.86 & 228.50 & I. 32 & 1.79 & 66.49 & I. & .79 & & 0.66 & 0.94 \\
\hline 510 & 22.21 & & 27.8 & & 2.86 & 229. & & 1 & 67. & 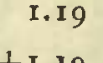 & .78 & 2 & 0.65 & 0.91 \\
\hline & 2 & to. 26 & 28. & $\begin{array}{r}-0.85 \\
0.86\end{array}$ & & & $\begin{array}{r}+1.30 \\
1.28\end{array}$ & & 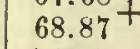 & $\begin{array}{l}-1.19 \\
\text { I. } 20\end{array}$ & & & +0.63 & \\
\hline & 22.72 & & 20 & 88 & & & $\begin{array}{l}1.28 \\
\text { I. } 28\end{array}$ & & & & & & & 0.8 \\
\hline & & & 30. & & 2 & 33 & & 1 & & & & & 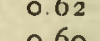 & 0.87 \\
\hline 514 & 2 & 0.26 & 31. & & 2. & 234.94 & $\begin{array}{l}1.27 \\
1.26\end{array}$ & 1. & 49 & $\begin{array}{l}1.21 \\
1.22\end{array}$ & 2. & 24 & $\begin{array}{l}0.60 \\
0.58\end{array}$ & 0.80 \\
\hline 15 & $\begin{array}{l}23.49 \\
23.75\end{array}$ & $\Gamma$ & $\begin{array}{l}32 . \\
33 .\end{array}$ & -0.91 & $\begin{array}{l}88 \\
88\end{array}$ & $6.20+$ & & 1.7 & 7 & 22 & 2.74 & 24 & + & 0.84 \\
\hline & & 0.25 & $\begin{array}{l}3 . \\
3\end{array}$ & 0.92 & & & 1. 24 & & & 1.23 & & & 0. & \\
\hline 518 & & & & 0.94 & & & & & & & & & 0. & \\
\hline 519 & 24.51 & 0. & 35.90 & $\begin{array}{l}0.95 \\
0.06\end{array}$ & 2.89 & 241.13 & I. 21 & 1.65 & 78.6 & I. & & & 0.53 & 0.81 \\
\hline 520 & 24.76 & & 36.8 & & 89 & 42 & & 1.63 & 79.8 & & & & & \\
\hline & & $\begin{array}{r}0.26 \\
0.25\end{array}$ & & & & & & & 8 & -1.25 & 2.71 & $34+$ & +0.50 & \\
\hline & & & & $\begin{array}{l}0.99 \\
1.00\end{array}$ & & & & & 82. & I. 25 & & 3 & 0 & 0.7 \\
\hline & & & & I.OY & & & & & 8 & & & & 0.47 & \\
\hline 52 & & 0. & 4 & $\begin{array}{l}1.01 \\
1.02\end{array}$ & 2. & .03 & & 1.5 & 84.88 & 1.26 & 2.67 & 249.76 & $\begin{array}{l}0.40 \\
0.45\end{array}$ & 0.75 \\
\hline 525 & 26. & -0. & 41.8 & -1.03 & $\begin{array}{l}2.89 \\
989\end{array}$ & 18. & 3 & 1.5 & 86. & & 2.66 & 50 . & & 0.71 \\
\hline & & 0. & & 1.04 & & & & & & & & & .43 & \\
\hline & & & & & & & & & & & & & & \\
\hline 529 & 27. & 0.24 & 46.04 & & 2.89 & 252.63 & & & & 1.28 & 2 & & $.4 \mathrm{I}$ & (1) 6 \\
\hline 530 & 2 & 0.24 & 47.1 & & 2.89 & $-\infty$ & & & & & & 51.86 & & \\
\hline & & +0.24 & & -1.09 & & & +1 & 49 & & $+\mathrm{r} .2$ & $\begin{array}{l}2.62 \\
2.61\end{array}$ & & $+0.3^{6}$ & 1.65 \\
\hline & & & & & & & 1.06 & & & 1.29 & & 0 & 5 & c \\
\hline & & & & & 2 & & 1.05 & & & & & & 34 & \\
\hline 534 & 28 & 0.23 & 51.53 & $\begin{array}{l}\text { I.II } \\
\text { I.I3 }\end{array}$ & 2.89 & 257.93 & 1.02 & 1.4 & 96.62 & 30 & 2.38 & 203.6 & $\begin{array}{l}0.32 \\
0.31\end{array}$ & 0.01 \\
\hline 535 & 28.42 & 22 & 52.6 & & 2.88 & 258.95 & +I.01 & 1.42 & & $-T_{0}$ & & & & \\
\hline & & o. & & $\mathrm{x}$. & & & & & & & & & 028 & \\
\hline & & & & - & & & o. & & & & & & & \\
\hline 0. & 29.33 & & 57.27 & & $\begin{array}{l}2.88 \\
2.88\end{array}$ & 26290 & 0.97 & & & I. 3 & & & & \\
\hline 40 & 29.55 & & 58.45 & & 2.88 & 263.85 & & & & & & & 0.2 & $0=54$ \\
\hline & & & & & & & & 1.34 & 105.56 & & 2.53 & 255.26 & & 0.5 \\
\hline
\end{tabular}




\begin{tabular}{|c|c|c|c|c|c|c|c|c|c|c|c|}
\hline \multirow[t]{2}{*}{ Arg. } & \multirow{2}{*}{$\frac{(v .8 .3)}{n}$} & \multirow{2}{*}{$\frac{(v . c .3)}{"}$} & \multirow{2}{*}{$\frac{(v .8 .4)}{11}$} & \multirow{2}{*}{$\frac{(v . c .4)}{\prime \prime}$} & \multirow[t]{2}{*}{ (p.c.0) } & \multirow[t]{2}{*}{$(0.8 .1)$} & \multirow[t]{2}{*}{ (p.c.1) } & \multirow[t]{2}{*}{ (p.8.2) } & \multirow[t]{2}{*}{ (p.c.2) } & \multirow[t]{2}{*}{$(p .8 .3)$} & \multirow[t]{2}{*}{ (p.c.3) } \\
\hline & & & & & & & & & & & \\
\hline 480 & 0.61 & 8.26 & 0.11 & 1.27 & 851 & 1091 & 168 & 556 & 640 & 156 & 130 \\
\hline 481 & 0.62 & 8.36 & 0.13 & 1.28 & 860 & 1076 & 173 & 559 & 639 & 156 & 129 \\
\hline 492 & 0.64 & 8.47 & 0.13 & 1.30 & 869 & 1063 & 179 & 561 & 637 & 156 & 128 \\
\hline 493 & 0.65 & 8.53 & 0.13 & 1.31 & 877 & 1049 & 194 & 564 & 636 & 156 & 128 \\
\hline 484 & 0.66 & 8.69 & 0.14 & 1.33 & 885 & 1035 & 190 & 566 & 634 & 156 & 127 \\
\hline 485 & 0.67 & 8.79 & 0.14 & 1.35 & 894 & 1021 & 196 & 569 & 633 & 156 & 126 \\
\hline 496 & 0.69 & 8.90 & 0. & 1.36 & 902 & 1007 & 202 & 571 & 632 & & 125 \\
\hline 487 & 0.71 & 9.01 & 0.15 & 1.37 & 911 & 934 & 208 & 574 & 30 & 156 & 124 \\
\hline 489 & 0.73 & 0.11 & 0.16 & 1.39 & 919 & 990 & 214 & 576 & 629 & 156 & 124 \\
\hline 489 & 0.75 & 9.23 & 0.17 & 1.10 & 927 & 966 & 220 & 579 & 627 & 156 & 123 \\
\hline 490 & 0.77 & 9.33 & 0.17 & 1.43 & 936 & 953 & 227 & 581 & 626 & 156 & 122 \\
\hline 491 & & 9. & 0 . & 1.43 & 945 & 940 & 234 & & 624 & & 121 \\
\hline 492 & 3 & 9.54 & 0.19 & 1.44 & 954 & 926 & 241 & 586 & 623 & 156 & 120 \\
\hline 493 & 0.85 & 9 & 0.19 & 1.46 & 963 & 913 & 243 & 589 & 621 & 156 & 120 \\
\hline 494 & 038 & 9.75 & 0.20 & 1.47 & 972 & 900 & 256 & 591 & 619 & 156 & 119 \\
\hline 495 & 0.91 & 9.86 & 0.21 & 1.49 & 981 & 887 & 264 & 594 & 618 & 156 & 118 \\
\hline 496 & & & & 1.50 & 990 & 874 & 272 & 5 & 616 & & 117 \\
\hline 497 & 0.99 & 10.07 & 0.23 & 1.51 & 999 & 861 & 280 & 599 & 615 & 156 & 117 \\
\hline 493 & 1.02 & 10.18 & 0.23 & 1.53 & 1008 & 848 & 288 & 601 & 613 & 155 & 116 \\
\hline 499 & 1.06 & 10.29 & 0.24 & 1.54 & 1017 & 835 & 296 & 604 & 611 & 155 & 115 \\
\hline 500 & 1.10 & 10.39 & 0.25 & 1.55 & 1026 & 822 & 304 & 606 & 610 & 155 & 115 \\
\hline 501 & 1 & 1 & & 1.57 & 1035 & & 312 & & & & 114 \\
\hline 502 & 1.19 & 10.60 & 7 & 1.58 & 1044 & 797 & 321 & 611 & 606 & 1 & 114 \\
\hline 503 & 1.24 & 10.70 & 0.29 & 1.59 & 1053 & 785 & 322 & 613 & 604 & 1 & 113 \\
\hline 504 & 1.29 & 10.81 & 0.29 & 1.60 & 1063 & 772 & 339 & 615 & 602 & 154 & 113 \\
\hline 505 & 1.34 & 10.91 & 30 & 1.62 & 1073 & 760 & 346 & 617 & 600 & 154 & 112 \\
\hline 506 & & 1 & & 1.63 & 1031 & 743 & 355 & 62 & 598 & & 112 \\
\hline 507 & 1.44 & 11.12 & 2 & 1.64 & 1090 & 736 & 364 & 622 & 596 & & 111 \\
\hline 508 & 1.50 & 11.22 & 3 & 1.65 & 1093 & 724 & 373 & 624 & 594 & & 110 \\
\hline 509 & 1.56 & 11.32 & 0.34 & 1.66 & 1108 & 712 & 382 & 626 & 592 & 153 & 110 \\
\hline 510 & 1.62 & 11.42 & 0.35 & 1.67 & 1117 & 700 & 391 & 628 & 590 & 153 & 109 \\
\hline 511 & 1 & 11 & 7 & 1. & 11 & $f$ & d & & & 1 & 11 \\
\hline 512 & 1. & 11.62 & 8 & 1.69 & 1135 & 7 & 410 & $6:$ & 5 & 15 & 108 \\
\hline 513 & 1.80 & 11.71 & 0.39 & 1.70 & $114 t$ & 666 & 420 & 63 & 58 & 153 & 108 \\
\hline 514 & 1 & 11.81 & 0 & 1.71 & 1153 & 654 & 430 & & 50 & 153 & 107 \\
\hline 515 & 1 & 11 & 0 & 1.72 & 1162 & $6+3$ & $\$ 40$ & 639 & 578 & 153 & 106 \\
\hline 516 & 2. & $1:$ & & 1.73 & 1171 & & & 6 & & & 106 \\
\hline 517 & 2. & 12.09 & 3 & 1.74 & 1180 & 1 & 46 & 64 & 573 & 1 & 106 \\
\hline 513 & 2 & 12.19 & 5 & 1.75 & 1189 & 6 & 471 & & 571 & & 105 \\
\hline 519 & 2.23 & 12.28 & 0.46 & 1.76 & 1197 & 599 & 481 & 648 & 569 & 152 & 105 \\
\hline 520 & 2.31 & 12.37 & 0.7 & 1.77 & 1206 & 588 & 493 & 650 & 566 & 152 & 104 \\
\hline & & 1 & & 1 & 1215 & 5 & & & 56 & & 104 \\
\hline 522 & 2.4 & 12.55 & & 1.79 & 1224 & 567 & 514 & & & & 103 \\
\hline & & 12.63 & 0.51 & 1.79 & 1233 & 557 & 525 & & 558 & & 103 \\
\hline 5 & 2.68 & 12.72 & 0.53 & 1.80 & 1242 & 546 & 536 & 658 & 556 & 151 & 103 \\
\hline 525 & 2.71 & 12.81 & & 1.81 & 1250 & 536 & $5+8$ & 660 & 553 & 151 & 102 \\
\hline & & & & & & & & & & & \\
\hline 5 & 2 & 1 & 0. & 1. & 12 & & 5 & & & & 1 \\
\hline & & 13.0 & & 1.83 & 12 & & 5 & & & & 101 \\
\hline 52 & 3.06 & 13.1 & 0.60 & 1.84 & 1284 & 498 & 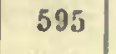 & & $0+2$ & 150 & 100 \\
\hline 530 & 15 & 1 & 0 & 1.8 & 1233 & 48 & 607 & 670 & 540 & 150 & 100 \\
\hline & & 13.3 & & 1.85 & 1301 & & & & & & 100 \\
\hline & & 13.3 & $0.6 t$ & 1.86 & 1310 & 470 & 630 & 673 & 535 & & 99 \\
\hline & 3.43 & 13.45 & 0.65 & 1.36 & 1313 & 461 & 642 & & 532 & 149 & 99 \\
\hline 53 & 3.5 & 13.52 & 0.67 & 1.87 & 1327 & 452 & 654 & 677 & 529 & 149 & 98 \\
\hline 535 & & 13.59 & 0.68 & 1.87 & 1335 & 443 & 666 & 678 & 527 & 149 & 98 \\
\hline & & & & & & & & & & & 98 \\
\hline & & 13.73 & & 1.88 & 1352 & 42 & 690 & 68 & 521 & 149 & 98 \\
\hline 53 & 3.9 & 13.80 & 0.73 & 1.89 & 1360 & 417 & 702 & 683 & 519 & 149 & 97 \\
\hline 532 & 4.03 & 13.97 & 0.74 & 1.89 & 1368 & 409 & 714 & $6 \$ 5$ & 516 & 148 & 97 \\
\hline 540 & 4.13 & 13.93 & 0.75 & 1.90 & 1376 & 401 & 726 & 686 & 513 & 147 & 97 \\
\hline
\end{tabular}


TABLE IX, Ara. 2.-Concluded.

\begin{tabular}{|c|c|c|c|c|c|c|c|c|c|c|c|c|c|c|}
\hline Arg. & $(v, c .0)$ & Diff. & $(v . s .1)$ & Diff. & Sec.var. & $\cdot(v, c .1)]$ & Diff. & Scc.var. & $(v . s .2)$ & DI & NeC. Valo & v.c.2) & 131. & 6.18 \\
\hline & " & $" \prime$ & $"$ & $"$ & $" \prime$ & "I &.$\prime \prime$ & $" \prime$ & $"$ & $"$ & $"$ & " & $" 1$ & $"$ \\
\hline 540 & 29.55 & +0.22 & 58.45 & I. 18 & 2.89 & 263.85 & & 1.34 & 105.56 & & 2.53 & 255.26 & & 0.54 \\
\hline 1 & & 0.22 & & I. 19 & $\begin{array}{l}2.38 \\
2.88\end{array}$ & $264.79^{\dagger}$ & $\begin{array}{r}+0.94 \\
0.92\end{array}$ & 1.33 & $106.88^{-1}$ & I. 31 & 1 & $9^{t}$ & 0.21 & 0.53 \\
\hline 2 & $\begin{array}{l}2 \\
3 \\
3\end{array}$ & 0.22 & 2 & I. 20 & $\begin{array}{l}88 \\
97\end{array}$ & 265.71 & 0.91 & 1.32 & & I. $3^{2}$ & & & & 2 \\
\hline 44 & 3 & 0.22 & 6 & 1.22 & $\begin{array}{l}87 \\
87\end{array}$ & 266.62 & 0.90 & 1.30 & 10 s & $1 \cdot 3^{2}$ & & & 0.18 & 0.51 \\
\hline 45 & 30 & $0.2 \mathrm{I}$ & 64.46 & I. 22 & & 267.52 & 0.88 & & 3 & I. $3^{2}$ & & 20 & 0.17 & 0.50 \\
\hline 70 & & $-0.2 \mathrm{~J}$ & $64.46+$ & +1.23 & $\begin{array}{l}87 \\
87\end{array}$ & $\begin{array}{l}263.40 \\
269.27\end{array}+$ & +0.87 & $\begin{array}{l}1.27 \\
1.26\end{array}$ & $112.15+$ & +1.32 & & $256.41+$ & +o.16 & \\
\hline 17 & 06 & 0.21 & 66.93 & I. 24 & 87 & 270.12 & 0. & & & I. 33 & & & o. & \\
\hline 8 & 27 & 0.21 & 68.18 & $\begin{array}{l}1.25 \\
.25\end{array}$ & 86 & 96 & & 1.23 & 11 & 1.3 & 4 & & & 6 \\
\hline 549 & 31 & $\begin{array}{l}0.20 \\
0.20\end{array}$ & & $\begin{array}{l}1.25 \\
1.26\end{array}$ & 2.86 & 2 & $\begin{array}{l}0.8 \\
0.8\end{array}$ & 1.22 & 11 & $\begin{array}{l}\text { I. } 33 \\
\text { I. } 32\end{array}$ & 3 & & $\begin{array}{l}0 . \\
0 .\end{array}$ & 5 \\
\hline 550 & 31.67 & -0.20 & & +1.28 & 2.86 & 272.60 & .80 & 1.21 & $113.77+$ & & 2.42 & .89 & & .44 \\
\hline 1 & & 0.19 & & 1.27 & 2.96 & & 0.78 & & $120.09^{\dagger}$ & 1.3 & & & & \\
\hline $\begin{array}{l}2 \\
3\end{array}$ & 6 & 0.19 & & I. 28 & 2 & 8 & 0.77 & & 2 & 1.3 & & & & 42 \\
\hline 54 & & 0.19 & 74.51 & I. 29 & 35 & 5 & 0.75 & 1. & & I. 3 & & & & 11 \\
\hline $\begin{array}{l}54 \\
55\end{array}$ & 3 & 0.19 & & I. 30 & & & 0.74 & & & 1. 3 & 8 & & 0.03 & \\
\hline 55 & 32. & -O. I 8 & 77. & & 2.84 & 276.44 & & 1.14 & $39+$ & & 2.36 & 257 & & .40 \\
\hline 56 & & 0.18 & & & & & & & & & & & & \\
\hline$b$ & & 0.18 & & $1.3^{2}$ & & & 0. & & & 1. & & & & \\
\hline & 33.35 & 0.18 & & I. $3^{2}$ & & & $\begin{array}{l}.09 \\
0.68\end{array}$ & & & 1.3 & & & 0. & \\
\hline 30 & Dar.o & 0.17 & & 1.33 & 2 & $0=00$ & 0.66 & & 13 & I. 34 & 2.32 & & 0.04 & 0.36 \\
\hline 9 & & -0.17 & $\begin{array}{l}83.6 \\
85.0\end{array}$ & +1.33 & & & & 1.06 & & $T=$ & 31 & 25 & & 0.35 \\
\hline 32 & & O.I 7 & 8 & $1 \cdot 34$ & & & 0.63 & & & $1.3^{2}$ & & & & \\
\hline 563 & & 0.17 & 87.69 & I. 34 & 01 & 1. 3 & $0.6 \mathrm{I}$ & & & 1.32 & & & 0.08 & \\
\hline 64 & 3 & 0.16 & 89.04 & I.35 & .80 & 82.38 & 0.60 & & 36 & I. 31 & & & 0. & \\
\hline 65 & & & 90. & $=26$ & 80 & 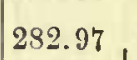 & 59 & 99 & 0 & I. 32 & & & 0. & \\
\hline 36 & & 0.16 & & $3^{6}$ & & & & 9 & &.+ \pm & & & & \\
\hline 30 & & 0.15 & 9 & $\begin{array}{l}\text { I. } 37 \\
\text { I. } 37\end{array}$ & & 9 & 0.55 & & & 1.31 & & & - $>$ - & \\
\hline & & 0.15 & 9 & $\begin{array}{l}1.37 \\
\text { I. } 38\end{array}$ & & & 0.54 & & & I. 32 & & & & \\
\hline & & 0.15 & 95. & $\begin{array}{l}1.3^{8} \\
\text { I. } 3^{8}\end{array}$ & 2.78 & & $\begin{array}{l}0.53 \\
0.51\end{array}$ & 0.94 & 43 & $\begin{array}{l}\text { I. } 3 \\
1.3\end{array}$ & 2.20 & 00.14 & & \\
\hline 0 & & -0.15 & & $-\mathbf{- 1} \cdot 3^{8}$ & 77 & 285.67 & & 0.93 & 145 & . & & 6 & & \\
\hline & & 0.14 & & 39 & & & & & & & & & & 26 \\
\hline & & 0.14 & 1 & (5) & & & & & & I. 2 & & & & \\
\hline & & 14 & 102.82 & 40 & & & & & & 1.3 & & & & \\
\hline & & O.I3 & & 1.40 & & & 0.43 & 0 & 150.35 & I. 29 & & 255 & & 0.25 \\
\hline 5 & & 13 & & -I & 2.74 & 287. & 2 & 0 & & I. $2 \quad \longrightarrow$ & & & & \\
\hline & & o. I 3 & & I & & & & & & 1. & & & & \\
\hline & & 0.13 & 1 & I. $4 \mathrm{I}$ & & & $0.3^{8}$ & & & $\begin{array}{l}1.29 \\
\text { I. } 28\end{array}$ & & & 0. & \\
\hline & 36. & 0.12 & 109 & I. $4 \mathrm{I}$ & & & 0.37 & & & 1.2 & & & . & \\
\hline & & 0 & 11118 & & 2.7 & & $0.3^{6}$ & & & 1.28 & & 25 & $0.3^{I}$ & 0 \\
\hline & & -0.12 & $\begin{array}{l}111.2 \\
112.6\end{array}$ & -1.42 & 2.71 & 28 & & 0 & & 7 & 2.05 & 53 & & 0.21 \\
\hline & & 2 & 1 & 1.42 & & & 0. & & & 1. & & & & \\
\hline & & 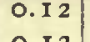 & II & I & & & 0. & & & & & & & \\
\hline 584 & 36. & it & 116. & I. & & & & & & I. & & & 0.3 & \\
\hline 585 & 3 & & & & & & 0 & & 163 & 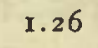 & & 252.12 & 0.37 & 0.19 \\
\hline & & & 119. & $-\mathbf{I}$ & & 2 & & 0.73 & 164 & & 1.98 & 5. & & \\
\hline & & & 121 & I. & & & & & & & & & & \\
\hline & & I I & 12 & & & & & & & & & & & \\
\hline 589 & 37 & I I & 124. & & 2.6 & & o. & & & I. 2 & & & & \\
\hline 30 & 37 & & 125 & & & & 0.2 & $0.0 \mathrm{~J}$ & 100.00 & I. 2 & & & 0.44 & 0 . \\
\hline & & 0 & & -1. 44 & $62>->$ & & & 0.68 & 170 & & 1 & & & 0.16 \\
\hline & & 0 & 128 & 43 & & & & & & & & & & \\
\hline & & 0.10 & & & & & & & & & & & & \\
\hline 594 & 37 & & $13 \mathrm{I}$ & I. 44 & & & & & & 1.22 & & & & \\
\hline 95 & & & & & & & & & & $.2 I$ & & 237.83 & & \\
\hline & & 0.10 & 134. & -1.4 & 2.58 & 993 & & 0.62 & 176 & & 7 & & & 0.1 \\
\hline & & & 135. & & & & & & & & & & & \\
\hline & & & 13 & 1.4 & & & 8 & & & & & & & \\
\hline (2) & 38. & & 138.50 & & & & 0.0 & & & I. I & & & & \\
\hline 600 & 38.5 & & 139.94 & & & & 0.05 & & 181 & I. I 8 & & 24 & & \\
\hline & & & & & 2.53 & 293.78 & & 0.56 & 182.62 & & 1.77 & 244.67 & & 0.12 \\
\hline
\end{tabular}


THFORBITOFUR $\mathrm{N}$ O US.

\begin{tabular}{|c|c|c|c|c|c|c|c|c|c|c|c|}
\hline Arg. & $(v .8 .3)$ & $(v . c .3)$ & $(v .8 .4)$ & (v.c.t) & $(p . c .0)$ & $(p .8 .1)$ & $(p, c .1)$ & $|(0.8 .2)|$ & (p.c. 2 & $|(\rho, k, 3)|$ & (p.c.3 \\
\hline & $"$ & $"$ & $"$ & $"$ & & & & & & & \\
\hline 540 & 4.13 & 13.93 & 0.75 & 1.90 & 1376 & 401 & 726 & 686 & 513 & 147 & 97 \\
\hline 541 & 4.23 & 13.99 & 0.76 & 1.90 & 1394 & 393 & 739 & 689 & 510 & 147 & 97 \\
\hline $5+3$ & 4.34 & 14.05 & 0.78 & 1.90 & 1392 & 336 & 751 & 689 & 507 & 147 & 96 \\
\hline 543 & 4.45 & 14.11 & 0.79 & 1.90 & 1400 & 878 & 763 & 691 & 503 & 146 & 96 \\
\hline $5+4$ & 4.56 & 14.17 & 0.81 & 1.91 & 1408 & 371 & 776 & 692 & 500 & 146 & 95 \\
\hline $5+5$ & 4.67 & 14.23 & 0.83 & 1.91 & 1416 & 363 & 788 & 6.93 & $49 \%$ & $1+6$ & $95^{\circ}$ \\
\hline 546 & 4.79 & 14.28 & 0.84 & 1.91 & 1424 & 356 & 801 & 695 & 491 & 146 & 95 \\
\hline 5.17 & 4.90 & 14.34 & 0.85 & 1.91 & 1431 & 349 & 813 & 696 & 491 & 146 & 24 \\
\hline 518 & .01 & 14.39 & 0.87 & 1.92 & 1439 & 343 & 826 & 698 & 187 & 145 & 24 \\
\hline 549 & 5.12 & 14.44 & 0.89 & 1.92 & 1446 & 336 & 838 & 699 & 484 & 145 & 94 \\
\hline 550 & 5.24 & 14.49 & 0.90 & 1.92 & 1454 & 329 & 851 & 700 & 481 & 145 & 93 \\
\hline 551 & 5.35 & 14.54 & 0.92 & 1.92 & 1461 & 323 & 864 & 702 & 478 & 145 & 93 \\
\hline 5502 & 5.47 & 14.59 & 0.93 & 1.92 & 1468 & 316 & 877 & 703 & 475 & 145 & 93 \\
\hline 553 & 5.58 & 14.63 & 0.95 & 1.92 & 1475 & 310 & 890 & 704 & 171 & 144 & 93 \\
\hline 554 & 5.70 & 11.67 & 0.96 & 1.02 & 1492 & 304 & 903 & 705 & 468 & 144 & 92 \\
\hline 555 & 5.82 & 14.71 & 0.98 & 1.92 & 1499 & 298 & 916 & 706 & 465 & 144 & 92 \\
\hline 556 & 5.9 .1 & 14.75 & 0.99 & 1.92 & 1496 & 292 & 929 & 708 & 462 & 144 & 92 \\
\hline 557 & 6.06 & 14.79 & 1.01 & 1.92 & 1502 & 287 & 943 & 703 & 458 & 144 & 92 \\
\hline 558 & 6.19 & 14.82 & 1.02 & 1.92 & 1503 & 281 & 956 & 710 & 455 & 143 & 92 \\
\hline 553 & 6.30 & 14.55 & 1.04 & 1.92 & 1515 & 276 & 970 & 711 & 452 & 143 & 91 \\
\hline 560 & 6.42 & 14.98 & 1.05 & 1.92 & 1522 & 270 & 983 & 712 & 149 & 143 & 91 \\
\hline 561 & 6.54 & 14.91 & 1.07 & 1.92 & 1528 & 265 & 996 & 713 & 445 & 143 & 91 \\
\hline 562 & 6.67 & 14.93 & 1.03 & 1.92 & 1534 & 260 & 1010 & 714 & 442 & 143 & 91 \\
\hline 563 & 6.79 & 14.96 & 1.10 & 1.91 & 1541 & 255 & 1023 & 715 & 439 & 142 & 91 \\
\hline 564 & 6.91 & 14.99 & 1.11 & 1.91 & 1547 & 250 & 1036 & 716 & 435 & 142 & 90 \\
\hline 565 & 7.04 & 15.01 & 1.13 & 1.21 & 1553 & 246 & 1049 & 716 & 431 & 112 & 90 \\
\hline 566 & 7.16 & 15.03 & 1.14 & 1.91 & 1559 & 242 & 1063 & 717 & 428 & 142 & 90 \\
\hline 567 & 7.29 & 15.05 & 1.16 & 1.90 & 1564 & 239 & 1076 & 717 & 424 & 142 & 90 \\
\hline 568 & 7.41 & 15.06 & 1.17 & 1.90 & 1570 & 234 & 1099 & 717 & 421 & 141 & 90 \\
\hline 569 & 7.54 & 15.08 & 1.18 & 1.90 & 1575 & 230 & 1102 & 718 & 417 & $14 I$ & 89 \\
\hline 570 & 7.66 & 15.09 & 1.20 & 1.99 & 1581 & 226 & 1116 & 718 & 413 & 141 & 89 \\
\hline 571 & 7.79 & 15.09 & 1.21 & 1.89 & 1586 & 223 & 1129 & 718 & 410 & 141 & 89 \\
\hline 572 & 7.91 & 15.10 & 1.23 & 1.89 & 1591 & 219 & 1142 & 718 & 406 & 141 & 89 \\
\hline 573 & 8.04 & 15.10 & 1.24 & 1.88 & 1596 & 210 & 1155 & 719 & 403 & 140 & 89 \\
\hline 574 & 8.16 & 15.11 & 1.26 & 1.88 & 1601 & 213 & 1169 & 719 & 399 & 140 & 89 \\
\hline 575 & 8.29 & 15.11 & 1.27 & 1.87 & 1605 & 210 & 1182 & 710 & 395 & 140 & 89 \\
\hline 576 & 8.42 & 15.12 & 1.29 & 1.86 & 1610 & 207 & 1195 & 710 & 392 & 140 & 88 \\
\hline 577 & 8.54 & 15.12 & 130 & 1.86 & 1614 & 205 & 1208 & 719 & 398 & 140 & 88 \\
\hline 578 & 8.67 & 15.11 & 1.32 & 1.85 & 1618 & 202 & 1221 & 719 & 395 & 140 & 89 \\
\hline 579 & 8.80 & 15.10 & 1.33 & 1.84 & 1622 & 200 & 1234 & 719 & 381 & 139 & 88 \\
\hline 590 & 8.93 & 15.09 & 1.34 & 1.84 & 1626 & 198 & 1348 & 719 & 378 & 139 & 89 \\
\hline 591 & 9.05 & 15.08 & 1.35 & 1.83 & 1630 & 196 & 1261 & 719 & 374 & 139 & 87 \\
\hline 592 & 9.18 & 15.07 & 1.36 & 1.83 & 1634 & 194 & 1274 & 510 & II & 139 & 87 \\
\hline 583 & 9.31 & 15.05 & 1.38 & 1.82 & 1637 & 192 & 1289 & 719 & 367 & 139 & 87 \\
\hline $58 t$ & 9.43 & 15.04 & 1.39 & 1.81 & 1641 & 191 & 1301 & 710 & 364 & 139 & 87 \\
\hline 595 & 9.56 & 15.02 & 1.40 & 1.81 & 1645 & 189 & 1315 & 718 & 360 & 139 & 87 \\
\hline 586 & 9.68 & .15 .00 & 1.42 & 1.80 & 1648 & 188 & 1329 & 719 & 357 & 139 & 87 \\
\hline 587 & 9.81 & 14.98 & 1.43 & 1.79 & 16.51 & 186 & 1342 & i1s & 353 & 139 & 87 \\
\hline 593 & 9.93 & 14.96 & 1.44 & 1.79 & 1654 & 185 & 1356 & 717 & 350 & 139 & 86 \\
\hline 589 & 10.06 & 14.93 & 1.45 & 1.78 & 1657 & 184 & 1369 & 717 & 346 & 138 & 86 \\
\hline 590 & 10.18 & 14.91 & 1.46 & 1.77 & 1660 & 183 & 1382 & 717 & $3+3$ & 138 & 86 \\
\hline 591 & 10.31 & 14.88 & 1.48 & 1.76 & 1662 & 182 & 1396 & 716 & 339 & 138 & 86 \\
\hline 592 & 10.43 & 14.85 & 1.49 & 1.75 & 1665 & 182 & 1409 & 716 & 335 & 138 & 86 \\
\hline 593 & 10.55 & 14.81 & 1.50 & 1.74 & 1667 & 181 & 1422 & 716 & 332 & 138 & 86 \\
\hline 594 & 10.68 & 14.78 & 1.51 & 1.73 & 1669 & 181 & 1436 & 715 & 328 & 139 & 85 \\
\hline 595 & 10.80 & 14.74 & 1.52 & 1.72 & 1671 & 181 & 1448 & 715 & 324 & 138 & 85 \\
\hline 596 & 10.92 & 14.71 & 1.53 & 1.72 & 1673 & 181 & 1461 & 714 & 321 & 138 & 85 \\
\hline 597 & 11.04 & 14.67 & 1.55 & 1.71 & 1674 & 181 & 1474 & 714 & 317 & 137 & 85 \\
\hline 598 & 11.16 & 14.6 .3 & 1.56 & 1.70 & 1676 & 151 & 1457 & 713 & 313 & 137 & 85 \\
\hline 599 & 11.28 & 14.59 & 1.57 & 1.69 & 1677 & 182 & 1500 & 712 & 310 & 137 & 85 \\
\hline 600 & 11.40 & 14.53 & 1.58 & 1.68 & 1678 & 182 & 1513 & 711 & 306 & 137 & 85 \\
\hline
\end{tabular}


TABle X, Ara. 3.-A ction of Neptune.

$\Lambda$ rg. $\mid$ (v.c.0) Diff. $\mid(v . s .1)$ Difr. $\mid$ (v.c.1) Diff. $\mid$ (v.s.2) Diff. (v.c.2) Diff. (v.s.3) (v.c.3) (v.s.4)|(v.c.4)

99.96

$99.67-0.29$

$\begin{array}{lll}92.38 & 0.29\end{array}$

0.87 +o. I I

34.22 " 7.42

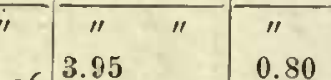

1.10 0.12

\begin{tabular}{l|l|l|l|l}
$34.77^{+0.55}$ & $7.3 i^{-0.06}$ & $3.82^{-0.13}$ & 0.81
\end{tabular}

\begin{tabular}{c|r|r|}
\hline$"$ & \multicolumn{1}{c}{ " } & \multicolumn{1}{c}{$"$} \\
1.15 & -0.85 & -1.13 \\
1.16 & 0.86 & 1.14
\end{tabular}

$\begin{array}{ll}91.77 & 0.30 \\ 91.31\end{array}$

$91.46-0.31$

\begin{tabular}{ll|l}
1.23 & 0.13 & 35.85
\end{tabular}

0.547 .30

\begin{tabular}{l|ll|l}
0.06 & 3.82 & 0.12 & 0.81 \\
0.07 & 3.70 & 0.12 & 0.83
\end{tabular}

\begin{tabular}{l|l|l|}
1.18 & 0.87 & 1.15
\end{tabular}

\begin{tabular}{ll}
1.38 & 0.15 \\
\hline & 0.15
\end{tabular}

36.39

0.547 .23

\begin{tabular}{lll|l}
0.07 & 3.58 & 0.12 & 0.84
\end{tabular}

\begin{tabular}{l|l|l}
1.20 & 0.89 & 1.16
\end{tabular}

$\begin{array}{rrr}91.15 & -0.3 \mathrm{I} \\ 90.84 & 0.3 \mathrm{I}\end{array}$

$1.53+0.17$

$36.99-0.53$ i

\begin{tabular}{ll|ll}
90.84 & $0.3 \mathrm{I}$ & 1.88 & 0.18 \\
00.59 & $0.3^{2}$ & 1.88 & 0.20
\end{tabular}

$37.44+0.52 \quad 7.01-0.08 \quad 3.36-0$. I I

0.88

1.22

$0.91 \quad 1.17$

\begin{tabular}{lll}
90.19 & 0.33 \\
\hline 89.86 & 0.33
\end{tabular}

2.29 0.21

$\begin{array}{lll}37.96 & 0.5^{2} & 6.92 \\ 38.48 & 0.52 & 0.92\end{array}$

0.09 \begin{tabular}{ll|}
3.25 & 0.11 \\
3.15 & 0.10
\end{tabular}

\begin{tabular}{lr|r|r}
0.88 & 1.23 & -0.93 & -1.18 \\
0.90 & 1.24 & 0.95 & 1.18
\end{tabular}

89.86

$2.51+0.24$

$\begin{array}{llll}38.48 & 0.5^{2} & 6.89\end{array}$

0.103 .15

\begin{tabular}{l|l}
0.10 & 0.91 \\
\hline 0.10 & 0.93
\end{tabular}

1.24

$89.53-0.33$

\begin{tabular}{ll}
2.75 & 0.24 \\
\hline & .99
\end{tabular}

39.51

$0.5^{2} 6.72$

0.103 .05

.09

12

$\begin{array}{ll}89.18 & 0.35 \\ 88.83 & 0.35\end{array}$

$\begin{array}{ll}3.26 & 0.27 \\ 3.54 & 0.28\end{array}$

$40.02+0.51 \quad 6.61-$

2.86

\begin{tabular}{ll|l}
1.25 & 0.97 & 1.19
\end{tabular}

$\begin{array}{ll}88.47 & 0.36 \\ 8.38 & 0.38\end{array}$

1

88.09

$\begin{array}{ll}3.54 & 0.28 \\ & 0.29\end{array}$

$\begin{aligned} & 3.83 \\ & 4.13\end{aligned}+0.30$

\begin{tabular}{ll|l|l}
40.52 & 0.50 & 6.40 \\
41.01 & 0.49 & 0.28
\end{tabular}

- $10 \quad 2.86-0.09$

0.97

1.27

\begin{tabular}{l|l}
1.00 & 1.19 \\
1.20
\end{tabular}

$16 \quad 87.71-0.3^{8}$

$\begin{array}{ll}87.31 & 0.40 \\ 8.41 & 0.4 \mathrm{I}\end{array}$

$\begin{array}{ll}4.13 & 0.32\end{array}$

$\begin{array}{ll}86.49 & 0.4 \mathrm{I} \\ 8.43\end{array}$

$\begin{array}{ll}4.78 & 0.33\end{array}$

4

$5.49+0.38$

$85.62-0.44$

$\begin{array}{lll}5.87 & 0.39 \\ 6.26 & 0.41\end{array}$

$6.67 \quad 0.41$

84.69

0.47

$7.09 \quad 0.42$

83.72

0.50

7.53

0.44

$83.22^{-0.50}$

$7.98+0.45$

89.17

0.52
0.53
0.55

$\begin{array}{ll}8.95 & 0.49\end{array}$

$\begin{array}{ll}81.62 & 0.55 \\ 81.05 & 0.57\end{array}$

$\begin{array}{ll}9.44 & 0.50 \\ & 0.51\end{array}$

$30 \quad 81.05$

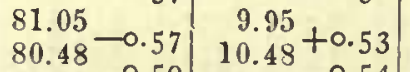

\begin{tabular}{l|l}
31 & 80.48 \\
32 & 79.89
\end{tabular}

79.29

\begin{tabular}{l|ll}
0.59 & 11.02 & 0.54 \\
0.60 & 11.58 & 0.56
\end{tabular}

78.68

$0.61 \quad 11.58$

78.05

0.63

35

36

37
38

39

76.76

76.10

$-0.64$

12.73

0.57
0.58

12.73

$+0.60$

75.42

\begin{tabular}{l|l}
0.65 & 13.94 \\
0.66 & 14.57
\end{tabular}

0.61
0.63

40

\begin{tabular}{l|l}
$74.73-0.69$ & 15.86 \\
74.04 & $16.52+0.66$
\end{tabular}

0.68
0.69

15.21

73.33

\begin{tabular}{l|l}
0.71 & 17.19 \\
0.72 & 17.87
\end{tabular}

72. 61

71.88

$0.73 \quad 17.87$

0.74

$\begin{array}{ll}18.56 & 0.69\end{array}$

70.39

$-0.75$

19.26

69.63

0.76

19.97

0.70

47

$48 \quad 68.8$

49

50

68.10

$\begin{array}{ll}1.40 & 0.72\end{array}$

67.32

0.78

5

52

5

55

$\begin{array}{rr}66.54 & -0.78 \\ 65.75 & 0.79 \\ 64.95 & 0.79\end{array}$

64.96

64.16

0.80
0.81

22.85

.

$\begin{array}{ll}24.32 & 0.74\end{array}$

$\begin{array}{ll}25.06 & 0.74 \\ 25.70 & 0.73\end{array}$

63.35

$\begin{array}{ll}25.79 & 0.73 \\ 2.74\end{array}$

$62.54-0.8$

26.53

0.74

57

58
59

61.730 .81

$27.27+0.74$
$27.99 \quad 0.72$

\begin{tabular}{ll|ll}
60.92 & 0.81 & 27.99 & 0.72 \\
\hline & 0.81 & 28.72 & 0.73
\end{tabular}

60.11

$0.8 \mathrm{I} \quad 29.72$

0.73
0.72

30.17

0.72

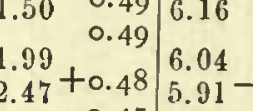

०. I 2.69

O. 12 2.61

0.08

$1.00 \quad 1.28-1.04-1.20$

\begin{tabular}{ll|l|l|}
0.07 & 1.05 & 1.29
\end{tabular}

\begin{tabular}{l|l|l}
1.29 & 1.08 & 1.19
\end{tabular}

\begin{tabular}{ll|l}
2.94 & 0.47 & 5.78
\end{tabular}

\begin{tabular}{l|l|l|}
0.13 & 2.41 \\
2.36
\end{tabular}

0.07
-0.06

\begin{tabular}{ll|l|l}
1.07 & 1.29 & 11 & 1.18
\end{tabular}

\begin{tabular}{ll|l}
3.86 & 0.46 & 5.50
\end{tabular}

0.142 .31

0.05

$29-1.13-1.17$

$44.31 \quad 0.45 \quad 5.36$

\begin{tabular}{l|l}
0.14 & 2.27 \\
0.14 &
\end{tabular}

$0.05 \quad 1.17 \quad 1.27$

$1.15 \quad 1.13$

$44.74+0.435 .22$

2.23

\begin{tabular}{l|l|l}
0.04 & 1.19 & 1.26
\end{tabular}

$1.16 \quad 1.12$

\begin{tabular}{ll|l}
45.16 & 0.42 & 5.08
\end{tabular}

$-0.142 .20-0.03$

$1.21 \quad 1.25-1.18-1.08$

\begin{tabular}{ll|l}
45.58 & 0.42 & 4.93
\end{tabular}

\begin{tabular}{l|ll|l}
0.15 & 2.18 & 0.02 & 1.25 \\
2.16 & -0.02 & 1.27
\end{tabular}

\begin{tabular}{l|l|l|}
1.23 & 1.19 & 1.06 \\
\hline
\end{tabular}

$\begin{array}{llll}45.98 & 0.40 & 4.78\end{array}$

$46.39+0.404 .63$

$0.152 .16 \quad 0.00$

$46.7 i^{+0.39} \mid \begin{array}{llll}4.48 & -0.15 & 2.16 & 0.00\end{array}$

\begin{tabular}{ll|l}
47.14 & 0.37 & 4.33
\end{tabular}

\begin{tabular}{lll|l}
47.49 & 0.35 & 4.18
\end{tabular}

47.83

48.1

$0.34 \quad 4.02$

$0.152 .16+0.01$

$\begin{array}{llll}0.15 & 2.17 & 0.02 & 1.35\end{array}$

3.87

$\begin{array}{lll}0.16 & 2.22 & 0.03\end{array}$

$48.47+0.3 \mathrm{~J} 3.72$

0.04

\begin{tabular}{ll|l}
49.04 & 0.28 & 3.58 \\
\hline & 3.43
\end{tabular}

\begin{tabular}{ll|l}
49.30 & 0.26 & 3.28 \\
\hline & 0.25 & 3.28
\end{tabular}

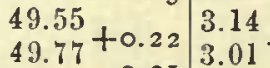

o. 5 $52.26+0.04$

\begin{tabular}{ll|ll}
49.98 & 0.21 & 2 & 87
\end{tabular}

50.16

\begin{tabular}{lll|l}
50.32 & 0.16 & 2.61
\end{tabular}

$\begin{array}{lll}0.14 & 3.35 & 0.05\end{array}$

$0.15 \quad 0.06 \quad 1.39$

\begin{tabular}{lll|l}
0.15 & 2.41 & 0.07 & 1.40 \\
2.48 & 0.07 & 1.40
\end{tabular}

$0.14\left[\begin{array}{ll}2.48 & 0.07\end{array}\right.$

$50.46+0.122 .49$

$-0.13 \frac{2.55}{2.64}+0.09$

1.40

0.1 32.73

०. 132.83

0.09
0.10

1.40

0.122 .94

O. I I

1.39

1.38

\begin{tabular}{|l|l|l}
$50.58+0.12$ & 2.38 \\
50.68 & 0.10 & 2.37
\end{tabular}

\begin{tabular}{ll|l}
50.76 & 0.08 & 2.27 \\
2.17
\end{tabular}

$50.81 \quad 0.05\left[\begin{array}{ll}2.17 \\ 2.07\end{array}\right.$

\begin{tabular}{ll|ll|ll|l}
50.83 & & 1.97 & & 3.72 & 0.14 & 1.28
\end{tabular}

\begin{tabular}{l|l|l}
$0.1 \mathrm{I}$ & 3.05 & 3.17 \\
& -0.12 & 1.37 \\
0.11 & & 1.35
\end{tabular}

\begin{tabular}{l|ll|l}
0.10 & 3.30 & 0.13 & 1.34
\end{tabular}

\begin{tabular}{l|ll|l}
0.10 & 3.44 & 0.14 & 1.32
\end{tabular}

1.22

1.19. 1.03

\begin{tabular}{ll|l}
1.20 & 1.20 & 1.00
\end{tabular}

\begin{tabular}{l|l|l|}
1.19 & 1.20 & 0.99
\end{tabular}

$50.81-0.021 .81$

$\begin{array}{lll}50.75 & 0.06 & 1.75\end{array}$

$50.670 .08 \quad 1.69$

50.57

10 1.69

$0.07 \mid$\begin{tabular}{ll|l}
3.87 & 0.15 & 1.25 \\
4.03 & 0.16 & 1.25
\end{tabular}

\begin{tabular}{l|ll|l}
0.06 & 4.19 & 0.16 & 1.20 \\
\hline 3.36 & 0.17 & 1.17
\end{tabular}

$4.53 \quad 0.17$

1.14

\begin{tabular}{ll|l}
50.29 & 0.15 & 1.58
\end{tabular}

$\begin{array}{llll}50.11 & 0.18 & 1.56\end{array}$

$\begin{array}{llll}49.90 & 0.21 & 1.55\end{array}$

0.034 .89

\begin{tabular}{l|ll}
0.02 & 5.07 & 0.18 \\
0.01 & 5.25 & 0.18 \\
0.00 & & 0.18
\end{tabular}

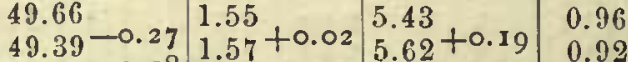

\begin{tabular}{|lll|ll|l|l|l|}
49 & 11 & 0.28 & 1.59 & 0.02 & 5.82 & 0.19 & 0.89 \\
\hline
\end{tabular}

$\begin{array}{llll}48.7 ! & 0.32 & 1.62\end{array}$

\begin{tabular}{ll|l}
48.41 & 0.35 & 1.66
\end{tabular}

0.035 .99

$0 . 0 4 \longdiv { 6 . 1 7 }$

0.18

0.85

\begin{tabular}{l|l}
48.07 & 1.72
\end{tabular}

6.36

0.77

$1.16-1.20-0.97$

\begin{tabular}{l|l|l}
1.14 & 1.19 & 0.95 \\
1.19 & 1.19 & 0.99
\end{tabular}

\begin{tabular}{ll|l|l}
1.10 & 1.18 & 0.91
\end{tabular}

\begin{tabular}{lll|l}
1.07 & 1.17 & 0.90
\end{tabular}

$1.05-1.17-0.89$

\begin{tabular}{l|l|l}
1.02 & 1.15 & 0.88
\end{tabular}

1.130 .86

\begin{tabular}{ll|l}
0.94 & 1.11 & 0.85 \\
0.09 & 0.84
\end{tabular}

$0.91-1.09-0.83$

\begin{tabular}{l|l|l|l|}
0.88 & 1.07 & 0.83 \\
\hline
\end{tabular}

\begin{tabular}{l|l|l}
\hline 0.85 & 1.05 & 0.82 \\
\hline
\end{tabular}

\begin{tabular}{ll|l}
0.79 & 0.99 & 0.82 \\
0.81
\end{tabular}

$0.76-0.99-0.81$ 
TA BHIE X, ARa. 3.-Conlinued.

\begin{tabular}{|c|c|c|c|c|c|c|c|c|c|c|c|c|c|c|}
\hline Arg. & $($ v.c. 0$)$ & l)ift. & $(v . s .1)$ & Diff. & v.c.1) & Difr. & 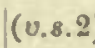 & it. & . & Difr. & $($ (v.8.3) & v.c.3) & $(v .8 .1)$ & $(v, c, 1)$ \\
\hline & $"$ & $" 1$ & $"$ & $"$ & $"$ & $"$ & $"$ & " & " & $"$ & $n$ & $"$ & " & " \\
\hline foo & 59.30 & & 30.17 & & 48.07 & & 1.72 & & 6.36 & & 0.77 & 0.49 & -0.83 & -1.09 \\
\hline 61 & 58.49 & -0.8 & 30.88 & -0.71 & 47.66 & 010 & $1.75^{-1}$ & +o.06 & $6.5 f^{-}$ & to.18 & 0.73 & 0.50 & 0.84 & 1.10 \\
\hline 62 & 57.67 & 0.52 & 31.59 & 0.71 & 47.24 & 0.42 & 1.56 & 0.08 & 6.71 & 0.17 & 0.69 & 0.52 & 0.85 & 11 \\
\hline 63 & 56.86 & 0.81 & 32.23 & $\begin{array}{l}0.70 \\
0.69\end{array}$ & 46.79 & 0.45 & 1.95 & $\begin{array}{l}0.09 \\
0.10\end{array}$ & 6.89 & 0.18 & & 0.54 & 0.87 & 1.13 \\
\hline 64 & 56.05 & 0.81 & 32. & 0.67 & 46.31 & $\begin{array}{l}0.48 \\
0.50\end{array}$ & 2.05 & $\begin{array}{l}0.10 \\
0.11\end{array}$ & 7.06 & $\begin{array}{l}0.17 \\
0.17\end{array}$ & 0.62 & 0.56 & 0.88 & 1.14 \\
\hline 65 & 55.24 & -0.80 & 33.61 & -0.66 & 45.81 & & 2.16 & +0.12 & 7.23 & -0.16 & .59 & 0.59 & -0.89 & -1.15 \\
\hline 66 & 54.44 & $\begin{array}{l}-0.80 \\
0.80\end{array}$ & 34.30 & 0.05 & 45.28 & 0.53 & 2.28 & 0,12 & 7.39 & 0.15 & & & 0.91 & \\
\hline 67 & 53.64 & 0.80 & 34.95 & 0.63 & 44.73 & 0.55 & 2.41 & 0.13 & 7.54 & 0.15 & 0 & 65 & 0.93 & 16 \\
\hline 68 & 53.84 & & 85.58 & 0.63 & 44.16 & 0.57 & 2.55 & & 7.69 & 0.15 & 0.50 & 8 & 0.95 & 17 \\
\hline 69 & 52.05 & $\begin{array}{l}0.79 \\
0.79\end{array}$ & 36.21 & $\begin{array}{l}0.03 \\
0.61\end{array}$ & 43.56 & $\begin{array}{l}0.00 \\
0.63\end{array}$ & 2.70 & $\begin{array}{l}0.15 \\
0.15\end{array}$ & 7.83 & $\begin{array}{l}0.14 \\
0.13\end{array}$ & 0.47 & 1 & 0.97 & \\
\hline 70 & 51.26 & -0.79 & 36.82 & .0 .59 & 42.93 & & 2.95 & to & 7.96 & . & 0.45 & 0.75 & -0.99 & -1.19 \\
\hline 71 &.+7 & $\begin{array}{l}0.79 \\
0.77\end{array}$ & 1 & $\begin{array}{r}0.59 \\
0.57\end{array}$ & & 0.66 & 3.02 & & 8.08 & 0.12 & 0.43 & 78 & 1.00 & 19 \\
\hline 73 & 49.70 & 0.77 & 37 & $\begin{array}{l}0.57 \\
0.55\end{array}$ & +1.62 & 0.68 & 3.19 & 0.18 & 8.20 & 0.18 & & 2 & 1.02 & 9 \\
\hline 73 & 48.93 & 0.76 & 34.53 & $\begin{array}{l}0.55 \\
0.53\end{array}$ & 40.94 & 0.71 & 3.37 & 0.19 & 8.2 & 0.09 & 39 & & 1.04 & \\
\hline 74 & 48.17 & 0.74 & 39.06 & 0.50 & 10.23 & 0.72 & 3.56 & 0.19 & 8.40 & 0.09 & 0.39 & 0.91 & 1.06 & 1.18 \\
\hline 75 & 47.43 & & 39.56 & & 39.51 & & 3.75 & & 8.49 & 0.08 & 0.37 & 0.96 & -1.08 & -1.18 \\
\hline 76 & 4 & -0.74 & 4 & -0.49 & & -0.74 & 3.96 & $\begin{array}{r}+0.21 \\
0.20\end{array}$ & 8.57 & -0.08 & 0.36 & .00 & 1.10 & \\
\hline 77 & .96 & 0.73 & 40.52 & 0.47 & 39.01 & 0.76 & 4.16 & 0.20 & 8.64 & 0.07 & 0 & 5 & 1.11 & 6 \\
\hline 79 & 45.24 & 0.72 & 40.96 & 0.44 & 37.23 & 0.78 & 4.37 & 0.21 & 8.69 & 0.05 & 6 & 10 & 1.12 & 15 \\
\hline 73 & 44.52 & $\begin{array}{l}0.72 \\
0.70\end{array}$ & 41. & $\begin{array}{l}0.42 \\
0.40\end{array}$ & 36.14 & $\begin{array}{l}0.79 \\
0.8 \mathrm{r}\end{array}$ & 4.59 & $\begin{array}{l}0.22 \\
0.21\end{array}$ & 8.73 & $\begin{array}{l}0.04 \\
0.03\end{array}$ & 0.36 & 1.14 & 1.13 & 1.13 \\
\hline 80 & 43.32 & & 41.78 & & 35.63 & & 4.90 & -0.22 & 8.76 & & 0.37 & 1.18 & -1.14 & -1.12 \\
\hline 81 & & -0.08 & & $-0.3^{8}$ & & -0.82 & 5.0 & $\begin{array}{r}+0.22 \\
0.22\end{array}$ & 8. & +0.0 & 0.39 & $2: 3$ & 1.15 & 10 \\
\hline 82 & 46 & $\begin{array}{l}0.05 \\
0.66\end{array}$ & 4: & 0.35 & 33 & 0.83 & 5.2 & 0.22 & $8.78^{-}$ & +0.01 & 0.40 & 1.29 & 1.16 & \\
\hline 83 & 30 & $0,6=$ & 42. & $\begin{array}{l}0.3^{2} \\
0.20\end{array}$ & 33.13 & $\begin{array}{l}0.85 \\
0.85\end{array}$ & 5.47 & $\begin{array}{l}0.23 \\
0.22\end{array}$ & 8.7 & -0.01 & 0.41 & 32 & 1.17 & 06 \\
\hline 84 & 41.15 & $\begin{array}{l}0.05 \\
0.63\end{array}$ & 43.12 & $\begin{array}{l}0.29 \\
0.26\end{array}$ & 32.28 & $\begin{array}{l}0.85 \\
0.86\end{array}$ & 5.69 & 0.22 & 8.75 & $\begin{array}{l}0.02 \\
0.03\end{array}$ & 0.43 & 1.36 & 1.17 & 1.04 \\
\hline 85 & 40.52 & 6 & 43.39 & & 31.12 & & 5.81 & -0.22 & 8.72 & & 0.46 & 1.41 & -1.18 & -1.02 \\
\hline 86 & 3 & $\begin{array}{l}-0.02 \\
0.60\end{array}$ & & $\begin{array}{r}+0.23 \\
0.21\end{array}$ & 30.55 & $\begin{array}{r}-0.87 \\
0.87\end{array}$ & 6.1 & $\begin{array}{r}0.22 \\
0.22\end{array}$ & 0.08 & & 49 & 15 & 1.18 & )1 \\
\hline 87 & 39.30 & $\begin{array}{l}0.00 \\
0.50\end{array}$ & 43 & 0.21 & 29.68 & 0.87 & 6.35 & $\begin{array}{l}0.22 \\
0.22\end{array}$ & 8.62 & 0.00 & 2 & 13 & 18 & 0.99 \\
\hline 89 & 71 & $\begin{array}{l}0.59 \\
0.57\end{array}$ & 4 & $\begin{array}{l}0.18 \\
0.16\end{array}$ & 29.80 & $\begin{array}{l}0.80 \\
0.80\end{array}$ & 6.57 & 0.22 & 8.55 & 0.08 & 5 & 2 & 1.17 & 0.97 \\
\hline 83 & 38. & $\begin{array}{l}0.57 \\
0.56\end{array}$ & & 0.12 & 27.91 & $\begin{array}{l}0.09 \\
0.90\end{array}$ & 6.79 & $0.2 \mathrm{I}$ & 8.47 & 0.09 & 0.59 & 1.56 & 116 & 0.95 \\
\hline 90 & 37.58 & & 44.28 & & 27.01 & $-0.8 ?$ & 7.00 & +0.21 & 8.39 & -0.1 & 0.62 & 1.59 & -1.16 & -0.94 \\
\hline 91 & & 0.54 & & 0.06 & 26.12 & 0.80 & 7.21 & 0.20 & 8.27 & 0.12 & 0.66 & ;1 & 1.15 & 32 \\
\hline 92 & 52 & $\begin{array}{l}0.5^{2} \\
0.50\end{array}$ & 44 & $\begin{array}{r}0.04 \\
+0.0\end{array}$ & 25.23 & 0.00 & 7.41 & 0.10 & 8.15 & & & 1. & 14 & 91 \\
\hline 93 & 36.02 & 0.40 & 4. & 0.00 & 24.33 & 0.00 & 7.60 & 0.19 & $8.02^{\circ}$ & 0.13 & 0 & if & 1.13 & 0.90 \\
\hline 94 & 35. & 0.46 & & -0.02 & 23 & 0.89 & 7.79 & 0.18 & 7.89 & 0.15 & 0.80 & 1.68 & 1.12 & 0.89 \\
\hline 95 & 35.07 & & 44.46 & -0.06 & 22.54 & -0.80 & 7.97 & & 7.74 & 0. & 0.85 & 1.70 & -1.11 & -0.88 \\
\hline 96 & & - & & 0.08 & 21.65 & $\begin{array}{l}-0.89 \\
0.88\end{array}$ & 8.14 & 0.16 & 7.58 & 0.17 & 0 & 1 & 0 & 0 \\
\hline 97 & 3 & $0.4^{2}$ & 44 & 0.12 & 20.77 & 0.87 & 8.3 & 0.15 & 7.41 & 0.17 & 0.95 & 1.72 & 8 & 0 \\
\hline 93 & 33.79 & $\begin{array}{l}0.41 \\
0.38\end{array}$ & 44. & 0.13 & 19.90 & 0.87 & 8.45 & 0.15 & 7.24 & 0.19 & 1 & 1.72 & 1. & 0 \\
\hline 99 & 33.41 & 0.37 & 44.07 & 0.17 & 19.03 & 0.87 & 8.60 & 0.13 & 70 & 0.20 & 1.05 & 1.73 & 1.0 .4 & 0.84 \\
\hline 100 & 33.04 & & 43.30 & -0.20 & 18.16 & 85 & 8.7 & 12 & 6.85 & & 1.10 & 1.72 & -1.08 & -0.83 \\
\hline 101 & & & 4 & 0.23 & 1 & & 8.8 & & 6.65 & & 1.15 & 82 & 1.02 & .83 \\
\hline 02 & 38 & 0.30 & 43.47 & 0.26 & 16. & 0.85 & 18.9 & 0.09 & 6.44 & 0.21 & 1. & . 0 & 1.00 & 0.83 \\
\hline 1 & 32.08 & 0.23 & 43 & 0.29 & 2 & 0.82 & 3.06 & 0.09 & 6.23 & 0.22 & 1.26 & 69 & 0.98 & 0.83 \\
\hline 1 & & 0.26 & & $0.3^{1}$ & & 0.81 & 9.15 & 0.08 & 6.01 & 0.22 & 1.31 & 1.67 & 0.96 & 0.83 \\
\hline 105 & 31.54 & & 42.61 & & 13.99 & & 9.23 & +0.07 & 5.79 & -0.2 & 1.35 & 1.65 & -0.95 & -0.83 \\
\hline 106 & & 0.23 & & 0.38 & & 0.78 & 9.30 & 0.05 & 5.57 & 0.23 & 1 & 1.63 & 0.94 & 0.84 \\
\hline 107 & & 0.10 & 41.89 & 0.40 & & 0.76 & 9.35 & 0.04 & 5.3 & 0.24 & 1. & 1.60 & 0. & 0.85 \\
\hline 108 & 30.92 & 0.16 & 41.49 & 0.41 & & 0.74 & 9.8 & 0.03 & 5. & 0.23 & & 1 & 0 . & 0.86 \\
\hline 109 & 30 & 0.14 & 11.08 & 0.44 & 10.92 & 0.73 & 9.42 & 0.01 & 4. & 0.23 & 1.53 & 1.53 & .89 & 0.87 \\
\hline 10 & 30.63 & & 40.64 & & 10.19 & & 9.43 & & 1.64 & & 1.56 & 1.49 & -0.88 & -0.88 \\
\hline & & & 40.17 & & 9.49 & $-0.7^{\circ}$ & & & 4. & -0.27 & 1. & 1.45 & 0.87 & 0.89 \\
\hline 115 & & 0.09 & 39.69 & 0.49 & & 0.09 & 9.42 & 0.02 & 4.17 & 0.23 & 1. & 1.41 & 0.86 & 0.91 \\
\hline 113 & & 0.07 & & $0.5^{2}$ & 8.14 & .00 & 9.40 & .02 & 3.9 & 0.23 & 1. & 1. & 0. & 0.93 \\
\hline 114 & 30. & 0.05 & 38. & 0.55 & 7.50 & $\begin{array}{l}0.04 \\
0.62\end{array}$ & 9.36 & & 3.71 & 0.23 & 1.67 & 1.32 & 0.85 & 0.95 \\
\hline 115 & 30.2 & & 38.05 & & C. 88 & & 0.31 & & 3. & & 1.70 & 1.27 & -0.84 & -0.96 \\
\hline & & .0 .01 & & $-0.5^{8}$ & 6.2 & .00 & 9.24 & & 3.26 & & 1. & 1.22 & $0.8 t$ & 0.97 \\
\hline & & 0.1 & 36.86 & & 5.71 & 57 & 9.1 & & 3. & & 1. & 1.17 & 0.84 & 0.99 \\
\hline & & & & 0.02 & 5.17 & 0.54 & 9.0 & 0.09 & 2.8 & $\begin{array}{l}0.22 \\
0.21\end{array}$ & 1.74 & 1.12 & 0.84 & 1.01 \\
\hline 18 & 30.46 & 0. & 00 & 0.65 & 2.00 & 0.49 & 0. & 0.11 & - & 0.20 & 1.74 & 1.07 & 0.84 & 1.02 \\
\hline 120 & 30.56 & & 34.95 & & 4.16 & & 8.88 & & 2.41 & & 1.74 & 1.02 & -0.84 & -1.04 \\
\hline
\end{tabular}




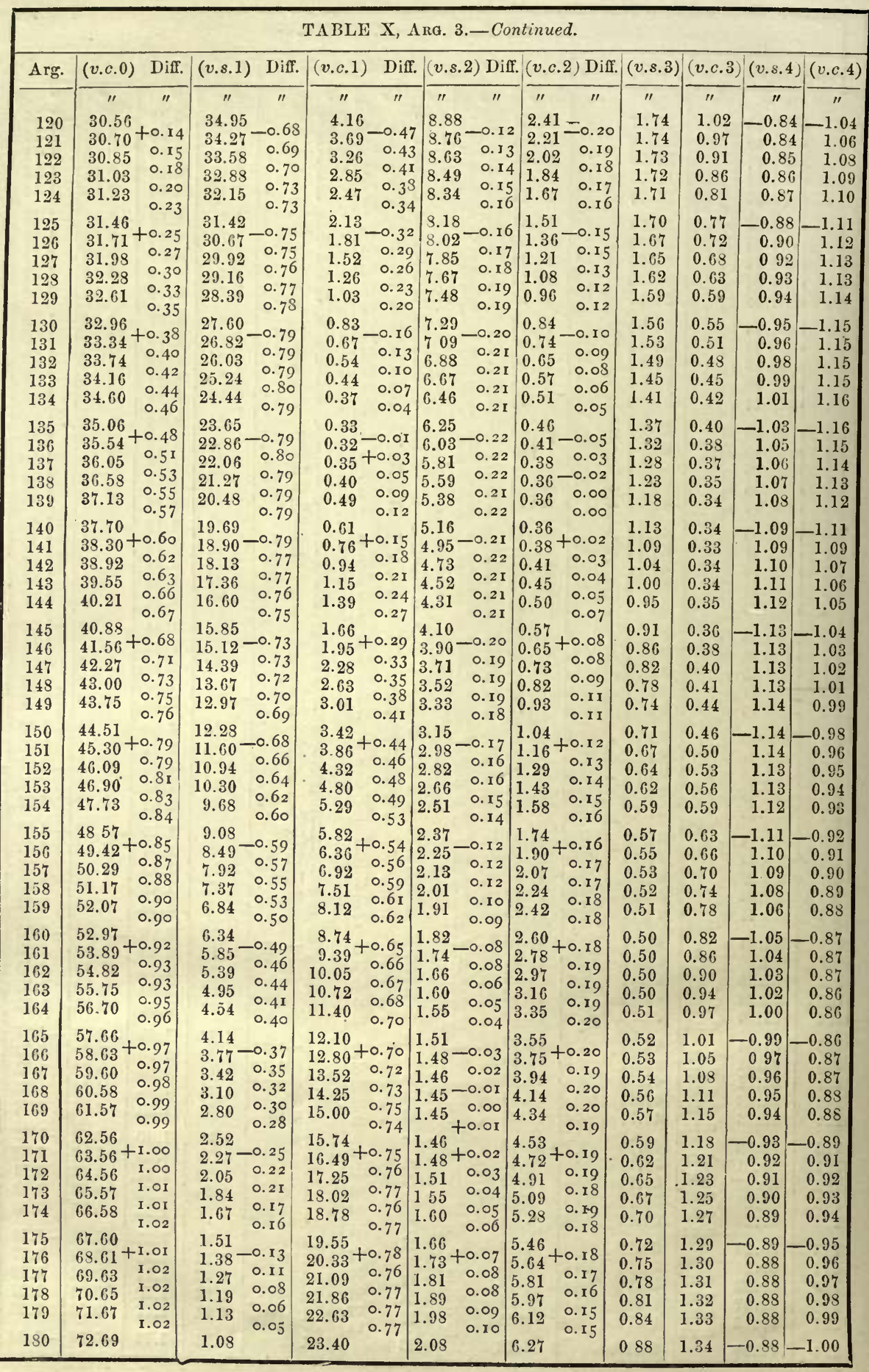




\begin{tabular}{|c|c|c|c|c|c|c|c|c|c|c|c|c|c|c|}
\hline \multirow[b]{2}{*}{ Arg. } & \multicolumn{14}{|c|}{ TALI.E X, Aro. 3.-Continued. } \\
\hline & $(v . c .0)$ & 1)ir. & $(v .8 .1)$ & wir. & $(v, c .1)$ & Diar. & $(0.8 .2)$ & ) Difr. & (u.c.3 & ) Dir. & $(v .8 .3)$ & (v.c.3) & $(0.8 .4)$ & $(v, c .4)$ \\
\hline & " & $"$ & " & " & " & " & $"$ & " & $"$ & " & $"$ & $"$ & . & - \\
\hline 180 & 72.69 & & 1.09 & & 23.40 & & 2.09 & & 0.27 & & 0.89 & 1.31 & -0.89 & -1.00 \\
\hline 15 & 73. & $\begin{array}{r}1.02 \\
1.02\end{array}$ & 1.07 & +0.01 & 24.17 & +0.77 & $2.20^{+}$ & $\begin{array}{r}+0.12 \\
0.11\end{array}$ & $6.42^{-1}$ & & & & 0.85 & 1.03 \\
\hline 15 & 3 & 1.02 & & 0.03 & 6.92 & 0.75 & 2.31 & $\begin{array}{l}0.11 \\
0.12\end{array}$ & 6.53 & 0.13 & & & 88 & \\
\hline 15 & & I.OI & & 0.05 & .68 & $\begin{array}{l}0.76 \\
0.75\end{array}$ & & 0.13 & 6.65 & 0.13 & & & & \\
\hline 18 & & 1.01 & & 0.08 & 3 & $\begin{array}{l}0.75 \\
0.74\end{array}$ & 2.5 & 0.13 & 6.81 & 0.12 & & & 0 & \\
\hline 185 & 77.77 & FI.0I & 1.24 & +0.10 & 7. & +0.73 & 2.69 & +0.14 & 1.93 & +0.11 & 1.03 & 1.33 & $-0.90 ?$ & -1.06 \\
\hline & & 1.00 & & 0.11 & & $\begin{array}{l}0.73 \\
\end{array}$ & & 0.13 & & & & & 2 & \\
\hline & & 0.99 & & 0.14 & & 0.71 & & 0.14 & & & & & & \\
\hline 89 & & 0.99 & & 0.15 & $\begin{array}{l}.31 .1 \\
.05\end{array}$ & 0.71 & $\begin{array}{l}3.1 \\
3.2\end{array}$ & 0.15 & $\begin{array}{l}7.22 \\
5\end{array}$ & 0.08 & & & & \\
\hline & & 0.99 & & 0.17 & & 0.70 & 1 & 0.15 & 7.30 & 0.07 & 1 & 1.28 & 0.95 & \\
\hline $\begin{array}{l}190 \\
191\end{array}$ & 82.75 & to. 99 & & +0.19 & - & +0.68 & $\begin{array}{l}3.40 \\
3.55\end{array}$ & +0.15 & $7.87+$ & +0.06 & 1.16 & 1.26 & -0.96 & -1.10 \\
\hline 2 & & 0.97 & & 0.22 & & 0.67 & $\begin{array}{l}3.55 \\
3.71\end{array}$ & 0.16 & & 0. & & & & \\
\hline 193 & & 0.95 & & 0.23 & 6 & 0.66 & 3.86 & & & 0.05 & & & & \\
\hline 194 & & 0 & 2.79 & 0.24 & $\begin{array}{l}76 \\
41\end{array}$ & 0.65 & 4.02 & .16 & 5 & 0.04 & & & 9 & \\
\hline 195 & 8 & 0 & & 0.26 & 11 & 0.64 & & 0.15 & ) & 0.03 & & & & \\
\hline & & 0.94 & $\begin{array}{l}3.05 \\
3.33\end{array}$ & +0.28 & & +0.62 & 4 & +0.16 & 7.60 & & 1. & 15 & -1.01 & 11 \\
\hline $19^{\circ}$ & & 0.5 & 3.62 & 0.29 & & 0.60 & & 0.16 & & & & & & \\
\hline 19 & & 0.92 & $4: 3$ & $0.3 \mathrm{i}$ & & 0.59 & & 0.15 & & & & & & \\
\hline 199 & 21. & 0 & 4.25 & 0.32 & & 0.57 & 4.79 & 0.15 & & & & & & \\
\hline 200 & 92.1 & & & 0.33 & 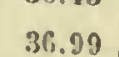 & $0.5^{6}$ & $-\infty$ & 0.15 & & 0.02 & & & & \\
\hline & & +0.83 & $4.82^{-1}$ & +0.34 & & +0.54 & 5. & +0.15 & & 8 & & & & \\
\hline 2 & & 0.87 & 5.29 & 0.36 & & 0.52 & 5.23 & 0.14 & & 0. & & & & \\
\hline 20 & & 0 & 5.65 & 0.37 & 6 & 0.51 & 5.37 & 0.14 & 7.50 & 0.04 & & & & \\
\hline 204 & 20. & ० & 6.03 & $0.3^{8}$ & 39.04 & 0.48 & 5.51 & 0.14 & 7.46 & 0.04 & 1.26 & 0.92 & 1.08 & 1.00 \\
\hline 205 & +1 & & 6.12 & & 39.51 & & 5.64 & & 7.41 & & 1.25 & 0.89 & 1.09 & 1 \\
\hline 2 & & +0.5 & $81^{-1}$ & +0.39 & & +0.45 & $5.77+$ & & 7 & & & & & \\
\hline 20 & & 0 & 22 & 0.41 & 4 & 0.44 & 5. & 12 & & & & & & \\
\hline-0 & & ० & & 0.42 & & 0.41 & 6.01 & 0.12 & & & & & & \\
\hline 209 & 9 & $\begin{array}{l}0.70 \\
0.75\end{array}$ & 806 & $\begin{array}{l}0.42 \\
0.43\end{array}$ & 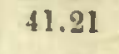 & $\begin{array}{l}0.40 \\
0.37\end{array}$ & 6.13 & $\begin{array}{l}0.11 \\
0.10\end{array}$ & 7.13 & 0.08 & 1.21 & 0.81 & 1.09 & 1 \\
\hline 210 & 100.32 & & 8.49 & & 41.58 & +0.36 & 0.29 & & 7.05 & & 1. & 79 & -1.10 & -1.00 \\
\hline 2 & & to. & & $\begin{array}{r}+0.43 \\
0.44\end{array}$ & & $\begin{array}{r}0.3^{0} \\
0.34\end{array}$ & & & 0.26 & & & & & \\
\hline 2 & 1 & & 9.36 & & & $\begin{array}{l}0.34 \\
0.31\end{array}$ & 6. & $?$ & 6.86 & & & & & \\
\hline 2 & 1 & 0 & 9.80 & $\begin{array}{l}0.44 \\
0.44\end{array}$ & $42.5 ?$ & 0.30 & 6.5 & 0.08 & 6.70 & 0.10 & & & & \\
\hline 214 & 103.1 & 0.67 & 10.24 & 0.45 & 4 & 0.29 & 6.58 & 0.07 & 6.66 & 0.10 & 1.1 & & 9 & \\
\hline 215 & 103.8 & to. & 10.69 & & 43.19 & +0.26 & 6.6 & +0.06 & 6.56 & 0.1 & 18 & 2 & -1.08 & -0 \\
\hline & & $0.6_{3}$ & & 0.45 & & 0.24 & & 0.06 & $6.4 i$ & 0.12 & & & & \\
\hline & & $0.6 \mathrm{t}$ & & 0.45 & & 0.22 & 6. & 0.05 & 6.6 & 0.11 & & & & \\
\hline 2 & & 0.60 & & 0.46 & & 0.21 & 6 & 0.05 & & 0.11 & & & & \\
\hline 219 & 10 & 0.57 & & 0.45 & & 0.18 & 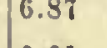 & 0.04 & 0.11 & 0.12 & 1.00 & 0.70 & 1.05 & 0.03 \\
\hline 220 & 106.9 & & $12.95+$ & 0 & 44.29 & -0.16 & 6.91 & & 5.99 & & 0 & 0.70 & -1.04 & -0 \\
\hline & & +0.55 & & & & 0.15 & & 3 & 5.87 & & & & & \\
\hline 2 & 1 & & 6 & & & 0.13 & 6. & 2 & 5.76 & 10 & & & & \\
\hline 2 & & & 0 & 0.45 & & 0.11 & 6.9 & -0.01 & 5. & 0.12 & & & & \\
\hline 0 & 102 & $\begin{array}{l}0.50 \\
0.4^{3}\end{array}$ & 14.75 & 0.44 & 44. & 0.10 & if ? & 0.00 & 0.00 & 0.11 & .8 & 0.71 & 1.01 & 0.92 \\
\hline 25 & 1 & & 15.1 & & 41.3 & & 6.98 & & 5.4 & & & & -1.00 & 9 \\
\hline & & & & & & & 6. & - & 5.31 & & & & & \\
\hline & & 0 & & 4 & & & & & 15 & 0.11 & & & & \\
\hline & & 0. & & 3 & & 4 & 6.9 & 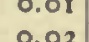 & 5 & 0. & & & & \\
\hline 229 & 111.18 & $\begin{array}{l}0.40 \\
0.37\end{array}$ & & $\begin{array}{l}0.43 \\
0.42\end{array}$ & $45.14+$ & 02 & 6.94 & 0.02 & 4.98 & II & 0.77 & 0.10 & 0.98 & 0.92 \\
\hline 230 & 1 & & 1 & & $\$ 5.15$ & & 6.91 & & 4.89 & & 0.76 & 0.78 & -0.97 & -0.93 \\
\hline & & Fo & & +0 & & 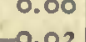 & & 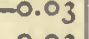 & 8 & ( & & & & \\
\hline 2 & & & & 0.41 & & & 6.8 & & 4 & & & & & \\
\hline & & & & & & & 6.80 & & 4.53 & & & & & \\
\hline 234 & 11 & & 18.96 & $\begin{array}{l}0.39 \\
0.38\end{array}$ & 45.0 & .05 & 6.75 & & 4.49 & & 0. & 9.00 & 0.94 & 0.96 \\
\hline 235 & 113 & & 19. & & 44.9 & & 6.70 & & 4.40 & & 0.1 & 0. & -0.93 & דים-0. \\
\hline & & & & fo. & & & $6.64^{\circ}$ & 0 & 4.31 & & & & 0.93 & \\
\hline & & 0.22 & & 0.37 & & & & & 4.23 & & & & & \\
\hline & & & & & & & 6.5 & 57 & 4.16 & 0 & & & 0. & \\
\hline 2 & 113 & $\begin{array}{l}0.18 \\
0.16\end{array}$ & 2 & $\begin{array}{l}0.35 \\
0.35\end{array}$ & 44.6 & 0.12 & & & 10 & .07 & 0.65 & 0.96 & 0.93 & 1.00 \\
\hline 240 & 114.11 & & 21.15 & & 14.47 & & 6.39 & & 4.02 & & 0.65 & 0.98 & -0.93 & - \\
\hline
\end{tabular}


TABLE X, Ara. 3.-Continued.

A rg. $\mid(v . c .0)$ Diff. $\mid(v . s .1)$ Diff. $\mid(v . c .1)$ Diff. $\mid(v . s .2)$ Diff. $(v . c .2)$ Diff. $|(v . s .3)|(v . c .3)(v . s .4) \mid(v . c .4)$

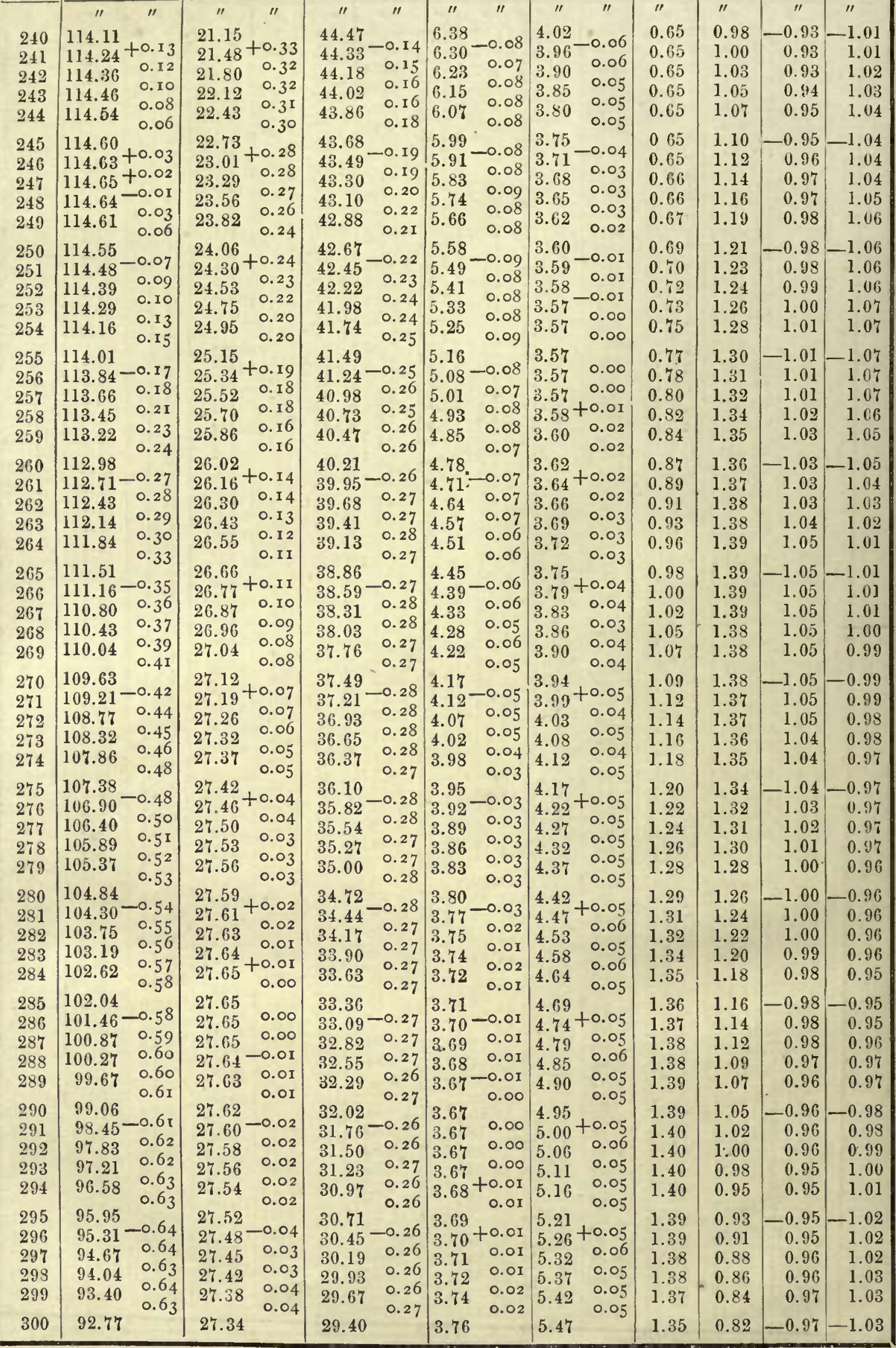


'A IBI.: X, А ка. 3.-Conlinved.

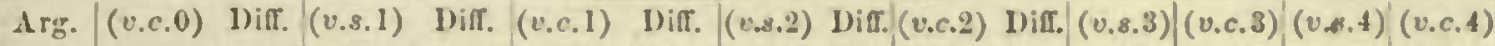

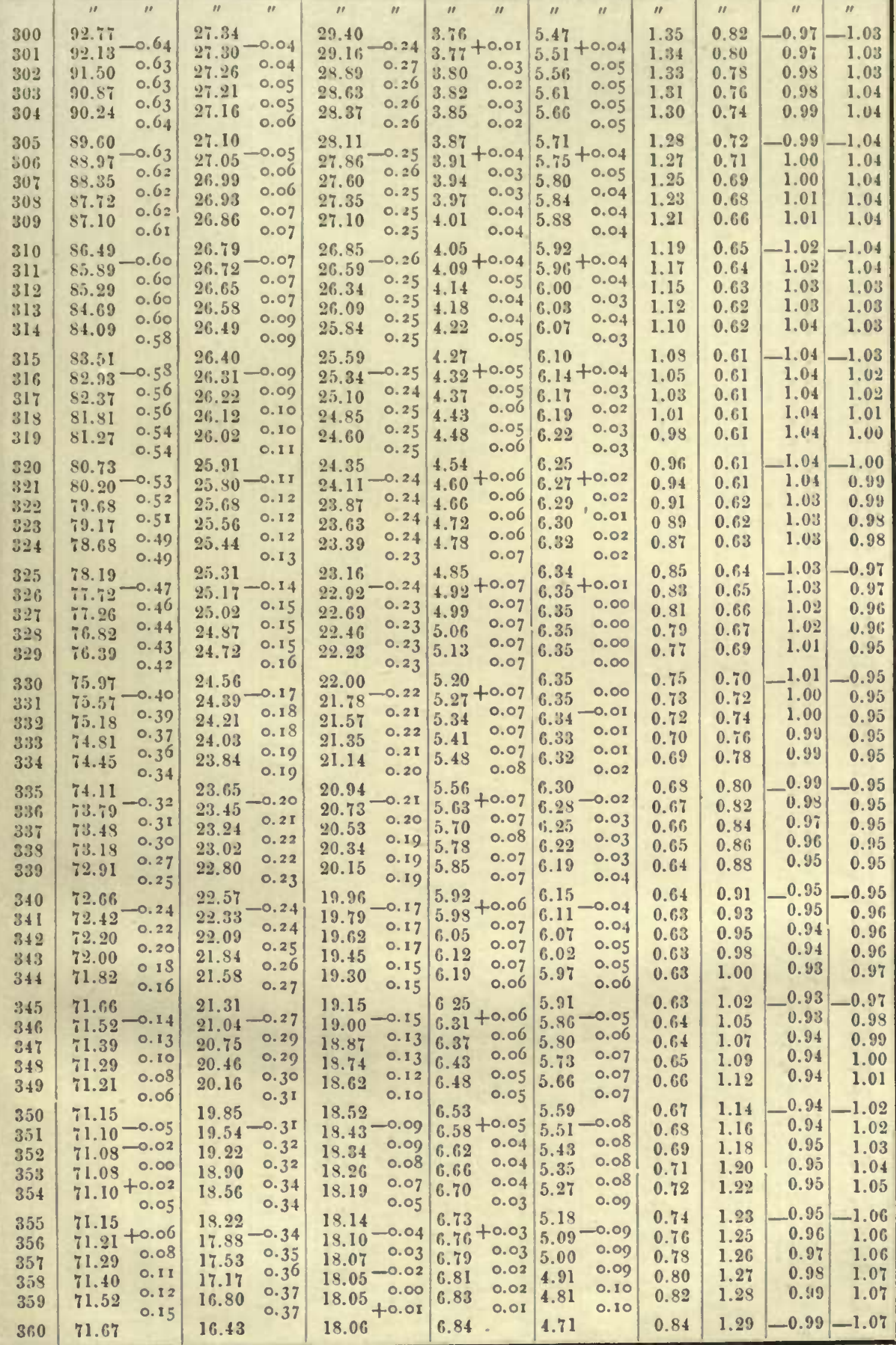




\begin{tabular}{|c|c|c|c|c|c|c|c|c|c|c|c|c|c|c|}
\hline Arg. & $(v . c .0)$ & Diff. & $(v . s .1)$ & Diff. & (v.c.1) & Diff. & $(v . s .2)$ & 2) Diff. & (v.c. 2 & 2) Diff. & $(v . s .3)$ & $($ v.c.3) & $(v .8 .4)$ & )$(v \cdot c .4)$ \\
\hline & " & $"$ & " & $"$ & " & " & $"$ & $"$ & " & " & " & $"$ & $" 1$ & " \\
\hline 360 & $71.67+$ & & 16.43 & & $18.06+$ & +0.02 & 6.84 & & 4.71 & & 0.84 & 1.29 & -0.99 & $9-1.07$ \\
\hline 361 & $71.84^{t}$ & to. I 7 & $16.05^{-}$ & $-0.3^{8}$ & $18.03^{t}$ & $\begin{array}{r}+0.02 \\
0.04\end{array}$ & 6.84 & $\begin{array}{l}0.00 \\
0.00\end{array}$ & $4.61^{-}$ & $\begin{array}{r}-0.10 \\
0.10\end{array}$ & 0.87 & 1.30 & 1.00 & 1.07 \\
\hline 362 & 72.02 & 0.18 & 15.67 & $0.3^{8}$ & 18.12 & $\begin{array}{l}0.04 \\
0.05\end{array}$ & 6.84 & $\begin{array}{l}0.00 \\
0.00\end{array}$ & 4.51 & 0.10 & 0.89 & 1.31 & 1.01 & 1.07 \\
\hline 363 & 72.24 & $\begin{array}{l}0.22 \\
0.24\end{array}$ & 15.28 & $\begin{array}{l}0.39 \\
0.30\end{array}$ & 18.17 & $\begin{array}{l}0.05 \\
0.07\end{array}$ & 6.84 & -0.01 & 4.41 & $\begin{array}{l}0.10 \\
0.10\end{array}$ & 0.91 & 1.31 & 1.02 & 1.07 \\
\hline 364 & 72.48 & $\begin{array}{l}0.24 \\
0.25\end{array}$ & 14.89 & $\begin{array}{l}0.39 \\
0.39\end{array}$ & 18.24 & 0.08 & $6.83^{-}$ & $\begin{array}{r}-0.01 \\
0.02\end{array}$ & 4.31 & 0.10 & 0.94 & 1.32 & 1.03 & 1.07 \\
\hline 365 & $72.73+$ & +0.27 & 14.50 & -0.39 & $18.32+$ & +0.10 & 6.81 & -0.02 & 4.21 & -0.10 & 0.96 & 1.32 & -1.03 & -1.07 \\
\hline 366 & $73.00^{\top}$ & 0.30 & 11 & 0.40 & 42 & O. I I & 6.79 & 0.03 & 4.11 & 0.10 & 0.98 & 1.32 & 1.03 & 1.06 \\
\hline 367 & $\begin{array}{l}73.30 \\
73.69\end{array}$ & 0.32 & 13.71 & 0.40 & 18.53 & 0.13 & 10.76 & 0.03 & $\begin{array}{l}4.01 \\
3.90\end{array}$ & O. I I & 1.01 & 1.32 & 1.04 & 1.00 \\
\hline 368 & $\begin{array}{l}73.62 \\
73.96\end{array}$ & 0.34 & 12.91 & 0.40 & $\begin{array}{l}18.00 \\
18.81\end{array}$ & 0.15 & 6.68 & 0.05 & $\begin{array}{l}3.90 \\
3.80\end{array}$ & 0.10 & 1.03 & 1.31 & 1.05 & 1.05 \\
\hline 369 & 73.96 & 0.37 & 13.91 & 0.40 & & o. 16 & & 0.05 & 3.80 & 0.10 & 1.06 & 1.31 & 1.06 & 1.05 \\
\hline 370 & 74.33 & $+0.3^{8}$ & 12.51 & $-0.4 \mathrm{I}$ & $18.97+$ & +o.18 & 6.63 & -0.05 & 3.70 & -0.10 & 1.08 & 1.30 & -1.07 & -1.05 \\
\hline $\begin{array}{l}371 \\
372\end{array}$ & & 0.41 & $\begin{array}{l}12.10 \\
11.70\end{array}$ & 0.40 & 19.15 & 0.20 & 6.58 & 0.06 & 3.60 & 0.09 & 1.10 & 1.29 & 1.07 & 1.05 \\
\hline $\begin{array}{l}372 \\
373\end{array}$ & $\begin{array}{l}75.12 \\
75.54\end{array}$ & 0.42 & 11.30 & 0.40 & 19.35 & 0.22 & $\begin{array}{l}6.52 \\
6.46\end{array}$ & 0.06 & 3.51 & o. Io & & 1.28 & 1.07 & 1.04 \\
\hline 374 & 75.99 & $\begin{array}{l}0.45 \\
0.46\end{array}$ & 10.90 & 0.40 & $\begin{array}{l}19.57 \\
19.80\end{array}$ & 0.23 & $\begin{array}{l}6.46 \\
6.39\end{array}$ & 0.07 & $\begin{array}{l}3.41 \\
3.32\end{array}$ & 0.09 & $\begin{array}{l}1.14 \\
1.16\end{array}$ & $\begin{array}{l}1.27 \\
1.25\end{array}$ & $\begin{array}{l}1.08 \\
1.08\end{array}$ & $\begin{array}{l}1.04 \\
1.03\end{array}$ \\
\hline 375 & 76.45 & & 10.50 & & 20.04 & & 6.32 & 0.07 & 3.23 & & 1.18 & 1.23 & -1.08 & -1.02 \\
\hline 376 & 76. & +0.49 & $10.10^{-}$ & -0.40 & $20.30+$ & +0.26 & $6.24-$ & -0.08 & 3.15 & -0.08 & 1.20 & 1.22 & 1.08 & 1.00 \\
\hline 377 & 77.44 & 0.50 & 9.71 & 0.39 & 20.59 & 0.28 & 6.15 & 0.09 & 3.07 & 0.08 & 1.22 & 1.20 & 1.08 & 0.99 \\
\hline 378 & 77.96 & 0.52 & 9.32 & 0.39 & 20.89 & $0.3^{I}$ & 6.06 & 0.09 & 2.99 & 0.08 & 1.23 & 1.19 & 1.08 & 0.98 \\
\hline 379 & 78.51 & $\begin{array}{l}0.55 \\
0.57\end{array}$ & 8.93 & $\begin{array}{l}0.39 \\
0.39\end{array}$ & 21.21 & $\begin{array}{l}0.32 \\
0.34\end{array}$ & 5.96 & $\begin{array}{l}0.10 \\
0.11\end{array}$ & 2.92 & $\begin{array}{l}0.07 \\
0.07\end{array}$ & 1.25 & 1.16 & 1.08 & 0.97 \\
\hline 380 & 79.08 & -0.58 & 8.54 & $-0.3^{8}$ & $21.55+$ & $\begin{array}{r}0.34 \\
+0.36\end{array}$ & 5.85 & & 2.85 & -0.07 & 1.26 & $1.13^{\circ}$ & -1.08 & -0.96 \\
\hline $\begin{array}{l}381 \\
382\end{array}$ & $\begin{array}{l}79.66 \\
80.27\end{array}$ & $0.6 \mathrm{r}$ & $\begin{array}{l}8.16 \\
7.79\end{array}$ & 0.37 & $\begin{array}{l}21.91 \\
22.28\end{array}$ & $\begin{array}{l}0.30 \\
0.37\end{array}$ & $\begin{array}{l}5.74 \\
5.63\end{array}$ & 0.11 & $\begin{array}{l}2.78 \\
9\end{array}$ & $\begin{array}{l}-0.07 \\
0.06\end{array}$ & 1.27 & 1.11 & 1.07 & 0.96 \\
\hline $\begin{array}{l}382 \\
383\end{array}$ & 80.90 & $0.6_{3}$ & 7.43 & 0.36 & 22.67 & 0.39 & $\begin{array}{l}5.63 \\
5.51\end{array}$ & 0.12 & 2.72 & 0.05 & 1. & 1.09 & 1.07 & 0.95 \\
\hline 384 & 81.54 & 0.64 & 7.07 & $0.3^{6}$ & 23.08 & $0.4 \mathrm{I}$ & $\begin{array}{l}5.51 \\
5.39\end{array}$ & 0.12 & 2.67 & 0.05 & 1. & 1.06 & 1.07 & 0.94 \\
\hline & 82.19 & 0.65 & 1.01 & 0.34 & & 0.43 & 5.39 & 0.12 & 2.62 & 0.04 & 1.29 & 1.04 & 1.06 & 0.93 \\
\hline $\begin{array}{l}385 \\
386\end{array}$ & 82.87 & +0.68 & $\begin{array}{l}6.73 \\
6.39\end{array}$ & -.34 & $\begin{array}{l}23.51 \\
23.95\end{array}+$ & -0.44 & 5.27 & -0.13 & 2.58 & -0.03 & 1.29 & 1.01 & -1.06 & -0.92 \\
\hline 387 & 83.56 & 0.69 & 6.06 & 0.33 & 24.41 & 0.46 & $\begin{array}{l}5.14 \\
5.01\end{array}$ & 0.13 & $\begin{array}{l}2.55 \\
253\end{array}$ & 0.02 & & 0. & 1.06 & 0.92 \\
\hline 388 & 84.27 & $0.7 \mathrm{I}$ & 5.74 & 0.32 & 24.89 & 0.48 & $\begin{array}{l}0.01 \\
4.87\end{array}$ & 0.14 & $\begin{array}{l}2.53 \\
2.51\end{array}$ & 0.02 & 1. & 0 & 1.05 & 0.92 \\
\hline 389 & 85.01 & 0.74 & 5.42 & 0.32 & 25.38 & 0.49 & 4.73 & O. I 4 & $\begin{array}{l}2.51 \\
2.49^{-}\end{array}$ & -0.02 & $\begin{array}{l}1.28 \\
1.28\end{array}$ & $\begin{array}{l}0.93 \\
0.91\end{array}$ & $\begin{array}{l}1.04 \\
1.03\end{array}$ & 0.91 \\
\hline 390 & 85.75 」 & L- 76 & 5.12 & 0.30 & 25.89 & $0.5 \mathrm{I}$ & 4.59 & 0.14 & 2.49 & 0.00 & 1.97 & 0.88 & 1.03 & 0.91 \\
\hline 391 & $86.51^{+}$ & $1-0.76$ & 4.84 & -0.28 & $26.42+$ & -0.53 & $4.45-$ & -0.14 & 2.49 & 0.00 & $\begin{array}{l}1.27 \\
1.26\end{array}$ & $\begin{array}{l}0.88 \\
0.86\end{array}$ & $\begin{array}{r}-1.02 \\
1.01\end{array}$ & -0.93 \\
\hline 392 & 87.29 & 0.78 & 4.56 & 0.28 & 26.96 & 0.54 & 4.31 & 0.14 & 2.50 & +0.01 & $\begin{array}{l}1.26 \\
1.25\end{array}$ & 0.83 & $\begin{array}{l}1.01 \\
1.00\end{array}$ & 0 \\
\hline 393 & 8808 & $\begin{array}{l}0.79 \\
0.81\end{array}$ & 4.30 & 0.20 & 27.51 & 0.55 & 4.16 & 0.15 & 2.52 & 0.02 & 1. & 0.81 & $\begin{array}{l}1.00 \\
0.99\end{array}$ & 0.90 \\
\hline 394 & 88.89 & 0.82 & 4.06 & $\begin{array}{l}0.24 \\
0.23\end{array}$ & 28.08 & $\begin{array}{l}0.57 \\
0.59\end{array}$ & 4.02 & $\begin{array}{l}\text { O.I } 4 \\
0.15\end{array}$ & 2.55 & $\begin{array}{l}0.03 \\
0.03\end{array}$ & 1.22 & 0.78 & $\begin{array}{l}0.99 \\
0.98\end{array}$ & $\begin{array}{l}0.90 \\
0.90\end{array}$ \\
\hline 395 & 89.71 & $-0.8_{3}$ & 3.83 & -0.22 & $28.67+$ & -0.60 & 3.87 & & 2.58 & 3 & 1.20 & 0.76 & -0.97 & .20 \\
\hline 396 & $90.54^{+}$ & 0.85 & 3.61 & 0.20 & $29.27^{+}$ & $\begin{array}{r}-0.00 \\
0.6 I\end{array}$ & 3.73 & -0.14 & $2.63^{-}$ & +0.05 & 1.1 & 0 . & 0.95 & 0.91 \\
\hline $\begin{array}{l}397 \\
398\end{array}$ & 91.39 & 0.86 & 3.41 & 0.19 & 29.88 & $\begin{array}{l}0.01 \\
0.62\end{array}$ & 3.58 & 0.15 & 2.68 & 0.05 & 1.1 & 0. & 0.94 & 0.91 \\
\hline 398 & $\begin{array}{l}92.25 \\
93.13\end{array}$ & 0.88 & 3.22 & 0.16 & 30.50 & 0.64 & 3.44 & $\begin{array}{l}0.14 \\
0.14\end{array}$ & 2.74 & 0.00 & 1.1 & 0.7 & 0.93 & 0.92 \\
\hline 399 & 93.13 & 0.89 & & 0.15 & 31.14 & $\begin{array}{l}0.64 \\
0.65\end{array}$ & 3.30 & $\begin{array}{l}0.14 \\
0.14\end{array}$ & 981 & $\begin{array}{l}0.07 \\
0.07\end{array}$ & 1. & 0. & 0.92 & 0.92 \\
\hline $\begin{array}{l}400 \\
401\end{array}$ & 94.02 & to.90 & 2.91 & -0.14 & $31.79+$ & -0.66 & 3.16 & -0.14 & 2.88 & to.08 & 1.08 & 0.68 & -0.91 & -0.93 \\
\hline 405 & $\begin{array}{l}94.92^{+} \\
95.83\end{array}$ & $0.9 \mathrm{r}$ & 5 & 0.12 & 32.45 & 0.66 & $\begin{array}{l}3.02 \\
9.80\end{array}$ & $\begin{array}{r}-0.14 \\
0.13\end{array}$ & $2.96^{-1}$ & +0.08 & 1.0 & 0.6 & 0.91 & 0.93 \\
\hline 403 & 96.75 & 0.92 & $\begin{array}{l}2.60 \\
2.56\end{array}$ & 0.09 & $\begin{array}{l}0.11 \\
33.79\end{array}$ & 0.68 & $\begin{array}{l}2.83 \\
276\end{array}$ & 0.13 & 3.06 & 0.10 & 1.0 & 0.6 & 0.90 & 0.94 \\
\hline 404 & 97.68 & 0.93 & 248 & 0.08 & 34.48 & 0.69 & 2.64 & 0.12 & 3.16 & $\begin{array}{l}\text { O.IO } \\
\text { O.I I }\end{array}$ & 0.99 & 0.65 & 0.90 & 0.95 \\
\hline 405 & 98.62 & 0.94 & 243 & 0.05 & & $0.6 y$ & & 0.12 & 3.27 & 0.12 & 0 & 0.65 & 0.89 & 096 \\
\hline 406 & $99.57-$ & +0.95 & $\begin{array}{l}2.43 \\
2.39\end{array}$ & -0.04 & $\begin{array}{l}35.17 \\
35.87+\end{array}$ & -0.70 & 2.52 & -O. I I & 3.39 & +0.1 2 & 0.93 & 0.64 & -0.89 & -0.37 \\
\hline 407 & 100.53 & 0.96 & $2.37-$ & -0.02 & 36.58 & 0.71 & 2.31 & 0.10 & $3.51^{-1}$ & $\begin{array}{r}+0.12 \\
0.13\end{array}$ & 0.90 & 0.64 & 0.89 & 0.99 \\
\hline 403 & 101.50 & 0.97 & 2.37 & 0.00 & 37.30 & 0.72 & 2.20 & O.II & $\begin{array}{l}3.64 \\
3.78\end{array}$ & 0.14 & 0 . & 0.65 & 0.89 & 1.01 \\
\hline 409 & 102.47 & 0.97 & 2.39 & +0.02 & 38.02 & 0.72 & 2.10 & o. 10 & $\begin{array}{l}3.78 \\
3.93\end{array}$ & 0.15 & 0 . & 0.65 & 0.90 & 1.02 \\
\hline 410 & 103.46 & & .4 & 0.05 & and & 0.72 & & 0.09 & 3. 35 & 0.15 & 6. & 0.0 & 0.90 & 1.03 \\
\hline 411 & $104.45^{+}$ & +0.99 & $\begin{array}{l}2.44 \\
2.52\end{array}$ & +0.08 & $\begin{array}{l}35.74 \\
39.47+c\end{array}$ & -0.73 & $\begin{array}{l}2.01 \\
1.93-\end{array}$ & -0.08 & $\begin{array}{l}4.08 \\
4.23\end{array}$ & to. 15 & 0.77 & 0.67 & -0.90 & -1.04 \\
\hline 412 & 105.44 & 0.99 & 2.61 & 0.09 & 40.19 & 0.72 & 1.86 & 0.07 & 4.39 & 0.16 & 0.74 & 0. & 0.91 & \\
\hline 413 & 106.44 & 1.00 & 2.72 & O. I I & 40.92 & 0.73 & 1.79 & 0.07 & 4.56 & 0.17 & 0.7 & 0. & 0. & 1 \\
\hline 414 & 107.44 & 1.00 & 2.85 & 0.13 & 41.66 & 0.74 & 1.73 & 0.06 & 4.73 & 0.17 & 0.69 & 0.72 & 0.92 & 1. \\
\hline 415 & 108 & & 3 & 0.15 & & 0.73 & & 0.04 & & 0.18 & 0.66 & 0.74 & .92 & 9 \\
\hline 416 & 109.45 & 1.01 & $3.18+$ & +o.18 & $\begin{array}{l}42.59 \\
43.12+\end{array}$ & -0.73 & 1.69 & -0.04 & 4.91 & 0.18 & 0.63 & 0.77 & -0.92 & -1.10 \\
\hline 417 & 110.46 & 1.01 & 3.38 & 0.20 & 43.84 & 0.72 & 1.65 & 0.03 & 5.09 & 0.18 & 0.61 & 0.79 & 0.93 & 1.10 \\
\hline 419 & 111.47 & I.OI & 3.60 & 0.22 & 44.57 & 0.73 & 1.62 & 0.02 & 5.27 & 0.10 & 0.59 & 0.82 & 0.94 & 1.10 \\
\hline 419 & 112.49 & .02 & 3.85 & 0.25 & 45.29 & 0.72 & 1759 & 0.01 & $\begin{array}{l}5.46 \\
5.55\end{array}$ & 0.19 & 0.56 & 0.85 & 0.96 & 1.11 \\
\hline 420 & 113.51 & & 4.12 & 0.27 & 4601 & & & o. & & 0.19 & & 0.88 & & 1.12 \\
\hline & & & & & & & 1.59 & & 5.84 & & 0.53 & 0.92 & -0.98 & -1.12 \\
\hline
\end{tabular}


'TAlil: X, A Iเo. 3.-Continued.

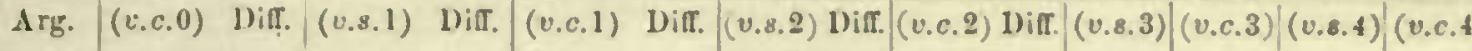

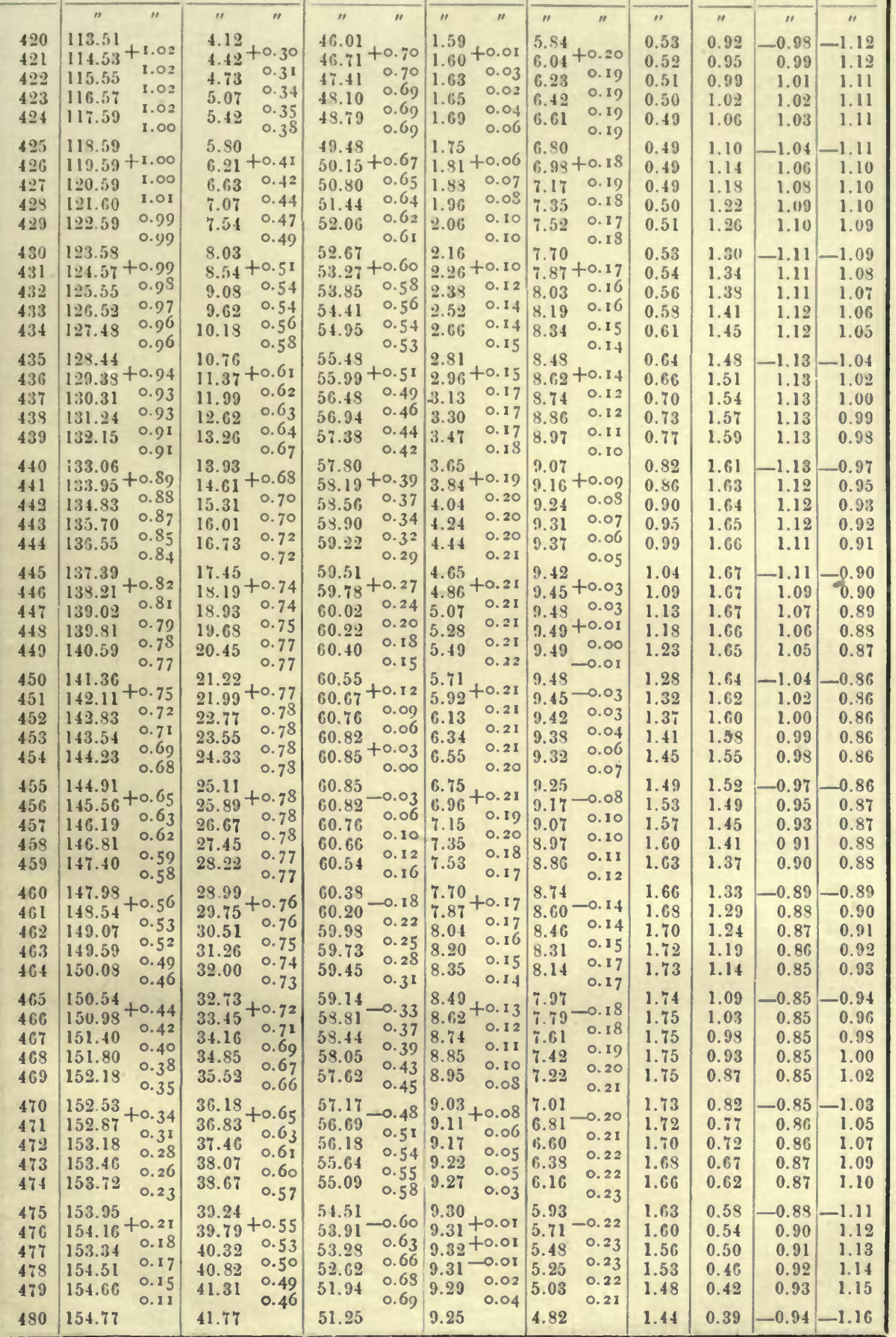


TABLE X, ARa. 3.-Continued.

Arg. (v.c.0) Diff. (v.s.1) Diff. $\mid(v . c .1)$ Diff. (v.s.2) Diff. (v.c.2) Diff. (v.s.3) (v.c.3) (v.s.4) (v.c.4)

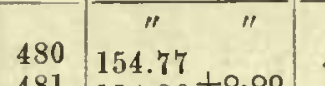

$481 \quad 154.86+0.09 \quad 42.20+0.43$

$\begin{array}{llll}482 & 154.93 & 0.07\end{array}$

\begin{tabular}{l|ll|ll|l}
483 & 154.98 & 0.05 & 42.61 & 0.41 & 50 \\
48 & 0.38 & 40
\end{tabular}

$484 \quad 154.99+0.01$

$\begin{array}{ll}42.99 & 0.3^{8} \\ 43.34 & 0.35\end{array}$

$485154.98^{-0.01}$

$\begin{array}{lll} & 0.33\end{array}$

\begin{tabular}{lll|l}
486 & $154.95^{-0.03}$ & 43.67 & 43.98 \\
+0.31
\end{tabular}

\begin{tabular}{lll|ll}
487 & 154.90 & 0.05 & 44.25 & 0.27
\end{tabular}

\begin{tabular}{l|ll|ll|}
488 & 154.82 & 0.08 & 44.49 & 0.24 \\
\hline
\end{tabular}

489 \begin{tabular}{lll|ll|}
154.82 & 0.10 & 44.49 & 0.22 \\
154.72 & 0.13 & 44.71 & 0.19
\end{tabular}

$\begin{array}{lll}490 & 154.59 & 0.13\end{array}$

$491154.45^{-0.14} \quad 45.05$ +o. I 5

$\begin{array}{llllll}492 & 154.28 & 0.17 & 45.18 & 0.13\end{array}$

\begin{tabular}{l|ll|ll}
493 & 154.08 & 0.20 & 45.28 & 0.10 \\
494 & 153.87 & 0.21 & 45.35 & 0.07
\end{tabular}

$494 \begin{array}{ll}153.87 & 0.21 \\ 15 & 0.24\end{array}$

$495 \quad 153.63=0.26$

$496 \quad 153.37-0.26$

\begin{tabular}{l|ll}
497 & 153.09 & 0.28 \\
498 & 159.79 & 0.30
\end{tabular}

\begin{tabular}{l|ll}
498 & 152.79 & 0.30 \\
499 & 152.46 & 0.33
\end{tabular}

$\begin{array}{lll}500 & 152.11 & 0.35\end{array}$

$501 \quad 151.74-0.37$

$\begin{array}{llll}502 & 151.36 & 0.3^{8}\end{array}$

\begin{tabular}{l|ll}
503 & 150.95 & $0.4 \mathrm{I}$
\end{tabular}

$\begin{array}{lll}504 & 150.52 & 0.43 \\ & & 0.44\end{array}$

\begin{tabular}{l|lr}
505 & 150.08 & 0.44 \\
508 & 149.61 & -0.47
\end{tabular}

$\begin{array}{lll}507 & 149.61 & 0.48\end{array}$

$\begin{array}{lll}508 & 148.63 & 0.50\end{array}$

$\begin{array}{lll}509 & 148.11 & 0.52\end{array}$

$\begin{array}{lll}510 & 147.58 & 0.53\end{array}$

$511 \quad 147.03^{-0.55}$

$\begin{array}{lll}512 & 146.46 & 0.57\end{array}$

\begin{tabular}{l|ll}
513 & 145.87 & 0.59 \\
514 & 145 & 0.60
\end{tabular}

\begin{tabular}{l|ll}
514 & 145.27 & 0.60 \\
0.61
\end{tabular}

$516 \quad 144.66-0.62$

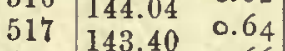

\begin{tabular}{l|ll}
518 & 142.74 & 0.66
\end{tabular}

\begin{tabular}{l|ll}
519 & 142.74 & 0.67 \\
142.07 & 0.68
\end{tabular}

45.35

$45.38+0.01$

$45.39+0.01$

$\begin{array}{rr}45.37^{-0.02} \\ 45.39 & 0.05\end{array}$

$\begin{array}{ll}45.23 & 0.09\end{array}$

45.12

0. I I

$44.98^{-0.1} 4$

$\begin{array}{ll}44.81 & 0.17\end{array}$

$44.60 \quad 0.21$

$\begin{array}{ll}44.38 & 0.22 \\ 0.26\end{array}$

44.12

$4: 3.8 t^{-0.2 S}$

$\begin{array}{lll}43.52 & 0.32\end{array}$

$\begin{array}{ll}43.18 & 0.34\end{array}$

42.89

42.42

0.40

$42.00^{-0.42}$

$\begin{array}{ll}41.56 & 0.44\end{array}$

41.09

0.47

40.09

0.51

$39.55-0.54$

$\begin{array}{ll}38.99 & 0.56 \\ 38.41 & 0.58\end{array}$

38.41

7.810 .60

\begin{tabular}{l|ll}
520 & 141.39 & -0.69
\end{tabular}

37.19

0.62

\begin{tabular}{l|l|l|}
51.25 & 0.25 & 4.82 \\
$50.53-0.72$ & $9.20-0.05$ & $4.60-0.22$
\end{tabular}

"

\begin{tabular}{llll|l|l|l|l|l|}
\hline 9.80 & 0.73 & 9.15 & 0.05 & +.39 & 0.21 & 1.39 \\
\hline
\end{tabular}

\begin{tabular}{ll|ll|ll|l}
49.04 & 0.76 & 9.08 & 0.07 & 4.17 & 0.22 & 1.30 \\
48.28 & 0.76 & 0.00 & 0.08 & 3.96 & 0.21 & 1.25
\end{tabular}

$0.2 \mathrm{I}$

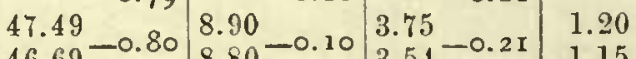

\begin{tabular}{rr|rr|r|r|}
$46.69^{-0.80}$ & 0.81 & $8.80^{-0.10}$ & 0.11 & $3.54^{-0.21}$ & 115 \\
\hline & 0.20 & 15
\end{tabular}

\begin{tabular}{ll|ll|l}
45.88 & $0.8 \mathrm{r}$ & 8.69 & $0 . \mathrm{II}$ & 3.54 \\
4 & 0.83 & 3.51 & 0.57
\end{tabular}

\begin{tabular}{ll|l}
45.05 & 0.83 & 8.57
\end{tabular}

0.123 .15

0.19

$\begin{array}{lllll}4.21 & 0.84 & 8.44\end{array}$

$43.37-0.84 \quad 8.30$

0.132 .28

. I 7

110

\begin{tabular}{l|l|l|l}
$2.51-0.86$ & $8.14-0.16$ & $2.64-0.17$ \\
0
\end{tabular}

\begin{tabular}{ll|l}
41.64 & 0.87 & 7.99
\end{tabular}

o.I $6 \begin{array}{ll}2.64 & 0.15\end{array}$

0.94

$\begin{array}{lll}0.77 & 0.87 & 7.81\end{array}$

39.90

\begin{tabular}{l|l}
0.87 & 7.6 \\
0.89 & 7.45
\end{tabular}

o. I 72.34

39.01

o. I 72.2

$38.12-0.897 .26$

\begin{tabular}{ll|l}
37.24 & 0.88 & 7.06 \\
36.35 & 0.89 & 6.86
\end{tabular}

$\begin{array}{lll}36.35 & 0.89 & 6.86\end{array}$

35.4

$$
0.8
$$

\begin{tabular}{ll|l}
34.57 & -0.89 & 6.45 \\
33.68 & 6.23
\end{tabular}

-. 2.07

0.15

\begin{tabular}{ll|l}
32.80 & 0.88 & 6.23 \\
31.92 & 0.88 & 5.80
\end{tabular}

$0.20\left[\begin{array}{ll}1.95 & -0.12 \\ 1.84 & 0.11\end{array}\right.$

31.9

\begin{tabular}{ll|l}
31.04 & 0.88 & 5.59
\end{tabular}

\begin{tabular}{ll|l}
30.18 & 0.86 & 5.37
\end{tabular}

\begin{tabular}{rr|r}
29.32 & -0.86 & 5.15 \\
28.46 & 0.86 & 4.93
\end{tabular}

\begin{tabular}{ll|l}
28.46 & 0.86 & 4.93
\end{tabular}

27.62

26.80

\begin{tabular}{ll|l}
0.82 & 4.72 \\
4.51
\end{tabular}

0.201 .74

$0.201 .74 \quad 0.08$

$25.98-0.814 .30$

$0.21 \quad \begin{array}{ll}1.66 & 0.08 \\ & 0.07\end{array}$

\begin{tabular}{rr|r}
25.17 & -0.81 & 4.09 \\
24.38 & 0.79 & 3.89
\end{tabular}

\begin{tabular}{lll|l}
23.61 & 0.77 & 3.69
\end{tabular}

22.8

$0.76 \quad 3.59$

0.221 .59

$-0.05$

22.11

$21.38-0.73 \quad 3.32$

$\begin{array}{lll}0.2 \mathrm{I} & 1.49 & 0.05\end{array}$

\begin{tabular}{l|ll}
$0.2 \mathrm{I}$ & 1.45 & 0.04 \\
0.22 & 1.49 & 0.03
\end{tabular}

$0.222^{1.42}-0.02$

$\begin{array}{llll}20.68 & 0.70 & 3.14\end{array}$

$\begin{array}{lll}-0.22 & 1.40 & 0.00 \\ 1.40 & 0.00\end{array}$

$0.22 \quad \begin{array}{ll}1.40 & 0.00\end{array}$

0.2 I $1.43+0.03$

$\begin{array}{lll}0.2 \mathrm{I} & 1.46 & 0.03 \\ 0.2 \mathrm{I} & & 0.05\end{array}$

19.99

\begin{tabular}{l|l}
0.70 & 2.98 \\
0.69 & 2.82
\end{tabular}

19.32

$0.67 \mid$\begin{tabular}{l|l}
2.82 \\
2.66
\end{tabular}

-0.2 I $\frac{1.51}{1.57}+0.06$

$36.56-0.63$

18.68

\begin{tabular}{l|l}
2.66 \\
.63
\end{tabular}

$\begin{array}{lll}522 & 140.00 & 0.70 \\ 523 & 139.29 & 0.71\end{array}$

\begin{tabular}{l|ll}
524 & 139.29 & 0.71 \\
138.57 & 0.72
\end{tabular}

$525 \quad 137.84^{0.73}$

$526 \quad \begin{aligned} & 137.84 \\ & 57.10\end{aligned}-0.74$

\begin{tabular}{l|ll}
527 & 136.35 & 0.75 \\
528 & 135.59 & 0.76
\end{tabular}

\begin{tabular}{l|ll}
528 & 135.59 & 0.76 \\
599 & 134.83 & 0.76
\end{tabular}

\begin{tabular}{l|ll}
529 & 134.83 & 0.76 \\
530 & 134.07 & 0.76
\end{tabular}

\begin{tabular}{l|ll}
530 & 134.07 & .76 \\
531 & $133.30-0.77$
\end{tabular}

\begin{tabular}{l|l}
531 & $133.30-0.77$
\end{tabular}

\begin{tabular}{l|ll}
533 & 132.52 & 0.78 \\
131.73 & 0.79
\end{tabular}

$\begin{array}{lll}534 & 130.94 & 0.79 \\ & 130.79\end{array}$

$\begin{array}{lll}535 & 130.15 & 0.79 \\ 536 & 199.35 & -0.80\end{array}$

$536 \quad 129.35-0.80$

$\begin{array}{lll}537 & 128.55 & 0.80\end{array}$

\begin{tabular}{l|ll}
538 & 127.75 & 0.80
\end{tabular}

$\begin{array}{lll}539 & 126.95 & 0.80 \\ 540 & & 0.80\end{array}$

35.91

35.25

34.57

0.65
0.66

\begin{tabular}{ll|l}
$18.05^{-0.63}$ & 2.51 \\
\hline & $0.60^{2}$ &
\end{tabular}

33.87

$0.68 \quad 16.87$

\begin{tabular}{l|l}
0.00 & 2.25 \\
0.58 & 2.13
\end{tabular}

0.70

16.32

32.15

$-0.72$

15.79

\begin{tabular}{l|l}
0.55 & 2.03 \\
0.53 &
\end{tabular}

$0 . 2 0 \longdiv { 1 . 6 4 } 0 . 0 7$

$0.20 \mid \begin{array}{ll}1.64 & 0.08\end{array}$

\begin{tabular}{l|ll}
0.19 & 1.81 & 0.09 \\
0.18 & 1.81 & 0.09
\end{tabular}

31.70

30.96

30.22

0.73

$15.98-0.5 \mathrm{I}$

0.181 .90

$+0.1 \mathrm{I}$

$29.46-0.76$

28.69

27.15

26.37

$25.58-0.79$

$\begin{array}{ll}24.81 & 0.77\end{array}$

14.79

$-0.5$

0.162 .01

0.162 .25

0.152 .39

0.13

2.53

0.14

.1 2.68 +o. 15

O.I 22.84

0.123 .00

0.16

16

$\begin{array}{lll}0.09 & 3.16 & 0.16 \\ & 0.17\end{array}$

0.88

0.78

0.69

0.65

0.60

0.56
0.53

0.49

0.46
0.43

0.41
0.39

0.37

0.36
0.35

0.34
0.34

0.34

0.34
0.35

0.36
0.37

0.39
0.41

0.41
0.43

0.46

0.49

0.52

0.55

0.62

0.66

0.70

0.74

0.78

3.91

\begin{tabular}{l|l|l|l|}
0.49 & 1.78 & 0.08 & 3.51 \\
3.69
\end{tabular}

18

13.51

$0.43 \quad 1.72 \cdot 0.06 \quad 3.87$

0.18

0.82

\begin{tabular}{l|ll|l|l}
$13.51-0.3^{8}$ & $1.63-0.03$ & 4.25 & 1.25 & 0.90
\end{tabular}

"
0.39
0.36

" - "

$0.96 \quad 1.16$

\begin{tabular}{l|l|l}
0.31 & 0.98 & 1.16
\end{tabular}

\begin{tabular}{ll|l}
0.30 & 1.01 & 1.16
\end{tabular}

$0.29-1.02-1.16$

$\begin{array}{llll}0.27 & 1.04 & 1.15\end{array}$

\begin{tabular}{l|l|l}
0.26 & 1.06 & 1.15 \\
0.26 & 1.08 & 1.15
\end{tabular}

\begin{tabular}{l|l|l}
0.26 & 1.09 & 1.14
\end{tabular}

$0.27-1.10-1.14$

\begin{tabular}{l|l|l}
0.28 & 1.11 & 1.13
\end{tabular}

\begin{tabular}{l|l|l}
0.29 & 1.12 & 1.12
\end{tabular}

\begin{tabular}{l|l|l|}
0.30 & 1.13 & 1.11 \\
0.32 & 1.14 & 1.10
\end{tabular}

$0.35-1.15-1.09$

$\begin{array}{llll}0.38 & 1.15 & 1.07\end{array}$

\begin{tabular}{l|l|l|}
0.41 & 1.15 & $\mathbf{1 . 0 5}$
\end{tabular}

\begin{tabular}{l|l|l|}
0.44 & 1.16 & 1.03 \\
0.47 & 1.16 & 1.01
\end{tabular}

$0.51-1.17-0.99$

$\begin{array}{lll}0.55 & 1.17 & 0.97\end{array}$

\begin{tabular}{ll|l}
0.59 & 1.16 & 0.95
\end{tabular}

\begin{tabular}{ll|l|}
0.68 & 1.15 & 0.91
\end{tabular}

$0.73-1.15-0.90$

\begin{tabular}{ll|l}
0.78 & 1.13 & 0.89
\end{tabular}

\begin{tabular}{ll|l}
0.83 & 1.11 & 0.87
\end{tabular}

\begin{tabular}{l|l|l}
0.87 & 1.10 & 0.86 \\
\hline
\end{tabular}

\begin{tabular}{ll|l|l|}
0.92 & 1.09 & 0.85
\end{tabular}

$0.97-1.08-0.84$

\begin{tabular}{lll|l}
1.02 & 106 & 0.84
\end{tabular}

\begin{tabular}{ll|l}
1.06 & 1.04 & 0.84
\end{tabular}

\begin{tabular}{l|l|l|}
1.11 & 1.02 & 0.83 \\
1.16 & 1.01 & 0.83
\end{tabular}

540

126.15

24.03

$\begin{array}{lll}23.25 & 0.78\end{array}$

12.78

2.46

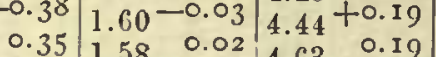

0.9

$1.20-1.00-0.82$

\begin{tabular}{ll|l}
1.24 & 0.98 & 0.82
\end{tabular}

1.32

1.35

$\begin{array}{lll}0.96 & 0.82\end{array}$

$1.39-0.90-0.84$

$0.99 \quad 0.83$

\begin{tabular}{l|l|l}
1.42 & 0.98 & 0.85
\end{tabular}

\begin{tabular}{l|l|l}
1.44 & 0.97 & 0.87
\end{tabular}

\begin{tabular}{l|l|l}
1.47 & 0.96 & 0.88 \\
1.49 & 0.95 & 0.89
\end{tabular}

$1.51-0.94-0.90$

\begin{tabular}{ll|l|l|}
1.53 & 0.94 & 0.91
\end{tabular}

\begin{tabular}{ll|l}
1.54 & 0.93 & 0.93
\end{tabular}

\begin{tabular}{l|l|l}
1.55 & 0.93 & 0.95 \\
1.55 & 0.92 & 0.97
\end{tabular}

$1.56-0.91-0.99$ 
TAIBIS X, Ano. 3.-Conlinued.

\begin{tabular}{|c|c|c|c|c|c|c|c|c|c|c|c|c|c|c|}
\hline A rg. & $($ (ᄂ.c.0) & Dift. & (v.8.1) & Dir. & (v.c.1) & Dia. ( & $(v .8 .2)$ & Dit. & (U.c. 2 & Did. & $($ (u.6.3) & (v.c.3) & $(v .8 .1)$ & $(u . c . t)$ \\
\hline & $"$ & " & " & $"$ & " & $"$ & $" 1$ & $"$ & " & " & " & $"$ & $"$ & $"$ \\
\hline 540 & 126.15 & & 22.45 & & 10.98 & & 1.82 & & 6.07 & & 1.26 & 1.43 & -0.59 & -1.15 \\
\hline 541 & & 0.79 & & -0.75 & & -0.10 & & 0.08 & $6.23^{-1}$ & -0.16 & & $1+1$ & 0.91 & \\
\hline 542 & 124.56 & 0.80 & & 0.77 & 10.80 & 0.08 & 1.98 & 0.03 & 6.39 & $\begin{array}{l}0.10 \\
0.15\end{array}$ & & 8 & 0.93 & \\
\hline 543 & 12 & $\begin{array}{l}0.80 \\
0.50\end{array}$ & & 0.77 & 10.75 & 0.05 & 2.0 & 0.10 & 6. & 0.15 & 3 & 36 & 35 & \\
\hline 544 & 129.96 & $\begin{array}{l}0.80 \\
0.79\end{array}$ & 19.10 & 0.75 & $3+$ & +0.02 & 2.18 & 0.11 & 6.6 & $\begin{array}{l}0.15 \\
0.14\end{array}$ & 1.35 & 1.83 & 0.96 & 1.17 \\
\hline 545 & 122.17 & -0.80 & 18.65 & -0.75 & 10.74 & & & -0.11 & 6.93 & -0.13 & 1.37 & 1.30 & -0.97 & -1.18 \\
\hline 5.16 & & 0.79 & 0 & 0.73 & & $\begin{array}{r}+0.05 \\
0.06\end{array}$ & 2. & 0.12 & & $\begin{array}{r}+0.13 \\
0.12\end{array}$ & & & 99 & \\
\hline 547 & & 0.78 & & $\begin{array}{l}0.73 \\
0.73\end{array}$ & & $\begin{array}{l}0.00 \\
0.09\end{array}$ & 2.5 & 0.13 & & $\begin{array}{l}0.12 \\
0.12\end{array}$ & & & 02 & \\
\hline 5. & 119 & 0.77 & & $\begin{array}{l}0.73 \\
0.71\end{array}$ & & 0.10 & 2.5 & 0.14 & 7 & $\begin{array}{l}0.12 \\
0.11\end{array}$ & & & 04 & \\
\hline $5+9$ & 119 & 0.77 & 15 & 0.70 & & 0.13 & 2.79 & 0.14 & 7.31 & 0.10 & $1 .+1$ & 1.17 & 1.06 & 1.18 \\
\hline 550 & & 0.76 & 15.03 & $-0.6 n$ & 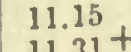 & +0.16 & $2.93+$ & -0.15 & 7.4 & +0.10 & 1.42 & 1.14 & $-1.0 \mathrm{~s}$ & -1.18 \\
\hline 551 & & 0.76 & & 0.63 & & 0.18 & 3.8 & 0.15 & & 0.08 & & 1 & 10 & \\
\hline 5.52 & & 0.75 & & 0.67 & & 0.20 & $\begin{array}{l}3.2 \\
3 .\end{array}$ & 0.15 & 7.5 & 0.08 & 42 & 8 & 2 & \\
\hline 553 & & 0.75 & 12 & 0.64 & & 0.22 & $\begin{array}{l}3 . \\
3 .\end{array}$ & 0.16 & 7.67 & 0.06 & ! & 4 & 3 & 5 \\
\hline 554 & 115 & 0.74 & 12.85 & 0.64 & & 0.25 & & 0.16 & 7. & 0.06 & 1.41 & 1.01 & 1.14 & 1.14 \\
\hline 555 & 114.50 & & .71 & -0.62 & 12.16 & & 3.70 & & 7.79 & & 1.41 & 0.98 & -1.15 & -1.13 \\
\hline 556 & & -0.73 & & $0.6 \mathrm{I}$ & & $\begin{array}{r}+0.27 \\
0.28\end{array}$ & & & & -0.04 & & & 15 & \\
\hline 7 & & $\begin{array}{l}0.7^{2} \\
0.70\end{array}$ & 1 & $\begin{array}{l}0.01 \\
0.59\end{array}$ & & 0.25 & 4. & 0.16 & 7. & 0.04 & & & & \\
\hline & & $\begin{array}{l}0.70 \\
0.70\end{array}$ & 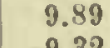 & $\begin{array}{l}0.59 \\
0.57\end{array}$ & $\begin{array}{l}02 \\
35\end{array}$ & $\begin{array}{l}0.31 \\
0.33\end{array}$ & 4. & 0. & 7. & 0.03 & & & 7 & 1.07 \\
\hline j9 & 11 & $\begin{array}{l}0.70 \\
0.69\end{array}$ & & 0.56 & 35 & $\begin{array}{l}0.33 \\
0.34\end{array}$ & 4.35 & $\begin{array}{l}0.10 \\
0.16\end{array}$ & 7.9 & 0.02 & 1.36 & 0.87 & 1.18 & 1.05 \\
\hline 560 & 110.96 & -0.67 & 8.76 & -0.53 & 13 & & 4.51 & 10.16 & 7.93 & . & 1.34 & 0.85 & -1.19 & -1.03 \\
\hline 1 & & $\begin{array}{l}-0.07 \\
0.66\end{array}$ & 8.23 & $\begin{array}{r}-0.53 \\
0.52\end{array}$ & & $\begin{array}{r}+0.35 \\
0.38\end{array}$ & 4. & & 7. & +0.01 & & 3 & 9 & \\
\hline 2 & 1 & 0.65 & 7. & 0.50 & & $0.3^{8}$ & 4. & $\begin{array}{l}0.17 \\
0.16\end{array}$ & & -0.01 & & 81 & 9 & 99 \\
\hline 3 & & 0.65 & & 0.48 & & 0.39 & 5. & $\begin{array}{l}0.16 \\
0.16\end{array}$ & & 0.01 & & 79 & 9 & 97 \\
\hline 564 & 108 & 0.63 & & 0.47 & & $\begin{array}{l}0.41 \\
0.42\end{array}$ & 5.7 & $\begin{array}{l}0.10 \\
0.16\end{array}$ & & $\begin{array}{l}0.02 \\
0.03\end{array}$ & 1.26 & .77 & .19 & 95 \\
\hline 565 & 107.70 & -0.62 & & - 0.44 & 15.64 & 0.43 & 5.32 & -0.15 & 7.87 & & 1.24 & .76 & .19 & -0.93 \\
\hline 6 & & 0.60 & & 0.43 & & $\begin{array}{l}0.43 \\
0.45\end{array}$ & & $\begin{array}{r}0.15 \\
0.15\end{array}$ & & & 1 & 74 & & \\
\hline 67 & 3 & 0.60 & & 0.41 & & $\begin{array}{l}0.45 \\
0.46\end{array}$ & 5. & 0.15 & & $\begin{array}{l}0.05 \\
0.05\end{array}$ & & & & \\
\hline 88 & & $0.5^{8}$ & & $0.3^{8}$ & & $\begin{array}{l}0.40 \\
0.47\end{array}$ & 5. & 0.14 & & $\begin{array}{l}0.05 \\
0.06\end{array}$ & & & & .87 \\
\hline 569 & 10 & $0.5^{6}$ & & 0.37 & .45 & 0.48 & 5.91 & 0.14 & 7.67 & $\begin{array}{l}0.00 \\
0.07\end{array}$ & 1.15 & 0.72 & 14 & 0.86 \\
\hline 570 & 104.74 & -0.5 & & $-0.3^{6}$ & & +0.49 & 6.0 & to.14 & 7.60 & -0.08 & 1.12 & 0.71 & -1.13 & -0.85 \\
\hline & & 0.54 & & 0.33 & & 0.50 & & 0.13 & & $\begin{array}{l}-0.08 \\
0.08\end{array}$ & & & 1.11 & 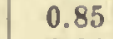 \\
\hline & & 0.52 & & $0.3^{2}$ & & 0.50 & & 0.12 & & $\begin{array}{l}0.08 \\
0.09\end{array}$ & & & & \\
\hline & $?$ & $0.5^{I}$ & $\begin{array}{l}2.93 \\
2.23\end{array}$ & 0.29 & 93 & 0.51 & & 0.12 & & $\begin{array}{l}0.09 \\
0.09\end{array}$ & & & & \\
\hline & & & & 0.28 & & $0.5^{2}$ & 6 & 0.12 & 26 & 0.09 & & 0.1 & 1.05 & 0.82 \\
\hline 575 & 102 & $-\infty$. & $\begin{array}{l}2.65 \\
2.39\end{array}$ & -0.26 & & +0.52 & & +0.11 & 7.17 & 2.1 & 0 & 0.71 & -1.04 & -0.81 \\
\hline 7 & & 0.48 & 5 & 24 & & 0.52 & & 0.10 & & 0.11 & & & & 15 \\
\hline 57 & 1 & 0.46 & 1.92 & o. & & 0.54 & & 0.09 & & 0.11 & & & 1.0 & 0.8 \\
\hline $\begin{array}{l}578 \\
579\end{array}$ & $\begin{array}{l}100.69 \\
100.25\end{array}$ & 0.43 & 1.71 & 0.21 & 22.58 & 0.55 & & 0.09 & & 0.11 & & & 0.98 & 0.8 \\
\hline & & .44 & & 0 & - & 0.54 & & 0.09 & & 0.12 & 91 & 0.74 & 0.36 & $0.2^{2}-10$ \\
\hline 80 & & 0.42 & $\begin{array}{l}1.53 \\
1.35\end{array}$ & -0.18 & $6+$ & +0.54 & 7.1 & +0.08 & & 0.1 & 89 & 76 & -0.94 & 80 \\
\hline & 98 & .41 & 1.19 & 0.16 & & 0.55 & 7 & 0.06 & & 0.13 & & & 0.92 & 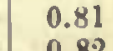 \\
\hline 583 & .57 & .41 & 1.06 & 0.13 & & 6 & 7.3 & 0.06 & & 0.12 & & & & \\
\hline 594 & 93.18 & 38 & 0.93 & $\begin{array}{l}0.13 \\
0.11\end{array}$ & 25.33 & 0.56 & 7.42 & 0.06 & $\begin{array}{l}10.20 \\
6.10\end{array}$ & 0.13 & & & & $\begin{array}{l}0.83 \\
0.84\end{array}$ \\
\hline 85 & 80 & & 0.82 & & 2588 & & 7.4 & 05 & 5.97 & & 0.81 & 0.84 & -0.86 & -0.85 \\
\hline & & 37 & & 0.09 & $1+$ & to. & 7. & & & 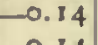 & & & & 0. \\
\hline 587 & & 36 & & 008 & & $0.5^{6}$ & & 0.03 & & 0.1. & & 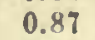 & 0.8 & 0.8 \\
\hline 588 & & 3 & 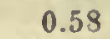 & 0.07 & & 0.56 & & 0.03 & & 0.13 & 0.7 & 8 & 0.8 & 0.91 \\
\hline 599 & 96.39 & -3 & 0.5 & .05 & & 6 & 7.0 & 0.03 & 5.12 & $\begin{array}{l}0.14 \\
0.14\end{array}$ & 0.7 & 0.92 & 0.83 & 0.93 \\
\hline 90 & 96.04 & & .50 & & 29.6 & & 7.6 & & 5.29 & & 7 & 0.94 & -0.82 & -0.94 \\
\hline & & ? & 7 & & & 0.56 & & 0.01 & & & 0.7 & 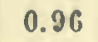 & $0 . x^{2}+x$ & 0. \\
\hline & & $3^{32}$ & & & & 0.55 & & 0.00 & 1 & & 0.7 &. & 0. & 0. \\
\hline 5 & 95.08 & 32 & 0 & . & & & & & 4. & 0.1 .4 & & & 0. & 1. \\
\hline 59. & 9. & & & $\begin{array}{l}0 . \\
0 .\end{array}$ & & $\begin{array}{l}0.56 \\
0.55\end{array}$ & & $\begin{array}{l}0.01 \\
0.02\end{array}$ & 4.7 & $\begin{array}{l}0.1 .1 \\
0.13\end{array}$ & : & 1.02 & 0.81 & 1.02 \\
\hline 595 & 94.46 & & 0.52 & & 31.4 & & 7.6 & & 4.60 & & 0.76 & 04 & -0.81 & -1.04 \\
\hline 00 & & -0.3 & 0.5 & $n^{\prime}$ & 01 & .55 & & & $4.47^{-}$ & 0.1. & & & 0. & 1. \\
\hline & & & 63 & 0.08 & 32.56 & 0.55 & & 0.03 & 1.34 & 0 & & & & 1.09 \\
\hline- & & & 0.70 & 0.0 & 33.11 & 0.55 & & .04 & 1.2 & 0.1 & 0.7 & 1.1 & 0.84 & 1.10 \\
\hline & 93.26 & 0.30 & 0. & & उ0 & & 7 & $\begin{array}{l}0.05 \\
0.04\end{array}$ & 1.08 & $\begin{array}{l}0.13 \\
0.13\end{array}$ & 0.7 & 1.13 & 0.85 & 1.12 \\
\hline 00 & 92.96 & & 0.97 & & 34.22 & & 7.42 & & 3.95 & & 0.80 & 1.15 & -0.85 & -1.18 \\
\hline
\end{tabular}


THE ORBIT OF URANUS.

TABLE X, ARG. 3.-Continued.

\begin{tabular}{|c|c|c|c|c|c|c|c|c|c|c|c|}
\hline Arg. & $(\rho . c .0)$ & (p.s.1) & (p.c.1) & $(\rho, s .2)$ & (p.c.2 & Arg. & $(\rho, c .0)$ & $(\rho . s .1)$ & $($ (p.c.1) & (p.s.2) & $(\rho . c .2)$ \\
\hline ( & 524 & 180 & 89 & 49 & 58 & 60 & 746 & 17 & 244 & 33 & \\
\hline
\end{tabular}

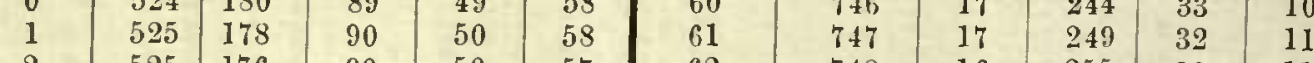

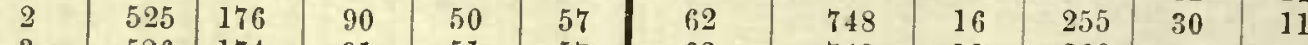

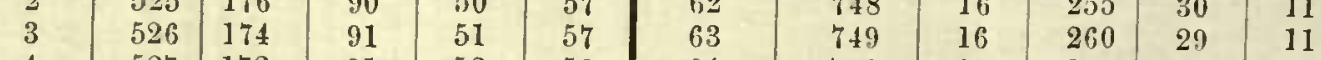

\begin{tabular}{l|l|l|l|l|l}
4 & 527 & 172 & 91 & 52 & 56
\end{tabular}

\begin{tabular}{l|l|l|l|l|l}
5 & 528 & 170 & 92 & 53 & 5 \\
6 & 529 & 168 & 92 & 54 & 55
\end{tabular}

\begin{tabular}{|l|l|l|l|l|l|}
\hline 7 & 529 & 168 & 92 & 54 & 55 \\
\hline & 531 & 165 & 92 & 55 & 55
\end{tabular}

\begin{tabular}{l|l|l|l|l|l}
8 & 533 & 163 & 93 & 55 & 5
\end{tabular}

\begin{tabular}{l|l|l|l}
9 & 535 & 161 & 93
\end{tabular}

\begin{tabular}{l|l|l|l|}
11 & 538 & 158 & 94 \\
1 & 540 & 156 & 94
\end{tabular}

\begin{tabular}{l|l|l|l|l|l}
12 & 543 & 154 & 94 & 57 & 5
\end{tabular}

\begin{tabular}{l|l|l|l|l|}
13 & 546 & 151 & 96 & 59
\end{tabular}

15

\begin{tabular}{l|l|l|r|r}
18 & 564 & 138 & 100
\end{tabular}

20

21

22

23

24

25

26

28

$611107 \quad 115$

30

31

32

33

35

36

37

38

39

\begin{tabular}{l|l|l|l}
40 & 69 & 115 \\
\hline & 674 & 65 & 149
\end{tabular}

\begin{tabular}{l|l|l|l}
41 & 680 & 65 & 119 \\
4 & 62 & 15
\end{tabular}

\begin{tabular}{l|l|l|l}
42 & 685 & 58 & 15
\end{tabular}

\begin{tabular}{l|l|l|l|}
43 & 689 & 55 & 160
\end{tabular}

\begin{tabular}{l|l|l|l}
44 & 694 & 52 & 16
\end{tabular}

\begin{tabular}{l|l|l|l}
45 & 699 & 49 & 169
\end{tabular}

\begin{tabular}{l|l|l|l}
46 & 703 & 46 & 173 \\
47 & 707 & 43 & 17
\end{tabular}

\begin{tabular}{ll|l|l}
48 & 711 & 43 & 17
\end{tabular}

\begin{tabular}{l|l|l|l}
49 & 715 & 37 & 18
\end{tabular}

\begin{tabular}{l|l|l|l}
50 & 719 & 35 & 19
\end{tabular}

\begin{tabular}{l|l|l|l}
51 & 722 & 32 & 196 \\
52 & 726 & 30 & 201
\end{tabular}

\begin{tabular}{l|l|l|l}
53 & 726 & 30 & 201 \\
53 & 729 & 29 & 206
\end{tabular}

\begin{tabular}{l|l|l|l}
54 & 732 & 26 & 21
\end{tabular}

\begin{tabular}{l|l|l|l}
55 & 735 & 24 & 21
\end{tabular}

\begin{tabular}{l|l|l|l}
56 & 737 & 22 & 22 \\
57 & 740 & 21 & 29
\end{tabular}

\begin{tabular}{l|l|l|l}
53 & 742 & 19 & 23 \\
\hline
\end{tabular}

\begin{tabular}{l|l|l|l}
59 & 744 & 18 & 238
\end{tabular}

60

746

17

244

120

\begin{tabular}{l|l|l|l|l}
749 & 16 & 266 & 27 & 11
\end{tabular}

\begin{tabular}{l|l|l|l|l}
750 & 16 & 271 & 26 & 12
\end{tabular}

\begin{tabular}{l|l|l|l|l}
750 & 16 & 277 & 24 & 13 \\
750 & 17 & 282 & 23 & 13
\end{tabular}

\begin{tabular}{l|l|l|l|l}
750 & 17 & 282 & 23 & 13 \\
74 & 287 & 22 & 14
\end{tabular}

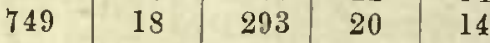

\begin{tabular}{l|l|l|l|l}
748 & 19 & 298 & 19 & 15
\end{tabular}

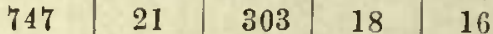

\begin{tabular}{l|l|l|l|l}
746 & 22 & 308 & 17 & 17
\end{tabular}

\begin{tabular}{l|l|l|l|l}
742 & 26 & 313 & 16 & 18 \\
& 26 & 14 & 19
\end{tabular}

\begin{tabular}{l|l|l|l|l}
740 & 29 & 323 & 13 & 20
\end{tabular}

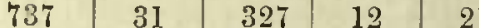

\begin{tabular}{l|l|l|l|l}
734 & 34 & 332 & 11 & 22 \\
731 & 37 & 336 & 10
\end{tabular}

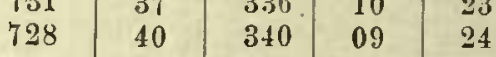

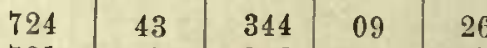

\begin{tabular}{llllll}
\hline 21 & 47 & 348 & 09 & 27
\end{tabular}

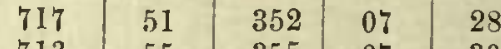

\begin{tabular}{l|l|l|l|l}
713 & 55 & 355 & 07 & 30 \\
708 & 59 & 359 & 06 & 31
\end{tabular}

\begin{tabular}{l|l|l|l|l}
703 & 63 & 362 & 06 & 33
\end{tabular}

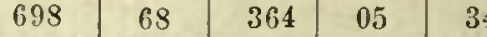

\begin{tabular}{l|l|l|l|l}
693 & 72 & 367 & 05 & 36
\end{tabular}

\begin{tabular}{l|l|l|l|l}
689 & 77 & 369 & 05 & 37 \\
682 & 82 & 372 & 05 & 39
\end{tabular}

\begin{tabular}{l|l|l|l|l}
676 & 87 & 373 & 05 & 41
\end{tabular}

\begin{tabular}{l|l|l|l|l}
670 & 92 & 375 & 05 & 42 \\
663 & 97 & 376 & 05 & 44
\end{tabular}

\begin{tabular}{l|l|l|l|l}
657 & 102 & 378 & 05 & 45
\end{tabular}

\begin{tabular}{l|l|l|l|l}
650 & 108 & 378 & 06 & 47
\end{tabular}

\begin{tabular}{l|l|l|l|l}
643 & 114 & 379 & 06 & 48
\end{tabular}

\begin{tabular}{l|l|l|l|l}
636 & 119 & 379 & 06 & 50
\end{tabular}

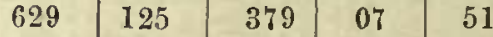

\begin{tabular}{l|l|l|l|l}
622 & 130 & 379 & 08 & 52 \\
614 & 136 & 379 & 09 & 54
\end{tabular}

\begin{tabular}{l|l|l|l|l}
606 & 141 & 378 & 09 & 55
\end{tabular}

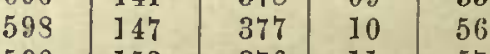

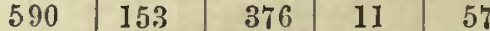

\begin{tabular}{l|l|l|l|l}
582 & 159 & 374 & 12 & 58 \\
574 & 164 & 373 & 13 & 60
\end{tabular}

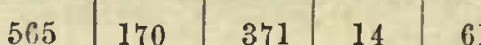

\begin{tabular}{l|l|l|l|l}
557 & 176 & 368 & 15 & 62
\end{tabular}

\begin{tabular}{l|l|l|l|l}
548 & 181 & 366 & 16 & 6
\end{tabular}

\begin{tabular}{l|l|l|l|l}
539 & 186 & 363 & 17 & 63
\end{tabular}

\begin{tabular}{l|l|l|l|l}
530 & 192 & 360 & 19 & 64 \\
521 & 197 & 357 & 20 & 64
\end{tabular}

\begin{tabular}{l|l|l|l|l}
512 & 202 & 353 & 21 & 65
\end{tabular}

\begin{tabular}{l|l|l|l}
503 & 207 & 350. & 23
\end{tabular}

\begin{tabular}{l|l|l|l|l}
494 & 212 & 346 & 24 & 66 \\
481 & 217 & 342 & 25 & 66
\end{tabular}

\begin{tabular}{l|l|l|l|l}
475 & 222 & 338 & 27 & 66
\end{tabular}

\begin{tabular}{l|l|l|l|l}
475 & 222 & 338 & 27 & 66 \\
465 & 227 & 333 & 29 & 67
\end{tabular}

\begin{tabular}{l|l|l|l|l}
456 & 231 & 329 & 30 & 67
\end{tabular}

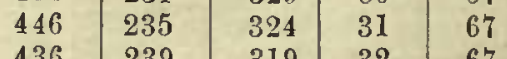

\begin{tabular}{l|l|l|l|l}
436 & 239 & 319 & 32 & 67
\end{tabular} 


\begin{tabular}{|c|c|c|c|c|c|c|c|c|c|c|c|}
\hline \multicolumn{12}{|c|}{ TABLE X, AвG. 3.-Continued. } \\
\hline Arg. & $(p . c .0)$ & $(p .8 .1)$ & (p.c.1) & $(\rho, s, 2)$ & $(\rho, c, 2)$ & Arg. & $(p \cdot e .0)$ & $(p .8 .1)$ & (p.c.1) & $(p .8 .2)$ & (p.e.2) \\
\hline 120 & 427 & 243 & 314 & 34 & 66 & 180 & 11 & 172 & 66 & 26 & 33 \\
\hline 121 & 417 & 247 & 309 & 35 & 66 & 181 & 10 & 168 & 66 & 25 & 33 \\
\hline 122 & 407 & 250 & 303 & 37 & 66 & 182 & 9 & 165 & 67 & 25 & 34 \\
\hline 123 & 397 & 254 & 298 & 38 & 65 & 183 & 9 & 162 & 67 & 24 & 35 \\
\hline 124 & 387 & 257 & 292 & 39 & 65 & 184 & 8 & 159 & 68 & 24 & 36 \\
\hline 125 & 378 & 260 & 286 & 41 & 64 & 185 & 8 & 156 & 69 & 24 & 37 \\
\hline 126 & 368 & 263 & 280 & 42 & 63 & 186 & 8 & 153 & 71 & 24 & 38 \\
\hline 127 & 359 & 266 & 274 & 43 & 63 & 187 & 8 & 150 & 72 & 23 & 39 \\
\hline 128 & 349 & 268 & 268 & 44 & 62 & 188 & 9 & 147 & 74 & 23 & 40 \\
\hline 129 & 339 & 270 & 262 & 45 & 61 & 189 & 9 & 144 & 75 & 23 & 41 \\
\hline 130 & 330 & 272 & 256 & 46 & 60 & 190 & 10 & 141 & 77 & 23 & 42 \\
\hline 131 & 320 & 274 & 250 & 47 & 59 & 191 & 11 & 138 & 79 & 24 & 43 \\
\hline 132 & 310 & 275 & 244 & 48 & 58 & 192 & 13 & 136 & 81 & 24 & 44 \\
\hline 133 & 301 & 277 & 238 & 49 & 57 & 193 & 14 & 133 & 84 & 24 & 45 \\
\hline 134 & 292 & 278 & 232 & 50 & 56 & 194 & 16 & 131 & 86 & 24 & 46 \\
\hline 135 & 282 & 279 & 225 & 50 & 55 & 195 & 18 & 129 & 89 & 25 & 47 \\
\hline 136 & 273 & 279 & 219 & 51 & 54 & 196 & 21 & 127 & 91 & 25 & 48 \\
\hline 137 & 263 & 280 & 213 & 52 & 52 & 197. & 23 & 125 & 94 & 26 & 49 \\
\hline 138 & 254 & 280 & 207 & 52 & 51 & 198 & 26 & 123 & 97 & 26 & 50 \\
\hline 139 & 245 & 280 & 201 & 52 & 50 & 199 & 29 & 121 & 99 & 27 & 51 \\
\hline 140 & 236 & 280 & 195 & 53 & 49 & 200 & 32 & 120 & 102 & 28 & 52 \\
\hline 141 & 227 & 280 & 189 & 53 & 47 & 201 & 36 & 118 & 1115 & 28 & 52 \\
\hline 142 & 218 & 280 & 183 & 53 & 46 & 202 & 39 & 117 & 108 & 29 & 53 \\
\hline 143 & 209 & 279 & 177 & 53 & 45 & 203 & 43 & 116 & 112 & 30 & 54 \\
\hline 144 & 201 & 278 & 171 & 53 & 44 & 204 & 47 & 114 & 115 & 31 & 54 \\
\hline 145. & 193 & 277 & 165 & 53 & 42 & 205 & 51 & 113 & 118 & 32 & 55 \\
\hline 146 & 184 & 275 & 160 & 53 & 41 & 206 & 55 & 112 & 122 & 33 & 55 \\
\hline 147 & 176 & 274 & 154 & 52 & 40 & 207 & 60 & 112 & 125 & 34 & 56 \\
\hline 148 & 168 & 272 & 149 & 52 & 39 & 208 & 65 & 111 & 129 & 35 & 56 \\
\hline 149 & 160 & 271 & 144 & 52 & 38 & 209 & 69 & 110 & 132 & 36 & 57 \\
\hline 150 & 152 & 269 & 139 & 51 & 36 & 210 & 75 & 110 & 135 & 37 & 57 \\
\hline 151 & 145 & 267 & 134 & 51 & 35 & 211 & 80 & 110 & 139 & 38 & 57 \\
\hline 152 & 137 & 265 & 129 & 50 & 35 & 212 & 85 & 110 & 143 & 39 & 57 \\
\hline 153 & 130 & 263 & 124 & 49 & 34 & 213 & 91 & 110 & 146 & 40 & 57 \\
\hline 154 & 123 & 260 & 120 & 49 & 33 & 214 & 96 & 110 & 150 & 41 & 57 \\
\hline 155 & 116 & 257 & 115 & 48 & 32 & 215 & 102 & 110 & 153 & 42 & 57 \\
\hline 156 & 109 & 255 & 111 & 47 & 31 & 216 & 108 & 110 & 157 & 43 & 57 \\
\hline 157 & 103 & 252 & 107 & 46 & 30 & 217 & 114 & 110 & 160 & 44 & 57 \\
\hline 158 & 96 & 249 & 103 & 45 & 30 & 218 & 120 & 111 & 164 & 45 & 57 \\
\hline 159 & 90 & 246 & 100 & 45 & 29 & 219 & 126 & 112 & 168 & 46 & 57 \\
\hline 160 & 84 & 243 & 96 & 44 & 29 & 220 & 133 & 113 & 171 & 47 & 57 \\
\hline 161 & 78 & 240 & 93 & 43 & 28 & 221 & 139 & 114 & 175 & 48 & 56 \\
\hline 162 & 73 & 236 & 90 & 42 & 28 & 222 & 145 & 115 & 178 & 49 & 56 \\
\hline 163 & 67 & 233 & 87 & 41 & 27 & 223 & 152 & 116 & 182 & 50 & 55 \\
\hline 164 & 62 & 229 & 84 & 40 & 27 & 224 & 159 & 117 & 185 & 50 & 55 \\
\hline 165 & 57 & 226 & 81 & 39 & 27 & 225 & 166 & 118 & 188 & 51 & 54 \\
\hline 166 & 53 & 222 & 79 & 38 & 27 & 226 & 173 & 119 & 192 & 52 & 53 \\
\hline 167 & 48 & 219 & 77 & 36 & 27 & 227 & 180 & 120 & 195 & 53 & 52 \\
\hline 168 & 44 & 215 & 75 & 35 & 27 & 228 & 187 & 122 & 198 & 54 & 52 \\
\hline 169 & 40 & 212 & 73 & 34 & 27 & 229 & 194 & 123 & 201 & 54 & 51 \\
\hline 170 & 36 & 208 & 71 & 33 & 27 & 230 & 201 & 125 & 204 & 55 & 51 \\
\hline 171 & 32 & 204 & $70^{\circ}$ & 32 & 27 & 231 & 208 & 127 & 207 & 55 & 50 \\
\hline 172 & 29 & 201 & 68 & 31 & 28 & 232 & 216 & 128 & 210 & 56 & 49 \\
\hline 173 & 26 & 197 & 67 & 30 & 28 & 233 & 223 & 130 & 213 & 56 & 49 \\
\hline 174 & 23 & 193 & 67 & 29 & 28 & 234 & 230 & 132 & 216 & 57 & 48 \\
\hline 175 & 21 & 190 & 66 & 28 & 29 & 235 & 238 & 134 & 218 & 57 & 47 \\
\hline 176 & 18 & 186 & 66 & 28 & 30 & 236 & 245 & 136 & 221 & 57 & 46 \\
\hline $17 i$ & 16 & 182 & 65 & 27 & 30 & 237 & 253 & 138 & 224 & 58 & 45 \\
\hline 178 & 14 & 179 & 65 & 27 & 31 & 238 & 260 & 140 & 226 & 58 & 45 \\
\hline 179 & 12 & 175 & 65 & 26 & 32 & 239 & 268 & 142 & 228 & 58 & 44 \\
\hline 180 & 11 & 172 & 66 & 26 & 33 & 240 & 275 & 144 & 231 & 58 & 43 \\
\hline
\end{tabular}


TABLE X, ARG. 3.-Continued.

\begin{tabular}{|c|c|c|c|c|c|c|c|c|c|c|c|}
\hline Arg. & $(\rho . c .0)$ & (p.s.1) & (p.c.1) & (p.s.2) & (p.c.2) & Arg. & $(\rho . c .0)$ & (..$s .1)$ & $(\rho . c .1)$ & $(p . s .2)$ & $(p .0 .2)$ \\
\hline 240 & 275 & 144 & 231 & 58 & 43 & 300 & 562 & 208 & 250 & 42 & 32 \\
\hline 241 & 283 & 146 & 233 & 58 & 42 & 301 & 562 & 208 & 250 & 42 & 32 \\
\hline 242 & 290 & 148 & 235 & 58 & 41 & 302 & 562 & 208 & 250 & 42 & 32 \\
\hline 243 & 298 & 150 & 237 & 58 & 40 & 303 & 561 & 208 & 250 & 42 & 32 \\
\hline 244 & 305 & 152 & 239 & 58 & 40 & 304 & 560 & 208 & 250 & 41 & 32 \\
\hline 245 & 313 & 154 & 240 & 57 & 39 & 305 & 559 & 208 & 250 & 41 & 31 \\
\hline 246 & 320 & 156 & 242 & 57 & 38 & 306 & 558 & 208 & 250 & 41 & 31 \\
\hline 247 & 328 & 159 & 244 & 57 & 38 & 307 & 557 & 208 & 250 & 41 & 31 \\
\hline 248 & 335 & 161 & 245 & 57 & 37 & 308 & 555 & 208 & 251 & 41 & 31 \\
\hline 249 & 342 & 163 & 246 & 57 & 36 & 309 & 553 & 208 & 251 & 40 & 30 \\
\hline 250 & 350 & 165 & 248 & 56 & 36 & 310 & 551 & 208 & 251 & 40 & 30 \\
\hline 251 & 357 & 167 & 249 & 56 & 35 & 311 & 549 & 209 & 251 & 40 & 30 \\
\hline 252 & 364 & 169 & 250 & 55 & 34 & 312 & 547 & 20,9 & 251 & 39 & 30 \\
\hline 253 & 371 & 171 & 251 & 55 & 34 & 313 & 544 & 209 & 251 & 39 & 29 \\
\hline 254 & 3 & 173 & 252 & 54 & 33 & 314 & 542 & 210 & 251 & 39 & 29 \\
\hline 255 & 385 & 175 & 253 & 54 & 33 & 315 & 539 & 210 & 251 & 38 & 29 \\
\hline 256 & 392 & 177 & 254 & 54 & 33 & 316 & 536 & 210 & 252 & 38 & 29 \\
\hline 257 & 399 & 179 & 254 & 53 & 32 & 317 & 532 & 211 & 252 & 38 & 28 \\
\hline 258 & 406 & 180 & 255 & 52 & 32 & 318 & 529 & 211 & 252 & 37 & 28 \\
\hline 259 & 412 & 182 & 256 & 52 & 32 & 319 & 525 & 212 & 252 & 37 & 28 \\
\hline 260 & 419 & 184 & 256 & 51 & 31 & 320 & 521 & 213 & 252 & 36 & 28 \\
\hline 261 & 426 & 185 & 256 & 51 & 21 & 321 & 517 & 213 & 252 & 36 & 28 \\
\hline 262 & 432 & 187 & 257 & 50 & 31 & 322 & 513 & 214 & 252 & 35 & 28 \\
\hline 263 & 438 & 189 & 257 & 50 & 31 & 323 & 509 & 215 & 252 & 34 & 28 \\
\hline 264 & 444 & 190 & 257 & 50 & 31 & 324 & 504 & 216 & 252 & 34 & 28 \\
\hline 265 & 450 & 192 & 257 & 49 & 31 & 325 & 500 & 217 & 252 & 33 & 28 \\
\hline 266 & 456 & 193 & 257 & 49 & 31 & 326 & 495 & 218 & 252 & 33 & 28 \\
\hline 267 & 462 & 194 & 257 & 48 & 31 & 327 & 490 & 219 & 252 & 32 & 28 \\
\hline 268 & 468 & 196 & 257 & 48 & 31 & 328 & 485 & 221 & 251 & 31 & 28 \\
\hline 269 & 473 & 197 & 257 & 47 & 31 & 329 & 480 & 222 & 251 & 31 & 28 \\
\hline 270 & 478 & 198 & 257 & 47 & 31 & 330 & 474 & 223 & 251 & 30 & 28 \\
\hline 271 & 484 & 199 & 257 & 46 & 31 & 331 & 469 & 224 & 250 & 30 & 28 \\
\hline 272 & 489 & 200 & 257 & 46 & 31 & 332 & 463 & 225 & 249 & 29 & 29 \\
\hline 273 & 494 & 201 & 257 & 45 & 31 & 333 & 457 & 227 & 249 & 29 & 29 \\
\hline $27 t$ & 499 & 202 & 256 & 45 & 31 & 334 & 451 & 228 & 248 & 28 & 29 \\
\hline 275 & 503 & 203 & 256 & 45 & 31 & 335 & 446 & 229 & 248 & 27 & 30 \\
\hline 276 & 509 & 203 & 256 & 45 & 31 & 336 & 439 & 231 & 247 & 27 & 30 \\
\hline 277 & 512 & 204 & 256 & 44 & 31 & 337 & 433 & 233 & 246 & 26 & 31 \\
\hline 278 & 517 & 205 & 255 & 44 & 31 & 338 & 427 & 234 & 245 & 25 & 31 \\
\hline 279 & 521 & 205 & 255 & 44 & 32 & 339 & 421 & 236 & 244 & 25 & 31 \\
\hline 280 & 521 & 206 & 255 & 44 & 32 & 340 & 414 & 238 & 243 & 24 & 32 \\
\hline 281 & 528 & 206 & 254 & 43 & 32 & 341 & 407 & 239 & 242 & 24 & 33 \\
\hline 282 & 531 & 207 & 254 & 43 & 32 & 342 & 401 & 241 & 241 & 24 & 33 \\
\hline 283 & 535 & 207 & 253 & 43 & 32 & 343 & 394 & 243 & 240 & 23 & 34 \\
\hline 284 & 538 & 207 & 253 & 43 & 32 & 344 & 387 & 244 & 238 & 23 & 35 \\
\hline 285 & 541 & 208 & 253 & 43 & 32 & 345 & 380 & 246 & 236 & 23 & 35 \\
\hline 286 & 544 & 208 & 252 & 43 & 33 & 346 & 373 & 248 & 235 & 22 & 36 \\
\hline 287 & 546 & 208 & 252 & 43 & 33 & 347 & 366 & 219 & 233 & 22 & 37 \\
\hline 288 & 549 & 208 & 252 & 43 & 33 & 348 & 35.9 & 251 & 231 & 22 & 38 \\
\hline 289 & 551 & 208 & 251 & 42 & 33 & 349 & 352 & 253 & 229 & 22 & 38 \\
\hline 290 & 553 & - 208 & 251 & 42 & 33 & 350 & 344 & 255 & 227 & 21 & 39 \\
\hline 291 & 555 & 208 & 251 & 42 & 33 & 351 & 337 & 256 & 225 & 21 & 40 \\
\hline 292 & 556 & 208 & 251 & 42 & 33 & 352 & $3: 30$ & 258 & 223 & 21 & 41 \\
\hline 293 & 558 & 208 & 251 & 42 & 33 & 853 & 323 & 260 & 221 & 21 & 42 \\
\hline 294 & 559 & 208 & 251 & 42 & 33 & 354 & 315 & 261 & 218 & 21 & 42 \\
\hline 295 & 560 & 208 & 250 & 42 & 33 & 355 & 308 & $2(i 3$ & 216 & 22 & 43 \\
\hline 296 & 561 & 208 & 250 & 42 & 33 & 356 & 300 & 264 & 213 & 22 & 44 \\
\hline 297 & 562 & 208 & 250 & 42 & 32 & 357 & 293 & 266 & 211. & 22 & 45 \\
\hline 298 & 562 & 208 & 250 & 42 & 32 & 358 & 285 & 267 & 208 & 22 & 46 \\
\hline 299 & 562 & 208 & 250 & 42 & 32 & 359 & 278 & 269 & 205 & 23 & 47 \\
\hline 300 & 562 & 208 & 250 & 42 & 32 & 360 & 270 & 270 & 203 & 23 & 47 \\
\hline
\end{tabular}




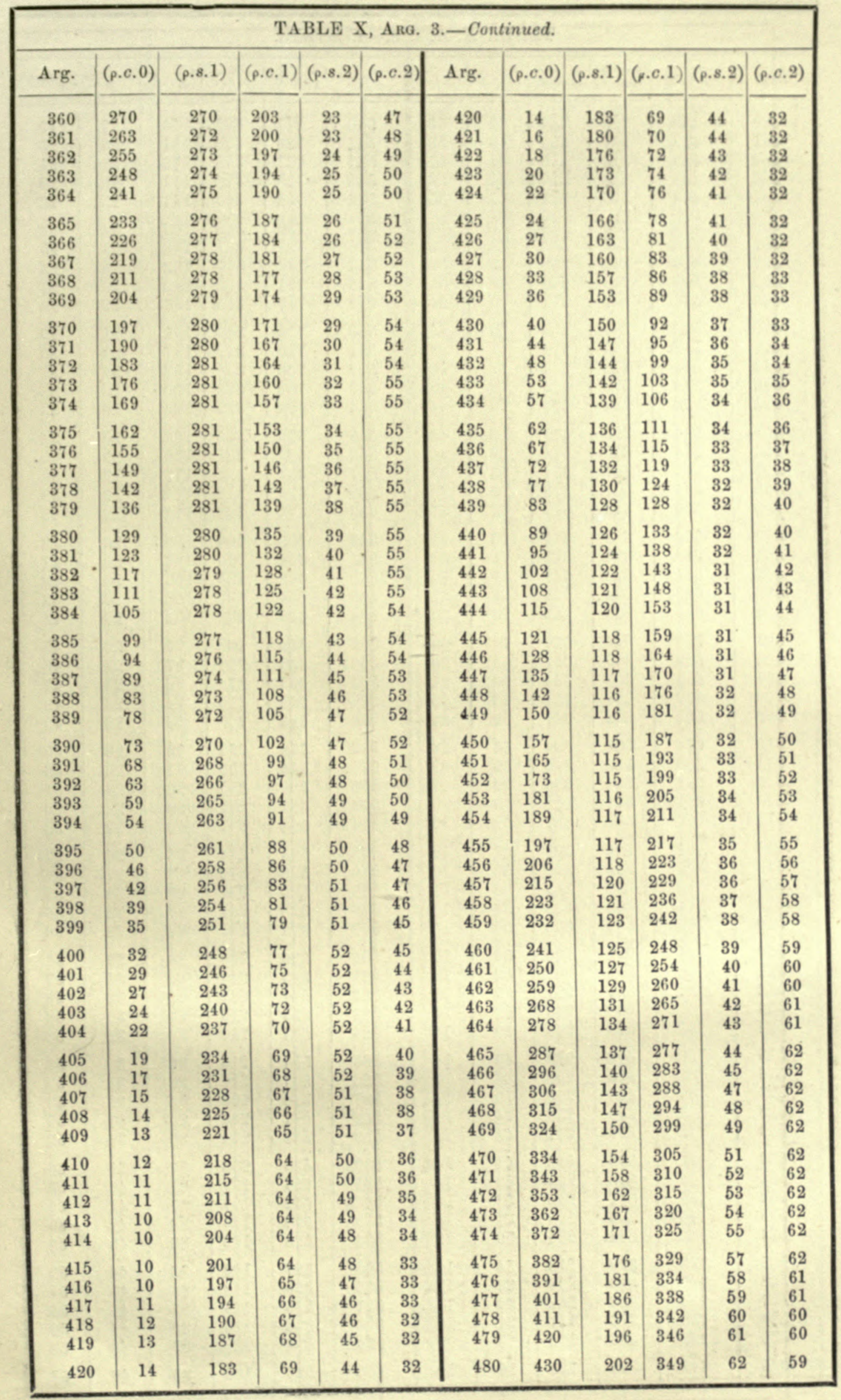


TABLE X, Ara. 3.-Concluded.

\begin{tabular}{|c|c|c|c|c|c|c|c|c|c|c|c|}
\hline Arg. & $(p . c .0)$ & (p.s.1) & (p.c.1) & (p.s.2, & $(p . c .2)$ & Arg. & $(\rho . c .0)$ & (p.s.1) & $(\rho . c .1)$ & $(\rho . s .2)$ & $(p . c .2)$ \\
\hline 480 & 430 & 202 & $349^{\circ}$ & 62 & 59 & 540 & $74 I$ & 381 & 186 & 29 & 19 \\
\hline 481 & 439 & 207 & 353 & 63 & 58 & 541 & 739 & 378 & 181 & 28 & 20 \\
\hline 482 & 449 & 213 & 356 & 64 & 57 & 542 & 737 & 375 & 176 & 27 & 21 \\
\hline 483 & 458 & 218 & 359 & 65 & 56 & 543 & 735 & 372 & 171 & 26 & 22 \\
\hline 484 & 468 & 223 & 361 & 66 & 55 & 544 & 732 & 368 & 167 & 25 & 23 \\
\hline 485 & 477 & 230 & 364 & 67 & 54 & 545 & 730 & 365 & 162 & 24 & 24 \\
\hline 486 & 486 & 236 & 366 & 68 & 53 & 546 & 727 & 361 & 158 & 24 & 25 \\
\hline 487 & 496 & 242 & 368 & 69 & 52 & 547 & 724 & 357 & 153 & 23 & 26 \\
\hline 488 & 505 & 248 & 370 & 69 & 51 & 548 & 721 & 353 & 149 & 22 & 27 \\
\hline 489 & 514 & 254 & 371 & 70 & 50 & 549 & 717 & 350 & 145 & 22 & 28 \\
\hline 490 & 523 & 260 & 373 & 70 & 49 & 550 & 714 & 346 & 141 & 21 & 29 \\
\hline 491 & 532 & 266 & 374 & 71 & 48 & 551 & 710 & 342 & 137 & 21 & 30 \\
\hline 492 & 540 & 272 & 374 & 71 & 46 & 552 & 706 & 338 & 133 & 21 & 31 \\
\hline 493 & 549 & 278 & 375 & 72 & 45 & 553 & 702 & 333 & 130 & 20 & 32 \\
\hline 494 & 558 & 284 & 375 & 72 & 44 & 554 & 698 & 329 & 126 & 20 & 33 \\
\hline 495 & 566 & 289 & 375 & 72 & 42 & 555 & 693 & 325 & 123 & 20 & 34 \\
\hline 496 & 574 & 295 & 374 & 72 & 41 & 556 & 689 & 321 & 120 & 20 & 35 \\
\hline 497 & 583 & 301 & 374 & 72 & 39 & 557 & 684 & 316 & 117 & 19 & 36 \\
\hline 498 & 591 & 307 & 373 & 72 & 38 & 558 & 670 & 312 & 114 & 19 & 38 \\
\hline 499 & 599 & 313 & 872 & 72 & 37 & 559 & 674 & 308 & 112 & 19 & 39 \\
\hline 500 & 606 & 318 & 371 & 72 & 35 & 560 & 669 & 303 & 109 & 20 & 40 \\
\hline 501 & 614 & 324 & 369 & 71 & 34 & 561 & 664 & 299 & 107 & 20 & 41 \\
\hline 502 & 621 & 329 & 367 & 71 & 33 & 562 & 659 & 295 & 105 & 20 & 42 \\
\hline 503 & 629 & 334 & 365 & 71 & 31 & 563 & 054 & 290 & 103 & 20 & 43 \\
\hline 504 & 636 & 339 & 363 & 70 & 30 & 564 & 649 & 286 & 101 & 20 & 44 \\
\hline 505 & 643 & 344 & 360 & 70 & 29 & 565 & 644 & 282 & 99 & 20 & 45 \\
\hline 506 & 649 & 349 & 357 & 69 & 28 & 566 & 638 & 278 & 97 & 21 & 46 \\
\hline 507 & 656 & 353 & 354 & 68 & 27 & 567 & 633 & 274 & 96 & 21 & 47 \\
\hline 508 & 662 & 358 & 351 & 68 & 25 & 568 & 628 & 270 & 94 & 22 & 49 \\
\hline 509 & 668 & 362 & 347 & 67 & 24 & 569 & 623 & 266 & 93 & 22 & 49 \\
\hline 510 & 674 & 366 & 344 & 66 & 23 & 570 & 618 & 262 & 92 & 23 & 50 \\
\hline 511 & 680 & 370 & 340 & 65 & 22 & 571 & 613 & 258 & 91 & 23 & 50 \\
\hline 512 & 686 & 374 & 336 & 64 & 21 & 572 & 608 & 255 & 90 & 24 & 51 \\
\hline 513 & 691 & 377 & 332 & 63 & 20 & 573 & 602 & 251 & 90 & 25 & 52 \\
\hline 514 & 696 & 380 & 327 & 62 & 19 & 574 & 598 & 247 & 89 & 25 & 53 \\
\hline 515 & 701 & 383 & 323 & 61 & 18 & 575 & 5.93 & 244 & 88 & 26 & 54 \\
\hline 516 & 706 & 386 & 318 & 59 & 18 & 576 & 588 & 240 & 88 & 27 & 54 \\
\hline 517 & 710 & 388 & 313 & 58 & 17 & 577 & 583 & 237 & 87 & 28 & 55 \\
\hline 518 & 714 & 390 & 308 & 57 & 16 & 578 & 578 & 234 & 87 & 28 & 56 \\
\hline 519 & 718 & 392 & 303 & 56 & 16 & 579 & 574 & 231 & 87 & 29 & 56 \\
\hline 520 & 721 & 394 & 298 & 54 & 15 & 580 & 570 & 228 & 86 & 30 & 57 \\
\hline 521 & 725 & 396 & 293 & 53 & 15 & 581 & 565 & 225 & 86 & 31 & 57 \\
\hline 522 & 728 & 397 & 287 & 52 & 14 & 582 & 561 & 222 & $8 \theta$ & 32 & 58 \\
\hline 523 & 731 & 398 & 282 & 50 & 14 & 583 & 557 & 219 & 86 & 32 & 58 \\
\hline 524 & 733 & 399 & 276 & 49 & 14 & 584 & 554 & 216 & 86 & 33 & 58 \\
\hline 525 & 736 & 400 & 270 & 48 & 14 & 585 & 551 & 214 & 86 & 34 & 58 \\
\hline 526 & 738 & 400 & 265 & 46 & 14 & 586 & 547 & 211 & 86 & 35 & 59 \\
\hline 527 & 740 & 400 & 259 & 45 & 14 & 587 & 544 & 209 & 86 & 36 & 59 \\
\hline 528 & 742 & 400 & 253 & 44 & 14 & 588 & 541 & 206 & 86 & 37 & 59 \\
\hline 529 & 743 & 399 & 247 & 42 & 14 & 589 & 539 & 204 & 86 & 38 & 59 \\
\hline 530 & 744 & 399 & 242 & 41 & 14 & 590 & 536 & 202 & 87 & 39 & 60 \\
\hline 531 & 745 & 398 & 2 & 40 & 14 & & & 19 & 87 & 40 & 60 \\
\hline 532 & 745 & 397 & 230 & 38 & 14 & 592 & 532 & 197 & 87 & 41 & 60 \\
\hline 533 & 146 & 396 & 225 & 37 & 15 & 593 & 530 & 195 & 88 & 42 & 60 \\
\hline 534 & 746 & 394 & 219 & 36 & 15 & 594 & 528 & 193 & 88 & 43 & 60 \\
\hline 535 & $\$ 45$ & 392 & 213 & 34 & 16 & 595 & 527 & 190 & 88 & 44 & 59 \\
\hline 536 & 745 & 390 & 208 & 33 & 17 & 596 & 526 & 188 & 88 & 45 & 59 \\
\hline 537 & 744 & 388 & 202 & 32 & 17 & 597 & 525 & 186 & 88 & 46 & 59 \\
\hline 538 & 743 & 386 & 197 & 31 & 18 & 598 & 525 & 184 & 89 & 47 & 59 \\
\hline 539 & 742 & 383 & 192 & 30 & 19 & 599 & 524 & 182 & 89 & 48 & 58 \\
\hline 540 & 741 & 381 & 186 & 29 & 19 & 600 & 524 & 180 & 89 & 49 & 58 \\
\hline
\end{tabular}




\begin{tabular}{|c|c|c|c|c|c|c|c|c|c|c|c|c|c|c|c|c|c|}
\hline ТАв. & \multicolumn{2}{|c|}{$X I$} & \multicolumn{3}{|c|}{ XII. } & \multicolumn{3}{|c|}{ xill. } & \multicolumn{3}{|c|}{ IIV. } & \multicolumn{3}{|c|}{ Xr. } & \multicolumn{3}{|c|}{ XII. } \\
\hline \multirow[t]{2}{*}{ A Ra } & \multicolumn{2}{|c|}{4.} & & 5. & & & 6. & & & 7. & & & 8. & & & 9. & \\
\hline & $(0.11)$ & (c.e.1) & (ne.e. 0 ) & (5.8.1) & $\underline{(\text { (s.e.1) }}$ & $(0.0 .0)$ & $(0.3 .1)^{\prime}$ & $(-a, 1)$ & $(0 . c .0)^{\prime}$ & $(0.81)\}$ & $(0.0 .1)$ & $(0.0 .0)$ & $(0.81)$ & $\left(\begin{array}{ll}10 & 0.1\end{array}\right)$ & $(0,0.0)$ & (0.0.1) & (v.e.1) \\
\hline & $"$ & 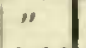 & $"$ & " & $1 "$ & $" \prime$ & $"$ & $"$ & " & " & " & 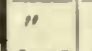 & " & $"$ & " & $"$ & " \\
\hline 0 & 0.67 & 0.13 & 1.11 & $0.03^{1}$ & 0.21 & 0.06 & 0.13 & 0.18 & 0.10 & 0.18 & 0.10 & 0.05 & 0.17 & 0.14 & 0.13 & 0.10 & 0.13 \\
\hline 10 & 0.64 & 0.13 & 1.12 & & & 0.06 & 0 & 8 & 0.10 & 81 & & 0.03 & & 0.19 & 0.12 & 0.10 & \\
\hline 20 & 0.61 & 0.14 & 1.12 & & & 0.07 & 0.15 & & 0.10 & & & 0.15 & 0.13 & & 0.12 & 9 & \\
\hline 30 & & 0.16 & 1.11 & 0.03 & 0.26 & & & & 0.10 & & & & & & 11 & 9 & \\
\hline 40 & 0.54 & 0.17 & 1.10 & 0.03 & 0.23 & & 61 & 0.16 & 0.10 & 0.18 & 0.19 & 0.30 & 0 & 0.37 & 11 & 9 & 9 \\
\hline 50 & 0.50 & 0.19 & 1.08 & 0.04 & 0.30 & 0 & 0.17 & 0.16 & 0.10 & 0.18 & 0.19 & 0.39 & 0.10 & 0.14 & 0.10 & 0.08 & 08 \\
\hline co & 0.47 & 0.21 & 1. & 4 & 0 . & & 0 & & 0. & 0.18 & & 0 & & & 09 & 8 & \\
\hline 70 & 0. & 0.24 & 1.04 & 05 & 2 & & & & & $i$ & & 18 & 3 & & 9 & & \\
\hline 80) & & 0.26 & & $0 \mathrm{c}$ & 0.34 & & & 0.13 & 0.09 & 0.17 & 0.18 & 4 & 15 & & 8 & & \\
\hline 90 & 8 & 0.29 & 0 & $0 i$ & 0.35 & 9 & 8 & 0.12 & 0.09 & 0.16 & 0.17 & 7 & 17 & 5 & 08 & 0.07 & 4 \\
\hline 100 & 0.31 & $0.3]$ & 0.83 & $0.0 \mathrm{~S}$ & 0.36 & $?$ & 0 & 0.12 & 0.09 & 0.16 & 17 & 1.00 & 0.21 & 0.83 & 0.07 & 0.07 & 03 \\
\hline 110 & 0.27 & 0.84 & 8 & 9 & i) & & 8 & & 0 & 50 & & & 5 & & 6 & 7 & \\
\hline 120 & 0.23 & 0.37 & 4 & 1] & 0. & & 9 & 0 & 0. & 40 & & 8 & 93 & & (c) & 6.) & \\
\hline 130 & 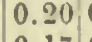 & 0.40 & 3 & 2 & 0.33 & & 0 & 0.09 & 0 & 14 & 1 & 2 & 0.34 & 1.06 & 0 & & \\
\hline 0 & .17 & 0.43 & 4 & c & 3 & 9 & ( & 0.08 & 0.06 & 0.150 & 3 & 6 & 0.40 & 1.13 & 0.05 & 6 & 01 \\
\hline 150 & 0.14 & $0.46)$ & 0.68 & 0.16 & 0.39 & 9 & 0 & 0 . & 0 & 0.12 & 2 & 1.70 & 0.45 & 19 & 05 & 06 & 1 \\
\hline 160 & 0.11 & 0.49 & 0.63 & & & 9 & 3 & & 0 . & & & & 2 & & 4 & & \\
\hline 170 & 0.09 & 0.51 & 0.57 & 91 & 0. & 8 & $i$ & 0 & 0 & 0 & ) & 6 & 8 & & 4 & 6) & \\
\hline 180 & 0.07 & 0.54 & 0.52 & 1 & 0.37 & 8 & 0. & 0 & 0. & 0.10 & 0. & 9 & 0.65 & 1.36 & 0.04 & (6) & \\
\hline 190 & 0. & 0.56 & 16 & 3 & 0.36 & 31 & 0.16 & 0 & 0.04 & $0.09 \mathrm{C}$ & 0.08 & 20 & 0.72 & 1.40 & 0.04 & 07 & 0 \\
\hline 200 & 0.04 & $0.5 ?$ & 0.41 & 0.25 & 0.35 & 0.08 & 0.16 & 4 & 0.03 & 0.03 & 0.07 & 2.31 & 0.78 & 43 & 0.04 & 0.07 & 0 \\
\hline 210 & $0.0: 3$ & 0.6 .1 & & & & & & & & & & & 5.1 & & 4 & & \\
\hline 220 & $0.0 \div$ & 0.633 & 2 & $\$$ & 0.32 & i & 4 & 0.02 & 0. & : & $0.0: 3$ & 8 & $92:$ & 8 & 4 & C & 1 \\
\hline $2: 30$ & 0.02 & 0.64 & 0.27 & 30 & 0.31 & 0.07 & 0.13 & 0.02 & 0.02 & 0.06 & 0.0 .5 & 2.55 & 0.98 & 0 & 0.04 & 10 & 11 \\
\hline 240 & 0.02 & 0.66 & 1 & 0.31 & 0.30 & 0. & 0.12 & 0.01 & 0.02 & 0.05 & 0.04 & 11 & 1.05 & 0 & 0.04 & 8 & 2 \\
\hline 250 & 0.03 & 0.67 & 0.20 & 0.32 & 0.23 & 0.06 & 0.11 & 0.01 & 0.01 & 0.04 & 0.03 & 2.65 & 1.10 & 1.50 & 0.05 & 0.08 & 2 \\
\hline 260 & 0. & 0.177 & & 1 & 0. & & 1 & 1 & 0. & 15 & 3 & 58 & & & $5)$ & $?$ & \\
\hline 270 & 0.06 & 0.63 & & 51 & 0.24 & 51 & 0.10 & 0.01 & 0.01 & 3 & 2 & 0 . & 1.21 & 6) & 06 & 0 & 4 \\
\hline $2 S 0$ & 0.08 & 0.65 & 0.12 & 0.35 & 0.22 & 0.0 .1 & 0.09 & 0.01 & 0.00 & 0.021 & 2 & 2.70 & 1.25 & 1.43 & 0.06 & 0 & D \\
\hline 290 & 0.10 & 0.68 & 0.10 & 0.36 & 0.21 & 1 & 0.08 & 0.01 & 0.00 & 0.02 & 0.01 & 2.68 & 1.29 & 1.40 & $0.06 \mid$ & 0.10 & 0.06 \\
\hline 300 & 0.13 & 0.67 & $\begin{array}{ll}0 & 0\end{array}$ & $0.3 \pi$ & 0.19 & 0.04 & 0.07 & 0.02 & 0.00 & 0.02 & 0.01 & 2.65 & 1.33 & 1.36 & 0.07 & 0.10 & 97 \\
\hline 310 & 0. & 0.67 & 0.09 & 7 & & & 6 & & 0 & 0.02 & 1 & & & & 88 & 0 & \\
\hline 320 & 0.19 & 0.66 & 0.08 & 0.37 & 0.16 & 3 & 0.03 & 0.02 & 0 & 0.02 & 0 & 5.1 & i.! & 5 & 0.08 & 0.11 & 9 \\
\hline 330 & 0.22 & 0.64 & 0.09 & 0.37 & 0.14 & 0.03 & 0.04 & 0.03 & 0.00 & 0.02 & 0. & 2.48 & 1.39 & ?) & $0.08 \mid$ & 0.11 & 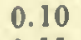 \\
\hline 340 & 0.26 & 0.133 & 0.10 & 0.37 & 0.12 & 0.02 & 0.04 & 0.04 & 0.00 & 0.02 & 0.01 & 2.40 & 1.40 & 1.13 & 0.09 & 0.11 & 0.11 \\
\hline 350 & 0.30 & 0.611 & 0.12 & 0.36 & 0.10 & 0.02 & 0.03 & 0.04 & 0.00 & 0.02 & 0.01 & 2.31 & 1.40 & 1.06 & 0.10 & 0.12 & .12 \\
\hline 360 & 0.33 & $0.5 ?$ & & 0.36 & & & & & & 0.02 & & 20 : & 90 & & 11 & 2 & \\
\hline 370 & $0.3 i$ & 0.56 & 0.16 & 35 & 0. & 1 & 0.02 & 0. & 0.01 & 0.03 & 0 & $?$ & 70 & 1 & ] & 0 & 4 \\
\hline 330 & 0.41 & 0.54 & 0.20 & 0.34 & 0.06 & 11 & 0.02 & 0.07 & 0.01 & 0.03 & 0.03 & $\left|1.9 c_{i}\right|$ & 1.350 & 3 & 0.12 & 0.13 & 5 \\
\hline 390 & 0.45 & 0.51 & 0.23 & 0.33 & 0.05 & $0.01\}$ & 0.02 & 0.08 & 0.01 & 0.04 & 0.03 & 1.83 & 1.32 & 0.75 & 0.12 & 0.13 & 0.16 \\
\hline 400 & $0.4 ?$ & 0.49 & 0.27 & 0.32 & 0.01 & 0.01 & 0.02 & 0.09 & 0.02 & 0.04 & 0.01 & 1.70 & 1.29 & $0.6,7$ & 0.13 & 0.13 & 17 \\
\hline$\$ 10$ & $0.5: 3$ & 0.46 & 0.32 & 0.31 & & & & & & $0.0 \div$ & & 60 & & & & & \\
\hline 420 & 0.57 & 0.43 & 0.36 & 0.29 & 0.03 & 11 & 0.01 & 0. & 0. & $0.0 t$ & 0 . & 12. & 1.21 & 2 & 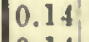 & c & \\
\hline 430 & 0.60 & 0.40 & 0.41 & 0.28 & $0.02\}$ & 0.01 & 0.02 & 0.11 & 0.03 & $0.0 f_{i}$ & 0. & 1.28 & 1.16 & 4 & 0.14 & 0 & \\
\hline 27 & 0.153 & 0.37 & $0.46 \mid$ & 0.26 & 0.02 & 0.01 & 0.02 & 0.12 & 0.04 & $0.07 \mathrm{C}$ & 0.07 & 1.14 & 1.10 & 0.37 & 0.15 & 0.14 & 0.19 \\
\hline 450 & 0.030 & 0.34 & 0.52 & 0.24 & 0.02 & 0.01 & 0.02 & 0.13 & 0.04 & 0.08 & 0.09 & 1.00 & 1.05 & 0.31 & 0.15 & 0.14 & 19 \\
\hline 460 & 0.69 & 0.31 & 578 & & & 1 & & & & $09 \mathrm{C}$ & & 87 & & & & & \\
\hline 4 & 0.71 & 0.29 & 0.63 & 0.21 & 0. & & 0. & 0. & 0 & 0.10 & 0. & & 2( & & if & c & 0 \\
\hline , & 0.73 & $0.26)$ & 0.68 & 0.19 & 0.03 & 2 & 0.03 & 0. & 0.06 & 0.10 & 0. & $0.61 \mid$ & 0.85 & 0.14 & 0.16 & 0.14 & 0.20 \\
\hline 490 & 0.75 & 0.24 & 0.74 & 0.17 & 0.04 & 0.02 & 0.04 & 0.16 & 0.07 & 0.11 & 0.12 & 0.50 & 0.78 & 0.10 & 0.16 & 0.13 & 0.20 \\
\hline 500 & 0.76 & 0.21 & 0.78 & 0.15 & 0.05 & 0.02 & 0.04 & 0.17 & 0.07 & 0.12 & 0.13 & 0.39 & 0.720 & 0.07 & 0.16 & 0.13 & 0.20 \\
\hline 5 & 7 ( & 0.19 & 4 & 11 & $6)$ & 3 & 5 & & 0.07 & 0.13 & & & & & & & \\
\hline 5 & 0.78 & 0.17 & 0.89 & 0.12 & 0.08 & 0.03 & 0.06 & 0.18 & 0. & 0.14 & & & 80 & & (i) & 0. & 0.19 \\
\hline 5 & 8 & 0.10 & 0.93 & 0.10 & 0.09 & 0.03 & 0.07 & 0.18 & 0.08 & 0.14 & 0.15 & 0.15 & 0.520 & 0.00 & 0.16 & 0.12 & 0.19 \\
\hline $3+0$ & 0.78 & 0.14 & 0.97 & 0.09 & 0.10 & 0.04 & 0.08 & 0.19 & 0.08 & 0.15 & 0.16 & 0.09 & 0.450 & 0.00 & 0.16 & 0.12 & 0.18 \\
\hline 550 & 0.77 & 0.13 & 1.00 & 0.09 & 0.12 & 0.04 & 0.08 & 0.19 & 0.02 & 0.16 & 0.17 & 0.05 & 0.40 & 0.00 & 0.15 & 0.12 & 0.18 \\
\hline & 76 & 0.13 & 04 & 0.07 & & 0.01 & & & 0.09 & 0.16 & & 0.02 & 0.34 & & 0.15 & & \\
\hline 57 & 0.74 & 0.12 & 1.06 & 0.06 & 0.16 & 0.05 & 0.10 & 0. & 0.09 & 0.17 & & 0.00 & 0.29 & 0.04 & 0.15 & 0.11 & 0.16 \\
\hline 59 & 0.72 & 0.12 & 1.09 & 0.05 & 0.18 & 0.05 & 0.11 & 0.13 & 0.10 & 0.17 & 0.18 & 0.00 & 0.25 & 0.07 & 0.14 & 0.11 & 0.15 \\
\hline D.10 & 0.70 & 0.12 & 1.10 & 0.04 & 0.19 & 0.06 & 11.12 & 0.13 & 0.10 & 0.19 & 0.19 & 0.02 & 0.21 & 0.10 & 0.14 & 0.10 & 0.14 \\
\hline 600 & 0.67 & 0.13 & 1.11 & 0.03 & 0.21 & 0.06 & 0.13 & 0.18 & 0.10 & 0.18 & 0.19 & 0.05 & 0.17 & 0.14 & 0.13 & 0.10 & 0.13 \\
\hline
\end{tabular}




\begin{tabular}{|c|c|c|c|c|c|c|c|c|c|c|}
\hline \multicolumn{6}{|c|}{ TABLE XVII $a}$. & \multicolumn{5}{|c|}{ TAIBLE XVII $b$. } \\
\hline Year. & $(v . s .1)$ & $(v . c .1)$ & Year. & (v.s.1) & $(v . c .1)$ & Year. & \multicolumn{2}{|l|}{ (v.s.1) } & \multicolumn{2}{|l|}{$(v . c .1)$} \\
\hline & $" \prime$ & " & & $"$ & $"$ & & $"$ & & $"$ & \\
\hline 1800 & -240.33 & -162.11 & 1850 & -183.57 & -189.69 & 1500 & -560.44 & & -96.24 & \\
\hline $18(1)$ & 239.19 & 162.61 & 1851 & 182.44 & 190.29 & 1510 & $550.85^{+}$ & $\begin{array}{r}9.59 \\
0.68\end{array}$ & $95.81^{t}$ & $\begin{array}{r}+0.43 \\
0.27\end{array}$ \\
\hline 1802 & 238.06 & 163.12 & 1852 & 181.30 & 190.89 & 1520 & 541.17 & 9.08 & 95.54 & 0.27 \\
\hline 1803 & 236.92 & 163.63 & 1853 & 180.17 & 191.50 & 1530 & 531.40 & $\begin{array}{l}9 \cdot 77 \\
9.86\end{array}$ & $95.44^{\top}$ & $\begin{array}{l}+0.10 \\
-0.07\end{array}$ \\
\hline 1804 & 235.79 & 164.14 & 1854 & 179.04 & 192.10 & 1540 & 521.54 & $9 \cdot 95$ & 95.51 & 0.24 \\
\hline 1805 & -234.65 & -164.65 & 1855 & -177.91 & -192.71 & 1550 & $-511.5 !$ & -10.04 & -95.75 & $0.4 T$ \\
\hline 1806 & 233.51 & 165.16 & 1856 & 176.78 & 193.32 & 1560 & $501.55^{+}$ & 10.13 & 96.16 & $\begin{array}{r}-0.41 \\
0.50\end{array}$ \\
\hline 1807 & 232.38 & 165.68 & 1857 & .65 & 193.93 & 1570 & 491.42 & 10.22 & 96.75 & 0.77 \\
\hline 1808 & 231.24 & 166.20 & 1858 & 174.51 & 194.54 & 1580 & 481.20 & 10.30 & 97.52 & 0.95 \\
\hline 1809 & 230.11 & 166.72 & 1859 & 173.38 & 195.15 & 1590 & 470.90 & $10.3^{8}$ & 98.47 & I. I 3 \\
\hline 1810 & -228.97 & -167.24 & 1860 & -172.25 & -195.77 & 1600 & -460.52 & & -99.60 & \\
\hline 1811 & 227.83 & 167.76 & 1861 & .12 & 196.39 & 1610 & 450. & -10.40 & 100.93 & -1.33 \\
\hline 1812 & 226.70 & 168.29 & 1862 & .99 & 197.01 & 1620 & 439.53 & 10.53 & 102.44 & I. $5^{I}$ \\
\hline 1813 & 225.56 & 168.82 & 1863 & 168.86 & 197.63 & 1630 & 428.92 & 68 & 104.14 & $\begin{array}{l}1.70 \\
.88\end{array}$ \\
\hline 1814 & 224.43 & 169.35 & 1864 & 167.72 & 198.25 & 1640 & 418.24 & I0 & 106.02 & $\begin{array}{l}1.88 \\
2.07\end{array}$ \\
\hline 1815 & -223.29 & -169.88 & 1865 & -166.59 & -198.88 & 1650 & -407.49 & & -108.09 & \\
\hline 1816 & 222.15 & 170.41 & 1866 & .46 & .51 & 1660 & $67^{+}$ & 10.88 & 11 & $\begin{array}{r}-2.20 \\
2.15\end{array}$ \\
\hline 1817 & 221.02 & 170.95 & 1867 & .33 & 200.14 & 1670 & 385.79 & 10 & 112.80 & $\begin{array}{l}2.45 \\
2.65\end{array}$ \\
\hline 1818 & 219.88 & 171.49 & 1868 & .20 & 200.77 & 1680 & 374.86 & $\begin{array}{l}10.93 \\
10.00\end{array}$ & 45 & $\begin{array}{l}2.05 \\
2.83\end{array}$ \\
\hline 1819 & 218.75 & 172.03 & 1869 & 162.07 & 201.40 & 1690 & 363.87 & II.04 & 118.28 & 3.03 \\
\hline 1820 & -217.61 & -172.57 & 1870 & -160.94 & -202.04 & 1700 & -352.83 & 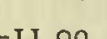 & -121.30 & \\
\hline 1821 & 216.47 & 173.11 & 1871 & 159.81 & 202.68 & 1710 & 341.74 & 1.0 & $124.52^{-}$ & -3.22 \\
\hline 1822 & 215.34 & 173.66 & 1872 & 158.68 & 203.32 & 1720 & 330.61 & II.13 3 & 127.93 & 3.41 \\
\hline 1823 & 214.20 & 174.21 & 1873 & 157.55 & 203.96 & 1730 & 319.43 & $\begin{array}{l}\text { I. } 18 \\
\end{array}$ & 13 & .00 \\
\hline 1824 & 213.07 & 174.76 & 1874 & 156.42 & 204.60 & 1740 & 308.22 & 11.21 & 135.32 & $\begin{array}{l}3.79 \\
2.08\end{array}$ \\
\hline 1825 & -211.93 & -175.31 & 1875 & -155.29 & -205.24 & 1750 & -296.97 & & -139.30 & \\
\hline 1826 & 210.80 & 175.86 & 1876 & 154.16 & 205.89 & 1760 & $285.69^{+}$ & +1 & 143.48 & -4.18 \\
\hline 1827 & 209.66 & 176.42 & 1877 & 153.03 & 206.54 & 1770 & 274.38 & II.3I & 147.85 & 4.37 \\
\hline 1828 & 208.53 & 176.97 & 1878 & 151.91 & 207.19 & 1780 & 263.04 & 11.34 & 152.42 & 4.57 \\
\hline 1829 & 207.39 & 177.53 & 1879 & 150.78 & 207.84 & 1790 & 251.69 & $\begin{array}{l}11.35 \\
\text { I I. } 36\end{array}$ & 157.17 & $\begin{array}{l}4.75 \\
4.04\end{array}$ \\
\hline 1830 & -206.26 & -178.09 & 1880 & -149.65 & -208.49 & 1800 & $-240.33+$ & $-I 1236$ & -162.11 & \\
\hline 1831 & 205.13 & 178.65 & 1881 & .52 & .15 & 1810 & 77 & 36 & 167.24 & $\begin{array}{r}5.33 \\
5.33\end{array}$ \\
\hline 1832 & 203.99 & 179.22 & 1882 & 147.39 & 209.81 & 1820 & 217.61 & $\begin{array}{l}11.30 \\
11.35\end{array}$ & 172.57 & $\begin{array}{l}5.33 \\
5.52\end{array}$ \\
\hline 1833 & 202.86 & 179.78 & 1883 & 146.27 & 210.47 & 1830 & 206.26 & $\begin{array}{l}1.35 \\
1.35\end{array}$ & 09 & 2.71 \\
\hline 1834 & 201.72 & 180.35 & 1884 & .14 & 211.13 & 1840 & 194.91 & $\begin{array}{l}11.35 \\
11.34\end{array}$ & 80 & 5.89 \\
\hline 1835 & -200.59 & -180.92 & 1885 & -144.01 & -211.79 & 1850 & -183.57 & & -189.69 & \\
\hline 1836 & 199.45 & 181.49 & 1886 & .89 & .45 & 1860 & $172.25+$ & FI & 195.77 & \\
\hline 1837 & 198.32 & 182.06 & 1887 & 141.76 & 213.12 & 1870 & 160.94 & I] & 202.04 & \\
\hline 1838 & 197.18 & 182.64 & 1888 & 140.64 & 213.79 & 1880 & 149.65 & $\begin{array}{l}11.29 \\
11.26\end{array}$ & 208.49 & $\begin{array}{l}0.45 \\
6.64\end{array}$ \\
\hline 1839 & 196.05 & 183.22 & 1889 & 139.51 & 214.46 & 1890 & 138.39 & $\begin{array}{l}11.20 \\
11.23\end{array}$ & 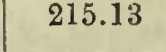 & $\begin{array}{l}0.04 \\
6.82\end{array}$ \\
\hline 1840 & -194.91 & -183.80 & 1890 & -138.39 & -215.13 & 1900 & -127.16 & & -221.95 & \\
\hline 1841 & 193.78 & 184.38 & 1891 & 137.27 & 215.80 & 1910 & $115.98+$ & I. I8 & 228.94 & 0.99 \\
\hline 1842 & 192.64 & 184.96 & 1892 & 136.14 & 216.48 & 1920 & 104.84 & 11.14 & 236.11 & 7.17 \\
\hline 1843 & $191.5 \mathrm{I}$ & 185.55 & 1893 & 135.02 & 217.17 & 1930 & 93.75 & II.09 & 243.46 & $7 \cdot 35$ \\
\hline 1844 & 190.37 & 186.13 & 1894 & 133.90 & 217.84 & 1940 & 82.72 & $\begin{array}{l}11.03 \\
10.98\end{array}$ & 251.00 & $\begin{array}{l}7.54 \\
7.72\end{array}$ \\
\hline 1845 & -189.24 & -186.72 & 1895 & -132.77 & -218.52 & 1950 & -71.74 & & -258.72 & \\
\hline 1846 & 188.10 & 187.31 & 1896 & 131.65 & 219.20 & 1960 & 60.82 & fio. & $266.61^{-}$ & -7. \\
\hline 1847 & 186.97 & 187.90 & 1897 & 130.53 & 219.88 & 1970 & 49.97 & 10.85 & 274.68 & \\
\hline 1848 & 185.84 & 188.50 & 1898 & 129.40 & 220.57 & 1980 & 39.19 & 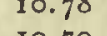 & 282.93 & \\
\hline 1849 & 184.70 & 189.09 & 1899 & 128.28 & 221.26 & 1990 & 28.49 & $\begin{array}{l}10.70 \\
10.6 \mathrm{I}\end{array}$ & 291.35 & $\begin{array}{l}8.42 \\
8.60\end{array}$ \\
\hline 1850 & -183.57 & -189.69 & 1900 & -127.16 & -221.95 & 2000 & -17.88 & & -299.95 & \\
\hline
\end{tabular}




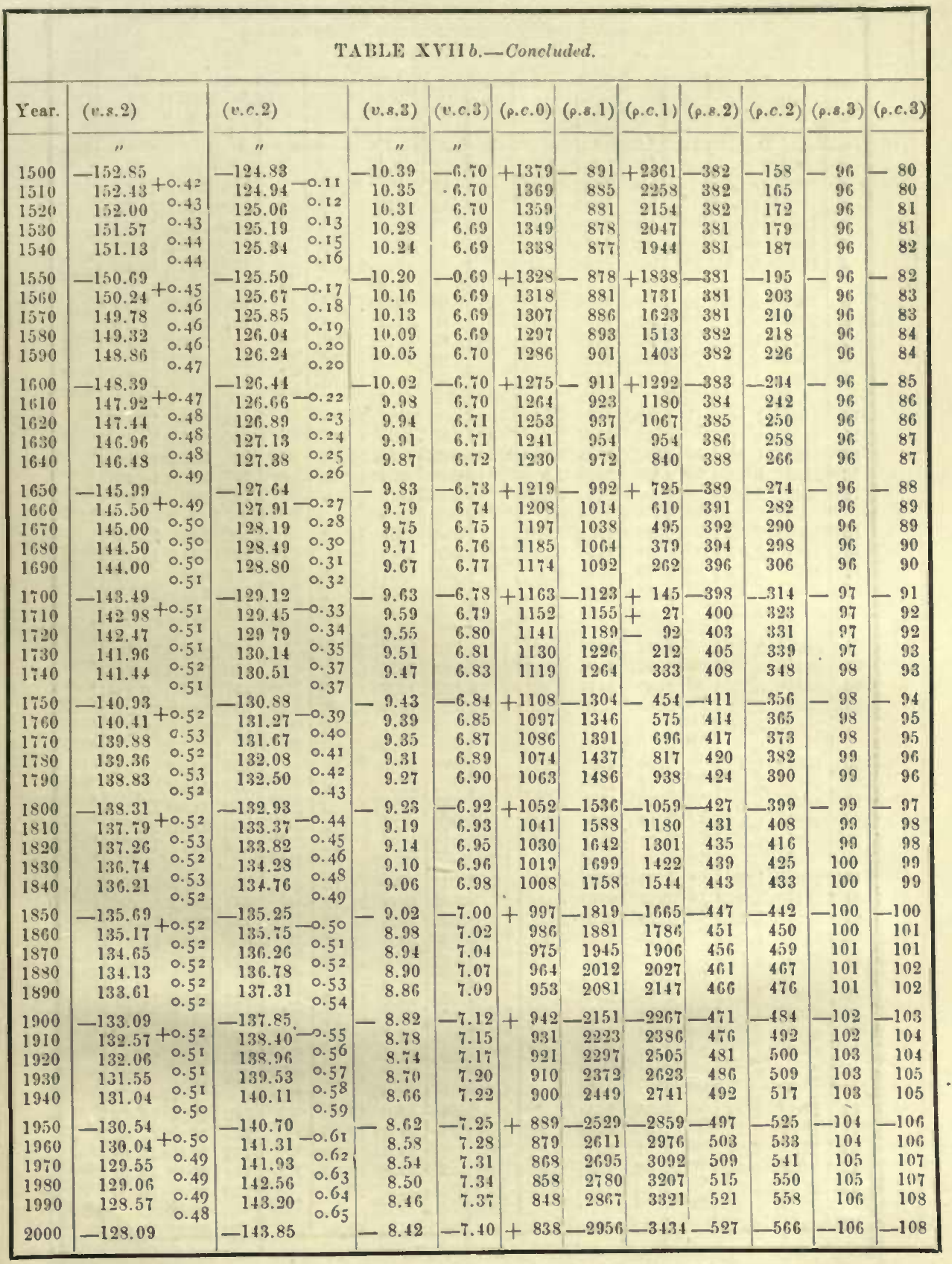


TAible XVhit.-lieduction to the Echiptio. Argunent $u$.

\begin{tabular}{|c|c|c|c|c|c|c|c|c|c|c|c|c|c|c|c|}
\hline$u$ & $u$ & $R$ & & $u$ & $u$ & $R$ & & $u$ & $u$ & $R$ & & $u$ & $u$ & $R$ & \\
\hline 。 & 。 & " & & 。 & 。 & " & & 。 & 。 & $"$ & & 。 & 。 & $"$ & - \\
\hline 0 & 180 & 10.00 & & 45 & 226 & 0.63 & & 90 & 270 & 10.00 & & 135 & 315 & 19.37 & \\
\hline 1 & 181 & 9.67 & $\begin{array}{r}-0.33 \\
0.32\end{array}$ & 46 & 226 & & $\begin{array}{r}+0.01 \\
0.01\end{array}$ & 91 & 271 & $10.33^{-}$ & $\begin{array}{r}+0.33 \\
0.32\end{array}$ & 136 & 316 & 19.36 & r \\
\hline 2 & 182 & 9.35 & & 47 & 227 & 0.65 & 0.03 & 92 & 272 & 10.65 & $\begin{array}{l}0.32 \\
0.33\end{array}$ & 137 & 317 & 19.35 & $\begin{array}{l}0.01 \\
0.03\end{array}$ \\
\hline 3 & 183 & 9.02 & 0. & 48 & & 0.68 & 0.04 & 93 & 273 & 18 & & 138 & 318 & 32 & 3 \\
\hline 4 & 184 & 8.70 & 0.33 & 49 & 29 & 0.72 & $\begin{array}{l}0.04 \\
0.05\end{array}$ & 94 & 274 & 11.30 & $\begin{array}{l}0.32 \\
0.33\end{array}$ & 139 & 319 & 19.28 & $\begin{array}{l}0.04 \\
0.05\end{array}$ \\
\hline 5 & 185 & 8.37 & & 50 & 230 & 0.77 & +0.0 & 95 & 275 & 11.63 & & 140 & 320 & 19.23 & \\
\hline 6 & 186 & 805 & 0.32 & 51 & 31 & 0.84 & 0.07 & 96 & 276 & 11.8 & $\begin{array}{r}0.32 \\
0.32\end{array}$ & 141 & 321 & 19.16 & $\begin{array}{l}0.07 \\
0.07\end{array}$ \\
\hline 7 & 187 & 3 & $0.3 \mathrm{I}$ & 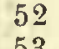 & 32 & 0.91 & 0.08 & 97 & 277 & . & 0.31 & 2 & - & & 0.08 \\
\hline 8 & $\begin{array}{l}188 \\
700\end{array}$ & 7.42 & a & 53 & 33 & 0.99 & 0.10 & 98 & 278 & 12.58 & 0.32 & 143 & 323 & 19.01 & \\
\hline 9 & 189 & 7.10 & $\begin{array}{l}.32 \\
0.30\end{array}$ & 54 & 234 & 1.09 & O.I I & 99 & 279 & 12.90 & $\begin{array}{l}0.32 \\
0.30\end{array}$ & 144 & 324 & 18.91 & $\begin{array}{l}0.10 \\
0.11\end{array}$ \\
\hline 10 & 190 & 6.80 & & 55 & 35 & 1.20 & & 100 & 280 & 13.20 & & 145 & 325 & 18.80 & \\
\hline 1 & 191 & 649 & $-0.3 I$ & 56 & 236 & $1.31^{-}$ & & 101 & 281 & $13.51+$ & -0.31 & 146 & 326 & & - I. II \\
\hline 12 & 192 & 6.19 & $\begin{array}{l}0.30 \\
0.30\end{array}$ & 57 & 237 & 1.44 & $\begin{array}{l}0.13 \\
0.14\end{array}$ & 102 & 282 & 13.81 & $\begin{array}{l}0.30 \\
0.30\end{array}$ & 147 & 327 & 18.56 & 0.13 \\
\hline 13 & 193 & 5.89 & 0.29 & 59 & 238 & 1.58 & $\begin{array}{l}0.14 \\
0.15\end{array}$ & 103 & 283 & 1 & $\begin{array}{l}0.30 \\
0.20\end{array}$ & 8 & 328 & 18.42 & 0.14 \\
\hline 14 & 194 & 5.60 & $\begin{array}{l}0.29 \\
0.28\end{array}$ & 59 & 239 & 1.73 & $\begin{array}{l}0.15 \\
0.15\end{array}$ & 104 & 284 & 14.40 & $\begin{array}{l}0.29 \\
0.28\end{array}$ & 149 & 329 & 18.27 & $\begin{array}{l}0.15 \\
0.15\end{array}$ \\
\hline 15 & 195 & 5.32 & & 60 & 240 & 1.88 & & 105 & 285 & 14.68 & & 150 & 330 & 18.12 & \\
\hline 16 & 196 & 5.04 & & 61 & 241 & $2.05^{-}$ & & 106 & 286 & $14.96+$ & & 151 & 331 & & (6) \\
\hline 17 & 197 & 4.76 & $\begin{array}{l}0.28 \\
0.27\end{array}$ & 62 & 242 & 2.23 & 0.18 & 107 & 287 & 15.24 & 0.28 & 152 & 332 & 17.77 & 0. \\
\hline 18 & 198 & 4.49 & 0.26 & 63 & 243 & 2.42 & 0.20 & 108 & 288 & 15.51 & $\begin{array}{l}0.27 \\
0.26\end{array}$ & 153 & 333 & 17.58 & $\begin{array}{l}0.19 \\
0.20\end{array}$ \\
\hline 19 & 199 & .23 & 0.25 & 64 & 244 & 2.62 & 0.20 & 109 & 289 & 15.7 & 0.25 & 154 & 334 & 17.38 & 0.20 \\
\hline 20 & 200 & 3.98 & & 65 & 245 & 2.82 & & 110 & 290 & 16. & & 155 & 335 & 17.18 & \\
\hline 21 & 20 & 3.73 & $\begin{array}{r}-0.25 \\
0.24\end{array}$ & 66 & 2 & $3.04^{t}$ & 0.22 & 111 & 291 & 16 & & 156 & 3 & & \\
\hline 22 & 20 & 9 & 0.24 & 67 & & 3.26 & $\begin{array}{l}0.22 \\
0.22\end{array}$ & $\because 3$ & 292 & 16.51 & $\begin{array}{l}0.24 \\
0.22\end{array}$ & 157 & & & 0.22 \\
\hline 23 & 203 & 3.26 & $\begin{array}{l}0.23 \\
0.22\end{array}$ & 68 & 248 & 3.49 & $\begin{array}{l}0.23 \\
0.24\end{array}$ & 113 & 293 & 16.74 & 0.22 & 158 & 338 & 16.51 & $\begin{array}{l}0.24 \\
0.24\end{array}$ \\
\hline 24 & 204 & 3.04 & 0.22 & 69 & 249 & 3.73 & $\begin{array}{l}0.24 \\
0.25\end{array}$ & 114 & 294 & 20.00 & $\begin{array}{l}0.22 \\
0.22\end{array}$ & 59 & 3 & 16 & 0.25 \\
\hline- & 205 & 2 & & 70 & 2 & 3.98 & & 115 & 295 & 17.18 & & 60 & 340 & 2 & \\
\hline 26 & 2 & 2.62 & 0.20 & 71 & 2 & $4.23^{\top}$ & 0.26 & 116 & 296 & 17.38 & 0.20 & 161 & 3 & & 0.26 \\
\hline 27 & 207 & 2.42 & 0.19 & 72 & 9. & 4.49 & 0.27 & & 297 & & 0.19 & & 3 & & 0. \\
\hline 28 & 208 & $\begin{array}{l}2.23 \\
2.05\end{array}$ & 0.18 & 73 & 253 & 4.76 & 0.28 & 118 & 298 & 17. & 0.18 & 16 & 343 & 15 & 0.28 \\
\hline & 209 & 2.00 & 0.17 & 74 & 254 & 5.04 & 0.28 & 119 & 299 & 17.95 & & 164 & 344 & 14.96 & 0.28 \\
\hline 30 & 21 & 1 & - & 75 & 2 & 5.32 & & I & 300 & 18 & & 16 & 345 & & \\
\hline 31 & & & 0.15 & 76 & 2 & 5.60 & 0.29 & 121 & 301 & 18. & 0.15 & 1 & 346 & 1 & 0.29 \\
\hline $\begin{array}{l}32 \\
33\end{array}$ & 219 & $\begin{array}{l}1.58 \\
144\end{array}$ & 0.14 & 778 & 257 & 589 & 0.30 & 122 & 302 & & 0.14 & & & & 0.3 \\
\hline 34 & $\begin{array}{l}213 \\
214\end{array}$ & $\begin{array}{l}1.78 \\
1.31\end{array}$ & 0.13 & 79 & $\begin{array}{l}258 \\
259\end{array}$ & $\begin{array}{l}6.19 \\
6.49\end{array}$ & 0.30 & $\begin{array}{l}123 \\
124\end{array}$ & 303 & $\begin{array}{l}18.56 \\
18.69\end{array}$ & 0.13 & $\begin{array}{l}168 \\
169\end{array}$ & 3 & $\begin{array}{l}13.81 \\
13.51\end{array}$ & o. \\
\hline 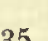 & 91 & & & & & & & & $50 \pm$ & & & & 349 & 1.5 .01 & 0.31 \\
\hline & 21 & & -0.11 & 80 & & & & & 25 & & 0 & & 35 & & \\
\hline 37 & 21 & $\begin{array}{l}1.09 \\
099\end{array}$ & 0.10 & $\begin{array}{l}81 \\
82\end{array}$ & 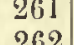 & 10 & 0.32 & 126 & 306 & $18.91^{\dagger}$ & & 17 & 3 & 12. & $0.3^{2}$ \\
\hline 38 & 218 & 0.91 & 0.08 & $\begin{array}{l}82 \\
83\end{array}$ & 26 & $\begin{array}{l}1.42 \\
7.73\end{array}$ & $0.3 I$ & $\begin{array}{l}127 \\
128\end{array}$ & $\begin{array}{l}307 \\
308\end{array}$ & 9 & 0.08 & $\begin{array}{l}172 \\
173\end{array}$ & $\begin{array}{l}352 \\
353\end{array}$ & & $0.3^{\mathrm{I}}$ \\
\hline 39 & 219 & 0.84 & 0.07 & 84 & 264 & 805 & $\begin{array}{l}0.3^{2} \\
0.3^{2}\end{array}$ & 129 & 309 & 19.16 & $\begin{array}{l}0.07 \\
0.07\end{array}$ & 174 & 354 & 11.95 & $\begin{array}{l}0.3^{2} \\
0.3^{2}\end{array}$ \\
\hline 40 & 22 & 0. & & 85 & 26 & 8. & & 1 & 310 & 1 & & 175 & 355 & 11 & \\
\hline 41 & 22 & 0.72 & o. & 86 & & & 0. & & 万11 & & 0.05 & 17 & 356 & & -0. \\
\hline 4 & 22 & & & 100 & & $9 .($ & & 13 & 312 & 19. & & 17 & & 10 & \\
\hline 4 & 22 & & o. & 88 & 26 & 9.3 & 0. & 133 & 313 & & & 178 & & & 3 \\
\hline 44 & 224 & 0.64 & 0. & 89 & 269 & 9.67 & $\begin{array}{l}0.32 \\
0.33\end{array}$ & 134 & 314 & 19.36 & $\begin{array}{l}0.01 \\
0.01\end{array}$ & 179 & 359 & 10.33 & $\begin{array}{l}0.32 \\
0.33\end{array}$ \\
\hline 45 & 225 & 0.63 & & 90 & 270 & 10.00 & & 135 & 315 & 19.37 & & 180 & 360 & 10.00 & \\
\hline
\end{tabular}




\begin{tabular}{|c|c|c|c|c|c|c|c|c|c|c|c|c|c|c|}
\hline$u$ & & $\beta$ & & & $u$ & & $\beta$ & & & $u$ & & $\beta$ & & \\
\hline $180^{\circ}$ & , & " & & $360^{\circ}$ & $190^{\circ}$ & , & " & & $350^{2}$ & $200^{\circ}$ & 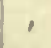 & $"$ & & $340^{\circ}$ \\
\hline $\begin{array}{l}0^{\circ} \\
10^{\prime} \\
20 \\
30 \\
40 \\
50\end{array}$ & $\begin{array}{l}0 \\
0 \\
0 \\
0 \\
0 \\
0\end{array}$ & $\begin{array}{r}0.00 \\
8.69 \\
16.18 \\
24.27 \\
32.35 \\
40.44\end{array}$ & $\begin{array}{l}8.09 \\
8.09 \\
8.09 \\
8.08 \\
8.09 \\
8.09\end{array}$ & $\begin{array}{c}180^{\circ} \\
50^{\prime} \\
40 \\
30 \\
20 \\
10\end{array}$ & $\begin{array}{l}10^{\prime} \\
10^{\prime} \\
20 \\
30 \\
40 \\
50\end{array}$ & $\begin{array}{l}8 \\
8 \\
8 \\
8 \\
8 \\
8\end{array}$ & $\begin{array}{r}2.82 \\
10.78 \\
18.74 \\
26.69 \\
34.64 \\
42.59\end{array}$ & $\begin{array}{l}7.96 \\
7.96 \\
7.95 \\
7.95 \\
7.95 \\
7.95\end{array}$ & $\begin{array}{c}170^{\circ} \\
60^{\prime} \\
10 \\
30 \\
20 \\
10\end{array}$ & $\begin{array}{c}20^{\circ} \\
10^{\prime} \\
20 \\
30 \\
40 \\
50\end{array}$ & $\begin{array}{l}15 \\
15 \\
16 \\
16 \\
16 \\
16\end{array}$ & $\begin{array}{r}50.98 \\
58.58 \\
6.17 \\
13.75 \\
21.32 \\
28.88\end{array}$ & $\begin{array}{l}7.60 \\
7.59 \\
7.58 \\
7.57 \\
7.56 \\
7.55\end{array}$ & $\begin{array}{c}160^{\circ} \\
50^{\prime} \\
40 \\
30 \\
20 \\
10\end{array}$ \\
\hline $\begin{array}{l}1^{\circ} \\
10^{\circ} \\
20 \\
30 \\
40 \\
50\end{array}$ & $\begin{array}{l}0 \\
0 \\
1 \\
1 \\
1 \\
1\end{array}$ & $\begin{array}{r}48.53 \\
56.62 \\
4.70 \\
12.79 \\
20.87 \\
23.96\end{array}$ & $\begin{array}{l}8.09 \\
8.09 \\
8.09 \\
8.08 \\
8.09 \\
8.03\end{array}$ & $\begin{array}{r}179^{\circ} \\
50^{\prime} \\
40 \\
30 \\
20 \\
10\end{array}$ & $\begin{array}{c}11^{\circ} \\
10^{\prime} \\
20 \\
30 \\
40 \\
50\end{array}$ & $\begin{array}{l}8 \\
8 \\
9 \\
9 \\
9 \\
9\end{array}$ & $\begin{array}{r}50.54 \\
58.48 \\
6.41 \\
14.34 \\
22.26 \\
30.18\end{array}$ & $\begin{array}{l}7.94 \\
7.93 \\
7.93 \\
7.92 \\
7.92 \\
7.91\end{array}$ & $\begin{array}{c}169^{\circ} \\
50^{\prime} \\
40 \\
30 \\
20 \\
10\end{array}$ & $\begin{array}{c}21^{\circ} \\
10^{\circ} \\
20 \\
30 \\
40 \\
50\end{array}$ & $\begin{array}{l}16 \\
16 \\
16 \\
16 \\
17 \\
17\end{array}$ & $\begin{array}{r}36.43 \\
43.98 \\
51.52 \\
59.05 \\
6.57 \\
14.05\end{array}$ & $\begin{array}{l}7.55 \\
7.54 \\
7.53 \\
7.52 \\
7.51 \\
7.50\end{array}$ & $\begin{array}{c}159^{\circ} \\
50^{\circ} \\
10 \\
30 \\
20 \\
10\end{array}$ \\
\hline $\begin{array}{l}2^{\circ} \\
10^{\prime} \\
20 \\
30 \\
40 \\
50\end{array}$ & $\begin{array}{l}1 \\
1 \\
1 \\
2 \\
2 \\
2\end{array}$ & $\begin{array}{r}37.04 \\
45.12 \\
53.20 \\
1.28 \\
9.36 \\
17.44\end{array}$ & \begin{tabular}{l|}
8.08 \\
8.05 \\
8.08 \\
8.08 \\
8.08 \\
8.08
\end{tabular} & $\begin{array}{c}178^{\circ} \\
50^{\prime} \\
40 \\
30 \\
20 \\
10\end{array}$ & $\begin{array}{l}12^{\circ} \\
10^{\prime} \\
20 \\
30 \\
40 \\
50\end{array}$ & $\begin{array}{r}9 \\
9 \\
9 \\
10 \\
10 \\
10\end{array}$ & $\begin{array}{r}38.09 \\
45.99 \\
53.89 \\
1.79 \\
9.68 \\
17.57\end{array}$ & $\begin{array}{l}7.90 \\
7.90 \\
7.90 \\
7.89 \\
7.89 \\
7.89\end{array}$ & $\begin{array}{c}188^{\circ} \\
50^{\circ} \\
40 \\
30 \\
20 \\
10\end{array}$ & $\begin{array}{l}22^{\circ} \\
10^{\circ} \\
20 \\
30 \\
40 \\
50\end{array}$ & $\begin{array}{l}17 \\
17 \\
17 \\
17 \\
17 \\
17\end{array}$ & $\begin{array}{l}21.58 \\
29.07 \\
36.55 \\
44.03 \\
51.50 \\
58.96\end{array}$ & $\begin{array}{l}7.49 \\
7.48 \\
7.48 \\
7.47 \\
7.46 \\
7.45\end{array}$ & $\begin{array}{c}158^{\circ} \\
50^{\circ} \\
40 \\
30 \\
20 \\
10\end{array}$ \\
\hline $\begin{array}{l}3 \\
10^{\circ} \\
20 \\
30 \\
40 \\
50\end{array}$ & $\begin{array}{l}2 \\
2 \\
3 \\
2 \\
2 \\
3\end{array}$ & $\begin{array}{r}25.52 \\
33.60 \\
41.67 \\
49.75 \\
57.82 \\
5.89\end{array}$ & \begin{tabular}{l|}
8.08 \\
8.07 \\
8.08 \\
8.07 \\
8.07 \\
8.07
\end{tabular} & $\begin{array}{c}177^{\circ} \\
50^{\circ} \\
40 \\
30 \\
20 \\
10\end{array}$ & $\begin{array}{c}13^{\circ} \\
10^{\prime} \\
20 \\
30 \\
411 \\
50\end{array}$ & $\begin{array}{l}10 \\
10 \\
10 \\
10 \\
10 \\
11\end{array}$ & $\begin{array}{r}25.46 \\
33.34 \\
41.21 \\
49.08 \\
56.94 \\
4.80\end{array}$ & $\begin{array}{l}7.88 \\
7.87 \\
7.87 \\
7.86 \\
7.86 \\
7.85\end{array}$ & $\begin{array}{c}187^{\circ} \\
50^{\prime} \\
40 \\
30 \\
20 \\
10\end{array}$ & $\begin{array}{l}23^{\circ} \\
10^{\circ} \\
20 \\
30 \\
40 \\
50\end{array}$ & $\begin{array}{l}18 \\
18 \\
18 \\
18 \\
18 \\
18\end{array}$ & $\begin{array}{r}6.41 \\
13.85 \\
21.28 \\
28.70 \\
36.11 \\
43.62\end{array}$ & $\begin{array}{l}7.43 \\
7.44 \\
7.43 \\
7.42 \\
7.41 \\
7.41 \\
7.40\end{array}$ & $\begin{array}{c}157^{\circ} \\
50^{\prime} \\
40 \\
30 \\
20 \\
10\end{array}$ \\
\hline $\begin{array}{l}4^{\circ} \\
10^{\prime} \\
20 \\
30 \\
40 \\
50 \\
50\end{array}$ & $\begin{array}{l}3 \\
3 \\
3 \\
3 \\
3 \\
3 \\
4\end{array}$ & $\begin{array}{l}13.96 \\
22.03 \\
30.09 \\
38.16 \\
46.22 \\
54.28 \\
9.31\end{array}$ & $\begin{array}{l}8.07 \\
8.06 \\
8.07 \\
8.06 \\
8.06 \\
8.06\end{array}$ & $\begin{array}{c}176^{\circ} \\
50^{\prime} \\
40 \\
30 \\
20 \\
10\end{array}$ & $\begin{array}{l}14^{\circ} \\
10^{\circ} \\
20 \\
30 \\
40 \\
50\end{array}$ & $\begin{array}{l}11 \\
11 \\
11 \\
11 \\
11 \\
11\end{array}$ & $\begin{array}{l}12.65 \\
20.50 \\
28.34 \\
36.17 \\
44.00 \\
51.82\end{array}$ & $\begin{array}{l}7.05 \\
7.85 \\
7.84 \\
7.83 \\
7.83 \\
7.82 \\
7.81\end{array}$ & $\begin{array}{r}166^{\circ} \\
50^{\circ} \\
10 \\
30 \\
20 \\
10\end{array}$ & $\begin{array}{c}24^{\circ} \\
10^{\circ} \\
20 \\
30 \\
40 \\
50\end{array}$ & $\begin{array}{l}18 \\
18 \\
19 \\
19 \\
19 \\
19 \\
10\end{array}$ & $\begin{array}{r}50.93 \\
58.31 \\
5.68 \\
13.05 \\
20.40 \\
27.75\end{array}$ & $\begin{array}{l}7 \cdot 39 \\
7 \cdot 37 \\
7 \cdot 37 \\
7 \cdot 35 \\
7 \cdot 35 \\
7 \cdot 33\end{array}$ & $\begin{array}{c}156^{\circ} \\
50^{\circ} \\
40 \\
30 \\
20 \\
10\end{array}$ \\
\hline $\begin{array}{l}5^{\circ} \\
10^{\prime} \\
20 \\
30 \\
40 \\
50\end{array}$ & $\begin{array}{l}4 \\
4 \\
4 \\
4 \\
4 \\
4\end{array}$ & $\begin{array}{r}2.34 \\
10.39 \\
18.45 \\
26.50 \\
34.56 \\
42.61\end{array}$ & $\begin{array}{l}8.05 \\
8.06 \\
8.05 \\
8.06 \\
8.05 \\
8.04\end{array}$ & $\begin{array}{c}175^{\circ} \\
50^{\circ} \\
40 \\
30 \\
20 \\
10\end{array}$ & $\begin{array}{c}15^{\circ} \\
10^{\circ} \\
20 \\
30 \\
40 \\
50\end{array}$ & $\begin{array}{l}11 \\
13 \\
12 \\
12 \\
12 \\
12\end{array}$ & $\begin{array}{r}59.63 \\
7.43 \\
15.2 .1 \\
23.04 \\
30.83 \\
38.61\end{array}$ & $\begin{array}{l}7.80 \\
7.81 \\
7.80 \\
7.79 \\
7.78 \\
7.79\end{array}$ & $\begin{array}{c}165^{\circ} \\
50^{\prime} \\
40 \\
30 \\
20 \\
10\end{array}$ & $\begin{array}{c}25 \\
10^{\circ} \\
20 \\
30 \\
40 \\
50\end{array}$ & $\begin{array}{l}19 \\
19 \\
19 \\
19 \\
20 \\
20\end{array}$ & $\begin{array}{r}35.09 \\
42.41 \\
49.72 \\
57.02 \\
4.32 \\
11.61\end{array}$ & $\begin{array}{l}7.33 \\
7.33 \\
7.31 \\
7 \cdot 30 \\
7.30 \\
7.29 \\
7.27\end{array}$ & $\begin{array}{c}155^{\circ} \\
50^{\prime} \\
40 \\
30 \\
20 \\
10\end{array}$ \\
\hline $\begin{array}{l}6^{\circ} \\
10^{\prime} \\
20 \\
30 \\
40 \\
50\end{array}$ & $\begin{array}{l}4 \\
4 \\
5 \\
5 \\
5 \\
5 \\
5\end{array}$ & $\begin{array}{r}50.65 \\
58.69 \\
6.73 \\
14.76 \\
22.79 \\
30.82\end{array}$ & $\begin{array}{l}8.04 \\
8.04 \\
8.03 \\
8.03 \\
8.03 \\
8.03\end{array}$ & $\begin{array}{c}174^{\circ} \\
50^{\prime} \\
40 \\
30 \\
20 \\
10\end{array}$ & $\begin{array}{c}16^{\circ} \\
10^{\circ} \\
20 \\
30 \\
40 \\
50\end{array}$ & $\begin{array}{l}12 \\
13 \\
13 \\
13 \\
13 \\
13\end{array}$ & $\begin{array}{r}46.40 \\
54.18 \\
1.95 \\
9.70 \\
17.45 \\
25.19\end{array}$ & $\begin{array}{l}7.78 \\
7.77 \\
7.75 \\
7.75 \\
7.74 \\
7.74\end{array}$ & $\begin{array}{r}164^{\circ} \\
50^{\prime} \\
40 \\
30 \\
20 \\
10\end{array}$ & $\begin{array}{c}26^{\circ} \\
10^{\prime} \\
20 \\
30 \\
40 \\
50\end{array}$ & $\begin{array}{l}20 \\
20 \\
20 \\
20 \\
20 \\
20\end{array}$ & $\begin{array}{l}18.88 \\
26.14 \\
33.40 \\
40.64 \\
47.89 \\
55.10\end{array}$ & $\begin{array}{l}7.26 \\
7.26 \\
7.24 \\
7.24 \\
7.22 \\
7.21\end{array}$ & $\begin{array}{c}154^{\circ} \\
50^{\prime} \\
40 \\
30 \\
20 \\
10\end{array}$ \\
\hline $\begin{array}{l}7^{\circ} \\
10^{\circ} \\
20 \\
30 \\
40 \\
50\end{array}$ & $\begin{array}{l}5 \\
5 \\
5 \\
6 \\
6 \\
6\end{array}$ & $\begin{array}{r}33.95 \\
46.89 \\
54.90 \\
2.92 \\
10.94 \\
18.95\end{array}$ & $\begin{array}{l}8.03 \\
8.02 \\
8.02 \\
8.02 \\
8.01 \\
8.01\end{array}$ & $\begin{array}{c}173^{\circ} \\
50^{\prime} \\
40 \\
30 \\
20 \\
10\end{array}$ & $\begin{array}{c}17^{\circ} \\
10^{\circ} \\
20 \\
30 \\
40 \\
50\end{array}$ & $\begin{array}{l}13 \\
13 \\
13 \\
13 \\
14 \\
14\end{array}$ & $\begin{array}{r}32.93 \\
40.66 \\
48.39 \\
56.11 \\
3.82 \\
11.52\end{array}$ & $\begin{array}{l}7.73 \\
7.73 \\
7.72 \\
7.71 \\
7.70 \\
7.69\end{array}$ & $\begin{array}{c}163^{\circ} \\
50^{\circ} \\
40 \\
30 \\
20 \\
10\end{array}$ & $\begin{array}{c}27^{\circ} \\
10^{\prime} \\
20 \\
30 \\
40 \\
50\end{array}$ & $\begin{array}{l}21 \\
21 \\
21 \\
21 \\
21 \\
21\end{array}$ & $\begin{array}{r}2.31 \\
9.51 \\
16.70 \\
23.88 \\
31.05 \\
38.21\end{array}$ & $\begin{array}{l}7.20 \\
7.19 \\
7.18 \\
7.17 \\
7.16 \\
7.14\end{array}$ & $\begin{array}{c}153^{\circ} \\
50^{\prime} \\
40 \\
30 \\
20 \\
10\end{array}$ \\
\hline $\begin{array}{l}8 \\
10^{\circ} \\
20 \\
30 \\
10 \\
50\end{array}$ & $\begin{array}{l}6 \\
6 \\
6 \\
6 \\
6 \\
i\end{array}$ & $\begin{array}{r}26.96 \\
34.97 \\
43.97 \\
50.97 \\
58.97 \\
6.96\end{array}$ & $\begin{array}{l}8.01 \\
8.00 \\
8.00 \\
8.00 \\
7.99 \\
8.00\end{array}$ & \begin{tabular}{|c|}
$172^{\circ}$ \\
$50^{\circ}$ \\
40 \\
30 \\
20 \\
10
\end{tabular} & $\begin{array}{c}18^{\circ} \\
10^{\prime} \\
20 \\
30 \\
40 \\
50\end{array}$ & $\begin{array}{l}14 \\
14 \\
14 \\
14 \\
14 \\
14\end{array}$ & $\begin{array}{l}19.21 \\
26.90 \\
31.59 \\
42.25 \\
19.92 \\
57.59\end{array}$ & $\begin{array}{l}7.69 \\
7.68 \\
7.67 \\
7.67 \\
7.66 \\
7.65\end{array}$ & $\begin{array}{c}162^{\circ} \\
50^{\prime} \\
40 \\
30 \\
20 \\
10\end{array}$ & $\begin{array}{c}28^{\prime} \\
10^{\prime} \\
20 \\
30 \\
40 \\
50\end{array}$ & $\begin{array}{l}21 \\
21 \\
21 \\
22 \\
22 \\
22\end{array}$ & $\begin{array}{r}45.35 \\
52.49 \\
59.61 \\
6.73 \\
13.83 \\
20.92\end{array}$ & $\begin{array}{l}7.14 \\
7.12 \\
7.12 \\
7.10 \\
7.09 \\
7.08\end{array}$ & $\begin{array}{c}152^{\circ} \\
50^{\circ} \\
40 \\
30 \\
20 \\
10\end{array}$ \\
\hline $\begin{array}{l}9^{\circ} \\
10^{\prime} \\
20 \\
30 \\
40 \\
50\end{array}$ & $\begin{array}{l}7 \\
i \\
i \\
i \\
7 \\
7\end{array}$ & $\begin{array}{l}14.96 \\
22.95 \\
30.93 \\
38.91 \\
46.88 \\
54.85\end{array}$ & $\begin{array}{l}7.99 \\
7.93 \\
7.98 \\
7.97 \\
7.97 \\
7.97\end{array}$ & $\begin{array}{c}171^{\circ} \\
50^{\prime} \\
40 \\
30 \\
20 \\
10\end{array}$ & $\begin{array}{c}19^{\circ} \\
10^{\prime} \\
20 \\
30 \\
40 \\
50\end{array}$ & $\begin{array}{l}15 \\
15 \\
15 \\
15 \\
15 \\
15\end{array}$ & $\begin{array}{r}5.23 \\
12.88 \\
20.52 \\
28.15 \\
35.77 \\
43.38\end{array}$ & $\begin{array}{l}7.0_{5} \\
7.6_{4} \\
7.6_{3} \\
7.62 \\
7.61 \\
7.60\end{array}$ & $\begin{array}{c}161^{\circ} \\
50^{\circ} \\
40 \\
30 \\
20 \\
10\end{array}$ & $\begin{array}{c}29^{\circ} \\
10^{\prime} \\
20 \\
30 \\
40 \\
50\end{array}$ & $\begin{array}{l}22 \\
22 \\
22 \\
22 \\
22 \\
23\end{array}$ & $\begin{array}{r}28.00 \\
85.07 \\
42.12 \\
49.17 \\
56.20 \\
8.23\end{array}$ & $\begin{array}{l}7.07 \\
7.05 \\
7.05 \\
7.03 \\
7.03 \\
7.01\end{array}$ & $\begin{array}{c}151^{\circ} \\
50^{\circ} \\
40 \\
30 \\
20 \\
10\end{array}$ \\
\hline $10^{\circ}$ & 8 & 2.82 & & $170^{\circ}$ & $20^{\circ}$ & 15 & 50.98 & & $160^{\circ}$ & $30^{\circ}$ & 23 & 10.24 & & $150^{\circ}$ \\
\hline $190^{\circ}$ & & $\beta$ & & $\begin{array}{c}350^{\circ} \\
u\end{array}$ & $200^{\circ}$ & & $\beta$ & & $\begin{array}{c}340^{\circ} \\
u\end{array}$ & $210^{\circ}$ & & $\beta$ & & $\begin{array}{c}330^{\circ} \\
u\end{array}$ \\
\hline
\end{tabular}




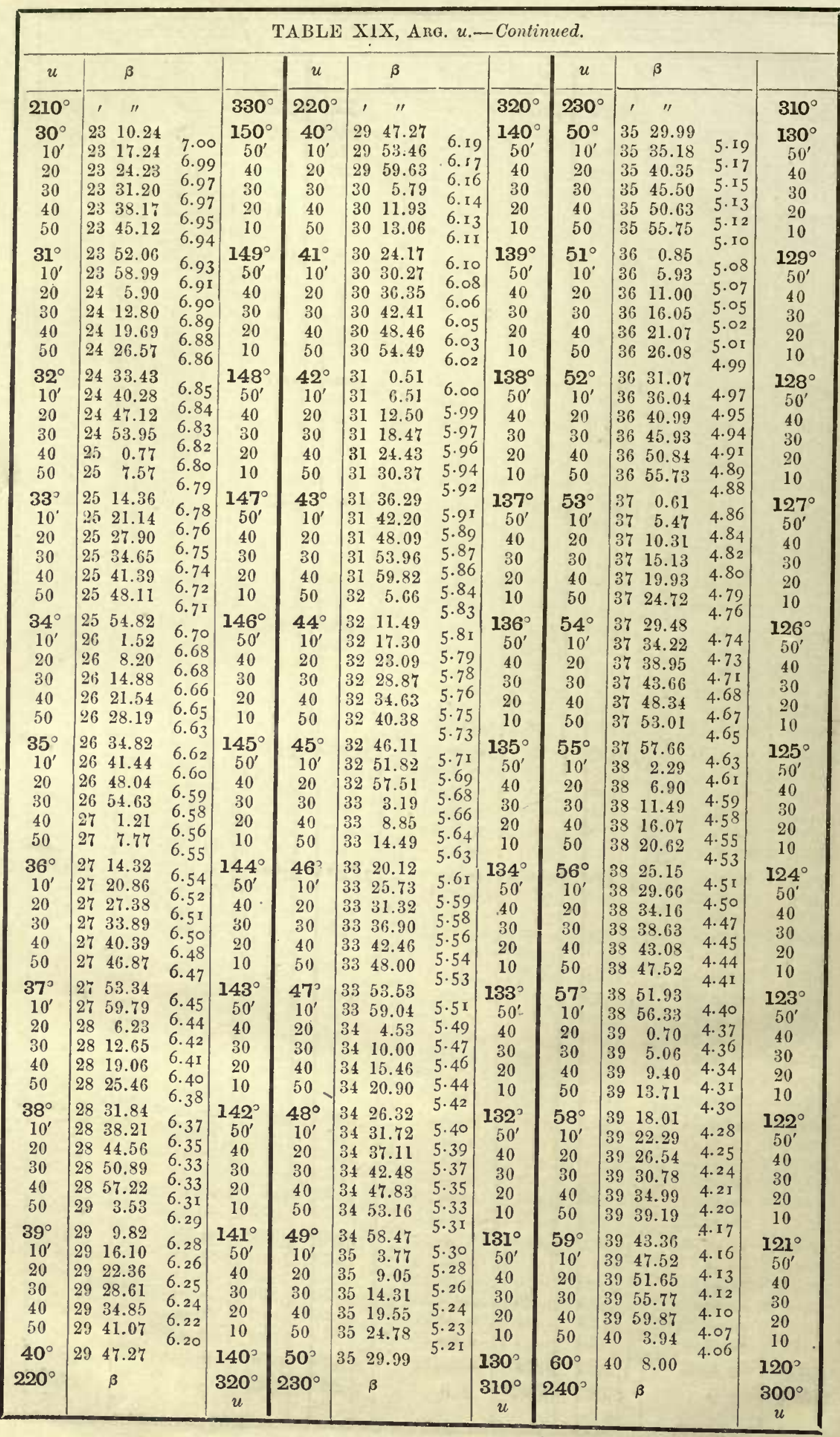




\begin{tabular}{|c|c|c|c|c|c|c|c|c|c|c|c|c|c|c|}
\hline & & & $=$ & & 101. & 2 &, 1 & t. & Cor & d. & & & & \\
\hline$u$ & & $\beta$ & & & $u$ & & $\beta$ & & & $u$ & & $\beta$ & & \\
\hline $240^{\circ}$ & ' & $"$ & & $300^{3}$ & $250^{\circ}$ & ' & $"$ & & $290^{\circ}$ & $260^{\circ}$ & ' & " & & $280^{\circ}$ \\
\hline $60^{\circ}$ & 40 & 8.00 & & $120^{\circ}$ & $70^{\circ}$ & 43 & 32.94 & & $110^{\circ}$ & $80^{\circ}$ & 45 & 38.29 & & $100^{\circ}$ \\
\hline $10^{\prime}$ & 40 & 12.03 & 4.03 & $50^{\prime}$ & $10^{\prime}$ & 43 & 35.60 & 2.76 & $50^{\circ}$ & $10^{\prime}$ & 45 & & 1.39 & $50^{\circ}$ \\
\hline 20 & 40 & 16.04 & 4.01 & 40 & 20 & 43 & 38.33 & 2.73 & +0 & 20 & 45 & +1.05 & 1.37 & 40 \\
\hline 30 & 10 & 20.04 & 4.00 & 30 & 30 & 43 & 41.04 & $\begin{array}{l}2.71 \\
2.60\end{array}$ & 30 & 30 & 45 & 42.40 & 1.35 & 30 \\
\hline 40 & 40 & 24.01 & 3.97 & 20 & 40 & 43 & 43.73 & $\begin{array}{l}2.09 \\
2.67\end{array}$ & 20 & 40 & 45 & 43.73 & 1.33 & 20 \\
\hline 50 & 40 & 27.96 & $\begin{array}{l}3.95 \\
3.03\end{array}$ & 10 & 50 & 43 & 46.40 & 2.64 & 10 & 50 & 45 & 15 & $\begin{array}{l}1.30 \\
1.27\end{array}$ & 10 \\
\hline $61^{\circ}$ & 40 & 31.89 & $\begin{array}{l}3.93 \\
2.0 x\end{array}$ & $119^{\circ}$ & $71^{\circ}$ & 43 & 49.04 & 2.62 & $109^{\circ}$ & $81^{\circ}$ & 45 & 46.30 & 1.26 & $99^{\circ}$ \\
\hline $10^{\prime}$ & 40 & 3.5 .80 & $\begin{array}{l}3.91 \\
3.89\end{array}$ & $50^{\prime}$ & $10^{\prime}$ & 43 & 51.66 & $\begin{array}{l}2.02 \\
2.60\end{array}$ & $50^{\circ}$ & $10^{\prime}$ & 45 & 47.56 & $\begin{array}{l}1.20 \\
1.23\end{array}$ & $50^{\prime}$ \\
\hline 20 & 40 & 39.69 & $\begin{array}{l}3.09 \\
3.87\end{array}$ & 40 & 20 & 43 & 54.26 & 2.58 & 40 & 20 & 45 & 48. & $\begin{array}{l}1.23 \\
1.21\end{array}$ & 40 \\
\hline 30 & 40 & 43.56 & $\begin{array}{l}3.07 \\
3.85\end{array}$ & 30 & 30 & 43 & 56.84 & 2.56 & 30 & 30 & 45 & 50. & 1.18 & 30 \\
\hline 40 & 40 & 47.41 & $\begin{array}{l}3.05 \\
3.83\end{array}$ & 20 & 40 & 43 & 59.40 & 2.53 & 20 & 40 & 45 & 51.18 & $\begin{array}{l}1.10 \\
1.16\end{array}$ & 20 \\
\hline 50 & 40 & 51.24 & 3.81 & 10 & 50 & 44 & 1.93 & 2.51 & 10 & 50 & 45 & 52.34 & 1.14 & 0 \\
\hline $62^{\circ}$ & 40 & 55.05 & 3.79 & $118^{\circ}$ & $72^{2}$ & 41 & 4.44 & 2.49 & $108^{\circ}$ & $82^{\circ}$ & 45 & 53.48 & $x, 11$ & $88^{\circ}$ \\
\hline $10^{\circ}$ & 40 & 58.81 & 3.76 & $50^{\prime}$ & $10^{\prime}$ & $4 f$ & 6.93 & 2.46 & $50^{\prime}$ & $10^{\prime}$ & 45 & 54 & 1.09 & $50^{\prime}$ \\
\hline 20 & 41 & $\begin{array}{r}2.60 \\
6.35\end{array}$ & 3.75 & 40 & 20 & 44 & 9.39 & 2.44 & 40 & 20 & 45 & 55. & $\begin{array}{l}1.09 \\
1.07\end{array}$ & 40 \\
\hline 30 & 41 & $\begin{array}{r}6.35 \\
\end{array}$ & $3-72$ & 30 & 30 & 14 & 11.83 & 2.42 & 30 & 30 & 45 & 56.75 & $\begin{array}{l}1.07 \\
1.04\end{array}$ & 30 \\
\hline 40 & $\$ 1$ & $\begin{array}{l}10.07 \\
13.79\end{array}$ & 3.71 & 20 & 40 & 44 & 14.25 & 2.40 & 20 & 40 & 45 & 57.79 & 1.02 & 20 \\
\hline 50 & $\$ 1$ & 13.73 & 3.68 & 10 & 50 & 41 & 16.65 & 2.38 & 10 & 50 & 45 & 58.81 & 0.99 & 10 \\
\hline $63^{\circ}$ & 41 & 17.46 & 3.67 & $117^{\circ}$ & $73^{\circ}$ & 44 & 19.03 & & $107^{\circ}$ & $83^{\circ}$ & 45 & 59.80 & & $97^{\circ}$ \\
\hline $10^{\prime}$ & 41 & 21.13 & $\begin{array}{l}3.07 \\
3.64\end{array}$ & $50^{\prime}$ & $10^{\prime}$ & 44 & 21.38 & $\begin{array}{l}2.35 \\
2.33\end{array}$ & $50^{\prime}$ & $10^{\prime}$ & 46 & & 0.98 & $50^{\prime}$ \\
\hline 20 & 41 & 24.77 & $\begin{array}{l}3.04 \\
3.62\end{array}$ & 40 & 20 & 44 & 23.71 & $\begin{array}{l}2.33 \\
2.31\end{array}$ & 40 & 20 & 46 & 1.73 & 0.95 & 40 \\
\hline 30 & 41 & 25.39 & $\begin{array}{l}3.02 \\
3.60\end{array}$ & 30 & 30 & 44 & 26.02 & $\begin{array}{l}2.31 \\
2.28\end{array} \mid$ & 30) & 30 & 46 & 2.66 & 0.93 & 30 \\
\hline 40 & +1 & 31.93 & $\begin{array}{l}3.00 \\
3.57\end{array}$ & 2 & 40 & 44 & 28.30 & $\begin{array}{l}2.20 \\
2.27\end{array}$ & 20 & 40 & 46 & 3.56 & $0.9 \circ$ & 20 \\
\hline 50 & 41 & 35.56 & $\begin{array}{l}3.57 \\
3.56\end{array}$ & 10 & 50 & 44 & 30.57 & $\begin{array}{l}2.27 \\
2.25\end{array}$ & 10 & 50 & 16 & 4.44 & $\begin{array}{l}0.88 \\
0.86\end{array}$ & 10 \\
\hline $64^{\circ}$ & 41 & 39.12 & 3.53 & $116^{\circ}$ & $74^{\circ}$ & 44 & 32.82 & & $106^{\circ}$ & $84^{\prime}$ & 46 & 5.30 & & $98^{3}$ \\
\hline $10^{\circ}$ & 41 & 42.65 & $\begin{array}{l}3.53 \\
3.52\end{array}$ & $50^{\prime}$ & $10^{\prime}$ & 44 & 35.04 & $\begin{array}{l}2.22 \\
2.19\end{array}$ & $50^{\prime}$ & $10^{\prime}$ & 46 & 6.14 & $\begin{array}{l}0.84 \\
0.81\end{array}$ & $50^{\prime}$ \\
\hline 20 & 41 & 46.17 & & 40 & 20 & 44 & 37.23 & $\begin{array}{l}2.19 \\
2.17\end{array}$ & 40 & 20 & 46 & 6.95 & 0.81 & 40 \\
\hline 30 & 41 & 49.66 & $\begin{array}{l}3 \cdot 49 \\
3 \cdot 47\end{array}$ & 30 & 30 & 44 & 39.40 & $\begin{array}{l}2.17 \\
2.15\end{array}$ & 30 & 30 & 46 & 7.74 & 9 & 30 \\
\hline 40 & 41 & 53.13 & $\begin{array}{l}3 \cdot 47 \\
3.45\end{array}$ & 2 & 40 & 4 & 41. & $\begin{array}{l}2.15 \\
2.13\end{array}$ & 2 & 40 & 46 & 8. & & 20 \\
\hline 50 & 41 & 56.58 & $\begin{array}{l}3.45 \\
3 \cdot 43\end{array}$ & 10 & 50 & 44 & 43.68 & 2.1 11 & 10 & 50 & 46 & 9.24 & 4 & 10 \\
\hline $65^{\circ}$ & 42 & 0.01 & & $115^{\circ}$ & $75^{\circ}$ & 44 & 45.79 & 2.09 & $105^{\circ}$ & $85^{\circ}$ & 46 & 9.96 & & $95^{\circ}$ \\
\hline $10^{\prime}$ & 42 & 3.42 & $\begin{array}{l}3 \cdot 41 \\
3 \cdot 3^{8}\end{array}$ & $50^{\circ}$ & $10^{\prime}$ & 44 & 47.87 & $\begin{array}{l}2.05 \\
2.06\end{array}$ & $50^{\prime}$ & $10^{\circ}$ & 46 & 10.65 & $\begin{array}{l}0.09 \\
0.67\end{array}$ & $50^{\prime}$ \\
\hline 20 & 42 & 6.80 & $\begin{array}{l}3 \cdot 3^{\circ} \\
3 \cdot 37\end{array}$ & 10 & 20 & 44 & 49.93 & 2.04 & 40 & 20 & 46 & 11.32 & & 40 \\
\hline 30 & 42 & 10.17 & $\begin{array}{l}3 \cdot 37 \\
3 \cdot 34\end{array}$ & 3 & 3( & 44 & 51. & 2.01 & 3 & 30 & 46 & 11.97 & $\begin{array}{l}0.05 \\
0.62\end{array}$ & 30 \\
\hline$\$ 0$ & 42 & $\begin{array}{l}13.51 \\
16.83\end{array}$ & $\begin{array}{l}3.34 \\
3.32\end{array}$ & 20 & 40 & $4 t$ & 53. & r.99 & 2 & 40 & 46 & 12. & $\begin{array}{l}0.02 \\
0.60\end{array}$ & 20 \\
\hline 50 & 42 & 16.83 & $3.3^{\circ}$ & 10 & 50 & 44 & 55.97 & 1.97 & 10 & 50 & 46 & 13.19 & 0.57 & 10 \\
\hline $66^{2}$ & 42 & $\begin{array}{l}20.13 \\
23.41\end{array}$ & 3.28 & $114^{\circ}$ & $76^{\circ}$ & 44 & 57.91 & I.95 & $104^{\circ}$ & $86^{\circ}$ & 46 & 13.76 & & $94^{\circ}$ \\
\hline $10^{\circ}$ & 42 & $\begin{array}{l}23.41 \\
26.67\end{array}$ & 3.26 & $50^{\prime}$ & $10^{\prime}$ & 14 & 59.89 & $\begin{array}{l}1.95 \\
1.92\end{array}$ & $50^{\prime}$ & $10^{\prime}$ & 46 & 14.31 & & $50^{\prime}$ \\
\hline 20 & 42 & 26.67 & 3.24 & 40 & 20 & 45 & 1.81 & & 40 & 20 & 46 & 1. & $\begin{array}{l}0.53 \\
0.51\end{array}$ & 40 \\
\hline 30 & 42 & 29.91 & 3.21 & 30 & 30 & 45 & 3.71 & 1. 88 & 3 & 30 & 46 & 15. & $\begin{array}{l}.5 \\
0.48\end{array}$ & 30 \\
\hline 40 & 42 & $\begin{array}{l}33.12 \\
36.33\end{array}$ & 3.20 & 20 & 40 & 45 & 5.59 & 1.85 & 20 & 40 & 46 & 15. & $\begin{array}{l}0.48 \\
0.46\end{array}$ & 20 \\
\hline 50 & 42 & 36.33 & 3.17 & 10 & 50 & 45 & 7.44 & 1.83 & 10 & 50 & 46 & 16.29 & $\begin{array}{l}0.40 \\
0.43\end{array}$ & 10 \\
\hline $67^{\circ}$ & 42 & 39.49 & & $113^{\circ}$ & $77^{\circ}$ & 45 & 9.27 & & $103^{\circ}$ & $87^{3}$ & 46 & 16.72 & & $93^{\circ}$ \\
\hline $10^{\prime}$ & 42 & 42.64 & $\begin{array}{l}3.15 \\
3.13\end{array}$ & $50^{\prime}$ & $10^{\prime}$ & 45 & 11.08 & & 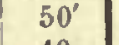 & 10 & 46 & 17 & $0.4 \mathrm{I}$ & $50^{\prime}$ \\
\hline 20 & 42 & $\begin{array}{l}45.77 \\
19.97\end{array}$ & $\begin{array}{l}3.13 \\
3.10\end{array}$ & 40 & 20 & 45 & 12.86 & 8 & 4 & 20 & 46 & 17.52 & 0.39 & 40 \\
\hline 30 & 42 & 48.87 & $\begin{array}{l}3.10 \\
3.09\end{array}$ & 30 & 30 & 45 & 14.62 & $\begin{array}{l}1.70 \\
7.71\end{array}$ & 3 & 30 & 46 & 17. & 0.37 & 30 \\
\hline 40 & 42 & $\begin{array}{l}51.96 \\
55.09\end{array}$ & $\begin{array}{l}3.09 \\
3.06\end{array}$ & 20 & 40 & 45 & 16.36 & 1.74 & 2 & 40 & 46 & 18. & ०. & 20 \\
\hline 50 & 42 & 55.02 & $\begin{array}{l}3.00 \\
3.04\end{array}$ & 10 & 50 & 45 & 18.08 & & 10 & 50 & 46 & 18.55 & o. & 10 \\
\hline $68^{\circ}$ & 42 & 58.06 & & $112^{\circ}$ & $78^{\circ}$ & 45 & 77 & & $102^{3}$ & $88^{\circ}$ & 46 & $18.8_{4}$ & & $92^{\circ}$ \\
\hline $10^{\prime}$ & 43 & 1.09 & $\begin{array}{l}3.02 \\
3.00\end{array}$ & $50^{\prime}$ & $10^{\prime}$ & 45 & 21.44 & 1.67 & $50^{\prime}$ & $10^{\prime}$ & 46 & 19. & 27 & $50^{\prime}$ \\
\hline 20 & 43 & 4.08 & $\begin{array}{l}3.00 \\
2.97\end{array}$ & 40 & 20 & 45 & 23.09 & 1.65 & 40 & 20 & 46 & 19.36 & o. & 40 \\
\hline 30 & 43 & 7.05 & $\begin{array}{l}2.97 \\
2.96\end{array}$ & 30 & 30 & 45 & 24.71 & 1.62 & 30 & 30 & 46 & 19.59 & 0.23 & 3 \\
\hline 40 & 43 & 10.01 & 2.90 & 20 & 40 & 45 & 26.31 & 1.60 & 2 & 40 & 46 & 19. & & 20 \\
\hline 50 & 43 & 12.94 & 2.93 & 10 & 50 & 45 & 27.89 & & 10 & 50 & 46 & 19.97 & 0.18 & 10 \\
\hline $69^{\circ}$ & 43 & 15.85 & & $111^{\circ}$ & $79^{\circ}$ & 45 & 29.45 & & $101^{\circ}$ & $89^{\circ}$ & 46 & 20.12 & & $91^{\circ}$ \\
\hline 10 & 43 & 18.74 & $\begin{array}{l}2.89 \\
2.86\end{array}$ & $50^{\prime}$ & $10^{\prime}$ & 45 & 30.98 & I. 53 & $50^{\prime}$ & $10^{\prime}$ & 46 & 20.25 & 0.13 & $50^{\prime}$ \\
\hline 20 & 43 & 21.60 & 2.86 & 40 & 20 & 45 & 32.49 & & 40 & 20 & 46 & 20.36 & 0.11 & 40 \\
\hline 30 & 43 & 24.45 & $\begin{array}{l}2.85 \\
2.82\end{array}$ & 30 & 30 & 45 & 33.97 & $\begin{array}{r}1.48 \\
1.16\end{array}$ & 30 & 30 & 46 & 20.44 & 0.08 & 30 \\
\hline 40 & 43 & 27.27 & 2.82 & 20 & 40 & 45 & 35.43 & 1.40 & 20 & 40 & 46 & 20.50 & & 20 \\
\hline 50 & 43 & 30.06 & $\begin{array}{l}2.79 \\
2.78\end{array}$ & 10 & 50 & 45 & 36.87 & $\begin{array}{l}1.44 \\
1.42\end{array}$ & 10 & 50 & 46 & 20.53 & $\begin{array}{l}0.03 \\
0.01\end{array}$ & 10 \\
\hline $70^{3}$ & 43 & 32.84 & & $110^{\circ}$ & $80^{\circ}$ & 45 & 38.29 & & $100^{\circ}$ & $90^{3}$ & 46 & $20.5 t$ & & $80^{\circ}$ \\
\hline $250^{\prime}$ & & $\boldsymbol{\beta}$ & & $\begin{array}{c}290^{\circ} \\
u\end{array}$ & $260^{\circ}$ & & $\boldsymbol{\beta}$ & & $\begin{array}{c}280^{\circ} \\
u\end{array}$ & $270^{\circ}$ & & $\beta$ & & $\begin{array}{c}270^{\circ} \\
u\end{array}$ \\
\hline
\end{tabular}




\begin{tabular}{|c|c|c|c|c|c|c|c|c|c|c|c|c|}
\hline \multirow{2}{*}{$\frac{\text { TABLE }}{\text { ARG. }}$} & \multicolumn{2}{|c|}{ XX. } & \multicolumn{5}{|c|}{ XXI. } & \multicolumn{5}{|c|}{ XXII. } \\
\hline & \multicolumn{2}{|c|}{1} & \multicolumn{5}{|c|}{2} & \multicolumn{5}{|c|}{3} \\
\hline & (b.s.1) & $(b . c .1)$ & $($ b.c.o) & $(b . s .1)$ & (b.c.1) & $(b . s .2)$ & (b.c.2) & $(b . c .0)$ & $(b . s .1)$ & $($ b.c.1) & $(b . s .2)$ & $(b . c .2)$ \\
\hline & " & " & " & " & " & $"$ & $m$ & " & " & $"$ & $"$ & $"$ \\
\hline 0 & 1.20 & 1.12 & 0.04 & 5.99 & 5.42 & 0.21 & 0.17 & 0.06 & 1.58 & 1.34 & 0.07 & 0.17 \\
\hline 10 & 1.15 & 1.17 & 0.03 & 6.13 & 5.23 & 0.19 & 0.18 & 0.06 & 1.63 & 1.14 & 0.08 & 0.23 \\
\hline 20 & 1.10 & 1.21 & 0.03 & 6.25 & 5.04 & 0.18 & 0.18 & 0.05 & 1.67 & 0.93 & 0.11 & 0.29 \\
\hline 30 & 1.04 & 1.25 & 0.02 & 6.34 & 4.84 & 0.17 & 0.18 & 0.04 & 1.68 & 0.72 & 0.15 & 0.32 \\
\hline 40 & 0.98 & 1.28 & 0.02 & 6.41 & 4.62 & 0.16 & 0.18 & 0.03 & 1.67 & 0.51 & 0.21 & 0.33 \\
\hline 50 & 0.92 & 1.31 & 0.112 & 6.45 & 4.40 & 0.15 & 0.18 & 0.03 & .60 & $\begin{array}{l}0.33 \\
\end{array}$ & 0.27 & 0.32 \\
\hline 60 & 0.86 & & 0.02 & & 4.18 & 0.15 & 0.18 & 0.02 & 8 & 0.18 & .32 & .28 \\
\hline 70 & 0.79 & 1.34 & 0.02 & 6.40 & 3.94 & 0.16 & 0.18 & 0.01 & $\begin{array}{l}1.32 \\
1.11\end{array}$ & 0.09 & .35 & 0.23 \\
\hline 80 & 0.72 & 1.35 & 0.02 & 6.32 & 3.69 & $\begin{array}{l}0.16 \\
0.17\end{array}$ & $\begin{array}{l}0.17 \\
0.16\end{array}$ & $\begin{array}{l}0.00 \\
0.00\end{array}$ & $\begin{array}{l}1.13 \\
0.94\end{array}$ & $\begin{array}{l}0.07 \\
0.13\end{array}$ & $\begin{array}{l}0.36 \\
0.35\end{array}$ & $\begin{array}{l}0.18 \\
0.13\end{array}$ \\
\hline $\begin{array}{r}90 \\
100\end{array}$ & $\begin{array}{l}0.66 \\
0.59\end{array}$ & $\begin{array}{l}1.30 \\
1.34\end{array}$ & $\begin{array}{l}0.03 \\
0.03\end{array}$ & $\begin{array}{l}6.19 \\
6.02\end{array}$ & $\begin{array}{l}3.43 \\
3.15\end{array}$ & 0.17 & 0.15 & 0.01 & 0.77 & 0.25 & 0.32 & $\begin{array}{l}0.13 \\
0.09\end{array}$ \\
\hline 110 & $\begin{array}{l}0.09 \\
0.52\end{array}$ & 1.32 & & 5.81 & 2.87 & 0.18 & 0.14 & 0.02 & & 0.41 & & 0.07 \\
\hline 120 & 0.46 & 1.30 & 0.04 & 5.56 & 2.57 & 0.19 & 0.12 & 0.04 & 62 & 0.60 & 5 & 0.06 \\
\hline 130 & 0.40 & 1.27 & 0.04 & 5.29 & 2.26 & 0.20 & 0.11 & 0.06 & 0.64 & 0.77 & 0.22 & 0.06 \\
\hline 140 & 0.34 & 1.24 & 0.05 & 4.99 & 1.96 & 0.20 & 0.10 & 0.08 & 0.72 & 0.91 & 0.20 & 0.07 \\
\hline 150 & 0.28 & 1. 20 & 0.05 & 4.68 & 1.66 & 0.20 & 0.09 & 0.09 & 0.83 & 1.00 & 0.18 & 0.08 \\
\hline 160 & 0.23 & 1.15 & 0.05 & 4.36 & 1.38 & 0.20 & 0.08 & 0.10 & 0.95 & 1.04 & 0.17 & 0.09 \\
\hline 170 & 0.19 & 1.10 & 0.06 & 4.03 & 1.11 & 0.20 & 0.08 & 0.11 & 1.04 & 1.04 & 0.17 & 0.10 \\
\hline 180 & 0.15 & 1.0 & 0.06 & 3.69 & 0.86 & 0.19 & 0.07 & 0.11 & 1.11 & 1.01 & 0.16 & 0.10 \\
\hline 190 & 0.12 & 0.98 & 0.06 & 3.36 & 0.65 & 0.17 & 0.07 & 0.11 & 1.14 & 0.97 & 0.14 & 0.11 \\
\hline 200 & 0.09 & 0.92 & 0.06 & 3.03 & 0.56 & 0.16 & 0.08 & 0.11 & 1.14 & 0.93 & 0.13 & 0.12 \\
\hline 210 & 0.07 & 0 & 0.06 & 2.71 & 0.3 & 0.14 & 0.08 & 0.11 & 1.11 & 0.91 & 0.11 & 0.13 \\
\hline 220 & 0.06 & 0. & 0.06 & 2.39 & 0.2 & 0.12 & 0.09 & 0.10 & 1.07 & 0.91 & 0.10 & 0.15 \\
\hline 230 & 0.05 & 0.72 & 0.06 & 2.08 & 0.12 & 0.11 & 0.11 & 0.10 & 1.02 & 0.92 & 0.10 & 0.18 \\
\hline 240 & 0.05 & 0. & 0.07 & 1.77 & 0.08 & 0.09 & 0.13 & 0.09 & 0.98 & 0.96 & 0 & 21 \\
\hline 250 & 0.06 & 0.59 & 0.07 & 1.48 & 0.0 & 0. & 0.15 & 0.09 & 0.96 & 1.00 & 0.12 & 0.23 \\
\hline 26 & & & & & & & & & & & & \\
\hline 270 & 0.10 & 0. & 0.08 & 1.05 & 0. & & 0.20 & 0.08 & 0.94 & 1.09 & 0.16 & 0.26 \\
\hline 280 & 0.13 & 0.40 & 0.08 & 0.72 & 0. & 0.02 & 0.22 & 0.07 & 0.95 & 1.13 & 0.19 & 0.26 \\
\hline 290 & 0.16 & 0.34 & 0.09 & 0.52 & 0.38 & 0.01 & 0.25 & 0.06 & .95 & 1.16 & 0.22 & 0.25 \\
\hline 300 & 0.20 & 0.28 & 0.10 & 0.35 & 0.54 & 0.01 & 0.27 & 0.06 & 0.94 & 1.18 & 0.23 & 0.23 \\
\hline 31 & 0.2 & & & & & & & & & & & \\
\hline 320 & 0.30 & 0.19 & 0.12 & 0.12 & 0 & 0 & 0.31 & 0.07 & 0.91 & 1.22 & 0.23 & 0.19 \\
\hline 330 & 0.36 & 0.15 & 0.13 & 0.06 & 1.1 & 0 & 0.33 & 0.08 & 0. & 1.25 & & 0.18 \\
\hline 340 & 0.42 & 0.12 & 0.14 & 0.04 & 1.44 & 0.05 & 0.35 & 0.09 & 0.86 & 1.28 & 0.20 & 0.17 \\
\hline 350 & 0.48 & 0.09 & 0.15 & 0.06 & 1.72 & 0.07 & 0.36 & 0.09 & 0.84 & 1.34 & 0.18 & 0.17 \\
\hline 360 & 0.5 & & 0. & 0 & 2 & & 0 & & & 0 & & 8 \\
\hline 37 & 0.61 & 0. & 0 . & 0 & 2. & 0 & 0 . & & & 1.46 & & 0 \\
\hline & 0.68 & 0.05 & 0.18 & 0.34 & 2.6 & 0.16 & 0.3 & 0.1 & 0. & 1.51 & 0.14 & 0 \\
\hline 390 & 0.74 & 0.05 & 0.19 & 0.50 & 2.9 & 0.19 & 0.36 & 2 & 8 & 1.54 & 0.13 & 0.21 \\
\hline 400 & 0.81 & 0.06 & 0.20 & 0.70 & 3.24 & 0.23 & 0.35 & 0.11 & 1.03 & 1.54 & 0.13 & 0.22 \\
\hline 41 & 0. & & & & & & & & & & & \\
\hline 420 & 0.94 & & 0.21 & 1.17 & 3.8 & 0.30 & 0.33 & 0 & 1. & 1.44 & & \\
\hline 430 & 1.00 & 0.13 & 0.21 & 1.45 & 4.14 & 0 . & 0.31 & 0. & 1.01 & 1.37 & 0.12 & 0.27 \\
\hline 440 & 1.06 & 0.16 & 0.21 & 1.75 & 4.42 & 0.36 & 0.29 & 0.04 & 0.91 & 1.30 & 0.12 & 0.29 \\
\hline 450 & 1.12 & 0.20 & 0.21 & 2. & 4.70 & & 0.2 & 0.03 & 0.77 & & 0.12 & \\
\hline 460 & 1.17 & 0. & & & & & & & & & & \\
\hline 470 & 1.21 & 0.30 & 0.20 & 2.72 & 5.2 & 0 & 0. & 0 & 0. & 1.40 & 0.17 & 0 \\
\hline 480 & 1.25 & 0.3 & 0.19 & 3.06 & 5.42 & & & & & 1.56 & & 0. \\
\hline 490 & 1.28 & 0.42 & 0.18 & 3.39 & 5.62 & 0.42 & 0.20 & 0.01 & 0.32 & 1.76 & 0.27 & 0.39 \\
\hline 500 & 1.3 & 0.48 & 0.17 & 3.71 & 5.79 & 0.42 & 0.18 & 0.01 & 0.36 & 1.96 & 0.32 & 0.37 \\
\hline & 1. & 0. & & & & & & & & & & \\
\hline 52 & 1.31 & 0.6 & 0.1 & 4.31 & 6.0 & & 0 . & & & & 0.40 & 0.27 \\
\hline 530 & 1.35 & 0.68 & & 4.58 & 6.0 & 0.38 & 0.16 & 0.02 & 0.81 & 2.35 & 0.40 & 5.20 \\
\hline 540 & 1.35 & 0.74 & 0.11 & 4.84 & 6.08 & 0.35 & 0.15 & 0.03 & 1.00 & 2.34 & 0.38 & 0.14 \\
\hline 550 & 1.34 & 0.81 & 0.10 & 5.07 & 6.04 & 0.33 & 0.15 & 0.03 & 1.18 & 2.26 & 0.33 & 0.08 \\
\hline 570 & 130 & 0.8 & 00 & 5. & & & 0. & 0.0 & 1. & 2.11 & 0.27 & 0.05 \\
\hline $\begin{array}{l}570 \\
580\end{array}$ & $\begin{array}{l}1.30 \\
1\end{array}$ & 0.9 & 0.0 & 5.48 & 5.8 & 0. & 0.1 & 0. & 1.41 & 1.94 & & 0.0 \\
\hline 590 & 1.24 & $\begin{array}{l}1.00 \\
1.06\end{array}$ & 0.06 & 5.67 & 5.75 & 0.26 & 0.16 & 0.05 & 1.48 & 1.74 & 0.14 & 0.07 \\
\hline 600 & 1.20 & 1.12 & 0.04 & 5.99 & 5.49 & $\begin{array}{l}0.23 \\
091\end{array}$ & 0.17 & 0.06 & 1.03 & $1.5 t$ & 00 & 0.11 \\
\hline & & & & & 0.45 & 0.21 & 0.17 & 0.06 & 1.58 & 1.34 & 0.07 & 0.17 \\
\hline
\end{tabular}


THE ORBIT-OF URA N U S.

TABLE XXII

\begin{tabular}{|c|c|c|c|c|c|c|c|c|c|c|c|}
\hline Year. & (b.c. 0$)$ & (b.8.1) & (b.c.1) & $(b .8 .2)$ & $(b, e, 2)$ & Year. & (b.c. 0$)$ & (b.8.1) & (b.e.1) & $($ b.8.2) & (b.c.2) \\
\hline & $" \prime$ & " & " & " & $" \prime$ & & " & & " & " & " \\
\hline 1300 & +0.66 & -3.85 & -12.74 & -0.34 & -0.97 & 1800 & +0.15 & -4.60 & -6.00 & -0.44 & -0.46 \\
\hline 1310 & 0.65 & 3.81 & 12.65 & 0.34 & 0.96 & 1810 & 0.14 & 4. 68 & 5.80 & 0.45 & 0.45 \\
\hline 1320 & 0.64 & 3.78 & 12.56 & 0.34 & 0.95 & 1820 & 0.13 & 4.75 & 5.61 & 0.45 & 0.44 \\
\hline 1330 & 0.62 & 3.74 & 12.46 & 0.34 & 0.94 & 1830 & 0.12 & 4.83 & 5.41 & 0.45 & 0.43 \\
\hline 1340 & 0.61 & 3.70 & 12.37 & 0.34 & 0.93 & 1840 & 0.11 & 4.92 & 5.21 & 0.46 & 0.42 \\
\hline 1350 & +0.60 & -3.66 & -12.27 & -0.34 & -0.92 & 1850 & +0.10 & -5.00 & -5.00 & -0.46 & -0.41 \\
\hline 1360 & 0.59 & 3.63 & 12.17 & 0.34 & $\quad 0.90$ & 1860 & 0.09 & 5.09 & 4.79 & 0.47 & 0.40 \\
\hline 1370 & 0.58 & 3.60 & 12.07 & 0.34 & 0.89 & 1870 & 0.08 & 5.18 & 4.58 & 0.47 & 0.39 \\
\hline 1380 & 0.57 & 3.57 & 11.97 & 0.34 & 0.88 & 1880 & 0.07 & 5.27 & 4.36 & 0.48 & 0.38 \\
\hline 1390 & 0.56 & 3.54 & 11.86 & 0.34 & 0.87 & 1890 & 0.06 & 5.36 & 4.14 & 0.48 & 0.37 \\
\hline 1400 & +0.55 & -3.51 & -11.76 & -0.35 & -0.86 & 1900 & +0.05 & -5.45 & -3.92 & -0.49 & -0.36 \\
\hline 1410 & 0.54 & 3.48 & 11.65 & 0.35 & $5 \quad 0.85$ & 1910 & 0.04 & 5.55 & 3.69 & 0.49 & 0.35 \\
\hline 1420 & 0.53 & 3.46 & 11.54 & 0.35 & 0.84 & 1920 & 0.03 & 5.64 & 3.46 & 0.50 & 0.34 \\
\hline 1430 & 0.52 & 3.44 & 11.43 & 0.35 & 0.83 & 1930 & 0.02 & 5.74 & 3.22 & 0.50 & 0.33 \\
\hline 1440 & 0.51 & 3.43 & 11.32 & 0.35 & 0.82 & 1940 & +0.01 & 5.84 & 2.97 & 0.50 & 0.32 \\
\hline 1450 & +0.50 & -3.42 & -11.21 & -0.35 & -0.80 & 1950 . & 0.00 & -5.94 & -2.73 & -0.51 & -0.80 \\
\hline 1460 & 0.49 & 3.41 & 11.10 & 0.35 & 0.79 & 1960 & -0.01 & 6.04 & 2.48 & 0.51 & 0.29 \\
\hline 1470 & 0.48 & 3.40 & 10.98 & 0.36 & 0.78 & 1970 & 0.02 & 6.14 & 2.24 & 0.52 & 0.28 \\
\hline 1480 & 0.46 & 3.40 & 10.86 & 0.36 & 0.77 & 1980 & 0.04 & 6.25 & 1.99 & 0.52 & 0.27 \\
\hline 1490 & 0.45 & 3.40 & 10.74 & 0.36 & 0.76 & 1990 & 0.05 & 6.36 & 1.73 & 0.52 & 0.26 \\
\hline 1500 & +0.44 & -3.40 & -10.62 & -0.36 & -0.75 & 2000 & -0.06 & -6.47 & -1.47 & -0.53 & -0.25 \\
\hline 1510 & 0.43 & 3.40 & 10.50 & 0.36 & 6 $\quad 0.74$ & 2010 & 0.07 & 6.58 & 1.21 & 0.53 & 0.24 \\
\hline 1520 & 0.42 & 3.41 & 10.38 & 0.36 & 0.73 & 2020 & 0.08 & 6.69 & 0.94 & 0.54 & 0.23 \\
\hline 1530 & 0.41 & 3.42 & 10.26 & 0.37 & 0.72 & 2030 & 0.09 & 6.80 & 0.66 & 0.54 & 0.22 \\
\hline 1540 & 0.40 & 3.43 & 10.14 & 0.37 & 0.71 & 2040 & 0.10 & 6.92 & 0.38 & 0.54 & 0.20 \\
\hline 1550 & +0.39 & -3.45 & -10.01 & -0.37 & $7-0.70$ & 2050 & -0.11 & -7.03 & -0.10 & -0.55 & -0.19 \\
\hline 1560 & 0.38 & 3.47 & 9.88 & 0.37 & 0.70 & 2060 & 0.12 & 7.14 & +0.18 & 0.55 & 0.18 \\
\hline 1570 & 0.37 & 3.49 & 9.75 & 0.37 & 0.69 & 2070 & 0.13 & 7.26 & 0.47 & 0.56 & 0.17 \\
\hline 1580 & 0.36 & 3.51 & 9.61 & 0.37 & 0.68 & 2080 & 0.15 & 7.37 & 0.76 & 0.56 & 0.16 \\
\hline 1590 & 0.35 & 3.53 & 9.47 & 0.38 & 0.67 & 2090 & 0.16 & 7.48 & 1.05 & 0.56 & 0.15 \\
\hline 1600 & +0.34 & -3.56 & -9.33 & -0.38 & $8-0.66$ & 2100 & -0.17 & -7.60 & +1.35 & -0.57 & -0.14 \\
\hline 1610 & 0.33 & 3.59 & 9.19 & 0.38 & 0.65 & 2110 & 0.18 & 7.72 & 1.65 & $\quad 0.57$ & 0.13 \\
\hline 1620 & 0.32 & 3.62 & 9.05 & 0.39 & 0.64 & 2120 & 0.19 & 7.84 & 1.96 & 0.57 & 0.12 \\
\hline 1630 & 0.32 & 3.65 & 8.90 & 0.39 & 0.63 & 2130 & 0.21 & 7.97 & 2.27 & 0.58 & $0: 10$ \\
\hline 1640 & 0.31 & 3.69 & 8.75 & 0.39 & 0.62 & 2140 & 0.22 & 8.09 & 2.58 & 0.58 & 0.09 \\
\hline 1650 & +0.30 & -3.73 & -8.60 & -0.39 & $9-0.61$ & 2150 & -0.23 & -8.21 & +2.90 & -0.58 & -0.08 \\
\hline 1660 & 0.29 & 3.77 & 8.44 & 0.40 & $\begin{array}{ll}0 & 0.60\end{array}$ & 2160 & 0.24 & 8.33 & 3.22 & 0.59 & 0.07 \\
\hline 1670 & 0.28 & 3.81 & 8.29 & 0.40 & 0.59 & 2170 & 0.25 & 8.44 & 3.54 & 0.59 & 0.06 \\
\hline 1680 & 0.27 & 3.85 & 8.13 & 0.40 & 0.58 & 2180 & 0.27 & 8.56 & 3.87 & 0.59 & $\begin{array}{r}0.05 \\
-0.03\end{array}$ \\
\hline 1690 & 0.26 & 3.90 & 7.96 & 0.41 & 0.57 & 2190 & 0.28 & 8.68 & 4.20 & 0.60 & 0.03 \\
\hline 1700 & +0.25 & -3.95 & -7.79 & -0.41 & $1-0.56$ & 2200 & -0.29 & -8.80 & +4.54 & -0.60 & -0.02 \\
\hline 1710 & 0.24 & 4.00 & 7.63 & 0.41 & 0.55 & 2210 & 0.30 & 8. 92 & 4.88 & 0.60 & -0.01 \\
\hline 1720 & 0.23 & 4.06 & 7.46 & 0.42 & 0.54 & 2220 & 0.31 & 9.04 & 5.23 & 0.61 & 0.00 \\
\hline 1730 & 0.22 & 4.12 & 7.29 & 0.42 & 0.53 & 2230 & 0.33 & 9.16 & 558 & 0.61 & +0.01 \\
\hline 1740 & 0.21 & 4.18 & 7.12 & 0.42 & 0.52 & 2240 & 0.34 & 9.28 & 5.94 & 0.61 & 0.03 \\
\hline 1750 & +0.20 & -4.25 & -6.95 & -0.43 & -0.51 & 2250 & -0.35 & -9.40 & +6.29 & -0.62 & +0.04 \\
\hline 1760 & 0.19 & 4.32 & 6.77 & 0.43 & $\begin{array}{l}3 \\
3\end{array}$ & 2260 & 0.36 & 9.52 & 6.65 & 0.62 & 0.05 \\
\hline 1770 & 0.18 & 4.38 & 6.58 & 0.43 & 0.49 & 2270 & 0.38 & 9.64 & 7.01 & 0.63 & 0.07 \\
\hline 1780 & 0.17 & 4.45 & 6.39 & 0.44 & 0.48 & 2280 & 0.39 & 9.76 & 7.37 & 0.63 & 0.08 \\
\hline 1790 & 0.16 & 4.53 & 6.20 & 0.44 & 0.47 & 2290 & 0.40 & 9.88 & 7.74 & 0.63 & 0.10 \\
\hline 1800 & +0.15 & -4.60 & -6.00 & -0.44 & -0.46 & 2300 & -0.42 & -10.00 & +8.10 & -0.64 & +0.11 \\
\hline
\end{tabular}




\section{TABLE FOR FORMING THE PRODUCTS OF GIVEN NUMBERS BY THE SINE OR COSINE OF A GIVEN ANGLE.}

THIs table is formed for the especial purpose of facilitating the formation of the products (v.s.3) $\sin 3 g$, (v.c. 3$) \cos 3$, etc., (..$s .1) \sin g$, (p.c.1) $\cos g$, for entire degrees of $g$. It is so arranged that the required products can be taken out at sight. Supposing the number to be given in seconds and decimal fractions of a second, we first seek the given angle at the top or bottom of the page, and then enter one of the first nine lines of the table with the fraction part of the second, interpolating for the hundredths. We then add the result mentally to the number corresponding to the entire seconds. The algebraic signs at the sides of the angles are those of the sines or cosines corresponding to the angle and to the column above or below. If the number does not exceed 3 " we can enter the table as if it were ten times greater, and remove the decimal point one place to the left in the result.

For example, to find the value of

$$
21^{\prime \prime} .67 \sin 280^{\circ}+2^{\prime \prime} .25 \cos 280^{\circ}
$$

we find the angle $280^{\circ}$ at the bottom of a pair of columns, the right hand one being the sine column. Entering this column with 0.67 as the argument, we find 0.66. Entering with 2.1 , we find 20.68 , to which adding 0.66 , we have $21^{\prime \prime} .34$ as the sine product. Entering the other column with 22.5, and moving the decimal point, we find $0^{\prime \prime} .39$ for the cosine product. Noticing the algebraic signs on each side of $280^{\circ}$, we find the result to be $-21^{\prime \prime} .34+0^{\prime \prime} .39=-20^{\prime \prime} .95$. 


\begin{tabular}{|c|c|c|c|c|c|c|c|c|c|c|c|}
\hline & \multicolumn{2}{|c|}{$\begin{array}{l}+1^{\circ}+ \\
+179- \\
-181- \\
-359+\end{array}$} & \multicolumn{2}{|c|}{$\begin{array}{l}+2^{\circ}+ \\
+178- \\
-182- \\
-358+\end{array}$} & \multicolumn{2}{|c|}{$\begin{array}{l}+3^{\circ}+ \\
+177- \\
-183- \\
-357+\end{array}$} & \multicolumn{2}{|c|}{$\begin{array}{l}+4^{\circ}+ \\
+176- \\
-184- \\
-356+\end{array}$} & \multicolumn{2}{|c|}{$\begin{array}{l}+5^{\circ}+ \\
+175- \\
-185- \\
-355+\end{array}$} & - \\
\hline & $\sin$ & $\cos$ & $\sin$ & $\cos$ & $\sin$ & $\cos$ & $\sin$ & $\cos$ & $\sin$ & $\cos$ & \\
\hline 0.1 & 0.00 & 0.10 & 0.00 & 0.10 & 0.01 & 0.10 & 0.01 & 0.10 & 0.01 & 0.10 & 0.1 \\
\hline 0.2 & 0.00 & 0.20 & 0.01 & 0.20 & 0.01 & 0.20 & 0.01 & 0.20 & 0.02 & 0.20 & 0.2 \\
\hline 0.3 & 0.01 & 0.30 & 0.01 & 0.30 & 0.02 & 0.30 & 0.02 & 0.30 & 0.03 & 0.30 & 0.3 \\
\hline 0.4 & 0.01 & 0.40 & 0.01 & 0.40 & 0.02 & 0.40 & 0.03 & 0.40 & 0.03 & 0.40 & 0.4 \\
\hline 0.5 & 0.01 & 0.50 & 0.02 & 0.50 & 0.03 & 0.50 & 0.03 & 0.50 & 0.04 & 0.50 & 0.5 \\
\hline 0.6 & 0.01 & 0.60 & 0.02 & 0.60 & 0.03 & 0.60 & 0.04 & 0.60 & 0.05 & 0.60 & 0.6 \\
\hline 0.7 & 0.01 & 0.70 & 0.02 & 0.70 & 0.04 & 0.70 & 0.05 & 0.70 & 0.06 & 0.70 & 0.7 \\
\hline 0.8 & 0.01 & 0.80 & 0.03 & 0.80 & 0.04 & 0.80 & 0.06 & 0.80 & 0.07 & 0.80 & 0.8 \\
\hline 0.9 & 0.02 & 0.90 & 0.03 & 0.90 & 0.05 & 0.90 & 0.06 & 0.90 & 0.08 & 0.90 & 0.9 \\
\hline 1.0 & 0.02 & 1.00 & 0.03 & 1.00 & 0.05 & 1.00 & 0.07 & 1.00 & 0.09 & 1.00 & 1.0 \\
\hline 2.0 & 0.03 & 2.00 & 0.07 & 2.00 & 0.10 & 2.00 & 0.14 & 2.00 & 0.17 & 1.99 & 2.0 \\
\hline 3.0 & 0.05 & 3.00 & 0.10 & 3.00 & 0.16 & 3.00 & 0.21 & 2.99 & 0.26 & 2.99 & 3.0 \\
\hline 4.0 & 0.07 & 4.00 & 0.14 & 4.00 & 0.21 & 3.99 & $0: 28$ & 3.99 & 0.35 & 3.98 & 4.0 \\
\hline 5.0 & 0.09 & 5.00 & 0.17 & 5.00 & 0.26 & 4.99 & 0.35 & 4.99 & 0.44 & 4.98 & 5.0 \\
\hline 6.0 & 0.10 & 6.00 & 0.21 & 6.00 & 0.31 & 5.99 & 0.42 & 5.99 & 0.52 & 5.98 & 6.0 \\
\hline 7.0 & 0.12 & 7.00 & 0.24 & 7.00 & 0.37 & 6.99 & 0.49 & 6.98 & 0.61 & 6.97 & 7.0 \\
\hline 8.0 & 0.14 & 8.00 & 0.28 & 8.00 & 0.42 & 799 & 0.56 & 7.98 & 0.70 & 7.97 & 8.0 \\
\hline 9.0 & 0.16 & 9.00 & 0.31 & 8.99 & 0.47 & 8.99 & 0.63 & 8.98 & 0.78 & 8.97 & 9.0 \\
\hline 10.0 & 0.17 & 10.00 & 0.35 & 9.99 & 0.52 & 9.99 & 0.70 & 9.98 & 0.87 & 9.96 & 10.0 \\
\hline 11.0 & 0.19 & 11.00 & 0.38 & 10.99 & 0.58 & 10.98 & 0.77 & 10.97 & 0.96 & 10.96 & 11.0 \\
\hline 12.0 & 0.21 & 12.00 & 0.42 & 11.99 & 0.63 & 11.98 & 0.84 & 11.97 & 1.05 & 11.95 & 12.0 \\
\hline 13.0 & 0.23 & 13.00 & 0.45 & 12.99 & 0.68 & 12.98 & 0.91 & 12.97 & 1.13 & 12.95 & 13.0 \\
\hline 14.0 & 0.24 & 34.00 & 0.49 & 13.99 & 0.73 & 13.98 & 0.98 & 13.97 & 1.22 & 13.95 & 14.0 \\
\hline 15.0 & 0.26 & 15.00 & 0.52 & 14.99 & 0.79 & 14.98 & 1.05 & 14.96 & 1.31 & 14.94 & 15.0 \\
\hline 16.0 & 0.28 & 16.00 & 0.56 & 15.99 & 0.84 & 15.98 & 1.12 & 15.96 & 1.39 & 15.94 & 16.0 \\
\hline 17.0 & 0.30 & 17.00 & 0.59 & 16.99 & 0.89 & 16.98 & 1.19 & 16.96 & 1.48 & 16.94 & 17.0 \\
\hline 18.0 & 0.31 & 18.00 & 0.63 & 17.99 & 0.94 & 17.98 & 1.26 & 17.96 & 1.57 & 17.93 & 18.0 \\
\hline 19.0 & 0.33 & 19.00 & 0.66 & 18.99 & 0.99 & 18.97 & 1.33 & 18.95 & 1.66 & 18.93 & 19.0 \\
\hline 20.0 & 0.35 & 20.00 & 0.70 & 19.99 & 1.05 & 19.97 & 1.40 & 19.95 & 1.74 & 19.92 & 20. \\
\hline 21.0 & 0.37 & 21.00 & 0.73 & 20.99 & 1.10 & 20.97 & 1.46 & 20.95 & 1.83 & 20.92 & 21. \\
\hline 22.0 & 038 & 22.00 & 0.77 & 21.99 & 1.15 & 21.97 & 1.53 & 21.95 & 1.92 & 21.92 & 22.0 \\
\hline 23.0 & 0.40 & 23.00 & 0.80 & 22.99 & 1.20 & 22.97 & 1.60 & 22.94 & 2.00 & 22.91 & 23.0 \\
\hline 24.0 & 0.42 & 24.00 & 0.84 & 23.99 & 1.26 & 23.97 & 1.67 & 23.94 & 2.09 & 23.91 & 24.0 \\
\hline 25.0 & 0.44 & 25.00 & 0.87 & 24.98 & 1.31 & 24.97 & 1.74 & 2494 & 2.18 & 24.90 & 25. \\
\hline 26.0 & 0.45 & 26.00 & 0.91 & 25.98 & 1.36 & 25.96 & 1.81 & 25.94 & 2.27 & 25.90 & 26 \\
\hline 27.0 & 0.47 & 27.00 & 0.94 & 26.98 & 1.41 & 26.96 & 1.88 & 26.93 & 2.35 & 26.90 & 27 \\
\hline 28.0 & 0.49 & 28.00 & 0.98 & 27.98 & 1.47 & 27.96 & 1.95 & 27.93 & 2.44 & 27.89 & 28.0 \\
\hline 29.0 & 0.51 & 29.00 & 1.01 & 28.98 & 1.52 & 28.96 & 2.02 & 28.93 & 2.53 & 28.89 & 29.0 \\
\hline \multirow[t]{3}{*}{30.0} & 0.52 & 30.00 & 1.05 & 29.98 & 1.57 & 29.96 & 2.09 & 29.93 & 2.61 & 29.89 & 20.0 \\
\hline & $\cos$ & $\sin$ & $\cos$ & $\sin$ & $\cos$ & $\sin$ & $\cos$ & $\sin$ & $\cos$ & $\sin$ & \\
\hline & \multicolumn{2}{|c|}{$\begin{array}{l}+271^{\circ}- \\
-269- \\
-91+ \\
+89+\end{array}$} & \multicolumn{2}{|c|}{$\begin{array}{l}+272- \\
-268- \\
-92+ \\
+88+\end{array}$} & \multicolumn{2}{|c|}{$\begin{array}{l}+273- \\
-267- \\
-93+ \\
+87+\end{array}$} & \multicolumn{2}{|c|}{$\begin{array}{l}+274- \\
-266- \\
-94+ \\
+86+\end{array}$} & \multicolumn{2}{|c|}{$\begin{array}{l}+275- \\
-265- \\
-95+ \\
+85+\end{array}$} & \\
\hline
\end{tabular}




\begin{tabular}{|c|c|c|c|c|c|c|c|c|c|c|c|}
\hline \multirow[t]{2}{*}{-. } & \multicolumn{2}{|c|}{$\begin{array}{l}+6^{\circ}+ \\
+174- \\
-186- \\
-354+\end{array}$} & \multicolumn{2}{|c|}{$\begin{array}{l}+7^{\circ}+ \\
+173- \\
-187- \\
-353+\end{array}$} & \multicolumn{2}{|c|}{$\begin{array}{l}+8^{\circ}+ \\
+172- \\
-188- \\
-352+\end{array}$} & \multicolumn{2}{|c|}{$\begin{array}{l}+9^{\circ}+ \\
+171- \\
-189- \\
-351+\end{array}$} & \multicolumn{2}{|c|}{$\begin{array}{l}+10^{\circ}+ \\
+170- \\
-190- \\
-350+\end{array}$} & \\
\hline & $\sin$ & $\cos$ & $\sin$ & $\cos$ & $\sin$ & $\cos$ & $\sin$ & $\cos$ & $\sin$ & $\cos$ & \\
\hline 0.1 & 0.01 & 0.10 & 0.01 & 0.10 & 0.01 & 0.10 & 0.02 & 0.10 & 0.02 & 0.10 & 0.1 \\
\hline 0.2 & 0.02 & 0.20 & 0.02 & 0.20 & 0.03 & 0.20 & 0.03 & 0.20 & 0.03 & 0.20 & 0.2 \\
\hline 0.3 & 0.03 & 0.30 & 0.04 & 0.30 & 0.04 & 0.30 & 0.05 & 0.30 & 0.05 & 0.30 & 0.3 \\
\hline 0.4 & 0.04 & 0.40 & 0.05 & 0.40 & 0.06 & 0.40 & 0.06 & 0.40 & 0.07 & 0.39 & 0.4 \\
\hline 0.5 & 0.05 & 0.50 & 0.06 & 0.50 & 0.07 & 0.50 & 0.08 & 0.49 & 0.09 & 0.49 & 0.5 \\
\hline 0.6 & 0.06 & 0.60 & 0.07 & 0.60 & 0.08 & 0.59 & 0.09 & 0.59 & 0.10 & 0.59 & 0.6 \\
\hline 0.7 & 0.07 & 0.70 & 0.09 & 0.69 & 0.10 & 0.69 & 0.11 & 0.69 & 0.12 & 0.69 & 0.7 \\
\hline 0.8 & 0.08 & 0.80 & 0.10 & 0.79 & 0.11 & 0.79 & 0.13 & 0.79 & 0.14 & 0.79 & 0.8 \\
\hline 0.9 & 0.09 & 0.90 & 0.11 & 0.89 & 0.13 & 0.89 & 0.14 & 0.89 & 0.16 & 0.89 & 0.9 \\
\hline 1.0 & 0.10 & 0.99 & 0.12 & 0.99 & 0.14 & 0.99 & 0,16 & 0.99 & 0.17 & 0.98 & 1.0 \\
\hline 2,0 & 0.21 & 1.99 & 0.24 & 1.99 & 0.28 & 1.98 & 0.31 & 1.98 & 0.35 & 1.97 & 2.0 \\
\hline 3.0 & 0.31 & 2.98 & 0.37 & 2.98 & 0,42 & 2.97 & 0,47 & 2.96 & 0.52 & 2.95 & 3.0 \\
\hline 4.0 & 042 & 3.98 & 0.49 & 3.97 & 0.56 & 3.96 & 0,63 & 3.95 & 0.69 & 3.94 & 4.0 \\
\hline 5.0 & 0.52 & 4.97 & 0.61 & 4.96 & 0.70 & 4.95 & 0.78 & 4.94 & 0.87 & 4.92 & 5.0 \\
\hline 6.0 & 0.63 & 5.97 & 0.73 & 5.96 & 0.84 & 5.94 & 0.94 & 5.93 & 1.04 & 5.91 & 6.0 \\
\hline 7.0 & 0.73 & 6.96 & 0.85 & 6.95 & 0.97 & 6.93 & 1.10 & 6.91 & 1.22 & 6.89 & 7.0 \\
\hline 8.0 & 0.84 & 7.96 & 0.97 & 7.94 & 1.11 & 7.92 & 1.25 & 7.90 & 1.39 & 7.88 & 8.0 \\
\hline 9.0 & 0.94 & 8.95 & 1.10 & 8.93 & 1.25 & 8.91 & 1.41 & 8.89 & 1.56 & 8.86 & 9.0 \\
\hline 10.0 & 1.05 & 9.95 & 1.29 & 9.93 & 1.39 & 9.90 & 1.56 & 9.88 & 1.74 & 9.85 & 10.0 \\
\hline 11.0 & 1.15 & 10.94 & 1.34 & 10.92 & 1.53 & 10.89 & 1.72 & 10.86 & 1.91 & 10.83 & 11.0 \\
\hline 12.0 & 1.25 & 11.93 & 1.46 & 11.91 & 1.67 & 11.88 & 1.88 & 11.85 & 2.08 & 11.82 & 12.0 \\
\hline 13.0 & 1.36 & 12.93 & 1.58 & 12.90 & 1.81 & 12.87 & 2.03 & 12.84 & 2.26 & 12.80 & 13.0 \\
\hline 14.0 & 1.46 & 13.92 & 1.71 & 13.90 & 1.95 & 13.86 & 2.19 & 13.83 & 2.43 & 13.79 & 14.0 \\
\hline 15.0 & 1.57 & 14.92 & 1.83 & 14.89 & 2.09 & 14.85 & 2.35 & 14.82 & 2.60 & 14.77 & 15.0 \\
\hline 16.0 & 1.67 & 15.91 & 1.95 & 15.88 & 2.23 & 15.84 & 2.50 & 15.80 & 2.78 & 15.76 & 16.0 \\
\hline 17.3 & 1.78 & 16.91 & 2.07 & 16.87 & 2.37 & 16.83 & 2.66 & 16.79 & 2.95 & 16.74 & 17.0 \\
\hline 18.0 & 1.88 & 17.90 & 2.19 & 17.87 & 2.51 & 17.82 & 2.82 & 17.78 & 3.13 & 17.73 & 18.0 \\
\hline 19.0 & 1.99 & 18.90 & 2.32 & 18.86 & 2.64 & 18.82 & 2.97 & 18.77 & 3.30 & 18.71 & 19.0 \\
\hline 20.0 & 2.09 & 19.89 & 2.44 & 19.85 & 2.78 & 19.81 & 3.13 & 19.75 & 3.47 & 19.70 & 20.0 \\
\hline 21.0 & 2.20 & 20.88 & 2.56 & 20.84 & 2.92 & 20.80 & 3.29 & 20.74 & 3.65 & 20.68 & 21.0 \\
\hline 22.0 & 2.30 & 21.88 & 2.68 & 21.84 & 3.06 & 21.79 & 3.44 & 21.73 & 3.82 & 21.67 & 22.0 \\
\hline 23.0 & 2.40 & 22.87 & 2.80 & 22.83 & 3.20 & 22.78 & 3,60 & 22.72 & 3.99 & 22.65 & 23.0 \\
\hline 24.0 & 2.51 & 23.87 & 2.92 & 23.82 & 3.34 & 23.77 & 3.75 & 23.70 & 4.17 & 23.64 & 24.0 \\
\hline 25.0 & 2.61 & 24.86 & 3.05 & 24.81 & 3.48 & 24.76 & 3.91 & 24.69 & 4.34 & 24.62 & 25. \\
\hline 26.0 & 2.72 & 25.86 & 3.17 & 25.81 & 3.62 & 25.75 & 4.07 & 25.68 & 4.51 & 25.61 & 26.0 \\
\hline 27.0 & 2.82 & 26.85 & 3.29 & 26.80 & 3.76 & 26.74 & 4.22 & 26.67 & 4.69 & 26.59 & 27.0 \\
\hline 28.0 & 2.93 & 27.85 & 3.41 & 27.79 & 3.90 & 27.73 & 4.38 & 27.66 & 4.86 & 27.57 & 28.0 \\
\hline 29.0 & 3.03 & 28.84 & 3.53 & 28.78 & 4.04 & 28.72 & 4.54 & 28.64 & 5.04 & 28.56 & 29.0 \\
\hline 30.0 & 3.14 & 29.84 & 3.66 & 29.78 & 4.18 & 29.71 & 4.69 & 29.63 & 5.21 & 29.54 & 30.0 \\
\hline & $\cos$ & $\sin$ & $\cos$ & $\sin$ & $\cos$ & $\sin$ & $\cos$ & $\sin$ & $\cos$ & $\sin$ & \\
\hline - & $\begin{array}{l}+27 \\
-26 \\
-8 \\
+8\end{array}$ & $\begin{array}{l}6- \\
4- \\
6+ \\
4+\end{array}$ & $\begin{array}{r}+27 \\
-26 \\
-9 \\
+8\end{array}$ & $\begin{array}{l}77- \\
33- \\
73+\end{array}$ & $\begin{array}{l}+2 \\
-2 \\
- \\
+\quad\end{array}$ & $\begin{array}{l}78- \\
62- \\
93+ \\
32+\end{array}$ & $\begin{array}{l}+2 \\
-2 \\
-2 \\
+\quad\end{array}$ & $\begin{array}{l}9- \\
31- \\
9+ \\
1+\end{array}$ & $\begin{array}{l}+29 \\
-26 \\
-10 \\
+\quad 8\end{array}$ & $\begin{array}{l}0- \\
0- \\
0+ \\
0+\end{array}$ & \\
\hline
\end{tabular}




\begin{tabular}{|c|c|c|c|c|c|c|c|c|c|c|c|}
\hline & \multicolumn{2}{|c|}{$\begin{array}{l}+11^{\circ}+ \\
+169- \\
-191- \\
-349+\end{array}$} & \multicolumn{2}{|c|}{$\begin{array}{l}+12^{\circ}+ \\
+168 .- \\
-192- \\
-348+\end{array}$} & \multicolumn{2}{|c|}{$\begin{array}{l}+13^{\circ}+ \\
+167- \\
-193- \\
-347+\end{array}$} & \multicolumn{2}{|c|}{$\begin{array}{l}+14^{\circ}+ \\
+166- \\
-194- \\
-346+\end{array}$} & \multicolumn{2}{|c|}{$\begin{array}{l}+15^{\circ}+ \\
+165- \\
-195- \\
-345+\end{array}$} & \\
\hline & $\sin$ & $\cos$ & $\sin$ & $\cos$ & $\sin$ & $\cos$ & $\sin$ & $\cos$ & $\sin$ & $\cos$ & \\
\hline 0.1 & 0.02 & 0.10 & 0.02 & 0.10 & 0.02 & 0.10 & 0.02 & 0.10 & 0.03 & 0.10 & 0.1 \\
\hline 0.2 & 0.04 & 0.20 & 0.04 & 0.20 & 0.04 & 0.19 & 0.05 & 0.19 & 0.05 & 0.19 & 0.2 \\
\hline 0.3 & 0.06 & 0.29 & 0.06 & 0.29 & 0.07 & 0.29 & 0.07 & 0.29 & 0.08 & 0.29 & 0.3 \\
\hline 0.4 & 0.08 & 0.39 & 0.08 & 0.39 & 0.09 & 0.39 & 0.10 & 0.39 & 0.10 & 0.39 & 0.4 \\
\hline 0.5 & 0.10 & 0.49 & 0.10 & 0.49 & 0.11 & 0.49 & 0.12 & 0.49 & 0.13 & 0.48 & 0.5 . \\
\hline 0.6 & 0.11 & 0.59 & 0.12 & 0.59 & 0.13 & 0.58 & 0.15 & 0.58 & 0.16 & 0.58 & 0.6 \\
\hline 0.7 & 0.13 & 0.69 & 0.15 & 0.68 & 0.16 & 0.68 & 0.17 & 0.68 & 0.18 & 0.68 & 0.7 \\
\hline 0.8 & 0.15 & 0.79 & 0.17 & 0.78 & 0.18 & 0.78 & 0.19 & 0.78 & 0.21 & 0.77 & 0.8 \\
\hline 0.9 & 0.17 & 0.88 & 0.19 & 0.88 & 0.20 & 0.88 & 0.22 & 0.87 & 0.23 & 0.87 & 0.9 \\
\hline 1.0 & 0.19 & 0.98 & 0.21 & 0.98 & 0.22 & 0.97 & 0.24 & 0.97 & 0.26 & 0.97 & 1.0 \\
\hline 2.0 & 0.38 & 1.96 & 0.42 & 1.96 & 0.45 & 1.95 & 0.48 & 1.94 & 0.52 & 1.93 & 2.0 \\
\hline 3.0 & 0.57 & 2.94 & 0.62 & 2.93 & 0.67 & 2.92 & 0.73 & 2.91 & 0.78 & 2.90 & 3.0 \\
\hline 4.0 & 0.76 & 3.93 & 0.83 & 3.91 & 0.90 & 3.90 & 0.97 & 3.88 & 1.04 & 3.86 & 4.0 \\
\hline 5.0 & 0.95 & 4.91 & 1.04 & 4.89 & 1.12 & 4.87 & 1.21 & 4.85 & 1.29 & 4.83 & 5.0 \\
\hline 6.0 & 1.14 & 5.89 & 1.25 & 5.87 & 1.35 & 5.85 & 1.45 & 5.82 & 1.55 & 5.80 & 6.0 \\
\hline 7.0 & 1.34 & 6.87 & 1.46 & 6.85 & 1.57 & 6.82 & 1.69 & 6.79 & 1.81 & 6.76 & 7.0 \\
\hline 8.0 & 1.53 & 7.85 & 1.66 & 783 & 1.80 & 7.79 & 1.94 & 7.76 & 2.07 & 7.73 & 8.0 \\
\hline 9.0 & 1.72 & 8.83 & 1.87 & 8.80 & 2.02 & 8.77 & 2.18 & 8.73 & 2.33 & 8.69 & 9.0 \\
\hline 10.0 & 1.91 & 9.82 & 2.08 & 9.78 & 2.25 & 9.74 & 2.42 & 9.70 & 2.59 & 9.66 & 10.0 \\
\hline 11.0 & 2.10 & 10.80 & 2.29 & 10.76 & 2.47 & 10.72 & 2.66 & 10.67 & 2.85 & 10.63 & 11.0 \\
\hline 12.0 & 2.29 & 11.78 & 2.49 & 11.74 & 2.70 & 11.69 & 2.90 & 11.64 & 3.11 & 11.59 & 12.0 \\
\hline 13.0 & 2.48 & 12.76 & 2.70 & 12.72 & 2.92 & 12.67 & 3.14 & 12.61 & 3.36 & 12.56 & 13.0 \\
\hline 14.0 & 2.67 & 13.74 & 2.91 & 13.69 & 3.15 & 13.64 & 3.39 & 13.58 & 3.62 & 13.52 & 14.0 \\
\hline 15.0 & 2.86 & 14.72 & 3.12 & 14.67 & 3.37 & 14.62 & 3.63 & 14.55 & 3.88 & 14.49 & 15.0 \\
\hline 16.0 & 3.05 & 15.71 & 3.33 & 15.65 & 3.60 & 15.59 & 3.87 & 15.52 & 4.14 & 15.45 & 16.0 \\
\hline 17.0 & 3.24 & 16.69 & 3.53 & 16.63 & 3.82 & 16.56 & 4.11 & 16.50 & 4.40 & 16.42 & 17.0 \\
\hline 18.0 & 3.43 & 17.67 & 3.74 & 17.61 & 4.05 & 17.54 & 4.35 & 17.47 & 4.66 & 17.39 & 18.0 \\
\hline 19.0 & 3.63 & 18.65 & 3.95 & 18.58 & 4.27 & 18.51 & 4.60 & 18.44 & 4.92 & 18.35 & 19.0 \\
\hline 20.0 & 3.82 & 19.63 & 4.16 & 19.56 & 4.50 & 19.49 & 4.84 & 19.41 & 5.18 & 19.32 & 20.0 \\
\hline 21.0 & 4.01 & 20.61 & 4.37 & 20.54 & 4.72 & 20.46 & 5.08 & 20.38 & 5.44 & 20.28 & 21.0 \\
\hline 22.0 & 4.20 & 21.60 & 4.57 & 21.52 & 4.95 & 21.44 & 5.32 & 21.35 & 5.69 & 21.25 & 22.0 \\
\hline 23.0 & 4.39 & 22.58 & 4.78 & 22.50 & 5.17 & 22.41 & 5.56 & 22.32 & 5.95 & 22.22 & 23.0 \\
\hline 24.0 & 4.58 & 23.56 & 4.99 & 23.48 & 5.40 & 23.38 & 5.81 & 23.29 & 6.21 & 23.18 & 24.0 \\
\hline 25.0 & 4.77 & 24.54 & 5.20 & 24.45 & 5.62 & 24.36 & 6.05 & 24.26 & 6.47 & 24.15 & 25.0 \\
\hline 26.0 & 4.96 & 25.52 & 5.41 & 25.43 & 5.85 & 25.33 & 6.29 & 25.23 & 6.73 & 25.11 & 26.0 \\
\hline 27.0 & 5.15 & 26.50 & 5.61 & 26.41 & 6.07 & 26.31 & 6.53 & 26.20 & 6.99 & 26.08 & 27.0 \\
\hline 28.0 & 5.34 & 27.49 & 5.82 & 27.39 & 6.30 & 27.28 & 6.77 & 27.17 & 7.25 & 27.05 & 28.0 \\
\hline 29.0 & 5.53 & 28.47 & 6.03 & 28.37 & 6.52 & 28.26 & 7.02 & 28.14 & 7.51 & 28.01 & 29.0 \\
\hline \multirow[t]{2}{*}{30.0} & 5.72 & 29.45 & 6.24 & 29.34 & 6.75 & 29.23 & 7.26 & 29.11 & 7.76 & 28.98 & 30.0 \\
\hline & $\cos$ & $\sin$ & $\cos$ & $\sin$ & $\cos$ & $\sin$ & $\cos$ & $\sin$ & $\cos$ & $\sin$ & \\
\hline - & \multicolumn{2}{|c|}{$\begin{array}{l}+281- \\
-259- \\
-101+ \\
+79+\end{array}$} & \multicolumn{2}{|c|}{$\begin{array}{l}+282- \\
-258- \\
-102+ \\
+\quad 78+\end{array}$} & \multicolumn{2}{|c|}{$\begin{array}{r}+283- \\
-257- \\
-103+ \\
+\quad 77+\end{array}$} & \multicolumn{2}{|c|}{$\begin{array}{l}+284- \\
-256- \\
-104+ \\
+76+\end{array}$} & \multicolumn{2}{|c|}{$\begin{array}{l}+285- \\
-255- \\
-105+ \\
+\quad 75+\end{array}$} & 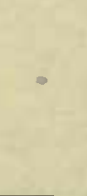 \\
\hline
\end{tabular}


TABLE OF PRODUCTS OF SINES AND COSINES.

\begin{tabular}{|c|c|c|c|c|c|c|c|c|c|c|c|}
\hline & \multicolumn{2}{|c|}{$\begin{array}{l}+16^{\circ}+ \\
+164- \\
-196- \\
-344+\end{array}$} & \multicolumn{2}{|c|}{$\begin{array}{l}+17^{\circ}+ \\
+163- \\
-197- \\
-343+\end{array}$} & \multicolumn{2}{|c|}{$\begin{array}{l}+18^{\circ}+ \\
+162- \\
-198- \\
-342+\end{array}$} & \multicolumn{2}{|c|}{$\begin{array}{l}+19^{\circ}+ \\
+161- \\
-199- \\
-341+\end{array}$} & \multicolumn{2}{|c|}{$\begin{array}{l}+20^{\circ}+ \\
+160- \\
-200- \\
-340+\end{array}$} & \\
\hline & $\sin$ & $\cos$ & $\sin$ & $\cos$ & $\sin$ & $\cos$ & $\sin$ & $\cos$ & $\sin$ & $\cos$ & \\
\hline 0.1 & 0.03 & 0.10 & 0.03 & 0.10 & 0.03 & 0.10 & 0.03 & 0.09 & 0.03 & 0.09 & 0.1 \\
\hline 0.2 & 0.06 & 0.19 & 0.06 & 0.19 & 0.06 & $\begin{array}{lll}0 & 19\end{array}$ & 0.07 & 0.19 & 0.07 & 0.19 & 0.2 \\
\hline 0.3 & 0.08 & 0.29 & 0.09 & 0.29 & 0.09 & 0.29 & 0.10 & 0.28 & 0.10 & 0.28 & 0.3 \\
\hline 0.4 & 0.11 & 0.38 & 0.12 & 0.38 & 0.12 & 0.38 & 0.13 & 0.38 & 0.14 & 0.38 & 0.4 \\
\hline 0.5 & 0.14 & 0.48 & 0.15 & 0.48 & 0.15 & 0.48 & 0.16 & 0.47 & 0.17 & 0.47 & 0.5 \\
\hline 0.6 & 0.17 & 0.58 & 0.18 & 0.57 & 0.19 & 0.57 & 0.20 & 0.57 & 0.21 & 0.56 & 0.6 \\
\hline 0.7 & 0.19 & 0.67 & 0.20 & 0.67 & 0.22 & 0.67 & 0.23 & 0.66 & 0.24 & 0.66 & 0.7 \\
\hline $\begin{array}{l}0.8 \\
0.9\end{array}$ & 0.22 & 0.77 & 0.23 & 0.77 & 0.25 & 0.76 & 0.26 & 0.76 & 0.27 & 0.75 & 0.8 \\
\hline 0.9 & 0.25 & 0.87 & 0.26 & 0.86 & 0.28 & 0.86 & 0.29 & 0.85 & 0.31 & 0.85 & 0.9 \\
\hline 1.0 & 0.28 & 0.96 & 0.29 & 0.96 & 0.31 & 0.95 & 0.33 & 0.95 & 0.34 & 0.94 & 1.0 \\
\hline 2.0 & 0.55 & 1,92 & 0.58 & 1.91 & 0.62 & 1.90 & 0.65 & 1.89 & 0.68 & 1.88 & 2.0 \\
\hline $3: 0$ & 0.83 & 2.88 & 0.88 & 2.87 & 0.93 & 2.85 & 0.98 & 2.84 & 1.03 & 2.82 & 3.0 \\
\hline 4.0 & 1.10 & 3.85 & 1.17 & 3.83 & 1.24 & 3.80 & 1.30 & 3.78 & 1.37 & 3.76 & 4.0 \\
\hline 5.0 & 1.38 & 4.81 & 1.46 & 4.78 & 1.55 & 4.76 & 1.63 & 4.73 & 1.71 & 4.70 & 5.0 \\
\hline 6.0 & 1.65 & 5.77 & 1.75 & 5.74 & 1.85 & 5.71 & 1.95 & 5.67 & 2. & 5.64 & 6.0 \\
\hline 7.0 & 1.93 & 6.73 & 2.05 & 6.69 & 2.16 & 6.66 & 2.28 & 6.62 & 2.39 & 6.58 & 7.0 \\
\hline 8.0 & 2.21 & 7.69 & 2.34 & 7.65 & 2.47 & 7.61 & 2.60 & 7.56 & 2.74 & 7.52 & 8.0 \\
\hline 9.0 & 2.48 & 8.65 & 2.63 & 8.61 & 2.78 & 8.56 & 2.93 & 8.51 & 3.08 & 8.46 & 9.0 \\
\hline 10.0 & 2.76 & 9.61 & 2.92 & 9.56 & 3.09 & 9.51 & 3.26 & 9.46 & 3.42 & 9.40 & 10.0 \\
\hline 11.0 & 3.03 & 10.57 & 3.22 & 10.52 & 3.40 & 10.46 & 3.58 & 10.40 & 3.76 & 10.34 & 11.0 \\
\hline 12.0 & 3.31 & 11.54 & 3.51 & 11.48 & 3.71 & 11.41 & 3.91 & 11.35 & 4.10 & 11.28 & 12.0 \\
\hline 13.0 & 3.58 & 12.50 & 3.80 & 12.43 & 4.02 & 12.36 & 4.23 & 12.29 & 4.45 & 12.22 & 13.0 \\
\hline 14.0 & 3.86 & 13.46 & 4.09 & 13.39 & 4.33 & 13.31 & 4.56 & 13.24 & 4.79 & 13.16 & 14.0 \\
\hline 15.0 & 4.13 & 14.42 & 4.39 & 14.34 & 4.64 & 14.27 & 4.88 & 14.18 & 5.13 & 14.10 & 15.0 \\
\hline 16.0 & 4.41 & 15.38 & 4.68 & 15.30 & 4.94 & 15.22 & 5.21 & 15.13 & 5.47 & 15.04 & 16.0 \\
\hline 17.0 & 4.69 & 16.34 & 4.97 & 16.26 & 5.25 & 16.17 & 5.53 & 16.07 & 5.81 & 15.97 & 17.0 \\
\hline 18.0 & 4.96 & 17.30 & 5.26 & 17.21 & 5.56 & 17.12 & 5.86 & 17.02 & 6.16 & 16.91 & 18.0 \\
\hline 19.0 & 5.24 & 18.26 & 5.56 & 18.17 & 5.87 & 18.07 & 6.19 & 17.96 & 6.50 & 17.85 & 19.0 \\
\hline 20.0 & 5.51 & 19.23 & 5.85 & 19.13 & 6.18 & 19.02 & 6.51 & 18.91 & 6.84 & 18.79 & 20.0 \\
\hline 21.0 & 5.79 & 20.19 & 6.14 & 20.08 & 6.49 & 19.97 & 6.84 & 19.86 & 7.18 & 19.73 & 21.0 \\
\hline 22.0 & 6.06 & 21.15 & 6.43 & 21.04 & 6.80 & 20.92 & 7.16 & 20.80 & 7.52 & 20.67 & 22.0 \\
\hline 23.0 & 6.34 & 22.11 & 6.72 & 22.00 & 7.11 & 21.87 & 7.49 & 21.75 & 7.87 & 21.61 & 23.0 \\
\hline 24.0 & 6.62 & 23.07 & 7.02 & 22.95 & 7.42 & 22.83 & 7.81 & 22.69 & 8.21 & 22.55 & 24.0 \\
\hline 25.0 & 6.89 & 24.03 & 7.31 & 23.91 & 7.73 & 23.78 & 8.14 & 23.64 & 8.55 & 23.49 & 250 \\
\hline 26.0 & $7.17^{\circ}$ & 24.99 & 7.60 & 24.86 & 8.03 & 24.73 & 8.46 & 24.58 & 8.89 & 24.43 & 26.0 \\
\hline 27.0 & 7.44 & 25.95 & 7.89 & 25.82 & 8.34 & 25.68 & 8.79 & 25.53 & 9.23 & 25.37 & 27.0 \\
\hline 28.0 & 7.72 & 26.92 & 8.19 & 26.78 & 8.65 & 26.63 & 9.12 & 26.47 & 9.58 & 26.31 & 28.0 \\
\hline 29.0 & 7.99 & 27.88 & 8.48 & 27.73 & 8.96 & 27.58 & 9.44 & 27.42 & 9.92 & 27.25 & 29.0 \\
\hline \multirow[t]{3}{*}{30.0} & 8.27 & 28.84 & 8.77 & 28.69 & 9.27 & 28.53 & 9.77 & 28.37 & 10.26 & 28.19 & 30.0 \\
\hline & $\cos$ & $\sin$ & $\cos$ & $\sin$ & $\cos$ & $\sin$ & $\cos$ & $\sin$ & $\cos$ & $\sin$ & \\
\hline & \multicolumn{2}{|c|}{$\begin{array}{l}+286- \\
-254- \\
-106+ \\
+74+\end{array}$} & \multicolumn{2}{|c|}{$\begin{array}{l}+287- \\
-253- \\
-107+ \\
+73+\end{array}$} & \multicolumn{2}{|c|}{$\begin{array}{l}+288- \\
-252- \\
-108+ \\
+\quad 72+\end{array}$} & \multicolumn{2}{|c|}{$\begin{array}{l}+289- \\
-251- \\
-109+ \\
+71+\end{array}$} & \multicolumn{2}{|c|}{$\begin{array}{l}+290- \\
-250- \\
-110+ \\
+70+\end{array}$} & \\
\hline
\end{tabular}




\begin{tabular}{|c|c|c|c|c|c|c|c|c|c|c|c|}
\hline & \multicolumn{2}{|c|}{$\begin{array}{l}+21^{\circ}+ \\
+159- \\
-201- \\
-339+\end{array}$} & \multicolumn{2}{|c|}{$\begin{array}{l}+22^{\circ}+ \\
+158- \\
-202- \\
-338+\end{array}$} & \multicolumn{2}{|c|}{$\begin{array}{l}+23^{\circ}+ \\
+157- \\
-203- \\
-337+\end{array}$} & \multicolumn{2}{|c|}{$\begin{array}{l}+24^{\circ}+ \\
+156- \\
-204- \\
-336+\end{array}$} & \multicolumn{2}{|c|}{$\begin{array}{l}+25^{\circ}+ \\
+155- \\
-205- \\
-335+\end{array}$} & \\
\hline & $\sin$ & $\cos$ & $\sin$ & $\cos$ & $\sin$ & $\cos$ & $\sin$ & $\cos$ & $\sin$ & $\cos$ & \\
\hline 0.1 & 0.04 & 0.09 & 0.04 & 0.09 & 0.04 & 0.09 & 0.04 & 0.09 & 0.04 & 0.09 & 0.1 \\
\hline 0.2 & 0.07 & 0.19 & 0.07 & 0.19 & 0.08 & 0.18 & 0.08 & 0.18 & 0.08 & 0.18 & 0.2 \\
\hline 0.3 & 0.11 & 0.28 & 0.11 & 0.28 & 0.12 & 0.28 & 0.12 & 0.27 & 0.13 & 0.27 & 0.3 \\
\hline 0.4 & 0.14 & 0.37 & 0.15 & 0.37 & 0.16 & 0.37 & 0.16 & 0.37 & 0.17 & 0.36 & 0.4 \\
\hline 0.5 & 0.18 & 0.47 & 0.19 & 0.46 & 0.20 & 0.46 & 0.20 & 0.46 & 0.21 & 0.45 & 0.5 \\
\hline 0.6 & & 0.56 & & 0.56 & 0.23 & 0.55 & 0.24 & 0.55 & 0.25 & 0.54 & 0.6 \\
\hline 0.7 & 0.2 & 0.65 & 0.26 & 0.65 & 0.27 & 0.64 & 0 & 0.64 & 0.3 & 0.63 & 0.7 \\
\hline 0.8 & 0.29 & 0.75 & 0.30 & 0.74 & 0.31 & 0.74 & 0.33 & 0.73 & 0.34 & 0.73 & 0.8 \\
\hline 0.9 & 0.32 & 0.84 & 0.34 & 0.83 & 0.35 & 0.83 & 0.37 & 0.82 & 0.38 & 0.82 & 0.9 \\
\hline 1.0 & 0.36 & 0.93 & 0.37 & 0.93 & 0.39 & 0.92 & 0.4 & 0.91 & 0.42 & 0.91 & 1 \\
\hline 2. & & 1.87 & & 1.85 & 0.78 & 1.84 & 0.81 & 1.83 & 0.8 & 1.81 & \\
\hline 3.0 & 1.08 & 2.80 & 1.12 & 2.78 & 1.17 & 2.76 & 1.2 & 2.74 & 1.2 & 2.72 & 3.0 \\
\hline 4.0 & 1.43 & 3.73 & 1.50 & 3.71 & 1.56 & 3.68 & 1.6 & 3.65 & 1.69 & 3.63 & 4.0 \\
\hline 5.0 & 1.79 & 4.67 & 1.87 & 4.64 & 1.95 & 4.60 & 2.03 & 4.57 & 2.11 & 4.53 & 5.0 \\
\hline 6.0 & 2.1 & 5.60 & 2.2 & & 2.34 & & 2. & & 9 & 5.44 & \\
\hline 7.0 & 2.51 & 6.54 & 2.6 & 6.49 & 2.74 & 6.44 & & 6.39 & 2. & 6.34 & 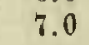 \\
\hline 8.0 & & 7.47 & 3.00 & $7: 42$ & 3.13 & 7.36 & 3.2 & 7.31 & 3. & 7.25 & 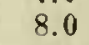 \\
\hline 9.0 & 3.23 & 8.40 & 3.37 & 8.34 & 3.52 & 8.28 & 3.66 & 8.22 & 3.80 & 8.16 & 9.0 \\
\hline 10. & 3.58 & 9.34 & 3. & 9.27 & 3.91 & 9.21 & 4.0 & 9.14 & 4. & 9.06 & 10. \\
\hline 11 & & 10.27 & & 10.20 & & 10 & & & & & \\
\hline 12.0 & 4.30 & 11.20 & 4.50 & .13 & 4.69 & 11.05 & & 6 & 5.07 & 8 & 12. \\
\hline 13.0 & 4.66 & 12.14 & 4.87 & 2.05 & 5.08 & 11.97 & 5.2 & 1.88 & 5.49 & 1.78 & 13. \\
\hline 14.0 & 5.02 & 13.177 & 5.24 & 12.98 & 5.47 & 12.89 & 5.69 & 12.79 & 5.92 & 12.69 & 14.0 \\
\hline 15. & 5.38 & .00 & 5. & 1 & 5 & & & & & & 15 \\
\hline 16 & & 1 & & .83 & 6 & & & & & & \\
\hline 17.0 & 6.09 & .87 & 6.37 & .76 & 6.64 & 15.65 & 6. & 3 & 7. & & 17 \\
\hline 18.0 & 6.45 & 16.80 & 6.74 & 16.69 & 7.03 & 16.57 & 7.8 & & 7. & 1 & 18 \\
\hline 19.0 & 6.81 & 17.74 & 7.12 & 17.62 & 7.42 & 17.49 & 7.73 & 17.36 & 8.03 & 17.22 & 19.0 \\
\hline 20. & 7. & 1 & 7. & 4 & 6 & & & & 8. & 3 & 20 \\
\hline & & 1 & & & & & & & & & \\
\hline & & 2 & 8. & & & & & & & & \\
\hline 23 & 8.24 & 21.47 & 8.62 & 21.33 & 8.99 & 21.17 & 9.3 & 21 & 9.72 & & 23 \\
\hline 24. & 8.60 & 22.41 & 8.99 & 22.25 & 9.38 & 22.09 & 9.76 & 21.93 & 10.14 & 21.75 & 24.0 \\
\hline 25. & 8.96 & 2 & & 8 & 7 & & & & & & 25 \\
\hline & 9.32 & 24.27 & & 24 & 1 & & 10 & & & & \\
\hline 27 & 9.68 & 25.21 & 10 & 25 & 10.55 & & 10. & & & & \\
\hline 28. & 10.03 & 26.14 & & 25.96 & 10.94 & 25.77 & 11. & & & & 28 \\
\hline 29.0 & 10.39 & 27.07 & 10.86 & 26.89 & 11.33 & 26.69 & 11.80 & 26.49 & 12.26 & 26.28 & 29.0 \\
\hline \multirow[t]{6}{*}{30.0} & 10.75 & 28.01 & $11.24^{\prime}$ & 27.82 & 11.72 & 27.62 & 12.20 & 27.41 & 12.68 & 27.19 & 30.0 \\
\hline & $\cos$ & $\sin$ & $\cos$ & $\sin$ & $\cos$ & $\sin$ & $\cos$ & $\sin$ & $\cos$ & $\sin$ & \\
\hline & \multirow{4}{*}{\multicolumn{2}{|c|}{$\begin{array}{l}+291- \\
-249- \\
-111+ \\
+69+\end{array}$}} & \multirow{4}{*}{\multicolumn{2}{|c|}{$\begin{array}{l}+292- \\
-248- \\
-112+ \\
+68+\end{array}$}} & \multirow{4}{*}{\multicolumn{2}{|c|}{$\begin{array}{l}+293- \\
-247- \\
-113+ \\
+67+\end{array}$}} & \multirow{4}{*}{\multicolumn{2}{|c|}{$\begin{array}{l}+294- \\
-246- \\
-114+ \\
+66+\end{array}$}} & \multirow{4}{*}{\multicolumn{2}{|c|}{$\begin{array}{l}+295- \\
-245- \\
-115+ \\
+65+\end{array}$}} & \\
\hline & & & & & & & & & & & \\
\hline & & & & & & & & & & & \\
\hline & & & & & & & & & & & \\
\hline
\end{tabular}




\begin{tabular}{|c|c|c|c|c|c|c|c|c|c|c|c|}
\hline & \multicolumn{2}{|c|}{$\begin{array}{l}+26^{\circ}+ \\
+154- \\
-206- \\
-334+\end{array}$} & \multicolumn{2}{|c|}{$\begin{array}{l}+27^{\circ}+ \\
+153- \\
-207- \\
-333+\end{array}$} & \multicolumn{2}{|c|}{$\begin{array}{l}+28^{\circ}+ \\
+152- \\
-208- \\
-332+\end{array}$} & \multicolumn{2}{|c|}{$\begin{array}{l}+29^{\circ}+ \\
+151- \\
-209- \\
-331+\end{array}$} & \multicolumn{2}{|c|}{$\begin{array}{l}+30^{\circ}+ \\
+150- \\
-210- \\
-330+\end{array}$} & \\
\hline & $\sin$ & $\cos$ & $\sin$ & $\cos$ & $\sin$ & $\cos$ & $\sin$ & $\cos$ & $\sin$ & $\cos$ & \\
\hline 0.1 & 0.04 & 0.09 & 0.05 & 0.09 & 0.05 & 0.09 & 0.05 & 0.09 & 0.05 & 0.09 & 0.1 \\
\hline 0.2 & 0.09 & 0.18 & 0.09 & 0.18 & 0.09 & 0.18 & 0.10 & 0.17 & 0.10 & 0.17 & 0.2 \\
\hline 0.3 & 0.13 & 0.27 & 0.14 & 0.27 & 0.14 & 0.26 & 0.15 & 0.26 & 0.15 & 0.26 & 0.3 \\
\hline 0.4 & 0.18 & 0.36 & 0.18 & 0.36 & 0.19 & 0.35 & 0.19 & 0.35 & 0.20 & 0.35 & 0.4 \\
\hline 0.5 & 0.22 & 0.45 & 0.23 & 0.45 & 0.23 & 0.44 & 0.24 & 0.44 & 0.25 & 0.43 & 0.5 \\
\hline 0.6 & 0.26 & 0.54 & 0.27 & 0.53 & 0.28 & 0.53 & 0.29 & 0.52 & 0.30 & 0.52 & 0.6 \\
\hline 0.7 & 0.31 & 0.63 & 0.32 & 0.62 & 0.33 & 0.62 & 0.34 & 0.61 & 0.35 & 0.61 & 0.7 \\
\hline 0.8 & 0.35 & 0.72 & 0.36 & 0.71 & 0.38 & 0.71 & 0.39 & 0.70 & 0.40 & 0.69 & 0.8 \\
\hline 0.9 & 0.39 & 0.81 & 0.41 & 0.80 & 0.42 & 0.79 & 0.44 & 0.79 & 0.45 & 0.78 & 0.9 \\
\hline 1.0 & 0.44 & 0.90 & 0.45 & 0.89 & 0.47 & 0.88 & 0.48 & 0.87 & 0.50 & 0.87 & 1.0 \\
\hline 2.0 & 0.88 & 1.80 & 0.91 & 1.78 & 0.94 & 1.77 & 0.97 & 1.75 & 1.00 & 1.73 & 2.0 \\
\hline 3.0 & 1.32 & 2.70 & 1.36 & 2.67 & 1.41 & 2.65 & 1.45 & 2.62 & 1.50 & 2.60 & 3.0 \\
\hline 4.0 & 1.75 & 3.60 & 1.82 & 3.56 & 1.88 & 3.53 & 1.94 & 3.50 & 2.00 & 3.46 & 4.0 \\
\hline 5.0 & 2.19 & 4.49 & 2.27 & 4.46 & 2.35 & 4.41 & 2.42 & 4.37 & 2.50 & 4.33 & 5.0 \\
\hline 6.0 & 2.63 & 5.39 & 2.72 & 5.35 & 2.82 & 5.30 & 2.91 & 5.25 & 3.00 & 5.20 & 6.0 \\
\hline 7.0 & 3.07 & 6.29 & 3.18 & 6.24 & 3.29 & 6.18 & 3.39 & 6.12 & 3.50 & 6.06 & 7.0 \\
\hline 8.0 & 3.51 & 7.19 & 363 & 7.13 & 3.76 & 7.06 & 3.88 & 7.00 & 4.00 & 6.93 & 8.0 \\
\hline 9.0 & 3.95 & 8.09 & 4.09 & 8.02 & 4.23 & 7.95 & 4.36 & 7.87 & 4.50 & 7.79 & 9.0 \\
\hline 10.0 & 4.38 & 8.99 & 4.54 & 8.91 & 4.69 & 8.83 & 4.85 & 8.75 & 5.00 & 8.66 & 10.0 \\
\hline 11.0 & 4.82 & 9.89 & 4.99 & 9.80 & 5.16 & 9.71 & 5.33 & 9.62 & 5.50 & 9.53 & 11.0 \\
\hline 12.0 & 5.26 & 10.79 & 5.45 & 10.69 & 5.63 & 10.60 & 5.82 & 10.50 & 6.00 & 10.39 & 12.0 \\
\hline 13.0 & 5.70 & 11.68 & 5.90 & 11.58 & 6.10 & 11.48 & 6.30 & 11.37 & 6.50 & 11.26 & 13.0 \\
\hline 14.0 & 6.14 & 12.58 & 6.36 & 12.47 & 6.57 & 12.36 & 6.79 & 12.24 & 7.00 & 12.12 & 14.0 \\
\hline 15.0 & 6.58 & 13.48 & 6.81 & 13.37 & 7.04 & 13.24 & 7.27 & 13.12 & 7.50 & 12.99 & 15.0 \\
\hline 16.0 & 7.01 & 14.38 & 7.26 & 14.26 & 7.51 & 14.13 & 7.76 & 13.99 & 8.00 & 13.86 & 16.0 \\
\hline 17.0 & 7.45 & 15.28 & 7.72 & 15.15 & 7.98 & 15.01 & 8.24 & 14.87 & 8.50 & 14.72 & 17.0 \\
\hline 18.0 & 7.89 & 16.18 & 8.17 & 16.04 & 8.45 & 15.89 & 8.73 & 15.74 & 9.00 & 15.59 & 18.0 \\
\hline 19.0 & 8.33 & 17.08 & 8.63 & 16.93 & 8.92 & 16.78 & 9.21 & 16.62 & 9.50 & 16.45 & 19.0 \\
\hline 20.0 & 8.77 & 17.98 & 9.08 & 17.82 & 9.39 & 17.66 & 9.70 & 17.49 & 10.00 & 17.32 & 20.0 \\
\hline 21.0 & 9.21 & 18.87 & 9.53 & 18.71 & 9.86 & 18.54 & 10.18 & 18.37 & 10.50 & 18.19 & 21.0 \\
\hline 22.0 & 9.64 & 19.77 & 9.99 & 19.60 & 10.33 & 19.42 & 10.67 & 19.24 & 11.00 & 19.05 & 22.0 \\
\hline .23 .0 & 10.08 & 20.67 & 10.44 & 20.49 & 10.80 & 20.31 & 11.15 & 20.12 & 11.50 & 19.92 & 23.0 \\
\hline 24.0 & 10.52 & 21.57 & 10.90 & 21.38 & 11.27 & 21.19 & 11.64 & 20.99 & 12.00 & 20.78 & 24.0 \\
\hline 25.0 & 10.96 & 22.47 & 11.35 & 22.28 & 11.74 & 22.07 & 12.12 & 21. & 12.50 & 21.65 & 250 \\
\hline 26.0 & 11.40 & 23.37 & 11.80 & 23.17 & 12.21 & 22.96 & 12.61 & 22.74 & 13.00 & 22.52 & 26.0 \\
\hline 27.0 & 11.84 & 24.27 & 12.26 & 24.06 & 12.68 & 23.84 & 13.09 & 23.61 & 13.50 & 23.38 & 27.0 \\
\hline 28.0 & 12.27 & 25.17 & 12.71 & 24.95 & 13.15 & 24.72 & 13.57 & 24.49 & 14.00 & 24.25 & 28.0 \\
\hline 29.0 & 12.71 & 26.07 & 13.17 & 25.84 & 13.61 & 25.61 & 14.06 & 25.36 & 14.50 & 25.11 & 29.0 \\
\hline \multirow[t]{3}{*}{30.0} & 13.15 & 26.96 & 13.62 & 26.73 & 14.08 & 26.49 & 14.54 & 26.24 & 15.00 & 25.98 & 30.0 \\
\hline & $\cos$ & $\sin$ & $\cos$ & $\sin$ & $\cos$ & $\sin$ & $\cos$ & $\sin$ & $\cos$ & $\sin$ & \\
\hline & \multicolumn{2}{|c|}{$\begin{array}{l}+296- \\
-244- \\
-116+ \\
+64+\end{array}$} & \multicolumn{2}{|c|}{$\begin{array}{l}+297- \\
-243- \\
-117+ \\
+\quad 63+\end{array}$} & \multicolumn{2}{|c|}{$\begin{array}{l}+298- \\
-242- \\
-118+ \\
+\quad 62+\end{array}$} & \multicolumn{2}{|c|}{$\begin{array}{l}+299- \\
-241- \\
-119+ \\
+\quad 61+\end{array}$} & \multicolumn{2}{|c|}{$\begin{array}{l}+300- \\
-240- \\
-120+ \\
+60+\end{array}$} & \\
\hline
\end{tabular}




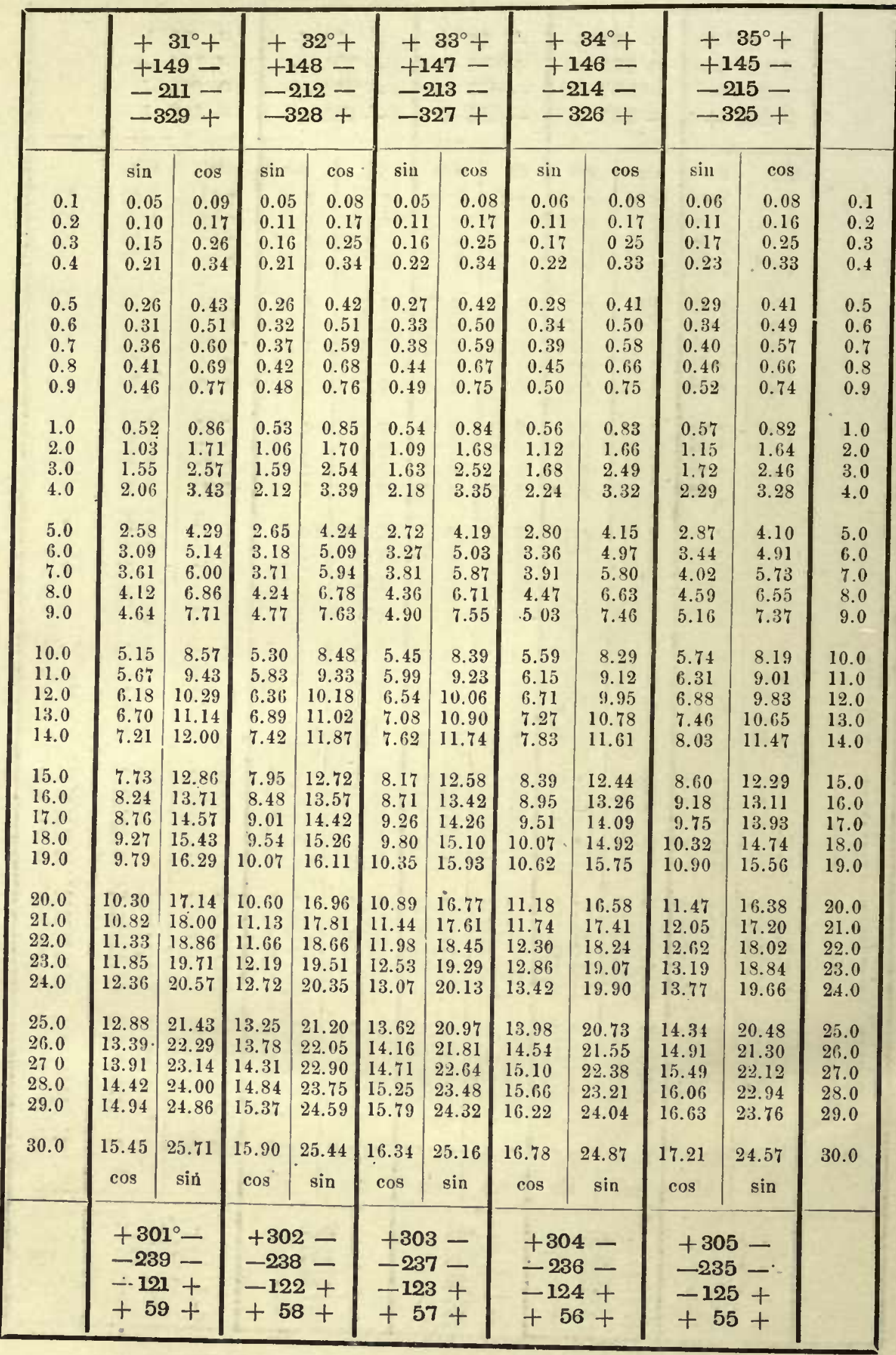




\begin{tabular}{|c|c|c|c|c|c|c|c|c|c|c|c|}
\hline & \multicolumn{2}{|c|}{$\begin{array}{l}+36^{\circ}+ \\
+144- \\
-216- \\
-324+\end{array}$} & \multicolumn{2}{|c|}{$\begin{array}{l}+37^{\circ}+ \\
+143- \\
-217- \\
-323+\end{array}$} & \multicolumn{2}{|c|}{$\begin{array}{l}+38^{\circ}+ \\
+142- \\
-218- \\
-322+\end{array}$} & \multicolumn{2}{|c|}{$\begin{array}{l}+39^{\circ}+ \\
+141- \\
-219- \\
-321+\end{array}$} & \multicolumn{2}{|c|}{$\begin{array}{l}+40^{\circ}+ \\
+140- \\
-220- \\
-320+\end{array}$} & \\
\hline & $\sin$ & $\cos$ & $\sin$ & $\cos$ & $\sin$ & $\cos$ & $\sin$ & $\cos$ & $\sin$ & $\cos$ & \\
\hline 0.1 & 0.06 & 0.08 & 0.06 & 0.08 & 0.06 & 0.08 & 0.06 & 0.08 & 0.06 & 0.08 & 0.1 \\
\hline 0.2 & 0.12 & 0.16 & 0.12 & 0.16 & 0.12 & 0.16 & 0.13 & 0.16 & 0.13 & 0.15 & 0.2 \\
\hline 0.3 & 0.18 & 0.24 & 0.18 & 0.24 & 0.18 & 0.24 & 0.19 & 0.23 & 0.19 & 0.23 & 0.3 \\
\hline 0.4 & 0.24 & 0.32 & 0.24 & 0.32 & 0.25 & 0.32 & 0.25 & 0.31 & 0.26 & 0.31 & 0.4 \\
\hline 0.5 & 0.29 & 0.40 & 0.30 & 0.40 & 0.31 & 0.39 & 0.31 & 0.39 & 0.32 & 0.38 & 0.5 \\
\hline 0.6 & 0.35 & 0.49 & 0.36 & 0.48 & 0.37 & 0.47 & 0.38 & 0.47 & 0.39 & 0.46 & 0.6 \\
\hline 0.7 & 0.41 & 0.57 & 0.42 & 0.56 & 0.43 & 055 & 0.44 & 0.54 & 0.45 & 0.54 & 0.7 \\
\hline 0.8 & 0.47 & 0,65 & 0.48 & 0.64 & 0.49 & 0.63 & 0.50 & 0.62 & 0.51 & 0.61 & 0.8 \\
\hline 0.9 & 0.53 & 0.73 & 0.54 & 0.72 & 0.55 & 0.71 & 0.57 & 0.70 & 0.58 & 0.69 & 0.9 \\
\hline 1.0 & 0.59 & 0.81 & 0.60 & 0.80 & 0.62 & 0.79 & 0.63 & 0.78 & 0.64 & 0.77 & 1.0 \\
\hline 2.0 & 1.18 & 1.62 & 1.20 & 1.60 & 1.23 & 1.58 & 1.26 & 1.55 & 1.29 & 1.53 & 2.0 \\
\hline 3.0 & 1.76 & 2.43 & 1.81 & 2.40 & 1.85 & 2.36 & 1.89 & 2.33 & 1.93 & 2.30 & 3.0 \\
\hline 4.0 & 2.35 & 3.24 & 2.41 & 3.19 & 2.46 & 3.15 & 2.52 & 3.11 & 2.57 & 3.06 & 4.0 \\
\hline 5.0 & 2.94 & 4.05 & 3.01 & 3.99 & 3.08 & 3.94 & 3.15 & 3.89 & 3.21 & 3.83 & 5.0 \\
\hline 6.0 & 3.53 & 4.85 & 3.61 & 4.79 & 3.6 & 4.73 & 3.78 & 4.66 & 3.86 & 4.60 & 6.0 \\
\hline 7.0 & 4.11 & 5.66 & 4.21 & 5.59 & 4.31 & 5.52 & 4.41 & 5.44 & 4.50 & 5.36 & 7.0 \\
\hline 8.0 & 4.70 & 6.47 & 4.81 & 6.39 & 4.93 & 6.30 & 5.03 & 6.22 & 5.14 & 6.13 & 8.0 \\
\hline 9.0 & 5.29 & 7.28 & 5.42 & 7.19 & 5.54 & 7.09 & 5.66 & 6.99 & 5.79 & 6.89 & 9.0 \\
\hline 10.0 & 5.88 & 8.09 & 6.02 & 7.99 & 6.16 & 7.88 & 6.29 & 7.77 & 6.43 & 7.66 & 10.0 \\
\hline 11.0 & 6.47 & 8.90 & 6.62 & 8.78 & 6.77 & 8.67 & 6.92 & 8.55 & 7.07 & 8.43 & 11.0 \\
\hline 12.0 & 7.05 & 9.71 & 7.22 & 9.58 & 7.39 & 9.46 & 7.55 & 9.33 & 7.71 & 9.19 & 12.0 \\
\hline 13.0 & 7.64 & 10.52 & 7.82 & 10.38 & 8.00 & 10.24 & 8.18 & 10.10 & 8.36 & 9.96 & 13.0 \\
\hline 14.0 & 8.23 & 11.33 & 8.43 & 11.18 & 8.62 & 11.03 & 8.81 & 10.88 & 9.00 & 10.72 & 14.0 \\
\hline 15.0 & 8.82 & 12.14 & 9.03 & 11.98 & 9.23 & 11.82 & 9.44 & 11.66 & 9.64 & 11.49 & 15.0 \\
\hline 16.0 & 9.40 & 12.94 & 9.63 & 12.78 & 9.85 & 12.61 & 10.07 & 12.43 & 10.28 & 12.26 & 16.0 \\
\hline 17.0 & 9.99 & 13.75 & 10.23 & 13.58 & 10.47 & 13.40 & 10.70 & 13.21 & 10.93 & 13.02 & 17.0 \\
\hline 18.0 & 10.58 & 14.56 & 10.83 & 14.38 & 11.08 & 14.18 & 11.33 & 13.99 & 11.57 & 13.79 & 18.0 \\
\hline 19.0 & 11.17 & 15.37 & 11.43 & 15.17 & 11.70 & 14.97 & 11.96 & 14.77 & 12.21 & 14.55 & 19.0 \\
\hline 20.0 & 11.76 & 16.18 & 12.04 & 15.97 & 12.31 & 15.76 & 12.59 & 15. & 12.86 & 15.32 & 20.0 \\
\hline 21.0 & 12.34 & 16.99 & 12.64 & 16.77 & 12.93 & 16.55 & 13.22 & 16.32 & 13.50 & 16.09 & 21.0 \\
\hline 22.0 & 12.93 & 17.80 & 13.24 & 17.57 & 13.54 & 17.34 & 13.85 & 17.10 & 14.14 & 16.85 & 22.0 \\
\hline 23.0 & 13.52 & 18.61 & 13.84 & 18.37 & 14.16 & 18.12 & 14.47 & 17.87 & 14.78 & 17.62 & 23.0 \\
\hline 24.0 & 14.11 & 19.42 & 14.44 & 19.17 & 14.78 & 18.91 & 15.10 & 18.65 & 15.43 & 18.39 & 24.0 \\
\hline 25.0 & 14.69 & 20.23 & 15.05 & 19.97 & 15.39 & 19.70 & 15.7 & 19. & 16.07 & 19.15 & 25.0 \\
\hline 26.0 & 15.28 & 21.03 & 15.65 & 20.76 & 16.01 & 20.49 & 16.36 & 20.21 & 16.71 & 19.92 & 26.0 \\
\hline 27.0 & 15.87 & 21.84 & 16.25 & 21.56 & 16.62 & 21.28 & 16.99 & 20.98 & 17.36 & 20.68 & 27.0 \\
\hline 28.0 & 16.46 & 22.65 & 16.85 & 22.36 & 17.24 & 22.06 & 17.62 & 21.76 & 18.00 & 21.45 & 28.0 \\
\hline 29.0 & 17.05 & 23.46 & 17.45 & 23.16 & 17.85 & 22.85 & 18.25 & 22.54 & 18.64 & 22.22 & 29.0 \\
\hline \multirow[t]{3}{*}{30.0} & 17.63 & 24.27 & 18.05 & 23.96 & 18.47 & 23.64 & 18.88 & 23.31 & 19.28 & 22.98 & 30.0 \\
\hline & $\cos$ & $\sin$ & $\cos$ & $\sin$ & $\cos$ & $\sin$ & $\cos$ & $\sin$ & $\cos$ & $\sin$ & \\
\hline & \multicolumn{2}{|c|}{$\begin{array}{l}+306- \\
-234- \\
-126+ \\
+54+\end{array}$} & \multicolumn{2}{|c|}{$\begin{array}{l}+307- \\
-233- \\
-127+ \\
+53+\end{array}$} & \multicolumn{2}{|c|}{$\begin{array}{l}+308- \\
-232- \\
-128+ \\
+\quad 52+\end{array}$} & \multicolumn{2}{|c|}{$\begin{array}{l}+309- \\
-231- \\
-129+ \\
+\quad 51+\end{array}$} & \multicolumn{2}{|c|}{$\begin{array}{l}+310- \\
-230- \\
-130+ \\
+50+\end{array}$} & \\
\hline
\end{tabular}




\begin{tabular}{|c|c|c|c|c|c|c|c|c|c|c|c|}
\hline & \multicolumn{2}{|c|}{$\begin{array}{l}+41^{\circ}+ \\
+139- \\
-221- \\
-319+\end{array}$} & \multicolumn{2}{|c|}{$\begin{array}{l}+42^{\circ}+ \\
+138- \\
-222- \\
-318+\end{array}$} & \multicolumn{2}{|c|}{$\begin{array}{l}+43^{\circ}+ \\
+137- \\
-223- \\
-317+\end{array}$} & \multicolumn{2}{|c|}{$\begin{array}{l}+44^{\circ}+ \\
+136- \\
-224- \\
-316+\end{array}$} & \multicolumn{2}{|c|}{$\begin{array}{l}+45^{\circ}+ \\
+135- \\
-225- \\
-315+\end{array}$} & \\
\hline & $\sin$ & $\cos$ & $\sin$ & $\cos$ & $\sin$ & $\cos$ & $\sin$ & $\cos$ & $\sin$ & $\cos$ & \\
\hline 0.1 & 0.07 & 0.08 & 0.07 & 0.07 & 0.07 & 0.07 & 0.07 & 0.07 & 0.07 & 0.07 & 0.1 \\
\hline 0.2 & 0.13 & 0.15 & 0.13 & 0.15 & 0.14 & 0.15 & 0.14 & 0.14 & 0.14 & 0.14 & 0.2 \\
\hline 0.3 & 0.20 & 0.23 & 0.20 & 0.22 & 0.20 & 0.22 & 0.21 & 0.22 & 0.21 & 0.21 & 0.3 \\
\hline 0.4 & 0.26 & 0.30 & 0.27 & 0.30 & 0.27 & 0.29 & 0.28 & 0.29 & 0.28 & 0.28 & 0.4 \\
\hline 0.5 & 0.33 & 0.38 & 0.33 & 0.37 & 0.34 & 0.37 & 0.35 & 0.36 & 0.35 & 0.35 & 0.5 \\
\hline 0.6 & 0.39 & 0.45 & & 0.45 & 0.41 & 0.44 & 0.42 & 0.43 & 0.42 & 0.42 & 0.6 \\
\hline 0.7 & 0.46 & 0.53 & 0.47 & 0.52 & 0.48 & 0.51 & 0.49 & 0.50 & 0.49 & 0.49 & 0.7 \\
\hline 0.8 & 0.52 & 0.60 & 0.54 & 0.59 & 0.55 & 0.59 & 0.56 & 0.58 & 0.57 & 0.57 & 0.8 \\
\hline 0.9 & 0.59 & 0.68 & 0.60 & 0.67 & 0.61 & 0.66 & 0.63 & 0.65 & 0.64 & 0.64 & 0.9 \\
\hline 1.0 & 0.66 & 0.75 & 0.67 & 0.74 & 0.68 & 0.73 & 0.69 & 0.72 & 0.71 & 0.71 & 1.0 \\
\hline 2.0 & 1.31 & 1.51 & 1.34 & 1.49 & 1.36 & 1.46 & 1.39 & 1.44 & $1.4 \mathrm{I}$ & 1.41 & 2.0 \\
\hline 3.0 & 1.97 & 2.26 & 2.01 & 2.23 & 2.05 & 2.19 & 2.08 & 2.16 & 2.12 & 2.12 & 3.0 \\
\hline 4.0 & 2.62 & 3.02 & 2.68 & 2.97 & 2.73 & 2.93 & 2.78 & 2.88 & 2.83 & 2.83 & 4.0 \\
\hline 5.0 & 3. & 3.77 & 3.35 & 3.72 & 3.41 & 3.66 & 3.47 & 3.60 & 3.54 & 3.54 & 5.0 \\
\hline 6.0 & 3. & 4.53 & & 4.46 & 4.09 & 4.39 & 4.17 & & 4.24 & 4.24 & 6.0 \\
\hline 7.0 & 4.59 & 5.28 & 4. & 5.20 & 4.77 & 5.12 & 4.86 & 5.04 & 4.95 & 4.95 & 7.0 \\
\hline 8.0 & 5.25 & 6.04 & 5. & 5.95 & 5.46 & 5.85 & 5.56 & 5.75 & 5.66 & 5.66 & 8.0 \\
\hline 9.0 & 5.90 & 6.79 & 6.02 & 6.69 & 6.14 & 6.58 & 6.25 & 6.47 & 6.36 & 6.36 & 9.0 \\
\hline 1 & 6 & 7.55 & 6. & 7.43 & 6.82 & 7.31 & 6.95 & 7.19 & 7.07 & 7.07 & 10.0 \\
\hline 1 & 7. & 8.30 & & 8. & 7. & 8.04 & 7.64 & 78 & 7.7 & 7.78 & 11.0 \\
\hline 12.0 & & 9.06 & 8 & 8.92 & 8.18 & 8.78 & 8.34 & 8. & 8.4 & 8.49 & 12.0 \\
\hline 13.0 & 8.53 & 9.81 & 8.70 & 9.66 & 8.87 & 9.51 & 9.03 & 9.35 & 9.19 & 9.19 & 13.0 \\
\hline 14.0 & 9.18 & 10.57 & 9.37 & 10.40 & 9.55 & 10.24 & 9.73 & 10.07 & 9.90 & 9.90 & 14.0 \\
\hline 15.0 & 9.84 & 1.32 & 10.04 & 5 & 10.23 & .97. & & & 10. & & 15.0 \\
\hline & 10 & 8 & i & & 10.91 & 70 & 11.1 & 11. & & & 1 \\
\hline 17.0 & 11.15 & 12.83 & 11.38 & 12.63 & 11.59 & 12.43 & 11.81 & 12. & 12. & 2 & 17.0 \\
\hline 18.0 & 11.81 & 13.58 & 12.04 & 3.38 & 12.28 & 13.16 & 12.50 & 12.95 & 12.73 & 2.73 & 18.0 \\
\hline 19.0 & 12.47 & 14.34 & 12.71 & 14.12 & 12.96 & 13.90 & 13.20 & 13.67 & 13.44 & 13.44 & 19.0 \\
\hline 20. & 12 & 15.09 & 13.38 & & & & & & & & 2 \\
\hline & & 1 & 14 & & 2 & & & & & & \\
\hline 22.0 & 14.43 & 16.60 & | 14.7 .2 & 16.35 & 15.00 & & & & & & 22.0 \\
\hline 23.0 & 15.09 & 17.36 & 15.39 & 17.09 & 15.69 & 16.82 & $15: 98$ & 16.54 & 16.26 & 16.26 & 23.0 \\
\hline 24.0 & 15.75 & 18.11 & 16.06 & 17.84 & 16.37 & 17.55 & 16.67 & 17.26 & 16.97 & 16.97 & 24.0 \\
\hline 25.0 & 16.40 & 18.87 & 16.73 & & 17.05 & & & & & & \\
\hline & & 19. & 17.40 & & & & & & & & \\
\hline & & 20 & 18.07 & & 1 & 19. & & & & & \\
\hline & & 21.13 & 18.74 & 20.81 & 19.10 & 20.48 & & & 19.80 & & 28.0 \\
\hline 29.0 & 19.03 & 21.89 & 19.40 & 21.55 & 19.78 & 21.21 & 20.15 & 20.86 & 20.51 & 20.51 & 29.0 \\
\hline \multirow[t]{6}{*}{30.0} & 19.68 & 22.64 & 20.07 & 22.29 & 20.46 & 21.94 & 20.84 & 21.58 & 21.21 & 21.21 & 30.0 \\
\hline & $\cos$ & $\sin$ & $\cos$ & $\sin$ & $\cos$ & $\sin$ & $\cos$ & $\sin$ & $\cos$ & $\sin$ & \\
\hline & \multirow{4}{*}{\multicolumn{2}{|c|}{$\begin{array}{l}+311- \\
-229- \\
-131+ \\
+49+\end{array}$}} & \multirow{4}{*}{\multicolumn{2}{|c|}{$\begin{array}{l}+312- \\
-228- \\
-132+ \\
+48+\end{array}$}} & \multirow{4}{*}{\multicolumn{2}{|c|}{$\begin{array}{l}+313- \\
-227- \\
-133+ \\
+47+\end{array}$}} & \multirow{4}{*}{\multicolumn{2}{|c|}{$\begin{array}{l}+314- \\
-226- \\
-134+ \\
+46+\end{array}$}} & \multirow{4}{*}{\multicolumn{2}{|c|}{$\begin{array}{l}+315- \\
-225- \\
-135+ \\
+45+\end{array}$}} & \\
\hline & & & & & & & & & & & \\
\hline & & & & & & & & & & & \\
\hline & & & & & & & & & & & \\
\hline
\end{tabular}




Whing

A.7.2. 


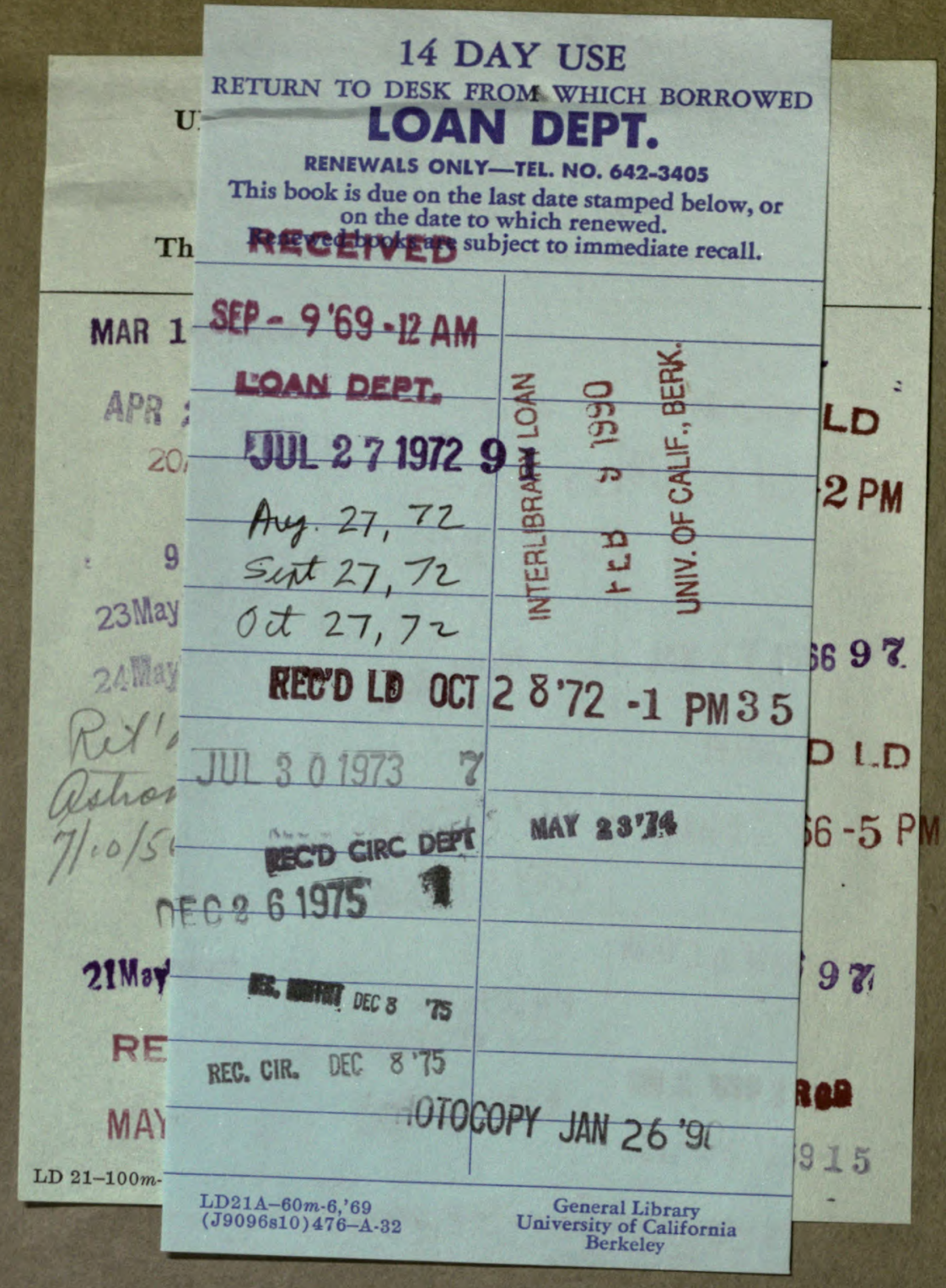


द्वी 